\title{
AIIIOZ 145644
}

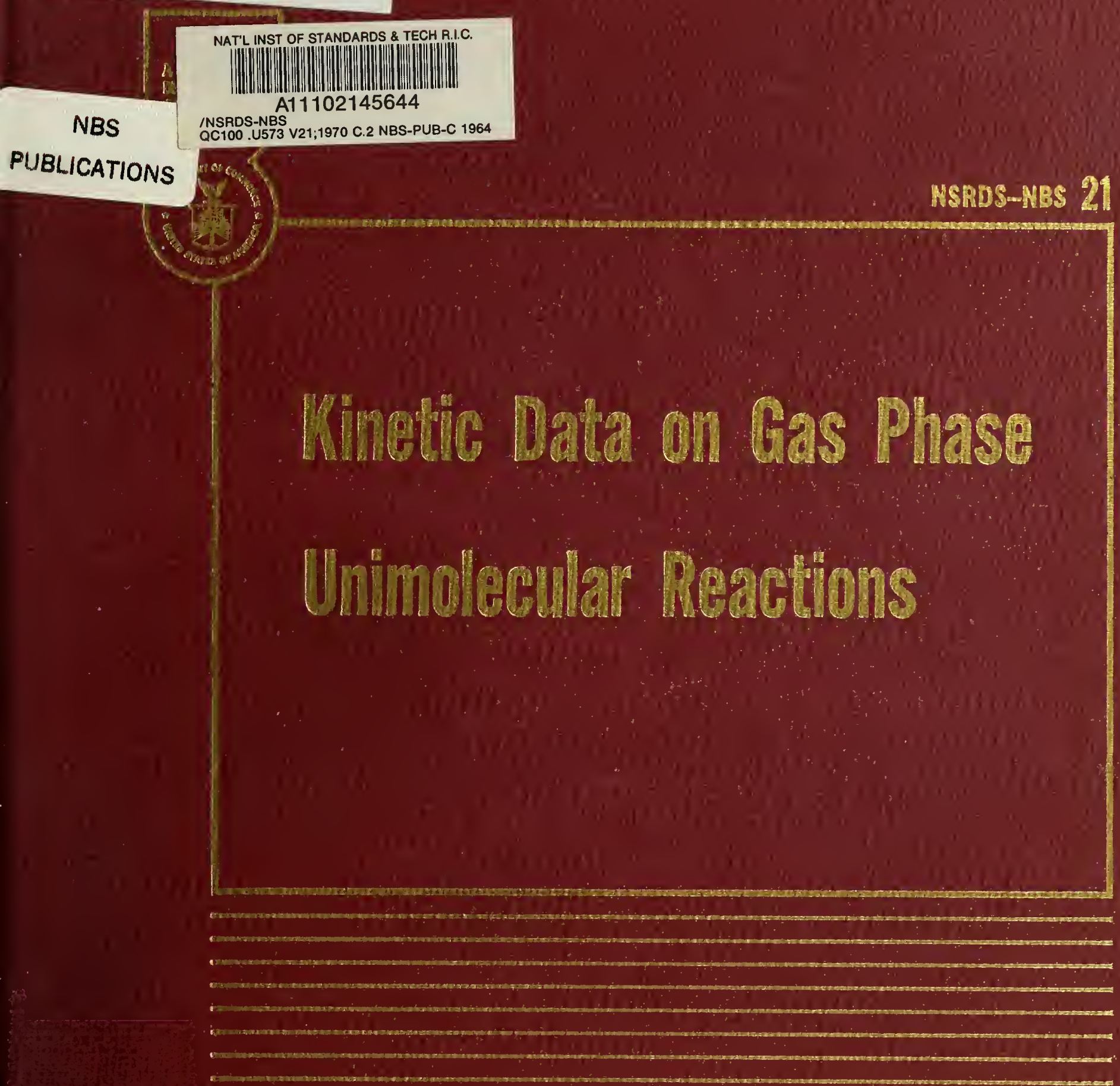

US. DEPARTEETI OF COMMERE

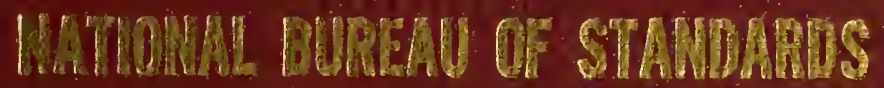

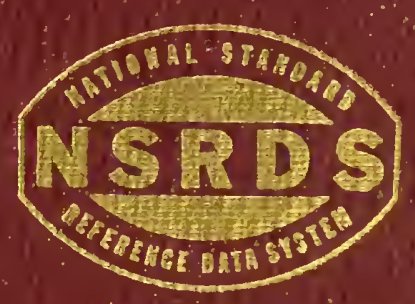







\title{
Kinetic Data on Gas Phase Unimolecular Reactions
}

\author{
Sidney W. Benson
}

Stanford Research Institute Menlo Park, California 94025

and

\section{H. Edward O'Neal}

San Diego State College San Diego, California 92115

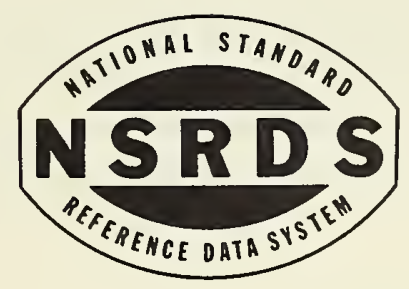

NSRDS-NBS 21

National Standard Reference Data Series, National Bureau of Standards (United States) 21, 645 pages (Feb. 1970) CODEN:NSRDA 
MAY 201970

147307

aC

\begin{abstract}
Available rate data on thermally induced, unimolecular, homogeneous gas phase reactions of molecules and free radicals have been reviewed and critically evaluated. Introductory discussion is given of theory and assumptions used in compiling the selected data. Mechanisms of reaction are discussed. The major portion ( 537 out of 617 pages) of the work presents selected data, with references, in the form of data sheets (one to two pages per molecule). Preferred values are indicated and discussed.
\end{abstract}

Key words: Arrhenius parameters; chemical kinetics; critical review data; gas phase; mechanisms; molecular reactions; rate constants; reaction rates.

copyright (C) 1970 by the Secretary of Comerco

on behalf of the U.S. Government

Library of Congress Catalog Card Number: 68-67395 
The National Standard Reference Data System provides effective access to the quantitative data of physical science, critically evaluated and compiled for convenience, and readily accessible through a variety of distribution channels. The System was established in 1963 by action of the President's Office of Science and Technology and the Federal Council for Science and Technology, with responsibility to administer it assigned to the National Bureau of Standards.

The System now comprises a complex of data centers and other activities, carried on in academic institutions and other laboratories both in and out of government. The independent operational status of existing critical data projects is maintained and encouraged. Dat centers that are components of the NSRDS produce compilations of critically evaluated data, critical reviews of the state of quantitative knowledge in specialized areas, and computations of useful functions derived from standard reference data. In addition, the centers and projects establish criteria for evaluation and compilation of data and make recommendations on needed improvements in experimental techniques. They are normally closely associated with active research in the relevant field.

The technical scope of the NSRDS is indicated by the principal categories of data compilation projects now active or being planned: nuclear properties, atomic and molecular properties, solid state properties, thermodynamic and transport properties, chemical kinetics, and colloid and surface properties.

The NSRDS receives advice and planning assistance from the National Research Council of the National Academy of Sciences-National Academy of Engineering. An overall Review Committee considers the program as a whole and makes recommendations on policy, long-term planning, and international collaboration. Advisory Panels, each concerned with a single technical area, meet regularly to examine major portions of the program, assign relative priorities, and identify specific key problems in need of further attention. For selected specific topics, the Advisory Panels sponsor subpanels which make detailed studies of users' needs, the present state of knowledge, and existing data resources as a basis for recommending one or more data compilation activities. This assembly of advisory services contributes greatly to the guidance of NSRDS activities.

The NSRDS-NBS series of publications is intended primarily to include evaluated reference data and critical reviews of long-term interest to the scientific and technical community.

Lewis M. Branscomb, Director. 
The NBS Office of Standard Reference Data, as administrator of the National Standard Reference Data System, has officially adopted the use of SI units for all NSRDS publications, in accordance with NBS practice. This publication does not use SI units because contractual commitments with the authors predate establishment of a firm policy on their use by NBS. We urge that specialists and other users of data in this field accustom themselves to SI units as rapidly as possible. 


\section{CONTENTS}

Abstract.

\section{INTRODUCTION.}

1.0. Purpose and objectives.

2.0. Scope and limitations.

3.0. Assumptions.

4.0. Thermodynamic relations of a unimolecular first-order reaction.

4.1. Thermodynamics of the transition state.

4.2. Transition state theory and the rate constant.

4.3. Transition state theory and the relations between the experimental activation energy and the enthalpy of activation.

4.4. Transition state theory and the relations between the Arrhenius $A$-factor and the entropy of activation.

4.5. Thermodynamics and the Arrhenius parameters of bond fission reactions............

5.0. Thermochemical data sources.

6.0. General index.

7.0. Reaction sheet information.

7.1. Reaction thermodynamics.

7.2. Rate constants..

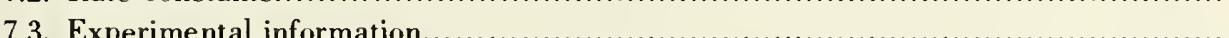

7.4. References.

7.5. Preferred values or evaluation

7.6. Comments.....

8.0. Glossary....

8.1. Standard thermodynamic and kinetic symbols

8.2. Notations used in illustrations of transition state calculations......

8.3. Symbols designating analytical methods and experimental rate measurement methods.

9.0. Experimental errors.

\section{COMPLEX FISSIONS - MOLECULAR ELIMINATION REACTIONS.}

1.0. Four-center reactions.

1.1. Transition state estimates of the $A$-factor of four-center elimination reactions......

2.0. Three-center elimination reactions....

3.0. Five-center elimination reactions.

3.1. Evaluation...

4.0. Six-center elimination reactions.

4.1. Evaluation-transition state estimates of the $A$-factors of six-center elimination reactions..

4.2. Ethyl acetate.

III. CYCLIC COMPOUND REACTIONS

1.0. Characteristics

2.0. The biradical mechanism

3.0. General remarks concerning transition state calculations...

4.0. Transition state estimates for cyclopropanes..

4.1. Cis-1,2-d $d_{2}$-cyclopropane.

4.2. Vinylcyclopropane.

4.3. Trans-1-methyl-2-vinylcyclopropane...

5.0. Transition state estimates for cyclobutanes.

5.1. Cyclobutane

5.2. $d_{2}$-methylene cyclobutane.

6.0. Polycyclic compounds.

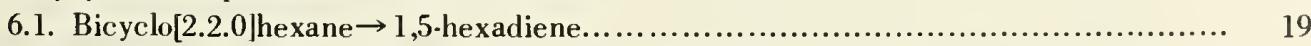

6.2. 1,3-Dimethylbicyclo[1.1.0]butane............................................. 20

IV. ISOMERIZATION REACTIONS (OTHER THAN CYCLIC COMPOUND REACTIONS).

1.0. Cope rearrangements.

2.0. "Ene" isomerizations

2.1. Transition state estimate of the $A$-factor for an "ene" isomerization. 


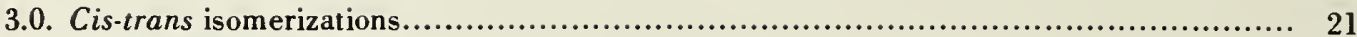

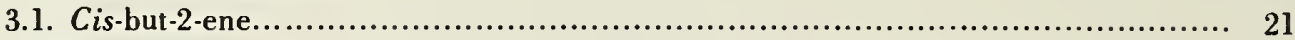

3.2. Isostilbene.................................................................. 22

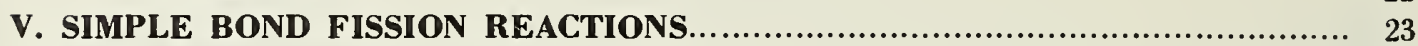

1.0. Characteristics................................................................... 23

2.0. Reliability of the experimental data................................................ 24

3.0. Kinetic and thermodynamic complications in radical-radical recombination reactions... 25

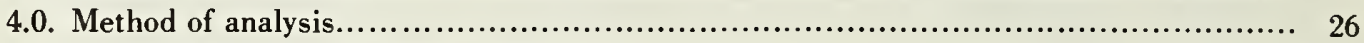

4.1. Illustrations of analysis methods............................................ 27

5.0. High and low $A$-factors in bond fission reactions................................... 28

6.0. Rotational barriers in radicals and single shock tube kinetic results.................... 29

6.1. Rotational barrier entropy corrections........................................ 30

7.0. Problems in the kinetics of azo compound decompositions............................. 31

8.0. Problems in the amine and hydrazine decompositions................................... 34

9.0. Summary of the calculated $A$-factors for radical-radical recombination reactions....... 36

VI. FREE RADICAL REACTIONS..................................................... 37

1.0. Characteristics and reliability of the experimental data............................. 37

2.0. Transition state estimates of the $A$-factors in radical decompositions..................... 39

APPENDIX A. THERMODYNAMIC GROUP ADDITIVITIES $\ldots \ldots \ldots \ldots \ldots \ldots \ldots \ldots \ldots . . \ldots \ldots$

Table A-1. Hydrocarbons............................................................ 40

Table A-2. Oxygen-containing compounds.............................................. 41

Table A-3. Nitrogen-containing compounds............................................ 44

Table A-4. Halogen-containing compounds............................................... 46

Table $A-5$. Free radicals............................................................... 48

APPENDIX B. FREQUENCIES USED IN TRANSITION STATE CALCULATIONS... 51

Table B-1. Bond stretching frequencies............................................. 51

Table B-2. Bond angle bending frequencies......................................... 51

Table B-3. Bond torsion frequencies................................................ 51

Table B-4. Approximate entropies and heat capacities of bond vibrations................. 52

APPENDIX C. INTERNAL ROTATIONS........................................ 53

Table C-1. Entropies of free internal rotors as a function of temperat ure................... 53

Table C-2. Some characteristic torsion barriers, $V$ (kcal/mole), to free rotation about single bonds for methyl groups.....

Table C-3. Some characteristic torsion barriers, $V$ (kcal/mole), to free rotation about single bonds for groups larger than methyl.....

Table C-4. Decrease in entropy of free rotor as functions of barrier height $(V)$, temperature $\left(T{ }^{\circ} \mathrm{K}\right)$, and partition function $\left(Q_{f}\right)$.

Table C-5. Molar heat capacity, $C_{p}^{\circ}(\mathrm{g} / \mathrm{mole})$ as a function of barrier $(V)$ temperature and partition function $\left(Q_{f}\right)$...

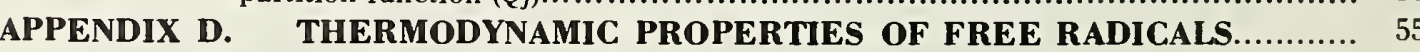

Table D-1. Preferred values of free radical heats of formation and their source.............. 55

Table D-2. Calculated thermodynamic properties of free radicals: entropies and heat capacities..

REFERENCES, to Sections I-VI and Appendices.

DATA SHEETS.

1. Complex fissions-molecular elimination reactions..

Four-center chlorides:

Chloroethane (ethyl chloride).

1-Chloropropane ( $n$-propyl chloride).

1-Chlorobutane ( $n$-butyl chloride).

1-Chloropentane ( $n$-pentyl chloride)

1-Chloro-2-methylpropane (isobutyl chloride)..

2-Chloropropane (isopropyl chloride).

2-Chloro-2-methylpropane ( $t$-butyl chloride).

2-Chlorobutane (sec-butyl chloride)

2-Chloro-2-methylbutane ( $t$-amyl chloride).

Chlorocyclopentane (cyclopentyl chloride).

Chlorocyclohexane (cyclohexyl chloride)..

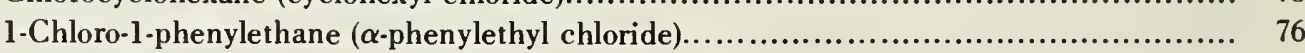

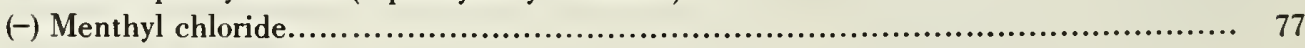

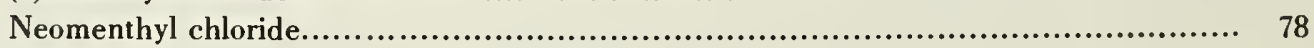


Endo-2-chlorobornane (bornyl chloride).

Exo-2-chlorobornane (isobornyl chloride).

1,1-Dichloroethane (ethylidene dichloride).

2,2-Dichloropropane.

1,1-Dichloropropane.

1,2-Dichloropropane.

1,1,1-Trichloroethane

1,

1-Chloro-lethoxyethane (a-chloroethyl ethyl ether)

( $\alpha$-chloroethyl ethyl ether)

Trichloromethyl chloromethanoate (trichloromethyl chloroformate).

Bromoethane (ethyl bromide)

1-Bromopropane ( $n$-propyl bromide).

l-Bromobutane ( $n$-butyl bromide).

l-Bromopentane ( $n$-pentyl bromide)

l-Bromohexane ( $n$-hexyl bromide).

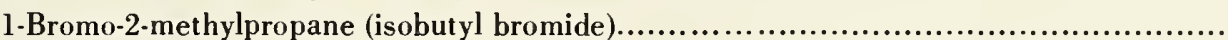

2-Bromopropane (isopropyl bromide)

2-Bromobutane (sec-butyl bromide)

4-Bromo-1-pentene.

Bromocyclopentane (cyclopentyl bromide)...

Bromocyclohexane (cyclohexyl bromide).

1-Bromo-1-phenylethane ( $\alpha$-phenylethyl bromide)..

2 -Bromo-2-methylpropane $(t$-but yl bromide)..

2-Bromo-2-methylbutane ( $t$-amyl bromide).

2,3-Dimethyl-2-bromobutane.

1,1-Dibromoethane (ethylidene dibromide).

Iodides:

Iodoethane (ethyl iodide)

2-Iodopropane (isopropyl iodide).

2-Iodobutane (sec-butyl iodide).

Others:

2-Iodo-2-methylpropane ( $t$-butyl iodide).

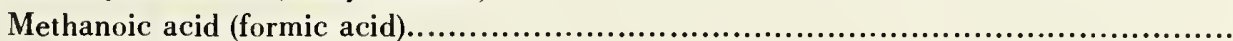

Ethanoic acid (acetic acid)........

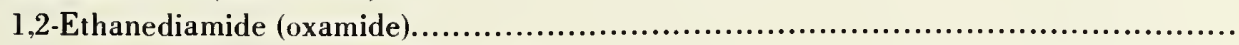

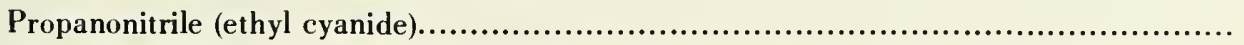

2-Methyl-2-aminopropane ( $t$-butyl amine)

Methanoloxime (formaldoxime)

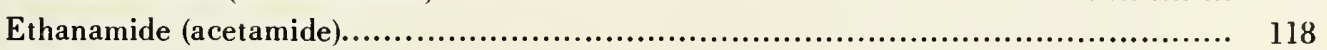

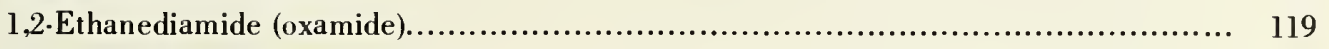

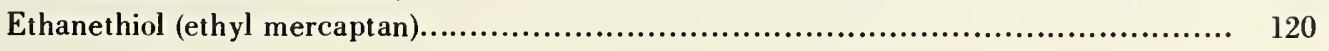

2-Methyl-2-propanethiol ( $t$-butyl mercaptan)....................................... 121

2,2 -Difluoroethyltrifluorosilane.................................................... 122

2-Chloroethyltrichlorosilane....................................................... 123

2-Chloroethylethyldichlorosilane................................................ 124

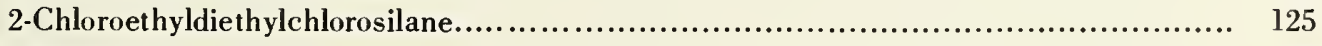

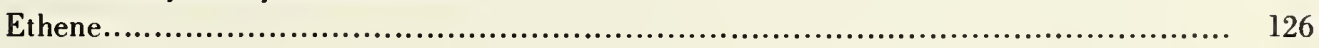

Three-center reactions:

Formyl fluoride..

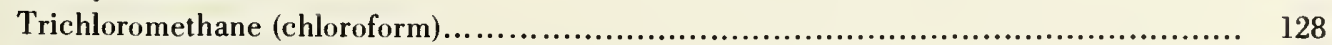

Chlorodifluoromethane.............................................................. 129

Five-center reactions:

Nitroethane....................................................................... 130

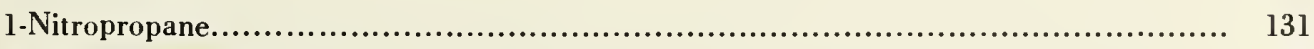

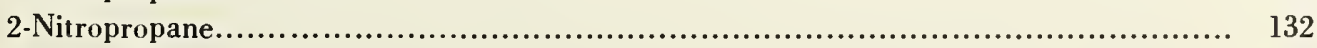

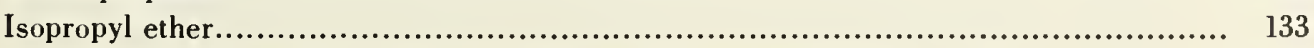

1,l-Dimethyl-1-ethanesulfonyl chloride (trimethylmethanesulfonyl chloride)............... 134 
Six-center eliminations:

Alcohols:

3-Butene-1-ol (allylcarbinol).

4-Pentene-2-ol (allylmethylcarbinol).

2-Methyl-4-pentene-2-ol (allyldimethylcarbinol)..................................... 137

3-Phenyl-3-butene-1-ol.

4-Phenyl-3-butene-1-ol.

1-Phenyl-4-ethyl-4-hydroxyhex-1-ene

3-Butenoic acid.

2,2-Dimethyl-3-butenoic acid

2,2-Dimethyl-trans-3-pentenoic acid

2,2,3-Trimethyl-3-butenoic acid.

2,2-Dimethyl-3-ethyl-3-pentenoic acid.

2-Methyl-2-(1-cyclopentyl) propionic acid.

2-Methyl-2-(1-cyclohexenyl) propionic acid.

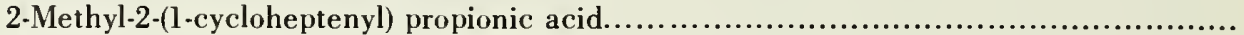

Esters:

Chloroformate esters:

Ethyl chloromethanoate (ethyl chloroformate)

Isopropyl chloromethanoate (isopropyl chloroformate)............................... 151

Isobutyl chloromethanoate (isobutyl chloroformate).................................. 152

sec-Butyl chloromethanoate (sec-butyl chloroformate) ............................... 153

Formic acid esters:

Ethyl methanoate (ethyl formate) .................................................. 154

Propyl methanoate ( $n$-propyl formate) ............................................ 155

Isopropyl methanoate (isopropyl formate)........................................ 156

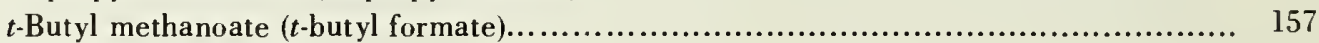

Primary acetic acid esters:

Ethyl ethanoate (ethyl acetate) ..................................................... 158

1-Propyl ethanoate $(n$-propyl acetate)........................................... 159

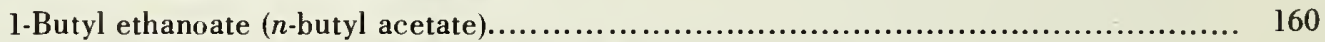

Isobutyl ethanoate (2-methylpropyl acetate; isobutyl acetate).......................... 161

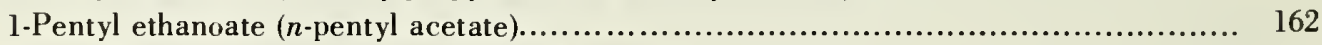

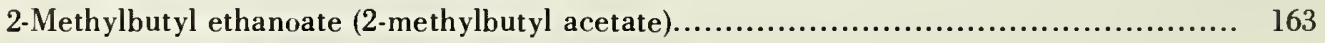

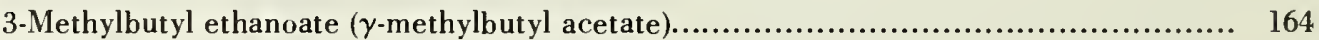

2-Ethylbutyl ethanoate $(\beta$-ethylbutyl acetate)....................................... 165

2-Methoxyethyl ethanoate $(\beta$-methoxyethyl acetate)................................... 166

2-Ethoxyethyl ethanoate $(\beta$-ethanoxyethyl acetate) ................................... 167

2-Aryethyl ethanoates (meta or para substituted 2-phenylethyl acetates)................ 168

Secondary acetic acid esters:

Isopropyl ethanoate (isopropyl acetate).

sec-Butyl et hanoate (sec-butyl acetate)............................................. 170

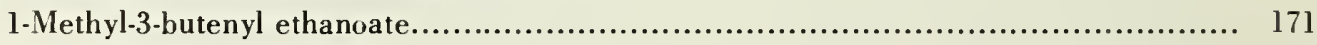

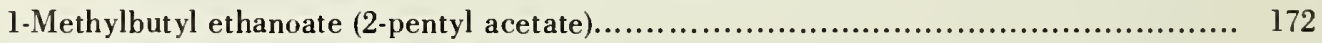

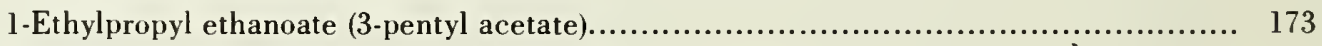

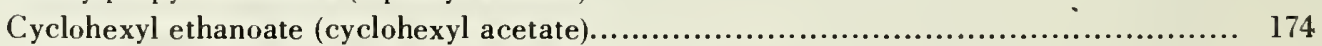

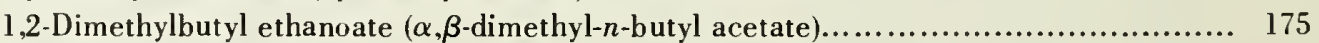

1-Isopropyl-2-methylpropyl ethanoate ( $\alpha$-isopropyl $\beta$-methyl- $n$-propyl acetate) $\ldots \ldots \ldots \ldots \ldots 176$

l-Methylhexyl ethanoate $(2$-heptyl acetate).......................................... 177

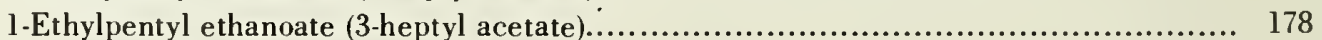

1-Propylbutyl ethanoate (4-heptyl acetate)........................................... 179

Endo-2-acetoxybornane (bornyl acetate)........................................... 180

Exo-2-acetoxybornane (isobornyl acetate)............................................ 181

1-Methyl-2-methoxyethyl ethanoate (1-methoxy-2-propyl acetate)....................... 182

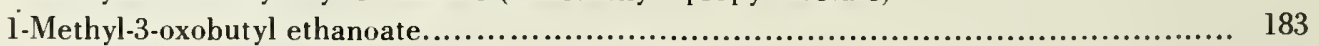

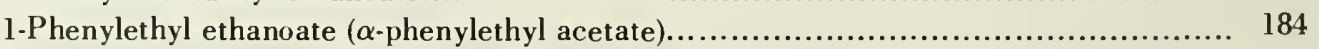

l-Arylethyl ethanoates (1-phenylethyl derivatives)..................................... 185

1,2-Diarylethyl ethanoates (meta or para substituted 1,2-diphenylethyl acetates)........... 186

1-Methyl-2-chloroethyl ethanoate (1-chloroisopropyl acetate)........................... 187 
1-Methyl-2-dimethylaminoethyl ethanoate; 2-dimethylamino-1-methyl ethyl ethanoate

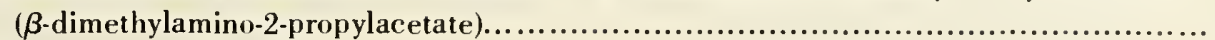

Tertiary acetic acid esters:

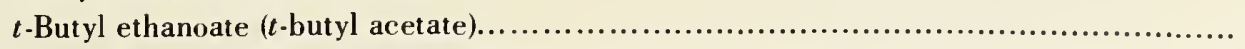

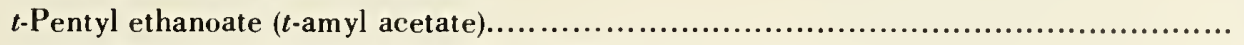

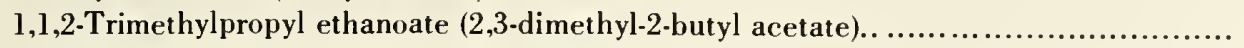

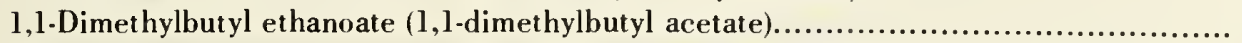

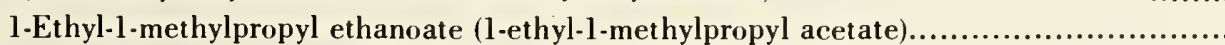

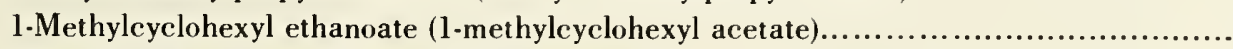

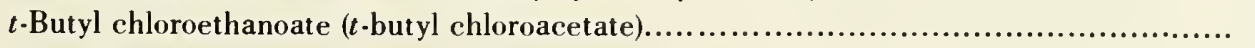

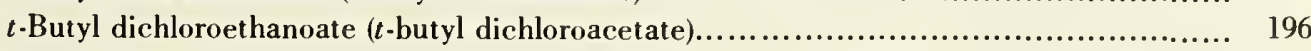

Propionic acid esters:

Ethyl propanoate (ethyl propionate).

$t$-Butyl propanoate ( $t$-butyl propionate)

Ethyl-2,2-dimethylpropanoate (ethyl trimethylacetate).................................. 199

$(-)$ Menthyl benzoate.............................................................. 200

Diesters:

Diacetic acid esters:

Methylene diethanoate (methylene diacetate).......................................... 201

Ethylidene diethanoate (ethylidene diacetate)...................................... 202

Butylidene diethanoate (butylidene diacetate).......................................... 203

Heptylidene diethanoate (heptylidene diacetate)...................................... 204

Crotonylidene diethanoate (crotonylidene diacetate)................................... 205

Furfurylidene diethanoate (furfurylidene diacetate)................................... 206

$2,2,2$-Trichloro-1-ethylidene diethanoate............................................. 207

Dipropionic acid esters:

Methylene dipropanoate.............................................................. 208

Ethylidene dipropanoate.......................................................... 209

Dibutyric acid esters:

Methylene dibutanoate........................................................... 210

Ethylidene dibutanoate........................................................... 211

$2,2,2$-Trichloro-1-ethylidene dibutanoate.......................................... 212

Carbonate esters:

Methyl ethyl carbonate.............................................................. 213

Diethyl carbonate.............................................................. 214

$X$-1-phenylethyl methyl carbonates, $X=$ ortho, meta, or para substitution.................. 215

Miscellaneous (ketones and anhydrides):

Ethanoic anhydride (acetic anhydride)............................................ 216

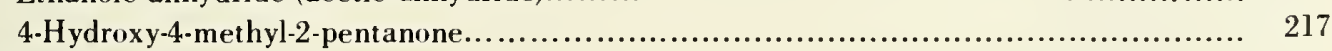

Ethers:

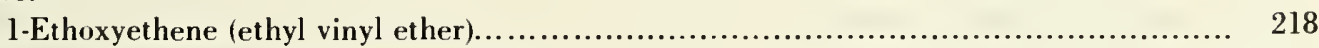

Isopropoxyethene (vinyl isopropyl ether)............................................ 219

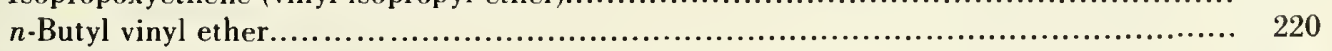

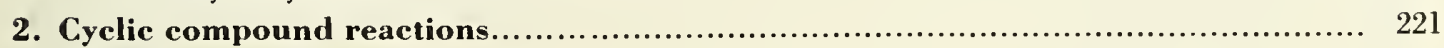

Three-membered rings

Alkylcyclopropanes:

Cyclopropane....................................................................... 223

trans-1,2-Dideutero cyclopropane.................................................... 224

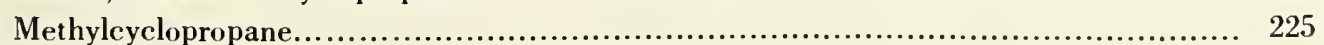

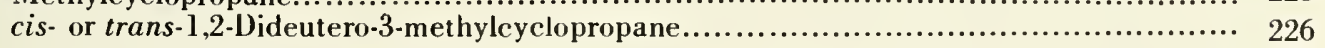

cis,trans-1,2-Dideutero-3-methylcyclopropane...................................... 227

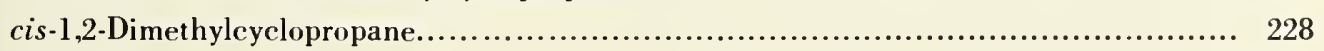

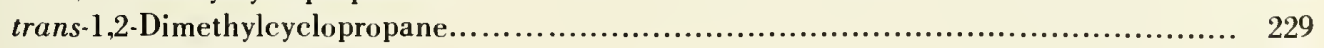

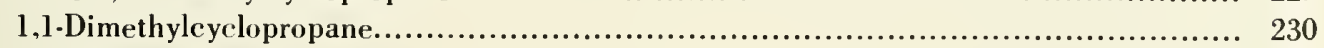

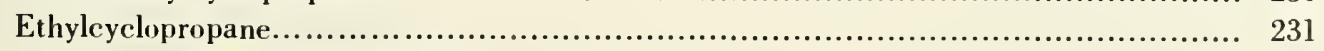

cis-1-Ethyl-2-methylcyclopropane................................................. 232

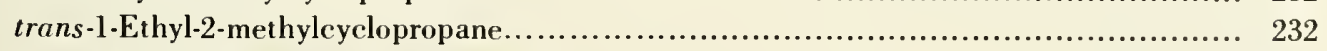

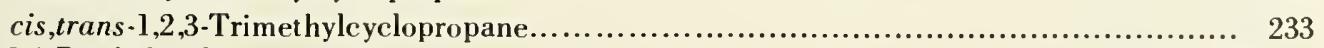

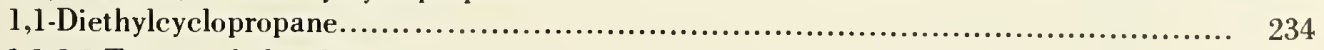

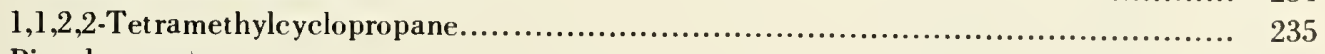

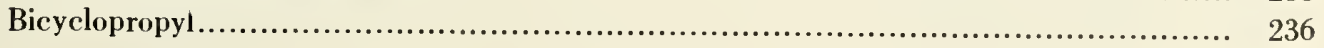


Vinylcyclopropane

cis-1-Methyl-2-vinylcyclopropane................................................. 238

trans-1-Methyl-2-vinylcyclopropane.................................................... 239

1-Methyl-1-vinylcyclopropane................................................... 240

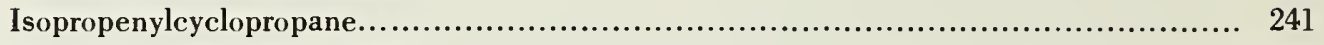

trans-1-Cyclopropyl-1-butene.................................................... 242

1-Cyclopropyl-2-methyl-1-propene.............................................. 243

1-Isopropenyl-1-methylcyclopropane........................................... 244

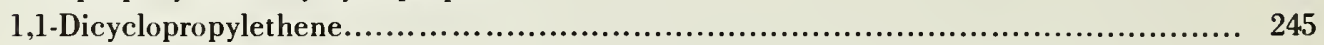

1-Cyclopropyl-1-cyclopentene................................................. 246

Halogen substituents:

Fluorocyclopropane........................................................... 247

1,1-Difluorocyclopropane........................................................... 248

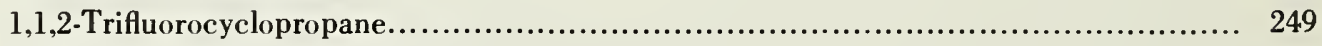

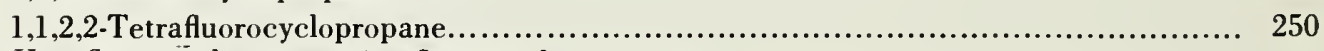

Hexafluorocyclopropane (perfluorocyclopropane)..................................... 251

Trifluoromethylcyclopropane........................................................ 252

$2,2,2$-Trifluoro-1-ethylcyclopropane.................................................. 253

Octafluorovinylcyclopropane (perfluorovinylcyclopropane)........................... 254

Decafluoroallylcyclopropane (perfluoroallylcyclopropane).............................. 255

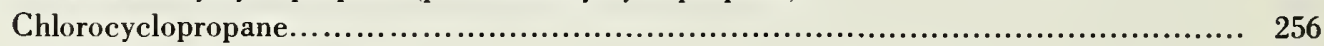

1,1-Dichlorocyclopropane...................................................... 257

2,2-Dichloro-1-methyl-1-vinylcyclopropane (1-methyl-1-vinyl-2,2-dichlorocyclopropane...... 258

Bromocyclopropane............................................................... 259

Diazirines:

Dimethyldiazirine.............................................................. 260

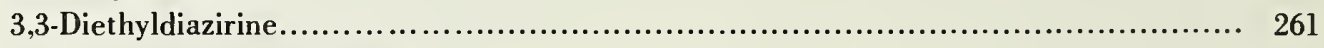

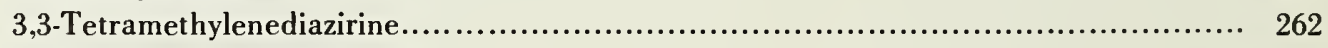

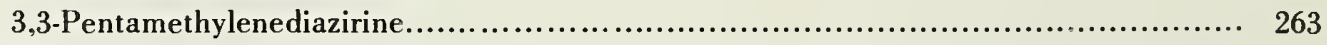

Difluorodiazirine...................................................................... 264

External double bonds:

Ethylidenecyclopropane........................................................... 265

Others:

2-Methyl-1-methylenecyclopropane............................................... 265

Oxirane (ethylene oxide).......................................................... 266

$\beta$, $\beta$-Dimethylcyclopropyl methyl ketone (2,2-dimethylcyclopropyl-1-ethanone)............. 267

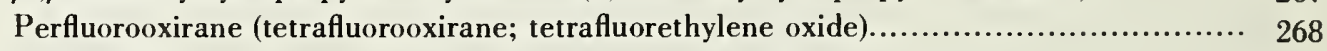

Cyclobutanes:

Cyclobutane....................................................................... 269

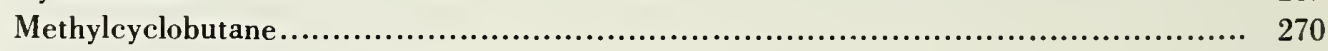

trans-1,2-Dimethylcyclobutane...................................................... 271

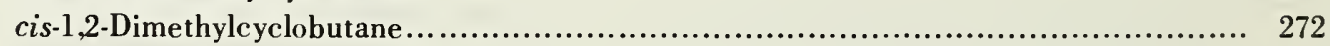

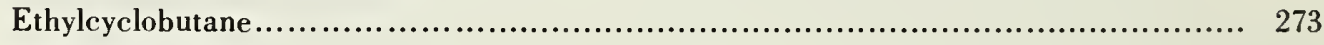

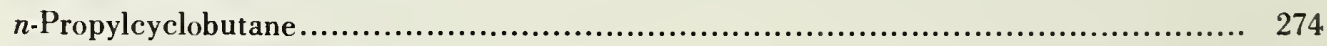

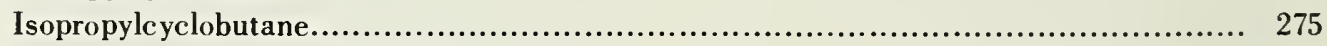

Isopropenylcyclobutane........................................................ 276

Oxysubstituents:

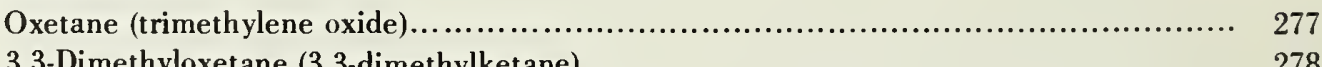

3,3-Dimethyloxetane (3,3-dimethylketane) ......................................... 278

Cyclobutane carboxaldehyde........................................................ 279

Ethanoylcyclobutane (methyl cyclobutyl ketone)..................................... 280

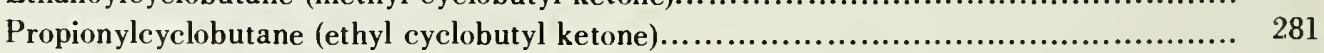

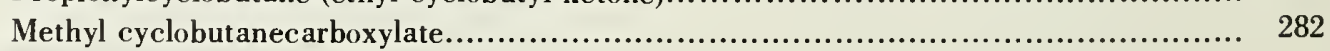

External Double bond:

Cyclobutanone.................................................................... 283

Methylenecyclobutane............................................................. 284

Cyclobutenes:

Cyclobutene.................................................................... 285

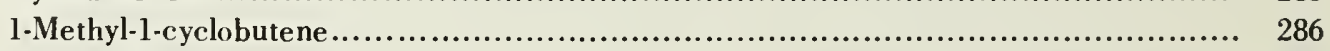

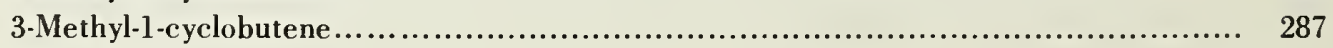

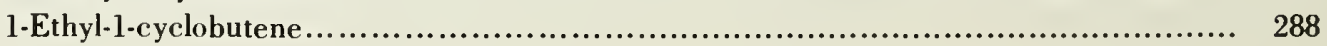


Page

1,2-Dimethyl-1-cyclobutene.................................................... 289

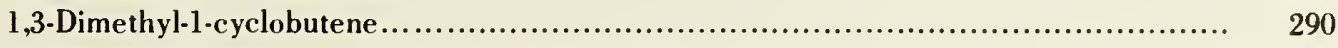

2,3-Dimethyl-1-cyclobutene ........................................................ 291

trans-1,2,3,4-tetramethyl-1-cyclobutene.............................................. 292

cis-1,2,3,4-tetramethyl-1-cyclobutene................................................. 293

Fluorine substituents:

Perfluorocyclobutene................................................................... 294

Perfluorocyclobutane................................................................. 295

cis-1,2-Dichlorohexafluorocyclobutane............................................ 296

(cis or trans)1,2-Dichlorohexafluorocyclobutane..................................... 297

cis-Hexafluoro-1, 2-bis-trifluoromethylcyclobutane................................. 298

trans-Hexafluoro-1, 2-bis-trifluoromethylcyclobutane................................. 299

Decafluoro-1,2-dimethylcyclobutene................................................... 300

Five-Membered Rings

Cyclopentene......................................................................... 301

1,5,5-Trimethyl-1,3-cyclopentadiene.............................................. 302

2,5,5-Trimethyl-1,3-cyclopent adiene................................................... 303

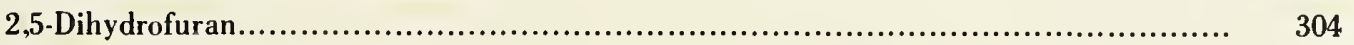

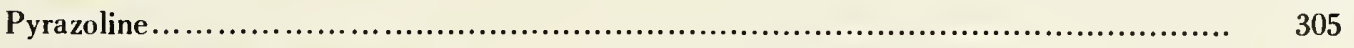

Methyl-substituted pyrazolines........................................................ 306

Six-Membered Rings

Cyclohexene........................................................................... 307

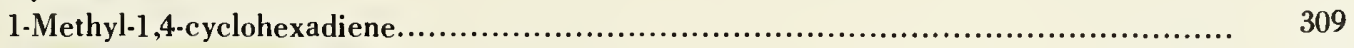

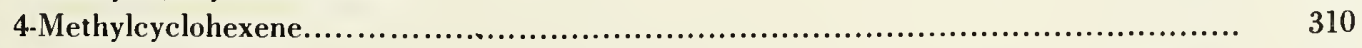

4-Vinylcyclohexene............................................................... 311

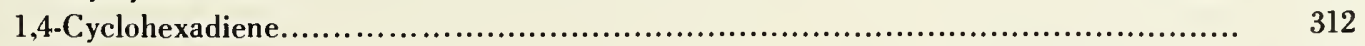

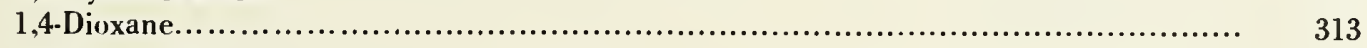

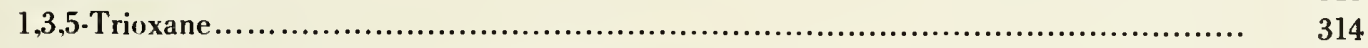

2,4,6-Trimethyl-1,3,5-trioxane (paraldehyde)....................................... 315

2,4,6-Tri- $n$-propyl-1,3,5-trioxane (para- $n$-butyraldehyde)............................. 316

2,4,6-Tri-isopropyl:1,3,5-trioxane (para-isobut yraldehyde)............................... 317

Other rings:

Cycloheptatriene..................................................................... 318

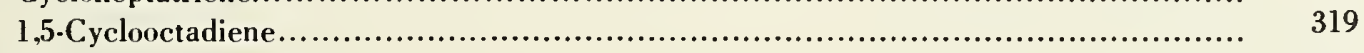

Polycyclics:

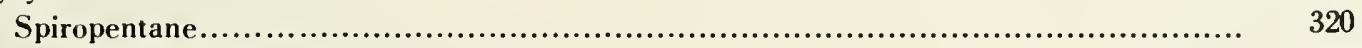

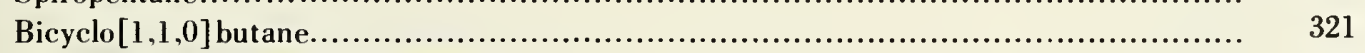

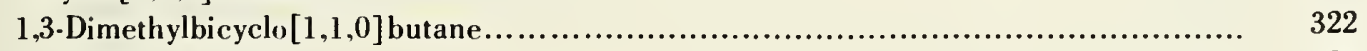

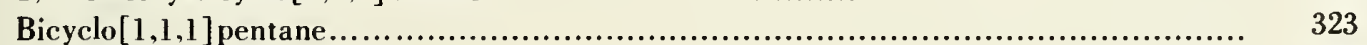

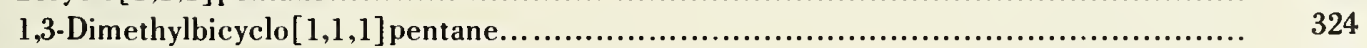

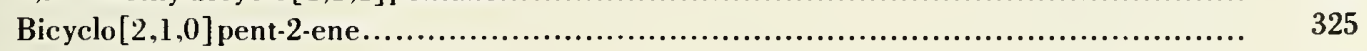

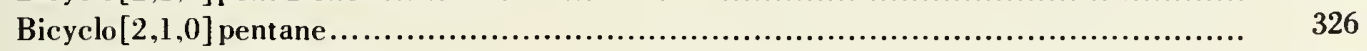

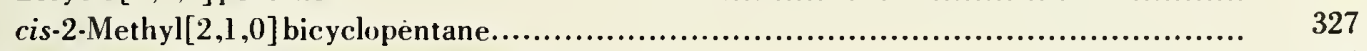

trans-2-Methyl[2,1,0]bicyclopentane................................................ 327

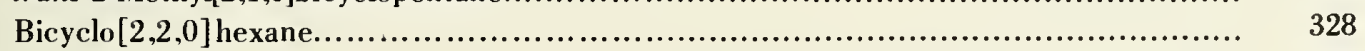

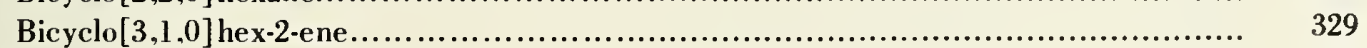

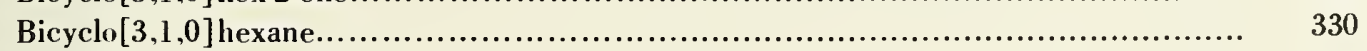

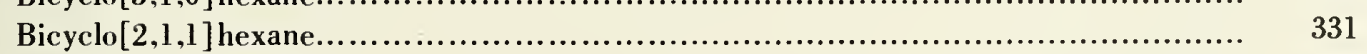

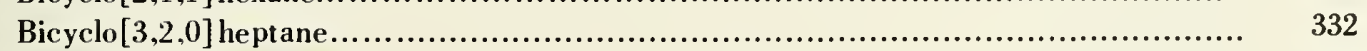

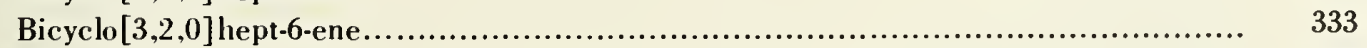

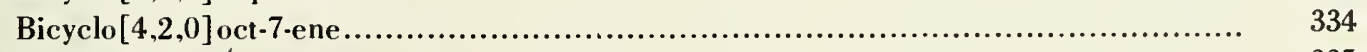

Bicyclo[2,2,1]-hepta-2,5-diene........................................................ 335

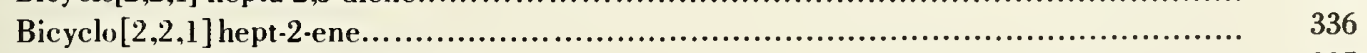

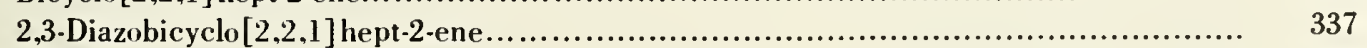

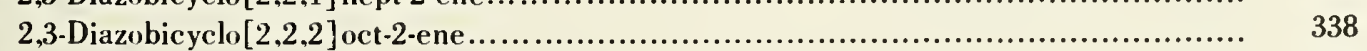

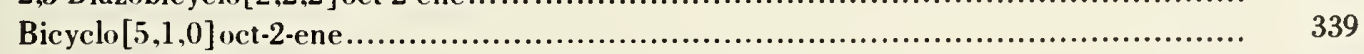

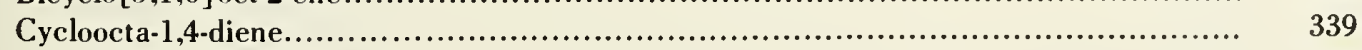

Tricyclo $\left[3,3,0,0^{2}, 6\right]$ octane.............................................................. 340

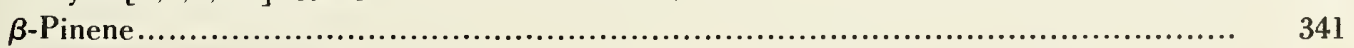

Endo-dicyclopentadiene........................................................... 342

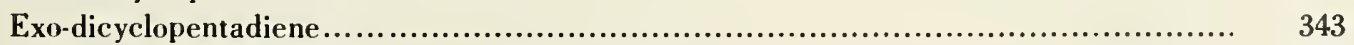

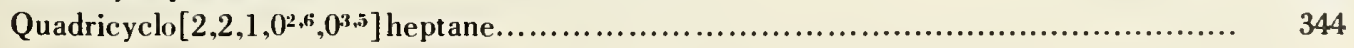


Endo-bicyclo[2,2,1] hept-5-ene-2-carboxaldehyde (endo-5-norbornene-2-carboxaldehyde; endo-methylene-2,5-tetrahydrobenzaldehyde ).

3. Isomerization Reactions (Other Than Cyclic Compound Reactions)

cis-1,2-Dideuteroethene.

trans-1,2-Dichloroethene.

cis-2-Butene

Perfluorobutadiene (hexafluorobutadiene).

trans-Perfluoro-2-butene.

cis-Perfluoro-2-butene.

cis-1,2-Diphenylethene (isostilbene).

Dimethyl-cis-1,2-ethendioate (dimethyl maleate)..

Methyl-cis-2-butenoate (methyl-cis crotonate).

Methyl-cis-cinnamate.

(cis-2-Butenenitrile) cis-crotonitrile.

cis- $\beta$-Cyanostyrene.

$1,1,6,6-d_{4}-1,5-$ Hexadiene

1,5-Heptadiene.

3-Methyl-1,5-hexadiene.

3-Vinyloxy-1-propene (vinyl allyl ether)..

3-Isopropenoxy-1-propene (isopropenyl allyl ether).

2-Methyl-3-vinyloxy-1-propene (2-methallyl vinyl ether).

1.Buten-3-yl vinyl ether.

1,2,6-Heptatriene.

cis-1,3,5-Hexatriene

cis-1,3-Hexadiene.

2-Methyl-cis-1,3-pent adiene.

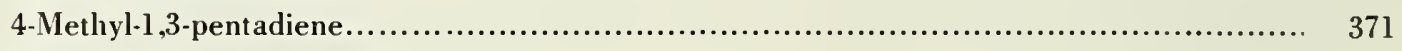

3,7-Dimethyl-1,6-octadiene.......................................................... 372

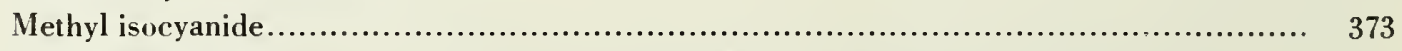

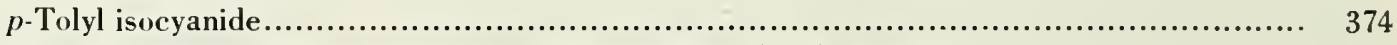

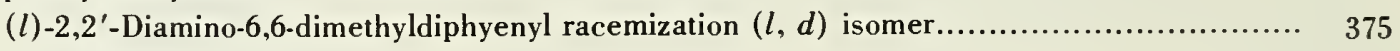

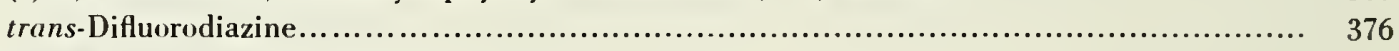

Nitrosomethane.................................................................. 377

4. Simple Bond Fission Reactions

Alkanes:

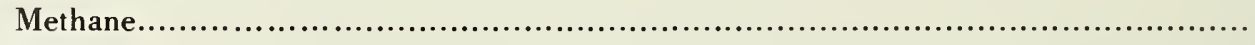

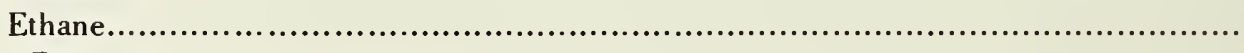

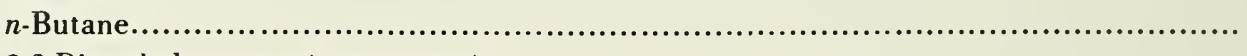

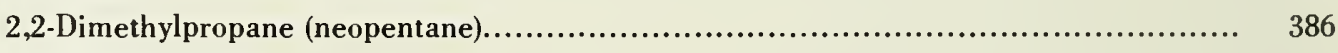

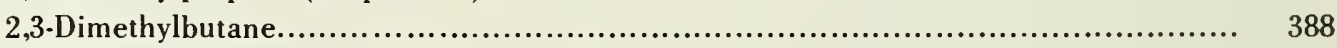

$2,2,3$-Trimethylbutane.......................................................... 389

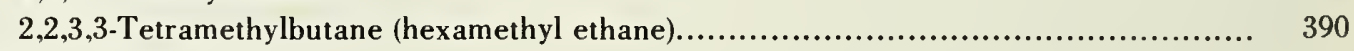

Alkenes and aromatics:

Propene (propylene)............................................................... 391

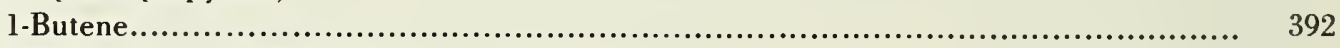

2-Methyl-1-butene (2-methylpropene; isobutene).................................... 393

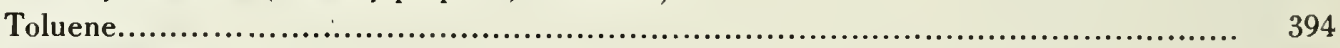

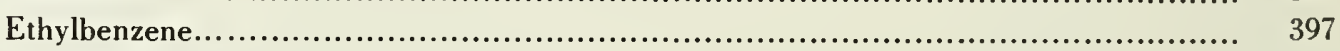

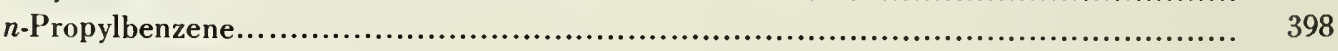

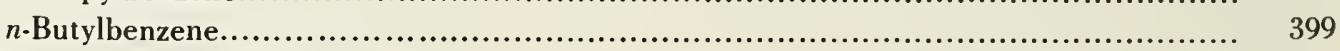

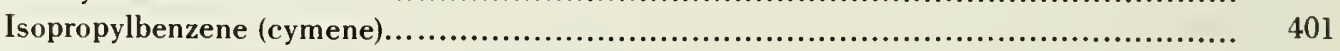

$t$.But ylbenzene.................................................................... 402

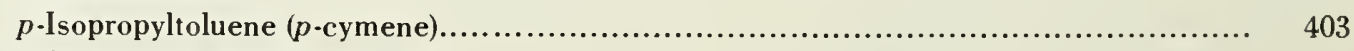

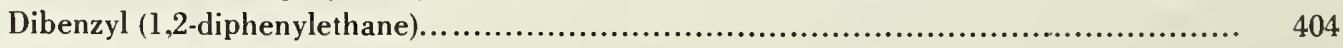

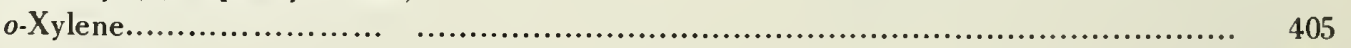

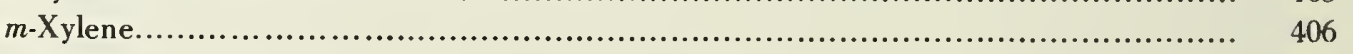

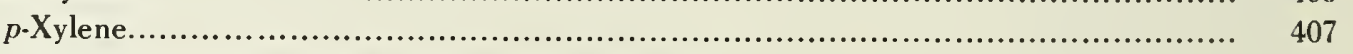

1,2-Methylnaphthalene ( $\alpha$ and $\beta$-methylnaphthalene)................................ 408

Para, ortho, and meta fluorotoluenes.................................................... 409

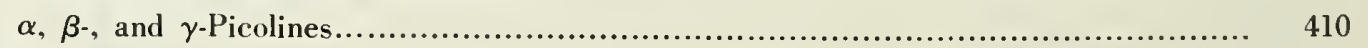

Oxygen compounds:

Acids:

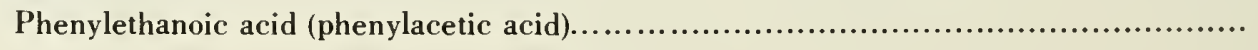


Diphenylethanoic acid (diphenylacetic acid).

Peracetic acid.

Ethers:

Dimethyl ether.

Diethyl ether.

Ketones:

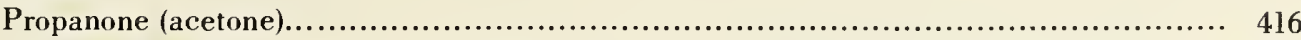

Acetophenone......................................................................... 417

l-Phenyl-2-propanone (benzyl methyl ketone)..................................... 418

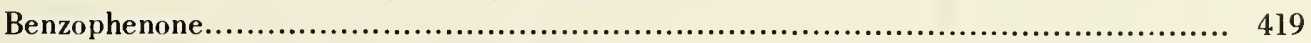

1,3-Diphenyl-1,2-propanone (dibenzil ketone)...................................... 420

1,2-Diphenyl-1,2-ethanedione (benzil)............................................... 421

1,1,1-Trifluoro-2-propanone (trifluoroacetone)..................................... 422

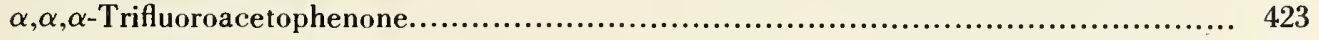

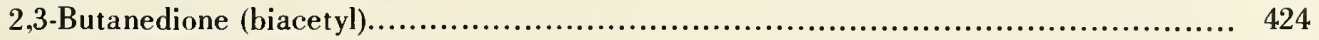

Esters:

Benzylethanoate (benzyl acetate) ................................................ 425

Benzylbenzoate.............................................................. 426

Peroxides:

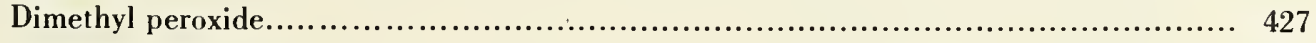

Diethyl peroxide................................................................. 428

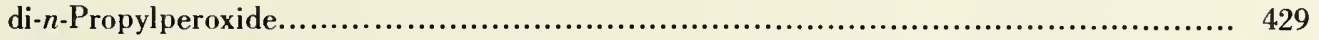

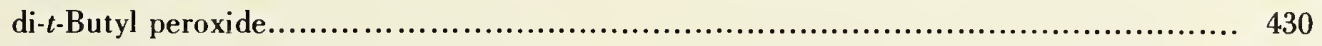

Diethanoyl peroxide (diacetyl peroxide) ............................................... 432

Dipropanoyl peroxide (dipropionyl peroxide)......................................... 433

Dibutanoyl peroxide (dibutyrl peroxide)............................................ 434

Hydroperoxides:

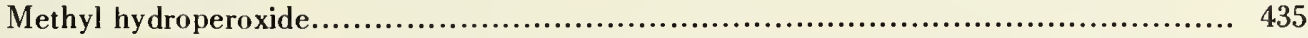

Ethyl hydroperoxide................................................................ 436

Isopropyl hydroperoxide........................................................... 437

$t$-Butyl hydroperoxide......................................................... 438

Amines:

Hydrazine........................................................................ 439

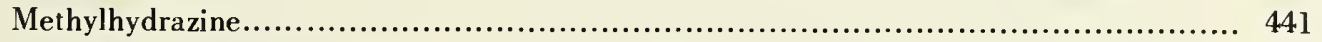

1,1 -Dimethylhydrazine.......................................................... 442

Benzylamine (phenylaminomethane) ............................................. 443

N-Methylbenzylamine........................................................... 444

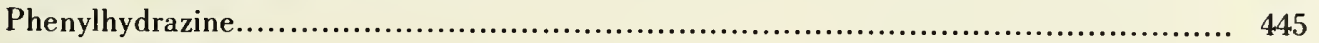

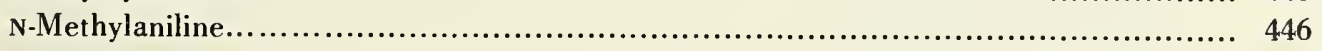

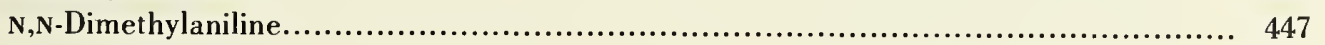

Primary azo compounds: $\quad 448$

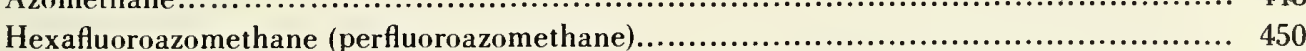

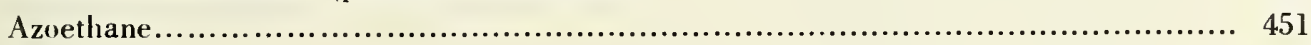

Azobutane......................................................................... 452

$1,1^{\prime}$-Azoisobutane................................................................ 453

Secondary azo compounds:

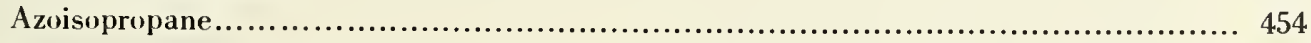

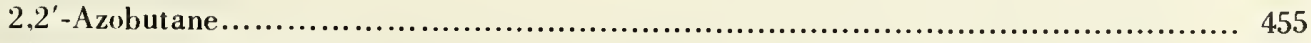

Methyl isopropyl diimide.......................................................... 456

Tertiary azo compound:
$\quad 2,2^{\prime}$-Azoisobutane............................................................. 457

Complex azo compounds:

Azotoluene........................................................................... 458

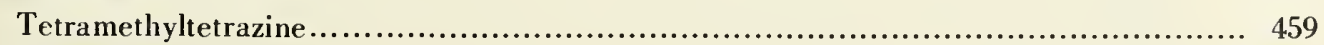

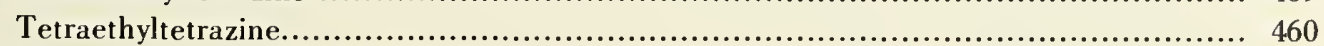

Cyanides:

Propanonitrile (ethyl cyanide).................................................... 461

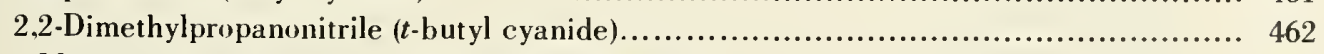

2-Methyl-2-phenylpropanonitrile (cumyl cyanide).................................... 463 


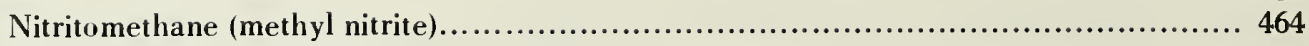

Nitritoethane (ethyl nitrite)....................................................... 466

l-Nitritopropane $(n$-propyl nitrite) ................................................. 467

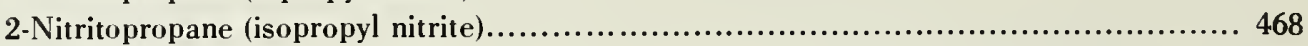

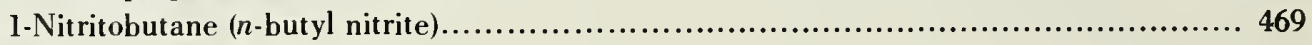

Nitrates:

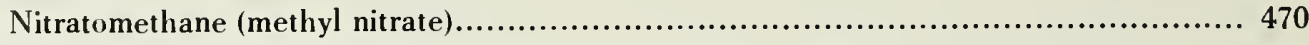

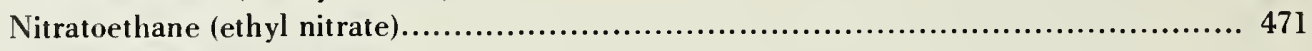

Collection of dinitrates, etc...................................................... 472

Nitro compounds: $\quad 473$

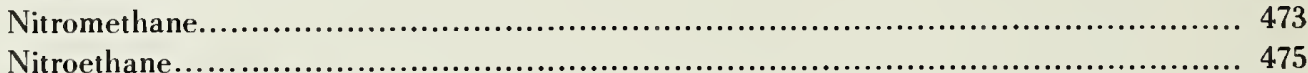

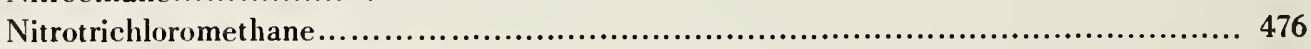

Tetranitromethane................................................................ 477

Other nitrogen compounds:

Ethanoldoxime (acetaldoxime) ................................................. 478

Dimeric nitrosomethane...................................................... 479

Dimeric-2-methyl-1-nitrosopropane (dimeric nitroisobutane)......................... 480

Diazomethane.................................................................. 481

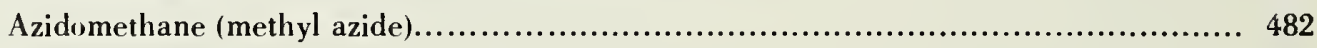

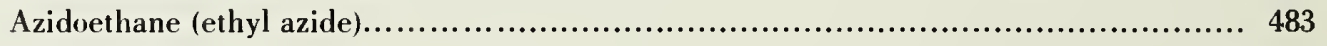

Sulfur compounds:

Methanethiol (methyl mercaptan) ................................................ 484

Ethanethiol (ethyl mercaptan)................................................... 485

Phenylmethanethiol (benzyl mercaptan) ........................................ 486

Thioanisole (phenylmethyl sulfide).............................................. 487

Methyl benzyl sulfide........................................................... 488

Methanylsulfonylphenylmethane (benzyl methyl sulfone) ............................. 489

Methanesulfonylmethane (dimethyl sulfone) ...................................... 490

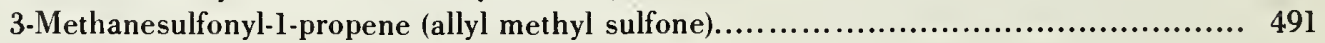

Halides:

Perfluoroethane...................................................................... 492

Perfluoropropene.................................................................... 493

Perfluoro-2-methyl-1-propene (perfluoroisobutene)....................................... 494

Bromomethane (methyl bromide) ................................................... 495

Dibromomethane (methylene dibromide) .......................................... 496

Tetrabromoethane (carbon tetrabromide)................................................ 497

Chlorobromomethane............................................................ 498

Dichlorobromomethane................................................................. 499

Trichlorobromomethane................................................................ 500

Trifluorobromomethane............................................................. 501

Tribromomethane.................................................................... 502

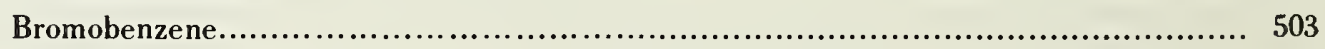

Substituted bromobenzenes............................................................. 504

2- and 3 -Bromopyridene........................................................... 505

1- and 2-Bromonaphthalene ( $\alpha$ - and $\beta$-bromonaphthalene)............................ 506

9-Bromophenanthrene and 9-bromoanthracene...................................... 507

2-Bromothiophene.................................................................... 508

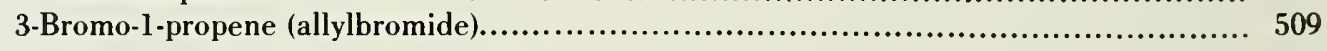

Bromophenylmethane (benzyl bromide) ............................................ 511

Substituted bromo phenylmethanes (substituted benzylbromides)....................... 512

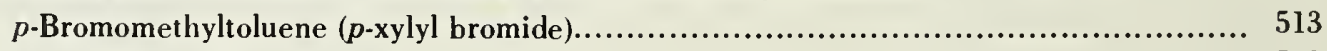

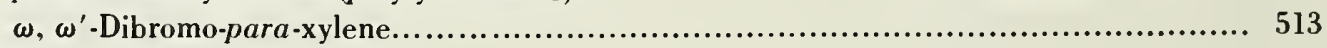

Benzoyl bromide....................................................................... 514

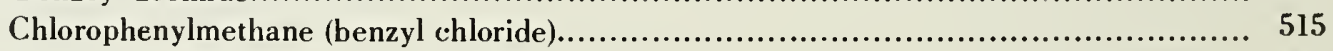

Benzoyl chloride.................................................................... 516

lodoethane (ethyl iodide)............................................................. 517

Metallic compounds:

Dimethylmercury............................................................... 518

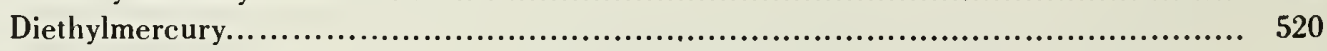

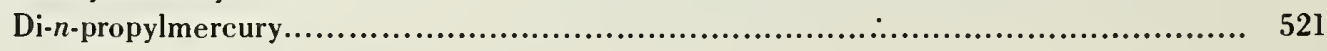


Di-n-butylmercury..

Diisopropylmercury

Divinylmercury.....

Diphenylmercury..

Phenylmercuric chloride............................................................. 526

Phenylmercuric bromide............................................................ 527

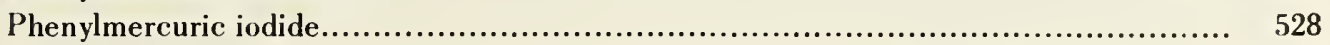

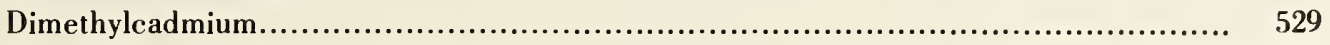

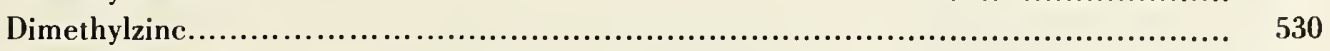

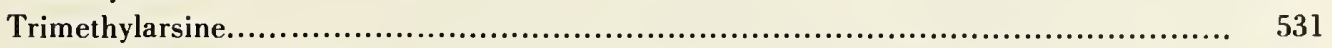

tris-(Trifluoromethyl)arsine..................................................... 532

Trimethylbismuth.................................................................. 533

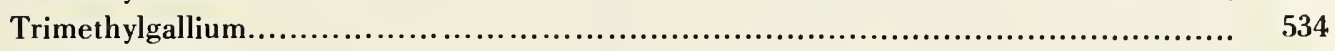

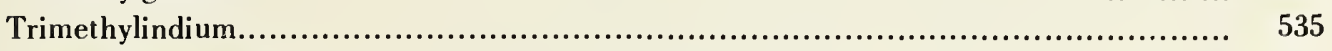

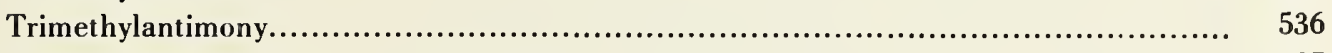

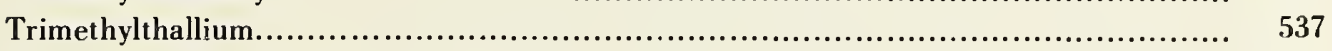

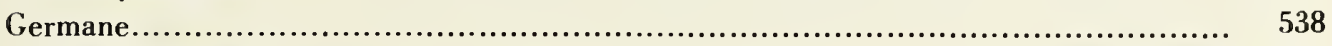

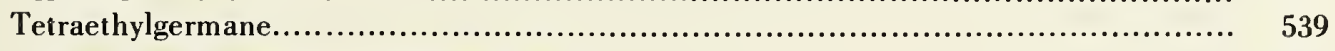

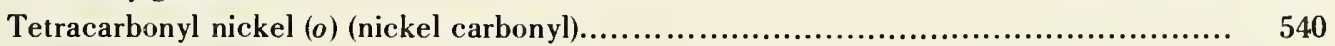

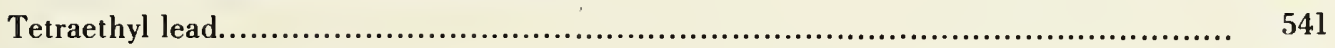

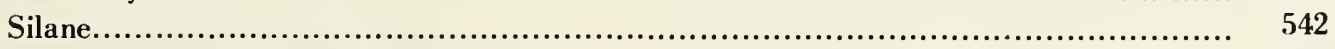

Tetramethylsilane (silicon tetramethyl)............................................. 543

Tetraethylsilane (silicon tetraethyl)................................................. 544

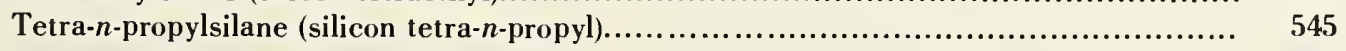

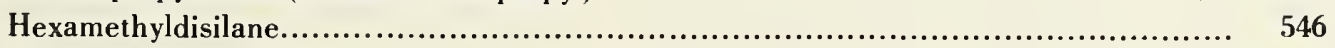

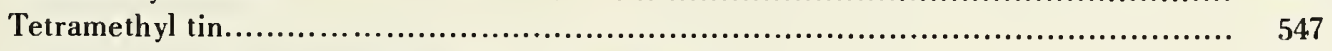

Boron:

Dichlorodimethylstannane (dimethyl tin dichloride)................................ 548

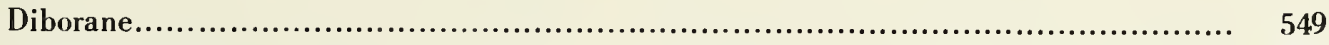

Borine carbonyl................................................................... 550

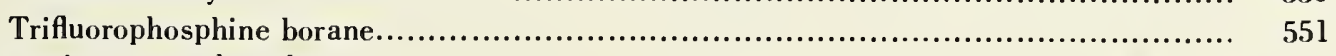

Inorganic nonmetal oxides:

Dinitrogen oxide (nitrous oxide) ...................................................... 553

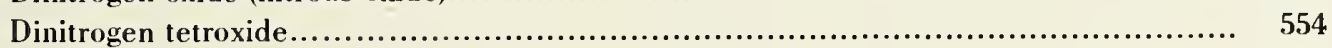

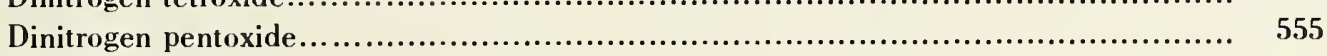

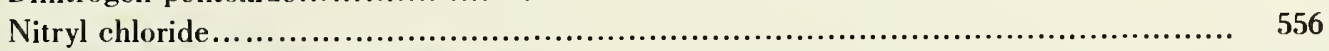

Nitroxylfluoride (fiuorine nitrate).................................................... 557

Chlorine nitrate (nitroxylchloride) ................................................. 558

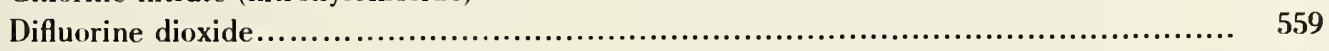

Chloryl fluoride ..................................................................... 561

Perchloryl fluoride................................................................... 562

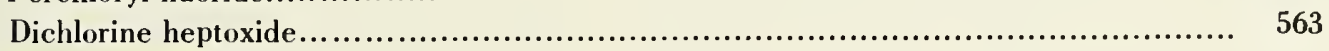

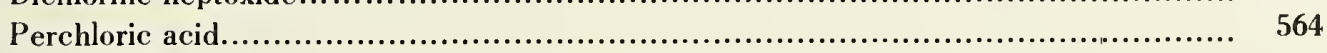

\section{Free radical reactions}

Ethyl radical ............................................................................. 567

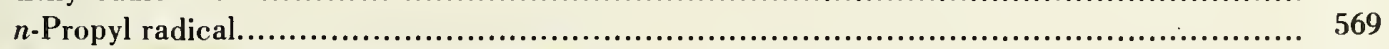

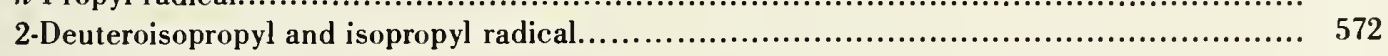

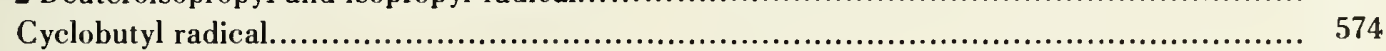

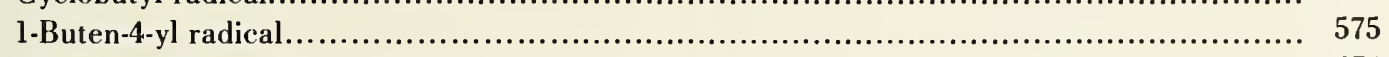

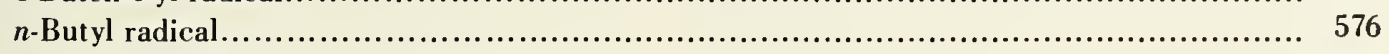

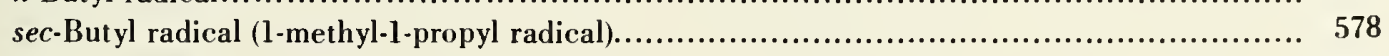

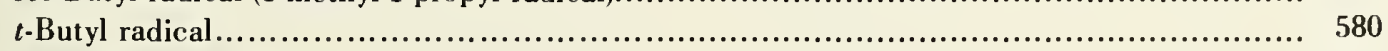

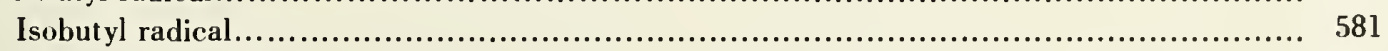

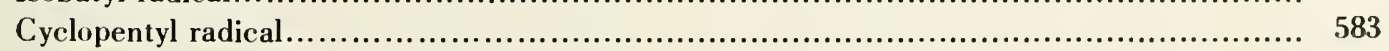

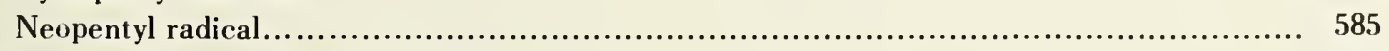

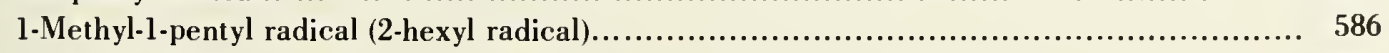

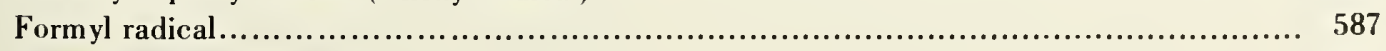

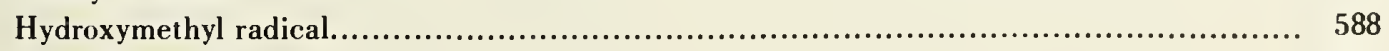

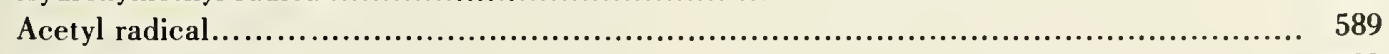

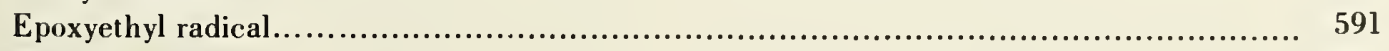

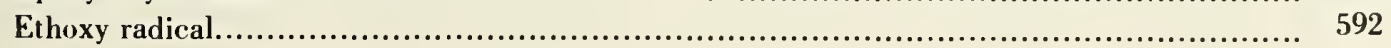


2-Oxo-1-propyl radical (acetonyl radical) ............................................ 593

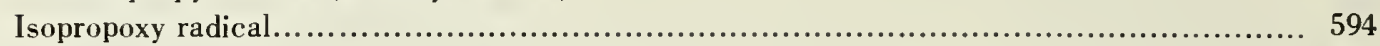

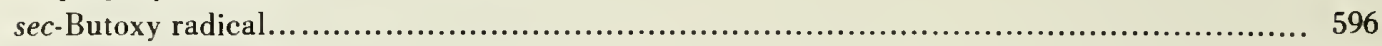

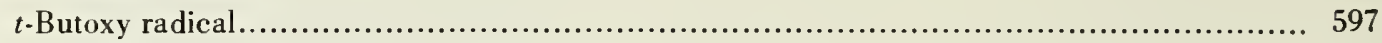

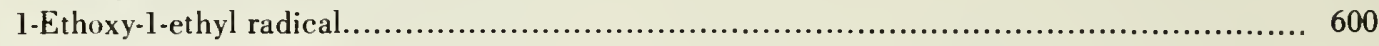

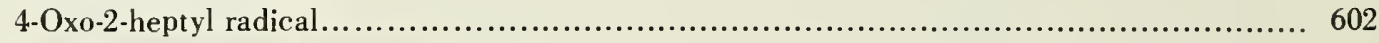

Methyl peroxymethyl radical.......................................................... 603

Perfluoro-t-butoxy radical........................................................... 604

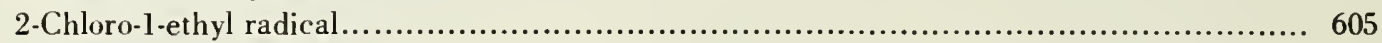

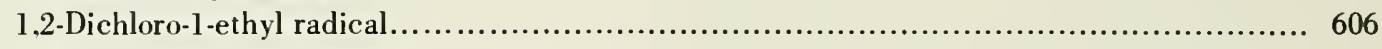

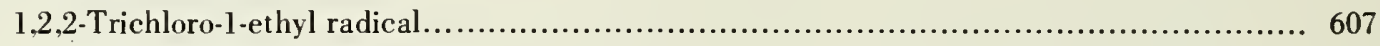

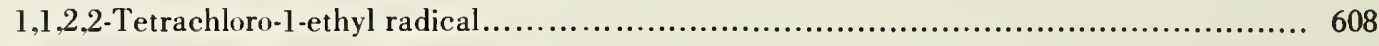

Pentachloroethyl radical.............................................................. 609

4-Chlorodifluoromethyl-2,5-cyclohexadienyl radical......................................... 610

2-Bromo-1-ethyl radical................................................................ 611

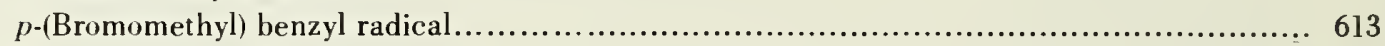

Dimethylgallium radical............................................................... 614

Methylindium diradical.................................................................. 615

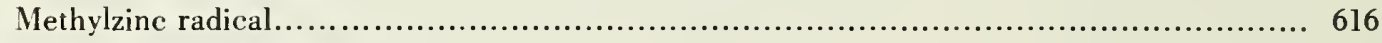

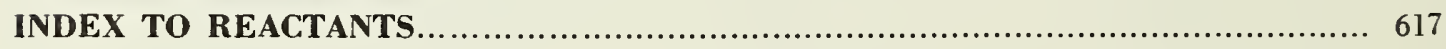




\title{
Kinetic Data on Gas Phase Unimolecular Reactions
}

\author{
Sidney W. Benson \\ Stanford Research Institute \\ Menlo Park, California 94025 \\ and \\ H. Edward O'Neal \\ San Diego State College \\ San Diego, California 92115
}

\section{Introduction}

\subsection{Purpose and Objectives}

This collection of chemical rate data has been made under the auspices of the National Standard Reference Data System. The purpose of the collection is to satisfy the following needs:

a. To provide in a convenient format a listing and referencing of all available reaction rate data for first-order, unimolecular, homogeneous, gas phase reactions.

b. To provide insofar as possible a critical evaluation of the reported kinetic parameters for each reaction.

c. To compile rate constants and Arrhenius parameters for each reaction and provide as much additional information as may be necessary for the reader to make an independent decision regarding the validity of the reported data.

d. To provide a primary rate data collection to which future kinetic results pertinent to unimolecular, homogeneous gas phase reactions may be conveniently added.

e. To indicate those areas in which theories of kinetics and existing rate data are nonconcordant, and by so doing provide a background with which future experimental investigations may be both planned and compared.

\subsection{Scope and Limitations}

Our aim has been to review all available rate data on thermally induced unimolecular, homogeneous, gas phase reactions of molecules and free radicals, and to present as comprehensive a collection of these data as possible. Literature through December 31, 1966, has been reviewed. Reactions of special interest appearing in print between January 1, 1967, and February 1, 1968 have also been included.
Although every effort has been made to effect as thorough and complete a survey as possible, it cannot be claimed with certainty that all reactions properly belonging to this monograph have been reviewed. Nor can it be claimed that all available data for any particular reaction listed have been presented. In some instances early literature was extensive, although not particularly reliable. Such literature has been intentionally omitted, or only selectively listed. (Many pyrolysis reactions originally listed in the literature as unimolecular have been omitted without comment where, in our opinion, the reactions were clearly complex chains.)

The initial collection (date of issue February 1970) has been limited to the reaction kinetics of thermally equilibrated molecules and free radicals. Ionic species have not been included. Also absent are the reaction kinetics of vibrationally and/or electronically energetic reactive intermediates produced photochemically, by chemical activation, or by high-energy radiation. "Unimolecular" reactions which are not first-order (i.e., those in their low-pressure or pressure-dependent regions) have been reviewed only in those cases where extrapolations or calculations were made to provide estimates of the limiting high-pressure first-order rate constants.

Evaluations of the reaction rate data necessarily reflect the opinions, views, and possible prejudices of the compilers. The reader should be well aware of the assumptions and ground rules followed by the authors in making decisions on the credibility of the reported rate constants and rate constant parameters. To this end, the discussions on mechanisms relative to each "reaction type" are pertinent. (See also sections I-3 and I-4). 


\subsection{Assumptions}

a. Gases are ideal.

b. The Arrhenius equation is a sufficiently accurate description of a rate constant

$$
\begin{array}{cc}
k=A e^{-E / R T} \mathrm{sec}^{-1} & \mathrm{I}-3.0-1 \\
\log k=\log A-E / \theta & \mathrm{I}-3.0-2
\end{array}
$$

with $\theta=2.303 R T$ in units of $\mathrm{kcal} / \mathrm{mole}$.

c. Forward and reverse rate constants of a reaction (even though determined under conditions far from the equilibrium state) are related through the equilibrium constant by the equation,

$$
K=k_{f} / k_{r}
$$

where $k_{f}$ and $k_{r}$ are the specific rate constants of a reaction in the forward and reverse directions, respectively.

d. In the high-pressure limit of a unimolecular reaction, molecules of the activated complex are in true equilibrium with reactant molecules. Transition state theory is accepted.

e. The activation energy for radical-radical recombination at $0^{\circ} \mathrm{K}$ is zero (i.e., $\Delta E_{\text {(rec) }}^{\stackrel{\ddagger}{*}}=0$ at $0^{\circ} \mathrm{K}$ ).

$\mathrm{f}$. The heat capacity of activation for radicalradical recombinations expressed in the concentration standard state is zero. This particular assumption is discussed in detail in the Bond Fission Reaction section (i.e., $\left\langle\Delta C_{v}^{+}\right\rangle_{\text {rec }}=0$ ).

\subsection{Thermodynamic Relations of a Unimolec- ular First-Order Reaction}

a. The general first-order reaction stoichiometry.

$$
a A \rightleftharpoons b B+c C \quad \quad \mathrm{I}-4.0-1
$$

b. Thermodynamic relations.

$$
\begin{aligned}
\Delta H^{\circ} & =\Sigma \Delta H_{f}^{\circ}(\text { products })-\Sigma \Delta H_{f}^{\circ} \text { (reactants) } \\
\Delta H^{\circ} & =b{\overline{\Delta H_{f}^{\circ}}}^{\mathrm{I}-4.0-2}+c{\overline{\Delta H_{f}^{\circ}}}^{\circ}(C)-a \overline{\Delta H_{f}^{\circ}}(A) \\
\Delta S^{\circ} & =\Sigma S^{\circ}(\text { products })-\Sigma S^{\circ} \text { (reactants) } \\
\Delta S^{\circ} & =b \bar{S}^{\circ}(B)+c \bar{S}^{\circ}(C)-a \bar{S}^{\circ}(A)
\end{aligned}
$$

.nd finally,

$$
\Delta G^{\circ}=\Delta H^{\circ}-T \Delta S^{\circ}=-R T \ln K_{p} \quad \mathrm{I}-4.0-6
$$

c. The 1 atm standard state is assumed through. out.

\subsection{Thermodynamics of the Transition State}

a. General activation reaction.

$$
\Sigma a_{i} A_{i} \rightleftarrows\left(T^{\ddagger}\right) \longrightarrow \text { products } \quad \text { I-4.1-1 }
$$

b. Thermodynamic relations.

$$
\begin{array}{rlr}
\Delta H^{\ddagger} & =\overline{\Delta H}_{f}^{\circ}(T)^{\mp}-\Sigma a_{i} \overline{\Delta H}_{f}^{\circ}\left(A_{i}\right) & \mathrm{I}-4.1-2 \\
\Delta S^{\ddagger} & =\bar{S}^{\circ}(T)^{\ddagger}-\Sigma a_{i} \bar{S}^{\circ}\left(A_{i}\right) & \mathrm{I}-4.1-3
\end{array}
$$

Note that the entropy of the transition state $\left(\bar{S}^{\circ}(T)^{\ddagger}\right)$ does not include the entropy contribution of the reaction coordinate. Finally,

$$
\Delta G^{\ddagger}=\Delta H^{\ddagger}-T \Delta S^{\ddagger}=-R T \ln K^{\ddagger} . \quad \mathrm{I}-4.1-4
$$

Again, the equilibrium constant $\left(K^{(\ddagger)}\right)$ between the transition state and the reactant excludes the contribution of the reaction coordinate to the transition state.

\subsection{Transition State Theory and the Rate Constant}

a. The transition state form of a rate constant is given by the equation

$$
k=\kappa \nu K^{\prime \ddagger} \text {. }
$$

Here $\kappa$ is the transmission coefficient, $\nu$ is the frequency with which the activated complex crosses the transition state to products, and $K^{\prime \neq}$ is the complete equilibrium constant between the reactants and the transition state. When the reaction coordinate is factored out of the equilibrium constant, either as a very loose vibration or as a translation, I-4.2-1 becomes (see, for example K. J. Laidler, Chemical Kinetics, McGraw-Hill Book Co., New York, 2d ed., p. 72)

$$
k=\frac{k T}{h} K^{\ddagger}
$$

In the above, the transmission coefficient has been set equal to unity and $K^{\ddagger}$ has been previously defined (I-4.1-4). In this form, transition state theory reduces kinetics to thermodynamics. As such, the thermodynamics of reactants, products, and transition states have been a primary concern of this monograph. In fact, the principal criterion used to evaluate the reported reaction rate data has been the estimated thermochemistry of transition states.

b. Classical form.

Writing $K^{\ddagger}$ in terms of entropy and enthalpy one 
obtains,

$$
k=\frac{k T}{h} e^{\Delta S_{T}^{\ddagger} / k} \times e^{-\Delta H_{T}^{\ddagger} / k T}
$$

c. Statistical form.

From statistical thermodynamics we have the following relations:

$$
\begin{gathered}
Z=\left(\frac{Q e V}{N}\right)^{. *} ; \\
S^{\circ}=\frac{\left(H_{T}^{\circ}-H_{0}^{\circ}\right)}{T}+k \ln (Z)
\end{gathered}
$$

Substitution of the above into $\mathrm{I}-4.2-3$ for the general activation reaction $(\mathrm{I}-4.1-1)$ gives,

$$
k=\frac{k T}{h} \frac{\Phi^{\ddagger}}{\prod_{i} \Phi_{i}^{\prime \prime i}}\left(\frac{e}{N}\right)^{\Delta n^{\ddagger}} e^{-\Delta n_{o}^{\ddagger} / k T} \quad \mathrm{I}-4.2-6
$$

or

$$
k=\frac{k T}{h} \frac{\bar{Q}^{\ddagger}}{\Pi_{i} Q_{l}^{a}}\left(\frac{V}{N}\right)^{\Delta n \ddagger} e^{-\Delta E_{0 / R T}^{\ddagger}}
$$

$\mathrm{I}-4.2-7$

In the above, $V$ has been factored from the translational partition functions (i.e., $\Phi=Q V$ ) in order to more clearly indicate the choice of thermodynamic standard states and also the units of the rate constant.

4.3. Transition State Theory and the Relations Between the Experimental Activation Energy and the Enthalpy of Activation

a. By definition, the experimental activation energy is given by,

$$
\begin{array}{r}
E=R T^{2} \frac{d \ln k_{\text {exp }}}{d T}=R T^{2}\left[\frac{d}{d T} \ln \frac{k T}{h}+\frac{d}{d T} \ln \left(\frac{Q^{\ddagger}}{\Pi_{i} Q_{i}^{a}}\right)\right. \\
\left.+\Delta n \frac{d}{d T} \ln \left(\frac{V}{N}\right)-\frac{d}{d T}\left(\frac{\Delta E_{o}^{\ddagger}}{R T}\right)\right] \quad \text { I-4.3-1 }
\end{array}
$$

b. For a unimolecular reaction $\left(\Delta n^{\ddagger}=0\right)$, $\Delta H_{T}^{\ddagger}=\Delta E_{T}^{\ddagger}$. Then from $\mathrm{I}-4.3-1$,

$$
\begin{aligned}
& E_{\text {(uni). }}=R T+\left(\Delta E_{T}^{\ddagger}-\Delta E_{o}^{\ddagger}\right)+\Delta E_{o}^{\ddagger} \text {, and } \\
& E_{\text {(uni). }}=\Delta E_{T}^{\ddagger}+R T=\Delta H_{T}^{\ddagger}+R T \quad \mathrm{I}-4.3-2
\end{aligned}
$$

c. For a bimolecular reaction $\left(\Delta n^{\ddagger}=-1\right)$; $\Delta H_{T}^{\ddagger}=\Delta E_{T}^{\ddagger}-R T$.
(1) With the rate constant expressed in concentration units, $k_{c}$ (e.g., $k$ (l/mole-sec),

$$
E_{c(\mathrm{bi})}=R T+\Delta \mathrm{E}_{T}^{\ddagger}=\Delta H_{T}^{\ddagger}+2 R T \quad \mathrm{I}-4.3-3
$$

(2) With $k$ expressed in pressure units, $k_{p}$, (e.g., $\left.k\left(\operatorname{atm}^{-1} \sec ^{-1}\right)\right)$.

(Note that $V=\frac{R T}{p}$ in $4.3-1$. )

$$
\begin{array}{cc}
E_{\nu(\mathrm{bi})}=R T+\Delta E_{T}^{\ddagger}-R T=\Delta E_{T}^{\ddagger} . & \mathrm{I}-4.3-4 \\
\Delta H_{T}^{\ddagger}=\Delta E_{T}^{\ddagger}-R T & \mathrm{I}-4.3-5 \\
\Delta E_{T}^{\ddagger}=\Delta E_{0}^{\ddagger}+T\left\langle\Delta C_{\mathrm{r}^{\ddagger}}\right\rangle, & \mathrm{I}-4.3-6
\end{array}
$$

Since $\quad \Delta H_{T}^{\ddagger}=\Delta E_{T}^{\ddagger}-R T$

and

where

$$
T\left\langle\Delta C_{1}^{\ddagger}\right\rangle \equiv \int_{0}^{T} \Delta C_{1}^{\ddagger} d T,
$$

one may also write,

$$
E_{p(\mathrm{bi})}=\Delta H_{T}^{\ddagger}+R T
$$

or,

$$
E_{p(\mathrm{bi})}=\Delta E_{o}^{\ddagger}+T\left\langle\Delta C_{1}^{\ddagger}\right\rangle \text {. }
$$

The latter relation $(\mathrm{I}-4.3-8)$ is discussed at some length with regard to radical-radical recombination reactions in the bond fission reaction section $(\mathrm{V}-3.0)$.

\subsection{Transition State Theory and the Relations Between} the Arrhenius $A$-Factor and the Entropy of Activation

a. Experimentally, the activation energies of reactions are usually obtained from Van't Hoff plots (i.e., $\log k$ versus $\frac{1}{T}$ ). Similarly, rate constants are usually reported in the Arrhenius form.

Thus,

$$
k=A e^{-E_{l} / R T}
$$

The relations between the experimental activation energy and transition state theory have been presented in section $\mathrm{I}-4.3$. From $\mathrm{I}-4.2-3$ and the appropriate activation energy relations of $\mathrm{I}-4.3$, the corresponding relations between the Arrhenius $A$-factors and the transition state entropies can be obtained.

1. Unimolecular reactions, units of $k\left(\mathrm{sec}^{-1}\right)$, (see I-4.3-2).

$$
A=\frac{e k T}{h} e^{\Delta s^{\ddagger} / k}\left(\sec ^{-1}\right) .
$$


2. Bimolecular reactions, units of $k(1 / \mathrm{mole}-\mathrm{sec})$, (see I-4.3-3).

$$
A=\frac{e^{2} k T}{h} e^{\Delta s^{\ddagger} / R}(1 / \text { mole-sec }) . \quad \mathrm{I}-4.4-3
$$

3. Bimolecular reactions, units of $k\left(\mathrm{~atm}^{-1} \mathrm{sec}^{-1}\right)$, (see I-4.3-7).

$$
A=\frac{e k T}{\mathrm{~h}} e^{\Delta S^{\ddagger} / R}\left(\mathrm{~atm}^{-1} \mathrm{sec}^{-1}\right) . \quad \mathrm{I}-4.4-4
$$

It should be emphasized that $\Delta S^{\ddagger}$ is always the entropy of activation for the pressure standard state and does not include the entropy contribution of the reaction coordinate in the transition state.

\subsection{Thermodynamics and the Arrhenius Parameters of Bond Fission Reactions}

a. For the reaction,

$$
R-R^{\prime} \underset{k_{b}}{\stackrel{k_{f}}{\rightleftarrows}} R+R^{\prime} \quad \quad \mathrm{I}-4.5-1
$$

the thermodynamics have been defined in section I-4.0 and the kinetic relations have been given in sections I-4.3 and I-4.4. Since $K=\left(k_{f} / k_{b}\right)$, we have

$$
K=e^{\Delta S_{T}^{\circ} / R} \times e^{-\Delta H_{T}^{\circ} / R T}=\frac{A_{f} e^{-E_{f}^{\prime} / R T}}{A_{b(p)} e^{-E_{b / R T}}} \cdot \mathrm{I}-4.5-2
$$

b. The activation energy for bond fission reactions.

1. For rate constants using the pressure standard state, the exponential terms are directly related to $\Delta H^{\circ}$. Thus,

$$
E_{p\left(f^{\prime}\right)}=\Delta H_{T}^{\circ}+E_{p(b)}
$$

Also, $\quad E_{p(f)}=\Delta H_{T}^{\circ}+\Delta E_{0}^{\ddagger}+(T)\left\langle\Delta C_{1^{\ddagger}}^{\ddagger}\right\rangle_{(b)}$

and since we have assumed $\Delta E_{0}^{\ddagger}=0$, this becomes

$$
E_{p(f)}=\Delta H_{T}^{\circ}+(T)\left\langle\Delta C_{V^{\circ}}^{\ddagger}\right\rangle_{(b) .} \quad \mathrm{I}-4.5-4
$$

2. For rate constants using the concentration standard state,

$$
\begin{aligned}
E_{c}=E_{p}-\Delta n^{\ddagger} R T=E_{p}+R T & =\Delta H_{T}^{\circ} \\
& +T\left\langle\Delta C_{v^{\prime}}^{\ddagger}\right\rangle+R T=\Delta E_{T}^{\circ}+2 R T . \quad \mathrm{I}-4.5-5
\end{aligned}
$$

c. The $A$-factor for bond fission reactions.

1. When the rate constant for recombination is in pressure units, the $A$-factor ratio is directly related to $\Delta S^{\circ}$. Thus,

and

$$
\begin{gathered}
A_{f\left(\mathrm{sec}^{-1}\right)}=A_{b} e^{\Delta S_{T}^{\circ} / R} \\
A_{f}=k_{b(\mu))} e^{\left\langle\Delta C_{r}^{\ddagger}\right\rangle_{b} / R} \times e^{{ }_{\varphi_{\tau}^{\circ} / R} / R} \quad \mathrm{I}-4.5-6
\end{gathered}
$$

2. When the rate constant for recombination is in concentration units, conversion to pressure units and equation $\mathrm{I}-4.5-6$ gives

$$
A_{f\left(\sec ^{-1}\right)}=\frac{k_{b(c)}}{R T} e^{\left\langle\Delta C_{r}^{\ddagger}\right)_{b} / R} e^{\Delta S_{\eta}^{\circ} / R} \quad \mathrm{I}-4.5^{-7}
$$

\subsection{Thermochemical Data Sources} See references [1-16], p. 61 .

\subsection{General Index}

a. Filing order.

In general each reaction is listed on a separate sheet. Exceptions to this rule are those reactions which have been studied collectively under identical experimental conditions for correlation purposes (e.g., the study of inductive, resonance, and steric effects of group substitutions located some distance from the reaction center(s)).

b. Classification and subclassification.

Molecular reactions are classified in one of four reaction types: (1) Molecular eliminations (or complex fissions); (2) Isomerizations of noncyclic compounds; (3) Cyclic compound reactions; and (4) Simple bond fissions. (See index for complete details.) Free radical reactions are treated as a fifth and separate group. Within each of the four major categories, reactions are subgrouped according to mechanistic and structural similarities. This offers the reader the distinct advantage of being able to rapidly scan the results for many reactions of the same kind.

c. Reactions have been indexed alphabetically in terms of the reactant(s). The section index lists the categories and subgroups into which each reactant molecule has been filed.

\subsection{Reaction Sheet Information}

\subsection{Reaction Thermodynamics}

Tabulated below the reaction are the standard state (1 atm, ideal gas) molar entropies, heats of formation, and specific heats of reactants and products at $25{ }^{\circ} \mathrm{C}$. In most cases the thermodynanic values listed are those calculated or estimated from group additivities (Appendix A). The probable errors in these values should rarely exceed 
$\pm 1.5 \mathrm{~g} / \mathrm{mole}$ in $\bar{S}^{\circ}$ and $\bar{C}_{p}^{\circ}$ and $\pm 1.5 \mathrm{kcal} / \mathrm{mole}$ in $\Delta \bar{H}_{f}^{\circ}$. Estimates which have greater uncertainty have generally been placed in parenthesis. For convenience the estimated reaction entropy, enthalpy, and heat capacity are also given.

\subsection{Rate Constants}

The Arrhenius parameters of each study are given as $\log A\left(\mathrm{sec}^{-1}\right)$ and $E(\mathrm{kcal} /$ mole). A rate constant for each study calculated at a mean reaction temperature is also listed. This will facilitate comparisons of the experimental reaction rates of all the studies for any given reaction.

\subsection{Experimental Information}

Experimental details and other pertinent observations for each study are given as follows under several headings: Conditions, System, Surface, and Experimental.

\section{a. Conditions.}

1. The temperature range of study (degrees Kelvin).

2. The pressure range of study ( $\mathrm{mm} \mathrm{Hg}$ or torr).

3. The specific pressure at which rate constants for the Arrhenius plot have been obtained (always indicated by parentheses, in units of $\mathrm{mm} \mathrm{Hg}$ ).

b. System.

Possibilities are: static, flow, stirred flow, shock tube, or single-pulse shock tube.

\section{c. Surface.}

Effects are summarized as:

1. None. This indicates that rate constants obtained in a packed reaction flask were the same as those obtained in the unpacked vessel within experimental error.

2. $<X \%$. Maximum contributions of surface reaction were obtained by comparing the rate constant increase to the surface/volume ratio increases and estimated from the equation,

$$
X \%=\left[\left(\frac{k_{s-k}}{k}\right) /\left(\frac{V S_{s}-S V_{s}}{S V_{s}}\right)\right] \times 100 .
$$

Here, $k_{s}, V_{s}$, and $S_{s}$ equal the rate constant volume and surface area appropriate to the packed reaction vessel and $k, S$ and $V$ are the rate constant, surface, and volume of the unpacked flask.

3. - A bar or blank indicates that no mention of any experimental test for surface effects was made.

\section{d. Experimental.}

1. Methods of product and reactant analysis.

2. Methods of analysis or experimental measurement(s) employed for obtaining the data from which the rate constants were evaluated.

3. Pertinent experimental observations and findings (e.g., abnormal surface effects, pressure falloff behavior, unusual reaction side products, effects of added gases with regard to catalysis, inhibition, collisional activation ( $3 \mathrm{~d}$ body effects), unusual mechanistic interpretations, significant theoretical calculations or observations).

\subsection{References}

References to each experimental study are numbered and listed on the same line as the Arrhenius parameters of that study.

\subsection{Preferred Values or Evaluation}

Preferred values will generally be those judged to be most consistent with the experimental reaction rate and transition state theory. In some instances, where experimental errors are believed to be large enough to accommodate changes in the reported Arrhenius parameters, preferred values are those estimated from transition state calculations.

\subsection{Comments}

Mechanistic interpretations by the authors are discussed here. In addition, results of transition state calculations are given for the purpose of comparison with the experimental values. Reactions reported to be unimolecular, but judged by the reviewers as questionable in this regard, have been cited as either suspect or unreliable. In all cases, under "Comments" the reasons for having made these evaluations are given and, where possible, other possibly more reasonable interpretations are suggested. 


\subsection{Glossary}

\subsection{Standard Thermodynamic and Kinetic Symbols}

\begin{tabular}{|c|c|c|}
\hline Symbol & Meaning & Value or Unit(s) \\
\hline$A$ & pre-experimental factor or $A$-factor (Arrhenius equation) & $\sec ^{-1}$ \\
\hline$E$ & activation energy (Arrhenius equation) & $\mathrm{kcal} / \mathrm{mole}$ \\
\hline$k$ & 1) specific rate constant & \\
\hline & 2) Boltzmann constant & 2) $1.3805 \times 10^{-16} \mathrm{ergs} / \mathrm{molec}-\mathrm{deg}$ \\
\hline$K_{p}$ & equilibrium constant in pressure units & at m$^{\Delta \prime \prime}$ \\
\hline$K_{c}$ & equilibrium constant in concentration units & $(\text { moles liter })^{\Delta \prime \prime}$ \\
\hline$k_{b}$ & rate constant for a reverse (back) reaction & $\left(\right.$ conc $\left.^{\Delta \prime \prime} \sec ^{-1}\right)$ \\
\hline$k_{\text {rec }}$ & rate constant for a recombination reaction & $\left(\operatorname{conc}^{\Delta \prime \prime} \sec ^{-1}\right)$ \\
\hline$\kappa$ & transmission coefficient & \\
\hline kcal & kilocalories & \\
\hline $\mathrm{g}$ & entropy unit (gibbs) & \\
\hline$S^{\circ}$ & molar standard state entropy ( 1 atm, ideal gas) & cal/deg-mole (gibbs/mole) \\
\hline$\Delta H_{f}^{\circ}$ & molar standard state heat of formation ( $1 \mathrm{~atm}$, ideal gas) & $\mathrm{kcal} / \mathrm{mole}$ \\
\hline$C_{p}^{0}$ & molar standard state specific heat ( 1 atm, ideal gas) & cal/deg-mole (gibbs/mole) \\
\hline$\left\langle\Delta C_{p}^{\circ}\right\rangle$ & $\begin{array}{l}\text { an average heat capacity change between two thermody- } \\
\text { namic states over a temperature range }\end{array}$ & gibbs/mole \\
\hline$\Delta G^{\circ}$ & molar gibbs free energy ( 1 atm, ideal gas) & $\mathrm{kcal} / \mathrm{mole}$ \\
\hline()$_{p}$ & sub $p$ signifies the 1 atm standard state & \\
\hline()$_{c}$ & sub $c$ signifies the concentration (moles/liter) standard state & \\
\hline()$_{T}$ & sub $T$ signifies ' at the temperature $T^{\prime}$ & \\
\hline()$_{0}$ & sub 0 signifies ' at $0^{\circ} \mathrm{K}^{\prime}$ & \\
\hline$N$ & Avogadro's number & \\
\hline$R$ & gas law constant & $1.987 \mathrm{cal} / \mathrm{mole}, \mathrm{deg}$ \\
\hline & & $0.08203 \mathrm{l}-\mathrm{atm} / \mathrm{mole}$, deg \\
\hline$h$ & Planck constant & $6.625 \times 10^{-27}$ ergs-sec \\
\hline$\alpha$ & collision efficiency of energy exchange & \\
\hline$\underline{T}$ & temperature & degrees Kelvin \\
\hline $\bar{T}$ & mean temperature & degrees Kelvin \\
\hline$t$ & time & seconds \\
\hline$\Delta n$ & mole change in a chemical reaction & moles \\
\hline$e$ & Naperian natural log base & 2.717 \\
\hline [ ] & 1) concentration & 1) moles/liter \\
\hline & $\begin{array}{l}\text { 2) a quantity whose value has been estimated on the basis } \\
\text { of the reported Arrhenius parameters }\end{array}$ & 2) variable \\
\hline ( ) & $\begin{array}{l}\text { 1) an estimated thermodynamic quantity which may en- } \\
\text { tail errors in excess of } 2 \text { gibbs } / \text { mole or } 2 \mathrm{kcal} / \mathrm{mole}\end{array}$ & \\
\hline & $\begin{array}{l}\text { 2) the pressure range of study employed for rate constant } \\
\text { measurements used to determine the Arrhenius para- } \\
\text { meters }\end{array}$ & 2) $\mathrm{mm} \mathrm{Hg}$ (torr) \\
\hline$\theta$ & energy equivalent of temperature $2.303(R) T$ & $\mathrm{kcal} / \mathrm{mole}$ \\
\hline()$^{\ddagger}$ & transition state & \\
\hline$\Delta S_{T}^{\ddagger}$ & $\begin{array}{l}\text { entropy of activation (excluding that of the reaction coor- } \\
\text { dinate) }\end{array}$ & cal/deg-mole \\
\hline$\Delta H_{T^{\prime}}^{\ddagger}$ & $\begin{array}{l}\text { enthalpy of activation (excluding that of the reaction coor- } \\
\text { dinate) }\end{array}$ & $\mathrm{kcal} / \mathrm{mole}$ \\
\hline r.c. & reaction coordinate & \\
\hline$\Phi$ & total molecular partition function & pure number \\
\hline$Q$ & $\begin{array}{l}\text { total molecular partition function with } V \text { factored out (i.e. } \\
\qquad Q V=\Phi)\end{array}$ & $V^{-1}$ \\
\hline$Q^{\ddagger}$ & $\begin{array}{l}\text { total molecular partition function of the transition state } \\
\text { (excluding the r.c.) }\end{array}$ & pure number \\
\hline
\end{tabular}



1) total molar partition function
1) pure number
2) kinetic theory collision frequency first-order rate constant
2) $\mathrm{sec}^{-1}$
$t_{c}$ contact time in a flow system
$\delta_{r}$
$P_{T}$ partial pressure of a reactant
RRK total pressure
RRKM
Rice-Ramsperger-Kassel theory of unimolecular reactions

\section{$M_{1 / 2}$}
Rice-Ramsperger-Kassel-Marcus theory of unimolecular

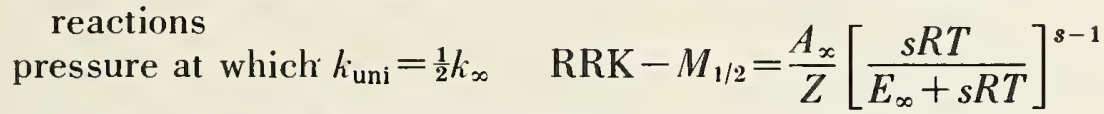
RRK number of effective oscillators exchanging energy with the reaction coordinate.

\subsection{Notations Used in Illustrations of Transition State Calculations}
$\left(X \in \mathcal{S}^{4}\right)_{\mathrm{z}}$
an internal rotation of group $X$ against group $Y$ around the $(X-Y)$ bond axis with a rotational barrier of $Z \mathrm{kcal} / \mathrm{mole}$ (for values of various rotations see appendix)
$\left(X \in \mathcal{f}^{x}\right)_{z}$
$\left(X_{4 e}\right)_{t}$
$\left(X_{3 e}\right) t$
$(X-Y)_{(Z)}$
$(X \cdot Y)$
$\left(X \cdot Y_{\backslash Z}\right)$
$\left(x^{-Y} Z\right)$
$\mathrm{X}^{\mathrm{Y} Z \mathrm{Z}}$
$D H^{\circ}(X-Y)$
$E_{(\text {Res) }}$
$E_{r_{x}}$
$E_{\mathrm{H} \text {-mir. }}$
an internal rotation of group $X$ against an infinitely heavy group
with rotational barrier of $Z \mathrm{kcal} / \mathrm{mole}$
a torsional motion about a 4 electron (normal double bond) as in the olefin $X$ (for definitions of $X$ see appendix)
a torsional motion about a 3 electron $(3 / 2$ order) bond as in the olefin $X$
a single bond stretch between groups (or atoms) $X$ and $Y$
a 1-electron (or half) bond stretch between groups or atoms $X$ and $Y$
a bond bend involving a single bond $(Y-Z)$ and a 1 -electron bond $(X \cdot Y)$ about the $X Y Z$ angle
a bond bend about the $X Y Z$ angle
a resonance conditions with kekule structures $(X=Y-Z \geqslant X-Y \leqslant Z)$
the $(X-Y)$ bond dissociation energy
resonance energy
the activation energy for an $X$ atom ring closing reaction
the activation energy for an $\mathrm{H}$-atom migration reaction quency in $\mathrm{cm}^{-1}$
is the frequency in
$\mathrm{kcal} / \mathrm{mole}$
$\mathrm{kcal} / \mathrm{mole}$
$\mathrm{kcal} / \mathrm{mole}$
$\mathrm{kcal} / \mathrm{mole}$

$Z$ is the vibration fre-

8.3. Symbols Designating Analytical Methods and Experimental Rate Measurement Methods

\section{Symbol}

M.S.

CHEM.

G.L.C.

O.R.

$\rho$

M.P.

U.V.

V

I.R.

N.M.R.

\section{Meaning}

Mass spectrometry

Standard chemical techniques

Gas-liquid phase partition chromatography

Optical rotation

Density

Melting points

Ultraviolet spectroscopy

Visible spectroscopy

Infrared spectroscopy

Nuclear magnetic resonance spectroscopy

\section{Symbol}

B.P. - P.V .

St.

Fl

$\mathrm{Sh}$

S P Shock

$\Delta P$

Pol.

$n_{r}$

\section{Meaning}

Boiling point separation pressure volume product Static

Flow

Shock tube

Single-pulse shock tube

Manometric measurements

Polarographic

Refractive Index 


\subsection{Experimental Errors}

Detailed discussions of the experimental errors involved in kinetic measurements are referenced in most physical chemistry and kinetics texts. We do not intend to reiterate or extend such treatments here. Rather, our purpose is to indicate the general magnitude of the average errors which might reasonably be expected in unimolecular reaction rate studies so that the reliability of kinetic studies may be easily evaluated.

It is well known that the most serious errors in kinetic studies are systematic ones. Further, it is recognized that the most frequent systematic errors arise from secondary reactions, heterogeneous effects, or other chemical complications. One of the principal objectives of this monograph has been to recognize the studies containing errors of this nature and subsequently to classify them as either unreliable or suspect. However, the kinds of errors of interest here are those, both systematic and random, which might be encountered in studies having no chemical complications. By careful experimental techniques and precautions, random errors in analysis and temperature control can be kept to a minimum. Thus very high precision in experimental rate constants can be realized and rate constant parameters are often reported to four or five significant figures on the basis of least squares analysis of the resulting Arrhenius plots. However, the errors reported in such cases reflect only very good control over random experimental errors and can not be interpreted to mean that the real experimental errors are necessarily small. Systematic errors could easily be present, and their magnitudes are almost impossible to estimate without prior knowledge of the "correct" behavior of the systems being studied.

Possible sources of error in the measurement of a rate constant are: (1) time measurements, (2) concentration measurements, and (3) temperature measurements. The last is generally the major source of error. Temperature control and temperature homogeneity of the reactor (as in static experiments) or of the reaction zone (as in flow experiments) are both very difficult and very important experimental problems. This is particularly true for gas phase homogeneous and thermally induced unimolecular reactions because they are most often studied in the $300-700{ }^{\circ} \mathrm{C}$ temperature range. The use of well-stirred liquid thermostats above $250{ }^{\circ} \mathrm{C}$ is uncommon and temperature fluctuations of the order of \pm 0.5 to $\pm 1{ }^{\circ} \mathrm{C}$ are the rule, rather than the exception, in air bath and solid core thermostats. At temperatures above $600{ }^{\circ} \mathrm{K}$ uncertainties in temperature con- trol and measurement probably exceed these values in most cases.

Most gas-phase kinetic system product analysis in recent years has been made using gas-liquid partition chromatographic techniques. Unless extreme care is exercised in the integration of product peaks and in the determination of product detector sensitivities, analytical precisions of better than \pm 2 percent are seldom achieved and errors of up to \pm 5 percent are not unusual. Other analytical methods such as M.S., I.R., U.V., etc., have very comparable reliabilities. Since systematic errors in analysis are probably as large as random errors, on the average, one would not expect to obtain analytical measurements of accuracy much better than \pm 4 to 5 percent.

Errors due to time measurements in static experiments are very small and may be ignored. In flow experiments, where residence times are not as well defined, time can be a more important source of error; however, it is generally less important than other uncertainties like temperature uniformity and control, or reaction zone volume.

In the completely general case of a dependent variable, $k=f\left(X_{1}, X_{2} \cdots X_{n}\right)$, which is a known function of $n$ independent variables $X_{1} \cdots X_{n}$, the relative error in $k$ due to errors in $X_{i}$ is given by,

$$
\left(\frac{\Delta k}{k}\right)^{2}=\sum_{i=1}^{n}\left(\frac{\partial \ln f}{\partial \ln X_{i}}\right)^{2}\left(\frac{\Delta X_{i}}{X_{i}}\right)^{2}
$$

With $k=f\left(A_{1}, A_{2}, t_{1}, t_{2}, T\right) ; E=g\left(k_{1}, k_{2}, T_{1}, T_{2}\right)$ and the relations,

$$
\begin{gathered}
k=\ln \left(\frac{N_{1}}{N_{2}}\right) /\left(t_{2}-t_{1}\right) \\
k=A e^{-E / R T} \\
E=\left[R T_{1} T_{2} /\left(T_{2}-T_{1}\right)\right] \ln \left(k_{2} / k_{1}\right)
\end{gathered}
$$

one can evaluate the necessary partial differential coefficients, and obtain,

$$
\begin{aligned}
\frac{\Delta k}{k}= & {\left[\left(\frac{1}{\ln \frac{N_{1}}{N_{2}}}\right)^{2}\left(\left(\frac{\Delta N_{1}}{N_{1}}\right)^{2}+\left(\frac{\Delta N_{2}}{N_{2}}\right)^{2}\right)\right.} \\
& +\left(\frac{t_{2}}{t_{2}-t_{1}}\right)^{2}\left(\frac{\Delta t_{2}}{t_{2}}\right)^{2} \\
& \left.+\left(\frac{t_{1}}{t_{2}-t_{1}}\right)^{2}\left(\frac{\Delta t_{1}}{t_{1}}\right)^{2}+\left(\frac{E}{R T}\right)^{2}\left(\frac{\Delta T}{T}\right)^{2}\right]^{1 / 2}
\end{aligned}
$$




$$
\begin{aligned}
\frac{\Delta E}{E}= & {\left[\left(\frac{T_{2}}{T_{2}-T_{1}}\right)^{2}\left(\frac{\Delta T_{1}}{T_{1}}\right)+\left(\frac{T_{1}}{T_{2}-T_{1}}\right)^{2}\left(\frac{\Delta T_{2}}{T_{2}}\right)^{2}\right.} \\
& \left.+\left(\frac{1}{\ln \frac{k_{i_{2}}}{k_{1}}}\right)^{2}\left(\left(\frac{\Delta k_{i_{2}}}{k_{22}}\right)^{2}+\left(\frac{\Delta k_{1}}{k_{1}}\right)^{2}\right)\right]^{1 / 2} \cdot(\mathrm{I}-9.0-6)
\end{aligned}
$$

In the above, $N_{1}, N_{2}$, and $t_{1}, t_{2}$ are the initial and final concentrations and initial and final time measurements respectively used to determine the rate constant in any given run (I-9.0-2), and $k_{1}, k_{2}$, and $T_{1}, T_{2}$ are the rate constants and temperatures used to calculate the activation energy (I-9.0-4).

An examination of the coefficients (in I-9.0-5) indicates that in order to measure the rate constant to an accuracy of $X \%^{1}=\Delta k / k \times 100$, it is necessary to

(1) measure the time with an accuracy of about

$$
\frac{\Delta t}{t} \times 100 \simeq\left(\frac{t_{2}-t_{1}}{t_{2}}\right) \frac{X \%}{(2)^{\frac{1}{2}}}=\left(\frac{\text { time interval }}{\text { longest time }}\right) \frac{(X \%)}{(2)^{\frac{1}{2}}}
$$

(2) measure temperature with an accuracy of about

$$
\frac{\Delta T}{T} \times 100=\frac{R T X \%}{E}
$$

(3) measure concentration with an accuracy of about

$$
\frac{\Delta N}{N} \times 100=\ln \left(\frac{N_{1}}{N_{2}}\right) \frac{X \%}{(2)^{1 / 2}} \cdot \quad(I-9.0-9)
$$

A very simple, straightforward, and reasonably reliable estimate of the error in the experimental activation energy can be made from (I-9.0-6). If one determines that errors in $k$, due to analysis and time variables, are some constant fraction ${ }^{2}(y)$ of the error in $k$, due to temperature measurements and control, then (I-9.0-5) becomes,

$$
\frac{\Delta k}{k}=(1+y)^{1 / 2}\left(\frac{E}{R T}\right)\left(\frac{\Delta T}{T}\right) .
$$

Substituting (I-9.0-10) and (I-9.0-4) ${ }^{2}$ into (I-9.0-6) gives

$$
\frac{\Delta E}{E}=(4+2 y)^{1 / 2}\left(\frac{\bar{T}}{T_{2}-T_{1}}\right)\left(\frac{\Delta T}{T}\right) \cdot(\mathrm{I}-9.0-11)
$$

For an "average" study with $\left(\frac{N_{1}}{N_{2}}\right) \simeq e, \frac{E}{R T} \simeq 35$, $\Delta T \simeq \pm 1{ }^{\circ} \mathrm{C}, T_{2}-T_{1} \simeq 60{ }^{\circ} \mathrm{C}, \bar{T} \simeq 600{ }^{\circ} \mathrm{K},(\Delta N / N)$ $\simeq 0.04$, and $(\Delta t / t) \simeq 0$, we find that analytical errors are approximately equal to errors arising from the temperature. Thus, $y \simeq 1$ and $\frac{\Delta E}{E}=6^{1 / 2} \times \frac{1.0}{60}$ $\simeq 0.040$. From the critical ratio of 35 , this "average" reaction would have an activation energy of 42 $\mathrm{kcal} / \mathrm{mole}$, and hence, a probable error in $E$ of $\Delta E \simeq 1.7 \mathrm{kcal} / \mathrm{mole}$. A quick perusal of reported activation energies for identical systems from different laboratories tends to confirm this estimate. Thus, as a first approximation of the probable errors in the activation energy for a thermally induced gas phase homogeneous kinetic reaction, (I-9.0-11) is extremely useful and fairly reliable Finally, from (I-9.0-3), the uncertainty in the Arrhenius $A$-factor can be calculated from the uncertainty in the activation energy.

With $\quad \Delta E=E(4+2 y)^{1 / 2}\left(\Delta T / T_{2}-T_{1}\right),(\mathrm{I}-9.0-12)$

$$
\Delta[\ln (A)]=\frac{\Delta E}{R T} \quad(\mathrm{I}-9.0-13)
$$

or

$$
\Delta[\log A]=\frac{\Delta E}{2.3 R T} \equiv \Delta E / \Theta . \quad(\mathrm{I}-9.0-14)
$$

$$
\begin{aligned}
& 2 y=\left[\left(\frac{1}{\ln \frac{N_{1}}{N_{2}}}\right)^{2}\left[\left(\frac{\Delta N_{1}}{N_{1}}\right)^{2}+\left(\frac{\Delta N_{2}}{N_{2}}\right)^{2}\right]\right. \\
& \left.+\left(\frac{t_{2}}{t_{2}-t_{1}}\right)^{2}\left(\frac{\Delta t_{2}}{t_{2}}\right)^{2}+\left(\frac{t_{1}}{t_{2}-t_{1}}\right)^{2}\left(\frac{\Delta t_{1}}{t_{1}}\right)^{2}\right] /\left(\frac{E}{R T}\right)^{2}\left(\frac{\Delta T}{T}\right)^{2}
\end{aligned}
$$




\section{Complex Fissions-Molecular Elimination Reactions}

\subsection{Four-Center Reactions}

Most four-center reactions involve hydrogen halide eliminations from alkyl halides. Reaction heats are 10 to $20 \mathrm{kcal}$ endothermic and reaction entropies range between 30 and $40 \mathrm{~g} /$ mole (positive). Surface catalysis is a characteristic of these reactions and "conditioning" of reaction cell" walls (by repeated decompositions of the reactant or by other methods) is generally required in order to achieve reproducible rate behavior.

Free radical, chain processes often provide competitive reaction paths to products. The general rules which seem to apply are that competing free radical chain process increase in the series $\mathrm{RCl}$, $\mathrm{RBr}, \mathrm{RI}$, and decrease with increasing alkyl substiution at the $\alpha$-carbon position. Thus primary halides have more chain decomposition than do secondary halides, which in turn are less unimolecular than tertiary halides. These behaviors have been explained in terms of the relative rates of chain propagation versus chain termination $[17,18]$.

Rate constant parameters for the homogeneous unimolecular contributions to the eliminations have generally been obtained for systems under "maximum inhibition" of chains. Additions of inhibitors or free radical traps (e.g., propylene, toluene, cyclohexene, etc.) decrease the overall reaction rates by lowering the concentrations of radicals which can propagate chains. At maximum inhibition, rate constants are invariant (with further additions of inhibitor) and are minimum values. Residual reactions are therefore assumed to be molecular. Support for the assumption of the absence of chain processes at maximum inhibition and for the molecularity of the residual reactions comes from several sources. None individually constitute proof of the assumption, but taken collectively they do offer a strong argument for it. The evidence in the case of ethyl bromide, which in the uninhibited system is estimated to be 90 percent chain and 10 percent molecular, is as follows:

1. Maximum inhibition by different inhibitors always reduces decomposition rates to identical first order values [19].

2. Absence of dibenyzl, hydrogen, and ethane in the toluene carrier flow experiments on ethyl bromide [20] suggests that the rate of radical production (i.e., chain initiation) is very slow relative to hydrogen bromide elimination. This in turn implies that free radical chains are long in the uninhibited decomposition and are therefore readily susceptible to inhibition through trapping of the chain carriers. Support for long chains also comes from results on the photochemically initiated ethyl bromide decomposition [21].
3. The hydrogen/deuterium isotope effect in the ethyl bromide uninhibited and in maximum inhibited systems [22] is very different. This suggests that different mechanisms are operative.

4. The hydrogen/deuterium isotope effect for the uninhibited decomposition of ethyl chloride [22] is very similar to that observed in the inhibited ethyl bromide system. This suggests similar decomposition mechanisms, and the ethyl chloride reaction is believed to be an uncomplicated fourcenter molecular elimination.

5. The rate of decomposition for ethyl bromide in the induction period, which precedes the onset of the well-characterized chain decomposition, has the same Arrhenius parameters as those of the inhibited decomposition [23].

6. Addition of free radical sensitizers (e.g., $\mathrm{Br}_{2}$ or allyl bromide) to maximally inhibited systems, or to those which apparently have no chain contribution (e.g., $t$-butyl bromide), temporarily accelerates rates, but as these catalysts are removed from the system by reaction, the rates return to those at maximum inhibition [24].

7. Theoretical calculations of the Arrhenius parameters for the reverse association reactions are consistent with the observed reaction rates at maximum inhibition.

In view of these observations and as a result of these reviewers' inability to propose any reasonable homogeneous chain mechanism in the presence of inhibitors which can account for the observed reaction rates, we feel that the residual reactions are essentially unimolecular processes.

\subsection{Transition State Estimates of the $A$-factor of Four- Center Elimination Reactions ${ }^{4}$}

Reasonable and fairly precise estimates of the Arrhenius $A$-factors for four-center elimination reactions have been made. The method, which involves rather straightforward thermodynamic considerations, is illustrated below for the ethyl chloride reaction. See reference [25] for a more complete discussion.

\section{Ethyl Chloride}

$$
\mathrm{CH}_{3}-\mathrm{CH}_{2} \mathrm{Cl} \rightarrow\left(\begin{array}{c}
\mathrm{H}-\mathrm{C}-\mathrm{C}_{-}^{-H} \\
\mathrm{H}^{-} \mathrm{C}-\mathrm{H} \\
\mathrm{H} \cdot \dot{\mathrm{Cl}}
\end{array}\right)^{\neq} \underset{\mathrm{HCl}}{\stackrel{\mathrm{C}_{2} \mathrm{H}_{4}}{+}}
$$

The transition state has been represented in terms of one-, two-, and three-electron bonds. Frequency assignments for the fractional bond order stretches have been assigned using Badger's rule and Pauling's equations relating bond order and bound length [26]. In the absence of any other data, $\frac{1}{2}$-order bond bends have been assigned a 
frequency of $\left(\frac{1}{2}\right)$ that for a unit order bend. In following the structural and vibrational frequency changes between the ground state and the transition state, it is clear that the largest entropy effect will

t Estimates of the $A$-factors reported on the data sheets have been made according to procedures outlined in ref. [25]. Methods illustrated here, however, entail some slight modifications of the earlier method which we feel constitutes an improvement. The modifications include some changes in bending frequencies (particularly for one electron bonds which are breaking in the reaction) and include changes in the choice of reaction coordinates. With regard to the latter modification, the reaction coordinate has been assigned to the lowest frequency motion in arise from the restriction in the internal rotation as the partial $\mathrm{Pi}$ bond forms. Other frequency changes are less important. Estimates are shown in table II-1.

the transition state. For all but three and four center reactions, the reaction coordinate so designated may be identified with an internal rotation of the ground state rather than a bond stretch (as in ref. [25]). It should be noted however, that the results ( $A$ factors) obtained from either method are almost identical for four- and six-center elimination reactions. Significant variations (and improvements) only arise in the cases of Cope and "ene" type isomerizations.

TABLE II-1. Transition state estimates-ethyl chloride reaction

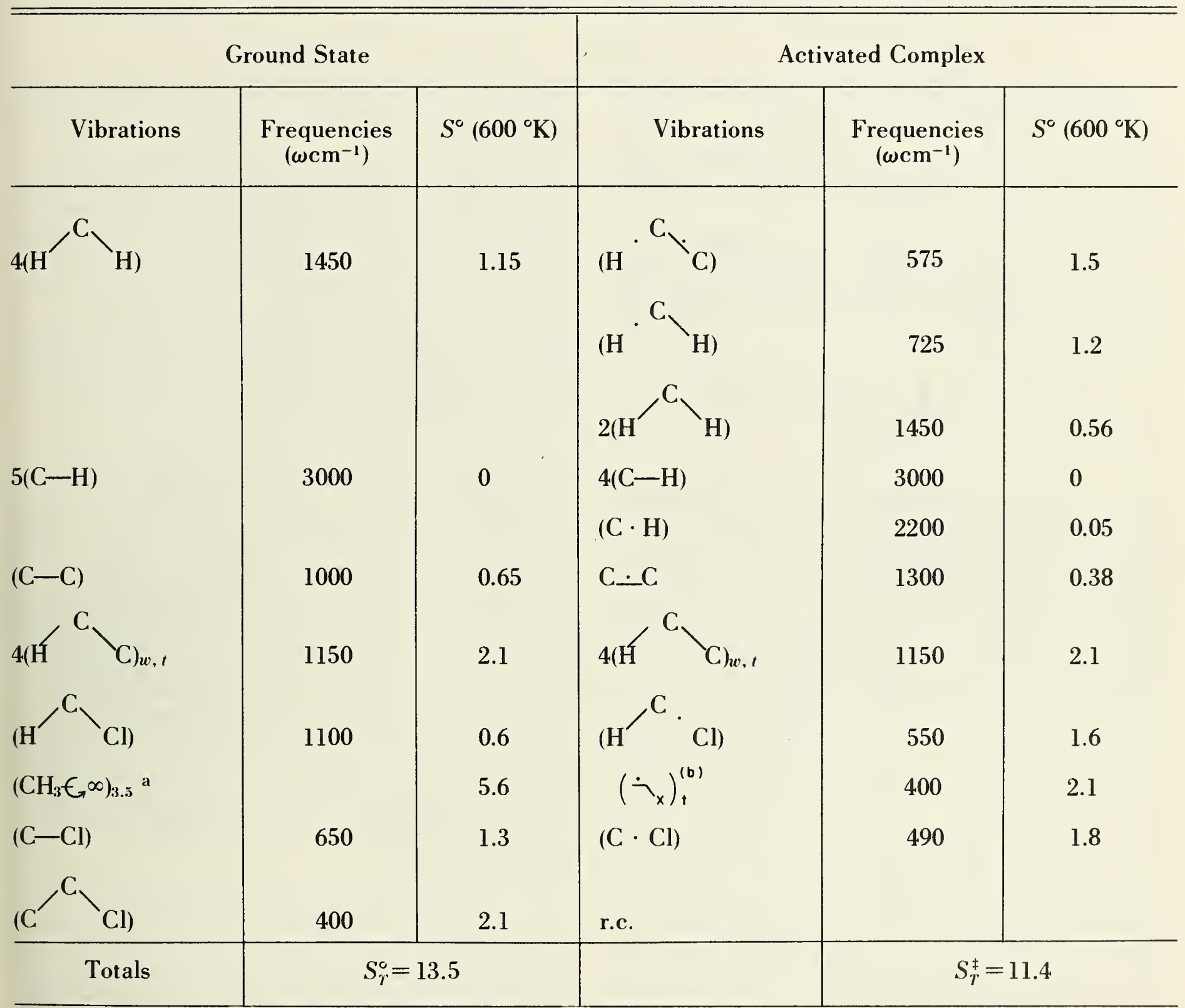

a The notation $\left(\mathrm{CH}_{3} \mathrm{C}_{\infty} \infty\right)_{3.5}$ indicates an internal rotation of a methyl group against a relatively large mass rotor with a barrier to rotation of $3.5 \mathrm{kcal} / \mathrm{mole}$.

$b\left(\mathcal{\neg}_{x}\right)$, indicates the propylene type torsion around a three-electron bond. Such torsion frequencies have been assigned values of $1 / 2$ the frequency of the corresponding torsion in the normal olefin (e.g., propylene in the above system). 
The intrinsic entropy of activation is therefore estimated to be

$$
\Delta S_{\mathrm{int}}^{\ddagger}=11.4-13.5=-2.1 \mathrm{~g} / \mathrm{mole} .
$$

The reaction coordinate degeneracy is 3 (i.e., there are three hydrogens on the methyl group which can react), and this gives a real entropy of activation of

$$
\Delta S^{\ddagger}=-2.1+R \ln 3=+0.1 \mathrm{~g} / \text { mole. }
$$

Thus we estimate $\left(600^{\circ} \mathrm{K}\right)$,

$$
A=\frac{e h T}{h} e^{د S \ddagger / R}=10^{13.6} \mathrm{sec}^{-1} ; A_{\mathrm{obs}}=10^{13.6 \pm 0.5} \mathrm{sec}^{-1} .
$$

The transition state calculations are for the most part relatively insensitive to the nature of the halogen. Note that the reaction coordinate has been assigned to that motion in the reactant which transforms into the lowest frequency motion in the transition state leading to reaction.

Estimated $A$-factors, in some reactions, seemed more reasonable than those reported. In these cases the preferred activation energy is the one calculated from the reported rate constant and the calculated $A$-factor.

\subsection{Three-Center Eliminations}

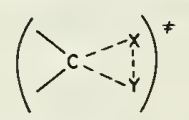

The $A$-factors of three-center elimination reactions have definite limits placed on them by thermodynamics and by transition state theory. Since no internal rotations are involved, entropies lost in activation cannot be very large. In the "tightest" possible activated complex, the entropy of activation may be equated to the negative of the sum of the entropies associated with the four bending and two stretching frequencies of the $X$ and $Y$ species in the reactant. It is unlikely that all six modes would contribute more than 5 to $7 \mathrm{~g} / \mathrm{mole}$ at decomposition temperatures. This sets a lower limit on the frequency factor, $A \geqslant 10^{12} \mathrm{sec}^{-1}$. An absolute upper limit is set by the overall reaction entropy and $Z$ (the collision frequency or maximum rate of the back reaction). Thus $A=A_{b} e^{\Delta S^{\circ} / R} \leqslant Z e^{\Delta S^{\circ} / R}$ Since usual values of $Z$ and $\Delta S^{\circ}$ are of the order of $10^{9} \mathrm{~atm}^{-1}$ and $35 \mathrm{~g} /$ mole, respectively, a maximum value for the frequency factor of $A \leqslant 10^{16.6} \mathrm{sec}^{-1}$ is obtained. It is unlikely that either of these limits are actually approached and we believe that threecenter elimination $A$-factors should be very close to normal (i.e., $A \simeq\left(\frac{e k T}{h}\right)$ ).

\subsection{Five-Center Elimination Reactions}

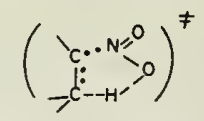

Decompositions of the nitroalkanes are the only known examples of reactions which probably proceed through five-center transition states. Reaction enthalpies and entropies are similar to the fourand six-center reactions and fall within the ranges of from 10 to $20 \mathrm{kcal}$ endothermic and from 30 to $40 \mathrm{~g} /$ mole (positive), respectively. The reaction rates are surface sensitive; therefore conditioning of reaction chamber walls is required. Product complexity clearly indicates the occurrence of competitive free radical and heterogeneous processes.

\subsection{Evaluation}

By the transition state methods used to estimate $A$-factors for the four- and six-center reactions, the $A$-factor estimates of the five-center reactions are between one and two orders-of-magnitude higher than those reported. Either the data are complicated by an incomplete suppression of chain decomposition, or transition state methods for estimating $A$ are inadequate. It should be noted that if the $A$-factors reported for the nitro compounds are correct, the reported entropies of activation correspond closely to the maximum possible entropy losses for activation.

\subsection{Six-Center Elimination Reactions}

Reaction thermodynamics are similar to those of other elimination reactions (i.e., $\Delta H^{\circ}=10-20$ kcal; $\Delta S^{\circ}=30-40 \mathrm{~g} /$ mole). Wall conditioning is generally required in the static systems before reproducible rate behavior can be achieved. In other respects the reactions are well behaved, going smoothly to the expected products with no apparent complications arising from competing chain processes. The only exceptions to the above seem to be the chloroformate esters, which are extremely surface sensitive and give some products which are most reasonably explained in terms of chain processes.

\subsection{Evaluation-Transition State Estimates of the} A-Factors of Six-Center Elimination Reactions ${ }^{*}$

The six-center elimination reactions have relatively large negative entropies of activation. Since at least three internal rotations are restricted in the transition state, a maximum of about $24 \mathrm{~g} /$ mole (about $8 \mathrm{~g} /$ mole per internal rotation at $600^{\circ} \mathrm{K}$ ) could be lost in forming the ring. Observed entropies of activation are about half this amount; therefore the 


\subsection{Ethyl Acetate}

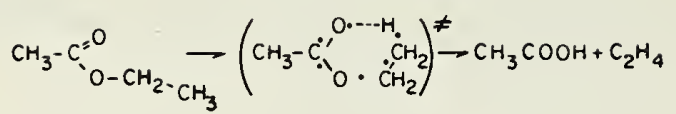

Frequency assignments in the ground state and activated complex are detailed in table II-2 (excluding the 12 acid methyl frequencies).

The intrinsic entropy of activation is therefore estimated to be

$$
\Delta S_{\text {int }}^{\ddagger}=24.4-31.7=-7.3 \mathrm{~g} / \text { mole. }
$$

The reaction path degeneracy is three, therefore the "real" entropy of activation is

$$
\Delta S^{\ddagger}=-7.3+R \ln 3=-5.1 \mathrm{~g} / \text { mole } .
$$

We therefore estimate the ethyl acetate $A$-factor $(700 \mathrm{~K})$ as

$$
A=\frac{e k T}{h} e^{\Delta S^{\ddagger} / K}=10^{12.5} \mathrm{sec}^{-1} ; A_{\mathrm{obs}}=10^{12.6} \mathrm{sec}^{-1} .
$$

We believe that transition state estimates of the above kind are probably reliable on the average to better than a factor of 2 .

\section{Cyclic Compound Reactions}

\subsection{Characteristics}

For convenience, cyclic compounds have been divided into several groups according to ring size (monocyclic reactants) and ring complexity (polycyclic reactants).

Experimental data for cyclic compound decompositions and isomerizations, especially cyclopropane and cyclobutane derivatives, are exceptionally good. The reactions are very well behaved kinetically, being for the most part free from surface effects and free from radical chain processes. Free radical chain inhibitors such as propylene, toluene, and nitric oxide are without effect on the observed reaction rates or reaction products. Also, rate constants determined using packed and unpacked reaction vessels are the same within experimental error. Many of the small ring (three- and four-membered rings) decompositions and isomerizations exhibit well behaved (i.e., consistent with theory) pressure falloff characteristics as predicted qualitatively by the old Lindemann theory (and quantitatively by the Rice-Ramsperger-Kassel-Marcus theory) of unimolecular reactions. There can be little doubt that these reactions are homogeneous, gas phase, unimolecular reactions.

\subsection{The Biradical Mechanism}

The mechanism of small ring compound decompositions has been the subject of controversy dating back to the first reaction of this type studied in any detail (cyclopropane). Two mechanisms were proposed, (1) a concerted process involving simultaneous $\mathrm{C}-\mathrm{C}$ bond rupture and formation with an $\mathrm{H}$-atom migration and (2) a consecutive step process proceeding through a biradical intermediate. In an elegant study of the cis-trans isomerization of 1,2-dideuterocyclopropane, Rabinovitch and Schlag showed that the rate of geometric isomerization was roughly 12 times faster than the structural isomerization to propylene and that the pressure falloff characteristics of the deuterated and undeuterated cyclopropanes were the same. The former result provided support for the biradical mechanism and the latter results indicated that the RRK (Rice-Ramsperger-Kassel) theory of unimolecular reactions was preferrable to the Slater theory. (See references [27], [28], and [29].) Differences of opinion, largely semantic, still exist with regard to the nature of the biradical intermediate. Whether the biradical is fully developed with little interaction between methylene ends or whether an appreciable interaction exists (an expanded ring) remains a subject of some debate.

The nature, and even the physical reality, of biradical intermediates in cyclic compound reactions has been argued extensively and will not be debated further here. However, there can be little question about the pragmatic value of the mechanism. From this review, our findings are that the kinetics of virtually all of the small ring compound reactions (with the exception of cyclobutenes) and the kinetics of the vast majority of the large ring and polycyclic compounds, are in excellent agreement with the biradical mechanism predictions, both qualitatively (with regard to reaction products) and quantitatively (with regard to transition state estimates of the Arrhenius parameters governing the formation of products).

Results of the transition state estimates made for these reactions are given on the data sheets with the preferred values. Thus, comparison, with the experimental parameters can readily be made. 


\subsection{General Remarks Concerning Transition State Calculations}

Estimates of activation entropies and enthalpies are based on the known or estimated thermodynamic properties of the reactants and biradical intermediates. For this purpose, the reader is referred to reference $[3]$ (Thermochemical Data Sources). A partial list of the more useful molecular and free radical group additivity values is given in Appendix B. Methods employed in the estimation of the radical group additivities and their use are not reviewed here. The reader is referred to the references indicated above. However additivity values in the appendix should be sufficient to enable the reader to follow the transition state calculations illustrated below.

To facilitate activation entropy and enthalpy estimates, thermodynamic paths from reactants to transition states have been used. These paths are not to be confused with the kinetic (or mechanistic) reaction path. Circled numbers indicate the calculational steps in the thermodynamic estimates. Step (1) is ring opening to the biradical at $298^{\circ} \mathrm{K}$; step (2) is incorporation of resonance (if any) in the biradical at $298^{\circ} \mathrm{K}$; step (3) is the correction to $\Delta S^{\circ}$ and $\Delta H^{\circ}$ for the average heat capacity change for steps (1)and(2)over the temperature range of $298^{\circ} \mathrm{K}$ to $\bar{T}$ experimental. Steps (4) and above are estimates of the activation entropies and enthalpies from the biradical to the various postulated transition states. These latter steps are detailed in terms of vibrational and rotational changes occurring. Enthalpy and entropy estimates, given below and above the arrows respectively, come from the additivity values. All entropy estimates are intrinsic entropies (i.e., symmetry numbers, optical isomer effects, and electron spin effects are not included). The activation entropy is calculated from the intrinsic entropy change by adding the entropy associated with the reaction path degeneracy, where reaction path degeneracy is related to the above by the equation

$$
K_{(\text {r.p.d. })}=\frac{\sigma n^{\ddagger}}{\sigma^{\ddagger} n} \text {. }
$$

In the above, $\sigma, n$, and $\sigma^{\ddagger}, n^{\ddagger}$ are the overall symmetry numbers and number of optical isomers in the ground state and transition state respectively. With regard to spin, only ground state singlets are important. For this reason, electron spin in the biradical need not be (and has not been) included. A big contribution to step(4,)insofar as $\Delta S^{\ddagger}$ is concerned, is the substitution of the contribution due to the reaction coordinate.

\subsection{Transition State Estimates for Cyclopropanes}

\subsection{Cis-1 2-d.-cyclopropane}

a. Mechanism

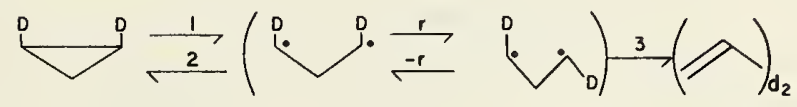

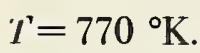

b. Transition state estimates

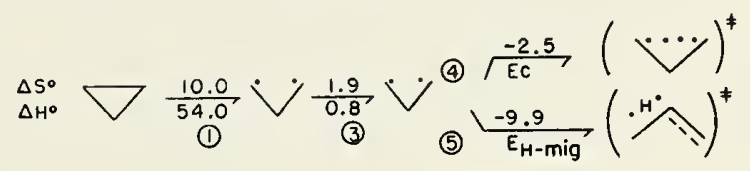

1. Cis-trans isomerization. $K_{\text {r.p.d. }}=3$

$$
\begin{aligned}
& \Delta S_{(C-t)}^{\ddagger}=10.0+1.9-2.5+R \ln 3=11.6 \mathrm{~g} / \mathrm{mole} \\
& A_{(C-t) \text { est }}^{\ddagger}=\frac{1}{2} \frac{e k t}{h} e^{\Delta S^{\ddagger} / R}=10^{15.9} \mathrm{sec}^{-1} ; \\
& A_{\text {obs }}=\frac{1}{2} A_{1}=10^{15.7}, 10^{16.1} \mathrm{sec}^{-1} \\
& E_{(C-t)}=64.2=54.8+E c_{3} \\
& E c_{3}=9.3 \mathrm{kcal} / \mathrm{mole}
\end{aligned}
$$

2. Propene formation. $K_{\text {r.p.d. }}=12$

$\Delta S_{(\text {prop })}^{\ddagger}=10.0+1.9-9.9+R \ln 12=7.0 \mathrm{~g} / \mathrm{mole}$

$A_{\text {(prop) est }}^{\ddagger}=10^{15.16} \mathrm{sec}^{-1}$;

$$
A_{\text {obs }}=10^{15.12} \mathrm{sec}^{-1}, 10^{15.20} \mathrm{sec}^{-1}
$$

$E_{\text {prop }}=65.6=54.0+0.8+E_{\mathrm{H}-\mathrm{mig}}$

$E_{\text {H-mig }}=10.8 \mathrm{kcal} / \mathrm{mole}$.

\section{c. Discussion}

Geometric isomerization was experimentally observed to be about 12 times faster than structural isomerization; therefore $k_{2} \gg k_{3}$. In addition, the rate constants for internal rotation can be shown to be much faster than either $k_{2}$ or $k_{3}$. This means that the rate-determining steps for cis-trans isnmerization and for propylene formation are ring opening and $\mathrm{H}$-migration, respectively. Thus the transition states indicated. Thermodynamics of the various steps in the calculation are:

$$
\begin{aligned}
\Delta X_{\oplus}^{\circ} & =\left[X^{\circ}(\grave{V})-X^{\circ}(\nabla)\right]_{2 s 8^{\circ} \mathrm{K}} \\
\left\langle\Delta C_{P}^{\circ}\right\rangle_{(3)} & =2.1 \mathrm{~g} / \text { mole } \\
\Delta S_{\oplus}^{\circ} & =-S^{\circ}\left(\mathrm{C}^{\mathrm{C}}\right)_{350 \mathrm{~cm}-1 \text { (r.c. })}
\end{aligned}
$$


The entropy of the transition state for ring opening is assumed to differ from that of the biradical (excluding spin) by the entropy of the reaction coordinate vibration for ring closing.

$\Delta S_{\circledast}^{\circ}=S^{\circ}\left[-2\left(. C_{2} \in E t\right)_{2.3}\right.$

$$
\left.+2\left(P_{40}\right)_{1}-(\mathrm{H}-\mathrm{C} \backslash \mathrm{C})_{1150 \text { r.c. }}\right]
$$

The entropy of the transition state for H-migration is fairly tight, and close to the entropy of the product olefin. Acitvation energies for $\mathrm{C}_{3}$ ring closing and 1,2-H-migration from the biradical have been obtained from the preferred values of the experimental activation energies of geometric and structural isomerization, respectively. Cyclopropane is the "standard" reaction for all three-membered ring reactions, and these activation energies have therefore been used to estimate the activation energies of other three-membered ring reactions.

\subsection{Vinyleyclopropane}

a. Mechanism

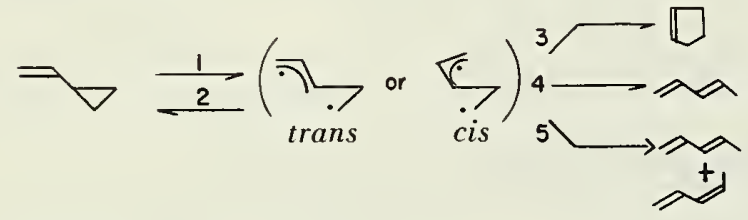

b. Transition State Estimates (cis only)

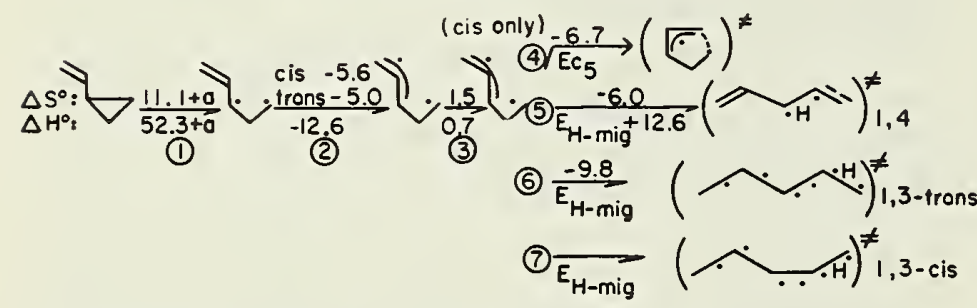

$a=1$ cis effect (cyclopentene formation) (see table A-2).

$a=0$ (all diolefin products).

See table A-2 for group correction for a cis effect.

1. Cyclopentene: $K_{\text {r.p.d. }}=2$

$$
\begin{aligned}
& \Delta S_{\text {C.P. }}^{\ddagger}=10.5-5.6+1.5-6.7+R \ln 2=1.1 \mathrm{~g} / \mathrm{mole} \\
& A_{\text {C.P. (est) }}=10^{13.8} \mathrm{sec}^{-1} ; \quad A_{\text {obs }}=10^{13.5}, 10^{13.6} \mathrm{sec}^{-1} \\
& E_{\text {C.P. }}=49.7=52.3+1.0-12.6+0.7+E c_{5} \\
& E_{C_{5}}=8.3 \mathrm{kcal} / \text { mole. }
\end{aligned}
$$

As in cyclopropane $k_{2}>k_{3}$. This has been experimentally confirmed by Willcott and Cargle [30]. who showed that geometric equilibration

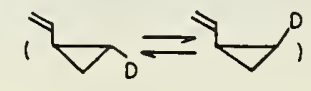

was at least five times faster than cyclopentene formation. Olefins are minor products: therefore $k_{3}>\left(k_{4}+k_{5}\right)$. Also, because of the development of allylic resonance in ring opening, only the cis biradical can close to cyclopentene. The activation eneroy deduced for $C_{\bar{s}}$ ring closing $(8.3 \mathrm{kcal} / \mathrm{mole})$ could be attributed to several effects: ring strain (probable). loss of allylic resonance in ring closing (most probable) and/or the barrier to rotation of the ethyl wroup as it moves to the conformation required for ring closing. Calculations based on low frequencies observed in the Raman spectra of the rotational barriers in various methyl-substituted pentanes (see Appendix C. table 3) sugrgest barriers ranging from 5 to $12 \mathrm{kcal} / \mathrm{mole}$. depending upon the degree of methyl substitution. Thus the latter effect could be important and could explain the higher than estimated activation energies observed for $>\nabla$ and for Because of their substitutions, these reactants might be expected to have higher than "normal" rotational barriers in the biradical. Note that $E_{C^{\prime} 3}$ must be less than $10.0 \mathrm{kcal}$ to account for the cis-trans results.

$$
\begin{aligned}
& \Delta x_{\oplus}^{\circ}=\left[x ^ { \circ } \left(\left[\sigma_{\text {cis }}-x^{\circ}(\forall)\right]\right.\right. \\
& \Delta S_{\oplus}^{\circ}=-S^{\circ}\left(E_{t} \epsilon_{.} . n P r\right)_{8.3(\text { r.c. })} \\
& \Delta S_{\overparen{Q}}^{c}=S^{\circ}\left[-\left(E t G_{i n} P r\right)_{2.6}+\left(C B_{3 e}\right)_{t}\right] .
\end{aligned}
$$

2. 1,4-pentadiene, $K_{\text {r.p.d. }}=2$

$\Delta S^{\ddagger}(1,4 P)=11.1-5.0+1.5-6.0+R \ln 2=3.0$

$A^{\ddagger}(1,4 P)=10^{14.2} \mathrm{sec}^{-1} ; A_{\text {obs }}=10^{14.4} \mathrm{sec}^{-1}$

$\dot{E}_{(1,4 P)}=52.3+0.7+10.8=63.8 \mathrm{kcal} / \mathrm{mole}$;

$E_{\text {obs }}=57.3 \mathrm{kcal} / \mathrm{mole}$.

Because all carbons and the migrating hydrogen are confined to lie in a plane, movement of the electron at the central carbon atom must remove much of the allylic resonance of the biradical. The observed activation energy implies a loss of about half the allylic resonance. This means that the estimated $A$-factor (based on no resonance) in the transition state is too high and should be closer to about $10^{14.0} \mathrm{sec}^{-1}$

\section{Trans-1,3-pentadiene, $K_{\text {r.p.d. }}=2$}

$$
\begin{aligned}
& X_{\text {(1) }}^{\circ}=\left[x^{\circ}\left(\mathcal{D}^{0}\right)-x^{\circ}(\bar{\nabla})\right] \text { for dienes } \\
& S_{\Im}^{\circ}=-\Delta S_{(2)}^{c}+S^{\circ}\left[-\left(\cdot \mathrm{CH}_{2} \in \infty\right)_{2 \cdot 3}-\left(E_{t} \in n P r\right)_{2 \cdot 6}+\right. \\
& \left(P_{4 e}\right)-\left(\mathrm{H}^{\mathrm{C}} \mathrm{C}\right)_{\text {r.c. }}
\end{aligned}
$$


$\Delta S_{(\text {trans } 1,3 P)}=11.1-5.0+1.5-9.8+R \ln 2=-0.8$ $\mathrm{g} / \mathrm{mole}$

$A_{(\text {trans } 1,3 P) \text { est }}^{\ddagger}=10^{13 \cdot 3} \mathrm{sec}^{-1} ; I_{\mathrm{obs}}=10^{13 \cdot 6)} \mathrm{sec}^{-1}$

$E_{\{\text {trans } 1,3 P)}=42.3-12.6+0.7+10.8=51.2$

$\mathrm{kcal} / \mathrm{mole}$

$E_{\mathrm{obs}}=53.6 \mathrm{kcal} / \mathrm{mole}$

In the transition states for 1,3-pentadiene formation, the allylic resonance may be freely maintained, or at worst, reduced to the resonance values of the 1,3 diene product. The above estimates for full allylic resonance in the transition state are in reasonable agreement with the facts.

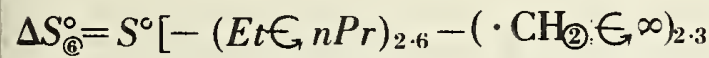

$$
\left.+\left(t B_{4 e}\right) t+\left(P_{4 e}\right)_{\ell}-\left(\mathrm{H}^{\mathrm{C}} \mathrm{C}\right)_{\text {r.c. }}\right]
$$

\subsection{Trans-1-Methyl-2-Vinyl Cýclopropane}

a. Mechanism for major path to cis-1,4-hexadiene:

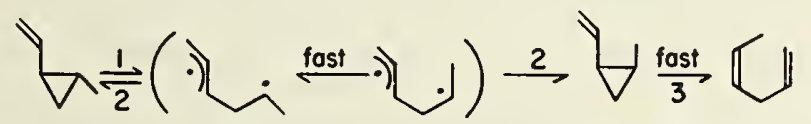

Transition State Estimates $\left(T=600^{\circ} \mathrm{K}\right)$ :

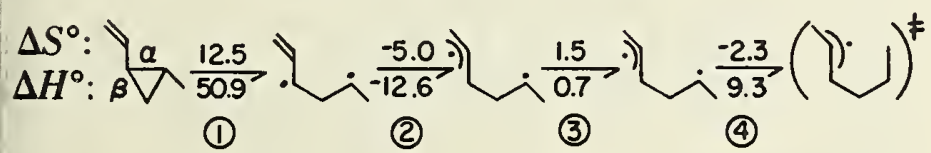

1. Cis-1,4-Hexadiene Formation (or cis $\rightarrow$ trans isomerization). $K_{\text {r.p.d. }}=1$.

$$
\begin{gathered}
\Delta S^{\ddagger}(1,4 H)=12.5-5.0+1.5-2.3=6.7 \mathrm{~g} / \mathrm{mole} \\
A^{\ddagger}(1,4 H)_{\mathrm{est}}=1 / 2 \frac{e k T}{h} e^{\Delta S^{\ddagger} / R}=10^{14.7} \mathrm{sec}^{-1} \\
A_{\mathrm{obs}}=10^{14.8} \mathrm{sec}^{-1}
\end{gathered}
$$

$$
\begin{gathered}
E(1,4)_{\text {est }}=50.9-12.6+0.7+9.3=48.3 \mathrm{kcal} / \mathrm{mole} \\
E_{\mathrm{obs}}=48.6 \mathrm{kcal} / \mathrm{mole}
\end{gathered}
$$

The dominant reaction here (in sharp contrast to vinylcyclopropane) is the formation of the open chain compound. Comparison of the mechanisms in the two systems suggests that this occurs via isomerization to the cis-1-methyl-2-vinylcyclopropane, which then rapidly isomerizes to cis-1,4-hexadiene by the fast "Ene" reaction. It is reasonable to suppose that this cis-trans isomerization occurs from the more stable ground-state conformation in which the vinyl group is trans to the ring. The conformation with the vinyl group gauche to the ring is the form required to produce cyclopentenes. There are two gauche forms possible, namely vinyl gauche to the $\alpha(\mathrm{C}-\mathrm{C})$ bond, and vinyl gauche to the $\beta(\mathrm{C}-\mathrm{C})$ bond. The former can only rupture the opposite, or $\beta(\mathrm{C}-\mathrm{C})$ bond, while the converse is true of the latter. Both produce cis biradicals.

It is observed that cis-1-methyl-2-vinylcyclopropane isomerizes very rapidly to cis-1,4-hexadiene (step 3). This is a concerted 1,5-H-migration reaction. Therefore, the rate determining step for cis-1,4hexadiene formation is ring opening (step 1), which leads to cis-1-methyl-2-vinyl-cyclopropane, which in turn reacts rapidly to form the diene. The factor of $1 / 2$ in $A$ arises from the fact that $\mathrm{C}_{3}$ ring closing to the cis and trans forms of the reactant occurs with equal probabilities.

$\Delta X_{\mathbb{1}}^{\circ}=\left[x^{\circ}\left(Y_{i}\right)-x^{\circ}(\bigsqcup)\right]$

$\Delta S^{\circ}=-S^{\circ}\left(E T G_{1} n P r\right)_{2.6}+\left(t B_{3 e}\right)_{i} ; \Delta H_{2}^{\circ}=-$ allyl

resonance

$\Delta S_{\oplus}^{\circ}=-S^{\circ}\left(C^{\mathrm{C}} \backslash\right.$ C) $350 \mathrm{~cm}^{-1}$ (as in cyclopropane)

b. 4-methyl-1-cyclopentene formation. $K_{\text {r.p.d. }}=1$.

If ring opening occurs with the vinyl group gauche to the $\beta(\mathrm{C}-\mathrm{C})$ bond, a resonance-stabilized cis biradical will result, which can either $\mathrm{C}_{3}$ ring close as above, or $C_{5}$ ring close to give a cyclopentene product. The latter process is slow compared to cis-trans isomerization; therefore, 4-methyl-1cyclopentene is a minor product. In the original study, the minor product was mistakenly reported as 3-methyl-1-cyclopentene. This would require $\beta(\mathrm{C}-\mathrm{C})$ cleavage. Since in terms of the biradical mechanism $\beta(\mathrm{C}-\mathrm{C})$ cleavage is about $1.4 \mathrm{kcal} /$ mole more endothermic than $\alpha(\mathrm{C}-\mathrm{C})$ cleavage, this was an unexpected result. It appears, however, that the expected mechanism shown below is undoubtedly operative.

Mechanism for 4-methyl-1-cyclopentene formation. $\left(4 M \mathrm{C}_{5}\right) . K_{\text {r.p.d. }}=1$.

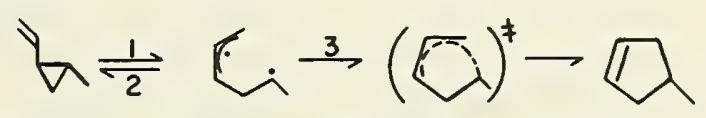

Transition State Estimates $\left(T=600^{\circ} \mathrm{K}\right)$

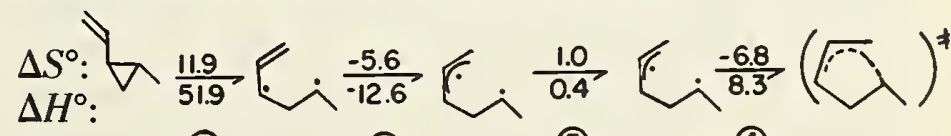

(1)

(2)

(3)

(4) 
$\Delta S_{(4)}^{\circ}=-S^{\circ}(n \operatorname{Pr} \in n P r)_{x .3}=-6.8 \mathrm{~g} /$ mole

$\Delta S^{\ddagger}\left(4 M C_{\text {. }}\right)=11.9-5.6+1.0-6.8=0.5 \mathrm{~g} / \mathrm{mole}$

$A^{\ddagger}\left(4 M C_{5}\right)_{\mathrm{est}}=10^{13 . \pi} \mathrm{sec}^{-1}$

$$
A_{\mathrm{obs}}=10^{13 . \tau} \mathrm{sec}^{-1}
$$

$E\left(4 M C_{5}\right)_{\mathrm{est}}=51.9-12.6+0.4+8.3=48.0 \mathrm{kcal} /$ mole

$$
E_{\mathrm{obs}}=48.6 \mathrm{kcal} / \mathrm{mole}
$$

\subsection{Transition State Estimates for Cyclobutanes}

\subsection{Cyclobutane}

a. Mechanism

$T=714^{\circ} \mathrm{K}$.

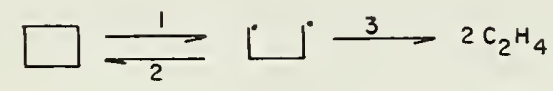

b. Transition State Estimates

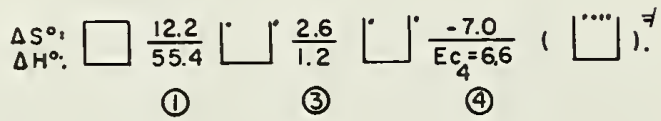

1. Ethene formation. $K_{\text {r.p.d. }}=4$

$\Delta S_{\text {(ethene) }}^{+}=12.2+2.6-7.0+R \ln 4=10.6 \mathrm{~g} / \mathrm{mole}$

$A_{\mathrm{est}}^{\ddagger}=\frac{1}{2} \frac{e k T}{h} e^{\Delta S^{\ddagger} / K}=10^{15.6} \mathrm{sec}^{-1} ; A_{\mathrm{obs}}=10^{15.6} \mathrm{sec}^{-1}$

$E_{\text {ethene }_{i}}=55.4+1.2+6.6=63.2 \mathrm{kcal} / \mathrm{mole}$.

\section{c. Discussion}

The biradical mechanism, when applied to the kinetics of cis- and trans-1,2-dimethylcyclobutane indicate that in the cyclobutane reactions, all processes from the biradical occur at very comparable rates. That is, $\mathrm{C}_{4}$ ring closing, decomposition and internal rotations of groups larger than $\left(\mathrm{CH}_{3}\right)$ and $\left(\cdot \mathrm{CH}_{2}\right)$ are all comparable. Thus $k_{3} \simeq k_{2}$, which accounts for the factor of $\left(\frac{1}{2}\right)$ in the $A$-factor estimate. The activation energy for $\mathrm{C}_{4}$ ring closing in the 1,2-dimethylcyclobutane systems was found to be $7.4 \mathrm{kcal} / \mathrm{mole}$. This includes two gauche repulsions in the conformation preceding ring closing. There is only one gauche repulsion in the ring closing conformation of cyclobutane, hence $E_{c_{4}} \simeq 7.4-0.8=6.6 \mathrm{kcal} / \mathrm{mole}$.
$\Delta x_{\mathbb{1}}^{0}=x^{0}\left(L^{0}\right)-x^{0}(\square)$

$\left\langle\Delta C_{p}^{\circ}\right\rangle_{3}=3.1 \mathrm{~g} / \mathrm{mole}$

$\Delta S_{\oplus}^{\circ}=-S^{\circ}\left[(E t \in E t)_{6.6} r . c.\right]$

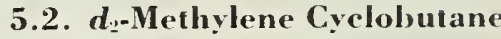

a. Mechanism

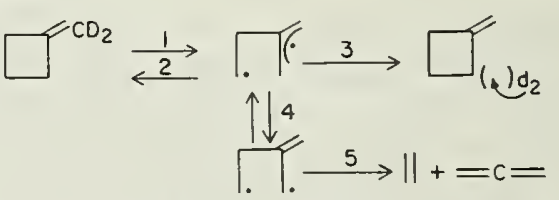

b. Transition State Estimates

$\Delta S^{\circ}:$

$\Delta H^{\circ}$ :

$\Delta S^{\circ}:$

$\Delta H^{\circ}$ :
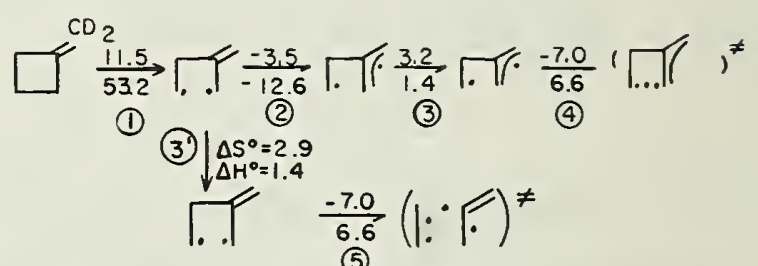

1. Isomerization. $K_{\text {r.p.d. }}=2\left(T \simeq 600^{\circ} \mathrm{K}\right)$

$$
\Delta S_{\text {isom }}^{\ddagger}=5.6 \mathrm{~g} / \mathrm{mole}
$$

$$
\begin{aligned}
& A_{\text {isom }}^{\stackrel{\ddagger}{\ddagger}}=\frac{1}{2} \frac{e h T}{h} e^{\Delta . S^{\ddagger} / R}=10^{14.5} \mathrm{sec}^{-1} \text {; } \\
& A_{\text {obs }}=(\text { not reported }) \\
& E_{\text {isom }}=48.6 \mathrm{kcal} / \mathrm{mole} \text {; } \\
& E_{\text {obs }}=49.5 \mathrm{kcal} / \mathrm{mole}
\end{aligned}
$$

\section{c. Discussion}

Full allylic resonance is achieved in the transition state. Steric inhibition of resonance in ring opening reactions does not seem to occur. The factor of $1 / 2$ in $A^{\ddagger}$ comes from the fact that ring closing can occur one of two ways with equal probability; only one incorporates $d_{2}$ in the ring.

2. Decomposition,-allene formation.

$$
\begin{aligned}
& K_{\text {r.p.d. }}=2\left(T \simeq 700^{\circ} \mathrm{K}\right) \\
& \Delta S_{\text {(allene) }}^{\ddagger}=9.6 \mathrm{~g} / \mathrm{mole} \\
& A_{\text {allene }}^{\ddagger}=\frac{e k T}{h} e^{\Delta . S^{\ddagger} / K}=10^{15.7} \mathrm{sec}^{-1} \\
& A_{\text {obs }}=10^{15.1}, 10^{15.7}, 10^{15.8} \mathrm{sec}^{-1} \\
& E_{\text {allene }}=61.2 \mathrm{kcal} / \mathrm{mole} ; \\
& E_{\text {obs }}=61.5,63.3,63.5 \mathrm{kcal} / \mathrm{mole} .
\end{aligned}
$$

\section{Discussion}

Decomposition cannot occur from the resonance stabilized biradical, since in order to develop the allene $\mathrm{Pi}$ bond, twisting of the conjugated resonance system is required. Hence the activation energy and $A$-factor for allene formation are similar to those for cyclobutane. 


$$
\begin{aligned}
& \left.\Delta X_{\circledast}^{c}=x^{\circ}(\Pi)-x^{\circ}, \square\right) \\
& \Delta S_{(2)}^{\circ}=S^{\circ}\left[-\left(. \mathrm{CH}_{2} \epsilon_{,} \infty\right)_{0.6}+\left(i B_{3 e}\right)_{t}\right] \text {; } \\
& \Delta H_{\text {C }}^{\mathrm{C}}=-E_{\text {res }} \text { (allyl) } \\
& \left\langle\Delta C_{p}^{0}\right\rangle_{(3)}=4.6 \mathrm{~g} / \mathrm{mole} \\
& \left\langle\Delta C_{p}^{\mathrm{c}}\right\rangle_{(3)}=3.4 \mathrm{~g} / \mathrm{mole} \\
& \Delta S_{\oplus}^{c} \quad=\Delta S_{\circledast}^{c}=-S^{c}\left(E t \epsilon_{i} i P r\right)_{6.6 .} \text {. }
\end{aligned}
$$

\subsection{Polycyclic Compounds}

Since the thermodynamic properties of almost all polycyclic compounds have not been experimentally determined, the thermodynamic estimates made in this monograph for the polycyclics are subject to greater uncertainties than are the estimates made for less elegant compounds. However, in view of the reasonably good agreements between the observed kinetic parameters and the calculated parameters (based on transition state estimates and the biradical mechanism) for most of the polycyclic reactions, it would be surprising if more than a few of the thermodynamic estimates were in error by more than $\pm 2 \mathrm{kcal} / \mathrm{mole}$ in $\Delta H_{f}^{\mathrm{c}}$, and $\pm 2 \mathrm{~g} /$ mole in $S^{\circ}$. Nevertheless, since errors of this magnitude are possible, transition state calculations have not been made as accurately as those for the small ring compounds. In particular, heat capacity effects have been ignored (although there is some indication that they may be small), entropy estimates have been made by analogy with the entropies of other cyclic compounds, and enthalpy estimates have been made relative to bond dissociation energies in alkanes. We believe that most transition state estimates given on the data sheets are good to about a factor of 3 in $A$ and $\pm 3 \mathrm{kcal} / \mathrm{mole}$ in $E$.

\subsection{Bicyclo[2.2.0]hexane $\longrightarrow 1,5$-hexadiene}

a. Mechanism

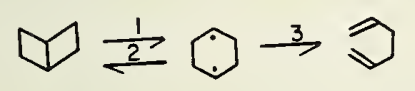

\section{b. Transition State Estimates}

\section{$1 A$-factor}

If one assumes, by analogy with the cyclobutane reaction, that $k_{3} \simeq k_{2}$, then the transition state for the reaction can be pictured as somewhere between the reactant and biradical, $(\hat{1})^{\neq}$. Since the intrinsic entropy of the reactant and cyclohexane is very similar $\left.\left(S_{\text {int }}^{\circ}, \square\right) \simeq 74.0 S_{\text {int }}^{\circ}(\bigcirc) \simeq 72.7\right)$, it is possible to place the entropy of the transition state about $S_{\text {int }}^{\circ}(\bigoplus)^{\ddagger} \simeq 73.4$. Thus with $K_{\text {r.p.d. }}=1$, $\Delta S^{\ddagger} \simeq-0.6$ and $A_{\text {est }}^{\ddagger}=\frac{1}{2} \frac{e k t}{h} e^{\Delta . l^{*} / R} \simeq 10^{13.0} \mathrm{sec}^{-1}$; $A_{\mathrm{obs}}=10^{13.4} \mathrm{sec}^{-1}$.

\section{Activation Energy}

Since the ring strain in $\left(\square_{\mid}\right)$) is known to be close to the sum of the $C_{3}$ and $C_{4}$ ring strains, $E \quad$ strain $\left(D_{1}\right) \simeq 2(26.2)=52.4 \quad \mathrm{kcal} / \mathrm{mole}$. The transition state is close to the boat form of cyclohexane with a strain energy of something like $4 \mathrm{kcal} / \mathrm{mole}$. Therefore, $\Delta H_{1.2}^{\circ} \simeq D H^{\circ}(\mathrm{C}-\mathrm{C})-$ $\Delta E$ strain $=78.7-52.4+4.0=30.3 \mathrm{kcal} / \mathrm{mole}$. From this and other transition state estimates, it appears that to a good approximation all internal ring closing reactions have activation energies of about $8 \mathrm{kcal} / \mathrm{mole}$ (i.e., $E_{3} \simeq 8 \mathrm{kcal} / \mathrm{mole}$ ). Thus, $E_{\text {est }} \simeq 38.3 \mathrm{kcal} / \mathrm{mole} ; E_{\mathrm{obs}}=36.0 \mathrm{kcal} / \mathrm{mole}$.

\subsection{1,3-Dimethyl bicyclo $[1,1,0]$ butane}

a. Mechanism

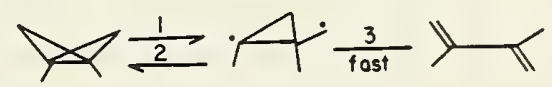

Process (3) must be faster than the decomposition of $((\cup))$ to $2(=)$ since it is much more exothermic, and process (2) is a normal $\mathrm{C}_{3}$ ring closing reaction. Therefore $k_{3} \gg k_{2}$ and the rate determining step is ring opening, step 1.

\section{1. $A$-factor Estimate}

Since $S_{\text {int }}^{\circ}((A))=85.5$, one estimates,

$$
\begin{gathered}
\Delta S_{1,2(\mathrm{int})}^{\circ}=S^{\circ}\left(1 A_{\mathrm{g}}\right)-S^{\circ}((\notin))=85.3-79.8 \\
=5.5 \mathrm{~g} / \mathrm{mole} . \\
\Delta S_{(2)}^{\ddagger}=-(\mathrm{C} \quad \mathrm{C}) 350 \mathrm{~cm}^{-1}=-2.2 \mathrm{~g} / \mathrm{mole}_{K_{\text {r.p.d. }}}=4
\end{gathered}
$$

$\Delta S_{\text {est }}^{\ddagger}=6.1 \mathrm{~g} / \mathrm{mole} ; A_{\text {est }}=10^{14.7} \mathrm{sec}^{-1} ;$

$A_{\mathrm{obs}}=10^{14.45} \mathrm{sec}^{-1}$

\section{Activation Energy Estimate}

The ring strain in the reactant has been measured to be $\left[311 F_{\text {ctrain }}=67.4 \mathrm{kcal} / \mathrm{mole}\right.$, therefore,

$$
\begin{array}{r}
\Delta H_{1,2}^{\circ}=D H^{\circ}(\mathrm{C}-\mathrm{C})-\Delta E_{\text {strain }}=77.3-(67.4 \\
-27.6)=37.5 \mathrm{kcal} / \mathrm{mole} .
\end{array}
$$

Thus, $\quad E_{\mathrm{act}} \simeq \Delta H_{1,2}^{\circ}+E_{2}=37.5+9.3=46.8 \mathrm{kcal} /$ mole. If there is a $4 \mathrm{kcal}$ resonance stabilization in the cyclopropyl biradical (see bicyclopropyl), then the estimated activation energy is lower by this amount, i.e., $E_{\text {act }} \simeq 42.8 \mathrm{kcal} /$ mole; $E_{\text {obs }}=43.3$ $\mathrm{kcal} / \mathrm{mole}$. The alternative mechanism is a concerted reaction breaking two opposing $\mathrm{C}-\mathrm{C}$ bonds at once. It is difficult to account for the $A$-factor on this basis. 


\section{Isomerization Reactions (Other Than Cyclic Compound Reactions)}

The isomerization reactions are of four basic types: (1) cis-trans olefin isomerizations, (2) structural allyl migration isomerizations, known commonly as "Claissen" or "Cope" rearrangements, (3) 1-5 H-migration isomerizations, known as "reverse ene" or simply "ene" reactions, and (4) cyanide isocyanide isomerizations, which are a distinct class by themselves.

\subsection{Cope Rearrangements}

Cope rearrangements are relatively well behaved reactions, not particularly surface sensitive nor subject to free radical chain reaction complications. Their activation energies are in the 30 to $35 \mathrm{kcal} /$ mole range and entropies of activation are negative. In the Cope isomerization, a 1,5-diene rearranges by forming a new sigma bond between the vinyl carbon atoms 1 and 6 with concurrent $\pi$ bond migration and scission of the 3.4 C-C bond. The transition state is six-centered and $A$ factor estimates of reasonable reliability (i.e., within about $\pm 2 \mathrm{~g} /$ mole) can be made by methods similar to those employed for the ester elimination reactions. An $A$-factor calculation is illustrated below.

1,1,6,6-(d-1,5-hexadiene:

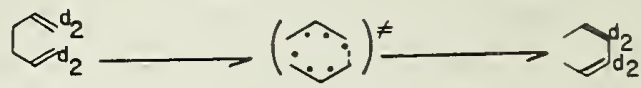

Frequency assignments of the ground state and transition state are shown in table IV-l. (Only those which change in the activation process have been listed.)

TABLE IV-1. Transition state estimates - 1,1,6,6- $\mathrm{d}_{4}-1,5$-hexadiene isomerization

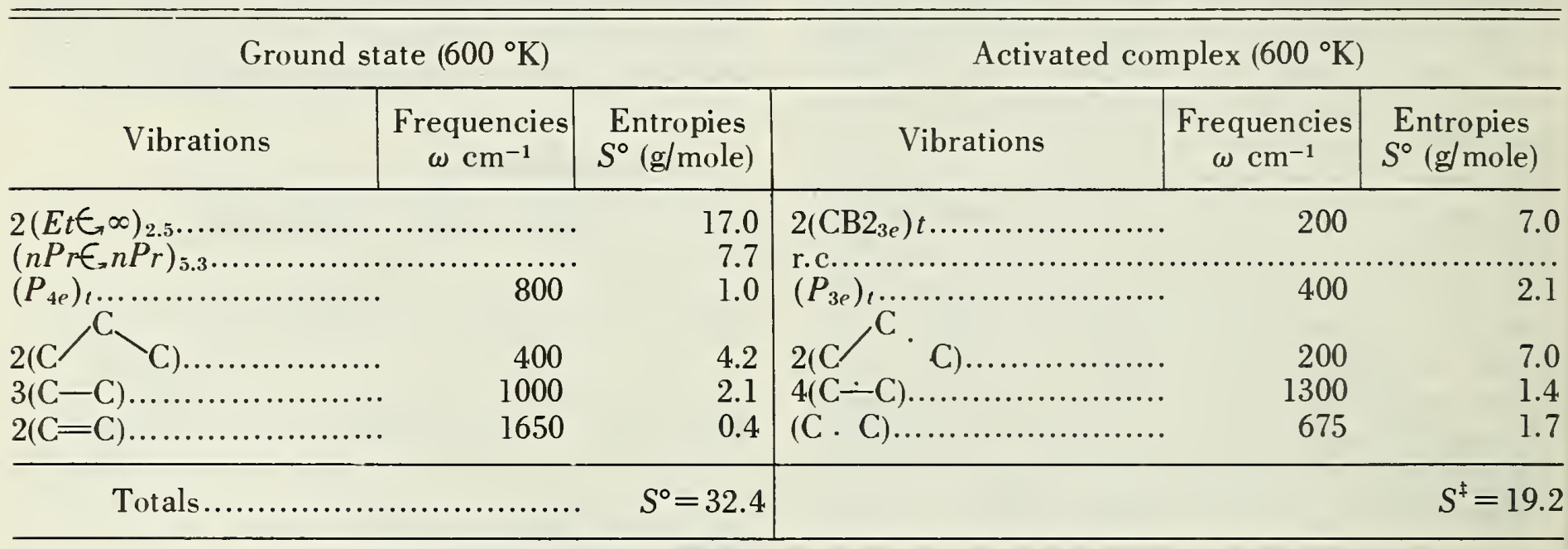

The intrinsic entropy of dctivation (equal to the real activation entropy, since the reaction path degeneracy is unity) is given as,

$$
\begin{aligned}
\Delta S^{\ddagger} & =19.2-32.4=-13.2 \mathrm{~g} / \mathrm{mole} \\
A_{\text {(est) }} & =10^{10.6} \mathrm{sec}^{-1} ; A_{\text {(obs) }}=10^{11.1} \mathrm{sec}^{-1}
\end{aligned}
$$

The kinetics of a number of other Cope rearrangements in solution (results below) have been measured by Foster, Cope, and Daniels [32]. 1-ethyl-propenyl allylmalonitrile $\longrightarrow$ l-ethyl-2-methyl4-pentenylidene-malonitrile $(\log k=10.94-25.78 / \theta$ between $392-413^{\circ} \mathrm{K}$ ); l-cyclohexenyl allyl malonitrile $\rightarrow 2$ allyl cyclohexylidene malonitrile (log $\left.k=10.8-26.16 / \theta, 392-413^{\circ} \mathrm{K}\right)$; ethyl (1,3-dimethyl. l-butenyl)-allylcyanoacetate $\rightarrow$ ethyl(1-methyl-2-isopropyl-4-pentenylidene)-cyanoacetate $(\log k=10.36$ $-28.62 / \theta, 453-473^{\circ} \mathrm{K}$ ).

\section{0. "Ene" Isomerizations}

In an "ene" reaction, a 1,5 hydrogen migration is accompanied either by migration of two $\pi$ bonds (giving an isomerization), or by one $\pi$ bond migration and a bond scission (giving a molecular elimination). Ester elimination reactions are examples of the latter reaction path. The former path ("ene" isomerizations) are similar in their behavior to the Cope rearrangements. They are well behaved experimentally, often proceed toward an equilibrium state in which reactants are still appreciable, and have activation energies in the 30 to $35 \mathrm{kcal} / \mathrm{mole}$ range with sizable (of the order of 10 to $15 \mathrm{~g} /$ mole) negative activation entropies. Transition state estimates seem consistently to predict $A$-factors of up to an order of magnitude higher than those observed experimentally. It is possible that the six-center "ene" 
isomerization transition states are less polar, and therefore tighter, than other "ene" reactions. Substituent effect studies, such as the sigma-rho relations observed for the acetate and benzoate esters by Smith, et al., would be interesting studies for these reactions.

\subsection{Transition State Estimate of the $\boldsymbol{A}$-Factor for an "Ene" Isomerization}

cis-2-methyl penta-1,3-diene

$\longrightarrow$ 4-methyl penta-1,3-diene

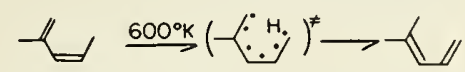

Frequencies of the ground and transition states are detailed in table IV-2.
The reaction path degeneracy is 3 ; therefore the estimated activation entropy is,

$$
\begin{aligned}
& \Delta S^{\ddagger}=14.6-25.3+R \ln 3=-8.5 \mathrm{~g} / \mathrm{mole} \\
& A_{\text {est }}\left(500^{\circ} \mathrm{K}\right)=10^{11.6} \mathrm{sec}^{-1} ; \\
& A_{\text {obs }}=10^{11.2} \mathrm{sec}^{-1} .
\end{aligned}
$$

\subsection{Cis-Trans Isomerizations}

The cis-trans isomerization reactions are particularly difficult reactions to study because they are so sensitive to wall and free radical catalysis. The low Arrhenius parameters of early studies were attributed to nonadiabatic (singlet $\rightarrow$ triplet $\rightarrow$ singlet) reaction paths. However, in every case subsequent studies have shown that the low parameters were a result of heterogeneity or free radical processes.

TABLE IV-2. Transition state estimates-cis-2-methyl penta-1,3-diene isomerization

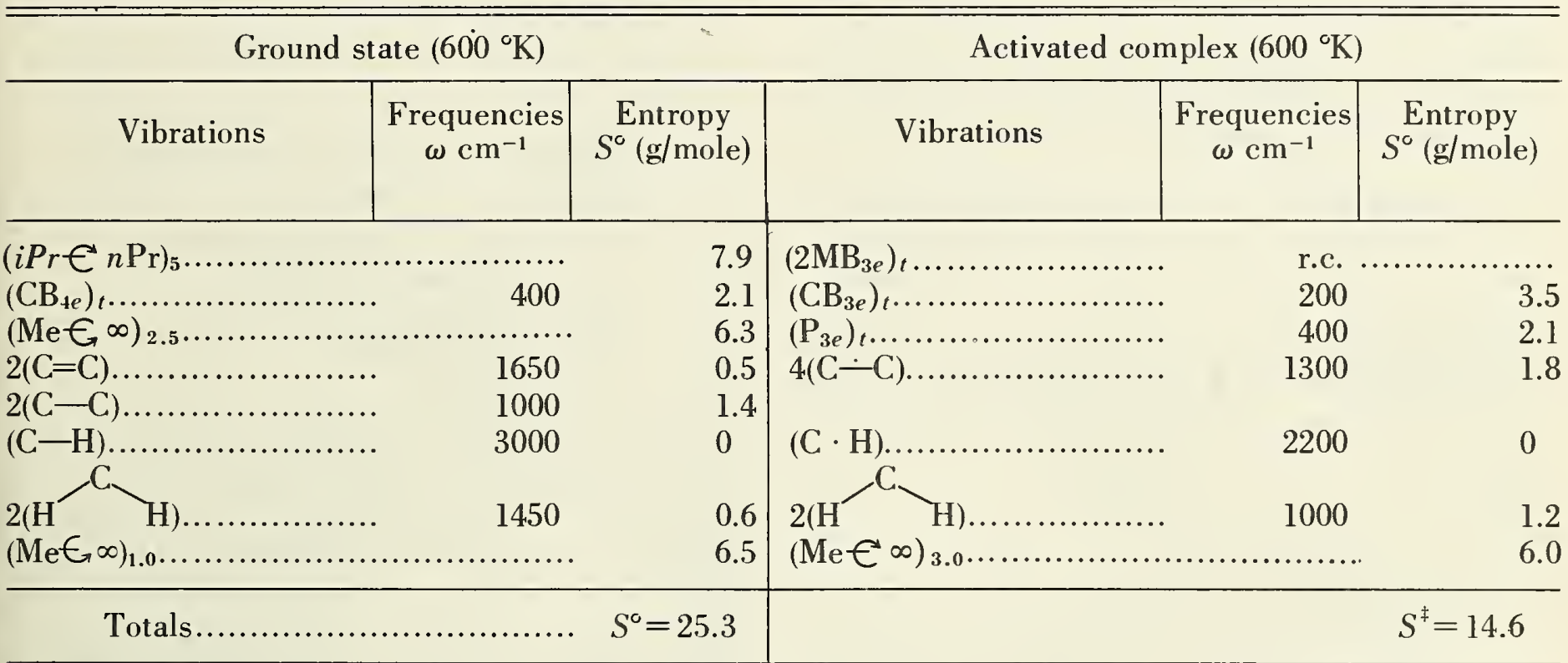

Transition state estimates of the rate constant parameters of the cis-trans isomerizations are in reasonable agreement with most of the data. Estimates of both the activation energy and the Arrhenius $A$-factors have been made. The method is illustrated below for cis-but-2-ene and for isostilbene.

\subsection{Cis-but-2-ene}

a. $A$-factor Estimate

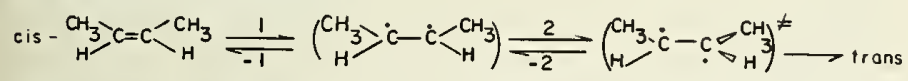

The reaction coordinate is taken as the torsion about the double bond. For calculation purposes, the mechanism is represented in terms of the intermediate formation of a flat biradical, followed by rotation about the remaining $\mathrm{sp}^{2}-\mathrm{sp}^{2}$ single bond with the latter rate determining. The more important frequency changes between the ground and transition states are given in table IV-3.
$\Delta S_{600}^{\ddagger}=+0.4-0.2=+0.2 \mathrm{~g} / \mathrm{mole}$

$A^{\ddagger}=10^{13.6+0.1}=10^{13.7} \mathrm{sec}^{-1} ; A_{\mathrm{obs}}=10^{13.8} \mathrm{sec}^{-1}$

\section{b. Activation Energy Estimate}

The activation energy is taken as the enthalpy of the reaction to the biradical, plus the energy required to rotate about the single bond to the perpendicular conformation. Thus,

$$
\begin{aligned}
& E_{a}=D(\mathrm{C}=\mathrm{C})^{5}+E_{2}(\text { rotation })^{6}=56.4+6=62.4 \\
& \quad \mathrm{kcal} / \mathrm{mole} \\
& E_{\mathrm{obs}}=62.8 \mathrm{kcal} / \mathrm{mole} .
\end{aligned}
$$

\subsection{Isostilbene}

a. $A$-Factor Estimate

cis

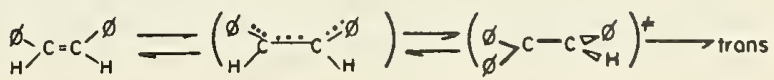


I'ABLE IV-3. Transition state estimates-cis-but-2-ene isomerization

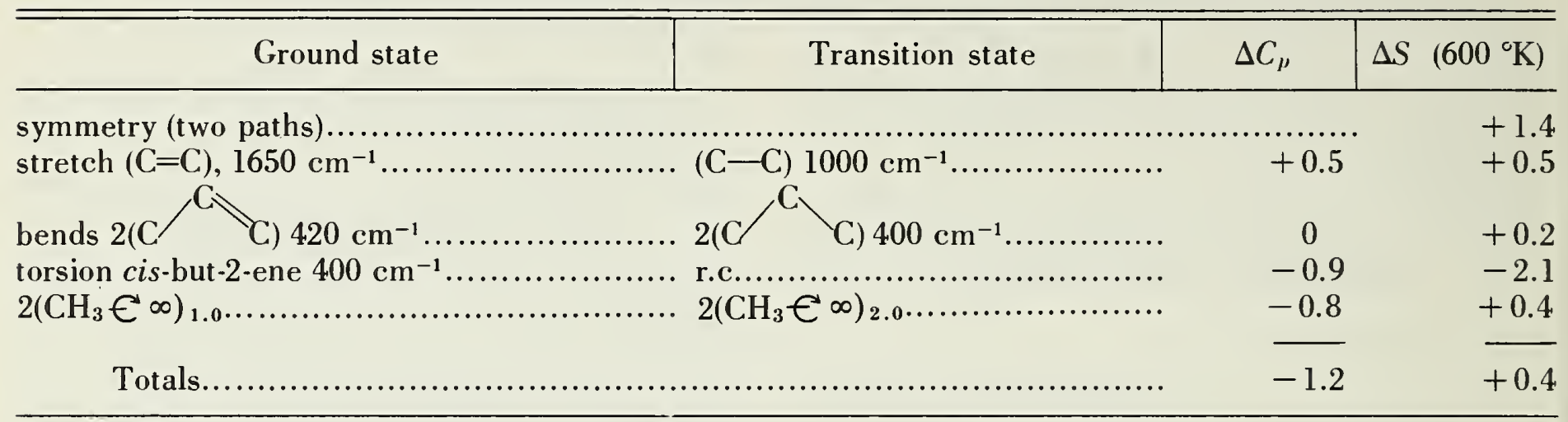

Vibrational frequencies and corresponding entropies for the ground and transition states are detailed in table IV-4.

TABLE IV-4. Transition state estimates-Isostilbene isomerization

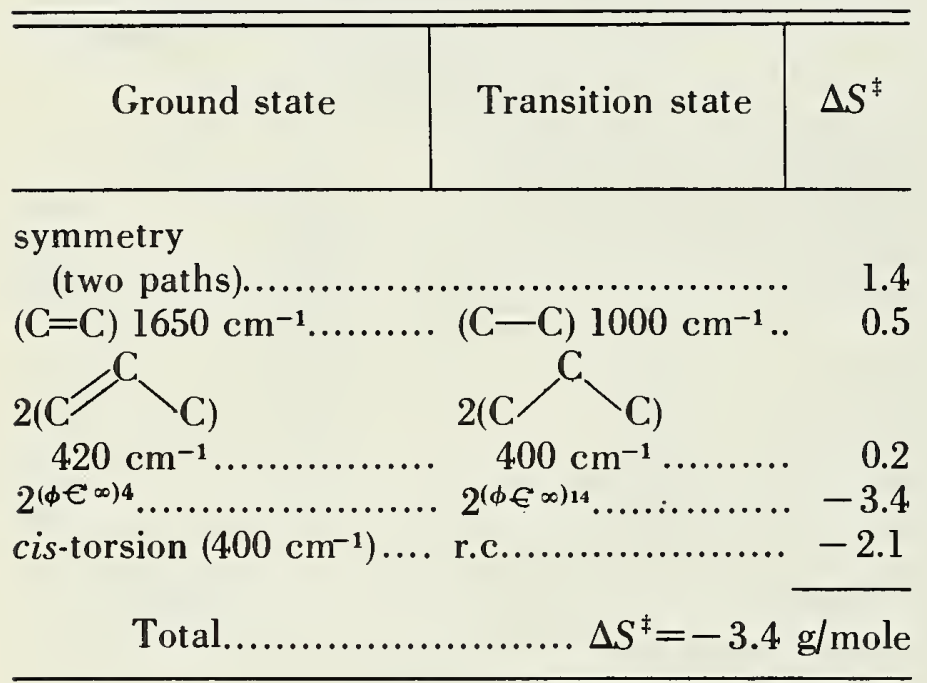

$$
\begin{aligned}
& \Delta S^{\ddagger}=-3.4 \mathrm{~g} / \mathrm{mole} \\
& A_{\text {est }}=10^{12.8} \mathrm{sec}^{-1} ; A_{\mathrm{obs}}=10^{12.7} \mathrm{sec}^{-1}
\end{aligned}
$$

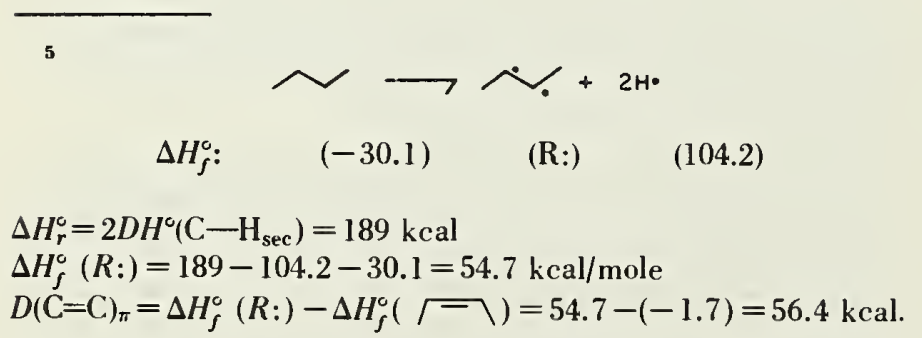

Since heat capacity effects are small, all reaction heats have been equated to those calculated at $T=298^{\circ} \mathrm{K}$.

${ }^{6} E_{2}$ (i.e., the barrier to rotation), is taken as about $6 \mathrm{kcal} / \mathrm{mole}$. This is the barrier for the cis-1,2-dideuterioethene isomerization, calculated in this manner, and has been used consistently for all reactions not involving resonance stabilized biradicals. b. Activation Energy Estimate<smiles>O=CC(O)C(=O)OCC(=O)O</smiles>

$$
\begin{aligned}
& 2 D H^{\circ}(\mathrm{C}-\mathrm{H})^{7}=\Delta H_{R}^{\circ}=163 \mathrm{kcal} / \mathrm{mole} \\
& \Delta H_{f}^{\circ}(R:)=163-104.2+34.3=93 \mathrm{kcal} / \mathrm{mole} \\
& E_{a}=\Delta H_{f}^{\circ}(R:)-\Delta H_{f}^{\circ} \text { (isostilbene) } \\
& \quad \quad+E(\text { barrier to rotation }) \\
& E_{a}=93-58+6=41 \mathrm{kcal} / \mathrm{mole} \\
& E_{\text {obs }}=42.8 \mathrm{kcal} / \mathrm{mole}
\end{aligned}
$$

In actual practice, since the rotational barrier around the $\mathrm{sp}^{2}-\mathrm{sp}^{2}$ bond cannot be known, instead of estimating the activation energies in these reactions, the observed activation energy (when $\left.A_{\text {calc }}=A_{\text {obs }}\right)$, or the corrected activation energy (using $A_{\text {calc }}$ to obtain $E_{\text {corr }}$ ) have been used to calculate rotational barriers. The rotational barriers and resonance energies for the various groups which have been used and have been found to give reasonable agreement with the observed kinetics are listed for each reaction. Further experiments

It is possible to rationalize the origin of this $6 \mathrm{kcal} / \mathrm{mole}$ barrier to rotation, as follows: The isomerization barrier of trans-1,3buta-diene to the cis form is about $4 \mathrm{kcal} / \mathrm{mole}$. This may be thought of in terms of the butadiene resonance, or simply as a resistance of the $s p^{2}-s^{2}$ carbon to torsion. If the latter is accepted, the flat biradical is stabilized by about $4 \mathrm{kcal} / \mathrm{mole}$. An additional steric barrier to rotation of the order of $2-4 \mathrm{kcal}$ may also exist. The iodine atom and NO catalyzed cis-trans isomerization reactions whose rates are rotationally limited, all involve activation energies of this order [33]. Thus, a 6 kcal barrier to rotation for nonresonant biradical species seems quite reasonable.

$$
\begin{aligned}
{ }^{7} \mathrm{DH}(\mathrm{C}-\mathrm{H})= & \mathrm{DH}^{\circ}(\mathrm{C}-\mathrm{H}), \text { as in } n \text {-butane }- \text { Resonance } \\
& =94.5-13.0=81.5 \mathrm{kcal} / \mathrm{mole}
\end{aligned}
$$


are needed to verify or discredit these estimates. It should be noted, however, that the resonance energies proposed are also quite consistent with the resonance energies calculated from transition state "fits" to the bond fission reactions involving corresponding groups (i.e., see also bond fissions), and with the resonance energies obtained from the kinetics of some small ring compound reactions (see cyclobutane derivatives).

For a review of cis,trans isomerization reactions, including solution results, see references $[15,33$, and 34$]$.

\section{Simple Bond Fission Reactions}

\subsection{Characteristics}

Although seemingly appropriate to this monograph, early studies of the decomposition kinetics of hydrocarbons, ethers, aldehydes, and ketones have been omitted. The reason for their omission is that these reactions are extremely complex and the experimental methods employed in their study, prior to about the 1950s (e.g., pressure-time determinations of reaction order, classical wet chemistry product analysis, etc.), did not permit estimation of the individual parameters for the elementary reactions in the mechanism. A reasonably complete review of this literature prior to 1953 has been made by Steacie [35]. With the advent of gasliquid phase partition chromatography, it soon became possible to perform mass balances as a function of time, detect trace quantities of products, and to begin to study the individual steps of the decomposition mechanism. Most of the studies reported here have been made since 1950 .

Bond fission reactions (i.e., the initiation reactions in decompositions) have always been of prime in. terest to kineticists and thermodynamicists. The kinetic literature in this area is extensive although, as will become apparent, not particularly reliable. Interest in bond fission reactions stems principally from the relations presumed valid between the Arrhenius parameters for dissociation and the thermodynamic properties of the free radical products. The most common assumption has been that the activation energy for radical-radical recombination reactions (concentration units) is zero. Often the difference between activation energies of recombination for rate constants measured in concentration units as opposed to the value obtained for rate constants expressed in pressure units (i.e., $E_{c}-E_{p}=R T$ ) has been ignored. In addition, heat capacity effects on the reaction enthalpy as well as on the activation energy for radical recombination have generally not been properly considered. The usual procedure has been to equate the experimental activation energy at the temperature $T$ to the reaction enthalpy at $T=298{ }^{\circ} \mathrm{K}$, and then to calculate the heat of formation of the product free radicals (i.e., $E_{(T)} \simeq \Delta H_{298}^{\circ}$ ). There are clearly some very grave oversimplifications involved in this procedure which could lead to erroneous results even when quite reliable kinetic data are available. Fortunately, it does appear on closer analysis that the assumption $E_{(T)} \simeq \Delta H_{298}^{\circ}$ should not be in error by much more than 1 or $2 R T$. However, for reactions carried out at temperatures in the neighborhood of $1000{ }^{\circ} \mathrm{K}$, an error of $1-2 R T$ will introduce uncertainties of the order of 2 to $4 \mathrm{kcal}$ in the pertinent free radical heats of formation.

In section $\mathrm{I}-4.5$, the thermodynamics of bond fission reactions and their relationship to the Arrhenius parameters of bond fission have been presented. The results are based on two assumptions: (1) that transition state theory is valid; (2) that the activation energy of radical recombination is zero at $0^{\circ} \mathrm{K}\left(\Delta E_{o}^{\ddagger}(\mathrm{rec})=0\right)$. Thus we have for the decomposition Arrhenius parameters:

$$
\begin{gathered}
E=\Delta H_{T}^{\circ}+T\left\langle\Delta C_{V^{\prime}(\mathrm{rec})}^{\ddagger}\right\rangle \\
4\left(\mathrm{sec}^{-1}\right)=\frac{k_{\text {rec }}(1 / \text { mole-sec })}{(R T)} \times \exp \left(\frac{\left\langle\Delta C_{V(\mathrm{rec})}^{\ddagger}\right\rangle}{R}\right) \\
\times \exp \left(\frac{\Delta S_{T}^{\circ}}{R}\right) \cdot(\mathrm{V}-1.0-1)
\end{gathered}
$$

The few radical-radical recombination rate constants which have been measured have generally been reported in concentration units. This is the reason for the above form of the $A$-factor equation. It is clear, from eq $(\mathrm{V}-1.0-1)$, that the activation energy of dissociation cannot be properly equated to the reaction enthalpy at $298^{\circ} \mathrm{K}$, but rather depends critically on the average heat capacity change for the recombination reaction $\left\langle\Delta C_{i}^{\ddagger}\right\rangle$. Only if both are zero is the usual assumption valid. In principle the reaction heat capacity change can be determined readily by direct experimental measurements or by statistical thermodynamic calculations. It therefore may be considered known or accessible. How ever, $\left\langle\Delta \mathrm{C}_{\mathrm{i}}^{\ddagger}(\mathrm{rec})\right\rangle$ can be obtained only by inference 
from the kinetics. If nature behaves in a particularly perverse and erratic manner, then each system could be unique and it would be quite impossible to generalize in any accurate way about the kineticthermodynamic relations of bond fission reactions.

If one assumes that nature is not so perverse, then one is forced to conclude that much, if not most, of bond fission rate data is not very reliable. This is, in fact, our present assessment of the data. This is not unreasonable since the experimental complexities in these systems render many of the results highly suspect. In addition, in those systems (hydrocarbons) for which apparently fairly reliable kinetic data on the reactions in both directions exist (i.e., dissociation and recombination), there are very serious discrepancies between the thermodynamics and kinetics which can only be resolved by making some rather questionable assumptions about the nature of the free radical products.

In the following sections we discuss these problems in some detail. Our purpose is to indicate the current discrepancies between the data and theory. Further studies will be needed before the problems can be satisfactorily resolved.

\subsection{Reliability of the Experimental Data}

A perusal of reported Arrhenius parameters for the same reaction from different laboratories quickly reveals the serious discrepancies which exist in the results. Large differences in the param. eters (i.e., up to three or four orders of magnitude in $A$ and up to or in excess of $10 \mathrm{kcal} /$ mole in $E$ ) are the rule rather than the exception. As examples, the extreme range of reported $A$ and $E$ values for the decompositions of ethane and azomethane are respectively, $10^{14.7-17.45} \mathrm{sec}^{-1}, \quad 79.3-91.7$ $\mathrm{kcal} / \mathrm{mole} ; 10^{14.2-17.3}, 46-55.4 \mathrm{kcal} / \mathrm{mole}$. The trend in results with time (presumably later studies are more reliable) is toward the higher parameters. The poor precision and lack of consistency between studies clearly indicate that the reactions are complex and that their study poses many experimental and interpretative problems.

There are basically two classes of simple bond fission reactions: those which occur with no or with negligible chain-stimulated decomposition; those which have long chain decomposition pathways. In the former class, which is small and in. cludes some of the azo and peroxide reactions, the rate-determining step for the reaction is bond rupture. This is the reaction of interest. Results on these reactions are generally fairly reliable. The most serious complicating feature encountered is surface catalysis, which can be estimated or eliminated experimentally. The vast majority of bond fission reactions involve free radical chain processes. These have been studied by one of two techniques. Both attempt to isolate the initiation (bond rupture) reaction from all subsequent chain processes.

The most common technique employed has been to carry out the decomposition in the presence of various free radical scavenwers. Toluene, propylene, aniline, and cyclopentene are a few of the substances employed for this purpose. It is assumed that the free radical products of the bond fission reactions react exclusively and metathetically with these additives (via $\mathrm{H}-\mathrm{atom}$ abstraction) to produce resonance-stabilized inert free radicals. It is further assumed that the stabilized radicals formed are unable to stimulate chain decomposition of the reactant and that their principal fate is radicalradical termination. In this case (i.e., complete chain suppression) the observed kinetic parameters will correspond to those of the bond fission reaction of interest. The same assumptions concerning the effectiveness of radical traps in suppressing chain reactions have been made in studying molecular elimination reactions. For molecular eliminations (which have lower activation energies and proceed at lower temperatures than bond fission reactions) it was concluded that the assumptions were probably valid. However, there is considerable evidence to show that the assumption of complete chain suppression in the presence of inhibitors is not valid in many bond fission systems. For example, McNesby and Gordon [36] showed that allyl radicals abstract hydrogen efficiently at temperatures above $500{ }^{\circ} \mathrm{K}$, and that they are capable of continuing chain processes. Bryce and Hardiman [37] confirmed this result, showing that allyl radicals sensitized the chain decomposition of $n$-pentane at $T \geqslant 400{ }^{\circ} \mathrm{C}$. The dual role of $\mathrm{NO}$ as a radical trap and as a radical chain sensitizer has also been demonstrated and discussed at length [38]. Finally, the importance of benzyl radical $\mathrm{H}$-abstraction reactions in the free radical chain decomposition of reactants in toluene carrier experiments has been demonstrated [39].

It seems clear, therefore, that the Arrhenius parameters obtained for reactions capable of chain decomposition must be viewed with caution when studied by the inhibitor technique. This is particularly true when the reaction of interest takes place at temperatures above $400{ }^{\circ} \mathrm{C}$ and forms the same products in the presence or absence of the inhibitor. The results of the toluene carrier technique are examples in point. Arrhenius parameters resulting from these studies are usually too low and the existence of some chain decomposition seems evident. Unfortunately, activation energies from these studies have historically provided erro- 
neously low estimates for many important free radicals. ${ }^{8}$

A second technique more recently used to give a direct measure of the kinetics of bond fission reactions uses the single pulse shock tube. In such studies the reactant and a comparative rate standard (i.e., a molecule whose unimolecular decomposition rate constant parameters are well known) at very low concentration in an inert gas medium are shocked to reaction temperatures. The reaction mixture is then quenched in a period short relative to the lifetime of the free radical chain processes of decomposition and also short relative to radical diffusion times to the walls. The observed kinetic parameters should therefore correspond to those of the homogeneous gas phase initiation (bond fission) reaction. Activation energies determined by the single pulse shock tube technique have all been in very reasonable agreement with estimated reaction enthalpies. However, the $A$-factors obtained in hydrocarbon systems predict anomalous values for the entropies of the free radical products and therefore must also be accepted with some caution. (See V-6.0.) Application of the S.P.Sh. technique to bond fission reactions has thus far been limited to a very few systems. However, the method does appear to have great promise.

\subsection{Kinetic and Thermodynamic Complica- tions in Radical-Radical Recombination Reactions}

In section I-4.3-8, it was shown that if the activation energy for radical-radical recombination at $0{ }^{\circ} \mathrm{K}$ was set equal to zero, the experimental activation energy for recombination when the rate constant is expressed in pressure units is given by $E_{p(\mathrm{rec})}=T\left\langle\Delta C_{l^{\prime}}^{\ddagger}\right\rangle_{(\mathrm{rec})}$. $^{9}$

The usual assumption of a zero activation energy for recombination is equivalent to setting $\left\langle\Delta C_{1}^{\ddagger}\right\rangle_{\text {rec }}$ $=0$. By applying simple equipartition of energy principles to the recombination reaction, it is possible to get some idea of the limiting values which $\left\langle\Delta C_{l(\text { rec })}^{\ddagger}\right\rangle$ can assume.

\footnotetext{
"Most of the heats of formation of free radicals used in this monograph have been determined either by competitive bromination techniques or by iodination experiments (see refs. [7] and [40]). The mechanism of these processes is now well understood, the errors are small, and the internal agreement between the two experimental systems is excellent. There can be little doubt that the values so obtained are the best ones presently available.

${ }^{9}\left\langle\Delta C_{V}^{\ddagger}\right\rangle$ represents the average heat capacity of activation over the temperature range of 0 to $T^{\circ} \mathrm{K}$. More exactly,
}

$$
T\left\langle\Delta C_{\ddagger}^{\ddagger}\right\rangle=\int_{0}^{T} \Delta C \ddagger d T .
$$

Case I-Simple fission into an atom + radical:

$$
R-X \rightleftharpoons(R---X)^{\ddagger} \rightleftharpoons R+X \text {. }
$$

The three additional translational degrees of freedom of the products originate from the reaction coordinate $(R-X)$ stretch, and the two bending modes of $X$ relative to the $R$ skeleton. The two extreme values for $\left\langle\Delta C_{i}^{\ddagger}\right\rangle$ then are:

a. $X$-bends very loose in the transition state $(h v \ll h T),\left\langle\Delta \mathrm{C}_{1}^{\ddagger}\right.$ rec $\rangle=2 R-\frac{3}{2} R=-\frac{1}{2} R$

b. $X$-bends very tight in the transition state $(h v \gg h T)\left\langle\Delta \mathrm{C}_{\mathrm{r} \text { rec }}\right\rangle=\frac{3}{2} R$

Of course one must also consider other vibrational and internal rotational changes which may occur between the free radicals and the transition state. The most serious of these would be changes in internal rotational barriers. However, following Hammond's thermic postulate [4.1], (which places the geometry of a very low activation energy, highly exothermic reaction close to the geometry of the reactants) it would seem reasonable to assume that such changes are small and may be ignored. Since one would expect that stretching of the reaction coordinate bond would result in some considerable loosening of the atom bending modes, condition (a) would seem more reasonable (i.e., $\left\langle\Delta \mathrm{C}_{V(\mathrm{rec})}^{\ddagger}\right\rangle=-\frac{1}{2} R$ ). Case II-Dissociation into two complex radical fragments:

$$
R-R^{1} \rightleftharpoons\left(R--R^{1}\right)^{\ddagger} \rightleftharpoons R+R^{1} .
$$

The six additional external degrees of freedom in the product radicals (i.e., three translations and three rotations) convert mainly to the $\left(R-R^{1}\right)$ stretch, the $\left(R \subseteq R^{1}\right)$ internal rotation, and the four rocking modes of $R$ and $R^{1}$ relative to each other in the transition state. The extreme values of $\Delta C_{V(\text { rec })}^{\ddagger}$ then are:

a. When the four rocks become low-frequency vibrations in the transition state,

$$
\Delta \mathrm{C}_{V(\mathrm{rec})}^{*}=\frac{9 R}{2}-3 R=\frac{3 R}{2} .
$$

b. When the four rocks have negligible restraining force and transform to rotations in the transition state, $\Delta C_{V(\mathrm{rec})}^{ \pm}=5 R / 2-3 R=-R / 2$.

Again, by Hammond's thermic postulate, the latter condition is probably the more probable case. Also, by the same argument, barriers to group rotations in the radicals and transition state should probably be equivalent. Thus, $\Delta C_{1}^{\ddagger}(\mathrm{rec})=-R / 2$.

Although heat capacities of activation within $R / 2$ of zero are favored here, it is clear that values differing from zero by up to $3 / 2 R$ are possible. 
Also, the exact relation between the bond fission reaction activation energy and the reaction enthalpy is not currently known and need not be the same for all reactions.

It is interesting to note that an extension of the Benson-Fueno [42] theory of atom recombination to a loosely coupled transition state in which free rotation still exists for recombining radicals yields,

$$
E_{\mathrm{rec}}=-\frac{1}{2} R T \text { and } \Delta C_{V(\mathrm{rec})}^{\ddagger}-\frac{1}{2} R,
$$

in agreement with case (b) above (standard states, 1 mole/liter).

\subsection{Method of Analysis}

Since the uncertainties associated with $\Delta C_{V(\mathrm{rec})}^{\ddagger}$ are not apt to be resolved soon, ${ }^{10}$ it seemed best at this time to follow convention and assign $\Delta C_{i(\mathrm{rec})}^{*}=0$ for all reactions. As previously indicated, this assumption is probably good to about $\pm \frac{1}{2} R$. It follows from $(I-4.5-4)$ and $(I-4.5-7)$ that for decomposition,

$$
E=\Delta H_{T}^{\circ}
$$

and

$$
A_{f}=\left(k_{c} / R T\right) e^{\frac{\Delta S_{T}^{o}}{R}}
$$

Agreement between the observed activation energy and the estimated reaction enthalpy at the mean reaction temperature was the single most important criterion used in evaluating the Arrhenius parameters reported for a reaction. This procedure was followed for all reactions whose product free radical heats of formation were reasonably well known. Since estimated reaction heat capacities both at $298{ }^{\circ} \mathrm{K}$ and the mean reaction temperature are probably reliable to about $\pm 1.0 \mathrm{~g} /$ mole and the usual errors in the free radical heats of formation are of the order of $0.5-1 \mathrm{kcal} / \mathrm{mole}$, the errors associated with the estimated activation energies (or reaction enthalpies at $T$ ) are probably not greater than \pm 2 or $3 \mathrm{kcal} / \mathrm{mole}$.

Often the estimated activation energy of a reaction indicated that the reported Arrhenius parameters were much too low to be reasonable. If the reported rate constants for these reactions were thought to be reliable, preferred "corrected" Arrhenius parameters were calculated by scaling the "low" parameters to the "corrected" activation energy.

\footnotetext{
${ }^{10}$ The rotating sector technique used for determining the absolute rates of radical-radical recombination is not presently accurate enough to measure very small activation energies (i.e., $0-2 \mathrm{kcal} / \mathrm{mole}$ ).
}

In all reactions, rate constants for radical-radical recombinations have been calculated from the estimated reaction entropies $[3-\mathrm{VI}]$ and the preferred $A$-factors. This was judged to be a particularly interesting thing to do, since a wealth of data on radical-radical recombination rate constants could determine what, if any, correlations exist between these rates and the geometry, resonance, and size of the recombining radicals. ${ }^{11}$ If the estimates of the error limits for the activation energies are valid, the recombination rate constants estimated in this way should be good to about a factor of five (or $0.7 \log _{10}$ units). The calculated recombination rate constants (see section V-9.0) do suggest some correlation between radical structure and the magnitude of the recombination rate constants. These are shown in table $\mathrm{V}-1$.

TABLE V-1. Radical-radical recombination rates

\begin{tabular}{l|c}
\hline \hline \multicolumn{1}{c|}{ Type of recombination } & Range of values ${ }^{\mathrm{a}}$ \\
\hline & \\
atom-radical & $k_{\mathrm{rec}}=10^{10.8 \pm 0.5} \mathrm{l} / \mathrm{mole}-\mathrm{sec}$ \\
diatomic radical-radical & $k_{\mathrm{rec}}=10^{10.0 \pm 0.5} \mathrm{l} / \mathrm{mole}-\mathrm{sec}$ \\
(alkyl) radical-radical & $k_{\mathrm{rec}}=10^{9.8 \pm 0.5} \mathrm{l} / \mathrm{mole-sec}$ \\
(oxy) radical-radical & $k_{\mathrm{rec}}=10^{9.1 \pm 0.5} \mathrm{l} / \mathrm{mole-sec}$ \\
(resonance stabilized) & $k_{\mathrm{rec}}=10^{8.4 \pm 0.5} \mathrm{l} / \mathrm{mole-sec}$ \\
& \\
\hline
\end{tabular}

a The error limits do not include uncertainties in rotational barriers in radicals or in activation energies of recombination. The latter two effects could add an additional uncertainty of perhaps another power of 10 in $k_{\text {rec }}$ (See sections $V-6.0$ and $\mathrm{V}-6.1$.

Small radicals tend to have values on the high ends of the ranges and the more complex radicals tend to have lower values. Thus, although not exceptionally important, steric effects do seem to play a minor role in the recombination process. It appears that resonance stabilization in the radicals tends to reduce the rate constants for recombination by about a factor of five.

The calculated recombination rate constants of radicals produced in the amine and hydrazine decompositions are about two orders of magnitude lower than the above (table $\mathrm{V}-9$ ). This could be a real effect peculiar to (RN.) radicals or it could mean that the Arrhenius parameters reported for

"Such generalizations are very common in the literature. The most unusual assumption is that all radicals recombine with rate constants of about

$$
h_{c}=10^{10} \mathrm{l} / \text { mole-sec. }
$$


these bond fission reactions are low. In the past the latter alternative has generally turned out to be correct. If the Arrhenius parameters are low, then the heats of formation currently accepted for the amine and anilino radicals are also too low (i.e., by about 4-5 kcal/mole) (see section $\mathrm{V}-8.0$ ).

\subsection{Illustrations of Analysis Methods}

The general method of analysis used for the bond fission reactions is illustrated below for three decomposition reactions: ethane, benzyl bromide, and azomethane.

a. Ethane Decomposition

$$
\mathrm{C}_{2} \mathrm{H}_{6} \rightarrow 2 \mathrm{CH}_{3} . \quad \bar{T}=855^{\circ} \mathrm{K} .
$$

Reaction thermodynamics are given in table $\mathrm{V}-2$.

TABLE V-2. Ethane deromposition thermodynamics

\begin{tabular}{l|r|r|r|l}
\hline \hline & $\mathrm{C}_{2} \mathrm{H}_{6}$ & $\mathrm{CH}_{3}$ & $X_{298}^{\circ}$ & $X_{T}^{\circ}=855^{\circ} \mathrm{K}$ \\
\hline & & & & \\
$S^{\circ}(\mathrm{g} / \mathrm{mole})$ & 54.9 & 46.4 & 37.9 & 40.0 \\
$\Delta \mathrm{H}_{f}^{\circ}(\mathrm{kcal} / \mathrm{mole})$ & -20.2 & 34.0 & 88.2 & 89.5 \\
$C_{p}^{\circ}(\mathrm{g} / \mathrm{mole})$ & 12.6 & 8.3 & 4.0 & \\
$C_{p(1000)}^{\circ}(\mathrm{g} / \mathrm{mole})$ & 29.6 & 14.4 & -0.8 & $\langle 2.0\rangle_{299 \times-\times .5}$ \\
\hline
\end{tabular}

The heats of formation for all pertinent species are well known. Entropies and heat capacities have been obtained from group additivities and from molecular thermodynamic calculations $[3,43]$.

Experimental parameters are (see data sheet for references):

$\begin{array}{cccccl}\log A: & 14.7 & 15.45 & 16.0 & 16.37 & 17.47 \\ E: & 79.3 & 87 & 86 & 88.0 & 91.74 \\ \log k: & 5.58 & 6.80 & 6.00 & 6.14 & 5.99\end{array}$

From thermochemistry we estimate at $855^{\circ} \mathrm{K}$ an activation energy of $89.5 \mathrm{kcal} / \mathrm{mole}$, which is within $R T$ of the latter two sets of parameters. Scaling the $A$-factor to the estimated activation energy gives

$$
\text { Preferred: } \log k=16.85-89.5 / \theta=-6.04 \text {. }
$$

From equation I-4.5-7, the reaction entropy at $855^{\circ} \mathrm{K}$, and the adjusted $A$-factor for decomposition, one can calculate a rate constant for methyl-methyl recombination of,

$$
k_{\text {rec }}(1 / \text { mole-sec })=R T(A) e^{-\Delta \varsigma^{\circ} / K}=10^{9.9} \mathrm{l} / \text { mole-sec. }
$$

The experimental rate constant for recombination determined at about $400{ }^{\circ} \mathrm{K}$ has been measured at values between $10^{10.3}$ and $10^{10.6} \mathrm{l} /$ mole-sec $[44,45$,
46]. The difference between the experimental and calculated recombination rate constants is about a factor of three, which is within experimental error and is regarded here as a reasonably gond agreement.

b. Benzyl-bromide

$$
\varnothing \mathrm{CH}_{2} \mathrm{Br} \rightarrow \emptyset \mathrm{CH}_{2} \cdot+\mathrm{Br} \cdot
$$

Heats of formation of all species are again known with reasonable certainty. Entropies and heat capacities are also believed to be fairly accurate. The thermodynamics of the reaction are shown in table $\mathrm{V}-3$.

The thermodynamics predict an activation energy of $56.7 \mathrm{kcal} / \mathrm{mole}$. The reported Arrhenius parameters are $\log k=13.0-50.5 / \theta$, which are certainly too low. Since the rate constants are probably representative of the decomposition, preferred parameters have been obtained by scaling to the thermodynamic activation energy.

TавцE V-3. Benzyl bromide decomposition thermodynamics

\begin{tabular}{l|r|r|r|r|c}
\hline \hline & $\begin{array}{c}\emptyset \mathrm{CH}_{2} \\
\mathrm{Br}\end{array}$ & $\varnothing \mathrm{CH}:$ & $\mathrm{Br}$. & $\Delta X_{298}^{\circ}$ & $\Delta X_{820}^{\circ}$ \\
\hline$S_{(298)}^{\circ}$ & 88.4 & 75.3 & 41.8 & 28.7 & 30.6 \\
$\Delta H^{\circ}(298)$ & 16.0 & 45.0 & 26.7 & 55.7 & 56.7 \\
$C_{p(298)}^{\circ}$ & 28.2 & 25.6 & 5.0 & 2.4 & \\
$C_{\mu(1000)}^{\circ}$ & 64.5 & 60.6 & 5.0 & 1.1 & $\langle 1.9\rangle_{298-820^{\circ} \mathrm{K}}$ \\
\hline
\end{tabular}

Preferred: $\log k=14.65-56.7 / \theta$

From eq I-4.5-7, the reaction entropy at $820^{\circ} \mathrm{K}$, and the adjusted $A$-factor for decomposition, one can calculate the rate constant for the benzylradical-bromine atom recombination.

$$
k_{\text {rec }}=(R T)(A) e^{-\Delta S^{\circ} / R}=10^{9.62} 1 / \text { mole-sec. }
$$

The kinetic theory collision frequency for bromine atoms and benzyl radicals, using $\sigma_{1,2} \simeq 4.7 \mathrm{~A}^{\circ}$, gives a second order rate constant of $Z=10^{11.35}$ $1 /$ mole-sec. An estimation of the $A$-factor for recombination from this value can be made from the following considerations. Statistically, only one in four collisions leads to the (attractive) singlet ground state (i.e., three out of four collisions form a repulsive triplet state and therefore do not lead to recombination). Empirically our results seen to 
show that resonance in the product radicals infers a "tighter" transition state and $A$-factors of recombination seem to be lower than "normal" for resonance stabilized radicals by about a factor of five. This might be considered to be a resonance induced steric factor. Thus one can predict, $A_{\text {rec }} \simeq 1 / 20 \times 10^{11.35} \simeq 10^{10.0} 1 /$ mole-sec. It has been shown (I-4.3-3) that for a recombination reaction in concentration units, the experimental activation energy should be, $\left[E_{c}=\Delta E_{T}^{\ddagger}+R T\right]$. When $\Delta E_{0}^{ \pm}=0$ and $\left\langle\Delta C_{I^{\prime}(\mathrm{rec})}^{\ddagger}=0\right.$, this gives $E_{c}=R T$. Thus our estimated recombination rate constant from collision theory is $k_{\text {rec }}=10^{10.0} \times e^{-1}=10^{9.57} \quad 1 /$ mole-sec. Although certainly fortuitous, the excellent agreement between the two calculated recombination rate constants is probably significant. First it tends to support the preferred Arrhenius parameters obtained by adjusting the reported values to the thermodynamic estimates of the activation energy, and second, it tends to support the usual assumption of high efficiency for radical-radical recombination reactions (i.e., that recombination rates may be estimated from kinetic theory collision frequencies).

\section{c. Azomethane $\mathrm{CH}_{3} \mathrm{~N}=\mathrm{NCH}_{3} \rightarrow \mathrm{CH}_{3} \mathrm{~N}=\mathrm{N}$.}

The heat of formation of the $\left(\mathrm{CH}_{3} \mathrm{~N}=\mathrm{N} \cdot\right)$ radical has not been measured by other methods, therefore the azomethane reaction is representative of reactions evaluated in terms of estimated $A$-factors of dissociation. The estimated reaction thermodynamics are shown in table $\mathrm{V}-4$.

TABLE V-4. Azomethane decomposition thermodynamics

\begin{tabular}{l|r|r|r|r|c}
\hline \hline & $\left(\mathrm{CH}_{3,} \mathrm{~N}\right)_{2}$ & $\left(\mathrm{CH}_{3}\right)$ & $\left(\mathrm{N}=\mathrm{NCH}_{3}\right)$ & $\Delta X_{298}^{\circ}$ & $\Delta X_{5,518}^{\circ}{ }^{\circ} \mathrm{K}$ \\
\hline$S_{(298)}^{\circ}$ & 71.0 & 46.4 & 64.2 & 39.6 & 40.0 \\
$\Delta H_{(298)}^{\circ}$ & 43.8 & 34.0 & 62.3 & 52.7 & 52.7 \\
$C_{(298)}^{\circ}$ & 20.1 & 8.3 & 4.0 & 1.9 & \\
$C_{p(800)}^{\circ}$ & 35.9 & 12.7 & 22.0 & 1.6 & $\langle 0.6\rangle_{298-550}$ \\
\hline
\end{tabular}

Experimental parameters are:

\begin{tabular}{rlllllll}
\multicolumn{10}{c}{ (see data sheet for references) } \\
$\log A:$ & 14.2 & 15.7 & 16.5 & 17.3 & 14.5 & 15.9 & 16.5 \\
$E:$ & 46 & 51.2 & 53.3 & 55.4 & 49.4 & 50.2 & 52.5
\end{tabular}

A recombination rate constant of the order of the methyl-methyl recombination should be good to about a factor of 2 or 3 . Thus we assume $k_{\text {rec }}=$ $10^{10.0 \pm 0.5} 1 /$ mole-sec. From eq $\mathrm{I}-4.5-7$ and the reaction entropy at $550^{\circ} \mathrm{K}$, one calculates

$$
A_{f}=\frac{k(c)}{R T} e^{\Delta S O / R}=10^{17.1 \pm 0.5} \mathrm{sec}^{-1}
$$

From the thermochemistry one obtains

$$
E_{\text {act(est) }}=52.7 \mathrm{kcal} \text {. }
$$

These estimates are in good agrecment with most of the data. Heats of formation of azomethane and the product radical are those obtained from the azo and amine data analysis (see $\mathrm{V}-7.0,8.0$ ).

\subsection{High and Low A-Factors in Bond Fission Reactions}

Most of the early kinetic studies of bond fission reactions reported $A$-factors in the range of $10^{13}$ $\mathrm{sec}^{-1}$. These values were consistent with the RiceRamsperger-Kassel theory of unimolecular reactions (which stated that all $A$-factors for such reactions should be in the range of molecular vibration frequencies) and were therefore termed "normal". Such confidence was placed in the constancy of "normal" frequency factors, that some experimentors were content to measure unimolecular reaction rate constants at one temperature, assume $A=10^{13} \mathrm{sec}^{-1}$, and then calculate the reaction activation energy.

As more data were accumulated, an increasing number of "abnormal" high-frequency factor reactions were found. Originally it appeared that the activation energies for many of these reactions were higher (e.g., mercury alkyls) or were lower (e.g., azoalkanes) than any single bond dissociation energy in the molecule. (This conclusion was usually based on erroneous values of the pertinent free radical heats of formation.) Simultaneous bond rupture of two or more bonds was therefore considered likely. A number of "multiple critical oscillator theories," which essentially formulated theoretical $A$-factor expressions for simultaneous multiple bond rupture, were proposed to explain the high $A$-factors $[47,48,49]$. As more reliable heat of formation data became available, it soon became apparent that the activation energies of most of these anomalous reactions were not really anomalous, but instead, were quite close to the bond dissociation energies of the weakest bonds. In addition, estimates of radical entropies and dissociation reaction entropies, in conjunction with reasonable assumptions about the rate constants of recombination, soon indicated that high $A$ factors (i.e., $10^{15-17} \mathrm{sec}^{-1}$ ) should be the rule rather than the exception $[27,50]$. This certainly is supported by the findings of this monograph. The more recent experimental results in all systems invariably and consistently support higher Arrhenius 
TABLE V-5. Hexamethylethane decomposition thermodynamics

\begin{tabular}{|c|c|c|c|c|c|c|c|c|c|c|}
\hline & \multicolumn{10}{|c|}{ hexamethylethane $\left(\left(\mathrm{CH}_{3}\right)_{3} \mathrm{C}-\mathrm{C}\left(\mathrm{CH}_{3}\right)_{3}\right) \stackrel{T=1050^{\circ} \mathrm{K}}{\longrightarrow} 2 t$-butyl $\left(\left(\mathrm{CH}_{3}\right)_{3} \mathrm{C} \cdot\right)$} \\
\hline & \multirow{2}{*}{ HME } & \multicolumn{3}{|c|}{$(t-\mathrm{Bu})$} & \multicolumn{2}{|c|}{$V=0$} & \multicolumn{2}{|c|}{$V=4$} & \multicolumn{2}{|c|}{$V=8$} \\
\hline & & $V=0$ & $V=4$ & $V=8$ & $\Delta X_{2 ! 18}^{\circ}$ & $\Delta X_{T}^{\circ}$ & $\Delta X_{29 \times}^{\circ}$ & $\Delta X_{r}^{\circ}$ & $\Delta X_{298}^{\circ}$ & $\Delta X_{T}^{\circ}$ \\
\hline$S_{298}^{\circ}$ & 93.2 & 75.4 & 70.3 & 67.6 & 57.6 & 47.4 & 47.4 & 44.7 & 42.0 & 40.1 \\
\hline$\Delta H_{j(298)}^{\circ}$ & -54.7 & 6.8 & 6.8 & 6.8 & 68.3 & 62.2 & 68.3 & 66.7 & 68.3 & 67.2 \\
\hline$C_{p(298)}^{\circ}$ & 45.9 & 18.7 & 22.6 & 21.7 & -8.5 & $(-82)$ & -0.7 & & -2.5 & \\
\hline$C_{p(10(\theta))}^{o}$ & 106.2 & 49.2 & 51.3 & 52.8 & -7.8 & $\langle-8.2\rangle$ & -3.6 & $\langle-2.2\rangle$ & -0.6 & $\langle-1.5\rangle$ \\
\hline $\log k_{(10,50)}$ & & & & & & 5.02 & & 3.50 & & 2.39 \\
\hline
\end{tabular}

parameters. We have found no reaction whose $A$-factor needed to be rationalized by a multiple reaction path process. The contrary seems true. High $A$-factors are more consistent with transition state estimates of the entropies of activation for single-bond rupture than are low $A$-factors. Also, recombination rate constants calculated for such reactions have values close to those one might predict from kinetic theory considerations, and are therefore intuitively reasonable. Thus, we feel that single bond rupture is the only important reaction path in gas phase bond dissociation reactions and that the "normal" low $A$-factors reported for many bond fission reactions will eventually be shown to be incorrect.

\subsection{Rotational Barriers in Radicals and Single Shock Tube Kinetic Results}

Single-pulse shock tube techniques have provided what appear to be fairly reliable measures of the rate constant parameters of bond dissociation in a number of hydrocarbons. In addition, absolute rate constant determinations for methyl, ethyl, isopropyl, and $t$-butyl radical recombinations have also been made. It is possible, therefore, to check the kinetics of the hydrocarbon decompositions with the estimated reaction thermodynamics. A quick perusal of the results indicates that the reported activation energies (ignoring heat capacity corrections for the moment) are in fairly good agreement with the estimated reaction enthalpies at the mean reaction temperatures. However, the $A$-factor entropy relations are somewhat surprising. Proper estimation of radical entropies could be the major problem. For example, the symmetries of the radicals are not known, although recent evidence seems to support $\mathrm{sp}^{2}$ hybridization (or flat radicals). In addition, the barriers to internal rotations are also not known, although E.S.R. results tend to indicate that they are generally low. It has usually been assumed that on geometric grounds, the methyl rotational barriers in the ethyl and $t$-butyl radicals are zero. However, the reaction kinetics suggest considerably higher barriers, as the calculations in table $\mathrm{V}-5$ illustrate.

The heat capacities and entropies of the $t$-butyl radical have been calculated by straightforward molecular considerations for three possible rotational barriers, $V=0, \quad V=4, V=8 \mathrm{kcal} / \mathrm{mole}^{1 \cdot 2}$ and $\sigma=6(3)^{3}$. It is quite apparent that the entropy of the $t$-butyl radical is very sensitive to the magnitude of the internal barriers to methyl rotation. This is also the case for the reaction enthalpy and entropy. From the kinetics. $k_{\text {rec }}=10^{9.5} 1 /$ mole-sec and $\log k_{\text {diss }}=16.3-68.5 / \theta$

$$
\begin{aligned}
& k_{\text {diss }(1050 \circ \mathrm{K})}=2.09 \\
& E=\Delta H_{T}^{\circ}=68.5 \mathrm{kcal} . \\
& \Delta S^{\circ}=2.303 R \log \left(\frac{\mathrm{R}^{\prime} \mathrm{TA}}{k_{\text {rec }}}\right)=40.0 \mathrm{~g} / \text { mole. }
\end{aligned}
$$

The $\log$ of the rate constant for dissociation calculated from the experimental recombination rate constants and the estimated thermodynamics are given in the last row of the above table. It is apparent that the best agreement between experimental and calculated rate constants, enthalpies, and entropies of reaction is obtained for rotational barriers of $8 \mathrm{kcal} / \mathrm{mole}$. The same conclusion is reached by similar analysis of the other hydrocarbon dissociation reactions.

As a second example, the dissociation reaction thermodynamics of 2,3-dimethylbutane to two isopropyl radicals are shown in table $\mathrm{V}-6$.

\footnotetext{
1: For details of the methods used in making these estimates from molecular and thermodynamic considerations, see ref. [3-VI] and appendices $A$ and $C$.
} 


\begin{tabular}{|c|c|c|c|c|c|c|c|c|c|c|}
\hline & $2,3-$ & imethyl & tane & 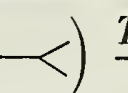 & $=1100$ & $\mathrm{~K} 2$ isopr & pyl & & & \\
\hline & & & & & & & & & & \\
\hline & 2,3DMB & $V=0$ & $V=4$ & $V=8$ & $\Delta X_{298}^{\circ}$ & $\Delta X_{T}^{\circ}$ & $\Delta X_{298}^{\circ}$ & $\Delta X_{T}^{\circ}$ & $\Delta X_{298}^{\circ}$ & $\Delta X_{T}^{\circ}$ \\
\hline$S_{298}^{\circ}$ & 87.4 & 69.0 & 65.4 & 63.8 & 50.6 & 46.3 & 43.4 & 43.4 & 40.2 & 41.8 \\
\hline$\Delta H_{f(298)}^{\circ}$ & -42.5 & 17.6 & & & 77.7 & 75.1 & 77.7 & 77.7 & 77.7 & 78.7 \\
\hline$C_{p(298)}^{\circ}$ & 33.6 & 15.1 & 17.7 & 17.1 & -3.4 & & 1.8 & & 0.6 & (1? \\
\hline$C_{p(1000)}^{\circ}$ & 79.1 & 37.9 & 39.1 & 40.3 & -3.3 & $(-3.3)$ & -0.9 & $(0)$ & 1.5 & $(1.2)$ \\
\hline $\log k_{(1100)}$ & & & & & & 3.27 & & 2.15 & & 1.55 \\
\hline
\end{tabular}

From the kinetics, $k_{\text {rec }}=10^{10.0} 1 /$ mole-sec $^{13}$ and $\log k_{\text {diss }}=16.1-76.0 / \theta=1.02$

$$
\begin{aligned}
& E=\Delta H_{T}^{\circ}=76.0 \mathrm{kcal} \\
& \Delta S^{\circ}=4.575 \log \left(R T \times \frac{10^{16.1}}{10^{10.0}}\right)=39.2 \mathrm{~g} / \text { mole } .
\end{aligned}
$$

Again the entropy of reaction and overall rate constant for dissociation estimated for the $V=8.0$ kcal barrier to rotation give the best agreement with the observed kinetics.

The discrepancy between the reaction entropies and the $A$-factors of dissociation and recombination means one of three things. The rate constants of dissociation are too low, the rate constants for recombination are too high, the entropies of the product radicals are lower than expected (i.e., rotational barriers are of the order of $8 \mathrm{kcal} / \mathrm{mole}$ ).

It is difficult to see how rotational barriers in radicals (e.g., $t$-butyl radical) can be significantly greater than zero. It is also difficult to believe that radical-radical recombination rates can be in error by the several orders of magnitude needed to reconcile the kinetic results with the thermodynamic estimates.

From the two representative reactions illustrated above, we see that (with the exception of the $V=0$ $\mathrm{kcal} / \mathrm{mole}$ rotational barrier model in hexamethylethane), the reaction enthalpies are all within \pm 2 $\mathrm{kcal} / \mathrm{mole}$ of the observed activation energies.

This experimental equivalence of $E_{a}$ and $\Delta H_{T}^{\circ}$ (within experimental error) strongly supports the validity of the single-pulse shock tube data (see $\mathrm{V}-3.0,4.0$ ). Thus the dilemma is posed and further study along these lines is needed.

1:3 The value of $h_{\text {rec }}=10^{10 . \times} \mathrm{l} /$ mole-sec reported in the literature [51] is higher than either methyl or ethyl radical recombinations and therefore seems too high.

\subsection{Rotational Barrier Entropy Corrections}

In table $\mathrm{V}-7$ are listed the entropy changes per rotor in going from the alkane $(\mathrm{RH})$ to the free radical (R.) as a function of barrier change and temperature. Values listed have been calculated from the data source for table C-5. A "normal" average barrier of $V=4.0 \mathrm{kcal} / \mathrm{mole}$ has been assumed for the rotations in the alkane. Other entropy changes such as electron spin and vibrational frequency differences are not included.

TABLE V-7. Free radical rotational barrier entropy corrections

\begin{tabular}{r|c|c|c}
\hline \hline$T{ }^{\circ} \mathrm{K}$ & $\begin{array}{c}\Delta S^{\circ} / \text { rotor } \\
V(4.0 \rightarrow 0)\end{array}$ & $\begin{array}{c}\Delta S^{\circ} / \text { rotor } \\
V(4.0 \rightarrow 2)\end{array}$ & $\begin{array}{c}\Delta S^{\circ} / \text { rotor } \\
V(4.0 \rightarrow 8)\end{array}$ \\
\hline 300 & +1.8 & +0.9 & -0.7 \\
500 & +1.3 & +0.7 & -0.7 \\
800 & +0.8 & +0.5 & -0.8 \\
1000 & +0.4 & +0.1 & -0.7 \\
\hline
\end{tabular}

All values are in units of $\mathrm{g} / \mathrm{mole}$.

If not otherwise indicated, the entropies of radicals given on the data sheets are for normal rotational barriers (i.e., no correction for barrier change in the radical relative to the alkane). This seemed the most reasonable procedure to follow since the true barriers are not known. However, one can readily convert the calculated reaction entropy at the reaction temperatures to the reaction entropy corresponding to 0,2 , and $8 \mathrm{kcal} / \mathrm{mole}$ rotational barriers with fairly good accuracy by applying the above corrections. For example, in the hexamethylethane decomposition (see table $\mathrm{V}-5$ ), the reaction entropy at $1050{ }^{\circ} \mathrm{K}$ for normal (i.e.. $4 \mathrm{kcal} /$ mole) rotational barriers in the product $t$-butyl radicals is $\Delta S^{\circ}(V=4)_{1050}=44.7 \mathrm{~g} /$ mole. To obtain the reaction entropy when rotational barriers are $8 \mathrm{kcal} / \mathrm{mole}$, one applies the corrections of table $\mathrm{V}-7$. Thus. 
$\Delta S^{\circ}(V-8)_{1050}=\Delta S^{\circ}(V=4)_{1050}+6(-0.7)=40.5$ $\mathrm{g} /$ mole. This may be compared to the value of 40.1 $\mathrm{g} / \mathrm{mole}$ which is obtained from the relation, $\Delta S^{\circ}(V=8)_{1050}=\Delta S^{\circ}(V=8)_{298}+\left\langle\Delta C_{p}^{\circ}\right\rangle_{1,=8} \underset{298-1050}{\times} \ln$ (1050/298).

When not otherwise indicated, the recombination rate constants given on the data sheets refer to values calculated for $4.0 \mathrm{kcal} / \mathrm{mole}$ barriers in the radicals. If recombination rate constants estimated for other barrier values are desired, they may readily be obtained from the corrected reaction entropies as indicated above. In general, "higher" than normal rotational barriers will raise the recombination rate constants while lower barriers will produce the opposite effect. For the hydrocarbon decompositions the calculated recombination rate constants have been listed with error limits. For example, $k_{\text {rec }}(t \mathrm{Bu} .+t \mathrm{Bu})=$ $10^{8.2}+4.01 /$ mole-sec. The high and low limits refer to estimated values based on rotational barriers in the radicals of 8 and $0 \mathrm{kcal} / \mathrm{mole}$, respectively. It should be remembered that similar uncertainties exist in the calculated recombination rate constants for all radicals with internal rotation barrier uncertainties.

\subsection{Problems in the Kinetics of Azo Compound Decompositions}

The kinetic data on azo compound reactions are extensive; however they are not easily analyzed by thermochemical considerations, since neither heats of formation of the product radicals nor heats of formation of the azo compounds themselves have been independently determined. The single exception to the above is the heat of formation of azoisopropane where combustion techniques give, $\Delta H_{f}^{\circ}(i \mathrm{PrN}=\mathrm{Ni} P \mathrm{Pr})_{\mathrm{gas}}=19.5 \pm 1.0 \mathrm{kcal} / \mathrm{mole}$.

It is possible to generate all of the unknown heats of formation (i.e., of both radicals and compounds) from the experimental activation energies for decomposition and from the azoisopropane heat of formation if we make the following assumptions: (1) Kinetics of the alkyl azo compound decompositions pertain to single bond rupture. (2) Group additivities are valid in the radicals as well as in the parent azo compounds. (3) The azo radical group value $\left[\mathrm{N}_{\mathrm{A}}-\left(\mathrm{N}_{\mathrm{A}} \cdot\right)(\mathrm{C})\right]$ is not affected by the nature of the alkyl group bonded to the nitrogen opposite the radical center. We will discuss the first of these assumptions later. The latter two are quite reasonable since one can envisage no real reason why any unusual next to nearest neighbor interracions should be important in the azo compounds or in the azo radicals. ${ }^{1+}$ In the following, the method of deriving

it The radical center is fairly localized on the end azo nitrogen and an interaction between that radical center and groups more than two atoms away does not seem reasonable. the pertinent enthalpy group additivities is given, and the "reasonableness" of the resulting groups is examined by comparison to well established group values of a similar kind.

Consider the single bond rupture of a linear chain, symmetrical, azo compound.

$$
\text { I. } \begin{aligned}
\mathrm{RN} & =\mathrm{NR} \longrightarrow \mathrm{R} \cdot+\cdot \mathrm{N}=\mathrm{NR} \\
\Delta \mathrm{H}^{\circ} & =\mathrm{DH}^{\circ}(\mathrm{RNN}-\mathrm{R}) .
\end{aligned}
$$

The reaction enthalpy written in terms of the various groups whose natures are changing is given by

$$
\begin{aligned}
\mathrm{E}_{l}=\Delta \mathrm{H}_{\mathrm{I}} & =\left[\mathrm{N}_{\mathrm{A}}-\left(\mathrm{N}_{\mathrm{A}} \cdot\right)(\mathrm{C})\right]+\left[\mathrm{C}-(\mathrm{H})_{\mathrm{X}}(\mathrm{C})_{3-\mathrm{x}}\right] \\
- & {\left[2\left(\mathrm{~N}_{\mathrm{A}}-\mathrm{C}\right)\right]+\left[\mathrm{C}-\left(\mathrm{N}_{\mathrm{A}}\right)(\mathrm{H})_{\mathrm{X}}(\mathrm{C})_{3-\mathrm{X}}\right]-n_{A} \mathrm{G} . }
\end{aligned}
$$

Here the group notations have their usual significance and $n_{t}$ is the number of gauche $(\mathrm{G})$ interractions around the $\left(\mathrm{C}-\mathrm{N}_{\mathrm{A}}\right)$ bond which is breaking. We assume from the kinetics that the reaction enthalpies for dissociation of azomethane, azoethane, azoisopropane, and azobutane are 52.5, $50.0,47.5$, and $43.5 \mathrm{kcal} / \mathrm{mole}$, respectively (see data sheets). The heat of formation of azoisopropane gives a fifth relation. There are a total of six group unknowns $\left(\left[\mathrm{N}_{\mathrm{A}}-\left(\mathrm{N}_{\mathrm{A}} \cdot\right)(\mathrm{C})\right],\left[\mathrm{C}-\mathrm{N}_{\mathrm{A}}\right]\right.$, and four $\left.\left[\mathrm{C}-\left(\mathrm{N}_{\mathrm{A}}\right)(\mathrm{H})_{\mathrm{X}}(\mathrm{C})_{3-\mathrm{X}}\right]\right)$. By making the usual assumption, that a methyl group in all environments is the same as the methyl in alkanes, the equations can be solved and the group values (column 2, table $\mathrm{V}-8$ ) are obtained. A comparison with the other group values in the table shows that the alkyl azo groups are very similar to the hydrocarbon groups and significantly less stabilizing than are the alkyl amine and alkyl oxygen groups. Such a variation is qualitatively expected since the $(-\mathrm{N}=\mathrm{N}-$ ) group should not be as effective in polarizing adjacent alkyl groups as should $\mathrm{O}$ and $\mathrm{N}$ atoms. The derived group additivities of table $\mathrm{V}-8$ are therefore not mnreasonable.

Using the groups, it is now possible to predict the activation energies of other azo compound reactions. Predictions and observed values are shown in table V-9. Since the estimates should be good to about $\pm 2 \mathrm{kcal} / \mathrm{mole}$, it is clear that there is very good agreement between observed and estimated activation energies. Note also that the transition state (biradical mechanism) estimated $A$-factors of the pyrazoline decompositions and azirine decompositions are in excellent agreement with those observed.

It can be argued, and legitimately so, that the assumption of single bond rupture in alkyl azo compounds coupled with the fitting of the thermodynamics to the kinetics is begring the real question 
TABLE V-8. Enthalpy (kcal/mole) group additivities

\begin{tabular}{l|r|l|l|l|r}
\hline \multicolumn{1}{c|}{ Group } & \multicolumn{1}{c|}{$\Delta H_{f}^{\circ}$} & \multicolumn{1}{c|}{ Group } & \multicolumn{1}{c}{$\Delta H_{f}^{\circ}$} & \multicolumn{1}{c}{ Group } & $\Delta H_{f}^{\circ}$ \\
\hline$\left[\mathrm{N}_{\mathrm{A}}-\left(\mathrm{N}_{\mathrm{A}} \cdot\right)(\mathrm{C})\right]$ & 72.4 & {$\left[\mathrm{C}-\left(\mathrm{C}_{d}\right)(\mathrm{H})_{2}(\mathrm{C})\right]$} & -4.8 & {$\left[\mathrm{C}-(\mathrm{N})(\mathrm{H})_{2}\right]$} & -6.6 \\
{$\left[\mathrm{~N}_{\mathrm{A}}-\mathrm{C}\right]$} & 32.0 & {$\left[\mathrm{C}-\left(\mathrm{C}_{d}\right)(\mathrm{H})\left(\mathrm{C} \cdot \mathrm{C}_{2}\right]\right.$} & -1.5 & {$[\mathrm{C}-(\mathrm{N})(\mathrm{H})(\mathrm{C})]$} & -5.2 \\
{$\left[\mathrm{C}-\left(\mathrm{N}_{\mathrm{A}}\right)(\mathrm{H})_{3}\right]$} & -10.1 & {$\left[\mathrm{C}-\left(\mathrm{C}_{d}\right)(\mathrm{C})_{3}\right]$} & +1.7 & {$\left[\mathrm{C}-(\mathrm{N})(\mathrm{C})_{2}\right]$} & -3.2 \\
{$\left[\mathrm{C}-\left(\mathrm{N}_{\mathrm{A}}\right)(\mathrm{H})_{2}(\mathrm{C})\right]$} & -5.8 & {$\left[\mathrm{C}-(\mathrm{C})_{2}(\mathrm{H})_{2}\right]$} & -5.0 & {$\left[\mathrm{C}-(\mathrm{O})(\mathrm{H})_{2}(\mathrm{C})\right]$} & -8.5 \\
{$\left[\mathrm{C}-\left(\mathrm{N}_{\mathrm{A}}\right)(\mathrm{H})(\mathrm{C})_{2}\right]$} & -2.1 & {$\left[\mathrm{C}-(\mathrm{C})_{3}(\mathrm{H})\right]$} & -1.9 & {$\left[\mathrm{C}-(\mathrm{O})(\mathrm{H})(\mathrm{C})_{2}\right]$} & -7.0 \\
{$\left[\mathrm{C}-\left(\mathrm{N}_{\mathrm{A}}\right)(\mathrm{C})_{3}\right]$} & +0.3 & {$\left[\mathrm{C}-(\mathrm{C})_{4}\right]$} & +0.5 & {$\left[\mathrm{C}-(\mathrm{O})(\mathrm{C})_{3}\right]$} & -6.6 \\
\hline
\end{tabular}

and necessarily "forcing" the good agreement between prediction and observation displayed in table $\mathrm{V}-9$. It is well known that the enthalpy of the second $\left(\mathrm{C}-\mathrm{N}_{\mathrm{A}}\right)$ bond rupture is very exothermic. Thus in azomethane,

II. $\mathrm{CH}_{3} \mathrm{~N}=\mathrm{NCH}_{3} \stackrel{\mathrm{l}}{\longrightarrow} \mathrm{CH}_{3} \mathrm{~N}=\mathrm{N} \cdot+\mathrm{CH}_{3}$. $D H^{\circ}\left(\mathrm{CH}_{3} \mathrm{~N}=\mathrm{N}-\mathrm{CH}_{3}\right)=D H_{1}^{\circ} \geqslant 52.5 \mathrm{kcal} / \mathrm{mole}$.

III. $\mathrm{CH}_{3} \mathrm{~N}=\mathrm{N} \cdot \stackrel{2}{\longrightarrow} \mathrm{CH}_{3} \cdot+\mathrm{N}$.2 $D H^{\circ}\left(\mathrm{CH}_{3} \mathrm{~N}=\mathrm{N} \cdot\right)=D H_{2}^{\circ} \leqslant-28.3 \mathrm{kcal} / \mathrm{mole}$.

Since,

$$
\begin{aligned}
D H_{1}^{\circ}-D H_{2}^{\circ}=2 \Delta H_{f}^{\circ}\left(\mathrm{CH}_{3}\right)-\Delta H_{f}^{\circ}\left(\mathrm{CH}_{3} \mathrm{~N}=\mathrm{NCH}_{3}\right) \\
=68.0-43.8=24.2 \mathrm{kcal} / \mathrm{mole} .
\end{aligned}
$$

Simultaneous stretching of the second $\left(\mathrm{C}-\mathrm{N}_{\mathrm{A}}\right)$ bond in the process of the first bond rupture, as a result of the $\mathrm{DH}_{2}^{\circ}$ exothermicity (which increases with the stabilization of the radical leaving group), could lead to a lower energy configuration of the transition state and therefore result in an activation energy significantly lower than the weakest bond dissociation energy in the molecule. Such a decomposition mode has been favored by many and has been termed simultaneous or concerted twobond rupture (see section $\mathrm{V}-5.0$ ). There is very good evidence to support two-bond rupture in some special systems studied in solution. Thus comparing the activation energies observed for the decomposition of the following compounds,

$\emptyset \mathrm{CH}\left(\mathrm{CH}_{3}\right) \mathrm{N}=\mathrm{NCH}_{3}[52](E=38.6)$, $\emptyset \mathrm{CH}\left(\mathrm{CH}_{3}\right) \mathrm{N}=\mathrm{NCH}\left(\mathrm{CH}_{3}\right)_{2}[53](E=36.5)$,

and

$$
\varnothing \mathrm{CH}\left(\mathrm{CH}_{3}\right) \mathrm{N}=\mathrm{NCH}\left(\mathrm{CH}_{3}\right) \emptyset[53](E=32.6)
$$

all in $\mathrm{kcal} / \mathrm{mole}$, we see a successive decrease in activation energies and increase in reaction rates as the stability of the second $R$ group is increased by $\mathrm{CH}_{33}$ and $\varnothing$ substitutions. ${ }^{1.5}$

On the other hand. the isotope studies of Seltzer [53] indicate that there is very little second $\left(\mathrm{C}-\mathrm{N}_{\mathrm{A}}\right)$ bond stretch in the transition state when the second $R$ leaving group is methyl. Thus for the compounds,

$$
\begin{aligned}
& \emptyset \mathrm{CH}\left(\mathrm{CH}_{33}\right) \mathrm{N}=\mathrm{NCH}_{3} \\
& \varnothing \mathrm{CD}\left(\mathrm{CH}_{33}\right) \mathrm{N}=\mathrm{NCH}_{3} \\
& \varnothing \mathrm{CH}\left(\mathrm{CH}_{3}\right) \mathrm{N}=\mathrm{NCD}_{3}
\end{aligned}
$$

and

decomposition rates at $161^{\circ} \mathrm{C}$ in solution were 1.16 . 1.04 , and $1.19 \times 10^{-4} \mathrm{sec}^{-1}$, respectively. The single deuterium substitution at the $\alpha$-carbon center involved in the first bond rupture, produced an appreciable secondary isotope effect; however, complete deuteration of the methyl (second $R$ ) leaving group had almost no effect on the reaction rate.

All these facts suggest that some two-bond rupture may occur in cases where substitution $\alpha$ to the $\mathrm{N}_{\mathrm{A}}$ can lead to appreciable stabilization of the second $R$ leaving group (i.e., the $R$ of the azo radical), but that one-bond rupiure is the principal decomposition mode for simpler azo compounds.

One bond rupture is, therefore, the favored reaction mode for all the gas phase azo compound decompositions in this monograph. The rather good agreement between the predicted and observed activation energies for the azotoluene reaction (where a benzyl resonance stabilization of the second $R$ leaving group is possible), lends further support to the above assertion.

\section{Thermodynamics of Single-Bond Rupture}

If one-bond rupture in the alkyl azo compounds is correct, the activation energies observed are necessarily direct measures of the $\left(\mathrm{C}-\mathrm{N}_{\mathrm{A}}\right)$ bond

\footnotetext{
1.5 Also, while azobenzene does not decompose 10 radicals thermally. $\emptyset \mathrm{N}=\mathrm{NC}(\varnothing)_{: 3}[54]$ decomposes at an appreciable rate at $50{ }^{\circ} \mathrm{C}$ while $(\emptyset)_{3:} \mathrm{CN}=\mathrm{NC}(\emptyset)_{3}[55]$ is so unstable it cannot be isolated even at $-40^{\circ} \mathrm{C}$.
} 
TABLE V-9. Summary of predicted and observed activation energies in azo compound decompositions.

\begin{tabular}{|c|c|c|c|c|}
\hline Reactant & $E_{\text {(calc) }}$ & $E_{\text {(obs) }}$ & $\log A_{\text {calc }}$ & $\log A_{\text {obs }}$ \\
\hline $\mathrm{CH}_{3} \mathrm{~N}=\mathrm{NCH}_{3}$ & “ 52.5 & 52.5 & & \\
\hline $\mathrm{C}_{2} \mathrm{H}_{5} \mathrm{~N}=\mathrm{NC}_{2} \mathrm{H}_{5}$ & 50.0 & $51.2,48.5$ & & $16.0,15.7$ \\
\hline$n \cdot \mathrm{C}_{4} \mathrm{H}_{9} \mathrm{~N}=\mathrm{NC}_{4} \mathrm{H}_{9}-n$ & 50.0 & $47.5,53.2$ & & $15.45,17.71$ \\
\hline$\left(\mathrm{CH}_{3}\right)_{2} \mathrm{CHCH}_{2} \mathrm{~N}=\mathrm{NCH}_{2} \mathrm{CH}\left(\mathrm{CH}_{3}\right)_{2}$ & 50.0 & 49.0 & & 16.2 \\
\hline$i \operatorname{PrN}=\mathrm{Ni} \operatorname{Pr}$ & 47.5 & 40.7 & & 13.75 \\
\hline $\mathrm{C}_{2} \mathrm{H}_{5}\left(\mathrm{CH}_{3}\right) \mathrm{CHN}=\mathrm{NCH}\left(\mathrm{CH}_{3}\right) \mathrm{C}_{2} \mathrm{H}_{5}$ & 47.5 & 48.4 & & 17.28 \\
\hline $\mathrm{CH}_{3} \mathrm{~N}=\mathrm{NCH}\left(\mathrm{CH}_{3}\right)_{2}$ & 47.5 & 47.8 & & 15.45 \\
\hline$t \mathrm{BuN}=\mathrm{N} t \mathrm{Bu}$ & 43.5 & $42.8,43.0$ & & $16.34,17.15$ \\
\hline & 44.0 & 42.4 & 16.0 & 15.9 \\
\hline & 44.0 & 41.0 & 16.0 & 15.83 \\
\hline & 41.5 & 41.0 & & 15.67 \\
\hline$=N$ & 37.5 & 40.0 & & 15.83 \\
\hline$y_{\text {cis }}$ & 41.5 & 40.3 & & 15.5 \\
\hline & 37.5 & 38.9 & & 15.42 \\
\hline & 37.5 & 37.7 & 14.6 & 14.48 \\
\hline & 30.7 & 33.17 & & 13.89 \\
\hline & 30.7 & 31.9 & 13.9 & 13.73 \\
\hline & 30.7 & 30.5 & & 13.4 \\
\hline & 30.7 & 30.87 & & 13.34 \\
\hline$\emptyset \mathrm{CH}_{2} \mathrm{~N}=\mathrm{NCH}_{2} \varnothing$ & 37.4 & 35.0 & & 14.1 \\
\hline
\end{tabular}

"Values assumed in order to obtain the radical and reactant thermochemical group values.

b Activation energies of the azo compounds have been obtained from the biradical mechanism,

example:

$$
\begin{aligned}
& x_{N=N} \underset{2}{\rightleftharpoons} \sim^{N} i_{N} \cdot \frac{1 \text { tost }}{3} \varkappa^{+N_{2}} \\
& E_{1}=\Delta E_{1,2}^{\circ}+E_{2}=D H^{\circ}(t \mathrm{BuN}=\mathrm{N} t \mathrm{Bu})-E_{\mathrm{strain}}-R T+E_{2}
\end{aligned}
$$

Assume

Then

$$
E_{2} \simeq \mathrm{V}_{0}(\text { internal rotation of } \mathrm{R} \in \mathrm{N}=\mathrm{N} \cdot) \simeq 1 \mathrm{kcal} / \mathrm{mole}
$$$$
E_{1}=D H^{\circ}(t \mathrm{BuN}=\mathrm{N} t \mathrm{Bu})-E_{\text {strain }} ; E_{\text {strain }} \simeq 6 \mathrm{kcal} / \mathrm{mole} \text {. }
$$ 
dissociation energies. The thermochemistry of azomethane, reactions II and III, gives the relations, $D H_{1}^{\circ}+D H_{2}^{\circ}=24.2 \mathrm{kcal} / \mathrm{mole}$. Also, the difference between the first and second dissociation energies should roughly equal the second pi bond energy in nitrogen. $D H_{1}^{\circ}-D H_{2}^{\circ}=\pi_{2(\mathrm{~N}=\mathrm{N})}$. From the reaction enthalpies one then obtains $\pi_{2(\mathrm{~N}=\mathrm{N})}=80.8 \mathrm{kcal} /$ mole. Similarly, two-bond rupture requires that $\pi_{2(\mathrm{~N}=\mathrm{N})}>80.8 \mathrm{kcal} / \mathrm{mole}$. By way of comparison we note that the pi bond energy in carbonyl compounds $\left(\pi_{(\mathrm{C} \equiv 0)}\right)$ is $75 \mathrm{kcal} / \mathrm{mole}$, and the pi bond energies in alkenes and alkynes are $\left(\pi_{2(\mathrm{c}=\mathrm{c})}\right)=73$ $\mathrm{kcal} / \mathrm{mole}$ and $\left(\pi_{1(\mathrm{c}=\mathrm{c})}\right)=60.0 \mathrm{kcal} / \mathrm{mole}$, respectively. A second pi bond energy in nitrogen much in excess of $81 \mathrm{kcal} / \mathrm{mole}$ does not therefore seem very likely.

\subsection{Some Problems in the Amine and Hydra- zine Decompositions}

The Arrhenius $A$-factors of the hydrazine and amine decompositions are all less than the normal $e k T / h$ value; some are significantly lower. Such a situation implies negative entropies of activation, which, in the case of simple bond fission reactions, is just not reasonable. We have chosen to discuss the thermal decompositions of the amines and hydrazines because their studies are typical ones in which the toluene carrier and aniline carrier techniques have been employed, and because the heats of formation of the product radicals have not as yet been determined by other methods.
There is and will be a natural tendency to use the activation energies in these studies to determine the heats of formation of the product amine radicals. Since the reported kinetic parameters are certainly too low, such procedures could lead to confusing the literature for a time with heats of formation of the amine radicals which are very probably too low by as much as 4 to $6 \mathrm{kcal} / \mathrm{mole}$. Such a history has already transpired in the cases of the alkyl, benzyl, and allyl radicals. A quick perusal of the dissociation reaction enthalpies of the hydrocarbons based on the more recent and reliable heats of formation of the above radicals quickly reveals that the original Arrhenius parameters reported in the hydrocarbon decompositions (via the toluene carrier technique) were too low. The present situation for the amines and hydrazines is almost certainly the same. Recombination rate constants of the product radicals have been estimated from the reported $A$-factors and the reaction entropies. These are compared with the structurally similar hydrocarbon radical recombination reactions in table $\mathrm{V}-10$.

It is seen that considerably lower recombination rates for amine radicals (with values lower by about 1.4 powers of 10 per nitrogen at the bond fission center) are calculated. If these recombination rate constants were correct, they would represent the lowest rates of their kind known. One would then have to conclude that there is something very peculiar about free radicals with the odd electron on nitrogen. It should be noted that the calculated

TABLE V-10. Recombination rate constants ( $\left.k_{\text {rec }} \mathrm{l} / \mathrm{mole}-\mathrm{sec}\right)$.

\begin{tabular}{|c|c|c|c|}
\hline \multicolumn{2}{|c|}{ Recombination rate constants for nitrogen compounds } & \multicolumn{2}{|c|}{ Hydrocarbon analog } \\
\hline Reaction & $\begin{array}{c}{ }^{a} \log k_{\text {rec }} \\
\text { (experimental) }\end{array}$ & $\begin{array}{c}\log k_{\text {rec }} \\
(\text { analog exp) }\end{array}$ & Reaction \\
\hline 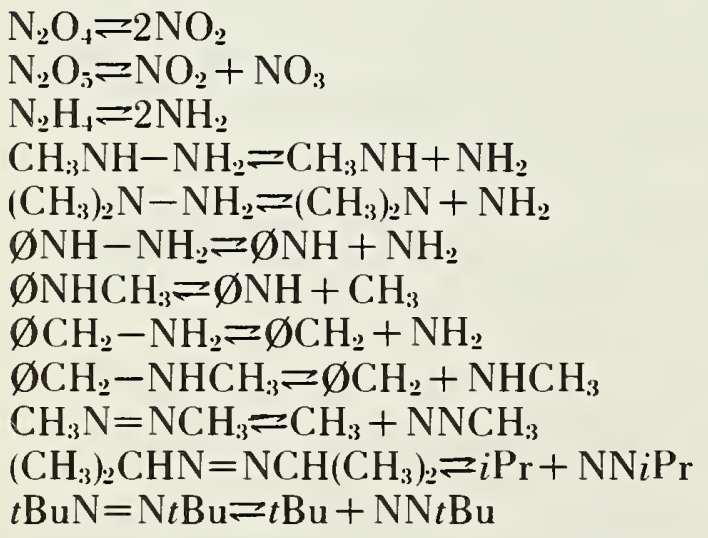 & $\begin{array}{l}8.2 \\
8.6 \\
6.2 \\
5.9 \\
6.1 \\
5.8 \\
7.5 \\
6.6 \\
5.9 \\
9.2 \\
9.4 \\
9.9\end{array}$ & $\begin{array}{l}9.8 \\
9.8 \\
9.8 \\
9.6 \\
9.6 \\
9.0 \\
9.0 \\
9.0 \\
8.7\end{array}$ & $\begin{array}{l}\mathrm{CH}_{3}+\mathrm{CH}_{3} \\
\mathrm{CH}_{3}+\mathrm{CH}_{3} \\
\mathrm{CH}_{3}+\mathrm{CH}_{3} \\
\mathrm{CH}_{3}+\mathrm{C}_{2} \mathrm{H}_{5} \\
\left(\mathrm{CH}_{3}\right)_{2} \mathrm{CH}+\mathrm{CH}_{3} \\
\varnothing \mathrm{CH}_{2}+\mathrm{CH}_{3} \\
\varnothing \mathrm{CH}_{2}+\mathrm{CH}_{3} \\
\emptyset \mathrm{CH}_{2}+\mathrm{CH}_{3} \\
\varnothing \mathrm{CH}_{2}+\mathrm{C}_{2 .} \mathrm{H}_{3}\end{array}$ \\
\hline
\end{tabular}

" Calculated from the observed $A$-factors for decomposition and the estimated decomposition reaction entropies. 
TABLE V-11. Amine radical thermochemistry via adjusted decomposition Arrhenius parameters

\begin{tabular}{|c|c|c|c|c|}
\hline Reaction & $\begin{array}{c}\log A \\
\text { (scaled) }\end{array}$ & $E$ (scaled) & $\Delta H_{2998}^{\circ}$ & $\Delta H_{f^{\circ}}^{\circ}\left(R^{\cdot}\right)$ \\
\hline $\mathrm{N}_{2} \mathrm{H}_{4} \rightarrow 2 \mathrm{NH}_{2}$ & 16.6 & 71.2 & 71.2 & $47.0\left(\mathrm{NH}_{2 .}\right)$ \\
\hline $\mathrm{CH}_{3} \mathrm{NHNH}_{2} \rightarrow \mathrm{CH}_{3} \mathrm{NH}+\mathrm{NH}_{2}$ & 16.7 & 64.8 & 63.8 & $40.0^{a}\left(\mathrm{CH}_{3} \mathrm{NH}\right)$ \\
\hline$\left(\mathrm{CH}_{3}\right)_{2} \mathrm{NNH}_{2} \rightarrow\left(\mathrm{CH}_{3}\right)_{2} \mathrm{~N}+\mathrm{NH}_{2}$ & 16.9 & 62.7 & 63.0 & $37.4^{a}\left[\left(\mathrm{CH}_{3}\right)_{2} \mathrm{~N}\right]$ \\
\hline$\emptyset \mathrm{NHNH}_{2} \rightarrow \emptyset \mathrm{NH}+\cdot \mathrm{NH}_{2}$ & 15.5 & 51.1 & 51.1 & $55.4^{\mathrm{a}}(\emptyset \mathrm{NH})$ \\
\hline$\emptyset \mathrm{NHCH}_{3} \rightarrow \emptyset \mathrm{NH}+\mathrm{CH}_{3}$. & 15.3 & 67.7 & 67.7 & $54.4(\emptyset \mathrm{NH})$ \\
\hline$\emptyset \mathrm{CH}_{2} \mathrm{NH}_{2} \rightarrow \emptyset \mathrm{CH}_{2}+\mathrm{NH}_{2}$ & 15.3 & 69.8 & 68.5 & $44.5\left(\mathrm{NH}_{2}\right)$ \\
\hline$\emptyset \mathrm{CH}_{2} \mathrm{NHCH}_{3} \rightarrow \emptyset \mathrm{CH}_{2}+\mathrm{HNCH}_{3}$ & 15.7 & 69.0 & 68.0 & $44.5\left(\mathrm{CH}_{3} \mathrm{NH}\right)$ \\
\hline$\emptyset \mathrm{N}\left(\mathrm{CH}_{3}\right)_{2} \rightarrow \emptyset \mathrm{NCH}_{3}+\mathrm{CH}_{3}$ & 15.0 & 65.2 & 64.8 & $53.5^{\mathrm{b}}\left(\emptyset \mathrm{NCH}_{3}\right)$ \\
\hline
\end{tabular}

a Obtained using the "best" value for $\Delta H_{f}^{\circ}\left(\mathrm{NH}_{2}\right)=45.0 \mathrm{kcal} / \mathrm{mole}$.

b There is good reason to suspect the group additivity values in this system. Methyl substitution at a nitrogen center in the alkyl amines produces a slight stabilization ( $1.8 \mathrm{kcal} / \mathrm{mole})$. One might expect a similar behavior for aryl amines. However the group values predict a slight decrease in stability in the aryl amines. If the group values are wrong for the latter, then $\Delta H_{f}^{\circ}\left(\emptyset \mathrm{NCH}_{3}\right)=49.6 \mathrm{kcal} / \mathrm{mole}$.

recombination rate constants for azo and alkyl radicals are very similar to the hydrocarbon analogs, although some question concerning the proper interpretation of the azo compound kinetics does exist (see $\mathrm{V}-7.0$ ).

By far the more reasonable alternative is that the recombination rate constants should be close to normal (i.e., like their hydrocarbon counterpart). Thus the preferred $A$-factors for the amine and hydrazine reactions should be higher than observed by about the differences indicated in table $\mathrm{V}-10$.

If the higher $A$-factors are assumed and the experimental rate constants are accepted, then "more reasonable" activation energies and heats of formation of the pertinent radicals can be estimated. The results of such estimates are summarized in table V-11.

Our "best" values for the various amine radical heats of formation are:

$$
\begin{aligned}
& \Delta H_{f}^{\circ}\left(\mathrm{NH}_{2}\right)=45.0 \mathrm{kcal} / \mathrm{mole} \\
& \Delta H_{f}^{\circ}\left(\mathrm{CH}_{3} \mathrm{NH}\right)=41.7 \mathrm{kcal} / \mathrm{mole} \\
& \Delta H_{f}^{\circ}\left(\mathrm{N}\left(\mathrm{CH}_{3}\right)_{2}\right)=37.4 \mathrm{kcal} / \mathrm{mole} \\
& \Delta H_{f}^{\circ}(\emptyset \mathrm{NH})=55.0 \mathrm{kcal} / \mathrm{mole} \\
& \Delta H_{f}^{\circ}\left(\emptyset \mathrm{NCH}_{3}\right)=49.6 \mathrm{kcal} / \mathrm{mole}
\end{aligned}
$$

The $D H^{\circ}(\mathrm{N}-\mathrm{H})$ bond dissociation energies (kcal/mole) in several compounds compared to analogous $D H^{\circ}(\mathrm{C}-\mathrm{H})$ and $D H^{\circ}(\mathrm{HO}-\mathrm{H})$ values are shown below.

$$
\begin{aligned}
& D H^{\circ}\left(\mathrm{CH}_{3}-\mathrm{H}\right)=104 \\
& D H^{\circ}\left(\mathrm{CH}_{3} \mathrm{CH}_{2}-\mathrm{H}\right)=98 \\
& D H^{\circ}\left(\mathrm{NH}_{2}-\mathrm{H}\right)=109.1 \\
& D H^{\circ}\left(\mathrm{CH}_{3} \mathrm{NH}-\mathrm{H}\right)=98.4 \\
& D H^{\circ}(\mathrm{HO}-\mathrm{H})=118.9 \\
& D H^{\circ}\left(\mathrm{CH}_{3} \mathrm{O}-\mathrm{H}\right)=104.1 \\
& D H^{\circ}(\mathrm{F}-\mathrm{H})=134
\end{aligned}
$$

The sequence of increasing bond strengths seems inherently reasonable. This sequence would not exist if the kinetics were accepted as reported (i.e., with $\Delta H_{f}^{\circ}\left(\mathrm{NH}_{2}\right)=41 \mathrm{kcal} / \mathrm{mole}$ ).

\subsection{Summary of the Calculated $\boldsymbol{A}$-Factors for Radical-Radical Recombination Reactions. ${ }^{\mathrm{a}}$}

\begin{tabular}{l|l}
\hline \hline \multicolumn{1}{c|}{ Reaction } & \multicolumn{1}{|c}{$\log k_{\text {rec }}$} \\
\hline & \\
atoms + radical & \\
$\varnothing \mathrm{CH}_{2} \cdot+\mathrm{H} \cdot$ & 10.5 \\
$\mathrm{CH}_{2}=\mathrm{CH}-\mathrm{CH}_{2} \cdot+\mathrm{H} \cdot$ & $10.4-11.7(10.7)$ \\
\hline
\end{tabular}

See fortnote at end of table. 


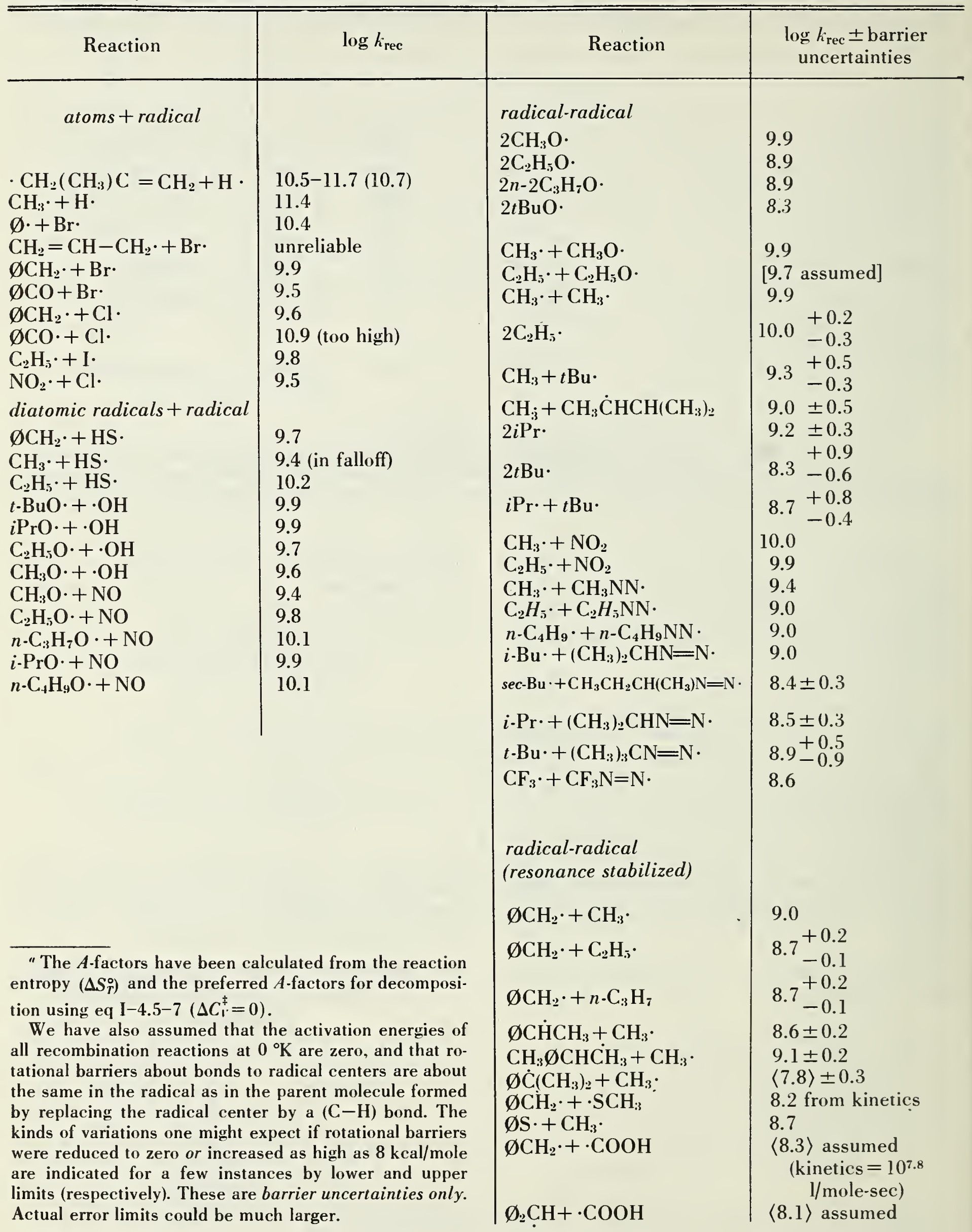




\begin{tabular}{|c|c|c|c|}
\hline Reaction & $\begin{array}{c}\log k_{\text {rec }} \pm \text { barrier } \\
\text { uncertainties }\end{array}$ & Reaction & $\begin{array}{c}\log k_{\text {rec }} \pm \text { barrier } \\
\text { uncertainties }\end{array}$ \\
\hline $\begin{array}{l}\emptyset \mathrm{CH}_{2} \mathrm{CO}+\emptyset \mathrm{CH}_{2} \cdot \\
\mathrm{CH}_{3} \mathrm{CO}+\emptyset \mathrm{CH}_{2} \cdot \\
\mathrm{CF}_{3} \cdot+\emptyset \mathrm{CO} \\
\emptyset \cdot \varnothing \mathrm{CO} \\
\mathrm{CH}_{3} \cdot+\varnothing \mathrm{CO} \\
2 \varnothing \mathrm{CO} \\
2{ }^{n} \mathrm{PrCO}_{2} \cdot \\
2 \mathrm{C}_{2} \mathrm{H}_{3} \mathrm{CO}_{2 \cdot} \cdot \\
2 \mathrm{CH}_{3} \mathrm{CO}_{2} \cdot \\
\mathrm{CH}_{3} \mathrm{CO}_{2} \cdot+\emptyset \mathrm{CH}_{2} \cdot\end{array}$ & $\begin{array}{l}8.7 \\
8.7 \\
8.4 \\
8.8 \\
8.9 \\
8.8 \\
8.7 \\
8.8 \\
8.7 \\
8.4\end{array}$ & 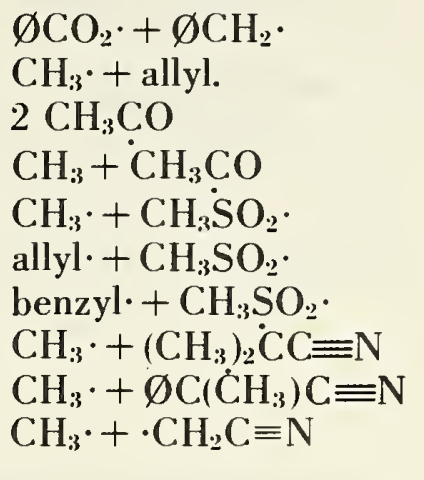 & $\begin{array}{l}8.9 \\
9.8 \\
8.5 \\
9.1 \\
7.8 \\
7.6 \\
8.0 \\
7.4 \\
7.4 \\
7.9\end{array}$ \\
\hline
\end{tabular}

\section{Free Radical Reactions}

\subsection{Characteristics and Reliability of the Experimental Data}

Direct experimental kinetic data on free radical unimolecular reactions (isomerizations and decompositions) are quite limited. Fewer than 50 such reactions are reported here. Some reactions appropriate to this monograph may have been overlooked; ${ }^{16}$ however, we believe their number to be few.

With the very substantial increase in reliable thermodynamic data on free radicals (i.e., experimentally determined heats of formation and third law entropy estimates) and with some fairly reliable measurements of the kinetics of the back reactions, it has been possible to calculate with reasonable confidence the kinetic parameters of the free radical reactions compiled here. Such calculations, as may be seen on the data sheets, are not often in agreement with the experimental results. We take this to mean that much of the unimolecular rate data on free radical reactions is not very reliable. This is really not surprising in view of the experimental difficulties and mechanistic complications which must be resolved before reliable kinetic results can be realized. It is perhaps instructive to briefly examine some of these problems.

To study the kinetics of a radical reaction, the following are necessary: (1) to generate unambiguously the radical of interest; (2) to identify

\footnotetext{
1" Pertinent kinetic results are often buried in the reaction scheme details of studies directed toward other objectives and have not been properly abstracted.
}

and analyze specifically the products of the reaction; and (3) to place the kinetics on an absolute basis.

Requirement No. 1. A number of techniques for generating radicals have been employed. These include $\mathrm{Hg}\left({ }^{3} \mathrm{P}_{1}\right)$ photosensitization of alkanes, photochemical decomposition of aldehydes and ketones, radical sensitized decompositions of aldehydes, and atom and radical additions to olefins (i.e., chemical activation). The $\mathrm{Hg}$ photosensitization studies are nonselective with regard to radical geometry. Thus with propane both isopropyl and $n$-propyl radicals are formed.

Requirement No. 2. In chemical activation processes addition can occur on either side of the double bond. This often leads to different radical species. Also, the radicals formed are "hot." This can lead to pressure dependent kinetics.

Photochemical and radical sensitized decompositions of aldehyde systems were designed to overcome the first requirement since it was believed, with some supporting evidence, that the only im. portant reactions in these systems were:

\section{Photochemical $\mathrm{RCHO}+h \nu \stackrel{\mathrm{a}}{\longrightarrow} \mathrm{R}+\mathrm{CHO}$}

followed by $\mathrm{CHO}+\mathrm{M} \stackrel{\text { fast }}{\longrightarrow} \mathrm{CO}+\mathrm{H}+\mathrm{M}$

$$
\begin{array}{r}
\text { Sensitized } \quad \mathrm{R}^{\prime}+\mathrm{RCHO} \stackrel{\mathrm{b}}{\longrightarrow} \mathrm{R}^{\prime} \mathrm{H}+\mathrm{RCO} \\
\mathrm{RCO} \stackrel{\text { fast }}{\longrightarrow} \mathrm{R}+\mathrm{CO} .
\end{array}
$$


However, H-abstractions at other positions lead to complications, particularly with regard to Requirement No. 2. For example, in the photochemical decomposition of isobutyraldehyde, the reactions of interest are:

$$
\begin{aligned}
&\left(\mathrm{CH}_{3}\right)_{2} \mathrm{CHCHO}+\mathrm{R} \cdot \stackrel{1}{\rightarrow}\left(\mathrm{CH}_{3}\right)_{2} \mathrm{CH} \dot{\mathrm{CO}}(\mathrm{RC} \mathrm{C})+\mathrm{RH} \\
& \mathrm{R}^{\prime} \dot{\mathrm{C}} \mathrm{O} \stackrel{2}{\rightarrow}\left(\mathrm{CH}_{3}\right)_{2} \dot{\mathrm{C}} \mathrm{H}(i \mathrm{Pr} \cdot)+\mathrm{CO} \\
& {\left[i \mathrm{Pr} \cdot \stackrel{3}{\rightarrow} \mathrm{H}+\mathrm{C}_{3} \mathrm{H}_{6}\right.} \\
& {\left[i \mathrm{Pr} \cdot \stackrel{4}{\rightarrow} \mathrm{CH}_{3}+\mathrm{C}_{2} \mathrm{H}_{4}\right] } \\
& 2 i \mathrm{Pr} \cdot \stackrel{5}{\rightarrow}(i \mathrm{Pr})_{2}
\end{aligned}
$$

Decompositions 3 and $4 *$ may be monitored by the rates of $\mathrm{H}_{2}$ or $\mathrm{C}_{3} \mathrm{H}_{6}$ production and of $\mathrm{CH}_{4}$ or $\mathrm{C}_{2} \mathrm{H}_{4}$ production, respectively. Complications are as follows: Down chain (type I) primary process fission (6) will lead to increased yields of methane

$$
\left(\mathrm{CH}_{3}\right)_{2} \mathrm{CHCHO} \stackrel{6}{\rightarrow}, \mathrm{CH}_{3} \dot{\mathrm{C}} \mathrm{HCHO}+\mathrm{CH}_{3}
$$

and, at higher temperatures, hydrogen (7). Calvert [56] has examined the data

$$
\mathrm{CH}_{3} \dot{\mathrm{C}} \mathrm{HCHO} \stackrel{7}{\rightarrow} \mathrm{H}+\mathrm{CH}_{3} \mathrm{CH}=\mathrm{C}=\mathrm{O}
$$

for this type of process and found it to be important.

Reactions (8) and (9) lead to increased propylene and $\mathrm{H}_{2}$ yields:

$$
\begin{aligned}
& \mathrm{R}+\mathrm{A} \stackrel{8}{\rightarrow} \cdot \mathrm{CH}_{2}-\mathrm{CH}\left(\mathrm{CH}_{3}\right) \mathrm{CHO}\left(\mathrm{R}^{\prime \prime} \mathrm{CO}\right)+\mathrm{RH} \\
& \mathrm{R}^{\prime \prime} \mathrm{CO} \stackrel{9}{\rightarrow} \mathrm{C}_{3} \mathrm{H}_{6}+\dot{\mathrm{C}} \mathrm{HO} .
\end{aligned}
$$

Corrections for these reactions have usually been made by determining the kinetics of reaction 8 at lower temperatures where reactions 3 and 4 are not important and subtracting the calculated propylene yields due to reaction 8 at higher temperature from the total propylene yields. The difference is then attributed to reaction 3 . A possible complication with this procedure, however, is that the nature of the radical $R$ (in reaction 8 ) undoubtedly changes with temperature. Thus at low temperatures, $R$ is principally $i \mathrm{Pr}$. while at high temperatures $R$ is

*Reaction 4 probably doesn't occur. principally $\mathrm{H}$ and $\mathrm{CH}_{3}$. This almost certainly will result in an increased importance to reactions 8 and 9 , since the $\mathrm{H}$-abstraction selectivity favoring the aldehydic hydrogen will no longer be very great. In fact, with $\mathrm{H}$-atoms, abstraction from the methyl groups of the aldehyde may even be favored. It is evident that identification of product yields with specific radical decomposition processes is not straightforward.

Requirement No. 3. Assuming that the radicals of interest are properly generated and their decomposition products can be unambiguously determined, the reaction rates must still be placed on an absolute basis. Generally decomposition and isomerization rates are measured relative to a monitoring or reference reaction whose kinetics are known. Radical-radical recombinations are the obvious choices. However, at the higher temperatures where the radicals are decomposing at an appreciable rate, the recombination reactions have ceased to be important and their products are not easily measured accurately. The usual procedure is to determine the relative rates of $\mathrm{H}$-abstraction to recombination at low temperatures (reactions 11 and 10 respectively).

$$
\begin{aligned}
& \mathrm{R} \cdot+\mathrm{R} \cdot \stackrel{10}{\rightarrow} \mathrm{R}_{2} \\
& \mathrm{R} \cdot+\mathrm{A} \stackrel{\mathrm{ll}}{\rightarrow} \mathrm{RH}+\mathrm{A}:_{-\mathrm{H}} .
\end{aligned}
$$

Corrections for the formation of $\mathrm{RH}$ by disproportionation (12) are also made.

$$
\mathrm{R} \cdot+\mathrm{R} \cdot \stackrel{12}{\rightarrow} \mathrm{RH}+\mathrm{R}_{-\mathrm{H}}
$$

The radical decomposition is then measured relative to the formation of $\mathrm{RH}$ via $\mathrm{H}$-abstraction from the parent compound.

Two difficulties are evident in this procedure. First, the $\mathrm{H}$-abstraction parameters may well change with temperature since abstraction at other positions on $A$ may become increasingly more important. Second, radical-radical recombination rates are not known very accurately. As a result, the values for reaction 10 are guessed, and generally range from $10^{10} \mathrm{l} /$ mole-sec to $10^{11} \mathrm{l} /$ mole-sec. This leads to an uncertainty of at least a factor of 3 in the absolute reaction rates.

It appears that all systems have complications and that a full understanding of all the secondary radical reactions taking place in any system is essential to a proper interpretation of kinetic data on any particular radical reaction. A good summary of the experimental approaches employed in the 
study of the alkyl radical reactions has been given by Kerr and Trotman-Dickenson [57].

In a relatively large fraction of the studies reviewed, no $A$-factors were reported. This is unfortunate, although understandable in view of the historic interest principally in activation energies. When such omissions occurred, given sufficient data in the paper, we have attempted to calculate the $A$-factors. These values are indicated by parenthesis on the data sheets. Assumptions required in these $A$-factor estimates are noted on the data sheets under "comments." We would like to sug. gest that considerably more work in the direct study of free radical unimolecular reactions is needed and should be pursued. Among the objec-

\section{A. H-atom elimination: $\left(T \sim 600^{\circ} \mathrm{K}\right)$}

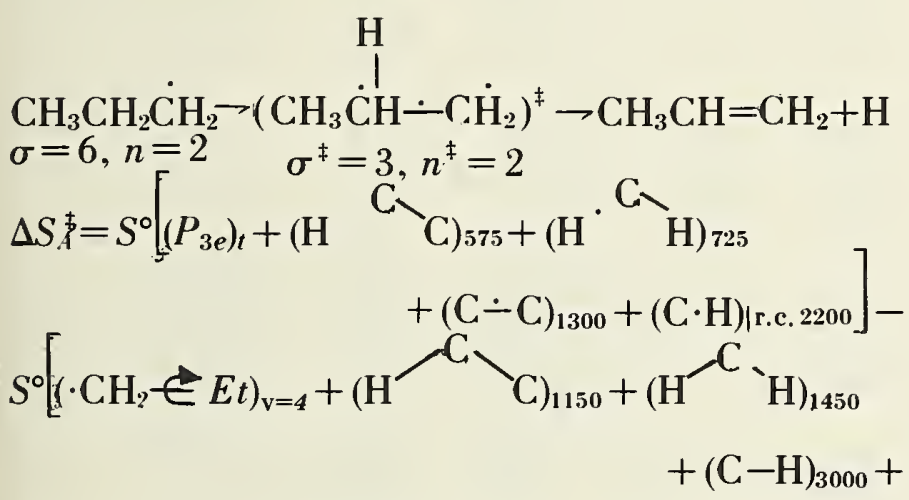

$\left.(\mathrm{C}-\mathrm{C})_{1000}\right]+R \ln \left(\frac{\sigma n^{\ddagger}}{\sigma^{\ddagger} n}\right)=[2.1+1.5+1.1+0.4$

+ r.c. $]-[5.6-0.9+0.5+0.3+0.7]+R \ln 4=+1.7$

$$
\begin{aligned}
& A_{\mathrm{H}-\mathrm{atom}} \\
& \qquad=\frac{e k \bar{T}}{h}\left\{\frac{\Delta S_{A}^{f}}{R}\right\}=10^{13.9} \mathrm{sec}^{-1}
\end{aligned}
$$

tives of such work should be the determining of both Arrhenius parameters $A$ and $E$, and their correla. tion with the reaction thermodynamics.

\subsection{Transition State Estimates of the $A$-factor for Radical Decompositions}

If one assumes that the transition states for the free radical reactions are relatively "tight" and that the vibrational frequencies of the various bonds are those suggested in Appendices B and C, then fairly reasonable estimates of the $A$-factors for these reactions can be made. Below are illustrated transition state calculations for decompositions of the $n$-propyl radical.

B. $\mathrm{CH}_{3}$ Elimination: $\left(T \sim 600^{\circ} \mathrm{K}\right)^{17}$

$\mathrm{CH}_{3} \mathrm{CH}_{2} \mathrm{CH}_{2} \cdot\left(\mathrm{CH}_{3} \cdot \mathrm{CH}_{2} \stackrel{-}{-} \mathrm{CH}_{2}\right)^{\ddagger} \rightarrow \mathrm{CH}_{3}+\mathrm{CH}_{2}=\mathrm{CH}_{2}$

$\begin{aligned} \sigma & =6, n=1 \quad \sigma^{\ddagger}=3, n^{\ddagger}=1 \\ \Delta S_{B}^{\ddagger} & =S^{\circ}\left[(\mathrm{C} \cdot \mathrm{C})_{\text {r.c. }}+(\mathrm{C}-\mathrm{C})+\left(P_{3 e}\right)_{t}+(\mathrm{C}\right.\end{aligned}$

$$
\begin{aligned}
-S^{\circ}\left[2(\mathrm{C}-\mathrm{C})+\left(\cdot \mathrm{CH}_{2}\right.\right. & \left.\in E_{t}\right)_{4}+(\mathrm{C}-\mathrm{C}) \\
& \left.+2\left(\mathrm{CH}_{3}\right)_{4 \text { e rocks }}+2\left(\mathrm{CH}_{3150}\right)_{3 e}{ }_{400}^{\text {rocks }}\right]+R \ln \left(\frac{6}{3}\right)
\end{aligned}
$$

$=[0.4+2.1+3.8+2(2.1)]-[2(0.7)+4.7$

$$
+2.4+2(0.5)]+1.4
$$

$\Delta S_{B}^{\ddagger}=+2.4$

$$
A_{\left.\mathrm{CH}_{3} \mathrm{elim}\right)} \simeq \frac{e k \bar{T}}{h}{ }_{e} \Delta S_{B}^{\ddagger} / R=10^{14.0} \mathrm{sec}^{-1}
$$

\footnotetext{
17 We have lowered the methyl rocks in the transition state by about a factor of three. See S. W. Benson, Thermochemical Kinetics, John Wiley \& Sons, New York (1968), p. 69.
} 


\section{Appendix A - Thermodynamic Group Additivities ${ }^{11}$}

TABLE A-1. Hydrocarbons. Group values for $\Delta \mathrm{H}_{f}^{\circ}, \mathrm{S}^{\circ}$, and $\mathrm{C}_{p}^{\circ}(1$ atm std state $)$.

\begin{tabular}{|c|c|c|c|c|c|c|c|c|c|}
\hline \multirow{2}{*}{ Group } & \multirow{2}{*}{$\Delta H_{f(2: ! x)}^{\circ}$} & \multirow{2}{*}{$S_{298}^{\circ}$} & \multicolumn{7}{|c|}{$C_{p}^{\circ}$} \\
\hline & & & 300 & 400 & 500 & 600 & 800 & 1000 & 1500 \\
\hline $\mathrm{C}-(\mathrm{H})_{3}(\mathrm{C})$ & -10.08 & 30.41 & 6.19 & 7.84 & 9.40 & 10.79 & 13.02 & 14.77 & 17.58 \\
\hline $\mathrm{C}-(\mathrm{H})_{2}(\mathrm{C})_{2}$ & -4.95 & 9.42 & 5.50 & 6.95 & 8.25 & 9.35 & 11.07 & 12.34 & 14.25 \\
\hline $\mathrm{C}-(\mathrm{H})(\mathrm{C})_{3}$ & -1.90 & -12.07 & 4.54 & 6.00 & 7.17 & 8.05 & 9.31 & 10.05 & 11.17 \\
\hline $\mathrm{C}-(\mathrm{C})_{4}$ & 0.50 & -35.10 & 4.37 & 6.13 & 7.36 & 8.12 & 8.77 & 8.76 & 8.12 \\
\hline $\mathrm{C}_{d}-(\mathrm{H})_{2}$ & 6.26 & 27.61 & 5.10 & 6.36 & 7.51 & 8.50 & 10.07 & 11.27 & 13.19 \\
\hline $\mathrm{C}_{d}-(\mathrm{H})(\mathrm{C})$ & 8.59 & 7.97 & 4.16 & 5.03 & 5.81 & 6.50 & 7.65 & 8.45 & 9.62 \\
\hline $\mathrm{C}_{d}-(\mathrm{C})_{2}$ & 10.34 & -12.70 & 4.10 & 4.61 & 4.99 & 5.26 & 5.80 & 6.08 & 6.36 \\
\hline $\mathrm{C}_{d}-\left(\mathrm{C}_{d}\right)(\mathrm{H})$ & 6.78 & 6.38 & 4.46 & 5.79 & 6.75 & 7.42 & 8.35 & 8.99 & 9.98 \\
\hline $\mathrm{C}_{d}-\left(\mathrm{C}_{d}\right)(\mathrm{C})$ & 8.88 & -14.6 & $(4.40)$ & (5.37) & (5.93) & $(6.18)$ & $(6.50)$ & $(6.62)$ & (6.72) \\
\hline $\mathrm{C}_{d}-\left(\mathrm{C}_{\mathrm{B}}\right)(\mathrm{H})$ & 6.78 & 6.38 & 4.46 & $5.7 \hat{9}$ & 6.75 & 7.42 & 8.35 & 8.99 & 9.98 \\
\hline$C_{d}-\left(C_{B}\right)(C)$ & 8.64 & $(-14.6)$ & $(4.40)$ & $(5.37)$ & $(5.93)$ & $(6.18)$ & $(6.50)$ & $(6.62)$ & (6.72) \\
\hline $\mathrm{C}_{d}-\left(\mathrm{C}_{t}\right)(\mathrm{H})$ & 6.78 & 6.38 & 4.46 & 5.79 & 6.75 & 7.42 & 8.35 & 8.99 & 9.98 \\
\hline $\mathrm{C}-\left(\mathrm{C}_{d}\right)(\mathrm{C})(\mathrm{H})_{2}$ & -4.76 & 9.80 & 5.12 & 6.86 & 8.32 & 9.49 & 11.22 & 12.48 & 14.36 \\
\hline $\mathrm{C}-\left(\mathrm{C}_{d}\right)_{2}(\mathrm{H})_{2}$ & -4.29 & $(10.2)$ & $(4.7)$ & $(6.8)$ & (8.4) & $(9.6)$ & (11.3) & $(12.6)$ & $(14.4)$ \\
\hline $\mathrm{C}-\left(\mathrm{C}_{d}\right)\left(\mathrm{C}_{\mathrm{B}}\right)(\mathrm{H})_{2}$ & -4.29 & $(10.2)$ & $(4.7)$ & (6.8) & (8.4) & $(9.6)$ & (11.3) & (12.6) & (14.4) \\
\hline $\mathrm{C}-\left(\mathrm{C}_{t}\right)(\mathrm{C})(\mathrm{H})_{2}$ & -4.73 & 10.30 & 4.95 & 6.56 & 7.93 & 9.08 & 10.86 & 12.19 & 14.20 \\
\hline $\mathrm{C}-\left(\mathrm{C}_{\mathrm{B}}\right)(\mathrm{C})(\mathrm{H})$ & -4.86 & 9.34 & 5.84 & 7.61 & 8.98 & 10.01 & 11.49 & 12.54 & 13.76 \\
\hline $\mathrm{C}-\left(\mathrm{C}_{d}\right)(\mathrm{C})_{2}(\mathrm{H})$ & -1.48 & $(-11.69)$ & $(4.16)$ & (5.91) & (7.34) & (8.19) & $(9.46)$ & $(10.19)$ & (11.28) \\
\hline $\mathrm{C}-\left(\mathrm{C}_{t}\right)(\mathrm{C})_{2}(\mathrm{H})$ & -1.72 & $(-11.19)$ & (3.99) & (5.61) & $(6.85)$ & (7.78) & (9.10) & $(9.90)$ & (11.12) \\
\hline $\mathrm{C}-\left(\mathrm{C}_{\mathrm{B}}\right)(\mathrm{C})_{2}(\mathrm{H})$ & -0.98 & $(-12.15)$ & $(4.88)$ & (6.66) & $(7.90)$ & $(8.75)$ & (9.73) & $(10.25)$ & (10.68) \\
\hline $\mathrm{C}-\left(\mathrm{C}_{d}\right)(\mathrm{C})_{3}$ & 1.68 & $(-34.72)$ & (3.99) & (6.04) & $(7.43)$ & $(8.26)$ & $(8.92)$ & $(8.96)$ & (8.23) \\
\hline $\mathrm{C}-\left(\mathrm{C}_{\mathrm{B}}\right)(\mathrm{C})_{3}$ & 2.81 & $(-35.18)$ & $(4.37)$ & (6.79) & (8.09) & (8.78) & (9.19) & $(8.96)$ & (7.63) \\
\hline $\mathrm{C}_{\mathrm{f}}-(\mathrm{H}) \mid$ & 26.93 & 24.7 & 5.27 & 5.99 & 6.49 & 6.87 & 7.47 & 7.96 & 8.85 \\
\hline $\mathrm{C}_{t}-(\mathrm{C})$ & 27.55 & 6.35 & 3.13 & 3.48 & 3.81 & 4.09 & 4.60 & 4.92 & 6.35 \\
\hline $\mathrm{C}_{t}-\left(\mathrm{C}_{d}\right)$ & 29.20 & $(6.43)$ & $(2.57)$ & (3.54) & $(3.50)$ & $(4.92)$ & (5.34) & $(5.50)$ & (5.80) \\
\hline $\mathrm{C}_{1}-\left(\mathrm{C}_{\mathrm{B}}\right)$ & $(29.20)$ & 6.43 & 2.57 & 3.54 & 3.50 & 4.92 & 5.34 & 5.50 & 5.80 \\
\hline $\mathrm{C}_{\mathrm{B}}-(\mathrm{H})$ & 3.30 & 11.53 & 3.24 & 4.44 & 5.46 & 6.30 & 7.54 & 8.41 & 9.73 \\
\hline$C_{B}-(C)$ & 5.51 & -7.69 & 2.67 & 3.14 & 3.68 & 4.15 & 4.96 & 5.44 & 5.98 \\
\hline $\mathrm{C}_{\mathrm{B}}-\left(\mathrm{C}_{d}\right)$ & 5.68 & -7.80 & 3.59 & 3.97 & 4.38 & 4.72 & 5.28 & 5.61 & 5.75 \\
\hline$C_{B}-\left(C_{\ell}\right)$ & 5.68 & -7.80 & 3.59 & 3.97 & 4.38 & 4.72 & 5.28 & 5.61 & 5.75 \\
\hline$C_{B}-\left(C_{B}\right)$ & 5.26 & -8.64 & 3.33 & 4.22 & 4.89 & 5.27 & 5.76 & 5.95 & (6.05) \\
\hline $\mathrm{C}_{a}$ & 34.20 & 6.0 & 3.9 & 4.4 & 4.7 & 5.0 & 5.3 & 5.5 & 5.7 \\
\hline
\end{tabular}

"These group values have been taken from ref. 3-Section 1-5.0.

TABLE A-1. Next-nearest neighbor corrections

\begin{tabular}{|c|c|c|c|c|c|c|c|c|c|}
\hline \multirow{2}{*}{ Group } & \multirow{2}{*}{$\Delta H_{f(29 \mathrm{y})}^{\circ}$} & \multirow{2}{*}{$S_{298}^{\circ}$} & \multicolumn{7}{|c|}{$C_{p}^{\circ}$} \\
\hline & & & 300 & 400 & 500 & 600 & 800 & 1000 & 1500 \\
\hline $\begin{array}{l}\text { alkane gauche correction } \\
\text { alkene gauche correction } \\
\text { cis correction } \\
\text { ortho correction }\end{array}$ & $\begin{array}{r}0.80 \\
0.50 \\
1.00 \\
0.57\end{array}$ & $\begin{array}{l}\left({ }^{b}\right) \\
-1.61\end{array}$ & $\begin{array}{r}-1.34 \\
1.12\end{array}$ & $\begin{array}{r}-1.09 \\
1.35\end{array}$ & $\begin{array}{r}-0.81 \\
1.30\end{array}$ & $\begin{array}{r}-0.61 \\
1.17\end{array}$ & $\begin{array}{r}-0.39 \\
0.88\end{array}$ & $\begin{array}{r}-0.26 \\
0.66\end{array}$ & $\begin{array}{c}0 \\
-0.05\end{array}$ \\
\hline
\end{tabular}

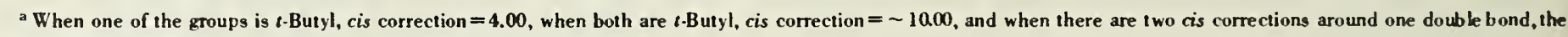
total correction is 3.00 .

${ }^{b}+1.2$ for but-2-ene, 0 for all other $2 \cdot e n e s$, and -0.6 for 3 -enes. 
TABLE A-1. Corrections to be applied to ring compound estimates

\begin{tabular}{|c|c|c|c|c|c|c|c|c|c|}
\hline \multirow{2}{*}{ Ring $(\sigma)$} & \multirow{2}{*}{$\Delta H_{f(: 9 x)}^{\circ}$} & \multirow{2}{*}{$S_{298}^{\circ}$} & \multicolumn{7}{|c|}{$C_{p}^{\circ}$} \\
\hline & & & 300 & 400 & 500 & 600 & 800 & 1000 & 1500 \\
\hline $\begin{array}{l}\text { cyclopropane }(6) \\
\text { cyclopropene (2) } \\
\text { cyclobutane (8) } \\
\text { cyclobutene (2) } \\
\text { cyclopentane (10) } \\
\text { cyclopentene (2) } \\
\text { cyclopentadiene } \\
\text { cyclohexane (6) } \\
\text { cyclohexene (2) } \\
\text { cyclohexadiene } 1,3 \\
\text { cyclohexadiene } 1,4 \\
\text { cycloheptane (1) } \\
\text { cycloheptene } \\
\text { cycloheptadiene } 1,3 \\
\text { cycloheptatriene } 1,3,5(1) \\
\text { cycloöctane }(8) \\
\text { cis-cycloöctene } \\
\text { trans-cycloöctene } \\
\text { cycloöctatriene } 1,3,5 \\
\text { cycloöctatetraene } \\
\text { cyclononane } \\
\text { cis-cyclononene } \\
\text { trans-cyclononene } \\
\text { spiropentane }(4) \\
\text { bicyclo(1,1,0)-butane }(2) \\
\text { bicyclo(2,1,0)-pentane } \\
\text { bicyclo(3,1,0)-hexane } \\
\text { bicyclo(4,1,0)-heptane } \\
\text { bicyclo(5,1,0)-octane } \\
\text { bicycloheptadiene }\end{array}$ & $\begin{array}{r}27.6 \\
53.7 \\
26.2 \\
29.8 \\
6.3 \\
5.9 \\
6.0 \\
0 \\
1.4 \\
4.8 \\
0.5 \\
6.4 \\
5.4 \\
6.6 \\
4.7 \\
9.9 \\
6.0 \\
15.3 \\
8.9 \\
17.1 \\
12.8 \\
9.9 \\
12.8 \\
63.5 \\
68.4 \\
55.3 \\
32.7 \\
28.9 \\
29.6 \\
29.7\end{array}$ & $\begin{array}{l}32.1 \\
33.6 \\
29.8 \\
29.0 \\
27.3 \\
25.8 \\
\\
18.8 \\
21.5 \\
\\
15.9 \\
\\
23.7 \\
16.5\end{array}$ & $\begin{array}{l}-3.05 \\
-4.61 \\
-2.53 \\
-6.5 \\
-5.98 \\
\\
-5.8 \\
-4.28\end{array}$ & $\begin{array}{l}-2.53 \\
-3.89 \\
-2.19 \\
-5.5 \\
-5.35 \\
-4.10 \\
-3.04\end{array}$ & $\begin{array}{l}-2.10 \\
-3.14 \\
-1.89 \\
-4.5 \\
-4.89 \\
-2.9 \\
-1.98\end{array}$ & $\begin{array}{l}-1.90 \\
-2.64 \\
-1.63 \\
-3.8 \\
-4.14 \\
-1.3 \\
-1.43\end{array}$ & $\begin{array}{l}-1.77 \\
-1.88 \\
-1.48 \\
-2.8 \\
-2.93 \\
\\
1.1 \\
-0.29\end{array}$ & $\begin{array}{r}-1.62 \\
-1.38 \\
-1.33 \\
-1.93 \\
-2.26 \\
\\
2.2 \\
0.08\end{array}$ & $\begin{array}{c}(-1.52) \\
-0.67 \\
-1.22 \\
-0.37 \\
-1.08 \\
\\
3.3 \\
0.81\end{array}$ \\
\hline
\end{tabular}

TABle A-2. Oxygen containing compounds. Group Contributions to $\Delta \mathrm{H}_{f}^{c}, \mathrm{~S}^{\circ}$, and $\mathrm{C}_{p}^{\circ}$

\begin{tabular}{|c|c|c|c|c|c|c|c|c|c|}
\hline \multirow{2}{*}{ Group } & \multirow{2}{*}{$\Delta H_{f(298)}^{\circ}$} & \multirow{2}{*}{$S_{29 \mathrm{~S}}^{\circ}$} & \multicolumn{7}{|c|}{$C_{p}^{\circ}$} \\
\hline & & & 300 & 400 & 500 & 600 & 800 & 1000 & 1500 \\
\hline $\begin{array}{l}\mathrm{CO}-(\mathrm{CO})\left(\mathrm{C}_{B}\right) \\
\mathrm{CO}-(\mathrm{CO})(\mathrm{C}) \\
\mathrm{CO}-(\mathrm{CO})(\mathrm{O}) \\
\mathrm{CO}-(\mathrm{O})\left(\mathrm{C}_{d}\right) \\
\mathrm{CO}-(\mathrm{O})\left(\mathrm{C}_{\mathrm{B}}\right)\end{array}$ & $\begin{array}{l}(-30.5) \\
-29.2 \\
(-32.6) \\
-33.5 \\
-42.4\end{array}$ & $(12.0)$ & $(5.5)$ & & & & & & \\
\hline $\mathrm{CO}-(\mathrm{O})(\mathrm{C})$ & -33.4 & 14.78 & 5.97 & 6.70 & 7.4 & 8.02 & 8.87 & 9.36 & \\
\hline $\mathrm{CO}-(\mathrm{O})(\mathrm{H})^{\mathrm{a}}$ & -29.5 & 34.93 & 7.03 & 7.87 & 8.82 & 9.68 & 11.16 & 12.20 & \\
\hline $\mathrm{CO}-\left(\mathrm{C}_{\mathrm{d}}\right)(\mathrm{H})$ & -31.7 & $(32.9)$ & $(6.0)$ & & & & & & \\
\hline $\mathrm{CO}-\left(\mathrm{C}_{\mathrm{B}}\right)_{2}$ & -39.1 & $(12.0)$ & & & & & & & \\
\hline $\mathrm{CO}^{-}-\left(\mathrm{C}_{\mathrm{B}}\right)(\mathrm{C})$ & -37.6 & (13.7) & (6.1) & & & & & & \\
\hline $\mathrm{CO}-\left(\mathrm{C}_{\mathrm{B}}\right)(\mathrm{H})^{\mathrm{b}}$ & -31.7 & $(32.9)$ & (6.5) & & & & & & \\
\hline $\mathrm{CO}-(\mathrm{C})_{2}$ & -31.5 & 15.01 & 5.59 & 6.32 & 7.09 & 7.76 & 8.89 & 9.61 & \\
\hline $\mathrm{CO}-(\mathrm{C})(\mathrm{H})$ & -29.6 & 34.93 & 7.03 & 7.87 & 8.82 & 9.68 & 11.16 & 12.20 & \\
\hline $\mathrm{CO}-(\mathrm{H})_{2}$ & -27.7 & 53.67 & 8.47 & 9.38 & 10.46 & 11.52 & 13.37 & 14.81 & \\
\hline
\end{tabular}


TABLE A-2. Oxygen containing compounds. Group Contributions to $\Delta \mathrm{H}_{f}^{\circ}, \mathrm{S}^{\circ}$, and $\mathrm{C}_{p}^{\circ}$-Continued

\begin{tabular}{|c|c|c|c|c|c|c|c|c|c|}
\hline \multirow{2}{*}{ Group } & \multirow{2}{*}{$\Delta H_{f(298)}^{\circ}$} & \multirow{2}{*}{$S_{298}^{\circ}$} & \multicolumn{7}{|c|}{$C_{p}^{\circ}$} \\
\hline & & & 300 & 400 & 500 & 600 & 800 & 1000 & 1500 \\
\hline $\begin{array}{l}\mathrm{O}-(\mathrm{CO})_{2} \\
\mathrm{O}-(\mathrm{CO})(\mathrm{O}) \\
\mathrm{O}-(\mathrm{CO})\left(\mathrm{C}_{\mathrm{d}}\right)^{c} \\
\mathrm{O}-(\mathrm{CO})(\mathrm{C}) \\
\mathrm{O}-(\mathrm{CO})(\mathrm{H}) \\
\mathrm{O}-(\mathrm{O})(\mathrm{C})\end{array}$ & $\begin{array}{r}-50.9 \\
-19.0 \\
-41.3 \\
-41.3 \\
-60.3 \\
-4.5\end{array}$ & $\begin{array}{r}(8.5) \\
(8.4) \\
\\
8.39 \\
24.52 \\
(9.0)\end{array}$ & $\begin{array}{r}(3.4) \\
(3.5) \\
(3.5) \\
3.81\end{array}$ & 4.98 & 5.80 & 6.34 & 7.19 & 7.75 & \\
\hline $\begin{array}{l}\mathrm{O}-(\mathrm{O})(\mathrm{H}) \\
\mathrm{O}-\left(\mathrm{C}_{\mathrm{d}}\right)_{2} \\
\mathrm{O}-\left(\mathrm{C}_{\mathrm{d}}\right)(\mathrm{C}) \\
\mathrm{O}-\left(\mathrm{C}_{\mathrm{B}}\right)_{2} \\
\mathrm{O}-\left(\mathrm{C}_{\mathrm{B}}\right)(\mathrm{C}) \\
\mathrm{O}-\left(\mathrm{C}_{\mathrm{B}}\right)(\mathrm{H})^{\mathrm{d}} \\
\mathrm{O}-(\mathrm{C})_{2} \\
\mathrm{O}-(\mathrm{C})(\mathrm{H})\end{array}$ & $\begin{array}{l}-16.27 \\
-32.8 \\
-31.3 \\
-19.3 \\
-22.6 \\
-37.9 \\
-23.7 \\
-37.88\end{array}$ & $\begin{array}{c}28.52 \\
8.1 \\
(8.5) \\
(8.1) \\
(8.3) \\
29.1 \\
8.68 \\
29.07\end{array}$ & $\begin{array}{c}5.17 \\
(4.2) \\
(3.5) \\
(4.2) \\
(3.8) \\
4.3 \\
3.4 \\
4.33\end{array}$ & $\begin{array}{l}4.5 \\
3.7 \\
4.45\end{array}$ & $\begin{array}{l}4.8 \\
3.7 \\
4.82\end{array}$ & $\begin{array}{l}5.2 \\
3.8 \\
5.23\end{array}$ & $\begin{array}{l}6.0 \\
4.4 \\
6.02\end{array}$ & $\begin{array}{l}6.6 \\
4.6 \\
6.61\end{array}$ & 8.17 \\
\hline $\begin{array}{l}\mathrm{C}_{\mathrm{d}}-(\mathrm{CO})(\mathrm{O}) \\
\mathrm{C}_{\mathrm{d}}-(\mathrm{CO})(\mathrm{C}) \\
\mathrm{C}_{d}-(\mathrm{CO})(\mathrm{H})^{\mathrm{e}} \\
\mathrm{C}_{\mathrm{d}}-(\mathrm{O})\left(\mathrm{C}_{\mathrm{d}}\right)^{\mathrm{f}} \\
\mathrm{C}_{\mathrm{d}}-(\mathrm{U})(\mathrm{C})^{\mathrm{g}} \\
\mathrm{C}_{\mathrm{d}}-(\mathrm{O})(\mathrm{H})^{\mathrm{h}}\end{array}$ & $\begin{array}{c}6.3 \\
9.4 \\
7.68 \\
8.9 \\
\\
10.3 \\
8.6\end{array}$ & 8.0 & 4.16 & 5.03 & 5.81 & 5.50 & 7.65 & 8.45 & 9.62 \\
\hline $\begin{array}{l}\mathrm{C}_{\mathrm{B}}-(\mathrm{CO}) \\
\mathrm{C}_{\mathrm{B}}-(\mathrm{O})\end{array}$ & $\begin{array}{r}9.7 \\
-1.8\end{array}$ & $\begin{array}{r}-9.6 \\
-10.2\end{array}$ & $\begin{array}{c}(3.9) \\
3.9\end{array}$ & 5.3 & 6.2 & 6.6 & 6.9 & 6.9 & \\
\hline $\mathrm{C}-(\mathrm{CO})_{2}(\mathrm{H})_{2}$ & -7.2 & & & & & & & & \\
\hline $\mathrm{C}-(\mathrm{CO})(\mathrm{C})_{2}(\mathrm{H})^{i}$ & -1.8 & $(-12.0)$ & $(5.0)$ & & & & & & \\
\hline $\mathrm{C}-(\mathrm{CO})(\mathrm{C})(\mathrm{H})_{2}$ & -5.0 & 9.6 & 6.2 & 7.7 & 8.7 & 9.5 & 11.1 & 12.2 & \\
\hline $\begin{array}{l}\mathrm{C}-(\mathrm{CO})(\mathrm{H})_{3}{ }^{\mathrm{j}} \\
\mathrm{C}-(\mathrm{O})_{2}(\mathrm{C})_{2}\end{array}$ & $\begin{array}{l}-10.08 \\
-18.1\end{array}$ & 30.41 & 6.19 & 7.84 & 9.40 & 10.79 & 13.02 & 14.77 & 17.58 \\
\hline $\mathrm{C}-(\mathrm{O})_{2}(\mathrm{C})(\mathrm{H})$ & -17.2 & & & & & & & & \\
\hline $\begin{array}{l}\mathrm{C}-(\mathrm{O})_{2}(\mathrm{H})_{2} \\
\mathrm{C}-(\mathrm{O})\left(\mathrm{C}_{\mathrm{B}}\right)(\mathrm{H})_{2}\end{array}$ & $\begin{array}{r}-17.7 \\
-6.6\end{array}$ & 9.7 & & & & & & & \\
\hline $\mathrm{C}-(\mathrm{O})\left(\mathrm{C}_{\mathrm{d}}\right)(\mathrm{H})_{2}$ & -6.9 & & & & & & & & \\
\hline $\mathrm{C}-(\mathrm{O})(\mathrm{C})_{3}$ & -6.60 & -33.56 & 4.33 & 6.19 & 7.25 & 7.70 & 8.20 & 8.24 & \\
\hline $\mathrm{C}-(\mathrm{O})(\mathrm{C})_{2}(\mathrm{H})$ & -7.00 & -11.35 & 4.80 & 6.64 & 8.10 & 8.73 & 9.81 & 10.40 & \\
\hline $\mathrm{C}-(\mathrm{O})(\mathrm{C})(\mathrm{H})_{2}$ & -8.5 & 10.3 & 4.99 & 6.85 & 8.30 & 9.43 & 11.11 & 12.33 & \\
\hline $\mathrm{C}-(\mathrm{O})(\mathrm{H})_{3}{ }^{\mathrm{k}}$ & -10.08 & 30.41 & 6.19 & 7.84 & 9.40 & 10.79 & 13.02 & 14.77 & 17.58 \\
\hline
\end{tabular}

Corrections to be Applied to Ring Compound Estimates
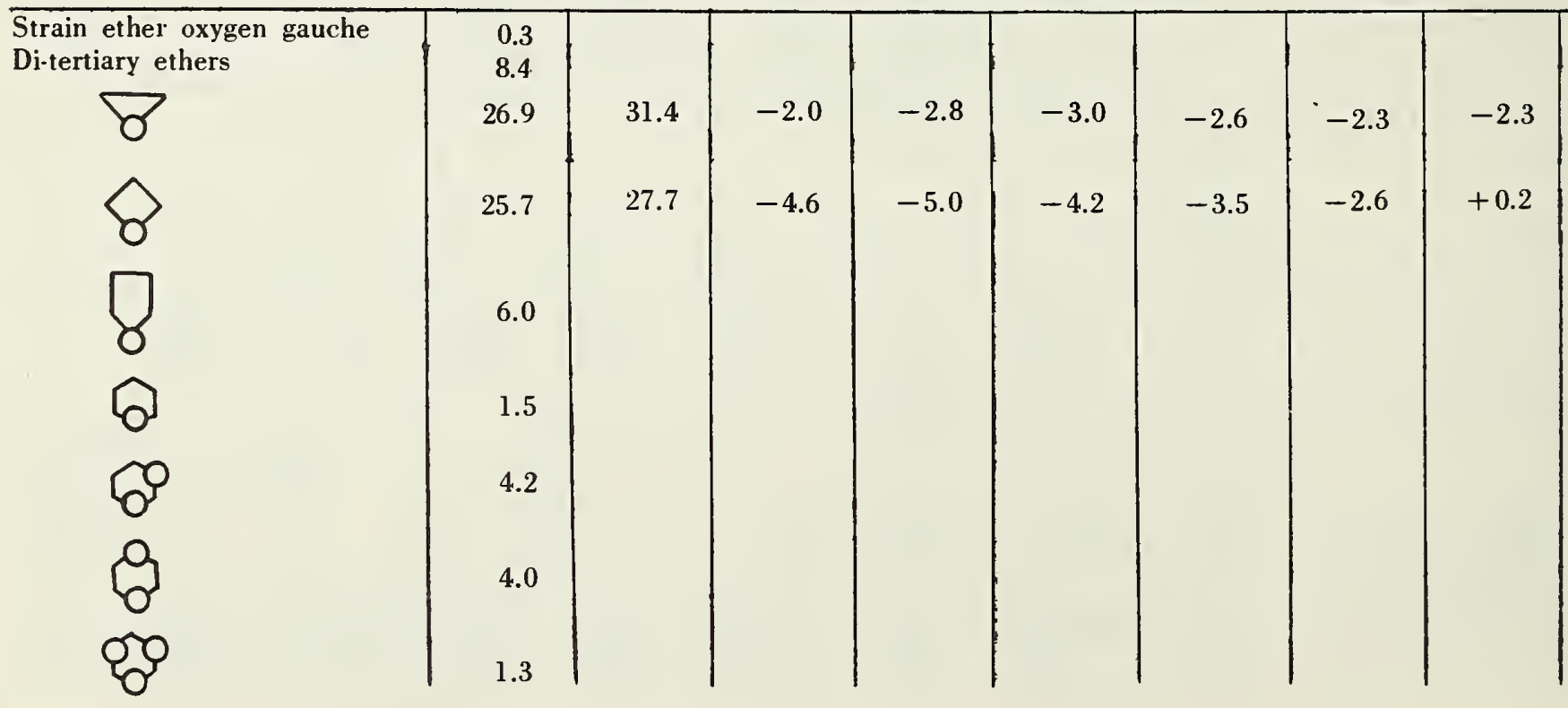
TABLE A-2. Oxygen containing compounds. Group Contributions to $\Delta \mathrm{H}_{f}^{\circ}, \mathrm{S}^{\circ}$, and $\mathrm{C}_{p}^{\circ}$-Continued

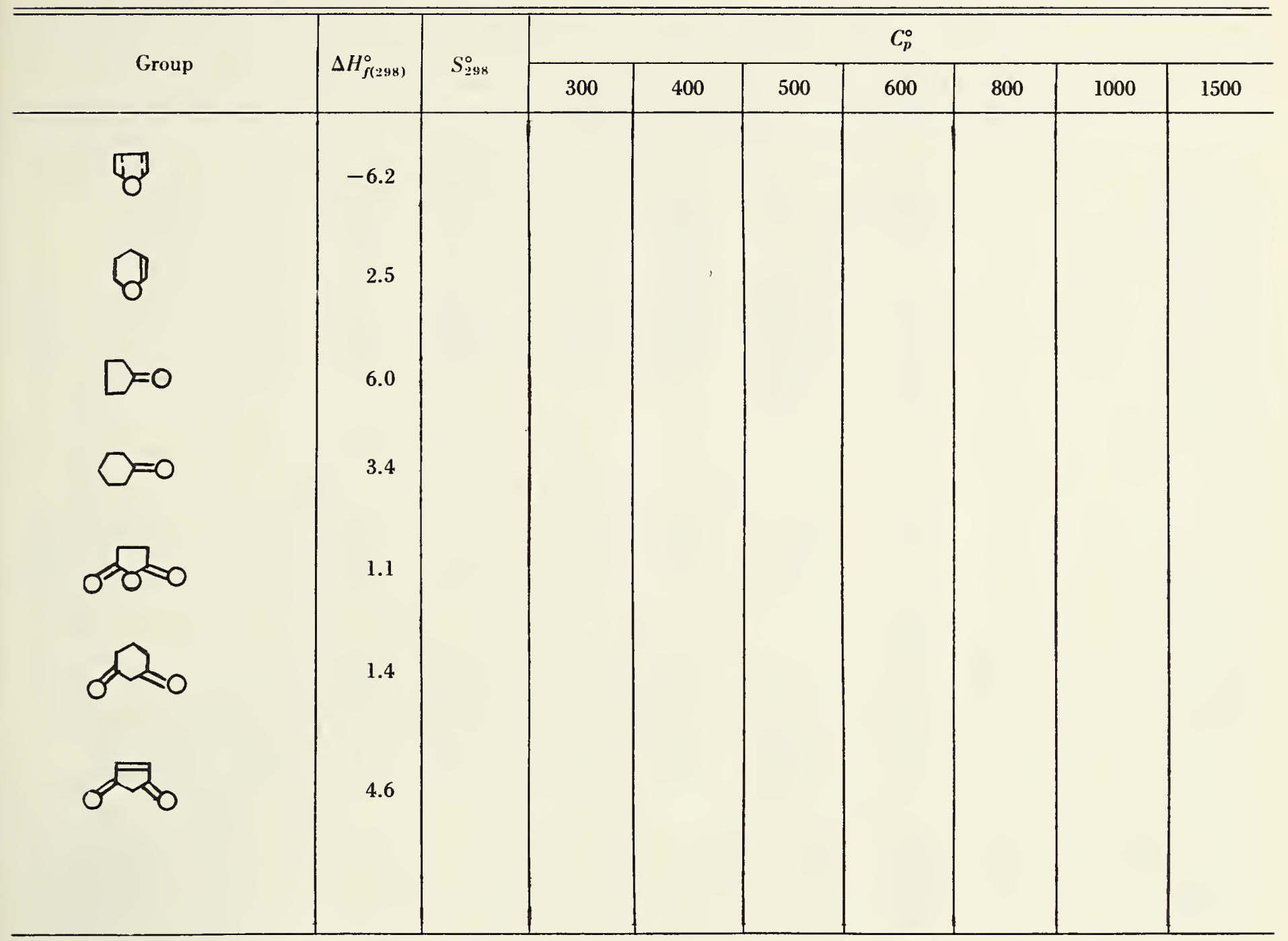

${ }^{a} \mathrm{CO}-(\mathrm{O})(\mathrm{H}) \equiv \mathrm{CO}-(\mathrm{C})(\mathrm{H})$, assigned.

${ }^{b} \mathrm{CO}-\left(\mathrm{C}_{\mathrm{B}}\right)(\mathrm{H}) \equiv \mathrm{CO}-\left(\mathrm{C}_{\mathrm{d}}\right)(\mathrm{H})$, assigned.

${ }^{\mathrm{c}} \mathrm{O}-(\mathrm{CO})\left(\mathrm{C}_{\mathrm{d}}\right) \equiv \mathrm{O}-(\mathrm{CO})(\mathrm{C})$, assigned.

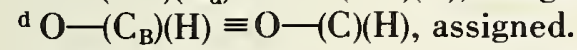

${ }^{e} \mathrm{C}_{d}-(\mathrm{CO})(\mathrm{H}) \equiv$ mean of $\mathrm{C}_{d}-\left(\mathrm{C}_{\mathrm{d}}\right)(\mathrm{H})$ and $\mathrm{C}_{\mathrm{d}}-(\mathrm{C})(\mathrm{H})$, assigned.

${ }^{\prime} \mathrm{C}_{\mathrm{d}}-(\mathrm{O})\left(\mathrm{C}_{\mathrm{d}}\right) \equiv \mathrm{C}_{\mathrm{d}}-\left(\mathrm{C}_{\mathrm{d}}\right)(\mathrm{C})$.

${ }^{g} \mathrm{C}_{\mathrm{d}}-(\mathrm{O})(\mathrm{C}) \equiv \mathrm{C}_{\mathrm{d}}-\mathrm{C}_{2}$.

${ }^{\text {h }} \mathrm{C}_{d}-(\mathrm{O})(\mathrm{H}) \equiv \mathrm{C}_{\mathrm{d}}-(\mathrm{C})(\mathrm{H})$, assigned.

${ }^{i} \mathrm{C}-(\mathrm{CO})(\mathrm{C})_{2}(\mathrm{H})$, estımated.

${ }^{\mathrm{j}} \mathrm{C}-(\mathrm{CO})(\mathrm{H})_{3} \equiv \mathrm{C}-(\mathrm{C})(\mathrm{H})_{3}$, assigned.

${ }^{\mathrm{k}} \mathrm{C}-(\mathrm{O})(\mathrm{H})_{3} \equiv \mathrm{C}-(\mathrm{C})(\mathrm{H})_{3}$, assigned. 
TABLE A-3. Group contributions to $\mathrm{C}_{\mu}^{\circ}, \mathrm{S}^{\circ}$, and $\Delta \mathrm{H}_{j}^{\circ} \mathrm{b}, \mathrm{c}(1$ atm std state) for nitrogen-containing compounds.

\begin{tabular}{|c|c|c|c|c|c|c|c|c|c|}
\hline \multirow{2}{*}{ Group } & \multirow{2}{*}{$\begin{array}{c}\Delta H^{\circ} \\
\text { (kcal/mole) }\end{array}$} & \multirow{2}{*}{$\begin{array}{c}S^{\circ} \\
\text { (e. u.) }\end{array}$} & \multicolumn{7}{|c|}{$C_{p}^{\circ}($ e. u. $)$} \\
\hline & & & 300 & 400 & 500 & 600 & 800 & 1000 & 1500 \\
\hline $\begin{array}{l}\mathrm{C}-(\mathrm{N})(\mathrm{H})_{3} \\
\mathrm{C}-(\mathrm{N})(\mathrm{C})(\mathrm{H})_{2} \\
\mathrm{C}-(\mathrm{N})(\mathrm{C})_{2}(\mathrm{H}) \\
\mathrm{C}-(\mathrm{N})(\mathrm{C})_{3} \\
\mathrm{~N}-(\mathrm{C})(\mathrm{H})_{2} \\
\mathrm{~N}-(\mathrm{C})_{2}(\mathrm{H}) \\
\mathrm{N}-(\mathrm{C})_{3} \\
\mathrm{~N}-(\mathrm{N})(\mathrm{H})_{2} \\
\mathrm{~N}-(\mathrm{N})(\mathrm{C})(\mathrm{H}) \\
\mathrm{N}-(\mathrm{N})(\mathrm{C})_{2} \\
\mathrm{~N}-(\mathrm{N})\left(\mathrm{C}_{\mathrm{B}}\right)(\mathrm{H}) \\
\mathrm{N}_{1}-(\mathrm{H})^{\mathrm{a}} \\
\mathrm{N}_{1}-(\mathrm{C}) \\
\left.\mathrm{N}_{1}-(\mathrm{C})_{\mathrm{B}}\right)^{\mathrm{e}} \\
\mathrm{N}_{\mathrm{A}}-(\mathrm{H})^{\mathrm{a}} \\
\mathrm{N}_{\mathrm{A}}-(\mathrm{C}) \\
\mathrm{N}-\left(\mathrm{C}_{\mathrm{B}}\right)(\mathrm{H})_{2} \\
\mathrm{~N}-\left(\mathrm{C}_{\mathrm{B}}\right)(\mathrm{C})(\mathrm{H}) \\
\mathrm{N}-(\mathrm{C})(\mathrm{C})_{2} \\
\mathrm{~N}-\left(\mathrm{C}_{\mathrm{B}}\right)_{2}(\mathrm{H}) \\
\mathrm{C}_{\mathrm{B}}-(\mathrm{N}) \\
\mathrm{N}_{\mathrm{A}}-(\mathrm{N}) \\
\mathrm{CO}-(\mathrm{N})(\mathrm{H}) \\
\mathrm{CO}-(\mathrm{N})(\mathrm{C}) \\
\mathrm{N}-(\mathrm{CO})(\mathrm{H})_{2} \\
\mathrm{~N}-(\mathrm{CO})(\mathrm{C})(\mathrm{H}) \\
\mathrm{N}-(\mathrm{CO})(\mathrm{C})_{2} \\
\mathrm{~N}-(\mathrm{CO})\left(\mathrm{C}_{\mathrm{B}}\right)(\mathrm{H}) \\
\mathrm{N}-(\mathrm{CO})_{2}(\mathrm{H}) \\
\mathrm{N}-(\mathrm{CO})_{2}(\mathrm{C}) \\
\mathrm{N}-(\mathrm{CO})_{2}\left(\mathrm{C}_{\mathrm{B}}\right)\end{array}$ & $\begin{array}{r}-10.08 \\
-6.6 \\
-5.2 \\
-3.2 \\
4.8 \\
15.4 \\
24.4 \\
11.4 \\
20.9 \\
29.2 \\
22.1 \\
\\
21.3 \\
16.7 \\
25.1 \\
32.0 \\
4.8 \\
14.9 \\
26.2 \\
16.3 \\
-0.5 \\
23.0 \\
-29.6 \\
-32.8 \\
-14.9 \\
-4.4 \\
+0.4 \\
-18.5 \\
-5.9 \\
-0.5\end{array}$ & $\begin{array}{r}30.41 \\
\mathrm{~d} 9.8 \\
\mathrm{~d}-11.7 \\
\mathrm{~d}-34.1 \\
29.71 \\
8.94 \\
-13.46 \\
29.13 \\
9.61 \\
-13.80\end{array}$ & $\begin{array}{r}6.19 \\
\text { d } 5.25 \\
\text { d } 4.67 \\
\text { d } 4.35 \\
5.72 \\
4.20 \\
3.48 \\
6.10 \\
4.82\end{array}$ & $\begin{array}{r}7.84 \\
\mathrm{~d} 6.90 \\
\mathrm{~d} 6.32 \\
\mathrm{~d} 6.16 \\
6.51 \\
5.21 \\
4.56 \\
7.38 \\
5.8\end{array}$ & $\begin{array}{r}9.40 \\
\text { d } 8.28 \\
\text { d } 7.64 \\
\text { d } 7.31 \\
7.32 \\
6.13 \\
5.43 \\
8.43 \\
6.5\end{array}$ & $\begin{array}{r}10.79 \\
\text { d } 9.39 \\
\text { d } 8.39 \\
\text { d } 7.91 \\
8.07 \\
6.83 \\
5.97 \\
9.27 \\
7.0\end{array}$ & $\begin{array}{r}13.02 \\
\text { d } 11.09 \\
\text { d } 9.56 \\
\text { d } 8.49 \\
9.41 \\
7.90 \\
6.56 \\
10.54 \\
7.8\end{array}$ & $\begin{array}{r}14.77 \\
\text { d } 12.34 \\
\text { d } 10.23 \\
\text { d } 8.50 \\
10.47 \\
8.65 \\
6.67 \\
11.52 \\
8.3\end{array}$ & $\begin{array}{r}17.58 \\
\\
\\
12.28 \\
9.55 \\
6.50 \\
13.19 \\
9.0\end{array}$ \\
\hline
\end{tabular}

a $\mathrm{N}_{\mathrm{I}}=$ double bonded nitrogen in Imines; $\mathrm{N}_{r}-\left(\mathrm{C}_{\mathrm{B}}\right)=$ pyridine $\mathrm{N}$.

$\mathrm{N}_{\mathrm{A}}=$ double bonded nitrogen in Azo compounds.

b No cis corrections applied to Imines or Azo compounds.

${ }^{c}$ Gauche corrections of $+0.8 \mathrm{kcal} / \mathrm{mole}$ to $\Delta H_{f}^{\circ}$ applied just as for hydrocarbons.

d Estimates by authors.

e For ortho or para substitution in pyridine add $-1.5 \mathrm{kcal} / \mathrm{mole}$ per group. 
TABLE A-3. Group contributions to $\mathrm{C}_{p}^{\circ}, \mathrm{S}^{\circ}$, and $\Delta \mathrm{H}_{f}^{\circ}$, e $(1$ atm std state $)$ for nitrogen-containing compounds. - Continued

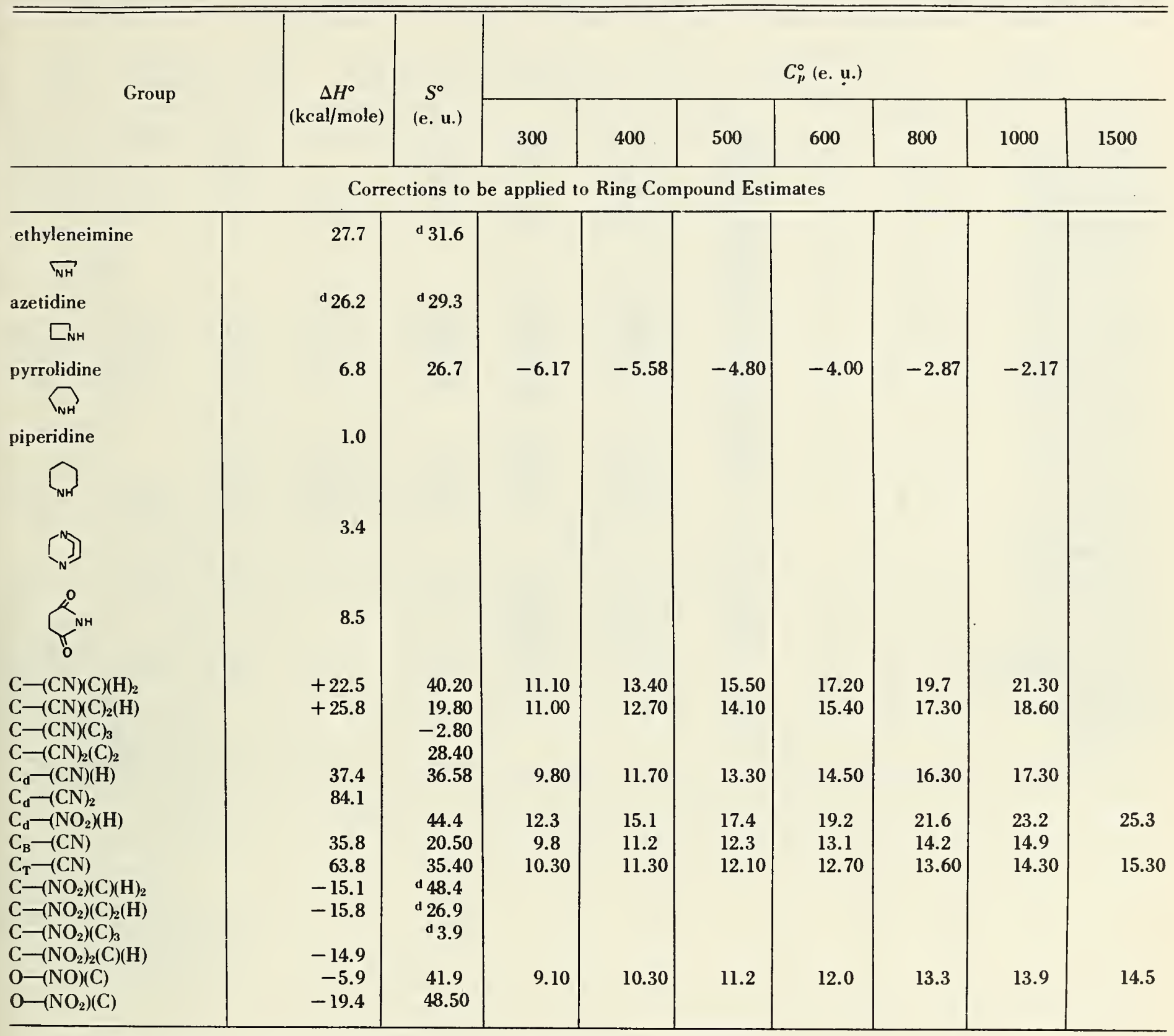

${ }^{\text {a }} \mathrm{N}_{\mathrm{l}}=$ double bonded nitrogen in Imines: $\mathrm{N}_{1}-\left(\mathrm{C}_{\mathrm{B}}\right)=$ pyridine $\mathrm{N}$.

$\mathrm{N}_{\mathrm{A}}$ double bonded nitrogen in Azo compounds.

b No cis corrections applied to Imines or Azo compounds.

c Gauche corrections of $+0.8 \mathrm{kcal} / \mathrm{mole}$ to $\Delta H_{f}^{\circ}$ applied just as for hydrocarbons.

d Estimates by authors.

e For ortho or para substitution in pyridine add $-1.5 \mathrm{kcal} / \mathrm{mole}$ per group. 
TABLE A-4. Halo-alkanes group contribution to $\Delta \mathrm{H}_{f(298)}^{\circ}, \mathrm{S}_{298}^{\circ}$ and $\mathrm{C}_{p}^{\circ}$, ideal gas at 1 atm std. state

\begin{tabular}{|c|c|c|c|c|c|c|c|c|}
\hline \multirow{2}{*}{ Group } & \multirow{2}{*}{$\Delta H_{f(298)}^{\circ}$} & \multirow{2}{*}{$S_{298}^{\circ}$} & \multicolumn{6}{|c|}{$C_{p}^{\circ}$} \\
\hline & & & 300 & 400 & 500 & 600 & 800 & 1000 \\
\hline $\begin{array}{l}\mathrm{C}-(\mathrm{F})_{3}(\mathrm{C}) \\
\mathrm{C}-(\mathrm{F}) \mathrm{F}_{2}(\mathrm{H})(\mathrm{C}) \\
\mathrm{C}-(\mathrm{F})(\mathrm{H})_{2}(\mathrm{C}) \\
\mathrm{C}-\mathrm{F}(\mathrm{F})_{2}(\mathrm{C})_{2} \\
\mathrm{C}-(\mathrm{F})(\mathrm{H})(\mathrm{C})_{2} \\
\mathrm{C}-(\mathrm{F})_{(\mathrm{C}))_{3}} \\
\mathrm{C}-(\mathrm{F})_{2}(\mathrm{Cl})(\mathrm{C}) \\
\mathrm{C}-(\mathrm{Cl})_{3}(\mathrm{C}) \\
\mathrm{C}-(\mathrm{Cl})_{2}(\mathrm{H})(\mathrm{C}) \\
\mathrm{C}-(\mathrm{Cl})(\mathrm{H})_{2}(\mathrm{C}) \\
\mathrm{C}-(\mathrm{Cl})_{2}(\mathrm{C})_{2} \\
\mathrm{C}-(\mathrm{Cl})(\mathrm{H})(\mathrm{C})_{2} \\
\mathrm{C}-(\mathrm{Cl})(\mathrm{C})_{3} \\
\mathrm{C}-(\mathrm{Br})_{3}(\mathrm{C}) \\
\mathrm{C}-(\mathrm{Br})(\mathrm{H})_{2}(\mathrm{C}) \\
\mathrm{C}-(\mathrm{Br})(\mathrm{H})(\mathrm{C})_{2} \\
\mathrm{C}-(\mathrm{I})(\mathrm{H})_{2}(\mathrm{C}) \\
\mathrm{C}-(\mathrm{I})(\mathrm{H})(\mathrm{C})_{2} \\
\mathrm{C}-(\mathrm{I})(\mathrm{C})_{3} \\
\mathrm{C}-(\mathrm{Cl})(\mathrm{Br})(\mathrm{H})(\mathrm{C}) \\
\mathrm{N}-(\mathrm{F})_{2}(\mathrm{C}) \\
\mathrm{N}-(\mathrm{F})\left(\mathrm{C}_{2}\right) \\
\mathrm{N}-(\mathrm{F})(\mathrm{C})\end{array}$ & $\begin{array}{c}-158.4 \\
(-109.3) \\
-51.8 \\
-97.0 \\
-48.4 \\
-43.9 \\
-106.3 \\
-20.7 \\
(-18.9) \\
-15.6 \\
(-19.5) \\
-12.8 \\
-12.8 \\
\\
-5.4 \\
-3.4 \\
7.95 \\
10.7 \\
13.0 \\
\\
-7.8 \\
(7) . \\
(-99)\end{array}$ & $\begin{array}{c}40.5 \\
50.4 \\
43.7 \\
37.8 \\
\\
17.6 \\
-5.4 \\
55.7 \\
40.8 \\
\\
42.5 \\
22.2 \\
(0.0) \\
45.7\end{array}$ & $\begin{array}{r}13.7 \\
16.3 \\
12.1 \\
8.9 \\
\\
9.0 \\
9.3 \\
16.7 \\
9.1 \\
\\
9.2 \\
(8.7) \\
(9.7) \\
12.4\end{array}$ & $\begin{array}{r}16.1 \\
18.0 \\
14.0 \\
10.7 \\
9.9 \\
10.5 \\
18.0 \\
11.0 \\
11.0\end{array}$ & $\begin{array}{l}17.5 \\
19.1 \\
15.4 \\
12.3 \\
\\
10.5 \\
11.0 \\
18.8 \\
12.6 \\
12.9\end{array}$ & $\begin{array}{l}19.8 \\
16.5 \\
13.4 \\
\\
11.2 \\
11.3 \\
19.4 \\
13.7 \\
13.9\end{array}$ & $\begin{array}{l}19.9 \\
15.5 \\
15.8\end{array}$ & $\begin{array}{l}20.3 \\
16.8 \\
17.2\end{array}$ \\
\hline
\end{tabular}


TABLE A-4. Halo-alkenes, alkynes and arenes group contributions to $\Delta \mathrm{H}_{f(298)}^{\circ}, \mathrm{S}_{298}^{\circ}$ and $\mathrm{C}_{p}^{\circ}$ ideal gas at 1 atm std. state

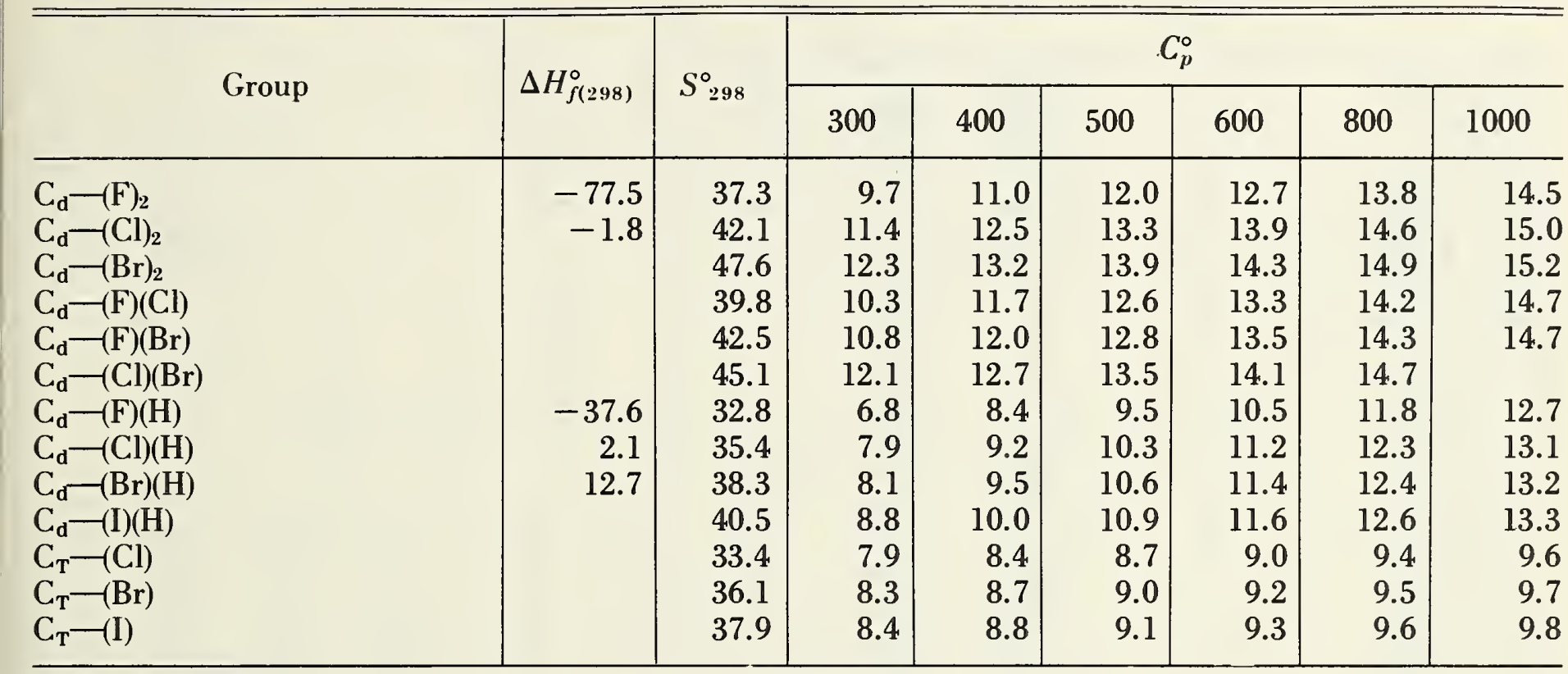

Arenes

\begin{tabular}{l|r|r|r|r|r|r|r|r}
\hline $\mathrm{C}_{\mathrm{B}}-(\mathrm{F})$ & -42.8 & 16.1 & 6.3 & 7.6 & 8.5 & 9.1 & 9.8 & 10.2 \\
$\mathrm{C}_{\mathrm{B}}-(\mathrm{Cl})$ & -3.8 & 18.9 & 7.4 & 8.4 & 9.2 & 9.7 & 10.2 & 10.4 \\
$\mathrm{C}_{\mathrm{B}}-(\mathrm{Br})$ & 10.7 & 21.6 & 7.8 & 8.7 & 9.4 & 9.9 & 10.3 & 10.5 \\
$\mathrm{C}_{\mathrm{B}}-(\mathrm{I})$ & 24.0 & 23.7 & 8.0 & 8.9 & 9.6 & 9.9 & 10.3 & 10.5 \\
$\mathrm{C}-\left(\mathrm{C}_{\mathrm{B}}\right)(\mathrm{F})_{3}$ & -162.7 & 42.8 & 12.5 & 15.3 & 17.2 & 18.5 & 20.1 & 21.0 \\
$\mathrm{C}-\left(\mathrm{C}_{\mathrm{B}}\right)(\mathrm{Br})(\mathrm{H})_{2}$ & -6.9 & & & & & & & \\
$\mathrm{C}-\left(\mathrm{C}_{\mathrm{B}}\right)(\mathrm{I})(\mathrm{H})_{2}$ & & & & & & & & \\
\hline
\end{tabular}

Corrections for Next Nearest Neighbors

\begin{tabular}{|c|c|c|c|c|c|c|c|c|}
\hline $\begin{array}{l}\text { ORTHO (F)(F) } \\
\text { ORTHO (Cl)(Cl) } \\
\text { ORTHO (Alk)(Halogen) } \\
\text { Cis (Halogen)(Halogen) } \\
\text { Cis (Halogen)(Alk) }\end{array}$ & $\begin{array}{l}5.0 \\
2.2 \\
0.6 \\
(0) \\
(0)\end{array}$ & 0 & 0 & 0 & 0 & 0 & 0 & 0 \\
\hline
\end{tabular}


TABLE A-5. Group contributions for estimation of thermochemical properties of free radicals ${ }^{\text {a }}(1$ atm std state).

\begin{tabular}{|c|c|c|c|c|c|c|c|c|c|}
\hline \multirow{2}{*}{ Radical } & \multirow{2}{*}{$\Delta H_{\mathcal{N}(298)}^{\circ \mathrm{b}}$} & \multirow{2}{*}{$S_{(298)}^{\circ}$} & \multicolumn{7}{|c|}{$C_{p}^{\circ}$} \\
\hline & & & 300 & 400 & 500 & 600 & 800 & 1000 & 1500 \\
\hline$\left[\cdot \mathrm{C}-(\mathrm{C})(\mathrm{H})_{2}\right]$ & 35.82 & 31.20 & 5.99 & 7.24 & 8.29 & 9.13 & 10.44 & 11.47 & 13.14 \\
\hline$\left[\cdot \mathrm{C}-(\mathrm{C})_{2}(\mathrm{H})\right]$ & 37.45 & 10.74 & 5.16 & 6.11 & 6.82 & 7.37 & 8.26 & 8.84 & 9.71 \\
\hline$\left[\cdot \mathrm{C}-(\mathrm{C})_{3}\right]$ & 37.00 & -10.77 & 4.06 & 4.92 & 5.42 & 5.75 & 6.27 & 6.35 & 6.53 \\
\hline$\left[\mathrm{C}-(\mathrm{C} \cdot)(\mathrm{H})_{3}\right]$ & -10.08 & 30.41 & 6.19 & 7.84 & 9.40 & 10.79 & 13.02 & 14.77 & 17.58 \\
\hline$\left[\mathrm{C}-(\mathrm{C} \cdot)(\mathrm{C})(\mathrm{H})_{2}\right]$ & -4.95 & 9.42 & 5.50 & 6.95 & 8.25 & 9.35 & 11.07 & 12.34 & 14.25 \\
\hline$\left[\mathrm{C}-(\mathrm{C} \cdot)(\mathrm{C})_{2}(\mathrm{H})\right]$ & -1.90 & -12.07 & 4.54 & 6.00 & 7.17 & 8.05 & 9.31 & 10.05 & 11.17 \\
\hline$\left[\mathrm{C}-(\mathrm{C} \cdot)(\mathrm{C})_{3}\right]$ & 1.50 & -35.10 & 4.37 & 6.13 & 7.36 & 8.12 & 8.77 & 8.76 & 8.12 \\
\hline$\left[\mathrm{C}-(\mathrm{O} \cdot)(\mathrm{C})(\mathrm{H})_{2}\right]$ & 6.1 & 36.4 & 7.9 & 9.8 & 10.8 & 12.8 & 15.0 & 16.4 & \\
\hline$\left[\mathrm{C}-(\mathrm{O} \cdot)(\mathrm{C})_{2}(\mathrm{H})\right]$ & 7.8 & 14.7 & 7.7 & 9.5 & 10.6 & 12.1 & 13.7 & 14.5 & \\
\hline$\left[\mathrm{C}-(\mathrm{O} \cdot)(\mathrm{C})_{3}\right]$ & 8.6 & -7.5 & 7.2 & 9.1 & 9.8 & 11.1 & 12.1 & 12.3 & \\
\hline$\left[\mathrm{C}-(\mathrm{S} \cdot)(\mathrm{C})(\mathrm{H})_{2}\right]$ & 32.4 & 39.0 & 9.0 & 10.6 & 12.4 & 13.6 & 15.8 & 17.4 & \\
\hline$\left[\mathrm{C}-(\mathrm{S} \cdot)(\mathrm{C})_{2}(\mathrm{H})\right]$ & 35.5 & 17.8 & 8.5 & 10.0 & 11.6 & 12.3 & 13.8 & 14.6 & \\
\hline$\left[\mathrm{C}-(\mathrm{S} \cdot)(\mathrm{C})_{3}\right]$ & 37.5 & -5.3 & 8.2 & 9.8 & 11.3 & 11.8 & 12.2 & 12.3 & \\
\hline$\left[\cdot \mathrm{C}-\left(\mathrm{H}_{2}\right)\left(\mathrm{C}_{d}\right)\right]$ & 23.2 & 27.65 & 5.39 & 7.14 & 8.49 & 9.43 & 11.04 & 12.17 & 14.04 \\
\hline$\left[\cdot \mathrm{C}-(\mathrm{H})(\mathrm{C})\left(\mathrm{C}_{d}\right)\right]$ & 25.5 & 7.02 & 4.58 & 6.12 & 7.19 & 8.00 & 9.11 & 9.78 & 10.72 \\
\hline$\left[\cdot \mathrm{C}-(\mathrm{C})_{2}\left(\mathrm{C}_{d}\right)\right]$ & 24.8 & -15.00 & 4.00 & 4.73 & 5.64 & 6.09 & 6.82 & 7.04 & 7.54 \\
\hline$\left[\mathrm{C}_{d}-(\mathrm{C} \cdot)(\mathrm{H})\right]$ & 8.59 & 7.97 & 4.16 & 5.03 & 5.81 & 6.50 & 7.65 & 8.45 & 9.62 \\
\hline$\left[\mathrm{C}_{d}-(\mathrm{C} \cdot)(\mathrm{C})\right]$ & 10.34 & -12.30 & 4.10 & 4.71 & 5.09 & 5.36 & 5.90 & 6.18 & 6.40 \\
\hline$\left[\cdot \mathrm{C}-\left(\mathrm{C}_{\mathrm{B}}\right)(\mathrm{H})_{2}\right]$ & 23.0 & 26.85 & 6.49 & 7.84 & 0.10 & 9.98 & 11.34 & 12.42 & 14.14 \\
\hline$\left[\cdot \mathrm{C}-\left(\mathrm{C}_{\mathrm{B}}\right)(\mathrm{C})(\mathrm{H})\right]$ & 24.7 & 6.36 & 5.30 & 6.87 & 7.85 & 8.52 & 9.38 & 9.84 & 10.12 \\
\hline$\left[\cdot \mathrm{C}-\left(\mathrm{C}_{\mathrm{B}}\right)(\mathrm{C})_{2}\right]$ & 24.5 & -15.46 & 4.72 & 5.48 & 6.20 & 6.65 & 7.09 & 7.10 & 6.94 \\
\hline$\left[\mathrm{C}_{\mathrm{B}}-\mathrm{C} \cdot\right]$ & 5.51 & -7.69 & 2.67 & 3.14 & 3.68 & 4.15 & 4.96 & 5.44 & 5.98 \\
\hline$\left[\mathrm{C}-(\cdot \mathrm{CO})(\mathrm{H})_{3}\right]$ & -5.4 & 66.6 & 12.74 & 14.63 & 16.47 & 18.17 & 21.14 & 23.27 & \\
\hline$\left[\mathrm{C}-(\cdot \mathrm{CO})(\mathrm{C})(\mathrm{H})_{2}\right]$ & -0.3 & 45.8 & 12.7 & 14.5 & 15.8 & 16.8 & 19.2 & 20.7 & \\
\hline$\left[\mathrm{C}-(\cdot \mathrm{CO})(\mathrm{C})_{2}(\mathrm{H})\right]$ & 2.6 & $(23.7)$ & (11.5) & $(12.8)$ & (14.3) & $(15.5)$ & $(17.4)$ & $(18.5)$ & \\
\hline$[\cdot \mathrm{N}-(\mathrm{H})(\mathrm{C})]$ & ${ }^{c}(51.8)$ & 30.23 & 5.38 & 5.67 & 5.89 & 6.09 & 6.60 & 6.97 & 7.74 \\
\hline$\left[\cdot \mathrm{N}-(\mathrm{C})_{2}\right]$ & ${ }^{c}(57.6)$ & 10.24 & 3.72 & 4.13 & 4.38 & 4.53 & 4.86 & 4.95 & 4.91 \\
\hline$\left[\mathrm{C}-(\cdot \mathrm{N})(\mathrm{C})(\mathrm{H})_{2}\right]$ & -6.6 & 9.8 & 5.25 & 6.90 & 8.28 & 9.39 & 11.09 & 12.34 & \\
\hline$\left[\mathrm{C}-(\cdot \mathrm{N})(\mathrm{C})_{2}(\mathrm{H})\right]$ & -5.2 & -11.7 & 4.67 & 6.32 & 7.64 & 8.39 & 9.56 & 10.23 & \\
\hline$\left[\mathrm{C}-(\cdot \mathrm{N})(\mathrm{C})_{3}\right]$ & $\mathrm{c}(-3.2)$ & 34.1 & 4.35 & 6.16 & 7.31 & 7.91 & 8.49 & 8.50 & \\
\hline$\left[\cdot \mathrm{C}-(\mathrm{H})_{2}(\mathrm{CN})\right]$ & ${ }^{d}(54.2)$ & 58.5 & 10.66 & 12.82 & 14.48 & 15.89 & 18.08 & 19.80 & \\
\hline$[\cdot \mathrm{C}-(\mathrm{H})(\mathrm{C})(\mathrm{CN})]$ & ${ }^{d}(52.8)$ & 40.0 & 9.1 & 11.4 & 13.1 & 14.4 & 16.3 & 17.4 & \\
\hline$\left[\cdot \mathrm{C}-(\mathrm{C})_{2}(\mathrm{CN})\right]$ & ${ }^{d}(52.1)$ & 19.6 & 8.8 & 10.4 & 11.3 & 12.3 & 13.7 & 14.5 & \\
\hline$\left[\cdot \mathrm{N}-(\mathrm{H})\left(\mathrm{C}_{\mathrm{B}}\right)\right]$ & & 27.3 & 4.6 & 5.4 & 6.0 & 6.4 & 7.2 & 7.7 & 8.6 \\
\hline$\left[\cdot \mathrm{N}-(\mathrm{C})\left(\mathrm{C}_{\mathrm{B}}\right)\right]$ & & $(6.5)$ & (3.9) & $(4.2)$ & (4.7) & $(5.0)$ & $(5.6)$ & $(5.8)$ & (5.9) \\
\hline$\left[\mathrm{C}_{\mathrm{B}}-\mathrm{N} \cdot\right]$ & -0.5 & -9.69 & 3.95 & 5.21 & 5.94 & 6.32 & 6.53 & 6.56 & \\
\hline$\left[\mathrm{C}-\left(\mathrm{CO}_{2} \cdot\right)(\mathrm{H})\right]$ & 49.7 & 71.4 & 14.4 & 17.8 & 20.4 & 23.1 & 27.1 & 29.6 & \\
\hline
\end{tabular}

See footnotes at end of table. 
TABLE A-5. Group contributions for estimates of thermochemical properties of free radicals a ( 1 atm std state). - Con.

\begin{tabular}{|c|c|c|c|c|c|c|c|c|c|}
\hline \multirow[b]{2}{*}{ Radical } & \multirow[b]{2}{*}{$\Delta H_{f(298)}^{\circ \mathrm{b}}$} & \multirow[b]{2}{*}{$S_{(298)}^{\circ}$} & \multicolumn{7}{|c|}{$C_{p}^{\circ}$} \\
\hline & & & 300 & 400 & 500 & 600 & 800 & 1000 & 1500 \\
\hline $\begin{array}{l}{\left[\mathrm{C}-\left(\mathrm{CO}_{2} \cdot\right)\left(\mathrm{H}_{\mathrm{I}_{2}(\mathrm{C})}\right]\right.} \\
{\left[\mathrm{C}-\left(\mathrm{CO}_{-} \cdot\right)(\mathrm{H})(\mathrm{C})_{2}\right]}\end{array}$ & $\begin{array}{l}-43.9 \\
-41.0\end{array}$ & 49.8 & 15.5 & 18.5 & 20.3 & 22.3 & 27.5 & 27.2 & \\
\hline$\left[\mathrm{C}-\left(\mathrm{N}_{\mathrm{A}}\right)(\mathrm{H})_{3}\right]$ & -10.08 & 30.41 & 6.19 & 7.84 & 9.40 & 10.79 & 13.02 & 14.77 & 17.58 \\
\hline$\left.\left[\mathrm{C}-\mathrm{N}_{\mathrm{A}}\right)(\mathrm{C})(\mathrm{H})_{2}\right]$ & -5.8 & 9.42 & 5.50 & 6.95 & 8.25 & 9.35 & 11.07 & 12.34 & 14.25 \\
\hline$\left[\mathrm{C}-\left(\mathrm{N}_{\mathrm{A}}\right)(\mathrm{C})_{2}(\mathrm{H})\right]$ & -2.3 & -12.07 & 4.54 & 6.00 & 7.17 & 8.05 & 9.31 & 10.05 & 11.17 \\
\hline$\left[\mathrm{C}-\left(\mathrm{N}_{\mathrm{A}}\right)(\mathrm{C})_{: 3}\right]$ & +0.5 & -35.10 & 4.37 & 6.13 & 7.36 & 8.12 & 8.77 & 8.76 & 8.12 \\
\hline$\left[\mathrm{N}_{\mathrm{A}}-\mathrm{C}\right]$ & 32.0 & 8.0 & 4.0 & 4.4 & 4.7 & 4.8 & 5.1 & 5.3 & 5.2 \\
\hline$\left.\left[N_{A} \longrightarrow N_{1} \cdot\right)(C)\right]$ & 72.4 & 36.1 & 7.8 & 8.2 & 8.4 & 8.6 & 8.9 & 9.0 & 9.0 \\
\hline
\end{tabular}

Internal Rotation Barrier Corrections (However see also V-6.0 and Appendix C)

\begin{tabular}{|c|c|c|c|c|c|c|c|c|}
\hline & $S^{\circ}$ & 300 & 400 & 500 & 600 & 800 & 1000 & 1500 \\
\hline $\begin{array}{l}\text { 1. } \mathrm{V}_{0}(\mathrm{RH}) \rightarrow 2 / 3 \mathrm{~V}_{0}(\mathrm{R} \cdot) \\
\left(\mathrm{CH}_{3} \mathrm{G}_{\mathrm{R}} \cdot \mathrm{R}\right) \sigma=3 \\
(\infty \mathrm{G} \cdot \mathrm{R})_{0}=3 \\
\text { 2. } \mathrm{V}_{0}\left(\mathrm{CH}_{3} \mathrm{G} \mathrm{RH}\right) \rightarrow \mathrm{V}_{0}^{\prime}\left(\mathrm{CH}_{3} G \mathrm{R} \cdot\right)\end{array}$ & $\begin{array}{l}+0.5 \\
+0.5\end{array}$ & $\begin{array}{l}0 \\
0.1\end{array}$ & $\begin{array}{r}-0.1 \\
0.1\end{array}$ & $\begin{array}{c}-0.2 \\
0\end{array}$ & $\begin{array}{l}-0.3 \\
-0.2\end{array}$ & $\begin{array}{l}-0.3 \\
-0.3\end{array}$ & $\begin{array}{l}-0.3 \\
-0.4\end{array}$ & $\begin{array}{l}-0.2 \\
-0.4\end{array}$ \\
\hline $\mathrm{V}_{0}=3.5, \mathrm{~V}_{0}^{\prime}=0$ & 1.5 & -1.1 & -1.1 & -1.1 & -1.0 & -0.8 & -0.6 & -0.4 \\
\hline
\end{tabular}

3. Mass corrections in conjugated systems

(i) If the masses on each side of a resonance stabilized bond in a radical have the same number of $\mathrm{C}$ atoms (i.e., are roughly equal), subtract $0.7 \mathrm{~g} / \mathrm{mole}$ from the entropy, e.g. $\left(\mathrm{CH}_{2}=\mathrm{C}-\mathrm{C} \nearrow_{\mathrm{H}}^{\mathrm{CH}_{3}}\right)_{m_{1}}=\mathrm{CH}_{2}, m_{2}=\left(\mathrm{CH}_{3}+\mathrm{H}\right)$.

(ii) If the masses (as above) differ by one $\mathrm{C}$ atom, subtract $0.3 \mathrm{~g} / \mathrm{mole}$ from $S^{\circ}$, e.g. $\left(\mathrm{CH}_{2}=\mathrm{C}-\mathrm{CH}_{2}\right) m_{1}=\mathrm{CH}_{2}, m_{2}=\mathrm{H}_{2}$.

a Units are $\Delta H_{f}^{\circ}(\mathrm{kcal} / \mathrm{mole}), S^{\circ}(\mathrm{g} / \mathrm{mole}), C_{p}^{\circ}(\mathrm{g} / \mathrm{mole})$. Entropies include electron degeneracy and conjugation effects. Corrections for changes in rotational barriers of groups bonded to the radical center relative to the saturated hydrocarbons are required.

${ }^{b}$ Heats of formation group values, with the exception of the alkoxy radicals, are those derived by assuming equivalent bond dissociation energies for all $(X-\mathrm{H})$ bonds of the same type (i.e., primary, secondary, or tertiary $(X-\mathrm{H})$ bonds).

c These heats of formation are based on the amine decomposition kinetics and are probably too low by up to 4 $\mathrm{kcal} / \mathrm{mole}$ (see V-7.0 and 8.0).

${ }^{d}$ These heats of formation have been calçulated assuming resonance stabilizations of $10.8 \mathrm{kcal} / \mathrm{mole}$ in $\left(\cdot \mathrm{CH}_{2} \mathrm{CN}\right)$, and $12.6 \mathrm{kcal} / \mathrm{mole}$ in $\left(\mathrm{CH}_{3} \mathrm{CHCN}\right.$ and $\left.\left(\mathrm{CH}_{3}\right)_{2} \mathrm{CCN}\right)$. 
To illustrate the use of the group table for radicals, the following examples are given:

I. Estimate $\Delta H_{f}^{\circ}, S^{\circ}$, and $C_{p}^{\circ}$ of the neopentyl radical $\left(\left(\mathrm{CH}_{3}\right)_{3} \mathrm{C}-\mathrm{CH}_{2} \cdot\right) \sigma=6$, assume $\left(\cdot \mathrm{CH}_{2}(t \mathrm{Bu})\right.$ has $\mathrm{V}=0$ or $\mathrm{V}=2$.

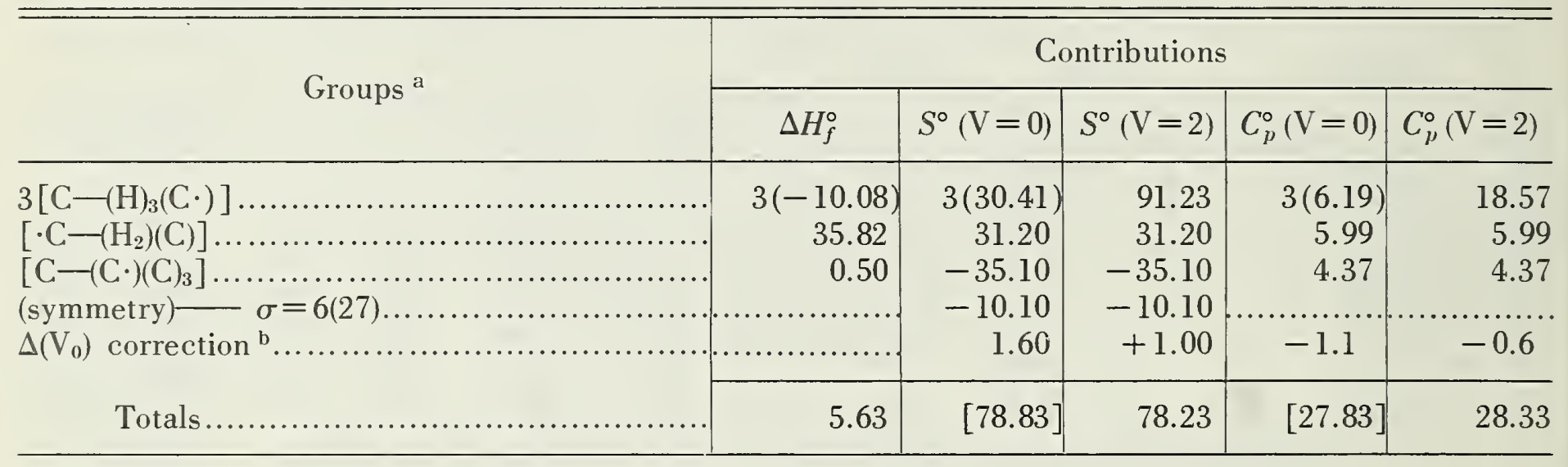

a Units are $\mathrm{kcal} / \mathrm{mole}\left(\Delta H_{f}^{\circ}\right) ; \mathrm{kcal} / \mathrm{mole}-\mathrm{deg}\left(S^{\circ}\right.$ and $\left.C_{p}^{\circ}\right)$.

Brackets denote preferred values.

${ }^{b}$ See Appendix C and V-6.0.

II. Estimate $\Delta H_{f}^{\circ}, S^{\circ}, C_{p}^{\circ}$ of the $t$-butoxy radical $\left(\mathrm{CH}_{3}\right)_{3} \mathrm{CO}$.

\begin{tabular}{|c|c|c|c|}
\hline \multirow{2}{*}{ Groups } & \multicolumn{3}{|c|}{ Contributions } \\
\hline & $\Delta H_{f}^{\circ}$ & $S^{\circ}$ & $C_{p}^{\circ}(300)$ \\
\hline $\begin{array}{l}3\left[\mathrm{C}-(\mathrm{C})(\mathrm{H})_{3}\right] \ldots \ldots \ldots \\
{\left[\mathrm{C}-(\mathrm{O} \cdot)(\mathrm{C})_{3}\right] \ldots \ldots \ldots} \\
\text { symmetry }\left(\sigma=(3)^{4}\right) \ldots\end{array}$ & $\begin{array}{c}-30.24 \\
8.6\end{array}$ & $\begin{array}{r}91.2 \\
-7.5 \\
-8.7\end{array}$ & $\begin{array}{r}18.57 \\
7.20 \\
\cdots\end{array}$ \\
\hline Totals... & -22.64 & 75.0 & 25.77 \\
\hline
\end{tabular}

III. Estimate $\Delta H_{f}^{\circ}, S^{\circ}$, and $C_{p}^{\circ}$ of the secondary butyl radical.

\begin{tabular}{|c|c|c|c|}
\hline \multirow{2}{*}{ Groups } & \multicolumn{3}{|c|}{ Contributions } \\
\hline & $\Delta H_{f}^{\circ}$ & $\begin{array}{c}S^{\circ} \\
\left(2 / 3 V_{0}\right)\end{array}$ & $C_{p}^{\circ}(300)$ \\
\hline$\left[\mathrm{C}-(\mathrm{H})_{3}(\mathrm{C} \cdot)\right]$. & -10.08 & 30.41 & 6.19 \\
\hline$\left[\mathrm{C}-(\mathrm{H})_{3}(\mathrm{C})\right]$ & -10.08 & 30.41 & 6.19 \\
\hline$\left[\mathrm{C}-(\mathrm{H})_{2}(\mathrm{C})(\mathrm{C} \cdot)\right.$ & -4.95 & 9.42 & 5.50 \\
\hline$\left[\cdot \mathrm{C}-(\mathrm{H})(\mathrm{C})_{2}\right] \ldots$ & 37.45 & 10.74 & 5.16 \\
\hline symmetry ..... & & -4.40 & \\
\hline$\Delta \mathrm{V}_{0}\left(\mathrm{CH}_{3} \mathrm{G} \cdot \mathrm{R}\right) .$. & & +0.50 & 0.0 \\
\hline$\Delta V_{0}(\infty \in \cdot R) \ldots$ & & +0.50 & 0.1 \\
\hline Totals. & 2.34 & 77.58 & 23.14 \\
\hline
\end{tabular}




\section{Appendix B-Frequencies Used in Transition \\ State Calculations}

TABLE B-1. Bond stretching frequencies

\begin{tabular}{|c|c|c|c|}
\hline Stretches & $\begin{array}{c}\omega\left(\mathrm{cm}^{-1}\right) \\
\text { frequencies }\end{array}$ & Stretches & $\begin{array}{c}\omega\left(\mathrm{cm}^{-1}\right) \\
\text { frequencies }\end{array}$ \\
\hline $\mathrm{C}=\mathrm{O}$ & 1700 & $\mathrm{C}-\mathrm{O}$ & 1400 \\
\hline $\mathrm{C}-\mathrm{O}$ ethers & 1100 & $(\mathrm{C} \cdot \mathrm{O})$ ethers & 710 \\
\hline $\begin{array}{c}\mathrm{C}-\mathrm{O} \text { acids, } \\
\text { esters }\end{array}$ & 1200 & $\begin{array}{c}(\mathrm{C} \cdot \mathrm{O}) \text { acids, } \\
\text { esters }\end{array}$ & 770 \\
\hline $\mathrm{C}=\mathrm{C}$ & 1650 & $\mathrm{C}-\mathrm{C}$ & 1300 \\
\hline $\mathrm{C}-\mathrm{C}$ & 1000 & $C \cdot C$ & 675 \\
\hline $\mathrm{C}-\mathrm{H}$ & 3000 & $\mathrm{C} \cdot \mathrm{H}$ & 2200 \\
\hline $\mathrm{C}-\mathrm{F}$ & 1100 & $C \cdot F$ & 820 \\
\hline $\mathrm{C}-\mathrm{Cl}$ & 650 & $\mathrm{C} \cdot \mathrm{Cl}$ & 490 \\
\hline $\mathrm{C}-\mathrm{I}$ & 500 & $C \cdot I$ & 375 \\
\hline $\mathrm{C}-\mathrm{Br}$ & 560 & $\mathrm{C} \cdot \mathrm{Br}$ & 420 \\
\hline
\end{tabular}

Notes to Table B-2:

a Note that the normal single bona bending frequencies are consistent with the relation, $\omega_{1} / \omega_{2}=\left(\mu_{2} / \mu_{1}\right)^{1 / 2}$. Deviations from this relation seldom exceed $50 \mathrm{~cm}^{-1}$.

$$
\mu=\left(\frac{\mathrm{M}_{\mathrm{A}} \mathrm{M}_{\mathrm{B}}}{\mathrm{M}_{\mathrm{A}}+\mathrm{M}_{\mathrm{B}}}\right) \text { for the bond }\left(\mathrm{A}^{\prime} \stackrel{\mathrm{R}}{\leftrightarrow}{ }_{\mathrm{B}}\right)
$$

b Methyl and methylene wags and twists, whose frequencies range from $1000-1300 \mathrm{~cm}^{-1}$ have been equated

with $\left(\mathrm{H}^{\mathrm{C}} \mathrm{C}\right)_{t, w}$ bends and assigned a mean frequency of $1150 \mathrm{~cm}^{-1}$. Methylene rocks have lower frequencies (i.e., $\sim 700 \mathrm{~cm}^{-1}$ ) which correspond closely to the out of

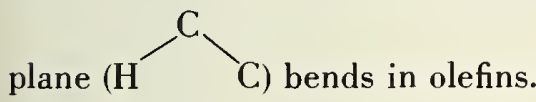

TABLE B-2. Bond angle bending frequencies

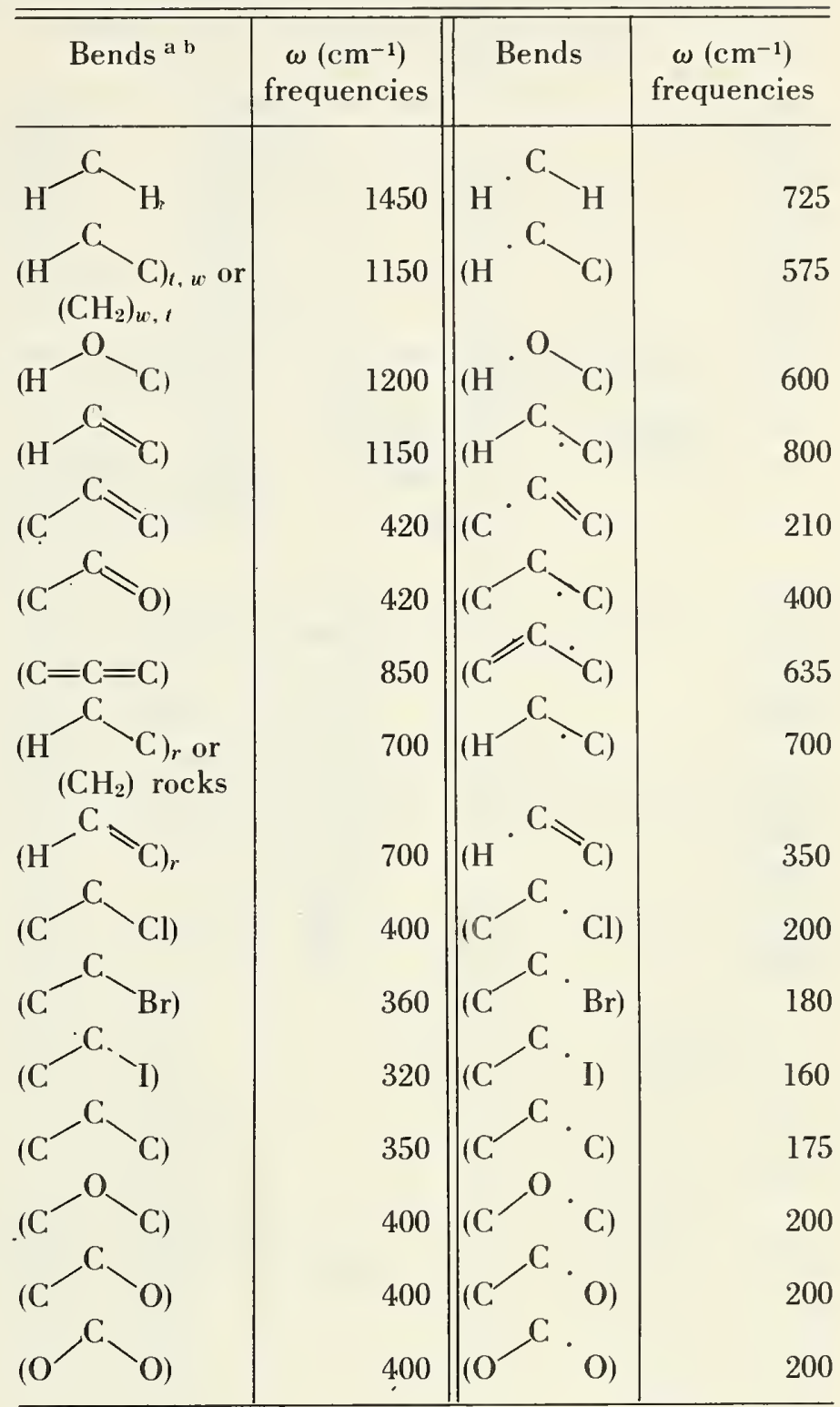

TABLE B-3. Bond Torsion Frequencies

\begin{tabular}{|c|c|c|c|c|}
\hline & Torsions & $\underset{F r e q u e n c i e s}{\omega}\left(\mathrm{cm}^{-1}\right)$, & Torsions & $\begin{array}{l}\omega\left(\mathrm{cm}^{-1}\right) \\
\text { Frequencies }\end{array}$ \\
\hline$=$ & ethylene $\left(E_{4} \mathrm{e}^{\prime}\right)$ & 1000 & $\left(\mathrm{E}_{3 \mathrm{e}}\right)_{\mathrm{t}} \quad-$ & 500 \\
\hline$\rightleftharpoons$ & propylene $\left(P_{4 e}\right)_{t}$ & BOO & 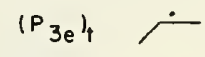 & 400 \\
\hline$\nu=i$ & isobutene $\left(\mathrm{iB}_{4 \mathrm{e}}\right)_{\mathrm{t}}$ & 700 & $\left(i B_{3 e}\right\}^{\prime}$ & 350 \\
\hline$\Leftrightarrow$ & cis - 2- butene $\left(\mathrm{CB}_{4 \mathrm{e}}\right)_{\mathrm{f}}$ & 400 & $\left(\mathrm{CB}_{3 e}\right)^{\prime}$ & 200 \\
\hline$\rightleftharpoons$ & trons-2-butene $\left(1 B_{4}\right)^{\prime} t$ & 300 & $\left(1 B_{3 e}\right)_{\uparrow}$ & 150 \\
\hline$>$ & 2-methylbut-2-ene $\left(2 \mathrm{MB}_{4} \mathrm{e}\right)_{\mathrm{f}}$ & 250 & $\left.\left(2 \mathrm{MB}_{3 \mathrm{e}}\right)_{t}\right) \cdot$ & 125 \\
\hline 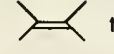 & tetromethylethylene (TME $\left.4 \mathrm{e}_{\mathrm{t}}\right)_{\mathrm{t}}$ & 210 & $\left.\left(T M E_{3 \mathrm{e}}\right)_{t}\right) \cdot \prec$ & 105 \\
\hline
\end{tabular}


TABLE B-4. Approximate entropies and heat capacities of bond vibrations.

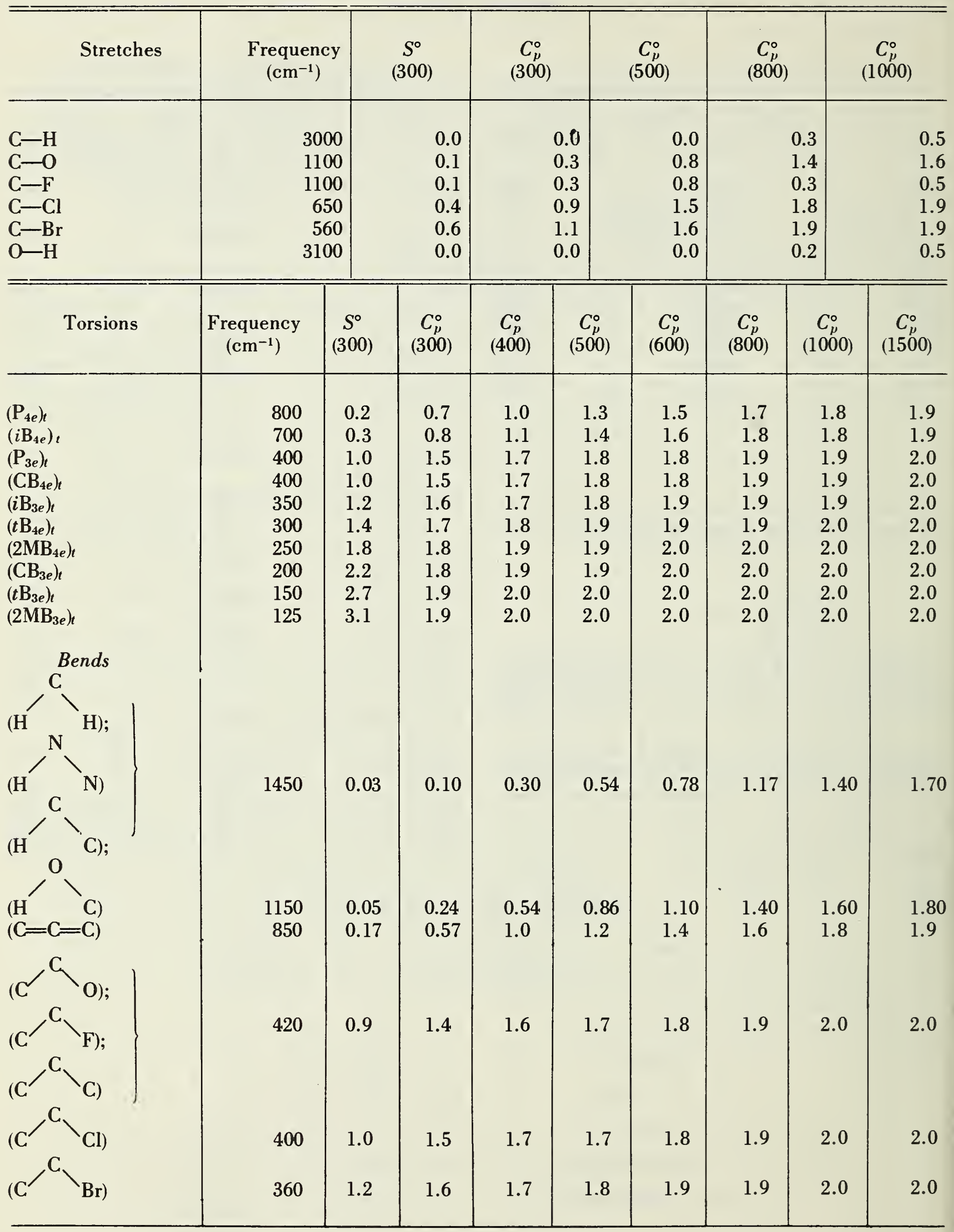


TABLE C-1. Entropies ${ }^{\mathrm{b}, \mathrm{c}}$ of free internal rotors as a function of temperature

\begin{tabular}{|c|c|c|c|c|c|c|c|}
\hline \multirow{2}{*}{ Free rotors } & \multirow{2}{*}{$\begin{array}{l}\text { Hybrid- } \\
\text { ization }\end{array}$} & \multirow{2}{*}{$Q_{\left(300{ }^{\circ}\right)^{a}}{ }^{\mathrm{a}}$} & \multicolumn{5}{|c|}{$T\left({ }^{\circ} \mathrm{K}\right)$} \\
\hline & & & 300 & 500 & 600 & 700 & 800 \\
\hline$(\infty) \in$ methyl.. & $S p^{3}$ & $11.6 / 3=3.7$ & 5.9 & 6.4 & 6.55 & 6.7 & 6.85 \\
\hline$(\infty) \in$ methylene. & $\mathrm{Sp}^{2}$ & $8.4 / 2=4.2$. & 5.2 & 5.75 & 5.9 & 6.1 & 6.25 \\
\hline$(\infty) \in$ Ethyl... & $\mathrm{Sp}^{3}$ & $37.0 \ldots \ldots \ldots$ & 8.2 & 8.7 & 8.9 & 9.0 & 9.15 \\
\hline$(\infty) \in i$-propyl. & $\mathrm{Sp}^{3}$. & $52.5 \ldots$ & 8.9 & 9.4 & 9.6 & 9.7 & 9.75 \\
\hline$(\infty) \in t$-butyl... & $\mathrm{Sp}^{3}$. & $64.5 \ldots$ & 9.3 & 9.8 & 9.95 & 10.1 & 10.25 \\
\hline$(\infty) \in\left({ }^{\prime} \mathrm{Et}\right) \ldots$. & $\mathrm{Sp}^{2}$. & $35.0 \ldots$ & 8.05 & 8.55 & 8.75 & 8.85 & 9.0 \\
\hline$(\infty) \in(\cdot i \operatorname{Pr})$. & $\mathrm{Sp}^{2}$. & $49.7 .$. & 8.75 & 9.25 & 9.45 & 9.55 & 9.6 \\
\hline
\end{tabular}

a The partition functions of free rotation were calculated from the relation,

$$
Q_{f}=\left(\frac{8 \Pi^{3} I k T}{h^{2}}\right)^{1 / 2}
$$

${ }^{b}$ With equal masses, change entropy by $-0.7 \mathrm{~g} /$ mole. For an opposing rotor heavier by 1 carbon group, change the free rotor entropy by $-0.3 \mathrm{~g} /$ mole.

${ }^{c}$ Entropies were estimated from tables prepared by Pitzer, et al., [10], pp. 441-446.

TABLE C-2. Some characteristic torsion barriers, V (kcal/mole) to rotation about single bonds for methyl groups

\begin{tabular}{|c|c|c|c|}
\hline Bond & $V$ & Bond & $V$ \\
\hline 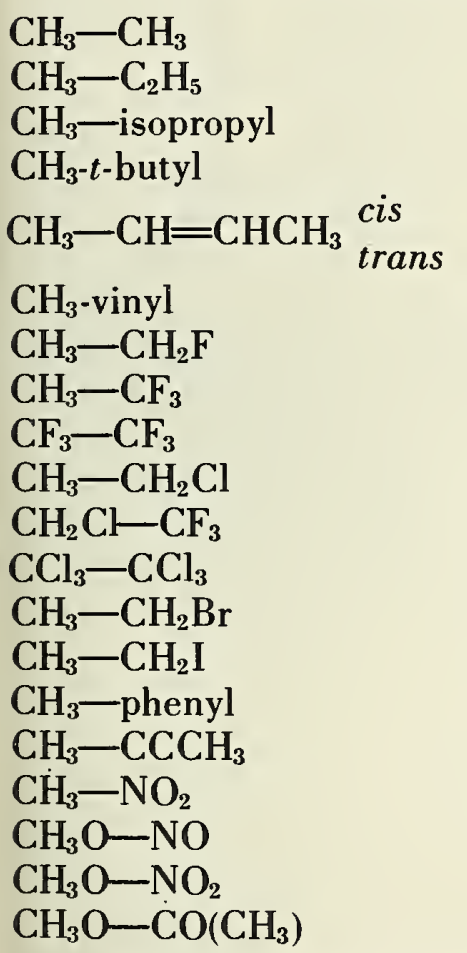 & $\begin{array}{c}2.9 \\
2.8 \\
3.6 \\
4.7 \\
0.75 \\
1.95 \\
2.0 \\
3.3 \\
3.5 \\
4.4 \\
3.7 \\
5.8 \\
10.8 \\
3.6 \\
3.2 \\
0 \\
0 \\
0 \\
9.0 \\
9.0 \\
13\end{array}$ & $\begin{array}{l}\mathrm{CH}_{3}-\mathrm{OH} \\
\mathrm{CH}_{3}-\mathrm{OCH}_{3} \\
\mathrm{CH}_{3}-\mathrm{NH}_{2} \\
\mathrm{CH}_{3}-\mathrm{NHCH}_{3} \\
\mathrm{CH}_{3}-\mathrm{N}\left(\mathrm{CH}_{3}\right)_{2} \\
\mathrm{CH}_{3}-\mathrm{SiH}_{3} \\
\mathrm{CH}_{3}-\mathrm{SiH}_{2}\left(\mathrm{CH}_{3}\right) \\
\mathrm{CH}_{3}-\mathrm{PH}_{2} \\
\mathrm{CH}_{3}-\mathrm{SH} \\
\mathrm{CH}_{3}-\mathrm{SCH} \\
\mathrm{CH}_{3}-\mathrm{CHO} \\
\mathrm{CH}_{3}-\mathrm{COCH} \\
\mathrm{CH}_{3}-\text { allene } \\
\mathrm{CH}_{3}-(\text { isobutene }) \\
\mathrm{CH}_{3}-\mathrm{CO}(\mathrm{OH}) \\
\mathrm{CH}_{3}-(\mathrm{epoxide} \text { ring) } \\
\left(\mathrm{CH}_{3}\right)_{2} \mathrm{~N}-\mathrm{COCH} 3 \\
\mathrm{CH}_{3}-\mathrm{OCHO} \\
\mathrm{CH}_{3}-\mathrm{ONO} \mathrm{OH}_{2} \\
\mathrm{CH}_{3}-\mathrm{O} \text { vinyl }\end{array}$ & $\begin{array}{r}1.1 \\
2.7 \\
1.9 \\
3.3 \\
4.4 \\
1.7 \\
1.7 \\
2.0 \\
1.3 \\
2.1 \\
1.2 \\
0.8 \\
1.6 \\
2.2 \\
0.5 \\
2.6 \\
20.0 \\
1.2 \\
2.3 \\
3.4\end{array}$ \\
\hline
\end{tabular}

For a more complete compilation, see J. Dale, Tetrahedron 22, 3373 (1966).

a Note: There seems to be a quite general "cis effect" such that $\mathrm{F}, \mathrm{CI}, \mathrm{CH}_{3}$, and $\mathrm{CN}$, cis to a $\mathrm{CH}_{3}$ lowers the $\mathrm{CH}_{3}$ barrier by $\sim 1.4 \mathrm{kcal}$. 
TABLE C-3. Some characteristic barriers, V (kcal/mole) to rotation about single bonds for groups larger than methyl ${ }^{\text {a }}$

\begin{tabular}{|c|c|}
\hline Group & $V$ \\
\hline$c_{2} \mathrm{H}_{5} \leftarrow \mathrm{c}_{3} \mathrm{H}_{7}(f)$ & $4.2,5.3$ \\
\hline $\mathrm{t} \mathrm{Bu}+\mathrm{nPr}(t)$ & 10.9 \\
\hline$\rightarrow \infty$ & 6.5 \\
\hline $7 x$ & 7.0 \\
\hline je & 8.9 \\
\hline$r x_{2}$ & $4.2,4.4$ \\
\hline 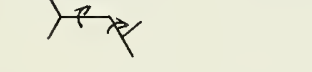 & $9.3,11.0$ \\
\hline tre & 10.8 \\
\hline$\rightarrow x_{2}$ & 7.8 \\
\hline
\end{tabular}

a U. A. Zirnet and M. M. Sushinskii, Optics and Spectroscopy 16, 489 (1964).

TABLE C-4. Decrease in entropy of free rotors as functions of barrier height $(\mathrm{V})$, temperature $\left(\mathrm{T},{ }^{\circ} \mathrm{K}\right)$ and partition function, $\mathrm{Q}_{\mathrm{f}}$

\begin{tabular}{r|c|c|c}
\hline \hline \multirow{2}{*}{$(V / R T)$} & \multicolumn{3}{|c}{$1 / Q_{f}$} \\
& \multicolumn{3}{|c}{} \\
\cline { 2 - 4 } & 0.0 & 0.2 & 0.4 \\
\hline & & & \\
0.0 & 0.0 & 0.0 & 0.0 \\
1.0 & 0.1 & 0.1 & 0.1 \\
2.0 & 0.4 & 0.4 & 0.4 \\
3.0 & 0.8 & 0.8 & 0.7 \\
4.0 & 1.1 & 1.1 & 1.0 \\
5.0 & 1.4 & 1.4 & 1.2 \\
6.0 & 1.7 & 1.6 & 1.4 \\
8.0 & 2.0 & 2.0 & 1.7 \\
10.0 & 2.3 & 2.2 & 1.9 \\
15.0 & 2.7 & 2.6 & 2.2 \\
20.0 & 3.1 & 2.9 & 2.4 \\
\hline
\end{tabular}

Note: Values listed are $\Delta S=S_{f}^{\circ}-S_{h}^{\circ}$ in $\mathrm{g} /$ mole. Taken from same source as table $\mathrm{C}-5$.
TABLE C-5. Molar heat, capacity, $\mathrm{C}_{p}^{\circ}(\mathrm{g} /$ mole $)$ of a hindered rotor as a function of barrier $(\mathrm{V})$, temperature, and partition function, $\left(\mathrm{Q}_{f}\right)$

\begin{tabular}{r|c|c|c|c|c}
\hline \hline \multirow{2}{*}{$V \mid R T$} & \multicolumn{5}{|c}{$\left(1 / Q_{f}\right)$} \\
\cline { 2 - 6 } & & & & & \\
& 0.0 & 0.2 & 0.4 & 0.6 & 0.8 \\
\hline & & & & & \\
0.0 & 1.0 & 1.0 & 1.0 & 1.0 & 1.0 \\
0.5 & 1.1 & 1.1 & 1.1 & 1.0 & 1.0 \\
1.0 & 1.2 & 1.2 & 1.2 & 1.1 & 1.1 \\
1.5 & 1.5 & 1.4 & 1.3 & 1.2 & 1.1 \\
2.0 & 1.7 & 1.7 & 1.5 & 1.4 & 1.2 \\
2.5 & 1.9 & 1.9 & 1.7 & 1.5 & 1.3 \\
3.0 & 2.1 & 2.0 & 1.8 & 1.6 & 1.3 \\
4.0 & 2.3 & 2.2 & 2.0 & 1.7 & 1.4 \\
6.0 & 2.3 & 2.2 & 1.9 & 1.5 & 1.2 \\
8.0 & 2.2 & 2.1 & 1.7 & 1.3 & 0.9 \\
10.0 & 2.1 & 2.0 & 1.5 & 1.0 & 0.7 \\
15.0 & 2.1 & 1.8 & 1.2 & 0.7 & 0.4 \\
20.0 & 2.0 & 1.7 & 1.0 & 0.5 & 0.2 \\
& & & & & \\
\hline
\end{tabular}

Tables from Thermodynamics, G. N. Lewis and M. Randall, revised by K. S. Pitzer and L. Brewer, McGrawHill Publishing Co., New York, N.Y. (1961).

$Q_{f}=\frac{3.6}{\sigma}\left\{\frac{I_{r} T}{100}\right\}^{1 / 2}$ with $I_{r}$ in AMU- ang $^{2}$ and $T$ in ${ }^{\circ} \mathrm{K}$.

$\sigma=$ symmetry of barrier (e.g., 3 for $-\mathrm{CH}_{3}, 2$ for $-\mathrm{CH}_{2}$, etc.) 


\section{Appendix $\mathbb{D}$. Thermodynamic Properties of Free Radicals}

\section{TABLE D-1. Preferred Values of Free Radical Heats of Formation and Their Source}

Uncertainty limits on $\Delta H_{f}^{\circ}$ of radicals are very hard to give. Even these $\Delta H_{f}^{\circ}$ values believed to be the best known are probably uncertain to $\pm 1 \mathrm{kcal} / \mathrm{mole}$. The uncertainties given below, therefore, are best guesses.

\section{Ketones \\ $\Delta H_{f}^{\circ}(\varnothing \mathrm{C} \mathrm{O})$}

$(21.0 \pm 2)$

$\Delta H_{f}^{\circ}\left(\mathrm{CF}_{3} \cdot\right)$

$(-112.5 \pm 2)$

$\Delta H_{f}^{\circ}\left(\varnothing \mathrm{CH}_{2} \cdot\right)$

$(45.0 \pm 1)$

$\Delta H_{f}^{\circ}\left(\emptyset \mathrm{CH}_{2} \mathrm{CO}\right)$

$(20.6 \pm 2)$

$\Delta H_{f}^{\circ}\left(\mathrm{CH}_{\dot{3}}\right)$

$(34.0 \pm 1)$

$\Delta H_{f}^{\circ}\left(\mathrm{CH}_{3} \dot{\mathrm{C} O}\right)$

$(-5.4 \pm 1)$

Esters

$\Delta H_{f}^{\circ}\left(\mathrm{CH}_{3} \mathrm{CO}_{2} \cdot\right)$

$(-49.7 \pm 2)$

$\Delta H_{f}^{\circ}\left(\emptyset \mathrm{CO}_{2} \cdot\right)$

$(-21.7 \pm 2)$

\section{Peroxides \\ $\Delta H_{f}^{\circ}\left(\mathrm{CH}_{3} \mathrm{O} \cdot\right)$ \\ $(3.9 \pm 1)$}

$\Delta H_{f}^{\circ}\left(\mathrm{C}_{2} \mathrm{H}_{5} \mathrm{O} \cdot\right)$

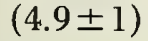

$\Delta H_{f}^{\circ}\left(n-\mathrm{C}_{3} \mathrm{H}_{7} \mathrm{O} \cdot\right)$ $(-9.7 \pm 1)$

$\Delta H_{f}^{\circ}(t \mathrm{BuO} \cdot)$ $(-21.5 \pm 1)$

$\Delta H_{f}^{\circ}\left(\mathrm{C}_{2} \mathrm{H}_{5} \mathrm{CO}_{2} \cdot\right)$ $(-55.0 \pm 2)$

$\Delta H_{f}^{\circ}\left(\mathrm{C}_{3} \mathrm{H}_{7} \mathrm{CO}_{2} \cdot\right)$ $(-60.0 \pm 2)$

$\Delta H_{f}^{\circ}(\cdot \mathrm{COOH})$ $(-53.3 \pm 3)$

From the observed activation energies for the decompositions of acetophenone, benzophenone, benzoyl bromide, and benzil decompositions one obtains 21.0, 21.0, 18.3 and 22.4 $\mathrm{kcal} / \mathrm{mole}$ respectively.

Bromination studies of Whittle (see $\mathrm{C}_{2} \mathrm{~F}_{6}$ decomposition).

Iodination studies by Benson, et al. (see toluene decomposition).

Estimated by additivity relations from $\Delta H_{f}^{\circ}$ (acetyl), see R. Walsh and S. W. Benson, J. Phys. Chem. 70, 3751 (1966).

Iodination studies on methane, see D. M. Golden, R. Walsh and S. W. Benson, J. Am. Chem. Soc. 87, 4053 (1965).

See $\Delta H_{f}^{\circ}\left(\varnothing \mathrm{CH}_{2} \dot{\mathrm{C}}\right)$.

From the decomposition kinetics of benzylacetate and diacetyl peroxide.

From the decomposition kinetics of benzylbenzoate and supported by additivity calculations based on $\left(\mathrm{CH}_{3} \mathrm{CO}_{2} \cdot\right)$ heat of formation.

From the decomposition kinetics of dimethyl peroxide.

Additivity calculation from dimethyl peroxide value. Kinetics of the $\left(\mathrm{C}_{2} \mathrm{H}_{5} \mathrm{O}_{2}\right.$ decomposition give $\Delta H_{f}^{\circ}\left(\mathrm{C}_{2} \mathrm{H}_{5} \mathrm{O} \cdot\right)=-4.9 \mathrm{kcal} /$ mole.

Kinetics of decomposition of $\left(\mathrm{C}_{3} \mathrm{H}_{7} \mathrm{O}\right)_{2}$ additivity relations based on $\left(\mathrm{CH}_{3} \mathrm{O}\right.$.)give $\Delta H_{f}^{\circ}\left(\mathrm{C}_{3} \mathrm{H}_{7} \mathrm{O} \cdot\right)$ $=-10.0 \mathrm{kcal} / \mathrm{mole}$.

From the decomposition kinetics of $(t \mathrm{BuO})_{2}$ which depend critically on the corrected heat of formation of the parent peroxide. The most recent value of $\Delta H_{f}^{\circ}(t \mathrm{BuO})_{2}=-80.5 \mathrm{kcal} /$ mole, G. Baker, J. H. Littlefield, R. Shaw, and J. C. J. Thynne, J. Chem. Soc. 6970 (1965) has been used here. Additivity calculations based on $\left(\mathrm{CH}_{3} \mathrm{O} \cdot\right)$ give $\Delta H_{f}^{\circ}(t \mathrm{BuO} \cdot)=-21.3$ $\mathrm{kcal} / \mathrm{mole}$.

Good agreement between the value from additivity based on $\Delta \mathrm{H}_{f}^{\circ}\left(\mathrm{CH}_{3} \mathrm{CO}_{2} \cdot\right)$ and tlie decomposition kinetics of $\left(\mathrm{C}_{2} \mathrm{H}_{5} \mathrm{CO}_{2}\right)_{2}$.

See comments for $\Delta H_{f}^{\circ}\left(\mathrm{C}_{2} \mathrm{H}_{5} \mathrm{CO}_{2} \cdot\right)$.

Determined from the decomposition kinetics of phenylacetic acid (ref. 2). This is considered the most reasonable result because the recombination rate constant calculated is close to that observed for the recombination of propyl+benzl radicals. Also, $\mathrm{D}(\mathrm{H}-\mathrm{COOH})=87.3 \mathrm{kcal} / \mathrm{mole}$, which is close to the corresponding bond dissociation energy in acetaldehyde $\left(\mathrm{D}\left(\mathrm{CH}_{3} \mathrm{CO}-\mathrm{H}\right)\right)$. 


\section{$\Delta H_{f}^{\circ}\left(\emptyset_{2} \mathrm{CH} \cdot\right)$ \\ $(59.3 \pm 6)$}

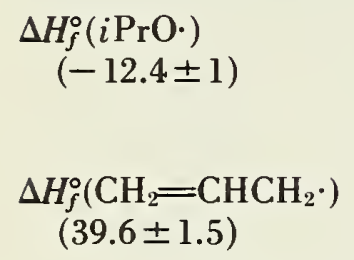

Based on an assumed recombination rate constant of $k_{\text {rec }} \simeq 10^{8.1} 1 /$ mole-sec. The heat of formation gives a total resonance energy which is lower by $4 \mathrm{kcal} / \mathrm{mole}$ than the sum of two benzyl resonances (i.e., Res $=22.8 \mathrm{kcal} / \mathrm{mole}$ compared to $2(13.5)=27 \mathrm{kcal} / \mathrm{mole})$. If the results of Back and Sehon on phenylacetic acid and diphenylacetic acid are accepted, one obtains $\Delta H_{f}^{\circ}\left(\mathrm{C}_{2} \mathrm{CH} \cdot\right) \approx 65.3 \mathrm{kcal} / \mathrm{mole}$ and a resonance energy of $16.7 \mathrm{kcal} / \mathrm{mole}$. This heat of formation is not presently established.

Based on $\Delta H_{f}^{\circ}\left(\mathrm{CH}_{3} \mathrm{O} \cdot\right)$ and additivity relations. The kinetics of the isopropylnitrite decomposition give $\Delta H_{f}^{\circ}(-16.7 \mathrm{kcal} / \mathrm{mole})$. The additivity estimate is believed to be the more reliable.

Measured by iodination studies on propylene as $\Delta H_{f}^{\circ}($ allyl. $)=40.6 \mathrm{kcal} / \mathrm{mole}$. A. S. Rogers, D. M. Golden and S. W. Benson, J. Am. Chem. Soc. 88, 3196 (1966) and from a study of the iodine catalyzed isomerization of 1-butene as 38.0, E. K. Egger, D. M. Golden and S. W. Benson, J. Am. Chem. Soc. 86, 5420 (1964). The kinetics of the allylmethyl sulphone de. composition support the lower value.

\section{$\Delta H_{f}^{\circ}\left(\cdot \mathrm{CH}_{2}\left(\mathrm{CH}_{3}\right) \mathrm{C}=\mathrm{CH}_{2}\right) \quad$ Calculated with additivity relations from $\Delta H_{f}^{\circ}($ allyl).}

$(29.6 \pm 2)$

$\Delta H_{f}^{\circ}\left(\mathrm{C}_{2} \mathrm{H}_{5} \cdot\right)$

$(25.7 \pm 1)$

$\Delta H_{f}^{\circ}\left(n-\mathrm{C}_{3} \mathrm{H}_{7} \cdot\right)$

$(20.7 \pm 1)$

$\Delta H_{f}^{\circ}(i \mathrm{Pr} \cdot)$

$(17.6 \pm 1)$

$\Delta H_{f}^{\circ}(t \mathrm{Bu} \cdot)$

$(6.8 \pm 1)$

$\Delta H_{f}^{\circ}(\emptyset \cdot)$

$(80.0 \pm 1)$

\section{$\Delta H_{f}^{\circ}\left(\varnothing \dot{\mathrm{C}}(\mathrm{H}) \mathrm{CH}_{3}\right)$}

$(36.6 \pm 3)$

\section{$\Delta H_{f}^{\circ}\left(\emptyset \dot{\mathrm{C}}\left(\mathrm{CH}_{3}\right)_{2}\right)$}

(26.3)

$\Delta H_{f}^{\circ}(\mathrm{HS} \cdot)$

$(35.5 \pm 3)$

$\Delta H_{f}^{\circ}(\emptyset \mathrm{S} \cdot)$

(49.5)

$\Delta H_{f}^{\circ}\left(\mathrm{CH}_{3} \mathrm{~S} \cdot\right)$

$(28.0 \pm 3)$

$\Delta H_{f}^{\circ}\left(\mathrm{CH}_{3} \mathrm{SO}_{2} \cdot{ }^{\cdot}\right)$

$(-60.5 \pm \overline{3})$
Iodination studies. D. B. Hartley and S. W. Benson, J. Chem. Phys. 39, 132 (1963).

Additivity relations based on $\Delta H_{f}^{\circ}\left(\mathrm{C}_{2} \mathrm{H}_{5} \cdot\right)$.

Iodination studies. P. S. Nangia and S. W. Benson, J. Am. Chem. Soc. 86, 2773 (1964).

Iodination studies. H. Teranishi and S. W. Benson, J. Am. Chem. Soc. 85, 2887 (1963).

Iodination studies. A. S. Rogers, D. M. Golden, and S. W. Benson, J. Am. Chem. Soc. 89, 4578 (1967). This gives $\mathrm{DH}^{\circ}(\emptyset-\mathrm{H})=112.3 \pm 1 \mathrm{kcal} / \mathrm{mole}$.

Based on the assumption of $\mathrm{D}(\mathrm{C}-\mathrm{H})$ secondary bond dissociation energy in $\varnothing-\mathrm{CH}_{2}-\mathrm{CH}_{3}$ $=95-13.5($ benzl resonance $)=81.5 \mathrm{kcal} / \mathrm{mole}$. The $\Delta H_{f}^{\circ}\left(\emptyset \mathrm{CHCH}_{3}\right)$ from the kinetics is $31.6 \mathrm{kcal} / \mathrm{mole}$.

Based on the assumption of $\mathrm{D}(\mathrm{C}-\mathrm{H})$ tertiary bond dissociation energy in $\emptyset \mathrm{CH}-\left(\mathrm{CH}_{3}\right)_{2}$ is $91-13.5=87.5 \mathrm{kcal} / \mathrm{mole}$.

Based on recent mass spectrometric studies on sulfides. J. A. Kerr, Chem. Revs. 66, 465 (1966).

Based on the kinetics of the phenyl methyl sulfide decomposition.

Based on the decomposition kinetics of $\emptyset \mathrm{CH}_{2} \mathrm{SCH}_{3}$ and on an assumed rate constant for recombination, $k_{\mathrm{rec}} \simeq 10^{8.8} \mathrm{l} / \mathrm{mole}$-sec (a mean between the rate constants of recombination for $\mathrm{CH}_{3}+\emptyset \mathrm{CH}_{2} \cdot$ and $\left.\mathrm{C}_{2} \mathrm{H}_{5} \cdot+\varnothing \mathrm{CH}_{2} \cdot\right)$. Appearance potential work places $\Delta H_{f}^{\circ}\left(\mathrm{CH}_{3} \mathrm{~S}\right)<31.8$ kcal/mole, T. F. Palmer and F. P. Lossing, J. Am. Chem. Soc. 84, 4661 (1962).

Based on the decomposition kinetics of dimethylsulfone, however see comments for this reaction. 
$\Delta H_{f}^{\circ}\left(\cdot \mathrm{CH}_{2}-\mathrm{C} \equiv \mathrm{N}\right)$

$(51.1 \pm 3)$

$\Delta H_{f}^{\circ}\left(\mathrm{CH}_{3}\right)_{2} \dot{\mathrm{C}}-\mathrm{C} \equiv \mathrm{N}$

$(33.8 \pm 3)$

$\Delta H_{f}^{\circ}\left(\varnothing \dot{\mathrm{C}}\left(\mathrm{CH}_{3}\right) \mathrm{CN}\right)$

$(54.4 \pm 3)$

$\Delta H_{f}^{\circ}\left(\mathrm{CF}_{2}:\right)$

$(-39.7 \pm 2)$

$\Delta H_{f}^{\circ}\left(\mathrm{CF}_{3} \mathrm{C}=\mathrm{CF}_{2}\right)$

$(-177.6)$

$\Delta H_{f}^{\circ}\left(\cdot \mathrm{CCl}_{3}\right)$

$(18.5 \pm 2)$

$\Delta H_{f}^{\circ}\left(\mathrm{CH}_{3} \mathrm{~N}_{2} \cdot\right)$

(66.0)

$\Delta H_{f}^{\circ}(\mathrm{R}-\mathrm{N}=\mathrm{N} \cdot)$

$(R=n$ alkyl $)$

$\Delta H_{f}^{\circ}\left(\mathrm{C}_{2} \mathrm{H}_{5} \mathrm{CH}\left(\mathrm{CH}_{3}\right) \mathrm{N}=\mathrm{N} \cdot\right)$

(47.0)

$\Delta H_{f}^{\circ}(t \mathrm{BuN}=\mathrm{N} \cdot)$

(43.5)

$\Delta H_{f}^{\circ}\left(\mathrm{NH}_{2} \cdot\right)$

$(43.3 \pm 3)$

$\Delta H_{f}^{\circ}\left(\mathrm{CH}_{3} \mathrm{NH}\right)$

(41.7)

$\Delta H_{f}^{\circ}\left[\left(\mathrm{CH}_{3}\right)_{2} \mathrm{~N} \cdot\right]$

(37.4)

$\Delta H_{f}^{\circ}(\varnothing \mathrm{NH})$

(52.8)

$\Delta H_{f}^{\circ}\left(\emptyset \mathrm{NCH}_{3}\right)$

(49.6) or (53.5)
Based on the decomposition kinetics of ethyl cyanide. Resonance energy equal $\simeq 12.5$ $\mathrm{kcal} / \mathrm{mole}$. Analysis of the activation energy for the cis-trans isomerization of $\beta$-cyanostyrene gives $10 \mathrm{kcal} / \mathrm{mole}$ for the resonance energy.

Based on the kinetics of the $t$-butylcyanide decomposition. Resonance energy obtained is $10.6 \mathrm{kcal} / \mathrm{mole}$.

Based on the decomposition kinetics of cumyl cyanide. Resonance energy $=22.1 \mathrm{kcal} / \mathrm{mole}$ which is close to the sum of the benzyl and cyano resonance energies (i.e., $\sim 13.5+10.6$ $=24.1 \mathrm{kcal} / \mathrm{mole}$, respectively).

Based on the equilibrium for the elimination reaction, $\mathrm{HCF}_{2} \mathrm{Cl} \rightleftarrows \mathrm{HCl}+\mathrm{CF}_{2}$ :, and the equilibrium for the tetrafluoroethylene decomposition, $\mathrm{C}_{2} \mathrm{~F}_{4} \rightleftarrows 2 \mathrm{CF}_{2}$ :

Based on the kinetics of the dissociation of perfluoroisobutene (not very reliable).

Based on the analysis of the bromination of chlororform, S. W. Benson, J. Chem. Phys. 43, 2044 (i965).

The decomposition kinetics of azomethane give $\Delta H_{f}^{\circ}=70.3 \mathrm{kcal} / \mathrm{mole}$.

See section V-8.0. Values in brackets below come directly from the kinetics.

Best compromise from the kinetics of all amine and hydrazine decompositions (see section V-8.1).

[41.0]

[34.9]

[48.6] 
TABle D-2. Calculated Thermodynamic Properties ${ }^{\mathrm{a}, \mathrm{b}, \mathrm{c}, \mathrm{d}}$ of Free Radicals: entropies and heat capacities

\begin{tabular}{|c|c|c|c|c|c|c|c|c|c|c|}
\hline Radical & $\sigma$ & $S^{\circ}$ & 300 & 400 & 500 & 600 & 800 & 1000 & 1500 & $V_{0}^{d}$ \\
\hline $\begin{array}{l}\mathrm{CH}_{3} \cdot \\
\mathrm{C}_{2} \mathrm{H}_{5} \cdot{ }^{\cdot a, b}\end{array}$ & $\begin{array}{l}6 \\
6\end{array}$ & $\begin{array}{r}46.4 \\
{[59.5} \\
58.9 \\
58.3 \\
57.9\end{array}$ & $\begin{array}{r}8.3 \\
11.1 \\
11.6 \\
11.4 \\
10.7\end{array}$ & $\begin{array}{r}9.1 \\
14.1 \\
14.6 \\
14.6 \\
14.0\end{array}$ & $\begin{array}{l}10.1 \\
16.8 \\
17.1 \\
17.3 \\
16.9\end{array}$ & $\begin{array}{l}10.9 \\
19.1 \\
19.3 \\
19.6 \\
19.4\end{array}$ & $\begin{array}{l}12.6 \\
22.9 \\
23.0 \\
23.3 \\
23.4\end{array}$ & $\begin{array}{l}14.0 \\
25.8 \\
25.9 \\
26.1 \\
26.3\end{array}$ & $\begin{array}{l}16.3 \\
30.5 \\
30.5 \\
30.6 \\
30.9\end{array}$ & $\begin{array}{l}0] \\
2 \\
4 \\
8\end{array}$ \\
\hline $\begin{array}{l}\mathrm{C}_{3} \mathrm{H}_{7} \\
n \text {-propyl }\end{array}$ & 6 & $\begin{array}{r}68.9 \\
{[68.1} \\
67.3 \\
66.6\end{array}$ & $\begin{array}{l}16.6 \\
17.7 \\
17.7 \\
17.3\end{array}$ & $\begin{array}{l}20.9 \\
21.8 \\
22.0 \\
21.8\end{array}$ & $\begin{array}{l}24.9 \\
25.5 \\
26.1 \\
25.9\end{array}$ & $\begin{array}{l}28.4 \\
28.9 \\
29.4 \\
29.5\end{array}$ & $\begin{array}{l}33.8 \\
34.1 \\
34.6 \\
35.0\end{array}$ & $\begin{array}{l}38.1 \\
38.3 \\
38.7 \\
39.3\end{array}$ & $\begin{array}{l}44.7 \\
44.9 \\
45.1 \\
45.7\end{array}$ & $\begin{array}{l}0 \\
2] \\
4 \\
8\end{array}$ \\
\hline $\begin{array}{l}i-\mathrm{C}_{3} \mathrm{H}_{7} \cdot \\
\text { isopropyl }\end{array}$ & 18 & $\begin{array}{r}68.8 \\
{[67.0} \\
65.6 \\
64.2\end{array}$ & $\begin{array}{l}15.3 \\
17.5 \\
17.5 \\
16.7\end{array}$ & $\begin{array}{l}19.6 \\
21.4 \\
21.8 \\
21.4\end{array}$ & $\begin{array}{l}23.6 \\
24.8 \\
26.0 \\
25.6\end{array}$ & $\begin{array}{l}27.1 \\
28.2 \\
29.2 \\
29.4\end{array}$ & $\begin{array}{l}32.9 \\
33.5 \\
34.5 \\
35.3\end{array}$ & $\begin{array}{l}37.4 \\
37.8 \\
38.6 \\
39.8\end{array}$ & $\begin{array}{l}44.3 \\
44.7 \\
45.1 \\
46.3\end{array}$ & $\begin{array}{l}0 \\
2] \\
4 \\
8\end{array}$ \\
\hline $\begin{array}{l}\mathrm{CH}_{3} \dot{\mathrm{C}} \mathrm{HCH}_{2} \mathrm{CH}_{3} \\
\text { sec-butyl } \\
\left(\mathrm{CH}_{3}\right)_{3} \mathrm{C} \cdot \\
\mathrm{V}_{0}\left(\text { iso }_{4} \mathrm{H}_{10}\right)=4.0\end{array}$ & $\begin{array}{r}9 \\
6(27)\end{array}$ & $\begin{array}{r}{[77.6} \\
{[74.6} \\
72.5 \\
70.4 \\
68.6\end{array}$ & $\begin{array}{l}23.1 \\
19.3 \\
21.7 \\
21.1 \\
19.3\end{array}$ & $\begin{array}{l}28.4 \\
25.1 \\
26.9 \\
27.5 \\
26.0\end{array}$ & $\begin{array}{l}33.3 \\
29.9 \\
31.4 \\
32.3 \\
31.4\end{array}$ & $\begin{array}{l}37.8 \\
35.1 \\
36.0 \\
37.5 \\
366.9\end{array}$ & $\begin{array}{l}44.9 \\
42.9 \\
43.5 \\
44.7 \\
45.3\end{array}$ & $\begin{array}{l}50.3 \\
48.9 \\
49.2 \\
50.4 \\
51.3\end{array}$ & $\begin{array}{l}58.9] \\
58.4 \\
58.7 \\
59.0 \\
60.2\end{array}$ & {$\left[\begin{array}{c}2 / 3 \mathrm{~V}_{0}^{\mathrm{e}} \\
0] \\
2 \\
4 \\
8\end{array}\right.$} \\
\hline $\begin{array}{l}\varnothing \cdot \\
\varnothing \mathrm{CH}_{2} \cdot \\
\varnothing \dot{\mathrm{C}} \mathrm{CCH}_{3}\end{array}$ & $\begin{array}{l}2 \\
2 \\
3\end{array}$ & $\begin{array}{r}69.1 \\
75.4 \\
{[85.1}\end{array}$ & $\begin{array}{l}18.96 \\
25.36 \\
30.4\end{array}$ & $\begin{array}{l}25.56 \\
33.18 \\
40.0\end{array}$ & $\begin{array}{l}31.01 \\
40.08 \\
47.8\end{array}$ & $\begin{array}{l}35.50 \\
45.63 \\
54.7\end{array}$ & $\begin{array}{l}42.20 \\
54.00 \\
64.8\end{array}$ & $\begin{array}{l}46.76 \\
59.91 \\
71.8\end{array}$ & $\begin{array}{l}53.74 \\
68.77 \\
82.2]\end{array}$ & $2 / 3 \mathrm{~V}_{0}^{\mathrm{e}, 13}$ \\
\hline $\begin{array}{l}\emptyset 0 . \\
\emptyset \mathrm{S} .\end{array}$ & $\begin{array}{l}2 \\
2\end{array}$ & $\begin{array}{l}73.7 \\
76.5\end{array}$ & $\begin{array}{l}22.5 \\
23.6\end{array}$ & $\begin{array}{l}29.8 \\
30.6\end{array}$ & $\begin{array}{l}35.8 \\
36.5\end{array}$ & $\begin{array}{l}40.6 \\
41.2\end{array}$ & $\begin{array}{l}47.5 \\
47.9\end{array}$ & $\begin{array}{l}52.3 \\
52.5\end{array}$ & & \\
\hline$\varnothing \dot{\mathrm{C}}\left(\mathrm{CH}_{3}\right)_{2}$ & 18 & {$[90.6$} & 36.0 & 46.1 & 55.4 & 63.3 & 75.2 & 83.7 & $96.5]$ & $2 / 3 \mathrm{~V}_{0}^{\mathrm{e}}, 13$ \\
\hline $\begin{array}{l}\mathrm{CH}_{3} \mathrm{~S} \cdot \\
\mathrm{CH}_{3} \mathrm{CH}_{2} \mathrm{~S} \cdot \\
\left(\mathrm{CH}_{3}\right)_{2} \mathrm{CHS} \cdot \\
\left(\mathrm{CH}_{3}\right)_{3} \mathrm{CS} \cdot\end{array}$ & & $\begin{array}{l}57.6 \\
67.2 \\
74.2 \\
77.2\end{array}$ & $\begin{array}{r}9.9 \\
15.2 \\
20.9 \\
26.8\end{array}$ & $\begin{array}{l}18.4 \\
25.7 \\
33.3\end{array}$ & $\begin{array}{l}13.4 \\
21.8 \\
30.4 \\
39.4\end{array}$ & $\begin{array}{l}24.4 \\
33.9 \\
44.2\end{array}$ & $\begin{array}{l}17.0 \\
28.8 \\
39.8 \\
51.3\end{array}$ & $\begin{array}{l}18.8 \\
32.2 \\
44.1 \\
56.6\end{array}$ & & \\
\hline $\begin{array}{l}\mathrm{CH}_{3} \mathrm{O} \cdot \\
\mathrm{CH}_{3} \mathrm{CH}_{2} \mathrm{O} \cdot \\
\left(\mathrm{CH}_{3}\right)_{2} \mathrm{CHO} \cdot \\
\left(\mathrm{CH}_{3}\right)_{3} \mathrm{CO} \cdot\end{array}$ & $\begin{array}{r}3 \\
3 \\
9 \\
81\end{array}$ & $\begin{array}{l}54.3 \\
64.6 \\
71.1 \\
75.0\end{array}$ & $\begin{array}{r}9.1 \\
14.1 \\
20.1 \\
25.8\end{array}$ & $\begin{array}{l}17.6 \\
25.2 \\
32.6\end{array}$ & $\begin{array}{l}12.6 \\
20.2 \\
29.4 \\
37.9\end{array}$ & $\begin{array}{l}23.6 \\
33.7 \\
43.5\end{array}$ & $\begin{array}{l}16.8 \\
28.0 \\
39.7 \\
51.2\end{array}$ & $\begin{array}{l}18.9 \\
31.2 \\
44.0 \\
56.6\end{array}$ & & \\
\hline $\begin{array}{l}\mathrm{CH}_{2}=\mathrm{CHCH}_{2} \cdot \\
\mathrm{CH}_{2}=\mathrm{C}\left(\mathrm{CH}_{3}\right) \mathrm{CH}_{2} \cdot \\
\mathrm{CH}_{2}=\mathrm{CHCHCH} \mathrm{CH}_{3}\end{array}$ & $\begin{array}{l}2 \\
6 \\
3\end{array}$ & $\begin{array}{l}62.1 \\
68.8 \\
70.8\end{array}$ & $\begin{array}{l}14.6 \\
20.8 \\
20.0\end{array}$ & $\begin{array}{l}18.5 \\
26.1 \\
25.4\end{array}$ & $\begin{array}{l}21.8 \\
30.5 \\
29.9\end{array}$ & $\begin{array}{l}24.4 \\
34.1 \\
33.8\end{array}$ & $\begin{array}{l}28.8 \\
40.0 \\
39.9\end{array}$ & $\begin{array}{l}31.9 \\
44.4 \\
44.3\end{array}$ & $\begin{array}{l}36.8 \\
51.2 \\
51.1\end{array}$ & $\begin{array}{r}13.0 \\
\left\{\begin{array}{r}15.0 \\
15.0 \\
3.0\end{array}\right.\end{array}$ \\
\hline
\end{tabular}


TABle D-2. Calculated Thermodynamic Properties ${ }^{\mathrm{a}, \mathrm{b}, \mathrm{c}, \mathrm{d}}$ of Free Radicals: entropies and heat capacities-Continued

\begin{tabular}{|c|c|c|c|c|c|c|c|c|c|c|}
\hline Radical & $\sigma$ & $S^{\circ}$ & 300 & 400 & 500 & 600 & 800 & 1000 & 1500 & $\mathrm{~V}_{0}$ \\
\hline $\mathrm{CH}_{3} \mathrm{CO}_{2}$ & 6 & 66.6 & 12.7 & 14.6 & 16.5 & 18.2 & 21.1 & 23.3 & & \\
\hline $\mathrm{CH}_{3} \mathrm{CH}_{2} \mathrm{CO}_{2} \cdot$ & 6 & 76.2 & 18.9 & 22.3 & 25.2 & 27.7 & 32.2 & 35.5 & & \\
\hline $\mathrm{NH}_{2}$ & 2 & 48.4 & 8.1 & & 9.1 & & 10.3 & 11.6 & & \\
\hline$\cdot \mathrm{CH}_{2} \mathrm{C} \equiv \mathrm{N}$ & 2 & 58.5 & 10.7 & 12.8 & 14.5 & 15.9 & 18.1 & 19.8 & & \\
\hline $\mathrm{CH}_{3} \dot{\mathrm{C}} \mathrm{HC} \equiv \mathrm{N}$ & 3 & {$[68.8$} & 15.3 & 19.1 & 22.2 & 24.9 & 29.0 & 31.9 & & ] $2 / 3 \mathrm{~V}_{\mathbf{0}}^{\mathrm{e}}$ \\
\hline$\left(\mathrm{CH}_{3}\right)_{2} \dot{\mathrm{C}}-\mathrm{C} \equiv \mathrm{N}$ & 18 & {$[75.8$} & 21.2 & 25.8 & 29.1 & 33.2 & 39.0 & 43.5 & & ] $2 / 3 \mathrm{~V}_{0}^{\mathrm{e}}$ \\
\hline $\mathrm{CH}_{3} \dot{\mathrm{NH}}$ & 3 & {$[59.0$} & 11.3 & 13.3 & 15.1 & 16.8 & 19.5 & 21.7 & $25.3]$ & $2 / 3 \mathrm{~V}_{0}^{\mathrm{e}}$ \\
\hline$\left(\mathrm{CH}_{3}\right)_{2} \mathrm{~N}$ & 18 & {$[66.2$} & 15.7 & 19.2 & 22.7 & 25.7 & 30.5 & 34.3 & $39.9]$ & $2 / 3 V_{0}^{e}$ \\
\hline$\emptyset \dot{\mathrm{NH}}$ & 1 & 75.3 & 24.8 & 32.8 & 39.2 & 44.2 & 51.4 & 56.3 & & 13 \\
\hline$\emptyset \mathrm{NCH}_{3}$ & 3 & {$[83.3$} & 30.2 & 39.3 & 47.0 & 53.3 & 62.6 & 69.0 & & i] $2 / 3 \mathrm{~V}_{0}^{\mathrm{e}}, 1$ \\
\hline$\cdot \mathrm{COOH}$ & 1 & 60.7 & 10.2 & 11.8 & 12.9 & 13.7 & 15.4 & 16.3 & & 13 \\
\hline \multirow[t]{4}{*}{$\left(\mathrm{CH}_{3}\right)_{3} \mathrm{CCH}_{2}$. } & \multirow[t]{4}{*}{$(3)^{3} 6$} & {$[78.8$} & 27.8 & 35.8 & 42.6 & 48.6 & 59.5 & 63.9 & 73.7 & $0]$ \\
\hline & & 78.2 & 28.3 & 36.3 & 42.9 & 48.8 & 59.6 & 64.0 & 73.7 & 2 \\
\hline & & 77.6 & 28.1 & 36.3 & 43.1 & 49.1 & 59.9 & 64.2 & 73.8 & 4 \\
\hline & & 77.2 & 27.4 & 35.7 & 42.7 & 48.9 & 60.0 & 64.4 & 74.1 & 8 \\
\hline \multirow[t]{4}{*}{$\mathrm{CH}_{3} \dot{\mathrm{C}} \mathrm{HCH}\left(\mathrm{CH}_{3}\right)_{2}$} & \multirow[t]{4}{*}{27} & 86.4 & 25.9 & 33.3 & 40.0 & 45.9 & 55.1 & 62.1 & 73.1 & 0 \\
\hline & & {$[84.7$} & 28.1 & 35.0 & 41.3 & 46.9 & 55.7 & 62.5 & 73.3 & 2] \\
\hline & & 83.0 & 28.5 & 35.7 & 42.5 & 48.0 & 56.8 & 63.4 & 73.7 & 4 \\
\hline & & 81.5 & 27.7 & 35.2 & 42.2 & 48.3 & 57.6 & 64.6 & 75.0 & 8 \\
\hline
\end{tabular}

"There is a small correction $(+0.3 \mathrm{~g} /$ mole) to the ethyl radical entropy obtained from the grou ss, due to the differences in reduced moments of inertia for roughly equivalent opposed mass rotors.

${ }^{b}$ Brackets indicate the values favored by the authors.

c Note that the values of this table represent our best estimates and have been generated over the period of preparation of this monograph (roughly 2 years). Values on some data sheets may be somewhat different but such differences should not exceed $\pm 1-2 \mathrm{~g} /$ mole in either the entropy or heat capacity.

${ }^{d} V_{0}$ denotes the barriers to rotation for the alkyl or aryl groups bonded to the radical centers.

${ }^{e} V_{0}$ for nonconjugated groups bonded to the radical center are assumed to be $2 / 3$ their value in the corresponding saturated compound. 


\section{、}




\section{REFERENCES TO SECTIONS I-VI AND APPENDICES}

[1] JANAF (United States Joint Army Navy Air Force) Interim Thermochemical Tables, Vols. 1 and 2 (Thermal Laboratory, The Dow Chemical Co., Midland, Mich., 1965).

[2] American Petroleum Institute Research Project 44 (API tables), Carnegie Institute of Technology (Chemical Thermodynamic Properties Center, Texas A\&M Univ., College Station, Tex., 1966).

[3] Additivity Rules for the Estimation of Thermochemical Properties:

I. Hydrocarbons, Benson, S. W., et al., Chem. Revs. 69 (1969).

II. Oxygen compounds, Benson, S. W., et al., Chem. Revs. 69 (1969).

III. Nitrogen containing compounds, Benson, S. W., et al., Chem. Revs. 69 (1969).

IV. Halogen substituted compounds, Benson, S. W., et al., Chem. Revs. 69 (1969).

V. Organometallic and organo-nonmetallic compounds, Benson, S. W., et al., Chem. Revs. 69 (1969).

VI. Free radicals and polycyclic compounds, O'Neal, H. E., and Benson, S. W., Int. J. Chem. Kinetics (1969).

[4] Janz, J. G., Estimation of the Thermodynamic Properties of Organic Molecules (Academic Press, New York, N.Y., 1958).

[5] Benson, S. W., The Foundations of Chemical Kinetics (McGraw-Hill Book Co., Inc., New York, N.Y., 1960).

[6] Benson, S. W., J. Chem. Ed. 42, 502 (1965).

[7] Kerr, J. A., Chem. Revs. 66, 465 (1966).

[8] Purnell, J. H., and Quinn, C. P., J. Chem. Soc., 4049 (1964).

[9] Mortimer, C. T., Reaction Heats and Bond Strengths (Pergamon Press, New York, N.Y., 1962).

[10] Lewis, G. N., and Randall, M., revised by Pitzer, K. S., and Brewer, L., Thermodynamics, 2d ed. (McGraw-Hill Book Co., Inc., New York, N.Y., 1961).

[11] Keller, R., Basic Tables in Chemistry (McGraw-Hill Book Co., Inc., New York, N.Y., 1967).

[12] The Handbook of Chemistry and Physics, 47th ed. (The Chemical Rubber Co., Cleveland, Ohio, 1966-67).

[13] NBS Tech. Note 270-3 (Jan. 1968).

[14] Bernstein, H. J., J. Phys. Chem. 69, 1550 (1965).

[15] Benson, S. W., Thermochemical Kinetics (John Wiley \& ' Sons, New York, N.Y., 1968).

[16] O'Neal, H. E., and Ring, M. A., Inorg. Chem. 5, 435 (1966).

[17] Maccoll, A., and Thomas, P. J., J. Chem. Soc. 979 (1955).

[18] Barton, D. H. R., et al., Trans. Faraday Soc. 45, 725 (1949).

[19] Thömas, P. J., J. Chem. Soc. 1192 (1959).

[20] Blades, A. T., and Murphy, G. W., J. Am. Chem. Soc. 74, 6219 (1952).

[21] Bárker, R., and Maccoll, A., J. Chem. Soc. 2839 (1963).

[22] Blades, A. T., Gilderson, P. W., and Wallbridge, M. G. H., Can. J. Chem. 40, 1526, 1533 (1962).

[23] Goldberg, A. E., and Daniels, F., J. Am. Chem. Soc. 79, 1314 (1957).

[24] Harden, G. D., and Maccoll, A., J. Chem. Soc. 2454 (1955).

[25] O’Neal, H. E., and Benson, S. W., J. Phys. Chem. 71, 2903 (1967).
[26] Pauling, L., The Nature of the Chemical Bond, 3d ed. (Cornell Univ. Press, Ithaca, N.Y., 1960), pp. 221-264.

[27] Trotman-Dickenson, A. F., Gas Kinetics (Butterworths Sci. Publ., London, 1955).

[28] Schlag, E., and Rabinovitch, B. S., J. Am. Chem. Soc. 82, 5996 (1960).

[29] Benson, S. W., J. Chem. Phys. 34, 521 (1961).

[30] Willicott, M. R., and Cargle, V. H., J. Am. Chem. Soc. 89, 723 (1967).

[31] Turner, R. B., Tet. Letters 15, 997 (1965).

[32] Foster, E. G., Cope, A. C., and Daniels, F., J. Am. Chem. Soc. 69, 1893 (1947).

[33] Benson, S. W., Egger, K. W., and Golden, D. M., J. Am. Chem. Soc. 87, 468 (1965).

[34] Cundall, R. B., Progress in Reaction Kinetics, Vol. 2 (The Macmillan Book Co., New York, N.Y., 1964), p. 167.

[35] Steacie, E. W. R., Atomic and Free Radical Reactions, Vol. 1 (Reinhold Publ. Corp., New York, N.Y., 1954).

[36] McNesby, J. R., and Gordon, A. S., J. Am. Chem. Soc. 79, 4593 (1957).

[37] Bryce, W. A., and Hardiman, M. S., Can. J. Chem. 40, 1031 (1962).

[38] Voevodski, V. V., Trans. Faraday Soc. 55, 65 (1959); Kinetika i Kataliz 5, 603 (1964). Wojcieckowski, B. W., and Laidler, K. J., Can. J. Chem. 38, 1027 (1960). Wojcieckowski, B. W., and Laidler, K. J., Trans. Faraday Soc. 59, 369 (1963). Norrish, R. G. W., and Pratt, G. L., Nature 197, 143 (1963). Ree, T., Yakn, K., and Eyring, H., Trans. Faraday Soc. 58, 2375 (1962). Gowenlock, B. G., Progress in Reaction Kinetics, Vol. 3 (Pergamon Press, New York, N.Y., 1965), p. 171.

[39] Blades, A. T., and Steacie, E. W. R., Can. J. Chem. 32, $1142(1954)$

[40] Fettis, G. C., and Knox, J. H., Progress in Reaction Kinetics, Vol. 2 (The Macmillan Book Co., New York, N.Y., 1964), p. 1.

[41] Hammond, G. S., J. Am. Chem. Soc. 77, 334 (1955).

[42] Fueno, T., and Benson, S. W., J. Chem. Phys. 36, 1597 (1962).

[43] Purnell, J. H., and Quinn, C. P. J. Chem. Soc. 4049 (1964).

[44] Mosley, F., and Robb, J. C., Proc. Roy. Soc. (London). A243, 130 (1957).

[45] Shepp, A., J. Chem. Phys. 24, 939 (1956).

[46] Kistiakowsky, G. B., and Roberts, E. K., J. Chem. Phys. 21 , 1637 (1953).

[47] Pritchard, H. O., J. Chem. Phys. 25, 267 (1956).

[48] Steel, C., J. Chem. Phys. 31, 899 (1959).

[49] Schlag, E. W. R., J. Chem. Phys. 35, 2117 (1961).

[50] Steel, C., and Laidler, K. J., J. Chem. Phys. 34, 1827 (1961).

[51] Metcalfe, E. L., and Trotman-Dickenson, A. F., J. Chem. Soc., 4260 (1962).

[52] Overberger, C. G., and DiGuilio, A. V., J. Am. Chem. Soc. 81,2154 (1959).

[53] Seltzer, S., and Dunne, F. T., J. Am. Chem. Soc. 87, 2628 (1965).

[54] Walling, C., Free Radicals in Solution John Wiley \& Sons, Inc., New York, N.Y., 1957), p. 576. 
[55] Welland, H., Hove, H., and Borner, K., Ann. Chem. 446, 31 (1926).

[56] Calvert, J., Chem. Revs. 59, 569 (1959).

[57] Kerr, J. A., and Trotman-Dickenson, A. F., Progress in Reaction Kinetics, Vol. 1 (Pergamon Press, New York, N.Y., 1961), p. 107.

[58] Dale, J., Tetrahedron 22, 3373 (1966).

[59] Zirnett, U. A., and Sushinskii, M. M., Optics and Spectroscopy 16, 489 (1964).

[60] Walsh, R., and Benson, S. W., J. Phys. Chem. 70, 3751 (1966).

[61] Golden, D. M., Walsh, R., and Benson, S. W., J. Am. Chem. Soc. 87, 4053 (1965).

[62] Baker, G., Littlefield, J. H., Shaw, R., and Thynne, J. C. J., J. Chem. Soc., 6970 (1965).
[63] Rogers, A. S., Golden, D. M., and Benson, S. W., J. Am. Chem. Soc. 88, 3196 (1966).

[64] Egger, E. K., Golden, D. M., and Benson, S. W., J. Am. Chem. Soc. 86, 5420 (1964).

[65] Hartley, D. B., and Benson, S. W.. J. Chem. Phys. 39, 132 (1963).

[66] Nangia, P. S., and Benson, S. W., J. Am. Chem. Soc. 86, 2773 (1964).

[67] Teranishi, H., and Benson, S. W., J. Am. Chem. Soc. 85, 2887 (1963).

[68] Rogers, A. S., Golden, D. M., and Benson, S. W., J. Am. Chem. Soc. 89, 4578 (1967).

[69] Palmer, T. F., and Lossing, F. P., J. Am. Chem. Soc. 84, 4661 (1962).

[70] Benson, S. W., J. Chem. Phys. 43, 2044 (1965). 
1. Complex Fissions -

Vlolecular Elimination Reactions 


\section{.}


Reaction: Chloroethane (ethyl chloride)

$\begin{array}{lrcr} & \mathrm{C}_{2} \mathrm{H}_{5} \mathrm{Cl}(\mathrm{I}) \rightarrow \mathrm{C}_{2} \mathrm{H}_{4}(\mathrm{II})+\mathrm{HCl}(\mathrm{III}) & \\ & \mathrm{I} & \text { II } & \text { III } \\ \Delta \mathrm{H}_{\mathcal{N}(298)}^{\circ} & -26.3 & 12.5 & -22.1 \\ S_{(298)}^{\circ} & 66.0 & 52.5 & 44.6 \\ C_{p(298)}^{\circ} & 15.1 & 10.4 & 7.0\end{array}$

$\Delta S^{\circ}=31.1 \mathrm{~g} / \mathrm{mole}$

$\Delta H^{\circ}=16.7 \mathrm{kcal} / \mathrm{mole}$

$\Delta C_{p}^{\circ}=2.3 \mathrm{~g} / \mathrm{mole}$

\begin{tabular}{|c|c|c|c|c|c|c|}
\hline $\log A$ & $\boldsymbol{E}$ & $\begin{array}{c}\log \boldsymbol{k}_{T} \\
(700)\end{array}$ & Conditions & System & Surface & References \\
\hline 13.16 & 56.46 & -4.46 & $\begin{array}{l}740-940{ }^{\circ} \mathrm{K} \\
1 \% \text { in } \mathrm{Ar}\end{array}$ & S.P. shock & - & $\begin{array}{l}\text { [1] W. Tsang, J. Chem. } \\
\text { Phys. 41, } 2487 \text { (1964). }\end{array}$ \\
\hline 14.2 & 59.5 & -4.37 & $\begin{array}{l}671-766^{\circ} \mathrm{K} \\
20-200 \text { torr }\end{array}$ & static & none & $\begin{array}{l}\text { [2] D. H. R. Barton and K. E. } \\
\text { Howlett, J. Chem. Soc., } \\
165 \text { (1949). }\end{array}$ \\
\hline 14.6 & 60.8 & -4.38 & $\begin{array}{l}685-729^{\circ} \mathrm{K} \\
20-200 \text { torr }\end{array}$ & static & & $\begin{array}{l}\text { [3] K. E. Howlett, J. Chem. } \\
\text { Soc. } 3695 \text { (1952). }\end{array}$ \\
\hline 13.51 & 56.61 & -4.16 & $\begin{array}{l}684-744{ }^{\circ} \mathrm{K} \\
136-210 \text { torr }\end{array}$ & static & none & $\begin{array}{l}\text { [4] H. Hartman, H. G. } \\
\text { Bosche, and H. Heydt- } \\
\text { mann, Zeit. für Physik. } \\
\text { Chemie, N.F. 42, } 329 \\
\text { (1964). }\end{array}$ \\
\hline 14.0 & 58.4 & -4.25 & $\begin{array}{l}675-794{ }^{\circ} \mathrm{K} \\
0.2-134 \text { torr }\end{array}$ & static & - & $\begin{array}{l}\text { [5] K. A. Holbrook and } \\
\text { A. R. W. Marsh, Trans. } \\
\text { Faraday Soc. 63, } 643 \\
\text { (1967). }\end{array}$ \\
\hline
\end{tabular}

Preferred:

$\log k=13.2-56.5 / \theta$.

$\log A_{\text {est }}=13.3$ (see section II-1.0).

\section{Experimental}

[1] Analysis is G.L.C. on ethylene. Comparative internal rate standard was 2-chloropropane.

[2] Rates by $\Delta \mathrm{P}$. Stoichiometry confirmed by $\mathrm{HCl}$ titration.

[3] Rates by $\triangle P$. Pressure dependence observed below 8 torr. Addition of $\mathrm{HCl}$ and ethylene did not affect the rate at pressures greater than 8 torr.

[4] Rates by HCl titration. 1,1,2,2d $d_{4}$ isotope effect studied by A. T. Blades, P. W. Gilderson, and M. G. H. Wallbridge, Can. J. Chem. 40, 5126 (1962). 
Reaction: 1-Chloropropane ( $n$-propyl chloride)

$\mathrm{CH}_{3} \mathrm{CH}_{2} \mathrm{CH}_{2} \mathrm{Cl}(\mathrm{I}) \rightarrow \mathrm{CH}_{3} \mathrm{CH}=\mathrm{CH}_{2}$ (II) $+\mathrm{HCl}$ (III)

$\begin{array}{lrcr} & \text { I } & \text { II } & \text { III } \\ \Delta H_{f(298)}^{\circ} & -31.3 & 4.8 & -22.1 \\ S_{(298)}^{\circ} & 75.4 & 63.6 & 44.6 \\ C_{p(298)}^{\circ} & 20.6 & 15.3 & 7.0\end{array}$

$\Delta S^{\circ}=32.8 \mathrm{~g} / \mathrm{mole}$

$\Delta H^{\circ}=14.0 \mathrm{kcal} / \mathrm{mole}$

$\Delta C_{p}^{\circ}=1.7 \mathrm{~g} / \mathrm{mole}$

\begin{tabular}{|c|c|c|c|c|c|c|}
\hline $\log A$ & $\boldsymbol{E}$ & $\begin{array}{c}\log \boldsymbol{k}_{T} \\
(700)\end{array}$ & Conditions & System & Surface & References \\
\hline 13.45 & 55.0 & -3.72 & $\begin{array}{l}693-751^{\circ} \mathrm{K} \\
50-155 \text { torr }\end{array}$ & static & none & $\begin{array}{l}\text { [1] D. H. R. Barton, A. J. } \\
\text { Head, and R. J. Wil- } \\
\text { liams, J. Chem. Soc. } \\
2039 \text { (1951). }\end{array}$ \\
\hline 13.50 & 55.08 & -3.69 & $\begin{array}{l}672-733^{\circ} \mathrm{K} \\
243-301 \text { torr }\end{array}$ & static & none & $\begin{array}{l}\text { [2] H. Hartman, H. G. Bosche, } \\
\text { and H. Heydtmann, Zeit } \\
\text { für Physik. Chemie, } \\
\text { N.F. } 42,329 \text { (1964). }\end{array}$ \\
\hline
\end{tabular}

Preferred:

$\log k=13.45-55.0 / \theta$

$\log A_{\text {est }}=13.2$ (see section II-1.0).

\section{Experimental}

[1] Rates by $\Delta \mathrm{P}$. Not inhibited by added propylene.

[2] Rates by $\mathrm{HCl}$ titration. 
Reaction:1-Chlorobutane ( $n$-butyl chloride)

$$
\begin{array}{lccc}
\mathrm{CH}_{3}\left(\mathrm{CH}_{2}\right)_{2} \mathrm{CH}_{2} \mathrm{Cl}(\mathrm{I}) \rightarrow \mathrm{CH}_{3} \mathrm{CH}_{2} \mathrm{CH}=\mathrm{CH}_{2}(\mathrm{II})+\mathrm{HCl}(\mathrm{III}) \\
& \text { I } & \text { II } & \text { III } \\
\Delta H_{f(298)}^{\circ} & -36.2 & 0 & -22.1 \\
S_{(2998)}^{\circ} & 84.8 & 73.6 & 44.6
\end{array}
$$

$\Delta S^{\circ}=33.4 \mathrm{~g} / \mathrm{mole}$

\begin{tabular}{|c|c|c|c|c|c|c|}
\hline $\log A$ & $\boldsymbol{E}$ & $\log \boldsymbol{k}_{T}$ & Conditions & System & Surface & References \\
\hline 14.0 & 57.0 & -3.79 & $\begin{array}{l}700-7444^{\circ} \mathrm{K} \\
10-135 \text { torr }\end{array}$ & static & none & $\begin{array}{l}\text { [1] D. H. R. Barton, A. J. } \\
\text { Head, and J. R. } \\
\text { Williams, J. Chem. } \\
\text { Soc. } 2039 \text { (1951). }\end{array}$ \\
\hline 13.63 & 55.15 & -3.58 & $\begin{array}{l}663-723{ }^{\circ} \mathrm{K} \\
116-308 \text { torr }\end{array}$ & static & none & $\begin{array}{l}\text { [2] H. Hartman, H. G. } \\
\text { Bosche, and H. } \\
\text { Heydtmann, Zeit für } \\
\text { Physik Chemie, N.F. } \\
\mathbf{4 2 ,} 329 \text { (1964). }\end{array}$ \\
\hline 14.5 & 57.9 & -3.57 & $\begin{array}{l}664-731{ }^{\circ} \mathrm{K} \\
80-200 \text { torr }\end{array}$ & static & & $\begin{array}{l}\text { [3] H. Hartman, H. } \\
\text { Heydtmann, and G. } \\
\text { Rinck, Zeit für } \\
\text { Physik Chemie, N.F. } \\
\mathbf{2 8 , 8 5}(1961) .\end{array}$ \\
\hline
\end{tabular}

$\begin{array}{llll}C_{p(298)}^{\circ} & 26.1 & 20.5 & 7.0\end{array}$

$\Delta H^{\circ}=14.1 \mathrm{kcal} / \mathrm{mole}$

$\Delta C_{p}^{\circ}=1.4 \mathrm{~g} / \mathrm{mole}$

\section{Preferred:}

$\log A=13.63-55.15 / \theta$

$\log A_{\text {est }}=13.2$ (see section II-1.0 and 1-chloropentane).

\section{Experimental}

[1] Rates by $\Delta P$; unaffected by added propylene.

[2] Rates by $\mathrm{HCl}$ titration.

[3] Arrhenius parameters of this study revised in|[2]. Rates by $\mathrm{HCl}$ titration. Confirmed stoichiometry $(\Delta \mathrm{P}=\Delta(\mathrm{HCl}))$. 
Reaction: 1-Chloropentane ( $n$-pentyl chloride)

$\begin{array}{cccc}\mathrm{CH}_{3}\left(\mathrm{CH}_{2}\right)_{3} \mathrm{CH}_{2} \mathrm{Cl}(\mathrm{I})-\mathrm{CH}_{3}\left(\mathrm{CH}_{2}\right)_{2} \mathrm{CH}=\mathrm{CH}_{2}(\mathrm{II}) & +\mathrm{HCl}(\mathrm{III}) \\ \Delta H_{f(298)}^{\circ} & \text { I } & \text { II } & \text { III } \\ S_{(298)}^{\circ} & -41.2 & -4.9 & -22.1 \\ C_{p(298)}^{\circ} & 94.2 & 82.8 & 44.6 \\ & 31.6 & 26.2 & 7.0\end{array}$

$\Delta S^{\circ}=33.2 \mathrm{~g} / \mathrm{mole}$

$\Delta H^{\circ}=14.2 \mathrm{kcal} / \mathrm{mole}$

$\Delta C_{p}^{\circ}=1.6 \mathrm{~g} / \mathrm{mole}$

\begin{tabular}{|c|c|c|c|c|c|c|}
\hline $\log A$ & $\boldsymbol{E}$ & $\begin{array}{c}\log \boldsymbol{k}_{T} \\
(700)\end{array}$ & Conditions & System & Surface & References \\
\hline 14.61 & 58.3 & -3.59 & $\begin{array}{l}669-729{ }^{\circ} \mathrm{K} \\
50-450 \text { torr }\end{array}$ & static & $\sim 5 \%$ & $\begin{array}{l}\text { [1] R. C. S. Grant and } \\
\text { E. S. Swinbourne, } \\
\text { J. Chem. Soc. } 4423 \\
\text { (1965). }\end{array}$ \\
\hline 13.81 & 55.33 & -3.46 & $\begin{array}{l}662-723{ }^{\circ} \mathrm{K} \\
103-211 \text { torr }\end{array}$ & static & negligible & $\begin{array}{l}\text { [2] H. Hartman, H. G. Bosch, } \\
\text { and H. Heydtmann, } \\
\text { Zeit für Physik Chemie, } \\
\text { N.F. 42, } 329 \text { (1964). }\end{array}$ \\
\hline
\end{tabular}

\section{Preferred:}

$\log A=13.81-55.33 / \theta$;

$\log A_{\text {est }}=13.2$ (see section II-1.0).

Comments: The $A$-factors appear to increase with the increasing size of the alkyl group in the $n$-alkylchloride series. Such an effect, if real, is difficult to rationalize. It seems more reasonable to consider this trend an apparent one only, especially in view of the rather sizable discrepancies in the rate constants reported for the same reactions by different investigators.

\section{Experimental}

[1] Rate by $\Delta P$. Checked stoichiometry by $\mathrm{HCl}$ titration and G.L.C. analysis.

Olefin composition: $96 \%$ l-pentene

$1 \%$ 2-pentene

[2] Rate by $\mathrm{HCl}$ titration.

$3 \%$ propene + butane + ethane. 
Reaction: 1-Chloro-2-methylpropane (isobutyl chloride)

\begin{tabular}{lccc}
$\left(\mathrm{CH}_{3}\right)_{2} \mathrm{CHCH}_{2} \mathrm{Cl}(\mathrm{I})$ & \multicolumn{3}{c}{$\mathrm{CH}_{2}=\mathrm{C}\left(\mathrm{CH}_{3}\right)_{2}(\mathrm{II})+\mathrm{HCl}(\mathrm{III})$} \\
& I & II & III \\
$\Delta H_{f(298)}^{\circ}$ & -37.5 & -4.0 & -22.1 \\
$S_{(298)}^{\circ}$ & 82.1 & 70.2 & 44.6 \\
$C_{p(298)}^{\circ}$ & 25.8 & 21.3 & 7.0
\end{tabular}

$\Delta S^{\circ}=32.7 \mathrm{~g} / \mathrm{mole}$

$\Delta \mathrm{H}^{\circ}=11.4 \mathrm{kcal} / \mathrm{mole}$

$\Delta C_{p}^{\circ}=2.5 \mathrm{~g} / \mathrm{mole}$

$\begin{array}{ccccccc}\log \boldsymbol{A} & \boldsymbol{E} & \begin{array}{c}\log \boldsymbol{k}_{T} \\ (700)\end{array} & \text { Conditions } & \text { System } & \text { Surface } & \text { References } \\ 14.02 & 56.85 & -3.72 & 690-717^{\circ} \mathrm{K} & \text { static } & \text { none } & \text { [1] K. E. Howlett, J. Chem. } \\ & & & & & & \text { Soc., } 4487 \text { (1952). }\end{array}$

Preferred:

$\log k_{\text {est }}=12.9-53.2 / \theta$ (see section II-1.0).

Comments: Rate constant is reliable but the Arrhenius parameters are probably high.

Since in the formation of the four-center transition state in this reaction a rather sizable internal rotation is lost, an $A$-factor greater than normal $(e k T / h)$ is not reasonable (particularly in this reaction where there is no reaction degeneracy). The transition state estimate of the $A$-factor is therefore preferred and the activation energy has been adjusted accordingly.

\section{Experimental}

[1] Rates by $\Delta \mathrm{P}$ and $\mathrm{HCl}$ titration. Pressure dependence observed below 6 torr total pressure. 
Reaction: 2-Chloropropane (isopropyl chloride)

\begin{tabular}{lrcr}
\multicolumn{4}{c}{$\left(\mathrm{CH}_{3}\right)_{2} \mathrm{CHCl}(\mathrm{I})-\mathrm{CH}_{2}=\mathrm{CHCH}_{3}(\mathrm{II})+\mathrm{HCl}(\mathrm{III})$} \\
& $\mathrm{I}$ & $\mathrm{II}$ & III \\
$\Delta \mathrm{H}_{f(298)}^{\circ}$ & -33.0 & 4.8 & -22.1 \\
$S_{(298)}^{\circ}$ & 74.0 & 63.6 & 44.6 \\
$C_{p(298)}^{\circ}$ & 21.4 & 15.3 & 7.0 \\
& & &
\end{tabular}

\begin{tabular}{|c|c|c|c|c|c|c|}
\hline $\log A$ & $\mathbb{E}$ & $\begin{array}{c}\log \boldsymbol{k}_{T} \\
(650)\end{array}$ & Conditions & System & Surface & References \\
\hline 13.64 & 51.1 & -3.53 & $740-940^{\circ} \mathrm{K}$ & S. P. shock & & $\begin{array}{l}\text { [1] W. Tsang, J. Chem. Phys. } \\
\text { 41, } 2487 \text { (1964). }\end{array}$ \\
\hline 13.4 & 50.5 & -3.58 & $\begin{array}{l}680^{\circ} \mathrm{K} \\
60-135 \text { torr }\end{array}$ & static & & $\begin{array}{l}\text { [2] K. E. Howlett, J. Chem. } \\
\text { Soc., } 3695 \text { (1952). }\end{array}$ \\
\hline 13.4 & 50.5 & -3.58 & $640-679^{\circ} \mathrm{K}$ & static & $\sim 12 \%$ & $\begin{array}{l}\text { [3] D. H. R. Barton and } \\
\text { A. J. Head, Trans. Fara- } \\
\text { day Soc. } 4 \mathbf{6}, 114(1950)\end{array}$ \\
\hline
\end{tabular}

Preferred:

$\log k=13.6-51.1 / \theta$

$\log A_{\text {est }}=13.7$ (see section II-1.0).

\section{Experimental}

[1] Rates by G.L.C. analysis on propylene. Comparative rate standard was $t$-butyl chloride and ethyl bromide.

[2] Rates by $\Delta \mathrm{P}$. Pressure dependence in rate constants observed at $\mathrm{P}=4$ torr.

[3] Rates by $\Delta \mathrm{P}$; unaffected by added propylene. 
Reaction: 2-Chloro-2-methylpropane ( $t$-butyl chloride)

\begin{tabular}{lrcr}
\multicolumn{4}{r}{$\left(\mathrm{CH}_{3}\right)_{3} \mathrm{CCl}(\mathrm{I}) \longrightarrow \mathrm{CH}_{2}=\mathrm{C}\left(\mathrm{CH}_{3}\right)_{2}(\mathrm{II})+\mathrm{HCl}(\mathrm{III})$} \\
& I & II & III \\
$\Delta H_{f}^{\circ}$ & $-43 . \mathrm{i}$ & -4.0 & -22.1 \\
$S_{(298)}^{\circ}$ & 77.4 & 70.2 & 44.6 \\
$C_{p(298)}^{\circ}$ & 27.9 & 21.3 & 7.0
\end{tabular}

$\Delta S^{\circ}=37.4 \mathrm{~g} / \mathrm{mole}$

$\Delta H^{\circ}=17.0 \mathrm{kcal} / \mathrm{mole}$

$\Delta C_{p}^{\circ}=10.4 \mathrm{~g} / \mathrm{mole}$

\begin{tabular}{cccc}
$\log A$ & $\mathbb{E}$ & $\begin{array}{c}\log k_{T} \\
(600)\end{array}$ & \multicolumn{1}{c}{ Conditio } \\
13.9 & 46.2 & -2.93 & $\begin{array}{l}750-950^{\circ} \mathrm{K} \\
1 \% \text { in Ar }\end{array}$ \\
13.74 & 44.69 & -2.53 & $\begin{array}{l}700-850{ }^{\circ} \mathrm{K} \\
1 \% \text { in Ar }\end{array}$
\end{tabular}

$13.77 \quad 45.0 \quad-2.62$

12.4

$\begin{array}{ll}41.4-2.68 & 563-614{ }^{\circ} \mathrm{K} \\ & 40-240 \text { torr }\end{array}$

14.2

$\begin{array}{lll}46.0-2.55 & 547-645^{\circ} \mathrm{K} \\ & 27-213 \text { torr }\end{array}$

$\begin{array}{lll}13.7 & 44.9 \quad-2.65 & 557-582{ }^{\circ} \mathrm{K} \\ & 38-61 \text { torr }\end{array}$
System Surface

S. P. shock

S. P. shock

static

small

static

small

Preferred:

$\log k=13.8=45.0 / \theta$

$\log A_{\text {est }}=13.8$ (see section II-1.0).

\section{Experimental}

[1] Rates from G.L.C. analysis on $i$-butene. Uncertainties in temperatures of the shock and therefore not as reliable as [2].

[2] Rates by G.L.C. Isopropyl bromide used as an internal comparative rate standard.

[4] Rates by $\Delta \mathrm{P}$; stoichiometry confirmed by $\mathrm{HCl}$ titration. Initial fast reactions found to be heterogeneous. Rates unaffected by added propylene.

[5] Rates by $\Delta \mathrm{P}$. Catalysis by surfaces decreased with surface "conditioning."

[6] Rates by $\Delta \mathrm{P}$; unaffected by large pressures of $\mathrm{SF}_{6}$. 
Reaction: 2-Chlorobutane (sec-butyl chloride)

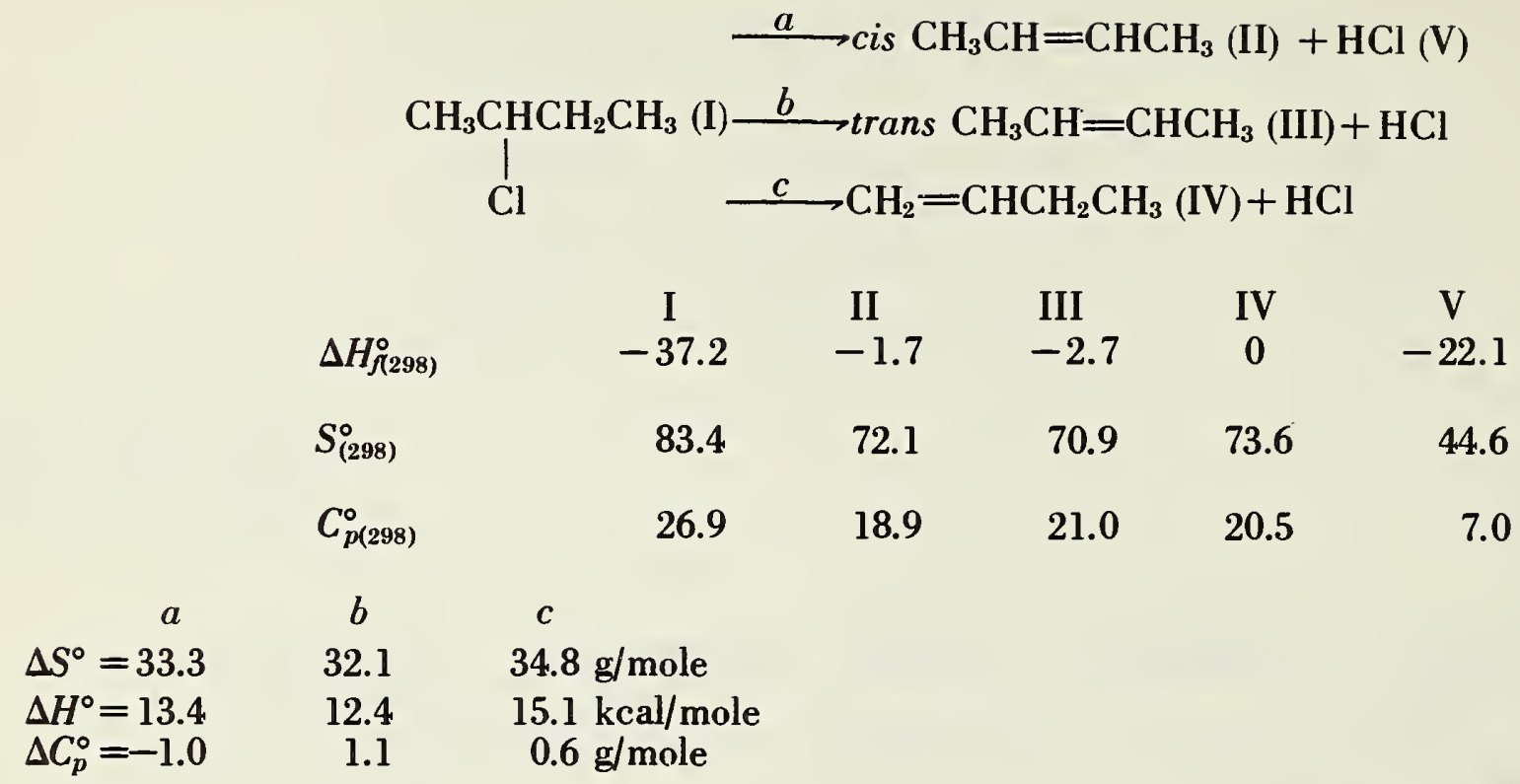

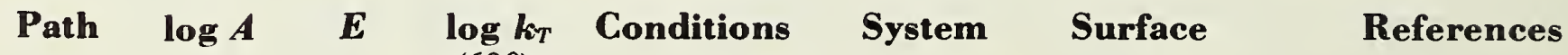

total $\quad 13.62 \quad 49.6 \quad-3.58 \quad 603-663^{\circ} \mathrm{K} \quad$ static $\quad<5 \%$
149-290 torr Stone, J. Chem.

[1] A. Maccoll and R. H. Soc., 2756 (1961).

$\begin{array}{rllllll}\text { total } & 14.0 & 50.6 & -3.55 & 589-666^{\circ} \mathrm{K} & \text { static } & \text { none } \\ a & 13.29 & 52.66 & & & & \\ b & 13.57 & 51.83 & & & \\ c & 13.64 & 51.65 & & & \end{array}$

total $\quad 14.07 \quad 50.75 \quad-3.53 \quad 571-696^{\circ} \mathrm{K} \quad$ static 74-225 torr

[3] H. Heydtmann and G. Rinck, Zeit. für Physik. Chemie 36, 75 (1963).

Preferred: $\quad \log k=14.0-50.6 / \theta$;

$\log k_{\text {est }}=14.0-50.6 / \theta$ (see section II-1.0).

$$
\begin{aligned}
\text { cis }+ \text { trans } \text { but-2-ene: } \log A_{\text {est }}=13.1 & E_{\text {est }}=49.2 \\
\text { but-1-ene: } \log A_{\text {est }}=13.4 & E_{\text {est }}=51.2
\end{aligned}
$$

\section{Experimental}

[1] Rates by $\Delta$ P. Product analysis by G.L.C. Cis/trans-but-2-ene ratios found to represent near equilibrium proportions. Cis/trans isomerization catalysed by $\mathrm{HCl}$ and carbonaceous surface. Olefin product distribution roughly given by 40 percent 1-butene; 60 percent 2 -butene.

[2] Rates by $\Delta \mathrm{P}$. Stoichiometry confirmed by $\mathrm{HCl}$ titration and G.L.C. analysis of olefin products. Product distributions roughly 50 percent 1-butene, 25 percent cis and 25 percent trans-2-butene.

[3] Study similar to that of [2]. G.L.C. analysis gave 43 percent trans and 20 percent cis-2-butene. This product distribution is in good agreement with that reported by Maccoll and Stone [1]. 
Reaction: 2-Chloro-2-methylbutane ( $t$-amyl chloride)

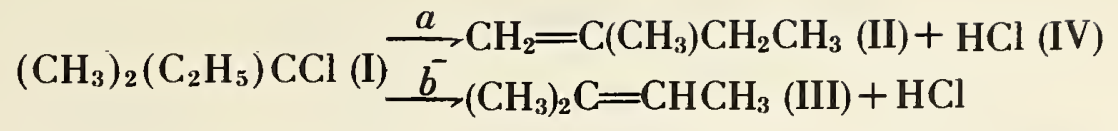

$\begin{array}{lrrrr} & \text { I } & \text { II } & \text { III } & \text { IV } \\ \Delta H_{f(298)}^{\circ} & -46.5 & -8.6 & -10.2 & -22.1 \\ S_{(298)}^{\circ} & 89.0 & 81.9 & 81.1 & 44.6 \\ C_{p(298)}^{\circ} & 33.4 & 26.7 & 25.1 & 7.0\end{array}$

$\begin{array}{cc}a & b \\ \Delta S^{\circ}=37.5 & 36.7 \mathrm{~g} / \mathrm{mole} \\ \Delta H^{\circ}=15.8 & 14.2 \mathrm{kcal} / \mathrm{mole} \\ \Delta C_{p}^{\circ}=0.3 & -1.3 \mathrm{~g} / \mathrm{mole}\end{array}$

\begin{tabular}{ccccccc}
$\log \boldsymbol{A}$ & $\boldsymbol{E}$ & $\begin{array}{c}\log \boldsymbol{k}_{T} \\
(570)\end{array}$ & Conditions & System & Surface & \multicolumn{1}{c}{ References } \\
14.65 & 46.0 & -2.98 & $543-600^{\circ} \mathrm{K}$ & static & none & [1] D. Brearley, G. B. \\
& & & & & & Kistiakowsky, and C. H. \\
& & & & & & Stauffer, J. Am. Chem. \\
& & & & & Soc. $\mathbf{5 8 , 4 3}(1956)$.
\end{tabular}

Preferred: $\log k=13.7-44.0 / \theta$. (see section II-1.0).

$\begin{array}{lr}\log A_{(a)}=13.8 & E_{\text {est }} \\ \log A_{(b)}=13.1 & 45.2 \\ & 43.2\end{array}$

Comments: The rate constant is reasonable, but the Arrhenius parameters seem high. Since the $t$-butyl and $t$-amyl bromide eliminations have almost identical $A$-factors, it is reasonable to expect similar behavior in the chloride system; $\log A(t$-butyl $)=13.7 ; \log A_{\text {est }}=13.8$. The estimated $A$-factor for $t$-amyl chloride is therefore preferred to the experimental value.

\section{Experimental}

[1] Rates by $\Delta \mathrm{P}$. Formation of both olefins assumed, although no product analyses were made. 
Reaction: Chlorocyclopentane (cyclopentyl chloride)

\begin{tabular}{|c|c|c|c|}
\hline$\Delta H_{f(298)}^{\circ}$ & $\begin{array}{c}\mathrm{I} \\
-26.3\end{array}$ & $\begin{array}{l}\text { II } \\
7.9\end{array}$ & $\begin{array}{c}\text { III } \\
-22.1\end{array}$ \\
\hline$S_{(298)}^{\circ}$ & 82.5 & 69.2 & 44.6 \\
\hline$C_{p(298)}^{\circ}$ & 23.5 & 18.1 & 7.0 \\
\hline
\end{tabular}

$\Delta S^{\circ}=31.3 \mathrm{~g} / \mathrm{mole}$

$\Delta H^{\circ}=12.1 \mathrm{kcal} / \mathrm{mole}$

$\Delta C_{p}^{\circ}=1.6 \mathrm{~g} / \mathrm{mole}$

\begin{tabular}{|c|c|c|c|c|c|c|}
\hline $\log A$ & $\boldsymbol{E}$ & $\log k_{T}$ & Conditions & System & Surface & References \\
\hline 13.47 & 48.3 & -4.11 & $\begin{array}{l}582-649^{\circ} \mathrm{K} \\
40-400 \text { torr }\end{array}$ & static & & $\begin{array}{l}\text { [1] E. S. Swinbourne, J. } \\
\text { Chem. Soc. } 4668 \text { (1960). }\end{array}$ \\
\hline
\end{tabular}

Preferred:

$\log k=13.1-47.4 / \theta$ (see section II-1.0).

Comments: The $A$-factors of the cyclopentyl chloride and cyclopentyl bromide decompositions should be very similar. The loss of pseudo rotation of the cyclopentane ring in the activated complex (evident in the cyclopentyl bromide results) is not evident here. However, we feel it should be, and therefore have lowered the $A$-factor.

\section{Experimental}

[1] Rates by $\Delta \mathrm{P}$ and by $\mathrm{HCl}$ titration. Cyclopentene identified by I.R. Added $\mathrm{Br}_{2}$ produced only slight rate accelerations. 
Reaction: Chlorocylclohexane (cyclohexyl chloride)

\begin{tabular}{lrrr} 
& \multicolumn{1}{c}{ (I) } & & \\
& $\mathrm{I}$ & $\mathrm{II}$ & $\mathrm{III}$ \\
$\Delta H_{f(298)}^{\circ}$ & -37.3 & -1.7 & -22.1 \\
$S_{(298)}^{\circ}$ & 83.1 & 74.3 & 44.6 \\
$C_{p(298)}^{\circ}$ & 29.1 & 25.3 & 7.0
\end{tabular}

$\Delta S^{\circ}=35.8 \mathrm{~g} / \mathrm{mole}$

$\Delta H^{\circ}=13.5 \mathrm{kcal} / \mathrm{mole}$

$\Delta C_{p}^{\circ}=3.2 \mathrm{~g} / \mathrm{mole}$

\begin{tabular}{|c|c|c|c|c|c|c|}
\hline $\log A$ & $E$ & $\begin{array}{l}\log \boldsymbol{k}_{T} \\
(600)\end{array}$ & Conditions & System & Surface & References \\
\hline 13.77 & 50.0 & -4.44 & $\begin{array}{l}591-658{ }^{\circ} \mathrm{K} \\
40-400 \text { torr }\end{array}$ & static & none & $\begin{array}{l}\text { [1] E. S. Swinbourne, Aus. } \\
\text { J. Chem. 11, 314 (1958). }\end{array}$ \\
\hline 13.88 & 50.2 & -4.40 & $623-749^{\circ} \mathrm{K}$ & stirred flow & & $\begin{array}{l}\text { [2] W. C. Herndon, M. B. } \\
\text { Henley, and J. M. } \\
\text { Sullivan, J. Phys. } \\
\text { Chem. 67, } 2842 \text { (1963). }\end{array}$ \\
\hline
\end{tabular}

Preferred:

$\log k=13.8-50.0 / \theta$

$\log A_{\text {est }}=13.5$ (see section II-1.0).

\section{Experimental}

[1] Rates by $\Delta \mathrm{P}$ and by $\mathrm{HCl}$ titration not affected by added propylene or cyclohexene. Possible pressure dependence below 5-10 torr pressure.

[2] Anaylsis by G.L.C. 
Reaction: 1-Chloro-1-phenylethane ( $\alpha$-phenylethyl chloride)

\begin{tabular}{cccc}
\multicolumn{4}{c}{$\emptyset \mathrm{CH}-\mathrm{CH}_{3}(\mathrm{I}) \longrightarrow \emptyset \mathrm{CH}=\mathrm{CH}_{2}(\mathrm{II})+\mathrm{HCl}(\mathrm{III})$} \\
& & & \\
& & & \\
$\Delta H_{f(298)}^{\circ}$ & -0.6 & 35.2 & -22.1 \\
$S_{(298)}^{\circ}$ & 94.4 & 82.5 & 44.6 \\
$C_{p(298)}^{\circ}$ & 34.4 & 29.2 & 7.0
\end{tabular}

$\Delta S^{\circ}=32.7 \mathrm{~g} / \mathrm{mole}$

$\Delta H^{\circ}=13.7 \mathrm{kcal} / \mathrm{mole}$

$\Delta C_{p}^{\circ}=1.8 \mathrm{~g} / \mathrm{mole}$

\begin{tabular}{|c|c|c|c|c|c|c|}
\hline $\log A$ & $\boldsymbol{E}$ & $\begin{array}{l}\log \boldsymbol{k}_{T} \\
(610)\end{array}$ & Conditions & System & Surface & References \\
\hline 10.78 & 39.3 & -3.30 & $583-638{ }^{\circ} \mathrm{K}$ & & & $\begin{array}{l}\text { [1] B. Stevenson, Ph. D. } \\
\text { Thesis, Univ. of } \\
\text { London (1957). }\end{array}$ \\
\hline 12.6 & 44.9 & -3.49 & & & & $\begin{array}{l}\text { [2] M. R. Bridge, Ph. D. } \\
\text { Thesis, Univ. of } \\
\text { London (1964). (Under } \\
\text { A. Maccoll.) }\end{array}$ \\
\hline
\end{tabular}

Preferred:

$\log k=12.9-45.4 / \theta$.

Comments: The $A$-factor of this reaction is much too low even if benzyl resonance (loss of the phenyl internal rotation) were developed. A maximum entropy loss can be estimated as $\Delta S_{\max }^{ \pm}=-S^{\circ}\left(\mathrm{CH}_{3}\right.$ rotation $+\varnothing$ rotation $)+R \ln \frac{\sigma}{\sigma \ddagger} \simeq-(5.9+8.4)+2.2=12.1 \mathrm{~g} /$ mole and $A_{\min }^{\ddagger}=10^{10.9} \mathrm{sec}^{-1}$.

However, most four-and six-center transition states are quite loose and a more reasonable estimate gives $\log A_{\text {est }}=12.9$ (with benzyl stiffening). It should be noted that the Arrhenius parameters of the corresponding bromide ( $\alpha$-phenylethyl bromide) are in reasonable agreement with the higher values predicted by the transition state calculation.

\section{Experimental}

[2] Private communication. No conditions given. Added to monograph after above comments. Note the excellent agreement between calculated and observed parameters. 
Reaction: (-)Menthyl chloride

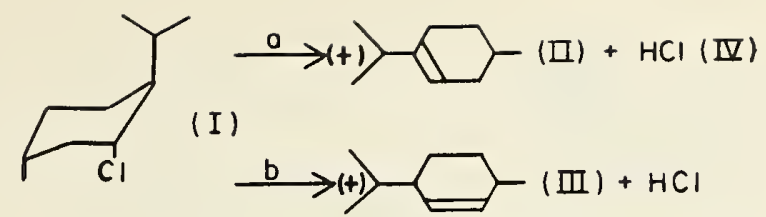

\begin{tabular}{crrrr} 
& \multicolumn{1}{c}{ I } & \multicolumn{1}{c}{ II } & III & \multicolumn{1}{c}{ IV } \\
$\Delta H_{f(298)}^{\circ}$ & -60.9 & -30.3 & -29.8 & -22.1 \\
$S_{(298)}^{\circ}$ & 112.8 & 104.3 & 102.5 & 44.6 \\
$C_{p(298)}^{\circ}$ & 50.2 & 47.0 & 47.3 & 7.0
\end{tabular}

$\begin{array}{cc}a & b \\ \Delta S^{\circ}=36.1 & 34.8 \mathrm{~g} / \mathrm{mole} \\ \Delta H^{\circ}=8.5 & 9.0 \mathrm{kcal} / \mathrm{mole} \\ \Delta C_{p}^{\circ}=3.8 & 4.1 \mathrm{~g} / \mathrm{mole}\end{array}$

$\begin{array}{lllllll}\text { Path } & \log A & E & \log k_{T} & \text { Conditions } & \text { System } & \text { Surface }\end{array}$ (600)

$a$

$\begin{array}{llllll}12.0 & 42.6 & -3.51 & 592-624{ }^{\circ} \mathrm{K} & \text { static } & \text { none }\end{array}$ $5.9-31.5$ torr

[1] D. H. R. Barton, A. J. Head, and R. J. Williams, J. Chem. Soc., 453 (1952). 12.6

$\begin{array}{llll}45.0 & -3.76 & 672-716^{\circ} \mathrm{K} \quad \text { flow }\end{array}$ $\sim 10$ torr

see below

[2] As above.

$a+b$

$11.9 \quad 42.4 \quad-3.52 \quad 573-623^{\circ} \mathrm{K}$

[3] T. Bamkole, Ph.D. Thesis, Univ. of London (1964).

Preferred:

$\log k_{a}=13.5-48.5 / \theta$

$\log k_{b}=13.5-50.0 / \theta$ (see section II-1.0).

Comments: The predicted isomer ratios are $(a: b) \simeq\left(\frac{3.5}{1}\right)$.

The rate constants are almost an order of magnitude larger $\left(600^{\circ} \mathrm{K}\right)$ than those for cyclohexyl chloride. A factor of 3 would be expected for path $a$ (i.e., the substituent effect on $E_{a}$ at the $\beta$-carbon). The factor of 3 remaining suggests some surface decomposition, which is in line with the low Arrhenius parameters. Transition state estimates of the gas phase reaction are preferred.

\section{Experimental}

[1] Rates by $\mathrm{HCl}$ titration. Product menthenes determined by optical rotation assuming no racemization and gave $\sim 30$ percent $p$-menth-2-ene independent of temperature $(3: 1$ of $a: b)$.

[2] Rates constant parameters determined using results of both dynamic and static methods. Surface effects were very large unless the walls were "conditioned." 
Reaction: Neomenthyl chloride

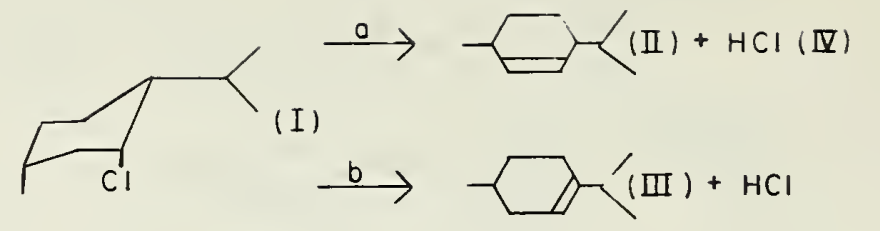

\begin{tabular}{crrrr} 
& \multicolumn{1}{c}{ I } & II & III & IV \\
$\Delta H_{f(298)}^{\circ}$ & -59.3 & -29.8 & -30.3 & -22.1 \\
$S_{(298)}^{\circ}$ & 112.8 & 102.5 & 104.3 & 44.6 \\
$C_{p(298)}^{\circ}$ & 50.2 & 47.3 & $47.0^{\circ} i$ & 7.0
\end{tabular}

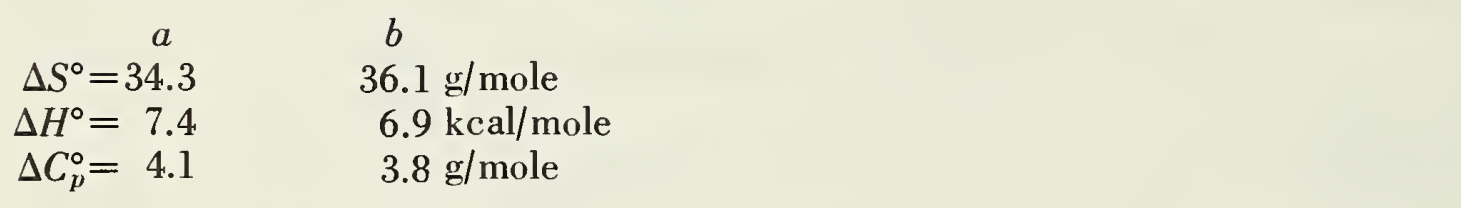

\begin{tabular}{|c|c|c|c|c|c|c|c|}
\hline Path & $\log A$ & $\boldsymbol{E}$ & $\begin{array}{c}\log \boldsymbol{k}_{T} \\
(600)\end{array}$ & Conditions & System & Surface & References \\
\hline$a+b$ & 11.04 & 40.9 & -3.89 & $573-623^{\circ} \mathrm{K}$ & & & $\begin{array}{l}\text { [1] T. Bamkole, Ph.D. } \\
\text { Thesis, Univ. of } \\
\text { London (1964). }\end{array}$ \\
\hline
\end{tabular}

Preferred:

Suspect. For reaction $a$, however, we prefer $\log k_{a}=13.5-50.0 / \theta$.

Comments: The Arrhenius parameters are far too low to be reasonable. One would expect rate constants and rate constant parameters to be similar to those of cyclohexyl chloride. Path $b$ should not occur at all unimolecularly in the gas phase, since the four-center $\mathrm{H} X$ eliminations occur only from cis comformations. Surface reactions and catalysis seem probable. This is supported by the fact that the observed decomposition rate for neomenthyl chloride is faster (factor of 3 ) than that for cyclohexyl chloride.

\section{Experimental}

[1] The ratio of product isomers was $\left(\frac{p \text {-menth-3-ene }}{p \text {-menth-2-ene }}\right) \simeq \frac{1}{5.7}$. 


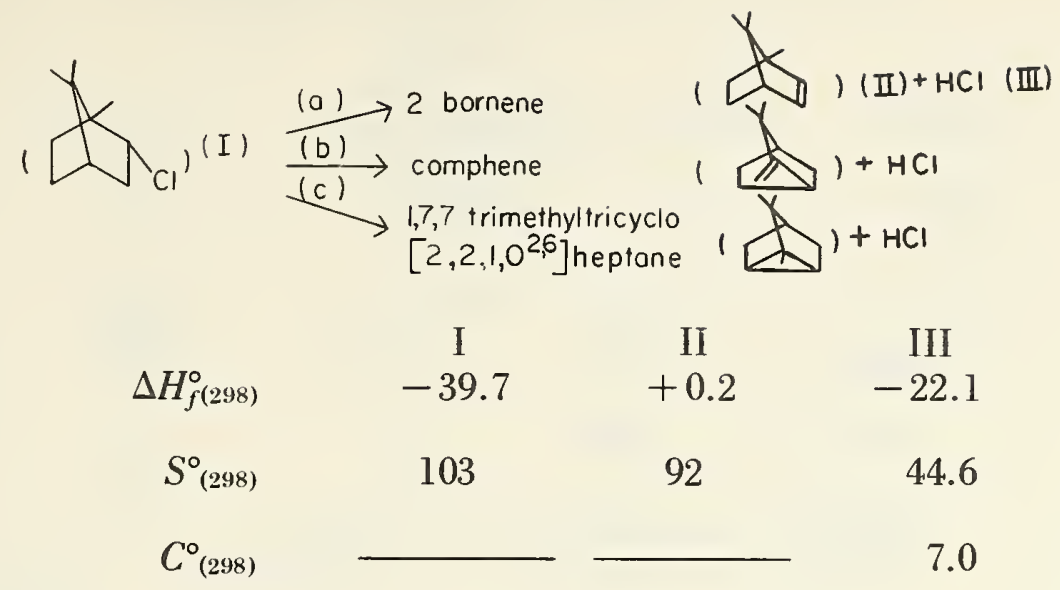

\section{$a$}

$\Delta S^{\circ}=33.6 \mathrm{~g} / \mathrm{mole}$

$\Delta H^{\circ}=17.8 \mathrm{kcal} / \mathrm{mole}$

$\Delta C_{p}^{\circ}=$

\section{Path $\log A \quad E \quad \log \mathbf{k}_{T}$ Conditions System Surface \\ $(600)$}

Total

$13.99 \quad 50.55-4.46$

none given

[1] R. C. Bicknell and Allan Maccoll, Chem. and Ind., 1912 (1961).

Total $\quad 13.78 \quad 50.6 \quad-4.59 \quad 593-663^{\circ} \mathrm{K}$

[2] R. C. Bicknell, $\mathrm{Ph} . \mathrm{D}$

Thesis, Univ. of

London (1962).

\section{Preferred:}

Suspect, although the rate parameters are reasonable. $\log A_{(a)}=13.5-50 / \theta$.

Comments: Paths $b$ and $c$ are not here considered reasonable for a gas phase reaction. Further confirmation of "carbonium ion" intermediates would seem necessary. Formation of camphene and tricyclene at the walls seems more likely. The reaction rates and parameters do, however, seem quite reasonable since they compare favorably to those of cyclohexyl chloride.

\section{Experimental}

[1] Rates hy G.I.C. analysis. Product distribution: tricyclene (55 percent), camphene (25 percent), bornylene (20 percent). Extensive rearrangements of the reactant structure, as in paths $b$ and $c$, were taken as evidence for the heterolytic nature of the $\mathrm{H} X$ elimination reactions. 
Reaction: Exo-2-chlorobornane (isobornyl chloride)

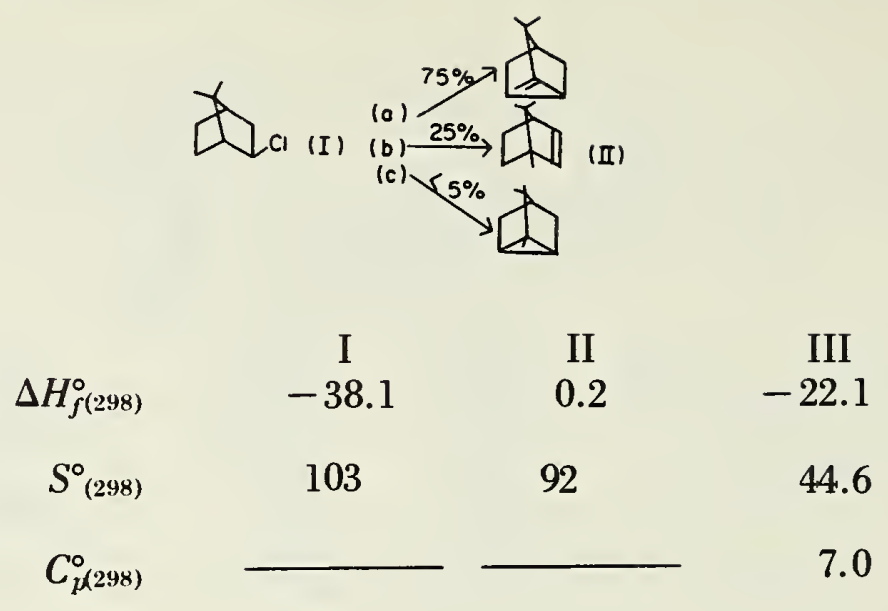

$\Delta S^{\circ}=33.6 \mathrm{~g} / \mathrm{mole}$

$\Delta H^{\circ}=16.2 \mathrm{kcal} / \mathrm{mole}$

$\Delta C_{p}^{\circ}=$

$\begin{array}{lccccc}\log A & \boldsymbol{E} & \begin{array}{c}\log \mathbf{k}_{T} \\ (605)\end{array} & \text { Conditions } & \text { System } & \text { Surface } \\ 14.78 & 49.7 & & 583-623^{\circ} \mathrm{K} & & \end{array}$

[1] R. C. Bicknell, Ph. D.

Thesis, Univ. of London (1962).

\section{Preferred:}

Suspect.

Comments: The $A$-factor appears to be much too high, particularly for path $a$, which would involve extensive structural rearrangement and a loss of one methyl rotation. The reaction rates also appear to be too high if cyclohexyl chloride is used as a comparative standard. A study of the individual reaction paths would be useful in establishing the unimolecularity of this reaction and its isomer, bornyl chloride.

\section{Experimental}

[1] Rates by G.L.C. analysis. Product distribution was 75 percent camphene, 25 percent bornylene, and less than 5 percent tricyclene. (See comments on bornyl chloride.) 
Reaction: 1,1-Dichloroethane (ethylidene dichloride)

\begin{tabular}{cccr}
\multicolumn{4}{r}{$\mathrm{C}_{2} \mathrm{H}_{4} \mathrm{Cl}_{2}(\mathrm{I}) \longrightarrow \mathrm{ClHC}=\mathrm{CH}_{2}(\mathrm{II})+\mathrm{HCl}(\mathrm{III})$} \\
& $\mathrm{I}$ & $\mathrm{II}$ & III \\
$\Delta H_{f(298)}^{\circ}$ & -28.2 & 8.9 & -22.1 \\
$S_{(298)}^{\circ}$ & 71.9 & 62.9 & 44.6 \\
$C_{p(298)}^{\circ}$ & 18.3 & 13.1 & 7.0
\end{tabular}

$\Delta S^{\circ}=35.6 \mathrm{~g} / \mathrm{mole}$

$\Delta H^{\circ}=15.0 \mathrm{kcal} / \mathrm{mole}$

$\Delta C_{p}^{\circ}=1.8 \mathrm{~g} / \mathrm{mole}$

\begin{tabular}{|c|c|c|c|c|c|c|}
\hline $\log A$ & $\boldsymbol{E}$ & $\begin{array}{c}\log k_{T} \\
(700)\end{array}$ & Conditions & System & Surface & References \\
\hline 12.08 & 49.5 & -3.37 & $\begin{array}{l}671-766^{\circ} \mathrm{K} \\
20-200 \text { torr }\end{array}$ & static & none & $\begin{array}{l}\text { [1] D. H. R. Barton and } \\
\text { K. E. Howlett, J. Chem. } \\
\text { Soc., } 165 \text { (1949). }\end{array}$ \\
\hline 11.65 & 48.3 & -3.43 & $\begin{array}{l}685-722^{\circ} \mathrm{K} \\
20-200 \text { torr }\end{array}$ & static & - & $\begin{array}{c}\text { [2] K. E. Howlett, J. Chem. } \\
\text { Soc., } 3695 \text { (1952). }\end{array}$ \\
\hline 13.45 & 53.5 & -3.25 & $\begin{array}{l}641-723^{\circ} \mathrm{K} \\
150-410 \text { torr }\end{array}$ & static & none & $\begin{array}{l}\text { [3] H. Hartman, H. Heydt- } \\
\text { mann, and G. Rinck, } \\
\text { Zeit. für Physik. } \\
\text { Chemie 28, } 71 \text { (1961). }\end{array}$ \\
\hline
\end{tabular}

Preferred:

$\log k=13.45-53.5 / \theta$.

$\log A_{\text {est }}=13.6$ (see section II-1.0).

Comments: The rate constants are in good agreement, although only the most recent study reports reasonable Arrhenius parameters.

\section{Experimental}

[1] Rates by $\Delta \mathrm{P}$ and $\mathrm{HCl}$ titration. No acetylene produced. Not inhibited by propylene. $P_{f} / P_{i} \simeq 2$.

[2] Rates fell off below 17 torr. High-pressure rate restored with added $\mathrm{HCl}$.

[3] Rates by $\Delta \mathrm{P}$ and $\mathrm{HCl}$ titration. No total pressure effect observed between 150 and 410 torr. 
Reaction: 2,2-Dichloropropane

\begin{tabular}{cccc}
\multicolumn{4}{r}{$\mathrm{CH}_{3} \mathrm{CCl}_{2} \mathrm{CH}_{3}(\mathrm{I}) \longrightarrow \mathrm{CH}_{2}=\mathrm{CClCH}_{3}(\mathrm{II})+\mathrm{HCl}(\mathrm{III})$} \\
$\Delta H_{f(298)}^{\circ}$ & $\mathrm{I}$ & II & III \\
$S_{(298)}^{\circ}$ & -32.3 & 0.9 & -22.1 \\
$C_{p(298)}^{\circ}$ & 78.2 & 71.0 & 44.6 \\
& 23.9 & 18.4 & 7.0
\end{tabular}

$\Delta S^{\circ}=37.4 \mathrm{~g} / \mathrm{mole}$

$\Delta H^{\circ}=11.1 \mathrm{kcal} / \mathrm{mole}$

$\Delta C_{p}^{\circ}=1.5 \mathrm{~g} / \mathrm{mole}$

\begin{tabular}{ccccccc}
$\log A$ & $E$ & $\begin{array}{c}\log k_{r} \\
(640)\end{array}$ & Conditions & System & Surface & \multicolumn{1}{c}{ References } \\
11.9 & 43.9 & -3.09 & $\begin{array}{l}603-683^{\circ} \mathrm{K} \\
7-40 \text { torr }\end{array}$ & static & none & {$[1]$ K. E. Howlett, J. Chem. } \\
Soc., 945 (1953).
\end{tabular}

Preferred:

$\log k=14.0-50 / \theta$.

Comments: The rate constant is reasonable, although the Arrhenius parameters seem low. By analogy with the rate constant parameters of ethyl chloride $\left(10^{13.2-56.5 / \theta}\right)$ and 1,1 -diethyl chloride $\left(10^{13.5-53 / \theta}\right)$, one would expect 2,2-dichloropropane to have parameters similar to those of isopropyl chloride (i.e., a somewhat higher $A$-factor and a lower activation energy by about $3 \mathrm{kcal} / \mathrm{mole}$. The transition state estimate of $A$ with adjusted $E$ is therefore preferred.

\section{Experimental}

[1] Rates by $\Delta \mathrm{P}$ and by $\mathrm{HCl}$ titration were unaffected by added propylene. $P_{f} / P_{i} \simeq 2$. 


$\begin{array}{cccc}\mathrm{CHCl}_{2} \mathrm{CH}_{2} \mathrm{CH}_{3} & (\mathrm{I}) \longrightarrow \mathrm{CHCl}= & \mathrm{CHCH}_{3}(\mathrm{II}) & +\mathrm{HCl}(\mathrm{III}) \\ & \text { I } & \text { II } & \text { III } \\ \Delta H_{f(298)}^{\circ} & -32.3 & 0.7 & -22.1 \\ S_{(298)}^{\circ} & 81.3 & 71.5 & 44.6 \\ C_{p(298)}^{\circ} & 23.8 & 18.3 & 7.0\end{array}$

$\Delta S^{\circ}=34.8 \mathrm{~g} / \mathrm{mole}$

$\Delta H^{\circ}=10.9 \mathrm{kcal} / \mathrm{mole}$

$\Delta C_{p}^{\circ}=1.5 \mathrm{~g} / \mathrm{mole}$

\begin{tabular}{ccccccc}
$\log \boldsymbol{A}$ & $\boldsymbol{E}$ & $\begin{array}{c}\log k_{T} \\
(700)\end{array}$ & \multicolumn{1}{c}{ Conditions } & System & Surface & References \\
12.76 & 51.2 & -3.22 & $\begin{array}{l}653-713^{\circ} \mathrm{K} \\
6-61 \text { torr }\end{array}$ & static & & [1] K. E. Howlett, J. Chem. \\
Soc., 945 (1953).
\end{tabular}

Preferred:

$\log k=13.5-53.5 / \theta$.

Comments: The reported Arrhenius parameters of this reaction seem low (see comments on 2,2-dichloropropane). Parameters similar to those of 1,1-dichloroethane would be expected. A transition state estimate of $A$ is therefore preferred (see section II-1.0).

\section{Experimental}

[1] Rated by $\Delta \mathrm{P}$ and by $\mathrm{HCl}$ titration determined under conditions of maximum inhibitions with isobutene. Rate constants appeared to be pressure dependent below 30 torr pressure. $P_{f} / P_{i} \simeq 2$. 
Reaction: 1,2-Dichloropropane

\begin{tabular}{|c|c|c|c|c|c|}
\hline \multirow[t]{3}{*}{$\mathrm{CH}_{2} \mathrm{ClCHClCH}_{3}(\mathrm{I})$} & $\vec{b}$ & \multicolumn{4}{|c|}{$\mathrm{ClCH}={ }^{\prime} \mathrm{CHCH}_{3}(\mathrm{III})+\mathrm{HCl}$} \\
\hline & $\stackrel{c}{\longrightarrow}$ & \multicolumn{4}{|c|}{$\mathrm{CH}_{2}=\mathrm{CHClCH}_{3}(\mathrm{IV})+\mathrm{HCl}$} \\
\hline & $\begin{array}{c}\mathrm{I} \\
-38.3\end{array}$ & $\begin{array}{l}\text { II } \\
-1.3\end{array}$ & $\begin{array}{l}\text { III } \\
0.7\end{array}$ & $\begin{array}{l}\text { IV } \\
0.9\end{array}$ & $\begin{array}{l}\mathrm{V} \\
-22.1\end{array}$ \\
\hline$S^{\circ}{ }_{(298)}$ & 83.6 & 73.3 & 71.5 & 71.0 & 44.6 \\
\hline$C_{(298)}^{\circ}$ & 24.1 & 18.2 & 18.3 & 18.4 & 7.0 \\
\hline
\end{tabular}

$b$

$\Delta S^{\circ}=32.5 \mathrm{~g} / \mathrm{mole}$

$\Delta H^{\circ}=16.9 \mathrm{kcal} / \mathrm{mole}$

$\Delta C_{p}^{\circ}=1.2 \mathrm{~g} / \mathrm{mole}$

$\begin{array}{cccccccc}\text { Path } & \log \boldsymbol{A} & \boldsymbol{E} & \begin{array}{c}\log \mathbf{k}_{T} \\ (700)\end{array} & \text { Conditions } & \text { System } & \text { Surface } \\ & & & & & & \\ \text { Total } & 13.8 & 54.9 & -3.34 & 689-725^{\circ} \mathrm{K} & \text { static } & <5 \% & {[1]}\end{array}$

\section{References}

Total

$\begin{array}{llllll}13.8 & 54.9 & -3.34 & 689-725^{\circ} \mathrm{K} & \text { static } & <5 \%\end{array}$

[1] D. H. Barton and

A. J. Head, Trans. Faraday Soc. 46, 114 (1950).

Preferred:

$\log k=13.8-54.9 / 0$

Estimated:

$$
\begin{array}{ll}
\log A_{(a)}=13.1 & E_{a} \simeq 54.2 \mathrm{kcal} / \mathrm{mole} \\
\log A_{(b)}=13.3 & E_{b} \simeq 53.2 \mathrm{kcal} / \mathrm{mole} \\
\log A_{(c)}=12.8 & E_{c} \simeq 53.2 \mathrm{kcal} / \mathrm{mole}
\end{array}
$$

Comments: (see section II-1.0).

\section{Experimental}

[1] Rates by $\triangle \mathrm{P}$. No induction periods and no effects by $\mathrm{Cl}_{2}$ or $\mathrm{O}_{2}$ in small amounts or or by added propylene. Thus the reaction is self-inhibiting. In the time period of the study $P_{f} P_{i} \simeq 2.0$. Over prolonged periods $P_{f} P_{i} \simeq 2.5$, indicatin a slow elimination of $\mathrm{HCl}$ from one of the olefin products. Analysis was not performed, but in a prior study (D. H. R. Bar ton, J. Chem. Soc., 148 (1949)), a mixture of olefins was observed. 
Reaction: 1,1,1-Trichloroethane

\begin{tabular}{cccc}
\multicolumn{4}{r}{$\mathrm{CCl}_{3} \mathrm{CH}_{3}(\mathrm{I}) \longrightarrow \mathrm{Cl}_{2} \mathrm{C}=\mathrm{CH}_{2}(\mathrm{II})+\mathrm{HCl}(\mathrm{III})$} \\
& I & II & III \\
$\Delta H_{f(298)}^{\circ}$ & -30.1 & 4.3 & -22.1 \\
$S_{(298)}^{\circ}$ & 75.0 & 68.3 & 44.6 \\
$C_{p(298)}^{\circ}$ & 22.5 & 16.5 & 7.0
\end{tabular}

$\Delta S^{\circ}=37.9 \mathrm{~g} / \mathrm{mole}$

$\Delta H^{\circ}=12.3 \mathrm{kcal} / \mathrm{mole}$

$\Delta C_{p}^{\circ}=1.0 \mathrm{~g} / \mathrm{mole}$

\begin{tabular}{|c|c|c|c|c|c|c|}
\hline $\log A$ & $\boldsymbol{E}$ & $\begin{array}{l}\log k_{T} \\
(700)\end{array}$ & Conditions & System & Surface & References \\
\hline 14.07 & 54.2 & -2.85 & $\begin{array}{l}683-713^{\circ} \mathrm{K} \\
70-80 \text { torr }\end{array}$ & static & $\sim 12 \%$ & $\begin{array}{l}\text { [1] P. F. Onyon and D. } \\
\text { Barton, J. Am. Che } \\
\text { Soc. 72, } 988(1950)\end{array}$ \\
\hline 14.0 & 54.0 & -2.85 & & flow & $\longrightarrow$ & $\begin{array}{l}\text { [2] N. Spokes and S. W. } \\
\text { Benson, to be } \\
\text { published. }\end{array}$ \\
\hline
\end{tabular}

Preferred:

$\log k=14.1-54.2 / \theta$.

$\log A_{\text {est }}=13.9$ (see section II-1.0).

\section{Experimental}

[1] Rates by $\Delta \mathrm{P}$ and $\mathrm{HCl}$ titration determined under maximum inhibition conditions using propylene. Under maximum inhibition, rates were independent of $P_{o}$ with no induction periods. $\left(P_{f} / P_{i} \sim 2.0\right)$. Free radical chain decomposition dominated in the pure trichloroethane decomposition.

[2] Analysis by M.S. 
Reaction: 1-Chloro-1-methoxyethane ( $\alpha$-chloroethyl methyl ether)

\begin{tabular}{|c|c|c|c|}
\hline$\Delta H_{f(298)}^{\circ}$ & $\begin{array}{c}\text { I } \\
-63.7\end{array}$ & $\begin{array}{c}\text { II } \\
-25.1\end{array}$ & $\begin{array}{c}\text { III } \\
-22.1\end{array}$ \\
\hline$S_{(298)}^{\circ}$ & 86.0 & 72.5 & 44.6 \\
\hline$C_{p(298)}^{\circ}$ & 24.4 & 18.5 & 7.0 \\
\hline
\end{tabular}

$\Delta S^{\circ}=31.1 \mathrm{~g} / \mathrm{mole}$
$\Delta H^{\circ}=16.5 \mathrm{kcal} / \mathrm{mole}$
$\Delta C_{p}^{\circ}=1.1 \mathrm{~g} / \mathrm{mole}$

$\begin{array}{ccccccc}\log A & E & \begin{array}{c}\log k_{T} \\ (480)\end{array} & \text { Conditions } & \text { System } & \text { Surface } & \text { References } \\ 11.46 & 33.3 & -3.70 & \begin{array}{l}453-523^{\circ} \mathrm{K} \\ 33-420 \text { torr }\end{array} & \text { static } & \sim 20 \% & \text { [1] P. J. Thomas, J. Chem. } \\ & & & & & \text { Soc., } 136 \text { (1961). }\end{array}$

Preferred: Suspect.

$\log k=13.1-36.9 / \theta$ (see section II-1.0).

Comments: The estimated equilibrium constant for the above reaction is about 20 atm at $480^{\circ} \mathrm{K}$ so that back reaction is appreciable at more than 50 percent decomposition. There must be unknown experimental complications, perhaps some polymerization of the vinyl ether or wall reactions or both. Although the reported rate constants may be fairly representative of the reaction indicated, the reported $A$-factor is certainly too low. The maximum loss in activation entropy for this reaction can be estimated in terms of the total loss of a methyl rotation, plus the symmetry change between transition and initial states:

$$
\begin{aligned}
\left(\Delta S_{\min }^{\ddagger}\right. & \simeq-6.4+R \ln 3)=-4.2 \mathrm{~g} / \text { mole. } \\
A_{\min }^{\ddagger} & =\frac{e k T}{h} \times 10\left(\frac{\Delta S^{\ddagger}}{4.575}\right) \sim 10^{12.5} \mathrm{sec}^{-1} .
\end{aligned}
$$

Thus

The transition state calculation of $A$ and adjusted $E_{\text {act }}$ are preferred, although $E$ still seems too low.

\section{Experimental}

[1]. Rates by $\Delta \mathrm{P}$; unaffected by added cyclohexene. $P_{f} / P_{i} \simeq 1.75$ was believed to be a result of the reverse process and the attainment of an equilibrium state (see discussion). $\mathrm{HCl}$ was titrated in situ with $\mathrm{NH}_{3}$. See also 1-chloro-1ethoxyethane. 
Reaction: 1-Chloro-1-ethoxyethane ( $\alpha$-chloroethyl ethyl ether)

\begin{tabular}{cccc}
$\mathrm{CH}_{3} \mathrm{CHClOCH}_{2} \mathrm{CH}_{3}(\mathrm{I}) \longrightarrow \mathrm{CH}_{2}=$ & \multicolumn{3}{c}{$\mathrm{CHOCH}_{2} \mathrm{CH}_{3}(\mathrm{II})+\mathrm{HCl}($ III $)$} \\
$\Delta H_{f(298)}^{\circ}$ & I & II & III \\
$S_{(298)}^{\circ}$ & -71.9 & -33.3 & -22.1 \\
$C_{p(298)}^{\circ}$ & 96.3 & 82.8 & 44.6 \\
& 29.8 & 23.9 & 7.0
\end{tabular}

$\Delta S^{\circ}=31.1 \mathrm{~g} / \mathrm{mole}$

$\Delta H^{\circ}=16.5 \mathrm{kcal} / \mathrm{mole}$

$\Delta C_{p}^{\circ}=1.1 \mathrm{~g} / \mathrm{mole}$

$\begin{array}{ccccccc}\log A & \boldsymbol{E} & \begin{array}{c}\log \boldsymbol{\varepsilon}_{T} \\ (450)\end{array} & \text { Conditions } & \text { System } & \text { Surface } & \text { References } \\ 10.52 & 30.3 & -4.20 & \begin{array}{c}164-221{ }^{\circ} \mathrm{C} \\ 20-280 \text { torr }\end{array} & \text { static } & \sim 15 \%(?) & {[1] \text { R. I. Failes and V. R. }} \\ & & & & & \begin{array}{c}\text { Stimson, Austr. J. Chem. } \\ \mathbf{2 0}, 1553 \text { (1967) }\end{array}\end{array}$

Preferred: Suspect.,

$\log k=13.2-36.0 / \theta$.

Comments: Independent measurements of the back reaction showed that it was predominantly heterogeneous, particularly at the lowest temperature $\left(144^{\circ} \mathrm{C}\right)$. This, of course, is not consistent with a homogeneous forward reaction. Reported Arrhenius parameters are much too low.

\section{Experimental}

[1]. Vessel required coating with allyl bromide pyrolysis products before reproducible results could be obtained. Only one set of runs was made at $194{ }^{\circ} \mathrm{C}$ with $S / V$ increased eight-fold to check homogeneity. Back reaction rate under similar conditions increased two-fold in rate for eight-fold $S / V$ increase. 
Reaction: Trichloromethyl chloromethanoate (trichloromethyl chloroformate)

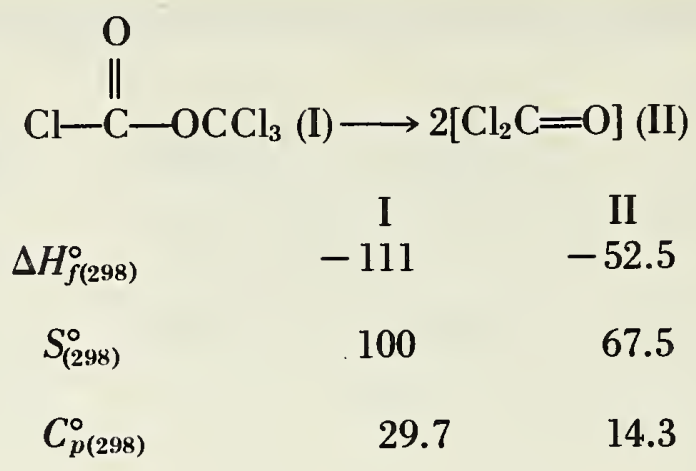

$\Delta S^{\circ}=35.0 \mathrm{~g} / \mathrm{mole}$
$\Delta H^{\circ}=6.0 \mathrm{kcal} / \mathrm{mole}$
$\Delta C_{p}^{\circ}=-1.1 \mathrm{~g} / \mathrm{mole}$

\begin{tabular}{ccccccc}
$\log \boldsymbol{A}$ & $\boldsymbol{E}$ & $\begin{array}{c}\log \boldsymbol{k}_{T} \\
(560)\end{array}$ & Conditions & System & Surface & \multicolumn{1}{c}{ References } \\
13.15 & 41.5 & -3.04 & $\begin{array}{l}533-583^{\circ} \mathrm{K} \\
4.5-16.4 \text { torr }\end{array}$ & static & none & [1] H. C. Ramsperger and \\
& & & & & & $\begin{array}{l}\text { G. Waddington, J. Am. } \\
\text { Chem. Soc. 55, 214 } \\
\text { (1933). }\end{array}$
\end{tabular}

Preferred:

$\log k=13.14-41.5 / \theta$.

Comments: A four-center transition state estimate gives $\log A_{\text {est }}=13.0$ (see section II-1.0).

\section{Experimental}

[1] Rate by $\Delta \mathrm{P} .\left(P_{f} / P_{o}\right)=2.0 \pm .02$. "Clean" surfaces accelerated the reaction; wall conditioning was essential for reproducibility. 
Reaction: Bromoethane (ethyl bromide)

$\begin{array}{cccc} & \mathrm{C}_{2} \mathrm{H}_{5} \mathrm{Br}(\mathrm{I}) \longrightarrow \mathrm{C}_{2} \mathrm{H}_{4}(\mathrm{II})+\mathrm{HBr}(\mathrm{III}) \\ \Delta H_{f(298)}^{\circ} & \mathrm{I} & \mathrm{II} & \mathrm{III} \\ S_{(299)}^{\circ} & -16.1 & 12.5 & -8.7 \\ C_{1(298)}^{\circ} & 69.0 & 52.5 & 47.4 \\ & 15.3 & 10.4 & 7.0\end{array}$

$\Delta S^{\circ}=30.9 \mathrm{~g} / \mathrm{mole}$

$\Delta H^{0}=19.9 \mathrm{kcal} / \mathrm{mole}$

$\Delta C_{p}^{0}=2.1 \mathrm{~g} / \mathrm{mole}$

\begin{tabular}{|c|c|c|c|c|c|c|}
\hline $\log A$ & $\boldsymbol{E}$ & $\begin{array}{c}\log \boldsymbol{k}_{T} \\
(700)\end{array}$ & Conditions & System & Surface & References \\
\hline 12.86 & 52.3 & 3.47 & $790-890{ }^{\circ} \mathrm{K}$ & flow & & $\begin{array}{l}\text { [1] A. T. Blades and G. W. } \\
\text { Murphy, J. Am. Chem. } \\
\text { Soc. 74, } 6219 \text { (1952). }\end{array}$ \\
\hline 13.45 & 53.9 & 3.37 & $\begin{array}{l}655-703^{\circ} \mathrm{K} \\
25-567 \text { torr }\end{array}$ & static & $<10 \%$ & $\begin{array}{l}\text { [2] P. J. Thomas, J. Chem. } \\
\text { Soc., } 1192 \text { (1959). }\end{array}$ \\
\hline 12.95 & 52.2 & 3.34 & $\begin{array}{l}796-906^{\circ} \mathrm{K} \\
6-44 \text { torr }\end{array}$ & flow & & $\begin{array}{l}\text { [3] A. T. Blades, Can. J. } \\
\text { Chem. 36, } 1043 \text { (1958). }\end{array}$ \\
\hline 12.85 & 52.0 & 3.38 & $\begin{array}{l}583-749{ }^{\circ} \mathrm{K} \\
50-300 \text { torr }\end{array}$ & static & & $\begin{array}{l}\text { [4] A. E. Goldberg and F. } \\
\text { Daniels, J. Am. Chem. } \\
\text { Soc. 79, 1314 (1957). }\end{array}$ \\
\hline 13.19 & 53.7 & 3.57 & $\begin{array}{l}740-940^{\circ} \mathrm{K} \\
1 \% \text { in } \mathrm{Ar}\end{array}$ & $\begin{array}{l}\text { S.P. } \\
\text { shock }\end{array}$ & & $\begin{array}{c}\text { [5] W. Tsang, J. Chem. Phys. } \\
4 \mathbf{1}, 2487 \text { (1964). }\end{array}$ \\
\hline
\end{tabular}

\section{Preferred:}

$\log k=13.45-53.9 / \theta$

$\log A_{\text {est }}=10^{13.3} \sec ^{-1}$ (see section II-1.0).

\section{Experimental}

[1] Analysis and rates from $\mathrm{HBr}$ titration. Toluene carrier technique. The absence of $\mathrm{C}_{2} \mathrm{H}_{6}, \mathrm{H}_{2}$ and $\left(\emptyset \mathrm{CH}_{2}\right)_{2}$ in the products was cited as evidence against a $(\mathrm{C}-\mathrm{Br})$ split as the initiation step of the concurrent free radical chain process.*

[2] Rates by $\Delta \mathrm{P}$ under conditions of maximum inhibition. The chain component of the decomposition was estimated at 90 percent.

[3] Toluene carrier technique. Rates from $\mathrm{HBr}$ titration. The ethyl $d_{5}$ isotope effect was studied.

[4] Rate constants were obtained from the initial reaction rates and from the rates in the presence of $n$-hexane.

[5] Single pulse shock. Rates by $\mathrm{HBr}$ titration. Isopropyl bromide was used as the comparative rate standard. $1,1,2,2 \cdot d_{4}$ isotope effect studied by A. T. Blades, P. W. Gilderson, and M. G. H. Wallbridge, Can. J. Chem. 40,5126 (1962).

*'This is not a valid conclusion if the chain length for ethyl bromide is long, which is indeed suggested by the photochemical data. See Barker and Maccoll, J. Chem. Soc., 2839 (1963). 
Reaction: 1-Bromopropane ( $n$-propyl bromide)

\begin{tabular}{cccc}
\multicolumn{4}{c}{$\mathrm{C}_{3} \mathrm{H}_{7} \mathrm{Br}(\mathrm{I}) \longrightarrow \mathrm{C}_{3} \mathrm{H}_{6}(\mathrm{II})+\mathrm{HBr}(\mathrm{III})$} \\
$\Delta H_{f(298)}^{\circ}$ & $\mathrm{I}$ & $\mathrm{II}$ & III \\
$S_{(298)}^{\circ}$ & -21.1 & 4.8 & -8.7 \\
$C_{p(298)}^{\circ}$ & 78.4 & 63.6 & 47.4 \\
& 20.8 & 15.3 & 7.0
\end{tabular}

$\Delta S^{\circ}=32.6 \mathrm{~g} / \mathrm{mole}$

$\Delta H^{\circ}=17.2 \mathrm{kcal} / \mathrm{mole}$

$\Delta C_{p}^{\circ}=1.5 \mathrm{~g} / \mathrm{mole}$

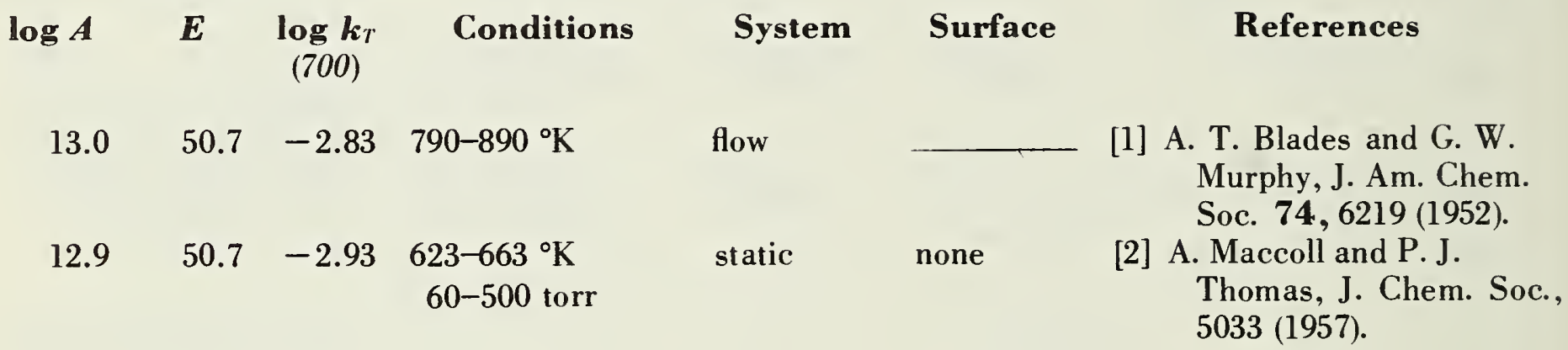

Preferred:

$\log k=13.0-50.7 / \theta$

$\log A_{\text {est }}=13.2$ (see section II-1.0).

\section{Experimental}

[1] Rates by $\mathrm{HBr}$ titration. Toluene carrier technique employed.

[2] Rates and analysis by $\mathrm{HBr}$ titration. Stoichiometry confirmed $(\Delta \mathrm{P}=\Delta(\mathrm{HBr}))$. Rates were accelerated by added $\mathrm{O}_{2}$. Relative inhibition efficiencies of propylene, cyclohexene, and 2,4-dimethyl-pent-2-ene were obtained. 
Reaction: 1-Bromobutane ( $n$-butyl bromide)

$\begin{array}{cccc}\mathrm{CH}_{3} \mathrm{CH}_{2} \mathrm{CH}_{2} \mathrm{CH}_{2} \mathrm{Br}(\mathrm{I}) \longrightarrow \mathrm{CH}_{2}=\mathrm{CHCH}_{2} \mathrm{CH}_{3}(\mathrm{II})+\mathrm{HBr}(\mathrm{III}) \\ \Delta H_{f(298)}^{\circ} & \text { I } & \text { II } & \text { III } \\ S_{(298)}^{\circ} & -26.0 & 0 & -8.7 \\ C_{p(298)}^{\circ} & 87.8 & 73.6 & 47.4 \\ & 26.3 & 20.5 & 7.0\end{array}$

$\Delta S^{\circ}=33.2 \mathrm{~g} / \mathrm{mole}$

$\Delta H^{\circ}=17.3 \mathrm{kcal} / \mathrm{mole}$

$\Delta C_{p}^{\circ}=1.2 \mathrm{~g} / \mathrm{mole}$

$\begin{array}{llclll}\log A & E & \begin{array}{c}\log k_{T} \\ (670)\end{array} & \text { Conditions } & \text { System } & \text { Surfa } \\ 13.18 & 50.9 & -3.42 & \begin{array}{l}64.4-692^{\circ} \mathrm{K} \\ 34-470 \text { torr }\end{array} & \text { static } & <10 \%\end{array}$

$12.92 \quad 50.5$ static

\section{References}

[1] A. Maccoll and P. J.

Thomas, J. Chem. Soc., 5033 (1957).

[2] M. R. Bridge and J. L.

Holmes, J. Chem. Soc.

B, 713 (1966).

\section{Preferred:}

$\log k=13.2-51.0 / \theta$

$\log A_{\text {est }}=13.2$ (see section II-1.0).

\section{Experimental}

[1] Rates determined by $\mathrm{HBr}$ titration $(\Delta(\mathrm{HBr})=\Delta \mathrm{P})$. Cyclohexene reduced the rate by less than 10 percent.

[2] Unpaired electron content and reactivity of carbons produced by pyrolysis of allylbromide, cis-but-2-ene isobutene $+\mathrm{EtBr}$ examined by E.S.R. showed that spin content of carbons does not catalyze elimination from $n \mathrm{BuBr}$ or

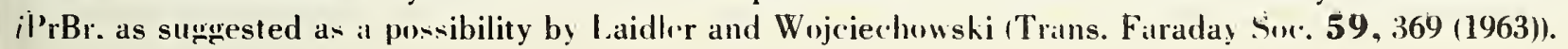


Reaction: 1-Bromopentane ( $n$-pentyl bromide)

$\begin{array}{cccc}\mathrm{CH}_{3}\left(\mathrm{CH}_{2}\right)_{3} \mathrm{CH}_{2} \mathrm{Br}(\mathrm{I}) \longrightarrow \mathrm{CH}_{2}= & \mathrm{CH}\left(\mathrm{CH}_{2}\right)_{2} \mathrm{CH}_{3}(\mathrm{II})+\mathrm{HBr}(\mathrm{III}) \\ \Delta H_{f(298)}^{\circ} & \text { I } & \text { II } & \text { III } \\ S_{(298)}^{\circ} & -31.0 & -4.9 & -8.7 \\ C_{p(298)}^{\circ} & 97.2 & 82.8 & 47.4 \\ & 31.8 & 26.2 & 7.0\end{array}$

$\Delta S^{\circ}=33.0 \mathrm{~g} / \mathrm{mole}$

$\Delta H^{\circ}=17.4 \mathrm{kcal} / \mathrm{mole}$

$\Delta C_{p}^{\circ}=1.4 \mathrm{~g} / \mathrm{mole}$

\begin{tabular}{|c|c|c|c|c|c|c|}
\hline $\log A$ & $\boldsymbol{E}$ & $\begin{array}{c}\log k_{T} \\
(670)\end{array}$ & Conditions & System & Surface & References \\
\hline 13.09 & 50.5 & -3.38 & $\begin{array}{l}653-703^{\circ} \mathrm{K} \\
22-338 \text { torr }\end{array}$ & static & $<10 \%$ & $\begin{array}{l}\text { [1] J. H. S. Green, A. Mac- } \\
\text { coll, and P. J. Thomas, } \\
\text { J. Chem. Soc., } 184 \\
\text { (1960). }\end{array}$ \\
\hline
\end{tabular}

Preferred:

$\log k=13.09-50.5 / \theta$.

$\log A_{\text {est }}=13.2$ (see section II-1.0).

\section{Experimental}

[1] Rates determined by $\mathrm{HBr}$ titration under maximum inhibition of chains with cyclohexene in seasoned reaction vessel. Chain component estimated at 46 percent.*

*With such a sizable chain component, the homogeneous unimolecular rate constants are subject to rather poor precision. 
Reaction: 1-Bromohexane ( $n$-hexyl bromide)

$\begin{array}{cccc} & \mathrm{C}_{6} \mathrm{H}_{13} \mathrm{Br}(\mathrm{I}) \longrightarrow \mathrm{C}_{6} \mathrm{H}_{12}(\mathrm{II})+\mathrm{HBr}(\mathrm{III}) & \\ & \mathrm{I} & \text { II } & \text { III } \\ \Delta H_{f(298)}^{\circ} & -35.9 & -9.9 & -8.7 \\ S_{(298)}^{\circ} & 106.6 & 92.2 & 47.4 \\ C_{p(298)}^{\circ} & 37.3 & 31.6 & 7.0\end{array}$

$\Delta S^{\circ}=33.0 \mathrm{~g} / \mathrm{mole}$

$\Delta H^{\circ}=17.3 \mathrm{kcal} / \mathrm{mole}$

$\Delta C_{p}^{\circ}=1.3 \mathrm{~g} / \mathrm{mole}$

\begin{tabular}{|c|c|c|c|c|c|c|}
\hline $\log A$ & $\boldsymbol{E}$ & $\begin{array}{c}\log \boldsymbol{k}_{T} \\
(670)\end{array}$ & Conditions & System & Surface & References \\
\hline 13.14 & 50.5 & -3.33 & $\begin{array}{c}653-703{ }^{\circ} \mathrm{K} \\
33-267 \text { torr }\end{array}$ & static & $<10 \%$ & $\begin{array}{l}\text { [1] J. H. S. Green, A. Mac- } \\
\text { coll, and P. J. Thomas, } \\
\text { J. Chem. Soc., 184 } \\
\text { (1960). }\end{array}$ \\
\hline
\end{tabular}

Preferred:

$\log k=13.14-50.5 / \theta$.

$\log A_{\text {est }} \simeq 13.2$ (see section II-1.0).

\section{Experimental}

[1] Rates determined by $\mathrm{HBr}$ titration under maximum inhibition with cyclohexene. Chain contribution about 37 percent, indicating rather poor precision for the unimolecular rate constant measurements. 
Reaction: 1-Bromo-2-methylpropane (isobutyl bromide)

$\begin{array}{cccc}\left(\mathrm{CH}_{3}\right)_{2} \mathrm{CHCH}_{2} \mathrm{Br}(\mathrm{I}) \longrightarrow & \mathrm{CH}_{2}=\mathrm{C}\left(\mathrm{CH}_{3}\right)_{2}(\mathrm{II})+\mathrm{HBr}(\mathrm{III}) \\ \Delta H_{f(298)}^{\circ} & \begin{array}{c}\mathrm{I} \\ \mathrm{II}\end{array} & \mathrm{III} \\ S_{(298)}^{\circ} & -26.7 & -4.0 & -8.7 \\ C_{p(298)}^{\circ} & 85.1 & 70.2 & 47.4 \\ & 26.0 & 21.3 & 7.0\end{array}$

$\Delta S^{\circ}=32.5 \mathrm{~g} / \mathrm{mole}$

$\Delta H^{\circ}=14.0 \mathrm{kcal} / \mathrm{mole}$

$\Delta C_{p}^{\circ}=2.3 \mathrm{~g} / \mathrm{mole}$

\begin{tabular}{|c|c|c|c|c|c|c|}
\hline $\log A$ & $\boldsymbol{E}$ & $\underset{(660)}{\log k_{T}}$ & Conditions & System & Surface & References \\
\hline 13.05 & 50.4 & -3.7 & $\begin{array}{l}509-565^{\circ} \mathrm{K} \\
48-100 \text { torr }\end{array}$ & static & $<10 \%$ & $\begin{array}{l}\text { [1] G. D. Harden and A. } \\
\text { Maccoll, J. Chem. Soc., } \\
1197 \text { (1959). }\end{array}$ \\
\hline
\end{tabular}

Preferred:

$\log k=13.05-50.4 / \theta$

Comments: Reliable as reported: $\log A_{\text {est }} \simeq 12.9$ (see section $\mathrm{II}-1.0$ ).

\section{Experimental}

[1] Rates obtained from pressure measurements under maximum inhibition with cyclohexene. Chain contribution to the uninhibited decomposition estimated at 90 percent. Relative inhibition efficiencies of cyclohexene, cyclopentadiene, and 2,4-dimethylpent-2-ene were obtained.*

\footnotetext{
*Because of the large chain component, rather poor precision in the measured unimolecular rate constants is expected.
} 
Reaction: 2-Bromopropane (isopropyl bromide)

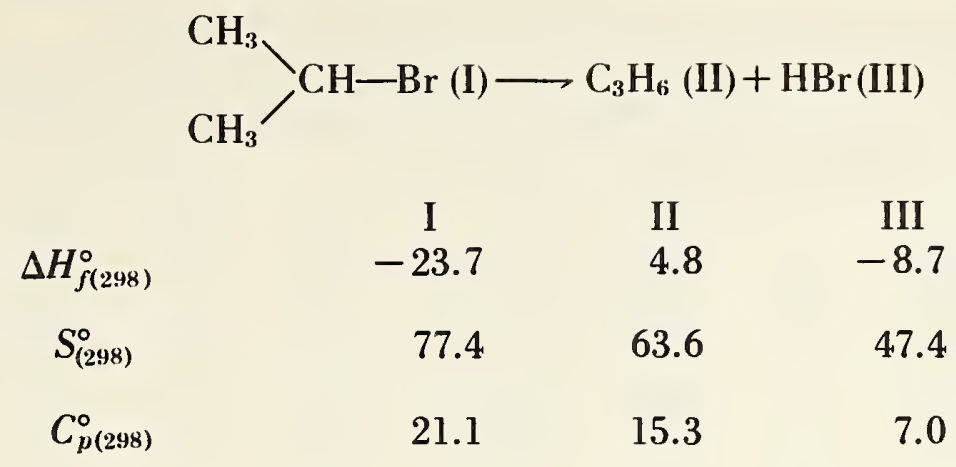

$\Delta S^{\circ}=33.6 \mathrm{~g} / \mathrm{mole}$
$\Delta H^{\circ}=19.8 \mathrm{kcal} / \mathrm{mole}$
$\Delta C^{\circ}=1.2 \mathrm{~g} / \mathrm{mole}$

$\begin{array}{llcccc}\log A & E & \begin{array}{l}\log k_{T} \\ (600)\end{array} & \text { Conditions } & \text { System } & \text { Surface } \\ & & & \end{array}$

$\begin{array}{llll}13.6 & 47.7 & -3.77 \quad 68 \dot{6}-760^{\circ} \mathrm{K} \quad \text { flow }\end{array}$

[1] A. T. Blades and G. W. Murphy, J. Am. Chem. Soc. 74, 6219 (1952).

13.62

$47.8-3.79 \quad 583-623^{\circ} \mathrm{K}$

static $\quad<10 \%$

26-120 torr

[2] A. Maccoll and P. J. Thomas, J. Chem. Soc., 969 (1955); A. Maccoll, J. Chem. Phys. 19, 977 (1951).

$12.74 \quad 47.0 \quad-4.38 \quad 347-497{ }^{\circ} \mathrm{K}$

[3] N. Semenov, D.A.N. SSSR, 301 (1955); G. B. Sergeev, Dok. Akad. Nauk. SSSR 106, 299 (1956).

Preferred:

$\log k=13.6-47.8 / \theta$

$\log A_{\text {est }}=13.7$ (see section II-1.0).

\section{Experimental}

[1] Rates determined by $\mathrm{HBr}$ titration. Toluene carrier technique.

[2] Rates determined by $\mathrm{HBr}$ titration $(\Delta \mathrm{P}=\Delta(\mathrm{HBr})$ ). Maximum inhibition with cyclohexene. Chain contribution less than 10 percent. 
Reaction: 2-Bromobutane (sec-butyl bromide)

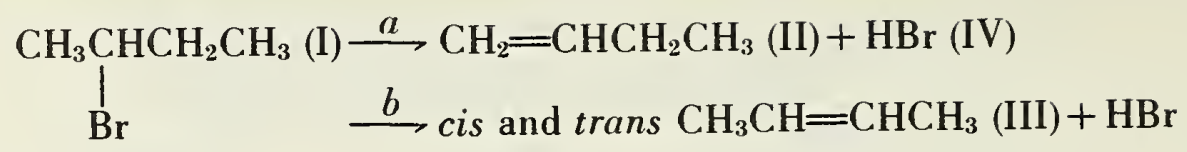

$\begin{array}{crcccc} & \text { I } & \text { II } & \text { III } & \text { III } & \text { IV } \\ \Delta H_{f(298)}^{\circ} & -27.9 & 0 & -1.7 & -2.7 & -8.7 \\ S_{(298)}^{\circ} & 86.8 & 73.6 & 72.1 & 70.9 & 47.4 \\ C_{p(298)}^{\circ} & 26.6 & 20.5 & 18.9 & 21.0 & 7.0\end{array}$

$a$
$\Delta S^{\circ}=34.2 \mathrm{~g} / \mathrm{mole}$
$\Delta H^{\circ}=19.2 \mathrm{kcal} / \mathrm{mole}$
$\Delta C_{p}^{\circ}=0.9 \mathrm{~g} / \mathrm{mole}$

\begin{tabular}{|c|c|c|c|c|c|c|c|}
\hline Path & $\log A$ & $\boldsymbol{E}$ & $\begin{array}{c}\log \boldsymbol{k}_{T} \\
(600)\end{array}$ & Conditions & System & Surface & References \\
\hline$a+b$ & 12.63 & 43.8 & -3.32 & $\begin{array}{l}573-623^{\circ} \mathrm{K} \\
25-343 \text { torr }\end{array}$ & static & $<10 \%$ & $\begin{array}{l}\text { [1] A. Maccoll and P. J. } \\
\text { Thomas, J. Chem. } \\
\text { Soc., } 2445 \text { (1955). }\end{array}$ \\
\hline$a+b$ & 13.53 & 46.47 & -3.39 & $\begin{array}{l}572-627^{\circ} \mathrm{K} \\
2-300 \text { torr }\end{array}$ & static & & $\begin{array}{l}\text { [2] M. N. Kale, A. Mac- } \\
\text { coll, and P. J. } \\
\text { Thomas, J. Chem. } \\
\text { Soc., } 3016 \text { (1958). }\end{array}$ \\
\hline$a+b$ & 13.0 & 45.5 & -3.55 & $\begin{array}{l}603-673^{\circ} \mathrm{K} \\
10-200 \text { torr }\end{array}$ & static & & $\begin{array}{l}\text { [3] G. B. Sergeev, Dok. } \\
\text { Akad. Nauk. SSSR } \\
\text { 106, } 299 \text { (1956). }\end{array}$ \\
\hline
\end{tabular}

Preferred: $\log k=13.5-46.5 / \theta$

Estimated:

$\log A_{a}=13.3 \quad E_{a}=47.8$

$\log A_{b}=13.1 \quad E_{b}=45.8$

$\log A_{a+b}=13.7-47.0 / \theta$ (see section II-1.0).

\section{Experimental}

[1] Rates determined under maximum inhibitions with cyclohexene. Chain contribution less than 5 percent. Analy. sis by $\mathrm{HBr}$ titration, $\Delta(\mathrm{HBr})=\Delta \mathrm{P}$.

[2] Revised Arrhenius parameters of [1] from repeated study under similar conditions. Since $\mathrm{HBr}$ catalyzes the isomerization of butenes, analysis of the isomeric butenes was not made. 
Reaction: 4-Bromo-1-pentene

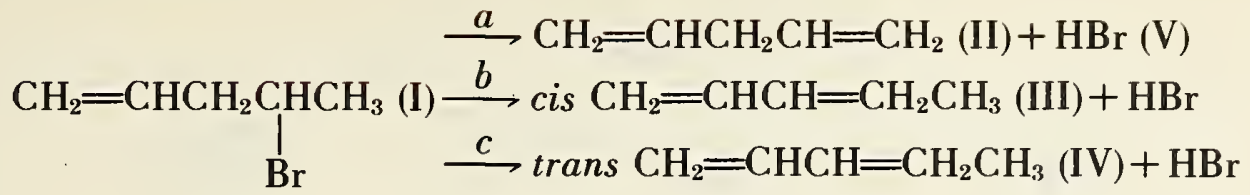

$$
\begin{aligned}
& \begin{array}{lccccc} 
& \text { I } & \text { II } & \text { III } & \text { IV } & \text { V } \\
\Delta H_{f(298)}^{\circ} & -2.6 & 25.2 & 18.7 & 18.6 & -8.7
\end{array} \\
& \begin{array}{llllll}
S_{(298)}^{\circ} & 94.4 & 79.7 & 77.5 & 76.4 & 47.4
\end{array} \\
& \begin{array}{llllll}
C_{p(298)}^{\circ} & 29.4 & 25.1 & 22.6 & 24.7 & 7.0
\end{array}
\end{aligned}
$$

\begin{tabular}{|c|c|c|c|c|c|c|c|}
\hline Path & $\log A$ & $\boldsymbol{E}$ & $\begin{array}{r}\log \boldsymbol{k}_{T} \\
(600)\end{array}$ & Conditions & System & Surface & References \\
\hline Total & 12.94 & 44.7 & -3.34 & $\begin{array}{l}573-623^{\circ} \mathrm{K} \\
112-206 \text { torr }\end{array}$ & static & $<10 \%$ & $\begin{array}{l}\text { [1] P. J. Thomas, J. } \\
\text { Chem. Soc., } 1192 \\
\text { (1959). }\end{array}$ \\
\hline
\end{tabular}

$$
\begin{aligned}
& \Delta S^{\circ}=32.7 \mathrm{~g} / \mathrm{mole} \\
& \Delta H^{\circ}=19.1 \mathrm{kcal} / \mathrm{mole} \\
& \Delta C_{p}^{\circ}=2.7 \mathrm{~g} / \mathrm{mole}
\end{aligned}
$$

Preferred: Rate constant reliable.

Estimated: (see section II-1.0).

$$
\begin{array}{lll}
\log A_{a}=13.3 & E_{a}=47.8 \\
\log A_{b+c}=13.1 & E_{b+c}=45.8 \\
\log A_{a+b+c}=13.7 & & E_{a+b+c}+47 . \theta .
\end{array}
$$

Comments: The overall reaction rate is comparable to that observed for sec-butyl bromide. This suggests that the allylic resonance possible for paths $b$ and $c$ does not appreciably develop in the transition state. The Arrhenius parameters, by comparison with sec-butyl bromide, look slightly low. The transition state estimate is preferred.

\section{Experimental}

[1] Rates were determined by $\Delta \mathrm{P}$ under conditions of maximum inhibition with cyclohexene. The normal decomposition was kinetically complex. Analysis by $\mathrm{HBr}$ titration. $\Delta \mathrm{P}=\Delta(\mathrm{HBr})$. No analysis of the olefin products was made. 
Reaction: Bromocyclopentane (cylcopentyl bromide)

\begin{tabular}{|c|c|c|c|}
\hline$\Delta H_{f(298)}^{\circ}$ & $\begin{array}{c}\text { I } \\
-17.0\end{array}$ & $\begin{array}{l}\text { II } \\
7.9\end{array}$ & $\begin{array}{c}\text { III } \\
-8.7\end{array}$ \\
\hline$S_{(298)}^{\circ}$ & 85.9 & 69.2 & 47.4 \\
\hline$C_{p(2,98)}^{\circ}$ & 23.7 & 18.1 & 1.0 \\
\hline
\end{tabular}

$\Delta S^{\circ}=30.7 \mathrm{~g} / \mathrm{mole}$

$\Delta H^{\circ}=16.2 \mathrm{kcal} / \mathrm{mole}$

$\Delta C_{p}^{\circ}=.1 .4 \mathrm{~g} / \mathrm{mole}$

\begin{tabular}{|c|c|c|c|c|c|c|}
\hline $\log A$ & $\boldsymbol{E}$ & $\begin{array}{c}\log \boldsymbol{k}_{T} \\
(600)\end{array}$ & Conditions & System & Surface & References \\
\hline 11.9 & 41.4 & -3.18 & $\begin{array}{l}573-633^{\circ} \mathrm{K} \\
38-106 \text { torr }\end{array}$ & static & $<10 \%$ & $\begin{array}{l}\text { [1] S. J. W. Price, R. } \\
\text { Shaw, and A. F. } \\
\text { Trotman-Dickinson, } \\
\text { J. Chem. Soc., } 3855 \\
\text { (1956). }\end{array}$ \\
\hline 12.84 & 43.7 & -3.07 & $\begin{array}{l}573-633^{\circ} \mathrm{K} \\
12-92 \text { torr }\end{array}$ & static & $<10 \%$ & $\begin{array}{l}\text { [2] M. N. Kale and A. } \\
\text { Mlaccoll J. Chem. Soc., } \\
5020 \text { (1957). }\end{array}$ \\
\hline
\end{tabular}

Preferred:

$\log k=12.8-43.7 / \theta$

$\log A_{\text {est }}=12.8$ (see section II-1.0).

Comments: The lower Arrhenius parameters of [1] could have resulted from a small chain component. Loss of the pseudo rotation of the cyclopentane ring in the transition state is consistent with the relatively low $A$-factor of [2].

\section{Experimental}

[1] Rates by $\Delta \mathrm{P}$. Stoichiometry confirmed, $\Delta(\mathrm{HBr})=\Delta \mathrm{P}$. Observed short induction periods. Inhibition with cyclohexene reduced rates by less than 10 percent.

[2] Rates by $\Delta \mathrm{P}$. No induction periods observed and no effect on the rate by added cyclohexene. 
Reaction: Bromocyclohexane (cyclohexyl bromide)

\begin{tabular}{|c|c|c|c|}
\hline$\Delta H_{f(298)}^{\circ}$ & $\underset{-28.0}{\text { I }}$ & $\begin{array}{r}\text { II } \\
-1.7\end{array}$ & $\begin{array}{c}\text { III } \\
-8.7\end{array}$ \\
\hline$S_{(298)}^{\circ}$ & 86.5 & 74.3 & 47.4 \\
\hline$C_{p(298)}^{\circ}$ & 28.8 & 25.3 & 7.0 \\
\hline
\end{tabular}

$\Delta S^{\circ}=35.2 \mathrm{~g} / \mathrm{mole}$

$\Delta H^{\circ}=17.6 \mathrm{kcal} / \mathrm{mole}$

$\Delta C_{p}^{\circ}=3.5 \mathrm{~g} / \mathrm{mole}$

$\log A \quad E \quad \log k$

(600)

13.52

$\begin{array}{rl}46.1-3.27 & 573-623^{\circ} \mathrm{K} \\ & 40-400 \text { torr }\end{array}$

System Surface

References

static none

[1] J. H. S. Green and A.

Maccoll, J. Chem. Soc., 2499 (1955).

\section{Preferred:}

$\log k=13.52-46.1 / \theta$

$\log A_{\text {est }}=13.5$ (see section II-1.0).

\section{Experimental}

[1] Rates obtained from pressure measurements. Stoichiometry confirmed by $H B r$ titration. $(\Delta \mathrm{P}=\Delta(\mathrm{HBr}))$. Rates unaffected by additions of cyclohexene or small amounts of allyl bromide or bromine, implying that the reaction is selfinhibiting and free from chain-induced decomposition. 
Reaction: 1-Bromo-1-phenylethane ( $\alpha$-phenylethyl bromide)

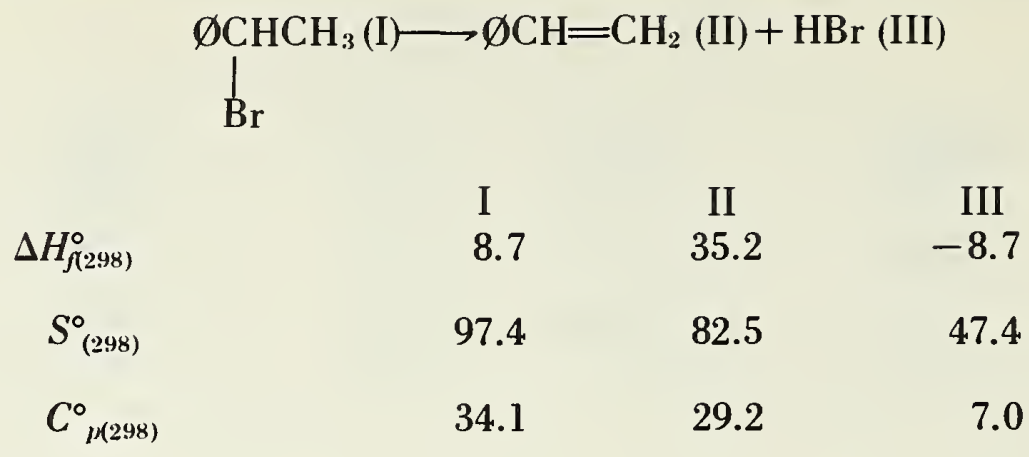

$\Delta S^{\circ}=32.5 \mathrm{~g} / \mathrm{mole}$

$\Delta \mathrm{H}^{\circ}=17.8 \mathrm{kcal} / \mathrm{mole}$

$\Delta C_{p}^{\circ}=2.1 \mathrm{~g} / \mathrm{mole}$

\begin{tabular}{|c|c|c|c|c|c|c|}
\hline $\log A$ & $\boldsymbol{E}$ & $\log \boldsymbol{k}_{T}$ & Conditions & System & Surface & References \\
\hline 12.18 & 38.8 & -2.7 & $528-558^{\circ} \mathrm{K}$ & & & $\begin{array}{l}\text { [1] B. Stevenson, Ph.D. } \\
\text { Thesis, Univ. of London } \\
\text { (1957). }\end{array}$ \\
\hline
\end{tabular}

Preferred:

$\log k=12.8-38.8 / \theta$.

Comments: Transition state estimate of $A$, assuming benzyl stiffening in the transition state, gives $\log A_{\text {est }}=$ 12.9. 
Reaction: 2-Bromo-2-methylpropane ( $t$-butyl bromide)

$\begin{array}{cccc} & \left(\mathrm{CH}_{3}\right)_{3} \mathrm{CBr}(\mathrm{I}) \longrightarrow \mathrm{C}_{4} \mathrm{H}_{9} \mathrm{Br}(\mathrm{II})+\mathrm{HBr}(\mathrm{III}) & \\ & \mathrm{I} & \mathrm{II} & \text { III } \\ \Delta H_{(298)}^{\circ} & -30.5 & -4.0 & -8.7 \\ S_{(298)}^{\circ} & 80.4 & 70.2 & 47.4 \\ C_{p(298)}^{\circ} & 27.9 & 21.3 & 7.0\end{array}$

$\Delta S^{\circ}=37.2 \mathrm{~g} / \mathrm{mole}$

$\Delta H^{\circ}=17.8 \mathrm{kcal} / \mathrm{mole}$

$\Delta C_{p}^{\circ}=0.4 \mathrm{~g} / \mathrm{mole}$

\begin{tabular}{|c|c|c|c|c|c|c|}
\hline $\log A$ & $\boldsymbol{E}$ & $\begin{array}{c}\log \mathbf{k}_{r} \\
\quad(525)\end{array}$ & Conditions & System & Surface & References \\
\hline 13.3 & 40.5 & -3.56 & $\begin{array}{l}509-565{ }^{\circ} \mathrm{K} \\
48-100 \text { torr }\end{array}$ & static & $<10 \%$ & $\begin{array}{l}\text { [1] G. B. Kistiakowsky and } \\
\text { C. H. Stauffer, J. Am. } \\
\text { Chem. Soc. 59, } 165 \\
\text { (1937). }\end{array}$ \\
\hline 14.0 & 42.0 & -3.48 & $\begin{array}{l}503-553^{\circ} \mathrm{K} \\
40-400 \text { torr }\end{array}$ & static & & $\begin{array}{l}\text { [2] G. D. Harden and A. } \\
\text { Maccoll, J. Chem. Soc., } \\
2455 \text { (1955). }\end{array}$ \\
\hline 13.5 & 41.5 & -3.77 & $\begin{array}{l}700-900^{\circ} \mathrm{K} \\
1 \% \text { in } \mathrm{Ar}\end{array}$ & $\begin{array}{l}\text { S.P. } \\
\text { shock }\end{array}$ & & $\begin{array}{l}\text { [3] W. Tsang, J. Chem. } \\
\text { Phys. 40, } 1498 \text { (1964). }\end{array}$ \\
\hline 13.24 & 41.0 & -3.84 & $\begin{array}{l}538-598^{\circ} \mathrm{K} \\
10-150 \text { torr }\end{array}$ & & & $\begin{array}{l}\text { [4] G. B. Sergeev, Dok. } \\
\text { Akad. Nauk. SSSR } \\
\text { 106, } 299 \text { (1956). }\end{array}$ \\
\hline 13.87 & 41.49 & -3.40 & $\begin{array}{l}700-900{ }^{\circ} \mathrm{K} \\
1 \% \text { in } \mathrm{Ar}\end{array}$ & $\begin{array}{l}\text { S.P. } \\
\text { shock }\end{array}$ & & $\begin{array}{l}\text { [5] W. Tsang, J. Chem. } \\
\text { Phys. 40, } 1171 \text { (1964). }\end{array}$ \\
\hline
\end{tabular}

Preferred:

$\log k=13.8-41.8 / \theta$

$\log A_{\text {est }}=13.8$ (see section II-1.0).

\section{Experimental}

[1] Reaction proceeds to an equilibrium state (i.e., $P_{f} \mid P_{i}<2$ ). Rates by pressure measurements.

[2] No inhibition observed with added cyclohexene or with cyclopentadiene. No appreciable rate acceleration observed with added bromine or allylbromide. The reaction is therefore self-inhibiting and free from radical-induced decomposition. Rates by pressure increase.

[3] Rates by G.L.C. analysis of isobutene. 
Reaction: 2-Bromo-2-methylbutane ( $t$-amyl bromide)

\begin{tabular}{|c|c|c|c|c|}
\hline \multirow{3}{*}{$\mathrm{C}_{2} \mathrm{H}_{5}($} & \multicolumn{4}{|c|}{$\stackrel{a}{\longrightarrow}\left(\mathrm{CH}_{3}\right)_{2} \mathrm{C}=\mathrm{CHCH}_{3}(\mathrm{II})+\mathrm{HBr}(\mathrm{IV})$} \\
\hline & \multicolumn{4}{|c|}{ I) $\stackrel{b}{\longrightarrow} \mathrm{CH}_{2}=\mathrm{C}\left(\mathrm{CH}_{3}\right) \mathrm{CH}_{2} \mathrm{CH}_{3}(\mathrm{III})+\mathrm{HBr}$} \\
\hline & $\begin{array}{c}\mathrm{I} \\
-33.9\end{array}$ & $\begin{array}{l}\text { II } \\
-10.2\end{array}$ & $\begin{array}{l}\text { III } \\
-8.6\end{array}$ & $\begin{array}{l}\text { IV } \\
-8.7\end{array}$ \\
\hline$S_{(298)}^{\circ}$ & 92.0 & 81.1 & 81.9 & 47.4 \\
\hline$C_{p(298)}^{\circ}$ & 33.4 & 25.1 & 26.7 & 7.0 \\
\hline
\end{tabular}

$$
\begin{aligned}
& \Delta S^{\circ}=36.5 \mathrm{~g} / \mathrm{mole} \\
& \Delta H^{\circ}=15.0 \mathrm{kcal} / \mathrm{mole} \\
& \Delta C_{p}^{\circ}=-1.3 \mathrm{~g} / \text { mole }
\end{aligned}
$$

\begin{tabular}{|c|c|c|c|c|c|c|c|}
\hline Path & $\log A$ & $\boldsymbol{E}$ & $\begin{array}{c}\log \boldsymbol{k}_{T} \\
(525)\end{array}$ & Conditions & System & Surface & References \\
\hline$a+b$ & 13.6 & 40.5 & -3.26 & $\begin{array}{l}493-543^{\circ} \mathrm{K} \\
\quad 80-363 \text { torr }\end{array}$ & static & $<10 \%$ & $\begin{array}{l}\text { [1] G. D. Hardin, J. } \\
\text { Chem. Soc., } 5024 \\
\text { (1957). }\end{array}$ \\
\hline
\end{tabular}

Preferred:

Reliable as reported: $\log k=13.6-40.5 / \theta$.

Comments:

Estimated: $\log A_{a}=13.1 \quad E_{a} \simeq 39.8 \mathrm{kcal} / \mathrm{mole}$

$\log A_{b}=13.8 \quad E_{b} \simeq 41.8 \mathrm{kcal} / \mathrm{mole}$

$\log A_{a+b}=13.7 \quad E_{a+b} \simeq 40.2 \mathrm{kcal} /$ mole (see section II-1.0).

\section{Experimental}

[1] Rates by pressure increase. Reaction proceeds to an equilibrium state $\left(P_{f} / P_{i}<2\right)$. Rates unaffected by addition of cyclohexene. Large pressures of added bromine produced only a slight acceleration. I.R. analysis indicated the major olefin formed to be 2-methylbut-2-ene. 
Reaction: 2,3-Dimethyl-2-bromobutane.

$$
\begin{aligned}
& \stackrel{a}{\longrightarrow} \mathrm{CH}_{2}=\mathrm{C}\left(\mathrm{CH}_{3}\right) \mathrm{CH}\left(\mathrm{CH}_{3}\right)_{2}(\mathrm{II})+\mathrm{HBr}(\mathrm{IV}) \\
& \left(\mathrm{CH}_{3}\right)_{2} \mathrm{BrCCH}\left(\mathrm{CH}_{3}\right)_{2}(\mathrm{I}) \\
& \stackrel{b}{\longrightarrow}\left(\mathrm{CH}_{3}\right)_{2} \mathrm{C}=\mathrm{C}\left(\mathrm{CH}_{3}\right)_{2}(\mathrm{III})+\mathrm{HBr} \\
& \begin{array}{ccccc} 
& \text { I } & \text { II } & \text { III } & \text { IV } \\
\Delta H_{f^{(298)}}^{\circ} & -39.5 & -14.8 & -15.9 & -8.7
\end{array} \\
& \begin{array}{lllll}
S_{(298)}^{\circ} & 98.9 & 87.4 & 86.7 & 47.4
\end{array} \\
& \begin{array}{lllll}
C_{p(298)}^{\circ} & 38.6 & 32.5 & 30.5 & 7.0
\end{array}
\end{aligned}
$$

$\begin{array}{cc}a & b \\ \Delta S^{\circ}=35.9 & 35.2 \mathrm{~g} / \text { mole } \\ \Delta H^{\circ}=16.0 & 14.9 \mathrm{kcal} / \mathrm{mole} \\ \Delta C^{\circ}=0.9 & -1.1 \mathrm{~g} / \mathrm{mole}\end{array}$

\section{$\begin{array}{lllllll}\text { Path } & \log \boldsymbol{A} & \boldsymbol{E} & \log \boldsymbol{k}_{T} & \text { Conditions } & \text { System } & \text { Surface }\end{array}$}

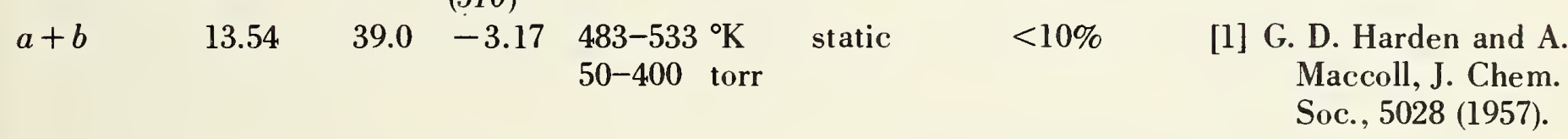

Preferred:

$\log k=13.54-39.0 / \theta$;

Estimated: $\log k_{a}=13.6-41.4 / \theta$

$\log k_{i}=12.8-38.4 / \theta$

$\log k_{a+b}=13.6-39.2 / \theta$ (see section II-1.0).

Comments: The calculated equilibrium constant for path $b$ gives $K_{\mathrm{eq}}=10^{3.82}$ torr, which corresponds to an equilibrium state at about 90 percent decomposition (in agreement with observation).

\section{Experimental}

[1] Rates by $\Delta P$. Reaction stated to proceed to an equilibrium state. Cyclohexene reduced the rate by 6 percent. Olefin products were not analyzed but the major product was believed to be 2,3-dimethyl-2-butene. 
Reaction: 1,1-Dibromoethane (ethylidene dibromide)

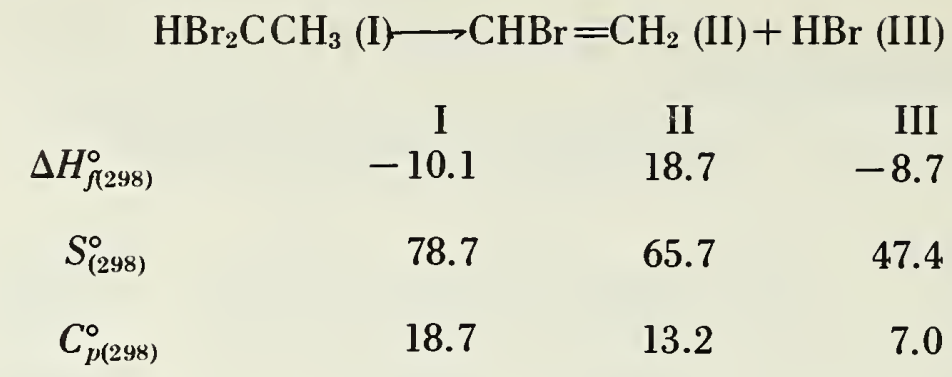

$\Delta S^{\circ}=34.4 \mathrm{~g} / \mathrm{mole}$

$\Delta H^{\circ}=20.1 \mathrm{kcal} / \mathrm{mole}$

$\Delta C_{p}^{\circ}=1.5 \mathrm{~g} / \mathrm{mole}$

\begin{tabular}{|c|c|c|c|c|c|c|}
\hline $\log A$ & $\boldsymbol{E}$ & $\underset{(660)}{\log k_{T}}$ & Conditions & System & Surface & References \\
\hline 12.9 & 49.5 & -3.5 & $623-703^{\circ} \mathrm{K}$ & & & $\begin{array}{l}\text { [1] P. T. Good, Ph.D. } \\
\text { Thesis. Univ. of } \\
\text { London (1956). }\end{array}$ \\
\hline
\end{tabular}

Preferred:

$\log k_{\text {est }}=13.5-51.3 / \theta$ (see section II-1.0).

Comments: Parameters seem slightly low, but not unreasonable. Prefer transition state estimate. 
Reaction: Iodoethane (ethyl iodide)

$\begin{array}{cccc} & \mathrm{C}_{2} \mathrm{H}_{5} \mathrm{I}(\mathrm{I}) \longrightarrow \mathrm{C}_{2} \mathrm{H}_{4}(\mathrm{II})+\mathrm{HI}(\mathrm{III}) \\ \Delta H_{f(298)}^{\circ} & \mathrm{I} & \text { II } & \text { III } \\ S_{(298)}^{\circ} & -2.2 & 12.5 & 6.2 \\ C_{p(298)}^{\circ} & 70.7 & 52.5 & 49.3 \\ & 15.4 & 10.4 & 7.0\end{array}$

$\Delta S^{\circ}=31.1 \mathrm{~g} / \mathrm{mole}$

$\Delta H^{\circ}=20.9 \mathrm{kcal} / \mathrm{mole}$

$\Delta C_{p}^{\circ}=2.0 \mathrm{~g} / \mathrm{mole}$

\begin{tabular}{|c|c|c|c|c|c|c|}
\hline $\log A$ & $\boldsymbol{E}$ & $\begin{array}{c}\log \boldsymbol{k}_{T} \\
(740)\end{array}$ & Conditions & System & Surface & References \\
\hline 14.1 & 52.8 & -1.49 & $\begin{array}{l}704-774{ }^{\circ} \mathrm{K} \\
0.6-4 \times 10^{-2} \text { torr } \\
P_{T} \simeq 2-18 \text { torr }\end{array}$ & flow & & 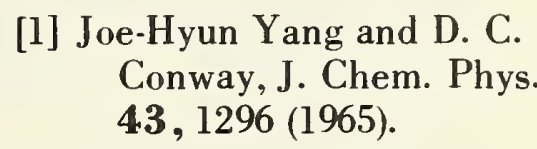 \\
\hline 13.36 & 50.0 & -1.40 & $\begin{array}{l}603-665^{\circ} \mathrm{K} \\
27-273 \text { torr }\end{array}$ & static & $<2 \%$ & $\begin{array}{l}\text { [2] A. N. Bose and S. W. } \\
\text { Benson, J. Chem. Phys. } \\
\text { 37, 2935 (1962). }\end{array}$ \\
\hline
\end{tabular}

Preferred:

$\log k=13.36-50.0 / \theta$

$\log A_{\text {est }}=13.3$ (see section II-1.0).

\section{Experimental}

[1] Toluene carrier technique. Determined rates from scintillation counter measurements of the $\mathrm{C}^{14}$ content of the ethylene formed. The ethyl iodide was $\mathrm{C}^{14}$ labeled. The $(\mathrm{C}-\mathrm{I})$ bond rupture reaction $\left(\mathrm{C}_{2} \mathrm{H}_{5} \mathrm{I} \longrightarrow \mathrm{C}_{2} \mathrm{H}_{5}+\mathrm{I}\right)$ was competitive (see fission reactions).

[2] Rates were followed by pressure change. The back reaction was also studied in the temperature range 562$604{ }^{\circ} \mathrm{K}, \log k_{b}=8.52-28.9 / \theta$ (with $k_{b}$ in units of $1 /$ mole-sec). Both rate constants are in good agreement with the thermodynamics. 
Reaction: 2-Iodopropane (isopropyl iodide)

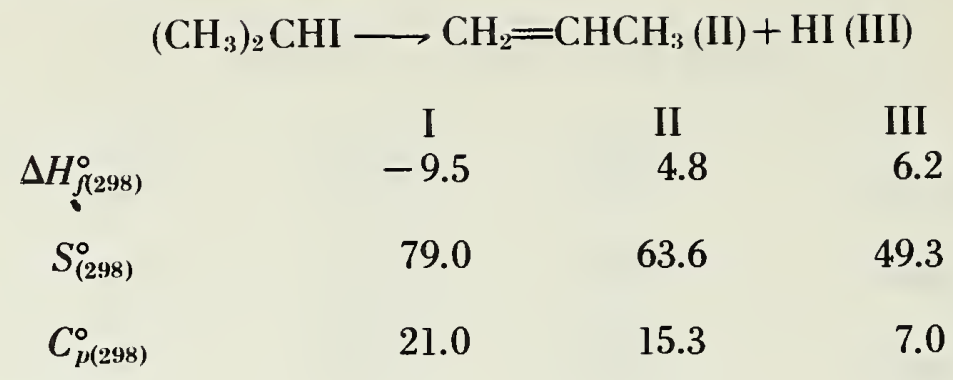

$\Delta S^{\circ}=33.9 \mathrm{~g} / \mathrm{mole}$

$\Delta H^{\circ}=20.5 \mathrm{kcal} / \mathrm{mole}$

$\Delta C_{p}^{\circ}=1.3 \mathrm{~g} / \mathrm{mole}$

\begin{tabular}{|c|c|c|c|c|c|c|}
\hline $\log A$ & $\boldsymbol{E}$ & $\log \boldsymbol{k}_{T}$ & Conditions & System & Surface & References \\
\hline 14.46 & 48.2 & $\begin{array}{l}(000) \\
-3.09\end{array}$ & $\begin{array}{l}558-630^{\circ} \mathrm{K} \\
8-150 \text { torr }\end{array}$ & static & & $\begin{array}{l}\text { [1] J. L. Holmes and A. } \\
\text { Maccoll Proc. Chem. } \\
\text { Soc., (London), } 175 \\
\text { (1957). }\end{array}$ \\
\hline 14.49 & 47.96 & -2.98 & $\begin{array}{l}563-630^{\circ} \mathrm{K} \\
8-87 \text { torr }\end{array}$ & static & none & $\begin{array}{l}\text { [2] J. L. Holmes and A. } \\
\text { Maccoll, J. Chem. } \\
\text { Soc., } 5919 \text { (1963). }\end{array}$ \\
\hline 12.96 & 43.5 & -2.88 & $\begin{array}{l}543-573^{\circ} \mathrm{K} \\
17.4-50 \text { torr }\end{array}$ & static & & $\begin{array}{l}\text { [3] H. Teranishi and S. W. } \\
\text { Benson, J. Chem. Phys. } \\
\text { 40,2946 (1964). }\end{array}$ \\
\hline 12.90 & 42.9 & -2.72 & $565-609^{\circ} \mathrm{K}$ & static & . & $\begin{array}{l}\text { [4] J. L. Jones and R. A. Ogg } \\
\text { J. Am. Chem. Soc. 59, } \\
1939 \text { (1937). }\end{array}$ \\
\hline 13.67 & 45.07 & -2.74 & $\begin{array}{l}680-850^{\circ} \mathrm{K} \\
<1 \% \text { in } \mathrm{Ar}\end{array}$ & S. P. shock & & $\begin{array}{c}\text { [5] W. Tsang, J. Chem. Phys. } \\
\text { 41, 2487 (1964). }\end{array}$ \\
\hline
\end{tabular}

Preferred:

$\log k_{a}=13.53-45.0 / \theta$

$\log A_{\text {est }}=13.3$ (see section II-1.0).

Comments: Kinetics of the back reaction (addition) have been measured by A. N. Bose and S. W. Benson, J. Chem. Phys. 37, 1081 (1962). They obtained $k_{b}=10^{7.89-23.4 / \theta}$ 1/mole-sec. With the reaction thermodynamics, this gives the preferred values for the elimination reaction.

\section{Experimental}

[1] Analysis by G.L.C. Autocatalytic below $558^{\circ} \mathrm{K}$. Arrhenius parameters revised in [2].

[2] Analysis by G.L.C. and spectrophotometry. Overall reaction $2 i \mathrm{PrI} \longrightarrow \mathrm{C}_{3} \mathrm{H}_{6}+\mathrm{C}_{3} \mathrm{H}_{8}+\mathrm{I}_{2}$. Rates were unaffected by $\mathrm{I}_{2}, \mathrm{NO}$, and $\mathrm{HI}$. Rates by $\Delta \mathrm{P}$ and by $\mathrm{I}_{2}$ titration. Rate constants reported were for $i$ PrI disappearance $(k)$ and $k_{\text {" }}=\frac{1}{2} k$.

[3] Rates followed spectrophotometrically. Some pressure fall-off below 30 torr pressure.

[4] Rates by $\Delta \mathrm{P}$ measurements. Iodine was titrated, giving $\Delta \mathrm{I}_{2}=\Delta \mathrm{P} ; 3$ percent $\mathrm{HI}$ was detected as an end product.

[5] Comparative internal rate standards were $t \cdot \mathrm{BuCl}$ and $t \cdot \mathrm{BuBr}$. 
Reaction: 2-Iodobutane (sec-butyl iodide)

$$
\begin{aligned}
& \begin{aligned}
\mathrm{CH}_{3} \mathrm{CHICH}_{2} \mathrm{CH}_{3}(\mathrm{I}) \stackrel{\frac{a}{b}}{\stackrel{c}{\longrightarrow}} \text { cis } \mathrm{CH}_{2}=\mathrm{CHCH}_{3} \mathrm{CH}=\mathrm{CHCH}_{3}(\mathrm{III})+\mathrm{HI} \\
\stackrel{\mathrm{C}}{\longrightarrow} \text { trans } \mathrm{CH}_{3} \mathrm{CH}=\mathrm{CHCH}_{3}(\mathrm{IV})+\mathrm{HI}
\end{aligned} \\
& \begin{array}{cccccc} 
& \text { I } & \text { II } & \text { III } & \text { IV } & \text { V } \\
\Delta H_{\text {(2298) }}^{\circ} & -14.4 & 0 & -1.7 & -2.7 & 6.2
\end{array} \\
& \begin{array}{llllll}
S_{(298)}^{\circ} & 89.8 & 73.6 & 72.1 & 70.9 & 49.3
\end{array} \\
& \begin{array}{llllll}
C_{p(298)}^{\circ} & 26.2 & 20.5 & 18.9 & 21.0 & 7.0
\end{array}
\end{aligned}
$$

$\Delta S^{\circ}=33.1 \mathrm{~g} / \mathrm{mole}$

$\Delta H^{\circ}=20.6 \mathrm{kcal} / \mathrm{mole}$

$\Delta C_{p}^{\circ}=1.3 \mathrm{~g} / \mathrm{mole}$

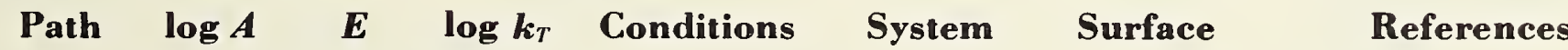

$\begin{array}{llllll}a+b+c \quad 14.9 & 47.9 & -3.18 & 523-603 & \\ & 8-120^{\circ} \mathrm{K}\end{array}$ static none
$8-120^{\circ} \mathrm{K} \quad$ A. Maccoll, J. Chem. Soc., 5919 (1963).

(overall reaction stoichiometry: $2 \mathrm{RI} \longrightarrow \mathrm{I}_{2}+\mathrm{C}_{4} \mathrm{H}_{10}+\mathrm{C}_{4} \mathrm{H}_{8}$ ) initial elimination followed by: $\mathrm{RI}+\mathrm{HI} \stackrel{\text { fast }}{\longrightarrow} \mathrm{RH}+\mathrm{I}_{2}$

Preferred: $\log k_{a+b+c}=13.65-44.7 / \theta$.

Comments: The reported Arrhenius parameters seem high. Transition state estimates (see section II-1.0) give:

$\log k_{4 \prime}=13.3-45.0 / \theta$

$\log k_{b+c}=13.1-43.0 / \theta$

The calculated values are similar to those of isopropyl idodide, as expected by comparison to the results of the analogous chloride and bromide reactions.

\section{Experimental}

[1] Rates by $\Delta \mathrm{P}$ in initial stages and corresponded to $\Delta\left(\mathrm{I}_{2}\right)$ titration. Olefin and alkene $\left(\mathrm{C}_{4} \mathrm{H}_{8}\right)$ production determined by G.L.C.

*Estimates of the decomposition reactions $(b+c)$ and $(a)$ have been obtained from a study of the back reactions (addition) in a static system between 565 and $606^{\circ} \mathrm{K}$ by P. S. Na:inia and S. W. Benson, J. Chem. Phys. 41, 530 (1964). Results given were:

$$
\begin{gathered}
k_{b+c}=10^{10.65} \times 10^{-37.5 / \theta} \mathrm{sec}^{-1} ; \\
k_{l l}=10^{12.61} \times 10^{1+2.27 / \theta} \mathrm{sec}^{-1} .
\end{gathered}
$$

Both of the above $A$-factors are probably too low. 
Reaction: 2-Iodo-2-methylpropane ( $t$-butyl iodide)

\begin{tabular}{cccc}
\multicolumn{4}{r}{$\left(\mathrm{CH}_{3}\right)_{3} \mathrm{CI}(\mathrm{I}) \longrightarrow \mathrm{CH}_{2}=\mathrm{C}\left(\mathrm{CH}_{3}\right)_{2}(\mathrm{II})+\mathrm{HI}(\mathrm{III})$} \\
$\Delta H_{f(298)}^{\circ}$ & I & II & III \\
$S_{(298)}^{\circ}$ & -17.3 & -3.7 & 6.2 \\
$C_{p(298)}^{\circ}$ & 82.4 & 70.4 & 49.3 \\
& 28.3 & 21.3 & 7.0
\end{tabular}

$\Delta S^{\circ}=37.3 \mathrm{~g} / \mathrm{mole}$

$\Delta H^{\circ}=19.8 \mathrm{kcal} / \mathrm{mole}$

$\Delta C_{p}^{\circ}=-0.0 \mathrm{~g} / \mathrm{mole}$

$\begin{array}{llll}\log A & E & \log k_{T} & \text { Conditions }\end{array}$ (700)

System Surface

References

13.73

38.08

$1.83650-760^{\circ} \mathrm{K}$

$<1 \%$ in $\mathrm{Ar}$

S. P. Sh.

[1] W. Tsang, J. Chem.

Phys. 41, 2487 (1964).

[2] See below.

Preferred:

$\log k=13.73-38.08 / \theta$

$\log A_{\text {est }}=13.8$ (see section II-1.0).

\section{Experimental}

[1] Comparative internal rate standard was isopropyl iodide.

[2] Reverse addition reaction measured in static system between $474-518{ }^{\circ} \mathrm{K}$ by A. N. Bose and S. W. Benson, J. Chem. Phys. 38, 878 (1963). Calculated rate constant for decomposition was $k=10^{12.52} \times 10^{-36.4 / \theta} \mathrm{sec}^{-1}$. These param. eters seem too low. 
Reaction: 2-Methyl-2-propanol ( $t$-butyl alcohol)

\begin{tabular}{cccc}
\multicolumn{4}{r}{$\left(\mathrm{CH}_{3}\right)_{3} \mathrm{COH}(\mathrm{I}) \longrightarrow\left(\mathrm{CH}_{3}\right)_{2} \mathrm{C}=\mathrm{CH}_{2}(\mathrm{II})+\mathrm{H}_{2} \mathrm{O}(\mathrm{III})$} \\
& $\mathrm{I}$ & $\mathrm{II}$ & III \\
$\Delta H_{f(298)}^{\circ}$ & -74.7 & -4.0 & -57.8 \\
$S_{(298)}^{\circ}$ & 77.9 & 70.2 & 45.1 \\
$C_{p(298)}^{\circ}$ & 27.3 & 21.3 & 8.0
\end{tabular}

$\Delta S^{\circ}=37.4 \mathrm{~g} / \mathrm{mole}$

$\Delta H^{\circ}=12.9 \mathrm{kcal} / \mathrm{mole}$

$\Delta C_{p}^{\circ}=2.0 \mathrm{~g} / \mathrm{mole}$

\begin{tabular}{|c|c|c|c|c|c|c|}
\hline $\log A$ & $\boldsymbol{E}$ & $\begin{array}{c}\log \boldsymbol{k}_{T} \\
(800)\end{array}$ & Conditions & System & Surface & References \\
\hline 14.68 & $65.5 \pm 7$ & -3.21 & $\begin{array}{l}778-824^{\circ} \mathrm{K} \\
3-325 \text { torr }\end{array}$ & static & none & $\begin{array}{l}\text { [1] R. F. Schultz and G. B. } \\
\text { Kistiakowsky, J. Am. } \\
\text { Chem. Soc. 56, } 395 \\
\text { (1934). }\end{array}$ \\
\hline 11.51 & 54.5 & -3.38 & $\begin{array}{l}760-893^{\circ} \mathrm{K} \\
20-400 \text { torr }\end{array}$ & static & none & $\begin{array}{l}\text { [2] J. A. Barnard, Trans. } \\
\text { Faraday Soc. } \mathbf{5 5}, 947 \\
\text { (1959). }\end{array}$ \\
\hline 13.4 & 61.6 & -3.43 & $\begin{array}{l}1050-1300^{\circ} \mathrm{K} \\
<1 \% \text { in } \mathrm{Ar}\end{array}$ & S. P. shock & 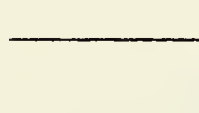 & $\begin{array}{l}\text { [3] W. Tsang, J. Chem. Phys. } \\
\text { 40, } 1498 \text { (1964). }\end{array}$ \\
\hline
\end{tabular}

\section{Preferred:}

$\log k=13.4-61.6 / \theta$

$\log A_{\text {est }}=13.6$ (see section II-1.0).

\section{Experimental}

[1] Rates determined by $\Delta P$. Stoichiometry confirmed by analysis of remaining alcohol by refractive index.

[2] Rates by $\Delta P$. Analysis by G.L.C. confirmed stoichiometry. Added NO had no effect on rates.

[3] Analysis by G.L.C. 
Reaction: 2-Methyl-2-butanol ( $t$-amyl alcohol)

\begin{tabular}{|c|c|c|c|c|}
\hline \multirow[t]{2}{*}{$\left(\mathrm{CH}_{\text {: }}\right.$} & \multicolumn{4}{|c|}{$\begin{array}{l}\mathrm{H} \underset{b}{\stackrel{a}{\longrightarrow}} 2 \text {-methyl-1-butene (II) }+\mathrm{H}_{2} \mathrm{O} \text { (IV) } \\
\stackrel{b}{ } \text {-methyl-2-butene (III) }+\mathrm{H}_{2} \mathrm{O}\end{array}$} \\
\hline & $\begin{array}{c}\mathrm{I} \\
-78.1\end{array}$ & $\begin{array}{l}\text { II } \\
-8.6\end{array}$ & $\begin{array}{l}\text { III } \\
-10.2\end{array}$ & $\begin{array}{l}\text { IV } \\
-57.8\end{array}$ \\
\hline$S_{(298)}^{\circ}$ & 89.5 & 81.9 & 81.1 & 45.1 \\
\hline$C_{p(298)}^{\circ}$ & 32.8 & 26.7 & 25.1 & 8.0 \\
\hline
\end{tabular}

$\begin{array}{cc}a & b \\ \Delta S^{\circ}=37.5 & 36.7 \mathrm{~g} / \mathrm{mole} \\ \Delta H^{\circ}=11.7 & 10.1 \mathrm{kcal} / \mathrm{mole} \\ \Delta C_{p}^{\circ}=1.9 & 0.3 \mathrm{~g} / \mathrm{mole}\end{array}$

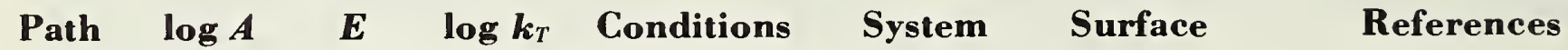
$\begin{array}{llllll}a+b & 13.52 & 60 & -3.51 & 757-799{ }^{\circ} \mathrm{K} & \text { static } \\ & & & & 19.4-324 \text { torr }\end{array}$ none $(770)$

[1] R. F. Schultz and G. B. Kistiakowsky, J. Am. Chem. Soc. 56, 395 (1934).

Preferred: $\log k=13.5-60.0 / \theta$

Estimated: (see section II-1.0).

$$
\begin{aligned}
\log A_{a}=13.5 & E_{a}-E_{b} \simeq 2 \mathrm{kcal} \\
\log A_{b}=12.6 & \log k_{a+b} \simeq 13.0-58.3 / \theta \\
& \text { Experimental }
\end{aligned}
$$

\section{Experimental}

[1] Rates by $\Delta \mathrm{P}$. Refractive index measurements on the remaining alcohol confirmed stoichiometry. 
Reaction: Methanoic acid (formic acid) (a)

$$
\mathrm{HCOOH}(\mathrm{I}) \stackrel{a}{\longrightarrow} \mathrm{H}_{2}(\mathrm{II})+\mathrm{CO}_{2}(\mathrm{III})
$$

Methanoic acid dimer (formic acid dimer) (b)

\begin{tabular}{crrrrr} 
& $(\mathrm{HCOOH})_{2} \stackrel{b}{r} 2 \mathrm{H}_{2} \mathrm{O}(\mathrm{IV})+2 \mathrm{CO}(\mathrm{V})$ & \\
$\Delta H_{f(298)}^{\circ}$ & \multicolumn{1}{c}{$\mathrm{I}$} & $\mathrm{II}$ & $\mathrm{III}$ & $\mathrm{IV}$ & IV \\
$S_{(298)}^{\circ}$ & -88.5 & 0 & -94.1 & -26.4 & -57.8 \\
$C_{p(298)}^{\circ}$ & 59.4 & 31.2 & 51.1 & 47.3 & 45.1 \\
& 10.8 & 7 & 8.9 & 7.0 & 8.0
\end{tabular}

$$
\begin{aligned}
a \\
\Delta S^{\circ}=22.9 \mathrm{~g} / \mathrm{mole} \\
\Delta \mathrm{H}^{\circ}=-5.6 \mathrm{kcal} / \mathrm{mole} \\
\Delta C_{p}^{\circ}=5.1 \mathrm{~g} / \mathrm{mole}
\end{aligned}
$$

\section{Path $\log A$}

\section{$\boldsymbol{E}$}

$\log \boldsymbol{k}_{T}$

Conditions

System

Surface

References (760)

$\begin{array}{llllll}a & 4.8 & 30.6 & -4.00 & 709-805{ }^{\circ} \mathrm{K} & \text { static none } \\ & 3-650 \text { torr }\end{array}$

13.6 $42.6 \quad 1.35$ (as ahove)

[1] Sir Cyril Hinshelwood, Proc. Roy. Soc. A255, 444 (1960).

(as above)

\section{Preferred:}

Not reliable.

Comments: The absence of any appreciable inhibition by isobutene and propylene might be expected, since formic acid would also be an efficient free radical scavenger. The pressure dependence leading to postulation of path $b$ would seem more reasonably explained in terms of a free radical chain. It is very difficult to visualize a geometry of the dimer leading readily to $\mathrm{H}_{2} \mathrm{O}$ and $\mathrm{CO}$. As for path $a$, the Arrhenius parameters are prohibitive for either a unimolecular or a chain process. The most reasonable explanation of the products is that they arise from reactions at the walls or from $\mathrm{H}$-atom chains or both.

\section{Experimental}

[1] Rates of both reactions obtained from $\Delta$ P. M.S. analysis of products. Rates were unaffected by added propylene and isobutene but slightly accelerated with NO. Surface effects in Pyrex vessels were large and gave 1/2 order kinetics. Rates reported were for carbon-coated reaction vessels. A linear dependence of the $\mathrm{CO}$ formation on the initial pressure was interpreted in terms of a unimolecular decomposition of the dimer. Based on the monomer pressure, $k(\mathrm{CO})=10^{7.46-28.5 / \theta} \mathrm{l} / \mathrm{mole}-\mathrm{sec}$. 
Reaction: Ethanoic acid (acetic acid)

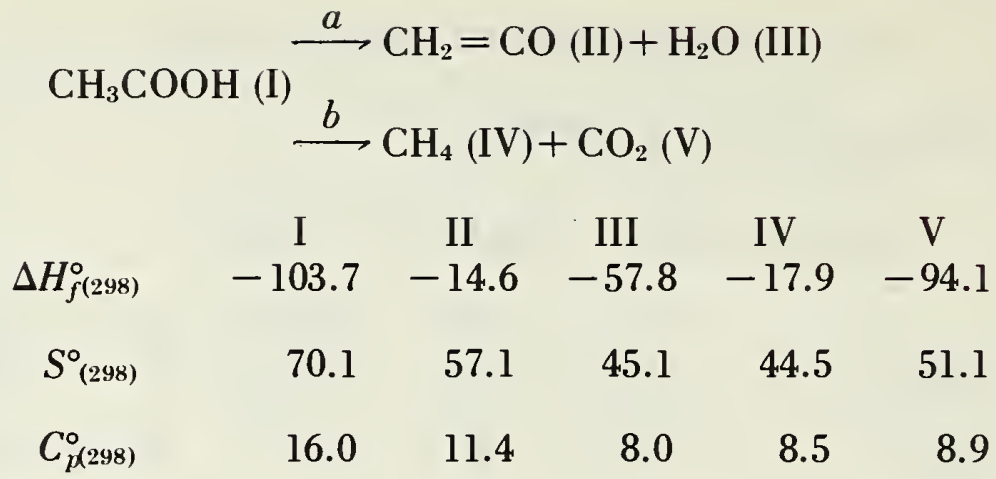

$\begin{array}{cc}a & b \\ \Delta S^{\circ}=32.1 & 25.5 \mathrm{~g} / \mathrm{mole} \\ \Delta H^{\circ}=31.3 & -8.3 \mathrm{kcal} / \mathrm{mole} \\ \Delta C_{p}^{\circ}=3.4 & 1.4 \mathrm{~g} / \mathrm{mole}\end{array}$

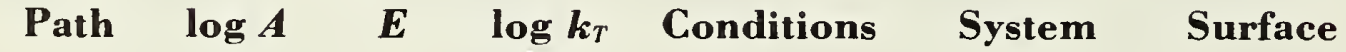
(870)

$a$

12.95

$67.5-4.00$

773-973 ${ }^{\circ} \mathrm{K} \quad$ flow

small $(\mathrm{C})$

[1]

C. H. Bamford and M. J. S. Dewar, J. Chem. Soc., 2877 (1949)

$\begin{array}{lllll}b & 11.90 & 62.0 & -3.67 \quad \text { (as above) }\end{array}$

(as above)

Evaluation:

a. Possibly reliable although suspect.

b. Not reliable, probably a free radical chain decomposition or surface reaction.

\section{Comments:}

$a$. The parameters for path $a$ are not unreasonable for a unimolecular four-center reaction; however any chain process propagated by $\mathrm{H}$-abstraction from the methyl group would lead to ketene $+\mathrm{H}_{2} \mathrm{O}$ formation.

$b$. This reaction $(b)$ would involve pentavalent carbon in the transition state and therefore appears highly improbable. Abstraction of the acidic-H would yield the observed products. A chain process therefore seems likely for both reaction paths. A study of this reaction in a mixture of normal and deuterated acetic acid would be useful for distinguishing the molecular and free radical paths of decomposition.

\section{Experimental}

[1] Rates determined by conventional gas analysis techniques on the products. Ketene was determined by adsorption in weakly acidic glycerol. (C) Carbon-coated walls were required for reproducibility. Reaction very surface-sensitive in clean reaction cells. 
Reaction: 1,2-Ethanedioic acid (oxalic acid)

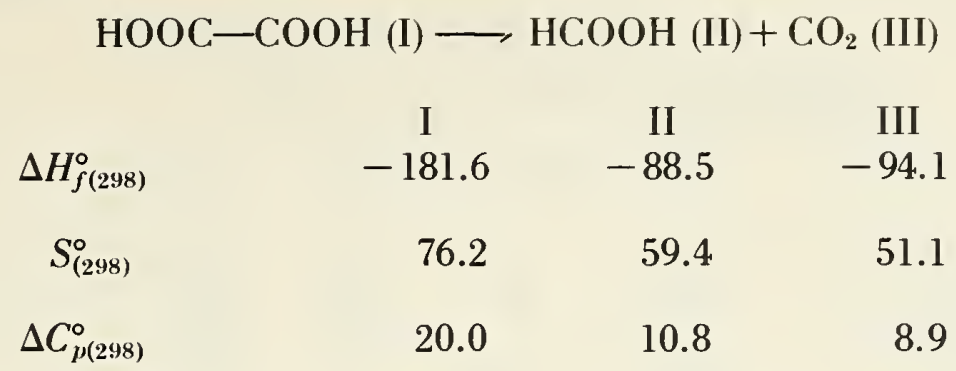
$\Delta S^{\circ}=34.3 \mathrm{~g} / \mathrm{mole}$
$\Delta H^{\circ}=-1.0 \mathrm{kcal} / \mathrm{mole}$
$\Delta C_{p}^{\circ}=-0.3 \mathrm{~g} / \mathrm{mole}$
$\log \boldsymbol{A} \quad \boldsymbol{E} \quad \log \boldsymbol{k}_{T}$ (405)
Conditions
System Surface
$\begin{array}{llll}11.9 & 30.0 & -4.29 & 390-420{ }^{\circ} \mathrm{K} \\ & & & 0.9 \text { torr }\end{array}$
static

References

[1] Gabriel Lapidus, Donald Barton, and Peter E. Yankwich, J. Phys. Chem. 68, 1863 (1964).

Preferred:

$\log k=10^{11.9}-30.0 / \theta$.

Comments: The transition state estimate of $A$ for the four-center elimination of $\mathrm{CO}_{2}$ gives $\log A_{\mathrm{est}}=12.4$ (see section II-1.0).

\section{Experimental}

[1] Rates determined by $\Delta \mathrm{P}$. Chemical analysis of products checked stoichiometry to \pm 1:4 percent. (Based on fourcenter transition state split out of $\mathrm{CO}_{2}$.) 
Reaction: Propanonitrile (ethyl cyanide)

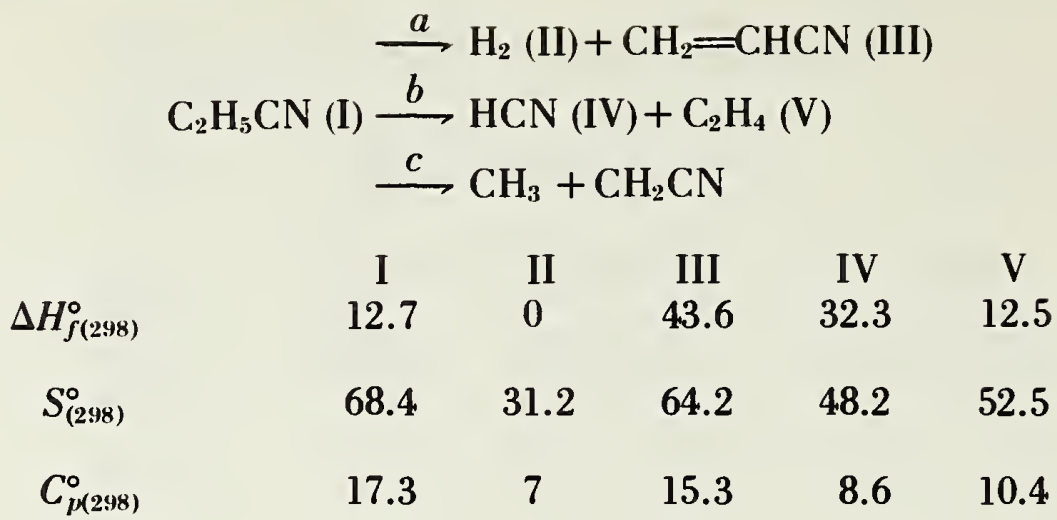

$\begin{array}{rlrl}a & b \\ \Delta S^{\circ}=27 & 32.3 \mathrm{~g} / \mathrm{mole} \\ \Delta H^{\circ}=30.9 & 32.1 \mathrm{kcal} / \mathrm{mole} \\ \Delta C^{\circ}=5.0 & 1.7 \mathrm{~g} / \mathrm{mole}\end{array}$

\begin{tabular}{|c|c|c|c|c|c|c|c|}
\hline Path & $\log A$ & $\boldsymbol{E}$ & $\begin{array}{c}\log k_{T} \\
(1000)\end{array}$ & Conditions & System & Surface & References \\
\hline & 12.4 & 64.6 & -1.72 & $\begin{array}{l}958-1038^{\circ} \mathrm{K} \\
6.5-15.8 \text { torr }\end{array}$ & flow & none & $\begin{array}{l}\text { [1] Margaret Hunt, } \\
\text { J. A. Kerr, and } \\
\text { A. F. Trotman- } \\
\text { Dickenson, J. } \\
\text { Chem. Soc. } 5074 \\
\text { (1965). }\end{array}$ \\
\hline & 15.0 & 77.3 & -1.90 & as above & & & \\
\hline & 14.1 & 72.7 & -1.79 & as above (see & ond fission & actions) & \\
\hline
\end{tabular}

Preferred:

Suspect.

Comments: Hydrogen has been observed as a product in all the aniline carrier studies, and its origin has not been satisfactorily explained. Yields of hydrogen were not significantly greater in this study as opposed to others. Path $a$ cannot, therefore, be taken too seriously. Path $b$ is a possible reaction path; however, the $A$-factor is too high for an elimination reaction. It is more reasonable that both path $a$ and $b$ products arise from free-radical-induced decompositions of ethyl cyanide:

$$
\begin{gathered}
\mathrm{R} \cdot+\mathrm{C}_{2} \mathrm{H}_{5} \mathrm{CN} \longrightarrow \mathrm{RH}+\cdot \mathrm{CH}_{2} \mathrm{CH}_{2} \mathrm{CN} \longrightarrow \mathrm{RH}+\mathrm{C}_{2} \mathrm{H}_{4}+\mathrm{CN} \\
\mathrm{R} \cdot+\mathrm{C}_{2} \mathrm{H}_{5} \mathrm{CN} \longrightarrow \mathrm{RH}+\mathrm{CH}_{3} \mathrm{CHCN} \longrightarrow \mathrm{RH}+\mathrm{CH}_{2}=\mathrm{CHCN}+\mathrm{H} . \\
\text { Experimental }
\end{gathered}
$$

[1] Aniline carrier technique. Rates were based on $\mathrm{C}_{2} \mathrm{H}_{4}$ formation (path $b$ ), and $\mathrm{H}_{2}$ production (path $a$ ). Analyses were performed by standard gasometric techniques and checked by G.L.C. (See bond fissions for a discussion of path $c$.) 
Reaction: 2-Methyl-2-aminopropane ( $t$-butyl amine)

\begin{tabular}{|c|c|c|c|}
\hline$\Delta H_{f(298)}^{\circ}$ & $\begin{array}{c}I \\
-28.6\end{array}$ & $\begin{array}{c}\text { II } \\
-4.0\end{array}$ & $\begin{array}{c}\text { III } \\
-11.0\end{array}$ \\
\hline$S_{(298)}^{\circ}$ & 78.1 & 70.2 & 46.0 \\
\hline$C_{1(298)}^{\circ}$ & 28.3 & 21.3 & 8.5 \\
\hline
\end{tabular}

\begin{abstract}
$\Delta S^{\circ}=38.1 \mathrm{~g} / \mathrm{mole}$
$\Delta H^{\circ}=13.6 \mathrm{kcal} / \mathrm{mole}$

$\Delta C_{p}^{\circ}=1.5 \mathrm{~g} / \mathrm{mole}$
\end{abstract}

\begin{tabular}{|c|c|c|c|c|c|c|}
\hline $\log A$ & $\boldsymbol{E}$ & $\begin{array}{c}\log \boldsymbol{k}_{T} \\
(790)\end{array}$ & Conditions & System & Surface & References \\
\hline 14.23 & 67.0 & $(-4.27)$ & $\begin{array}{l}771-814^{\circ} \mathrm{K} \\
50-180 \text { torr }\end{array}$ & static & none & $\begin{array}{l}\text { [1] H. O. Pritchard, R. G. } \\
\text { Sowden, and A. F. } \\
\text { Trotman-Dickenson, } \\
\text { J. Chem. Soc. } 546 \\
\text { (1954). }\end{array}$ \\
\hline
\end{tabular}

\title{
Preferred:
}

Suspect (see also $t$ - $\mathrm{BuNH}_{2} \longrightarrow\left(\mathrm{CH}_{3}\right)_{2} \mathrm{C}=\mathrm{NH}+\mathrm{CH}_{4}$ ).

Comments: Since a chain process seems likely for the parallel decomposition mode of $t$-butyl amine, a chain process propagated by $\mathrm{NH}_{2}$ radicals seems highly probable for this reaction also. The "molecular split" proposed requires confirmation. The chain processes would be:

Chain initiation:

$$
t-\mathrm{BuNH}_{2} \longrightarrow t-\mathrm{Bu} \cdot+\mathrm{NH}_{2} \cdot
$$

Chain (1) $\mathrm{R}+t-\mathrm{BuNH}_{2} \stackrel{(1)}{\longrightarrow} \mathrm{RH}+\cdot \mathrm{CH}_{2}-\mathrm{C}\left(\mathrm{CH}_{3}\right)_{2} \mathrm{NH}_{2}$

$$
\cdot \mathrm{CH}_{2} \mathrm{C}\left(\mathrm{CH}_{3}\right)_{2} \mathrm{NH}_{2} \longrightarrow \mathrm{CH}_{2}=\mathrm{C}\left(\mathrm{CH}_{3}\right)_{2}+\mathrm{NH}_{2} \text {. }
$$

Chain (2) $\mathrm{R} \cdot+t-\mathrm{BuNH}_{2} \stackrel{(2)}{\longrightarrow} \mathrm{RH}+t \cdot \mathrm{Bu} \dot{\mathrm{NH}}$

$$
t-\mathrm{Bu} \dot{\mathrm{N}} \mathrm{H} \longrightarrow\left(\mathrm{CH}_{3}\right)_{2} \mathrm{C}=\mathrm{NH}+\mathrm{CH}_{3} \text {. }
$$

\section{Experimental}

[1] Rates followed by $\Delta \mathrm{P}$ and by chemical analysis of the products. A parallel reaction path to form $\mathrm{CH}_{4}$ and $\mathrm{C}_{3} \mathrm{H}_{7} \mathrm{~N}$ also occurred. The ratio of ammonia/methane $\simeq 1 / 2.5 \pm 0.1$ over the entire temperature range. Added toluene had no effect. 
Reaction: 2-Methyl-2-aminopropane ( $t$-butyl amine)

\begin{tabular}{cccc}
\multicolumn{4}{r}{$\left(\mathrm{CH}_{3}\right)_{3} \mathrm{CNH}_{2}(\mathrm{I}) \longrightarrow$} \\
& I & II & III \\
$\Delta H_{f(298)}^{\circ}$ & -28.6 & 2.5 & -17.9 \\
$S_{(298)}^{\circ}$ & 78.1 & 71.3 & 44.5 \\
$C_{p(298)}^{\circ}$ & 28.3 & 20.1 & 8.5
\end{tabular}

$\Delta S^{\circ}=37.7 \mathrm{~g} / \mathrm{mole}$

$\Delta H^{\circ}=13.2 \mathrm{kcal} / \mathrm{mole}$

$\Delta C_{p}^{\circ}=0.3 \mathrm{~g} / \mathrm{mole}$

\begin{tabular}{|c|c|c|c|c|c|c|}
\hline $\log A$ & $\boldsymbol{E}$ & $\begin{array}{c}\log \boldsymbol{k}_{T} \\
(790)\end{array}$ & Conditions & System & Surface & References \\
\hline 14.64 & 67.0 & -3.86 & $\begin{array}{l}771-814{ }^{\circ} \mathrm{K} \\
50-180 \text { torr }\end{array}$ & static & none & $\begin{array}{l}\text { [1] H. O. Pritchard, R. G. } \\
\text { Sowden, and A. F. } \\
\text { Trotman-Dickenson, J. } \\
\text { Chem. Soc. } 546 \text { (1954). }\end{array}$ \\
\hline
\end{tabular}

Preferred:

Suspect.

Comments: This elimination (if unimolecular) would involve pentavalent carbon in the transition state, which seems unreasonable. A free radical chain propagated by $\mathrm{CH}_{3}$ radicals would yield the expected products. (See $t$-butyl amine $\longrightarrow \mathrm{NH}_{3}+$ isobutene.) The absence of an appreciable effect on the rate by toluene does not exclude a chain process, since the olefin products would also be good radical traps. Chain processes in the absence of toluene would therefore be the same as those with added toluene. One should note the similarity of this reaction to the decomposition of neopentane, which is known to be a chain process: $k=10^{11.7-51.5 / \theta}(1 / \mathrm{moles})^{1 / 2} \mathrm{sec}^{-1}$, which gives a pseudo first-order rate constant: $k=10^{10.4-51.5 / \theta} \mathrm{sec}^{-1}$, and $\log k(790)=-3.8$.

\section{Experimental}

[1] Exact nature of the product $\mathrm{C}_{3} \mathrm{H}_{7} \mathrm{~N}$ was not determined. Acetone diphenylhydrazone was obtained from a solution of $\mathrm{C}_{3} \mathrm{H}_{7} \mathrm{~N}$ in dilute sulfuric acid. Rates determined by $\Delta \mathrm{P}$ and chemical analysis were unaffected by added toluene. 
Reaction: Methanoloxime (formaldoxime)

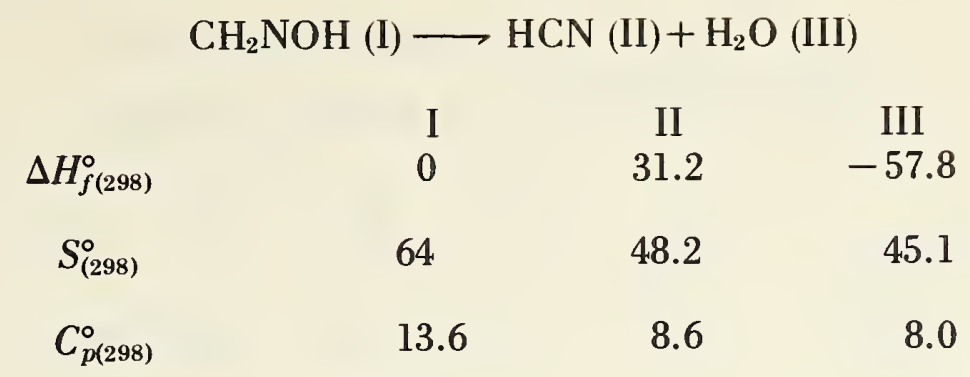

$\Delta S^{\circ}=29.3 \mathrm{~g} / \mathrm{mole}$

$\Delta H^{\circ}=-26.6 \mathrm{kcal} / \mathrm{mole}$

$\Delta C_{p}^{\circ}=3.0 \mathrm{~g} / \mathrm{mole}$

$\begin{array}{rcccccc}\log \boldsymbol{A} & \boldsymbol{E} & \begin{array}{c}\log \boldsymbol{k}_{T} \\ (650)\end{array} & \text { Conditions } & \text { System } & \text { Surface } & \text { References } \\ 9.5 & 39.0 & -3.61 & \begin{array}{l}623-688^{\circ} \mathrm{K} \\ 69-104 \text { torr }\end{array} & \text { static } & \begin{array}{c}\text { appreciable } \\ \text { on clean } \\ \text { surfaces }\end{array} & \begin{array}{c}\text { [1] A. Taylor and H. } \\ \text { Bender, J. Chem. Phys. } \\ 9,761 \text { (1941). }\end{array}\end{array}$

\section{Preferred:}

Not reliable as a unimolecular process.

Comments: The Arrhenius parameters are prohibitively low for a unimolecular elimination reaction. Observations of strong catalysis by $\mathrm{O}_{2}$ and of surface effects suggest both heterogeneous and radical contributions to the rate of decomposition. Acetaldoxime decomposition has been shown to be a free radical reaction. (G. L. Pratt and J. H. Purnell, Trans. Faraday Soc. 58, 692 (1962).)

\section{Experimental}

[1] Rates by $\Delta \mathrm{P}$ with assumed stoichiometry of $1 \rightarrow 2$. Observed $\left(P_{f} / P_{o}\right) \simeq 1.8 \rightarrow 1.9$. Marked catalysis by NO and by $\mathrm{O}_{2}$. Initial rates were used to obtain the rate constants since the products reacted further to give $\mathrm{CO}, \mathrm{NH}_{3}$, and small amounts of $\mathrm{N}_{2}, \mathrm{H}_{2}$, and $\mathrm{C}_{2} \mathrm{H}_{6}$. The reaction was heterogeneous on cleaned surfaces. 
Reaction: Ethanamide (acetamide)

$$
\begin{aligned}
& \mathrm{CH}_{3}-\stackrel{\mathrm{O}}{\mathrm{C}}-\mathrm{NH}_{2}(\mathrm{I}) \underset{b}{a}\left(\mathrm{CH}_{3} \mathrm{CO}(\mathrm{IV})+\cdot \mathrm{NH}_{2}(\mathrm{~V})\right. \\
& \begin{array}{cccccc} 
& \text { I } & \text { II } & \text { III } & \text { IV } & \text { V } \\
\Delta H_{f(298)}^{\circ} & -57.8 & -14.6 & -11.0 & -5.6 & (41) \\
S^{\circ}{ }_{(298)} & 69 & 57.8 & 46.0 & 64.2 & 46.4
\end{array} \\
& \begin{array}{llllll}
C_{p(298)}^{\circ} & 15.7 & 12.4 & 8.5 & 12.3 & 8.1
\end{array}
\end{aligned}
$$

$\begin{array}{rl}a & b \\ \Delta S^{\circ}=34.8 & 41.6 \mathrm{~g} / \mathrm{mole} \\ \Delta H^{\circ}=32.2 & 93.2 \mathrm{kcal} / \mathrm{mole} \\ \Delta C_{p}^{\circ}=5.2 & 4.7 \mathrm{~g} / \mathrm{mole}\end{array}$

Path $\quad \log A \quad E \quad \log k_{T} \quad$ Conditions $\quad$ System $\quad$ Surface (1250)

$\begin{array}{llllll}a+b & 14.7 & 73.4 & 1.87 & 1209-1293{ }^{\circ} \mathrm{K} & \text { flow }\end{array}$

9.5-12.0 torr

References

[1] Margaret Hunt, J. A. Kerr, and A. F. Trotman-Dickenson, J. Chem. Soc. 5074 (1965).

Preferred:

Suspect.

Comments: At the very high reaction temperatures employed, a free radical chain decomposition of ethanamide propogated by the anilino radical $(\emptyset N H)$ should occur. Preferrential $\mathrm{H}$-abstraction from the methyl group (expected on bond energy considerations) would lead to the major reaction products, ketene and ammonia. This seems a more likely reaction path than the unimolecular four-center process proposed. The thermodynamics of reaction $b$ indicate that the $(\mathrm{CO}-\mathrm{N})$ split proposed is rather improbable. An initiation reaction involving a $(\mathrm{CO}-\mathrm{C})$ split seems more likely. A four-center elimination with an $A$-factor of about $10^{13.0} \mathrm{sec}^{-1}$ is probable. In this case $E \sim 68 \mathrm{kcal} / \mathrm{mole}$.

\section{Experimental}

[1] Aniline carrier technique. Rates were based on the $\mathrm{NH}_{3}$ production. Small amounts of $\mathrm{H}_{2}, \mathrm{CH}_{4}$, and $\mathrm{CO}$ were also formed. Methane and CO were found in near equal amounts, indicating path $b$ as a minor process. Data were not accurate enough to treat path $b$ quantitatively. 
Reaction: 1,2-Ethanediamide (oxamide)

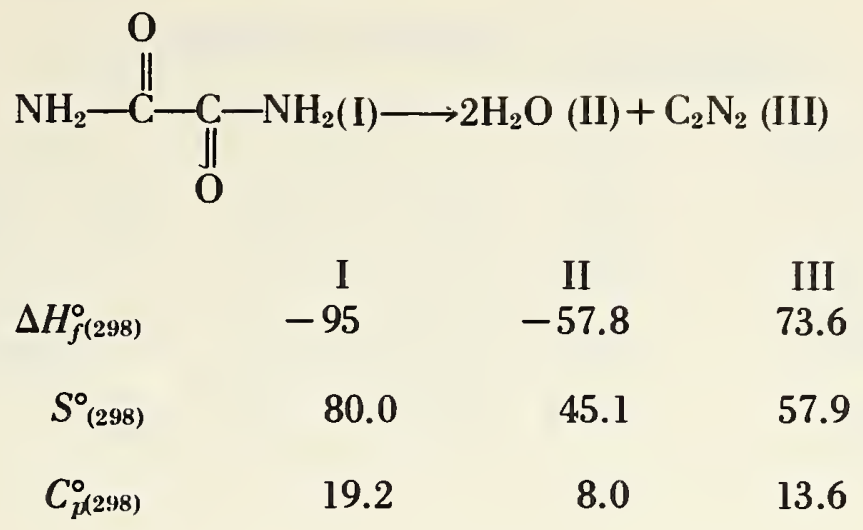

$\Delta S^{\circ}=68.1 \mathrm{~g} / \mathrm{mole}$
$\Delta H^{\circ}=53.0 \mathrm{kcal} / \mathrm{mole}$
$\Delta C_{p}^{\circ}=10.4 \mathrm{~g} / \mathrm{mole}$

\begin{tabular}{|c|c|c|c|c|c|c|}
\hline $\log A$ & $\boldsymbol{E}$ & $\begin{array}{l}\log k_{T} \\
(680)\end{array}$ & Conditions & System & Surface & References \\
\hline 10.25 & 41.4 & -3.05 & $\begin{array}{l}593-763^{\circ} \mathrm{K} \\
140-300 \text { torr }\end{array}$ & static & none & $\begin{array}{l}\text { [1] F. M. Taylor and L. L. } \\
\text { Bircumshaw, J. Chem. } \\
\text { Soc. } 3405 \text { (1956). }\end{array}$ \\
\hline
\end{tabular}

Preferred:

Not reliable.

Comments: Arrhenius parameters are much too low for a homogenous unimolecular reaction. The heat of the reaction exceeds the observed activation energy. The reaction cannot be unimolecular if the thermodynamics are even close to correct. A free radical chain decomposition or heterogeneous decomposition are the only reasonable prospects.

\section{Experimental}

[1] Rates determined by $\Delta \mathrm{P} .\left(P_{x} / P_{0}\right)=3.0 \pm 0.06$. Addition of products did not affect the rate. Added nitrogen gave unusual effects: small amounts decreased the rate and larger amounts increased it to a constant value. Cyanogen was estimated by conductometric titrations of the cyanate-cyanide with silver nitrate. (Z. Anal. Chem. 99, 415 (1934).) 
Reaction: Ethanethiol (ethyl mercaptan)

\begin{tabular}{|c|c|c|c|c|c|}
\hline \multirow[b]{2}{*}{$\Delta H_{f(298)}^{\circ}$} & $\mathrm{C}_{2} \mathrm{H}_{5} \mathrm{SH}(\mathrm{I})$ & \multicolumn{3}{|c|}{$\stackrel{a}{\longrightarrow} \mathrm{C}_{2} \mathrm{H}_{4}(\mathrm{II})+\mathrm{H}_{2} \mathrm{~S}(\mathrm{III})$} & \\
\hline & $\begin{array}{c}\mathrm{I} \\
-11.0\end{array}$ & $\begin{array}{c}\text { II } \\
12.5\end{array}$ & $\begin{array}{c}\text { III } \\
-4.8\end{array}$ & $\begin{array}{c}\text { IV } \\
25.7\end{array}$ & $\begin{array}{c}\mathrm{V} \\
(35.5)\end{array}$ \\
\hline$S_{(298)}^{\circ}$ & 71.8 & 52.5 & 49.2 & 57.8 & 46.7 \\
\hline$C_{p(298)}^{\circ}$ & 17.2 & 10.4 & 8.1 & 12.1 & 7.1 \\
\hline
\end{tabular}

$\begin{array}{cc}a & b \\ \Delta S^{\circ}=29.9 & 32.7 \mathrm{~g} / \mathrm{mole} \\ \Delta H^{\circ}=18.7 & 72.2 \mathrm{kcal} / \text { mole } \\ \Delta C_{p}^{\circ}=1.3 & 2.0 \mathrm{~g} / \mathrm{mole}\end{array}$

Path $\quad \log A \quad E \quad \log k_{T} \quad$ Condition

System Surface

References

$a$

13.0

$51.2-0.09$

$785-938^{\circ} \mathrm{K} \quad$ flow $\quad$ (see below)
$\delta P \simeq 0.7 \mathrm{~mm} \mathrm{Hg}$
$P_{T} \sim 12 \mathrm{~mm} \mathrm{Hg}$

[1]

A. H. Sehon and B. deB. Darwent, J. Am. Chem. Soc. 76, 4806 (1954).

$\begin{array}{lllll}b & 13.48 & 63 \pm 1 & -2.53 & \text { (as above) }\end{array}$

Comments: Path $a: \log k_{a}=13.0-51.5 / \theta ; \log A_{\text {est }}=13.1$ (see section II-1.0).

Path $b$ : Reported parameters are low. We prefer, $\log k=15.5-72.2 / \theta$.

\section{Experimental}

[1] The elimination reaction was predominant at the lower temperatures. At the higher temperatures, appreciable dibenzl was produced, indicating radical production (path $b$ ). Some surface dependence was observed in path $a$ at the extreme low temperatures. The $A$-factors reported were assumed. (See bond fissions for evaluation of path $b$ parameters.) 
Reaction: 2-Methyl-2-propanethiol ( $t$-butyl mercaptan)

\begin{tabular}{cccc}
\multicolumn{4}{r}{$\left(\mathrm{CH}_{3}\right)_{3} \mathrm{CSH}(\mathrm{I}) \longrightarrow\left(\mathrm{CH}_{3}\right)_{2}=\mathrm{CH}_{2}(\mathrm{II})+\mathrm{H}_{2} \mathrm{~S}(\mathrm{III})$} \\
& I & II & III \\
$\Delta H_{f(298)}^{\circ}$ & -26.4 & -4.0 & -4.8 \\
$S_{(298)}^{\circ}$ & 79.2 & 70.2 & 49.2 \\
$C_{p(298)}^{\circ}$ & 28.9 & 21.3 & 8.1
\end{tabular}

$\begin{aligned} & \Delta S^{\circ}=40.2 \mathrm{~g} / \mathrm{mole} \\ & \Delta H^{\circ}=17.6 \mathrm{kcal} / \mathrm{mole} \\ & \Delta C_{p}^{\circ}=0.5 \mathrm{~g} / \mathrm{mole}\end{aligned}$

$\begin{array}{llll}\log A & \boldsymbol{E} & \log \boldsymbol{k}_{T} & \text { Conditions }\end{array}$ (1090)

System Surface

References

13.3

55.0

$\begin{array}{rl}2.27 & 950-1230^{\circ} \mathrm{K} \\ & <1 \% \text { in } \mathrm{Ar}\end{array}$

S. P. shock

[1] W. Tsang, J. Chem.

Phys. 40, 1498 (1964).

\section{Preferred:}

$\log k=13.4-55.3 / \theta$.

$\log A_{\text {est }}=13.6$ (see section II-1.0).

\section{Experimental}

[1] Rates by G.L.C. analysis. 
Reaction: 2,2-Difluoroethyltrifluorosilane

\begin{tabular}{cccr}
\multicolumn{4}{r}{$\mathrm{CHF}_{2} \mathrm{CH}_{2} \mathrm{SiF}_{3}(\mathrm{I}) \longrightarrow \mathrm{CHF}^{2}=\mathrm{CH}_{2}(\mathrm{II})+\mathrm{SiF}_{4}(\mathrm{III})$} \\
$\Delta \mathrm{H}_{\mathcal{f}^{2}(98)}^{\circ}$ & -406 & -31.5 & -384 \\
$S_{(298)}^{\circ}$ & 95.0 & 60.4 & 67.4 \\
$C_{p(298)}^{\circ}$ & 31.6 & 11.9 & 17.6
\end{tabular}

$\Delta S^{\circ}=32.8 \mathrm{~g} / \mathrm{mole}$

$\Delta H^{\circ}=-9.5 \mathrm{kcal} / \mathrm{mole}$

$\Delta C_{p}^{\circ}=-2.1 \mathrm{~g} / \mathrm{mole}$

\begin{tabular}{|c|c|c|c|c|c|c|}
\hline $\log A$ & $\boldsymbol{E}$ & $\begin{array}{c}\log k_{T} \\
(460)\end{array}$ & Conditions & System & Surface & References \\
\hline 12.27 & 32.72 & -3.27 & $\begin{array}{l}424-494^{\circ} \mathrm{K} \\
10-180 \text { torr }\end{array}$ & static & $<5 \%$ & $\begin{array}{l}\text { [1] R. N. Haszeldine, P. J. } \\
\text { Robinson, and R. F. } \\
\text { Simmons, J. Chem. Soc. } \\
1890 \text { (1964). }\end{array}$ \\
\hline
\end{tabular}

Preferred:

$\log k=12.27-32.72 / \theta$.

Comments: Transition state calculation gives $\log A_{\text {est }}=12.3$ (see section II-1.0).

This cannot be a chain reaction: the $A$-factor is too high and the activation energy too low; therefore the four-center molecular interpretation above seems correct.

\section{Experimental}

[1] Rates determined by $\Delta P$. Chemical and I.R. analysis of products confirmed the stoichiometry. Rates unaffected by additions of $\mathrm{NO}$ and cyclohexene. 
Reaction: 2-Chloroethyltrichlorosilane

$\mathrm{ClCH}_{2} \mathrm{CH}_{2} \mathrm{SiCl}_{3}(\mathrm{I}) \longrightarrow \mathrm{C}_{2} \mathrm{H}_{4}(\mathrm{II})+\mathrm{SiCl}_{4}$ (III)

$\begin{array}{cccr}\Delta H_{(298)}^{\circ} & \text { I } & \text { II } & \text { III } \\ S_{(298)}^{\circ} & \left(\begin{array}{r}-14.1 \\ 102.6 \\ \\ C_{p(298)}^{\circ}\end{array}\right) & 12.5 & -152 \\ 33.2 & 52.5 & 79.1 \\ & 10.4 & 21.6\end{array}$

$\Delta S^{\circ}=29 \mathrm{~g} / \mathrm{mole}$

$\Delta \mathrm{H}^{\circ}=1.5 \mathrm{kcal} / \mathrm{mole}$

$\Delta C_{p}^{\circ}=-1.2 \mathrm{~g} / \mathrm{mole}$

\begin{tabular}{|c|c|c|c|c|c|c|}
\hline $\log A$ & $\boldsymbol{E}$ & $\begin{array}{c}\log k_{T} \\
(650)\end{array}$ & Conditions & System & Surface & References \\
\hline 11.26 & 45.5 & $(-4.04]$ & $\begin{array}{l}629-690{ }^{\circ} \mathrm{K} \\
37-50 \text { torr }\end{array}$ & static & none & $\begin{array}{l}\text { [1] I. M. T. Davidson, C. } \\
\text { Eaborn, and M. N. } \\
\text { Lilly, J. Chem. Soc. } \\
2624 \text { (1964). }\end{array}$ \\
\hline
\end{tabular}

\section{Preferred:}

$\log k=12.3-48.5 / \theta$ (from transition-state calculation of the $A$-factor; see section II-1.0).

Comments: The rate of formation of the minor product, vinyltrichlorosilane, via a four-center molecular elimination of $\mathrm{HCl}$ can be estimated (by analogy with the $n \cdot \mathrm{PrCl}$ reaction rate) to be an order of magnitude slower than the observed rate. This is consistent with the experimental findings and tends to support the molecular mechanism proposed for the overall reaction.

One cannot exclude the possibility that this reaction is a free-radical process, however.

$$
\text { Chain: } \begin{aligned}
\mathrm{SiCl}_{3}+ & \mathrm{ClCH} \mathrm{CH}_{2} \mathrm{SiCl}_{3} \longrightarrow \mathrm{SiCl}_{4}+\cdot \mathrm{CH}_{2} \mathrm{CH}_{2} \mathrm{SiCl}_{3} \\
& \cdot \mathrm{CH}_{2} \mathrm{CH}_{2} \mathrm{SiCl}_{3} \longrightarrow \mathrm{C}_{2} \mathrm{H}_{4}+\mathrm{SiCl}_{3} \cdot
\end{aligned}
$$

Such a chain is consistent with the fact that the $(\mathrm{Si}-\mathrm{Cl})$ and $(\mathrm{C}-\mathrm{H})$ bonds are stronger than the $(\mathrm{C}-\mathrm{Cl})$ and $(\mathrm{Si}-\mathrm{H})$ bonds, respectively. Olefins could therefore be very poor traps for silyl radicals, while halogen abstraction could be very fast and specific.

\section{Experimental}

[1] Rates followed by $\Delta$ P. Analysis of products by G. L. C. No effect of NO or cyclohexene. Two parallel first-order reactions detected by product analysis occurred, producing $\mathrm{HCl}+$ vinyltrichlorosilane and trichlorosilane + vinylchloride. Arrhenius parameters could not be obtained for these reactions, although one would expect the former to have parameters similar to $n$-propylchloride. 
Reaction: 2-Chloroethylethyldichlorosilane

\begin{tabular}{lrcr}
$\mathrm{ClCH}_{2} \mathrm{CH}_{2}-\mathrm{SiCl}_{2}\left(\mathrm{C}_{2} \mathrm{H}_{5}\right)$ & $(\mathrm{I})$ & \multicolumn{3}{c}{$\mathrm{C}_{2} \mathrm{H}_{4}(\mathrm{II})+\mathrm{Cl}_{3} \mathrm{SiC}_{2} \mathrm{H}_{5}(\mathrm{III})$} \\
$\Delta H_{p(298)}^{\circ}$ & $\mathrm{I}$ & $\mathrm{II}$ & III \\
$S_{(298)}^{\circ}$ & -128 & 12.5 & -135 \\
& 115 & 52.5 & 93.0 \\
$C_{p(298)}^{\circ}$ & 41.6 & 10.4 & 29.8
\end{tabular}

$\Delta S^{\circ}=30.5 \mathrm{~g} / \mathrm{mole}$

$\Delta H^{\circ}=5.5 \mathrm{kcal} / \mathrm{mole}$

$\Delta C_{p}^{\circ}=-1.4 \mathrm{~g} / \mathrm{mole}$

\begin{tabular}{lllllll}
$\log A$ & $\boldsymbol{E}$ & $\begin{array}{c}\log \boldsymbol{k}_{T} \\
(650)\end{array}$ & \multicolumn{1}{c}{ Conditions } & System & Surface & \multicolumn{1}{c}{ References } \\
12.26 & 46.5 & -3.37 & $\begin{array}{l}629-670^{\circ} \mathrm{K} \\
6-25 \text { torr }\end{array}$ & static & none & [1] I. M. Davidson and \\
C. J. L. Metcalfe, J. \\
Chem. Soc. 2630 (1964).
\end{tabular}

Preferred:

$\log k=12.26-46.5 / \theta$

$\log A_{\text {est }}=12.3$ (see section II-1.0; however, also see comments on 2-chloroethyltrichlorosilane.

\section{Experimental}

[1] Rates by $\Delta P$ and G.L.C. analysis of products were unaffected by added propylene. A parallel reaction producing $\mathrm{HCl}$ and $\mathrm{CH}_{2}=\mathrm{CHSiCl}_{2}\left(\mathrm{C}_{2} \mathrm{H}_{5}\right)$ accounted for 10 percent of products. Arrhenius parameters for this elimination were not obtained. 
Reaction: 2-Chloroethyldiethylchlorosilane

$$
\mathrm{ClCH}_{2} \mathrm{CH}_{2}-\mathrm{Si}\left(\mathrm{C}_{2} \mathrm{H}_{5}\right)_{2} \mathrm{Cl}(\mathrm{I}) \longrightarrow \mathrm{C}_{2} \mathrm{H}_{4}(\mathrm{II})+\left(\mathrm{C}_{2} \mathrm{H}_{5}\right)_{2} \mathrm{SiCl}_{2}(\mathrm{III})
$$

$$
\begin{aligned}
& \Delta H_{f(298)}^{\circ} \\
& S_{(298)}^{\circ} \\
& C_{p(298)}^{\circ}
\end{aligned} \quad\left(\begin{array}{c}
-111.6 \\
127 \\
50.0
\end{array}\right) \quad \begin{array}{cc}
\text { I } \\
12.5 \\
52.5 \\
10.4
\end{array}\left(\begin{array}{c}
-118.3 \\
105 \\
38.2
\end{array}\right)
$$

$\Delta S^{\circ}=30.5 \mathrm{~g} / \mathrm{mole}$

$\Delta H^{\circ}=5.8 \mathrm{kcal} / \mathrm{mole}$

$\Delta C_{p}^{\circ}=-1.4 \mathrm{~g} / \mathrm{mole}$

\begin{tabular}{lllllll}
$\log \boldsymbol{A}$ & $\boldsymbol{E}$ & $\begin{array}{c}\log \boldsymbol{k}_{T} \\
(600)\end{array}$ & Conditions & System & Surface & \multicolumn{1}{c}{ References } \\
11.88 & 41.1 & -3.09 & $571-625^{\circ} \mathrm{K}$ & static & none & [1] I. M. T. Davidson and \\
M. R. Jones, J. Chem. \\
\end{tabular}

\section{Preferred:}

$\log k=12.40-42.7 / \theta$. However, see comments on 2-chloroethyltrichlorosilane.

\section{Experimental}

[1] Rates determined by $\Delta P^{\prime}$ and G.L.C. analysis and were unaffected by added NO or propylene in a greaseless system. Stopcock grease was observed to promote a heterogeneous reaction. 
Reaction: Ethene

$\begin{array}{cccc} & \mathrm{C}_{2} \mathrm{H}_{4}(\mathrm{I}) \longrightarrow \mathrm{C}_{2} \mathrm{H}_{2}(\mathrm{II})+\mathrm{H}_{2}(\mathrm{III}) & \\ \Delta H_{f(298)}^{\circ} & \mathrm{I} & \text { II } & \text { III } \\ S_{(298)}^{\circ} & 12.5 & 53.9 & 0 \\ C_{p(298)}^{\circ} & 52.5 & 48.0 & 31.2 \\ & 10.4 & 10.5 & 7\end{array}$

$\Delta S^{\circ}=26.7 \mathrm{~g} / \mathrm{mole}$

$\Delta \mathrm{H}^{\circ}=41.4 \mathrm{kcal} / \mathrm{mole}$

$\Delta C_{p}^{\circ}=7.1 \mathrm{~g} / \mathrm{mole}$

\begin{tabular}{|c|c|c|c|c|c|c|}
\hline $\log A$ & $\boldsymbol{E}$ & $\begin{array}{c}\log \boldsymbol{k}_{T} \\
(1550)\end{array}$ & Conditions & System & Surface & References \\
\hline 8.87 & 46.5 & 2.31 & $1300-1800^{\circ} \mathrm{K}$ & S.P. shock & & $\begin{array}{l}\text { [1] G. B. Skinner and } \\
\text { E. M. Sokolski, J. Phys. } \\
\text { Chem. 64, } 1028 \text { (1960). }\end{array}$ \\
\hline 11.95 & 65.0 & $\begin{array}{r}(1300) \\
1.03\end{array}$ & $1170-1425^{\circ} \mathrm{K}$ & S.P. shock & & (as above) \\
\hline
\end{tabular}

\section{Preferred:}

The reaction must be a chain process.

Comments: Haugen and Benson (J. Phys. Chem. 71, 1735 (1967)) have recently proposed a free radical mechanism which accounts for all the experimental data. Such a large change in Arrhenius parameters for any molecular reaction is unreasonable.

\section{Experimental}

[1] Arrhenius parameters changed with the temperature range studied. Analysis by G.L.C. Since the authors were unable to propose a free radical mechanism with Arrhenius parameters of the order observed, and since they believed a free radical mechanism could not account for the constant product ratios observed over the wide range of decompositions studied (i.e., 2 to 95 percent ethylene decomposition), they proposed a molecular split. 
Reaction: Formyl fluoride

$\mathrm{HCOF}(\mathrm{I} \longrightarrow \mathrm{HF}(\mathrm{II})+\mathrm{CO}(\mathrm{III})$

\begin{tabular}{crrr}
$\Delta H_{(298)}^{\circ}$ & I & II & \multicolumn{1}{c}{ III } \\
$S_{(298)}^{\circ}$ & ( & -64.2 & -26.4 \\
& & 41.5 & 47.3 \\
$C_{p(298)}^{\circ}$ & & 7.0 & 7.0
\end{tabular}

$\Delta S^{\circ}=$
$\Delta H^{\circ}=$
$\Delta C_{p}^{\circ}=$

$\log A \quad E \quad \log k_{T}$

Conditions

System

Surface

References

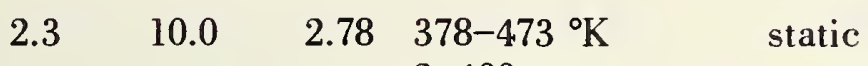

none, in coated

[1] G. Fischer and A. S. 2-400 torr reaction cells. Buchanan, Trans. Faraday Soc. 60, 378 (1964).

\section{Preferred:}

Not reliable; probably heterogeneous.

Comments: The Arrhenius parameters of this reaction are much too low to be reasonable. Reinvestigations of reactions for molecules of moderate complexity in which electronic state crossover paths have been proposed have invariably found that the reaction was heterogeneous. (e.g., cis $\longrightarrow$ trans isomerisation but-2-ene).

\section{Experimental}

[1] Rates based on the production of CO. Added $\mathrm{CO}, \mathrm{O}_{2}$, and $\mathrm{HF}$ did not affect the reaction rates although $\mathrm{SiF}_{4}$ reduced the rates somewhat. A nonadiabatic reaction (singlet $\longrightarrow$ triplet $\longrightarrow$ singlet) of low energy and probability was proposed. 
Reaction: *Trichloromethane (chloroform)

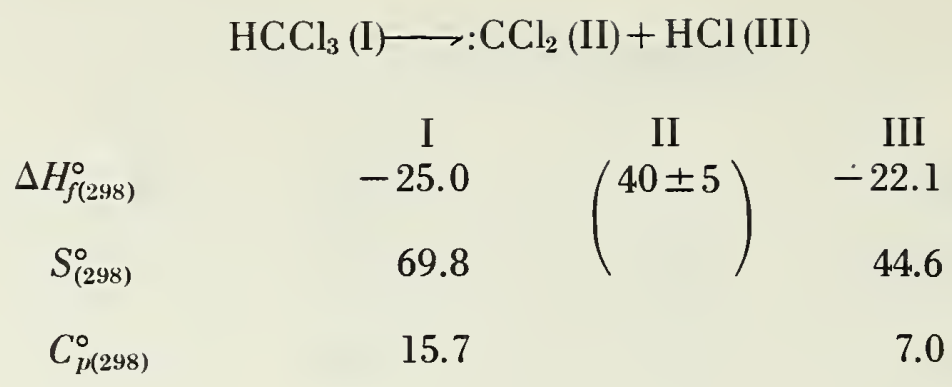

$\Delta S^{\circ}=$
$\Delta H^{\circ}=43 \pm 5 \mathrm{kcal} / \mathrm{mole}$
$\Delta C_{p}^{\circ}=$

\begin{tabular}{|c|c|c|c|c|c|c|}
\hline $\log A$ & $\boldsymbol{E}$ & $\begin{array}{c}\log \boldsymbol{k}_{T} \\
(820)\end{array}$ & Conditions & System & Surface & References \\
\hline 11.42 & 47.0 & -1.10 & $\begin{array}{l}783-857^{\circ} \mathrm{K} \\
1.18 \longrightarrow 35.9 \text { torr } \\
P_{t}>15 \text { torr }\end{array}$ & flow & $<10 \%$ & $\begin{array}{l}\text { [1] A. E. Shilov and R. D. } \\
\text { Sabirova, Russ. J. Phys. } \\
\text { Chem. 34, } 408 \text { (1960). }\end{array}$ \\
\hline
\end{tabular}

Preferred:

Suspect. Free radical chain processes are indicated.

Comments: The Arrhenius parameters of this reaction are too low for a three-center molecular elimination. At least a partial free radical contribution to the rate seems reasonable, not only on the basis of the kinetic parameters, but also on the basis of the products. Decomposition of $\mathrm{DCCl}_{3}$ in toluene gave only $\simeq 35$ percent $\mathrm{DCl}$ although the ratio was unaffected by wide changes in temperature, contact time, and $\mathrm{DCCl}_{3} / \mathrm{toluene}$ ratios. One should have 100 percent $\mathrm{DCl}$ if the decomposition is entirely molecular. Semeluk and Bernstein, J. Am. Chem. Soc 79, 46 (1957); J. Am. Chem. Soc. 76, 3793 (1954) have interpreted their results on this reaction in terms of a free radical mechanism, although some of their mechanistic details are known to be wrong. A transition state $A$-factor estimate gives $\log A_{\text {est }}=13.7$.

\section{Experimental}

[1] Rates determined by formation of $\mathrm{HCl}$. Added toluene had no effect. Almost no dibenzyl was detected. First order above 15 torr pressure. Decomposition of $\mathrm{DCCl}_{3}$ also studied with and without toluene.

*Reaction products: $\mathrm{HCl}, \mathrm{C}_{2} \mathrm{Cl}_{4}, \mathrm{C}_{2} \mathrm{Cl}_{6}$, and smaller amounts of other chlorinated ethanes. 
Reaction: Chlorodifluoromethane

\begin{tabular}{cccc}
\multicolumn{4}{c}{$\mathrm{CF}_{2} \mathrm{HCl}(\mathrm{I}) \longrightarrow \mathrm{CF}_{2}:(\mathrm{II})+\mathrm{HCl}(\mathrm{III})$} \\
$\Delta H_{\mathcal{N}^{298)}}^{\circ}$ & $\mathrm{I}$ & $\mathrm{II}$ & $\mathrm{III}$ \\
$S_{(298)}^{\circ}$ & -112.3 & $(-39.7)$ & -22.1 \\
$C_{p(298)}^{\circ}$ & 66.4 & 59.6 & 44.6 \\
& 13.1 & 9.1 & 7.0
\end{tabular}

$\Delta S^{\circ}=37.8 \mathrm{~g} / \mathrm{mole}$

$\Delta H^{\circ}=50.5 \mathrm{kcal} / \mathrm{mole}$

$\Delta C_{p}^{\circ}=3.0 \mathrm{~g} / \mathrm{mole}$

\begin{tabular}{|c|c|c|c|c|c|c|}
\hline $\log A$ & $\boldsymbol{E}$ & $\begin{array}{c}\log \boldsymbol{k}_{T} \\
(890)\end{array}$ & Conditions & System & Surface & References \\
\hline 13.84 & $\begin{array}{r}55.8 \\
\\
51.4 \\
\pm 2.5\end{array}$ & $(0.14)$ & $\begin{array}{l}803-973^{\circ} \mathrm{K} \\
\left(943-1023^{\circ} \mathrm{K}\right)\end{array}$ & static & & $\begin{array}{l}\text { [1] J. W. Edwards and P. A. } \\
\text { Small, Nature 202, } \\
1329 \text { (1964). } \\
\text { [2] F. Gozzo and C. R. } \\
\text { Patrick, Nature 202, } \\
80 \text { (1964). }\end{array}$ \\
\hline
\end{tabular}

Preferred:

$\log k=13.84-55.8 / \theta$.

Comments: Transition state estimation of $A$ suggests approximate minimum and maximum values of: $\log A_{\min }=10^{13 \cdot 1} ; \log A_{\max }=10^{14.2}$ (see section II-2.0).

Assuming the reaction is correct as reported, and that the back reaction $\left(\mathrm{CF}_{2}:+\mathrm{HCl} \rightarrow\right)$ has $E=X$ $\mathrm{kcal} / \mathrm{mole}$, it is possible to obtain a limit for the heat of formation of the $\mathrm{CF}_{2}$ : biradical. $\Delta H_{f}^{\circ}\left(\mathrm{CF}_{2}:\right)=$ $(-34.4-X)$. The tetrafluoroethylene decomposition results place $\Delta H_{f}^{\circ}\left(\mathrm{CF}_{2}:\right)=-39.7$, which makes $E_{b}=X=$ $5.3 \mathrm{kcal} / \mathrm{mole}$. This is a reasonable value for $\mathrm{CF}_{2}$ : addition to $\mathrm{HCl}$.

\section{Experimental}

[1] and [2] No experimental details given. The $\mathrm{CF}_{2}$ : radicals dimerize, producing $\mathrm{C}_{2} \mathrm{~F}_{4}$. 
Reaction: *Nitroethane

\begin{tabular}{crcc}
\multicolumn{4}{r}{$\mathrm{CH}_{3} \mathrm{CH}_{2} \mathrm{NO}_{2}(\mathrm{I}) \longrightarrow \mathrm{C}_{2} \mathrm{H}_{4}(\mathrm{II})+\mathrm{HONO}$ (III) } \\
$\Delta H_{\text {f(298) }}^{\circ}$ & I & II & III \\
$S_{(298)}^{\circ}$ & -24.4 & 12.5 & -18.5 \\
$C_{p(298)}^{\circ}$ & 75.6 & 52.5 & 58 \\
& 21.6 & 10.4 & 9.6
\end{tabular}

$\Delta S^{\circ}=34.9 \mathrm{~g} / \mathrm{mole}$

$\Delta H^{\circ}=18.4 \mathrm{kcal} / \mathrm{mole}$

$\Delta C_{p}^{\circ}=-1.6 \mathrm{~g} / \mathrm{mole}$

\begin{tabular}{|c|c|c|c|c|c|c|}
\hline $\log A$ & $\boldsymbol{E}$ & $\begin{array}{c}\log \boldsymbol{k}_{T} \\
(650)\end{array}$ & Conditions & System & Surface & References \\
\hline 11.35 & 41.4 & -2.57 & $\begin{array}{l}687-715{ }^{\circ} \mathrm{K} \\
5-10 \% \text { reactant }\end{array}$ & flow & $\longrightarrow$ & $\begin{array}{l}\text { [1] K. A. Wilde, Ind. Eng. } \\
\text { Chem. 48, } 769 \text { (1956). }\end{array}$ \\
\hline 11.53 & 41.5 & -2.42 & $\begin{array}{l}583-713{ }^{\circ} \mathrm{K} \\
4-40 \text { torr }\end{array}$ & static & $<10 \%$ & $\begin{array}{l}\text { [2] deM. Claude Frejacques, } \\
\text { Compt. Rend. 231, } \\
1061 \text { (1950). }\end{array}$ \\
\hline 10.83 & 39.7 & -2.52 & $593-714^{\circ} \mathrm{K}$ & $\begin{array}{l}\text { static \& } \\
\text { flow }\end{array}$ & & $\begin{array}{l}\text { [3] K. A. Wilde, J. Phys. } \\
\text { Chem. 61, } 385 \text { (1957). }\end{array}$ \\
\hline 13.0 & 47.0 & -2.80 & $\begin{array}{l}628-678{ }^{\circ} \mathrm{K} \\
50-300 \text { torr }\end{array}$ & static & $\sim 10 \%$ & $\begin{array}{l}\text { [4] T. L. Cottrell, T. E. } \\
\text { Graham, and T. J. } \\
\text { Reid, Trans. Faraday } \\
\text { Soc. 47, } 1089 \text { (1951). }\end{array}$ \\
\hline 12.4 & 45 & -2.62 & (by reevaluation & data) & & $\begin{array}{l}\text { [5] G. N. Spokes and S. W. } \\
\text { Benson, J. Am. Chem. } \\
\text { Soc. 89, } 6030 \text { (1967). }\end{array}$ \\
\hline
\end{tabular}

\section{Preferred:}

$\log k=12.4-45 / \theta$.

Comments: The complexities of this reaction (partial chain contribution to the rate) are such that appreciable uncertainty in the parameters is possible. Nitrous acid can decompose $\left(2 \mathrm{HONO} \rightleftharpoons \mathrm{H}_{2} \mathrm{O}+\mathrm{NO}+\mathrm{NO}_{2}\right)$, thus providing a ready source of radicals for a radical-induced decomposition of nitroethane; $\mathrm{NO}_{2}$ is a powerful chain initiator. The experimental $A$-factors require an extremely tight activated complex (i.e., almost total loss of both internal rotations.) By analogy with six-center transition state reactions, one would expect an $A$-factor in excess of $10^{13} \mathrm{sec}^{-1}$ (see also 2-nitropropane).

\section{Experimental}

[1] Polarographic analysis of nitroalkane for rate determinations. I.R. used for qualitative product identification. Increased rate at $T>715^{\circ} \mathrm{K}$ attributed to concurrent $(\mathrm{C}-\mathrm{N})$ bond split. Additions of $\mathrm{NO}_{2}$ and $\mathrm{H}_{2} \mathrm{O}$ did not affect the decomposition rates.

[2] Rates determined from $\Delta P$. Stoichiometry of the reaction independent of initial pressure and almost independent

*Final products: $\mathrm{C}_{2} \mathrm{H}_{4}, \mathrm{NO}, \mathrm{H}_{2} \mathrm{O}$ (major); $\mathrm{HCN}, \mathrm{NO}_{2}, \mathrm{CO}_{2}, \mathrm{RCHO}$ (minor).

(Continued) 
Nitroethane (Continued)

of the temperature.

[3] Rate by I.R. analysis on nitroethane. Kinetics of the high-temperature reaction believed to be initiated by the $(\mathrm{C}-\mathrm{N})$ bond fission (see bond fissions) were also studied.

[4] Rates were obtained from the initial slopes of the pressure-time curves.

\section{Reaction: *1-Nitropropane}

$\begin{array}{cccc}\mathrm{CH}_{3} \mathrm{CH}_{2} \mathrm{CH}_{2} \mathrm{NO}_{2}(\mathrm{I}) \longrightarrow \mathrm{CH}_{3} \mathrm{CH}=\mathrm{CH}_{2}(\mathrm{II})+\mathrm{HONO}(\mathrm{III}) \\ \Delta H_{f^{298)}}^{\circ} & \text { I } & \text { II } & \text { III } \\ S_{(298)}^{\circ} & -29.4 & 4.8 & -18.5 \\ C_{p(298)}^{\circ} & 85.0 & 63.6 & 58 \\ & 27.1 & 15.3 & 9.6\end{array}$

$$
\begin{aligned}
& \Delta S^{\circ}=36.6 \mathrm{~g} / \mathrm{mole} \\
& \Delta H^{\circ}=15.7 \mathrm{kcal} / \mathrm{mole} \\
& \Delta C_{p}^{\circ}=-2.2 \mathrm{~g} / \mathrm{mole}
\end{aligned}
$$

\begin{tabular}{|c|c|c|c|c|c|c|}
\hline $\log A$ & $\boldsymbol{E}$ & $\begin{array}{c}\log \boldsymbol{k}_{T} \\
(650)\end{array}$ & Conditions & System & Surface & References \\
\hline 11.74 & 41.5 & -2.21 & $\begin{array}{l}583-713^{\circ} \mathrm{K} \\
4-40 \text { torr }\end{array}$ & static & $<10 \%$ & $\begin{array}{l}\text { [1] deM. Claude } \\
\text { Frejacques, Compt. } \\
\text { Compt. Rend. } 321, \\
1061 \text { (1950). }\end{array}$ \\
\hline 13.4 & 47.7 & -2.63 & $687-733^{\circ} \mathrm{K}$ & flow & & $\begin{array}{l}\text { [2] K. A. Wilde, Ind. Eng. } \\
\text { Chem. 48, } 769 \text { (1956). }\end{array}$ \\
\hline 11.5 & 42 & -2.63 & $700-1100^{\circ} \mathrm{K}$ & $\begin{array}{l}\text { VLPP } \\
\text { (very low } \\
\text { pressure } \\
\text { pyrolysis) }\end{array}$ & & $\begin{array}{l}\text { [3] G. N. Spokes and S. W. } \\
\text { Benson, J. Am. } \\
\text { Chem. Soc. 89, } \\
6030 \text { (1967). }\end{array}$ \\
\hline
\end{tabular}

\section{Preferred: \\ $\log k=13.4-47.7 / \theta$.}

Comments: Transition state calculation of $A$ gives $\log A_{\text {est }}=13.2$ (See comments on nitroethane).

\section{Experimental}

[1] Rates by $\Delta \mathrm{P}$.

[2] Rates by polarographic analysis on nitropropane.

[3] Reaction products were complex: propylene, $\mathrm{NO}, \mathrm{H}_{2} \mathrm{O}$ (principal products); $\mathrm{HCN}, \mathrm{NO}_{2}, \mathrm{CO}_{2}$, and aldehydes (minor products).

*Final products are complex (see [3] above). 
Reaction: ${ }^{2}$-Nitropropane

$\begin{array}{cccc}\mathrm{CH}_{3} \mathrm{CHNO}_{2} \mathrm{CH}_{3}(\mathrm{I}) \longrightarrow \mathrm{CH}_{3} \mathrm{CH}=\mathrm{CH}_{2}(\mathrm{II})+\mathrm{HONO}(\mathrm{III}) & \text { I } & \text { II } & \text { III } \\ \Delta H_{f(298)}^{\circ} & -34.0 & 4.8 & -18.5 \\ S_{(298)}^{\circ} & 83.4 & 63.6 & 58 \\ C_{p(298)}^{\circ} & 26.8 & 15.3 & 9.6\end{array}$

$\Delta S^{\circ}=38.2 \mathrm{~g} / \mathrm{mole}$

$\Delta H^{\circ}=20.3 \mathrm{kcal} / \mathrm{mole}$

$\Delta C_{p}^{\circ}=-1.9 \mathrm{~g} / \mathrm{mole}$

\begin{tabular}{|c|c|c|c|c|c|c|}
\hline $\log A$ & $\boldsymbol{E}$ & $\begin{array}{c}\log \boldsymbol{k}_{T} \\
(700)\end{array}$ & Conditions & System & Surface & References \\
\hline 11.34 & 39.0 & -0.83 & $\begin{array}{l}683-713{ }^{\circ} \mathrm{K} \\
4-40 \text { torr }\end{array}$ & static & $<10 \%$ & $\begin{array}{l}\text { [1] deM. Claude Fre- } \\
\text { jacques, Compt. Rend. } \\
\mathbf{2 3 1}, 1061 \text { (1950). }\end{array}$ \\
\hline 11.05 & 39.0 & -1.12 & $683-733^{\circ} \mathrm{K}$ & flow & & $\begin{array}{l}\text { [2] K. A. Wilde, Ind. } \\
\text { Eng. Chem. 48, } 769 \\
\text { (1956). }\end{array}$ \\
\hline 11.05 & 39.3 & -1.07 & $\begin{array}{l}523-610^{\circ} \mathrm{K} \\
16 \text { torr }\end{array}$ & static & negligible & $\begin{array}{l}\text { [3] T. E. Smith and J. G. } \\
\text { Calvert, J. Phys. Chem } \\
\text { 63, } 1305 \text { (1959). }\end{array}$ \\
\hline 11.3 & 40 & -1.09 & $800-1000^{\circ} \mathrm{K}$ & VLPP & & $\begin{array}{l}\text { [4] G. N. Spokes and S. W. } \\
\text { Benson, J. Am. Chem. } \\
\text { Soc. 89, } 6030 \text { (1967). }\end{array}$ \\
\hline
\end{tabular}

\section{Preferred:}

Rate constants are reliable; parameters seem low.

$\log k=11.3-40 / \theta$ (however, see below).

Comments: Arrhenius parameters appear unreasonably low. The minimum possible $A$-factor would correspond to total loss of two internal rotations. With symmetry corrections one obtains $\Delta S^{\ddagger} \simeq(-6.9-8.4+R$ $\ln 12)=-10.3 \mathrm{~g} / \mathrm{mole}$ and $A_{\mathrm{min}}^{\ddagger}=10^{13.5-2.2}=10^{11.3} \mathrm{sec}^{-1}$. More realistic estimates give $\Delta S^{\ddagger} \simeq 0$ and $\log A_{\text {est }}=$ 13.5. See comments on nitroethane.

\section{Experimental}

[1] Rates by $\Delta \mathrm{P}$. Stoichiometry independent of initial pressure and almost independent of temperature.

[2] Rate by polarographic analysis on nitropropane.

[3] Rate by formation of propylene. Analysis by M.S. Identification of products by G.L.C. S/V large effcct on overall 2-nitropropane decomposition but negligible effect on propylene production. Also neither the addition of propylene nor NO had any effect on the rate of propylene formation.

*Products: Propylene, $\mathrm{NO}, \mathrm{H}_{2} \mathrm{O}$ roughly equivalent amounts and approximately 65-70 percent of products; acetone, acetonitrile, and formaldehyde approximately 15-25 percent; hydrocarbons, nitroparaffins, $\mathrm{CO}$, and $\mathrm{CO}_{2}$ approximately 10 percent. 
Reaction: Isopropyl ether

\begin{tabular}{|c|c|c|c|}
\hline$\Delta H_{f(298)}^{\circ}$ & $\begin{array}{c}\text { I } \\
-77.0\end{array}$ & $\begin{array}{l}\text { II } \\
4.9\end{array}$ & $\begin{array}{c}\text { III } \\
-65.1\end{array}$ \\
\hline$S_{(298)}^{\circ}$ & 97.4 & 63.8 & 74.1 \\
\hline$C_{p(298)}^{\circ}$ & 37.8 & 15.3 & 21.5 \\
\hline
\end{tabular}

$\Delta S^{\circ}=40.5 \mathrm{~g} / \mathrm{mole}$

$\Delta H^{\circ}=16.8 \mathrm{kcal} / \mathrm{mole}$

$\Delta C_{p}^{\circ}=-1.0 \mathrm{~g} / \mathrm{mole}$

\begin{tabular}{|c|c|c|c|c|c|c|}
\hline $\log A$ & $\boldsymbol{E}$ & $\begin{array}{c}\log \boldsymbol{k}_{T} \\
(730)\end{array}$ & Conditions & System & Surface & References \\
\hline 14.62 & 63.5 & -3.69 & $\begin{array}{l}696-760^{\circ} \mathrm{K} \\
\sim 10-600 \text { torr }\end{array}$ & static & & $\begin{array}{l}\text { [1] N. J. Daly and V. R. } \\
\text { Stimson, Aust. J. Chem. } \\
\text { 19, } 239 \text { (1966). }\end{array}$ \\
\hline
\end{tabular}

\section{Preferred:}

Suspect.

Comments: Evidence for first-order reaction is ambiguous. Until more definite evidence is available, it is preferable to treat this as a chain reaction.

\section{Experimental}

[1] Initial rates followed manometrically. Analysis by G.L.C. Major products were propylene and 2-propanol (70-90 percent), and propane and acetone (30-10 percent). No permanent gases were formed. In the presence of 2 -methyl propene $(20 \mathrm{~cm})$, propane production was about 80 percent slower and propene production about 30 percent slower. Rates were not significantly surface sensitive. A complex free radical chain process was proposed to explain the minor reaction products and to account for the somewhat larger than overall first-order kinetics observed for the isopropanol and propene formation. The principal source of the latter, however, was thought to be unimolecular. 
Reaction: 1,1-Dimethyl-1-ethanesulfonyl chloride (trimethylmethanesulfonyl chloride)

$$
\begin{aligned}
& \stackrel{a}{\longrightarrow}\left(\mathrm{CH}_{3}\right)_{3} \mathrm{CCl} \text { (II) }+\mathrm{SO}_{2} \text { (III) } \\
& \left(\mathrm{CH}_{3}\right)_{3} \mathrm{CSO}_{2} \mathrm{Cl}(\mathrm{I}) \\
& \stackrel{b}{\longrightarrow}\left(\mathrm{CH}_{3}\right)_{2} \mathrm{C}=\mathrm{CH}_{2}(\mathrm{IV})+\mathrm{SO}_{2}+\mathrm{HCl}(\mathrm{V}) \\
& \Delta H_{f(298)}^{\circ} \\
& \text { I II III IV } \\
& S_{(298)}^{\circ} \\
& \begin{array}{llll}
77.4 & 59.3 & 70.2 & 44.6
\end{array} \\
& C_{p(298)}^{\circ}
\end{aligned}
$$

\begin{tabular}{|c|c|c|c|c|c|}
\hline $\log A$ & $\boldsymbol{E}$ & $\begin{array}{c}\log k_{T} \\
(470)\end{array}$ & Conditions & System & Surface \\
\hline
\end{tabular}

$\Delta S^{\circ}=$

$\Delta H^{\circ}=$

$\Delta C_{p}^{\circ}=$

\section{References}

$$
4.88 \quad 15.3 \pm .8 \quad-2.24 \quad 363-578^{\circ} \mathrm{K} \quad \text { static }
$$

[1] R. T. Van Aller, R. B.

Scott, Jr., and E. L.

Brockelbank, J. Org.

Chem. 31, 2357 (1966).

Preferred:

Unreliable.

Comments: A low $A$-factor is inconsistent with any unimolecular reaction transition state proposed. The maximum entropy loss in activation would correspond to the entropy of two large internal rotations $\left(\Delta S_{\max } \simeq-18 \mathrm{~g} /\right.$ mole $)$. This reaction is probably heterogeneous.

\section{Experimental}

[1] Static system. Rate was followed manometrically, and also by G.L.C. determinations of products. It was found that (isobutene/t-butyl chloride) $=2.0$ at all temperatures. There was no effect on the rates by added toluene or $\mathrm{O}_{2}$. NO accelerated the decomposition. Absence of isobutyl chloride was taken as evidence against the presence of free radicals. Wall effects were observed and the possibility of a heterogeneous wall-catalyzed reaction was not excluded. The solution reaction was also studied and found to be strongly solvent dependent. 
Reaction: 3-Butene-1-ol (allylcarbinol)

$\begin{array}{cccc}\mathrm{CH}_{2}=\mathrm{CHCH}_{2} \mathrm{CH}_{2} \mathrm{OH}(\mathrm{I}) \longrightarrow \mathrm{CH}_{3} \mathrm{CH}=\mathrm{CH}_{2}(\mathrm{II})+\mathrm{H}_{2} \mathrm{CO}(\mathrm{III}) \\ \Delta H_{\text {f(298) }}^{\circ} & \text { I } & \text { II } & \text { III } \\ S_{(298)}^{\circ} & -36.3 & 4.9 & -27.7 \\ C_{p(298)}^{\circ} & 84.8 & 63.8 & 52.3 \\ & 23.7 & 15.3 & 8.5\end{array}$

$\Delta S^{\circ}=31.3 \mathrm{~g} / \mathrm{mole}$

$\Delta \mathrm{H}^{\circ}=13.5 \mathrm{kcal} / \mathrm{mole}$

$\Delta C_{p}^{\circ}=0.1 \mathrm{~g} / \mathrm{mole}$

\begin{tabular}{|c|c|c|c|c|c|c|}
\hline $\log A$ & $\boldsymbol{E}$ & $\begin{array}{c}\log \boldsymbol{k}_{r} \\
(665)\end{array}$ & Conditions & System & Surface & References \\
\hline 11.65 & 41.0 & -1.82 & $643-685^{\circ} \mathrm{K}$ & static & None & $\begin{array}{l}\text { [1] G. G. Smith and B. L. } \\
\text { Yates, J. Chem. Soc., } \\
7242(1965) \text {. }\end{array}$ \\
\hline
\end{tabular}

Preferred:

$\log k=11.65-41.0 / \theta$.

Comments: Transition state estimate gives $\log A_{\text {est }}=11.7$ (see section II-4.0). Note that the back reaction has an $A$-factor of $10^{7} \mathrm{l} / \mathrm{mole}$-sec and an activation energy of $26.4 \mathrm{kcal} / \mathrm{mole}$. It should be measurable at about $550^{\circ} \mathrm{K}$; so should $\mathrm{H}-\mathrm{D}$ exchange between products.

\section{Experimental}

[1] Rates by $\Delta$ P. Analysis by G.L.C. 
Reaction: 4-Pentene-2-ol (allylmethylcarbinol)

$$
\mathrm{CH}_{2}=\mathrm{CHCH}_{2}\left(\mathrm{CH}_{3}\right) \mathrm{CHOH}(\mathrm{I}) \longrightarrow \mathrm{CH}_{3} \mathrm{CH}=\mathrm{CH}_{2}(\mathrm{II})+\mathrm{CH}_{3} \mathrm{CHO}
$$

\begin{tabular}{crrr}
$\Delta \mathrm{H}_{f(298)}^{\circ}$ & \multicolumn{1}{c}{ I } & II & III \\
$S_{(298)}^{\circ}$ & -44.8 & 4.9 & -39.8 \\
$C_{p(298)}^{\circ}$ & 92.8 & 63.8 & 63.2 \\
& 29.7 & 15.3 & 13.1
\end{tabular}

$\Delta S^{\circ}=34.2 \mathrm{~g} / \mathrm{mole}$

$\Delta H^{\circ}=9.9 \mathrm{kcal} / \mathrm{mole}$

$\Delta C_{p}^{\circ}=-1.3 \mathrm{~g} / \mathrm{mole}$

\begin{tabular}{ccccccc}
$\log A$ & $\boldsymbol{E}$ & $\begin{array}{c}\log k_{T} \\
(645)\end{array}$ & Conditions & System & Surface & \multicolumn{1}{c}{ References } \\
11.93 & 40.9 & -1.93 & $625-663^{\circ} \mathrm{K}$ & static & none & [1] G. G. Smith and B. L. \\
Yates, J. Chem. Soc. \\
7242 (1965).
\end{tabular}

Preferred:

$\log k=11.93-40.9 / \theta$.

Comments: The activation energies for the elimination reactions of the alcohols do not change appreciably with $\beta$-(to the double bond)-alkyl substitution. This is in marked contrast to the sizable substituent effect found in the ester eliminations. However, reaction enthalpies decrease with methyl substitution (by about $4 \mathrm{kcal} / \mathrm{mole}$ ) for both alcohols and ester eliminations. This means that the back reaction activation energies for the ester reactions are essentially constant with $\mathrm{CH}_{3}$ substitution, but increase by about 4 kcal with successive $\mathrm{CH}_{3}$ substitution in the $\beta$-hydroxy alcohol systems. Transition state estimates give $\log A_{\text {est }}=11.7$ (see section II-4.0).

\section{Experimental}

[1] Rates by $\Delta$ P. Analysis by G.L.C. 
Reaction: 2-Methyl-4-pentene-2-ol (allyldimethylcarbinol)

$$
\begin{array}{crcc}
\mathrm{CH}_{2}=\mathrm{CHCH}_{2} \mathrm{C}\left(\mathrm{CH}_{3}\right)_{2} \mathrm{OH}(\mathrm{I}) \longrightarrow & \multicolumn{3}{c}{\mathrm{CH}_{3} \mathrm{CH}=\mathrm{CH}_{2}(\mathrm{II})+\mathrm{CH}_{3} \mathrm{COCH}_{3}(\mathrm{III})} \\
\Delta H_{(298)}^{\circ} & -54.1 & \text { II } & \text { III } \\
S_{(298)}^{\circ} & 97.4 & 63.9 & -51.7 \\
C_{p(298)}^{\circ} & 35.4 & 15.3 & 70.5
\end{array}
$$

$\Delta S^{\circ}=36.9 \mathrm{~g} / \mathrm{mole}$

$\Delta H^{\circ}=7.3 \mathrm{kcal} / \mathrm{mole}$

$\Delta C_{p}^{\circ}=-2.2 \mathrm{~g} / \mathrm{mole}$

$\begin{array}{lcccccc}\log \boldsymbol{A} & \boldsymbol{E} & \begin{array}{c}\log \boldsymbol{k}_{T} \\ (625)\end{array} & \text { Conditions } & \text { System } & \text { Surface } & \text { References } \\ 12.14 & 40.7 & -2.09 & 607-643^{\circ} \mathrm{K} & \text { static } & \text { none } & \text { [1] G. G. Smith and Brian L. } \\ & & & & & & \begin{array}{l}\text { Yates, J. Chem. Soc. } \\ 7242 \text { (1965). }\end{array}\end{array}$

\section{Preferred:}

$\log k=12.14-40.7 / \theta$ (see comments on 4-pentene-2-ol).

Transition state estimate gives $\log A_{\text {est }}=11.9$ (see section II-4.0).

\section{Experimental}

[1] Rates by $\Delta P$. Analysis by G.L.C. 
Reaction: 3-Phenyl-3-butene-1-ol

$\begin{array}{cccc}\mathrm{CH}_{2}=\mathrm{C}(\emptyset) \mathrm{CH}_{2} \mathrm{CH}_{2} \mathrm{OH}(\mathrm{I}) \rightarrow \emptyset\left(\mathrm{CH}_{3}\right) \mathrm{C}=\mathrm{CH}_{2}(\mathrm{II})+\mathrm{CH}_{2} \mathrm{O}(\mathrm{III}) \\ \Delta H_{\text {f(298) }}^{\circ} & -15.7 & \text { II } & \text { III } \\ S_{(298)}^{\circ} & 109.4 & 88.2 & -27.7 \\ \mathrm{C}_{p(298)}^{\circ} & 44.1 & 35.9 & 82.3\end{array}$

$\Delta S^{\circ}=31.1 \mathrm{~g} / \mathrm{mole}$

$\Delta H^{\circ}=13.7 \mathrm{kcal} / \mathrm{mole}$

$\Delta C_{p}^{\circ}=0.3 \mathrm{~g} / \mathrm{mole}$

$\begin{array}{ccccccc}\log \boldsymbol{A} & \boldsymbol{E} & \begin{array}{c}\log \boldsymbol{k}_{T} \\ (620)\end{array} & \text { Conditions } & \text { System } & \text { Surface } & \text { References } \\ 11.80 & 38.9 & -1.91 & 605-631^{\circ} \mathrm{K} & \text { static } & \text { none } & \text { [1] G. G. Smith and B. L. } \\ \text { Yates, J. Chem. Soc., } \\ \text { 7a (1965). }\end{array}$

Preferred:

$\log k=11.80-38.9 / \theta$.

Transition state estimate gives $\log A_{\text {est }}=11.7$ (see section II-4.0).

Experimental

[1] Rates by $\Delta$ P. Analysis by G.L.C. 
Reaction: 4-Phenyl-3-butene-1-ol

\begin{tabular}{|c|c|c|c|}
\hline$\Delta H_{f(298)}^{\circ}$ & $\begin{array}{c}I \\
-13.5\end{array}$ & $\begin{array}{l}\text { II } \\
32.0\end{array}$ & $\begin{array}{c}\text { III } \\
-27.7\end{array}$ \\
\hline$S_{(298)}^{\circ}$ & 112.3 & 93.6 & 52.3 \\
\hline$C_{p(298)}^{\circ}$ & 43.1 & 33.7 & 8.5 \\
\hline
\end{tabular}

\begin{abstract}
$\Delta S^{\circ}=33.6 \mathrm{~g} / \mathrm{mole}$
$\Delta H^{\circ}=17.8 \mathrm{kcal} / \mathrm{mole}$

$\Delta C^{\circ}=-0.9 \mathrm{~g} / \mathrm{mole}$
\end{abstract}

\begin{tabular}{|c|c|c|c|c|c|c|}
\hline $\log A$ & $\boldsymbol{E}$ & $\begin{array}{c}\log \boldsymbol{k}_{T} \\
(680)\end{array}$ & Conditions & System & Surface & References \\
\hline 11.58 & 42.8 & -2.17 & $661-700^{\circ} \mathrm{K}$ & static & none & $\begin{array}{l}\text { [1] G. G. Smith and B. L. } \\
\text { Yates, J. Chem. Soc. } \\
7242 \text { (1965). }\end{array}$ \\
\hline
\end{tabular}

Preferred:

$\log k=11.58-42.8 / \theta$.

Transition state estimate gives $\log A_{\text {est }}=11.6$ (see section II-4.0).

\title{
Experimental
}

[1] Rates by $\Delta P$. Analysis by G.L.C. 
Reaction: 1-Phenyl-4-ethyl-4-hydroxyhex-l-ene

$\begin{array}{cccc}\emptyset \mathrm{CH}=\mathrm{CHCH}_{2} \mathrm{C}\left(\mathrm{C}_{2} \mathrm{H}_{5}\right)_{2} \mathrm{OH} & (\mathrm{I}) \longrightarrow \emptyset \mathrm{CH}_{2} \mathrm{CH}=\mathrm{CH}_{2}(\mathrm{II})+\mathrm{C}_{2} \mathrm{H}_{5} \mathrm{COC}_{2} \mathrm{H}_{5}(\mathrm{III}) \\ \Delta H_{(298)}^{\circ} & \text { I } & \text { II } & \text { III } \\ S_{(298)}^{\circ} & -36.0 & 32.0 & -62.2 \\ C_{p(298)}^{\circ} & 143.3 & 93.6 & 88.4 \\ & 65.8 & 33.7 & 31.3\end{array}$

$\Delta S^{\circ}=38.7 \mathrm{~g} / \mathrm{mole}$

$\Delta H^{\circ}=5.8 \mathrm{kcal} / \mathrm{mole}$

$\Delta C_{p}^{\circ}=-0.8 \mathrm{~g} /$ mole

\begin{tabular}{|c|c|c|c|c|c|c|}
\hline $\log A$ & $\boldsymbol{E}$ & $\begin{array}{c}\log \boldsymbol{k}_{T} \\
(600)\end{array}$ & Conditions & System & Surface & References \\
\hline 12.04 & 41.75 & -3.16 & $579-607^{\circ} \mathrm{K}$ & static & & $\begin{array}{l}\text { [1] G. G. Smith and R. } \\
\text { Taylor, Chem. Ind. } \\
949(1961) .\end{array}$ \\
\hline 12.00 & 41.80 & -3.22 & $650^{\circ} \mathrm{K}$ & static & none & $\begin{array}{l}\text { [2] G. G. Smith and B. L. } \\
\text { Yates, J. Chem. Soc. } \\
7242 \text { (1965). }\end{array}$ \\
\hline
\end{tabular}

Preferred:

$\log k=12.0-41.8 / \theta$.

Transition state calculation gives $\log A_{\text {est }}=11.6$ (see section II-4.0).

\section{Experimental}

[1] Rates by $\Delta \mathrm{P}$ unaffected by $\mathrm{N}_{2}$. First-order kinetics were obtained to 99 percent reaction.

[2] Rates by $\Delta$ P. Analysis by G.L.C. Single temperature point used with prior data of [1] to obtain revised Arrhenius parameters. 
Reaction: 3-Butenoic acid

$\begin{array}{cccc}\mathrm{CH}_{2}=\mathrm{CHCH}_{2} \mathrm{COOH}(\mathrm{I}) \longrightarrow \mathrm{CH}_{3} \mathrm{CH}=\mathrm{CH}_{2}(\mathrm{II})+\mathrm{CO}_{2}(\mathrm{III}) \\ \Delta H_{f(298)}^{\circ} & \text { I } & \text { II } & \text { III } \\ S_{(298)}^{\circ} & -84.1 & 4.9 & -94.1 \\ C_{p(298)}^{\circ} & 84.4 & 63.8 & 51.1 \\ & 25.8 & 15.3 & 8.9\end{array}$

$\Delta S^{\circ}=30.5 \mathrm{~g} / \mathrm{mole}$

$\Delta H^{\circ}=-5.1 \mathrm{kcal} / \mathrm{mole}$

$\Delta C_{p}^{\circ}=-1.6 \mathrm{~g} / \mathrm{mole}$

$\begin{array}{ccccccc}\log \boldsymbol{A} & \boldsymbol{E} & \begin{array}{c}\log \boldsymbol{k}_{T} \\ (620)\end{array} & \text { Conditions } & \text { System } & \text { Surface } & \text { References } \\ 11.34 & 40.6 & -2.97 & 587-651^{\circ} \mathrm{K} & \text { static } & <5 \% & \text { [1] G. G. Smith and S. E. } \\ & & & & & & \begin{array}{l}\text { Blau, J. Phys. Chem. } \\ \text { 68, } 1231 \text { (1964). }\end{array}\end{array}$

\section{Preferred:}

$\log k=11.34-40.6 / \theta$.

Transition state estimate gives $\log A_{\text {est }}=11.1$ (see section II-4.0).

\section{Experimental}

[1] Rate by $\Delta$ P. Analysis by G.L.C. No effect on rate with added cyclohexene. 
Reaction: 2,2-Dimethyl-3-butenoic acid

\begin{tabular}{|c|c|c|c|}
\hline$\Delta H_{f(298)}^{\circ}$ & $\begin{array}{c}\text { I } \\
-94.7\end{array}$ & $\begin{array}{c}11 \\
-10.3\end{array}$ & $\begin{array}{c}\text { III } \\
-94.1\end{array}$ \\
\hline$S_{(298)}^{\circ}$ & 96.2 & 80.4 & 51.1 \\
\hline$C_{p(298)}^{\circ}$ & 35.8 & 25.5 & 8.9 \\
\hline
\end{tabular}

$\Delta S^{\circ}=35.3 \mathrm{~g} / \mathrm{mole}$

$\Delta H^{\circ}=-9.7 \mathrm{kcal} / \mathrm{mole}$

$\Delta C_{p}^{\circ}=-1.4 \mathrm{~g} / \mathrm{mole}$

\begin{tabular}{|c|c|c|c|c|c|c|}
\hline $\log A$ & $\boldsymbol{E}$ & $\begin{array}{c}\log \boldsymbol{k}_{T} \\
(530)\end{array}$ & Conditions & System & Surface & References \\
\hline 11.13 & 36.6 & -3.95 & $\begin{array}{l}511-548{ }^{\circ} \mathrm{K} \\
150-250 \text { torr }\end{array}$ & static & & $\begin{array}{l}\text { [1] D. B. Bigley and R. W. } \\
\text { May, J. Chem. Soc. } \\
\text { B, } 557 \text { (1967). }\end{array}$ \\
\hline
\end{tabular}

\section{Preferred:}

$\log k=11.13-36.6 / \theta$.

Comments: Transition states estimates for $A$ of this and related reactions are all similar to that for 3-butenoic acid (i.e., $\sim A \simeq 10^{11.1} \mathrm{sec}^{-1}$ ); therefore, this reaction seems quite reasonable. Rate constants are reliable.

\section{Experimental}

[1] Rates were followed by rates of $\mathrm{CO}_{2}$ produced. Analysis of olefin was by G.L.C., using tetralin as an internal standard. Gogd mass balances. Relative rates to but-3-enoic acid at $500^{\circ} \mathrm{K}$ were calculated. 
Reaction: 2,2-Dimethyl-trans-3-pentenoic acid

\begin{tabular}{|c|c|c|c|}
\hline$\Delta H_{f(298)}^{\circ}$ & $\begin{array}{c}I \\
-102.5\end{array}$ & $\begin{array}{c}\text { II } \\
-15.1\end{array}$ & $\begin{array}{c}\text { III } \\
-94.1\end{array}$ \\
\hline$S_{(298)}^{\circ}$ & 104.8 & 90.2 & 51.1 \\
\hline$C_{p(298)}^{\circ}$ & 41.1 & 30.6 & 8.9 \\
\hline
\end{tabular}

$$
\begin{aligned}
& \Delta S^{\circ}=36.5 \mathrm{~g} / \mathrm{mole} \\
& \Delta \mathrm{H}^{\circ}=-6.7 \mathrm{kcal} / \mathrm{mole} \\
& \Delta C_{p}^{\circ}=-1.6 \mathrm{~g} / \mathrm{mole}
\end{aligned}
$$

\begin{tabular}{|c|c|c|c|c|c|c|}
\hline $\log A$ & $\boldsymbol{E}$ & $\begin{array}{c}\log k_{T} \\
(545)\end{array}$ & Conditions & System & Surface & References \\
\hline 11.74 & 40.3 & -4.43 & $\begin{array}{l}526-564^{\circ} \mathrm{K} \\
150-250 \text { torr }\end{array}$ & static & & $\begin{array}{l}\text { [1] D. B. Bigley and R. W. } \\
\text { May, J. Chem. Soc. B, } \\
557 \text { (1967). }\end{array}$ \\
\hline
\end{tabular}

\section{Preferred:}

Reliable.

Comments: Transition state estimates for $A$ of this and related reactions are all similar to that for 3-butenoic acid (i.e., $\sim A \approx 10^{11.1} \mathrm{sec}^{-1}$ ); therefore, this reaction seems quite reasonable. Rate constants are probably reliable.

\section{Experimental}

[1] Rates were followed by rates of $\mathrm{CO}_{2}$ produced. Analysis of olefin was by G.L.C., using tetralin as an internal standard. Good mass balances. Relative rates to but-3-enoic acid at $500{ }^{\circ} \mathrm{K}$ were calculated. 
Reaction: 2,2,3-Trimethyl-3-butenoic acid

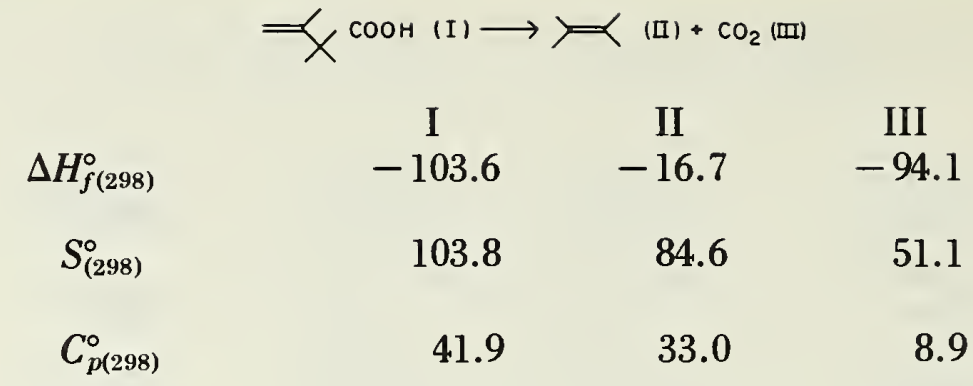

$\Delta S^{\circ}=31.9 \mathrm{~g} / \mathrm{mole}$

$\Delta H^{\circ}=-7.2 \mathrm{kcal} / \mathrm{mole}$

$\Delta C_{p}^{\circ}=0 \mathrm{~g} / \mathrm{mole}$

\begin{tabular}{|c|c|c|c|c|c|c|}
\hline $\log A$ & $\boldsymbol{E}$ & $\begin{array}{c}\log k_{T} \\
(465)\end{array}$ & Conditions & System & Surface & References \\
\hline 10.85 & 32.9 & -4.62 & $\begin{array}{l}447-488{ }^{\circ} \mathrm{K} \\
150-250 \text { torr }\end{array}$ & static & & $\begin{array}{l}\text { [1] D. B. Bigley and R. W. } \\
\text { May, J. Chem. Soc. B, } \\
557 \text { (1967). }\end{array}$ \\
\hline
\end{tabular}

\section{Preferred:}

Reliable.

Comments: Transition state estimates for $A$ of this and related reactions are all similar to that for 3-butenoic acid (i.e., $\sim A \simeq 10^{11.1} \mathrm{sec}^{-1}$ ); therefore, this reaction seems quite reasonable. Rate constants are probably reliable.

\section{Experimental}

[1] Rates were followed by rates of $\mathrm{CO}_{2}$ produced. Analysis of olefin was by G.L.C., using tetralin as an internal standard. Good mass balances. Rates relative to but-3-enoic acid at $500{ }^{\circ} \mathrm{K}$ were calculated. 
Reaction: 2,2-Dimethyl-3-ethyl-3-pentenoic acid

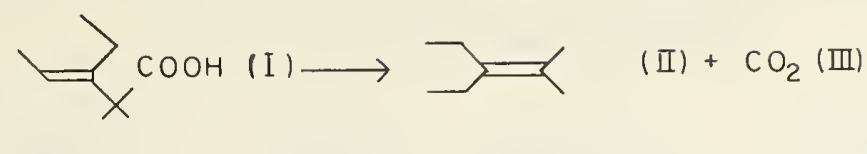

\begin{tabular}{crrr} 
& \multicolumn{1}{c}{ I } & \multicolumn{1}{c}{ II } & \multicolumn{1}{c}{ III } \\
$\Delta H_{f(298)}^{\circ}$ & -113.3 & -26.3 & -94.1 \\
$S_{(298)}^{\circ}$ & 123.0 & 105.6 & 51.1 \\
$C_{p(298)}^{\circ}$ & 52.3 & 43.2 & 8.9
\end{tabular}

$\Delta S^{\circ}=33.7 \mathrm{~g} / \mathrm{mole}$

$\Delta H^{\circ}=-7.1 \mathrm{kcal} / \mathrm{mole}$

$\Delta C_{p}^{\circ}=0.2 \mathrm{~g} / \mathrm{mole}$

\begin{tabular}{|c|c|c|c|c|c|c|}
\hline $\operatorname{Iog} A$ & $\boldsymbol{E}$ & $\begin{array}{c}\log \boldsymbol{k}_{T} \\
(485)\end{array}$ & Conditions & System & Surface & References \\
\hline 11.68 & 36.0 & -4.54 & $\begin{array}{l}468-502{ }^{\circ} \mathrm{K} \\
150-250 \text { torr }\end{array}$ & static & & $\begin{array}{l}\text { [1] D. B. Bigley and R. W. } \\
\text { May, J. Chem. Soc., } \\
\text { B, } 557 \text { (1967) }\end{array}$ \\
\hline
\end{tabular}

\section{Preferred:}

Reliable.

Comments: Transition state estimates for $A$ of this and related reactions are all similar to that for 3-butenoic acid (i.e., $\sim A \simeq 10^{11.1} \mathrm{sec}^{-1}$ ), therefore, this reaction seems quite reasonable. Rate constants are probably reliable.

\section{Experimental}

[1] Rates were followed by rates of $\mathrm{CO}_{2}$ produced. Analysis of olefin was by G.L.C., using tetralin as an internal standard. Good mass balances. Rates relative to but-3-enoic acid at $500^{\circ} \mathrm{K}$ were calculated. 
Reaction: 2-Methyl-2-(1-cyclopentyl) propionic acid

\begin{tabular}{|c|c|c|c|}
\hline$\Delta \mathrm{H}_{f(298)}^{\circ}$ & $\begin{array}{c}\mathrm{I} \\
-100.0\end{array}$ & $\begin{array}{c}\text { II } \\
-10.8\end{array}$ & $\begin{array}{c}\text { III } \\
-94.1\end{array}$ \\
\hline$S_{(298)}^{\circ}$ & 110.7 & 93.3 & 51.1 \\
\hline$C_{p(298)}^{\circ}$ & 44.7 & 34.3 & \\
\hline
\end{tabular}

$\Delta S^{\circ}=33.7 \mathrm{~g} / \mathrm{mole}$

$\Delta H^{\circ}=-4.9 \mathrm{kcal} / \mathrm{mole}$

$\Delta C_{p}^{\circ}=-1.5 \mathrm{~g} / \mathrm{mole}$

\begin{tabular}{|c|c|c|c|c|c|c|}
\hline $\log A$ & $\boldsymbol{E}$ & $\begin{array}{c}\log \boldsymbol{k}_{T} \\
(485)\end{array}$ & Conditions & System & Surface & References \\
\hline 8.95 & 29.9 & -4.52 & $\begin{array}{l}470-502^{\circ} \mathrm{K} \\
150-250 \text { torr }\end{array}$ & static & & $\begin{array}{l}\text { [1] D. B. Bigley and R. W. } \\
\text { May, J. Chem. Soc., } \\
\text { B } 557 \text { (1967) }\end{array}$ \\
\hline
\end{tabular}

\section{Preferred:}

Suspect.

Comments: Transition state estimates for $A$ of this and related reactions are all similar to that for 3-butenoic acid (i.e., $\sim A \simeq 10^{11.1} \mathrm{sec}^{-1}$ ), therefore the $A$-factors seem too low by at least an order of magnitude. Rate constants are probably reliable.

\section{Experimental}

[1] Rates were followed by rates of $\mathrm{CO}_{2}$ produced. 
Reaction: 2-Methyl-2-(1-cyclohexenyl) propionic acid

$\Delta S^{\circ}=33.1 \mathrm{~g} / \mathrm{mole}$

\begin{tabular}{|c|c|c|c|}
\hline$\Delta H_{f(298)}^{\circ}$ & $\begin{array}{c}\text { I } \\
-110.7\end{array}$ & $\begin{array}{c}\text { II } \\
-21.8\end{array}$ & $\begin{array}{c}\text { III } \\
-94.1\end{array}$ \\
\hline$S_{(298)}^{\circ}$ & 114.3 & 96.3 & 51.1 \\
\hline$C_{p(298)}^{\circ}$ & 51.7 & 43.0 & 8.9 \\
\hline
\end{tabular}

$\Delta H^{\circ}=-5.2 \mathrm{kcal} / \mathrm{mole}$

$\Delta C_{p}^{\circ}=+0.2 \mathrm{~g} / \mathrm{mole}$

\begin{tabular}{|c|c|c|c|c|c|c|}
\hline $\log A$ & $\boldsymbol{E}$ & $\log k_{T}$ & Conditions & System & Surface & References \\
\hline 10.04 & 33.3 & -4.51 & $\begin{array}{l}487-517^{\circ} \mathrm{K} \\
150-250 \text { torr }\end{array}$ & static & & $\begin{array}{l}\text { [1] D. B. Bigley and R. W. } \\
\text { May, J. Chem. Soc. B, } \\
557 \text { (1967). }\end{array}$ \\
\hline
\end{tabular}

Preferred:

Suspect.

Comments: Transition state estimates for $A$ of this and related reactions are all similar to that for 3-butenoic acid (i.e., $\sim A \simeq 10^{11.1} \mathrm{sec}^{-1}$ ). The $A$-factors seem too low by at least an order of magnitude. Rate constants are probably reliable.

\section{Experimental}

[1] Rates were followed by rates of $\mathrm{CO}_{2}$ produced. 
Reaction: 2-Methyl-2-(1-cycloheptenyl) propionic acid

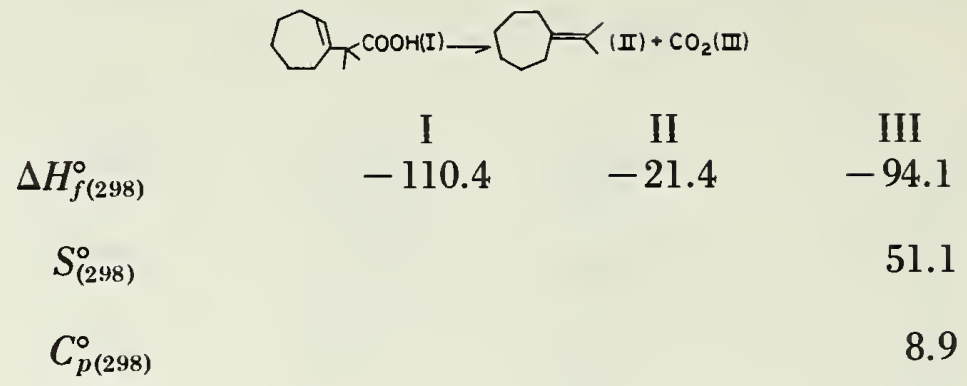

$\Delta S^{\circ}=(33) \mathrm{g} / \mathrm{mole}$

$\Delta H^{\circ}=-4.1 \mathrm{kcal} / \mathrm{mole}$

$\Delta C_{p}^{\circ}=()$

\begin{tabular}{|c|c|c|c|c|c|c|}
\hline $\log A$ & $\boldsymbol{E}$ & $\begin{array}{c}\log \boldsymbol{k}_{T} \\
(485)\end{array}$ & Conditions & System & Surface & References \\
\hline 8.50 & 28.6 & -4.39 & $\begin{array}{l}468-502^{\circ} \mathrm{K} \\
150-250 \text { torr }\end{array}$ & static & & $\begin{array}{l}\text { [1] D. B. Bigley and R. W. } \\
\text { May, J. Chem. Soc. B, } \\
557 \text { (1967). }\end{array}$ \\
\hline
\end{tabular}

\section{Preferred:}

Suspect.

Comments: Transition state estimates for $A$ of this and related reactions are all similar to that for 3-butenoic acid (i.e., $\sim A \simeq 10^{11.1} \mathrm{sec}^{-1}$ ). The $A$-factors seem too low by at least an order of magnitude. Rate constants are probably reliable.

\section{Experimental}

[1] Rates were followed by rates of $\mathrm{CO}_{2}$ produced. 
Reaction: Ethyl chloroethanoate (ethyl chloroformate)

$$
\begin{array}{crcr}
\multicolumn{4}{c}{\mathrm{ClCOOC}_{2} \mathrm{H}_{5}(\mathrm{I}) \longrightarrow \mathrm{C}_{2} \mathrm{H}_{5} \mathrm{Cl}(\mathrm{II})+\mathrm{CO}_{2}(\mathrm{III})} \\
\Delta H_{f(298)}^{\circ} & \mathrm{I} & \mathrm{II} & \text { III } \\
S_{(298)}^{\circ} & -108.3 & -26.3 & -94.1 \\
C_{p(298)}^{\circ} & 90.4 & 66.0 & 51.1 \\
& 24.7 & 15.1 & 8.9
\end{array}
$$

$\Delta S^{\circ}=26.7 \mathrm{~g} / \mathrm{mole}$

$\Delta H^{\circ}=-12.1 \mathrm{kcal} / \mathrm{mole}$

\begin{tabular}{|c|c|c|c|c|c|c|}
\hline $\log A$ & $\boldsymbol{E}$ & $\begin{array}{c}\log \boldsymbol{k}_{T} \\
(500)\end{array}$ & Conditions & System & Surface & References \\
\hline 10.74 & 29.41 & -2.11 & $\begin{array}{l}383-473^{\circ} \mathrm{K} \\
10-700 \text { torr }\end{array}$ & static & none & $\begin{array}{l}\text { [1] A. R. Choppin and G. F. } \\
\text { Kirby, J. Am. Chem. } \\
\text { Soc. 61, } 3176 \text { (1939). }\end{array}$ \\
\hline 8.91 & a 32.5 & -5.29 & $513-552{ }^{\circ} \mathrm{K}$ & $\begin{array}{c}\text { stirred } \\
\text { flow }\end{array}$ & $\left({ }^{b}\right)$ & $\begin{array}{l}\text { [2] E. S. Lewis and W. C. } \\
\text { Herndon, J. Am. Chem. } \\
\text { Soc. 83, } 1955 \text { (1961). }\end{array}$ \\
\hline
\end{tabular}

$\Delta C_{p}^{\circ}=-0.7 \% /$ mole

Preferred:

Unreliable.

Comments: The Arrhenius parameters for this reaction are extremely low for a unimolecular elimination reaction. In addition, the mechanism proposed involves either pentavalent carbon or an appreciable development of a very polar (essentially ionic) transition state. Both seem quite unreasonable for a gas phase reaction. The large rate discrepancies (factor of $10^{3}$ ) between the two experimental studies also suggest serious complications (probably surface catalysis) for this reaction. A free radical mechanism propagated by ethyl radicals with chlorine abstraction would lead to the observed products. Heterogeneous reactions are also supported by the abnormally low activation energies and $A$-factor.

Fast chains which produce the observed products:

(1) $R+1$
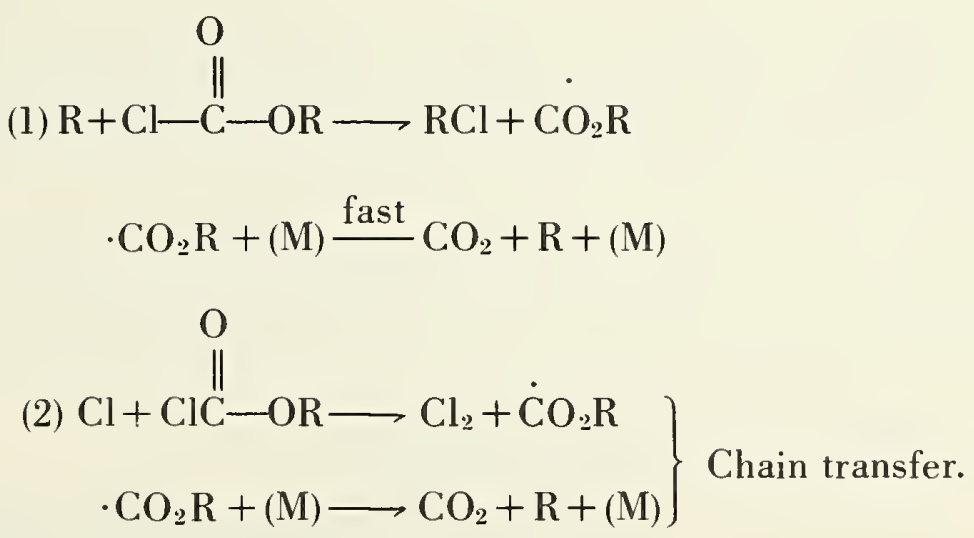
Ethyl chloroethanoate (Continued)

J. C. J. Thynne, Trans. Faraday Soc. 58, 676 (1962) has shown that decomposition of the $\cdot \mathrm{CO}_{2} \mathrm{R}$ radical gives exclusively $\mathrm{CO}_{2}$ and $\mathrm{R} \cdot$, not $\mathrm{CO}$ and $\cdot \mathrm{OR}$. Thus the proposed chain accounts nicely for the products observed. The $\mathrm{Cl}$ abstraction by $\mathrm{R}$ - would also be favored relative to the $\mathrm{H}$-abstraction by several powers of 10 in the rate.

\section{Experimental}

[1] Rates by $\Delta \mathrm{P}$. Chemical analysis for $\mathrm{CO}_{2}$ and ethyl chloride. No effect by the addition of a variety of foreign gases. (Choppin and Kirby, J. Am. Chem. Soc. 62, 1592 (1940).)

$[2]^{\text {a }}$ The Arrhenius factors have been recalculated from the rate constant data since the quoted transition state parameters are not consistent with the rate constants. Rate by G.L.C. analysis of the products.

${ }^{b}$ The first runs in a series were often characterized by much faster rates. This was attributed to surface catalysis. 
Reaction: Isopropyl chloromethanoate (isopropyl chloroformate)

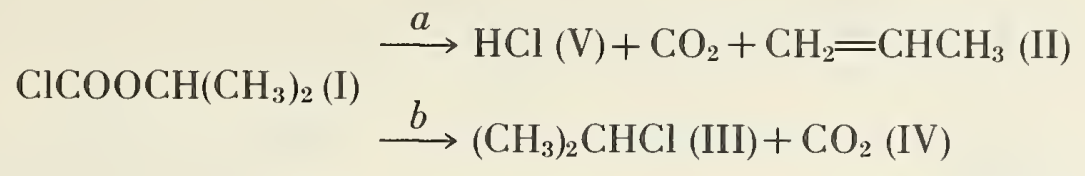

\begin{tabular}{crrrrr} 
& \multicolumn{1}{c}{ I } & II & III & IV & \multicolumn{1}{c}{ V } \\
$\Delta H_{f(298)}^{\circ}$ & -116.1 & 4.9 & -33.0 & -94.1 & -22.1 \\
$S_{(298)}^{\circ}$ & 96.8 & 63.8 & 74.1 & 51.1 & 44.6 \\
$C_{p(298)}^{\circ}$ & 30.7 & 15.3 & 21.4 & 8.9 & 7.0
\end{tabular}

$\begin{array}{rl}a & b \\ \Delta S^{\circ}=62.7 & 28.4 \mathrm{~g} / \mathrm{mole} \\ \Delta H^{\circ}=4.8 & -11.0 \mathrm{kcal} / \mathrm{mole} \\ \Delta C_{p}^{\circ}=0.5 & -0.4 \mathrm{~g} / \mathrm{mole}\end{array}$

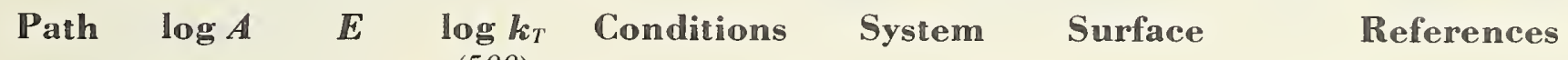

$a$

$9.11 \quad 25.8 \quad-2.21 \quad 453-493^{\circ} \mathrm{K}$

static and some*

flow

[1] A. R. Choppin and

E. L. Compere, J. Am. Chem. Soc. 70, 3797 (1948).

[2] E. S. Lewis and W.

C. Herndon, J. Am. Chem. Soc. 83, 1955 (1961).

[1]

[2]

Preferred:

$\log k_{a}=12.9-39.7 / \theta$ (see below).

path $b$, unreliable.

Comments: Since such serious discrepancies in rates exist for these reactions, there must be serious complications with the reactions themselves. Surface effects observed by Choppin and Compere and fast initial rates observed by Lewis and Herndon strongly suggest heterogeneous catalysis. The unreasonably low $A$-factors in all cases except one also suggest wall catalysis. See ethyl chloroformate for a more complete discussion.

Path $a$ appears reasonable for the elimination reaction. The rate and rate parameters compare favorably with those of the substituted phenyl ethyl methyl carbonates, as one might expect. The transition state estimate (see section II-4.0) is: $\log A_{(a)}=10^{12.34} ; \log k_{a}=12.34-38.3 / \theta$. Path $b$ is probably not a unimolecular reaction.

\section{Experimental}

[1] Rate by $\Delta \mathrm{P}$. Qualitative chemical analysis of the products.

[2] Rate by G.L.C. analysis of products.

*Packing the static reaction cell with glass wool doubled the observed rate of decomposition. Increased surface in the How experiment decreased the reaction rate.

**Parameters were calculated from the rate constants reported. 
Reaction: Isobutyl chloromethanoate (isobutyl chloroformate)

$$
\begin{array}{crccc}
\mathrm{ClCOOCH}_{2} \mathrm{CH}\left(\mathrm{CH}_{3}\right)_{2}(\mathrm{I}) \longrightarrow \mathrm{CH}_{2}=\mathrm{C}\left(\mathrm{CH}_{3}\right)_{2}(\mathrm{II})+\mathrm{HCl}(\mathrm{III})+\mathrm{CO}_{2}(\mathrm{IV}) \\
\Delta H_{f(298)}^{\circ} & \text { I } & \text { II } & \text { III } & \text { IV } \\
S^{\circ}{ }_{(298)} & -119.5 & -4.0 & -22.1 & -94.1 \\
\Delta C_{{ }^{(298)}}^{\circ} & 106.5 & 70.2 & 44.6 & 51.1 \\
& 35.7 & 21.3 & 7.0 & 8.9
\end{array}
$$

$\Delta S^{\circ}=59.4 \mathrm{~g} / \mathrm{mole}$

$\Delta H^{\circ}=-0.7 \mathrm{kcal} / \mathrm{mole}$

\begin{tabular}{|c|c|c|c|c|c|c|}
\hline $\log A$ & $\mathbb{E}$ & $\begin{array}{c}\log \boldsymbol{k}_{T} \\
(560)\end{array}$ & Conditions & System & Surface & References \\
\hline 13.0 & 40.0 & -2.61 & $540-573^{\circ} \mathrm{K}$ & static & & $\begin{array}{l}\text { [1] Calculated by A. R. } \\
\text { Choppin and E. L. } \\
\text { Compere, J. Am. } \\
\text { Chem. Soc. 70, } 3797 \\
\text { (1948) from the data } \\
\text { of E. T. Lessig, J. } \\
\text { Phys. Chem. 36, } \\
\text { 2325 (1932). }\end{array}$ \\
\hline
\end{tabular}

$\Delta C_{p}^{\circ}=1.5 \mathrm{~g} / \mathrm{mole}$

Preferred:

$\log k=11.9-37.2 / \theta$.

Transition state estimate is preferred (see section II-4.0).

\section{Experimental}

[1] The rate constants were calculated from reported 3/4 lifetimes measured manometrically. It is inferred that the rate constants are representative of the above elimination reaction. Initial products are probably isobutene + $\mathrm{ClCOOH}$. 
Reaction: sec-Butyl chloromethanoate (sec-butyl chloroformate)

$$
\begin{aligned}
& \stackrel{a}{\longrightarrow} \mathrm{CH}_{2}=\mathrm{CHCH}_{2} \mathrm{CH}_{3}(\mathrm{II})+\mathrm{HCl}+\mathrm{CO}_{2} \\
& \operatorname{ClCOOCH}\left(\mathrm{CH}_{3}\right) \mathrm{CH}_{2} \mathrm{CH}_{3}(\mathrm{I}) \stackrel{b}{\longrightarrow} \text { cis }-\mathrm{CH}_{3} \mathrm{CH}=\mathrm{CHCH}_{3} \text { (III) }+\mathrm{HCl}+\mathrm{CO}_{2} \\
& \stackrel{c}{\longrightarrow} \text { trans }-\mathrm{CH}_{3} \mathrm{CH}=\mathrm{CHCH}_{3}(\mathrm{IV})+\mathrm{HCl}(\mathrm{VI})+\mathrm{CO}_{2}(\mathrm{VII}) \\
& \stackrel{d}{\longrightarrow} \mathrm{CH}_{3} \mathrm{CHClCH}_{2} \mathrm{CH}_{3}(\mathrm{~V})+\mathrm{CO}_{2} \\
& \begin{array}{crcccrrr} 
& \text { I } & \text { II } & \text { III } & \text { IV } & \text { V } & \text { VI } & \text { VII } \\
\Delta H_{f(298)}^{\circ} & -120.3 & 0 & -1.7 & -2.7 & -37.2 & -22.1 & -94.1 \\
S_{(298)}^{\circ} & 106.2 & 73.6 & 72.1 & 70.9 & 83.4 & 44.6 & 51.1 \\
C_{p(298)}^{\circ} & 36.5 & 20.5 & 18.9 & 21.0 & 26.7 & 7.0 & 8.9
\end{array}
\end{aligned}
$$

$a$

$\Delta S^{\circ}=63.1 \mathrm{~g} / \mathrm{mole}$

$\Delta H^{\circ}=4.1 \mathrm{kcal} / \mathrm{mole}$

$\Delta C_{p}^{\circ}=-0.1 \mathrm{~g} / \mathrm{mole}$

Path $\quad \log A \quad E \quad \log k_{T}$ Conditions System Surface References

$\begin{array}{lllllll}a & 10.21 & 31.5 & -3.19 & 513-528^{\circ} \mathrm{K} & \text { stirred flow } & \text { [1] E. S. Lewis and }\end{array}$

W. C. Herndon, J. Am. Chem. Soc. 83, 1955 (1961).

$\begin{array}{lrrrr}c & 13.7 & 40.0 & -3.32 & 513-528^{\circ} \mathrm{K} \\ b & 13.8 & 40.4 & -3.34 & 513-528^{\circ} \mathrm{K} \\ d & 9.7 & 28.6 & -2.46 & 513-528^{\circ} \mathrm{K} \\ a+b+c & 12.9 & 37.0 & -2.80 & 513-528^{\circ} \mathrm{K}\end{array}$

Preferred:

Path $a \log k=12.1-36.0 / \theta \quad \log A_{\text {est }}=12.6$

Path $b \log k=11.9-35.8 / \theta \quad \log A_{\text {est }}=12.1$

Path $c \log k=11.9-35.9 / \theta \quad \log A_{\text {est }}=12.1$ (see section II-4.0).

Path $d$ Not reliable.

Comments: Paths $a, b$, and $c$ are probably unimolecular elimination reactions, although the Arrhenius parameters appear somewhat unexpected by comparison with the formates. Path $d$ is probably not a unimolecular reaction and probably has a heterogeneous component. (See discussion on ethyl chloroformate.) The small temperature range (i.e., $\Delta T \sim 15^{\circ} \mathrm{K}$ ) would be quite conducive to large errors in the Arrhenius parameters. $(\Delta E \pm 4 \mathrm{kcal}$.$) The transition state calculation of the A$-factors is preferred. Eliminations via primary and secondary $\mathrm{H}$-atom transfers were almost statistical.

\section{Experimental}

[1] Nitrogen carrier. Analysis by G.L.C. Deuterium isotope effects have also been studied for this reaction by these authors (J. Am. Chem. Soc. 83, 1959 (1959)). 
Reaction: Ethyl methanoate (ethyl formate)

\begin{tabular}{cccc}
\multicolumn{4}{r}{$\mathrm{HCOOCH}_{2} \mathrm{CH}_{3}(\mathrm{I}) \longrightarrow \mathrm{C}_{2} \mathrm{H}_{4}(\mathrm{II})+\mathrm{HCOOH}(\mathrm{III})$} \\
$\Delta \mathrm{H}_{f(298)}^{\circ}$ & $\mathrm{I}$ & $\mathrm{II}$ & $\mathrm{III}$ \\
$S_{(298)}^{\circ}$ & -88.9 & 12.5 & -88 \\
$C_{p(298)}^{\circ}$ & 82.0 & 52.5 & 60.1 \\
& 21.7 & 10.4 & 9.1
\end{tabular}

$\Delta S^{\circ}=30.6 \mathrm{~g} / \mathrm{mole}$

$\Delta H^{\circ}=13.4 \mathrm{kcal} / \mathrm{mole}$

$\Delta C_{p}^{\circ}=-2.2 \mathrm{~g} / \mathrm{mole}$

\begin{tabular}{|c|c|c|c|c|c|c|}
\hline $\log A$ & $\boldsymbol{E}$ & $\begin{array}{c}\log \boldsymbol{k}_{T} \\
(865)\end{array}$ & Conditions & System & Surface & References \\
\hline 11.33 & 44.14 & 0.18 & $\begin{array}{l}810-920^{\circ} \mathrm{K} \\
15-21 \text { torr }\end{array}$ & flow & 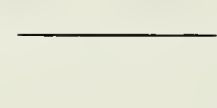 & $\begin{array}{l}\text { [1] A. T. Blades, Can. J. } \\
\text { Chem. 32, } 366 \text { (1954). }\end{array}$ \\
\hline 9.41 & 40.01 & $\begin{array}{l}(673) \\
-3.58\end{array}$ & $648-698^{\circ} \mathrm{K}$ & static & (see below) & $\begin{array}{l}\text { [2] R. F. Makens and W. G. } \\
\text { Eversole, J. Am. Chem. } \\
\text { Soc. 61, } 3203 \text { (1939). }\end{array}$ \\
\hline
\end{tabular}

Preferred:

$\log k=12.4-48.3 / \theta$.

Comments: Rate parameters for both studies are certainly low. In [2] surface effects were observed and it is possible that similar complications existed in the high-temperature flow experiments. If rate constants in the middle of the two temperature ranges are used to calculate the Arrhenius parameters, one obtains approximately $\log k=13.3-52.1 / \theta$.

If the calculated $A$-factor and the activation energy of ethyl acetate are adopted, then the calculated and observed rates of reaction at the higher temperature agree within 30 percent, while at the lower tempreatures, the observed rates are a factor of 5 slower. This could reflect an erroneous interpretation of the manometric data. The estimated parameters are preferred.

\section{Experimental}

[1] Rates by acid titration. Toluene flow technique.

[2] Rates by gasometric analysis for ethylene. Ethylene formation was not particularly surface sensitive, although subsequent decomposition of formic acid was strongly surface sensitive. 
Reaction: Propyl methanoate ( $n$-propyl formate)

$\mathrm{HCOOCH}_{2} \mathrm{CH}_{2} \mathrm{CH}_{3}$ (I) $\longrightarrow \mathrm{C}_{3} \mathrm{H}_{6}$ (II) $+\mathrm{HCOOH}$ (III)

$\begin{array}{rrrr}\Delta H_{f(298)}^{\circ} & \text { I } & \text { II } & \text { III } \\ S_{(298)}^{\circ} & -93.9 & 4.8 & -88 \\ C_{p(298)}^{\circ} & 91.4 & 63.6 & 60.1 \\ & 27.2 & 15.3 & 9.1\end{array}$

$\Delta S^{\circ}=32.3 \mathrm{~g} / \mathrm{mole}$

$\Delta H^{\circ}=10.7 \mathrm{kcal} / \mathrm{mole}$

$\Delta C_{p}^{\circ}=-2.8 \mathrm{~g} /$ mole

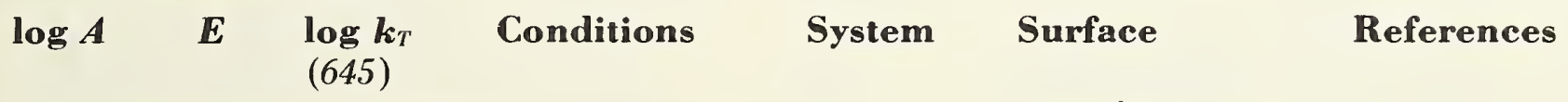
$9.4 \quad 39.66-4.04$
$613-673^{\circ} \mathrm{K} \quad$ static

[1] R. B. Anderson and H. H.

Rowley, J. Phys. Chem. 47, 454 (1943).

\section{Preferred:}

$\log k=12.1-47.7 / \theta$ (see below).

Comments: Unreliable; Arrhenius parameters are much too low for the molecular elimination reaction. The estimated Arrhenius parameters are preferred. As in the ethyl formate system, the reported reaction rates are appreciably lower than the calculated rates. A systematic error in both studies is indicated.

\section{Experimental}

[1] Rates based on propene formation analyzed by absorption in sulfuric acid. Stoichiometry confirmed by a subsequent surface decomposition of formic acid. 
Reaction: Isopropyl methanoate (isopropyl formate)

$\Delta S^{\circ}=35.3 \mathrm{~g} / \mathrm{mole}$

$\mathrm{HCOOCH}\left(\mathrm{CH}_{3}\right)_{2}(\mathrm{I}) \longrightarrow \mathrm{CH}_{2}=\mathrm{CHCH}_{3}(\mathrm{II})+\mathrm{HCOOH}(\mathrm{III})$

$\Delta H^{\circ}=13.5 \mathrm{kcal} / \mathrm{mole}$

$\Delta C_{p}^{\circ}=-3.3 \mathrm{~g} / \mathrm{mole}$

$\begin{array}{crcr}\Delta \mathrm{H}_{f(298)}^{\circ} & \mathrm{I} & \mathrm{II} & \text { III } \\ S_{(298)}^{\circ} & -96.7 & 4.8 & -88 \\ C_{p(298)}^{\circ} & 88.4 & 63.6 & 60.1 \\ & 27.7 & 15.3 & 9.1\end{array}$

\begin{tabular}{|c|c|c|c|c|c|c|}
\hline $\log A$ & $\boldsymbol{E}$ & $\begin{array}{c}\log \boldsymbol{k}_{T} \\
(600)\end{array}$ & Conditions & System & Surface & References \\
\hline 12.58 & 44.0 & -3.44 & $\begin{array}{l}721-811^{\circ} \mathrm{K} \\
10.6-21.5 \text { torr }\end{array}$ & flow & & $\begin{array}{l}\text { [1] A. T. Blades, Can. J. } \\
\text { Chem. 32, } 366 \text { (1954). }\end{array}$ \\
\hline 12.58 & 44.23 & -3.53 & $596-608^{\circ} \mathrm{K}$ & static & & $\begin{array}{l}\text { [2] R. B. Anderson and } \\
\text { H. H. Rowley, J. Phys. } \\
\text { Chem. 47, 454 (1943). }\end{array}$ \\
\hline
\end{tabular}

Preferred:

$\log k=12.6-44.0 / \theta$.

Transition state estimate of $A$ gives $\log A_{\text {est }}=12.7$ (see section II-4.0).

\section{Experimental}

[1] Rates by acid titration. Toluene carrier technique.

[2] Rates determined on basis of propene formation analyzed by absorption in 83 percent sulfuric acid. Subsequent decomposition of the formic acid complicated the stoichiometry since $\left(P_{\infty} / P_{0}\right)$ was temperature and surface sensitive. Rate of propene formation was relatively insensitive to change of surface. 
Reaction: $t$-Butyl methanoate ( $t$-butyl formate)

$\begin{array}{crcr}\mathrm{HCOOC}\left(\mathrm{CH}_{3}\right)_{3}(\mathrm{I}) \longrightarrow \mathrm{CH}_{2}=\mathrm{C}\left(\mathrm{CH}_{3}\right)_{2}(\mathrm{II})+\mathrm{HCOOH}(\mathrm{III}) \\ \Delta H_{f(298)}^{\circ} & \mathrm{I} & \text { II } & \text { III } \\ S_{(298)}^{\circ} & -105.6 & -4.0 & -88 \\ C_{p(298)}^{\circ} & 92.2 & 70.2 & 60.1 \\ & 33.4 & 21.3 & 9.1\end{array}$

$\Delta S^{\circ}=38.1 \mathrm{~g} / \mathrm{mole}$

$\Delta \mathrm{H}^{\circ}=13.6 \mathrm{kcal} / \mathrm{mole}$

$\Delta C_{p}^{\circ}=-3.0 \mathrm{~g} / \mathrm{mole}$

\begin{tabular}{|c|c|c|c|c|c|c|}
\hline $\log A$ & $\boldsymbol{E}$ & $\begin{array}{c}\log \boldsymbol{k}_{T} \\
(550)\end{array}$ & Conditions & System & Surface & References \\
\hline 11.1 & 34.6 & $(-2.65)$ & $\begin{array}{l}503-573^{\circ} \mathrm{K} \\
38-103 \text { torr }\end{array}$ & static & none & $\begin{array}{l}\text { [1] E. Gordon, S. J. W. } \\
\text { Price, and A. F. Trot- } \\
\text { man-Dickenson, J. } \\
\text { Chem. Soc. } 2813 \text { (1957). }\end{array}$ \\
\hline
\end{tabular}

\section{Preferred:}

$\log k=12.9-39.2 / \theta$. Rate constants are probably reliable around $\bar{T}$.

Comments: One would expect the $t$-butyl derivative to have an $A$-factor very close to the isopropyl derivative; the Arrhenius parameters are therefore probably low. A transition state estimate of the $A$-factor gives log $A_{\text {est }}=12.9$ and is preferred (see section II -4.0 ).

\section{Experimental}

[1] Rates by $\Delta \mathrm{P}$ were unaffected by added cyclohexene. The decomposition rates based on isobutene formation agreed with the observed pressure changes within (3-20 percent). 
Reaction: Ethyl ethanoate (ethyl acetate)

\begin{tabular}{cccc}
\multicolumn{4}{r}{$\mathrm{CH}_{3} \mathrm{COOC}_{2} \mathrm{H}_{5}(\mathrm{I}) \longrightarrow \mathrm{C}_{2} \mathrm{H}_{4}(\mathrm{II})+\mathrm{CH}_{3} \mathrm{COOH}(\mathrm{III})$} \\
$\Delta H_{f(298)}^{\circ}$ & I & II & III \\
$S_{(298)}^{\circ}$ & -103.4 & 12.5 & -103.7 \\
$C_{p(298)}^{\circ}$ & 90.1 & 52.5 & 67.6 \\
& 26.7 & 10.4 & 16.0
\end{tabular}

$\Delta S^{\circ}=30.0 \mathrm{~g} / \mathrm{mole}$

$\Delta H^{\circ}=12.2 \mathrm{kcal} / \mathrm{mole}$

$\Delta C_{p}^{\circ}=-0.3 \mathrm{~g} / \mathrm{mole}$

\begin{tabular}{|c|c|c|c|c|c|c|}
\hline $\log A$ & $\boldsymbol{E}$ & $\begin{array}{c}\log \boldsymbol{k}_{T} \\
\quad(830)\end{array}$ & Conditions & System & Surface & References \\
\hline 12.48 & 47.75 & -0.09 & $\begin{array}{l}787-883^{\circ} \mathrm{K} \\
11.9-18.8 \text { torr }\end{array}$ & flow & & $\begin{array}{l}\text { [1] A. T. Blades, Can. J. Chem. } \\
\text { 32, } 366 \text { (1954). }\end{array}$ \\
\hline 12.59 & 48.0 & -0.05 & $773-876^{\circ} \mathrm{K}$ & flow & & $\begin{array}{l}\text { [2] A. T. Blades and P. W. } \\
\text { Gilderson, Can. J. } \\
\text { Chem. 38, } 1407 \text { (1960). }\end{array}$ \\
\hline 12.54 & 48.0 & -0.10 & $725-810^{\circ} \mathrm{K}$ & flow & & $\begin{array}{l}\text { [3] J. C. Scheer, E. C. } \\
\text { Kooyman, and F. L. J. } \\
\text { Sixma, Rec. Trav. } \\
\text { Chim. des Pays-Bas } \\
\mathbf{8 2}, 1123 \text { (1963). }\end{array}$ \\
\hline
\end{tabular}

Preferred:

$\log k=12.59-48.0 / \theta$.

$\log A_{\text {est }} \simeq 12.6$ (see section II-4.0).

\section{Experimental}

[1] Toluene carrier technique. Rate determined by titration of acetic acid.

[2] (as above) Ethyl $\left(d_{5}\right)$ acetate decomposition also determined: $\left(k_{H} / k_{d}\right) \simeq 0.8 \times 10^{1.515 / \theta}$ interpreted as a primary isotope effect with $\beta(\mathrm{C}-\mathrm{H})$ bond rupture playing a major role in the transition state. Other relative rate isotope studies on ethyl acetate include: ethyl acetate $\left(d_{3}\right)$, [2] above; and 1,1,2,2(d $\left.d_{4}\right)$ ethyl acetate, A. T. Blades and P. W. Gilderson, Can. J. Chem. 38, 1401 (1960). In the isotope studies, the olefin products were determined by M. S.

[3] Rates by G. L. C. (see comments on $t$-butyl acetate). 
Reaction: 1-Propyl ethanoate ( $n$-propyl acetate)

\begin{tabular}{rrrr}
$\mathrm{CH}_{3} \mathrm{COOCH}_{2} \mathrm{CH}_{2} \mathrm{CH}_{3}(\mathrm{I}) \longrightarrow \mathrm{CH}_{3} \mathrm{COOH}(\mathrm{II})+\mathrm{CH}_{2}=$ & \multicolumn{3}{c}{$\mathrm{CHCH}_{3}(\mathrm{III})$} \\
$\Delta H_{f(298)}^{\circ}$ & I & II & III \\
$S_{(298)}^{\circ}$ & -108.4 & -103.7 & 4.8 \\
$C_{(298)}^{\circ}$ & 99.5 & 67.6 & 63.6 \\
& 32.2 & 16.0 & 15.3
\end{tabular}

$\Delta S^{\circ}=31.7 \mathrm{~g} / \mathrm{mole}$

$\Delta H^{\circ}=9.5 \mathrm{kcal} / \mathrm{mole}$

$\Delta C_{p}^{\circ}=-0.9 \mathrm{~g} /$ mole

\begin{tabular}{|c|c|c|c|c|c|c|}
\hline $\log A$ & $\mathbf{E}$ & $\begin{array}{c}\log \boldsymbol{k}_{T} \\
(760)\end{array}$ & Conditions & System & Surface & References \\
\hline $\begin{array}{l}12.40 \\
11.27 \\
12.27\end{array}$ & $\begin{array}{l}47.7 \\
43.7 \\
48.3\end{array}$ & $\begin{array}{l}-1.29 \\
-1.27 \\
-1.59\end{array}$ & $725-810^{\circ} \mathrm{K}$ & flow & & $\begin{array}{l}\text { [1] J. C. Scheer, E. C. } \\
\text { Kooyman, and F. L. J. } \\
\text { Sixma, Rec. Trav. } \\
\text { Chim. des Pays-Bas } 82, \\
1123 \text { (1963). }\end{array}$ \\
\hline
\end{tabular}

Preferred:

$\log k=12.4-47.7 / \theta$

$\log A_{\text {est }}=12.3 \quad E \simeq 47.4 \mathrm{kcal} /$ mole (see section II-4.0).

\section{Experimental}

[1] Rates by G.L.C. analysis on product olefins and reactant. It is apparent that the experimental errors in the method of study are such that the Arrhenius parameters reported are not reliable to better than an order of magnitude. (For comments on the method see $t$-butyl acetate.) 
Reaction: 1-Butyl ethanoate ( $n$-butyl acetate)

$\begin{array}{crrc}\mathrm{CH}_{3} \mathrm{COO}\left(\mathrm{CH}_{2}\right)_{3} \mathrm{CH}_{3}(\mathrm{I}) \longrightarrow \mathrm{CH}_{3} \mathrm{COOH}(\mathrm{II})+\mathrm{CH}_{2}=\mathrm{CHCH}_{2} \mathrm{CH}_{3}(\mathrm{III}) \\ \Delta H_{f(298)}^{\circ} & -113.3 & -103.7 & \text { II } \\ S_{(298)}^{\circ} & 108.9 & 67.6 & 73.6 \\ C_{p(298)}^{\circ} & 37.7 & 16.0 & 20.5\end{array}$

$\Delta S^{\circ}=32.3 \mathrm{~g} / \mathrm{mole}$
$\Delta H^{\circ}=9.6 \mathrm{kcal} / \mathrm{mole}$
$\Delta C_{p}^{\circ}=-1.2 \mathrm{~g} / \mathrm{mole}$
$\begin{array}{llllll}\log A & \boldsymbol{E} & \log \boldsymbol{k}_{T} & \text { Conditions } & \text { System } & \text { Surface }\end{array}$ (760)
$12.19 \quad 46.0 \quad-1.01 \quad 725-810^{\circ} \mathrm{K} \quad$ flow
[1] J. C. Scheer, E. C.
$13.28 \quad 49.3 *-0.89$
Kooyman, and F. L. J. Sixma, Rec. Trav. Chim. des Pays-Bas 82, 1123 (1963).

Preferred:

$\log k=12.19-46.0 / \theta$

$\log A_{\text {est }}=12.2$ (see section II-4.0).

Comments: The rate constants should be very similar to those for $n$-propyl and $n$-pentyl acetates. Rather large experimental errors are evident.

[1] Rates by G.L.C. analysis for 1-butene.

\section{Experimental}

${ }^{*}$ Reported $A$-factor of $10^{11.2} \mathrm{sec}^{-1}$ was assumed to be a misprint. Experimental errors were large. (See comments on $t$-but yl acetate.) 
Reaction: Isobutyl ethanoate (2-methylpropyl acetate; isobutyl acetate)

$$
\begin{array}{crcc}
\mathrm{CH}_{3} \mathrm{COOCH}_{2} \mathrm{CH}\left(\mathrm{CH}_{3}\right)_{2}(\mathrm{I}) \longrightarrow \mathrm{CH}_{3} \mathrm{COOH}(\mathrm{II})+\mathrm{CH}_{2} & =\mathrm{C}\left(\mathrm{CH}_{3}\right)_{2}(\mathrm{III}) \\
\Delta H_{f(298)}^{\circ} & \text { I } & \text { II } & \text { III } \\
S_{(298)}^{\circ} & 114.6 & -103.7 & -4.0 \\
C_{p(298)}^{\circ} & 37.5 & 16.0 & 21.3
\end{array}
$$

\begin{tabular}{|c|c|c|c|c|c|c|}
\hline $\log A$ & $\boldsymbol{E}$ & $\begin{array}{c}\log \boldsymbol{k}_{T} \\
(760)\end{array}$ & Conditions & System & Surface & References \\
\hline $\begin{array}{l}11.06 \\
12.38\end{array}$ & $\begin{array}{l}44.1 \\
48.8\end{array}$ & $\begin{array}{l}(-1.59) \\
(-1.64)\end{array}$ & $725-810^{\circ} \mathrm{K}$ & flow & & $\begin{array}{l}\text { [1] J. C. Scheer, E. C. } \\
\text { Kooyman, and F. L. J. } \\
\text { Sixma, Rec. Trav. } \\
\text { Chim. des Pays-Bas } \\
\mathbf{8 2 , ~} 1123 \text { (1963). }\end{array}$ \\
\hline
\end{tabular}

$\Delta S^{\circ}=32.6 \mathrm{~g} / \mathrm{mole}$

$\Delta H^{\circ}=6.9 \mathrm{kcal} / \mathrm{mole}$

$\Delta C_{p}^{\circ}=-0.2 \mathrm{~g} / \mathrm{mole}$

Preferred:

Estimated Arrhenius parameters are preferred (see section II-4.0).

$\log k=11.9-47.3 / \theta$.

\section{Experimental}

[1] Rates by G.L.C. analysis of isobutene. See comments on $t$-butyl acetate. 
Reaction: 1-Pentyl ethanoate ( $n$-pentyl acetate)

$\begin{array}{rrrr}\mathrm{CH}_{3} \mathrm{COO}\left(\mathrm{CH}_{2}\right)_{4} \mathrm{CH}_{3}(\mathrm{I}) \longrightarrow \mathrm{CH}_{3} \mathrm{COOH}(\mathrm{II})+\mathrm{CH}_{2}=\mathrm{CHCH}_{2} \mathrm{CH}_{2} \mathrm{CH}_{3} \text { (III) } \\ \Delta H_{f(298)}^{\circ} & \begin{array}{c}\mathrm{I} \\ \mathrm{II}\end{array} & \text { III } \\ S_{(298)}^{\circ} & 118.3 & -103.7 & -4.9 \\ C_{p(298)}^{\circ} & 43.2 & 16.0 & 26.2\end{array}$

$\Delta S^{\circ}=32.1 \mathrm{~g} /$ mole

$\Delta H^{\circ}=9.7 \mathrm{kcal} / \mathrm{mole}$

$\Delta C_{p}^{\circ}=-1.0 \mathrm{~g} / \mathrm{mole}$

\begin{tabular}{|c|c|c|c|c|c|c|}
\hline $\log A$ & $\boldsymbol{E}$ & $\begin{array}{c}\log \boldsymbol{k}_{T} \\
(760)\end{array}$ & Conditions & System & Surface & References \\
\hline
\end{tabular}

$12.2 \quad 46.4 \quad(-1.14) \quad 725-810^{\circ} \mathrm{K} \quad$ flow

[1] J. C. Scheer, E. C.

Kooyman, and F. L. J.

Sixma, Rec. Trav.

Chim. des Pays-Bas

82, 1123 (1963).

Preferred:

$\log k=12.2-46.4 / \theta$.

$\log A_{\text {est }}=12.2$ (see section II-4.0).

\section{Experimental}

[1] Rates by G.L.C. analysis of 1-pentene. See comments on $t$-butyl acetate. 
Reaction: 2-Methylbutyl ethanoate (2-methylbutyl acetate)

$\begin{array}{rrrc}\mathrm{CH}_{3} \mathrm{COOCH}_{2} \mathrm{CH}\left(\mathrm{CH}_{3}\right) \mathrm{CH}_{2} \mathrm{CH}_{3}(\mathrm{I}) \longrightarrow \mathrm{CH}_{3} \mathrm{COOH}(\mathrm{II})+\mathrm{CH}_{2}=\mathrm{C}\left(\mathrm{CH}_{3}\right) \mathrm{C}_{2} \mathrm{H}_{5}(\mathrm{III}) \\ \Delta H_{f(298)}^{\circ} & -118.8 & -103.7 & \text { III } \\ S_{(298)}^{\circ} & 115.6 & 67.6 & 81.9 \\ C_{p(298)}^{\circ} & 43.0 & 16.0 & 26.7\end{array}$

$\Delta S^{\circ}=33.9 \mathrm{~g} / \mathrm{mole}$

$\Delta \mathrm{H}^{\circ}=6.5 \mathrm{kcal} / \mathrm{mole}$

$\Delta C_{p}^{\circ}=-0.3 \mathrm{~g} / \mathrm{mole}$

\begin{tabular}{|c|c|c|c|c|c|c|}
\hline $\log A$ & $\boldsymbol{E}$ & $\begin{array}{c}\log \boldsymbol{k}_{T} \\
(760)\end{array}$ & Conditions & System & Surface & References \\
\hline $\begin{array}{l}12.61 \\
11.60\end{array}$ & $\begin{array}{l}49.0 \\
45.7\end{array}$ & $\begin{array}{l}(-1.48) \\
(-1.52)\end{array}$ & $725-810^{\circ} \mathrm{K}$ & flow & & $\begin{array}{l}\text { [1] J. C. Scheer, E. C. } \\
\text { Kooyman, and F. L. J. } \\
\text { Sixma, Rec. Trav. } \\
\text { Chim. des Pays-Bas 82, } \\
1123 \text { (1963). }\end{array}$ \\
\hline
\end{tabular}

Preferred:

$\log k=11.9-46.7 / \theta$. Rate constants are reliable.

Comments: Rate constants should be close to those for 2-methylpropyl acetate, as observed. The estimated Arrhenius parameters are preferred (see section II-4.0).

\section{Experimental}

[1] Rates by G.L.C. analysis. See comments on $t$-butyl acetate. 
Reaction: 3-Methylbutyl ethanoate ( $\gamma$-methylbutyl acetate)

$\begin{array}{crcc}\mathrm{CH}_{3} \mathrm{COO}\left(\mathrm{CH}_{2}\right)_{2} \mathrm{CH}\left(\mathrm{CH}_{3}\right)_{2} & (\mathrm{I}) \longrightarrow \mathrm{CH}_{3} \mathrm{COOH}(\mathrm{II})+\mathrm{CH}_{2}=\mathrm{CHCH}\left(\mathrm{CH}_{3}\right)_{2}(\mathrm{III}) \\ \Delta H_{f(298)}^{\circ} & -119.5 & -103.7 & -6.9 \\ S_{(298)}^{\circ} & 115.6 & 67.6 & 79.7 \\ C_{p(298)}^{\circ} & 43.0 & 16.0 & 25.8\end{array}$

$\Delta S^{\circ}=31.7 \mathrm{~g} / \mathrm{mole}$

$\Delta H^{\circ}=8.9 \mathrm{kcal} / \mathrm{mole}$

$\Delta C_{p}^{\circ}=-1.2 \mathrm{~g} / \mathrm{mole}$

\begin{tabular}{|c|c|c|c|c|c|c|}
\hline $\log A$ & $\boldsymbol{E}$ & $\begin{array}{c}\log \boldsymbol{k}_{T} \\
(760)\end{array}$ & Conditions & System & Surface & References \\
\hline 12.63 & 47.9 & $(-1.11)$ & $\left(725-810^{\circ} \mathrm{K}\right)$ & flow & & $\begin{array}{l}\text { [1] J. C. Scheer, E. C. } \\
\text { Kooyman, and F. L. J. } \\
\text { Sixma, Rec. Trav. } \\
\text { Chim. des Pays-Bas } 82, \\
1123 \text { (1963). }\end{array}$ \\
\hline
\end{tabular}

Preferred:

$\log k=12.2-46.4 / \theta$. Rate constants are reliable.

Comments: The Arrhenius parameters seem slightly high. Rate constant agrees with that of pentyl ethanoate, as one might expect.

\section{Experimental}

[1] Rates by G.L.C. analysis. See comments on $t$-butyl acetate. 
Reaction: 2-Ethylbutyl ethanoate ( $\beta$-ethylbutyl acetate)

$$
\begin{array}{crcc}
\mathrm{CH}_{3} \mathrm{COOCH}_{2} \mathrm{CH}\left(\mathrm{C}_{2} \mathrm{H}_{5}\right)_{2}(\mathrm{I}) \longrightarrow & \multicolumn{4}{c}{\mathrm{CH}_{3} \mathrm{COOH}(\mathrm{II})+\mathrm{CH}_{2}=\mathrm{C}\left(\mathrm{C}_{2} \mathrm{H}_{5}\right)_{2}(\mathrm{III})} \\
\Delta H_{f(298)}^{\circ} & -122.9 & -103.7 & -13.6 \\
S_{(298)}^{\circ} & 125.0 & 67.6 & 89.9 \\
C_{p(298)}^{\circ} & 48.5 & 16.0 & 32.2
\end{array}
$$

\begin{tabular}{|c|c|c|c|c|c|c|}
\hline $\log A$ & $\boldsymbol{E}$ & $\begin{array}{c}\log \boldsymbol{k}_{T} \\
(760)\end{array}$ & Conditions & System & Surface & References \\
\hline 13.04 & 49.7 & $(-1.23)$ & $725-810^{\circ} \mathrm{K}$ & flow & & $\begin{array}{l}\text { [1] J. C. Scheer, E. C. } \\
\text { Kooyman, and F. L. J. } \\
\text { Sixma, Rec. Trav. Chim. } \\
\text { des Pays-Bas 82, } 1123 \\
\text { (1963). }\end{array}$ \\
\hline
\end{tabular}

$\Delta S^{\circ}=32.5 \mathrm{~g} / \mathrm{mole}$

$\Delta H^{\circ}=\lceil 5.6 \mathrm{kcal} / \mathrm{mole}$

$\Delta C_{p}^{\circ}=-0.3 \mathrm{~g} / \mathrm{mole}$

Preferred:

$\log k=11.9-45.8 / \theta$. Rate constants are reliable.

Comments: Estimated Arrhenius parameters are preferred (see section II-4.0). Replacement of a methyl group by an ethyl group at the $\beta$-carbon position seems to produce a slight rate acceleration.

\section{Experimental}

[1] Rates obtained from G.L.C. analysis. See comments on $t$-butyl acetate. 
Reaction: 2-Methoxyethyl ethanoate ( $\beta$-methoxyethyl acetate)

\begin{tabular}{crrc}
$\mathrm{CH}_{3} \mathrm{COOCH}_{2} \mathrm{CH}_{2} \mathrm{OCH}_{3}(\mathrm{I}) \longrightarrow \mathrm{CH}_{3} \mathrm{COOH}(\mathrm{II})+\mathrm{CH}_{2}$ & $=\mathrm{CHOCH}_{3}(\mathrm{III})$ \\
$\Delta H_{f(298)}^{\circ}$ & \multicolumn{1}{l}{$\mathrm{I}^{\circ}$} & II & III \\
$S_{(298)}^{\circ}$ & 109.3 & 67.6 & 71.1 \\
$C_{p(298)}^{\circ}$ & 35.1 & 16.0 & 18.8
\end{tabular}

$\Delta S^{\circ}=29.4 \mathrm{~g} / \mathrm{mole}$

$\Delta H^{\circ}=6.6 \mathrm{kcal} / \mathrm{mole}$

$\Delta C_{p}^{\circ}=-0.3 \mathrm{~g} / \mathrm{mole}$

\begin{tabular}{|c|c|c|c|c|c|c|}
\hline $\log A$ & $\boldsymbol{E}$ & $\begin{array}{c}\log \boldsymbol{k}_{T} \\
(760)\end{array}$ & Conditions & System & Surface & References \\
\hline 11.96 & 47.8 & $(-1.76)$ & $725-810^{\circ} \mathrm{K}$ & flow & & $\begin{array}{l}\text { [1] J. C. Scheer, E. C. } \\
\text { Kooyman, and F. L. J. } \\
\text { Sixma, Rec. Trav. Chim. } \\
\text { des Pays-Bas, 82, } 1123 \\
\text { (1963). }\end{array}$ \\
\hline
\end{tabular}

Preferred:

$\log k=12.2-48.6 / \theta$. Rate constants are reliable.

$\log A_{\text {est }}=12.2$ and is preferred (see section II-4.0).

Comments: The calculated Arrhenius $A$-factor is the same as that for $n$-butyl acetate.

\section{Experimental}

[1] Rates by G.L.C. analysis. See comments on $t$-butyl acetate. 
Reaction: 2-Ethoxyethyl ethanoate ( $\beta$-ethanoxyethyl acetate)

$\begin{array}{crcc}\mathrm{CH}_{3} \mathrm{COOCH}_{2} \mathrm{CH}_{2} \mathrm{OC}_{2} \mathrm{H}_{5}(\mathrm{I}) \longrightarrow \mathrm{CH}_{3} \mathrm{COOH}(\mathrm{II})+\mathrm{CH}_{2} & =\mathrm{CHOC}_{2} \mathrm{H}_{5}(\mathrm{III}) \\ \Delta H_{f(298)}^{\circ} & -143.9 & -103.7 & -33.6 \\ S_{(298)}^{\circ} & 119.8 & 67.6 & 81.6 \\ C_{p(298)}^{\circ} & 40.1 & 16.0 & 23.8\end{array}$

$\Delta S^{\circ}=29.4 \mathrm{~g} / \mathrm{mole}$

$\Delta H^{\circ}=6.6 \mathrm{kcal} / \mathrm{mole}$

$\Delta C_{p}^{\circ}=-0.3 \mathrm{~g} / \mathrm{mole}$

\begin{tabular}{|c|c|c|c|c|c|c|}
\hline $\log A$ & $\boldsymbol{E}$ & $\begin{array}{c}\log k_{T} \\
(760)\end{array}$ & Conditions & System & Surface & References \\
\hline 12.09 & 47.9 & $(-1.67)$ & $725-810^{\circ} \mathrm{K}$ & flow & & $\begin{array}{l}\text { [1] J. C. Scheer, E. C. } \\
\text { Kooyman, and F. L. J. } \\
\text { Sixma, Rec. Trav. } \\
\text { Chim. des Pays-Bas } 82, \\
1123 \text { (1963). }\end{array}$ \\
\hline
\end{tabular}

\section{Preferred:}

$\log k=12.09-47.9 / \theta$.

$\log A_{\text {est }}=12.2$ (see section II-4.0).

Comments: Rate constant should be similar to that for 2-methoxyethyl ethanoate.

\section{Experimental}

[1] Rates based on G.L.C. analysis. See comments on $t$-butyl acetate. 
Reaction: 2-Arylethyl ethanoates (meta- or para-substituted 2-phenylethyl acetates)

$\begin{array}{cccc}\mathrm{X}-\emptyset \mathrm{CH}_{2} \mathrm{CH}_{2} \mathrm{OCOCH}_{3}(\mathrm{I}) \longrightarrow \mathrm{X}-\varnothing \mathrm{CH}=\mathrm{CH}_{2}(\mathrm{II})+\mathrm{CH}_{3} \mathrm{COOH} \\ \Delta H_{f(298)}^{\circ} & \mathrm{I}(\mathrm{X}=\mathrm{H}) & \mathrm{II}(\mathrm{X}=\mathrm{H}) & \mathrm{III} \\ S_{(298)}^{\circ} & 120 & 35.4 & -103.7 \\ \Delta S_{p(299)}^{\circ} & 45.6 & 29.6 & 67.6\end{array}$

$\Delta S^{\circ}=30.1 \mathrm{~g} / \mathrm{mole}$

$\Delta H^{\circ}=9.0 \mathrm{kcal} / \mathrm{mole}$

$\Delta C_{p}^{\circ}=0 \mathrm{~g} / \mathrm{mole}$

\begin{tabular}{ccccccc}
$\log \boldsymbol{A}$ & $\boldsymbol{E}$ & $\begin{array}{c}\log \boldsymbol{k}_{T} \\
(380)\end{array}$ & Conditions & System & (X-substituents) & \multicolumn{1}{c}{ References } \\
12.37 & 45.4 & -2.82 & $343-409^{\circ} \mathrm{C}$ & static & $\mathrm{H}$ & [1] R. Taylor, G. G. \\
12.27 & 44.8 & & $344-409^{\circ} \mathrm{C}$ & & $m \cdot \mathrm{Cl}$ & Smith, and W. H. \\
12.38 & 45.4 & & $341-409^{\circ} \mathrm{C}$ & & $p-\mathrm{Me}$ & Wetzel, J. Am. Chem. \\
12.46 & 45.9 & & $343-405^{\circ} \mathrm{C}$ & & $p-\mathrm{MeO}$ & Soc. 84, $4817(1962)$.
\end{tabular}

Preferred:

2-arylethyl acetate: $\log k=12.37-45.4 / \theta$.

Comments: Transition state estimates of $A$ for all compounds above gives $\log A_{\text {est }}=12.2$ (see section II-4.0).

\section{Experimental}

[1] Rates were obtained from $\Delta \mathrm{P}$ and initial slopes. These compounds fit a Hammett $\rho-\sigma^{+}$plot (H. C. Brown and Y. Okamoto, J. Am. Chem. Soc. 80, 4979 (1958)), giving a $\rho$ factor of about 0.3 . This $\rho$ is numerically smaller than that obtained for the l-arylethyl acetates, suggesting that substituents in the 2 -aryl ring affect the rates of decomposition less than substituents in the l-aryl ring. 
Reaction: Isopropyl ethanoate (isopropyl acetate)

\begin{tabular}{crcc}
$\mathrm{CH}_{3} \mathrm{COOCH}\left(\mathrm{CH}_{3}\right)_{2}(\mathrm{I})$ & \multicolumn{3}{c}{$\mathrm{CH}_{3} \mathrm{CH}=\mathrm{CH}_{2}(\mathrm{II})+\mathrm{CH}_{3} \mathrm{COOH}(\mathrm{III})$} \\
$\Delta H_{f(298)}^{\circ}$ & -111.2 & II & III \\
$S_{(298)}^{\circ}$ & 96.5 & 63.6 & -103.7 \\
$C_{p(298)}^{\circ}$ & 32.7 & 15.3 & 67.6 \\
& & & 16.0
\end{tabular}
$\Delta S^{\circ}=34.7 \mathrm{~g} /$ mole
$\Delta H^{\circ}=12.3 \mathrm{kcal} / \mathrm{mole}$
$\Delta C_{p}^{\circ}=-1.4 \mathrm{~g} / \mathrm{mole}$

\begin{tabular}{|c|c|c|c|c|c|c|}
\hline $\log A$ & $\boldsymbol{E}$ & $\begin{array}{l}\log \boldsymbol{k}_{T} \\
(610)\end{array}$ & Conditions & System & Surface & References \\
\hline 13.0 & 45.0 & $(-3.12)$ & $\begin{array}{l}715-801^{\circ} \mathrm{K} \\
10.3-22.8 \text { torr }\end{array}$ & flow & & $\begin{array}{l}\text { [1] A. T. Blades, Can. J. } \\
\text { Chem. 32, } 366 \text { (1954). }\end{array}$ \\
\hline 13.4 & 46.34 & $(-3.20)$ & $\begin{array}{l}586-635^{\circ} \mathrm{K} \\
152-189 \text { torr }\end{array}$ & static & & $\begin{array}{l}\text { [2] E. U. Emovan, Allan } \\
\text { Maccoll, J. Chem. Soc. } \\
335 \text { (1962). }\end{array}$ \\
\hline 13.38 & 46.6 & $(-3.25)$ & $650-710^{\circ} \mathrm{K}$ & flow & & $\begin{array}{l}\text { [3] J. C. Scheer, E. C. } \\
\text { Kooyman, and F. L. J. } \\
\text { Sixma, Rec. Trav. } \\
\text { Chim. des Pays-Bas } \\
\text { 82, 1123 (1963). }\end{array}$ \\
\hline
\end{tabular}

Preferred:

$\log k=13.0-45.0 / \theta$.

Comments: All studies agree within experimental error.

$\log A_{\text {est }} \simeq 12.9$ (see section II-4.0).

\section{Experimental}

[1] Toluene carrier technique. Rate by acid titration. No bibenzyl in products taken as evidence for the molecular nature of the elimination.

[2] Rate by $\Delta P$. Stoichiometry confirmed by G.L.C. analysis.

[3] Rates by G.L.C. analysis. See comments on $t$-butyl acetate. 
Reaction: sec-Butyl ethanoate (sec-butyl acetate)

$$
\begin{aligned}
& \stackrel{a}{\longrightarrow} \text { cis } \mathrm{CH}_{3} \mathrm{CH}=\mathrm{CHCH}_{3}(\mathrm{II})+\mathrm{CH}_{3} \mathrm{COOH}(\mathrm{V}) \\
& \mathrm{CH}_{3} \mathrm{COOCH}\left(\mathrm{CH}_{3}\right) \mathrm{CH}_{2} \mathrm{CH}_{3}(\mathrm{I}) \quad \stackrel{b}{\longrightarrow} \text { trans } \mathrm{CH}_{3} \mathrm{CH}=\mathrm{CHCH}_{3}(\mathrm{III})+\mathrm{CH}_{3} \mathrm{COOH} \\
& \stackrel{c}{\longrightarrow} \mathrm{CH}_{2}=\mathrm{CHCH}_{2} \mathrm{CH}_{3}(\mathrm{IV})+\mathrm{CH}_{3} \mathrm{COOH}
\end{aligned}
$$

\begin{tabular}{|c|c|c|c|c|c|c|c|}
\hline Path & $\log A$ & $\boldsymbol{E}$ & $\begin{array}{c}\log \boldsymbol{k}_{T} \\
(600)\end{array}$ & Conditions & System & Surface & References \\
\hline total & 13.3 & 46.6 & $(-3.67)$ & $\begin{array}{l}576-632{ }^{\circ} \mathrm{K} \\
126-341 \text { torr }\end{array}$ & static & none & $\begin{array}{l}\text { [1] E. U. Emovan and } \\
\text { A. Maccoll, J. } \\
\text { Chem. Soc. } \mathbf{3 3 5} \\
\text { (1962). }\end{array}$ \\
\hline total & 14.15 & 48.6 & $(-3.58)$ & $650-710^{\circ} \mathrm{K}$ & flow & & $\begin{array}{l}\text { [2] J. C. Scheer, E. C. } \\
\text { Kooyman, and } \\
\text { F. L. J. Sixma, Rec. } \\
\text { Trav. Chim. des } \\
\text { Pays-Bas } \mathbf{8 2}, 1123 \\
\text { (1963). }\end{array}$ \\
\hline
\end{tabular}

$\begin{array}{crrrrr} & \text { I } & \text { II } & \text { III } & \text { IV } & \text { V } \\ \Delta H_{f(298)}^{\circ} & -115.4 & -1.7 & -2.7 & 0 & -103.7 \\ S_{(298)}^{\circ} & 105.9 & 72.1 & 70.9 & 73.6 & 67.6 \\ C_{p(298)}^{\circ} & 38.2 & 18.9 & 21.0 & 20.5 & 16.0\end{array}$

$$
\begin{aligned}
& \Delta S^{\circ}=35.3 \mathrm{~g} / \mathrm{mole} \\
& \Delta H^{\circ}=11.7 \mathrm{kcal} / \mathrm{mole} \\
& \Delta C_{p}^{\circ}=-1.7 \mathrm{~g} / \mathrm{mole}
\end{aligned}
$$

Preferred:

Estimated: $\log k=13.3-46.6 / \theta$.

$\log A_{1 \text {-butene }}=12.6$

$\log A_{2 \text {-butene }}=12.4$ (see section II-4.0).

\section{Experimental}

[1] Rate by $\Delta$ P. Olefin analysis by G.L.C. indicating $\sim 57$ percent 1-butene, (trans/cis) 2-butenes $\simeq 0.64$. Wall conditioning was essential. Stoichiometry confirmed by titration of acid.

[2] Rates by G.L.C. Olefin product ratio was: 1-butene/2-butene $=1.32$ or about 57 percent but-1-ene. See comments on $t$-butyl acetate. 
Reaction: 1-Methyl-3-butenyl ethanoate

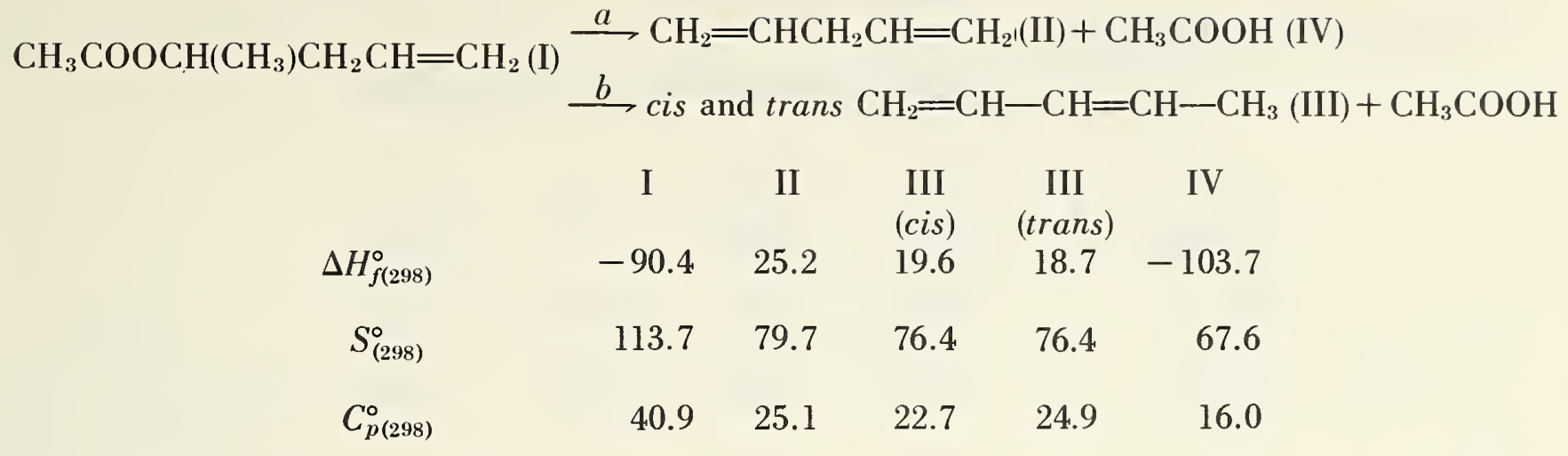

$$
\begin{aligned}
& \Delta S^{\circ}=33.6 \mathrm{~g} / \mathrm{mole} \\
& \Delta H^{\circ}=11.9 \mathrm{kcal} / \mathrm{mole} \\
& \Delta C_{p}^{\circ}=0.2 \mathrm{~g} / \text { mole }
\end{aligned}
$$

\begin{tabular}{cccccccc} 
Path & $\log \boldsymbol{A}$ & $\boldsymbol{E}$ & $\begin{array}{c}\log \boldsymbol{k}_{T} \\
(600)\end{array}$ & Conditions & System & Surface & References \\
\hline$+b$ & 13.0 & 44.41 & -3.17 & $\begin{array}{l}564-628^{\circ} \mathrm{K} \\
82-245 \text { torr }\end{array}$ & static & $\begin{array}{c}<\% \%(\text { con- } \\
\text { ditioned) }\end{array}$ & [1] A. Maccoll, J. Chem. \\
Soc. $227(1964)$.
\end{tabular}

Preferred: $\log k=13.0-44.4 / \theta$

Estimated: $\log A=12.6$

$\log A_{b}=12.4$ (see section II-4.0).

\section{Experimental}

[1] Rates by $\Delta \mathrm{P} .\left(P_{f} / P_{i}\right) \sim 1.68 \rightarrow 1.88$ taken as an indication of olefin polymerization. G.L.C. analysis on olefin gave (trans/cis) $\sim 7 / 3$ and $(1,4$-pentadiene/1,3-pentadiene) $\simeq 1 / 2$. The latter ratio changed (increased) with time after completion of the reaction indicating polymerization of the 1,3 diolefin. 
Reaction: 1-Methylbutyl ethanoate (2-pentyl acetate)

$$
\begin{aligned}
& \stackrel{a}{\longrightarrow} \mathrm{CH}_{3} \mathrm{COOH}+\text { (cis and trans) } \mathrm{CH}_{3} \mathrm{CH}=\mathrm{CHCH}_{2} \mathrm{CH}_{3} \text { (II) } \\
& \mathrm{CH}_{3} \mathrm{COOCH}\left(\mathrm{CH}_{3}\right) \mathrm{C}_{3} \mathrm{H}_{7} \text { (I) } \stackrel{b}{\longrightarrow} \mathrm{CH}_{3} \mathrm{COOH}(\mathrm{IV})+\mathrm{CH}_{2}=\mathrm{CH}\left(\mathrm{CH}_{2}\right)_{2} \mathrm{CH}_{3} \text { (III) } \\
& \begin{array}{ccccc} 
& \text { I } & \text { II (cis) } & \text { III } & \text { IV } \\
\Delta H_{f(298)}^{\circ} & -120.4 & -6.7 & -4.9 & -103.7
\end{array} \\
& \begin{array}{lllll}
S_{p(298)}^{\circ} & 115.3 & 82.8 & 82.8 & 67.6
\end{array} \\
& \begin{array}{lllll}
C_{p(298)}^{\circ} & 43.7 & 24.2 & 26.2 & 16.0
\end{array}
\end{aligned}
$$

\begin{tabular}{|c|c|c|c|c|c|c|c|}
\hline Path & $\log A$ & $\boldsymbol{E}$ & $\underset{(684)}{\log \boldsymbol{k}_{T}}$ & Conditions & System & Surface & References \\
\hline
\end{tabular}

\footnotetext{
$b$

$\Delta S^{\circ}=35.1 \mathrm{~g} / \mathrm{mole}$

$\Delta H^{\circ}=11.8 \mathrm{kcal} / \mathrm{mole}$

$\Delta C^{\circ}=-1.5 \mathrm{~g} /$ mole
}

$\begin{array}{llllll}a+b & 12.73 \quad 43.7 & -1.21 & 650-710^{\circ} \mathrm{K} & \text { flow }\end{array}$

[1] J. C. Scheer, E. C. Kooyman, and F. L. J. Sixma, Rec. Trav. Chim. des Pays-Bas 82, 1123 (1963).

Preferred:

$\log k=12.7-43.7 / \theta$

Comments: Estimated: $\log A_{(a)}=12.35$

$\log A_{(b)}=12.6$ (see section II-4.0).

Experimental

[1] See $t$-butyl acetate. The observed olefin product ratio was: $(1$-ene/2-ene $) \simeq 1.2$, or about 55 percent pent-1-ene. 
Reaction: 1-Ethylpropyl ethanoate (3-pentyl acetate)

$\begin{array}{rrrr}\mathrm{CH}_{3} \mathrm{COOCH}\left(\mathrm{C}_{2} \mathrm{H}_{5}\right)_{2}(\mathrm{I}) \longrightarrow \mathrm{CH}_{3} \mathrm{COOH}(\mathrm{II})+\mathrm{CH}_{3} \mathrm{CH}=\mathrm{CHCH}_{2} \mathrm{CH}_{3}(\mathrm{II}) \\ \Delta H_{f(298)}^{\circ} & -119.5 & -103.7 & \text { II } \\ S_{(298)}^{\circ} & 115.3 & 67.6 & -7.6 \\ C_{p\left(298^{\prime}\right.}^{\circ} & 43.7 & 16.0 & 25.9\end{array}$

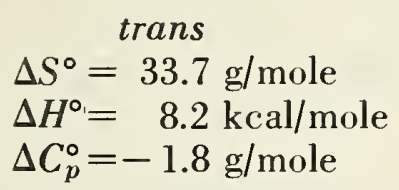

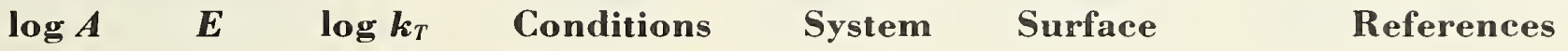

$13.09 \quad 44.7 \quad-1.20 \quad 650-710^{\circ} \mathrm{K} \quad$ flow

[1] J. C. Scheer, E. C.

Kooyman, and F. L. J.

Sixma, Rec. Trav. Chim. des Pays-Bas 82, 1123 (1963).

Preferred:

$\log k=13.09-44.7 / \theta$

$\log A_{\text {est }}=12.9$ (see section II-4.0).

\section{Experimental}

[1] (see $t$-butyl acetate.) Rates based on G.L.C. analysis. 
Reaction: Cyclohexyl ethanoate (cyclohexyl acetate)

\begin{tabular}{|c|c|c|c|}
\hline$\Delta H_{f(298)}^{\circ}$ & $\begin{array}{c}\mathrm{I} \\
-116.3\end{array}$ & $\begin{array}{c}\text { II } \\
-103.7\end{array}$ & $\begin{array}{l}\text { III } \\
-1.7\end{array}$ \\
\hline$S_{(298)}^{\circ}$ & 105.6 & 67.6 & 74.3 \\
\hline$C_{p(298)}^{\circ}$ & 40.7 & 16.0 & 25.3 \\
\hline
\end{tabular}

$\Delta S^{\circ}=36.3 \mathrm{~g} / \mathrm{mole}$

$\Delta H^{\circ}=10.9 \mathrm{kcal} / \mathrm{mole}$

$\Delta C_{p}^{\circ}=0.6 \mathrm{~g} / \mathrm{mole}$

\begin{tabular}{|c|c|c|c|c|c|c|}
\hline $\log A$ & $\boldsymbol{E}$ & $\begin{array}{c}\log \boldsymbol{k}_{T} \\
(700)\end{array}$ & Conditions & System & Surface & References \\
\hline 11.56 & 40.3 & -1.01 & $\begin{array}{l}623-773^{\circ} \mathrm{K} \\
7-70 \text { torr }\end{array}$ & flow & & $\begin{array}{l}\text { [1] M. Kraus, N. Vavruska, } \\
\text { and V. Bǎzant, Collec- } \\
\text { tion Czechoslov. Chem. } \\
\text { Commun. 22, 484 (1957). }\end{array}$ \\
\hline
\end{tabular}

Preferred:

$\log k=13.0-44.9 / \theta$. Rate constants are reliable.,

Comments: The $A$-factor reported seems about an order of magnitude too low. Estimated parameters are preferred (see section II-4.0).

\section{Experimental}

[1] Rates by chemical analysis on cyclohexene. 
Reaction: 1,2-Dimethylbutyl ethanoate $(\alpha, \beta$-dimethyl- $n$-butyl acetate)

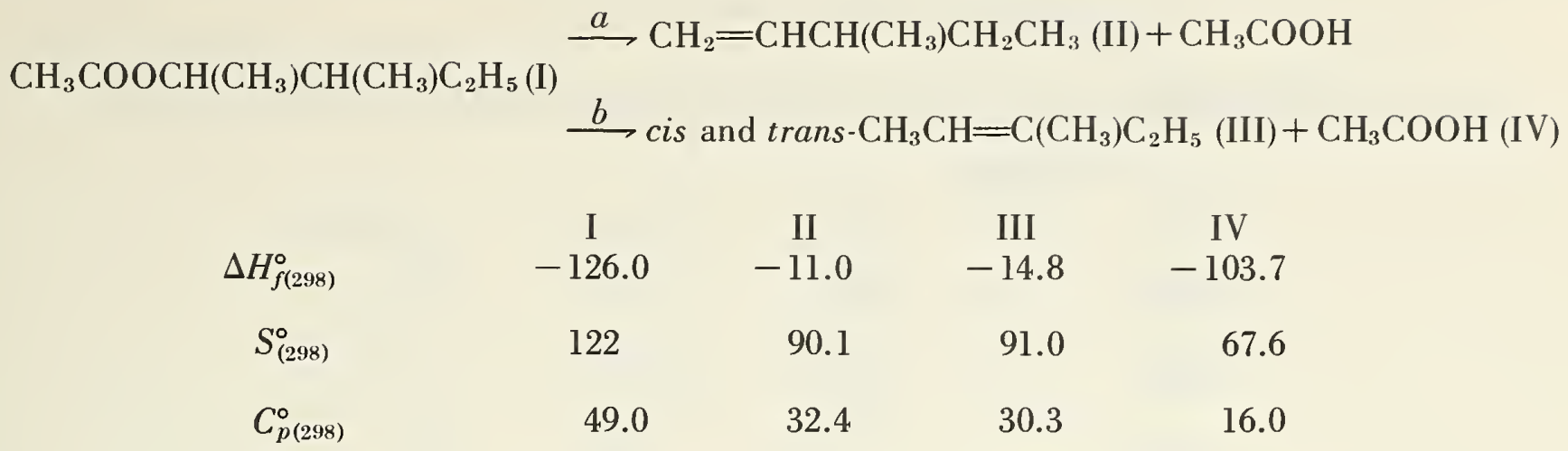

$$
\begin{gathered}
a \\
\Delta S^{\circ}=35.7 \mathrm{~g} / \mathrm{mole} \\
\Delta H^{\circ}=11.3 \mathrm{kcal} / \mathrm{mole} \\
\Delta C_{p}^{\circ}=-0.6 \mathrm{~g} / \mathrm{mole}
\end{gathered}
$$

\begin{tabular}{|c|c|c|c|c|c|c|}
\hline $\log A$ & $\boldsymbol{E}$ & $\underset{(684)}{\log \boldsymbol{k}_{T}}$ & Conditions & System & Surface & References \\
\hline 12.60 & 43.2 & -1.21 & $650-710^{\circ} \mathrm{K}$ & flow & & $\begin{array}{l}\text { [1] J. C. Scheer, E. C. } \\
\text { Kooyman, and F. L. J. } \\
\text { Sixma, Rec. Trav. } \\
\text { Chim. des Pays-Bas } 82, \\
1123 \text { (1963). }\end{array}$ \\
\hline
\end{tabular}

Preferred:

$\log k=12.6-43.2 / \theta$.

Estimated: $\log A_{(a)}=12.6$

$\log A_{(b)}=12.0$ (see section II-4.0).

\section{Experimental}

[1] Rates based on G.L.C. analysis. Isomer ratio: $\left(\frac{1 \text {-ene }}{2 \cdot \text { enes }}\right) \simeq 3.2$, or about 76 percent 3-methyl-1-pentene. 
Reaction: 1-Isopropyl-2-methylpropyl ethanoate ( $\alpha$-isopropyl $\beta$-methyl- $n$-propyl acetate)

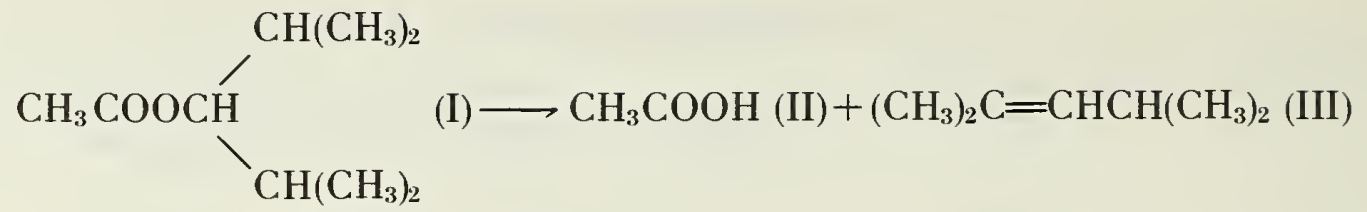

\begin{tabular}{crrr} 
& \multicolumn{1}{c}{ I } & \multicolumn{1}{c}{ II } & \multicolumn{1}{c}{ III } \\
$\Delta H_{f(298)}^{\circ}$ & -132.0 & -103.7 & -22.6 \\
$S_{(298)}^{\circ}$ & 128.7 & 67.6 & 95.3 \\
$C_{p(298)}^{\circ}$ & 54.3 & 16.0 & 38.6
\end{tabular}

$\Delta S^{\circ}=34.2 \mathrm{~g} / \mathrm{mole}$
$\Delta H^{\circ}=5.7 \mathrm{kcal} / \mathrm{mole}$
$\Delta C_{p}^{\circ}=0.3 \mathrm{~g} /$ mole

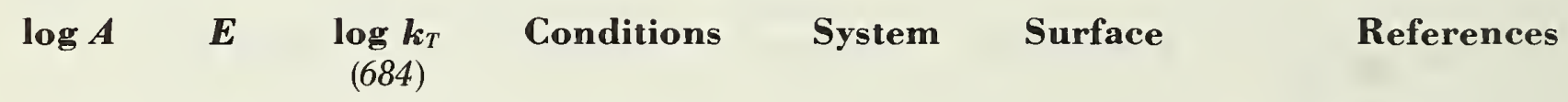

12.84 $44.7 \quad-1.43 \quad 650-710^{\circ} \mathrm{K} \quad$ flow

[1] J. C. Scheer, E. C.

Kooyman, and F. L. J. Sixma, Rec. Trav.

Chim. des Pays-Bas 82, 1123 (1963).

Preferred:

$\log k=12.84-44.7 / \theta$.

$\log A_{\text {est }}=12.3$ (see section II-4.0).

\section{Experimental}

[1] Rates based on G.L.C. analysis (see $t$-butyl acetate). 
Reaction: 1-Methylhexyl ethanoate (2-heptyl acetate)

$$
\begin{aligned}
& \begin{array}{ccccc}
\mathrm{CH}_{3} \mathrm{COOCH}\left(\mathrm{CH}_{3}\right)\left(\mathrm{CH}_{2}\right)_{4} \mathrm{CH}_{3}(\mathrm{I}) & \stackrel{(a)}{\longrightarrow} \mathrm{CH}_{3} \mathrm{COOH}+\mathrm{CH}_{2}=\mathrm{CH}\left(\mathrm{CH}_{2}\right)_{4} \mathrm{CH}_{3} \text { (II) } \\
\cline { 2 - 2 } & \stackrel{(b)}{\longrightarrow} \mathrm{CH}_{3} \mathrm{COOH}(\mathrm{IV})+\text { cis } & \text { and trans } & \mathrm{CH}_{3} \mathrm{CH}= \\
& \text { I } & \text { II } & \text { III cis } & \text { IV } \\
\Delta H_{f(298)}^{\circ} & -130.5 & -14.8 & -16.6 & -103.7
\end{array} \\
& \begin{array}{lllll}
S_{(298)}^{\circ} & 134.1 & 101.6 & 101.6 & 67.6
\end{array} \\
& \begin{array}{lllll}
C_{p(298)}^{\circ} & 54.7 & 37.2 & 35.2 & 16.0
\end{array}
\end{aligned}
$$

\begin{tabular}{|c|c|c|c|c|c|c|c|}
\hline Path & $\log A$ & $\boldsymbol{E}$ & $\begin{array}{c}\log \boldsymbol{k}_{T} \\
(684)\end{array}$ & Conditions & System & Surface & References \\
\hline$a+b$ & 13.32 & 45.3 & -1.15 & $650-710^{\circ} \mathrm{K}$ & flow & & $\begin{array}{l}\text { [1] J. C. Scheer, E. C. } \\
\text { Kooyman, and } \\
\text { F. L. J. Sixma, Rec. } \\
\text { Trav. Chim. des } \\
\text { Pays-Bas 82, } 1123 \\
\text { (1963). }\end{array}$ \\
\hline
\end{tabular}

$$
\begin{aligned}
& \Delta S^{\circ}=35.1 \mathrm{~g} / \mathrm{mole} \\
& \Delta H^{\circ}=12.0 \mathrm{kcal} / \mathrm{mole} \\
& \Delta C_{p}^{\circ}=-1.5 \mathrm{~g} / \mathrm{mole}
\end{aligned}
$$

Preferred:

$\log k=12.73-43.7 / \theta$.

Estimated: $\log A_{(a)}=12.6$

$\log A_{(b)}=12.3$ (see section II-4.0).

Comments: Arrhenius parameters seem slightly high although the rate constant agrees well with 2-pentyl acetate as one would expect. The 2-pentyl actate parameters are preferred.

\section{Experimental}

[1] Rates and analysis by G.L.C. Isomer ratios: $\left(\frac{1 \text {-heptene }}{2 \text {-heptene }}\right)=1.2$, or 58 percent 1 -heptene. (See comments on $t$-butyl acetate.) 
Reaction: 1-Ethylpentyl ethanoate (3-heptyl acetate)

$\mathrm{CH}_{3} \mathrm{COOCH}\left(\mathrm{C}_{2} \mathrm{H}_{5}\right) \mathrm{C}_{4} \mathrm{H}_{9}$ (I)

$\stackrel{(a)}{\longrightarrow} \mathrm{CH}_{3} \mathrm{COOH}(\mathrm{IV})+\mathrm{CH}_{3} \mathrm{CH}=\mathrm{CH}\left(\mathrm{CH}_{2}\right)_{3} \mathrm{CH}_{3}$ (II) (cis, trans)

$\stackrel{(b)}{\longrightarrow} \mathrm{CH}_{3} \mathrm{COOH}+\mathrm{CH}_{3} \mathrm{CH}_{2} \mathrm{CH}=\mathrm{CH}\left(\mathrm{CH}_{2}\right)_{2} \mathrm{CH}_{3}$ (III) (cis, trans)

$\begin{array}{crccr} & \text { I } & \text { II cis } & \text { III trans } & \text { IV } \\ \Delta H_{f(298)}^{\circ} & -129.5 & -16.6 & -17.6 & -103.7 \\ S_{(298)}^{\circ} & 134.1 & 101.6 & 100.6 & 67.6 \\ C_{p(298)}^{\circ} & 54.7 & 35.2 & 36.5 & 16.0\end{array}$

$\begin{aligned} & a, \text { cis } \\ & \Delta S^{\circ}=35.1 \mathrm{~g} / \mathrm{mole} \\ & \Delta H^{\circ}=9.2 \mathrm{kcal} / \mathrm{mole} \\ & \Delta C_{p}^{\circ}=-3.5 \mathrm{~g} / \mathrm{mole}\end{aligned}$

$\begin{array}{llllll}\log A & E & \log k_{r} & \text { Conditions } & \text { System } & \text { Surface }\end{array}$ (684)

$13.93 \quad 46.9 \quad-1.05 \quad 650-710^{\circ} \mathrm{K} \quad$ flow
[1] J. C. Scheer, E. C.

Kooyman, and F. L. J. Sixma, Rec. Trav. Chim. des Pays-Bas 82, 1123 (1963).

Preferred:

$\log k=12.75-43.2 / \theta$.

Estimated: $\log A_{a}=12.35$

$\log A_{b}=12.3$ (see section II-4.0).

Comments: Arrhenius parameters are certainly high.

\section{Experimental}

[1] Rates based on G.L.C. analysis. Isomer ratio $\left(\frac{3 \text {-heptene }}{2 \text {-heptene }}\right) \simeq 1.0$ or close to 50 percent 3 -heptene (See $t$-buty] acetate). 
Reaction: 1-Propylbutyl ethanoate (4-heptyl acetate)

$\mathrm{CH}_{3} \mathrm{COOCH}\left(\mathrm{C}_{3} \mathrm{H}_{7}\right)_{2}$ (I) $\longrightarrow \mathrm{CH}_{3} \mathrm{COOH}(\mathrm{III})+$ cis and trans $\mathrm{CH}_{3} \mathrm{CH}_{2} \mathrm{CH}=\mathrm{CH}\left(\mathrm{CH}_{2}\right)_{2} \mathrm{CH}_{3}(\mathrm{II})$

\begin{tabular}{crrrr} 
& \multicolumn{1}{c}{ I } & II cis & II trans & III \\
$\Delta H_{f(298)}^{\circ}$ & -129.4 & -16.6 & -17.6 & -103.7 \\
$S_{(298)}^{\circ}$ & 134.1 & 101.6 & 100.6 & 67.6 \\
$C_{p(298)}^{\circ}$ & 54.7 & 35.2 & 36.5 & 16.0
\end{tabular}

$$
\begin{gathered}
\text { trans } \\
\Delta S^{\circ}=34.1 \mathrm{~g} / \mathrm{mole} \\
\Delta H^{\circ}=8.1 \mathrm{kcal} / \mathrm{mole} \\
\Delta C_{p}^{\circ}=-3.2 \mathrm{~g} / \mathrm{mole}
\end{gathered}
$$

\begin{tabular}{|c|c|c|c|c|c|c|}
\hline $\log A$ & $\boldsymbol{E}$ & $\begin{array}{c}\log \boldsymbol{k}_{T} \\
(684)\end{array}$ & Conditions & System & Surface & References \\
\hline 13.32 & 44.9 & -1.01 & $650-710^{\circ} \mathrm{K}$ & flow & & $\begin{array}{l}\text { [1] J. C. Scheer, E. C. } \\
\text { Kooyman, and F. L. J. } \\
\text { Sixma, Rec. Trav. } \\
\text { Chim. des Pays-Bas } \\
\text { 82, } 1123 \text { (1963). }\end{array}$ \\
\hline
\end{tabular}

Preferred:

$\log k=12.6-42.7 / \theta$.

Comments: Arrhenius parameters are slightly high although the rate constant seems reasonable (compare with 3-heptyl acetate). The transition state estimate of $\log A$ is preferred (see section II-4.0).

\section{Experimental}

[1] Rates based on G.L.C. analysis. (See $t$-butyl acetate.) 
Reaction: Endo-2-acetoxybornane (bornyl acetate)

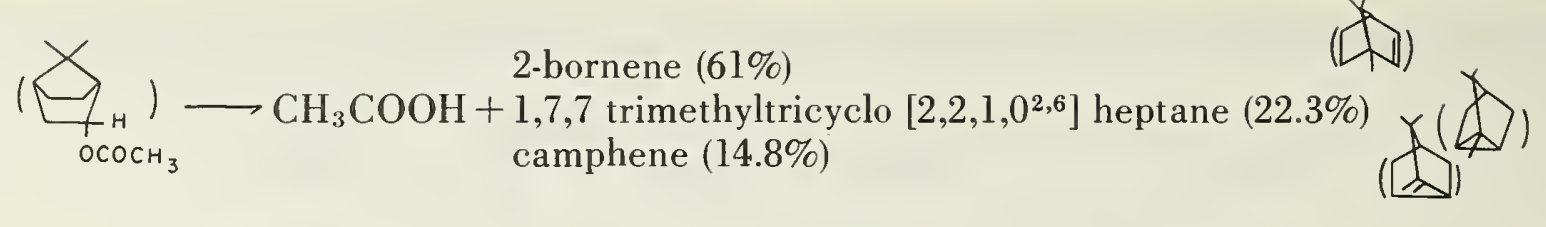

$$
\left.\begin{array}{c}
\Delta H_{f(298)}^{\circ} \\
S_{(298)}^{\circ} \\
C_{p(298)}^{\circ}
\end{array}\right\} \text { see exo-2-acetoxybornane (isobornyl acetate) }
$$

$\Delta S^{\circ}=$

$\Delta H^{\circ}=$

$\Delta C_{p}^{\circ}=$

\begin{tabular}{|c|c|c|c|c|c|c|}
\hline $\log A$ & $\boldsymbol{E}$ & $\begin{array}{c}\log \boldsymbol{k}_{T} \\
(620)\end{array}$ & Conditions & System & Surface & References \\
\hline 11.99 & 45.3 & -3.96 & $\begin{array}{l}603-643^{\circ} \mathrm{K} \\
132-242 \text { torr }\end{array}$ & static & none & $\begin{array}{l}\text { [1] E. U. Emovan, J. Chem. } \\
\text { Soc. (B), } 588 \text { (1966). }\end{array}$ \\
\hline
\end{tabular}

Preferred: (See exo-2-acetoxybornane, isobornyl acetate).

\section{Experimental}

[1] Products were analyzed by G.L.C. Rates were followed by $\Delta \mathrm{P}$ measurements and the rate constants were based on titration of the acetic acid product. Added cyclohexene reduced the reaction rates by about 3-8 percent. Products were 61 percent bornylene, 23.3 percent tricyclene, and 14.8 percent camphene. See discussion for bornyl chloride. 
Reaction: Exo-2-acetoxybornane (isobornyl acetate)

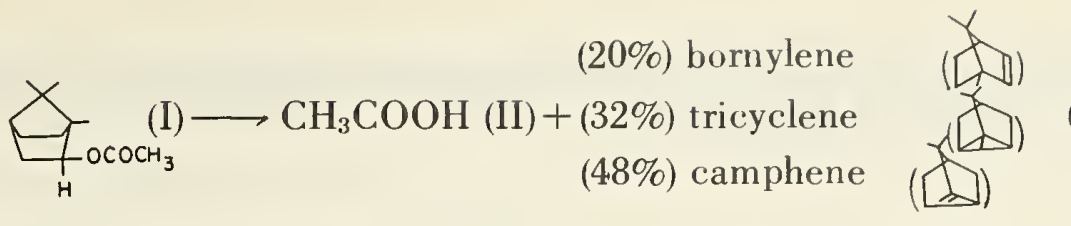

\begin{tabular}{crrr} 
& I & II & III \\
$\Delta H_{f(298)}^{\circ}$ & -117.9 & -103.7 & 0.2 \\
$S_{(298)}^{\circ}$ & 125.4 & 67.6 & 92 \\
$C_{p(298)}^{\circ(29)}$ & & 16.0 & \\
\hline
\end{tabular}

$\Delta S^{\circ}=34.2 \mathrm{~g} / \mathrm{mole}$

$\Delta H^{\circ}=14.4 \mathrm{kcal} / \mathrm{mole}$

$\Delta C_{p}^{\circ}=$
$\log A \quad E \quad \log k_{T}$
Conditions
System
Surface
References
(595.)
11.64 $42.02-4.06 \quad 571-618^{\circ} \mathrm{K} \quad$ static none 104-216 torr
[1] E. U. Emovan, J. Chem.
Soc. (B), 588 (1966).

\section{Preferred:}

Suspect.

Comments: The rate constant parameters appear much too low. A transition state calculation gives $\log A_{\text {est }}=13.0$ for the bornylene product. Heterogeneous effects may well be appreciable.

\section{Experimental}

[1] Products were analyzed by G.L.C. and were about 20 percent bornylene, 32 percent tricyclene, and 48 percent camphene. Rate constants were obtained from titration of the acetic acid. Added cyclohexene lowered the rates by about 3 percent. See discussion for bornyl chloride. 
Reaction: 1-Methyl-2-methoxyethyl ethanoate (1-methoxy-2-propyl acetate)

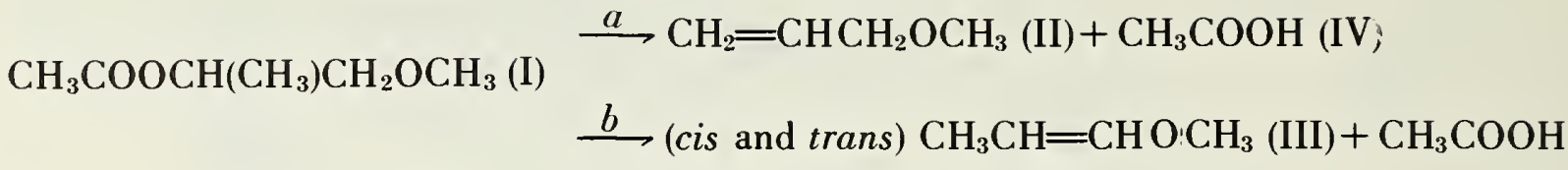

\begin{tabular}{crrrr} 
& \multicolumn{1}{c}{ I } & II & III cis & \multicolumn{1}{c}{ IV } \\
$\Delta H_{f(298)}^{\circ}$ & -142.4 & -27.2 & -31.4 & -103.7 \\
$S_{(298)}^{\circ}$ & 115.7 & 82.8 & 82.2 & 67.6 \\
$C_{p(298)}^{\circ}$ & 41.1 & 23.7 & 22.3 & 16.0
\end{tabular}

\footnotetext{
$\Delta S^{\circ}=34.7 \mathrm{~g} / \mathrm{mole}$

$\Delta H^{\circ}=11.5 \mathrm{kcal} / \mathrm{mole}$

$\Delta C_{p}^{\circ}=-1.4 \mathrm{~g} / \mathrm{mole}$
}

\begin{tabular}{|c|c|c|c|c|c|c|}
\hline $\log A$ & $\boldsymbol{E}$ & $\begin{array}{c}\log \boldsymbol{k}_{T} \\
(648)\end{array}$ & Conditions & System & Surface & References \\
\hline 13.05 & 46.6 & -2.67 & $650-710^{\circ} \mathrm{K}$ & flow & & $\begin{array}{l}\text { [1] J. C. Scheer, E. C. } \\
\text { Kooyman, and F. L. J. } \\
\text { Sixma, Rec. Trav. } \\
\text { Chim. des Pays-Bas } 82, \\
1123 \text { (1963). }\end{array}$ \\
\hline
\end{tabular}

Preferred:

$\log k=13.05-46.6 / \theta$.

Estimated: $\log A_{(a)}=12.6$

$\log A_{(b)}=12.35$ (see section II-4.0).

\section{Experimental}

[1] Rates based on G.L.C. analysis. Isomer product ratios (unrecorrected for detector sensitivity) were $\left(\frac{3-\text { methoxypropene }}{1 \text {-methoxypropene }}\right) \simeq 1.35$ or about 58 percent 3 -methoxypropene. 
Reaction: 1-Methyl-3-oxobutyl ethanoate

$\mathrm{CH}_{3} \mathrm{COOCH}\left(\mathrm{CH}_{3}\right) \mathrm{CH}_{2} \mathrm{COCH}_{3}$ (I) $\longrightarrow \mathrm{CH}_{3} \mathrm{COCH}=\mathrm{CHCH}_{3}$ (II) $+\mathrm{CH}_{3} \mathrm{COOH}$ (III)

\begin{tabular}{crrr} 
& \multicolumn{1}{c}{ I } & \multicolumn{1}{c}{ II } & \multicolumn{1}{c}{ III } \\
$\Delta H_{f(298)}^{\circ}$ & -148.1 & -41.4 & -103.7 \\
$S_{(298)}^{\circ}$ & 120.8 & 86.2 & 67.6 \\
$C_{p(298)}^{\circ}$ & 45.0 & 26.8 & 16.0
\end{tabular}

$\Delta S^{\circ}=33.0 \mathrm{~g} / \mathrm{mole}$

$\Delta H^{\circ}=3.0 \mathrm{kcal} / \mathrm{mole}$

$\Delta C_{p}^{\circ}=-2.2 \mathrm{~g} / \mathrm{mole}$

\begin{tabular}{ccccccc}
$\log \boldsymbol{A}$ & $\boldsymbol{E}$ & $\begin{array}{c}\log \boldsymbol{k}_{T} \\
(550)\end{array}$ & \multicolumn{1}{c}{ Conditions } & System & \multicolumn{1}{c}{ Surface } & \multicolumn{1}{c}{ References } \\
11.88 & 37.4 & -3.00 & $\begin{array}{l}529-570 \circ \mathrm{K} \\
89-302 \text { torr }\end{array}$ & static & $\begin{array}{r}<5 \% \text { (con- } \\
\text { ditioned) }\end{array}$ & $\begin{array}{r}\text { [1] A. Maccoll, J. Chem. } \\
\text { Soc., 227 (1964). }\end{array}$
\end{tabular}

\section{Preferred:}

$\log k=11.9-37.4 / 0$.

Comments: Transition state calculation (assuming appreciable enolic form (or conjugation) of the ketone in the transition state) gives $\log A_{\text {est }} \simeq 11.6$ (see section II-4.0).

\section{Experimental}

[1] Rates from $\Delta \mathrm{P}$. Analysis of olefin product by G.L.C. The low $A$-factor and exclusive secondary-H-elimination observed were taken as evidence for polarity enhancement in the transition state by the $\beta$-carbonyl group.*

*This conclusion is supported by the lowered $A$-factor (i.e., relative to isopropyl acetate) and suggests the loss of one additional internal rotation in the transition state. The activation energy lowering of about $7 \mathrm{kcal} / \mathrm{mole}$ relative to sec-butyl acetate also supports the highly polar transition state proposed. 
Reaction: 1-Phenylethyl ethanoate ( $\alpha$-phenylethyl acetate)

$$
\mathrm{CH}_{3} \mathrm{COOCHCH}_{3}(\varnothing)(\mathrm{I}) \longrightarrow \varnothing \mathrm{CH}=\mathrm{CH}_{2}(\mathrm{II})+\mathrm{CH}_{3} \mathrm{COOH}(\mathrm{III})
$$

\begin{tabular}{cccr} 
& I & II & \multicolumn{1}{c}{ III } \\
$\Delta H_{f(298)}^{\circ}$ & -79.1 & 35.4 & -103.7 \\
$S_{(298)}^{\circ}$ & 116.5 & 82.5 & 67.6 \\
$C_{p(298)}^{\circ}$ & 45.4 & 29.6 & 16.0
\end{tabular}

$\Delta S^{\circ}=33.6 \mathrm{~g} / \mathrm{mole}$

$\Delta H^{\circ}=10.8 \mathrm{kcal} / \mathrm{mole}$

$\Delta C_{p}^{\circ}=0.2 \mathrm{~g} / \mathrm{mole}$

\begin{tabular}{|c|c|c|c|c|c|c|}
\hline $\log A$ & $\boldsymbol{E}$ & $\begin{array}{c}\log \boldsymbol{k}_{T} \\
(610)\end{array}$ & Conditions & System & Surface & References \\
\hline 12.83 & 43.7 & -2.83 & $\begin{array}{l}585-641^{\circ} \mathrm{K} \\
6-200 \text { torr }\end{array}$ & static & 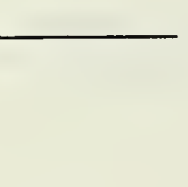 & $\begin{array}{l}\text { [1] R. Taylor, G. G. } \\
\text { Smith, W. H. Wetzel, } \\
\text { J. Am. Chem. Soc. 84, } \\
4817 \text { (1962). }\end{array}$ \\
\hline
\end{tabular}

Preferred:

$\log k=12.8-43.7 / \theta$.

Comments: Assuming no benzyl resonance in the transition state gives $\log A_{\text {est }}=12.6$. This is consistent with the fact that the activation energy has not been lowered appreciably from the ethyl acetate value by the phenyl substitution. The actual lowering is more comparable to that of alkyl substitution. (See ethyl and isopropyl acetate.)

\section{Experimental}

[1] Rates by $\Delta \mathrm{P}$. Identification of products by I.R. 
Reaction: 1-Arylethyl ethanoates (1-phenylethyl derivatives)

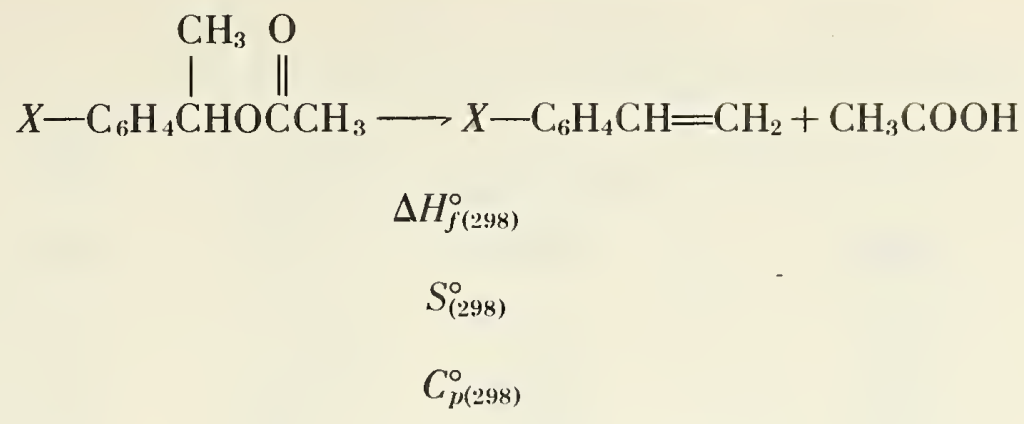

$\Delta S^{\circ}=$
$\Delta H^{\circ}=$
$\Delta C_{p}^{\circ}=$

$\begin{array}{llll}\log A & \boldsymbol{E} & \log \boldsymbol{k}_{T} & \text { Conditions }\end{array}$ (600)

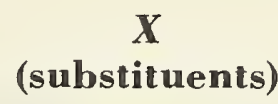

Surface

$p$ - $\mathrm{MeO}$

$\begin{array}{llll}12.52 & 41.7 & -2.80 & 571-617\end{array}$

$\begin{array}{llll}13.08 & 43.4 & -2.34 & 588-638\end{array}$

$13.03 \quad 43.6 \quad-2.59 \quad 581-640$

$13.17 \quad 44.1 \quad-2.50 \quad 591-642$

$12.92 \quad 43.4 \quad-2.50 \quad 589-642$

$12.97 \quad 43.6 \quad-2.52 \quad 587-642$

$12.80 \quad 43.2 \quad-2.68 \quad 585-637$

$12.94 \quad 43.8 \quad-2.63 \quad 587-642$

$12.98 \quad 43.8 \quad-2.59 \quad 587-642$

$\begin{array}{llll}12.80 & 43.6 & -2.33 & 603-657\end{array}$

$\begin{array}{llll}12.83 & 43.7 & -2.33 & 603-658\end{array}$

$12.84,44.1 \quad-2.96 \quad 585-637$

$12.92 \quad 44.6 \quad-2.56 \quad 603-658$

$\begin{array}{llll}12.87 & 44.1 & -2.43 & 603-659\end{array}$

$\begin{array}{llll}12.84 & 44.4 & -2.57 & 604-658\end{array}$

$12.83 \quad 44.4 \quad-2.70 \quad 603-653$

$12.89 \quad 44.7 \quad-2.62 \quad 599-657$

12.69

$\begin{array}{lll}44.5 & -2.75 & 601-657\end{array}$

\section{References}

[1] R. Taylor, G. G.

Smith, and W. H.

Wetzel, J. Am. Chem.

Soc. 84, 4817 (1962).

\section{Preferred:}

Reliable as reported.

Comments: The heterolytic nature of the transition state in the ester eliminations is strongly supported by these results. Transition state estimate of $A$ for all the above compounds gives $\log A_{\text {est }}=12.7$ (see section II-4.0).

\section{Experimental}

[1] All rates obtained from $\Delta \mathrm{P}$ measurements. Meta and para compounds were found to fit a linear free energy $\rho$ - $\sigma^{+}$plot very well, giving $\rho=-0.66$ at $600^{\circ} \mathrm{K}$, where the $\sigma^{+}$constants are those given by H. C. Brown and Y. Okamoto, J. Am. Chem. Soc. 80, 4979 (1958).

${ }^{*} 1$-(2-Fluorenyl)-ethyl acetate

**1-( $\alpha$-Naphthyl)-ethyl acetate 
Reaction: 1,2-Diarylethyl ethanoates (meta or para substituted 1,2-diphenylethyl acetates)

\begin{tabular}{|c|c|c|c|}
\hline$\Delta H_{f(298)}^{\circ}$ & $\begin{array}{c}\mathrm{I} \\
X=Y=H \\
-56.6\end{array}$ & $\begin{array}{c}\text { trans II } \\
X=Y=H \\
58.6\end{array}$ & $\begin{array}{c}\text { III } \\
-103.7\end{array}$ \\
\hline$S_{(\mathbf{2 9 8})}^{\circ}$ & 146.2 & 108.3 & 67.6 \\
\hline$C_{p(\mathbf{2 9 8})}^{\circ}$ & 64.4 & 48.9 & 16.0 \\
\hline
\end{tabular}

$\Delta S^{\circ}=29.7 \mathrm{~g} /$ mole

$\Delta H^{\circ}=11.5 \mathrm{kcal} / \mathrm{mole}$

$\Delta C_{p}^{\circ}=0.5 \mathrm{~g} / \mathrm{mole}$

\begin{tabular}{|c|c|c|c|c|c|c|c|}
\hline \multirow[b]{2}{*}{$\log A$} & \multirow[b]{2}{*}{$\boldsymbol{E}$} & \multirow[b]{2}{*}{$\begin{array}{c}\log k_{T} \\
(600)\end{array}$} & \multicolumn{5}{|c|}{ Substituents } \\
\hline & & & Conditions & System & $\mathbf{X}$ & $\mathbf{Y}$ & References \\
\hline 13.05 & 43.3 & -2.72 & $575-625^{\circ} \mathrm{K}$ & static & $\mathrm{H}$ & $\mathrm{H}$ & [1] G. G. Smith, F. D. \\
\hline 12.56 & 40.6 & -2.23 & $15-150$ torr & & $p-\mathrm{MeO}$ & $p \cdot \mathrm{Cl}$ & Bagley, and R. \\
\hline 12.54 & 40.6 & -2.25 & & & $p-\mathrm{MeO}$ & $\mathrm{H}$ & Taylor, J. Am. Chem. \\
\hline 12.67 & 41.7 & -2.52 & & & $p-\mathrm{Me}$ & $\mathrm{H}$ & Soc. 83, 3647 (1961). \\
\hline 13.07 & 43.3 & -2.70 & & & $\mathrm{H}$ & $m-\mathrm{Cl}$ & \\
\hline 13.06 & 43.3 & -2.71 & & & $\mathrm{H}$ & $p \cdot \mathrm{Cl}$ & \\
\hline 12.94 & 43.0 & -2.72 & & & $\mathrm{H}$ & $p$-Me & \\
\hline 12.58 & 42.1 & -2.76 & & & $\mathrm{H}$ & $p \cdot \mathrm{MeO}$ & \\
\hline 12.56 & 42.1 & -2.78 & & & $p-\mathrm{Cl}$ & $\mathrm{H}$ & \\
\hline 12.87 & 43.2 & -2.87 & & & $p$-Cl & $p \cdot \mathrm{MeO}$ & \\
\hline 12.96 & 43.8 & -3.00 & & & $m \cdot \mathrm{Cl}$ & $\mathrm{H}$ & \\
\hline
\end{tabular}

\section{Preferred:}

Reliable as reported.

Comments: For 1,2-diarylethyl acetate: $\log k=13.05-43.3 / \theta$. Transition state calculation of $A$ for all the above compounds gives $\log A_{\text {est }}=12.4$ (see section II-4.0).

\section{Experimental}

[1] Rates by $\Delta P$. Analysis by N.M.R. Various linear free energy $\sigma-\rho$ plots were made to show: (a) that substituents in the l-aryl ring have a greater effect on the rate than do substituents in the 2 -aryl ring; (b) that the rate is most strongly influenced by electron release to the $(\mathrm{C}-\mathrm{O})$ bond. Effects on the bonds being broken (i.e., the $\mathrm{C}-\mathrm{O}$ and $\beta-(\mathrm{C}-\mathrm{H})$ ) and not on those being formed (i.e., the olefinic double bond and the acid $\mathrm{O}-\mathrm{H}$ ) are most import ant in determining the rate of elimination. 
Reaction: 1-Methyl-2-chloroethyl ethanoate (1-chloroisopropyl acetate)

\begin{tabular}{|c|c|c|c|c|}
\hline \multirow{2}{*}{$\begin{array}{c}\mathrm{CH}_{3} \mathrm{COOCH}\left(\mathrm{CH}_{3}\right) \mathrm{CH}_{2} \mathrm{CJ}(\mathrm{I}) \\
\Delta H_{f(298)}^{\circ}\end{array}$} & \multicolumn{4}{|c|}{$\stackrel{b}{\longrightarrow} \mathrm{CH}_{3} \mathrm{COOH}+\mathrm{CH}_{2}=\mathrm{CHCH}_{2} \mathrm{Cl}$ (III) } \\
\hline & $\begin{array}{c}\text { I } \\
-116.5\end{array}$ & $\begin{array}{l}\text { II } \\
0.7\end{array}$ & $\begin{array}{l}\text { III } \\
-1.3\end{array}$ & $\begin{array}{c}\text { IV } \\
-103.7\end{array}$ \\
\hline$S_{(298)}^{\circ}$ & 106.1 & 71.5 & 73.2 & 67.6 \\
\hline$C_{p(298)}^{\circ}$ & 35.7 & 18.3 & 18.7 & 16.0 \\
\hline
\end{tabular}

$$
\begin{array}{cc}
a & b \\
\Delta S^{\circ}=33.0 & 34.7 \mathrm{~g} / \mathrm{mole} \\
\Delta H^{\circ}=13.5 & 11.5 \mathrm{kcal} / \mathrm{mole} \\
\Delta C_{p}^{\circ}=-1.4 & -1.0 \mathrm{~g} / \mathrm{mole}
\end{array}
$$

$\begin{array}{lllllll}\log \boldsymbol{A} & \boldsymbol{E} & \log \boldsymbol{k}_{T} & \text { Conditions } & \text { System } & \text { Surface } & \text { References }\end{array}$ (684)

$\begin{array}{llll}11.90 & 43.8 \quad-2.09 & 650-710^{\circ} \mathrm{K} \quad \text { flow }\end{array}$

[1] J. C. Scheer, E. C.

Kooyman, and F. L. J. Sixma, Rec. Trav. Chim. des Pays-Bas 82, 1123 (1963).

Preferred:

$\log k=12.8-46.6 / \theta$.

Comments: Estimated parameters are preferred (see section II-4.0).

Estimated: $\log A_{a}=12.6$

$\log A_{b}=12.6$.

\section{Experimental}

[1] Rates based on G.L.C. analysis. Ratio. of isomers: $\left(\frac{1 \text {-chloropropene }}{3 \text {-chloropropene }}\right) \approx_{1} 1.15$ or 153.4 percent 1-chloropropene (see $t$-butyl acetate). 
Reaction: 1-Methyl-2-dimethylaminoethyl ethonoate (2-dimethylamino-1-methyl ethyl ethanoate $\beta$-dimethylamino-2-propylacetate)

\begin{tabular}{|c|c|c|c|c|}
\hline \multirow[t]{2}{*}{$\mathrm{CH}_{3} \mathrm{COOCH}\left(\mathrm{CH}_{3}\right) \mathrm{CH}_{2}$} & \multicolumn{4}{|c|}{$\begin{array}{l}\stackrel{a}{\longrightarrow} \mathrm{CH}_{3} \mathrm{COOH}(\mathrm{IV})+\mathrm{CH}_{2}=\mathrm{CHCH}_{2} \mathrm{~N}\left(\mathrm{CH}_{3}\right)_{2}(\mathrm{II}) \\
\stackrel{b}{\longrightarrow} \mathrm{CH}_{3} \mathrm{COOH}+\text { cis, trans } \mathrm{CH}_{3} \mathrm{CH}=\mathrm{CHN}\left(\mathrm{CH}_{3}\right)_{2} \text { (III) }\end{array}$} \\
\hline & $\begin{array}{c}I \\
-101.9\end{array}$ & $\begin{array}{c}\text { II } \\
13.5\end{array}$ & $\begin{array}{c}\text { III trans } \\
11.6\end{array}$ & $\begin{array}{c}\text { IV } \\
-103.7\end{array}$ \\
\hline$S_{(298)}^{\circ}$ & 120.7 & 88.5 & 87.2 & 67.6 \\
\hline$C_{p(298)}^{\circ}$ & 47.2 & 30.1 & 32.6 & 16.0 \\
\hline
\end{tabular}

$\Delta S^{\circ}=35.4 \mathrm{~g} / \mathrm{mole}$

$\Delta H^{\circ}=11.7 \mathrm{kcal} / \mathrm{mole}$

$\Delta C_{p}^{\circ}=-1.1 \mathrm{~g} / \mathrm{mole}$

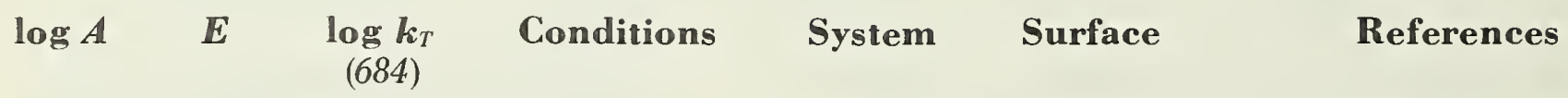

$12.14 \quad 42.2-1.34 \quad 650-710^{\circ} \mathrm{K} \quad$ flow

[1] J. C. Scheer, E. C.

Kooyman, and F. L. J.

Sixma, Rec. Trav.

Chim. des Pays-Bas 82, 1123 (1963).

Preferred:

$\log k=12.8-44.2 / \theta$.

Comments: Estimated parameters are preferred. Rate constants are taken as reliable.

Estimated: $\log A_{a}=12.6$

$\log A_{b}=12.35$ (see section II-4.0).

\section{Experimental}

[1] Rates based on G.L.C. analysis (see $t$-butyl acetate). No isomer product ratios reported. 
Reaction: $t$-Butyl ethanoate ( $t$-butyl acetate)

$\begin{array}{crcc}\mathrm{CH}_{3} \mathrm{COOC}\left(\mathrm{CH}_{3}\right)_{3}(\mathrm{I}) \longrightarrow \mathrm{CH}_{2}=\mathrm{C}\left(\mathrm{CH}_{3}\right)_{2}(\mathrm{II})+\mathrm{CH}_{3} \mathrm{COOH}(\mathrm{III}) \\ \Delta H_{f(298)}^{\circ} & -120.1 & -4.0 & \text { III } \\ S_{(298)}^{\circ} & 100.3 & 70.2 & 67.6 \\ C_{p(298)}^{\circ} & 38.4 & 21.3 & 16.0\end{array}$

$\Delta S^{\circ}=37.9 \mathrm{~g} / \mathrm{mole}$

$\Delta H^{\circ}=12.4 \mathrm{kcal} / \mathrm{mole}$

$\Delta C_{p}^{\circ}=-1.1 \mathrm{~g} / \mathrm{mole}$

\begin{tabular}{|c|c|c|c|c|c|c|}
\hline $\log A$ & $E$ & $\begin{array}{c}\log \boldsymbol{k}_{T} \\
(545)\end{array}$ & Conditions & System & Surface & References \\
\hline 13.34 & 40.5 & -2.90 & $\begin{array}{l}516-576^{\circ} \mathrm{K} \\
5-275 \text { torr }\end{array}$ & static & $\begin{array}{l}\text { none on } \\
\text { conditioned } \\
\text { surfaces. }\end{array}$ & $\begin{array}{l}\text { [1] C. E. Rudi, Jr., and P. } \\
\text { Fugassi, J. Phys. Chem. } \\
\text { 52, } 357 \text { (1948). }\end{array}$ \\
\hline 13.15 & 40.0 & -2.89 & $\begin{array}{l}514-564{ }^{\circ} \mathrm{K} \\
135-172 \text { torr }\end{array}$ & static & $\longrightarrow$ & $\begin{array}{l}\text { [2] E. U. Emovan and A. } \\
\text { Maccoll, J. Chem. Soc., } \\
335 \text { (1962). }\end{array}$ \\
\hline 14.52 & 43.6 & -2.96 & $560-610^{\circ} \mathrm{K}$ & flow & & $\begin{array}{l}\text { [3] J. C. Scheer, E. C. } \\
\text { Kooyman, and F. L. J. } \\
\text { Sixma, Rec. Trav. Chim. } \\
\text { des Pays-Bas 82, } 1123 \\
\text { (1963). }\end{array}$ \\
\hline
\end{tabular}

Preferred:

$\log k=13.15-40.0 / \theta$

$\log A_{\text {est }}=13.1$ (see section II-4.0).

\section{Experimental}

[1] Rates by titration of the acid.

[2] Rates by $\Delta P$. Stoichiometry confirmed by G.L.C.

[3] Analysis by G.L.C. The experimental method for determining the reaction rates was unusual. The temperat ure of the reactor was allowed to cool slowly during which time 10-14 runs were performed. The first runs constituted $\sim 80$ percent decomposition in the period of contact, while at the lower temperatures, $\sim 20$ percent decomposition occurred. Constant-temperature conditions for the runs were assumed. The Arrhenius parameters obtained by the method would not be expected to be accurate to better than a power of 10 in $A$ and about $3-4 \mathrm{kcal} /$ mole in $E$. See for example $n$-propyl ethanoate, $n$-butyl ethanoate, and 2-methylbutyl ethanoate. 
Reaction: $t$-Pentyl ethanoate ( $t$-amyl acetate)

$$
\begin{gathered}
\mathrm{CH}_{3} \mathrm{COOC}\left(\mathrm{CH}_{3}\right)_{2} \mathrm{CH}_{2} \mathrm{CH}_{3}(\mathrm{I}) \stackrel{a}{\longrightarrow} \mathrm{CH}_{2}=\mathrm{C}\left(\mathrm{CH}_{3}\right) \mathrm{CH}_{2} \mathrm{CH}_{3} \text { (II) }+\mathrm{CH}_{3} \mathrm{COOH}(\mathrm{IV}) \\
\stackrel{b}{\longrightarrow}\left(\mathrm{CH}_{3}\right)_{2} \mathrm{C}=\mathrm{CHCH}_{3} \text { (III) }+\mathrm{CH}_{3} \mathrm{COOH}
\end{gathered}
$$

\begin{tabular}{crrrr} 
& I & II & III & \multicolumn{1}{c}{ IV } \\
$\Delta H_{f(298)}^{\circ}$ & -123.5 & -8.6 & -10.2 & -103.7 \\
$S_{(298)}^{\circ}$ & 112.0 & 81.9 & 81.1 & 67.6 \\
$C_{p(298)}^{\circ}$ & 43.9 & 26.7 & 25.1 & 16.0
\end{tabular}

$\begin{array}{rl}a & b \\ \Delta S^{\circ}=37.5 & 36.7 \mathrm{~g} / \mathrm{mole} \\ \Delta H^{\circ}=11.2 & 9.6 \mathrm{kcal} / \mathrm{mole} \\ \Delta C_{p}^{\circ}=-1.2 & -2.8 \mathrm{~g} / \mathrm{mole}\end{array}$

$\begin{array}{lllllll}\text { Path } & \log \boldsymbol{A} & \boldsymbol{E} & \log \boldsymbol{k}_{T} & \text { Conditions } & \text { System } & \text { Surface }\end{array}$

$\begin{array}{lllllll}a+b & 13.43 & 40.26 & -3.17 & 501-562{ }^{\circ} \mathrm{K} & \text { static } & \text { none. }\end{array}$ 127-284 torr (conditioned)

[1] E. U. Emovan, A. Maccoll, J. Chem. Soc., 335 (1962).

$\begin{array}{llllll}a+b & 15.17 & 44.3 & -3.06 & 560-610^{\circ} \mathrm{K} & \text { flow }\end{array}$

[2] J. C. Scheer, E. C. Kooyman, F. L. J. Sixma, Rec. Trav. Chim. des Pays-Bas 82, 1123 (1963).

Preferred:

$\log k=13.4-40.3 / \theta$

Estimated: $\log A_{1 \text {-ene }}=12.95$

$\log A_{2 \text {-ene }}=12.5$ (see section II-4.0).

\section{Experimental}

[1] Rates by $\Delta$ P. Olefin product analysis by G.L.C. gave 75 percent 2 -methylbut-1-ene. Stoichiometry confirmed by acid titration.

[2] Rates by G.L.C. Product olefin ratio: $\frac{\text { but-1-ene }}{\text { but-2-ene }}=3.2$ or about 75 percent 2 -methylbut-1-ene (see comments for $t$-butyl ethanoate). 
Reaction: 1,1,2-Trimethylpropyl ethanoate (2,3-dimethyl-2-butyl acetate)

\begin{tabular}{|c|c|c|c|c|}
\hline \multirow[t]{2}{*}{$\mathrm{CH}_{3} \mathrm{COOC}\left(\mathrm{CH}_{3}\right)_{2} \mathrm{C}$} & \multicolumn{4}{|c|}{$\stackrel{a}{\longrightarrow}\left(\mathrm{CH}_{3}\right)_{2} \mathrm{C}=\mathrm{C}\left(\mathrm{CH}_{3}\right)_{2}(\mathrm{II})+\mathrm{CH}_{3} \mathrm{COOH}(\mathrm{IV})$} \\
\hline & $\begin{array}{c}\text { I } \\
-128.9\end{array}$ & $\begin{array}{c}\text { II } \\
-15.9\end{array}$ & $\begin{array}{c}\text { III } \\
-14.8\end{array}$ & $\begin{array}{c}\text { IV } \\
-103.7\end{array}$ \\
\hline$S_{(298)}^{\circ}$ & 118.6 & 86.7 & 87.4 & 67.6 \\
\hline$C_{p(298)}^{\circ}$ & 49.1 & 30.5 & 32.5 & 16.0 \\
\hline
\end{tabular}

$$
\begin{gathered}
a \\
\Delta S^{\circ}=35.7 \mathrm{~g} / \mathrm{mole} \\
\Delta H^{\circ}=9.3 \mathrm{kcal} / \mathrm{mole} \\
\Delta C_{p}^{\circ}=-2.6 \mathrm{~g} / \mathrm{mole}
\end{gathered}
$$

\begin{tabular}{|c|c|c|c|}
\hline $\log A$ & $\boldsymbol{E}$ & $\begin{array}{c}\log \boldsymbol{k}_{T} \\
(584)\end{array}$ & Surface \\
\hline
\end{tabular}

$14.22 \quad 41.3 \quad-1.24 \quad 560-610^{\circ} \mathrm{K} \quad$ flow

[1] J. C. Scheer, E. C.

Kooyman, and F. L. J.

Sixma, Rec. Trav.

Chim. des Pays-Bas

82,1123 (1963).

Preferred:

$\log k=13.1-37.9 / \theta$.

Comments: Arrhenius parameters are certainly too high. Estimated parameters are preferred.

Estimated: $\log A_{a}=12.0$

$\log A_{b}=12.9$ (see section II-4.0).

\section{Experimental}

[1] Rates based on G.L.C. analysis (see comments on $t$-butyl ethanoate). Product isomer ratio: $\frac{\text { path } b}{\text { path } a} \simeq \frac{9}{1}$, or 90 percent 2,3-dimethyl-1-butene. 
Reaction: 1,1-Dimethylbutyl ethanoate (1,1-dimethyl butyl acetate)

$$
\begin{aligned}
& \begin{array}{c}
\mathrm{CH}_{3} \mathrm{COOC}\left(\mathrm{CH}_{3}\right)_{2} \mathrm{CH}_{2} \mathrm{CH}_{2} \mathrm{CH}_{3} \text { (I) } \stackrel{a}{\longrightarrow} \mathrm{CH}_{2}=\mathrm{C}\left(\mathrm{CH}_{3}\right) \mathrm{CH}_{2} \mathrm{CH}_{2} \mathrm{CH}_{3} \text { (II) }+\mathrm{CH}_{3} \mathrm{COOH}(\mathrm{IV}) \\
\longrightarrow
\end{array} \\
& \begin{array}{ccccc} 
& \text { I } & \text { II } & \text { III } & \text { IV } \\
\Delta H_{f(298)}^{\circ} & -128.5 & -13.6 & -15.2 & -103.7
\end{array} \\
& \begin{array}{lllll}
S_{(298)}^{\circ} & 121.3 & 91.3 & 89.3 & 67.6
\end{array} \\
& \begin{array}{lllll}
C_{p(298)}^{\circ} & 49.4 & 32.2 & 30.2 & 16.0
\end{array}
\end{aligned}
$$

\begin{tabular}{|c|c|c|c|c|c|c|}
\hline $\log A$ & $E$ & $\begin{array}{c}\log \boldsymbol{k}_{T} \\
(584)\end{array}$ & Conditions & System & Surface & References \\
\hline 13.85 & 40.6 & -1.33 & $560-610^{\circ} \mathrm{K}$ & flow & & $\begin{array}{l}\text { [1] J. C. Scheer, E. C. } \\
\text { Kooyman, and F. L. J. } \\
\text { Sixma, Rec. Trav. } \\
\text { Chim. des Pays-Bas 82, } \\
1123 \text { (1963). }\end{array}$ \\
\hline
\end{tabular}

$$
\begin{gathered}
a \\
\Delta S^{\circ}=37.6 \mathrm{~g} / \mathrm{mole} \\
\Delta H^{\circ}=11.2 \mathrm{kcal} / \mathrm{mole} \\
\Delta C_{p}^{\circ}=-1.2 \mathrm{~g} / \mathrm{mole}
\end{gathered}
$$

Preferred:

$\log k=13.17-38.8 / \theta$.

Estimated: $\log A_{a}=12.9$

$\log A_{b}=12.35$.

Comments: Estimated parameters are preferred (see section II-4.0).

\section{Experimental}

[1] Rates based on G.L.C. analysis (see $t$-butyl ethanoate). Olefin product ratio: (path $a /$ path $b$ ) $\simeq 2.57$ or about 72 percent 2-methyl pent-1-ene. 
Reaction: 1-Ethyl-1-methylpropyl ethanoate (1-ethyl-1-methylpropyl acetate)

$$
\stackrel{a}{\longrightarrow} \text { cis and trans } \mathrm{CH}_{3} \mathrm{CH}=\mathrm{C}\left(\mathrm{CH}_{3}\right) \mathrm{CH}_{2} \mathrm{CH}_{3}(\mathrm{II})+\mathrm{CH}_{3} \mathrm{COOH}
$$

$\mathrm{CH}_{3} \mathrm{COOC}\left(\mathrm{C}_{2} \mathrm{H}_{5}\right)_{2} \mathrm{CH}_{3}$ (I)

$$
\stackrel{b}{\longrightarrow} \mathrm{CH}_{2}=\mathrm{C}\left(\mathrm{CH}_{2} \mathrm{CH}_{3}\right)_{2}(\mathrm{III})+\mathrm{CH}_{3} \mathrm{COOH}(\mathrm{IV})
$$

\begin{tabular}{crrrr} 
& \multicolumn{1}{c}{ I } & II trans & III & \multicolumn{1}{c}{ IV } \\
$\Delta H_{f(298)}^{\circ}$ & -127.0 & -14.3 & -13.6 & -103.7 \\
$S_{(298)}^{\circ}$ & 121.3 & 89.6 & 90.3 & 67.6 \\
$C_{p(298)}^{\circ}$ & 49.4 & 30.3 & 31.8 & 16.0
\end{tabular}

$$
\begin{aligned}
& \Delta S^{\circ}=35.9 \mathrm{~g} / \mathrm{mole} \\
& \Delta H^{\circ}=9.0 \mathrm{kcal} / \mathrm{mole} \\
& \Delta C_{p}^{\circ}=-3.1 \mathrm{~g} / \mathrm{mole}
\end{aligned}
$$

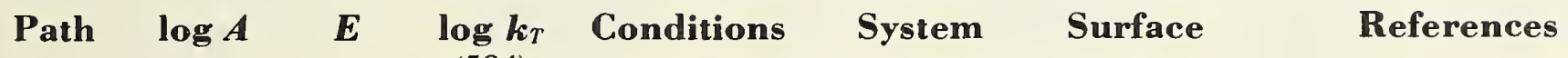
(584)

$\begin{array}{llllll}a+b & 14.60 & 42.4 & -1.27 & 560-610^{\circ} \mathrm{K} & \text { flow }\end{array}$

[1] J. C. Scheer, E. C. Kooyman, and F. L. J. Sixma, Rec. Trav. Chim. des Pays-Bas 82 , 1123 (1963).

\section{Preferred:}

$\log k=13.14-38.5 / \theta$.

Comments: Arrhenius parameters are certainly too high. Estimated parameters are preferred.

Estimated: $\log A_{(a)}=12.7$

$\log A_{(b)}=12.6$ (see section II-4.0).

\section{Experimental}

[1] Rates based on G.L.C. analysis (see $t$-butyl ethanoate). Isomer product ratios: $\left(\frac{\text { path } b}{\text { path } a}\right)=1.8$ or about 65 percent 2-ethylbut-1-ene. 
Reaction: 1-Methylcyclohexyl ethanoate (1-methylcyclohexyl acetate)

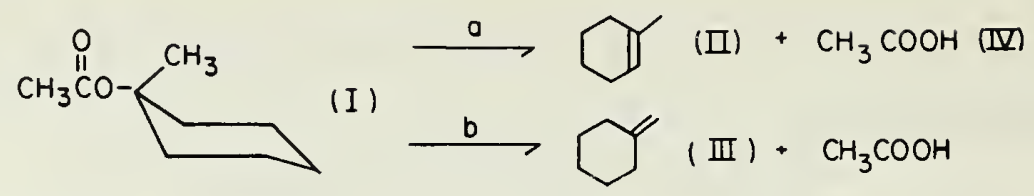

\begin{tabular}{crrrr} 
& I & II & III & \multicolumn{1}{c}{ IV } \\
$\Delta H_{f(298)}^{\circ}$ & -124.4 & -9.6 & -8.2 & -103.7 \\
$S_{(298)}^{\circ}$ & 111.6 & 81.4 & 79.8 & 67.6 \\
$C_{p(298)}^{\circ}$ & 46.4 & 30.4 & 28.5 & 16.0
\end{tabular}

$\Delta S^{\circ}=37.4 \mathrm{~g} / \mathrm{mole}$

$\Delta H^{\circ}=11.1 \mathrm{kcal} / \mathrm{mole}$

$\Delta C_{p}^{\circ}=0 \mathrm{~g} / \mathrm{mole}$

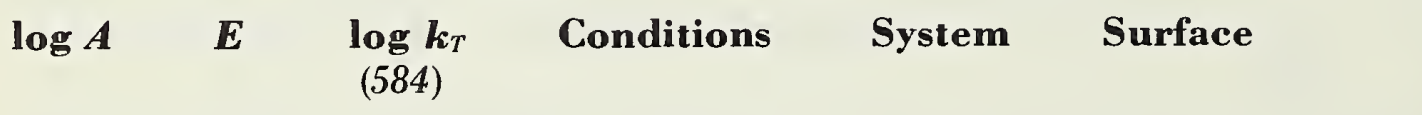

References

$15.36 \quad 45.2 \quad-1.54 \quad 560-610^{\circ} \mathrm{K} \quad$ flow

[1] J. C. Scheer, E. C.

Kooyman, and F. L.J.

Sixma, Rec. Trav.

Chim. des Pays-Bas,

82,1123 (1963).

Preferred:

$\log k=13.1-39.1 / \theta$.

Comments: Arrhenius parameters are certainly high. Transition state estimates are preferred. Note that the isomer ratio is close to that predicted by the calculated $A$-factors.

Estimated: $\log A_{(a)}=13.0$

$\log A_{(b)}=12.6($ see section II-4.0).

\section{Experimental} [1] Rates based on G.L.C. analysis (see $t$-butyl ethanoate). Product ratios: $\left(\frac{\text { path } a}{\text { path } b}\right) \simeq 3$ or about 75 percent 1 -methyl
cyclohexene.

(See discussion of cyclohexyl acetate.) 
Reaction: $t$-Butyl chloroethanoate $(t$-butyl chloroacetate)

\begin{tabular}{cccc}
$\mathrm{CH}_{2} \mathrm{ClCOOC}\left(\mathrm{CH}_{3}\right)_{3}(\mathrm{I})$ & \multicolumn{3}{c}{$\mathrm{CH}_{2}=\mathrm{C}\left(\mathrm{CH}_{3}\right)_{2}(\mathrm{II})+\mathrm{CH}_{2} \mathrm{ClCOOH}(\mathrm{III})$} \\
$\Delta H_{f(298)}^{\circ}$ & -124 & II & III \\
$S_{(298)}^{\circ}$ & 109.7 & 70.2 & 76.6 \\
$C_{p(298)}^{\circ}$ & 41.3 & 21.3 & 18.9
\end{tabular}

$\Delta S^{\circ}=37.1 \mathrm{~g} / \mathrm{mole}$

$\Delta H^{\circ}=13.5 \mathrm{kcal} / \mathrm{mole}$

$\Delta C_{p}^{\circ}=-1.1 \mathrm{~g} / \mathrm{mole}$

\begin{tabular}{ccccccc}
$\log A$ & $\boldsymbol{E}$ & $\begin{array}{c}\log k_{T} \\
(515)\end{array}$ & Conditions & System & Surface & \multicolumn{1}{c}{ References } \\
13.09 & 38.11 & -3.08 & $\begin{array}{l}489-540^{\circ} \mathrm{K} \\
100-300 \text { torr }\end{array}$ & static & $\begin{array}{l}\text { (none when } \\
\text { conditioned) }\end{array}$ & $\begin{array}{l}\text { [1] E. U. Emovan, J. } \\
\text { Chem. Soc., } 1246\end{array}$ \\
& & & & & & (1963).
\end{tabular}

Preferred:

$\log k=13.09-38.11 / \theta$

$\log A_{\text {est }}=13.1$ (see section II-4.0).

\section{Experimental}

[1] Rates by $\Delta$ P. Stoichiometry confirmed by G.L.C. No effect on rate by added cyclohexene. 
Reaction: $t$-Butyl dichloroethanoate ( $t$-butyl dichloroacetate)

$\mathrm{CHCl}_{2} \mathrm{COOC}\left(\mathrm{CH}_{3}\right)_{3}(\mathrm{I}) \longrightarrow \mathrm{CH}_{2}=\mathrm{C}\left(\mathrm{CH}_{3}\right)_{2}(\mathrm{II})+\mathrm{CHCl}_{2} \mathrm{COOH}(\mathrm{III})$

\begin{tabular}{crcr} 
& \multicolumn{1}{c}{ I } & II & \multicolumn{1}{c}{ III } \\
$\Delta H_{f(298)}^{\circ}$ & -125.7 & -4.0 & -109.3 \\
$S_{(298)}^{\circ}$ & 115.8 & 70.2 & 83.4 \\
$C_{p(298)^{\prime}}^{\circ}$ & 44.2 & 21.3 & 21.8
\end{tabular}

$\Delta S^{\circ}=37.8 \mathrm{~g} / \mathrm{mole}$

$\Delta H^{\circ}=12.4 \mathrm{kcal} / \mathrm{mole}$

$\Delta C_{p}^{\circ}=-1.1 \mathrm{~g} / \mathrm{mole}$

$\begin{array}{ccccccc}\log \boldsymbol{A} & \boldsymbol{E} & \begin{array}{c}\log \boldsymbol{k}_{T} \\ (500)\end{array} & \text { Conditions } & \text { System } & \text { Surface } & \text { References } \\ 12.77 & 36.09 & -3.00 & \begin{array}{l}487-520^{\circ} \mathrm{K} \\ 100-300 \text { torr }\end{array} & \text { static } & \begin{array}{l}\text { (none when } \\ \text { conditioned) }\end{array} & \begin{array}{c}\text { [1] E. U. Emovan, J. Chem. } \\ \text { Soc., } 1246 \text { (1963). }\end{array}\end{array}$

Preferred:

$\log k=12.8-36.1 / \theta$

$\log A_{\text {est }}=13.1$ (see section II-4.0).

\section{Experimental}

[1] Rates by $\Delta$ P. Stoichiometry confirmed by G.L.C. No effect on rate by added cyclohexene. 
Reaction: Ethyl propanoate (ethyl propionate)

\begin{tabular}{crcc}
$\mathrm{CH}_{3} \mathrm{CH}_{2} \mathrm{COOC}_{2} \mathrm{H}_{5}(\mathrm{I}) \longrightarrow \mathrm{C}_{2} \mathrm{H}_{4}(\mathrm{II})+\mathrm{CH}_{3} \mathrm{CH}_{2} \mathrm{COOH}(\mathrm{III})$ \\
& \multicolumn{1}{c}{} & $\mathrm{II}$ & III \\
$\Delta H_{f(298)}^{\circ}$ & -108.7 & 12.5 & -109.8 \\
$S_{(298)}^{\circ}$ & 99.1 & 52.5 & 76.6 \\
$C_{p(298)}^{\circ}$ & 33.4 & 10.4 & 22.7
\end{tabular}

$\Delta S^{\circ}=30.0 \mathrm{~g} / \mathrm{mole}$

$\Delta H^{\circ}=11.4 \mathrm{kcal} / \mathrm{mole}$

$\Delta C_{p}^{\circ}=-0.3 \mathrm{~g} / \mathrm{mole}$

\begin{tabular}{|c|c|c|c|c|c|c|}
\hline $\log A$ & $\boldsymbol{E}$ & $\begin{array}{c}\log \boldsymbol{k}_{T} \\
(830)\end{array}$ & Conditions & System & Surface & References \\
\hline 12.72 & 48.5 & -0.05 & $\begin{array}{l}778-875^{\circ} \mathrm{K} \\
15-20 \text { torr }\end{array}$ & flow & 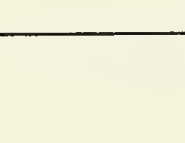 & $\begin{array}{l}\text { [1] A. T. Blades and P. W. } \\
\text { Gilderson, Can. J. } \\
\text { Chem. 38, } 1412 \text { (1960). }\end{array}$ \\
\hline
\end{tabular}

Preferred:

$\log k=12.72-48.5 / \theta$.

$\log A_{\text {est }} \simeq 12.6$ (see section II-4.0).

\section{Experimental}

[1] Rates by titration of the acid. Toluene carrier technique. Copyrolysis of ethylpropionate with ethyl $d_{5}$ acetate and comparison of the ethyl $d_{5}$ acetate to the unlabeled acetate gave an activation energy difference between propionate and acetate esters of $20 \pm 65 \mathrm{cal} / \mathrm{mole}$. 
Reaction: $t$-Butyl propanoate ( $t$-butyl propionate)

$\mathrm{CH}_{3} \mathrm{CH}_{2} \mathrm{COOC}\left(\mathrm{CH}_{3}\right)_{3}(\mathrm{I}) \longrightarrow \mathrm{CH}_{2}=\mathrm{C}\left(\mathrm{CH}_{3}\right)_{2}(\mathrm{II})+\mathrm{CH}_{3} \mathrm{CH}_{2} \mathrm{COOH}$ (III)

\begin{tabular}{crrr} 
& \multicolumn{1}{c}{ I } & \multicolumn{1}{c}{ II } & III \\
$\Delta H_{f(298)}^{\circ}$ & -125.4 & -4.0 & -109 \\
$S_{(298)}^{\circ}$ & 109.2 & 70.2 & 76.6 \\
$C_{p(298)}^{\circ}$ & 45.1 & 21.3 & 22.7
\end{tabular}

$\Delta S^{\circ}=37.6 \mathrm{~g} / \mathrm{mole}$

$\Delta H^{\circ}=12.4 \mathrm{kcal} / \mathrm{mole}$

$\Delta C_{p}^{\circ}=-1.1 \mathrm{~g} / \mathrm{mole}$

$\begin{array}{lcccccc}\log \boldsymbol{A} & \boldsymbol{E} & \begin{array}{c}\log \boldsymbol{k}_{T} \\ (540)\end{array} & \text { Conditions } & \text { System } & \text { Surface } & \text { References } \\ 12.79 & 39.16 & -3.06 & \begin{array}{l}513-569{ }^{\circ} \mathrm{K} \\ 17-125 \text { torr }\end{array} & \text { static } & \text { none } & \text { [1] E. Warrick, P. } \\ \text { Fugassi, J. Phys. } \\ \text { Chem. 52, 1314 (1948). }\end{array}$

Preferred:

$\log k=12.8-39.2 / \theta$.

Comments: Note that $t$-butyl acetate and $t$-butyl propionate have identical rates of reaction, as do ethyl acetate and ethyl propionate.

$\log A_{\text {est }}=13.1$ (see section II-4.0).

\section{Experimental}

[1] Rates by $\Delta \mathrm{P}$ and titration of the acid. Surface conditioning required. 
Reaction: Ethyl 2,2-dimethylpropanoate (ethyl trimethylacetate)

$\Delta S^{\circ}=30.0 \mathrm{~g} / \mathrm{mole}$

$\left(\mathrm{CH}_{3}\right)_{3} \mathrm{CCOOC}_{2} \mathrm{H}_{5}(\mathrm{I}) \longrightarrow\left(\mathrm{CH}_{3}\right)_{3} \mathrm{CCOOH}(\mathrm{II})+\mathrm{C}_{2} \mathrm{H}_{4}(\mathrm{III})$

\begin{tabular}{crrc} 
& \multicolumn{1}{c}{ I } & II & III \\
$\Delta H_{f(298)}^{\circ}$ & -121.4 & -121.7 & 12.5 \\
$S_{(298)}^{\circ}$ & 109.2 & 86.7 & 52.5 \\
$C_{p(298)}^{\circ}$ & 43.5 & 32.8 & 10.4
\end{tabular}

$\Delta H^{\circ}=12.2 \mathrm{kcal} / \mathrm{mole}$

$\Delta C_{p}^{\circ}=-0.3 \mathrm{~g} / \mathrm{mole}$

$\begin{array}{llll}\log A & \boldsymbol{E} & \log \boldsymbol{k}_{T} & \text { Condition }\end{array}$

System Surface

References

$11.24 \quad 44.00$

$635-694{ }^{\circ} \mathrm{K}$

30-370 torr

static none

[1] J. T. D. Cross and V. R.

Stimson, Aus. J.

Chem. 20, 177 (1967).

Preferred:

$\log k=12.6-48.1 / \theta$.

$\log A_{\text {est }}=12.6$.

Comments: The parameters are somewhat low in terms of transition state theory. They should correspond closely to those of ethylacetate. The authors checked their experimental conditions by studying also the ethylacetate decomposition. Although rate constants were not greatly different from those of previous workers, an Arrhenius plot of their data gives $E \simeq 44.2 \mathrm{kcal} / \mathrm{mole}$. Since the accepted value is $48.0 \mathrm{kcal} / \mathrm{mole}$, one may conclude that the reported parameters of the ethyl trimethylacetate reaction entail a similar systematic error.

\section{Experimental}

Rates were followed manometrically and agreed well with product analysis (i.e., titration of the acid). Identification of ethylene by G.L.C. Rates were unaffected by additions of cyclohexene and isobutene. 
Reaction: (-) Menthyl benzoate

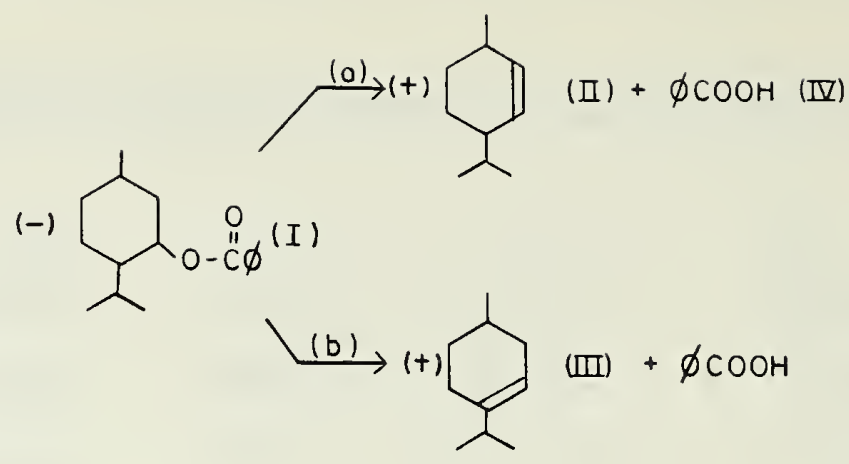

\begin{tabular}{lrrrr} 
& \multicolumn{1}{c}{ I } & II & III & IV \\
$\Delta H_{f(298)}^{\circ}$ & -115.6 & -24.8 & -26.1 & -79.1 \\
$S_{(298)}^{\circ}$ & 155.8 & 106.2 & 106.4 & 87.8 \\
$C_{p(298)}^{\circ}$ & 75.5 & 46.5 & 47.0 & 28.7
\end{tabular}

$\begin{array}{cc}a & b \\ \Delta S^{\circ}=38.2 & 38.4 \mathrm{~g} / \mathrm{mole} \\ \Delta H^{\circ}=11.7 & 10.4 \mathrm{kcal} / \mathrm{mole} \\ \Delta C_{p}^{\circ}=-0.3 & 0.2 \mathrm{~g} / \text { mole }\end{array}$

\begin{tabular}{|c|c|c|c|c|c|c|c|}
\hline Path & $\log A$ & $\boldsymbol{E}$ & $\begin{array}{c}\log \boldsymbol{k}_{T} \\
(595)\end{array}$ & Conditions & System & Surface & References \\
\hline$a+b$ & 11.0 & 38.1 & -2.99 & $\begin{array}{l}569-615^{\circ} \mathrm{K} \\
2.7-27.1 \text { torr }\end{array}$ & static & none & $\begin{array}{l}\text { [1] D. H. R. Barton, A. J } \\
\text { Head, and R. J. } \\
\text { Williams, J. Chem. } \\
\text { Soc. } 1715 \text { (1953). }\end{array}$ \\
\hline
\end{tabular}

Preferred:

Rate constants are reliable; however, the parameters look low (see below).

Comments: Transition state estimates give for $(+) p$-menth-2-ene: $\log A \simeq 13.0 ;(+) p$-menth-3-ene: $\log$ $A=12.7$. The authors were unwilling to attach error limits to the reported Arrhenius parameters due to the difficulty of working with a compound of such low volatility. The calculated parameters are therefore preferred.

\section{Experimental}

[1] Rates by benzoic acid titration. Flow experiments were also made which gave slightly faster rates but the same activation energy. $p$-menth-2-ene $\simeq$ const $\simeq 35$ percent from $628 \longrightarrow 647^{\circ} \mathrm{K}$ in flow experiments. 
Reaction: Methylene diethanoate (methylene diacetate)

\begin{tabular}{crcr}
\multicolumn{4}{c}{$\mathrm{CH}_{2}\left(\mathrm{OCOCH}_{3}\right)_{2}(\mathrm{I}) \longrightarrow \mathrm{CH}_{2} \mathrm{O}(\mathrm{II})+\left(\mathrm{CH}_{3} \mathrm{CO}\right)_{2} \mathrm{O}(\mathrm{III})$} \\
$\Delta H_{f(298)}^{\circ}$ & I & II & \multicolumn{1}{c}{ III } \\
$S_{(298)}^{\circ}$ & -187.3 & -27.7 & -141.8 \\
$C_{p(298)}^{\circ}$ & 111.9 & 52.3 & 93.2 \\
& 36.6 & 8.5 & 27.6
\end{tabular}

$\Delta S^{\circ}=33.6 \mathrm{~g} / \mathrm{mole}$

$\Delta H^{\circ}=17.8 \mathrm{kcal} / \mathrm{mole}$

$\Delta C_{p}^{\circ}=-0.5 \mathrm{~g} / \mathrm{mole}$

$\begin{array}{ccccccc}\log \boldsymbol{A} & \boldsymbol{E} & \begin{array}{c}\log \boldsymbol{k}_{T} \\ (535)\end{array} & \text { Conditions } & \text { System } & \text { Surface } & \text { References } \\ 9.23 & 33.0 & -4.25 & \begin{array}{l}493-578{ }^{\circ} \mathrm{K} \\ \sim 0.4-4 \mathrm{~atm}\end{array} & \text { static } & \text { none } & \text { [1] C. C. Coffin and W. B. } \\ \text { Beazley, Can. J. Res. } \\ \text { B 15, 229 (1937). }\end{array}$

\section{Preferred:}

$\log k=10.7-36.4 / \theta$. Rate constants around $\bar{T}$ are probably reliable.

Comments: At $550 \%$, the equilibrium constant for methylene diacetate from the thermodynamics is about $K_{\mathrm{eq}}=1.9 \mathrm{~atm}$. Thus equilibrium would be reached at about 80 percent decomposition (at $P_{t} \sim 1 \mathrm{~atm}$ ). This agrees with observation. Arrhenius parameters seem too low. Prefer calculated values of $\log A=10.7$.

\section{Experimental}

[1] Rates were followed manometrically and also by titration of the hydrolised anhydride. The reactions were believed to proceed to an equilibrium condition since, with increasing initial pressures (i.e., up to 5 atm), the reaction rate decreased. Because of a secondary reaction, the equilibrium condition could not be reached. 
Reaction: Ethylidene diethanoate (ethylidene diacetate)<smiles>CC(=O)OC(C)CCCCC(C)OC(C)=O</smiles>

$\begin{array}{cccc} & \text { I } & \text { II } & \text { III } \\ \Delta H_{f(298)}^{\circ} & -196.2 & -141.8 & -39.8 \\ S_{(298)}^{\circ} & 120 & 93.2 & 63.2 \\ C_{p(298)}^{\circ} & 42.1 & 27.6 & 13.1\end{array}$

$\Delta S^{\circ}=36.4 \mathrm{~g} / \mathrm{mole}$

$\Delta H^{\circ}=14.6 \mathrm{kcal} / \mathrm{mole}$

$\Delta C_{p}^{\circ}=-1.4 \mathrm{~g} / \mathrm{mole}$

\begin{tabular}{rrrrrrr}
$\log \boldsymbol{A}$ & $\boldsymbol{E}$ & $\begin{array}{c}\log \boldsymbol{k}_{T} \\
(520)\end{array}$ & \multicolumn{1}{c}{ Conditions } & System & Surface & \multicolumn{1}{c}{ References } \\
10.27 & 32.9 & -3.55 & $\begin{array}{l}493-541^{\circ} \mathrm{K} \\
110-460 \text { torr }\end{array}$ & static & none & [1] C. C. Coffin, Can. J. \\
& & & & & Res. 5, 636 (1931).
\end{tabular}

Preferred:

$\log k=10.27-32.9 / \theta$.

Comments: It has been noted (S. W. Benson, Foundations of Chemical Kinetics, McGraw-Hill Book Co., New York (1960), p. 257) that the acetic anhydride product decomposes at a rate of about an order of magnitude faster than the diacetate at the temperatures of study. This interesting observation can be resolved by examining the thermodynamics of the acetic anhydride decomposition. The estimated equilibrium constant for decomposition at $520^{\circ} \mathrm{K}$ is $K_{\text {eq }} \simeq 0.8$ torr.

For initial diacetate pressures of 300 torr, this $K_{\text {eq }}$ gives about 5 percent decomposition of the anhydride at 100 percent reaction.

The errors in $K_{\mathrm{eq}}$ would accommodate about a factor of three in these estimates. The stoichiometry and products observed are therefore consistent with the thermodynamics. The limited temperature range of study will allow errors of $2-3 \mathrm{kcal} / \mathrm{mole}$ in $E$ and about one $\log$ unit in $A$. Thus the very low $A$-factor reported for this reaction (and the other diacetates) definitely rules out the four-center transition states that have been proposed (see S. W. Benson, loc. cit. and A. F. Trotman-Dickenson, Gas Kinetics, Butterworths Scientific Publications, London (1955), p. 130). Two transition states are consistent with the large negative entropy of activation. An eight-center transition state

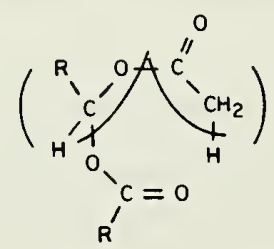

leading to $\mathrm{CH}_{3} \mathrm{COOH}+\mathrm{CH}_{2} \mathrm{CO}+\mathrm{CH}_{3} \mathrm{CHO}$, followed by rapid equilibration of ketene and acetic acid to form the anhydride; and a six-center transition state

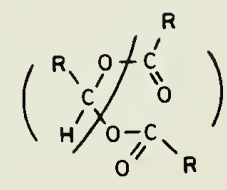

(Continued) 
proceeding directly to the observed products. The latter seems the more reasonable because of the insensitivity of the reaction activation energies to varied $R$ group substitution. $A$-factor estimates for both transition states are about a factor of ten higher than observed. At the higher temperatures the formation of vinyl acetate undoubtedly arises from the "normal" six-center elimination where the reactive $\beta$-hydrogens are those of the central alkyl group.

\section{Experimental}

[1] Rates were by $\Delta \mathrm{P}$ with an assumed stoichiometry of $P_{f} / P_{0} \simeq 2.0$. Chemical analysis showed the major products to be acetic anhydride and $\mathrm{CH}_{3} \mathrm{CHO}$ in a $1 / 1$ ratio. At higher temperatures acetic acid and vinyl acetate were also formed.

Reaction: Butylidene diethanoate (butylidene diacetate)

$$
\begin{array}{cccc}
\mathrm{CH}_{3}\left(\mathrm{CH}_{2}\right)_{2} \mathrm{CH} \\
\mathrm{OCOCH}_{3} \\
\Delta H_{f(298)}^{\circ} & -205.4 & -50.9 & -141.8 \\
S_{(298)}^{\circ} & 138.8 & 82.0 & 93.2 \\
C_{p(298)}^{\circ} & 53.1 & 24.0 & 27.6
\end{array}
$$

$\Delta S^{\circ}=36.4 \mathrm{~g} / \mathrm{mole}$

$\Delta H^{\circ}=12.7 \mathrm{kcal} / \mathrm{mole}$

$\Delta C_{p}^{\circ}=-1.5 \mathrm{~g} / \mathrm{mole}$

\begin{tabular}{rlcllll}
$\log \boldsymbol{A}$ & $\boldsymbol{E}$ & $\begin{array}{c}\log \boldsymbol{k}_{T} \\
(510)\end{array}$ & \multicolumn{1}{c}{ Conditions } & System & Surface & \multicolumn{1}{c}{ References } \\
10.47 & 32.9 & -3.63 & $\begin{array}{l}484-538^{\circ} \mathrm{K} \\
50-560 \text { torr }\end{array}$ & static & none & [1] C. C. Coffin, Can. J. \\
Res. 6, 417 (1932).
\end{tabular}

\section{Preferred:}

Probably reliable. See comments on ethylidene diacetate.

$\log A_{\text {est }}=11.0$.

\section{Experimental}

[1] Rates by $\Delta \mathrm{P}$. Quantitative analysis of products. Brown film deposits observed presumed to be polymer formation. Always somewhat less than 100 percent of anhydride obtained. 
Reaction: Heptylidene diethanoate (heptylidene diacetate)

$$
\begin{array}{cccc}
\mathrm{CH}_{3}\left(\mathrm{CH}_{2}\right)_{5} \mathrm{CH}\left(\mathrm{OCOCH}_{3}\right)_{2}(\mathrm{I}) \longrightarrow & \multicolumn{4}{c}{\left(\mathrm{CH}_{3} \mathrm{CO}\right)_{2} \mathrm{O}(\mathrm{II})+\mathrm{CH}_{3}\left(\mathrm{CH}_{2}\right)_{5} \mathrm{CHO}} \\
\Delta H_{f(2998)}^{\circ} & -220 & -141.8 & -65.9 \\
S_{(2998)}^{\circ} & 167 & 93.2 & 109.8 \\
C_{p(299)}^{\circ} & 69.6 & 27.6 & 41.8
\end{array}
$$

\begin{tabular}{|c|c|c|c|c|c|c|}
\hline $\log A$ & $\boldsymbol{E}$ & $\begin{array}{c}\log \boldsymbol{k}_{T} \\
(520)\end{array}$ & Conditions & System & Surface & References \\
\hline 10.48 & 33.0 & -3.37 & $\begin{array}{l}473-573^{\circ} \mathrm{K} \\
88-185 \text { torr }\end{array}$ & static & none & $\begin{array}{l}\text { [1] C. C. Coffin, J. R. Dacey, } \\
\text { and N. A. D. Parlee, } \\
\text { Can. J. Res. B15, } \\
247 \text { (1937). }\end{array}$ \\
\hline
\end{tabular}

$\Delta S^{\circ}=36.0 \mathrm{~g} / \mathrm{mole}$

$\Delta H^{\circ}=12.3 \mathrm{kcal} / \mathrm{mole}$

$\Delta C_{p}^{\circ}=0.1 \mathrm{~g} / \mathrm{mole}$

Preferred:

Probably reliable. See comments on ethylidene diacetate.

$\log A_{\text {est }}=11.0$.

\section{Experimental}

[1] Rates were obtained from pressure measurements. 
Reaction: Crotonylidene diethanoate (crotonylidene diacetate)

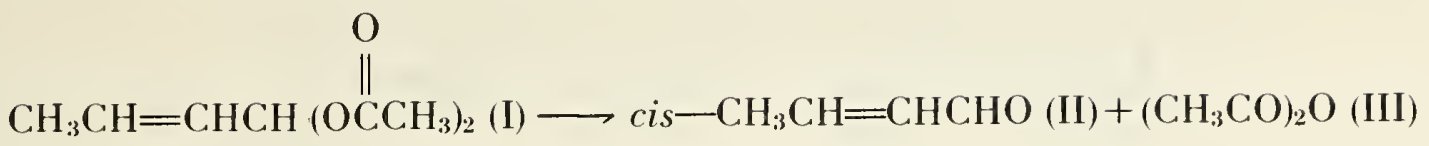

\begin{tabular}{crrr} 
& \multicolumn{1}{c}{ I } & \multicolumn{1}{c}{ II } & \multicolumn{1}{c}{ III } \\
$\Delta H_{f(298)}^{\circ}$ & -178.5 & -25.5 & -141.8 \\
$S_{(298)}^{\circ}$ & 135.5 & 75.5 & 93.2 \\
$C_{p(298)}^{\circ}$ & 50.4 & 22.9 & 27.6
\end{tabular}

$\Delta S^{\circ}=33.2 \mathrm{~g} / \mathrm{mole}$

$\Delta H^{\circ}=11.2 \mathrm{kcal} / \mathrm{mole}$

$\Delta C_{p}^{\circ}=0.1 \mathrm{~g} / \mathrm{mole}$

\begin{tabular}{|c|c|c|c|c|c|c|}
\hline $\log A$ & $\boldsymbol{E}$ & $\begin{array}{c}\log \boldsymbol{k}_{T} \\
(510)\end{array}$ & Conditions & System & Surface & References \\
\hline 11.1 & 33.0 & -3.00 & $\begin{array}{l}492-533^{\circ} \mathrm{K} \\
44-420 \text { torr }\end{array}$ & static & & $\begin{array}{l}\text { [1] J. R. Dacey and C. C. } \\
\text { Coffin, Can. J. Res. } \\
\text { B15,260 (1937). }\end{array}$ \\
\hline
\end{tabular}

Preferred:

Probably reliable. $\log A_{\text {est }}=11.0$. See comments on ethylidene diacetate.

\section{Experimental}

[1] Rates obtained manometrically. 
Reaction: Furfurylidene diethanoate (furfurylidene diacetate)

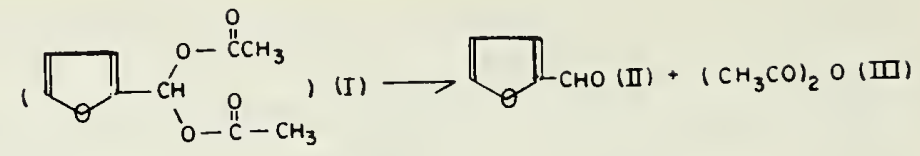

$$
\begin{aligned}
& \begin{array}{cccc} 
& \text { I } & \text { II } & \text { III } \\
\Delta H_{f(298)}^{\circ} & -187 & -38.5 & -141.8
\end{array} \\
& \begin{array}{llll}
S_{(298)}^{\circ} & 129.8 & 70.7 & 93.2
\end{array} \\
& \begin{array}{llll}
C_{\text {(2)!16) }}^{\circ} & 53 & 21.9 & 27.6
\end{array}
\end{aligned}
$$

$\Delta S^{\circ}=34.1 \mathrm{~g} / \mathrm{mole}$

\begin{tabular}{|c|c|c|c|c|c|c|}
\hline $\log A$ & $\boldsymbol{E}$ & $\begin{array}{c}\log \boldsymbol{k}_{T} \\
(520)\end{array}$ & Conditions & System & Surface & References \\
\hline 11.1 & 33.0 & -2.75 & $\begin{array}{l}493-553^{\circ} \mathrm{K} \\
\sim 20-175 \text { torr }\end{array}$ & static & & $\begin{array}{l}\text { [1] J. R. Dacey and C. C. } \\
\text { Coffin, Can. J. Res. } \\
\text { B15, } 260 \text { (1937). }\end{array}$ \\
\hline
\end{tabular}

$\Delta H^{\circ}=6.7 \mathrm{kcal} / \mathrm{mole}$

$\Delta C_{\mu}^{\circ}=-3.5 \mathrm{~g} / \mathrm{mole}$

Preferred:

Probably reliable.

$\log A_{\text {est }}=11.0$. See comments on ethylidene diacetate.

Experimental

[1] Rates followed manometrically. 
Reaction: 2,2,2-Trichloro-1-ethylidene diethanoate

$\begin{array}{cccc}\mathrm{CCl}_{3} \mathrm{CH}\left(\mathrm{OCOCH}_{3}\right)_{2}(\mathrm{I}) \longrightarrow \mathrm{CCl}_{3} \mathrm{CHO}(\mathrm{II})+\left(\mathrm{CH}_{3} \mathrm{CO}\right)_{2} \mathrm{O}(\mathrm{III}) \\ \Delta H_{f(298)}^{\circ} & \mathrm{I} & \mathrm{II} & \mathrm{III} \\ S_{(298)}^{\circ} & -203 & -49.7 & -141.8 \\ C_{p(298)}^{\circ} & 140.0 & 83.2 & 93.2 \\ & 52.2 & 23.2 & 27.6\end{array}$

$\Delta S^{\circ}=36.4 \mathrm{~g} / \mathrm{mole}$

$\Delta H^{\circ}=11.3 \mathrm{kcal} / \mathrm{mole}$

$\Delta C_{p}^{\circ}=-1.4 \mathrm{~g} / \mathrm{mole}$

\begin{tabular}{|c|c|c|c|c|c|c|}
\hline $\log A$ & $\boldsymbol{E}$ & $\begin{array}{c}\log \boldsymbol{k}_{T} \\
(530)\end{array}$ & Conditions & System & Surface & References \\
\hline 10.11 & 33.0 & -3.52 & $\begin{array}{l}503-563^{\circ} \mathrm{K} \\
50-360 \text { torr }\end{array}$ & static & & $\begin{array}{l}\text { [1] N. A. D. Parlee, J. R. } \\
\text { Dacey, and C. C. Coffin, } \\
\text { Can. J. Res. B15, 254 } \\
\text { (1937). }\end{array}$ \\
\hline
\end{tabular}

Preferred:

Probably reliable.

$\log A_{\text {est }}=11.0$. See comments on ethylidene diacetate.

\section{Experimental}

[1] Rates were obtained from $\Delta \mathrm{P}$ measurements. Solution decompositions gave rate constants in agreement with the gas phase results. 
Reaction: Methylene dipropanoate

\begin{tabular}{cccc}
\multicolumn{4}{c}{$\mathrm{CH}_{2}\left(\mathrm{OCOC}_{2} \mathrm{H}_{5}\right)_{2}(\mathrm{I}) \longrightarrow \mathrm{CH}_{2} \mathrm{O}(\mathrm{II})+\left(\mathrm{C}_{2} \mathrm{H}_{5} \mathrm{CO}\right)_{2} \mathrm{O}(\mathrm{III})$} \\
$\Delta H_{f(298)}^{\circ}$ & $\mathrm{I}$ & $\mathrm{II}$ & \multicolumn{1}{l}{ III } \\
$S_{(298)}^{\circ}$ & -197.3 & -27.7 & -151.8 \\
$C_{p(298)}^{\circ}$ & 131.1 & 52.3 & 112.4 \\
& 49.0 & 8.5 & 40.0
\end{tabular}

$\Delta S^{\circ}=33.6 \mathrm{~g} / \mathrm{mole}$

$\Delta H^{\circ}=17.8 \mathrm{kcal} / \mathrm{mole}$

$\Delta C_{p}^{\circ}=-0.5 \mathrm{~g} / \mathrm{mole}$

\begin{tabular}{ccccccc}
$\log \boldsymbol{A}$ & $\boldsymbol{E}$ & $\begin{array}{c}\log \boldsymbol{k}_{T} \\
(535)\end{array}$ & Conditions & System & Surface & \multicolumn{1}{c}{ References } \\
9.23 & 33.0 & -4.25 & $\begin{array}{l}493-578^{\circ} \mathrm{K} \\
0.4-4 \mathrm{~atm}\end{array}$ & static & none & [1] C. C. Coffin and W. B. \\
Beazley, Can. J. Res. \\
B 15, 229 (1937).
\end{tabular}

Preferred:

$\log k=10.7-36.4 / \theta$.

Comments: See methylene and ethylidene diacetates.

\section{Experimental}

[1] See methylene diacetate. 
Reaction: Ethylidene dipropanoate

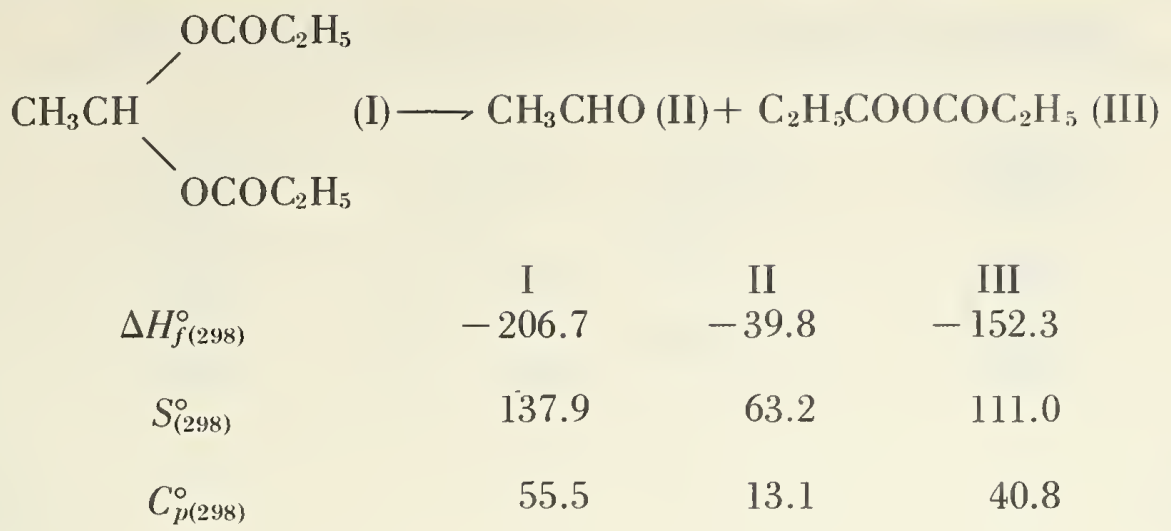

$\Delta S^{\circ}=36.3 \mathrm{~g} / \mathrm{mole}$

$\Delta H^{\circ}=14.6 \mathrm{kcal} / \mathrm{mole}$

$\Delta C_{p}^{\circ}=-1.6 \mathrm{~g} / \mathrm{mole}$

$\begin{array}{lllllll}\log \boldsymbol{A} & \boldsymbol{E} & \begin{array}{c}\log \boldsymbol{k}_{T} \\ (510)\end{array} & \text { Conditions } & \text { System } & \text { Surface } & \text { Reference } \\ 10.4 & 32.9 & -3.63 & 484-538^{\circ} \mathrm{K} & \text { static } & \text { none } & \text { [1] C. C. Coffin, Can. J. } \\ \text { Res. 6, } 417 \text { (1932). }\end{array}$

Preferred:

Probably reliable. $\log A_{\text {est }}=11.0$.

Comments: See ethylidene diacetate.

\section{Experimental}

[1] Rates by $\Delta \mathrm{P}$. Products analyzed chemically. Always less than 100 percent yield of the anhydride was realized. Stoichiometry of $1 \rightarrow 2$ within \pm 2 percent fairly well established. 
Reaction: Methylene dibutanoate

\begin{tabular}{crcc}
$\mathrm{CH}_{2}\left(\mathrm{OCO}\left(\mathrm{CH}_{2}\right)_{2} \mathrm{CH}_{3}\right)_{2}(\mathrm{I}) \longrightarrow \mathrm{CH}_{2} \mathrm{O}(\mathrm{II})+\left(\mathrm{C}_{3} \mathrm{H}_{7} \mathrm{CO}\right)_{2} \mathrm{O}(\mathrm{III})$ \\
$\Delta H_{f(298)}^{\circ}$ & \multicolumn{1}{c}{$\mathrm{I}$} & II & III \\
$S_{(298)}^{\circ}$ & -207.3 & -27.7 & -161.8 \\
$C_{p(298)}^{\circ}$ & 149.9 & 52.3 & 131.2 \\
& 60.0 & 8.5 & 51.0
\end{tabular}

$$
\begin{aligned}
& \Delta S^{\circ}=33.6 \mathrm{~g} / \mathrm{mole} \\
& \Delta H^{\circ}=17.8 \mathrm{kcal} / \mathrm{mole} \\
& \Delta C_{p}^{\circ}=-0.5 \mathrm{~g} / \mathrm{mole}
\end{aligned}
$$

\begin{tabular}{|c|c|c|c|c|c|c|}
\hline $\log A$ & $\boldsymbol{E}$ & $\begin{array}{c}\log \boldsymbol{k}_{T} \\
(535)\end{array}$ & Conditions & System & Surface & References \\
\hline 9.23 & 33.0 & -4.25 & $\begin{array}{l}493-578^{\circ} \mathrm{K} \\
0.4-4 \text { atm }\end{array}$ & static & none & $\begin{array}{l}\text { [1] C. C. Coffin and } \\
\text { W. B. Beazley, Can. J. } \\
\text { Res. B15, } 229 \text { (1937). }\end{array}$ \\
\hline
\end{tabular}

\section{Preferred:}

$\log k=10.7-36.4 / \theta$.

Comments: See methylene and ethylidene diacetates.

\section{Experimental}

[1] See methylene diacetate. 
Reaction: Ethylidene dibutanoate

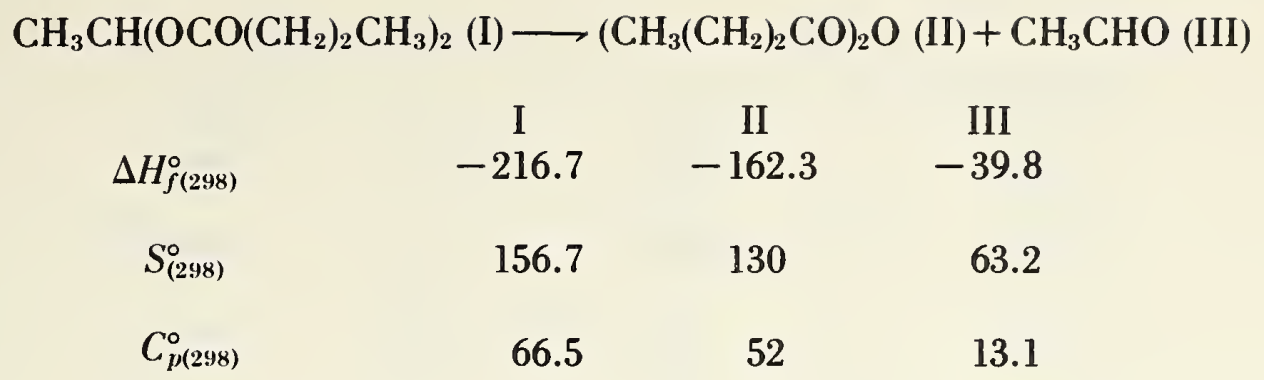

$\Delta S^{\circ}=36.5 \mathrm{~g} / \mathrm{mole}$

$\Delta H^{\circ}=14.6 \mathrm{kcal} / \mathrm{mole}$

$\Delta C_{p}^{\circ}=-1.4 \mathrm{~g} / \mathrm{mole}$

$\begin{array}{ccccccc}\log \boldsymbol{A} & \boldsymbol{E} & \begin{array}{c}\log \boldsymbol{k}_{T} \\ (520)\end{array} & \text { Conditions } & \text { System } & \text { Surface } & \text { References } \\ 10.25 & 33.0 & -3.60 & \begin{array}{l}473-573^{\circ} \mathrm{K} \\ 27-103 \text { torr } \\ (\sim 100 \text { torr })\end{array} & \text { static } & \text { none } & \text { [1] C. C. Coffin, J. R. } \\ & & & & & \text { Dacey, and N. A. D. } \\ & & & & & \text { Parlee, Can. J. Res. } \\ & & & & & \text { B15, 247 (1937). }\end{array}$

Preferred:

Probably reliable.

$\log A_{\text {est }}=11.0$.

Comments: See ethylidene diacetate.

\section{Experimental}

[1] Rates obtained from $\Delta \mathrm{P}$ measurements. 
Reaction: 2,2,2-Trichloro-1-ethylidene dibutanoate

$\begin{array}{crcc}\mathrm{CCl}_{3} \mathrm{CH}\left(\mathrm{OCO}\left(\mathrm{CH}_{2}\right)_{2} \mathrm{CH}_{3}\right)_{2} & (\mathrm{I}) \longrightarrow \mathrm{CCl}_{3} \mathrm{CHO}(\mathrm{II})+\left(\mathrm{CH}_{3}\left(\mathrm{CH}_{2}\right)_{2} \mathrm{CO}\right)_{2} \mathrm{O}(\mathrm{III}) \\ \Delta H_{f^{(298)}}^{\circ} & \begin{array}{c}\mathrm{I} \\ \text { II }\end{array} & \text { III } \\ S_{(298)}^{\circ} & 176.7 & 83.2 & 130 \\ C_{p(298)}^{\circ} & 76.6 & 23.2 & 52\end{array}$

$\Delta S^{\circ}=36.5 \mathrm{~g} / \mathrm{mole}$

$\Delta H^{\circ}=11.5 \mathrm{kcal} / \mathrm{mole}$

$\Delta C_{p}^{\circ}=-1.4 \mathrm{~g} /$ mole

\begin{tabular}{|c|c|c|c|c|c|c|}
\hline $\log A$ & $\boldsymbol{E}$ & $\begin{array}{c}\log \boldsymbol{k}_{T} \\
(540)\end{array}$ & Conditions & System & Surface & References \\
\hline 10.11 & 33.0 & -3.77 & $\begin{array}{l}514-563^{\circ} \mathrm{K} \\
53-253 \text { torr } \\
(\sim 200 \text { torr })\end{array}$ & static & & $\begin{array}{l}\text { [1] N. A. D. Parlee, J. R. } \\
\text { Dacey, and C. C. Coffin, } \\
\text { Can. J. Res. B 15, } 254 \\
\text { (1937). }\end{array}$ \\
\hline
\end{tabular}

Preferred:

Probably reliable.

$\log A_{\text {est }}=11.0$.

Comments: See ethylidene diacetate.

\section{Experimental}

[1] Rates followed by $\Delta \mathrm{P}$. Rate constants in solution (obtained from the titration of the hydrolized anhydride) agreed with the gas phase results. 
Reaction: Methyl ethyl carbonate

\begin{tabular}{|c|c|c|c|c|}
\hline$\Delta H_{f(298)}^{\circ}$ & I & $\begin{array}{c}\text { II } \\
-94.1\end{array}$ & $\begin{array}{c}\text { III } \\
12.5\end{array}$ & $\begin{array}{c}\text { IV } \\
-48.1\end{array}$ \\
\hline$S_{(298)}^{\circ}$ & & 51.1 & 52.5 & 57.3 \\
\hline$C_{p(298)}^{o}$ & & 8.9 & 10.4 & 10.5 \\
\hline
\end{tabular}

$\Delta S^{\circ}=$

$\Delta H^{\circ}=$

$\Delta C_{p}^{\circ}=$

\begin{tabular}{ccccccc}
$\log \boldsymbol{A}$ & $\boldsymbol{E}$ & $\begin{array}{c}\log \boldsymbol{k}_{T} \\
(610)\end{array}$ & Conditions & System & Surface & \multicolumn{1}{c}{ References } \\
13.7 & $46 \pm 2$ & -2.78 & $\begin{array}{l}573-648^{\circ} \mathrm{K} \\
20 \text { torr }\end{array}$ & static & none & {$[1]$ A. S. Gordon and W. P. } \\
& & & & & & $\begin{array}{l}\text { Norris, J. Phys. Chem. } \\
\mathbf{6 9}, 3013 \text { (1965). }\end{array}$
\end{tabular}

\section{Preferred:}

$\log k=12.6-43.0 / \theta$. Rate constants are reliable.

Comments: The Arrhenius parameters are certainly high. Transition state estimate of $A$ gives $\log A=12.6$, which is supported by the experimental values found for the methyl-1-aryl ethyl carbonates.

\section{Experimental}

[1] Analysis by M.S. and G.L.C. Added $\mathrm{CO}_{2}$ had no effect on the rate. 
Reaction: Diethyl carbonate

$$
\begin{aligned}
& \mathrm{C}_{2} \mathrm{H}_{5} \mathrm{OCOC}_{2} \mathrm{H}_{5}(\mathrm{I}) \longrightarrow \mathrm{CO}_{2}(\mathrm{II})+\mathrm{C}_{2} \mathrm{H}_{4}(\mathrm{III})+\mathrm{C}_{2} \mathrm{H}_{5} \mathrm{OH}(\mathrm{IV}) \\
& \Delta H_{f(298)}^{\circ} \\
& S_{(298)}^{\circ} \\
& C_{p(298)}^{\circ}
\end{aligned}
$$

\begin{tabular}{|c|ccc} 
I & II & III & IV \\
& -94.1 & 12.5 & -56.5 \\
& & & \\
& 51.1 & 52.5 & 67.6 \\
& 8.9 & 10.4 & 15.5
\end{tabular}

$\Delta S^{\circ}=$
$\Delta H^{\circ}=$
$\Delta C_{p}^{\circ}=$

$\begin{array}{ccccccc}\log \boldsymbol{A} & \boldsymbol{E} & \begin{array}{c}\log \boldsymbol{k}_{T} \\ (610)\end{array} & \text { Conditions } & \text { System } & \text { Surface } & \text { References } \\ 13.9 & 46 & -2.58 & 573-648^{\circ} \mathrm{K} & \text { static } & \text { none } & \text { [1] A. S. Gordon and W. P. } \\ \text { Norris, J. Phys. Chem. } \\ \text { 69, 3013 (1965). }\end{array}$

Preferred:

$\log k=12.9-43.3 / \theta$. Rate constants $\bar{T}$ reliable.

Comments: As in the methyl ethyl carbonate decomposition, the Arrhenius parameters are high. A negative entropy of activation is more reasonable. Transition state estimates of $A$ are therefore preferred.

\section{Experimental}

[1] Analysis by M.S. and G.L.C. No effect on the rate by $\mathrm{CO}_{2}$. The unimolecular path probably forms<smiles>CCCCC(=O)O</smiles>

as initial products and the semi-carbonate probably rearranges at the wall. 
Reaction: $X$-1-phenylethyl methyl carbonates, $X=$ ortho, meta, or para substitution

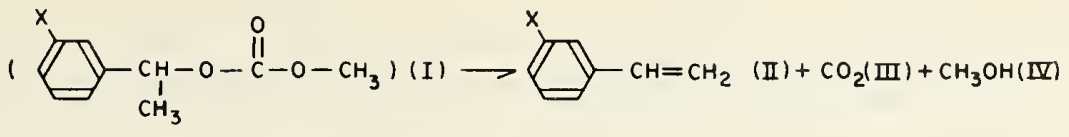

\begin{tabular}{|c|c|c|c|c|}
\hline & $\mathrm{I}(X=\mathrm{H})$ & $\mathrm{II}(X=\mathrm{H})$ & III & IV \\
\hline$\Delta H_{f(298)}^{\circ}$ & & 35.4 & -94.1 & -48.1 \\
\hline$S_{(298)}^{\circ}$ & & 82.5 & 51.1 & 57.3 \\
\hline$C_{p(298)}^{o}$ & & 29.6 & 8.9 & 10.5 \\
\hline
\end{tabular}

$$
\begin{aligned}
& \Delta S^{\circ}= \\
& \Delta H^{\mathrm{c}}= \\
& \Delta C_{p}^{\circ}=
\end{aligned}
$$

\begin{tabular}{|c|c|c|c|c|c|c|}
\hline $\log A$ & $\boldsymbol{E}$ & $\begin{array}{c}\log \boldsymbol{k}_{T} \\
(600)\end{array}$ & Conditions & System & $\begin{array}{c}\text { (substituent } \\
X=\text { ) }\end{array}$ & References \\
\hline 12.33 & 39.9 & -2.20 & $307-341.4^{\circ} \mathrm{C}$ & static & $\mathrm{H}$ & [1] G. G. Smith and B. L. \\
\hline 12.71 & 41.9 & -2.55 & & & $o-\mathrm{Cl}$ & Yates, J. Org. Chem. \\
\hline 12.66 & 41.5 & -2.46 & & & $m-\mathrm{Cl}$ & $\mathbf{3 0}, 434(1965)$ \\
\hline 12.22 & 38.8 & -1.91 & & & $p-\mathrm{Me}$ & \\
\hline
\end{tabular}

\section{Preferred:}

All reliable as reported. Transition state calculation of $A$ for all compounds gives $\log A_{\text {est }}=12.6$ (see section II-4.0).

\section{Experimental}

[1] Rates by $\Delta$ P. Analysis by G.L.C. 
Reaction: Ethanoic anhydride (acetic anhydride)

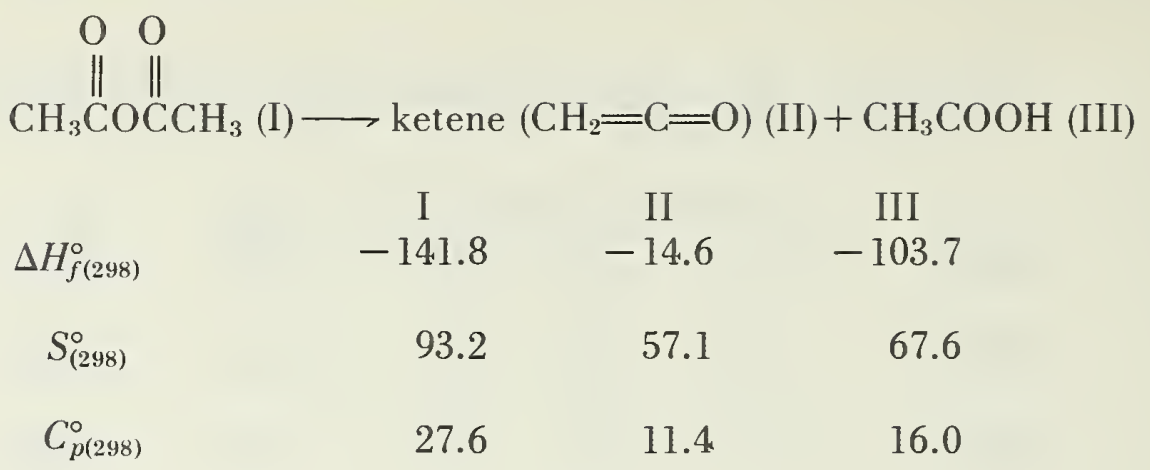

$\Delta S^{\circ}=31.5 \mathrm{~g} / \mathrm{mole}$

$\Delta H^{\circ}=23.5 \mathrm{kcal} / \mathrm{mole}$

$\Delta C_{p}^{\circ}=-0.2 \mathrm{~g} / \mathrm{mole}$

\begin{tabular}{|c|c|c|c|c|c|c|}
\hline $\log A$ & $\boldsymbol{E}$ & $\begin{array}{c}\log \boldsymbol{k}_{T} \\
(600)\end{array}$ & Conditions & System & Surface & References \\
\hline 12.0 & 34.5 & -2.56 & $\begin{array}{l}553-646{ }^{\circ} \mathrm{K} \\
(6-13 \text { torr total } \\
\text { pressure })\end{array}$ & flow & $\sim 10 \%$ & $\begin{array}{l}\text { [1] J. Murawski and M. } \\
\text { Szwarc, Trans. Faraday } \\
\text { Soc. 47, } 269 \text { (1951). }\end{array}$ \\
\hline
\end{tabular}

Preferred:

$\log k=12.0-34.5 / \theta$

$\log A_{\text {est }}=12.6$ (see section II-4.0).

\section{Experimental}

[1] Rates based on ketene formation. Stoichiometry confirmed by titration of the acid. Toluene carrier technique. If the lowest temperature points of the data are used, the "best" line for the Arrhenius plot gives $\log A_{\text {est }}=12.3$. 
Reaction: 4-Hydroxy-4-methyl-2-pentanone

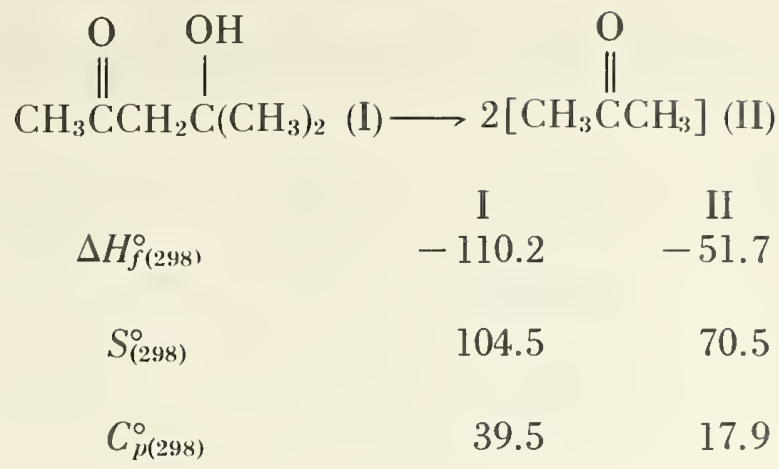

$\Delta S^{\circ}=36.5 \mathrm{~g} / \mathrm{mole}$

$\Delta H^{\circ}=6.8 \mathrm{kcal} / \mathrm{mole}$

$\Delta C_{p}^{\circ}=-3.7 \mathrm{~g} / \mathrm{mole}$

$\begin{array}{ccccccc}\log \boldsymbol{A} & \boldsymbol{E} & \begin{array}{c}\log \boldsymbol{k}_{T} \\ (510)\end{array} & \text { Conditions } & \text { System } & \text { Surface } & \text { References } \\ 11.63 & 32.3 & -2.21 & 495-528^{\circ} \mathrm{K} & \text { static } & \text { none } & \text { [1] G. G. Smith and B. L. } \\ & & & & & & \begin{array}{l}\text { Yates, J. Org. Chem. } \\ \mathbf{3 0 , 2 0 6 7}(1965) .\end{array}\end{array}$

Preferred:

$\log k=11.63-32.3 / \theta$.

Transition state estimate gives $\log A=11.7$ (see section II-4.0).

\section{Experimental}

[1] Rates by $\Delta$ P. Analysis by G.L.C., $n_{r}$ and I.R. 
Reaction: 1-Ethoxyethene (ethyl vinyl ether)

$\begin{array}{cccc}\mathrm{CH}_{2}=\mathrm{CHOCH}_{2} \mathrm{CH}_{3}(\mathrm{I}) \longrightarrow \mathrm{C}_{2} \mathrm{H}_{4}(\mathrm{II})+\mathrm{CH}_{3} \mathrm{CHO}(\mathrm{III}) \\ \Delta H_{f(298)}^{\circ} & \mathrm{I} & \mathrm{II} & \text { III } \\ S_{(298)}^{\circ} & -33.6 & 12.5 & -39.8 \\ C_{p(298)}^{\circ} & 82.6 & 52.5 & 63.2 \\ & 23.8 & 10.4 & 13.1\end{array}$

$\Delta S^{\circ}=33.1 \mathrm{~g} / \mathrm{mole}$

$\Delta H^{\circ}=6.3 \mathrm{kcal} / \mathrm{mole}$

$\Delta C_{p}^{\circ}=-0.3 \mathrm{~g} / \mathrm{mole}$

\begin{tabular}{|c|c|c|c|c|c|c|}
\hline $\log A$ & $\boldsymbol{E}$ & $\begin{array}{c}\log \boldsymbol{k}_{T} \\
(750)\end{array}$ & Conditions & System & Surface & References \\
\hline 11.43 & 43.8 & -1.30 & $770-859^{\circ} \mathrm{K}$ & flow & & $\begin{array}{l}\text { [1] A. T. Blades and G. W. } \\
\text { Murphy, J. Am. Chem. } \\
\text { Soc. 74, } 1039 \text { (1952). }\end{array}$ \\
\hline 11.60 & 44.4 & -1.31 & $\begin{array}{l}640-721^{\circ} \mathrm{K} \\
66-754 \text { torr }\end{array}$ & static & & $\begin{array}{l}\text { [2] S. Wang and C. A. Wink- } \\
\text { ler, Can. J. Res. 21B, } \\
97 \text { (1943). }\end{array}$ \\
\hline
\end{tabular}

Preferred:

$\log k=11.6-44.4 / \theta$ (see below).

Comments: The Arrhenius parameters of both studies seem slightly low, particularly when compared to the vinyl isopropyl ether decomposition. The transition state calculation of $A$ gives $\log A_{\text {est }}=12.2$. However, both studies are in excellent agreement over a large temperature range, which makes it difficult not to accept the parameters reported.

\section{Experimental}

[1] Toluene carrier technique. Rates based on U.V. analysis for acetaldehyde. Above $810^{\circ} \mathrm{K}$, some free radical split was evident.

[2] Rates by $\Delta \mathrm{P}$ and by chemical analysis of the products. Subsequent decomposition of acetaldehyde producing $\mathrm{CH}_{4}, \mathrm{C}_{2} \mathrm{H}_{6}$, and $\mathrm{CO}$ was observed. 
Reaction: Isopropoxyethene (vinyl isopropyl ether)

\begin{tabular}{cccc}
$\mathrm{CH}_{2}=\mathrm{CHOCH}\left(\mathrm{CH}_{3}\right)_{2}(\mathrm{I})$ & \multicolumn{3}{c}{$\longrightarrow \mathrm{CH}_{2}=\mathrm{CHCH}_{3}(\mathrm{II})+\mathrm{CH}_{3} \mathrm{CHO}(\mathrm{III})$} \\
$\Delta H_{f(298)}^{\circ}$ & I & II & III \\
$S_{(298)}^{\circ}$ & -41.4 & 4.9 & -39.8 \\
$C_{p(298)}^{\circ}$ & 88.9 & 63.8 & 63.2 \\
& 29.8 & 15.3 & 13.1
\end{tabular}

$\Delta S^{\circ}=38.1 \mathrm{~g} / \mathrm{mole}$

$\Delta H^{\circ}=6.5 \mathrm{kcal} / \mathrm{mole}$

$\Delta C_{p}^{\circ}=-1.4 \mathrm{~g} / \mathrm{mole}$

\begin{tabular}{lclll}
$\log A$ & $\boldsymbol{E}$ & $\begin{array}{l}\log k_{T} \\
(755)\end{array}$ & \multicolumn{1}{c}{ Conditions } & Syst \\
12.58 & 43.56 & -0.03 & $\begin{array}{l}720-794{ }^{\circ} \mathrm{K} \\
17.4-21.5 \text { torr }\end{array}$ & flow
\end{tabular}

Surface

References

Preferred:

$\log k=12.58-43.56 / \theta$

Transition state estimate of $A$ gives $\log A_{\text {est }}=12.5$ (see section II -4.0 ).

\section{Experimental}

[1] Toluene flow technique. Rates based on U.V. analysis for acetaldehyde. Above $843^{\circ} \mathrm{K}$ a minor free radical induced decomposition was evident. 
Reaction: $n$-Butyl vinyl ether

$\begin{array}{rrrr}\mathrm{CH}_{2}=\mathrm{CHOC}_{4} \mathrm{H}_{9}(\mathrm{I}) \longrightarrow \mathrm{CH}_{3} \mathrm{CHO}(\mathrm{II})+\mathrm{CH}_{2}=\mathrm{CHCH}_{2} \mathrm{CH}_{3}(\mathrm{III}) \\ \Delta H_{f(298)}^{\circ} & -43.5 & -39.8 & 0 \\ S_{(298)}^{\circ} & 102.2 & 63.2 & 73.6 \\ C_{p(298)}^{\circ} & 34.8 & 13.1 & 20.5\end{array}$

$\Delta S^{\circ}=34.6 \mathrm{~g} / \mathrm{mole}$

$\Delta H^{\circ}=3.7 \mathrm{kcal} / \mathrm{mole}$

$\Delta C_{p}^{\circ}=1.2 \mathrm{~g} / \mathrm{mole}$

\begin{tabular}{|c|c|c|c|c|c|c|}
\hline $\log A$ & $\boldsymbol{E}$ & $\begin{array}{c}\log \boldsymbol{k}_{T} \\
(620)\end{array}$ & Conditions & System & Surface & References \\
\hline 11.15 & 42.38 & -3.79 & $\begin{array}{l}590-650^{\circ} \mathrm{K} \\
60-345.5 \text { torr } \\
\quad \text { (in presence } \\
\text { of cyclohexene) }\end{array}$ & static & $\begin{array}{l}\text { None in an } \\
\text { aged reac- } \\
\text { tion } \\
\text { vessel }\end{array}$ & $\begin{array}{l}\text { [1] T. O. Bamkole and E. U. } \\
\text { Emovon, J. Chem. Soc., } \\
\text { B, } 523 \text { (1967). }\end{array}$ \\
\hline
\end{tabular}

Preferred:

$\log k=11.15-42.38 / \theta$

$\log A_{\text {est }}=11.0$.

\section{Experimental}

Products were analyzed by G.L.C. and titrimetric determination of the aldehyde. Rates by $\Delta$ P. Stoichiometry was confirmed $\left(\Delta \mathrm{P}=\Delta\left(\mathrm{CH}_{3} \mathrm{CHO}\right)\right)$. Addition of cyclohexene resulted in a significant rate suppression, suggesting the presence of a chain reaction. Reaction products in the presence of $\mathrm{HBr}$ and acetaldehyde were complex, confirming the importance of free radicals in the uninhibited system. 
2. Cyclic Compound Reactions 


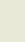


Reaction: Cyclopropane

$\begin{array}{ccc}\mathrm{C}_{3} \mathrm{H}_{6}(\nabla)(\mathrm{I}) \longrightarrow \mathrm{CH}_{2}=\mathrm{CHCH}_{3}(\mathrm{II})( \\ \Delta H_{f(298)}^{\circ} & \mathrm{I} & \mathrm{II} \\ S_{(298)}^{\circ} & 12.7 & 4.8 \\ C_{p(298)}^{\circ} & 56.8 & 63.6 \\ C_{p(700)}^{\circ} & 13.3 & 15.3\end{array}$

$\Delta S^{\circ}=6.8 \mathrm{~g} / \mathrm{mole}$
$\Delta H^{\circ}=-7.9 \mathrm{kcal} / \mathrm{mole}$
$\Delta C_{p}^{\circ}=2.0 \mathrm{~g} / \mathrm{mole}$

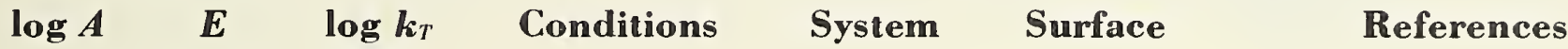
(750)

$\begin{array}{llll}15.17 & 65 & -3.77 \quad 772 \pm 29^{\circ} \mathrm{K} & \text { static none }\end{array}$ $1.27-76.5$ torr

[1] T. S. Chambers and G. B. Kistiakowsky, J. Am. Chem. Soc. 56, 399 (1934).

$\begin{array}{cccc}14.89 \quad 65.2 \quad-4.11 \quad & 753 \pm 40^{\circ} \mathrm{K} \quad \text { static } \\ & 10-900 \\ & (600)\end{array}$

[2] E. S. Corner and R. N. Pease, J. Am. Chem. Soc. 67, 2067 (1945).

$15.45 \quad 65.6 \quad-3.67 \quad 750 \pm 57^{\circ} \mathrm{K} \quad$ static

[3] W. E. Falconer, T. F. Hunter, and A. F. Trotman-Dickenson, J. Chem. Soc. 609 (1961).

Preferred:

$\log k=15.2-65.5 / \theta$.

Comments: Transition state estimates for the biradical mechanism are in good agreement with the kinetics.

Mechanism: $\triangle \frac{1}{2} \bumpeq \frac{3}{2} \wedge ; k_{2}>k_{3}$

Estimates give: $\log k=\log \left(\frac{k_{1} k_{3}}{k_{2}}\right)=15.16-65.4 / \theta$. (See discussion on small ring compounds, section III-4.0, and also 1,2-dideuteriocyclopropane.)

\section{Experimental}

[1] Rate determined by chemical analysis on propylene; $k_{\infty}$ obtained by extrapolation of $k_{\text {uni }}$ versus $1 / P$.

[2] Rate determined by chemical analysis on propylene. Added $\mathrm{H}_{2}, \mathrm{C}_{2} \mathrm{H}_{4}$, and $\mathrm{C}_{3} \mathrm{H}_{6}$ had no effect on the rate in the high-pressure region.

[3] Rate based on G.L.C. analysis. Used the Slater equation $\left(\log p=\frac{n}{2} \log T_{2} / T_{1}\right)$ to determine $k_{x}$. 


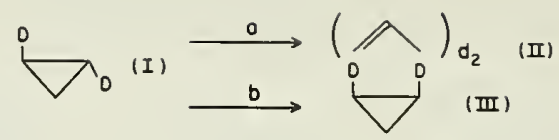

$\begin{array}{cccc} & \text { I } & \text { II } & \text { III } \\ \Delta H_{f(298)}^{\circ} & 10.9 & 3.0 & 10.9 \\ S_{(2.98)}^{\circ} & 59.0 & 65.0 & 60.4 \\ C_{p(298)}^{\circ} & 13.9 & 15.9 & 13.9\end{array}$

\begin{tabular}{|c|c|}
\hline & $b$ \\
\hline$\Delta S^{\circ}=6.0$ & $1.4 \mathrm{~g} / \mathrm{mo}$ \\
\hline $\begin{array}{l}\Delta H^{\circ}=-7.9 \\
\Delta C_{p}^{\circ}=2.0\end{array}$ & $\begin{array}{ll}0 & \mathrm{kcal} / \mathrm{mole} \\
0 & \mathrm{~g} / \mathrm{mole}\end{array}$ \\
\hline
\end{tabular}

\begin{tabular}{|c|c|c|c|c|c|c|c|}
\hline Path & $\log A$ & $\boldsymbol{E}$ & $\begin{array}{c}\log k_{T} \\
(717)\end{array}$ & Conditions & System & Surface & References \\
\hline & 15.12 & 65.4 & -4.82 & $\begin{array}{l}723 \pm 48^{\circ} \mathrm{K} \\
0.01 \longrightarrow 630 \\
\quad \text { torr }(100)\end{array}$ & static & & $\begin{array}{l}\text { [1] E. W. Schlag and } \\
\text { B. S. Rabinovitch, } \\
\text { J. Am. Chem. Soc. } \\
\mathbf{8 2}, 5996 \text { (1960). }\end{array}$ \\
\hline
\end{tabular}

$a$

$\begin{array}{cccc}15.20 & 65.5 & -4.77 & 717 \pm 30 \% \\ & & 0.03 \underset{1}{\longrightarrow} 76 \\ & & \text { torr }(56)\end{array}$

[2] E. W. Schlag,

B. S. Rabinovitich, and $\mathrm{K}$. Wiberg, J. Chem. Phys. 28, 504 (1958).

$\begin{array}{lllll}b & 16.11 & 65.1 & -3.57 & \text { (as in [1]) } \\ b & 15.7 & 64.2 & -3.87 & \text { (as in [2]) }\end{array}$

Preferred:

$\log k_{a}=15.2-65.5 / \theta$.

$\log k_{b}=15.8-64.1 / \theta$.

Comments: By the biradical mechanism (see cyclopropane), the rate-determining step for geometric (trans cis) isomerization is ring opening. We estimate $\log A_{1}=\log A_{b}=15.8$ (see section III-4.0). Individual rate constants of the mechanism have also been deduced (see discussion on small ring compound reactions). Activation energies for ring closing and 1,2-H-migration from the biradical are estimated to be $E_{2\left(C_{3} \text { ring close) }\right.}$ $=9.3 \mathrm{kcal} / \mathrm{mole}$ and $E_{3(\mathrm{H}-\mathrm{migration})}=10.7 \mathrm{kcal} / \mathrm{mole}$ respectively.

\section{Experimental}

[1] Analysis by G.L.C. Falloff occurred near 200 torr for path $(a) ; n \simeq 14$. Falloff near 1000 torr for path $(b) ; n \simeq 14$. Rates were not affected by 0.2 torr of $\mathrm{O}_{2}$. The shift in falloff with temperature was not consistent with the Slater theory.

[2] Analysis by I.R. 
Reaction: Methylcyclopropane

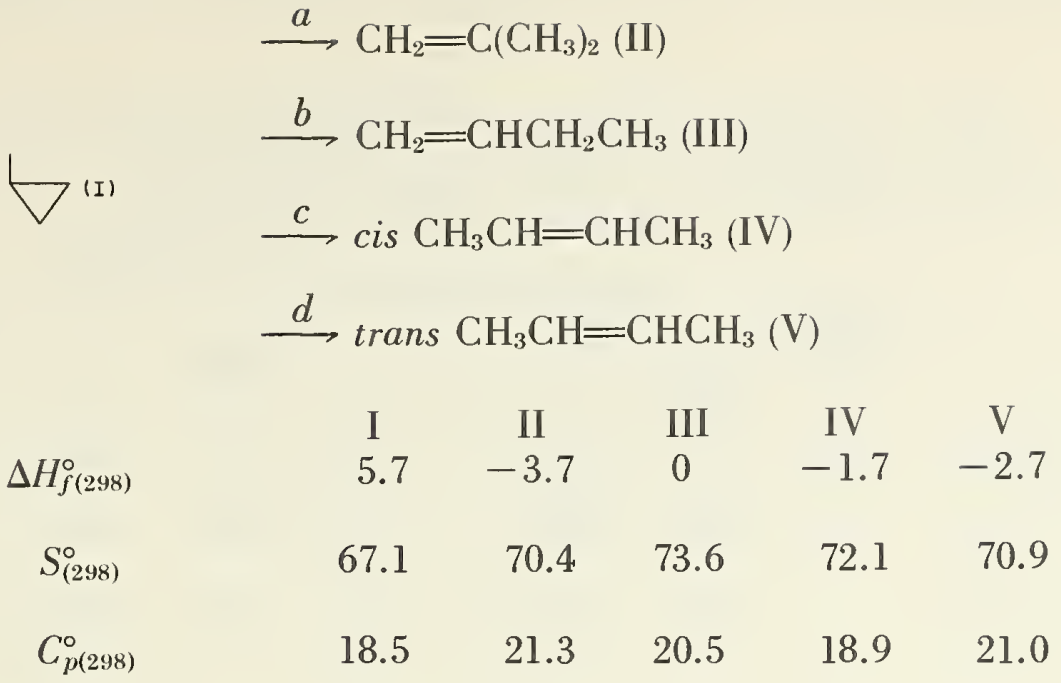

$$
\begin{gathered}
a \\
\Delta S^{\circ}=3.3 \mathrm{~g} / \mathrm{mole} \\
\Delta H^{\circ}=-9.4 \mathrm{kcal} / \mathrm{mole} \\
\Delta C_{p}^{\circ}=2.8 \mathrm{~g} / \mathrm{mole}
\end{gathered}
$$

\section{$\begin{array}{llllll}\text { Path } & \log \boldsymbol{A} \quad \boldsymbol{E} \quad \log \boldsymbol{k}_{T} & \text { Conditions } & \text { System } & \text { Surface } & \text { References }\end{array}$ (738)}

[1] D. W. Placzek and B. S. Rabinovitch, J. Chem. Phys. 69, 2141 (1965).

$\begin{array}{llllll}a & 14.06 & 64.3 & -4.99 & \text { (as above) } & \text { static } \\ b & 14.14 & 62.0 & -4.22 & & \\ c & 13.97 & 61.9 & -4.37 & & \\ d & 14.32 & 64.4 & -4.76 & & \\ & & & & & \\ \text { total } & 15.45 & 65.0 & -3.80 & \text { (as above) } & \text { static }\end{array}$

D. W. Setzer and B. S. Rabinovitch, J. Am. Chem. Soc. 86, 564 (1964).

[3] J. P. Chesick, J. Am. Chem. Soc. 82 , 3277 (1960).

\section{Preferred:}

Rate constant parameters of [1] are preferred.

Comments: The range of values reported in the two studies for any single process are of the order of the experimental errors associated with the data. The agreement between observed Arrhenius parameters and those obtained by transition state estimates for a biradical mechanism similar to that for cyclopropane is therefore good.

Estimates are $\log k_{a}=14.4-65.8 / \theta$

$$
\begin{aligned}
& \log k_{b}=14.7-63.0 / \theta \\
& \log k_{c}=14.3-63.0 / \theta
\end{aligned}
$$

$\log k_{d}=14.5-63.0 / \theta$ (see section III-4.0).

\section{Experimental}

[1] and [2] Calculated from the data of [3].

[3] Analysis by G.L.C. $\left(\mathrm{C}_{2}+\mathrm{C}_{3}+\mathrm{C}_{5}\right.$ compounds represented less than 2 percent of products.) Falloff observed near 100 torr. Relative collision efficiencies determined for $\mathrm{He}, \mathrm{Ar}, \mathrm{N}_{2}, \mathrm{CH}_{4}, \mathrm{C}_{2} \mathrm{H}_{6}$, and toluene. Pressure and temperature dependencies in the butene isomer product distributions were reported. 
Reaction: cis or trans-1,2-Dideutero-3-methylcyclopropane

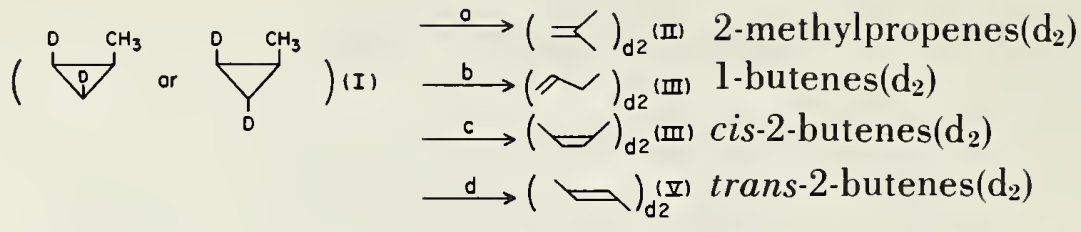

$\begin{array}{crrccc} & \text { I(cis) } & \text { II } & \text { III } & \text { IV } & \text { V } \\ \Delta H_{f(298)}^{\circ} & 3.9 & -5.8 & -1.8 & -3.5 & -4.5 \\ S_{(298)}^{\circ} & 68.5 & 71.6 & 75.0 & 73.5 & 72.3 \\ C_{p(298)}^{\circ} & 19.1 & 21.9 & 21.1 & 19.5 & 21.6\end{array}$

$\Delta S^{\circ}=3.1 \mathrm{~g} / \mathrm{mole}$

$\Delta H^{\circ}=-9.7 \mathrm{kcal} / \mathrm{mole}$

$\Delta C_{p}^{\circ}=2.8 \mathrm{~g} / \mathrm{mole}$

$\begin{array}{lllllll}\text { Path } & \log A & \boldsymbol{E} & \log k_{T} & \text { Conditions } & \text { System } & \text { Surface }\end{array}$

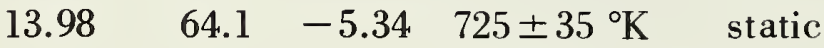

(10) torr

[1] D. W. Setzer and

B. S. Rabinovitch,

J. Am. Chem. Soc.

86, 564 (1964).

$\begin{array}{llll}b & 13.87 & 61.6 & -4.70 \\ c & 13.76 & 61.5 & -4.78 \\ d & 14.26 & 64.5 & -5.19 \\ & & & \\ \text { total } & 14.43 & 62.3 & -4.35\end{array}$

Preferred:

Within experimental error, all parameters are reliable as reported. (Compare to methylcyclopropane results.)

\section{Experimental}

[1] Analysis by G.L.C. Rates are very comparable to those of methylcyclopropane as calculated by these authors. Primary and secondary deuterium isotope effects for the formation of the various olefins were measured. All products expected on the basis of a biradical mechanism were observed. 
Reaction: Cis-1,2-dideutero-3-methylcyclopropane

Trans-1,2-dideutero-3-methylcyclopropane
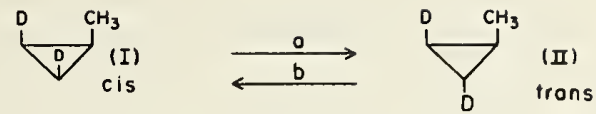

$\begin{array}{crc} & \text { I cis } & \text { II trans } \\ \Delta H_{f(298)}^{\circ} & 3.9 & 3.9 \\ S^{\circ}{ }_{(298)} & 68.5 & 68.5 \\ C_{p(298)}^{\circ} & 19.1 & 19.1\end{array}$

$$
\begin{aligned}
& a \\
& \Delta S^{\circ}= 0 \mathrm{~g} / \mathrm{mole} \\
& \Delta H^{\circ}= 0 \mathrm{kcal} / \mathrm{mole} \\
& \Delta C_{p}^{\circ}=0 \mathrm{~g} / \mathrm{mole}
\end{aligned}
$$

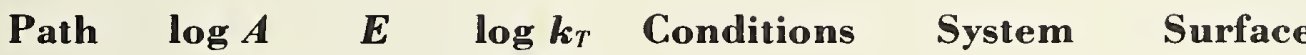
(725)

$\begin{array}{lllll}a & 15.35 & 60.5 & -2.89 & \begin{array}{l}725 \pm 35^{\circ} \mathrm{K} \\ 10 \text { torr }\end{array}\end{array}$

$$
10 \text { torr }
$$

[1] D. W. Setzer and

B. S. Rabinovitch, J. Am. Chem. Soc. 86, $564(1964)$
$b$
$15.35 \quad 60.5 \quad-2.89$

Preferred:

$\log k_{a}=\log k_{b}=15.35-60.5 / \theta$.

Transition state estimates give: $\log k_{\text {est }}=15.4-62.1 / \theta$ (see section III-4.0).

\section{Experimental}

[1] Analysis by I.R. 
Reaction: cis-1,2-Dimethylcyclopropane

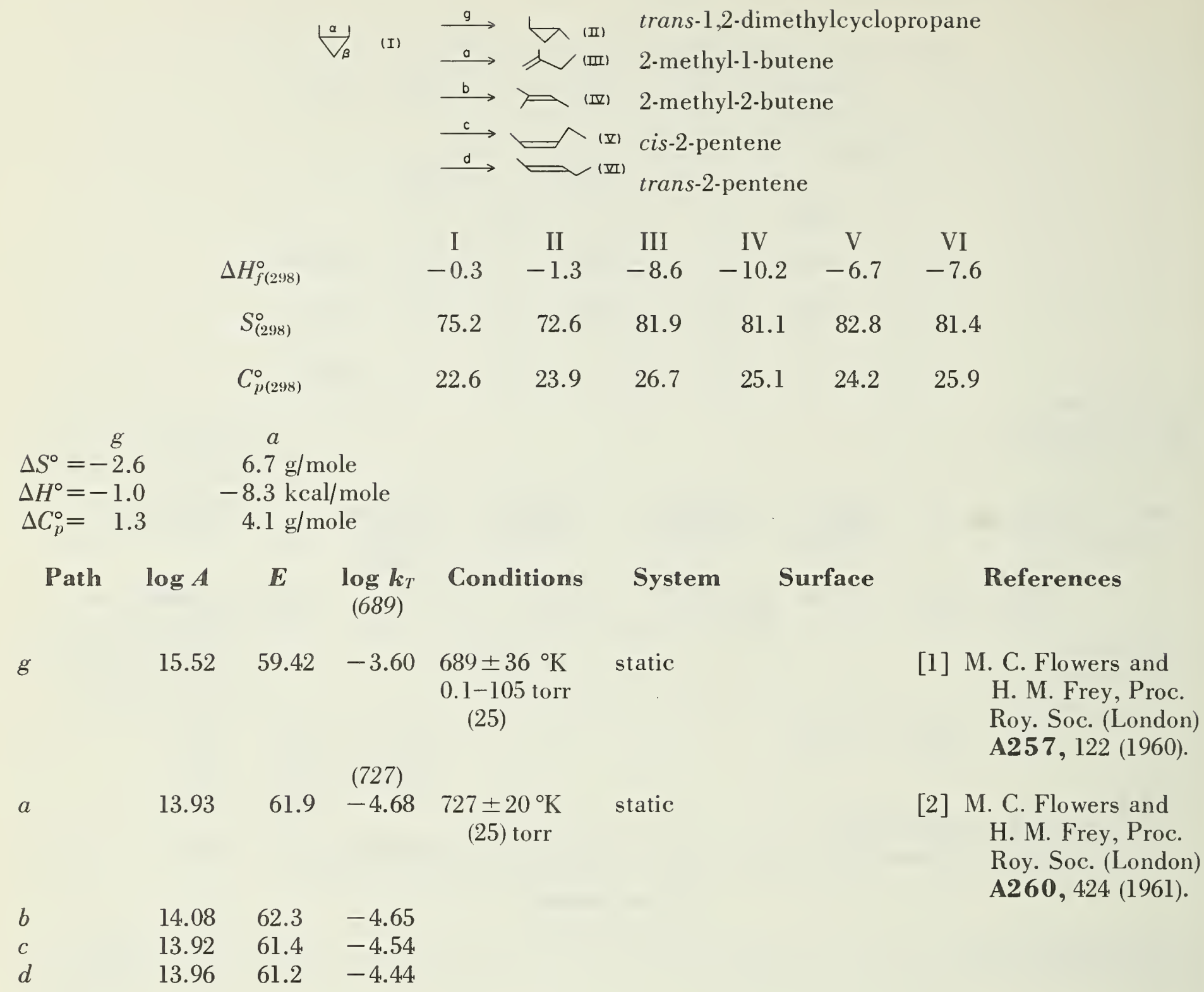

Preferred:

All rate constants are reliable as reported.

Comments: Transition state estimates for the biradical mechanism (see section III-4.0) are in reasonable agreement with the observed parameters.

Estimated: $\log k_{y}=15.5-61.2 / \theta$

$\log k_{c}=14.1-61.7 / \theta$.

(See also trans-1,2-dimethylcyclopropane.)

\section{Experimental}

[1] Analysis by G.L.C. Falloff observed near 16 torr.

[2] Analysis by G.L.C. Structural isomerization is slow relative to geometric isomerization at $727 \pm 20^{\circ} \mathrm{K}$. All the products expected are observed. Paths $c$ and $d$ by $\alpha(\mathrm{C}-\mathrm{C})$ rupture; paths $a$ and $b$ by $\beta(\mathrm{C}-\mathrm{C})$ rupture. 
Reaction: trans-1,2-Dimethylcyclopropane

\begin{tabular}{|c|c|c|c|c|c|c|c|}
\hline & & $\begin{array}{ll}\stackrel{a}{a} & \\
\stackrel{g}{\longrightarrow}\end{array}$ & $\forall$ & $\begin{array}{l}\text { ㅁ) } c i s-1 \\
\text { ताI) } 2-\mathrm{m}\end{array}$ & -Dimeth & $\begin{array}{l}\text { cyclop } \\
\text { ne }\end{array}$ & pane \\
\hline & (.) $\stackrel{a}{\longrightarrow}(I)$ & $b$ & & (D) $2-r$ & hyl-2-bl & & \\
\hline & & & & VII) $\operatorname{trc}$ & $s-2-$ pent & & \\
\hline & $\Delta H_{f(298)}^{\circ}$ & $\begin{array}{c}\text { I } \\
-1.3\end{array}$ & $\begin{array}{c}\text { II } \\
-0.3\end{array}$ & $\begin{array}{c}\text { III } \\
-8.6\end{array}$ & $\begin{array}{c}\text { IV } \\
-10.2\end{array}$ & $\begin{array}{c}\mathrm{V} \\
-6.7\end{array}$ & $\begin{array}{c}\text { VI } \\
-7.6\end{array}$ \\
\hline & $S^{\circ}{ }_{(298)}$ & 72.6 & 75.2 & 81.9 & 81.1 & 82.8 & 81.4 \\
\hline & $C_{p(298)}^{\circ}$ & 23.9 & 22.6 & 26.7 & 25.1 & 24.2 & 25.9 \\
\hline$b$ & $c$ & & & & & & \\
\hline$\Delta S^{\circ}=8.5$ & $10.2 \mathrm{~g} / \mathrm{mole}$ & & & & & & \\
\hline$\Delta H^{\circ}=-8.9$ & $-5.4 \mathrm{kcal} / \mathrm{mole}$ & & & & & & \\
\hline$\Delta C_{p}^{\circ}=1.2$ & $0.3 \mathrm{~g} / \mathrm{mole}$ & & & & & & \\
\hline
\end{tabular}

\begin{tabular}{|c|c|c|c|c|c|c|c|}
\hline Path & $\log A$ & $\boldsymbol{E}$ & $\begin{array}{l}\log \boldsymbol{k}_{T} \\
(689)\end{array}$ & Conditions & System & Surface & References \\
\hline$g$ & 15.97 & 60.49 & -3.22 & $\begin{array}{l}689 \pm 36^{\circ} \mathrm{K} \\
0.1-105 \\
\quad(25)\end{array}$ & static & & $\begin{array}{l}\text { [1] M. C. Flowers and } \\
\text { H. M. Frey, Proc. } \\
\text { Roy. Soc. (London) } \\
\text { A25 7, } 122(1960) .\end{array}$ \\
\hline$a$ & 13.93 & 61.9 & $\begin{array}{l}(727) \\
-4.68\end{array}$ & $\begin{array}{c}727 \pm 20^{\circ} \mathrm{K} \\
(25)\end{array}$ & static & & $\begin{array}{l}\text { [2] M. C. Flowers and } \\
\text { H. M. Frey, Proc. } \\
\text { Roy. Soc. (London) } \\
\text { A260, } 424 \text { (1961). }\end{array}$ \\
\hline $\begin{array}{l}b \\
c \\
d\end{array}$ & $\begin{array}{l}14.08 \\
14.40 \\
14.30\end{array}$ & $\begin{array}{l}62.3 \\
63.6 \\
62.9\end{array}$ & $\begin{array}{l}-4.65 \\
-5.02 \\
-4.61\end{array}$ & & & & \\
\hline
\end{tabular}

Preferred:

All rate constants are reliable as reported.

Comments: Estimated Arrhenius parameters (see section III-4.0) for the biradical mechanism are in reasonable agreement with observations: $\log k_{g}=15.8-62.2 / \theta ; \log k_{c}=14.3-62.7 / \theta$. A comparison of the cis and trans-1,2-dimethylcyclopropane parameters for the various reaction paths shows quite clearly that the rate-determining steps for the structural isomerizations are $\mathrm{H}$-migrations and that internal rotation and ring closing in the biradical are fast. Thus paths $a$ and $b$ involving $\beta(\mathrm{C}-\mathrm{C})$ rupture for both cis and trans reactants have identical parameters, indicating rapid equilibrium between rotational conformations in the biradical. Also, paths $c$ and $d$ involving $\alpha(\mathrm{C}-\mathrm{C})$ rupture are in the same ratio to one another in both systems and differ absolutely only by the differences in entropy and heats of formation of the reactants (i.e., by a cis methyl effect).

\section{Experimental}

[1] and [2] See comments on cis-1,2-dimethylcyclopropane. 
Reaction: 1,1-Dimethylcyclopropane

\begin{tabular}{|c|c|c|c|}
\hline$\lambda_{\beta}^{a}(I)$ & $\begin{array}{l}\stackrel{a}{\longrightarrow} \rightleftharpoons< \\
\stackrel{b}{\longrightarrow}\end{array}$ & $\begin{array}{l}\text { (II) } 3 \text {-methyl } \\
\text { (III) 2-methyl }\end{array}$ & $\begin{array}{l}\text { ene } \\
\text { ene }\end{array}$ \\
\hline$\Delta H_{f(298)}^{\circ}$ & $\begin{array}{c}\text { I } \\
-1.9\end{array}$ & $\begin{array}{c}\text { II } \\
-6.9\end{array}$ & $\begin{array}{c}\text { III } \\
-10.2\end{array}$ \\
\hline$S_{(298)}^{\circ}$ & 70.9 & 79.7 & 81. \\
\hline$C_{p(298)}^{\circ}$ & 24.7 & 28.1 & 25 \\
\hline
\end{tabular}

$\begin{array}{ccc} & a & b \\ \Delta S^{\circ}= & 8.8 & 10.2 \mathrm{~g} / \mathrm{mole} \\ \Delta H^{\circ}=-5.0 & -8.3 \mathrm{kcal} / \text { mole } \\ \Delta C_{p}^{\circ}=3.4 & 0.4 \mathrm{~g} / \mathrm{mole}\end{array}$

\begin{tabular}{|c|c|c|c|c|c|c|c|}
\hline Path & $\log A$ & $\boldsymbol{E}$ & $\begin{array}{c}\log \boldsymbol{k}_{T} \\
(750)\end{array}$ & Conditions & System & Surface & References \\
\hline & $\begin{array}{l}14.75 \\
14.75\end{array}$ & $\begin{array}{l}62.6 \\
62.6\end{array}$ & $\begin{array}{l}-3.49 \\
-3.49\end{array}$ & $\begin{array}{l}752 \pm 32{ }^{\circ} \mathrm{K} \\
0.1-550 \text { torr } \\
\quad(100)\end{array}$ & static & none & $\begin{array}{l}\text { [1] M. C. Flowers and } \\
\text { H. M. Frey, J. } \\
\text { Chem. Soc., } 3953 \\
\text { (1959). }\end{array}$ \\
\hline$-b$ & 15.05 & 62.6 & -3.13 & & & & \\
\hline$-b$ & 15.37 & 63.6 & -3.13 & $\begin{array}{l}735 \pm 20^{\circ} \mathrm{K} \\
0.0075-35 \text { torr } \\
\quad(35)\end{array}$ & static & & $\begin{array}{l}\text { [2] M. C. Flowers and } \\
\text { H. M. Frey, J. } \\
\text { Chem. Soc., } 1157 \\
\text { (1962). }\end{array}$ \\
\hline
\end{tabular}

Preferred:

Rate constants are reliable as reported.

Comments: All products expected by the biradical mechanism were observed: $\alpha(\mathrm{C}-\mathrm{C})$ split, 3 -methyl-1butene and 2-methyl-2-butene; $\beta(\mathrm{C}-\mathrm{C})$ split cannot lead to products. Transition state estimates are in excellent agreement with the observed parameters.

Estimated: $\log A_{a}=14.7$

$\log A_{b}=14.7$

$\log E_{a}=E_{b}=61.3 \mathrm{kcal} / \mathrm{mole}$ (see section III-4.0).

\section{Experimental}

[1] Analysis by G.L.C. Falloff observed near 4-8 torr ( $\left.743^{\circ} \mathrm{K}\right)$. 2-methyl-1-butene amounted to 1-2 percent of products. Slight rate acceleration with added NO.

[2] Analysis by G.L.C. Temperature dependence of falloff gave $n \simeq 19$ from the Slater equation $(\log P=n / 2 \log$ $\left.T_{2} / T_{1}\right)$ gave $n \simeq 29$. Thus the Slater equation is not consistent with the data. Relative third-body collision efficiencies determined for $\mathrm{CH}_{4}, \mathrm{H}_{2}, \mathrm{~N}_{2}, \mathrm{Ar}, \mathrm{He}, \mathrm{C}_{2} \mathrm{H}_{4}, \mathrm{CF}_{4}, \mathrm{SF}_{6}, \mathrm{CO}_{2}$, and toluene. In another pertinent study, M. C. Flowers and H. M. Frey [J. Phys. Chem. 65, 373 (1961)] found that $k_{x}$ did not decrease at very high pressures, contrary to theoretical predictions. [See D. J. Wilson, J. Phys. Chem. 64, 323 (1960).] 
Reaction: Ethylcyclopropane

$\begin{aligned} \Delta S^{\circ}= & 5.4 \mathrm{~g} / \mathrm{mole} \\ \Delta H^{\circ}= & -9.3 \mathrm{kcal} / \mathrm{mol}\end{aligned}$

$\nabla$ (I)

$\stackrel{a}{\longrightarrow}$ cis and trans $\cdot \mathrm{CH}_{3} \mathrm{CH}=\mathrm{CHCH}_{2} \mathrm{CH}_{3}$ (II)

$\stackrel{b}{\longrightarrow} \mathrm{CH}_{2}=\mathrm{C}\left(\mathrm{CH}_{3}\right) \mathrm{CH}_{2} \mathrm{CH}_{3}(\mathrm{III})$

$\stackrel{c}{\longrightarrow} \mathrm{CH}_{2}=\mathrm{CHCH}=\mathrm{CH}_{2}(\mathrm{IV})+\mathrm{CH}_{4}(\mathrm{~V})$

$\stackrel{d}{\longrightarrow} \mathrm{CH}_{2}=\mathrm{CH}\left(\mathrm{CH}_{2}\right)_{2} \mathrm{CH}_{3}(\mathrm{VI})$

$\Delta H_{f(298)}^{\circ}$

(cis) (trans)

$$
\begin{aligned}
& S_{(298)}^{\circ} \\
& C_{p(298)}^{\circ}
\end{aligned}
$$

76.5

$-6.7$

$-7.6$

III

IV

$\begin{array}{ll}\mathrm{V} & \mathrm{V} \text { I }\end{array}$

0.7

82.8

81.4

81.9

66.6

$-17.9$

$-5.0$

24.0

24.2

25.9

26.7

19.0

44.5

83.3

$8.5 \quad 26.3$

$\Delta H^{\circ}=-9.3 \mathrm{kcal} / \mathrm{mole}$

$\Delta C_{p}^{\circ}=2.7 \mathrm{~g} / \mathrm{mole}$

\section{$\begin{array}{lllllll}\text { Path } & \log A & \boldsymbol{E} & \log k_{T} & \text { Conditions } & \text { System } & \text { Surface }\end{array}$}

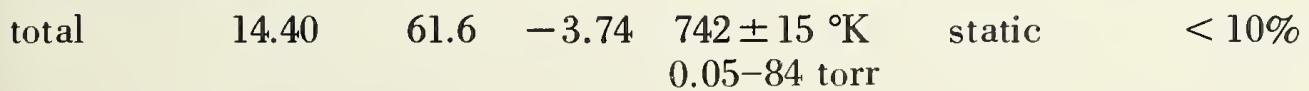

[1] M. L. Halberstadt and J. P. Chesick, J. Phys. Chem. 69, 429 (1965).

\section{Preferred:}

Paths $a, b$, and $d$ are reliable; path $c$ is highly suspect.

Comments: Path $a, b$, and $d$, by analogy with other cyclopropane ring isomerization reactions, are undoubtedly unimolecular and surface independent reactions. The surface dependence observed in the pentene/butadiene ratio probably comes from path $c$. Reaction $c$ is not likely to be unimolecular since any reasonable transition state to products involves a highly concerted process with pentavalent carbon (i.e., $\stackrel{H}{H}^{+}$). A heterogeneous free radical mechanism seems more plausible. (See comments on 1,1-diethylcyclopropane.) The calculated Arrhenius parameters for the various structural isomerization reactions are the same as those obtained for methylcyclopropane except that all activation energies are lower by $0.8 \mathrm{kcal} / \mathrm{mole}$. This is a result of the gauche repulsion of the ethyl group with the ring which is relieved in the biradical and transition states.

Estimated: $\log k_{\text {est }}=15.1-63.2 / \theta$ (see section III-4.0).

\section{Experimental}

[1] Analysis by G.L.C. Rates of pentenes/butadiene increased with increasing $(s / v)$. Fallout observed near 2 torr $\left(740^{\circ} \mathrm{K}\right)$. An RRK- $s=22$ fit the falloff. The lifetime of the activated molecule was found to be a factor of 10 longer than that for methylcyclopropane. 
Reaction: cis-1-Ethyl-2-methylcyclopropane $(a)$

trans-1-Ethyl-2-methylcyclopropane $(b)$

\begin{tabular}{|c|c|c|}
\hline$\Delta H_{f(298)}^{\circ}$ & $\begin{array}{c}\text { I } \\
-5.3\end{array}$ & $\begin{array}{c}\text { II } \\
-6.3\end{array}$ \\
\hline$S_{(298)}^{\circ}$ & 82.8 & 83.2 \\
\hline$C_{p(298)}^{\circ}$ & 28.1 & 29.4 \\
\hline
\end{tabular}

$a$
$\Delta S^{\circ}=0.4 \mathrm{~g} / \mathrm{mole}$
$\Delta H^{\circ}=-1.0 \mathrm{kcal} / \mathrm{mole}$
$\Delta C_{p}^{\circ}=1.3 \mathrm{~g} / \mathrm{mole}$

Path $\log A \quad E \quad \log k_{T}$ Conditions System Surface $\quad$ References (694)

$\begin{array}{lllllll}a & 15.08 & 58.87 & -3.46 & 694 \pm 25^{\circ} \mathrm{K} & \text { static } & \text { none }\end{array}$ 3-100 torr

[1] C. S. Elliot and H. M.

Frey, J. Chem. Soc. 900 (1964).

$\begin{array}{llll}b & 15.08 & 60.07 & -3.84\end{array}$

Preferred:

$\log k_{a}=15.08-58.87 / \theta ; \log k_{b}=15.08-60.07 / \theta$

Comments: Transition state estimates for the biradical mechanism (see section III-4.0) with ring opening as rate determining give: $\log A_{a}=\log A_{b}=15.5 ; E_{a}=60.4, E_{b}=61.4$.

\section{Experimental}

[1] Analysis by G.L.C. Slow structural isomerizations to $C_{6}$ olefins were also observed. Olefin products identified were cis and trans-2-hexene, 3-emthyl-2-pentene, and 2-methyl-1-pentene. Measurement of the cis $\rightleftharpoons$ trans equilibrium over the temperature range of study gave $K_{e q}=10^{0.5 / 4.575-1.2 / \theta}$. 
Reaction: (a) cis,cis-1,2,3-Trimethylcyclopropane

(b) cis,trans-1,2,3-Trimethylcyclopropane

\begin{tabular}{|c|c|c|}
\hline$\Delta H_{f(298)}^{\circ}$ & $\begin{array}{c}\text { I } \\
-5.3\end{array}$ & $\begin{array}{c}\text { II } \\
-7.3\end{array}$ \\
\hline$S_{(298)}^{\circ *}$ & 82.5 & 81.7 \\
\hline$C_{p(298)}^{\circ}$ & 25.2 & 28.0 \\
\hline
\end{tabular}

$$
\begin{aligned}
a & \\
\Delta S^{\circ} & =-0.8 \mathrm{~g} / \mathrm{mole} \\
\Delta H^{\circ} & =-2.0 \mathrm{kcal} / \mathrm{mole} \\
\Delta C_{p}^{\circ}= & 2.8 \mathrm{~g} / \mathrm{mole}
\end{aligned}
$$

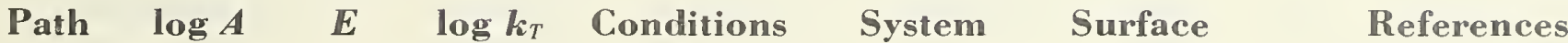 (696)}

$a$

$$
60.95-3.36
$$

$696 \pm 28^{\circ} \mathrm{K}$ static none

[1] H. M. Frey and D. C. Marshall, J. Chem. Soc. 5717 (1963).

$b$

$63.25-4.56$

Preferred:

$\log k_{a}=15.78-60.95 / \theta$

$\log k_{b}=15.30-63.25 / \theta$.

Comments: The agreement between the observed Arrhenius parameters and those calculated by transition state methods for a biradical mechanism (see section III-4.0) is acceptable.

Estimated: $* \log A_{a}=16.0$

${ }^{*} \log A_{b}=16.2$

$E_{a}=61.3$

$E_{b}=63.3$.

\section{Experimental}

[1] Analysis by G.L.C. A concurrent slow structural isomerization produced cis and trans-3-methyl-2-pentene. Small yields of 2-ethyl-1-butene were observed at runs carried to near completion. Falloff observed near 1 torr.

*Entropy estimates (which do not agree with the kinetics) are not considered to be particularly accurate (i.e., $\pm 2 \mathrm{~g} / \mathrm{mole}$ ). 
Reaction: 1,1-Diethylcyclopropane

\begin{tabular}{crrrrr} 
& \multicolumn{1}{c}{} & II & III & IV & \multicolumn{1}{c}{ V } \\
$\Delta H_{f(298)}^{\circ}$ & -11.8 & -16.0 & -19.3 & 13.1 & -17.9 \\
$S_{(298)}^{\circ}$ & 89.7 & 98.5 & 100.3 & 85.2 & 44.5 \\
$C_{p(298)}^{\circ}$ & 35.7 & 37.5 & 37.2 & 30.1 & 8.5
\end{tabular}

$$
\begin{gathered}
a \\
\Delta S^{\circ}=8.8 \mathrm{~g} / \mathrm{mole} \\
\Delta H^{\circ}=-4.2 \mathrm{kcal} / \mathrm{mole} \\
\Delta C_{p}^{\circ}=1.8 \mathrm{~g} / \mathrm{mole}
\end{gathered}
$$

\begin{tabular}{|c|c|c|c|c|c|c|c|}
\hline Path & $\log A$ & $\boldsymbol{E}$ & $\begin{array}{c}\log \boldsymbol{k}_{T} \\
\quad(723)\end{array}$ & Conditions & ¿System & Surface & References \\
\hline & 14.95 & 63.8 & -4.34 & $\begin{array}{l}723 \pm 25^{\circ} \mathrm{K} \\
0.2-16 \text { torr } \\
(4.5)\end{array}$ & static & $<10 \%$ & $\begin{array}{l}\text { [1] H. M. Frey and D. C. } \\
\text { Marshall, J. Chem. } \\
\text { Soc. } 191 \text { (1965). }\end{array}$ \\
\hline & 14.84 & 63.4 & $\begin{array}{l}-4.33 \\
-4.48\end{array}$ & & & & \\
\hline
\end{tabular}

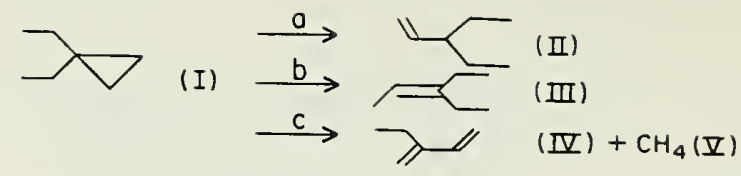

Preferred:

Reactions $a$ and $b$ are reliable as reported; reaction $c$ is unreliable.

Comments: By the biradical mechanism one would expect the parameters of paths $a$ and $b$ to be the same as those for 1,1-dimethylcyclopropane (which is the case within experimental error). The unimolecularity of path $c$ is suspect. The observed Arrhenius parameters imply a large entropy increase $(\Delta S \ddagger=+9 \mathrm{~g} / \mathrm{mole}$ ) in going to the transition state. However, the concerted process required would, by transition state estimates, involve a zero or negative entropy of activation. Thus, path $c$ would appear to be most reasonably explained in terms of a surface sensitive free radical process propagated by the reactions:

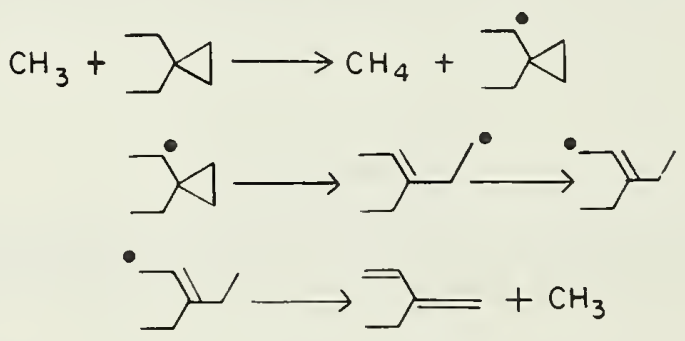

(See also comments on ethylcyclopropane.)

\section{Experimental}

[1] Analysis by G.L.C. Rate falloff observed near 1 torr. 
Reaction: 1,1,2,2-Tetramethylcyclopropane

\begin{tabular}{|c|c|c|}
\hline$\Delta H_{f(298)}^{\circ}$ & $\begin{array}{c}\text { I } \\
-14.7\end{array}$ & $\begin{array}{c}\text { II } \\
-22.3\end{array}$ \\
\hline$S_{(298)}^{\circ}$ & 85.1 & 95.3 \\
\hline$C_{p(298)}^{o}$ & 36.0 & 38.6 \\
\hline
\end{tabular}

$$
\begin{gathered}
a \\
\Delta S^{\circ}=9.2 \mathrm{~g} / \mathrm{mole} \\
\Delta H^{\circ}=-7.6 \mathrm{kcal} / \mathrm{mole} \\
\Delta C_{p}^{\circ}=2.6 \mathrm{~g} / \mathrm{mole}
\end{gathered}
$$

\begin{tabular}{|c|c|c|c|c|c|c|}
\hline $\log A$ & $\boldsymbol{E}$ & $\begin{array}{c}\log \boldsymbol{k}_{\boldsymbol{T}} \\
(733)\end{array}$ & Conditions & System & Surface & References \\
\hline 15.83 & $* 64.4$ & -3.38 & $\begin{array}{l}733 \pm 25^{\circ} \mathrm{K} \\
2-22.5 \text { torr } \\
\quad(5)\end{array}$ & static & none & $\begin{array}{l}\text { [1] H. M. Frey and D. C. } \\
\text { Marshall, J. Chem. } \\
\text { Soc. } 3052 \text { (1962). }\end{array}$ \\
\hline
\end{tabular}

Preferred:

$\log k=14.4-59.6 / \theta$. Rate constants around $\bar{T}$ are probably reliable.

Comments: The single product expected on the basis of the free radical mechanism (i.e., by $\alpha(\mathrm{C}-\mathrm{C})$ rupture) was the only primary product reported. By comparison to the Arrhenius parameters of other alkyl substituted cyclopropanes, the values for tetramethylcyclopropane appear high. The rather narrow temperature range of study and the probable errors in $E$ tend to confirm this suspicion. We prefer the transition state estimate of the $A$-factor and have adjusted the activation energy accordingly (see section III-4.0).

\section{Experimental}

[1] Analysis by G.L.C. Falloff observed near 0.5 torr. A slow subsequent isomerization and decomposition of the product was observed.

*Estimated error in $E$ is $\pm 2.4 \mathrm{kcal} / \mathrm{mole}$. 
Reaction: Bicyclopropyl

$$
\begin{aligned}
\stackrel{a}{\beta} \perp_{\text {(I) }} \stackrel{a}{\longrightarrow} \text { cyclohexerie } \mathbb{C} \text { (II) } \\
\stackrel{b}{\longrightarrow} \text { all products (see below) }
\end{aligned}
$$

$\begin{array}{ccc}\Delta H_{f(298)}^{\circ} & \text { I } & \text { II } \\ S_{(298)}^{\circ} & 31.6 & -1.7 \\ C_{p(298)}^{\circ} & 76.3 & 74.3\end{array}$

$\Delta S^{\circ}=-2.0 \mathrm{~g} / \mathrm{mole}$

$\Delta H^{\circ}=-33.3 \mathrm{kcal} / \mathrm{mole}$

$\Delta C_{p}^{\circ}=0.1 \mathrm{~g} / \mathrm{mole}$

\begin{tabular}{|c|c|c|c|c|c|c|c|}
\hline Path & $\log A$ & $\boldsymbol{E}$ & $\begin{array}{c}\log \boldsymbol{k}_{T} \\
(714)\end{array}$ & Conditions & System & Surface & References \\
\hline 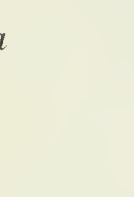 & 16.8 & 70.8 & -4.87 & $714 \pm 33^{\circ} \mathrm{K}$ & static & 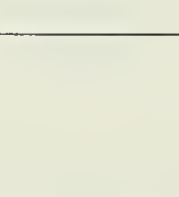 & $\begin{array}{l}\text { [1] M. C. Flowers and } \\
\text { H. M. Frey, J. } \\
\text { Chem. Soc., } 1689 \\
\text { (1962). }\end{array}$ \\
\hline
\end{tabular}

Preferred:

Reliable as reported.

Comments: The biradical mechanism is able to identify the 17 products formed. Thus, $\alpha(\mathrm{C}-\mathrm{C})$ split leads to four primary products, three of which may decompose to give eight more products.

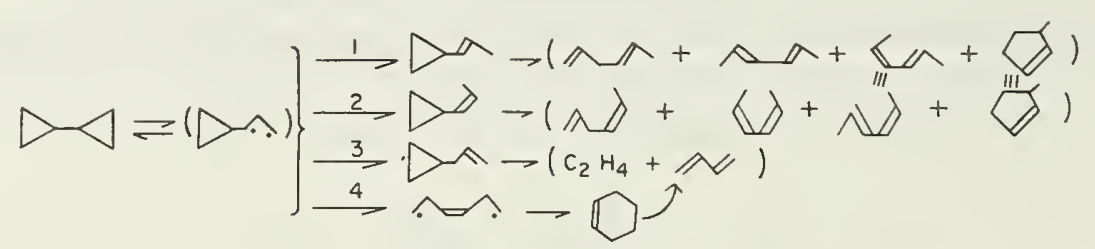

$\beta(\mathrm{C}-\mathrm{C})$ split leads to only one primary product which has four secondary products.

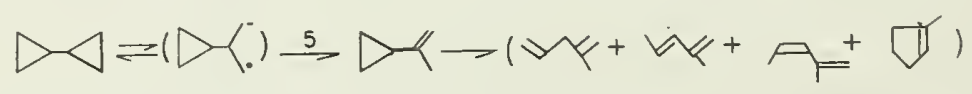

Thus we have five primary products and 12 secondary products for a total of 17 products (as observed).

Quantitatively, all primary processes involve H-migrations with the exception of that forming cyclohexene. Parameters similar to those reactions producing olefins from methylcyclopropane are therefore predicted from transition state calculations. Thus $\log k_{b(\text { est })}=15.5-64.2 / \theta$. A factor of 2 in $A$ has been used to account for isomerization from each cyclopropane ring. Since $E_{b(\mathrm{obs})}=60.4$ (i.e., $\sim 4 \mathrm{kcal} / \mathrm{mole}$ lower than the methylcyclopropane reaction activation energy), a resonance energy of this size could exist in the 1 cyclopropyl-1,3-n-propyl biradical ( $>$ i .

\section{Experimental}

[1] Analysis by G.L.C. Secondary decomposition produced $C_{6}$ dienes. At least 17 products were formed. The most important were: 1,3-butadiene, ethylene, isopropenylcyclopropane, cis and trans-1-cyclopropylpropene. 
Reaction: Vinylcyclopropane

$\begin{array}{cccccc} & \text { I } & \text { II } & \text { III } & \text { IV } & \text { V } \\ \Delta H_{f(298)}^{\circ} & 30.0 & 7.9 & 25.2 & 19.7 & 18.6 \\ S_{(298)}^{\circ} & 74.2 & 69.2 & 79.7 & 76.5 & 76.4 \\ C_{p(298)}^{\circ} & 24.0 & 18.1 & 25.1 & 22.6 & 24.4\end{array}$

$\begin{array}{rc}a & b \\ \Delta S^{\circ}=-5.0 & 5.5 \mathrm{~g} / \mathrm{mole} \\ \Delta H^{\circ}=-22.1 & -4.8 \mathrm{kcal} / \mathrm{mole} \\ \Delta C_{p}^{\circ \prime}=-5.9 & 1.1 \mathrm{~g} / \mathrm{mole}\end{array}$

\begin{tabular}{|c|c|c|c|c|c|c|}
\hline Path & $\log A$ & $\boldsymbol{E}$ & $\begin{array}{c}\log \boldsymbol{k}_{T} \\
(630)\end{array}$ & Conditions & System & Surface \\
\hline & 13.61 & 49.7 & -3.61 & $\begin{array}{l}631 \pm 33^{\circ} \mathrm{K} \\
0.14-24.7\end{array}$ & static & $<10 \%$ \\
\hline & 14.43 & 57.3 & -5.42 & (10) & & \\
\hline & 13.90 & 56.2 & -5.57 & \multicolumn{3}{|c|}{ - same as above- } \\
\hline & 13.00 & 53.6 & -5.57 & & & \\
\hline & 13.50 & 49.6 & -3.67 & $639 \pm 26^{\circ} \mathrm{K}$ & & \\
\hline
\end{tabular}

\section{Preferred:}

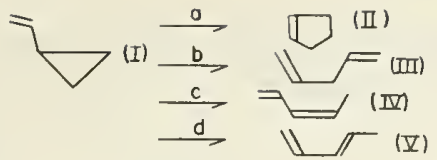

Reliable as reported with the experimental error limits noted below.

Comments: Mechanism:

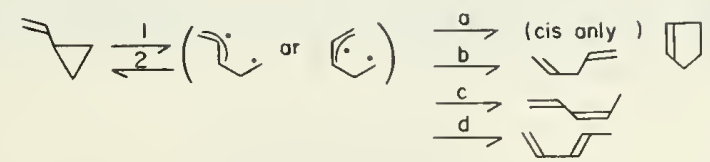

with $k_{2}>k_{a}+k_{b}+k_{c}+k_{d}$.

Transition state estimates (see discussion on small ring compound reactions, section III-4.2) give:

$$
\begin{aligned}
& \log k_{a}=13.8-49.7 / \theta \\
& \log k_{b}=14.5-63.8(-) / \theta \quad \text { (57.5 with half allylic resonance) } \\
& \log k_{c}=13.9-57.5 / \theta \\
& \log k_{d}=13.3-51.2 / \theta .
\end{aligned}
$$

[2] M. C. Flowers and H. M. Frey, J. Chem. Soc. 3547 (1961).

References J. Phys. Chem. 66, 1671 (1962).

The activation energy for $\mathrm{C}_{5}$ ring closing in vinylcyclopropane systems is estimated to be: $E_{a}=E_{\mathrm{C}_{5} \text { r.c. }}=8.3$ $\mathrm{kcal} / \mathrm{mole}$.

\section{Experimental}

[1] Analysis by G.L.C. Product identification with I.R. Falloff observed near 8 torr. No effect on the rate with added NO.

[2] Analysis by G.L.C. Other olefin products less than 1 percent. Products are those predicted by the biradical mechanism. Since products other than cyclopentene are minor ones, their rate constant parameters are not exceptionally reliable (i.e., $\Delta E \simeq 2-4 \mathrm{kcal} / \mathrm{mole}$ ). 
Reaction: cis-1-Methyl-2-vinylcyclopropane

$\begin{array}{ccc} & \text { cis-1,4-hexadiene } \\ \Delta H_{f(298)}^{\circ} & \text { II } & \text { II } \\ S_{(298)}^{\circ} & 80.5 & 19.0 \\ C_{p(298)}^{\circ} & 28.0 & 29.3 \\ & & 29.2\end{array}$

$\Delta S^{\circ}=7.3 \mathrm{~g} / \mathrm{mole}$

$\Delta H^{\circ}=-5.2 \mathrm{kcal} / \mathrm{mole}$

$\Delta C_{p}^{\circ}=1.2 \mathrm{~g} / \mathrm{mole}$

\begin{tabular}{|c|c|c|c|c|c|c|}
\hline $\log A$ & $\boldsymbol{E}$ & $\begin{array}{c}\log \boldsymbol{k}_{T} \\
(457)\end{array}$ & Conditions & System & Surface & References \\
\hline 11.03 & 31.2 & -3.89 & $\begin{array}{l}457 \pm 28^{\circ} \mathrm{K} \\
3-40 \text { (3) torr }\end{array}$ & static & none & $\begin{array}{c}\text { [1] R. J. Ellis and H. M. } \\
\text { Frey, Proc. Chem. } \\
\text { Soc., 221 (1964) } \\
\text { (preliminary). } \\
\text { [2] R. J. Ellis and H. M. } \\
\text { Frey, J. Chem. Soc } \\
5578 \text { (1964). }\end{array}$ \\
\hline
\end{tabular}

Preferred:

Reliable as reported.

Comments: The Arrhenius parameters are extraordinarily low and indicate that this reaction cannot be a biradical process. If the parameters are compared to those for the "ene" type isomerization of 4-methyl-1,3-

pentadiene and its inverse $\left(>\frac{1}{2}-\angle ; \log k_{1}=11.72-36.19 / \theta ; \log k_{2}=11.24-32.76 / \theta\right)$ it is apparent that the cis-1-methyl-2-vinylcyclopropane reaction is concerted and proceeds through a six-centered transition state.

\section{Experimental}

[1] and [2] Analysis by G.L.C. Identification of products by I.R. The trans isomer was not formed. 
Reaction: trans-1-Methyl-2-vinylcyclopropane

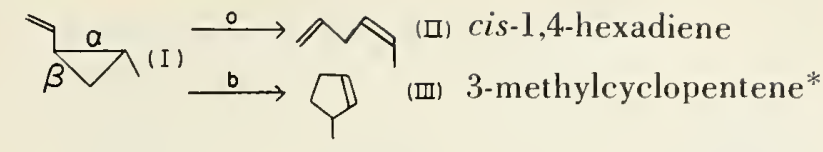

$\begin{array}{cccc} & \text { I } & \text { II } & \text { III } \\ \Delta H_{f(298)}^{\circ} & 23.0 & 19.0 & 0.0 \\ S_{(298)}^{\circ} & 80.9 & 89.3 & 79.0 \\ C_{p(298)}^{\circ} & 29.3 & 29.2 & 25.9\end{array}$
$a \quad b$
$\Delta S^{\circ}=6.9$
$\Delta H^{\circ}=-4.0$
$-3.4 \mathrm{~g} / \mathrm{mole}$
$\Delta C_{p}^{\circ}=-0.1$
$-23.0 \mathrm{kcal} / \mathrm{mole}$
$-3.4 \mathrm{~g} / \mathrm{mole}$

$\begin{array}{llllll}\text { Path } & \log A & \boldsymbol{E} & \log k_{T} & \text { Conditions } & \text { System }\end{array}$ (585)

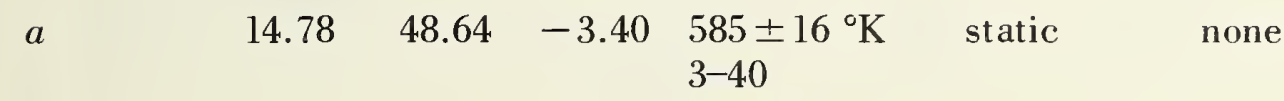

same as above

\section{References}

[1] R. J. Ellis and H. M. Frey, J. Chem. Soc., 5578 (1964).

$b$

13.67

$48.64-4.51$

same as above $\longrightarrow$

Preferred:

Both reactions reliable as reported.

Comments: Reaction $a$ undoubtedly involves geometric isomerization to the cis isomer (with ring opening as rate determining) followed by the rapid concerted isomerization of cis-1-methyl-2-vinylcyclopropane to cis1,4-hexadiene. By the biradical mechanism reaction, $b$ must proceed via $\beta(\mathrm{C}-\mathrm{C})$ cleavage and have as its rate-determining step $\mathrm{C}_{5}$ ring closing. 4-methyl-1-cyclopentene was not observed; therefore, $\alpha(\mathrm{C}-\mathrm{C})$ cleavage (which would be expected to be the favored reaction path) leads to hexadiene formation, and not $\mathrm{C}_{5}$ ring formation.*

Mechanism:

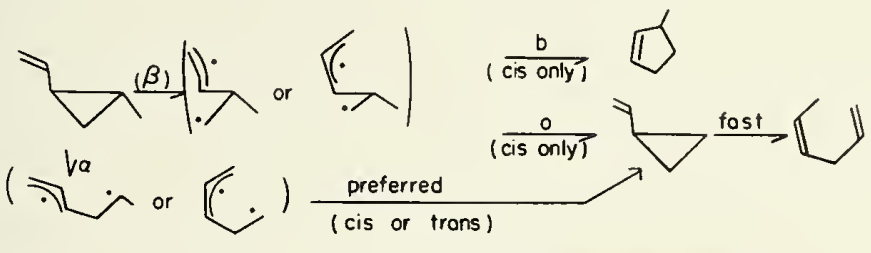

\section{Experimental}

[1] Analysis by G.L.C. Identification confirmed with I.R.

*NOTE added in proof: The cyclopentene product was incorrectly reported and should be 4-methylcyclopentene (private communication, H. M. Frey). Thus the "expected" product via the energetically favored $\alpha(C-C)$ rupture was the product formed. The discussion of this reaction in section III-4.3 is consistent with the "real" facts. 
Reaction: 1-Methyl-1-vinylcyclopropane

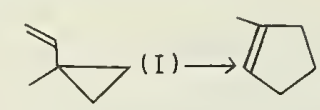

$\Delta H_{f(298)}^{\circ}$

$S_{(298)}^{\circ}$

$C_{p(298)}^{\circ}$ (II)

23.0

79.4

78.0

26.2

$\Delta S^{\circ}=-1.4 \mathrm{~g} / \mathrm{mole}$

$\Delta H^{\circ}=-23.3 \mathrm{kcal} / \mathrm{mole}$

$\Delta C_{p}^{\circ}=-2.0 \mathrm{~g} / \mathrm{mole}$

$\begin{array}{cccccc}\log A & \boldsymbol{E} & \begin{array}{c}\log \boldsymbol{k}_{T} \\ (608)\end{array} & \text { Conditions } & \text { System } & \text { Surf } \\ 14.11 & 49.35 & -3.64 & \begin{array}{l}608 \pm 22 \\ 2-30 \text { torr }\end{array} & \text { static } & \text { none }\end{array}$

\section{Preferred:}

Reliable as reported.

Transition state estimate gives, $\log A_{\text {est }}=14.1$ (see section III-4.2).

\section{Experimental}

[1] Analysis by G.L.C. Rate constants were not pressure dependent. 
Reaction: Isopropenylcyclopropane

\begin{tabular}{ccc} 
& \multicolumn{2}{c}{ 1-methylcyclopentene } \\
$\Delta H_{(\mathrm{I}(298)}^{\circ}$ & $\mathrm{II}$ & $\mathrm{II}$ \\
$S_{(298)}^{\circ}$ & 22.0 & -0.3 \\
$C_{p(298)}^{\circ}$ & 81.7 & 78.0 \\
& 30.1 & 24.2
\end{tabular}

$$
\begin{aligned}
& \Delta S^{\circ}=-3.7 \mathrm{~g} / \mathrm{mole} \\
& \Delta H^{\circ}=-22.3 \mathrm{kcal} / \mathrm{mole} \\
& \Delta C_{p}^{\circ}=-5.9 \mathrm{~g} / \mathrm{mole}
\end{aligned}
$$

\begin{tabular}{|c|c|c|c|c|c|c|}
\hline $\log A$ & $\boldsymbol{E}$ & $\begin{array}{c}\log \boldsymbol{k}_{T} \\
(605)\end{array}$ & Conditions & System & Surface & References \\
\hline 13.89 & 50.9 & -3.72 & $\begin{array}{l}605-659^{\circ} \mathrm{K} \\
0.5-21 \text { torr } \\
\quad(5)\end{array}$ & static & none & $\begin{array}{l}\text { [1] H. M. Frey and D. C. } \\
\text { Marshall, J. Chem. } \\
\text { Soc., } 3981 \text { (1962). }\end{array}$ \\
\hline
\end{tabular}

Preferred:

Reliable as reported.

Comments: Transition state estimates based on the biradical mechanism (see vinylcyclopropane) are in good agreement with the observed parameters: $\log A_{\text {est }}=14.1 ; E=49.7 \mathrm{kcal} / \mathrm{mole}$.

\section{Experimental}

[1] Analysis by G.L.C. 
Reaction: trans-1-Cyclopropyl-1-butene

$\begin{array}{ccc}\longrightarrow(\mathrm{I}) \longrightarrow \text { (II) } & \text { 3-ethylcyclopentene } \\ \Delta H_{\text {f(298) }}^{\circ} & \mathrm{I} & \mathrm{II} \\ S_{(298)}^{\circ} & 17.5 & -3.3 \\ C_{p(298)}^{\circ} & 92.6 & 88.4 \\ & 34.2 & 29.4\end{array}$

$\Delta S^{\circ}=-4.2 \mathrm{~g} / \mathrm{mole}$

$\Delta H^{\circ}=-20.8 \mathrm{kcal} / \mathrm{mole}$

$\Delta C_{p}^{\circ}=-4.8 \mathrm{~g} / \mathrm{mole}$

\begin{tabular}{lrlllll}
$\log \boldsymbol{A}$ & $\boldsymbol{E}$ & $\begin{array}{c}\log \boldsymbol{k}_{T} \\
(626)\end{array}$ & Conditions & System & Surface & \multicolumn{1}{c}{ References } \\
13.79 & 49.98 & -3.60 & $\begin{array}{l}626 \pm 20^{\circ} \mathrm{K} \\
2-25 \text { torr }\end{array}$ & static & none & [1] R. J. Ellis and H. M. \\
Frey, J. Chem. Soc., \\
\end{tabular}

Preferred:

Reliable as reported.

Comments: Biradical mechanism transition state estimates give: $\log k_{\mathrm{est}}=13.8-49.7 / \theta$ (see section III-4.2).

\section{Experimental}

[1] Analysis by G.L.C. The rate constants were independent of pressure. At the highest temperatures very small yields of various dienes were also detected. 
Reaction: 1-Cyclopropyl-2-methyl-1-propene

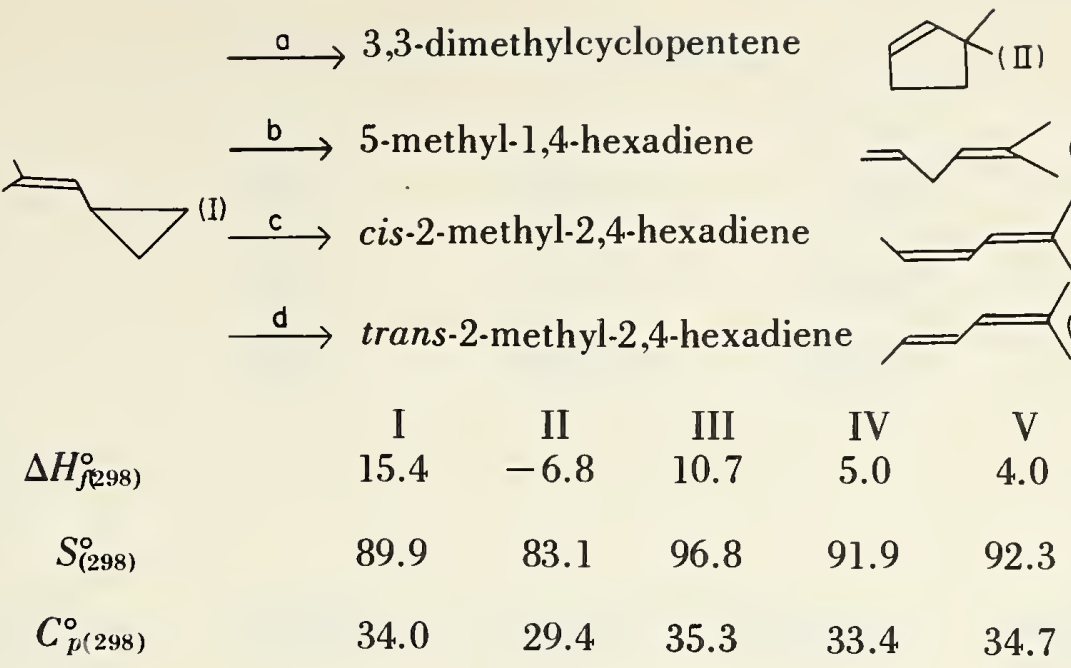

$\begin{array}{ccc} & a & b \\ \Delta S^{\circ}= & -6.8 & 6.9 \mathrm{~g} / \mathrm{mole} \\ \Delta H^{\circ}= & -22.2 & -4.7 \mathrm{kcal} / \mathrm{mole} \\ \Delta C_{p}^{\circ}= & -4.6 & 1.3 \mathrm{~g} / \mathrm{mole}\end{array}$

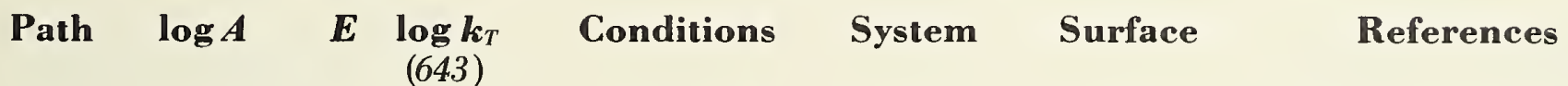

$\begin{array}{lllllll}a & 14.0 & 54.6 & -4.56 & 643 \pm 20^{\circ} \mathrm{K} & \text { static } & <10 \%\end{array} \quad$ [1] C. S. Elliott and 2-17 torr H. M. Frey, J.

Chem. Soc., 345

(1965).

$\begin{array}{llll}b & 14.61 & 56.65 & -4.65 \\ c & 13.33 & 53.0 & -4.68 \\ d & 13.25 & 52.1 & -4.46\end{array}$

Preferred:

Reliable as reported.

Comments: Transition state estimates:

$$
\begin{aligned}
& \log k_{a}=14.0-49.7 / \theta \\
& \log k_{b}=14.6-57.2 / \theta \quad \text { (half-allylic resonance) } \\
& \log k_{c}=13.0-51.2 / \theta \\
& \log k_{d}=13.2-51.2 / \theta
\end{aligned}
$$

The discrepancy between the estimated and observed activation energy in the cyclopentene formation could be a result of steric effects caused by the methyl substitutions at the position for ring closing and in the cis form of the biradical. (See the discussion on vinylcyclopropane and section III-4.2).

\section{Experimental}

[1] Analysis by G.L.C. Identification with M.S. and I.R. The diene products are those predicted by the biradical mechanism. Absence of 2,4-dimethyl-1,3-pentadiene in the products would be expected on the bsais of energy considerations (i.e., no allylic resonance stabilization of the biradical intermediate formed via $\beta(C-C)$ rupture). 
Reaction: 1-Isopropenyl-1-methylcyclopropane

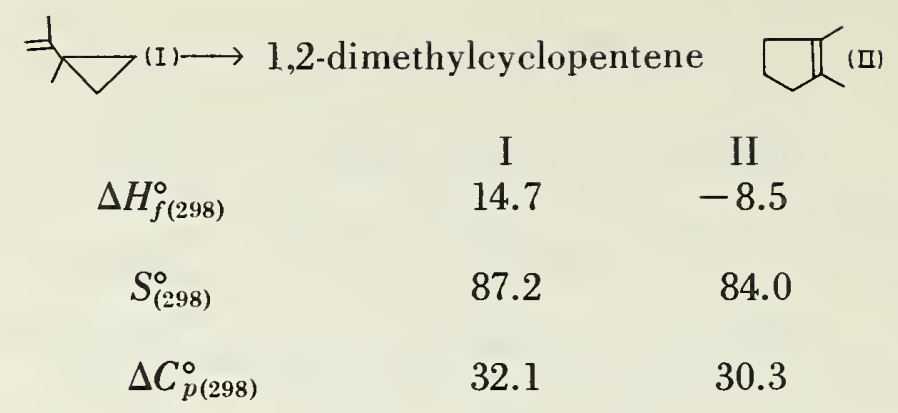

$\Delta S^{\circ}=-3.2 \mathrm{~g} / \mathrm{mole}$

$\Delta H^{\circ}=-23.2 \mathrm{kcal} / \mathrm{mole}$

$\Delta C_{p}^{\circ}=-1.8 \mathrm{~g} / \mathrm{mole}$

\begin{tabular}{|c|c|c|c|c|c|c|}
\hline $\log A$ & $\boldsymbol{E}$ & $\begin{array}{c}\log \boldsymbol{k}_{T} \\
(620)\end{array}$ & Conditions & System & Surface & References \\
\hline 14.14 & 50.5 & -3.64 & $\begin{array}{l}620 \pm 22^{\circ} \mathrm{K} \\
0.5-3.2 \text { torr } \\
\quad(5)\end{array}$ & static & none & $\begin{array}{l}\text { [1] C. S. Elliott and H. M. } \\
\text { Frey, J. Chem. Soc., } \\
4289 \text { (1965). }\end{array}$ \\
\hline
\end{tabular}

\section{Preferred:}

Reliable as reported.

Comments: Transition state estimates for the biradical mechanism (see vinylcyclopropane) give: $\log A_{\text {est }}$ $=14.1 ; E=48.1 \mathrm{kcal} /$ mole. (See discussion on small ring compound reactions, section III-4.2.

\section{Experimental}

[1] Analysis by G.L.C. Identification with M.S. At the highest temperatures a diene was formed in small yields $(\sim 1.5 \%)$. 
Reaction: 1,1-Dicyclopropylethene

\begin{tabular}{ccc}
$\Delta=(\mathrm{I}) \longrightarrow D(\mathrm{D})$ & \multicolumn{2}{c}{ 1-cyclopropylcyclopentene } \\
$\Delta H_{f(298)}^{\circ}$ & I & II \\
$S_{(298)}^{\circ}$ & 51.0 & 26.6 \\
$C_{p(298)}^{\circ}$ & 93.6 & 88.7
\end{tabular}
$\Delta S^{\circ}=-4.9 \mathrm{~g} / \mathrm{mole}$
$\Delta H^{\circ}=-24.4 \mathrm{kcal} / \mathrm{mole}$
$\Delta C_{p}^{\circ}=-5.8 \mathrm{~g} / \mathrm{mole}$

\begin{tabular}{ccccccc}
$\log \boldsymbol{A}$ & $\boldsymbol{E}$ & $\begin{array}{c}\log \boldsymbol{k}_{T} \\
(640)\end{array}$ & Conditions & System & Surface & \multicolumn{1}{c}{ References } \\
14.29 & 51.06 & -3.15 & $\begin{array}{l}615-663^{\circ} \mathrm{K} \\
(1-5 \mathrm{~mm} \mathrm{Hg})\end{array}$ & static & none & [1] G. R. Branton and H. M. \\
Frey, J. Chem. Soc. A, \\
(1342 (1966).
\end{tabular}

Preferred:

$\log k=14.29-51.06 / \theta$.

Comments: Transition state estimates on the basis of a biradical' mechanism give $A_{\mathrm{est}}=10^{14.1} \mathrm{sec}^{-1}$ and $E_{\text {est }}=49.7 \mathrm{kcal} / \mathrm{mole}$ in good agreement with the reported parameters.

\section{Experimental}

[1] Rates were based on G.L.C. analysis of the products. Added $\mathrm{N}_{2}$ up to $200 \mathrm{~mm} \mathrm{Hg}$ did not affect the kinetics. 1-cyclopropylcyclopentene never exceeded 53 percent of the products because of its own subsequent decomposition. Reaction paths:

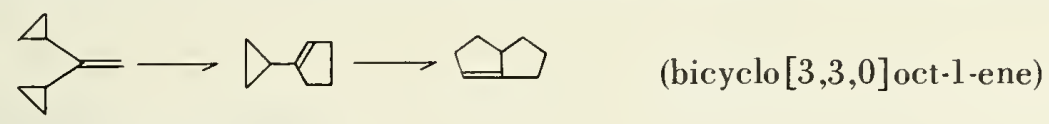

Other products were less than 2 percent. 
Reaction: 1-Cyclopropyl-1-cyclopentene

$\begin{array}{ccc}D \backslash \text { (I) } \longrightarrow \text { (II) } & {[3,3,0 \text {-bicyclo-1-octene }]} \\ \Delta H_{f(298)}^{\circ} & \text { I } & \text { II } \\ S_{(298)}^{\circ} & 26.6 & 4.2 \\ C_{p(298)}^{\circ} & 88.7 & 85.3 \\ & 32.8 & 28.3\end{array}$

$\Delta S^{\circ}=-3.4 \mathrm{~g} / \mathrm{mole}$

$\Delta H^{\circ}=-22.4 \mathrm{kcal} / \mathrm{mole}$

$\Delta C_{p}^{\circ}=-4.5 \mathrm{~g} / \mathrm{mole}$

\begin{tabular}{|c|c|c|c|c|c|c|}
\hline $\log A$ & $\boldsymbol{E}$ & $\begin{array}{c}\log \boldsymbol{k}_{T} \\
(640)\end{array}$ & Conditions & System & Surface & References \\
\hline 14.01 & 51.29 & -3.51 & $\begin{array}{l}615-663^{\circ} \mathrm{K} \\
1-15 \text { torr }\end{array}$ & static & none & $\begin{array}{l}\text { [1] G. R. Branton and H. M. } \\
\text { Frey, J. Chem. Soc. A, } \\
1342(1966) .\end{array}$ \\
\hline
\end{tabular}

Preferred:

$\log k=14.01-51.29 / \theta$.

Comments: Transition state estimates based on a biradical mechanism give $A_{\text {est }}=10^{14.1}$ and $E \simeq 49.9 \mathrm{kcal} /$ mole (see 1,1-dicyclopropylethene for related reaction and section III-4.2 for estimation methods).

\section{Experimental}

[1] (See 1,1-dicyclopropylethene.) 
Reaction: Fluorocyclopropane

\begin{tabular}{rcc}
$\bigsqcup_{\beta}^{(I)}(\lambda)_{F_{1}}$ (I) & \multicolumn{3}{c}{ monofluoropropenes (see below) } \\
$\Delta H_{f(298)}^{\circ}$ & -30.9 & II \\
$S_{(298)}^{\circ}$ & 65.4 & 69.8 \\
$C_{p(298)}^{\circ}$ & 15.5 & 16.9
\end{tabular}

$\Delta S^{\circ}=\quad 4.4 \mathrm{~g} / \mathrm{mole}$

$\Delta H^{\circ}=-13.4 \mathrm{kcal} / \mathrm{mole}$

$\Delta C_{p}^{\circ}=1.4 \mathrm{~g} / \mathrm{mole}$

\section{$\log \boldsymbol{A} \quad \boldsymbol{E} \quad \log k_{T}$ \\ (730) \\ $14.58 \quad 61.01 \quad-3.69$ \\ $0.017-46$ torr \\ static none}

(46)
Conditions System Surface References

[1] F. Casas, J. A. Kerr, and

A. F. Trotman-

Dickinson, J. Chem.

Soc., 3655 (1964).

\section{Preferred:}

Reliable: $\log k=14.58-61.01 / \theta$.

Comments: S. Pavlou and B. S. Rabinovitch (private communication) report some heterogeneity for this reaction. Thus the (cis/trans) 1-propene ratio was found to be total pressure dependent at low pressures. Comparison of the activation energy with that for cyclopropane suggests a destabilization of the $C_{3}$ ring of about $4 \mathrm{kcal} /$ mole by the $\mathrm{F}$ atom substitution.

\section{Experimental}

[1] Analysis by G.L.C. Fluoropropene distribution: cis-1-propene $\sim 18.5$ percent, trans-1-propene $\sim 60.9$ percent, 2-propene $\sim 9.1$ percent, 3 -propene $\sim 11.4$ percent. Falloff observed near 100 torr at $748{ }^{\circ} \mathrm{K}$. RRK-s $\simeq 12-14$. 
Reaction: 1,1-Difluorocyclopropane

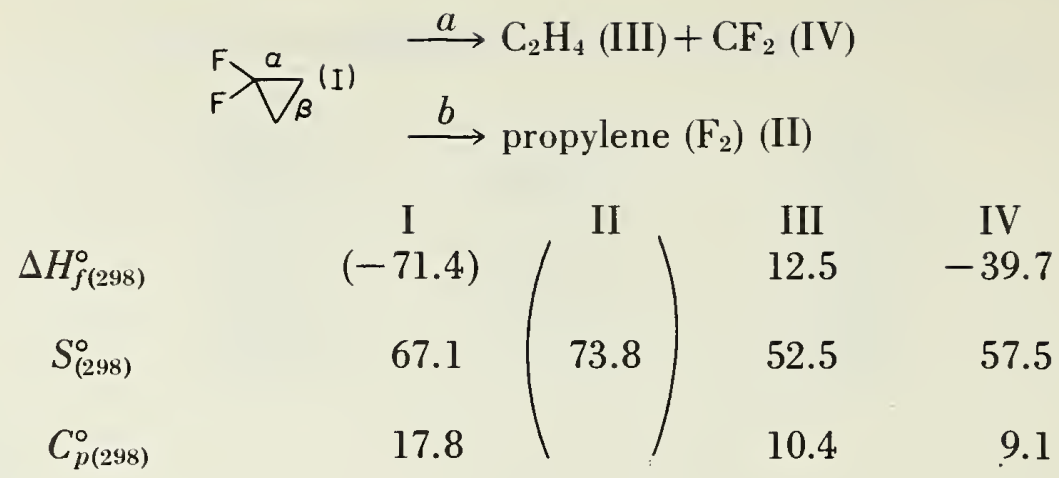

Path a

$\Delta S^{\circ}=42.9 \mathrm{~g} / \mathrm{mole}$

$\Delta H^{\circ}=4.4 .2 \mathrm{kcal} / \mathrm{mole}$

$\Delta C_{p}^{\circ}=1.7 \mathrm{~g} / \mathrm{mole}$

\begin{tabular}{|c|c|c|c|c|c|c|c|}
\hline Path & $\log A$ & $\boldsymbol{E}$ & $\begin{array}{c}\log \boldsymbol{k}_{T} \\
(696)\end{array}$ & Conditions & System & Surface & References \\
\hline$a+b$ & 14.09 & 56.35 & -3.61 & $\begin{array}{l}696 \pm 22^{\circ} \mathrm{K} \\
0.007-280 \text { torr } \\
\quad(280)\end{array}$ & static & none & $\begin{array}{c}\text { [1] F. P. Herbert, J. A } \\
\text { Kerr, and A. F. } \\
\text { Trotman-Dick- } \\
\text { enson, J. Chem. } \\
\text { Soc., } 5710 \text { (1965) }\end{array}$ \\
\hline
\end{tabular}

Preferred:

Parameters and rate constants are probably reliable. Proposed products and reaction path $b$ are suspect.

Comments: Atkinson and McKeagan (see perfluorocyclopropane) report that $\mathrm{CF}_{2}$ : addition to $\mathrm{C}_{2} \mathrm{H}_{4}\left(530^{\circ} \mathrm{K}\right)$ is slow but detectable relative to its addition to perfluoropropylene. The latter has an activation energy of 8 $\mathrm{kcal} / \mathrm{mole}$ (for $k$ in pressure units); therefore a reasonable guess for the former would be about $11-12 \mathrm{kcal} /$ mole. Thus for a total strain of $35.6 \mathrm{kcal} /$ mole (i.e., a normal $\mathrm{C}_{3}$ ring strain (27.6) plus an additional $8 \mathrm{kcal} / \mathrm{mole}$ destabilization for the two $\mathrm{F}$ atom substitutions) one obtains, $(\stackrel{\mathrm{F}}{\mathrm{F}}\rangle)=-71.4 \mathrm{kcal} / \mathrm{mole}, \Delta H_{a}^{\circ}=44.2$ $\mathrm{kcal} / \mathrm{mole}$, and an estimated $E_{a}=44.2+12.0=56.2 \mathrm{kcal} / \mathrm{mole}$, in very good agreement with the observed activation energy. The extensive polymerization of the product olefins would then also be readily understandable in terms of polymerization initiated by $\mathrm{CF}_{2}$ :. Olefin formation via $\mathrm{H}$-migration from the biradical (path $b$ ), according to these thermodynamics, should probably be competitive. The enthalpy change for ring opening

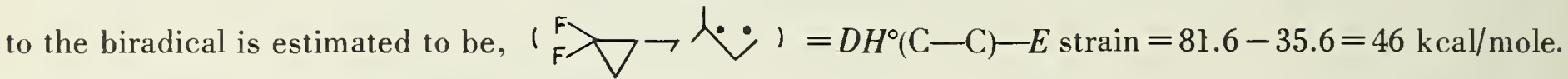
Thus, $E_{b} \simeq 46.0+10.8\left(\mathrm{H}\right.$-mig) $=56.8 \mathrm{kcal} / \mathrm{mole}$. The $A$-factor for decomposition of the biradical to $\mathrm{CF}_{2}$ : would be expected to be slightly favored over H-migration. If our estimates are correct, paths $a$ and $b$ should have similar Arrhenius parameters and should be competitive processes. Path $a$ could also be a concerted elimination without proceeding via a biradical.

\section{Experimental}

[1] Analysis by G.L.C. Falloff observed near 100 torr; RRK-s 17. Olefin products polymerized and therefore were not analyzed. Rates were determined from the disappearance of reactant. The reaction proposed was path $b$. 
Reaction: 1,1,2-Trifluorocyclopropane

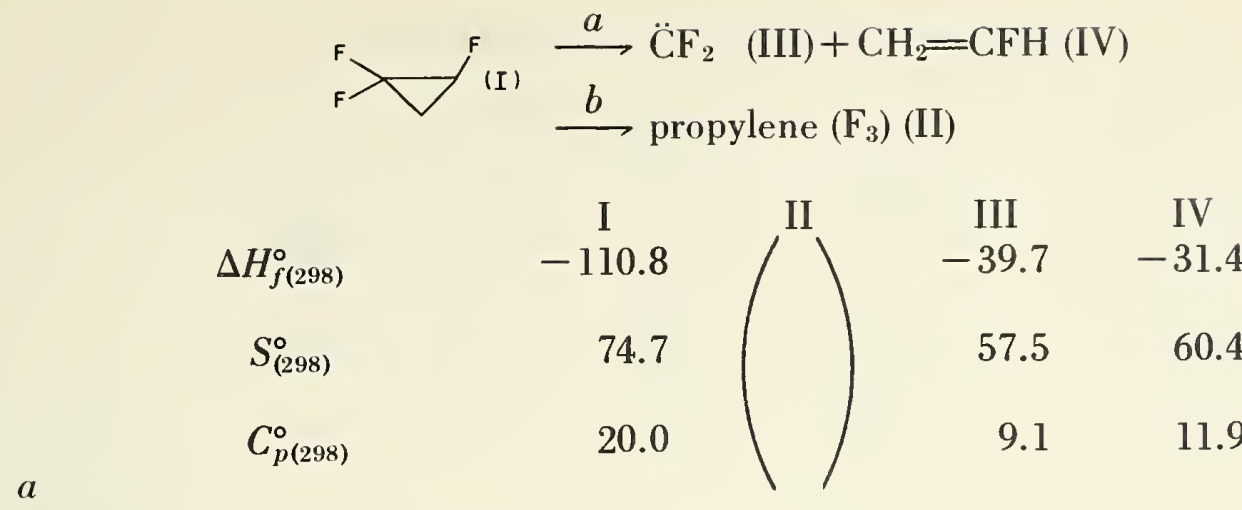

$\Delta S^{\circ}=43.2 \mathrm{~g} / \mathrm{mole}$

$\Delta H^{\circ}=39.7 \mathrm{kcal} / \mathrm{mole}$

$\Delta C_{p}^{\circ}=1.0 \mathrm{~g} / \mathrm{mole}$

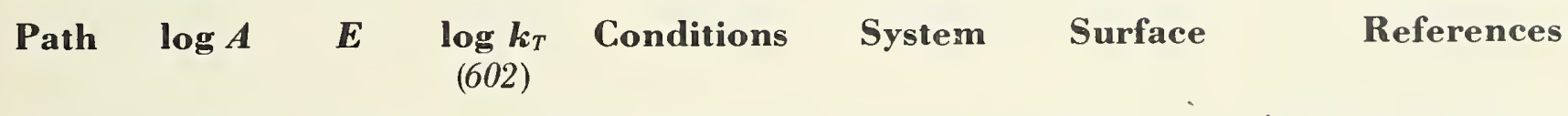

total $\quad 14.43 \quad 50.52-3.91 \quad 602 \pm 12^{\circ} \mathrm{K}$ static none

0.007-200 torr

[1] F. P. Herbert, J. A.

Kerr, and A. F.

Trotman-Dicken-

son, J. Chem.

Soc., 5710 (1965).

\section{Preferred:}

Rate constants and parameters are reliable. Reaction products and path are suspect (see also 1,1difluorocyclopropane).

Comments: The observed activation energy and estimated reaction enthalpy are consistent with a reverse activation energy of $E_{-a}=11 \mathrm{kcal} /$ mole, which is close to that estimated for $\mathrm{CF}_{2}:+\mathrm{C}_{2} \mathrm{H}_{4}$. A total strain energy of $39.6 \mathrm{kcal} / \mathrm{mole}$ has been used for $(\nabla)_{\mathrm{F}_{3}}$. By our thermodynamics, ring opening to the biradical is estimated as: $D H^{\circ}(\mathrm{C}-\mathrm{C})-E$ strain $=81.6-39.6=42.0 \mathrm{kcal} / \mathrm{mole}$ endothermic. With $E_{\mathrm{H}-\mathrm{mig}}=10.8 \mathrm{kcal} / \mathrm{mole}$, one obtains $E_{b} \simeq 52.8 \mathrm{kcal} /$ mole. Paths $a$ and $b$ should therefore be competitive processes, with path $a$ slightly favored.

\section{Experimental}

[1] Analysis by G.L.C. Falloff observed near 100 torr; RRK $-s \sim 18$. Olefin products polymerized. The reaction assumed was that of path $b$. 
Reaction: 1,1,2,2-Tetrafluorocyclopropane

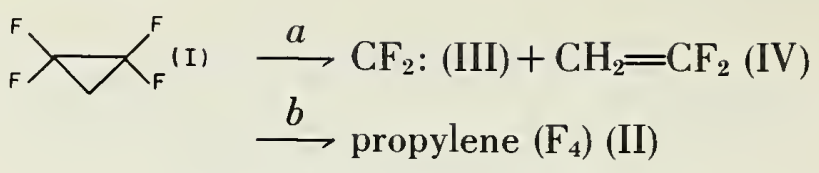

\begin{tabular}{|c|c|c|c|}
\hline$\Delta H_{f(298)}^{\circ}$ & $\begin{array}{c}\mathrm{I} \\
(-155.5)\end{array}$ & $\begin{array}{c}\text { III } \\
-39.7\end{array}$ & $\begin{array}{c}\text { IV } \\
-80.8 *\end{array}$ \\
\hline$S_{(298)}^{\circ}$ & 75.3 & 57.5 & \\
\hline$C_{p(298)}^{\circ}$ & 22.2 & 9.1 & \\
\hline
\end{tabular}

$\Delta S^{\circ}=($
$\Delta H^{\circ}=(35.0)$
$\Delta C_{p}^{\circ}=($

$\begin{array}{llllll}\text { Path } & \log A & \boldsymbol{E} & \log \boldsymbol{k}_{T} & \text { Conditions } & \text { System }\end{array}$

References (543)

$\begin{array}{lllllll}\text { total } & 15.27 & 48.48 & -4.25 & 543 \pm 18^{\circ} \mathrm{K} & \text { static }\end{array}$ $0.005-210$ torr

[1] F. P. Herbert, J. A. (210)

Kerr, and A. F.

Trotman-Dickenson, J. Chem.

Soc., 5710 (1965).

Preferred:

Rate constants and parameters are reliable. Reaction path $b$ and products are suspect (see also 1,1difluorocyclopropane).

Comments: There is considerable uncertainty in the thermodynamics. Additivity gives $\Delta H_{f}^{\circ}\left(\mathrm{CH}_{2}=\mathrm{CF}_{2}\right)$ $=-70.7 \mathrm{kcal} / \mathrm{mole}$. We have used a total ring strain of $43.6 \mathrm{kcal} / \mathrm{mole}$, although this could well be larger (see perfluorocyclopropane). However, with the strain indicated, we estimate:

$$
\text { path } a: E_{a}=\Delta H_{a}^{\circ}+E_{-a} \simeq 35+10=45 \mathrm{kcal} / \mathrm{mole}
$$

path $b: E_{b}=D H^{\circ}(\mathrm{C}-\mathrm{C})-E_{\mathrm{st}}+E_{\mathrm{H}-\mathrm{mig}}=81.6-43.6+10.8=48.8 \mathrm{kcal} / \mathrm{mole}$.

Both processes, therefore, seem possible and are probably competitive.

\section{Experimental}

[1] Analysis by G.L.C. Falloff observed near 100 torr; RRK $-s \sim 21$. Olefin product polymerized. The reaction assumed was that of path $b$.

*An experimental value. 
Reaction: Hexafluorocyclopropane (perfluorocyclopropane)

$\begin{array}{cccc} & 1 \nabla_{\mathrm{F}_{6}} \longrightarrow \mathrm{C}_{2} \mathrm{~F}_{4}(\mathrm{II})+\mathrm{CF}_{2}:(\mathrm{III}) & \\ \Delta H_{f(298)}^{c} & \mathrm{I} & \mathrm{II} & \text { III } \\ S_{(298)}^{c} & {[228]} & -155.0 & -39.6 \\ C_{p(298)}^{c} & 80.2 & 71.8 & 57.5 \\ & 26.6 & 19.4 & 9.1\end{array}$

$\Delta S^{\mathrm{c}}=49.1 \mathrm{~g} / \mathrm{mole}$

$\Delta H^{\circ}=33.4 \mathrm{kcal} / \mathrm{mole}$

$\Delta C_{p}^{c}=1.9 \mathrm{~g} / \mathrm{mole}$

\begin{tabular}{|c|c|c|c|c|c|c|}
\hline $\log A$ & $\boldsymbol{E}$ & $\begin{array}{c}\log k_{T} \\
(535)\end{array}$ & Conditions & System & Surface & References \\
\hline 13.25 & 38.6 & -4.50 & $\begin{array}{l}526-549^{\circ} \mathrm{K} \\
2-16 \mathrm{~mm} \mathrm{Hg}\end{array}$ & static & 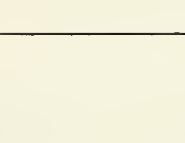 & $\begin{array}{l}\text { [1] B. Atkinson and D. } \\
\text { McKeagan, Chem. } \\
\text { Comm. } 189 \text { (1966) }\end{array}$ \\
\hline
\end{tabular}

Preferred:

$\log k=15.0-43.0 / \theta$. Rate constants are reliable. .

Comments: The temperature range of study was only $23{ }^{\circ} \mathrm{C}$, and with a 2 -percent error in the rate constants, could produce an error in $E$ of $\pm 2.6 \mathrm{kcal} / \mathrm{mole}$. By analogy with the similar reaction, perfluoroallylcyclopropane. and as a result of transition state estimates of the $A$-factor, slightly higher than observed parameters are preferred. With the activation energy suggested for the back reaction $\left(E_{b(p)}=8.0 \mathrm{kcal} / \mathrm{mole}\right)$. the heat of formation of perfluorocyclopropane in brackets is obtained. This is consistent with a strain energy in $\left(\nabla_{F_{6}}\right)$ of $63 \mathrm{kcal} / \mathrm{mole}$. By the biradical mechanism, decomposition of the biradical is rate determining with an áctivation energy about $23 \mathrm{kcal} / \mathrm{mole}$.

$$
\text { Mechanism: } \begin{aligned}
(\nabla)_{\mathrm{F}_{6}} \underset{2}{\stackrel{1}{\rightleftarrows}}\left(\bigvee^{\circ}\right)_{\mathrm{F}_{6}} \stackrel{3}{\longrightarrow} \mathrm{C}_{2} \mathrm{~F}_{4}+\mathrm{CF}_{2}: \\
\Delta H_{1,2}^{\mathrm{c}}=18.6 \mathrm{kcal} / \mathrm{mole} \quad E_{3} \simeq 23 \mathrm{kcal} / \mathrm{mole} \\
E_{-3}=8.0 \mathrm{kcal} / \mathrm{mole} .
\end{aligned}
$$

Since some addition of $\mathrm{CF}_{2}:$ to $\mathrm{C}_{2} \mathrm{H}_{4}$ did occur. although very slow relative to the addition tol $\widehat{N}_{6}{ }^{\text {an }}$ activation energy for addition to ethylene of around $12 \mathrm{kcal} / \mathrm{mole}$ seems reasonable.

\section{Experimental}

[1] Rates were obtained from $\Delta \mathrm{P}$ measurements. Material balance $\left(2 \mathrm{C}_{3} \mathrm{~F}_{6} \longrightarrow 3 \mathrm{C}_{2} \mathrm{~F}_{4}\right)$ was checked by analysis (U.V., I.R., G.I.C.). With added perfluoropropene, perfluoromethylcyclopropane was formed. With added ethylene. small amounts of $(\nabla / F_{2}$ were formed. Thus $C_{2}$ : adds more rapidly to $\overbrace{F_{6}}$ than $t_{0}\left(\mathrm{C}_{2} \mathrm{H}_{4}\right)$. The mechanism proposed was,

$$
\begin{gathered}
\nabla^{\prime} \mathrm{F}_{6} \stackrel{\frac{1}{\rightleftharpoons}}{\rightleftharpoons} \mathrm{C}_{2} \mathrm{~F}_{4}+\mathrm{CF}_{2}: \\
2 \mathrm{CF}_{2}: \stackrel{3}{\longrightarrow} \mathrm{C}_{2} \mathrm{~F}_{4}
\end{gathered}
$$

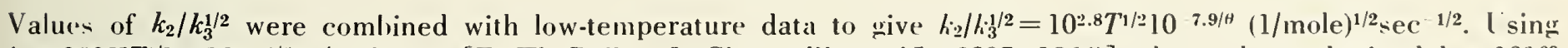
$k_{i 3}=1\left(^{6.57} T^{1 / 2} \times 10^{-1.2 / t} 1 /\right.$ mole-sec $\left[F\right.$. W. Dalby, J. Chem. Phys. 41, 2297 (1964)], the authors obtained $k_{2}=10^{6.09}$ $T^{1 / 2} \times 10^{-8.5 / \theta} 1 /$ mole-sec. 
Reaction: Trifluoromethylcyclopropane

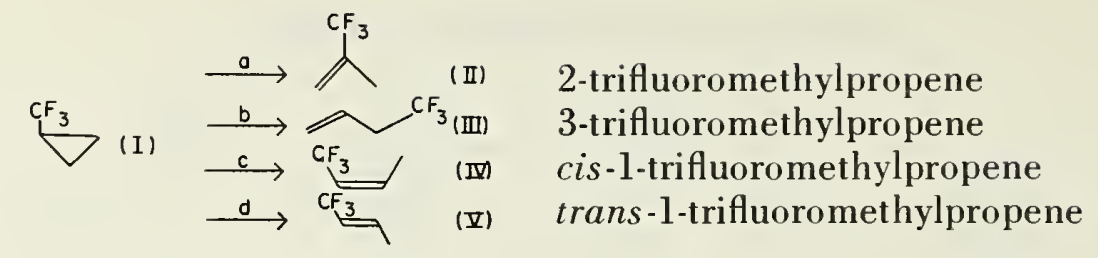

\begin{tabular}{crrrrr} 
& I & II & III & IV & \multicolumn{1}{c}{ V } \\
$\Delta H_{f(298)}^{\circ}$ & -138.5 & -148.2 & -145.8 & -147.5 & -148.5 \\
$S_{(298)}^{\circ}$ & 79.3 & 83.7 & 85.7 & 84.4 & 84.4 \\
$C_{p(298)}^{\circ}$ & 25.0 & 27.8 & 27.0 & 25.4 & 27.5
\end{tabular}

$$
\begin{aligned}
& a \\
\Delta S^{\circ}= & 4.4 \mathrm{~g} / \mathrm{mole} \\
\Delta H^{\circ} & =-9.7 \mathrm{kcal} / \mathrm{mole} \\
\Delta C_{p}^{\circ}= & 2.8 \mathrm{~g} / \mathrm{mole}
\end{aligned}
$$

\begin{tabular}{|c|c|c|c|c|c|c|c|}
\hline Path & $\log A$ & $\boldsymbol{E}$ & $\underset{(758)}{\log \boldsymbol{k}_{T}}$ & Conditions & System & Surface & References \\
\hline & 13.87 & 69.5 & -6.17 & $\begin{array}{c}758 \pm 45^{\circ} \mathrm{K} \\
(82) \text { torr }\end{array}$ & static & & $\begin{array}{l}\text { [1] D. W. Placzek and } \\
\text { B. S. Rabinovitch, } \\
\text { J. Phys. Chem. } \\
\text { 69, 2141 (1965). }\end{array}$ \\
\hline
\end{tabular}

\section{Preferred:}

All rate constants reliable as reported. (Compare with results on methylcyclopropane.)

Comments: The biradical mechanism is consistent with the kinetics. The products observed are those expected. Paths $b, c$, and $d$ occur by $\alpha(\mathrm{C}-\mathrm{C})$ rupture and path $a$ by $\beta(\mathrm{C}-\mathrm{C})$ rupture. Transition state estimates (see section III-4.0) are the same as those for methylcyclopropane and predict somewhat higher $A$-factors. It is apparent that the fluorine substitution outside the ring produces a rate decrease. It is not clear whether the effect is a result of increased activation energies or (as the overall parameters imply) lowered $A$-factors. We favor an activation energy increase with fluorine substitution.

\section{Experimental}

[1] Analysis by G.L.C.; N.M.R. used for identification purposes. 
Reaction: 2-2-2-Trifluoro-1-ethylcyclopropane

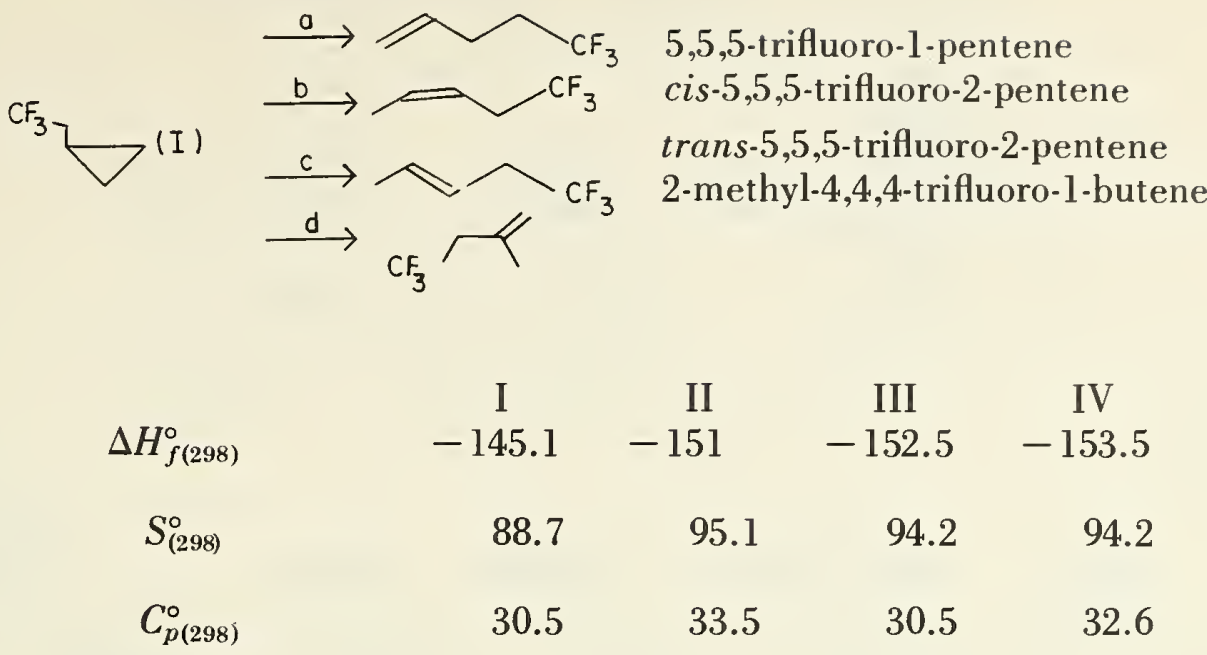

$\Delta S^{\circ}=6.4 \mathrm{~g} / \mathrm{mole}$

$\Delta H^{\circ}=-5.9 \mathrm{k} / \mathrm{cal} / \mathrm{mole}$

$\Delta C_{p}^{\circ}=3.0 \mathrm{~g} / \mathrm{mole}$

$\log \mathbf{k}_{T}$

Conditions

System Surface

References

747)

(

13.88

63.

$-4.67$

$747 \pm 68^{\circ} \mathrm{K}$

static

(82) torr

[1] D. W. Placzek and B. S. Rabinovitch, J. Phys. Chem. 69, 2141 (1965)

$\begin{array}{llll}b & 13.84 & 63.3 & -4.68 \\ c & 13.69 & 63.1 & -4.77 \\ \text { total } & 14.39 & 63.6 & -4.22\end{array}$

\section{Preferred:}

Rate constants are reliable as reported.

Comments: All products expected by the biradical mechanism were observed. The reactions are analogous to those of ethylcyclopropane. As in the trifluoromethyl cyclopropane reaction, it is apparent that fluorine substitution decreases the reaction rate and comparison of the parameters for the two overall reactions suggests that the rate decrease is a result of generally higher activation energies.

\section{Experimental}

[1] Analysis by G.L.C. N.M.R. used for identification. Rate constant parameters for 2-trifluoroethylpropene (path $d)$ were not obtained. However, it was estimated that $\left(k_{d} / k_{a}+k_{b}\right)=0.1$. 
Reaction: Octafluorovinylcyclopropane (perfluorovinylcyclopropane)

\begin{tabular}{|c|c|c|}
\hline$\Delta H_{f(298)}^{\circ}$ & $\left(\begin{array}{l}\mathrm{I} \\
\end{array}\right)$ & $\left(\begin{array}{l}\text { II } \\
()\end{array}\right.$ \\
\hline$S_{(298)}^{\circ}$ & 108.7 & 103.4 \\
\hline$C_{p(298)}^{\circ}$ & 41.6 & 37.9 \\
\hline
\end{tabular}

$\Delta S^{\circ}=-5.3 \mathrm{~g} / \mathrm{mole}$

$\Delta H^{\circ}=$

$\Delta C_{p}^{\mathrm{c}}=-3.7 \mathrm{~g} / \mathrm{mole}$

$\begin{array}{ccccccc}\log \boldsymbol{A} & \boldsymbol{E} & \begin{array}{c}\log \boldsymbol{k}_{T} \\ (430)\end{array} & \text { Conditions } & \text { System } & \text { Surface } & \text { References } \\ 13.9 & 34.6 & -3.69 & \begin{array}{l}404-456^{\circ} \mathrm{K} \\ 1-90 \text { torr }\end{array} & \text { static } & \text { none } & \text { [1] R. A. Mitsch and } \\ \text { E. W. Neuvar, J. Phys. } \\ \text { Chem. 70, 546 (1966). }\end{array}$

Preferred:

Reliable. $\log k=13.9-34.6 / \theta$.

Comments: The $A$-factor should be similar to that for vinylcyclopropane, and this is as observed. With a strain energy of $46.5 \mathrm{kcal} / \mathrm{mole}$ (see perfluoroallylcyclopropane) and a "normal" allyl resonance, an enthalpy of ring opening to the biradical of $\Delta H_{1,2}^{\circ}=21.1 \mathrm{kcal} / \mathrm{mole}\left(\right.$ at $298{ }^{\circ} \mathrm{K}$ ) is obtained. An activation energy for $\mathrm{C}_{5}$ ring closing of about $13.5 \mathrm{kcal} / \mathrm{mole}$ is therefore suggested by the observed activation energy. [Compare to $E_{\mathrm{C}_{5}}=8.3 \mathrm{kcal} / \mathrm{mole}(\nabla)$ and $\left.E_{\mathrm{C}_{5}}=13.2 \mathrm{kcal} / \mathrm{mole}(\checkmark) \cdot\right]$ Steric effects of the fluorine could be responsible for this higher value.

\section{Experimental}

[1] Analysis by G.L.C. First-order plots were linear to 97 percent reaction. Rates were unaffected by 10 percent NO. The decrease in activation energy for the perfluoro compound relative to the hydrocarbor analogue was attributed to increased ring strain, a reasonable conclusion. 
Reaction: Decafluoroallycyclopropane (perfluoroallylcyclopropane)
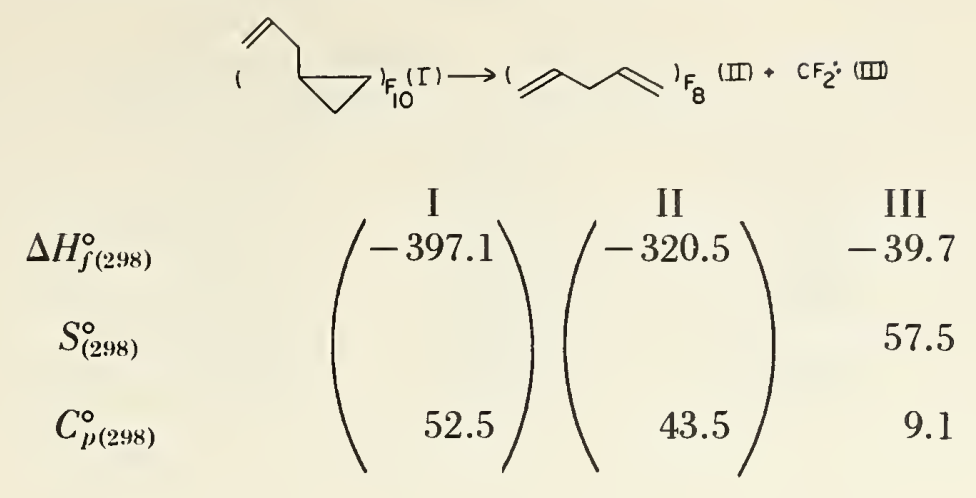

$\Delta S^{\circ}=49.9 \mathrm{~g} / \mathrm{mole}$

$\Delta H^{\circ}=[33.7 \mathrm{kcal} / \mathrm{mole}]$

$\Delta C_{p}^{\circ}=0.1 \mathrm{~g} / \mathrm{mole}$

\begin{tabular}{rlrllll}
$\log \boldsymbol{A}$ & $\boldsymbol{E}$ & $\begin{array}{c}\log \boldsymbol{k}_{T} \\
(496)\end{array}$ & Conditions & System & Surface & \multicolumn{1}{c}{ References } \\
14.8 & 42.7 & -4.02 & $\begin{array}{l}469-523^{\circ} \mathrm{K} \\
1-40 \text { torr }\end{array}$ & static & none & [1] R. A. Mitsch and \\
E. W. Neuvar, J. Phys. \\
Chem. 70, 546 (1966).
\end{tabular}

Preferred:

$\log k=14.8-42.7 / \theta$.

Comments: If an activation energy of $\mathrm{CF}_{2}$ : addition to the olefin product of $9.0 \mathrm{kcal} / \mathrm{mole}$ is assumed [see

$\left.{ }^{\prime} \nabla^{\prime} F_{6}\right]$, the reaction enthalpy in brackets is obtained. This gives a ring strain in perfluoroallycyclopropane of $46.5 \mathrm{kcal} / \mathrm{mole}$.

\section{Experimental}

[1] Analysis and rate by G.L.C. $\mathrm{C}_{2} \mathrm{~F}_{4}$ was added in order to quantitatively trap $\mathrm{CF}_{2}$ : [as ${ }^{\prime} \nabla^{\prime} \mathrm{F}_{6}$ ]. NO had no effect on the reaction rate. 
Reaction: Chlorocyclopropane

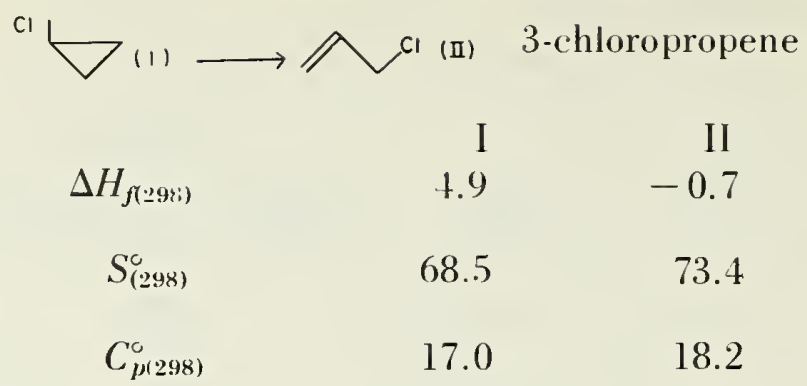

$\Delta S^{c}=4.9 \mathrm{~g} / \mathrm{mole}$

$\Delta H^{\circ}=-5.5 \mathrm{kcal} / \mathrm{mole}$

$\Delta C_{p}=1.2 \mathrm{~g} / \mathrm{mole}$

\begin{tabular}{|c|c|c|c|c|c|c|}
\hline $\begin{array}{r}\log A \\
14.8\end{array}$ & $\begin{array}{l}\boldsymbol{E} \\
56.2\end{array}$ & $\log k_{T}$ & $\begin{array}{l}\text { Conditions } \\
613-6933^{\circ} \mathrm{K} \\
35-440 \text { torr }\end{array}$ & System & Surface & $\begin{array}{l}\text { References } \\
\text { [1] R.C.S. (irant and E. S } \\
\text { Swinbourne, Chem. } \\
\text { Comm. } 620 \text { (1966). }\end{array}$ \\
\hline
\end{tabular}

Preferred:

Unimolecularity of this reaction needs verification.

Comments: Transition state estimates of low $A$ for the normal biradical mechanism of a cyclopropane isomerization give $\log A_{\text {est }}=14.7$ in good agreement with the value observed. However, the low activation energy suggests either an appreciable increase in ring strain, due to the chlorine substitution, or an unexpected radical stabilization by chlorine. Thus from $E_{\text {act }}$ one can estimate that $D H^{\circ}(\mathrm{RCHCl}-\mathrm{H})=91 \mathrm{kcal} / \mathrm{mole}$ compared to $D H^{c}\left(\mathrm{R}\left(\cdot \mathrm{H}_{2}-\mathrm{H}\right)=98 \mathrm{kcal} / \mathrm{mole}\right.$.

A 7-kcal stabilization (or increased ring strain) is not very reasonable. A more plausible explanation of the experimental results is that the reaction is a free radical process with a $\beta-\mu$ radical recombination termination (see bromocyclopropane for the proposed mechanism). Estimated parameters are slightly lower than those observed (giving faster predicted rates) suggesting that the radical process is certainly feasible. With estimates:

$$
\begin{aligned}
& \log k_{i i} \simeq 13.5-80.2 / \theta \\
& \log k_{t} \simeq 11.2 \\
& \log _{2} k_{1} \simeq 10.7-4 / \theta \\
& \log k_{3} \simeq 13.5-14 / \theta
\end{aligned}
$$

one obtains, $\log l_{\text {i.st }} \simeq 13.2-49.2 / \theta$.

\section{Experimental}

[1] Analysis by (:L.C. The only product, 3-chloropropene. decomposed at $T>400^{\circ} \mathrm{C}$. 
Reaction: 1.1-Dichlorocyclopropane

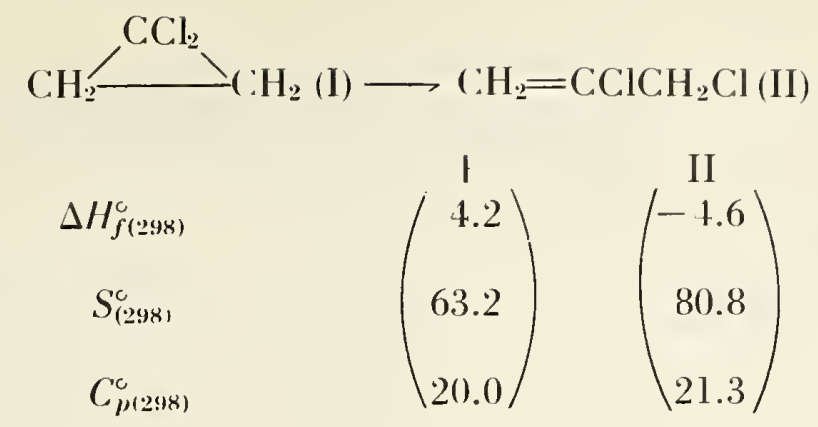

$\Delta S^{\circ}=17.6 \mathrm{~g} / \mathrm{mole}$
$\Delta H^{\circ}=-8.8 \mathrm{kcal} / \mathrm{mole}$
$\Delta C_{p}^{c}=1.3 \mathrm{~g} / \mathrm{mole}$

\begin{tabular}{|c|c|c|c|c|c|c|}
\hline $\log A$ & $\boldsymbol{E}$ & $\underset{(700)}{\log k_{T}}$ & Conditions & System & Surface & References \\
\hline 15.13 & 57.8 & -2.95 & $\begin{array}{l}615-714^{\circ} \mathrm{K} \\
20-120 \mathrm{torr}\end{array}$ & static & No effect & $\begin{array}{l}\text { [1] K. A. W. Parry and P. J. } \\
\text { Robinson, Chem. } \\
\text { Comm. } 1083 \text { (1967). }\end{array}$ \\
\hline
\end{tabular}

Preferred:

$\log k=1.5 .13-57.8 / \theta^{\prime}$ sec $\left.^{11}\right)$ seems reasonable.

Comments: There is about 5 percent sicondary decomposition of product at one half-life. Although the authors suggest that the mechanism does not involve biradicals and propose a roncerted path. it seems much more plausible that the biradical path is operative. The $C_{2} H_{2} C_{2} H_{2} C_{1} l_{2}$ biradical is favored over the $i: H_{2}$ (:Cl.2 $: \mathrm{H}_{2}$ biradical by only $1.8 \pm 2 \mathrm{kral}$. but lhe $\mathrm{Cl}$ mioration in the latter is undoubtedly faster than the $\mathrm{H}$ migration in the former and so makı's the abserved path the favored one. A low activation energy for 1-2-Cl migration makes ring opening the rate-determining step. $A_{\text {est }}$ from transition state theory is $10^{15.5} \mathrm{sec}^{-1}$. If each $\mathrm{Cl}$ introduces $3.0 \mathrm{hcal}$ of additional rinw strain. $E_{\text {est }}-.88 \mathrm{kcal} / \mathrm{mole}$.

\section{Experimental}

[1] Analysis by G.L.C. No effect on the rates with $\mathrm{NO}, \mathrm{C}_{3} \mathrm{H}_{6}$, or the product. $S / V$ increased a factor of sixteen. 
Reaction: 2,2-Dichloro-1-methyl-1-vinylcyclopropane (1-methyl-1-vinyl-2,2-dichlorocyclopropane)

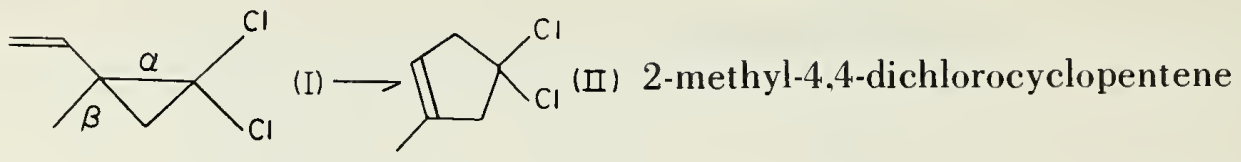

$\begin{array}{ccc} & \text { I } & \text { II } \\ \Delta H_{f^{(}(298)}^{c} & 10.5 & -14.7 \\ S_{(298)}^{c} & 92.6 & 91.2 \\ C_{\mu(298)}^{c} & 32.2 & 30.2\end{array}$

$\Delta S=(-1.4) \mathrm{g} / \mathrm{mole}$

$\left.\Delta H^{\circ}=-2.5 .2\right) \mathrm{kcal} / \mathrm{mole}$

$\Delta C_{\nu} \doteq|-2.0| \mathrm{g} / \mathrm{mole}$

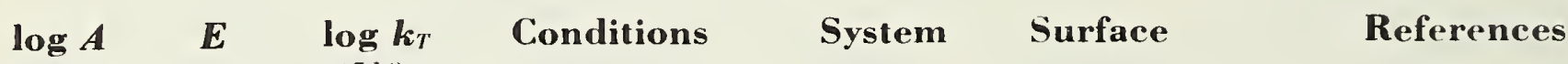

$11.7 \quad 34.6 \quad-3.13 \quad 473-548^{\circ} \mathrm{K} \quad$ static
[1] A. D. Ketley, A. J.

Berlin, E. Gorman, and L. P. Fisher, J. Org. ('hem. 3 1, 305 1 1966).

Irreferred:

Suspect.

Comments: Transition state estimalres give log $l_{i}=13.9-39.7 / \theta$. The parameters are too lon. The hadly behaved Arrhenius plot (with high rates at the lower temperatures) suggests some surface catalysis.

\section{Expr-rimental}

[1] Static sy-tem. Inalysis hy G.L.C. 0.5- $\mathrm{ml}$ samples werr sealed in $2.5-\mathrm{ml}$ pyrex ampules. Parameters above are maximum values (alculated from reported rate constants at 200 ), 212, and $234^{\circ} \mathrm{C}$. The Arrhenius plot showed had curvature. The $\beta\left(C-()\right.$ split product $\left(Q_{C 1}^{c I}\right)$ expected from a biradical mechanism was not observed. A number

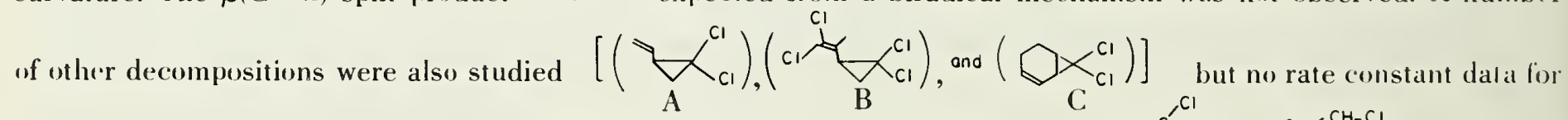
these were reported. However, the exclusive products formed were $\mathbb{C}_{\mathrm{Cl}}^{\mathrm{Cl}}$ from $\mathrm{A}$ and $\mathrm{C}_{\mathrm{Cl}}^{\mathrm{Cl}}$, and ${ }^{\mathrm{CH}_{2} \mathrm{Cl}}$ from $\mathrm{C}$. 
Reaction: Bromocyclopropane

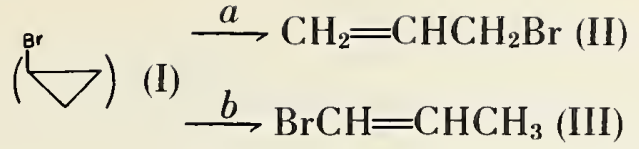

$\begin{array}{cccc} & \text { I } & \text { II } & \text { III } \\ \Delta H_{f(298)}^{c} & 18.7 & 9.5 & 11.2 \\ S_{(298)}^{c} & 70.2 & 76.4 & 74.5 \\ C_{p(298)}^{c} & 16.8 & 19.4 & 18.5\end{array}$

$\Delta S^{\circ}=6.2 \mathrm{~g} / \mathrm{mole}$

$\Delta H^{\circ}=-9.2 \mathrm{kcal} / \mathrm{mole}$

$\Delta C_{p}=2.6 \mathrm{~g} / \mathrm{mole}$

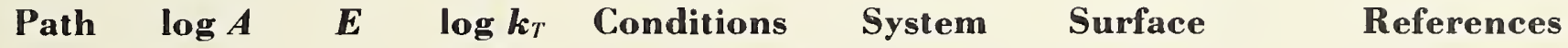

(610)

$\begin{array}{llllll}a+b & 13.5 & 47.3 & -3.45 & 583-633^{\circ} \mathrm{K} & \text { static }\end{array}$

[1] R.C.S. Grant and E. S. Swinborne, Chem. Comm. 620 (1966).

Preferred:

Suspect.

Comments: Both Arrhenius parameters are low in terms of transition state estimates for a biradical threemembered ring isomerization. However, they are very similar to those for the sec-butyl bromide four-center elimination reaction (i.e., $k_{\text {elim }}=10^{13.5-46.5 / \theta}$ which is known to have an appreciable free radical component in the uninhibited system. It is most likely, then, that this reaction is a free radical process with the following mechanism:

Estimates:

$l_{i}^{\prime}=10^{13.6-58, \theta}$

$\log k_{i} \simeq 14.6-70 / \theta$

$\log h_{i} \simeq 11.0$

$\log k_{1} \simeq 10.7-16.2 / \theta$
Units:

$k_{i}, k_{3} \sec ^{-1}$

$k_{1}, k_{1}(1 /$ mole-sec $)$

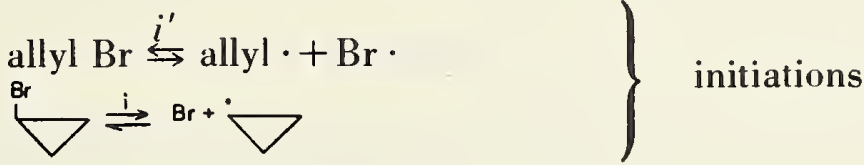

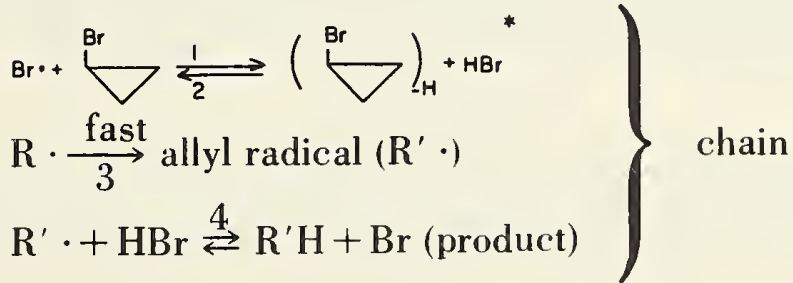

$$
\begin{aligned}
& R^{\prime} \cdot+B r \cdot \stackrel{B}{\longrightarrow} \bigodot^{8 r} \text { termination }
\end{aligned}
$$

*Alternatively, the ring-opening reaction:

$$
\mathrm{Br}+c-\mathrm{C}_{3} \mathrm{H}_{5} \mathrm{Br} \stackrel{l^{\prime}}{\rightleftarrows} \quad \dot{\mathrm{C}_{2}} \mathrm{CHBrCH}_{2} \mathrm{Br} \stackrel{\text { fast }}{\longrightarrow} \mathrm{CH}_{2}=\mathrm{CHCH}_{2} \mathrm{Br}+\mathrm{Br}
$$




\section{Experimental}

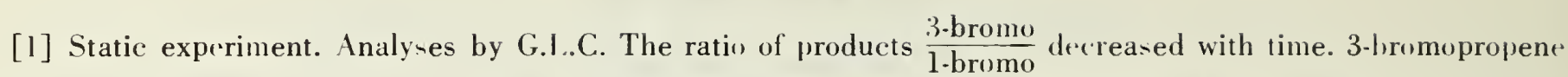
was found to isomerize to l-bromopropene at roughly $(1 / 2)$ the rate of the bromocyclopropane isomerization. The olefin isomerization was catalyzed by $\mathrm{HBr}$. Surface effects were not studied.

Reaction: Dimethyldiazirine

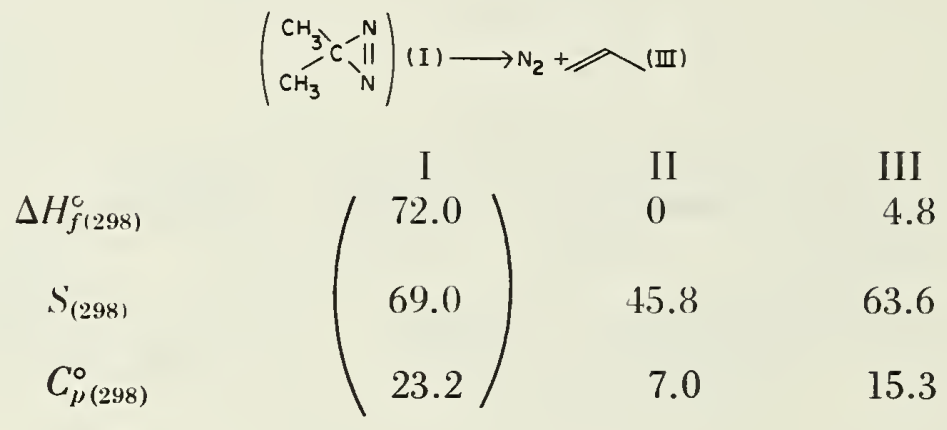

$\Delta S^{\circ}=-40.1 \mathrm{~g} / \mathrm{mole}$

$\Delta H^{\mathrm{c}}=-68.2 \mathrm{kcal} / \mathrm{mole}$

$\Delta C_{p}^{c}=-0.9 \mathrm{~g} / \mathrm{mole}$

\begin{tabular}{|c|c|c|c|c|c|c|}
\hline $\log A$ & $\boldsymbol{E}$ & $\begin{array}{c}\log _{1422)} \boldsymbol{k}_{T} \\
\end{array}$ & Conditions & System & Surface & References \\
\hline 13.89 & 3.3 .17 & -3.31 & $\begin{array}{l}422 \pm 25^{\circ} \mathrm{K} \\
2.3-100 \text { torr } \\
\quad \text { (4) }\end{array}$ & static & & $\begin{array}{l}\text { [1] H. M. Frey and I. D. R. } \\
\text { Stevens. J. Chem. Soc.. } \\
3865 \text { (1962). }\end{array}$ \\
\hline
\end{tabular}

Preferred:

Reliable. $\log l_{i}=13.89-33.17 / \theta$

Comments: The reaction very likely proceeds through a hot diazo compound intermediate.

Mechanism:

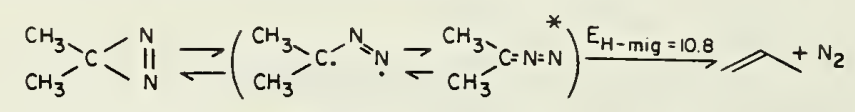

If the $D H^{\circ}(C-\mathrm{N})$ hond is taken as the activation energy observed for the decomposition of $2.2^{\prime}$-azobutane

$\left(\checkmark-\mathrm{N}=\mathrm{N} \longrightarrow\right.$ ) (see section $\mathrm{V}-7.0$ ) and a normal ('yclopropane strain is assumed, then $E_{\text {est }} \simeq 47.5-27.6$ $+10.8=30.7 \mathrm{kcal} /$ mole, in reasonable agreement with the observed value.

\section{Experimental}

[1] Analysis by G.L.C. $\left(\Delta \mathrm{N}_{2}=\Delta \mathrm{P}\right)$ Falloff observed below 100 torr. The rate constant parameters in the falloff region at 4 torr; therefore, in the high-pressure region somewhat higher values would pertain. Propylene did not affect the rate. 
Reaction: 3,3-Diethyldiazirine

\begin{tabular}{|c|c|c|c|c|}
\hline$\Delta H_{f(298)}^{\circ}$ & $\begin{array}{c}\text { I } \\
63.1\end{array}$ & $\begin{array}{c}\text { II } \\
-7.7\end{array}$ & $\begin{array}{l}\text { III } \\
0.7\end{array}$ & $\begin{array}{c}\text { IV } \\
0 .\end{array}$ \\
\hline$S_{(298)}^{c}$ & 87.8 & 82.2 & 76.5 & 45.8 \\
\hline$C_{p(298)}^{c}$ & 33.2 & 25.8 & 24.0 & 7 \\
\hline
\end{tabular}

$$
\begin{array}{rl} 
& a(\text { trans }) \\
\Delta S^{c} & 40.2 \mathrm{~g} / \mathrm{mole} \\
\Delta H^{\circ}= & -70.8 \mathrm{kcal} / \mathrm{mole} \\
\Delta C_{p}= & 0.4 \mathrm{~g} / \mathrm{mole}
\end{array}
$$

\section{$\begin{array}{llllll}\text { Path } & \log \boldsymbol{A} \quad \boldsymbol{E} \quad \log \boldsymbol{k}_{T} & \text { Conditions } & \text { System } & \text { Surface } & \text { References }\end{array}$ (405)}

$\begin{array}{rllll}(a+b) \quad 13.73 \quad 31.89 \quad-3.48 \quad & 391-422^{\circ} \mathrm{K} \quad \text { static } \\ & & 1-20 \text { torr } \\ & & \\ & & & \end{array}$

[1] H. M. Frey and A. W. Scaplehorn, J. Chem. Soc. A, 968 (1966).

Preferred:

Reliable. $\quad \log k=13.73-31.89 / \theta$.

Comments: Transition state estimates give, $E \simeq D H^{\circ}(\mathrm{C}-\mathrm{N})-\Delta_{\text {st }}+E_{\text {r.c. }} \simeq 47.5-27.6+10.8=30.7 \mathrm{kcal} / \mathrm{mole}$ and $A_{\mathrm{est}} \simeq 10^{13.9} \mathrm{sec}^{-1}$ (see dimethyldiazirine). The ethylcyclopropane product probably forms from a $1,3-\mathrm{H}$ atom shift in an intermediate hot olefin:

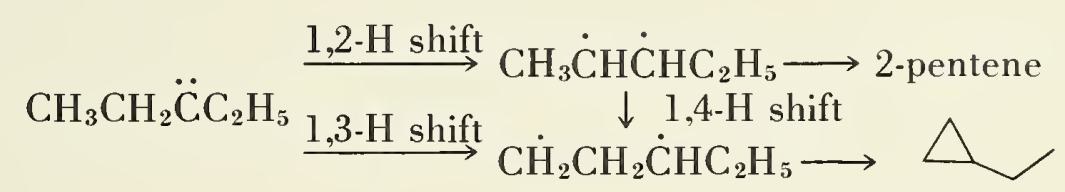

\section{Experimental}

[1] Static system. Analysis by G.L.C. The ratio of products was (cis/trans/ $(\nabla)=130.6 / 67.5 / 1.61$ independent of temperature and percent decomposition. The problem of differences in product ratios between thermal and photochemical diazirine systems is discussed relative to hot carbene intermediates in the photochemical systems. Results are inconclusive. 
Reaction: 3,3-Tetramethylenediazirine

$\begin{array}{cccc}\quad\left(\bigcirc \frac{N}{N}\right)(I) \longrightarrow(\square)\left([)+N_{2}(\mathrm{II})\right. & \\ & \text { I } & \text { II } & \text { III } \\ \Delta H_{f(298)}^{c} & 79.7 & 8.6 & 0 \\ S_{(298)}^{c} & 77.6 & 69.2 & 45.8 \\ C_{p(298)}^{c} & 25.3 & 18.1 & 7.0\end{array}$

$\Delta S^{c}=-37.4 \mathrm{~g} / \mathrm{mole}$

$\Delta H^{\prime \prime}=-71.1 \mathrm{kcal} / \mathrm{mole}$

$\Delta C_{p}^{\mathrm{c}^{\prime}}=-0.2 \mathrm{~g} / \mathrm{mole}$

\begin{tabular}{|c|c|c|c|c|c|c|}
\hline $\log A$ & $\boldsymbol{E}$ & $\begin{array}{c}\log \boldsymbol{k}_{T} \\
(390)\end{array}$ & Conditions & System & Surface & References \\
\hline 13.4 & 30.5 & -3.70 & $\begin{array}{l}373-412{ }^{\circ} \mathrm{K} \\
P_{T} \sim 1-150 \text { torr } \\
\text { (7 torr) }\end{array}$ & $\begin{array}{l}\text { static } \\
\text { no }(\mathrm{SN}) \\
\text { effect }\end{array}$ & None & $\begin{array}{l}\text { [1] H. M. Frey and A. W. } \\
\text { Scaplehorn, J. Chem } \\
\text { Soc. A, } 968 \text { (1966). }\end{array}$ \\
\hline
\end{tabular}

Preferred:

$\log l_{i}=13.4-30.5 / \theta$.

Comments: Transition state estimates for a biradical mechanism (see dimethyldiazirine) are in good agreement with the observed parameters.

\section{Experimental}

[1] Analysis by G.L.C. 
Reaction: 3,3-Pentamethylenediazirine

\begin{tabular}{|c|c|c|c|}
\hline$\Delta H_{f(298)}^{c}$ & $\begin{array}{c}\text { I } \\
68.4\end{array}$ & $\begin{array}{c}\text { II } \\
-0.8\end{array}$ & $\begin{array}{c}\text { III } \\
0\end{array}$ \\
\hline$S_{(298)}^{c}$ & 78.5 & 74.3 & 45.8 \\
\hline$C_{p(298)}^{c}$ & 31.9 & 25.3 & 7.0 \\
\hline
\end{tabular}

$\Delta S=41.6 \mathrm{~g} / \mathrm{mole}$

$\Delta H^{\circ}=-69.2 \mathrm{kcal} / \mathrm{mole}$

$\Delta C_{p}^{\circ}=0.4 \mathrm{~g} / \mathrm{mole}$

\begin{tabular}{|c|c|c|c|c|c|c|}
\hline $\log A$ & $\boldsymbol{E}$ & $\begin{array}{c}\log \boldsymbol{k}_{T} \\
(.390)\end{array}$ & Conditions & System & Surface & References \\
\hline 13.34 & 30.87 & -3.96 & $\begin{array}{l}374-410^{\circ} \mathrm{K} \\
P \sim 1-150 \text { torr } \\
(5 \text { torr })\end{array}$ & static & & $\begin{array}{l}\text { [1] H. M. Frey and A. W. } \\
\text { Scaplehorn, J. Chem. } \\
\text { Soc. B, } 968 \text { (1966). }\end{array}$ \\
\hline
\end{tabular}

Preferred:

$\log k=13.34-30.87 / \theta$.

Comments: A biradical mechanism is consistent with the observed parameters (see dimethyldiazirine).

\section{Experimental}

[1] Analysis hy G.L.C. on cyclohexene. 
Reaction: Difluorodiazirine

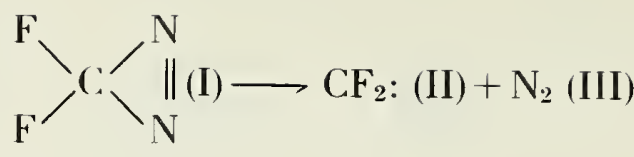

\begin{tabular}{|c|c|c|c|}
\hline & I & II & III \\
\hline$\Delta H_{f(298)}^{(}$ & & -39.7 & 0 \\
\hline$S_{(298)}^{\circ}$ & 69 & 57.5 & 45.8 \\
\hline$C_{p(298)}^{\circ}$ & & 9.1 & 7.0 \\
\hline
\end{tabular}

$\Delta S^{\circ}=(34.3) \mathrm{g} /$ mole

$\Delta H^{\circ}=$

$\Delta C_{p}^{\circ}=$

\begin{tabular}{|c|c|c|c|c|c|c|}
\hline $\log A$ & $\boldsymbol{E}$ & $\begin{array}{c}\log \boldsymbol{k}_{T} \\
(424)\end{array}$ & Conditions & System & Surface & References \\
\hline 13.1 & 32.2 & -3.12 & $\begin{array}{l}400-447{ }^{\circ} \mathrm{K} \\
100-800 \text { torr } \\
(200)\end{array}$ & static & none & $\begin{array}{l}\text { [1] E. W. Neuvar and } \\
\text { R. A. Mitsch, J. Phys. } \\
\text { Chem. 71, 1229 (1967) }\end{array}$ \\
\hline
\end{tabular}

\section{Preferred:}

$\log k=13.9-33.2 / \theta$.

Comments: Since the reaction was in its falloff region, the reported parameters are probably low. (See, for comparison, dimethyldiazirine decomposition.) We have assumed $k_{\exp } \simeq 1 / 2 k_{\infty}$ and raised $A$ to the calculated value.

\section{Experimental}

[1] Analysis by G.L.C. on $\mathrm{CF}_{2} \mathrm{~N}_{2}$ disappearance. Pyrolysis performed in excess $\mathrm{C}_{2} \mathrm{~F}_{4}$ to trap $\mathrm{CF}_{2}$ : as $(\nabla)_{F 6}$. Reaction is in its falloff region; therefore parameters are slightly low; however, it was estimated that $E_{200 \mathrm{~mm}} \simeq E_{x}$. 
Reaction: Ethylidenecyclopropane (I)

2-Methyl-1-methylenecyclopropane (II)

\begin{tabular}{|c|c|c|}
\hline$\Delta H_{f(298)}^{\circ}$ & $\begin{array}{c}\text { I } \\
39.1\end{array}$ & $\begin{array}{c}\text { II } \\
38.3\end{array}$ \\
\hline$S_{(298)}^{\circ}$ & 74.7 & 74.5 \\
\hline$C_{p(298)}^{\circ}$ & 21.6 & 24.2 \\
\hline
\end{tabular}

$\Delta S^{\circ}=-0.2 \mathrm{~g} / \mathrm{mole}$

$\Delta H^{\circ}=-0.8 \mathrm{kcal} / \mathrm{mole}$

$\Delta C_{p}^{\circ}=2.6 \mathrm{~g} / \mathrm{mole}$

\begin{tabular}{|c|c|c|c|c|c|c|c|}
\hline Path & $\log A$ & $\boldsymbol{E}$ & $\begin{array}{c}\log \boldsymbol{k}_{T} \\
(488)\end{array}$ & Conditions & System & Surface & References \\
\hline & 14.0 & 40.76 & -4.25 & $\begin{array}{c}488 \pm 18^{\circ} \mathrm{K} \\
\text { (25 torr) }\end{array}$ & static & $<10 \%$ & $\begin{array}{c}\text { [1] J. P. Chesick, J. Am. } \\
\text { Chem. Soc. 85, } \\
2720 \text { (1963). }\end{array}$ \\
\hline
\end{tabular}

$\begin{array}{llll}b & 13.9 & 40.26 & -4.13\end{array}$

Preferred:

Reliable as reported.

Comments: The observed kinetics are consistent with the biradical mechanism with ring opening (assisted by one allylic resonance) rate determining. Transition state estimates give:

$$
\begin{aligned}
& \log k_{a l}=14.1-39.3 / \theta \\
& \log k_{b}=14.2-38.8 / \theta \text { (see section III-4.2). }
\end{aligned}
$$

\section{Experimental}

[1] Analysis by G.L.C. Both reactions proceeded to the equilibrium state. The equilibrium constant for the isomerization was:

$$
\begin{aligned}
& K_{a, b}=10^{0.64 / 4.575-0.57 / \theta} \\
& K_{a, b}=0.77 \text { at } 210^{\circ} \mathrm{C} .
\end{aligned}
$$


Reaction: Oxirane (ethylene oxide)

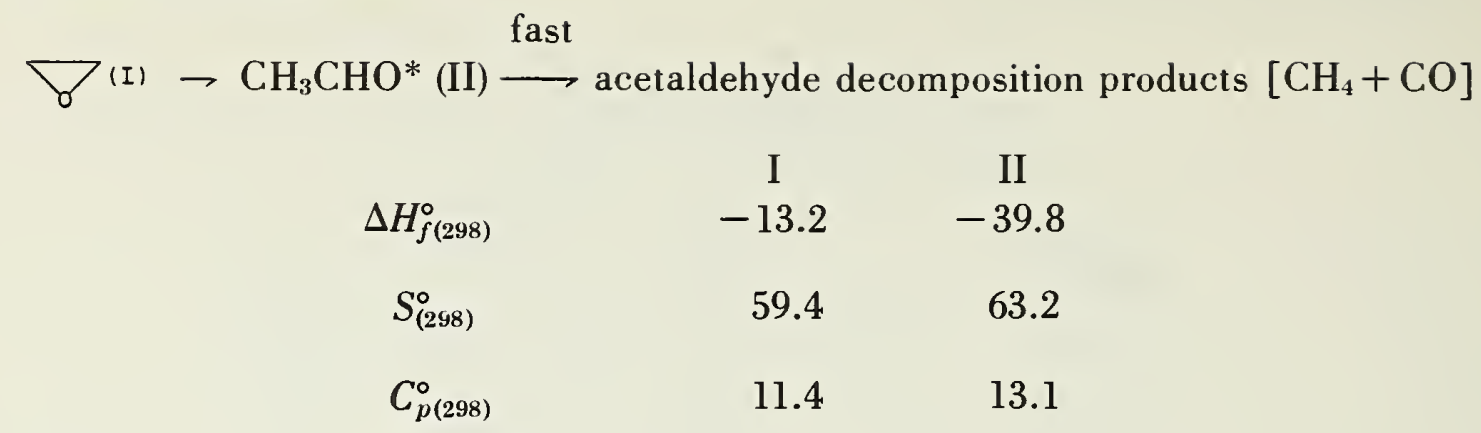

$\Delta S^{\circ}=3.8 \mathrm{~g} /$ mole

$\Delta H^{\circ}=-26.6 \mathrm{kcal} / \mathrm{mole}$

$\Delta C_{p}^{\circ}=\quad 1.7 \mathrm{~g} / \mathrm{mole}$

$\begin{array}{llccll}\log \boldsymbol{A} & \boldsymbol{E} & \begin{array}{c}\log \boldsymbol{k}_{T} \\ (670)\end{array} & \text { Conditions } & \text { System } & \text { Surface } \\ 14.13 & 56.9 & -4.39 & \begin{array}{c}673 \pm 30^{\circ} \mathrm{K} \\ (30) \text { torr }\end{array} & \text { static } & \\ & & & & & \\ 14.34 & 57.4 & -4.34 & \begin{array}{c}668 \pm 45^{\circ} \mathrm{K} \\ 180-440 \text { torr }\end{array} & \text { static } & <10 \% \\ & & & & & \\ & & & & & \\ & & & & & \\ & & & & & \end{array}$

[1] M. L. Neufeld and A. T. Blades, Can. J. Chem. 41, 2956 (1963).

[2] K. H. Mueller and W. D. Walters, J. Am. Chem. Soc. 73, 1458 (1951); J. Am. Chem. Soc. 76, 330 (1954).

[3] L. Crocco, I. Glassman, and I. E. Smith, J. Chem. Phys. 31, 506 (1959).

\section{Preferred:}

$\log k=14.34-57.4 / \theta$.

Comments: The "hot" acetaldehyde intermediate decomposes to $\mathrm{CH}_{4}+\mathrm{CO}$ at low pressures and is stabilized at high pressures. For a biradical mechanistic interpretation and review of this reaction, see S. W. Benson, J. Chem. Phys. 40, 105 (1964). The H-migration activation energy from the kinetics is estimated to be about $4.4 \mathrm{kcal} / \mathrm{mole}$ (i.e., appreciably lower than the $1,2-\mathrm{H}$-migration in cyclopropane). Transition state estimate gives: $\log A_{\text {est }}=14.5$ (see section III-4.0).

\section{Experimental}

[1] Ảnalysis by G.L.C. Rates obtained from initial rates under maximum inhibition with propylene.

[2] Analysis by G.L.C. Rates obtained under "maximum inhibition" conditions with propylene.

[3] Rates determined by "temperature profile history." Short chain mechanism proposed from which the activation energy was deduced.

*Indicates vibrationally excited acetaldehyde. 
Reaction: $\beta, \beta$-Dimethylcyclopropyl methyl ketone (2,2-dimethylcyclopropyl-1-ethanone)

$\begin{array}{ccc}\longrightarrow & \text { (II) } & \text { 2-methyl-5-one-1-hexene } \\ \Delta H_{f(298)}^{\circ} & -40.8 & -46.7 \\ S_{(298)}^{\circ} & 93.9 & 105.5 \\ C_{p(298)}^{\circ} & 36.0 & 39.0\end{array}$

$\Delta S^{\circ}=11.6 \mathrm{~g} / \mathrm{mole}$

$\Delta H^{\circ}=-5.9 \mathrm{kcal} / \mathrm{mole}$

$\Delta C_{p}^{\circ}=3.0 \mathrm{~g} / \mathrm{mole}$

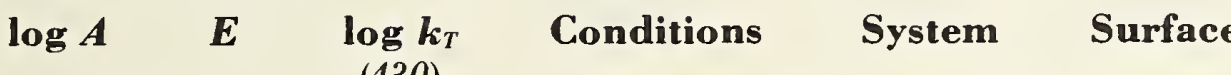
$(430)$

$12.6 \quad 33 \quad-4.15 \quad 425-436^{\circ} \mathrm{K} \quad$ static

[1] R. M. Roberts and R. G. Landolt, J. Am. Chem. Soc. 87, 2281 (1965).

Preferred:

$\log k=12.6-33 / \theta$.

Comments: Note the similarity in mechanism between the diolefin isomerization, cis-2-methyl penta-1,3diene $\longrightarrow$ 4-methyl penta-1,3-diene. The initial product is probably enol which rearranges on the walls to the ketone.

\section{Experimental}

[1] Rates based on G.L.C. analysis. I-Acetyl-trans-2-methylcyclopropane was stable up to $460^{\circ} \mathrm{K}$, indicating a sixcenter, concerted, "ene" reaction. 
Reaction: Perfluorooxirane (tetrafluorooxirane; tetrafluoroethylene oxide)
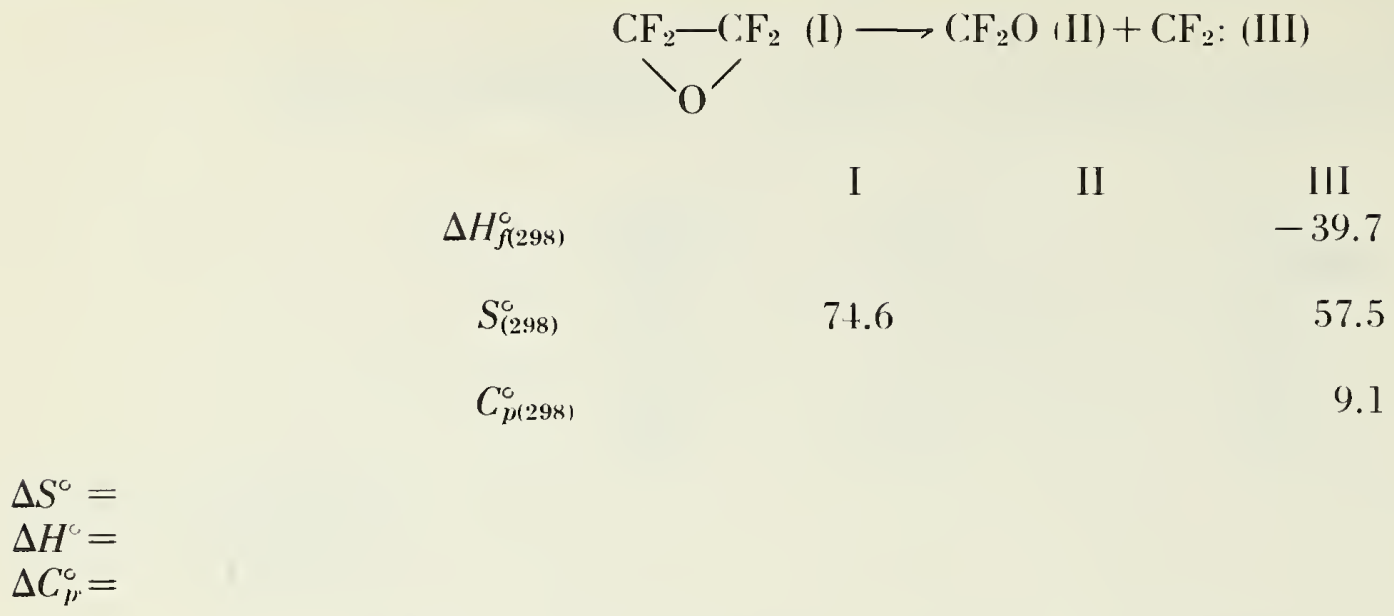

\begin{tabular}{|c|c|c|c|c|c|c|}
\hline $\log A$ & $\boldsymbol{E}$ & $\begin{array}{c}\log \boldsymbol{k}_{T} \\
(36.3)\end{array}$ & Conditions & System & Surface & References \\
\hline 13.7 & 31.6 & -5.32 & $\begin{array}{l}363 \pm 50^{\circ} \mathrm{K} \\
0.13-785 \text { torr } \\
\quad(785)\end{array}$ & static & $<10 \%$ & $\begin{array}{l}\text { [1] M. Lenzi and A. Mele. } \\
\text { J. Chem. Phys. 43, } \\
\text { 1974 (1965). }\end{array}$ \\
\hline
\end{tabular}

\section{Preferred:}

Reliable. $\log k=13.7-31.6 / \theta$

\section{Experimental}

[1] Rate and analysis by (;.I.C. and I.R. A free radical non-chain mechanism was proposed with the above initiation rate determining step. Falloff observed near 780 torr. The RRK-s $\simeq 8.9$ fit the falloff data. The ( $F_{2}$ : hiradicals gave $\mathrm{C}_{2} \mathrm{~F}_{4}$ llow percent conversion) and perfluorocyclopropane thigh percent conversions). An isomerization on the watls leading to trifluornacetylfluoride was also observed. 
Reaction: Cyclobutane

$\Delta S^{\circ}=41.5 \mathrm{~g} / \mathrm{mole}$

$\Delta \ddot{H}^{\circ}=18.7 \mathrm{kcal} / \mathrm{mole}$

$\Delta C_{p}^{\circ}=3.4 \mathrm{~g} / \mathrm{mole}$

\begin{tabular}{|c|c|c|c|c|c|c|}
\hline $\log A$ & $\boldsymbol{E}$ & $\log \boldsymbol{k}_{T}$ & Conditions & System & Surface & References \\
\hline 15.6 & 62.5 & -3.45 & $\begin{array}{l}717 \pm 24{ }^{\circ} \mathrm{K} \\
1-996 \text { torr } \\
(120)\end{array}$ & static & & $\begin{array}{l}\text { [1] C. T. Genaux, F. Kern, } \\
\text { and W. D. Walters, } \\
\text { J. Am. Chem. Soc. 75, } \\
6196 \text { (1953). }\end{array}$ \\
\hline 15.62 & 62.5 & -3.47 & $\begin{array}{l}728 \pm 45^{\circ} \mathrm{K} \\
240-1500 \text { torr } \\
(650-790)^{\circ} \mathrm{K} \\
(1250-1500) \text { torr }\end{array}$ & static & none & $\begin{array}{l}\text { [2] R. W. Carr, Jr., and } \\
\text { W. D. Walters, J. Phys. } \\
\text { Chem. 67, } 1370 \\
\text { (1963). }\end{array}$ \\
\hline
\end{tabular}

\section{Preferred:}

$\log k=15.6-62.5 / \theta$.

$\begin{array}{ccc}\Delta H_{f(298)} & \text { I } & \text { II } \\ S_{(298)}^{\circ} & 6.3 & 12.5 \\ C_{p(298)}^{\circ} & 63.5 & 52.5 \\ & 17.4 & 10.4\end{array}$

$\stackrel{\text { (I) }}{\longrightarrow} 2\left(\mathrm{C}_{2} \mathrm{H}_{4}\right)$ (II)

2.5

2.5

0.4 
Reaction: Methylcyclobutane

\begin{tabular}{cccc}
\multicolumn{4}{c}{$\sqcup(\mathrm{I}) \longrightarrow \mathrm{C}_{3} \mathrm{H}_{6}(\mathrm{II})+\mathrm{C}_{2} \mathrm{H}_{4}(\mathrm{III})$} \\
$\Delta H_{f(298)}^{\circ}$ & I & II & III \\
$S_{(298)}^{\circ}$ & -0.7 & 4.8 & 12.5 \\
$C_{p(298)}^{\circ}$ & 74.4 & 63.6 & 52.5 \\
$C_{p(700)}^{\circ}$ & 22.6 & 15.3 & 10.4 \\
& 49.0 & 28.4 & 18.8
\end{tabular}

$\Delta S^{\circ}=31.7 \mathrm{~g} / \mathrm{mole}$

$\Delta H^{\circ}=18.0 \mathrm{kcal} / \mathrm{mole}$

$\Delta C_{p}^{\circ}=3.1 \mathrm{~g} / \mathrm{mole}$

\begin{tabular}{|c|c|c|c|c|c|c|}
\hline $\log A$ & $\boldsymbol{E}$ & $\begin{array}{c}\log \boldsymbol{k}_{T} \\
(703)\end{array}$ & Conditions & System & Surface & References \\
\hline 15.38 & 61.2 & -3.65 & $\begin{array}{l}703 \pm 20^{\circ} \mathrm{K} \\
7-417 \text { torr } \\
(10),(200)\end{array}$ & static & none & $\begin{array}{l}\text { [1] M. N. Das and W. D. } \\
\text { Walters, Zeit. fur } \\
\text { Physik. Chemie } \\
\text { N.F.15, } 23 \text { (1958). }\end{array}$ \\
\hline
\end{tabular}

Preferred:

Reliable as reported.

Comments: By the biradical mechanism (see cyclobutane) products are those expected and $\log k_{\text {est }}=15.5-$ $62.1 / \theta$ (see section III-5.0).

\section{Experimental}

[1] Analysis by I.R. and M. S. Rate by $\Delta P$. Rate unaffected by added NO, toluene, and propylene and by $\mathrm{KCl}$ surface. Falloff observed near 10 torr $\left(700^{\circ} \mathrm{K}\right)$ with an RRK-s $\simeq 23$. (See A. F. Pataracchia and W. D. Walters, J. Phys. Chem. 68, 3894 (1964).) 
Reaction: trans-1,2-Dimethylcyclobutane

\begin{tabular}{|c|c|c|c|c|c|c|}
\hline \multirow[b]{2}{*}{$\Delta H_{f(298)}^{\circ}$} & \multicolumn{6}{|c|}{$\begin{array}{l}\stackrel{b}{\longrightarrow} 2 \mathrm{C}_{3} \mathrm{H}_{6}(\mathrm{III}) \\
\stackrel{\mathrm{c}}{\longrightarrow} \mathrm{C}_{2} \mathrm{H}_{4}(\mathrm{IV})+\text { cis } \text { and trans } \mathrm{CH}_{3} \mathrm{CH}=\mathrm{CHCH}_{3}(\mathrm{~V})\end{array}$} \\
\hline & $\begin{array}{c}\mathrm{I} \\
-7.7\end{array}$ & $\begin{array}{l}\text { II } \\
-6.7\end{array}$ & $\begin{array}{l}\text { III } \\
4.8\end{array}$ & $\begin{array}{c}\text { IV } \\
12.5\end{array}$ & $\begin{array}{l}\mathrm{V} \text { cis } \\
-1.7\end{array}$ & $\begin{array}{l}\text { V trans } \\
-2.7\end{array}$ \\
\hline$S_{(298)}^{\circ}$ & 81.1 & 82.3 & 63.6 & 52.5 & 72.1 & 70.9 \\
\hline$C_{p(298)}^{\circ}$ & 27.9 & 26.5 & 15.3 & 10.4 & 18.9 & 21.0 \\
\hline$C_{p(700)}^{\circ}$ & 59.4 & 58.9 & 28.4 & 18.8 & 37.6 & 38.4 \\
\hline
\end{tabular}

$\begin{array}{rlrl} & a & b \\ \Delta S^{\circ}= & 1.2 & 46.1 \mathrm{~g} / \mathrm{mole} \\ \Delta H^{\circ}= & 1.0 & 17.3 \mathrm{kcal} / \mathrm{mole} \\ \Delta C_{p}^{\circ}=-1.4 & 2.7 \mathrm{~g} / \mathrm{mole}\end{array}$

$\begin{array}{lllllll}\text { Path } & \log A & \boldsymbol{E} & \log \boldsymbol{k}_{T} & \text { Conditions } & \text { System } & \text { Surface }\end{array}$

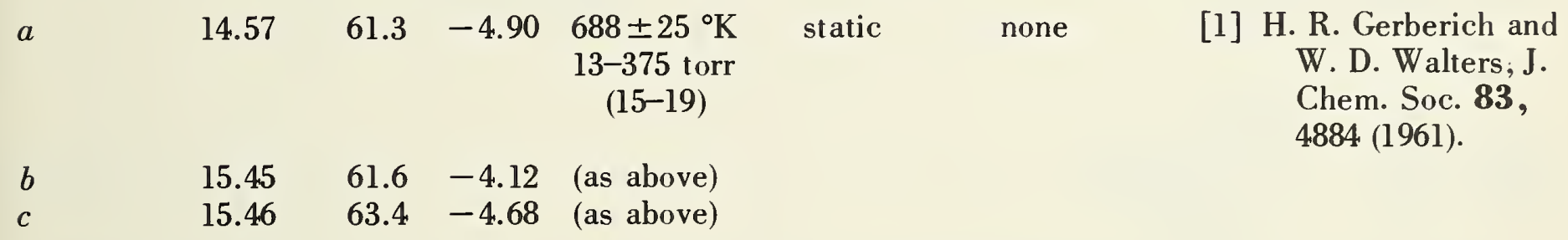

Preferred:

Reliable as reported.

Comments: Products are those expected by the biradical mechanism. The biradical mechanism is shown below.

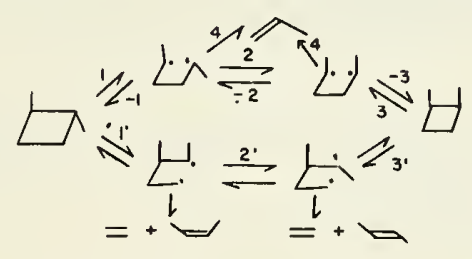

Rates of the competitive reactions from the biradical intermediates (i.e., internal rotations, ring closings, and decompositions) must all be competitive processes with comparable rate constants and rate constant parameters. Since the (cis/trans) but-2-ene ratios differ depending on the starting isomer, these reactions are rotationally controlled.

By applying steady state considerations to the above mechanism and by making reasonable assumptions regarding the relative rates of three of the pertinent reactions, it has been possible to obtain estimates of the rate constants for decomposition, internal rotation, and $\mathrm{C}_{4}$ ring closing from the biradical intermediates. (See E. O’Neal and S. W. Benson, J. Phys. Chem. 72, 1866 (1968).)

Activation energies for ring closing and decomposition in the analysis are both comparable. $E_{\text {r.c. }} \simeq E_{\text {dec }} \simeq 7.4 \mathrm{kcal} / \mathrm{mole}$.

Estimated:

$$
\begin{aligned}
& \log k_{a}=14.4-61.1 / \theta \\
& \log k_{b}=14.9-61.4 / \theta \\
& \log k_{c}=15.3-63.2 / \theta \text { (see secton III-5.0). }
\end{aligned}
$$




\section{Experimental}

[1] Analysis by I.R. and G.L.C. Rate by (..I.C. Rates unaffected by added NO. The (trans/cis) but-2-ene ratio $\simeq 9$. (See also results on cis-1,2-dimethylcyclobutane.) Geometric isomerization was slow relative to structural isomerization.

Reaction: cis-1,2-Dimethylcyclobutane

$$
\begin{aligned}
& \Xi_{(\mathrm{I})} \stackrel{0}{\longrightarrow} \sum_{2 \mathrm{C}_{3} \mathrm{H}_{6}}(\mathrm{III}) \\
& \stackrel{c}{\longrightarrow} \text { cis and trans } \mathrm{CH}_{3} \mathrm{CH}=\mathrm{CHCH}_{3}(\mathrm{IV})+\mathrm{C}_{2} \mathrm{H}_{4}(\mathrm{~V}) \\
& \begin{array}{lllll} 
& \text { I } & \text { II } & \text { III } & \text { IV cis IV trans }
\end{array} \\
& \begin{array}{rrrrrrr}
S_{(298)}^{\circ} & 82.3 & 81.1 & 63.6 & 72.1 & 70.9 & 52.5
\end{array} \\
& \begin{array}{lllllll}
C_{p(298)}^{\circ} & 26.5 & 27.9 & 15.3 & 18.9 & 21.0 & 10.4
\end{array} \\
& \begin{array}{lllllll}
C_{p(700)}^{\circ} & 58.9 & 59.4 & 28.4 & 37.6 & 38.4 & 18.8
\end{array} \\
& \begin{array}{cl}
a & c(\text { cis }) \\
\Delta S^{\circ}=-1.2 & 42.3 \mathrm{~g} / \text { mole } \\
\Delta H^{\circ}=-1.0 & 17.5 \mathrm{kcal} / \text { mole } \\
\Delta C_{p}^{\circ}=1.4 & 2.8 \mathrm{~g} / \text { mole }
\end{array}
\end{aligned}
$$

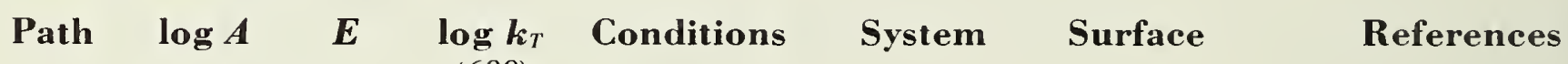

$$
\begin{aligned}
& \begin{array}{lllllll}
a & 14.81 & 60.1 & -4.28 & 688 \pm 25^{\circ} \mathrm{K} & \text { static none }
\end{array} \\
& \text { (15-19) } \\
& \begin{array}{llllll}
b & 15.48 & 60.4 & -3.51 & 678 \pm 25^{\circ} \mathrm{K} & \text { static none }
\end{array} \\
& \begin{array}{lllll}
c & 15.57 & 63.4 & -4.57 & \text { (as above) }
\end{array}
\end{aligned}
$$

\section{Experimental}

[1] Analysis by I.R. and G.L.C. Rate by G.L.C.

[2] Analysis by I.R. and G.L.C. Rates by G.L.C. Rates were not affected by added NO and propylene. The ratio of (cis/trans) but-2-ene was $\simeq 1.8$. The geometric isomerization to trans-1,2-dimethylcyclobutane was slow relative to the structural isomerizations. 
Reaction: Ethylcyclobutane

\begin{tabular}{|c|c|c|c|}
\hline$\Delta H_{f(298)}^{\circ}$ & $\begin{array}{c}\text { I } \\
-5.2\end{array}$ & $\begin{array}{c}\text { II } \\
12.5\end{array}$ & $\begin{array}{c}\text { III } \\
0\end{array}$ \\
\hline$S_{(298)}^{\circ}$ & 83.8 & 52.5 & 73.6 \\
\hline$C_{p(298)}^{\circ}$ & 28.1 & 10.4 & 20.5 \\
\hline$C_{p(700)}^{\circ}$ & 59.2 & 18.8 & 38.7 \\
\hline
\end{tabular}

$\Delta S^{\circ}=42.3 \mathrm{~g} / \mathrm{mole}$

$\Delta H^{\circ}=17.7 \mathrm{kcal} / \mathrm{mole}$

$\Delta C_{p}^{\circ}=2.8 \mathrm{~g} / \mathrm{mole}$

$\begin{array}{llclll}\log A & \boldsymbol{E} & \begin{array}{c}\log k_{T} \\ (713)\end{array} & \text { Conditions } & \text { System } & \text { Surfac } \\ 15.56 & 62.0 & -3.45 & \begin{array}{l}713 \pm 20^{\circ} \mathrm{K} \\ 7-400 \text { torr } \\ (10,70,200)\end{array} & \text { static } & \text { none } \\ & & & & \end{array}$

\section{References}

[1] R. E. Wellman and W. D. Walters, J. Am. Chem. Soc. 79, 1542 (1957).

\section{Preferred:}

$\log k=15.56-62.0 / \theta$.

Comments: By the biradical mechanism, the parameters should be about the same as those for methylcyclobutane. Products are those expected. Transition state estimates for the biradical mechanism (see section III-5.0) give: $\log k_{\text {est }}=15.3-61.6 / \theta$.

\section{Experimental}

[1] Analysis by I.R., M.S. Rates by $\Delta$ P. Rates were unaffected by added NO, propylene, and toluene. 
Reaction: $n$-Propylcyclobutane

\begin{tabular}{cccc}
\multicolumn{4}{c}{$Z(\mathrm{I}) \longrightarrow \mathrm{CH}_{2}=\mathrm{CHCH}_{2} \mathrm{CH}_{3}(\mathrm{II})+\mathrm{C}_{2} \mathrm{H}_{4}$ (III) } \\
& I & II & III \\
$\Delta H_{f(298)}^{\circ}$ & -10.1 & -4.9 & 12.5 \\
$S_{(298)}^{\circ}$ & 93.2 & 82.8 & 52.5 \\
$C_{p(298)}^{\circ}$ & 33.6 & 26.2 & 10.4 \\
$C_{p(700)}^{\circ}$ & 69.4 & 49.1 & 18.8
\end{tabular}

\begin{abstract}
$\Delta S^{\circ}=42.1 \mathrm{~g} / \mathrm{mole}$
$\Delta H^{\circ}=17.7 \mathrm{kcal} / \mathrm{mole}$

$\Delta C_{p}^{\circ}=3.0 \mathrm{~g} / \mathrm{mole}$
\end{abstract}

\begin{tabular}{|c|c|c|c|c|c|c|}
\hline $\log A$ & $E$ & $\begin{array}{c}\log \boldsymbol{k}_{T} \\
(710)\end{array}$ & Conditions & System & Surface & References \\
\hline 15.53 & 61.6 & -3.68 & $\begin{array}{l}701 \pm 28^{\circ} \mathrm{K} \\
5.4-495 \text { torr } \\
(5.4,117)\end{array}$ & static & none & $\begin{array}{l}\text { [1] S. M. E. Kellner and } \\
\text { W. D. Walters, J. Phys. } \\
\text { Chem. 65, } 466 \text { (1961). }\end{array}$ \\
\hline
\end{tabular}

Preferred:

Reliable.

Comments: Transition state estimates are the same as those for ethylcyclobutane (see section III-5.0).

\title{
Experimental
}

[1] Analysis by I.R., M.S., G.L.C. Rates by $\Delta \mathrm{P}$; unaffected by added NO, propylene, and toluene. Side products less than 2 percent of total products. 
Reaction: Isopropylcyclobutane

\begin{tabular}{crcr}
$\sum(\mathrm{I}) \longrightarrow \mathrm{C}_{2} \mathrm{H}_{4}(\mathrm{II})+\mathrm{CH}_{2}=$ & \multicolumn{3}{c}{$\mathrm{CHCH}\left(\mathrm{CH}_{3}\right)_{2}(\mathrm{II})$} \\
$\Delta H_{f(298)}^{\circ}$ & $\begin{array}{c}\mathrm{I} \\
\text { II }\end{array}$ & III \\
$S_{(298)}^{\circ}$ & -11.1 & 12.5 & -6.9 \\
$C_{p(298)}^{\circ}$ & 90.5 & 52.5 & 79.7 \\
$C_{p(\mathbf{7 0 0})}^{\circ}$ & 33.4 & 10.4 & 28.5 \\
& 69.6 & 18.8 & 50.2
\end{tabular}

$\Delta S^{\circ}=41.7 \mathrm{~g} / \mathrm{mole}$

$\Delta H^{\circ}=16.7 \mathrm{kcal} / \mathrm{mole}$

$\Delta C_{p}^{\circ}=5.5 \mathrm{~g} / \mathrm{mole}$

$\begin{array}{ccccccc}\log \boldsymbol{A} & \boldsymbol{E} & \begin{array}{c}\log \boldsymbol{k}_{T} \\ (708)\end{array} & \text { Conditions } & \text { System } & \text { Surface } & \text { References } \\ 15.63 & & & & \\ & & & & & \end{array}$

Preferred:

Reliable.

Comments: Transition state estimates for the biradical mechanism give: $\log k=15.3-61.4 / \theta$, in good agreement with the observed parameters (see section III-5.0).

\section{Experimental}

[1] Analysis by I.R., M.S., G.L.C. Rate by $\Delta$ P. Less than 1 percent side products. 
Reaction: Isopropenylcyclobutane

\begin{tabular}{|c|c|c|c|c|}
\hline$\Delta H_{f(298)}$ & $\begin{array}{c}\text { I } \\
16.9\end{array}$ & $\begin{array}{c}\text { II } \\
12.5\end{array}$ & $\begin{array}{c}\text { III } \\
18.1\end{array}$ & $\begin{array}{c}\text { IV } \\
-10.5\end{array}$ \\
\hline$S_{(298)}^{\circ}$ & 89.0 & 52.5 & 75.4 & 82.3 \\
\hline$C_{p(298)}^{o}$ & 34.0 & 10.4 & 25.0 & 31.4 \\
\hline$C_{p(600)}^{\circ}$ & 59.4 & 17.1 & 41.4 & 40.1 \\
\hline
\end{tabular}

$\begin{array}{rl}a & b \\ \Delta S^{\circ}=38.9 & -6.7 \mathrm{~g} / \mathrm{mole} \\ \Delta H^{\circ}=13.7 & -27.4 \mathrm{kcal} / \mathrm{mole} \\ \Delta C_{p}^{\circ}=1.4 & -2.6 \mathrm{~g} / \mathrm{mole}\end{array}$

\begin{tabular}{|c|c|c|c|c|c|c|c|}
\hline Path & $\log A$ & $\boldsymbol{E}$ & $\begin{array}{c}\log \boldsymbol{k}_{\boldsymbol{T}} \\
(599)\end{array}$ & Conditions & System & Surface & References \\
\hline & 14.64 & 51.03 & -3.98 & $\begin{array}{l}599 \pm 24{ }^{\circ} \mathrm{K} \\
1-40 \text { torr } \\
(3.5)\end{array}$ & static & none & $\begin{array}{l}\text { [1] R. J. Ellis and H. M. } \\
\text { Frey, Trans } \\
\text { Faraday Soc. 59, } \\
2076 \text { (1963). }\end{array}$ \\
\hline
\end{tabular}

$b$

$14.53 \quad 51.03-4.09$

$\longleftarrow$ as above $\longrightarrow$

\section{Preferred:}

$\log k_{a}=14.64-51.03 / \theta ; \log k_{b}=14.53-51.03 / \theta$

Comments: Products are those expected from the biradical mechanism (see section III-5.2). We estimate: $\log k_{a}=\log k_{b}=14.2-49.3 / \theta$. If the rate constant parameters are compared to those of isopropylcyclobutane (i.e., $k=10^{15.6-62.6 / \theta} \mathrm{sec}^{-1}$ ), it appears that close to a full allylic resonance (i.e., $E_{\text {res }} \simeq 12.6 \mathrm{kcal} / \mathrm{mole}$ ) is achieved in the transition state.

\section{Experimental}

[1] Analysis by G.L.C. In clean reaction cells (unconditioned walls) other products were produced. The ratio of paths $a$ and $b$ products was essentially temperature independent. 
Reaction: Oxetane (trimethylene oxide)

\begin{tabular}{|c|c|c|c|}
\hline$\Delta H_{f(298)}^{\circ}$ & $\begin{array}{c}\text { I } \\
-19.3\end{array}$ & $\begin{array}{c}\text { II } \\
12.5\end{array}$ & $\begin{array}{c}\text { III } \\
-27.7\end{array}$ \\
\hline$S_{(298)}^{\circ}$ & 63.5 & 52.5 & 53.7 \\
\hline$C_{p(298)}^{\circ}$ & 14.3 & 10.4 & 8.5 \\
\hline
\end{tabular}

$\Delta S^{\circ}=42.7 \mathrm{~g} / \mathrm{mole}$

$\Delta H^{\circ}=4.1 \mathrm{kcal} / \mathrm{mole}$

$\Delta C_{p}^{\circ}=4.6 \mathrm{~g} / \mathrm{mole}$

$\begin{array}{ccccccc}\log \boldsymbol{A} & \boldsymbol{E} & \begin{array}{c}\log \boldsymbol{k}_{T} \\ (713)\end{array} & \text { Conditions } & \text { System } & \text { Surface } & \text { References } \\ 14.78 & 60 & -3.61 & \begin{array}{l}713 \pm 20^{\circ} \mathrm{K} \\ 54-330 \text { torr } \\ (100)\end{array} & \text { static } & <10 \% & \text { [1] D. A. Bittker and W. D. } \\ & & & & & \text { Walters, J. Am. Chem. } \\ & & & & & \text { Soc. 77, 1429 (1955). }\end{array}$

Preferred:

$\log k=14.78-60 / \theta$.

Comments: Transition state estimates for the biradical mechanism (see cyclobutane and section III-5.0). with ring opening via an $\alpha(\mathrm{C}-\mathrm{O})$ split gives: $\log A_{\mathrm{est}}=14.7 ; E_{\mathrm{est}}=59.0 \mathrm{kcal} / \mathrm{mole}$. The activation energy for $\beta \mathrm{C}-\mathrm{C}$ ) split is estimated to be more than $3 \mathrm{kcal} /$ mole higher and is therefore probably not important.

\section{Experimental}

[1] Analysis by I.R., M.S., Chem., P.O.L. Stoichiometry confirmed in the initial stages. Rates were determined by $\Delta \mathrm{P}$ in the presence of added NO, propylene, or toluene. The uninhibited rate was 10 percent faster. The origin of the free radical component to the decomposition was attributed to the subsequent decomposition of the formaldehyde product. 
Reaction: 3,3-Dimethyloxetane (3,3-dimethylketane)

\begin{tabular}{lrcr} 
& \multicolumn{3}{c}{} \\
& I & II & III \\
$\Delta H_{f(298)}^{\circ}$ & -34.0 & -4.0 & -27.7 \\
$S_{(298)}^{\circ}$ & 75.4 & 70.2 & 53.7 \\
$C_{p(298)}^{\circ}$ & 25.6 & 21.4 & 8.5
\end{tabular}

$\Delta S^{\circ}=48.5 \mathrm{~g} / \mathrm{mole}$

$\Delta H^{\circ}=2.3 \mathrm{kcal} / \mathrm{mole}$

$\Delta C_{p}^{\circ}=4.3 \mathrm{~g} / \mathrm{mole}$

\begin{tabular}{ccccccc}
$\log \boldsymbol{A}$ & $\boldsymbol{E}$ & $\begin{array}{c}\log \boldsymbol{k}_{T} \\
(700)\end{array}$ & Conditions & System & Surface & \multicolumn{1}{c}{ References } \\
15.58 & 60.7 & -3.37 & $\begin{array}{l}698 \pm 25^{\circ} \mathrm{K} \\
6-70 \text { torr }\end{array}$ & static & none & [1] G. F. Cohoe and W. D. \\
W alters, J. Phys. Chem. \\
$71,2326(1967)$.
\end{tabular}

Preferred:

Reliable. $\log k=15.58-60.7 / \theta$.

Comments: Transition state calculations indicate that the reaction should proceed almost exclusively via $\beta(\mathrm{C}-\mathrm{C})$ cleavage rather than by an $\alpha(\mathrm{C}-\mathrm{C})$ split as in trimethylene oxide. Thus one estimates (for a mechanism'like cyclobutane):

$$
\begin{gathered}
\beta(\mathrm{C}-\mathrm{C}) \text { split: } \log k=16.2-60.3 / \theta \\
\alpha(\mathrm{C}-\mathrm{C}) \text { split: } \log k=14.7-59.8 / \theta \\
\text { Experimental }
\end{gathered}
$$

[1] Analysis by G.L.C. Good mass balances were obtained. Rates were unaffected by additions of $\mathrm{C}_{3} \mathrm{H}_{6}$, and up to 50 percent NO. 
Reaction: Cyclobutane carboxaldehyde

$\begin{array}{cccc}\square{ }_{\mathrm{CH}=0}(\mathrm{II}) & \mathrm{C}_{2} \mathrm{H}_{4}(\mathrm{II})+\mathrm{CH}_{2}=\mathrm{CH}-\mathrm{CH}=\mathrm{O}(\mathrm{III}) \\ \Delta H_{f(298)}^{\circ} & \mathrm{I} & \mathrm{II} & \mathrm{III} \\ S_{(298)}^{\circ} & -19.6 & 12.5 & -16.6 \\ C_{p(298)}^{\circ} & 80.7 & 52.5 & (67.3) \\ C_{p(600)}^{\circ} & 23.9 & 10.4 & 17.1 \\ & 42.4 & 17.1 & 26.0\end{array}$

$\Delta S^{\circ}=39.1 \mathrm{~g} / \mathrm{mole}$

$\Delta H^{\circ}=15.5 \mathrm{kcal} / \mathrm{mole}$

$\Delta C_{p}^{\circ}=3.6 \mathrm{~g} / \mathrm{mole}$

\begin{tabular}{|c|c|c|c|c|c|c|}
\hline $\log A$ & $\boldsymbol{E}$ & $\begin{array}{c}\log \boldsymbol{k}_{T} \\
(653)\end{array}$ & Conditions & System & Surface & References \\
\hline 14.43 & 53.3 & -3.41 & $\begin{array}{l}653 \pm 20^{\circ} \mathrm{K} \\
9-35 \text { torr } \\
(9-12)\end{array}$ & static & none & $\begin{array}{l}\text { [1] B. C. Roquitte and W. D. } \\
\text { Walters, J. Am. Chem. } \\
\text { Soc. 84, } 4049 \text { (1962). }\end{array}$ \\
\hline
\end{tabular}

\section{Preferred:}

Reliable as reported. $\log k=14.43-53.3 / \theta$

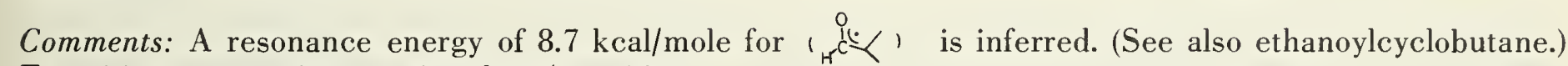
Transition state estimates give: $\log A_{\text {est }}=14.2$.

\section{Experimental}

[1] Analysis by I.R., M.S., G.L.C., P.O.L., U.V. Rates by $\Delta$ P. Rates were unaffected by additions of NO, propylene, toluene, and by a $\mathrm{KCl}$ surface. 
Reaction: Ethanoylcyclobutane (methyl cyclobutyl ketone)

$\begin{array}{rccc}\mathrm{CH}_{3} \mathrm{CO}-\square(1) & \longrightarrow \mathrm{C}_{2} \mathrm{H}_{4}(\mathrm{II})+\mathrm{CH}_{3} \mathrm{CO}-\mathrm{CH}=\mathrm{CH}_{2}(\mathrm{III}) \\ \Delta H_{f(298)}^{\circ} & \mathrm{I} & \mathrm{II} & \text { III } \\ S_{(298)}^{\circ} & -30.9 & 12.5 & -28.6 \\ C_{p(298)}^{\circ} & 89.3 & 52.5 & 76.0 \\ C_{p(600)}^{\circ} & 28.7 & 10.4 & 21.5\end{array}$

$\Delta S^{\circ}=39.2 \mathrm{~g} / \mathrm{mole}$

$\Delta H^{\circ}=14.8 \mathrm{kcal} / \mathrm{mole}$

$\Delta C_{p}^{\circ}=3.2 \mathrm{~g} / \mathrm{mole}$

\begin{tabular}{|c|c|c|c|c|c|c|}
\hline $\log A$ & $\boldsymbol{E}$ & $\begin{array}{c}\log \boldsymbol{k}_{T} \\
(658)\end{array}$ & Conditions & System & Surface & References \\
\hline 14.53 & 54.5 & -3.56 & $\begin{array}{l}658 \pm 25^{\circ} \mathrm{K} \\
10-65 \text { torr } \\
\quad(5 \mathrm{C})\end{array}$ & static & none & $\begin{array}{l}\text { [1] L. G. Daignault and W } \\
\text { D. Walters, J. Am. } \\
\text { Chem. Soc. 80, } 541 \\
\text { (1958). }\end{array}$ \\
\hline
\end{tabular}

Preferred:

$\log k=14.53-54.5 / \theta$.

Comments: Biradical mechanism estimates: $\log k=14.4-54.4 / \theta$. Comparison of the rate constant parameters with those of isopropyl cyclobutane suggest a carbonyl resonance $\mathrm{kcal} / \mathrm{mole}$.

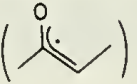

stabilization of about 7.9

\section{Experimental}

[1] Analysis by I.R., M.S., U.V. Rates by $\Delta$ P. Rates were unaffected by NO, toluene, and propylene. 
Reaction: Propionylcyclobutane (ethyl cyclobutyl ketone)

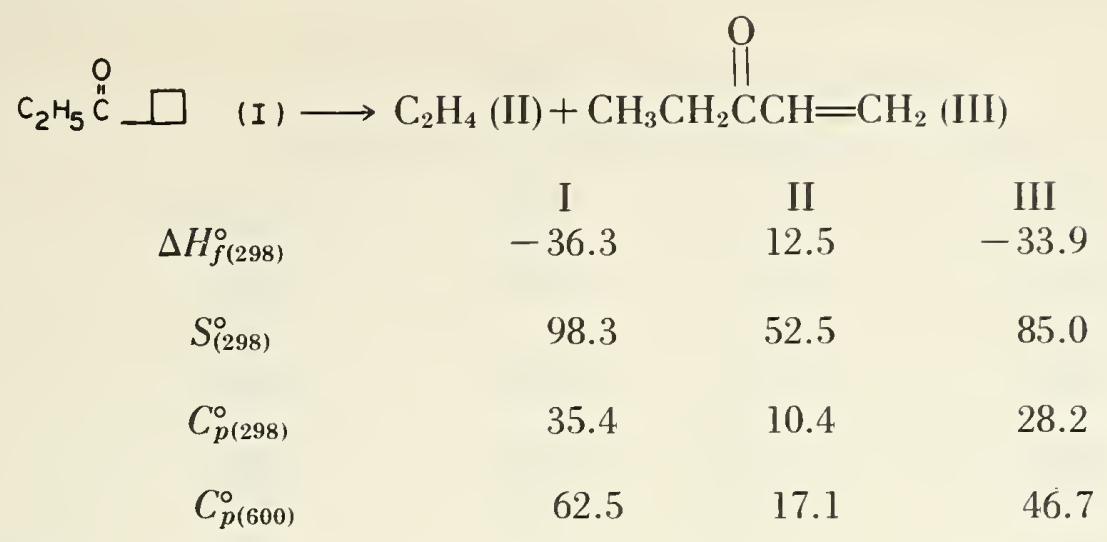

$\Delta S^{\circ}=39.2 \mathrm{~g} / \mathrm{mole}$

$\Delta H^{\circ}=14.9 \mathrm{kcal} / \mathrm{mole}$

$\Delta C_{p}^{\circ}=3.2 \mathrm{~g} / \mathrm{mole}$

\begin{tabular}{|c|c|c|c|c|c|c|}
\hline $\log A$ & $\boldsymbol{E}$ & $\begin{array}{c}\log \boldsymbol{k}_{T} \\
(663)\end{array}$ & Conditions & System & Surface & References \\
\hline 14.51 & 54.2 & -3.36 & $\begin{array}{l}663 \pm 20^{\circ} \mathrm{K} \\
5.48-17.47 \text { torr } \\
(5.4-7.4)\end{array}$ & static & none & $\begin{array}{l}\text { [1] B. C. Roquitte and W. D. } \\
\text { Walters, J. Phys. } \\
\text { Chem. 68, } 1606 \text { (1964). }\end{array}$ \\
\hline
\end{tabular}

\section{Preferred:}

Reliable as reported. $\log k=14.51-54.2 / \theta$.

Comments: The products are those expected on the basis of the biradical mechanism. The activation energy suggest a resonance energy for
estimates give $\log A_{\text {est }}=14.5$.

\section{Experimental}

[1] Analysis by U.V., I.R., G.L.C. Rates by $\Delta$ P. Rates unaffected by NO and propylene additions. 
Reaction: Methyl cyclobutanecarboxylate

\begin{tabular}{|c|c|c|c|}
\hline$\beth^{\prime \prime}{ }_{-}^{\prime \prime}, \mathrm{CH}_{3}(\mathrm{I})-$ & $\mathrm{C}_{2} \mathrm{H}_{4}(\mathrm{II})+\mathrm{CH}_{2}=$ & $\stackrel{\mathrm{O}}{\| \mathrm{C}}-\mathrm{O}$ & ${ }_{3}$ (III) \\
\hline$\Delta H_{f(298)}^{\circ}$ & $\begin{array}{c}\mathrm{I} \\
-74.2\end{array}$ & $\begin{array}{c}\text { II } \\
12.5\end{array}$ & $\begin{array}{c}\text { III } \\
-70.5\end{array}$ \\
\hline$S_{(298)}^{\circ}$ & 97.2 & 52.5 & $(84.4)$ \\
\hline$C_{p(298)}^{\circ}$ & 32.6 & 10.4 & 26.0 \\
\hline$C_{p(700)}^{\circ}$ & 64.4 & 18.8 & 44.8 \\
\hline
\end{tabular}

$\Delta S^{\circ}=39.7 \mathrm{~g} / \mathrm{mole}$

$\Delta H^{\circ}=16.2 \mathrm{kcal} / \mathrm{mole}$

$\Delta C_{p}^{\circ}=3.8 \mathrm{~g} / \mathrm{mole}$

\begin{tabular}{lllllll}
$\log \boldsymbol{A}$ & $\boldsymbol{E}$ & $\begin{array}{c}\log \boldsymbol{k}_{T} \\
(673)\end{array}$ & \multicolumn{1}{c}{ Conditions } & System & Surface & \multicolumn{1}{c}{ References } \\
14.84 & 57.3 & -3.77 & $\begin{array}{l}673 \pm 20^{\circ} \mathrm{K} \\
6-12 \text { torr } \\
(10)\end{array}$ & static & none & 11] M. Zupan and W. D. \\
Walters, J. Am. Chem. \\
\end{tabular}

Preferred:

Reliable. $\log k=14.84-57.3 / \theta$.

Comments: Transition state estimates for the biradical mechanism coupled with the observed activation energy suggest a carboxylic resonance stabilization of the free radical. (See also ethanoylcyclobutane.) ) of about $4.7 \mathrm{kcal} / \mathrm{mole}$.

\section{Experimental}

[1] Analysis by M.S., G.L.C. Rates by $\Delta$ P. Rates were unaffected by NO and propylene. 
Reaction: Cyclobutanone

\begin{tabular}{|c|c|c|c|}
\hline$\Delta H_{f(298)}^{\circ}$ & $\begin{array}{c}\text { I } \\
-20.5\end{array}$ & $\begin{array}{c}\text { II } \\
12.5\end{array}$ & $\begin{array}{c}\text { III } \\
-14.6\end{array}$ \\
\hline$S_{(298)}^{\circ}$ & 71.3 & 52.5 & 57.1 \\
\hline$C_{p(298)}^{\circ}$ & 20.0 & 10.4 & 11.4 \\
\hline$C_{p(600)}^{\circ}$ & 33.2 & 17.1 & $(17.2)$ \\
\hline
\end{tabular}

$\Delta S^{\circ}=38.3 \mathrm{~g} / \mathrm{mole}$

$\Delta H^{\circ}=18.4 \mathrm{kcal} / \mathrm{mole}$

$\Delta C_{p}^{\circ}=1.8 \mathrm{~g} / \mathrm{mole}$

\begin{tabular}{|c|c|c|c|c|c|c|}
\hline $\log A$ & $\boldsymbol{E}$ & $\begin{array}{c}\log \boldsymbol{k}_{T} \\
(626)\end{array}$ & Conditions & System & Surface & References \\
\hline 14.56 & 52.0 & -3.60 & $\begin{array}{c}626+20^{\circ} \mathrm{K} \\
10-88 \text { torr } \\
(15-30)\end{array}$ & static & $<10 \%$ & $\begin{array}{l}\text { [1] M. N. Das, F. Kern, } \\
\text { T. D. Coyle, and } \\
\text { W. D. Walters, J. Am. } \\
\text { Chem. Soc. 76, } 6271 \\
\text { (1954). }\end{array}$ \\
\hline
\end{tabular}

Preferred:

Reliable as reported. $\log k=14.56-52.0 / \theta$.

Comments: The biradical mechanism is consistent with the kinetics with ring opening being rate determining (see section III-5.2).

Estimated parameters are: $\log A_{\text {est }}=14.7 ; E_{\text {act }}=52.6 \mathrm{kcal} /$ mole. The activation energy was calculated using the resonance energy obtained in the ethanoylcyclobutane reaction and the normal $\mathrm{C}_{4}$ ring closing activation energy. Evidently the resonance enerwy of the biradical need not be lost in the decomposition step (see for comparison, methylene cyclobutane). The two paths, $\alpha(\mathrm{C}-\mathrm{C})$ fission and $\beta 1 \mathrm{C}-\mathrm{C}$ ) fission have about the same parameters.

\section{Experimental}

[1] Analysis by I.R., M.S., Chem. Rates by $\Delta$ P. Rates were unaffected by NO, toluene, and propylene. 
Reaction: Methylenecyclobutane

$\begin{array}{cccc} & \square(\mathrm{I}) & \mathrm{C}_{2} \mathrm{H}_{4}(\mathrm{II})+\mathrm{CH}_{2}=\mathrm{C}=\mathrm{CH}_{2} \text { (III) } \\ \Delta H_{f(298)}^{\circ} & \text { I } & \text { II } & \text { III } \\ S_{(298)}^{\circ} & 29.8 & 12.5 & 45.9 \\ C_{p(298)}^{\circ} & 72.5 & 52.5 & 58.3 \\ C_{p(700)}^{\circ} & 20.3 & 10.4 & 14.1 \\ & 43.2 & 18.8 & 23.8\end{array}$

$\Delta S^{\circ}=38.3 \mathrm{~g} /$ mole

$\Delta H^{\circ}=28.6 \mathrm{kcal} / \mathrm{mole}$

$\Delta C_{p}^{\circ}=4.2 \mathrm{~g} / \mathrm{mole}$

$\begin{array}{lllllll}\log A & \boldsymbol{E} & \begin{array}{c}\log \boldsymbol{k}_{T} \\ (723)\end{array} & \text { Conditions } & \text { System } & \text { Surface } & \\ 15.08 & 61.5 & -3.52 & \begin{array}{l}708 \pm 25^{\circ} \mathrm{K} \\ 2.3-10.4\end{array} & \text { static } & \end{array}$

[1] R. L. Brandaur. B. Short, and S. M. E. Kellner, J. Pliys. Chem. 65, 2269 (1961).

$\begin{array}{llll}15.68 & 63.3 & -3.46 & 723 \pm 20^{\circ} \mathrm{K} \quad \text { static }\end{array}$

[2] J. P. Chesick. J. Phys. $0.1-25$ torr Chem. 65, 2170 (1961).

(6)

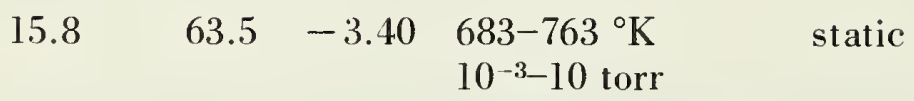

[3] P. J. Burkhardt, Ph. D. Diss., Univ. of Oregon (1962).

Preferred:

$\log k=15.8-63.5 / \theta$.

Comments: Comparison of these kinetics with those of dideuteromethylenecyclobutane (unpublished results of $\mathrm{W}$. von $\mathrm{E}$. Doering) provides a very forceful argument for biradical intermediates in small ring compound reactions. The latter reaction proceeds readily at about $600{ }^{\circ} \mathrm{K}$ with an activation enerery of $49.5 \mathrm{kcal} / \mathrm{mole}$. Thus ring closing from the biradical is very fast relative to decomposition (see below), and ring opening occurs with an allylic resonance assist (see also methylenecyclopropane). Apparently decomposition of methylenecyclobutane has an activation energy close to that of cyclobutane because in twisting of the biradical toward the allenic $\pi$ bond conformation, the allylic resonance is lost.

Mechanism:

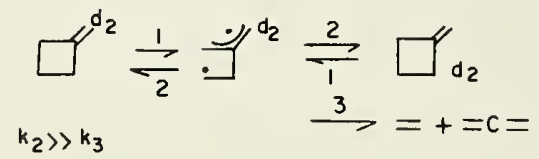

Transition state estimates give: $\log k=\log \left(\frac{k_{1} k_{3}}{k_{2}}\right)=15.7-61.9 / \theta$ (decomposition)

$\log k_{1}=14.5-48.3 / \theta$ (isomerization)

\section{Experimental}

[1] Analysis by G.L.C.., I.R., and M.S. Rates by $\Delta P$.

[2] Analysis and rates by G.L.C. Falloff observed near $6 \operatorname{torr}\left(732^{\circ} \mathrm{K}\right)$. Added nitrogen accelerated the rate in the low-pressure region.

[3] Analysis by M.S. and (.I..C. Falloff over the entire pressure range was observed and high-pressure rate constants were obtained from fits of the data to the Kassel integral. 


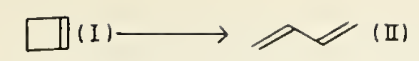

$\begin{array}{ccc}\Delta H_{f(298)}^{\circ} & \text { I } & \text { II } \\ S_{(298)}^{\circ} & 36.0 & 26.3 \\ C_{p(298)}^{\circ} & 63.0 & 66.6 \\ C_{p(400)}^{\circ} & 16.0 & 19.0 \\ & 21.6 & 24.3\end{array}$

$\Delta S^{\circ}=3.6 \mathrm{~g} / \mathrm{mole}$ $\Delta H^{\circ}=-9.7 \mathrm{kcal} / \mathrm{mole}$ $\Delta C_{p}^{\circ}=3.0 \mathrm{~g} / \mathrm{mole}$

\begin{tabular}{|c|c|c|c|c|c|c|}
\hline $\log A$ & $\boldsymbol{E}$ & $\begin{array}{c}\log _{(426)} k_{T} \\
(4)\end{array}$ & Conditions & System & Surface & References \\
\hline 13.08 & 32.5 & -3.60 & $\begin{array}{l}425 \pm 22^{\circ} \mathrm{K} \\
5-50 \text { torr } \\
(8-14)\end{array}$ & static & $<10 \%$ & $\begin{array}{l}\text { [1] W. Cooper and W. D. } \\
\text { Walters, J. Am. Chem. } \\
\text { Soc. } \mathbf{8 0 ,} 4220 \text { (1958). }\end{array}$ \\
\hline 13.18 & 32.7 & -3.60 & $\begin{array}{l}426 \pm 23{ }^{\circ} \mathrm{K} \\
0.01-23 \text { torr } \\
(0.055,0.22,5.4)\end{array}$ & static & $<10 \%$ & $\begin{array}{l}\text { [2] W. P. Hauser and W. D. } \\
\text { Walters, J. Phys. } \\
\text { Chem. 67, } 1328 \text { (1963). }\end{array}$ \\
\hline 13.40 & 32.9 & -3.48 & $\begin{array}{l}426 \pm 23^{\circ} \mathrm{K} \\
48-1521 \text { torr } \\
(100,1500)\end{array}$ & static & $<10 \%$ & $\begin{array}{l}\text { [3] R. W. Carr, Jr., and } \\
\text { W. D. Walters, J. Phys. } \\
\text { Chem. 69, } 1073 \text { (1965). }\end{array}$ \\
\hline
\end{tabular}

Preferred:

All reliable. $\log k=13.4-32.9 / \theta$.

Comments: A biradical mechanism would require a resonance energy in the transition state of about 26 hcal/mole which is greater than two allylic resonances. The reaction is therefore "concerted." For a review of cyclobutene isomerizations see R. Criegie, D. Seeback, R. E. Winter, B. Börretzen, and H. A. Brune. Chemische Berichte 98, 2339 (1965).

\section{Experimental}

[1] Analysis by I.R., M.S., U.V. Rate by U.V. No inhibition with NO and propylene. At 0.5 torr, rate constant was about $0.7 k_{x}$.

[2] Analysis and rate by U.V. Falloff ubserved near 10 torr at $423^{\circ} \mathrm{K}$. Collision efficiencies of He. $\mathrm{N}_{2} . \mathrm{H}_{2},\left(\mathrm{O}_{2}\right.$. $\mathrm{C}_{4}, \mathrm{C}_{2} \mathrm{H}_{2}, \mathrm{C}_{2} \mathrm{H}_{6}$. and $\mathrm{C}_{3} \mathrm{H}_{8}$ were determined.

[3] Rates by U.V. 
Reaction: 1-Methyl-1-cyclobutene

$\begin{array}{ccc} & \square(\mathrm{II}) & \\ & \mathrm{I} & \mathrm{II} \\ \Delta H_{f(298)}^{\circ} & 27.8 & 18.1 \\ S_{(298)}^{\circ} & 72.0 & 75.4 \\ C_{p(298)}^{\circ} & 22.2 & 25.0 \\ C_{p(500)}^{\circ} & 33.0 & 37.1\end{array}$

$\Delta S^{\circ}=3.4 \mathrm{~g} / \mathrm{mole}$

$\Delta H^{\circ}=-9.7 \mathrm{kcal} / \mathrm{mole}$

$\Delta C_{p}^{\circ}=2.8 \mathrm{~g} / \mathrm{mole}$

$\begin{array}{ccccccc}\log \boldsymbol{A} & \boldsymbol{E} & \begin{array}{c}\log \boldsymbol{k}_{T} \\ (446)\end{array} & \text { Conditions } & \text { System } & \text { Surface } & \text { References } \\ 13.79 & 35.1 & -3.42 & \begin{array}{l}446 \pm 25^{\circ} \mathrm{K} \\ 5-150 \text { torr }\end{array} & \text { static } & <10 \% & \text { [1] H. M. Frey, Trans. } \\ \text { Faraday Soc. } \mathbf{5 8 , 9}, 957 \\ \text { (1962). }\end{array}$

\section{Preferred:}

Reliable. (see cyclobutene) $\log k=13.79-35.1 / \theta$.

\section{Experimental}

[1] Analysis by G.L.C. Not inhibited by added NO and propylene. Rate constants were pressure independent. 
Reaction: 3-Methyl-1-cyclobutene

$\begin{array}{ccc} & \square(\mathrm{I}) \longrightarrow & \\ & \mathrm{I} & \mathrm{II} \\ \Delta H_{f(298)}^{\circ} & 28.2 & 18.6 \\ S_{(298)}^{\circ} & 70.5 & 76.4 \\ C_{p(298)}^{\circ} & 23.9 & 24.7 \\ C_{p(400)}^{\circ} & 30.7 & 31.2\end{array}$

$\Delta S^{\circ}=5.9 \mathrm{~g} / \mathrm{mole}$

$\Delta H^{\circ}=-9.6 \mathrm{kcal} / \mathrm{mole}$

$\Delta C_{p}^{\circ}=0.8 \mathrm{~g} / \mathrm{mole}$

$\begin{array}{ccccccc}\log \boldsymbol{A} & \boldsymbol{E} & \begin{array}{c}\log \boldsymbol{k}_{T} \\ (408)\end{array} & \text { Conditions } & \text { System } & \text { Surface } & \text { References } \\ 13.53 & 31.55 & -3.37 & \begin{array}{l}408 \pm 2{ }^{\circ} \mathrm{K} \\ 0.4 \rightarrow 25 \text { torr (7) }\end{array} & \text { static } & <10 \% & \text { [1] H. M. Frey, Trans. } \\ & & & & & \begin{array}{l}\text { Faraday Soc. 60, } 83 \\ \text { (1964). }\end{array}\end{array}$

Preferred:

Reliable. (see cyclobutene) $\log k=13.53-31.55 / \theta$.

Comments: Note that the intrinsic $A$-factors of all the "cyclobutene" reactions are the same (i.e., very close to normal with $\Delta S^{*}=0$ ).

\section{Experimental}

[1] Analysis by G.L.C. Rates unaffected by added propylene. Falloff observed near 25 torr and $k_{x}$ (reported) was obtained from a $\log k$ versus $\mathrm{P}^{-1 / 2}$ plot. Falloff curve fit by RRK $-s=15$. (See H. M. Frey and D. C. Marshall, Trans. Faraday Soc. 61, 1715 (1965). 
Reaction: 1-Ethyl-1-cyclobutene

$\begin{array}{ccc}\square(\mathrm{I}) \longrightarrow \mathrm{CH}_{2}=\mathrm{C}\left(\mathrm{C}_{2} \mathrm{H}_{5}\right) \mathrm{CH}=\mathrm{CH}_{2}(\mathrm{II}) \\ \Delta H_{f(298)}^{\circ} & \mathrm{I} & \mathrm{II} \\ S_{(298)}^{\circ} & 22.8 & 13.1 \\ C_{p(298)}^{\circ} & 81.8 & 85.2 \\ C_{p(400)}^{\circ} & 27.3 & 30.1 \\ & 35.9 & 38.7\end{array}$

$\Delta S^{\circ}=3.4 \mathrm{~g} / \mathrm{mole}$

$\Delta H^{\circ}=-9.7 \mathrm{kcal} / \mathrm{mole}$

$\Delta C_{p}^{\circ}=2.8 \mathrm{~g} / \mathrm{mole}$

$\begin{array}{lcclll}\log \boldsymbol{A} & \boldsymbol{E} & \begin{array}{c}\log \boldsymbol{k}_{T} \\ (446)\end{array} & \text { Conditions } & \text { System } & \text { Surface } \\ 13.76 & 34.83 & -3.34 & \begin{array}{l}446 \pm 22 \\ 1-20 \text { torr (2) }\end{array} & \text { static } & <10 \%\end{array}$

\section{References}

[1] H. M. Frey and R. F.

Skinner, Trans. Faraday Soc. 61, 1918 (1965).

Preferred:

Reliable. (see cyclobutene) $\log k=13.76-34.88 / \theta$.

\section{Experimental}

[1] Analysis by G.L.C. Rate constants were pressure independent. 
Reaction: 1,2-Dimethyl-1-cyclobutene

\begin{tabular}{ccc}
\hline (I $) \longrightarrow \mathrm{CH}_{2}=\mathrm{C}\left(\mathrm{CH}_{3}\right) \mathrm{C}\left(\mathrm{CH}_{33}\right)=\mathrm{CH}_{2}(\mathrm{II})$ \\
$\Delta H_{f(298)}^{\circ}$ & $\mathrm{I}$ & $\mathrm{II}$ \\
$S_{(294)}^{\circ}$ & 20.6 & 11.8 \\
$C_{p(298)}^{\circ}$ & 79.4 & 81.4 \\
$C_{p(500)}^{\circ}$ & 27.1 & 30.8 \\
& 38.7 & 41.0
\end{tabular}

$$
\begin{aligned}
& \Delta S^{\circ}=2.0 \mathrm{~g} / \mathrm{mole} \\
& \Delta H^{\circ}=-8.8 \mathrm{kcal} / \mathrm{mole} \\
& \Delta C_{p}^{\circ}=3.7 \mathrm{~g} / \mathrm{mole}
\end{aligned}
$$

\begin{tabular}{|c|c|c|c|c|c|c|}
\hline $\log A$ & $\boldsymbol{E}$ & $\begin{array}{c}\log \boldsymbol{k}_{T} \\
(446)\end{array}$ & Conditions & System & Surface & References \\
\hline 13.84 & 36.04 & -3.83 & $\begin{array}{l}446 \pm 240^{\circ} \mathrm{K} \\
1-100 \text { torr }(3)\end{array}$ & static & none & $\begin{array}{l}\text { [1] H. M. Frey, Trans. } \\
\text { Faraday Soc. 59, } \\
1619(1963)\end{array}$ \\
\hline
\end{tabular}

\section{Preferred:}

Reliable. (see cyclobutene) $\log k=13.84-36.04 / \theta$.

\section{Experimental}

[1] Analysis by G.L.C. No inhibition of the rates with added NO and propylene. 
Reaction: 1,3-Dimethyl-1-cyclobutene

$\begin{array}{ccc} & \square[(1) \longrightarrow & \\ & & \\ \Delta H_{f(298)}^{\circ} & \text { I II }) & \text { II } \\ S_{(298)}^{\circ} & 20.0 & 11.5 \\ C_{p(298)}^{\circ} & 78.1 & 86.1 \\ & 30.0 & 30.5\end{array}$

$\Delta S^{\circ}=8.0 \mathrm{~g} / \mathrm{mole}$

$\Delta H^{\circ}=-8.5 \mathrm{kcal} / \mathrm{mole}$

$\Delta C_{p}^{\prime}=0.5 \mathrm{~g} / \mathrm{mole}$

\begin{tabular}{|c|c|c|c|c|c|c|}
\hline $\log A$ & $\boldsymbol{E}$ & $\begin{array}{c}\log \boldsymbol{k}_{T} \\
(420)\end{array}$ & Conditions & System & Surface & References \\
\hline 13.65 & 33.0 & -3.52 & $\begin{array}{l}420 \pm 27^{\circ} \mathrm{K} \\
0.25-140 \text { torr }(3)\end{array}$ & static & none & $\begin{array}{l}\text { [1] H. M. Frey, D. C. } \\
\text { Marshall, and R. F. } \\
\text { Skinner. Trans. } \\
\text { Faraday Soc. 61, } \\
861 \text { (1965). }\end{array}$ \\
\hline
\end{tabular}

\section{Preferred:}

Reliable. (see cyclobutene) $\log k=13.65-33.0 / \theta$.

\section{Experimental}

[1] Analysis by G.L.C. The vapor pressure of 1,3-dimethyl-1-cyclobutene was found to be $\log p m m=7.856-1633 / T^{\circ} \mathrm{K}$ between -4.35 and $22.25^{\circ} \mathrm{C}$. 
Reaction: 2,3-Dimethyl-1-cyclobutene

\begin{tabular}{ccc} 
& \multicolumn{1}{c}{$\longrightarrow(\mathrm{I}) \longrightarrow$} & \\
$\Delta H_{f(298)}^{\circ}$ & $\mathrm{I}$ & $\mathrm{II}$ \\
$S_{(298)}^{\circ}$ & 20.0 & 12.5 \\
$C_{p(298)}^{\circ}$ & 78.0 & 87.3 \\
& 30.0 & 29.2
\end{tabular}

$\Delta S^{\circ}=9.3 \mathrm{~g} / \mathrm{mole}$

$\Delta H^{\circ}=-7.5 \mathrm{kcal} / \mathrm{mole}$

$\Delta C_{p}^{\circ}=-0.8 \mathrm{~g} / \mathrm{mole}$

\begin{tabular}{|c|c|c|c|c|c|c|}
\hline $\log A$ & $\boldsymbol{E}$ & $\underset{(433)}{\log \boldsymbol{k}_{T}}$ & Conditions & System & Surface & References \\
\hline 13.52 & 33.39 & -3.34 & $\begin{array}{l}433 \pm 21{ }^{\circ} \mathrm{K} \\
0.7-32.5 \text { torr } \\
\quad(3)\end{array}$ & static & none & $\begin{array}{l}\text { [1] H. M. Frey, D. C. } \\
\text { Marshall, and R. F. } \\
\text { Skinner, Trans. } \\
\text { Faraday Soc. 61, } \\
861 \text { (1965). }\end{array}$ \\
\hline
\end{tabular}

\section{Preferred:}

Reliable. (see cyclobutene). $\log l_{i}=13.52-33.39 / \theta$.

\section{Experimental}

[1] Rates and analysis by G.L.C. 
Reaction: trans-1,2,3,4-Tetramethyl-1-cyclobutene

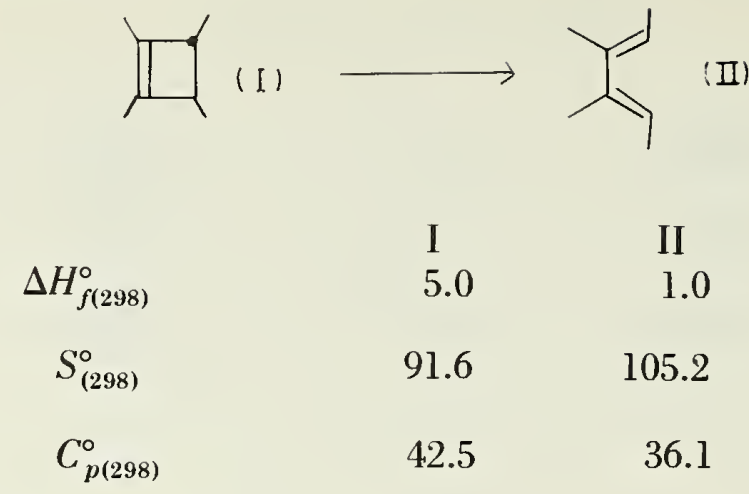

$\Delta S^{\circ}=13.6 \mathrm{~g} / \mathrm{mole}$

$\Delta H^{\circ}=-4.0 \mathrm{kcal} / \mathrm{mole}$

$\Delta C_{p}^{\circ}=-6.4 \mathrm{~g} / \mathrm{mole}$

\begin{tabular}{|c|c|c|c|c|c|c|}
\hline $\log A$ & $\boldsymbol{E}$ & $\begin{array}{c}\log \boldsymbol{k}_{T} \\
(419)\end{array}$ & Conditions & System & Surface & References \\
\hline 13.85 & 33.59 & -3.67 & $\begin{array}{l}398-440^{\circ} \mathrm{K} \\
1-76 \text { torr }\end{array}$ & static & & $\begin{array}{l}\text { [1] G. R. Branton, H. M. Frey, } \\
\text { and R. F. Skinner, } \\
\text { Trans. Faraday Soc. 62, } \\
1546 \text { (1966). }\end{array}$ \\
\hline 13.99 & 33.2 & -3.37 & $408-428^{\circ} \mathrm{K}$ & (see below) & & $\begin{array}{l}\text { [2] R. Criegie, D. Seebach, } \\
\text { R. E. Winter, } \\
\text { B. B̈̈rretzen, and } \\
\text { H. A. Brune, Chemische } \\
\text { Berichte 98, } 2339 \text { (1965). }\end{array}$ \\
\hline
\end{tabular}

Preferred:

$\log k=13.85-33.59 / \theta$.

Comments: Absence of the trans-cis-3,4-dimethyl-2,4-hexadiene indicates that the mechanism does not involve biradicals, but rather is a conrotary concerted process.

\section{Experimental}

[1] Rates were obtained by G.L.C. analysis on the cyclobutene remaining. The product was observed to polymerize rapidly presumably on the surface. Reaction in solution gave exclusively and quantitatively the indicated product.

[2] Reaction was studied in neat and in solution and the rate was followed by U.V. absorption of the diene. 
Reaction: cis-1,2,3,4-Tetramethyl-1-cyclobutene

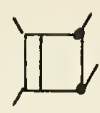

(I)

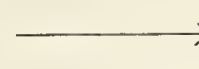<smiles>CC=C(C)/C(C)=C\C</smiles>

$$
\begin{gathered}
\Delta H_{f(298)}^{\circ} \\
S_{(298)}^{\circ} \\
C_{p(298)}^{\circ}
\end{gathered}
$$

$\Delta S^{\circ}=9.4 \mathrm{~g} / \mathrm{mole}$

$\Delta H^{\circ}=-6.7 \mathrm{kcal} / \mathrm{mole}$

$\Delta C_{p}^{\circ}=-5.2 \mathrm{~g} / \mathrm{mole}$

$\begin{array}{rcclll}\log \boldsymbol{A} & \boldsymbol{E} & \begin{array}{c}\log \boldsymbol{k}_{T} \\ (452)\end{array} & \text { Conditions } & \text { System } & \text { Surface } \\ 14.1 & 37.36 & -3.95 & \begin{array}{l}431-474{ }^{\circ} \mathrm{K} \\ 1-76 \text { torr }\end{array} & \text { static } & <5 \%\end{array}$

1.0

103.6

36.1 
Reaction: Perfluorocyclobutene

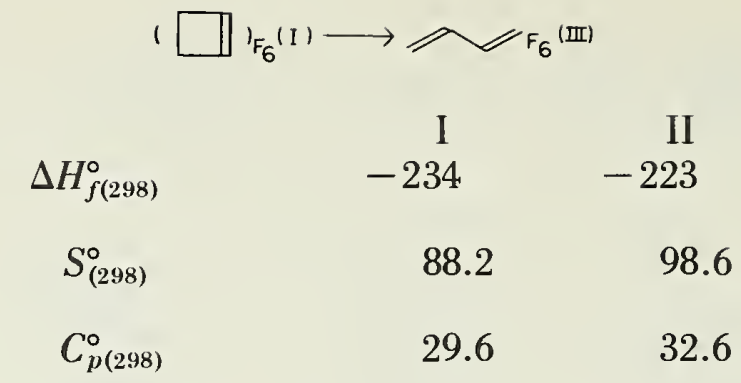

$\Delta S^{\circ}=10.4 \mathrm{~g} / \mathrm{mole}$

$\Delta H^{\circ}=11.0 \mathrm{kcal} / \mathrm{mole}$

$\Delta C_{p}^{\circ}=3.0 \mathrm{~g} / \mathrm{mole}$

\begin{tabular}{|c|c|c|c|c|c|c|}
\hline $\log A$ & $\boldsymbol{E}$ & $\begin{array}{c}\log \boldsymbol{k}_{T} \\
(503)\end{array}$ & Conditions & System & Surface & References \\
\hline 14.12 & 47.08 & -6.34 & $\begin{array}{l}503 \pm 36^{\circ} \mathrm{K} \\
1-18 \text { torr }\end{array}$ & static & $<10 \%$ & $\begin{array}{l}\text { [1] E. W. Schlag and W. B. } \\
\text { Peatman, J. Am. Chem. } \\
\text { Soc. 86, } 1676 \text { (1964). }\end{array}$ \\
\hline
\end{tabular}

Preferred:

Reliable. $\log k=14.12-47.08 / \theta$.

\section{Experimental}

[1] Analysis and rate by G.L.C. The reverse reaction was also studied: $K_{\mathrm{eq}}=10^{2.07} \times 10^{-11.7 / \theta}$. The equilibrium constant parameters $\left[\Delta S^{\circ}=9.5 \mathrm{~g} / \mathrm{mole}\right.$ and $\left.\Delta H^{\circ}=-11.7 \mathrm{kcal} / \mathrm{mole}\right]$ are in reasonably good agreement with the thermodynamic estimates. 
Reaction: Perfluorocyclobutane

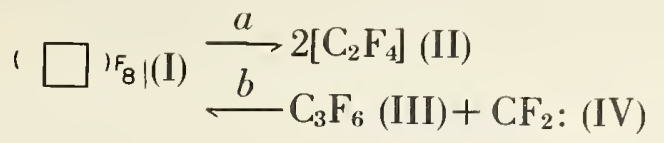

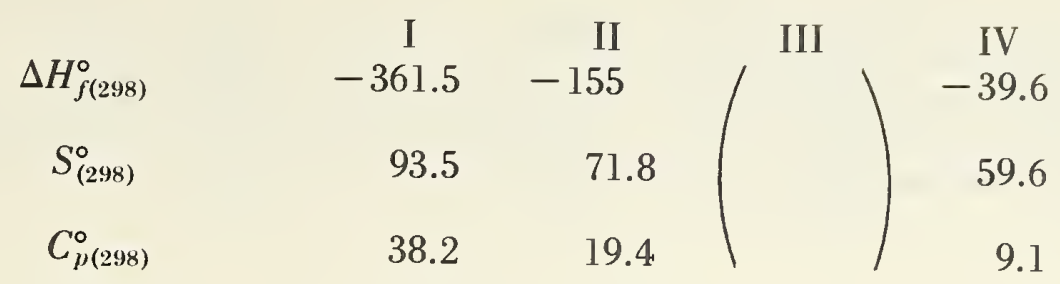

$$
\begin{aligned}
& \Delta S^{\circ}=50.1 \mathrm{~g} / \mathrm{mole} \\
& \Delta H^{\circ}=51.5 \mathrm{kcal} / \mathrm{mole} \\
& \Delta C_{p}^{\circ}=0.6 \mathrm{~g} / \mathrm{mole}
\end{aligned}
$$

\section{$\begin{array}{llllll}\text { Path } & \log \boldsymbol{A} & \log \boldsymbol{k}_{T} & \text { Conditions } & \text { System } & \text { Surface }\end{array}$}

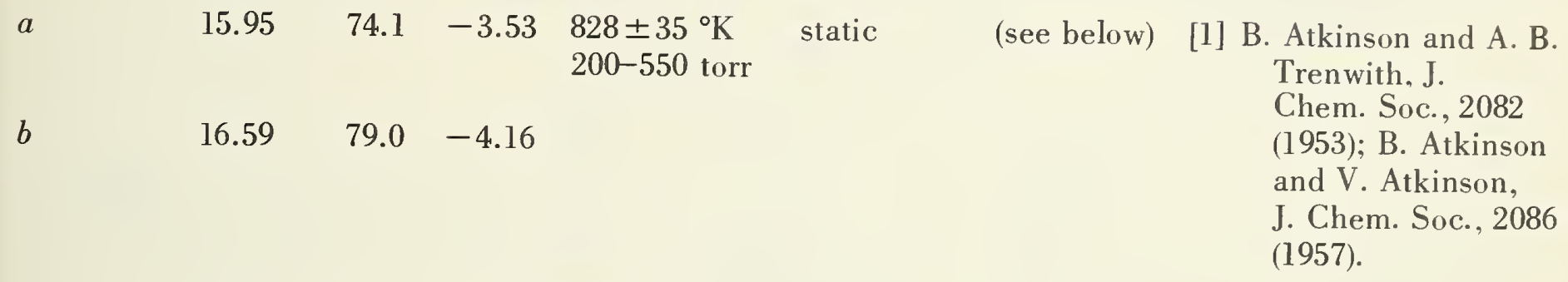
$a$
16.0
$74.3-3.53 \quad 683 \pm 50^{\circ} \mathrm{K} \quad$ static
$0.01-300$. torr
[2] J. N. Butler, J. Am. Chem. Soc. 84, $(10-100)$
$b$
17.2
$87.0-5.67$
$787 \pm 51^{\circ} \mathrm{K}$
static
20-600 torr
$16.32 \quad 74.3-3.21 \quad 1343 \pm 300^{\circ} \mathrm{K} \quad$ S.P. shock
$1 \%$ in 500 torr
of $\mathrm{Ar}$
[3] A. Lifshitz, H. F. Carroll, and S. H. Bauer, J. Chem. Phys. 39, 1661 (1963).

Preferred:

$\log k_{a}=16.0-74.3 / \theta$; process $b-$ suspect.

Comments: The observed equilibrium constant for reaction $(a)$ is in good agreement with the estimated thermodynamics. From $K_{\text {eq }}, \Delta S^{\circ}=49.5 \mathrm{~g} /$ mole. Reaction path $(b)$ is probably not a unimolecular first-order process. With the parameters reported in either [1] or [2], one would expect path $(b)$ to dominate at the temperatures of the S.P. shock study. This was not the case. In addition, the fluorine migration required is not very probable.

Mechanism, path $a$ :

$$
\square \frac{1}{2} \Pi_{0} \stackrel{3}{-} 2 \mathrm{C}_{2} \mathrm{~F}_{4} \quad E_{\mathrm{act}} \simeq \Delta H_{1,2}^{\circ}+E_{3} \simeq D H^{\circ}(\mathrm{C}-\mathrm{C})-E_{\mathrm{est}}+E_{3} \simeq 81.6-28+E_{3}
$$

$E_{3} \simeq 20.7 \mathrm{kcal} / \mathrm{mole}$. The high value of $E_{3}$ compared to that for cyclobutane $\left(E_{3} \simeq 5.8 \mathrm{kcal} / \mathrm{mole}\right)$ is probably 
a result of the lower exothermicity of step 3 for the fluorocarbon caused by fluorine destablization of the $(\mathrm{C} \pi-\mathrm{C} \pi)$ bond.

\section{Experimental}

[1] Analysis by I.R. Rates by $\Delta \mathrm{P}$. Surface effects apparent in pyrex cell, but well-behaved kinetics in steel cell. Kinetics of the reverse reaction also studied. $\left(\log k_{\mathrm{rec}}=8.01-25.4 / \theta\right.$, with $k$ in $1 /$ mole-sec.)

[2] Analysis and rates by G.L.C. Falloff (path $a$ ) observed near 10 torr with an RRK-s $\simeq 20$. At pressures below $0.05 \mathrm{~mm}$, the rate was pressure independent due to a slow heterogeneous reaction. Path $(b)$ rate unaffected by $\mathrm{O}_{2}$. Reaction $(a)$ catalyzed by $\mathrm{O}_{2}$. The equilibrium constant for path $(a)$ was found to be $K_{a}=10^{8.6-49.9 / \theta} \mathrm{moles} / \mathrm{l}$.

[3] Analysis by G.L.C.

Reaction: cis-1,2-Dichlorohexafluorocyclobutane

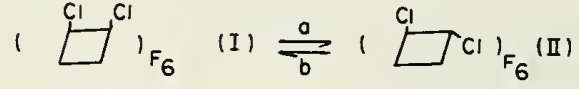

$$
\begin{aligned}
& \Delta H_{f(298)}^{\circ} \quad-265 \quad-265 \\
& \begin{array}{lll}
S_{(298)}^{\circ} & 101.3 & 99.9
\end{array} \\
& \begin{array}{lll}
C_{p(298)}^{\circ} & 40.8 & 40.8
\end{array}
\end{aligned}
$$

$\Delta S^{\circ}=-1.4 \mathrm{~g} / \mathrm{mole}$

\begin{tabular}{|c|c|c|c|c|c|c|c|}
\hline Path & $\log A$ & $\boldsymbol{E}$ & $\begin{array}{c}\log \boldsymbol{k}_{T} \\
(720)\end{array}$ & Conditions & System & Surface & References \\
\hline$a$ & 15.1 & 60.2 & -3.18 & $\begin{array}{l}698-742^{\circ} \mathrm{K} \\
60 \text { torr }\end{array}$ & static & none & $\begin{array}{l}\text { [1] B. Atkinson and M. } \\
\text { Stedman, J. Chem. } \\
\text { Soc., } 512 \text { (1962). }\end{array}$ \\
\hline & 14.88 & 60.2 & -3.40 & (as above) & & none & (as above) \\
\hline
\end{tabular}

$\Delta H^{\circ}=0 \mathrm{kcal} / \mathrm{mole}$

$\Delta C_{p}^{\circ}=0 \mathrm{~g} / \mathrm{mole}$

Preferred

Reliable. $\log k_{a}=15.1-60.2 / \theta$

$\log k_{b}=14.88-60.2 / \theta$.

\section{Experimental}

[1] Analysis by G.L.C. A slow formation of dichlorohexafluorobut-2-ene occurred at the higher temperatures. The equilibrium constant for the reaction was temperature-independent and given by $K_{a, b}=1.67$. 
Reaction: (cis or trans)-1,2-Dichlorohexafluorocyclobutane

\begin{tabular}{|c|c|c|}
\hline$\Delta \bar{H}_{f(298)}^{\circ}$ & $\frac{I}{-267.7}$ & $\underset{-113.5}{\text { II }}$ \\
\hline $\bar{S}_{(298)}^{\circ}$ & $104 \pm 2$ & 77.1 \\
\hline $\bar{C}_{p(298)}^{\circ}$ & 37.6 & 20.0 \\
\hline
\end{tabular}

$\Delta S^{\circ}=50.2 \mathrm{~g} / \mathrm{mole}$ $\Delta H^{\circ}=40.7 \mathrm{kcal} / \mathrm{mole}$ $\Delta C_{p}^{\circ}=2.4 \mathrm{~g} / \mathrm{mole}$

\begin{tabular}{|c|c|c|c|c|c|c|}
\hline $\log A$ & $\boldsymbol{E}$ & $\begin{array}{c}\log \boldsymbol{k}_{T} \\
(743)\end{array}$ & Conditions & System & Surface & References \\
\hline 15.4 & 65.3 & -3.82 & $\begin{array}{l}698-787^{\circ} \mathrm{K} \\
30-140 \text { torr }\end{array}$ & static & & $\begin{array}{l}\text { [1] B. Atkinson and M. Sted- } \\
\text { man, J. Chem. Soc., } \\
512(1962) .\end{array}$ \\
\hline
\end{tabular}

Preferred:

Reliable. $\log k=15.4-65.3 / \theta$.

\section{Experimental}

[1] Rates by $\Delta \mathrm{P}$. Analysis by G.L.C. The reverse reaction kinetics were also studied giving: $k_{b}=4.3 \times 10^{7} \times 10^{-26.6 / \theta}$ $1 /$ mole-(sec), from which $K_{a, b}=10^{\circ .76-38.7 / \theta}$ moles/l. The observed equilibrium data do not agree very well with the entropy estimates. (From $K_{e q}, \Delta S^{\circ} \simeq 45.7 \mathrm{~g} /$ mole.) 
Reaction: cis-Hexafluoro-1,2-bis-trifluoromethylcyclobutane

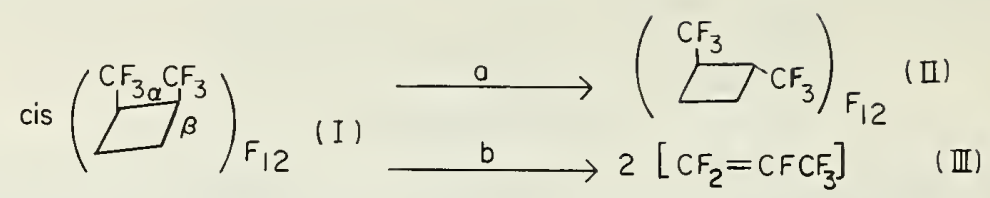

$$
\begin{gathered}
\Delta H_{f(298)}^{\circ} \\
S_{(298)}^{\circ} \\
C_{p(298)}^{\circ}
\end{gathered}
$$

$\Delta S^{\circ}=$

\begin{tabular}{|c|c|c|c|c|c|c|c|}
\hline Path & $\log A$ & $\boldsymbol{E}$ & $\log k_{T}$ & Conditions & System & Surface & References \\
\hline & 15.36 & 64.2 & -3.86 & $\begin{array}{l}683-773{ }^{\circ} \mathrm{K} \\
25-125 \text { torr }\end{array}$ & static & none & $\begin{array}{l}\text { [1] B. Atkinson and } \\
\text { P. B. Stockwell, } \\
\text { J. Chem. Soc. B, } \\
984 \text { (1966). }\end{array}$ \\
\hline
\end{tabular}

$\Delta H^{\circ}=$

$\Delta C_{p}=$

Preferred:

Reliable, as reported. $\log k_{a}=15.36-64.2 / \theta$

$\log k_{b}=15.08-64.2 / \theta$

Comments: Parameters are consistent with a biradical mechanism (see cyclobutane and perfluorocyclobutane) with $k_{2} \approx k_{3}$ and $E_{2} \simeq E_{3}$. Absence of $\beta(\mathrm{C}-\mathrm{C})$ split products is understandable since from the perfluorocyclobutane results and those above, one can guess that

$$
\begin{aligned}
& \text { (П.) } F_{F_{9}} \stackrel{3}{\rightarrow} 2(=)_{F_{4}} \quad E_{3} \simeq 20.7 \quad \mathrm{kcal} / \mathrm{mole} \\
& (\Omega)_{F_{12}} \stackrel{3^{\prime}}{\longrightarrow} 2\left({ }_{F_{6}} \quad E_{3} \simeq 10.6 \quad \mathrm{kcal} / \mathrm{mole}\right. \\
& \left(\prod\right)_{F_{12}} \stackrel{3^{\prime \prime}}{\rightarrow}\left(\text { or } \int\right)_{F_{8}}+(=)_{F_{4}} E_{3^{4}} \simeq 15.6 \quad \mathrm{kcal} / \mathrm{mole}
\end{aligned}
$$

This is consistent with a decrease in olefin stability with increasing fluorine atom substitution at the Pi bond centers.

\section{Experimental}

[1] Analysis by G.L.C. and by NMR (for isomers). No (S/V) ratio effect in aged pyrex or nickel vessels. Dissociation to $\mathrm{C}_{2} \mathrm{~F}_{4}$ and 2 -butenes was not observed. In the temperature range studied, $K_{\mathrm{eq}}\left(\frac{\text { trans }}{\text { cis }}\right) \simeq 0.28$. (See also trans isomer results.) 
Reaction: Trans-Hexafluoro-1,2-bis-trifluoromethylcyclobutane
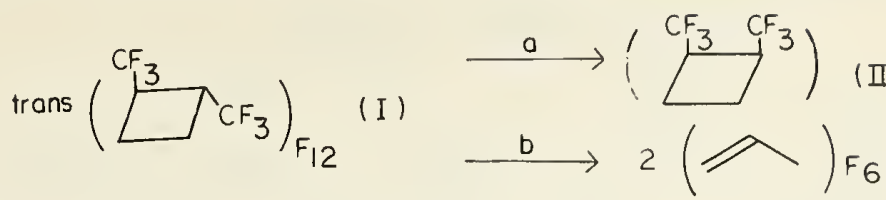

(II)

$\Delta S^{\circ}=$

$\Delta H^{\circ}=$

$\Delta C_{p}^{\circ}=$
Path

$a$

$\begin{array}{lll}15.77 \quad 64.2-3.45 \quad & 683-773^{\circ} \mathrm{K} \\ & 25-125 \text { torr }\end{array}$

static

none
Preferred:

$\log k_{a}=15.77-64.2 / \theta$

$\log k_{b}=15.63-64.2 / \theta$.
$b$
$\begin{array}{lll}15.63 & 64.2 & -3.59\end{array}$

System Surface

[1] B. Atkinson and P. B. Stockwell, J. Chem. Soc. B 984 (1966).
References

\section{Experimental}

[1] See cis-isomer. 
Reaction: Decafluoro-1,2-dimethylcyclobutene

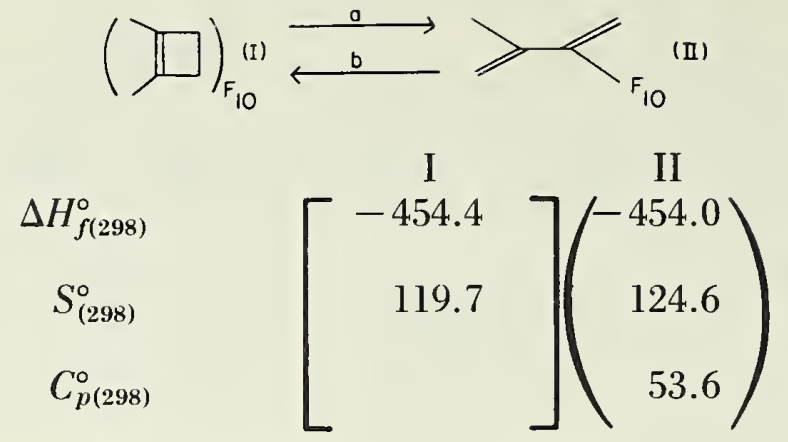

$\Delta S^{\circ}=4.9 \mathrm{~g} / \mathrm{mole}$

$\Delta H^{\circ}=0.40 \mathrm{kcal} / \mathrm{mole}$

$\Delta C_{p}^{\circ}=$

\begin{tabular}{|c|c|c|c|c|c|c|c|}
\hline Path & $\log A$ & $\boldsymbol{E}$ & $\begin{array}{c}\log \boldsymbol{k}_{T} \\
(595)\end{array}$ & Conditions & System & Surface & References \\
\hline & 13.64 & 46.0 & -3.26 & $\begin{array}{l}563-625^{\circ} \mathrm{K} \\
40-1500 \mathrm{~mm} \\
\mathrm{Hg}\end{array}$ & static & none & $\begin{array}{l}\text { [1] J. P. Chesick, } \\
\text { J. Am. Chem. Soc. } \\
\text { 88, } 4800 \text { (1966). }\end{array}$ \\
\hline & 12.57 & 45.6 & -4.18 & & & & \\
\hline
\end{tabular}

Preferred:

$\log k_{a}=13.64-46.0 / \theta$

$\log k_{b}=12.57-45.6 / \theta$.

Comments: Conclusions regarding the effect of fluorine atom on the stability of $\left(\mathrm{C}_{\pi}-\mathrm{C}_{\pi}\right)$ bonds also seem valid.

\section{Experimental}

[1] Analysis on product and reactant by G.L.C. The equilibrium constant was measured over the temperature range of $564-658{ }^{\circ} \mathrm{K}$ and found to be, $\log K_{a, b}=1.06-0.40 / \theta$. Also, from the method of opposing reaction rates, the data gave $\log \left(k_{a}+k_{b}\right)=13.62-45.8 \pm 0.6 / \theta$. In view of the greater exothermicity of this isomerization, as opposed to the perfluorocyclobutene isomerization (i.e., $\square, \Delta H_{1,2}^{\circ}=11.7 \mathrm{kcal} / \mathrm{mole}$ ), the data support the theory of Pi bond destabilization by fluorine atom substitution (E. W. Schlag and E. W. Kaiser, Jr., J. Am. Chem. Soc. 87, 1171 (1965)). 
Reaction: Cyclopentene

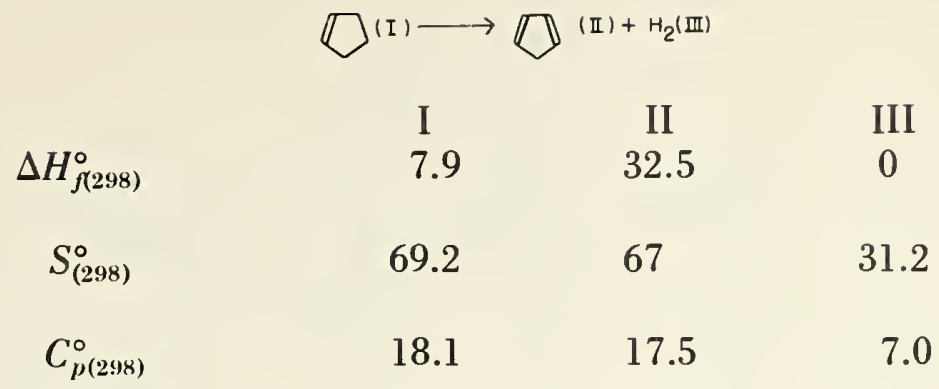

$\Delta S^{\circ}=29.0 \mathrm{~g} / \mathrm{mole}$

$\Delta H^{\circ}=24.6 \mathrm{kcal} / \mathrm{mole}$

$\Delta C_{p}^{\circ}=6.4 \mathrm{~g} / \mathrm{mole}$

\begin{tabular}{ccccccc}
$\log \boldsymbol{A}$ & $\boldsymbol{E}$ & $\begin{array}{c}\log \boldsymbol{k}_{T} \\
(790)\end{array}$ & Conditions & System & Surface & \multicolumn{1}{c}{ References } \\
13.04 & 58.8 & -3.23 & $\begin{array}{l}789 \pm 33^{\circ} \mathrm{K} \\
38-249 \mathrm{~mm} \mathrm{Hg}\end{array}$ & static & none & [1] D. W. Vanas and W. D. \\
& & & & & Walters, J. Am. Chem. \\
& & & & & Soc. 70, 4035 (1948)
\end{tabular}

Preferred:

$\log k=13.04-58.8 / \theta ; \log A_{\text {est }} \simeq 13.4$

Comments: The entropy of the transition state should be close to that of the product. The mechanism appears to be a $1,4 \mathrm{H}_{2}$ elimination.

\section{Experimental}

[1] Chemical and U.V. analysis. Rates by $\Delta P$. Stoichiometry confirmed in the initial stages of reaction. No inhibition by added propylene. 
Reaction: 1,5,5-Trimethyl-1,3-cyclopentadiene

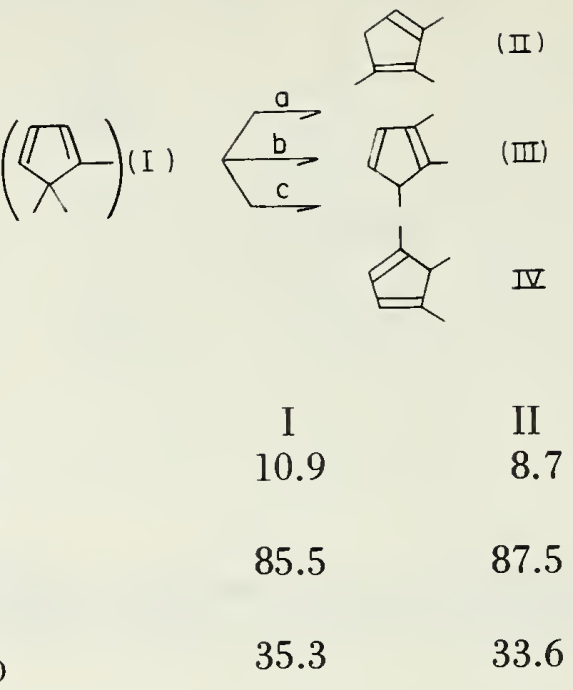

$\Delta S^{\circ}=2.0 \mathrm{~g} / \mathrm{mole}$

$\Delta H^{\circ}=-2.2 \mathrm{kcal} / \mathrm{mole}$

$\Delta C_{p}^{\circ}=-1.7 \mathrm{~g} / \mathrm{mole}$

$\begin{array}{cccccll}\text { Path } & \log \boldsymbol{A} & \boldsymbol{E} & \begin{array}{c}\log k_{T} \\ (650)\end{array} & \text { Conditions } & \text { System } & \text { Surface } \\ (a+b+c) & 12.7 & 41.6 & -1.29 & \begin{array}{l}623-673^{\circ} \mathrm{K} \\ \text { not reported }\end{array} & \begin{array}{l}\text { flow, } \\ t_{c} \simeq 5-50 \\ \text { sec }\end{array} & \begin{array}{l}\text { not } \\ \text { studied }\end{array}\end{array}$

References

Preferred: Probably not reliable as unimolecular process! (see note).
[1] J. W. DeHaan and
H. Kloosterziel, Rec. des Trav. Chim. des Pays-Bas 84, 1594 (1965)

Comments: This appears to be one of the very few examples (see MeNC pyrolysis) of a $\mathrm{CH}_{3}$ migration which could involve pentavalent carbon. For this reason the interpretation must remain tentative. The test for radicals with $d t \mathrm{BP}$ was not reliable since its half-life at these temperatures would be a few milliseconds. A possible radical path is:

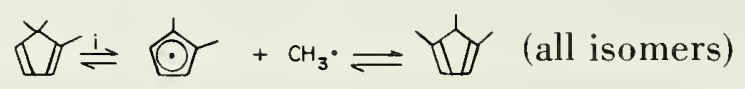

The initiation step $i$ has $E_{\text {act }}$ between $50-56$ kcal. The chain is:

$$
\begin{aligned}
& \mathrm{CH}_{3}+\llbracket \frac{1}{-1} y_{\text {or }} \ll \\
& \text { y) } \frac{2}{-2} \forall+\mathrm{CH}_{3}
\end{aligned}
$$

The other isomers could also be formed from 1.5 concerted $\mathrm{H}$-shifts in the 1,2,3 product. Also. mitigating against a $\mathrm{CH}_{3}$ shift is the absence of isomers, such as

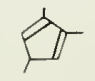

If termination is by $\mathrm{Me}+$ allyl, this leads to a first order rate constant $\sim 10^{14-47 / \theta} \mathrm{sec}^{-1}$ in good agreement with the reported rate constant. Alternatively, $\mathrm{Me}-$ Me termination yields a $3 / 2$ order rate law $\sim 10^{11-30 / \theta}$ $1^{1 / 2} /$ mole $^{1 / 2}$-sec. At 10 torr of cyclopentadiene, this also gives rates very close to the reported values.

\section{Experimental}

[1] Analysis on products by M.S., U.V., IR, and N.M.R. Products were also hydrogenated and compared to known samples of trimethylcyclopentanes. A 1,5-intramolecular shift of the methyl group followed by rapid equilibration of product pentadienes by intramolecular $\mathrm{H}$-shifts was postulated. A free radical catalyzed reaction path was excluded since the rate was not accelerated by the addition of di-t-butyl peroxide. 
Reaction: 2,5,5-Trimethyl-1,3-cyclopentadiene

$\begin{array}{ccc} & & \\ & & \text { II } \\ \Delta H_{f(298)}^{\circ} & 10.3 & 8.7 \\ S_{(298)}^{\circ} & 85.5 & 87.5 \\ C_{p(298)}^{\circ} & 35.3 & 34.6\end{array}$

$\Delta S^{\circ}=2.0 \mathrm{~g} / \mathrm{mole}$

$\Delta H^{\circ}=-1.6 \mathrm{kcal} / \mathrm{mole}$

$\Delta C_{p}^{\circ}=-0.7 \mathrm{~g} / \mathrm{mole}$

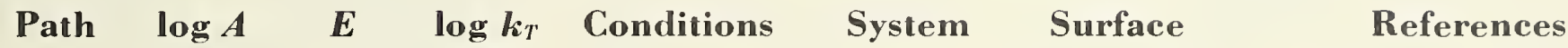

(650)

[1] J. W. DeHaan and

H. Kloosterzeil,

Rec. des Trav. Chim. des Pays-Bas 84, 1594 (1965).

$\begin{array}{llll}b & 14.22 & 45.7 & -1.15\end{array}$

Preferred:

Suspect. Probably chain.

Comments and Experimental:

(See 1,5,5-trimethylcyclopenta-1,3-diene). 
Reaction: 2,5-Dihydrofuran*

$\begin{array}{cccc} & P(\mathrm{I}) \longrightarrow & \\ \Delta H_{f(298)}^{\circ} & \mathrm{I} & \mathrm{II} & \mathrm{III} \\ S_{(298)}^{\circ} & -17.8 & -1.2 & 0 \\ C_{p(298)}^{\circ} & 67.7 & 66.1 & 31.2 \\ & 16.2 & 12.5 & 7.0\end{array}$

$\Delta S^{\circ}=29.6 \mathrm{~g} / \mathrm{mole}$

$\Delta \mathrm{H}^{\circ}=16.6 \mathrm{kcal} / \mathrm{mole}$

$\Delta C_{p}^{\circ}=3.3 \mathrm{~g} / \mathrm{mole}$

\begin{tabular}{|c|c|c|c|c|c|c|}
\hline $\log A$ & $\boldsymbol{E}$ & $\begin{array}{c}\log \boldsymbol{k}_{T} \\
(650)\end{array}$ & Conditions & System & Surface & References \\
\hline 12.72 & 48.5 & -3.59 & $\begin{array}{l}649 \pm 34 \\
5-46 \mathrm{~mm} \mathrm{Hg} \\
\quad(10)\end{array}$ & static & none & $\begin{array}{l}\text { [1] C. A. Wellington and } \\
\text { W. D. Walters, J. Am. } \\
\text { Chem. Soc. 83, } \\
4888 \text { (1961) }\end{array}$ \\
\hline
\end{tabular}

\section{Preferred:}

Reliable as reported. $\log k=12.72-48.5 / \theta$.

\section{Experimental}

[1] Analysis by M.S., G.L.C., I.R. Rates by $\Delta$ P. No effect on the rates with added NO, propylene or toluene. Rate constants were pressure independent.

*Note that 2,3-dihydrofuran does not eliminate $\mathrm{H}_{2}$ to go to furan, but rather decomposes to $\mathrm{CO}+\mathrm{C}_{3} \mathrm{H}_{6}$ via a complex biradical path. This is consistent with the Woodward Hoffman rule predictions. (See also 1,3 cyclohexadiene and 1,4-cyclohexadiene.) 


\section{Reaction: Pyrazoline}

$\begin{array}{ccccc} & \widehat{N}_{\mathrm{N}=\mathrm{N}} & \text { (I) } \underset{\mathrm{b}}{\stackrel{\mathrm{b}}{\longrightarrow}} \mathrm{N}_{2}+\mathrm{N}_{2}+\mathrm{C}_{3} \mathrm{H}_{6} \text { (II) } & \\ & & & & \\ & \mathrm{I} & \mathrm{II} & \mathrm{III} & \mathrm{IV} \\ \Delta H_{f(298)}^{\circ} & 56.3 & 0 & 12.7 & 4.8 \\ S_{(298)}^{\circ} & 68.7 & 45.8 & 56.8 & 63.6 \\ C_{p(2998)}^{\circ} & 17.7 & 7.0 & 13.3 & 15.3\end{array}$

a

$$
b
$$

$\Delta S^{\circ}=33.9 \quad 40.7 \mathrm{~g} / \mathrm{mole}$

$\Delta H^{\circ}=-43.6 \quad-51.5 \mathrm{kcal} / \mathrm{mole}$

$\Delta C_{p}^{\circ}=2.1 \quad 4.6 \mathrm{~g} / \mathrm{mole}$

$\begin{array}{rlrlll}\log \boldsymbol{A} & \boldsymbol{E} & \log \boldsymbol{k}_{T} & \text { Conditions } & \text { System } & \text { Surfac } \\ 15.9 & 42.4 & & 443-563^{\circ} \mathrm{K} & \text { static } & \text { none } \\ & & & 100-200 \text { torr } & & \end{array}$

\section{References}

[1] R. J. Crawford, R. J.

Dummel, and A.

Mishra, J. Am. Chem.

Soc. 87, 3024 (1965)

Preferred:

$\log k=15.9-42.4 / \theta$.

Comments: The kinetics are. consistent with a biradical mechanism. (See methyl substituted pyrazolines and section $\mathrm{V}-7.0$.)

$$
\begin{gathered}
\widehat{\mathrm{N}_{\mathrm{N}}} \frac{1}{2}\left(\widehat{\mathrm{N}_{\mathrm{N}}^{\circ}}\right) \frac{3}{\text { fost }} \mathrm{N}_{2}+\widehat{ } \\
E_{\mathrm{est}} \simeq D H^{\circ}\left(\mathrm{C}-\mathrm{N}_{\mathrm{A}}\right)-E_{\text {strain }}+E_{2}=(50.0-1.0)-6.0+1.0=44.0 \mathrm{kcal} / \mathrm{mole} .
\end{gathered}
$$

Transition state estimates of the $A$-factor give $\log A_{\text {est }}=16.0$.

\section{Experimental}

[1] Rates were followed by pressure change. First order kinetics observed to better than 95 percent conversion. 
Reaction: Methyl-substituted pyrazolines

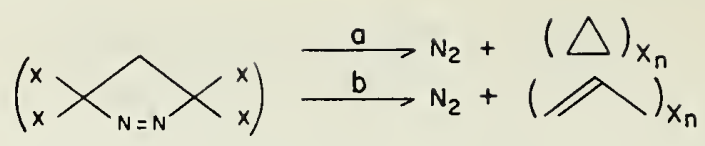

$\Delta S^{\circ}=$

$\Delta H^{\circ}=$

$\Delta C_{p}^{\circ}=$

Path $\log A^{*} \quad E^{*} \quad \log k_{T} \quad$ Conditions $\quad$ System Compounds

$$
\begin{aligned}
& 443-563^{\circ} \mathrm{K} \quad \text { static } \\
& 100-200 \mathrm{~mm} \mathrm{Hg}
\end{aligned}
$$

$a+b \quad 15.67$

15.83

15.83

15.53

15.66

15.42

14.48
41.0

42.2

40.0

40.3

40.2

38.9

37.7 $\log A_{\text {est }} \quad E_{\text {est }}$

41.6

44.1

37.8

41.6

41.6

37.8

$14.6 \quad 37.8$
References

[1] R. J. Crawford, R. J. Dummel, and A. Mishra, J. Am. Chem. Soc. 87, 3023 (1965).

\section{Preferred:}

Reliable: all values as reported.

Comments: The biradical mechanism (see estimates above) and the observed kinetic parameters tend to support the single-bond rupture hypothesis in alkyl azo compound decompositions (see section V-7.0).

\section{Experimental}

[1] Rates were obtained from $\Delta \mathrm{P}$ measurements. Change in surface from stainless steel to glass did not affect the rates. First-order plots were obtained to greater than 95 percent reaction.

*Calculated on the basis that the reported entropies and energies of activation were obtained from Arrhenius parameters such that $k=\left(\frac{e h T}{h} e^{\Delta s} \ddagger_{R}\right) \times 10^{-\mathrm{E} / \theta}$.

A study of the products in the thermal decomposition of 4-methyl diazirene gave isobutene $+\mathrm{N}_{2}$ only, which was taken as evidence for a biradical intermediate as opposed to a "hot" methylcyclopropane intermediate. The latter would give other olefin products prefer: entially (e.g., $\sim, \curvearrowright$, and $\rightleftharpoons+\mathrm{N}_{2}$ ). 


\section{Reaction: Cyclohexene}

\begin{tabular}{|c|c|c|c|}
\hline$\Delta H_{f(298)}^{\circ}$ & $\begin{array}{c}\mathrm{I} \\
-1.7\end{array}$ & $\begin{array}{c}\text { II } \\
26.3\end{array}$ & $\begin{array}{c}\text { III } \\
12.5\end{array}$ \\
\hline$S_{(298)}^{\circ}$ & 74.3 & 66.6 & 52.5 \\
\hline$C_{p(298)}^{\circ}$ & 25.1 & 19.0 & 10.4 \\
\hline
\end{tabular}

$\Delta S^{\circ}=44.8 \mathrm{~g} / \mathrm{mole}$

$\Delta H^{\circ}=40.5 \mathrm{kcal} / \mathrm{mole}$

$\Delta C_{p}^{\circ}=4.3 \mathrm{~g} / \mathrm{mole}$

\begin{tabular}{|c|c|c|c|c|c|c|}
\hline $\log A$ & $\boldsymbol{E}$ & $\underset{(860)}{\log \boldsymbol{k}_{T}}$ & Conditions & System & Surface & References \\
\hline 12.95 & 57.5 & -1.67 & $\begin{array}{l}798 \pm 40{ }^{\circ} \mathrm{K} \\
10-200 \text { torr }\end{array}$ & static & $<10 \%$ & $\begin{array}{l}\text { [1] L. Küchler, Trans. } \\
\text { Faraday Soc. 35, } 874 \\
\text { (1939). }\end{array}$ \\
\hline 12.13 & 55.1 & -1.88 & $\begin{array}{l}938-1018^{\circ} \mathrm{K} \\
7-70 \mathrm{~mm} \mathrm{Hg}\end{array}$ & flow & & $\begin{array}{l}\text { [2] M. Kraus, M. Vavruska } \\
\text { and V. Bazant, Collec- } \\
\text { tion Czech. Chem. } \\
\text { Commun. 22, 484 (1957) }\end{array}$ \\
\hline 17.15 & 72.7 & -1.33 & $753 \pm 55^{\circ} \mathrm{K}$ & static & $<10 \%$ & $\begin{array}{l}\text { [3] S. R. Smith and A. S. } \\
\text { Gordon, J. Phys. Chem. } \\
\text { 65, 1124. (1961). }\end{array}$ \\
\hline 15.18 & 66.2 & -1.65 & $\begin{array}{l}858 \pm 44^{\circ} \mathrm{K} \\
25 \mathrm{~mm} \mathrm{Hg} \\
t_{c}=0.57-5.4 \\
\quad \text { sec. }\end{array}$ & flow & none & $\begin{array}{l}\text { [4] M. Uchiyama, T. Tomioka } \\
\text { and A. Amano, J. Phys. } \\
\text { Chem. 68, } 1878 \text { (1964). }\end{array}$ \\
\hline 15.02 & 66.7 & -1.93 & $\begin{array}{l}1025 \pm 125^{\circ} \mathrm{K} \\
1 \% \text { in } \mathrm{Ar}\end{array}$ & S. P. Sh. & none & $\begin{array}{l}\text { [5] W. Tsang, J. Chem. Phys. } \\
\text { 4.2, 1805 (1961). }\end{array}$ \\
\hline
\end{tabular}

\section{Preferred:}

$\log k=15.18-66.2 / \theta .^{*}$

Comments: $k_{\text {back }}=10^{7.19-27.5 / \theta} 1 /$ mole-sec [D. Rowley and H. Steiner, Disc. Faraday Soc. 10, $\left.198(1951)\right]$. The reaction is probably concerted.

\section{Experimental}

[1] Rate by $\Delta \mathrm{P}$. No effect on the rate by added $\mathrm{NO}$ and $\mathrm{H}_{2}$.

[2] Rates were based on butadiene formation.

[3] Analysis by G.L.C. and M.S. Decomposition in $\mathrm{D}_{2}$ produced no D incorporation in the products.

[4] Analysis by G.L.C.

[5] Method of comparative rates with 2-chloropropane decomposition as the internal standard.

${ }^{*}$ Reaction is undoubtedly unimolecular and homogeneous. The large discrepancies in Arrhenius parameters apparently arise from large systematic errors since rate constants in the middle of the experimental temperature ranges are in good agreement. Only the latter two sets of results agree thermodynamically with the rate constant for the association reaction. 
Reaction: Cyclohexene

\begin{tabular}{|c|c|c|c|}
\hline$\Delta H_{f(298)}^{\circ}$ & $\begin{array}{c}\mathrm{I} \\
-1.7\end{array}$ & $\begin{array}{c}\text { II } \\
26.3\end{array}$ & $\begin{array}{c}\text { III } \\
0\end{array}$ \\
\hline$S_{(298)}^{\circ}$ & 74.2 & 72.1 & 31.2 \\
\hline$C_{p(298)}^{\circ}$ & 25.1 & 23.4 & 7.0 \\
\hline
\end{tabular}

$\Delta S^{\circ}=29.1 \mathrm{~g} / \mathrm{mole}$

$\Delta H^{\circ}=28.0 \mathrm{kcal} / \mathrm{mole}$

$\Delta C_{p}^{\circ}=5.3 \mathrm{~g} / \mathrm{mole}$

$\begin{array}{ccccccc}\log \boldsymbol{A} & \boldsymbol{E} & \begin{array}{c}\log \boldsymbol{k}_{T} \\ (755)\end{array} & \text { Conditions } & \text { System } & \text { Surface } & \text { References } \\ 16.28 & 71.2 & -4.33 & 753 \pm 55 & \text { static } & <10 \% & \text { [1] } \begin{array}{c}\text { S. R. Smith and A. S. } \\ \text { Gordon, J. Phys. Chem. }\end{array} \\ & & & & & & \mathbf{6 5}, 1124 \text { (1961) }\end{array}$

Preferred:

$\log k=13.5-62.0 / \theta$. Rate constants around $\bar{T}$ are probably reliable; however, see comments.

Comments: Absence of $\mathrm{D}$ in the products with added $\mathrm{D}_{2}$ does not exclude the possibility of a free radical mechanism for the above reaction since $\mathrm{H} \cdot+\mathrm{O} \longrightarrow+\mathrm{H}_{2}$ is very fast and $\mathrm{H}+\mathrm{D}_{2} \longrightarrow \mathrm{HD}+\mathrm{D}$ has a 9 $\mathrm{kcal} / \mathrm{mole}$ activation energy. If the reaction is molecular, the $A$-factor should be closer to normal, possibly with a slightly negative $\Delta S^{\ddagger}$.

\section{Experimental}

[1] Analysis by G.L.C. and M.S. No incorporation of $\mathrm{D}$ in products with added $\mathrm{D}_{2}$. 
Reaction: 1-Methyl-1,4-cyclohexadiene

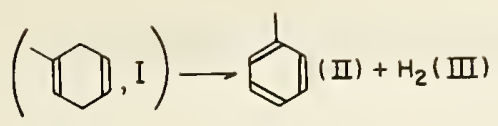

$\begin{array}{cccc}\Delta H_{f(298)}^{\circ} & \text { I } & \text { II } & \text { III } \\ S_{(298)}^{\circ} & 16.0 & 12.0 & 0 \\ C_{p(298)}^{\circ} & 81.8 & 76.4 & 31.2 \\ & 29.5 & 24.8 & 7.0\end{array}$

$\Delta S^{\circ}=25.8 \mathrm{~g} / \mathrm{mole}$

$\Delta H^{\circ}=-4.0 \mathrm{kcal} / \mathrm{mole}$

$\Delta C_{p}^{\circ}=2.3 \mathrm{~g} / \mathrm{mole}$

$\begin{array}{ccccccc}\log \boldsymbol{A} & \boldsymbol{E} & \begin{array}{c}\log \boldsymbol{k}_{T} \\ (610)\end{array} & \text { Conditions } & \text { System } & \text { Surface } & \text { References } \\ 12.69 & 44.69 & -3.32 & \begin{array}{l}587-635^{\circ} \mathrm{K} \\ 1-20 \text { torr }\end{array} & \text { static } & \begin{array}{c}\text { none in } \\ \text { aged re- } \\ \text { actor }\end{array} & \begin{array}{l}\text { [1] H. M. Frey and D. H. } \\ \text { Lister, J. Chem. Soc. } \\ \text { A, 509 (1967) }\end{array}\end{array}$

Preferred:

$\log k=12.69-44.69 / \theta$. (See also 1,4-cyclohexadiene.)

\section{Experimental}

[1] It was demonstrated that the reaction was molecular and did not involve free radical chains (as does the thermal decomposition of 1,3-cyclohexadiene) by pyrolyzing a mixture of the reactant and di-t-butyl peroxide (30 percent) at $619^{\circ} \mathrm{K}$. Rates of decomposition were the same as in the absence of the peroxide sensitizer. Molecular elimination is also consistent with the Woodward-Hoffmann rules. The peroxide test is not a useful one, since the peroxide half-life is only 3 milliseconds at $619^{\circ} \mathrm{K}$. 
Reaction: 4-Methylcyclohexene

$\begin{array}{cccc}\text { D (I) } \longrightarrow \mathrm{CH}_{2}=\mathrm{CHCH}=\mathrm{CH}_{2}(\mathrm{II})+\mathrm{CH}_{2}=\mathrm{CHCH}_{3}(\mathrm{III}) \\ \Delta H_{f(298)}^{\circ} & -8.8 & 26.3 & \text { III } \\ S_{(298)}^{\circ} & 81.0 & 66.6 & 4.8 \\ C_{p(298)}^{\circ} & 30.3 & 19.0 & 15.3\end{array}$

$\Delta S^{\circ}=49.2 \mathrm{~g} / \mathrm{mole}$

$\Delta H^{\circ}=39.9 \mathrm{kcal} / \mathrm{mole}$

$\Delta C_{p}^{\circ}=4.0 \mathrm{~g} / \mathrm{mole}$

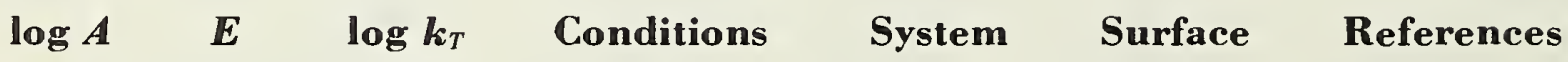

(1025)

$15.13 \quad 66.6+1.03 \quad 1025 \pm 125 \quad$ S.P.Sh.

[1] W. Tsang, J. Chem.

$\sim 1 \%$ in $\mathrm{Ar}$

Phys. 42, 1805 (1965)

\section{Preferred:}

Reliable as reported. $\log k=15.13-66.6 / \theta$.

Comments: The reaction is probably concerted.

\section{Experimental}

[1] Analysis by G.L.C. Comparative rate study with the decomposition of 2-chloropropane as the internal standard. Small amounts of methane and ethylene were also detected. 
Reaction: 4-Vinylcyclohexene

$\begin{array}{ccc}\mathrm{O}_{(\mathrm{I})} \longrightarrow 2\left[\mathrm{CH}_{2}=\mathrm{CHCH}=\mathrm{CH}_{2}\right](\mathrm{II}) \\ \Delta H_{f(298)}^{\circ} & \mathrm{I} & \mathrm{II} \\ S_{(298)}^{\circ} & 15.6 & 26.3 \\ C_{p(298)}^{\circ} & 88.6 & 66.6 \\ & 35.6 & 19.0\end{array}$

$\Delta S^{\circ}=44.6 \mathrm{~g} / \mathrm{mole}$

$\Delta H^{\circ}=37.0 \mathrm{kcal} / \mathrm{mole}$

$\Delta C_{p}^{\circ}=2.4 \mathrm{~g} / \mathrm{mole}$

\begin{tabular}{|c|c|c|c|c|c|c|}
\hline $\log A$ & $\boldsymbol{E}$ & $\begin{array}{l}\log k_{T} \\
(1025)\end{array}$ & Conditions & System & Surface & References \\
\hline 15.20 & 62.0 & +1.98 & $\begin{array}{l}1025 \pm 125 \\
\sim 1 \% \text { in Ar }\end{array}$ & S.P.Sh. & & $\begin{array}{l}\text { [1] W. Tsang, J. Chem. } \\
\text { Phys. 42, } 1805 \text { (1965) }\end{array}$ \\
\hline 8.37 & 36.0 & & $\begin{array}{l}783-978^{\circ} \mathrm{K} \\
760 \mathrm{~mm} \mathrm{Hg}\end{array}$ & flow & & $\begin{array}{l}\text { [2] T. F. Doumani, R. F. } \\
\text { Deering, and A. C. } \\
\text { McKinnis, Ind. Eng. } \\
\text { Chem. 39, } 89 \text { (1947) }\end{array}$ \\
\hline
\end{tabular}

Preferred:

$\log k=15.20-62.0 / \theta$.

Comments: The thermodynamics and the forward rate constant parameters, with an average $\left\langle\overline{\Delta C}_{p}^{\circ}\right\rangle \simeq 1.0$ $\mathrm{g} / \mathrm{mole}$, gives an estimate of the back reaction: $k_{\text {back }}=10^{7.5-25.4 / \theta} \mathrm{l} / \mathrm{mole}-\mathrm{sec}\left(800^{\circ} \mathrm{K}\right)$. The observed addition rate constants are: $k_{\text {back }}=10^{8.14-26.8 / \theta} \mathrm{l} / \mathrm{mole}-\mathrm{sec}$ and $k_{\mathrm{back}}=10^{6.95-23.69 / \theta} \mathrm{l} / \mathrm{mole}-\mathrm{sec}$ obtained by D. Rowley and H. Steiner, Disc. Faraday Soc. 10, 198 (1951) and G. B. Kistiakowsky and W. W. Ransom, J. Chem. Phys. 7, 725 (1939), respectively. The rate constant for association is predicted well by the thermodynamics, but no decision with regard to the parameters can be made since the estimated value is an intermediate one. The reaction is consistent with a biradical mechanism (see 1,5-cyclooctadiene).

\section{Experimental}

[1] Analysis by G.L.C. Comparative rate method with 2-chloropropane decomposition as standard.

[2] Rates were based on the "volume" of gases produced. No detailed analyses were made. 
Reaction: 1,4-Cyclohexadiene

\begin{tabular}{cccc}
\multicolumn{5}{c}{ D(I) $($ II $)+\mathrm{H}_{2}$ (III) } \\
$\Delta H_{f(298)}^{\circ}$ & I & II & III \\
$S_{(298)}^{\circ}$ & 26.3 & 19.8 & 0 \\
$C_{p(298)}^{\circ}$ & 72 & 64.3 & 31.2 \\
& 23.4 & 19.5 & 7.0
\end{tabular}

$\Delta S^{\circ}=23.5 \mathrm{~g} / \mathrm{mole}$

$\Delta H^{\circ}=-6.5 \mathrm{kcal} / \mathrm{mole}$

$\Delta C_{p}^{\circ}=3.1 \mathrm{~g} / \mathrm{mole}$

\begin{tabular}{|c|c|c|c|c|c|c|}
\hline $\log A$ & $\boldsymbol{E}$ & $\begin{array}{c}\log \boldsymbol{k}_{T} \\
(635)\end{array}$ & Conditions & System & Surface & References \\
\hline 12.02 & 42.69 & -2.68 & $576-616^{\circ} \mathrm{K}$ & static & none & $\begin{array}{l}\text { [1] R. J. Ellis and H. M. } \\
\text { Frey, J. Chem. Soc. A, } \\
553(1966) .\end{array}$ \\
\hline 12.36 & 43.8 & -2.72 & $603-663^{\circ} \mathrm{K}$ & static flow & $<15 \%$ & $\begin{array}{l}\text { [2] S. W. Benson and } \\
\text { R. Shaw, Trans. } \\
\text { Faraday Soc. 63, } 985 \\
\text { (1967). }\end{array}$ \\
\hline
\end{tabular}

Preferred:

Reliable. $\log k=12.36-43.8 / \theta$.

\section{Experimental}

[1] Rates were based on G.L.C. analysis. Propylene had no effect on the reaction rate. Cyclohexa-1.3-diene does not eliminate $\mathrm{H}_{2}$ molecularly, but rather decomposes via a free radical chain process. (See also $\mathrm{S}$. W. Benson and $\mathrm{R}$. Shaw, J. Am. Chem. Soc. 89, 5351 (1967).)

[2] Rates were based on G.L.C. analysis of $\mathrm{C}_{6}$ products and mass-spectrometric analysis of $\mathrm{H}_{2}$. No $\mathrm{H}, \mathrm{D}$ exchange was observed with an equal amount of added hexadeuterobenzene and no methane was produced with added toluene; therefore no $\mathrm{H}$-atoms are involved in the reaction. 
Reaction: 1,4-Dioxane

$\begin{array}{crrr}\left.C_{0}^{0}\right](\mathrm{I}) & \longrightarrow 2\left[\mathrm{CH}_{2} \mathrm{O}\right](\mathrm{II})+\mathrm{C}_{2} \mathrm{H}_{4}(\mathrm{III}) & \\ \Delta H_{f(298)}^{\circ} & \mathrm{I} & \mathrm{II} & \mathrm{III} \\ S_{(298)}^{\circ} & -76.0 & -27.7 & 12.5 \\ C_{p(298)}^{\circ} & 76.5 & 52.3 & 52.5 \\ & 19.4 & 8.5 & 10.4\end{array}$

$\Delta S^{\circ}=80.6 \mathrm{~g} / \mathrm{mole}$

$\Delta H^{\circ}=43.1 \mathrm{kcal} / \mathrm{mole}$

$\Delta C_{p}^{\circ}=8.0 \mathrm{~g} / \mathrm{mole}$

\begin{tabular}{|c|c|c|c|c|c|c|}
\hline \multirow[t]{2}{*}{$\log A$} & $\boldsymbol{E}$ & $\log \boldsymbol{k}_{T}$ & Conditions & System & Surface & References \\
\hline & 71 & & & & & $\begin{array}{l}\text { [1] L. Küchler and J. D. } \\
\text { Lambert, Z. Physik. } \\
\text { Chemie B42, } 359 \\
\text { (1939). }\end{array}$ \\
\hline
\end{tabular}

Preferred:

Suspect. A free radical chain process is likely.

\section{Experimental}

[1] Reaction rates were strongly inhibited by NO, but were not catalyzed by acetaldehyde decomposition. No para-ortho hydrogen conversion.

Products: $\mathrm{CH}_{2} \mathrm{O}+\mathrm{C}_{2} \mathrm{H}_{4}$. 
Reaction: 1,3,5-Trioxane

\begin{tabular}{|c|c|c|}
\hline$\Delta H_{f(298)}^{\circ}$ & $\begin{array}{c}\mathrm{I} \\
-120.8\end{array}$ & $\begin{array}{c}\text { II } \\
-27.7\end{array}$ \\
\hline$S_{(298)}^{\circ}$ & 73.8 & 52.5 \\
\hline$C_{p(298)}^{\circ}$ & 18.4 & 8.5 \\
\hline
\end{tabular}

$\Delta \mathrm{S}^{\circ}=83.7 \mathrm{~g} / \mathrm{mole}$

$\Delta H^{\circ}=37.7 \mathrm{kcal} / \mathrm{mole}$

$\Delta C_{p}^{\circ}=7.1 \mathrm{~g} / \mathrm{mole}$

\begin{tabular}{|c|c|c|c|c|c|c|}
\hline $\log A$ & $\boldsymbol{E}$ & $\begin{array}{c}\log \boldsymbol{k}_{T} \\
(585)\end{array}$ & Conditions & System & Surface & References \\
\hline 15.0 & 47.4 & -2.71 & $\begin{array}{l}583 \pm 38^{\circ} \mathrm{K} \\
0.1-100 \mathrm{~mm} \mathrm{Hg} \\
\quad(100)\end{array}$ & static & $<10 \%$ & $\begin{array}{l}\text { [1] W. Hogg, D. M. } \\
\text { McKinnon, and A. F. } \\
\text { Trotman-Dickenson, and } \\
\text { G. J. O. Verbeke, J. Chem. } \\
\text { Soc., } 1403 \text { (1961). }\end{array}$ \\
\hline 14.8 & 47.4 & -2.91 & $\begin{array}{l}582 \pm 37^{\circ} \mathrm{K} \\
0.1-600 \mathrm{~mm} \mathrm{Hg}\end{array}$ & static & none & $\begin{array}{l}\text { [2] R. LeG. Burnett, R. P. } \\
\text { Bell, Trans. Faraday } \\
\text { Soc. 34, } 420 \text { (1938). }\end{array}$ \\
\hline
\end{tabular}

Preferred:

$\log k=15.0-47.4 / \theta$.

Comments: The reaction must be concerted. The enthalpy of ring opening can be estimated at $\Delta H^{\circ} \approx 80$ $\mathrm{kcal} / \mathrm{mole}$.

\section{Experimental}

[1] Rates by $\Delta P$. Falloff observed over the entire pressure range. Arrhenius parameters also obtained at $0.1,1$ and $10 \mathrm{~mm} \mathrm{Hg}$. At $100 \mathrm{~mm} \mathrm{Hg}$ the reaction was close to its high-pressure limit. Falloff was too severe to be attributable to a normal unimolecular reaction pressure dependence.

[2] Rates by $\Delta \mathrm{P}$. Pressure dependent below $15 \mathrm{~mm} \mathrm{Hg}$. 
Reaction: 2,4,6-Trimethyl-1,3,5-trioxane (paraldehyde)

\begin{tabular}{|c|c|c|}
\hline$\Delta H_{f(298)}^{\circ}$ & $\begin{array}{c}\text { I } \\
-152\end{array}$ & $\begin{array}{c}\text { II } \\
-39.8\end{array}$ \\
\hline$S_{(298)}^{\circ}$ & 94.8 & 63.2 \\
\hline$C_{p(298)}^{\circ}$ & 34.9 & 13.1 \\
\hline
\end{tabular}

$\Delta S^{\circ}=94.8 \mathrm{~g} / \mathrm{mole}$

$\Delta \mathrm{H}^{\circ}=32.6 \mathrm{kcal} / \mathrm{mole}$

$\Delta C_{p}^{\circ}=4.4 \mathrm{~g} / \mathrm{mole}$

\begin{tabular}{cccllll}
$\log \boldsymbol{A}$ & $\boldsymbol{E}$ & $\begin{array}{c}\log \boldsymbol{k}_{T} \\
(515)\end{array}$ & Conditions & System & Surface & \multicolumn{1}{c}{ References } \\
15.1 & 44.16 & -3.64 & $\begin{array}{l}482-543^{\circ} \mathrm{K} \\
11.8-520 \mathrm{~mm} \mathrm{Hg}\end{array}$ & static & none & [1] C. C. Coffin, Can. J. \\
& & & & & Res. 7, 75 (1932).
\end{tabular}

Preferred:

Reliable. (see 1,3,5-trioxane) $\log k=15.1-44.16 / \theta$.

\section{Experimental}

[1] Rates by $\Delta \mathrm{P}$ measurements. In the packed reaction vessel, polymerization of the product aldehyde was observed. 
Reaction: 2,4,6-Tri- $n$-propyl-1,3,5-trioxane (para- $n$-butyraldehyde)

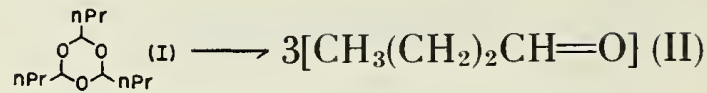

$$
\begin{aligned}
& \begin{array}{ccc} 
& \text { I } & \text { II } \\
\Delta H_{f(298)}^{\circ} & -179.6 & -49.8
\end{array} \\
& \begin{array}{lll}
S^{\circ}{ }_{(298)} & 151.2 & 82.0
\end{array} \\
& \begin{array}{lll}
C^{\circ}(298) & 67.9 & 24.1
\end{array}
\end{aligned}
$$

$\Delta S^{\circ}=94.8 \mathrm{~g} / \mathrm{mole}$

\begin{tabular}{|c|c|c|c|c|c|c|}
\hline $\log A$ & $\boldsymbol{E}$ & $\begin{array}{l}\log \boldsymbol{k}_{T} \\
(510)\end{array}$ & Conditions & System & Surface & References \\
\hline 14.35 & 42.0 & -3.65 & $\begin{array}{l}488-534{ }^{\circ} \mathrm{K} \\
13-550 \mathrm{~mm} \mathrm{Hg}\end{array}$ & static & & $\begin{array}{l}\text { [1] C. C. Coffin, Can. J. Res. } \\
\text { 9, } 603(1933)\end{array}$ \\
\hline
\end{tabular}

$\Delta H^{\circ}=30.2 \mathrm{kcal} / \mathrm{mole}$

$\Delta C_{p}^{\circ}=4.4 \mathrm{~g} / \mathrm{mole}$

Preferred:

Reliable. (see 1,3,5-trioxane) $\log k=14.35-42.0 / \theta^{\prime}$

\section{Experimental}

[1] Rates followed manometrically. $\Delta \mathrm{P}_{\infty}=2 P_{0}$; therefore, no side reactions occurred. 
Reaction: 2,4,6-Tri-isopropyl-1,3,5-trioxane (para-isobutyraldehyde)

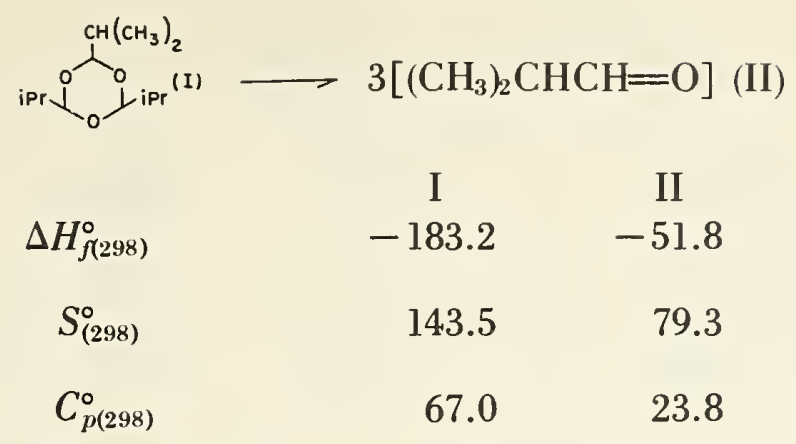

$\Delta S^{\circ}=94.4 \mathrm{~g} / \mathrm{mole}$ $\Delta H^{\circ}=27.8 \mathrm{kcal} / \mathrm{mole}$ $\Delta C_{p}^{\circ}=4.4 \mathrm{~g} / \mathrm{mole}$

\begin{tabular}{ccccccc}
$\log \boldsymbol{A}$ & $\boldsymbol{E}$ & $\begin{array}{c}\log \boldsymbol{k}_{T} \\
(510)\end{array}$ & \multicolumn{1}{c}{ Conditions } & System & Surface & \multicolumn{1}{c}{ References } \\
14.75 & 42.8 & -3.60 & $\begin{array}{l}488-534^{\circ} \mathrm{K} \\
13-550 \mathrm{~mm} \mathrm{Hg}\end{array}$ & static &
\end{tabular}

\section{Preferred:}

Reliable (see 1,3,5-trioxane).

$\log k=1 \dot{4} .75-42.8 / \theta$.

\section{Experimental}

[1] Rates followed manometrically. The reaction stoichiometry was accurately 1 mole to 3 moles; therefore, side reactions were not important. 
Reaction: Cycloheptatriene

$\begin{array}{ccc} & \text { II } & \\ \Delta H_{f(298)}^{\circ} & 44.1 & 12.0 \\ S_{(298)}^{\circ} & 75.4 & 76.4 \\ C_{p(298)}^{\circ} & 24.2 & 24.8\end{array}$

$\Delta S^{\circ}=\quad 1.0 \mathrm{~g} / \mathrm{mole}$

$\Delta H^{\circ}=-32.1 \mathrm{kcal} / \mathrm{mole}$

$\Delta C_{p}^{\circ}=0.6 \mathrm{~g} / \mathrm{mole}$

\begin{tabular}{clcccc}
$\log \boldsymbol{A}$ & $\boldsymbol{E}$ & $\log \boldsymbol{k}_{T}$ & \multicolumn{1}{c}{ Conditions } & System & Surface \\
& & & $658 \pm 23^{\circ} \mathrm{K}$ & static & $<10 \%$ \\
13.54 & 51.1 & & $0.9-19.7 \mathrm{~mm} \mathrm{Hg}$ & &
\end{tabular}

$\begin{array}{llll}13.90 & 52.2 & 661 \pm 44^{\circ} \mathrm{K} & \text { stirred } \\ \text { flow }\end{array}$

[2] W. C. Herndon and

L. L. Lowey, J. Am.

Chem. 86, 1922 (1964).

Preferred:

$\log k=13.90-52.2 / \theta$.

Comments: Results agree within experimental error and are reliable as reported. The conclusion regarding the biradical mechanism (see below) seems valid since the enthalpy of ring opening alone is estimated to be about $79 \mathrm{kcal} / \mathrm{mole}$. Norcaradiene is formed, which then converts to toluene via biradical.

\section{Experimental}

[1] Analysis by G.L.C. No effect by NO on the rate, or by a 20-fold increase in the pressure through added nitrogen. A transition state similar to norcaradiene, or $(0,1,4)$ bicyclohepta-2-4.diene, was suggested, and the biradical mechanism was dismissed on energy considerations.

[2] Analysis by G.L.C. 
Reaction: 1,5-Cyclooctadiene

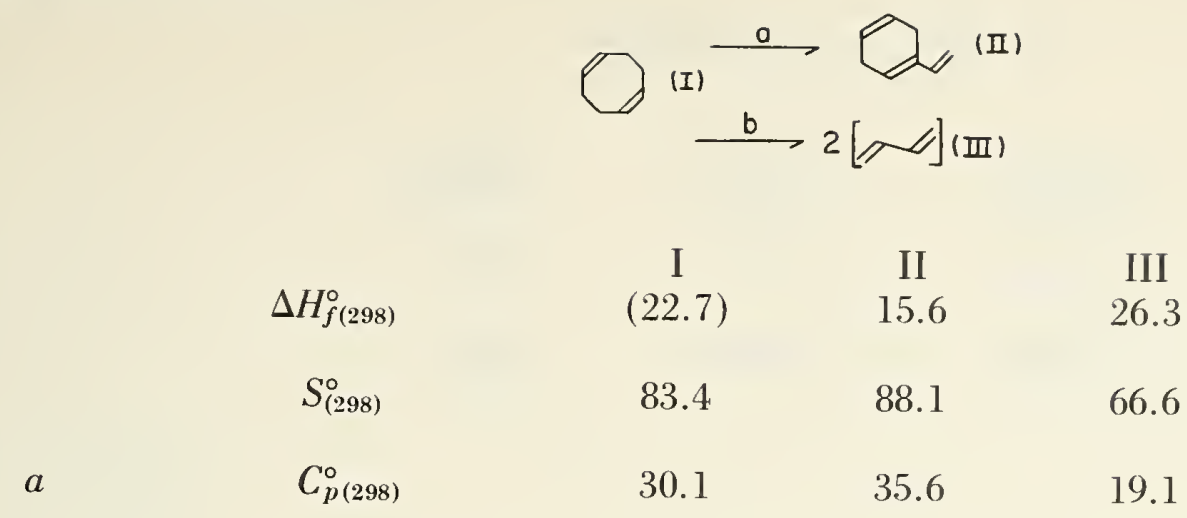

$\Delta S^{\circ}=4.7 \mathrm{~g} / \mathrm{mole}$

$\Delta H^{\circ}=-7.1 \mathrm{kcal} / \mathrm{mole}$

$\Delta C_{p}^{\circ}=5.5 \mathrm{~g} / \mathrm{mole}$

Path $\quad \log A \quad E \quad \log k_{T} \quad$ Conditions System Surface

$\begin{array}{llllll}14.46 & 49 & -3.85 & 586 \pm 14^{\circ} \mathrm{K} & \text { static } & <10 \%\end{array}$ $1.2 \mathrm{~mm} \mathrm{Hg}$

[1] R. Srinivasan and

A. A. Levi, J. Am. Chem. Soc. 86, 3756 (1964).

$b \quad * 15.7 \quad * 55 \quad-4.85$

Preferred:

Reliable. $\log k_{a}=14.46-49 / \theta ; \log k_{b}=15.7-55 / \theta$.

Comments: A biradical mechanism has been proposed for this reaction which unites the kinetics and thermodynamics of three reactions: the decomposition of 4-vinyl-cyclohexene, the decompositions of 1,2-divinylcyclobutanes, and the dimerization of butadiene. (See S. W. Benson, J. Chem. Phys. 46, 4920 (1967).)

\section{Experimental}

[1] Analysis by G.L.C. Surface effects appreciable at $T>327^{\circ}$ C. No effect on the rate by added propylene. 
Reaction: Spiropentane

\begin{tabular}{ccc} 
& \multicolumn{1}{c}{$\square($ I $)$} & \\
& I & II \\
$\Delta H_{f(298)}^{\circ}$ & 44.2 & 29.7 \\
$S_{(298)}^{\circ}$ & 67.5 & 72.5 \\
$C_{p(298)}^{\circ}$ & 21.1 & 20.3
\end{tabular}

$\Delta S^{\circ}=5.0 \mathrm{~g} / \mathrm{mole}$

$\Delta H^{\circ}=-14.5 \mathrm{kcal} / \mathrm{mole}$

$\Delta C_{p}^{\circ}=-0.8 \mathrm{~g} / \mathrm{mole}$

\begin{tabular}{|c|c|c|c|c|c|c|}
\hline $\log A$ & $\boldsymbol{E}$ & $\begin{array}{c}\log \boldsymbol{k}_{T} \\
(675)\end{array}$ & Conditions & System & Surface & References \\
\hline 15.86 & 57.57 & -2.78 & $\begin{array}{l}658 \pm 25^{\circ} \mathrm{K} \\
25-350 \mathrm{~mm} \mathrm{Hg}\end{array}$ & static & & $\begin{array}{l}\text { [1] M. C. Flowers and } \\
\text { H. M. Frey, J. Chem. } \\
\text { Soc., } 5550 \text { (1961). }\end{array}$ \\
\hline 15.2 & 55.5 & -2.77 & $\begin{array}{l}643-703{ }^{\circ} \mathrm{K} \\
10^{-3}-5 \mathrm{~mm} \mathrm{Hg}\end{array}$ & static & & $\begin{array}{l}\text { [2] P. J. Burkhardt and } \\
\text { D. F. Swinehart, Ph.D. } \\
\text { diss., University of } \\
\text { Oregon, } 1962 .\end{array}$ \\
\hline
\end{tabular}

Preferred:

$\log k=15.2-55.5 / \theta$.

Comments: The reaction is consistent with a biradical mechanism.

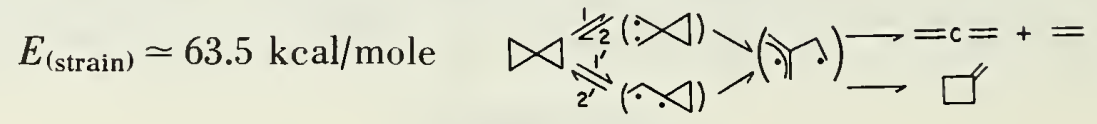

\section{Experimental}

[1] Analysis by G.L.C. Falloff observed near $13.5 \mathrm{~mm} \mathrm{Hg}$. A decomposition to allene t ethylene was also observed $\left(\sim 9\right.$ percent of reaction at $408{ }^{\circ} \mathrm{C}$ ) with an activation energy in excess of $58 \mathrm{kcal} / \mathrm{mole}$. Allene did not seem to be formed from the subsequent decomposition of methylenecyclobutane. Estimated error was $\pm 0.6 \mathrm{kcal} / \mathrm{mole}$.

[2] Analysis by G.L.C. and M.S. Falloff observed over the entire pressure range. High pressure rate constants were obtained from a fit of the data to the Kassel integral. 
Reaction: Bicyclo[ $[1,1,0]$ butane

$\begin{array}{ccc} & \text { II } & \\ & \text { I } & \text { II } \\ \Delta H_{f(298)}^{\circ} & (55.3) & 26.3 \\ S_{(298)}^{\circ} & 62.4 & 66.6 \\ C_{p(298)}^{\circ} & 16.0 & 19.1\end{array}$

$\Delta S^{\circ}=4.2 \mathrm{~g} / \mathrm{mole}$

$\Delta H^{\circ}=-29.0 \mathrm{kcal} / \mathrm{mole}$

$\Delta C_{p}^{\circ}=3.1 \mathrm{~g} / \mathrm{mole}$

\begin{tabular}{|c|c|c|c|c|c|c|}
\hline $\log A$ & $\boldsymbol{E}$ & $\begin{array}{c}\log \boldsymbol{k}_{T} \\
(490)\end{array}$ & Conditions & System & Surface & References \\
\hline 14.23 & 40.58 & -3.87 & $\begin{array}{l}496 \pm 28{ }^{\circ} \mathrm{K} \\
6-100 \text { torr }(20)\end{array}$ & static & $<10 \%$ & $\begin{array}{l}\text { [1] H. M. Frey and I. D. R } \\
\text { Stevens, Trans. } \\
\text { Faraday Soc. 61, } 90 \\
\text { (1965). }\end{array}$ \\
\hline 14.52 & 41.4 & -3.95 & $\begin{array}{l}489 \pm 13^{\circ} \mathrm{K} \\
6-14 \text { torr }\end{array}$ & static & $10-20 \%$ & $\begin{array}{l}\text { [2] R. Srinivasan, A. A. } \\
\text { Levi and I. Haller, } \\
\text { J. Phys. Chem. 69, } \\
1775 \text { (1965). }\end{array}$ \\
\hline
\end{tabular}

\section{Preferred:}

Reliable. $\log k=14.5-41.4 / \theta ; \log A_{\text {est }}=14.9$.

Comments: It has been shown that bicyclo[1,1,0]butane-2-єxo- $d_{1}$ does not isomerize to the $d_{1}$ endo form at reaction temperatures (K. B. Wiberg and J. M. Lavanish, J. Am. Chem. Soc. 88, 5272 (1966)). In terms of a biradical mechanism, this means that the rate-determining step must be ring opening.

A concerted mechanism has been proposed for this reaction because of its low activation energy and because of its similarity to the cyclobutene reaction which is known to be concerted.

However, the biradical mechanism cannot be ruled out on the basis of the energetics. For the mechanism,

$$
\begin{aligned}
E=\Delta H_{1,2}^{\circ}+E_{2} & =D H^{\circ}(\mathrm{C}-\mathrm{C})-\Delta E_{1,2} \text { strain }-\left(E_{\text {resonance }}\right)+E_{2} \\
= & 80.1-69.2+27.6-(4)+9.3=43.8 \mathrm{kcal} / \text { mole. }
\end{aligned}
$$

This estimate of $E$ (which is within experimental error) depends critically on a $4 \mathrm{kcal} /$ mole resonance energy in the $(D \cdot)$ radical. This value comes from the kinetics of the bicyclopropyl reaction.

If the reaction is concerted, then the reported $A$-factor will be the highest of its kind known. For this reason, we favor a biradical mechanism.

\section{Experimental}

[1] Analysis by G.L.C. Falloff observed near $20 \mathrm{~mm} \mathrm{Hg}$ (estimated to be within 5 percent of the high-pressure region).

[2] Analysis by G.L.C. 
Reaction: 1,3-Dimethylbicyclo[1,1,0]butane

\begin{tabular}{ccc} 
& \multicolumn{2}{c}{$\longrightarrow$ (I) (II) } \\
& I & II \\
$\Delta H_{(298)}^{\circ}$ & 39.3 & 11.0 \\
$S_{(298)}^{\circ}$ & 75.0 & 80.4 \\
$C_{p(298)}^{\circ}$ & 26.8 & 30.1
\end{tabular}

$\Delta S^{\circ}=\quad 5.4 \mathrm{~g} / \mathrm{mole}$

$\Delta H^{\circ}=-29.3 \mathrm{kcal} / \mathrm{mole}$

$\Delta C_{p}^{\circ}=\quad 3.3 \mathrm{~g} / \mathrm{mole}$

$\begin{array}{ccccccc}\log \boldsymbol{A} & \boldsymbol{E} & \begin{array}{c}\operatorname{kog} k_{T} \\ (550)\end{array} & \text { Conditions } & \text { System } & \text { Surface } & \text { References } \\ 14.45 & 43.3 & -2.76 & \begin{array}{l}547 \pm 24^{\circ} \mathrm{K} \\ 0.2-15 \\ (2-4)\end{array} & \text { static } & <10 \% & \text { [1] J. P. Chesick, J. Phys. } \\ \text { Chem. 68, 2033 (1964). }\end{array}$

Preferred:

$\log k=14.45-43.3 / \theta$.

Comments: The reported parameters are consistent with a biradical mechanism,

$$
\begin{aligned}
& E_{\text {est }}=\left.D H^{\circ} \mathrm{C}-\mathrm{C}\right)-\Delta E_{\text {strain }}-E_{\text {res }}+E_{2} \\
&= 77.3-(67.4-27.6)-(4)+9.3=42.8 \mathrm{kcal} / \mathrm{mole} \\
& \log A_{\text {est }}= 14.6(\text { see bicyclo }[1,1,0] \text { (butane) } \\
& \text { Experimental }
\end{aligned}
$$

[1] Analysis by G.L.C. Heterogeneity was significant at $T<531^{\circ} \mathrm{K}$. Rates were unaffected by high pressures of Side product production at the walls decreased with increasing temperature (i.e., from 5.4 percent $\left(250^{\circ} \mathrm{C}\right.$ ) to 1.3 percent $\left.\left(279^{\circ} \mathrm{C}\right)\right)$. 
Reaction: Bicyclo[1,1,1]pentane

$\begin{array}{ccc} & \bigotimes(\mathrm{I}) \rightarrow \mathrm{II}^{(\mathrm{II})} & \\ \Delta H_{(298)}^{\circ} & (34.3 \text { to } 44.3) & 25.4 \\ S_{(298)}^{\circ} & 67.1 & 79.7 \\ C_{p(298)}^{\circ} & & \\ \end{array}$

$\Delta S^{\circ}=12.6 \mathrm{~g} / \mathrm{mole}$

$\Delta H^{\circ}=$

$\Delta C_{p}^{\circ}$

$\begin{array}{llc}\log A & \boldsymbol{E} & \begin{array}{ll}\log \boldsymbol{k}_{T} \\ (565)\end{array}\end{array}$

Conditions

System Surface

References

15.24

$$
\begin{array}{ccc}
49.0 & -3.72 & 553-582{ }^{\circ} \mathrm{K} \\
& & 0.25-35 \text { torr } \\
& (5 \text { torr })
\end{array}
$$

static none

Preferred:

Reliable as reported.

$\log k=15.24-49.0 / \theta$.

Comments: Parameters at $P_{\infty}$ may be slightly higher. Although the $A$-factor is "high," suggesting a biradical mechanism, the activation energy is low and favors a concerted process. Thus, for the biradical mechansim,

$$
\begin{gathered}
\text { Mech } \Delta \underset{2}{\stackrel{\prime}{\rightleftharpoons}} \circlearrowright \stackrel{\text { fost }}{\rightleftharpoons}= \\
E_{\text {biradical }} \simeq D H^{\circ}(\mathrm{C}-\mathrm{C})-\Delta E_{\text {strain }}+E_{2} ; 49 \simeq 77-\left(E_{\text {st }}-26\right)+8
\end{gathered}
$$

This suggests $\varepsilon_{\mathrm{st}}(\bigotimes) \simeq 162 \mathrm{kcal} / \mathrm{mole}$, which seems a bit too high.

\section{Experimental}

[1] Analysis by G.L.C. No effect by change in (S/V) ratio. The decomposition was total pressure dependent and $k_{(2.5 \text { torr) }} \simeq 1 / 2 k_{(35 \text { torr })}$. 
Reaction: 1,3-Dimethylbicyclo[1,1,1] pentane

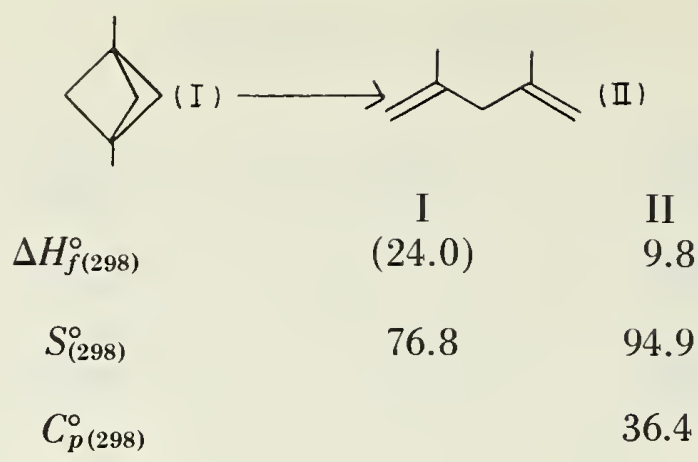

$\Delta S^{\circ}=18.1 \mathrm{~g} / \mathrm{mole}$

$\Delta H^{\circ}=(-14.2) \mathrm{kcal} / \mathrm{mole}$

$\Delta C_{p}^{\circ}$

\begin{tabular}{|c|c|c|c|c|c|c|}
\hline $\log A$ & $\boldsymbol{E}$ & $\begin{array}{c}\log \boldsymbol{k}_{T} \\
(580)\end{array}$ & Conditions & System & Surface & References \\
\hline 16.2 & 53.0 & -3.77 & $\begin{array}{l}568-594^{\circ} \mathrm{K} \\
3 \text { torr }\end{array}$ & static & none & $\begin{array}{l}\text { [1] R. Srinivasan, J. Am. } \\
\text { Chem. Soc. 90, } 2752 \\
\text { (1968). }\end{array}$ \\
\hline
\end{tabular}

Preferred:

Reliable, as reported. $\quad \log k=16.2-53.0 / \theta$.

Comments: The high $A$-factor suggests a biradical mechanism, and the observed activation energy is in fair agreement with thermodynamic estimates (see bicyclo[ $[1,1,1]$ for the mechanism).

$$
\begin{aligned}
E_{\mathrm{act}} & \simeq D H^{\circ}(\mathrm{C}-\mathrm{C})-\Delta E_{\text {strain }}+E_{2} \\
& \simeq 77.3-(58(?)-26)+8 \simeq 53.3 \mathrm{kcal} / \mathrm{mole} \\
A_{\mathrm{est}} & \simeq 10^{15.6} \mathrm{sec}^{-1} ; E_{\mathrm{st}} \sim 58 \mathrm{kcal} / \mathrm{mole} . \\
& \text { Experimental }
\end{aligned}
$$

[1] Analysis by G.L.C. No S/V dependence. 
Reaction: Bicyclo $[2,1,0]$ pent-2-ene

\begin{tabular}{ccc} 
& \multicolumn{1}{|l}{$\longrightarrow(\mathrm{I})$} & $\mathrm{II}$ \\
$\Delta H_{f(298)}^{\circ}$ & 67.6 & 32.5 \\
$S_{(298)}^{\circ}$ & 67.7 & 67.0 \\
$C_{p(298)}^{\circ}$ & $(18.6)$ & 17.5
\end{tabular}

$\Delta S^{\circ}=-0.7 \mathrm{~g} / \mathrm{mole}$

$\Delta H^{\circ}=-35.1 \mathrm{kcal} / \mathrm{mole}$

$\Delta C_{p}^{\circ}=-1.1 \mathrm{~g} / \mathrm{mole}$

\begin{tabular}{|c|c|c|c|c|c|c|}
\hline $\log A$ & $E$ & $\begin{array}{c}\log \boldsymbol{k}_{T} \\
(340)\end{array}$ & Conditions & System & Surface & References \\
\hline 14.2 & 26.9 & -2.83 & $\begin{array}{l}299-381{ }^{\circ} \mathrm{K} \\
0.2-760 \text { torr }\end{array}$ & & & $\begin{array}{l}\text { [1] D. M. Golden and } \\
\text { J. I. Brauman, J. Am. } \\
\text { Chem. Soc., 90, } 1920 \\
\text { (1968). }\end{array}$ \\
\hline
\end{tabular}

Preferred:

$\log k=14.2-26.9 / \theta$.

Comments: Transition state estimates based on the biradical mechanism give $\log A \simeq 14.2 ; \Delta H_{1.2}^{\circ}=26.3$

$-E_{\text {resonance. }}$

\section{Experimental}

[1] Analysis by G.L.C. Pressure falloff observed at temperatures above $346{ }^{\circ} \mathrm{K} . \mathrm{M}_{1 / 2}\left(382{ }^{\circ} \mathrm{K}\right) \sim 25$ torr. Highpressure rates obtained with added $\mathrm{Ar}$ or $\mathrm{C}_{3} \mathrm{H}_{8}$. An RRK- $\approx 10$ fit the data. 
Reaction: Bicyclo $[2,1,0$,$] pentane$

\begin{tabular}{|c|c|c|c|}
\hline$\Delta H_{f(298)}^{\circ}$ & $\begin{array}{c}\text { I } \\
36.0\end{array}$ & $\begin{array}{l}\text { II } \\
7.9\end{array}$ & $\begin{array}{r}\text { III } \\
25.2\end{array}$ \\
\hline$S_{(298)}^{\circ}$ & 68.5 & 69.2 & 79.7 \\
\hline$C_{p(298)}^{\circ}$ & 17.9 & $18.1^{\prime}$ & 25.1 \\
\hline
\end{tabular}

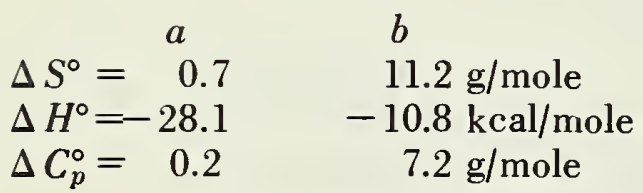

\begin{tabular}{|c|c|c|c|c|c|c|c|}
\hline Path & $\log A$ & $\boldsymbol{E}$ & $\log \boldsymbol{k}_{T}$ & Conditions & System & Surface & References \\
\hline$a$ & 14.58 & 46.6 & -4.64 & $\begin{array}{l}572 \pm 111^{\circ} \mathrm{K} \\
0.04-66.7\end{array}$ & static & $<10 \%$ & $\begin{array}{l}\text { [1] M. L. Halberstadt and } \\
\text { J. P. Chesick, J. Am } \\
\text { Chem. Soc. 84, 2688 } \\
\text { (1962) }\end{array}$ \\
\hline$a$ & 14.1 & 45.6 & -4.70 & $\begin{array}{l}531 \pm 33^{\circ} \mathrm{K} \\
400 \mathrm{~mm} \mathrm{Hg}\end{array}$ & static- & none & $\begin{array}{l}\text { [2] C. Steel, R. Zand, } \\
\text { P. Hurwitz, and }\end{array}$ \\
\hline$b$ & 14.4 & 52.3 & -6.39 & $\begin{array}{l}547 \pm 42^{\circ} \mathrm{K} \\
400 \text { torr }\end{array}$ & static & none & $\begin{array}{l}\text { Chem. Soc. 86, } 679 \\
\text { (1964) }\end{array}$ \\
\hline
\end{tabular}

Preferred:

$\log k_{a}=14.1-45.6 / \theta ; \log k_{b}=14.4-52.3 / \theta$.

Comments: The kinetics are in reasonable agreement with a biradical mechanism.

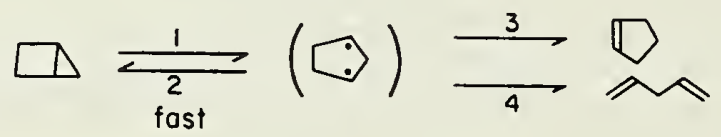

From the cis, trans isomerization of 2-methyl $[2,1,0]$ bicyclopentane, $k_{2}>\left(k_{3}+k_{4}\right)$.

$$
\begin{aligned}
& \Delta H_{1,2}^{\circ}=D H^{\circ}(\mathrm{C}-\mathrm{C})-\Delta E_{\text {strain }}=78.7-53.8+6.3=31.2 \mathrm{kcal} / \mathrm{mole} \\
& \log A_{a(\text { est })}=14.0 ; \log A_{b(\text { est })}=14.5
\end{aligned}
$$

The H-migration and ring opening activation energies obtained from the observed activation energies (i.e., $14.4 \mathrm{kcal} / \mathrm{mole}$ and $21.1 \mathrm{kcal} / \mathrm{mole}$, respectively) seem high, but not unreasonably so.

\section{Experimental}

[1] Analysis by G.L.C. Falloff observed near $3 \mathrm{~mm} \mathrm{Hg}$. An RRK-s $\approx 18 \pm 2$ fit the falloff behaviour. Added nitrogen increased the rates in the low-pressure region with a collision efficiency of 0.1 relative to the reactant.

[2] Analysis by G.L.C. Reaction performed in sealed ampoules with estimated pressures of about $400 \mathrm{~mm} \mathrm{Hg}$. The energetics of the reaction and others are discussed in detail with regard to the biradical intermediates. 
Reaction: cis 2-Methyl $[2,1,0]$ bicyclopentane trans-2-Methyl $[2,1,0]$ bicyclopentane

\begin{tabular}{|c|c|c|}
\hline & $\mathrm{I}($ cis $)$ & $\mathrm{II}($ trans $)$ \\
\hline$\Delta H_{f(298)}^{\circ}$ & 28.3 & 28.8 \\
\hline$S_{(298)}^{\circ}$ & 74.9 & 75.3 \\
\hline$C_{p(298)}^{\circ}$ & 22.7 & 24.0 \\
\hline
\end{tabular}

$\Delta S^{\circ}=0.4 \mathrm{~g} / \mathrm{mole}$

$\Delta H^{\circ}=0.5 \mathrm{kcal} / \mathrm{mole}$

$\Delta C_{p}=1.3 \mathrm{~g} / \mathrm{mole}$

\begin{tabular}{|c|c|c|c|c|c|c|c|}
\hline \multirow[t]{2}{*}{ Path } & $\log A$ & $\boldsymbol{E}$ & $\begin{array}{c}\log \boldsymbol{k}_{T} \\
(490)\end{array}$ & Conditions & System & Surface & References \\
\hline & 14.36 & 39.15 & -3.10 & $\begin{array}{l}491 \pm 15^{\circ} \mathrm{K} \\
0.08-1.7\end{array}$ & static & $<10 \%$ & $\begin{array}{l}\text { [1] J. P. Chesick, J. Am. } \\
\text { Chem. Soc. 84, } 3250 \\
\text { (1962). }\end{array}$ \\
\hline
\end{tabular}

$\begin{array}{llll}b & 13.90 \quad 38.65 & -3.34\end{array}$

Preferred:

$\log k_{a}=14.36-39.15 / \theta$

$\log k_{b}=13.90-38.65 / \theta$

Comments: The kinetics are consistent with a biradical mehcanism:

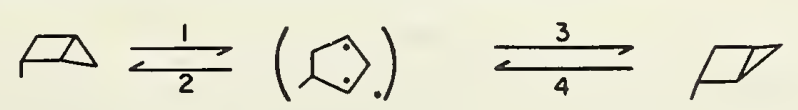

$\log A_{a(\mathrm{est})}=14.3 ; \log A_{b(\mathrm{est})}=14.0$.

$E_{\text {est }}=\Delta H_{1,2}+E_{2}=31.2+E_{2} ; E_{2} \simeq 8 \mathrm{kcal} / \mathrm{mole}$.

\section{Experimental}

[1] Analysis by I.R. and G.L.C. Rates from G.L.C. analysis. A clear distinction between the identity of the two isomers could not be made. Tentatively, the cis isomer was identified as the one of lower energy and entropy. 
Reaction: Bicyclo[2,2,0] hexane

$\begin{array}{ccc} & \text { II } & \text { II } \\ \Delta H_{(298)}^{\circ} & \text { I } & \text { II } \\ S_{(298)}^{\circ} & 29.6 & 19.8 \\ C_{p(298)}^{\circ} & 72.6 & 89.4 \\ & 24.6 & 28.8\end{array}$

$\Delta S^{\circ}=16.8 \mathrm{~g} / \mathrm{mole}$

$\Delta H^{\circ}=-9.8 \mathrm{kcal} / \mathrm{mole}$

$\Delta C_{p}^{\circ}=4.2 \mathrm{~g} / \mathrm{mole}$

\begin{tabular}{|c|c|c|c|c|c|c|}
\hline $\log A$ & $\boldsymbol{E}$ & $\begin{array}{r}\log \boldsymbol{k}_{T} \\
(445)\end{array}$ & Conditions & System & Surface & References \\
\hline 13.4 & 36.0 & -4.28 & $\begin{array}{l}443 \pm 40^{\circ} \mathrm{K} \\
400 \mathrm{~mm} \mathrm{Hg}\end{array}$ & static & & $\begin{array}{l}\text { [1] C. Steel, R. Zand, } \\
\text { P. Hurwitz, and S. G. } \\
\text { Cohen, J. Am. Chem. } \\
\text { Soc. 86, } 679 \text { (1964). }\end{array}$ \\
\hline
\end{tabular}

Preferred:

$\log k=13.4-36.0 / \theta$.

Comments: The kinetics are in agreement with a biradical mechanism. It seems quite likely that $k_{2} \simeq k_{3}$.

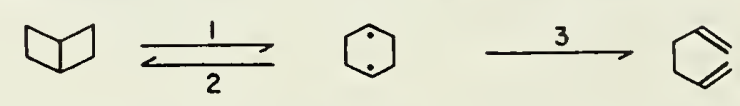

$\log \mathrm{A}_{\text {est }}=13.0 ; \Delta H_{1,2}^{\circ} \simeq 27 ; E_{\text {r.c. }} \simeq 9 \mathrm{kcal} /$ mole (see section III-6.0).

\section{Experimental}

[1] Analysis by G.L.C. Keaction performed in sealed ampoules at estimated pressures of about $400 \mathrm{~mm} \mathrm{Hg}$. 


\section{Reaction: Bicyclo[3,1,0]hex-2-ene}

\begin{tabular}{|c|c|c|c|}
\hline$\Delta H_{f(298)}^{\circ}$ & $\begin{array}{c}\text { I } \\
37.6\end{array}$ & $\begin{array}{c}\text { II } \\
26.0\end{array}$ & $\begin{array}{c}\text { III } \\
26.3\end{array}$ \\
\hline$S_{(298)}^{\circ}$ & 73.0 & 72.1 & 72 \\
\hline$C_{p(298)}^{\circ}$ & 19.9 & 23.4 & 23.4 \\
\hline
\end{tabular}

$$
\begin{aligned}
& \because \\
\Delta S^{\circ} & =-0.9 \mathrm{~g} / \mathrm{mole} \\
\Delta H^{\circ} & =-11.6 \mathrm{kcal} / \mathrm{mole} \\
\Delta C_{p}^{\circ} & =+3.5 \mathrm{~g} / \mathrm{mole}
\end{aligned}
$$

\begin{tabular}{|c|c|c|c|c|c|c|c|}
\hline Path & $\log A$ & $\boldsymbol{E}$ & $\begin{array}{c}\log \boldsymbol{k}_{T} \\
(605)\end{array}$ & Conditions & System & Surface & References \\
\hline$+b$ & 14.5 & 50.2 & -3.64 & $\begin{array}{l}586-620^{\circ} \mathrm{K} \\
3-30 \mathrm{~mm} \mathrm{Hg}\end{array}$ & static & none & $\begin{array}{l}\text { [1] R. J. Ellis and H. M. } \\
\text { Frey, J. Chem. } \\
\text { Soc. A, } 553 \text { (1966). }\end{array}$ \\
\hline & 14.28 & 50.2 & -3.86 & & & & \\
\hline & 14.10 & 50.2 & -4.04 & (as above) & & & (as above) \\
\hline
\end{tabular}

Preferred:

Reliable.

Comments: Reactions fit a biradical mechanism with H-migration as rate determining. Transition state estimates give, $A_{(a+b)}=10^{14.3} \mathrm{sec}^{-1}$ and $E \simeq 48.5 \mathrm{kcal} / \mathrm{mole}$ (see section III-6.0).

\section{Experimental}

[1] Rates were based on G.L.C. analysis of products. A subsequent decomposition of cyclohexa-1,4-diene to benzene and hydrogen was also observed (see cyclohexa-1.3-diene). The following added gases had no effect on the reaction rates: propylene ( 8 fold excess), nitric oxide, $t$-butyl peroxide. Product ratios were constant over the temperature range of study, ( ), ( $)=1.5$. 
Reaction: Bicyclo $[3,1,0]$ hexane

\begin{tabular}{|c|c|c|c|c|c|c|c|}
\hline & & \multicolumn{2}{|c|}{$\Delta H_{f(298)}^{\circ}$} & $\begin{array}{c}\mathrm{I} \\
10.3\end{array}$ & $\begin{array}{c}\text { II } \\
-1.7\end{array}$ & $\begin{array}{c}\text { III } \\
-0.3\end{array}$ & \\
\hline & & \multicolumn{2}{|c|}{$S_{(298)}^{\circ}$} & 73.0 & 74.3 & 78.0 & \\
\hline & & \multicolumn{2}{|c|}{$C_{p(298)}^{\circ}$} & 23.9 & 25.3 & 24.2 & \\
\hline $\begin{aligned} \Delta S^{\circ} & = \\
\Delta H^{\circ} & =-1 \\
\Delta C_{p}^{\circ} & =\end{aligned}$ & $\begin{array}{l}a \\
1.3 \\
2.0 \\
1.4\end{array}$ & \multicolumn{2}{|c|}{$\begin{array}{c}b \\
5.0 \mathrm{~g} / \mathrm{mole} \\
-10.6 \mathrm{kcal} / \mathrm{mole} \\
0.3 \mathrm{~g} / \mathrm{mole}\end{array}$} & \multirow[b]{2}{*}{ Conditions } & \multirow[b]{2}{*}{ System } & \multirow[b]{2}{*}{ Surface } & \multirow[b]{2}{*}{ References } \\
\hline Path & $\log A$ & $\boldsymbol{E}$ & $\begin{array}{c}\log \boldsymbol{k}_{T} \\
(730)\end{array}$ & & & & \\
\hline$a$ & 13.29 & 57.4 & -3.90 & $\begin{array}{l}728 \pm 37^{\circ} \mathrm{K} \\
5-70 \mathrm{~mm} \mathrm{Hg}\end{array}$ & static & $<10 \%$ & $\begin{array}{l}\text { [1] H. M. Frey and R. C. } \\
\text { Smith, Trans. } \\
\text { Faraday Soc. 58, } \\
697 \text { (1962). }\end{array}$ \\
\hline$b$ & 13.89 & 61.17 & -4.42 & (as above) & & & (as above). \\
\hline
\end{tabular}

Preferred:

$\log k_{a}=13.29-57.4 / \theta ; \log k_{b}=13.89-61.17 / \theta$.

Comments: The kinetics favor biradical mechanisms (see section III-6.0).

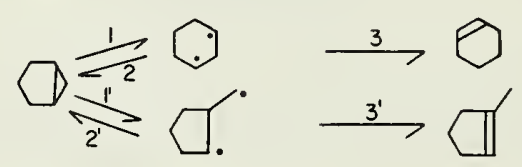

$\log A_{u(\text { est })}=13.5 ; \Delta H_{1,2(\text { est })}^{\circ} \simeq 46 \mathrm{kcal} / \mathrm{mole} ; E_{a(\mathrm{est})}=56.5^{\circ}$

$\log A_{b(\text { est })}=14.2 ; \Delta H_{\left(1^{\prime}, 2^{\prime}\right) \text { est }} \simeq 52 \mathrm{kcal} / \mathrm{mole} ; E_{b(\text { est })}=62.5$.

\section{Experimental}

[1] Analysis by G.L.C. At the highest temperatures, small amounts ( 1-4 percent) of methylenecyclopentane and benzene were detected. Yields increased with increasing (S/V). 
Reaction: Bicyclo[2,1,1]hexane

$\begin{array}{ccc}\& \text { (I) } & \text { I } & \text { II } \\ \Delta H_{f(298)}^{\circ} & 15.4 & 20.2 \\ S_{(298)}^{\circ} & 69.4 & 88.4 \\ C_{\text {(I298) }}^{\circ-} & 23.8 & 28.8\end{array}$

$$
\begin{aligned}
& \Delta S^{\circ}=19.0 \mathrm{~g} / \mathrm{mole} \\
& \Delta H^{\circ}=4.8 \mathrm{kcal} / \mathrm{mole} \\
& \Delta C_{p}^{\circ}=5.0 \mathrm{~g} / \mathrm{mole}
\end{aligned}
$$

\begin{tabular}{lcccccc}
$\log \boldsymbol{A}$ & $\boldsymbol{E}$ & $\begin{array}{c}\log \boldsymbol{k}_{T} \\
(620)\end{array}$ & \multicolumn{1}{c}{ Conditions } & System & Surface & \multicolumn{1}{c}{ References } \\
15.17 & 55 & -4.21 & $\begin{array}{l}618 \pm 18^{\circ} \mathrm{K} \\
0.2-20 \mathrm{~mm} \mathrm{Hg}\end{array}$ & static & $<10 \%$ & [1] R. Srinivasan and A. A. \\
& & & & & Levi, J. Am. Chem. Soc. \\
$\mathbf{8 5}, 3363$ (1963).
\end{tabular}

\section{Preferred:}

$\log k=15.17-55.0 / \theta$.

Comments: The kinetics favor a biradical mechanism (see III-6.0).

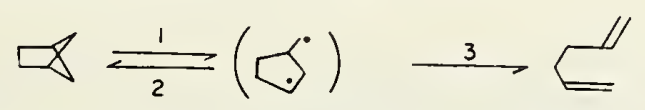

Very likely $k_{2} \simeq k_{3}$. With a strain energy for the reactant of $39 \mathrm{kcal} / \mathrm{mole}$,

$$
\begin{aligned}
& E \simeq D H^{\circ}(\mathrm{C}-\mathrm{C})-\Delta E_{\text {strain }}+E_{2}\left(\text { or } E_{3}\right) \\
& 55 \simeq 80.1-39+6.3+E_{2} ; E_{2} \simeq 7.6 \mathrm{kcal} / \mathrm{mole} \\
& \log A_{\text {est }} \simeq 15.2
\end{aligned}
$$

\section{Experimental}

[1] Analysis by G.L.C. NO and propylene had no effect on the reaction rates. 
Reaction: Bicyclo[3,2,0]heptane

$\begin{array}{cccccc} & \text { (I) } & \text { II } & \text { III } & \text { IV } \\ & & \text { I } & \mathrm{C}_{2} \mathrm{H}_{4} \text { (II) } & \text { (II) } & \\ \Delta H_{f(298)}^{\circ} & 3.8 & 12.5 & 7.9 & 14.8 \\ S_{(298)}^{\circ} & 78.9 & 52.5 & 69.2 & 98.8 \\ C_{p(298)}^{\circ} & 24.5 & 10.4 & 17.5 & 34.3\end{array}$

$\begin{array}{cc}a & b \\ \Delta S^{\circ}=42.8 & 19.9 \mathrm{~g} / \mathrm{mole} \\ \Delta H^{\circ}=16.6 & 11.0 \mathrm{kcal} / \mathrm{mole} \\ \Delta C_{p}^{\circ}=3.4 & 9.8 \mathrm{~g} / \mathrm{mole}\end{array}$

\begin{tabular}{|c|c|c|c|c|c|c|c|}
\hline Path & $\log A$ & $\boldsymbol{E}$ & $\begin{array}{c}\log _{6} \boldsymbol{k}_{T} \\
(735)\end{array}$ & Conditions & System & Surface & References \\
\hline & 14.84 & 60.74 & -3.22 & $\begin{array}{l}737 \pm 38^{\circ} \mathrm{K} \\
2-20 \mathrm{~mm} \mathrm{Hg}\end{array}$ & static & $<10 \%$ & $\begin{array}{l}\text { [1] R. J. Ellis and H. M. } \\
\text { Frey, J. Chem. } \\
\text { Soc., 4184 (1964). }\end{array}$ \\
\hline
\end{tabular}

$\begin{array}{llll}b & 15.40 & 63.97 & -3.62\end{array}$

Preferred:

$\log k_{a}=14.84-60.74 / \theta ; \log k_{b}=15.40-63.97 / \theta$.

Comments: The kinetics favor biradical mechanisms (see section III-6.0).

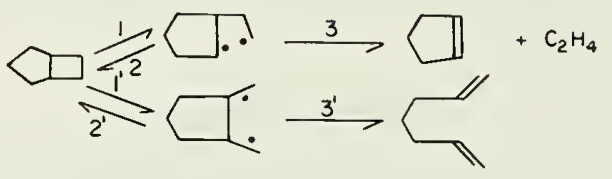

Probably, $k_{2} \simeq k_{3} ; k_{2^{\prime}} \simeq k_{3^{\prime}}$ as in cyclobutane decompositions.

Transition state estimates give:

$$
\begin{aligned}
& \log A_{a(\text { est })} \simeq 15.0 ; \Delta H_{1,2}^{\circ} \simeq 55 \mathrm{kcal} / \mathrm{mole} ; \quad E_{a(\text { est })}=61 \mathrm{kcal} / \mathrm{mole} \\
& \log A_{b(\text { est })} \simeq 15.3 ; \Delta H_{1^{\prime}, 2^{\prime}}^{\circ} \simeq 56.5 \mathrm{kcal} / \mathrm{mole} ; E_{b(\text { est })}=62.5 \mathrm{kcal} / \mathrm{mole}
\end{aligned}
$$

\section{Experimental}

[1] Analysis by G.L.C. Rates were corrected for subsequent decomposition of 1,6-heptadiene to butadiene and propylene. 
Reaction: Bicyclo[3,2,0]hept-6-ene

\begin{tabular}{|c|c|}
\hline & I \\
\hline$\Delta H_{f(298)}^{\circ}$ & 33.3 \\
\hline$S_{(298)}^{\circ}$ & 74.6 \\
\hline$C_{p(298)}^{o}$ & 23.0 \\
\hline
\end{tabular}

$$
\begin{aligned}
\Delta S^{\circ}= & 2.7 \mathrm{~g} / \mathrm{mole} \\
\Delta H^{\circ}= & -12.5 \mathrm{kcal} / \mathrm{mole}^{\circ} \\
\Delta C_{p}^{\circ}= & 2.1 \mathrm{~g} / \mathrm{mole}^{\prime}
\end{aligned}
$$

\begin{tabular}{|c|c|c|c|c|c|c|}
\hline $\log A$ & $\boldsymbol{E}$ & $\underset{(575)}{\log \boldsymbol{k}_{T}}$ & Conditions & System & Surface & References \\
\hline 14.31 & 45.51 & -2.99 & $\begin{array}{l}547-600{ }^{\circ} \mathrm{K} \\
0.5-6 \mathrm{~mm} \mathrm{Hg}\end{array}$ & static & none & $\begin{array}{l}\text { [1] G. R. Branton, H. M. } \\
\text { Frey, D. C. Montague, } \\
\text { and I. D. R. Stevens, } \\
\text { Trans. Faraday Soc. } \\
\text { 62, } 659(1966) .\end{array}$ \\
\hline
\end{tabular}

Preferred:

$\log k=14.31-45.51 / \theta$.

Comments: The reaction may be compared to cyclobutene decompositions which proceed with "normal" $A$-factors, much lower activation energies, and are concerted processes. The exclusive con-rotary isomerizations of the cyclobutenes as predicted by the Woodward-Hoffman rules is here sterically inhibited. The result is a disrotary (or biradical) mechanism.

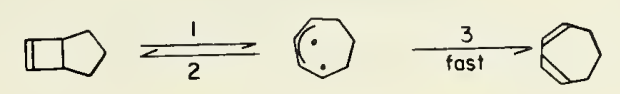

Transition state estimates give,

$$
\begin{gathered}
\log A_{\text {est }}=14.3 ; \Delta H_{1.2}^{\circ} \simeq 35 \mathrm{kcal} / \text { mole } ; E_{2} \simeq 10.5 \mathrm{kcal} / \mathrm{mole} ; E_{\text {strain }} \simeq(6.3+29.8) \mathrm{kcal} / \mathrm{mole} \\
\text { Experimental }
\end{gathered}
$$

\section{Experimental}

[1] Analysis and rate by G.L.C. Reaction in solution gave $\log k=14.65-45.86 / \theta$. 
Reaction: Bicyclo[4,2,0] oct-7-ene

$\begin{array}{ccc} & \text { II } & \text { II } \\ & 21.0 & 19.7 \\ \Delta H_{f(298)}^{\circ} & 82.2 & 83.7 \\ S_{(298)}^{\circ} & 33.9 & (31) \\ \Delta C_{p(298)}^{\circ} & \text { III }\end{array}$

$\Delta S^{\circ}=1.5 \mathrm{~g} / \mathrm{mole}$

$\Delta H^{\circ}=-1.3 \mathrm{kcal} / \mathrm{mole}$

$\Delta C_{p}^{\circ}=-2.9 \mathrm{~g} / \mathrm{mole}$

$\begin{array}{lcccccc}\log \boldsymbol{A} & \boldsymbol{E} & \begin{array}{c}\log \boldsymbol{k}_{T} \\ (535)\end{array} & \text { Conditions } & \text { System } & \text { Surface } & \text { References } \\ 14.13 & 43.18 & -3.51 & \begin{array}{l}509-558^{\circ} \mathrm{K} \\ 0.5-4.0 \mathrm{~mm} \mathrm{Hg}\end{array} & \text { static } & \text { none } & \text { [1] G. R. Branton, H. M. } \\ & & & & & \begin{array}{l}\text { Frey, and R. F. } \\ \text { Skinner, Trans. }\end{array} \\ & & & & & \text { Faraday Soc. 62, 1546 } \\ & & & & & \end{array}$

Preferred:

$\log k=14.13-43.18 / \theta$.

Comments: The activation energy for this reaction is lower than one would expect for a biradical mechanism (i.e., $\Delta H_{\text {to biradical }}^{\circ} \simeq 42 \mathrm{kcal} / \mathrm{mole}$ ). However, the observed $A$-factor is quite close to the estimated value, $\log A_{\text {est }}=13.9$. (See bicyclo[3,2.0] hept-6-ene.)

\section{Experimental}

[1] Rates were obtained from G.L.C. analysis on reactant and product. 
Reaction: Bicyclo[2,2,1]hepta-2,5-diene

\begin{tabular}{|c|c|c|c|c|c|}
\hline & I & II & III & IV & V \\
\hline$\Delta H_{f(298)}$ & & 44.1 & 54.2 & 32.5 & 12.0 \\
\hline$S_{(298)}^{\circ}$ & 71.3 & 75.4 & 48.0 & 66.2 & 76.4 \\
\hline$C_{p(298)}^{\circ}$ & 26.1 & 24.2 & 10.5 & 17.5 & 24.8 \\
\hline
\end{tabular}

\begin{abstract}
$a$
$\Delta S^{\circ}=4.1 \mathrm{~g} / \mathrm{mole}$

$\Delta H^{\circ}=-13 \mathrm{kcal} / \mathrm{mole}$

$\Delta C_{p}^{\circ}=-1.9 \mathrm{~g} / \mathrm{mole}$
\end{abstract}

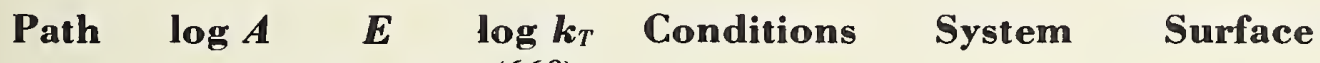

References

$(660)$

S. Flow

$\begin{array}{lllll}14.68 & 50.61 & -2.08 & 661 \pm 44^{\circ} \mathrm{K} & \text { S. Flow } \\ 14.62 & 50.19 & -2.00 & \\ 14.23 & 53.14 & -3.37\end{array}$

[1] W. C. Herndon and and L. L. Lowry, J. Am. Chem. Soc. 86, 1922 (1964).

$\begin{array}{llllllll}a & 14.91 & 51.6 & -2.18 & 615 \pm 15^{\circ} \mathrm{K} & \text { static } & \sim 10 \% & \text { [2] J. H. Birely and J. P. } \\ b & 14.72 & 50.5 & -2.00 & 0.8-2 \text { torr } & & <10 \% & \begin{array}{l}\text { Chesick, J. Phys. } \\ \text { Chem 66, } 668 \\ \end{array} \\ & & & & & & (1962) .\end{array}$

$\begin{array}{cccccccc}a & 13.94 & 49.8 & -2.55 & 613 \pm 20^{\circ} \mathrm{K} & \text { static } & <10 \% & \text { [3] B. C. Roquite, Can. } \\ b & 14.30 & 49.8 & -2.19 & 4.4-24.7 \text { torr } & & <10 \% & \begin{array}{l}\text { J. Chem. 42, } 2134 \\ \text { (1964). }\end{array}\end{array}$

Preferred:

$$
\begin{aligned}
& \log k_{a}=14.68-50.61 / \theta \\
& \log k_{b}=14.62-50.19 / \theta \\
& \log k_{c}=14.23-53.14 / \theta
\end{aligned}
$$

Comments: The estimated enthalpy for ring opening, $\alpha(\mathrm{C}-\mathrm{C})$ split - path $b$, is about $57 \mathrm{kcal} / \mathrm{mole}$, therefore path $b$ must be a concerted process. The estimated enthalpy of ring opening, $\beta(\mathrm{C}-\mathrm{C}) \mathrm{split}-$ paths $a$ and $c$, is about $38 \mathrm{kcal} / \mathrm{mole}$, thus paths $a$ and $c$ are consistent with the biradical mechanism.

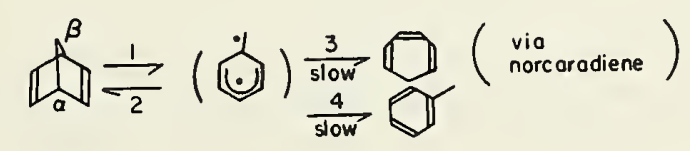

$$
\begin{aligned}
& \Delta H_{1,2}^{\circ}=D H^{\circ}(\mathrm{C}-\mathrm{C})-\Delta E_{\text {strain }}-R_{\text {allyl }}=80.1-(31.5-2)-12.6=38 \mathrm{kcal} / \mathrm{mole} \\
& \log A_{c(\text { est })}=14.2 \\
& \log A_{a(\text { est })}=14.5 .
\end{aligned}
$$




\section{Experimental}

[1] Analysis by I.R. and G.L.C. Rates by G.L.C. Toluene was also believed to be a product of the subsequent decomposition of cycloheptatriene. This is consistent with the observations of [3].

[2] Analysis by G.L.C. Source of toluene was uncertain.

[3] Analysis by G.L.C. Toluene yields were very low in the initial stages. No effect on reaction rates by added $\mathrm{O}_{2}$ and NO. The ratio of toluene to cycloheptatriene increased with increasing percent decomposition (see [1]).

Reaction: Bicyclo[2,2,1]hept-2-ene:

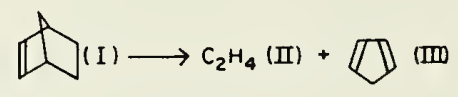

$\begin{array}{cccc} & \text { I } & \text { II } & \text { III } \\ \Delta H_{f(298)}^{\circ} & 22.3 & 12.5 & 32.5 \\ S_{(298)}^{\circ} & 76.0 & 52.5 & 67.0 \\ C_{p(298)}^{\circ} & 27.3 & 10.4 & 17.5\end{array}$

$\Delta S^{\circ}=43.5 \mathrm{~g} / \mathrm{mole}$

$\Delta H^{\circ}=22.7 \mathrm{kcal} / \mathrm{mole}$

$\Delta C_{p}^{\circ}=0.6 \mathrm{~g} / \mathrm{mole}$

\begin{tabular}{|c|c|c|c|c|c|c|}
\hline $\log A$ & $\boldsymbol{E}$ & $\begin{array}{c}\log \boldsymbol{k}_{T} \\
(560)\end{array}$ & Conditions & System & Surface & References \\
\hline 13.84 & 43.47 & -3.13 & $\begin{array}{l}558 \pm 19^{\circ} \mathrm{K} \\
5-43 \mathrm{~mm} \mathrm{Hg}\end{array}$ & static & $<10 \%$ & $\begin{array}{l}\text { [1] B. C. Roquite, J. Phys. } \\
\text { Chem. 69, } 1351 \text { (1965). }\end{array}$ \\
\hline 13.78 & 42.75 & -2.91 & $\begin{array}{l}625 \pm 48^{\circ} \mathrm{K} \\
673 \pm 43^{\circ} \mathrm{K}\end{array}$ & $\begin{array}{l}\text { stirred flow } \\
\text { flow }\end{array}$ & $<10 \%$ & $\begin{array}{l}\text { [2] W. C. Herndon, W. B. } \\
\text { Cooper, Jr., and M. } \\
\text { J. Chambers, J. Phys. } \\
\text { Chem. 68, 2016 (1964). }\end{array}$ \\
\hline
\end{tabular}

Preferred:

$\log k=13.84-43.47 / \theta$.

Comments: A biradical mechanism ( D) $\frac{1}{2}$ (c) $\stackrel{3}{\longrightarrow}$ (products) gives $\Delta H_{1,2}^{\circ}=D H^{\circ}(\mathrm{C}-\mathrm{C})-E_{\text {strain }}$

$-\mathrm{R}_{\mathrm{ally} 1}=80.1-24.5+6-12.5=49.1 \mathrm{kcal} / \mathrm{mole}$. This exceeds the experimental activation energy by much more than experimental error. There seems to be little chance of extra stabilization of the transition state for ring closing in this system (as opposed to endo-dicyclopentadiene), thus the reaction is undoubtedly a concerted process.

\section{Experimental}

[1] Analysis by G.L.C. Rates were unaffected by added $\mathrm{O}_{2}$, NO, propylene and toluene. A reaction product estimated as less than 2 percent of the total was not identified.

[2] Analysis by G.L.C. Both flow methods were consistent and the data from both were combined to obtain the reported Arrhenius parameters. 
Reaction: 2,3-Diazobicyclo[2,2,1]hept-2-ene

$\begin{array}{cccc} & & & \\ & & & \\ & \text { I } & \text { II } & \text { III } \\ \Delta H_{f(298)}^{\circ} & (62) & 36.0 & 0 \\ S_{(298)}^{\circ} & 75.9 & 70.4 & 45.8 \\ C_{p(298)}^{\circ} & 27.3 & 17.9 & 7.0\end{array}$

$\Delta S^{\circ}=40.3 \mathrm{~g} / \mathrm{mole}$

$\Delta H^{\circ}=-26 \mathrm{kcal} / \mathrm{mole}$

$\Delta C_{p}^{\circ}=-2.4 \mathrm{~g} / \mathrm{mole}$

\begin{tabular}{|c|c|c|c|c|c|c|}
\hline $\log A$ & $\boldsymbol{E}$ & $\log k_{T}$ & Conditions & System & Surface & References \\
\hline 14.86 & 37.3 & & $\begin{array}{l}429 \pm 25^{\circ} \mathrm{K} \\
(35 \mathrm{~mm} \mathrm{Hg})\end{array}$ & static & $<10 \%$ & $\begin{array}{l}\text { [1] S. G. Cohen, R. Zand, and } \\
\text { C. Steel, J. Am. Chem. } \\
\text { Soc. 83, } 2895 \text { (1961). }\end{array}$ \\
\hline 14.78 & 36.9 & & $\begin{array}{l}443-563^{\circ} \mathrm{K} \\
100-200 \mathrm{~mm} \mathrm{Hg}\end{array}$ & static & none & $\begin{array}{l}\text { [2] R. J. Crawford, R. J. } \\
\text { Dummel, and A. Mishra, } \\
\text { J. Am. Chem. Soc. 87, } \\
3023 \text { (1965). }\end{array}$ \\
\hline
\end{tabular}

Preferred:

Reliable, $\log k=14.78-36.9 / \theta$.

Comments: Using the activation energy for azoisopropane $(\sim 47.5 \mathrm{kcal} / \mathrm{mole})$ as a measure of the $\left(\mathrm{C}-\mathrm{N}_{\mathrm{azo}}\right)$ bond energy, and assuming the same strain as in norbornane [ $\$, E_{\mathrm{st}} \simeq 17.6 \pm 2.5 \mathrm{kcal} / \mathrm{mole}$ ], one obtains an enthalpy of ring opening to the biradical of, $\Delta H_{1,2}^{\circ}=30.0 \mathrm{kcal} / \mathrm{mole}$. A biradical mechanism, therefore, is consistent with the kinetics with $E_{2}=7.0 \mathrm{kcal} / \mathrm{mole}$. Also, $\log A_{\text {est }}=14.4$ (see section III-7.0).

Mechanism:

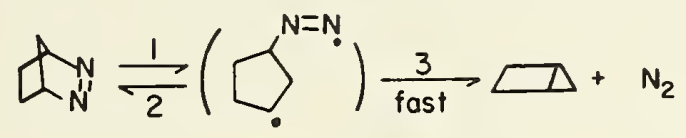

\section{Experimental}

[1] Analysis by G.L.C. Reactions performed in sealed tubes at approximate pressure reported and in the presence of $1 \mathrm{~atm}$ pressure of isooctane.

[2] Rates followed by pressure change. First order kinetics observed to greater than 95 percent reaction. 
Reaction: 2,3-Diazobicyclo[2,2,2] oct-2-ene

\begin{tabular}{|c|c|c|c|}
\hline$\Delta H_{f(299}^{\circ}$ & $\begin{array}{c}\text { I } \\
(45.6)\end{array}$ & $\begin{array}{c}\text { II } \\
19.8\end{array}$ & $\begin{array}{c}\text { III } \\
0\end{array}$ \\
\hline$S^{\circ}{ }_{(298)}$ & 81.8 & 88.4 & 45.8 \\
\hline$C_{p(\mathbf{2 9 8})}^{\circ}$ & 32.8 & 28.8 & 7.0 \\
\hline
\end{tabular}

$\Delta S^{\circ}=52.4 \mathrm{~g} / \mathrm{mole}$

$\Delta H^{\circ}=-25.8 \mathrm{kcal} / \mathrm{mole}$

$\Delta C_{p}^{\circ}=3.0 \mathrm{~g} / \mathrm{mole}$

\begin{tabular}{|c|c|c|c|c|c|c|}
\hline $\log A$ & $\boldsymbol{E}$ & $\log k_{T}$ & Conditions & System & Surface & References \\
\hline 15.3 & 44.6 & & $\begin{array}{l}503 \pm 30^{\circ} \mathrm{K} \\
25 \mathrm{~mm} \mathrm{Hg}\end{array}$ & static & $<10 \%$ & $\begin{array}{l}\text { [1] S. G. Cohen and R. Zand, } \\
\text { J. Am. Chem. Soc. 84, } \\
586(1962)\end{array}$ \\
\hline
\end{tabular}

\section{Preferred:}

$\log k=15.3-44.6 / \theta$.

Comments: The kinetics are consistent with a biradical process. The activation energy suggests a strain energy of about $7 \mathrm{kcal} /$ mole in the reactant if there is a $4 \mathrm{kcal}$ back activation energy for the biradical (boat form of cyclohexane). This seems reasonable. $\log A_{\text {est }}=15.5$. (See section III-7.0.)

\section{Experimental}

[1] Analysis by U.V. Reaction performed in sealed tubes at 1 atm pressure of isooctane or toluene. 
Reaction: Bicyclo[ $[5,1,0]$ oct-2-ene

Cycloocta-1,4-diene

\begin{tabular}{|c|c|c|}
\hline$\Delta H_{(298)}^{\circ}$ & $\begin{array}{c}I \\
25 \pm 2\end{array}$ & $\begin{array}{c}\text { II } \\
25.0\end{array}$ \\
\hline$S^{\circ}{ }_{(298)}$ & 77.0 & 83.4 \\
\hline$C_{p(298)}^{\circ}$ & 29.3 & 30.0 \\
\hline
\end{tabular}

$\Delta S^{\circ}=(6.4) \mathrm{g} / \mathrm{mole}$

$\Delta H^{\circ}=(0) \mathrm{kcal} / \mathrm{mole}$

$\Delta C_{p}^{\circ}=0.7 \mathrm{~g} / \mathrm{mole}$

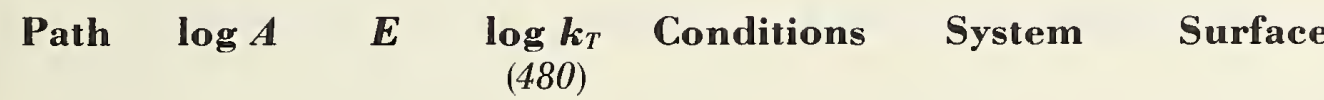

References

$\begin{array}{llllll}a & 13.36 & 38.6 & -4.22 & 468-489^{\circ} \mathrm{K} & \text { static }\end{array}$

$\begin{aligned} & b \\ & b\end{aligned} \quad \mathbf{1 1 . 8 0} \quad 38.6 \quad-5.76$

[1] W. Grimme. Chem.

Ber. 98, 756 (1965).

Preferred:

$\log k_{a}=13.36-38.6 / \theta$.

$\log k_{b}=11.80-38.6 / \theta$.

\section{Experimental}

[1] Rates were followed by G.L.C. analysis. The equilibrium constant for the reaction was found to be about $K_{\text {eq }} \simeq 37$ at $473{ }^{\circ} \mathrm{K}$ with a temperature dependence $\Delta H_{R}^{\circ}=0 \pm 0.3 \mathrm{kcal} / \mathrm{mole}$. The sum of rate constants was reported $\left(k_{a}+k_{b}\right)$ $=2.2 \times 10^{13-38.6 \pm .6 / \theta} \mathrm{sec}^{-1}$. Parameters above have been calculated from these data. Above $325^{\circ} \mathrm{C}$ equilibrium is displaced irreversibly to form

$\checkmark$ from (II) in a "vinylcyclopropane type" reaction. 
Reaction: Tricyclo[3,3,0,0, 6 octane

$\begin{array}{ccr} & \square(\mathrm{I}) \longrightarrow(\mathrm{II}) & \\ & \mathrm{I} & \mathrm{II} \\ \Delta H_{f(298)}^{\circ} & (14.2) & (23) \\ S_{(298)}^{\circ} & 74.6 & 83.4 \\ C_{p(298)}^{\circ} & 30.1 & 30.1\end{array}$

$\Delta S^{\circ}=8.8 \mathrm{kcal} / \mathrm{mole}$

$\Delta H^{\circ}=8.8 \mathrm{kcal} / \mathrm{mole}$

$\Delta C_{p}^{\circ}=0 \mathrm{~g} / \mathrm{mole}$

\begin{tabular}{|c|c|c|c|c|c|c|}
\hline $\log A$ & $\boldsymbol{E}$ & $\begin{array}{c}\log \boldsymbol{k}_{T} \\
(615)\end{array}$ & Conditions & System & Surface & References \\
\hline 15.51 & 55.9 & -4.35 & $\begin{array}{l}616 \pm 16 \\
1.5 \text { torr }\end{array}$ & static & $\sim 10 \%$ & $\begin{array}{l}\text { [1] R. Srinivasan and A. A. } \\
\text { Levi, J. Am. Chem. 86, } \\
3756 \text { (1964). }\end{array}$ \\
\hline
\end{tabular}

Preferred:

$\log k=15.51-55.9 / \theta$.

Comments: Transition state estimates based on a biradical mechanism give $\Delta S \ddagger=7.3 \mathrm{~g} / \mathrm{mole}$ and $\log A_{\text {est }}$ $=15.2$. A total strain of about $40 \mathrm{kcal} / \mathrm{mole}$ is estimated.

\section{Experimental}

[1] Analysis by I.R. and G.L.C. Rate by G.L.C. 1,5-cyclo-octadiene was an unstable product which decomposed by two paths to butadiene and to 4 -vinyl cyclohexane. The rates were unaffected by propylene and decreased slightly with increased $(S / V)$. 
Reaction: $\beta$-Pinene

$\begin{array}{cccc} & \text { I } & \text { II } & \text { III } \\ \Delta H_{f(298)}^{\circ} & 4.0 & 17.5 & -1.2 \\ S_{(298)}^{\circ} & 94.2 & 118.5 & 101.8 \\ C_{p(298)}^{\circ} & 41.3 & 49.9 & 45.1\end{array}$

$\begin{array}{cc}a & b \\ \Delta S^{\circ}=24.3 & 7.6 \mathrm{~g} / \text { mole } \\ \Delta H^{\circ}=13.5 & -5.2 \mathrm{kcal} / \mathrm{mole} \\ \Delta C^{\circ}=8.6 & 3.8 \mathrm{~g} / \mathrm{mole}\end{array}$

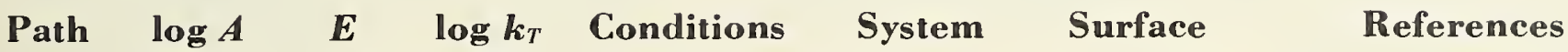
(650)

$a$

$17.7 \quad 49.9+1.09 \quad 648 \pm 25^{\circ} \mathrm{C} \quad$ flow 20-80 tor

[1] E. Hawkins and J. Vogh, J. Phys. Chem. 57, 902 (1953).

$b$

$15.3 \quad 45.1+0.14$

Preferred:

$\log k_{a}=17.7-49.9 / \theta$

$\log k_{b}=15.3-45.1 / \theta$.

Comments: A biradical mechanism is consistent with both processes.

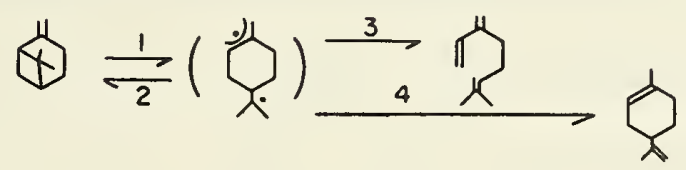

The enthalpy for biradical formation is about, $\Delta H_{1,2}^{\circ}=39 \mathrm{kcal} / \mathrm{mole}$ using $E_{\text {strain }}=26.2 \mathrm{kcal} / \mathrm{mole}$. $\log A_{\text {est }}$ $=16.9$.

\section{Experimental}

[1] Analysis and rate by $\rho$ and O.R. Two minor products were also formed, but were not identified. 
Reaction: Endo-dicyclopentadiene

\begin{tabular}{ccc} 
& \multicolumn{1}{l}{$\longrightarrow$ I } & II \\
& 45.4 & 32.5 \\
$\Delta H_{f(298)}^{\circ}$ & & \\
$S_{(298)}^{\circ}$ & 86.3 & 67.0 \\
$C_{p(298)}^{\circ}$ & 36.1 & 17.5
\end{tabular}

$\Delta S^{\circ}=47.7 \mathrm{~g} / \mathrm{mole}$

$\Delta H^{\circ}=19.6 \mathrm{kcal} / \mathrm{mole}$

$\Delta C_{p}^{\circ}=-1.1 \mathrm{~g} / \mathrm{mole}$

\begin{tabular}{|c|c|c|c|c|c|c|}
\hline $\log A$ & $\boldsymbol{E}$ & $\underset{(455)}{\log \boldsymbol{k}_{T}}$ & Conditions & System & Surface & References \\
\hline 13.0 & 33.7 & -3.19 & $\begin{array}{l}462 \pm 33^{\circ} \mathrm{K} \\
17-106 \mathrm{~mm} \mathrm{Hg}\end{array}$ & static & none & $\begin{array}{l}\text { [1] J. B. Harkness, G. B. } \\
\text { Kistiakowsky, and } \\
\text { W. H. Mears, J. Chem. } \\
\text { Phys. 5, } 682 \text { (1937) }\end{array}$ \\
\hline 13.01 & 33.97 & -3.31 & $\begin{array}{l}426-484^{\circ} \mathrm{K} \\
\mathrm{N}_{2} \text { carrier }\end{array}$ & (stirred flow) & & $\begin{array}{l}\text { [2] W. C. Herndon, C. R. } \\
\text { Grayson, and J. M. } \\
\text { Manion, J. Org. Chem. } \\
\text { 32, } 526 \text { (1967) }\end{array}$ \\
\hline
\end{tabular}

Preferred:

$\log k=13.01-33.97 / \theta$.

Comments: The estimated thermodynamics are in good agreement with the parameters of Kistiakowsky, et al. There is considerable literature on the subject of the mechanism of the cyclopentadiene decomposition and dimerization (see [2] for other references). Generally a concerted process has been favored because of the low activation energy. However, the biradical mechanism cannot be excluded on this basis. Thus for the process,

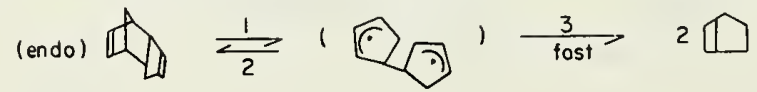

$$
\begin{aligned}
& E=D H^{\circ}(\mathrm{C}-\mathrm{C})-\Delta E_{\text {strain }}-2 R_{\text {allyl }}-\left(R_{\text {overlap }}\right)+E_{2} \\
& =78.7-(24.5+6-2(6))-2(12.6)-R_{0}+2(?) \\
& 34=37.0-R_{0}
\end{aligned}
$$

If in the transition state for ring closing some allylic overlap between rings leads to extra stabilization (i.e. $\sim 3 \mathrm{kcal} / \mathrm{mole}$ is required), then the biradical process would be possible. The low $A$-factor observed would also be expected with such an interaction. Note that the activation energy observed for the exo dimer decomposition is just about what would be expected with no allylic overlap stabilization.

\section{Experimental}

[1] Analysis by $\Delta \mathrm{P}$ and $\eta_{\tau}$. Rates by $\Delta \mathrm{P}$. Rates unaffected by small amounts of $\mathrm{O}_{2}$. The association reaction was also studied. Rate constant parameters were $\log k_{b}(1 /$ mole-sec $)=4.93-14.9 / \theta^{*}{ }^{*}$

[2] Analysis was by G.L.C. Rates were unaffected by addition of propylene or by surface conditioning. Isomerization to the exo form was not detectable even at 95 percent conversion suggesting that the rate constant for isomerization is at least a factor of $10^{4}$ times slower than decomposition.

*The back reaction has also been studied by G. A. Benford and A. Wasserman, J. Chem. Soc. 362 (1939) and by Schultze (Oel. u. Kohle, 6, 113 (1938), who obtained, respectively, $\log k_{b}=6.1-16.7 / \theta$ and $\log k_{b}=6.8-16.9 / \theta$. 
Reaction: Exo-dicyclopentadiene

$\begin{array}{ccc} & 2\left[\prod\right](I) \longrightarrow & \\ & \text { I } & \text { II } \\ \Delta H_{f(298)}^{\circ} & 45.4 & 32.5 \\ S_{(298)}^{\circ} & 86.3 & 67.0 \\ C_{p(298)}^{\circ} & 36.1 & 17.5\end{array}$

$\Delta S^{\circ}=47.7 \mathrm{~g} / \mathrm{mole}$

$\Delta H^{\circ}=19.6 \mathrm{kcal} / \mathrm{mole}$

$\Delta C_{p}^{\circ}=-1.1 \mathrm{~g} / \mathrm{mole}$

\begin{tabular}{|c|c|c|c|c|c|c|}
\hline $\log A$ & $\boldsymbol{E}$ & $\begin{array}{c}\log \boldsymbol{k}_{T} \\
(550)\end{array}$ & Conditions & System & Surface & References \\
\hline 13.72 & 38.49 & -1.58 & $\begin{array}{l}474-625^{\circ} \mathrm{K} \\
\mathrm{N}_{2} \text { carrier }\end{array}$ & stirred flow & none & $\begin{array}{l}\text { [1] W. C. Herndon, C. R. } \\
\text { Grayson, and J. M. } \\
\text { Manion, J. Org. Chem. } \\
\text { 32, } 526 \text { (1967). }\end{array}$ \\
\hline
\end{tabular}

\section{Preferred:}

Reliable. $\log k=13.72-38.49 / \theta$.

Comments: An estimate of the activation energy for the exo decomposition via the biradical mechanism gives $E=37.5 \mathrm{kcal} / \mathrm{mole}$ (see endo-dicyclopentadiene).

\section{Experimental}

[1] Analysis by G.L.C. Rates were unaffected by added propylene or the nature of the surface. Isomerization to the endo form was not observed to compete with the decomposition to cyclopentadiene. 
Reaction: Quadricyclo $\left[2,2,1,0,2,60^{3}, 5\right]$ heptane

\begin{tabular}{|c|c|c|}
\hline$\Delta H_{f(298)}^{\circ}$ & $\left({ }^{I}\right)$ & $\begin{array}{c}\text { II } \\
57.1\end{array}$ \\
\hline$S_{(298)}^{\circ}$ & 71.8 & 71.3 \\
\hline$C_{p(298)}^{\circ}$ & 26 & 26.1 \\
\hline
\end{tabular}

$\Delta S^{\circ}=-0.5 \mathrm{~g} / \mathrm{mole}$

$\Delta H^{\circ}=\mathrm{kcal} / \mathrm{mole}$

$\Delta C_{p}^{\circ}=0.1 \mathrm{~g} / \mathrm{mole}$

\begin{tabular}{|c|c|c|c|c|c|c|}
\hline $\log A$ & $\boldsymbol{E}$ & $\begin{array}{c}\log k_{T} \\
(445)\end{array}$ & Conditions & System & Surface & References \\
\hline 12.81 & 33.51 & -3.65 & $\begin{array}{l}443 \pm 20^{\circ} \mathrm{K} \\
1-18 \text { torr } \\
(3-4)\end{array}$ & static & $<10 \%$ & $\begin{array}{l}\text { [3] H. M. Frey, J. Chem., 365, } \\
\text { (1964). }\end{array}$ \\
\hline
\end{tabular}

Preferred:

$\log =12.81-33.5 / \theta$.

\section{Experimental}

[1] Analysis by G.L.C. Rates unaffected by propylene and other added third bodies. 
Reaction: Endo-bicyclo[2,2,1]hept-5-ene-2-carboxaldehyde (endo-5-norbornene-2-carboxaldehyde) (endo-methylene-2,5-tetrahydrobenzaldehyde)

\begin{tabular}{|c|c|c|c|}
\hline$\Delta H_{f(298)}^{\circ}$ & $\begin{array}{c}\text { I } \\
-4.2\end{array}$ & $\begin{array}{c}\text { II } \\
32.5\end{array}$ & $\begin{array}{c}\text { III } \\
-18.1\end{array}$ \\
\hline$S^{\circ}{ }_{(298)}$ & 89.7 & 67.0 & $(65.9)$ \\
\hline$C_{p(\mathbf{2 9 8})}^{\circ}$ & 35.3 & 17.5 & 15.7 \\
\hline
\end{tabular}

$\Delta S^{\circ}=43.2 \mathrm{~g} / \mathrm{mole}$

$\Delta H^{\circ}=18.6 \mathrm{kcal} / \mathrm{mole}$

$\Delta C_{p}^{\circ}=-2.1 \mathrm{~g} / \mathrm{mole}$

\begin{tabular}{|c|c|c|c|c|c|c|}
\hline $\log A$ & $\boldsymbol{E}$ & $\begin{array}{c}\log k_{\varepsilon_{T}} \\
(490)\end{array}$ & Conditions & System & Surface & References \\
\hline 12.34 & 33.6 & -2.65 & $490 \pm 26$ & static & none & $\begin{array}{l}\text { [1] G. B. Kistiakowsky and } \\
\text { J. R. Lacher, J. Am. } \\
\text { Chem. Soc. } \mathbf{5 8 , 1 2 3} \\
\text { (1936). }\end{array}$ \\
\hline
\end{tabular}

Preferred:

$\log k=13.3-35.8 / \theta$.

Comments: The forward and reverse rate constants give $\Delta H^{\circ}=19.4 \mathrm{kcal} / \mathrm{mole}$ and $\Delta S^{\circ}=37.2 \mathrm{~g} / \mathrm{mole}$, ignoring heat capacity effects. This is not in very good agreement with the estimated entropy. We prefer a slightly higher $A$-factor analogous to endo-dicyclopentadiene.

The low activation energy reported favors a concerted mechanism for this reaction unless some stabilization of overlapping allylic centers in the transition state occurs. (See dicyclopentadiene.) For the biradical mechanism:

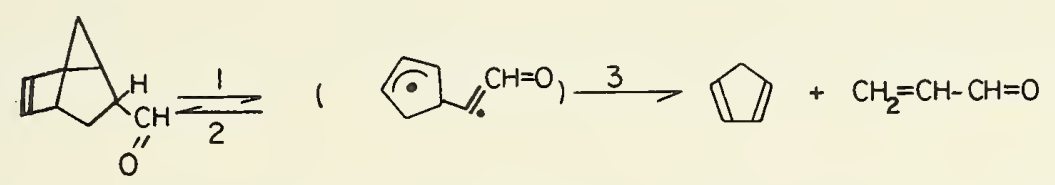

$E=D H^{\circ}(\mathrm{C}-\mathrm{C})-R_{\text {allyl }}-R_{\text {carbonyl }}-\Delta \mathrm{E}_{\text {strain }}-R_{0}+E_{2}=77.9-12.6-8.7-24.5+6.0-R_{0}+2=40.1-R_{0}$. It would be necessary to have an overlap stabilization of about $5 \mathrm{kcal} /$ mole in order for the reaction to involve biradicals.

\section{Experimental}

[1] Rate by $\Delta \mathrm{P}$. Reverse reaction was studied in detail. Rate constant parameters were, $k_{b}=10^{6.18-15.2 / \theta} 1 / \mathrm{mole}$-sec. 
3. Isomerization Reactions

(Other Than Cyclic Compound Reactions) 
Reaction: cis-1,2-Dideuteroethene<smiles>[2H]C=C[2H]</smiles><smiles>[2H]C=CCl</smiles>

$$
\Delta H_{f(298)}^{\circ}
$$

$S_{(298)}^{\circ}$

$C_{p(298)}^{\circ}$

\section{I}

10.3

55.3

11.0
(II)

II

10.3

55.3

11.0

$\Delta S^{\circ}=0 \mathrm{~g} / \mathrm{mole}$

$\Delta H^{\circ}=0 \mathrm{kcal} / \mathrm{mole}$

$\Delta C_{p}^{\circ}=0 \mathrm{~g} / \mathrm{mole}$

\begin{tabular}{|c|c|c|c|c|c|c|}
\hline $\log A$ & $\boldsymbol{E}$ & $\begin{array}{c}\log \boldsymbol{k}_{T} \\
(775)\end{array}$ & Conditions & System & Surface & References \\
\hline 12.48 & 61.3 & -4.80 & $\begin{array}{l}723-823^{\circ} \mathrm{K} \\
9-310 \text { torr }\end{array}$ & static & (a) & $\begin{array}{l}\text { [1] B. S. Rabinovitch, } \\
\text { J. E. Douglas, and } \\
\text { F. S. Looney, J. Chem. } \\
\text { Phys. 20, } 1807 \text { (1952). }\end{array}$ \\
\hline$\sim 13.0$ & $\sim 65.0$ & -5.32 & $\begin{array}{l}723-823^{\circ} \mathrm{K} \\
9-310 \text { torr }\end{array}$ & static & $<2 \%$ & $\begin{array}{l}\text { [2] J. E. Douglas, } \\
\text { B. S. Rabinovitch, and } \\
\text { F. S. Looney, J. Chem. } \\
\text { Phys. 23, } 315 \text { (1955). }\end{array}$ \\
\hline
\end{tabular}

Preferred:

$\log k=13.0-65.0 / \theta$.

$\log A_{\text {est }}=13.6$ (see section IV-3.0).

Comments: A classical calculation of the pressure at which this reaction is halfway into the falloff region gives $M_{1 / 2}=1 / 3 \mathrm{~atm}$. An effective $s=6$ can be estimated from the heat capacity of dideuteroethene.

\section{Experimental}

[1] Analysis by I.R.

[2] Analysis by I.R. Rate was pressure dependent over the entire pressure range. No effect on the rate by traces of $\mathrm{O}_{2}$.

"Appreciable polymerization observed at high pressures. At low pressures the reaction was relatively free from surface effects. 
Reaction: trans-1,2-Dichloroethene<smiles>ClC=CCl</smiles>

(I) $\stackrel{a}{b}$

$\Delta H_{f(298)}^{\circ}$

$S_{(298)}^{\circ}$

$C_{p(298)}^{\circ}$

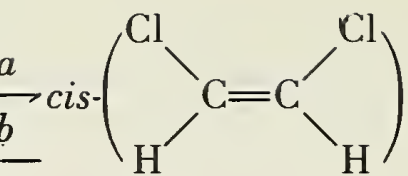

II

5.4

6.4

69.2

69.2

15.8

14.4
$\Delta S^{\circ}=0 \mathrm{~g} / \mathrm{mole}$
$\Delta H^{\circ}=1.0 \mathrm{kcal} / \mathrm{mole}$
$\Delta C_{p}^{\circ}=-1.4 \mathrm{~g} / \mathrm{mole}$

Path $\quad \log A$

$\boldsymbol{E}$

$\log \boldsymbol{k}_{T} \quad$ Conditions

(826)

$a$

12.69

$41.9+1.60$

$560-608^{\circ} \mathrm{K}$

static

200-760 $\mathrm{mm} \mathrm{Hg}$

System

Surface

[1] J. L. Jones and R. L.

$b$

$16.0-1.90$

$473-523^{\circ} \mathrm{K}$ $40 \mathrm{~mm} \mathrm{Hg}$

static

.

$a$

12.68

$55.3-2.12$

$806-846^{\circ} \mathrm{K} \quad$ flow $39 \mathrm{~mm} \mathrm{Hg}$

$\left({ }^{b}\right)$ Taylor, J. Am. Chem. Soc. 62 , 3480 (1940).

\section{References}

[2] B. Tamamuski, H. Akiyama, and $\mathrm{K}$. Ishii, Zeit. Elektrochem. 47, 340 (1941).

[3] L. D. Hawton and G. P. Semeluk, Can. J. Chem. 44, 2143 (1966).

$\begin{array}{lll}b & 12.76 \quad 56.0\end{array}$

Preferred:

$\log k=12.68-55.3 / \theta ; \log k_{b}=12.76-56.0 / \theta$.

$\log A_{\text {est }}=13.0$ (see section IV-3.0).

Comments: The extreme sensitivity of this isomerization to impurities, free radical catalysis, and heterogeneity has been shown in other studies (see B. S. Rabinovitch and M. J. Hulatt, J. Chem. Phys. 27, 592 (1957); C. Steel, J. Phys. Chem. 64, 1588 (1960)). It is possible that some catalysis still exists in the preferred study, although the low parameters could well indicate a pressure falloff. From $C_{v}^{\circ}, s \simeq 8$ one can calculate an $M_{1 / 2} \simeq 20 \mathrm{~mm} \mathrm{Hg}$.

\section{Experimental}

[1] Analysis by refractive index. ${ }^{\mathrm{a}}$

[2] Analysis by dielectric constant measurements. High-pressure region above $150 \mathrm{~mm} \mathrm{Hg}$.

[3] Toluene carrier technique. Analysis by G.L.C. ${ }^{b}$

a Rates showed some surface dependence and also some variation with total pressure. The reverse reaction was highly surface sensitive and could not be studied. A side reaction was detected which increased with $(S / V)$.

b The reaction was surface sensitive. Extrapolation of the rate constants to zero $(S / V)$ ratio was made to obtain the homogeneous component rate constant of the reaction. 


\begin{tabular}{ccc} 
& \multicolumn{1}{c}{$\longrightarrow$ (II) } \\
& $\begin{array}{l}\text { I I }) \\
\Delta H_{f(298)}^{\circ}\end{array}$ & -2.7 \\
$S_{(298)}^{\circ}$ & 72.1 & 70.9 \\
$C_{p(298)}^{\circ}$ & 19.4 & 20.7 \\
$C_{p(700)}^{\circ}$ & 37.6 & 38.4
\end{tabular}

$\Delta S^{\circ}=-1.2 \mathrm{~g} / \mathrm{mole}$

$\Delta H^{\circ}=-1.0 \mathrm{kcal} / \mathrm{mole}$

$\Delta C_{p}^{\circ}=1.3 \mathrm{~g} / \mathrm{mole}$

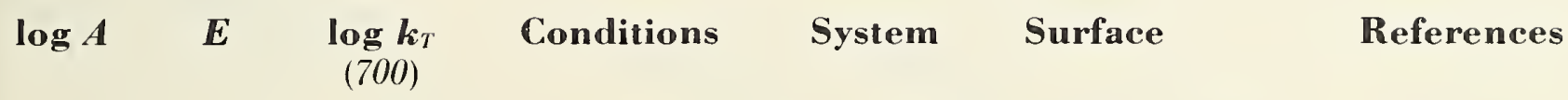

$\begin{array}{lllccc}0.3 & -5.32 & 663-690^{\circ} \mathrm{K} & \text { static } & S / V \times 6 & \text { [1] G. B. Kistiakowsky and } \\ & 102-1440 \text { torr } & & \text { "Essentially } & \text { W. R. Smith, J. Am. } \\ & & \text { homoge- } & \text { Chem. Soc. 58, 766 } \\ & & \text { neous" } & \text { (1936). }\end{array}$
$\begin{array}{lll}11.0 & 52.0 \quad-5.23 \quad & 665-690{ }^{\circ} \mathrm{K} \\ & 200-400 \text { torr }\end{array}$
(a)
[2] W. F. Anderson, J. A.
Bell, J. M. Diamond, and K. R. Wilson, J. Am. Chem. Soc. 80, 2384 (1958).
$13.78 \quad 62.8 \quad-5.82 \quad 686-742^{\circ} \mathrm{K} \quad$ static none

$$
\begin{gathered}
10^{-3}-2 \times 10^{3} \\
\text { torr (N.) } \\
(2-12 \text { torr) }
\end{gathered}
$$
14.00
$62.4-5.48$
$683-749^{\circ} \mathrm{K}$
$0.015-400$ torr
static
$14.54 \quad 65.0 \quad-5.76 \quad 1000-1250^{\circ} \mathrm{K} \quad$ S.P.Sh. $1-6 \%$ in $\mathrm{Ar}$

(a)

[3] B. S. Rabinovitch and

K. W. Michel, J. Am. Chem. Soc. 81, 5065 (1959).

[4] R. B. Cundall and T. F. Palmer, Trans. Faraday Soc. 57, 1936 (1961).
[5] A. Lifshitz, S. H. Bauer, and E. L. Resler, Jr., J. Chem. Phys. 38, 2056 (1963).

Preferred:

$\log k=13.78-62.8 / \theta$

$\log A_{\text {est }} \simeq 13.3$ (see section IV-3.0).

\section{Experimental}

[1] Analysis by M.P. of mixture. Because of the unreasonably low $A$-factor, authors postulated a chain mechanism. Each run was first order, but the rate was dependent on initial pressure.

[2] Analysis by I.R. In falloff region; RRK- $\mathrm{M}_{1 / 2} \simeq 310$ torr at $400^{\circ} \mathrm{C}$.

(Continued)

a Propane observed as a side product probably formed heterogeneously. 


\section{Cis-2-butene (Continued)}

[3] Analysis by G.L.C. Identification by M.S. Falloff below 2 torr, at high pressures (above 10 torr) an autocatalytic reaction occurred. Side products increased with decreasing temperature and increased $S / V$.

[4] Analysis by (.L.C. Confirmed autocatalytic high-pressure reaction and pressure falloff region (i.e., $\sim 4$ torr) reported in [3].

[5] Analysis by G.L.C. The parameters were obtained from the low-temperature rate constants and those obtained at the shock temperatures. Parameters could not be obtained from the shock tube results alone because of a rather sizable scatter in the data.

Reaction: Perfluorobutadiene (hexafluorobutadiene)

\begin{tabular}{|c|c|c|}
\hline$\Delta H_{f(298)}^{\circ}$ & $\begin{array}{c}\text { I } \\
-225\end{array}$ & $\begin{array}{r}\text { II } \\
-237\end{array}$ \\
\hline$S_{(298)}^{\circ}$ & 101 & 93.8 \\
\hline$C_{p(298)}^{\circ}$ & 32.6 & 29.6 \\
\hline
\end{tabular}

$\Delta S^{\circ}=-7.2 \mathrm{~g} / \mathrm{mole}$

$\Delta H^{\circ}=-12 \mathrm{kcal} / \mathrm{mole}$

$\Delta C_{p}^{\circ}=-3.0 \mathrm{~g} / \mathrm{mole}$

$\begin{array}{lccccl}\log A & \boldsymbol{E} & \begin{array}{c}\log k_{T} \\ (500)\end{array} & \text { Conditions } & \text { System } & \text { Surface } \\ 12.03 & 35.38 & -3.43 & \begin{array}{l}467-539^{\circ} \mathrm{K} \\ 1.0-18 \text { torr }\end{array} & \text { static } & \text { none }\end{array}$

\section{Experimental}

[1] Analysis by G.L.C. Added $\mathrm{O}_{2}$ and NO had no effect on the rate. The equilibrium constant for the above reaction was determined to be: $K_{\mathrm{eq}}=10^{-2.07} \times 10^{11.70 / \theta}$. This is in reasonably good agreement with the estimated thermodynamics. 
Reaction: trans-Perfluoro-2-butene<smiles>FC(=C(F)C(F)(F)F)C(F)(F)F</smiles>

(I)<smiles>CC(F)(F)C(F)=C(F)C(F)(F)F</smiles>

I

$\Delta H_{f(298)}^{\circ}$

$S_{(298)}^{\circ}$

$C_{p(298)}^{\circ}$
$(-382.6)$

107.5

31.6
107.0

30.3

$a$

$\Delta S^{\circ}=-0.5 \mathrm{~g} / \mathrm{mole}$

$\Delta H^{\circ}=0.8 \mathrm{kcal} / \mathrm{mole}$

$\Delta C_{p}^{\circ}=-1.3 \mathrm{~g} / \mathrm{mole}$

Path $\log A \quad E \quad \log k_{T}$ Conditions System Surface

(740)

a

13.53

$56.4-3.12$

$698-783^{\circ} \mathrm{K}$ 5 torr

none

static

References

[1] E. W. Schlag and E.

W. Kaiser, Jr., J.

Am. Chem. Soc.

87, 1171 (1965).
$b$
13.64. $55.6-2.78$
$\leftarrow$ same as above $\rightarrow$

\section{Preferred:}

Reliable as reported.

$\log A_{\text {est }} \simeq 13.3$ (see section IV-3.0).

\section{Experimental}

[1] Analysis by G.L.C. No effect on the rate by added NO. Reaction proceeds to an equilibrium state with $K_{\text {eq }}$ $($ trans $\rightarrow$ cis $)=10^{-0.107} \times 10^{-0.817 / \theta}$. The lower activation energy observed relative to the corresponding hydrocarbon isomerization (i.e., $\sim \Delta E_{\text {act }} \sim 6-8 \mathrm{kcal} / \mathrm{mole}$ ) was attributed to the destabilization of the double bond by fluorine substitution.*

*This conclusion appears to be a reasonable one and is supported by the lower activation energies produced by fluorine substitution in some cyclopropane isomerizations. 
Reaction: cis-1,2-Diphenylethene (isotilbene)

\begin{tabular}{|c|c|c|}
\hline$\Delta H_{f(298)}^{\circ}$ & $\begin{array}{c}\mathrm{I} \\
59.7\end{array}$ & $\begin{array}{c}\text { II } \\
58.7\end{array}$ \\
\hline$S_{(298)}^{\circ}$ & 106.9 & 108.3 \\
\hline$C_{p(298)}^{\circ}$ & 47.9 & 49.2 \\
\hline$C_{p(600)}^{\circ}$ & 86.9 & 87.5 \\
\hline
\end{tabular}

$\Delta S^{\circ}=1.4 \mathrm{~g} / \mathrm{mole}$

$\Delta H^{\circ}=-1.0 \mathrm{kcal} / \mathrm{mole}$

$\Delta C_{p}^{\circ}=1.3 \mathrm{~g} / \mathrm{mole}$

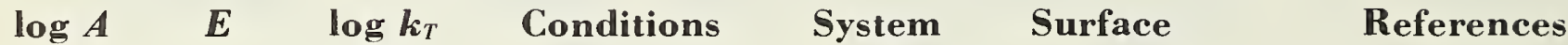

\begin{tabular}{llclll}
$\lg \boldsymbol{A}$ & $\boldsymbol{E}$ & $\begin{array}{c}\log \boldsymbol{k}_{T} \\
(585)\end{array}$ & \multicolumn{1}{c}{ Conditions } & System & Surfar \\
12.78 & 42.8 & -3.21 & $\begin{array}{l}553-615^{\circ} \mathrm{K} \\
4-3588 \mathrm{~mm} \mathrm{Hg}\end{array}$ & static & none
\end{tabular}
[1] G. B. Kistiakowsky and W. R. Smith, J. Am. Chem. Soc. 56, 638 (1934).

\section{Preferred:}

Reliable as reported.

$\log k=12.78-42.8 / \theta$.

Comments: The reaction is undoubtedly unimolecular. Transition state estimates give $\log A_{\text {est }}=12.5$ (see section IV-3.0).

\section{Experimental}

[1] Analysis by miscibility of product in Nujol (trans form was more soluble). The equilibrium constant (cis $\rightleftharpoons$ trans) was found to be about 13.3 at $614^{\circ} \mathrm{K}$, from which a heat of reaction of $\Delta H_{\mathrm{eq}}^{\circ} \simeq-3 \mathrm{kcal} / \mathrm{mole}$ was inferred assuming $\Delta S_{\mathrm{eq}}^{\circ}=0 .^{*}$

*These gas phase equilibrium measurements imply that the cis repulsion is somewhat larger than additivity predictions, but should not exceed $3 \mathrm{kcal} /$ mole. R. B. Williams (J. Am. Chem. Soc. 64, 1395 (1942)), has measured the $\Delta H^{\circ}$ (hydrogenation) of cis stilbene to be $\Delta H_{\left(\mathrm{H}_{2}\right)}^{\circ} \simeq 26.3 \mathrm{kcal} / \mathrm{mole}$, which gives $\Delta H^{\circ}$ (cis $\longrightarrow$ trans) stilbene $=-5.7 \mathrm{kcal} / \mathrm{mole}$. The discrepancy can be rationalized as follows. Styrene is flat in solid and in solution while it is not flat in the gas phase. This is due to $\mathrm{H}$. . H repulsions:

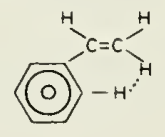

The result is that $\Delta H_{f}^{\circ}$ (gaseous styrene) does not show a butadiene type stabilization. Cis-stilbene cannot be flat even in solution and as a result it probably cannot solvate as well as trans-stilbene (which is flat in solution). Thus it is quite feasible that $\Delta H_{\text {isom }}^{\circ}$ (sol' $n$ ) $=-5.7$ $\mathrm{kcal} /$ mole (as measured by Williams) while $\Delta H_{\text {mas }}^{\circ} \simeq-3.0 \mathrm{kcal} /$ mole as estimated by Kistiakowsky and $\mathrm{Smith}$. 
Reaction: Dimethyl-cis-1,2-ethenedioate (dimethyl maleate)<smiles>COC(=O)/C=C\C(=O)OCC(=O)OC</smiles>

\begin{tabular}{crr} 
& \multicolumn{1}{c}{ I } & \multicolumn{1}{c}{ II } \\
$\Delta H_{f(298)}^{\circ}$ & -147.2 & -148.2 \\
$S_{(298)}^{\circ}$ & 109.6 & 111.0 \\
$C_{p(298)}^{\circ}$ & 39.3 & 40.6
\end{tabular}

$\Delta S^{\circ}=1.4 \mathrm{~g} / \mathrm{mole}$

$\Delta H^{\circ}=-1.0 \mathrm{kcal} / \mathrm{mole}$

$\Delta C_{p}^{\circ}=1.3 \mathrm{~g} / \mathrm{mole}$

\begin{tabular}{|c|c|c|c|c|c|c|}
\hline $\log A$ & $\boldsymbol{E}$ & $\begin{array}{c}\log \boldsymbol{k}_{T} \\
(583)\end{array}$ & Conditions & System & Surface & References \\
\hline 5.11 & 26.5 & -4.82 & $\begin{array}{l}573-593{ }^{\circ} \mathrm{K} \\
732-4060 \text { torr }\end{array}$ & static & & $\begin{array}{l}\text { [1] M. Nelles and G. B. } \\
\text { Kistiakowsky, J. Am. } \\
\text { Chem. Soc. 54, } 2208 \\
\text { (1932); Zeit. physik. } \\
\text { Chemie. (Bodenstein } \\
\text { Festband) } \mathbf{1 5 2 , 3 6 9} \\
\text { (1931). }\end{array}$ \\
\hline
\end{tabular}

Preferred:

Unreliable.

Comments: Arrhenius parameters are much too low to be reasonable. It is likely that similar complications occurred in this reaction as those sometimes found in the cis $\rightarrow$ trans but-2-ene reaction (i.e., heterogeneity and autocatalysis) probably promoted by'small amounts of free acid.

\section{Experimental}

[1] Analysis of mixture by M.P. Rate constants were pressure dependent. A slow decomposition of the fumaric ester was observed. 
Reaction: Methyl cis-2-butenoate (methyl-cis-crotonate)

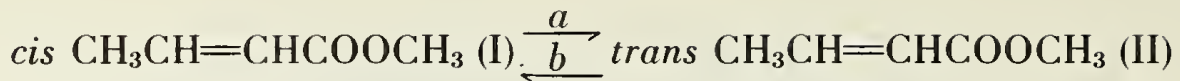

$$
\begin{aligned}
& \begin{array}{ccc} 
& \text { I } & \text { II } \\
\Delta H_{f(298)}^{\circ} & -74.4 & -75.4 \\
S_{(298)}^{\circ} & (91.9)^{*} & 92.3 \\
C_{p(298)}^{\circ} & 29.4 & 30.7
\end{array}
\end{aligned}
$$

$\Delta S^{\circ}=0.4 \mathrm{~g} / \mathrm{mole}$

$\Delta H^{\circ}=-1.0 \mathrm{kcal} / \mathrm{mole}$

$\Delta C_{p}^{\circ}=1.3 \mathrm{~g} / \mathrm{mole}$

\begin{tabular}{|c|c|c|c|c|c|c|c|}
\hline \multirow[t]{3}{*}{ Path } & $\log A$ & $\boldsymbol{E}$ & $\begin{array}{c}\log \boldsymbol{k}_{T} \\
(750)\end{array}$ & Conditions & System & Surface & References \\
\hline & 13.2 & $57.8 \pm 1.5$ & -3.64 & $\begin{array}{l}673-833^{\circ} \mathrm{K} \\
0.1 \rightarrow 10 \mathrm{~mm} \\
(10)\end{array}$ & static & none & $\begin{array}{l}\text { [1] J. N. Butler and } \\
\text { G. J. Small, } \\
\text { Can. J. Chem. 41, } \\
2492 \text { (1963). }\end{array}$ \\
\hline & 12.6 & 58.0 & -4.30 & (as above) & & & (as above) \\
\hline
\end{tabular}

Preferred:

Reliable as reported. $\log A_{\text {est }}=13.2$; est $E_{\text {act }}=57.6 \mathrm{kcal} / \mathrm{mole}$ using a resonance stabilization in the biradical of $4 \mathrm{kcal} / \mathrm{mole}$ (see section IV-3.0).

\section{Experimental}

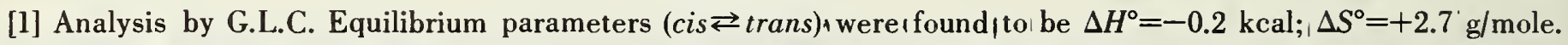
Up to 1 percent added oxygen did not affect the rate. Simultaneous free radical reactions were also detected leading to vinylacetate, $\mathrm{CO}_{2}$, butenes, $\mathrm{CO}, \mathrm{CH}_{4}$, and propylene.

*The estimated thermodynamics are in poor agreement with the observed equilibrium constant. There are obviously some poorly understood steric effects here. (See also cis-2-methyl penta-1,3-diene.) 
Reaction: Methyl-cis-cinnamate<smiles>COC(=O)/C=C/C=C/C=O</smiles>

$\Delta S^{\circ}=1.4 \mathrm{~g} / \mathrm{mole}$

$\Delta H^{\circ}=-1.0 \mathrm{kcal} / \mathrm{mole}$

$\Delta C_{p}^{\circ}=1.3 \mathrm{~g} / \mathrm{mole}$

\begin{tabular}{|c|c|c|c|c|c|c|}
\hline $\log A$ & $\boldsymbol{E}$ & $\begin{array}{c}\log \boldsymbol{k}_{T} \\
(610)\end{array}$ & Conditions & System & Surface & References \\
\hline 10.54 & $\begin{array}{r}41.6 \\
\pm 2.0\end{array}$ & -4.36 & $\begin{array}{l}563-660^{\circ} \mathrm{K} \\
5-500 \text { torr }\end{array}$ & static & none & $\begin{array}{l}\text { [1] G. B. Kistiakowsky and } \\
\text { W. R. Smith, J. Am. } \\
\text { Chem. Soc. 57, } 269 \\
\text { (1935). }\end{array}$ \\
\hline
\end{tabular}

Preferred:

$\log k=12.6-47.4 / \theta$. (see section IV-3.0).

\section{Comments:}

Arrhenius parameters seem too low. Experimental errors were fairly large; therefore the calculated parameters are preferred. They are compatible with a $6 \mathrm{kcal}$ resonance energy in the carbonyl system:<smiles>[R]OC(=O)C([R])C</smiles>

Experimental

[1] Analysis by mixed M.P. Some falling off of the rate constant at pressures below 5 torr appeared to occur. 
Reaction: cis-2-Butenenitrile(cis-crotonitrile)

\begin{tabular}{ccc} 
cis $\cdot \mathrm{CH}_{3} \mathrm{CH}=\mathrm{CHCN}(\mathrm{I})$ & \multicolumn{3}{c}{ trans $-\mathrm{CH}_{3} \mathrm{CH}=\mathrm{CHCN}(\mathrm{II})$} \\
$\Delta H_{f(298)}^{\circ}$ & 37.0 & I \\
$S_{(298)}^{\circ}$ & 72.4 & 72.8 \\
$C_{p(298)}^{\circ}$ & 19.3 & 20.6
\end{tabular}

$* \Delta S^{\circ}=+0.4 \mathrm{~g} /$ mole

$\Delta H^{\circ}=-1.0 \mathrm{kcal} / \mathrm{mole}$

$\Delta C_{p}^{\circ}=1.3 \mathrm{~g} / \mathrm{mole}$

\begin{tabular}{|c|c|c|c|c|c|c|}
\hline $\log A$ & $\boldsymbol{E}$ & $\begin{array}{c}\log \boldsymbol{k}_{T} \\
(750)\end{array}$ & Conditions & System & Surface & References \\
\hline 11.0 & 51.3 & -3.96 & $\begin{array}{l}573-633^{\circ} \mathrm{K} \\
0.2-20 \text { torr }\end{array}$ & static & $\left({ }^{a}\right)$. & $\begin{array}{l}\text { [1] J. N. Butler and R. D. } \\
\text { McAlpine, Can. J. } \\
\text { Chem. 41, 2487 (1963). }\end{array}$ \\
\hline
\end{tabular}

Preferred:

$\log k=12.7-55.7 / \theta$ (see section IV-3.0).

Comments: Both rate constant parameters seem low, $A$ possibly by as much as 2 orders of magnitude. Transition state calculations are preferred and are consistent with a $(>\mathrm{C}-\mathrm{C} \equiv \mathrm{N})$ resonance energy of about $9 \mathrm{kcal} / \mathrm{mole}$.

\section{Experimental}

[1] Analysis by G.L.C.

*Predicted thermodynamics are slightly different from those observed.

a The only appreciable side reaction was surface polymerization. Experimental equilibrium parameters between $573-633^{\circ} \mathrm{K}$ were $\Delta H^{\circ}=0.17 \mathrm{kcal}$ and $\Delta S^{\circ}=-0.39 \mathrm{~g} / \mathrm{mole}$. Rate constants showed small pressure dependences. Error limits for the activation energy were placed at $\pm 3.7 \mathrm{kcal} / \mathrm{mole}$. 
Reaction: cis- $\beta$-Cyanostyrene<smiles>N#CC=CO</smiles>

$\Delta H_{f(298)}^{\circ}$

$S_{(298)}^{\circ}$

$C_{p(298)}^{\circ}$<smiles>N#CC=CO</smiles>

II

I

68.4

90.0

33.5

91.4

34.8

$\Delta S^{\circ}=1.4 \mathrm{~g} / \mathrm{mole}$

$\Delta H^{\circ}=-1.0 \mathrm{kcal} / \mathrm{mole}$

$\Delta C_{p}^{\circ}=1.3 \mathrm{~g} / \mathrm{mole}$

$\begin{array}{lll}\log A & E \quad \begin{array}{l}\log k_{T} \\ (615)\end{array}\end{array}$

11.6

\section{$-4.74$}

Conditions System

static (a)

\section{References}

[1] G. B. Kistiakowsky and W. R. Smith, J. Am. Chem. Soc. 58, 2428 (1936).

\section{Preferred:}

$\log k=12.3-48.0 / \theta$.

Comments: Wall effects were indicated both from the (S/V) dependence and the initial pressure dependence. However the reported parameters are in reasonable agreement with transition state estimates. The latter are preferred.

A $10 \mathrm{kcal}$ resonance energy for $(>\dot{\mathrm{C}}-\mathrm{C} \equiv \mathrm{N})$ is calculated from $E$.

\section{Experimental}

[1] Analysis by refractive index $\left(N_{r}\right)$. The equilibrium constant was found to be $K($ trans/cis $)=1.7$ and temperature independent.

\footnotetext{
a $A$ marked increase in rate at the low pressures was observed with increased (S/V). Rates also increased at the lower pressures.
} 
Reaction: $1,1^{\prime}, 6,6^{\prime}-d_{4}-1,5-$ Hexadiene

\begin{tabular}{|c|c|c|}
\hline$\Delta H_{f(298)}^{\circ}$ & $\begin{array}{c}\text { I } \\
16.5\end{array}$ & $\begin{array}{c}\text { II } \\
16.5\end{array}$ \\
\hline$S_{(298)}^{\circ}$ & 92.2 & 92.2 \\
\hline$C_{p(298)}^{\circ}$ & 29.9 & 29.9 \\
\hline
\end{tabular}

$$
\begin{aligned}
& \Delta S^{\circ}=0 \\
& \Delta H^{\circ}=0 \\
& \Delta C_{p}^{\circ}=0
\end{aligned}
$$

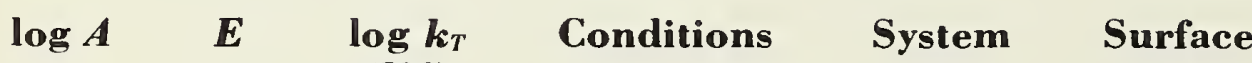

\section{References} (530)
11.1
35.5
$-3.50$
$(530 \%)$

[1] V. Toscano and W. von E. Doering, unpublished work.

Preferred: Reliable.

$\log k=11.1-35.5 / \theta$.

Comments: Transition state estimate of $A$ gives $\log A_{\text {est }}=10.7$ (see section IV-1.0).

\section{Experimental}

[1] Unpublished work. "Cope" rearrangement. 
Reaction: 1,5-Heptadiene

\begin{tabular}{|c|c|c|}
\hline & I & II \\
\hline$\Delta H_{f(298)}^{\circ}$ & 12.4 & 12.0 \\
\hline$S_{(298)}^{\circ}$ & 99.4 & 96.9 \\
\hline$C_{p(298)}^{\circ}$ & 34.0 & 36.6 \\
\hline$C_{p(500)}^{\circ}$ & 49.0 & 51.1 \\
\hline
\end{tabular}

$\Delta S^{\circ}=-2.5 \mathrm{~g} / \mathrm{mole}$
$\Delta H^{\circ}=-0.4 \mathrm{kcal} / \mathrm{mole}$
$\Delta C_{p}^{\circ}=2.6 \mathrm{~g} / \mathrm{mole}$

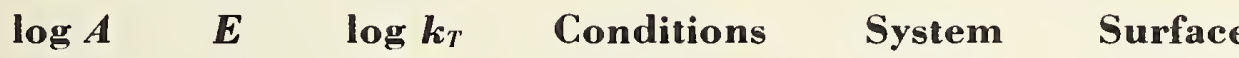
(485)

$\begin{array}{rlll}9.09 & 32.5 \quad-5.55 & 451-523{ }^{\circ} \mathrm{K} \quad \text { static } \\ & 2 \text { torr }\end{array}$

[1] A. Amano and M. Uchiyama, J. Phys. Chem. 69, 1278 (1965).

Preferred:

$\log A_{\text {est }}=11.1$

Comments: Arrhenius parameters are certainly too low, although rate constants are reasonable. See comments on the reverse reaction and also. section III-2.0.

\section{Experimental}

[1] Analysis by G.L.C. Radical-induced decomposition was not detected. 
Reaction: 3-Methyl-1,5-hexadiene

$\begin{array}{lcc}\Delta H_{f(298)}^{\circ} & \text { II } & \text { II } \\ S_{(298)}^{\circ} & 12.0 & 12.4 \\ C_{p(298)}^{\circ} & 96.9 & 99.4 \\ C_{p(500)}^{\circ} & 36.6 & 34.0\end{array}$

$\Delta S^{\circ}=2.5 \mathrm{~g} / \mathrm{mole}$

$\Delta H^{\circ}=0.4 \mathrm{kcal} / \mathrm{mole}$

$\Delta C_{p}^{\circ}=-2.6 \mathrm{~g} / \mathrm{mole}$

\begin{tabular}{|c|c|c|c|c|c|c|}
\hline $\log A$ & $\boldsymbol{E}$ & $\begin{array}{c}\log \boldsymbol{k}_{T} \\
(485)\end{array}$ & Conditions & System & Surface & References \\
\hline 9.84 & 32.5 & -4.80 & $\begin{array}{l}451-523^{\circ} \mathrm{K} \\
2 \text { torr }\end{array}$ & static & & $\begin{array}{l}\text { [1] A. Amano and M. } \\
\text { Uchiyama, J. Phys. } \\
\text { Chem. 69, } 1278 \text { (1965). }\end{array}$ \\
\hline 10.85 & $* 34.95$ & & & & & $\begin{array}{l}\text { [2] H. M. Frey (private } \\
\text { communication)* to be } \\
\text { published. }\end{array}$ \\
\hline
\end{tabular}

\section{Preferred:}

$\log k=10.85-34.95 / \theta$.

Comments: Cope rearrangement. This reaction is probably unimolecular under the conditions studied but the Arrhenius parameters are unreasonably low. The transition state estimate of $A$ gives $\log A_{\text {est }}=11.3$. Further evidence against the reported $A$-factor may be found in the analogous isomerization

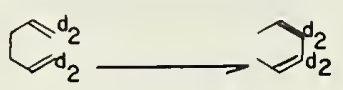

whose $A$-factor is $\log A=11.1$.

\section{Experimental}

[1] Rate and analysis by G.L.C. The isomerization proceeded to the equilibrium state, giving an equilibrium constant for the reaction (as written) of $K_{\mathrm{eq}} \simeq 5.6$, independent of the temperature. The absence of propylenes and butenes in the products was cited as evidence against the formation of allylic radicals in the primary act (i.e., bond fission followed by recombination).

[2] The system is well represented by,

$$
\text { 3-methylhexa-1,5-diene } \begin{array}{rl}
1 & \geqslant 2 \\
2 & \text { trans hepta-1,5-diene } \\
4 & 3 \text { cis hepta-1,5-diene. }
\end{array}
$$

All processes are important.

Arrhenius parameters are:

$\log k_{1}=10.55-34.20 / \theta$

$\log k_{2}=10.39-35.34 / \theta$

$\log k_{3}=10.54-35.72 / \theta$

$\log k_{4}=10.66-36.72 / \theta$.

* Results added to data sheet after its original preparation. Comments have been left in their orixinal form to illustrate the degree of reliability inherent in the transition state estimates. 
Reaction: 3-Vinyloxy-1-propene (vinyl allyl ether)

\begin{tabular}{|c|c|c|}
\hline$\Delta H_{f(298)}^{\circ}$ & $\begin{array}{c}I \\
-8.7\end{array}$ & $\begin{array}{c}\text { II } \\
-25.8\end{array}$ \\
\hline$S_{(298)}^{\circ}$ & 90.0 & 89.3 \\
\hline$C_{p(298)}^{\circ}$ & 26.9 & 28.1 \\
\hline
\end{tabular}

$\Delta S^{\circ}=-0.7 \mathrm{~g} / \mathrm{mole}$

$\Delta H^{\circ}=-17.1 \mathrm{kcal} / \mathrm{mole}$

$\Delta C_{p}^{\circ}=1.2 \mathrm{~g} / \mathrm{mole}$

\begin{tabular}{|c|c|c|c|c|c|c|}
\hline $\log A$ & $\boldsymbol{E}$ & $\begin{array}{r}\log \boldsymbol{k}_{\boldsymbol{T}} \\
(455)\end{array}$ & Conditions & System & Surface & References \\
\hline 11.70 & 30.6 & -2.99 & $\begin{array}{l}440-473^{\circ} \mathrm{K} \\
150-400 \text { torr }\end{array}$ & static & none & $\begin{array}{l}\text { [1] F. W. Schuler and G. W. } \\
\text { Murphy, J. Am. Chem. } \\
\text { Soc. 72, } 3155 \text { (1950). }\end{array}$ \\
\hline
\end{tabular}

Preferred:

$\log k=11.70-30.6 / \theta$.

Comments: Reliable. Transition state calculation of the $A$-factor gives $\log A_{\text {est }}=11.1$ (see section IV-1.0).

\section{Experimental}

[1] Analysis by U.V. absorption of the aldehyde. Reaction was first order out to 99 percent reaction. Cope rearrangement. 
Reaction: 3-Isopropenoxy-1-propene (isopropenyl allyl ether)<smiles>C=C(C)OCC=CCC(=O)CCC=CC</smiles>

\begin{tabular}{ccc} 
& \multicolumn{1}{c}{ I } & II \\
$\Delta H_{f(298)}^{\circ}$ & -18.1 & -37.0 \\
$S_{(298)}^{\circ}$ & 95.9 & 98.0 \\
$C_{p(298)}^{\circ}$ & 33.1 & 32.9
\end{tabular}

$\Delta S^{\circ}=2.1 \mathrm{~g} / \mathrm{mole}$

$\Delta H^{\circ}=-18.9 \mathrm{kcal} / \mathrm{mole}$

$\Delta C_{p}^{\circ}=-0.2 \mathrm{~g} / \mathrm{mole}$

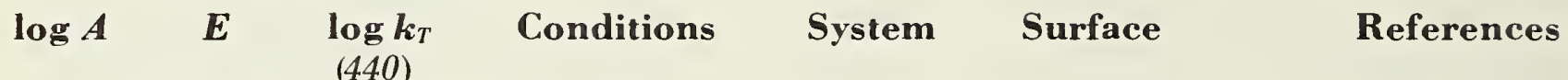

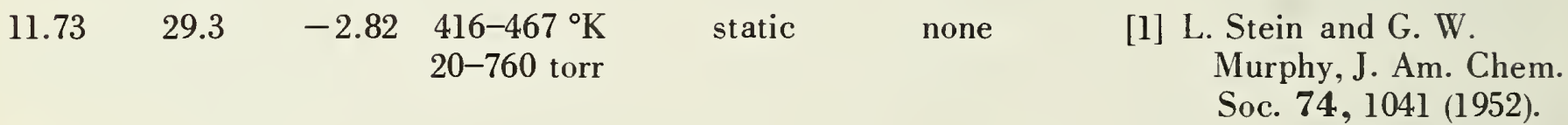

Preferred:

$\log k=11.73-29.3 / \theta$.

Comments: Transition state estimate gives, $\log A=11.3$ (see section IV-1.0).

\section{Experimental}

[1] Analysis by U.V. on the ketone. Unconditioned surfaces produced rate acceleration. Cope rearrangement. 
Reaction: 2-Methyl-3-vinyloxy-1-propene (2-methallyl vinyl ether)

\begin{tabular}{|c|c|c|}
\hline$\Delta H_{f(298)}^{\circ}$ & $\begin{array}{c}\text { I } \\
-16.4\end{array}$ & $\begin{array}{c}\text { II } \\
-32.4\end{array}$ \\
\hline$S_{(298)}^{\circ}$ & 96.4 & 97.4 \\
\hline$C_{p(\mathbf{2 9 8 )}}^{\circ}$ & 32.9 & 33.7 \\
\hline
\end{tabular}

$\Delta S^{\circ}=1.0 \mathrm{~g} / \mathrm{mole}$

$\Delta H^{\circ}=-16.0 \mathrm{kcal} / \mathrm{mole}$

$\Delta C_{p}^{\circ}=0.8 \mathrm{~g} / \mathrm{mole}$

\begin{tabular}{|c|c|c|c|c|c|c|}
\hline $\log A$ & $\boldsymbol{E}$ & $\begin{array}{l}\log \boldsymbol{k}_{T} \\
(440)\end{array}$ & Conditions & System & Surface & References \\
\hline 11.15 & 29.1 & -3.30 & $\begin{array}{l}423-461^{\circ} \mathrm{K} \\
6-100 \text { torr } \\
(10-15)\end{array}$ & static & none & $\begin{array}{l}\text { [1] H. M. Frey and B. M. } \\
\text { Pope, J. Chem. Soc. B, } \\
209(1966) .\end{array}$ \\
\hline
\end{tabular}

Preferred:

$\log k=11.15-29.1 / \theta$.

Comments: Transition state estimation of $A$ gives $\log A_{\text {est }}=11.3$ (same as vinyl allyl ether and vinyl isopropenyl allyl ether, see section IV-1.0).

\section{Experimental}

[1] Analysis by G.L.C. It was concluded, very reasonably, that a six-centered ring complex was involved in the transition state, since a four-centered ring complex would lead to 3-methylpent-4-enal (not observed) and since the observed $A$-factor is quite similar to those found for allyl and isopropenyl allyl ethers. Cope rearrangement.

*The product of this reaction was mistakenly reported as trans-hex-4-enal. 
Reaction: 1-Buten-3-yl vinyl ether

$\begin{array}{lcr} & \text { I } & \text { II } \\ & -18.0 & -33.2 \\ \Delta H_{f(298)}^{\circ} & 96.3 & 97.8 \\ S_{(298)}^{\circ} & 33.0 & 33.4 \\ C_{p(298)}^{\circ} & & \end{array}$

$\Delta S^{\circ}=\quad 1.5 \mathrm{~g} / \mathrm{mole}$

$\Delta H^{\circ}=-15.2 \mathrm{kcal} / \mathrm{mole}$

$\Delta C_{p}^{\circ}=0.4 \mathrm{~g} / \mathrm{mole}$

\begin{tabular}{|c|c|c|c|c|c|c|c|}
\hline \multirow[t]{2}{*}{ Path } & $\log A$ & $\boldsymbol{E}$ & $\begin{array}{r}\log k \\
(440)\end{array}$ & Conditions & System & Surface & Referen \\
\hline & 11.32 & 27.87 & & $\begin{array}{l}423-461^{\circ} \mathrm{K} \\
6-100 \text { torr }\end{array}$ & $\begin{array}{l}\text { static, } \\
\text { analysis } \\
\text { G.L.C. }\end{array}$ & $\begin{array}{l}\text { No effect } \\
(14 \times \mathrm{x} / \mathrm{V}) \\
\text { in aged } \\
\text { vessels }\end{array}$ & $\begin{array}{l}\text { H. M. Frey, } \\
\text { unpublished } \\
\text { results }\end{array}$ \\
\hline
\end{tabular}

Preferred:

$\log k=11.32-27.87 / \theta$

Comments: Reliable. Y. Pocker, Proc. Chem. Soc., 141 (1961), has shown that the rate of loss of optical activity of active ether is nearly identical with its rate of conversion to aldehyde, thus verifying a single, irriversible intramolecular reaction path.

\section{Experimental}

[1] Analysis was by G.L.C. Product confirmation by N.M.R. and I.R. 


\begin{tabular}{|c|c|c|}
\hline$\Delta H_{f(298)}^{\circ}$ & $\begin{array}{c}\text { I } \\
57.3\end{array}$ & $\begin{array}{c}\text { II } \\
26.4\end{array}$ \\
\hline$S_{(298)}^{\circ}$ & 96.8 & 94.6 \\
\hline$C_{p(298)}^{\circ}$ & 32.6 & 34.0 \\
\hline
\end{tabular}

$\Delta S^{\circ}=-2.2 \mathrm{~g} / \mathrm{mole}$

$\Delta H^{\circ}=-20.9 \mathrm{kcal} / \mathrm{mole}$

$\Delta C_{p}^{\circ}=1.4 \mathrm{~g} / \mathrm{mole}$

$\begin{array}{ccccccc}\log \boldsymbol{A} & \boldsymbol{E} & \begin{array}{c}\log \boldsymbol{k}_{T} \\ (470)\end{array} & \text { Conditions } & \text { System } & \text { Surface } & \text { References } \\ 9.97 & 28.47 & -3.27 & \begin{array}{l}445-491^{\circ} \mathrm{K} \\ 0.5-20 \text { torr }\end{array} & \text { static } & \text { none } & \text { [1] H. M. Frey and D. H. } \\ \text { Lister, J. Chem. Soc. A, } \\ \end{array}$

Preferred:

$\log k=9.97-28.47 / \theta$.

Comments: Cope rearrangement. Reliable study, although the Arrhenius parameters are somewhat lower than those estimated by transition state methods. $\log A_{\text {est }}=10.8$ (see section IV-1.0).

\section{Experimental}

[1] Analysis was by G.L.C. Product confirmation by N.M.R. and I.R. 
Reaction: cis-1,3,5-Hexatriene

\begin{tabular}{ccc} 
& \multicolumn{1}{c}{$\longrightarrow$ (I) } & \\
$\Delta H_{f(298)}^{\circ}$ & 40.8 & 26.3 \\
$S_{(298)}^{\circ}$ & 78.0 & 72.1 \\
$C_{p(298)}^{\circ}$ & 27.3 & 23.4
\end{tabular}

$\Delta S^{\circ}=-5.9 \mathrm{~g} / \mathrm{mole}$

$\Delta H^{\circ}=-14.5 \mathrm{kcal} / \mathrm{mole}$

$\Delta C_{p}^{\circ}=-3.9 \mathrm{~g} / \mathrm{mole}$

\begin{tabular}{|c|c|c|c|c|c|c|}
\hline $\log A$ & $\boldsymbol{E}$ & $\begin{array}{c}\log \boldsymbol{k}_{T} \\
\left(425^{\circ}\right)\end{array}$ & Conditions & System & Surface & References \\
\hline 11.85 & 29.9 & -3.52 & $\begin{array}{l}390-463^{\circ} \mathrm{K} \\
60-200 \text { torr }\end{array}$ & static & none & $\begin{array}{l}\text { [1] K. E. Lewis and H. } \\
\text { Steiner, J. Chem. Soc. } \\
3080 \text { (1964). }\end{array}$ \\
\hline
\end{tabular}

Preferred:

$\log k=11.85-29.9 / \theta$

$A_{\text {est }}=10^{11.5} \mathrm{sec}^{-1}$ (see section IV-1.0).

\section{Experimental}

[1] Rates followed by U.V. spectroscopy. Calculated $E_{a}$ using the Polanyi-Evans method agreed with the observed value. $A$-factor calculations based on cyclic and linear activated complexes gave the best agreement with the cyclic complex.

$A \neq($ cyclic $) \simeq 4-8 \times 10^{11} \mathrm{sec}^{-1}$

$A \ddagger($ linear $)=1-4 \times 10^{13} \mathrm{sec}^{-1}$. 
Reaction: cis-1,3-Hexadiene

$\begin{array}{ccc}r & \Gamma_{(\mathrm{I})} & \mathrm{I}^{\text {(II) }} \\ H_{f(298)}^{\circ} & 14.6 & \mathrm{II} \\ S_{(298)}^{\circ} & 84.9 & 83.6 \\ C_{p(298)}^{\circ} & 28.5 & 28.6\end{array}$

$\Delta S^{\circ}=-1.3 \mathrm{~g} / \mathrm{mole}$

$\Delta H^{\circ}=-2.5 \mathrm{kcal} / \mathrm{mole}$

$\Delta C_{p}^{\circ}=0.1 \mathrm{~g} / \mathrm{mole}$

\begin{tabular}{|c|c|c|c|c|c|c|}
\hline $\log A$ & $\boldsymbol{E}$ & $\begin{array}{c}\log \boldsymbol{k}_{T} \\
\left(500^{\circ}\right)\end{array}$ & Conditions & System & Surface & References \\
\hline 10.8 & 32.5 & -3.42 & $\begin{array}{l}474-518{ }^{\circ} \mathrm{K} \\
2-70 \text { torr }\end{array}$ & static & none & $\begin{array}{l}\text { [1] H. M. Frey and B. M. } \\
\text { Pope, J. Chem. Soc. A, } \\
1701 \text { (1966). }\end{array}$ \\
\hline
\end{tabular}

Preferred:

$\log k=10.8-32.5 / \theta$.

Comments: $\log A_{\text {est }}=11.9$. The non-polar "ene" reactions seem to have $A$-factors about an order of magnitude lower than calculated. (See also cis-2-methyl penta-1,3-diene and its reverse.)

\section{Experimental}

[1] Rates were based on G.L.C. analysis. The equilibria were found to be too far on the product side to measure. (This is not reflected in the thermodynamic estimates which suggest $K_{\text {eq }}\left(500^{\circ} \mathrm{K}\right) \simeq 6.5$.) 
Reaction: 2-Methyl-cis-1,3-pentadiene

$\begin{array}{ccc} & \rangle(\mathrm{I}) & \\ & \mathrm{I} & \mathrm{II} \\ \Delta H_{f(298)}^{\circ} & 11.7 & 11.4 \\ S_{(298)}^{\circ} & * 83.6 & 83.7 \\ C_{p(298)}^{\circ} & 29.1 & 29.5 \\ C_{p(509)}^{\circ} & 40.0 & 40.6\end{array}$

$\Delta S^{\circ}=0.1 \mathrm{~g} / \mathrm{mole}^{*}$

$\Delta H^{\circ}=-0.3 \mathrm{kcal} / \mathrm{mole}^{*}$

$\Delta C_{p}^{\circ}=0.4 \mathrm{~g} / \mathrm{mole}$

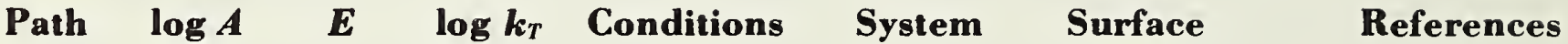

$a$

$\begin{array}{llll}11.24 & 32.76 \quad-3.22 & 473-510^{\circ} \mathrm{K} \quad \text { static } \\ 2-15 \mathrm{~mm}\end{array}$

none

[1] H. M. Frey and R. J. Ellis, J. Chem Soc. 4770 (1965).

Preferred:

$\log k=11.24-32.76 / \theta$.

Comments: The transition state calculation of $A$ for a concerted "ene"-six-center reaction gives $\log A_{\text {est }}=11.1$.

\section{Experimental}

[1] Analysis by G.L.C. The reaction proceeds to an equilibrium state. The equilibrium constant found for the reaction as written above was $K_{e q}=10^{-0.477+3.43 / \theta}$. (A correction to the sign of the entropy change as reported has been made.)

*These values indicate interesting cis-cis methyl-methyl next to nearest neighbor interactions (i.e., an entropy enhancement of $2 \mathrm{~g} / \mathrm{mole}$ and a steric repulsion of $3.4 \mathrm{kcal} / \mathrm{mole}$ in the reactant. Note that the estimated thermochemistry using group values (nearest neighbor interactions only) do not show this cis-cis effect. A similar (but opposite) effect is apparent in the equilibrium constant found for $c i s \rightleftarrows \rightleftarrows$ trans methyl crotonate. This strain energy is about the same as the double bond interaction in butadiene ( $3.6 \mathrm{kcal})$, and this would be compatible with a non-planar configuration of the double bonds in the cis-diene. Such a structure would have about $2 \mathrm{~g} / \mathrm{mole}$ more entropy from optical isomerism, plus a lower frequency in the $(=\mathrm{CH} \mathrm{CH}=)$ torsion. 
Reaction: 4-Methyl-1,3-pentadiene

\begin{tabular}{|c|c|c|}
\hline$\Delta H_{f(298)}^{\circ}$ & $\begin{array}{c}\text { I } \\
11.4\end{array}$ & $\begin{array}{c}\text { II } \\
11.7\end{array}$ \\
\hline$S_{(298)}^{\circ}$ & 83.7 & 83.6 \\
\hline$C_{p(298)}^{\circ}$ & 29.5 & 29.1 \\
\hline
\end{tabular}

$\Delta S^{\circ}=-0.1 \mathrm{~g} / \mathrm{mole}$

$\Delta H^{\circ}=0.3 \mathrm{kcal} / \mathrm{mole}$

$\Delta C_{p}^{\circ}=-0.4 \mathrm{~g} / \mathrm{mole}$

\begin{tabular}{ccccccc}
$\log \boldsymbol{A}$ & $\boldsymbol{E}$ & $\begin{array}{c}\log \boldsymbol{k}_{T} \\
(495)\end{array}$ & Conditions & System & Surface & \multicolumn{1}{c}{ References } \\
11.72 & 36.19 & -4.25 & $\begin{array}{l}473-510^{\circ} \mathrm{K} \\
2-15 \text { torr }\end{array}$ & static & none & [1] H. M. Frey and R. J. \\
Ellis, J. Chem. Soc. \\
\end{tabular}

Preferred:

$\log k=11.72-36.16 / \theta$.

Comments: Estimated thermodynamic properties are not in very good agreement with the kinetic observations on the equilibrium properties of the reaction. (See cis-2-methylpenta-1,3-diene.)

$\log A_{\text {est }}=12.5$ (see section IV-2.0).

\section{Experimental}

[1] See cis-2-methylpenta-1,3-diene. 
Reaction: 3,7-Dimethyl-1,6-octadiene

\begin{tabular}{|c|c|c|}
\hline$\Delta H_{f(298)}^{\circ}$ & $\begin{array}{c}\mathrm{I} \\
-17.6\end{array}$ & $\begin{array}{c}\text { II } \\
-23.6\end{array}$ \\
\hline$S_{(298)}^{\circ}$ & 122.0 & 113.8 \\
\hline$C_{p(298)}^{\circ}$ & 52.2 & 51.7 \\
\hline$C_{p(700)}^{\circ}$ & 94.5 & 94.2 \\
\hline
\end{tabular}

$\Delta S^{\circ}=-8.2 \mathrm{~g} / \mathrm{mole}$

$\Delta H^{\circ}=-6.0 \mathrm{kcal} / \mathrm{mole}$

$\Delta C_{p}^{\circ}=-0.5 \mathrm{~g} / \mathrm{mole}$

$\begin{array}{llllll}\log A & \boldsymbol{E} & \begin{array}{c}\log \boldsymbol{k}_{T} \\ (670)\end{array} & \text { Conditions } & \text { System } & \text { Surface } \\ 9.06 & 35.2 & -2.42 & 656-682^{\circ} \mathrm{K} & \text { flow }\end{array}$
[1] W. D. Huntsman and T. H. Curry, J. Am. Chem. Soc. 80, 2252 (1958).

\section{References}

Preferred:

$\log k=9.06-35.2 / \theta$.

Comments: Transition state estimates of the $A$-factor suggest that the rate constant parameters may be slightly low.

$$
\log A_{\text {est }} \simeq 9.6 \text { (see section IV-1.0). }
$$

\section{Experimental}

[1] Analysis by I.R. and quantitative hydrogenation. The rate was unaffected by radical chain sensitizers (ethylene oxide and $t$-butyl peroxide) and by NO. It was suggested that the reaction proceeded through a bicyclic (five-center and six-center) transition state with $(C-C)$ and $\left(C^{\prime} \backslash_{C}\right)$ bridges. The energetics exclude a biradical mechanism. The reaction must be concerted. 
Reaction: Methyl isocyanide

\begin{tabular}{ccc} 
& $\mathrm{CH}_{3} \mathrm{NC}(\mathrm{I})$ & \multicolumn{2}{c}{$\mathrm{CH}_{3} \mathrm{CN}(\mathrm{II})$} \\
& $\mathrm{I}$ & $\mathrm{II}$ \\
$\Delta H_{f(298)}^{\circ}$ & 35.9 & 19.2 \\
$S_{(298)}^{\circ}$ & 59.1 & 58.7 \\
$C_{p(298)}^{\circ}$ & 12.8 & 12.5
\end{tabular}

$\Delta S^{\circ}=-0.4 \mathrm{~g} / \mathrm{mole}$

$\Delta H^{\circ}=-16.7 \mathrm{kcal} / \mathrm{mole}$

$\Delta C_{p}^{\circ}=-0.3 \mathrm{~g} / \mathrm{mole}$

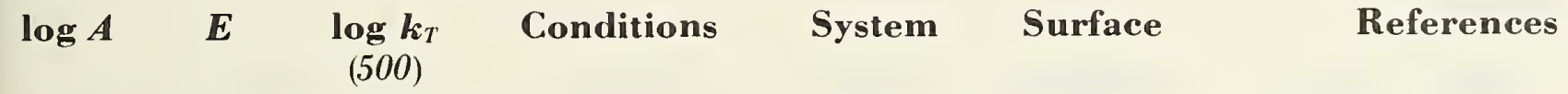

$\begin{array}{lll}13.6 \quad 38.4 \quad-3.18 \quad \begin{array}{l}472-533^{\circ} \mathrm{K} \\ 10^{-2.5}-10^{5} \text { torr }\end{array} & \text { static none }\end{array}$

[1] F. W. Schneider and B. S. Rabinovitch, J. Am. Chem. Soc. 84, 4215 (1962).

Preferred:

$\log k=13.6-38.4 / \theta$.

\section{Experimental}

[1] Analysis by G.L.C. Added air $(\sim 5$ percent) and $<0.15$ percent $t$-butyl peroxide gave no rate acceleration. Falloff behavior studied in detail. Rates began to falloff at about 1000 torr at $200{ }^{\circ} \mathrm{C}$. The methyl- $d_{1}$ and methyl- $d_{3}$ isotope effects for the methyl isocyanide have been studied by B. S. Rabinovitch, P. W. Gilderson, and F. W. Schneider, J. Am. Chem. Soc. 87, 158 (1965) and·by F. W. Schneider and B. S. Rabinovitch, J. Am. Chem. Soc. 85, 2365 (1963) respectively. This reaction has also been studied at high temperatures behind reflected shocks in the falloff region by A. Lifshitz, H. F. Caroll, and S. H. Bauer, J. Am. Chem. Soc. 86, 1488 (1964). 
Reaction: $p$-Tolyl isocyanide

$\begin{array}{ccc} & -\square-\mathrm{CN}(\mathrm{II}) & \\ \Delta \mathrm{H}_{f(298)}^{\circ} & 61 & \text { II } \\ S_{(298)}^{\circ} & 87 & 84.4 \\ C_{p(298)}^{\circ} & 33 & 33\end{array}$

$\Delta S^{\circ}=0 \mathrm{~g} /$ mole

$\Delta H^{\circ}=-16.6 \mathrm{kcal} / \mathrm{mole}$

$\Delta C_{p}^{\circ}=0 \mathrm{~g} / \mathrm{mole}$

\begin{tabular}{|c|c|c|c|c|c|c|}
\hline $\log A$ & $\boldsymbol{E}$ & $\begin{array}{c}\log \boldsymbol{k}_{T} \\
(475)\end{array}$ & Conditions & System & Surface & References \\
\hline 12.5 & 33.8 & -3.05 & $\begin{array}{l}453-493^{\circ} \mathrm{K} \\
17-30 \text { torr }\end{array}$ & static & & $\begin{array}{l}\text { [1] G. Kohlmaier and } \\
\text { B. S. Rabinovitch, J. } \\
\text { Phys. Chem. 63, } 1793 \\
\text { (1959). }\end{array}$ \\
\hline
\end{tabular}

Preferred:

$\log k=13.7-36.8 / \theta$. Rate constants are reliable.

Comments: The solution results suggest that the Arrhenius parameters reported for the gas phase isomerization may be slightly low. Solution parameters are preferred.

\section{Experimental}

[1] Analysis by I.R. Rates in solution of nujol gave $k=10^{13.7} \times 10^{-36.8} \theta \mathrm{sec}^{-1}$. 
Reaction: (l)2,2'-Diamino-6,6' -dimethyldiphenyl

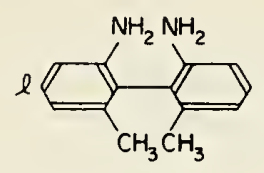

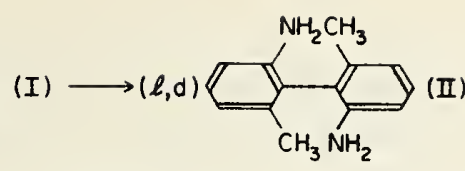

$\Delta H_{f(298)}^{\circ}$

$S_{(298)}^{\circ}$

$C_{p(298)}^{\circ}$

$$
\begin{aligned}
& \Delta S^{\circ}= \\
& \Delta H^{\circ}= \\
& \Delta C_{p}^{\circ}=
\end{aligned}
$$

\begin{tabular}{|c|c|c|c|c|c|c|}
\hline $\log A$ & $\boldsymbol{E}$ & $\begin{array}{c}\log k_{T} \\
(635)\end{array}$ & Conditions & System & Surface & References \\
\hline 10.37 & 45.1 & -5.15 & $\begin{array}{l}615-660{ }^{\circ} \mathrm{K} \\
50-150 \mathrm{~mm} \mathrm{Hg}\end{array}$ & static & & $\begin{array}{l}\text { [1] G. B. Kistiakowsky and } \\
\text { W. R. Smith, J. Am. } \\
\text { Chem. Soc. 58, } 1043 \\
\text { (1936). }\end{array}$ \\
\hline
\end{tabular}

Preferred:

$\log k=11.0-46.9 / \theta$.

Comments: The solution $A$-factor seems the more reasonable.

\section{Experimental}

[1] Rates of racemization were followed by optical rotation measurements. The racemization kinetics were also followed in solution. Activation energies were identical but the liquid phase $A$-factor was higher by a factor of about 2.3 . 
<smiles>FN=NF</smiles>

$\begin{array}{ccc} & \text { I } & \text { II } \\ \Delta H_{f(298)}^{\circ} & 19.4 & 16.4 \\ S_{(298)}^{\circ} & (64.0) & (64.0) \\ C_{p(298)}^{\circ} & (13.2) & (13.2)\end{array}$

$\Delta S^{\circ}=0 \mathrm{~g} / \mathrm{mole}$

$\Delta H^{\circ}=-3.0 \mathrm{kcal} / \mathrm{mole}$

$\Delta C_{p}=0 \mathrm{~g} / \mathrm{mole}$

$\begin{array}{clcccc}\log A & \boldsymbol{E} & \begin{array}{c}\log k_{T} \\ (590)\end{array} & \text { Conditions } & \text { System } & \text { Surface } \\ 14.0 & 32.2 & +2.07 & \begin{array}{l}570-615^{\circ} \mathrm{K} \\ P \sim 1.6 \mathrm{~atm}\end{array} & \text { S. P. Shock None }\end{array}$

\section{References}

[1] J. Binenboym, A.

Burcat, A. Lifshitz, J.

Shamir, J. Am. Chem.

Soc. 88, 5039 (1966).

Preferred:

Suspect.

Comments: Although the $A$-factor is of the right order for a cis-trans rotation, the energy is far too low. For the process $t-\mathrm{N}_{2} \mathrm{~F}_{2} \longrightarrow \mathrm{N}_{2}+2 \mathrm{~F}, \Delta H^{\circ}=19 \mathrm{kcal}$. It is very unlikely that $\mathrm{F}$ atoms are not produced in the temperature range studied. This is supported by the observation that $\mathrm{F}_{2}+\mathrm{N}_{2}$ was produced at higher temperatures. The chain reaction would be:

$$
\begin{aligned}
& \mathrm{N}_{2} \mathrm{~F}_{2}+\mathrm{M} \longrightarrow \mathrm{N}_{2}+2 \mathrm{~F}+\mathrm{M} \\
& \mathrm{F}+t \cdot \mathrm{N}_{2} \mathrm{~F}_{2} \rightleftharpoons \mathrm{N}_{2} \mathrm{~F}_{3} \rightleftharpoons \text { cis }-\mathrm{N}_{2} \mathrm{~F}_{2}+\mathrm{F} \\
& \mathrm{F}+\mathrm{F}+\mathrm{M} \longrightarrow \mathrm{F}_{2}+\mathrm{M} .
\end{aligned}
$$

Thermochemical estimates of the Pi-bond strength in $\mathrm{N}_{2} \mathrm{~F}_{2}$ range from $57 \mathrm{kcal}$ to $84 \mathrm{kcal}$, depending on the choice of N-F bond strength. A value closer to $60 \mathrm{kcal}$ is more likely.

\section{Experimental}

[1] Single-pulse shock tube (1/70) in argon. The authors suggested a linear transition state because of thermodynamic considerations. Alster and Burnelle have performed extended Huckel calculations for the perpendicular transition state, J. Am. Chem. Soc. 88, 5039 (1966), and concluded that the Pi-bond in difluorodiazine could be weak and that a perpendicular transition state was possible; however, see comments. 


\section{Reaction: Nitrosomethane}

\begin{tabular}{lcc}
\multicolumn{2}{r}{$\mathrm{CH}_{3} \mathrm{NO}(\mathrm{I}) \longrightarrow \mathrm{CH}_{2}=\mathrm{NOH}(\mathrm{II})$} \\
& $\mathrm{I}$ & $\mathrm{II}$ \\
$\Delta H_{f(298)}^{\circ}$ & 17 & 0 \\
$S_{(298)}^{\circ}$ & 63.6 & 64.6 \\
$C_{p(298)}^{\circ}$ & 14.3 & 13
\end{tabular}

$\Delta S^{\circ}=\quad 1.0 \mathrm{~g} / \mathrm{mole}$

$\Delta H^{\circ}=-17 \mathrm{kcal} / \mathrm{mole}$

$\Delta C_{p}^{\circ}=-1.3 \mathrm{~g} / \mathrm{mole}$

\begin{tabular}{|c|c|c|c|c|c|c|}
\hline $\log A$ & $\boldsymbol{E}$ & $\begin{array}{c}\log k_{T} \\
(665)\end{array}$ & Conditions & System & Surface & References \\
\hline 8.9 & 27.1 & 0.00 & $\begin{array}{l}633-698{ }^{\circ} \mathrm{K} \\
P_{t}=4.5 \text { torr }\end{array}$ & flow & $\begin{array}{l}\text { a large } \\
\text { effects }\end{array}$ & $\begin{array}{l}\text { [1] L. Batt and B. G. } \\
\text { Gowenlock, Trans. } \\
\text { Faraday Soc. } \mathbf{5 6}, 682 \\
\text { (1960). }\end{array}$ \\
\hline
\end{tabular}

\section{Preferred: \\ $\log k=12.9-39.3 / \theta$.}

Comments: It seems unlikely that the conditions employed to isolate the homogeneous part of the reaction would really do so. The large negative entropy of activation (i.e., $\Delta S^{\ddagger}=-20 \mathrm{~g} / \mathrm{mole}$ ) would not be consistent with the four-center transition state proposed. One may conclude that heterogenity was still a problem in this system.

If we accept the observed reaction rates as representative (within a factor of 2-4) of the homogeneous isomerization and estimate the $A$-factor by transition state methods, the preferred parameters are obtained.

\section{Experimental}

[1] Analysis made spectrophotometrically on $\left(\mathrm{CH}_{3} \mathrm{NO}\right)$ remaining (nitrosmethane when warmed from $-190{ }^{\circ} \mathrm{C}$ to $-78^{\circ} \mathrm{C}$ forms the cis-dimer exclusively.)

a Heterogeneous effects were large and the first order rate constant was divided into homogeneous and heterogeneous components. The homogeneous parameters were those obtained at the lowest $(\mathrm{S} / \mathrm{V})$ ratio and in the presence of $\mathrm{C}_{6} \mathrm{~F}_{12}(0.5$ torr $)$. 

4. Simple Bond Fission Reactions 
Reaction: Methane

$$
\begin{aligned}
& \begin{array}{r}
\mathrm{CH}_{4}\left(\mathrm{II} \stackrel{a}{\stackrel{b}{\longrightarrow}} \mathrm{CH}_{3} \cdot(\mathrm{II})+\mathrm{H} \cdot(\mathrm{III})\right. \\
\longrightarrow \mathrm{CH}_{2}:(\mathrm{IV})+\mathrm{H}_{2}(\mathrm{~V})
\end{array} \\
& \begin{array}{cccccc} 
& \text { I } & \text { II } & \text { III } & \text { IV } & \text { V } \\
\Delta H_{f(298)}^{\circ} & -17.9 & 34.0 & 52.1 & 88 & 0
\end{array} \\
& \begin{array}{llllll}
S_{(298)}^{\circ} & 44.5 & 46.5 & 27.4 & 49.0 & 31.2
\end{array} \\
& \begin{array}{llllll}
C_{p(298)}^{\circ} & 8.5 & 8.8 & 5.0 & (8.1)
\end{array} \\
& \begin{array}{llll}
C_{p(1000)}^{\circ} & 17.2 & 14.5 & 5.0
\end{array}
\end{aligned}
$$

$\Delta S^{\circ}=29.4 \mathrm{~g} / \mathrm{mole}$

$\Delta H^{\circ}=104 \mathrm{kcal} / \mathrm{mole}$

$\Delta C_{p}^{\circ}=5.3 \mathrm{~g} / \mathrm{mole}$

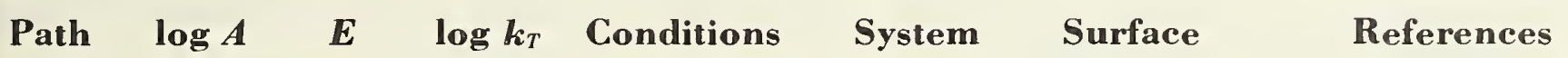
(1500)

$a$

$14.71 \quad 100.1$

$0.12 \quad 1200-1800^{\circ} \mathrm{K}$ shock $1-12 \% \mathrm{CH}_{4}$ in

Ar with total

$P=5 \mathrm{~atm}$

$b$
$0.39 \quad 1670-2070{ }^{\circ} \mathrm{K} \quad$ S.P.Sh. $2-10 \%$ in $\mathrm{Ar}$ $P_{\mathrm{T}}=4$ atm

$\begin{array}{llll}14.12 & 93.0 & 0.57 \quad 1656-1965^{\circ} \mathrm{K} & \text { S.P.Sh. }\end{array}$

$10 \%$ in Ar

$P_{\mathrm{CH}_{4}}=290$ torr

$a+b$

14.88
$103.0-0.13 \quad 1148-1398^{\circ} \mathrm{K}$ flow
[1] G. B. Skinner and R. A. Ruehwein, J. Phys. Chem. 63, 1736 (1959).

[2] G. I. Kozlov and V. G. Knore, Russ. J. Phys. Chem. 37, 1128 (1963).

[3] V. Kevkorian, C. E. Heath, and M. Boudart, J. Phys. Chem. 64, 964 . (1960).

[4] H. B. Palmer and T. J. Hirt, J. Phys. Chem. 67, 709 (1963).

Preferred:

$\log k_{a}=15.3-104 / \theta$.

Comments: One might at first expect such a small molecule to exhibit appreciable pressure falloff behavior. However, from the thermodynamics (which are well known) and the kinetic theory maximum recombination rate constant $\left(k_{\mathrm{rec}}=10^{11.5} \mathrm{l} / \mathrm{mole}-\mathrm{sec}=1 / 4 Z\right)$ high pressure rate constants close to those reported are obtained. The $R R K-M_{1 / 2}=16$ atm $n=6$. Thus at least seven of the nine oscillators must be active. The initiation reaction in the methane pyrolysis is one of conjecture. There is no way of distinguishing between paths $a$ and $b$, although path $a$ would seem the more likely reaction. All rate constants reported are reasonably consistent with the rates calculated from the preferred high-pressure limiting Arrhenius parameters for 
path $a$. The mechanism is probably [1],

$$
\begin{gathered}
\mathrm{CH}_{4} \longrightarrow \mathrm{CH}_{3} \cdot+\mathrm{H} \cdot \\
\mathrm{H} \cdot+\mathrm{CH}_{4} \rightleftharpoons \mathrm{H}_{2}+\mathrm{CH}_{3} \cdot \\
\mathrm{CH}_{3} \cdot+\mathrm{CH}_{3} \cdot \rightleftharpoons \mathrm{C}_{2} \mathrm{H}_{6}
\end{gathered}
$$

Ethane is unstable at high temperatures and will go on to form principally ethylene and hydrogen. From the entropy and $A$-factor one obtains $k_{\text {rec }}=10^{11.4} \mathrm{l} / \mathrm{mole}$-sec.

For earlier work on the $\mathrm{CH}_{4}$ pyrolysis see:

L. S. Kassel, J. Am. Chem. Soc. 54, 3949 (1932)

H. H. Storch, Ind. Eng. Chem. 26, 56 (1934)

F. deRudder and H. Biedermann, Bull. Soc. Chim. France 47, 704 (1930)

A. S. Gordon, J. Am. Chem. Soc. 70, 395 (1948)

P. S. Shantorovitch and B. V. Pavlov, Zhur. Fiz. Khim. 30,811 (1956)

J. E. Germain and C. Vaniscotte, Bull. Soc. Chim. France, 692 (1957); 319 (1958)

\section{Experimental}

[1] Rates equated to $\mathrm{CH}_{4}$ disappearance. Product yields of $\mathrm{C}_{2} \mathrm{H}_{6}, \mathrm{C}_{2} \mathrm{H}_{4}, \mathrm{C}_{2} \mathrm{H}_{2}$, and $\mathrm{H}_{2}$ were determined. A chain length of 2 was inferred from the mechanism.

[2] Products analyzed were $\mathrm{CH}_{4}, \mathrm{C}_{2} \mathrm{H}_{6}, \mathrm{C}_{2} \mathrm{H}_{4}, \mathrm{C}_{2} \mathrm{H}_{2}$, and $\mathrm{H}_{2}$. Rates based on disappearance of $\mathrm{CH}_{4}$ were equated to path $b$. The absence of appreciable effects of different additives (i.e., $\mathrm{C}_{2} \mathrm{H}_{4}, \mathrm{H}_{2}, \mathrm{C}_{2} \mathrm{H}_{6}, \mathrm{C}_{2} \mathrm{H}_{2}$ ) was taken as evidence of very short reaction chains. The overall rate was attributed to the first step in the $\mathrm{CH}_{4}$ decomposition, which was assumed to be an $\mathrm{H}_{2}$ molecular elimination because of the "low" activation energy.

[3] Rates were based on the disappearance of methane. First-order rate constants were independent of methane partial pressures and total pressure at all temperatures studied. The reaction was not inhibited by $\mathrm{H}_{2}$. Path $a$ was thought to be dominant.

[4] Rates were determined by resistance changes in the carbon deposited on a carbon rod. Data of prior studies (covering a $1400^{\circ} \mathrm{K}$ range) were summarized to give the parameters given above. 
Reaction: Ethane

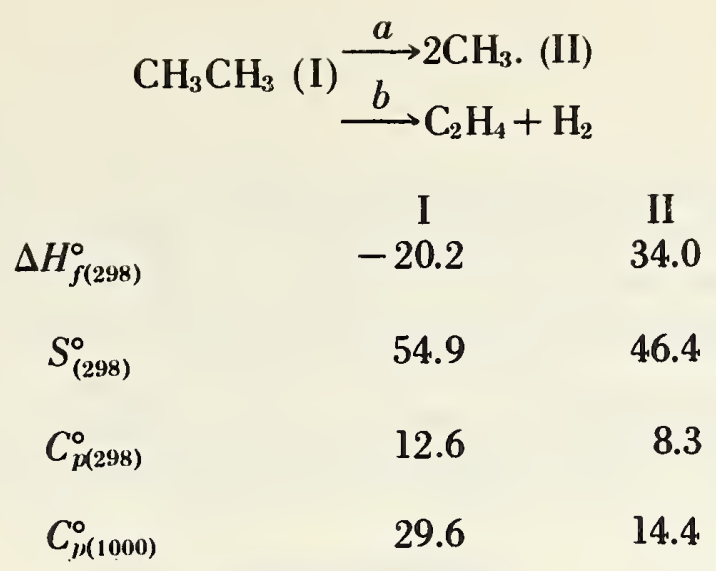

Path a

$\Delta S^{\circ}=37.9 \mathrm{~g} / \mathrm{mole}$

$\Delta H^{\circ}=88.2 \mathrm{kcal} / \mathrm{mole}$

$\Delta C_{p}^{\circ}=4.0 \mathrm{~g} / \mathrm{mole}$

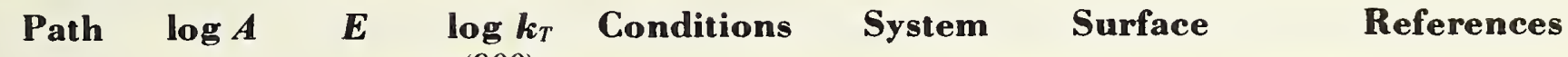
(900)

$a$
14.7
79.3 . $-4.56 \quad 1057-14.18^{\circ} \mathrm{K}$ $\delta P(1-6 \%$ in
Ar)

$a$

17.45
$\begin{array}{lll}91.74-4.83 & 838-872^{\circ} \mathrm{K} \\ 61-202 \text { torr }\end{array}$

$a$

$16.37 \quad 88.0 \cdot-5.00$

$a$

$14.7 \quad 79.3-4.56$

$a$
$15.45 \quad 87 \pm 2 \quad-5.68 \quad 1039-1109^{\circ} \mathrm{K} \quad$ flow $\delta P=0.1$ torr $P_{T}=10$ torr

$a$ 16.0
86.0 $823-893^{\circ} \mathrm{K}$ 40-600 torr
S. P. Shock

static

[2] C. P. Quinn, Proc. Roy. Soc. A2 75, 190 (1963).

[3] R. W. Dexter and A. B. Trenwith, Proc. Chem. Soc., 392 (1964).

[4] V. G. Knorre and G. I. Kozlov, Rus. J. Phys. Chem. 11, 1427 (1964).

[5] C. H. Leigh, M. Szwarc and J. Bigeleisen, J. Am. Chem. Soc. 77, 2193 (1955).

st atic none
[6] M. C. Lin and M. H. Back, Can. J.

Chem. 44, 2357 (1967).

[7] H. G. Davis and K. D. Williamson, World Petr. Cong. Proc., 5th, Vol. IV, 37 New York (1959).

(Continued) 
Preferred:

$\log k_{a}=16.75-89.5 / \theta$.

Comments: Preferred parameters are those calculated from the reaction enthalpy, an average heat capacity of $\bar{\Delta} C_{p}^{\circ}=2.3 \mathrm{~g} / \mathrm{mole}\left(298-858^{\circ} \mathrm{K}\right)$ and the rate constant of [6]. The $A$-factor and reaction entropy set the methyl radical recombination rate constant at $k_{\mathrm{rec}}=10^{9.9} \mathrm{l} / \mathrm{mole}-\mathrm{sec}\left(850^{\circ} \mathrm{K}\right)$. This is in fair agreement with the recombination rate of methyl radicals measured by sector techniques at lower temperatures (i.e., $300-400^{\circ} \mathrm{K}$, $k_{\text {rec }}=10^{10.3} 1 . /$ mole-sec).

Earlier studies on the ethane pyrolysis, concerned more with the overall decomposition kinetics, are summarized in, E. W. R. Steacie, Atomic and Free Radical Reactions, 2d ed., Reinhold Publ. Corp., New York, N.Y. (1954).

\section{Experimental}

[1] G.L.C. analysis for $\mathrm{CH}_{4}, \mathrm{H}_{2}, \mathrm{C}_{2} \mathrm{H}_{6}, \mathrm{C}_{2} \mathrm{H}_{4}, \mathrm{C}_{2} \mathrm{H}_{2}, \mathrm{C}_{3} \mathrm{H}_{8}, \mathrm{C}_{3} \mathrm{H}_{6}$, and $\mathrm{C}_{4} \mathrm{H}_{10}$. Assumed Rice-Herzfeld mechanism and that the formation of $\mathrm{CH}_{4}$ was a measure of path $a$. Rate constants were calculated from the rate of ethane pyrolysis divided by the chain length (i.e., $2 \times \mathrm{C}_{2} \mathrm{H}_{4} / \mathrm{CH}_{4}$ ).

[2] Rate by initial rates of methane formation. Complete product analysis by G.L.C. An additional source of $\mathrm{CH}_{4}$ was found and attributed to the $n$-butyl radical decomposition in the latter stages of reaction

$$
\left(\mathrm{C}_{2} \mathrm{H}_{4}+\dot{\mathrm{C}}_{2} \mathrm{H}_{5} \cdot \longrightarrow \mathrm{C}_{4} \mathrm{H}_{9} \cdot ; \mathrm{C}_{4} \mathrm{H}_{9} \cdot \longrightarrow \mathrm{C}_{3} \mathrm{H}_{6}+\mathrm{CH}_{3} \cdot\right) \text {. This seems unlikely. }
$$

[3] Rates were obtained from $\mathrm{CH}_{4}$ production. Assumed $E_{a}=88 \mathrm{kcal} / \mathrm{mole}$. Observed pressure dependence at $P<100$ torr.

[4] Used the rate constant of [2] in a Rice-Herzfeld mechanism on a computer to calculate the rate of ethane pyrolysis. Calculated rates agreed with observed rates, except at the high temperatures (where the calculated rates were high).

[5] Toluene carrier technique. $\mathrm{C}^{14}$ labeled ethane was used to distinguish between $\mathrm{CH}_{4}$ produced from the toluene carrier decomposition and that arising from ethane. Amount of decomposition was less than 1 percent. Rates were independent of the ethane partial pressure.

[6] Rates were based on the formation of $\mathrm{CH}_{4}$ in the initial stages, which was equated with the initiation reaction. Chain termination was attributed to the combination and disproportionation of ethyl radicals. Analysis for methane was made by standard gas analysis techniques. A surface dependence of the overall rate of the ethane pyrolysis was attributed to absorption of $\mathrm{H}$-atoms at the surface leading to $\mathrm{H}$-atom chain termination with ethyl radicals. 


\section{$\mathrm{CH}_{3} \mathrm{CH}_{2} \mathrm{CH}_{2} \mathrm{CH}_{3}$}

(I)

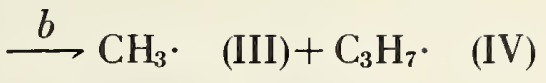

\begin{tabular}{|c|c|c|c|c|c|c|}
\hline \multirow[b]{2}{*}{$\Delta H_{f(298)}^{\circ}$} & I & \multicolumn{2}{|c|}{ II } & III & \multicolumn{2}{|c|}{ IV } \\
\hline & -30.2 & $\begin{array}{c}V=4.0 \\
25.7\end{array}$ & $V=0$ & 34.0 & $\begin{array}{c}V=4.0 \\
20.7\end{array}$ & $V=0$ \\
\hline$S_{(298)}^{\circ}$ & 74.1 & 57.8 & 59.6 & 46.4 & 67.2 & 69.0 \\
\hline$C_{p(298)}^{\circ}$ & 23.3 & 12.1 & 10.9 & 8.3 & 17.6 & 16.4 \\
\hline$C_{p(800)}^{\circ}$ & 48.2 & 24.0 & 23.0 & 12.7 & 35.1 & 34.1 \\
\hline
\end{tabular}

$\begin{array}{cc}V=4.0^{*} & V=4.0^{*} \\ a & b \\ \Delta S^{\circ}=41.5 & 39.5 \mathrm{~g} / \mathrm{mole} \\ \Delta H^{\circ}=81.6 & 84.9 \mathrm{kcal} / \mathrm{mole} \\ \Delta C_{p}^{\circ}=0.9 & 2.6 \mathrm{~g} / \mathrm{mole}\end{array}$

$\begin{array}{lllllll}\text { Path } & \log A & \boldsymbol{E} & \log k_{T} & \text { Conditions } & \text { System } & \text { Surface }\end{array}$

$\begin{array}{llllll}a+b & 18.58 & 86.3 & -6.64 & 693-803^{\circ} \mathrm{K} & \text { static }\end{array}$

10-150 torr

[1] J. H. Purnell and C. P. Quinn, Proc.

Roy. Soc. A2 70, 267 (1962); Nature

(L) 189, 656

(1961); J. H. Purnell and C. P. Quinn, Can. J. Chem. 43, 721

Preferred:

$\log k_{a}=17.27-81.8 / \theta$;

(1965).

$\log k_{b} \simeq 17.6-85.9 / \theta$ (estimated).

Comments: The thermodynamics indicate that path $b$ should be about a factor of 10 slower than path $a$. Adjustment of the rate constant parameters to the expected energy change of the reaction seems warranted considering the mechanistic complexities of this system. From the reaction entropies and the preferred parameters, one obtains, $k_{-a}=10^{10.0+0.2} \mathrm{l} /$ mole-sec; $k_{-b}=10^{10.5} \mathrm{l} / \mathrm{mole}-\mathrm{sec}$. The value reported for ethyl radical recombination rate from sector measurements is $k_{\text {rec }}=10^{10.2} \mathrm{l} /$ mole-sec.

\section{Experimental}

[1] Total product analysis by G.L.C. as a function of the extent of reaction. Rates of formation of $\mathrm{C}_{2} \mathrm{H}_{6}, \mathrm{CH}_{4}$, and $\mathrm{C}_{2} \mathrm{H}_{4}$ were followed. Parameters of the above initiation steps were obtained from ethane formation using:

$$
\begin{gathered}
2 \mathrm{C}_{2} \mathrm{H}_{5} \cdot \stackrel{t}{\longrightarrow} \mathrm{C}_{4} \mathrm{H}_{10}, k_{t}=1.57 \times 10^{10} \mathrm{l} / \text { mole-sec } \quad \text { and } \\
\mathrm{C}_{2} \mathrm{H}_{5} \cdot+\mathrm{C}_{4} \mathrm{H}_{10} \stackrel{4}{\longrightarrow} \mathrm{C}_{2} \mathrm{H}_{6}+n \mathrm{Bu} \cdot, \quad k_{4}=5.9 \times 10^{8} \times 10^{-15.2 / \theta} \mathrm{l} / \mathrm{mole}-\mathrm{sec} .
\end{gathered}
$$

Pressure dependence of the ethyl radical decomposition was demonstrated.

\footnotetext{
*See discussion preceeding bond fission reactions concerning radical entropies and rotational barriers.
} 
Reaction: 2,2-Dimethylpropane (neopentane)

\begin{tabular}{|c|c|c|c|c|c|}
\hline \multirow[b]{2}{*}{$\Delta H_{f(298)}^{\circ}$} & \multirow[b]{2}{*}{$\begin{array}{c}\text { I } \\
-39.7\end{array}$} & \multirow[b]{2}{*}{$\begin{array}{c}\text { II } \\
34.0\end{array}$} & \multicolumn{3}{|c|}{ III } \\
\hline & & & $\begin{array}{c}V=4.0 \\
6.7\end{array}$ & $V=0$ & $V=8$ \\
\hline$S_{(298)}^{\circ}$ & 73.2 & 46.4 & *70.3 & 75.4 & 67.6 \\
\hline$C_{p(\mathbf{2 9 8 )}}^{\circ}$ & 29.1 & 8.3 & 22.6 & 18.7 & 21.7 \\
\hline
\end{tabular}

$$
\begin{aligned}
V=4 \\
\Delta S^{\circ}=43.5 \mathrm{~g} / \mathrm{mole} \\
\Delta H^{\circ}=80.4 \mathrm{kcal} / \mathrm{mole} \\
\Delta C_{p}=1.8 \mathrm{~g} / \mathrm{mole} \\
\left\langle\Delta \bar{C}_{p}^{\circ}=-0.3\right\rangle
\end{aligned}
$$

\begin{tabular}{|c|c|c|c|c|}
\hline $\log A$ & $\boldsymbol{E}$ & $\begin{array}{c}\log k_{T} \\
(400)\end{array}$ & Conditions & System \\
\hline 17.4 & $83 \pm 5$ & 0.91 & $\begin{array}{l}803-840^{\circ} \mathrm{K} \\
80-450 \text { torr }\end{array}$ & static \\
\hline 16.1 & 78.2 & 0.56 & $\begin{aligned} & \sim 1000-1200^{\circ} \mathrm{K} \\
& \sim 0.7 \% \text { in } 4-\text { atm } \\
& \text { Ar with } 1 \% \\
& \text { toluene }\end{aligned}$ & S.P. șhock \\
\hline
\end{tabular}

Surface

References
[1] J. Engel, A. Combe, M. Letort and M. Niclause, Compt. Rend. 244, 453 (1957).

[2] W. Tsang, J. Chem. Phys. 44, 4283 (1966).

\section{Preferred:}

$\log k=16.7-80.4 / \theta$.

Comments: The activation energy of [2] is in reasonable agreement with the reaction thermodynamics. Recombination rate constants calculated for the various rotational barriers to the methyl groups in the $t$-butyl radical give: $V=4.0, k_{\text {rec }}=10^{9.3} \mathrm{l} / \mathrm{mole-sec} ; \quad V=0, k_{\text {rec }}=10^{9.0} \mathrm{l} / \mathrm{mole}-\mathrm{sec} ; \quad V=8, k_{\mathrm{rec}}=10^{9.8} \mathrm{l} / \mathrm{mole}$-sec. Rate constants obtained by sector technique for $\left(\mathrm{CH}_{3}+\mathrm{CH}_{3}\right)$ and $(t \mathrm{Bu} \cdot+t \mathrm{Bu} \cdot)$ are, respectively: $10^{10.3}$ 1/mole-sec [A. Shepp, J. Chem. Phys. 24, 939 (1956)], and 109.5 l/mole-sec [E. Metcalfe, J. Chem. Soc., 3560 (1963)]. Assuming $k_{\left(\mathrm{CH}_{3}+t \mathrm{Bu}\right)}=2\left(k_{\mathrm{CH}_{3}+\mathrm{CH}_{3}} \times k_{t \mathrm{Bu}+t \mathrm{Bu}}\right)^{1 / 2}$, one obtains $k_{\mathrm{CH}_{3}+t \mathrm{Bu}}=10^{10.2} \mathrm{l} / \mathrm{mole}$-sec. The recombination rate constant calculated from the dissociation reaction kinetics and the estimated thermodynamics is closest to the expected value when the $t$-butyl radical entropy, based on $8 \mathrm{kcal}$ rotational barriers, is used. However, such a barrier is quite unreasonable.

\section{Experimental}

[1] Rates were obtained from pressure-time curves. Kinetics were deduced from the mechanism:

$$
k=k_{3}\left(\frac{k_{1}}{k_{4}}\right)^{1 / 2}=1.5 \times 10^{13} \times 10 \frac{-51.5}{\theta} \quad \sec ^{-1}\left(\frac{\mathrm{cc}}{\text { moles }}\right)^{1 / 2} .
$$

${ }^{*}$ See section $\mathrm{V}-6.0$. 
2,2-Dimethylpropane (Continued)

$$
\begin{aligned}
& \text { initiation }\left\{\begin{array}{l}
\mathrm{C}_{5} \mathrm{H}_{12} \stackrel{\mathrm{l}}{\longrightarrow} \mathrm{C}_{4} \mathrm{H}_{9} \cdot+\mathrm{CH}_{3} . \\
\mathrm{C}_{4} \mathrm{H}_{9} \cdot+\mathrm{C}_{5} \mathrm{H}_{12} \longrightarrow \mathrm{C}_{4} \mathrm{H}_{10}+\mathrm{C}_{5} \mathrm{H}_{11}
\end{array}\right. \\
& \text { chain }\left\{\begin{array}{l}
\mathrm{C}_{55} \mathrm{H}_{11} \cdot \stackrel{2}{\longrightarrow} \mathrm{C}_{4} \mathrm{H}_{8}+\mathrm{CH}_{3} . \\
\mathrm{CH}_{3} \cdot+\mathrm{C}_{5} \mathrm{H}_{12} \stackrel{3}{\longrightarrow} \mathrm{CH}_{4}+\mathrm{C}_{5} \mathrm{H}_{11}
\end{array}\right. \\
& \text { termination } 2 \mathrm{CH}_{3} \stackrel{4}{\longrightarrow} \mathrm{C}_{2} \mathrm{H}_{6} \text {. }
\end{aligned}
$$

[2] Comparative rate standard was 2,3-dimethylbutane. G.L.C. analysis. Products were $i$-butene and $\mathrm{CH}_{4}$. $\mathrm{Small}$ amounts of $\mathrm{C}_{2} \mathrm{H}_{6}$ and propylene were also detected. 
Reaction: 2,3-Dimethylbutane

\begin{tabular}{|c|c|c|c|c|c|c|c|c|c|}
\hline \multicolumn{2}{|l|}{$\Delta H_{f(298)}^{\circ}$} & $\begin{array}{c}\mathrm{I} \\
-42.5\end{array}$ & $\| \begin{array}{c}V=4 \\
-17.6\end{array}$ & $\begin{array}{c}\mathrm{II} \\
V=0^{*}\end{array}$ & $V=8$ & $\begin{array}{c}V=4 \\
5.6\end{array}$ & $\begin{array}{c}\mathrm{III} \\
V=0^{*}\end{array}$ & $V=8$ & $\begin{array}{c}\text { IV } \\
34.0\end{array}$ \\
\hline \multicolumn{2}{|l|}{$S_{(298)}^{\circ}$} & 87.4 & 65.4 & 69.0 & 63.8 & 83.9 & 86.5 & 81.3 & 46.5 \\
\hline \multicolumn{2}{|l|}{$C_{p(298)}^{\circ}$} & 33.6 & 17.7 & 15.1 & 17.1 & 28.4 & 25.8 & 27.8 & 8.8 \\
\hline \multicolumn{2}{|l|}{$C_{p(1000)}^{\circ}$} & 79.1 & 39.1 & 37.9 & 40.3 & 64.0 & 62.8 & 65.2 & 14.5 \\
\hline \multicolumn{2}{|c|}{$\begin{aligned} & V=4.0 \\
& a \\
= & * 43.4 \\
= & 77.7 \\
= & +1.8\end{aligned}$} & \multicolumn{2}{|c|}{$\begin{array}{l}V=4.0 \\
b \\
* 43.0 \mathrm{~g} / \mathrm{mole} \\
82.1 \mathrm{kcal} / \mathrm{mole} \\
3.6 \mathrm{~g} / \mathrm{mole}\end{array}$} & & & & & & \\
\hline Path & $\log A$ & $\boldsymbol{E}$ & $\begin{array}{c}\log \boldsymbol{k}_{T} \\
(1100)\end{array}$ & Conditions & System & & ce & Refert & \\
\hline$a$ & 16.1 & 76.0 & 1.00 & $\begin{array}{l}1000-1200{ }^{\circ} \mathrm{K} \\
1 \% \text { in } \mathrm{Ar}, \\
\text { with } 1 \% \\
\text { toluene }\end{array}$ & S.P.Sh. & & & $\begin{array}{l}\text { 1] W. Tsang } \\
\text { Phys. } 4 \\
\text { (1965). }\end{array}$ & $\begin{array}{l}\text { Chem. } \\
352\end{array}$ \\
\hline$b$ & 16.6 & 81.1 & 0.49 & (as above) & & & & as above) & \\
\hline
\end{tabular}

Preferred:

$\log k_{a}=16.5-78.0 / \theta$.

$\log k_{b}=17.0-83.1 / \theta$.

Comments: The thermodynamics predict activation energies of $E_{a}=78.0$ and $E_{b}=83.1 \mathrm{kcal} / \mathrm{mole}$. Both are in reasonable agreement with the reported values. The recombination rate constants calculated from the thermodynamics and $A$-factors are:

$$
\begin{gathered}
k_{-a}=10^{9.0 \pm 0.5} \cdot 1 / \mathrm{mole}-\mathrm{sec} \text { and } k_{-b}=10^{9.2 \pm 0.3} \cdot 1 / \mathrm{mole}-\mathrm{sec} \text {. } \\
\text { Experimental }
\end{gathered}
$$

[1] Comparative rate technique with cyclohexene decomposition as the standard. Analysis of products by G.L.C. Path $a$ products: $\mathrm{C}_{3} \mathrm{H}_{6}+\mathrm{H}_{2}, \mathrm{C}_{2} \mathrm{H}_{4} * *+\mathrm{CH}_{4}$

Path $b$ products:

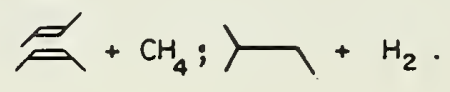

*See section V-6.0.

**Production of $\mathrm{C}_{2} \mathrm{H}_{4}$ from the decomposition of the $\left(\mathrm{CH}_{3} \mathrm{CHCH}_{3}\right)$ radical (as proposed by the author) does not seem reasonable. 
Reaction: $2,2^{\circ}$ methylbutane

$$
\left(\mathrm{CH}_{3}\right)_{3} \mathrm{C}-\mathrm{CH}\left(\mathrm{CH}_{3}\right)_{2} \quad \text { (I) } \longrightarrow\left(\mathrm{CH}_{3}\right)_{3} \mathrm{C} \cdot \quad \text { (II) }+\left(\mathrm{CH}_{3}\right)_{2} \mathrm{CH} \cdot \quad \text { (III) }
$$

\begin{tabular}{|c|c|c|c|c|c|c|c|}
\hline$\Delta H_{f(298)}^{\circ}$ & $\begin{array}{c}\text { I } \\
-48.6\end{array}$ & $\begin{array}{c}V=4 \\
6.8\end{array}$ & $\begin{array}{c}\text { II } \\
V=8^{*}\end{array}$ & $V=0$ & $\begin{array}{c}V=4 \\
17.6\end{array}$ & $\begin{array}{l}\text { III } \\
V=8^{*}\end{array}$ & $V=2$ \\
\hline$S_{(298)}^{\circ}$ & 91.6 & 70.3 & 67.6 & 75.4 & 65.4 & 63.6 & 66.2 \\
\hline$C_{p(298)}^{\circ}$ & 39.9 & 22.6 & 21.7 & 18.7 & 17.7 & 17.1 & 17.5 \\
\hline$C_{p(1000)}^{\circ}$ & 92.7 & 51.3 & 52.8 & 49.2 & 39.1 & 40.3 & 38.1 \\
\hline $\begin{array}{r}\Delta S^{\circ}= \\
\Delta H^{\circ}= \\
\Delta C_{p}^{\circ}= \\
C_{p(1000)}^{\circ}=\end{array}$ & $\begin{array}{l}\text { mole } \\
\text { al/mole } \\
\text { mole } \\
\text { mole }\end{array}$ & & & & & & \\
\hline
\end{tabular}

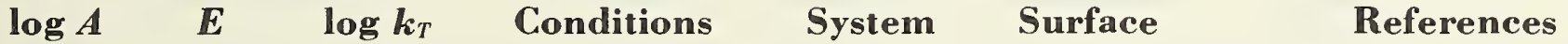
(1133)
$16.2 \quad 73.0$
$2.12 \quad 1069-1197^{\circ} \mathrm{K}$
$0.01 \%$ in $4 \mathrm{~atm}$
Ar with $1 \%$
toluene

\section{Preferred: \\ $\log k=16.05-72.2 / \theta$.}

Comments: The thermodynamics predict an activation energy of about $72 \mathrm{kcal} / \mathrm{mole}$, which is within experimental error. Recombination rate constants obtained from the reaction entropies are $\log k_{\text {rec }}(1 / \mathrm{mole}$-sec) $=8.7_{-0.4}^{+0.8}$ (depending on the radical models assumed). The high barrier model seems to be the more consistent with reported parameters and the observed recombination rates. However, it is not reasonable.

\section{Experimental}

[1] The comparative rate standard was cyclohexene. Rates were based on G.L.C. analysis of products which were found to be primarily isobutene, propylene, and 2-methylbut-2-ene. Ethylene and 2,3-dimethylbut-2-ene were also detected.

* See section V-6.0 for a discussion of rotational barriers in free radicals. 
Reaction: 2,2,3,3-Tetramethylbutane (hexamethyl ethane)

$$
\left(\mathrm{CH}_{3}\right)_{3} \mathrm{CC}\left(\mathrm{CH}_{3}\right)_{3}(\mathrm{I}) \longrightarrow 2\left[\left(\mathrm{CH}_{3}\right)_{3} \mathrm{C} \cdot\right] \text { (II) }
$$

\begin{tabular}{|c|c|c|c|c|c|c|c|}
\hline & & & I & & II & & \\
\hline & & & & $V=8^{*}$ & $V=4^{*}$ & $V=0^{*}$ & \\
\hline & & $\Delta H_{f(298)}^{P}$ & -54.7 & n.............. & 6.8 & ........... & ...... \\
\hline & & $S_{(298)}^{\circ}$ & 93.2 & 67.6 & 70.3 & 75.4 & \\
\hline & & $C_{p(\mathbf{2 9 8})}^{\circ}$ & 45.9 & 21.7 & 22.6 & 18.7 & \\
\hline & & $C_{p(1000}^{\circ}$ & 106.2 & 52.8 & 51.3 & 49.2 & \\
\hline $\begin{array}{r}V=8 \\
\Delta S^{\circ}=42.0 \\
\Delta H^{\circ}=68.3 \\
\Delta C_{p}^{\circ}=-2.5\end{array}$ & & $\begin{array}{r}V=4 \\
47.4 \\
68.3 \\
-0.7\end{array}$ & $\begin{array}{l}V=0 \\
57.6 \mathrm{~g} / \mathrm{mole} \\
68.3 \mathrm{kcal} / \mathrm{mole} \\
-8.5 \mathrm{~g} / \mathrm{mole}\end{array}$ & & & & \\
\hline $\log A$ & $\boldsymbol{E}$ & $\begin{array}{r}\log \boldsymbol{k}_{T} \\
(1052)\end{array}$ & Conditions & System & Surface & & References \\
\hline 16.3 & 68.5 & 2.07 & $\begin{array}{c}985-1119^{\circ} \mathrm{K} \\
<0.1 \% \text { in } 4 \text { atm } \\
\text { Ar with } 1 \% \\
\text { toluene }\end{array}$ & S. P. Sh. & & [1] $\nabla$ & $\begin{array}{l}\text { W. Tsang, J. Chem. } \\
\text { Phys. 44, } 4283 \text { (1966). }\end{array}$ \\
\hline
\end{tabular}

Preferred:

$\log k=16.07-67.2 / \theta$

$\log k_{\text {rec }}(1 /$ mole-sec $)=8.3_{-0.6}^{+0.9}$

Comments: The thermodynamics predict an activation energy of about $67.2 \mathrm{kcal} / \mathrm{mole}$ which is just within the limits of experimental error. The back recombination rate constant has been measured. The value reported is $k_{\text {rec }}=10^{9.5} \mathrm{l} /$ mole-sec, E. L. Metcalfe, J. Chem. Soc., 3560 (1963). The kinetics of the decomposition reactions seem to support the low entropy, high rotational barrier model of the $t$-butyl radical. This, however, is not very reasonable.*

\section{Experimental}

[1] Comparative rate standard was cyclohexene. Rates were based on G.L.C. analysis of the products: $i$-butene, propylene, 2,3-dimethylbut-2-ene, benzene, methane and $\mathrm{C}_{2}$ hydrocarbons. Isobutene represented at least 90 percent of the decomposition product. It was assumed that all chain decomposition was quenched by the toluene.

${ }^{*}$ See section $\mathrm{V}-6.0$ for a discussion of this reaction. 
Reaction: Propene (propylene)

$$
\mathrm{CH}_{3} \mathrm{CH}=\mathrm{CH}_{2}(\mathrm{I}) \longrightarrow \mathrm{CH}_{2}=\mathrm{CHCH}_{2} \cdot(\mathrm{II})+\mathrm{H} \cdot
$$

$\begin{array}{cccc}\Delta H_{f(298)}^{\circ} & \text { I } & \text { II } & \text { III } \\ S_{(298)}^{\circ} & 4.8 & (38.0) & 52.1 \\ C_{p(298)}^{\circ} & 63.7 & 61.4 & 27.4 \\ C_{p(1000)}^{\circ} & 15.5 & 16.8 & 5.0 \\ & 34.5 & 33.5 & 5.2\end{array}$

$\Delta S^{\circ}=25.1 \mathrm{~g} / \mathrm{mole}$

$\Delta H^{\circ}=87.9 \mathrm{kcal} / \mathrm{mole}$

$\Delta C_{p}^{\circ}=6.3 \mathrm{~g} /$ mole

$\left\langle\Delta \bar{C}_{p}^{\circ}=5.2\right\rangle$

\begin{tabular}{|c|c|c|c|c|c|c|}
\hline $\log A$ & $\boldsymbol{E}$ & $\begin{array}{r}\log \boldsymbol{k}_{T} \\
(1048)\end{array}$ & Conditions & System & Surface & References \\
\hline 13.40 & 78.0 & -2.87 & $\begin{array}{l}953-1143^{\circ} \mathrm{K} \\
2-14 \text { torr }\end{array}$ & flow & none & $\begin{array}{l}\text { [1] M. Szwarc, J. Chem. } \\
\text { Phys., 17, 284 (1949). }\end{array}$ \\
\hline
\end{tabular}

\section{Preferred:}

$\log k \simeq 15.3-89.2 / \theta$. Reported parameters are unreliable. Rate constants are probably roughly reliable.

Comments: The discrepancy between the reported activation energy and the thermodynamic estimate of the energy is too large to be reconciled. Certainly the mechanistic assumptions are questionable. If the overall decomposition rate constant is scaled to the preferred activation energy, one obtains $\log k_{\max }=16.6-$ 89.9/ $\theta$. This includes the chain length and therefore gives a maximum value for the $A$-factor. From $A$ and the reaction entropy, a maximum value for the recombination rate constant is obtained, $k_{\text {rec }}(\max )=10^{11.7}$ $1 /$ mole-sec. A reasonable estimate of the recombination rate constant from kinetic theory considerations is about $1 / 4$ (spin restrictions) $\times 1 / 5$ (resonance steric factor) $\times$ (collision frequency rate constant) $Z \simeq 10^{12.0}$ $1 /$ mole-sec $) \simeq 10^{10.7} \mathrm{l} /$ mole-sec. Thus a chain length of about 10 is inferred. This is equal to the value assumed by Szwarc. Reaction is probably not at high-pressure limit.

\section{Experimental}

[1] Rate constants were calculated from the rates of $\left(\mathrm{CH}_{4}+\mathrm{H}_{2}\right)$ formation. Unpyrolysed propylene was found to decompose at a slightly lower rate than prepyrolysed propylene. Prepyrolysed propylene decomposed at a constant rate. The latter was used for rate constant determinations. Products observed were $\mathrm{CH}_{4}, \mathrm{H}_{2}, \mathrm{C}_{2} \mathrm{H}_{4}$, and allene $\left(\mathrm{C}_{3} \mathrm{H}_{4}\right)$ in ratio of $\left(\mathrm{CH}_{4} / \mathrm{H}_{2}\right) \simeq 2,\left(\mathrm{C}_{2} \mathrm{H}_{4} / \mathrm{CH}_{4}\right) \simeq 1,\left(\mathrm{C}_{3} \mathrm{H}_{4} / \mathrm{H}_{2}+\mathrm{CH}_{4}\right) \simeq 1$. Added allene had no effect on the rate; 1-4 percent diallyl accelerated the rate and changed $\left(\mathrm{CH}_{4} / \mathrm{H}_{2}\right) \rightarrow 1.0$.

$$
\begin{aligned}
& \mathrm{CH}_{2}=\mathrm{CHCH}_{3} \stackrel{1}{\longrightarrow} \mathrm{CH}_{2}=\mathrm{CHCH}_{2}+\mathrm{H} \cdot \\
& \mathrm{CH}_{2}=\mathrm{CHCH}_{2} \cdot \stackrel{5}{\longrightarrow} \mathrm{CH}_{2}=\mathrm{C}=\mathrm{CH}_{2}+\mathrm{H} \cdot \\
& \mathrm{CH}_{2}=\mathrm{CHCH}_{3}+\mathrm{H} \cdot \stackrel{2}{\longrightarrow} \mathrm{CH}_{2}=\mathrm{CHCH}_{2} \cdot+\mathrm{H}_{2} \\
& \mathrm{CH}_{2}=\mathrm{CHCH}_{3}+\mathrm{H} \cdot \stackrel{3 a}{\longrightarrow} \mathrm{C}_{2} \mathrm{H}_{4}+\mathrm{CH}_{3} \cdot \\
& \mathrm{CH}_{2}=\mathrm{CHCH}_{3}+\mathrm{CH}_{3} \cdot \stackrel{3 b}{\longrightarrow} \mathrm{CH}_{2}=\mathrm{CHCH}_{2} \cdot+\mathrm{CH}_{4} \\
& \mathrm{CH}_{2}=\mathrm{CHCH}_{2} \cdot+\mathrm{H} \cdot \stackrel{6}{\longrightarrow} \mathrm{CH}_{2}=\mathrm{CHCH}_{3}
\end{aligned}
$$

From the steady state relations, $k_{d e c}=\left(k_{1} k_{5} k_{2} / k_{6}\right)^{1 / 2}=1.1 \times 10^{13-72 / \theta} \mathrm{sec}^{-1} . k$ was calculated by assuming a chain length of 10 and equating $k_{2}=k_{6}$. 
Reaction: 1-Butene

$$
\mathrm{CH}_{2}=\mathrm{CHCH}_{2} \mathrm{CH}_{3}(\mathrm{I}) \stackrel{(+\mathrm{M})}{\longrightarrow} \mathrm{CH}_{2}=\mathrm{CHCH}_{2} \cdot \text { (II) }+\mathrm{CH}_{3} \text {. }
$$

$\begin{array}{cccc}\Delta H_{f(298)}^{\circ} & \text { I } & \text { II } & \text { III } \\ S_{(298)}^{\circ} & 0 & (38.0) & 34.0 \\ C_{p(298)}^{\circ} & 73.6 & 61.4 & 46.4 \\ C_{p(1000)}^{\circ} & 20.5 & 16.8 & 8.3 \\ & 47.0 & 33.5 & 14.4\end{array}$

$$
\begin{aligned}
\Delta S^{\circ} & =34.2 \mathrm{~g} / \mathrm{mole} \\
\Delta H^{\circ} & =72.0 \mathrm{kcal} / \mathrm{mole} \\
\Delta C_{p}^{\circ} & =4.6 \mathrm{~g} / \mathrm{mole} \\
\left\langle\Delta C_{p}^{\circ}\right\rangle & =2.7 \mathrm{~g} / \mathrm{mole}
\end{aligned}
$$

\begin{tabular}{|c|c|c|c|c|c|c|}
\hline $\log A$ & $\boldsymbol{E}$ & $\begin{array}{l}\log \\
(945)\end{array}$ & Conditions & System & Surface & References \\
\hline 13.0 & 61.5 & -1.23 & $\begin{array}{l}935-1051 \% \mathrm{~K} \\
0.1-0.6 \text { torr } \\
P_{T} \simeq 15 \text { torr }\end{array}$ & flow & none & $\begin{array}{l}\text { [1] A. H. Sehon and M. } \\
\text { Szwarc, Proc. Roy. } \\
\text { Soc. A2 02, } 263 \text { (1950) } \\
\text { J. Chem. Phys. 18, } \\
237 \text { (1950) }\end{array}$ \\
\hline $12.7 !$ & 59.1 & -0.97 & $\begin{array}{l}900-990^{\circ} \mathrm{K} \\
4-21.3 \text { torr }\end{array}$ & & & $\begin{array}{l}\text { [2] J. A. Kerr, R. Spencer, } \\
\text { and A. F. Trotman- } \\
\text { Dickenson, J. Chem. } \\
\text { Soc., } 6652(1965)\end{array}$ \\
\hline
\end{tabular}

\section{Preferred:}

Rate constants are low; parameters are unreliable.

Comments: The reported parameters apply to the pressure dependent region of decomposition [2] and are therefore unreliable. It is apparent from the reaction thermodynamics that both $A$ and $E$ should be considerably higher. We estimate $\log k_{\infty}=16.0-73.3 / \theta$ and $k_{\text {rec }}=10^{9.8} 1 / \mathrm{mole}-\mathrm{sec}$.

\section{Experimental}

[1] Toluene carrier technique. Rates of reaction were equated to rates of $\mathrm{CH}_{4}$ formation. Pressure dependence of the rate constants was evident at the high temperatures. Hydrogen and 1,3-butadiene were also found in the products. Ratio $\left(\emptyset \mathrm{CH}_{2}\right)_{2} /\left(\mathrm{CH}_{4}+\mathrm{H}_{2}\right) \simeq 0.3$ reasonably explained on basis of $\emptyset \mathrm{CH}_{2} \cdot+\mathrm{CH}_{2}=\mathrm{CHCH}_{2} \cdot$ reactions.

[2] Aniline carrier technique. Rates were based on $\mathrm{CH}_{4}$ formation and the usual mechanism. A strong pressure dependence of the rate constants was observed. A high-pressure activation energy of about $71.5 \mathrm{kcal} / \mathrm{mole}$ was estimated on the basis of 19 effective oscillators (analogous to methyl cyclopropane). A high-pressure rate constant of $\log k=15.0-71.5 / \theta$ was estimated. 
Reaction: 2-Methyl-1-butene (2-methylpropene) (isobutene)

$\begin{array}{cccc}\left(\mathrm{CH}_{3}\right)_{2} \mathrm{C}=\mathrm{CH}_{2}(\mathrm{I}) \longrightarrow & \mathrm{CH}_{2} \\ & \mathrm{C} & \mathrm{II} & \mathrm{CH}=\mathrm{CH}_{2}(\mathrm{II})+\mathrm{H} \cdot(\mathrm{III}) \\ \Delta H_{f(298)}^{\circ} & -4.0 & (29.6) & 52.1 \\ S_{(298)}^{\circ} & 70.2 & 69.0 & 27.4 \\ C_{p(298)}^{\circ} & 21.4 & 23.0 & 5.0 \\ C_{p(1000)}^{\circ} & 50.6 & 46.0 & 5.2\end{array}$

$\Delta S^{\circ}=26.2 \mathrm{~g} / \mathrm{mole}$

$\Delta H^{\circ}=85.7 \mathrm{kcal} / \mathrm{mole}$

$\Delta C_{p}^{\circ}=6.6 \mathrm{~g} / \mathrm{mole}$

$\left\langle\Delta C_{p}^{\circ}=3.6\right\rangle_{298-1000}$
$\log A$
$\boldsymbol{E}$
$\log \boldsymbol{k}_{T}$
Conditions
System
Surface
References
$13.7 \quad 76 \pm 4 \quad-2.81 \quad 930-1082^{\circ} \mathrm{K}$ 1-20 torr
none
flow
none
[1] M. Szwarc, J. Chem.
Phys. 17, 292 (1949).

\section{Preferred:}

Unreliable (see comments on propylene decomposition).

Comments: From the thermodynamics, we estimate $\log k_{\max }=17.0-88.2 / \theta ; k_{\mathrm{rec}} \simeq 10^{12.3} 1 / \mathrm{mole}$-sec (apparent). Using the same "real" recombination rate constant deduced in the propylene decomposition system $\left(k_{\text {rec }}=10^{10.7} \mathrm{l} /\right.$ mole-sec $)$, one obtains a chain length of about 40 .

\section{Experimental}

[1] Toluene carrier technique. Rate constant parameters were deduced from the mechanism and the rate of formation of $\left(\mathrm{H}_{2}+\mathrm{CH}_{4}\right)$. Products of the decomposition were $\mathrm{H}_{2}, \mathrm{CH}_{4}$, and allene. The ratio $\mathrm{CH}_{4} / \mathrm{H}_{2} \simeq 9$ was taken as the value of the chain length. The mechanism assumed was similar to that proposed for the propylene pyrolysis and by $\mathrm{F}$. O. Rice and W. S. Haynes, J. Am. Chem. Soc. 70, 964 (1948). The experimental first order rate constant obtained for the reaction (including the chain contribution) was $k_{(\exp )}=10^{12.7} \times 10^{-67 \pm 4 / \theta} \mathrm{sec}^{-1}$. 


$\begin{array}{cccc} & \varnothing \mathrm{CH}_{3}(\mathrm{I}) \longrightarrow \varnothing \mathrm{CH}_{2} \cdot(\mathrm{II})+\mathrm{H} \cdot \operatorname{atom}(\mathrm{III}) \\ \Delta H_{f(298)}^{\circ} & \mathrm{I} & \mathrm{II} & \text { III } \\ S_{(298)}^{\circ} & 12.0 & 45.0 & 52.1 \\ C_{p(298)}^{\circ} & 76.4 & 75.3 & 27.4 \\ C_{p(1000)}^{\circ} & 24.8 & 25.6 & 5.0 \\ & 62.5 & 60.6 & 5.0\end{array}$

$\Delta S^{\circ}=26.3 \mathrm{~g} / \mathrm{mole}$
$\Delta H^{\circ}=85.1 \mathrm{kcal} / \mathrm{mole}$
$\Delta C_{p}^{\circ}=5.8 \mathrm{~g} / \mathrm{mole}$

$\begin{array}{llllll}\log A & \boldsymbol{E} & \log k_{T} & \text { Conditions } & \text { System } & \text { Surface }\end{array}$

$\begin{array}{lllll}13.3 & 77.5 & -2.50 & 1007-1137^{\circ} \mathrm{K} \quad \text { flow none } & \end{array}$

[1] M. Szwarc, Disc.

Faraday Soc. 10, 222

(1951); Disc. Faraday

Soc. 2, 39 (1947);

Nature 160, 403 (1947);

J. Chem. Phys. 16, 128, 637 (1948).

(See Experimental) $\quad 1133-1213^{\circ} \mathrm{K} \quad$ flow

[2] H. Blades, A. T. Blades and E. W. R. Steacie, Can. J. Chem. 32, 298 (1954).

(See Experimental) $\quad-2.53 \quad 1010-1226^{\circ} \mathrm{K} \quad$ flow

[3] M. Takahasi, Bull. Chem.

Soc. (Japan) 33, 801

(1960); Ibid. 33, 808

(1960).

$14.8 \quad 85.0$

913-1143 $\mathrm{K} \quad$ flow

signif-

icant.

[4] S. J. Price, Can. J. Chem. 40, 1310 (1962).

\section{Preferred:}

$\log k=15.5-88.3 / \theta$.

Comments: Accepting the rate constants of [4] as those appropriate to the homogeneous decomposition reaction, and scaling the parameters to fit the thermodynamic enthalpy, one obtains the preferred parameters. From the reaction entropy one then can calculate $k_{\text {rec }}=10^{10.5} \mathrm{l} / \mathrm{mole}$-sec in agreement with estimates based on kinetic theory collision frequencies. The results of Price [4] are in reasonable agreement with the thermodynamics of the reaction, where the benzyl radical heat of formation has been determined by two separate kinetic measurements to be $\Delta H_{f}^{\circ}\left(\varnothing \mathrm{CH}_{2} \cdot\right)=45 \pm 1 \mathrm{kcal} / \mathrm{mole}(\mathrm{R}$. Walsh, D. M. Golden, and S. W. Benson, J. Am. Chem. Soc. 88, 650 (1966); G. L. Esteban, J. A. Kerr, and A. F. Trotman-Dickenson, J. Chem. Soc., 3879 (1963)).

One of the complications of this reaction, which makes it difficult to accept the parameters determined in any of the studies, concerns the fate of the "nonreactive" benzyl radicals. The mechanism proposed as- 
sumes complete recombination of benzyl radicals to form dibenzyl. The thermodynamics, however, clearly show that at temperatures above $1000^{\circ} \mathrm{K}$ and at relatively low toluene pressures and percent reaction, the concentration of benzyl radicals in equilibrium with dibenzyl must be high.

At $1000^{\circ} \mathrm{K}$ the equilibrium constant for the reaction $\left(\varnothing \mathrm{CH}_{2}\right)_{2} \rightleftarrows 2 \varnothing \mathrm{CH}_{2} \cdot$ equals $\mathrm{Keq}=10^{-1.1}$ torr. Thus for a toluene pressure of about 10 torr and a 1 percent decomposition, the ratio of $\left.\left(\varnothing \mathrm{CH}_{2} \cdot / \varnothing \mathrm{CH}_{2}\right)_{2}\right)$ would be close to unity. Clearly, benzyl radicals cannot be "dead" in the system but must be involved in secondary reactions. The pertinent question is whether the secondary reactions of benzyl radicals contribute to the $\mathrm{H}_{2}$ or $\mathrm{CH}_{4}$ used to measure the bond breaking step. The answer seems to be "yes" in at least one instance. Blades and Steacie showed that styrene was an important nonvolatile product. Styrene probably results from molecular elimination of $\mathrm{H}_{2}$ from dibenzyl. In a pyrolysis of dibenzyl in toluene, Blades and Steacie also showed that $\mathrm{H}_{2}$ was an important product. Rate constants could, then, be in error by as much as a factor of two. Fortunately $\mathrm{CH}_{4}$ was not found in the dibenzyl-toluene system; thus $\mathrm{H}$-atoms, which could be chain propagators if they were formed from dibenzyl, were probably not present. In spite of the wealth of data for this reaction, the exact nature of the decomposition (i.e., the elementary reactions involved) has not been satisfactorily elucidated and the assignment of the overall kinetics to a particular bond-breaking reaction must also be considered somewhat suspect. Nevertheless, the agreement of the parameters of [4] with those estimated from the thermodynamics tends to support their validity.

\section{Experimental}

[1] Rate constants were based on $\left(\mathrm{H}_{2}+\mathrm{CH}_{4}\right)$ yields analyzed by standard gasometric methods. Products were $\mathrm{H}_{2}$, $\mathrm{CH}_{4}$, and dibenzyl in the following proportions: $\left(\mathrm{H}_{2} / \mathrm{CH}_{4}\right)=3 / 2,\left(\mathrm{H}_{2}+\mathrm{CH}_{4}\right)=\left(\varnothing \mathrm{CH}_{2}\right)_{2}$. The mechanism proposed was:

$$
\begin{aligned}
& \emptyset \mathrm{CH}_{3} \stackrel{\mathrm{l}}{\longrightarrow} \emptyset \mathrm{CH}_{2} \cdot+\mathrm{H} \cdot \quad \text { rate determining } \\
& \mathrm{H} \cdot+\varnothing \mathrm{CH}_{3} \stackrel{2}{\longrightarrow} \mathrm{H}_{2}+\varnothing \mathrm{CH}_{2} \text {. } \\
& \mathrm{H} \cdot+\varnothing \mathrm{CH}_{3} \stackrel{3}{\longrightarrow} \emptyset \mathrm{H}+\mathrm{CH}_{3} \text {. } \\
& \mathrm{CH}_{3}+\emptyset \mathrm{CH}_{3} \stackrel{4}{\longrightarrow} \mathrm{CH}_{4}+\emptyset \mathrm{CH}_{2} \text {. } \\
& 2 \varnothing \mathrm{CH}_{2} \cdot \stackrel{5}{\longrightarrow}\left(\emptyset \mathrm{CH}_{2}\right)_{2}
\end{aligned}
$$

[2] This study repeated the toluene pyrolysis over a broader range of conditions than those of [1]. It was found that the rate constants based on yields of $\left(\mathrm{H}_{2}+\mathrm{CH}_{4}\right)$ varied with contact time, initial pressure, and surface conditioning. In addition, as much as 50 percent of the nonvolatile products were found to be styrene and dimethyldiphenyls rather than dibenzyl. Since the reaction order was not unity, and since the activation energy seemed to depend on the experimental conditions (e.g., with $t_{c}=0.068 \mathrm{sec}, E_{\text {act }}=90 \mathrm{kcal} / \mathrm{mole}$ ), it was concluded that a correlation of activation energy with the bond dissociation energy could not be made.

[3] Rate constants were calculated as in [1] and [2]. Analysis was by M.S. Arrhenius parameters were shown to depend on the temperature range of study. Between $\left(764-843{ }^{\circ} \mathrm{C}\right), E_{\text {act }} \simeq 78 \mathrm{kcal} / \mathrm{mole}$ and between $\left(850-898^{\circ} \mathrm{C}\right)$ $E_{\text {act }} \simeq 90 \mathrm{kcal} / \mathrm{mole}$. The conclusion was that the curvature was a result of competitive reactions rather than variable experimental conditions.

[4] Rates were determined as in [1] and [2]. The reaction was found to be strongly surface dependent at temperatures below $1050{ }^{\circ} \mathrm{K}$. Isolation of the homogeneous reaction gave the parameters reported. Results of [3] were con. cluded to arise from heterogeneous effects at the lower temperatures. The best straight line through all high temperature results of [2] and [3] gave $E_{\text {act }}=86 \mathrm{kcal} / \mathrm{mole}$. 
Reaction: Toluene

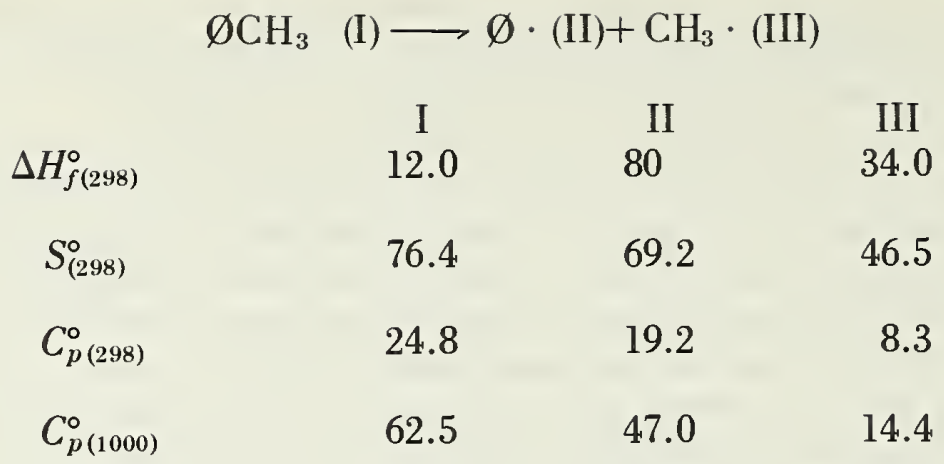

$\Delta S^{\circ}=41.3 \mathrm{~g} / \mathrm{mole}$

$\Delta H^{\circ}=102.0 \mathrm{kcal} / \mathrm{mole}$

$\Delta C_{p}^{\circ}=2.7 \mathrm{~g} / \mathrm{mole}$

\begin{tabular}{lrrrrrr}
$\log \boldsymbol{A}$ & $\boldsymbol{E}$ & $\begin{array}{c}\log \boldsymbol{k}_{T} \\
(1223)\end{array}$ & Conditions & System & Surface & \multicolumn{1}{c}{ References } \\
14.53 & 80.0 & 0.23 & $1073-1373^{\circ} \mathrm{K}$ & flow & none & [1] L. A. Errede and F. De \\
Maria, J. Phys. Chem. \\
\end{tabular}

Preferred:

Not reliable.

Comments: This reaction is thermodynamically unsound. If a reasonable recombination rate is assumed, $k_{\text {rec }} \geqslant 10^{9.6} \mathrm{l} /$ mole-sec, one obtains an estimate of the reaction of interest: $k \geqslant 10^{15.5} \times 10^{-102 / \theta} \mathrm{sec}^{-1}$. This is almost three orders of magnitude slower than the reported rate.

\section{Experimental}

[1] Rate constant parameters were deduced from the rate of benzene formation in the $p$-xylene decomposition. It was assumed that the mechanism for benzene formation from toluene was the same as the mechanism of toluene formation from xylene. The parameters were not definitely attributed to the above reaction, although it was considered the most likely initiation (and rate determining) process. 
Reaction: Ethylbenzene

\begin{tabular}{cccc}
\multicolumn{4}{r}{$\varnothing \mathrm{CH}_{2} \mathrm{CH}_{3}(\mathrm{I}) \longrightarrow \mathrm{CH}_{2} \cdot(\mathrm{II})+\mathrm{CH}_{3} \cdot(\mathrm{III})$} \\
& $\mathrm{I}$ & $\mathrm{II}$ & $\mathrm{III}$ \\
$\Delta H_{f(298)}^{\circ}$ & 7.2 & 45.0 & 34.0 \\
$S_{(298)}^{\circ}$ & 86.2 & 75.3 & 46.4 \\
$C_{p(298)}^{\circ-}$ & 30.9 & 25.6 & 8.3 \\
$C_{p(1000)}^{\circ}$ & 74.8 & 60.6 & 14.4
\end{tabular}

$\Delta S^{\circ}=35.5 \mathrm{~g} / \mathrm{mole}$

$\Delta H^{\circ}=71.8 \mathrm{kcal} / \mathrm{mole}$

$\Delta C_{p}^{\circ}=3.0 \mathrm{~g} / \mathrm{mole}$

\begin{tabular}{|c|c|c|c|c|c|c|}
\hline $\log A$ & $\boldsymbol{E}$ & $\begin{array}{c}\log k_{k} \\
(938)\end{array}$ & Conditions & System & Surface & References \\
\hline 13.0 & 63.2 & -1.73 & $\begin{array}{l}888-1018^{\circ} \mathrm{K} \\
0.1-0.35 \text { torr }\end{array}$ & flow & & $\begin{array}{l}\text { [1] M. Szwarc, J. Chem. } \\
\text { Phys. 17, } 431 \text { (1949). }\end{array}$ \\
\hline 14.6 & 70.1 & -1.74 & $876-1000{ }^{\circ} \mathrm{K}$ & flow & none & $\begin{array}{l}\text { [2] G. L. Esteban, J. A. } \\
\text { Kerr, and A. F. } \\
\text { Trotman-Dickenson, } \\
\text { J. Chem. Soc., } 3873 \\
\text { (1963). }\end{array}$ \\
\hline
\end{tabular}

Preferred:

$\log k=15.3-73.0 / \theta$.

Comments: From the thermodynamics and $\overline{\Delta C}_{p}^{\circ}=1.9$, the estimated activation energy is $73.0 \mathrm{kcal}$, which is probably within experimental error. The heat of formation of the benzyl radical used above is therefore supported. The methyl-benzyl radical recombination constant is calculated to be $k_{\text {rec }}=10^{9.0} 1 /$ mole-sec.

\section{Experimental}

The rate constants of both studies were calculated on the basis of the rate of $\mathrm{CH}_{4}$ formation. Standard gas analysis techniques were employed.

[1] Toluene carrier technique. Basic mechanism assumed: $\varnothing \mathrm{CH}_{2} \mathrm{CH}_{3} \longrightarrow{ }^{\longrightarrow} \varnothing \mathrm{CH}_{2} \cdot+\mathrm{CH}_{3} \cdot ; \mathrm{CH}_{3} \cdot{ }+\emptyset \mathrm{CH}_{3} \stackrel{2}{\longrightarrow}$ $\mathrm{CH}_{4}+\emptyset \mathrm{CH}_{2} \cdot ; 2 \emptyset \mathrm{CH}_{2} \cdot \stackrel{\text { s }}{\longrightarrow}\left(\emptyset \mathrm{CH}_{2}\right)_{2}$. Products observed: $\left(\mathrm{CH}_{4} /\left(\varnothing \mathrm{CH}_{2}\right)_{2}\right) \simeq 1$ with a gas composition of 2 percent $\mathrm{C}_{2}$, 15-25 percent $\left(\mathrm{H}_{2}+\mathrm{CH}_{4}\right)$. Most of the $\mathrm{H}_{2}$ formed was attributed to a heterogeneous decomposition: $\emptyset \mathrm{CH}_{2} \mathrm{CH}_{3} \longrightarrow$ $\emptyset \mathrm{CH}=\mathrm{CH}_{2}+\mathrm{H}_{2}$.

[2] Aniline carrier technique employed. Basic mechanism: $\emptyset \mathrm{CH}_{2} \mathrm{CH}_{3} \longrightarrow \emptyset \mathrm{CH}_{2} \cdot+\mathrm{CH}_{3} \cdot ; \mathrm{CH}_{3} \cdot \emptyset \mathrm{NH}_{2} \longrightarrow \mathrm{CH}_{4}$ $+\emptyset \mathrm{NH} ; \emptyset \mathrm{NH} \longrightarrow$ polymer. Main products were $\mathrm{CH}_{4}, \mathrm{H}_{2}$, and $\mathrm{C}_{2} \mathrm{H}_{4}$ roughly produced in the ratio of 20/10/1, respectively. Benzene was a minor product.

The origin of the $\mathrm{H}_{2}$ in both studies has not been satisfactorily explained, but could well arise from a molecular elimination from $\left(\emptyset \mathrm{CH}_{2}\right)_{2}$ or $\left(\emptyset \mathrm{NH}_{2}\right)_{2}$ (see comments on toluene). 
Reaction: $n$-Propylbenzene

\begin{tabular}{|c|c|c|c|}
\hline$\Delta H_{f(298)}^{\circ}$ & $\begin{array}{l}\mathrm{I} \\
2.3\end{array}$ & $\begin{array}{c}\text { II } \\
45.0\end{array}$ & $\begin{array}{c}\text { III }\left(V=4.5^{*}\right) \\
25.7\end{array}$ \\
\hline$S_{(298)}^{\circ}$ & 95.6 & 75.3 & 57.8 \\
\hline$C_{p(299)}^{\circ}$ & 36.4 & 25.6 & 12.1 \\
\hline$C_{p(1000)}^{\circ}$ & 87.1 & 60.6 & 27.2 \\
\hline
\end{tabular}

$\Delta S^{\circ}=37.5 \mathrm{~g} / \mathrm{mole}$

$\Delta H^{\circ}=68.4 \mathrm{kcal} / \mathrm{mole}$

$\Delta C_{p}^{\circ}=1.3 \mathrm{~g} / \mathrm{mole}$

\begin{tabular}{|c|c|c|c|c|c|c|}
\hline $\log A$ & $\boldsymbol{E}$ & $\begin{array}{c}\log \boldsymbol{k}_{T} \\
\quad(934)\end{array}$ & Conditions & System & Surface & References \\
\hline 12.5 & 57.5 & -0.96 & $\begin{array}{l}883-1033^{\circ} \mathrm{K} \\
0.3-1.5 \text { torr }\end{array}$ & flow & none & $\begin{array}{l}\text { [1] C. H. Leigh and M. } \\
\text { Szwarc, J. Chem. Phys. } \\
\text { 20, } 403 \text { (1952). }\end{array}$ \\
\hline 14.9 & 68.6 & -1.15 & $860-1008^{\circ} \mathrm{K}$ & flow & none & $\begin{array}{l}\text { [2] G. L. Esteban, J. A. Kerr, } \\
\text { and A. F. Trotman- } \\
\text { Dickenson, J. Chem. } \\
\text { Soc. } 3873 \text { (1963). }\end{array}$ \\
\hline
\end{tabular}

Preferred:

$\log k=14.9-68.6 / \theta$.

Comments: From the thermodynamics, $E=69.0 \mathrm{kcal} / \mathrm{mole}$ in good agreement with [2]. This gives a recombination rate constant of $k_{\text {rec }}=10^{8.7} \mathrm{l} /$ mole-sec.

\section{Experimental}

[1] Rate constants were based on $\mathrm{C}_{2}$ hydrocarbon formation (i.e. $\left[\mathrm{C}_{2} \mathrm{H}_{4}+\mathrm{C}_{2} \mathrm{H}_{6}\right]$ ). Analysis was by standard gasometric techniques. Products observed were: $\mathrm{C}_{2} \mathrm{H}_{4}, \mathrm{H}_{2}, \mathrm{CH}_{4},\left(\varnothing \mathrm{CH}_{2}\right)_{2}$ and minor amounts of $\mathrm{C}_{2} \mathrm{H}_{6}$ in the approximate ratios:

$$
\left(\mathrm{H}_{2}+\mathrm{CH}_{4}\right) / \mathrm{C}_{2} \mathrm{H}_{4} \simeq 0.85 ;\left(\mathrm{H}_{2} / \mathrm{CH}_{4}\right) \simeq 3 / 2 ; \frac{\left(\mathrm{C}_{2} \mathrm{H}_{4}\right)}{\mathrm{C}_{2} \mathrm{H}_{4}+\mathrm{C}_{2} \mathrm{H}_{6}} \simeq 0.9
$$

Decomposition of the ethyl radical relative to trapping with toluene was the preferred reaction mode. First-order rate constants were independent of $t_{c}, P_{T}$, and $\delta P$ reactant.

[2] Aniline carrier technique. Rate constants were based on $\mathrm{C}_{2} \mathrm{H}_{6}$ and $\mathrm{C}_{2} \mathrm{H}_{4}$ analyzed by G.L.C. Mechanism:

$$
\begin{aligned}
& \emptyset \mathrm{C}_{3} \mathrm{H}_{7} \stackrel{1}{\longrightarrow} \emptyset \mathrm{CH}_{2} \cdot+\mathrm{C}_{2} \mathrm{H}_{5} \cdot ; \mathrm{C}_{2} \mathrm{H}_{5} \cdot \stackrel{2}{\longrightarrow} \mathrm{C}_{2} \mathrm{H}_{4}+\mathrm{H} \cdot ; \\
& \mathrm{C}_{2} \mathrm{H}_{5} \cdot+\emptyset \mathrm{NH}_{2} \stackrel{3}{\longrightarrow} \mathrm{C}_{2} \mathrm{H}_{6}+\emptyset \dot{\mathrm{NH}} ; \mathrm{H} \cdot+\emptyset \mathrm{NH}_{2} \stackrel{4}{\longrightarrow} \mathrm{H}_{2}+\emptyset \mathrm{NH} .
\end{aligned}
$$

Main products were; $\mathrm{C}_{2} \mathrm{H}_{4}, \mathrm{H}_{2}, \mathrm{C}_{2} \mathrm{H}_{6}$ and $\mathrm{CH}_{4}$. Methane production was attributed to the reaction:

$$
\varnothing \mathrm{C}_{3} \mathrm{H}_{7} \longrightarrow \varnothing \mathrm{C}_{2} \mathrm{H}_{5} \cdot+\mathrm{CH}_{3} .
$$

\footnotetext{
*(See discussion on rotational barriers in radicals preceding bond breaking reactions.)
} 


\begin{tabular}{|c|c|c|c|c|c|}
\hline \multirow[t]{2}{*}{$\emptyset(\mathrm{C}$} & \multicolumn{5}{|c|}{ 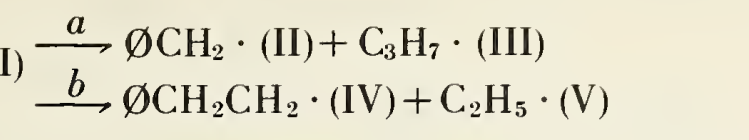 } \\
\hline & I & II & $\begin{array}{c}\text { III } \\
(V=4.0\end{array}$ & $\begin{array}{c}\text { IV } \\
V=4.0\end{array}$ & $\begin{array}{c}\mathrm{V} \\
\left.V=4.5^{*}\right)\end{array}$ \\
\hline$\Delta H_{f(298)}^{\circ}$ & -2.9 & 45.0 & 20.7 & 52.6 & 25.7 \\
\hline$S_{(298)}^{\circ}$ & 105.0 & 75.3 & 67.2 & 89.2 & 57.8 \\
\hline$C_{p(298)}^{\circ}$ & 41.9 & 25.6 & 17.6 & 30.7 & 12.1 \\
\hline$C_{p(1000)}^{\circ}$ & 99.4 & 60.6 & 39.5 & 72.3 & 27.2 \\
\hline
\end{tabular}

$\begin{array}{rl}a & b \\ \Delta S^{\circ}=37.5 & 42.0 \mathrm{~g} / \text { mole } \\ \Delta H^{\circ}=68.6 & 81.2 \mathrm{kcal} / \mathrm{mole} \\ \Delta C_{p}^{\circ}=1.3 & 0.9 \mathrm{~g} / \text { mole }\end{array}$

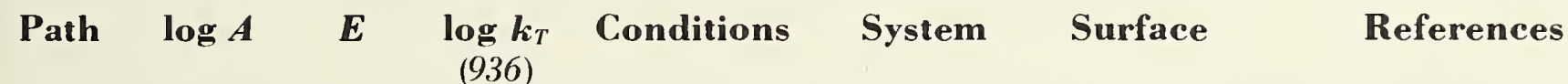

$a$

$\begin{array}{lllll}14.5 & 65.0 & -0.69 & 879-947^{\circ} \mathrm{K} & \text { flow none }\end{array}$ 0.2-1 torr

[1] C. H. Leigh and M. Szwarc, J. Chem. Phys. 20, 407

(1952).

$a$

$14.5 \quad 67.1-1.18 \quad 863-1008 \%$ flow

[2] G. L. Esteban, J. A. Kerr, and A. F. Trotman-Dickenson, J. Chem. Soc., 3873 (1963).

Preferred:

$\log k_{a}=14.5-67.1 / \theta$.

Comments: Thermodynamics give $\Delta H_{T}^{\circ}=E_{a}=69.2 \mathrm{kcal} /$ mole. From the entropy one obtains, $k_{-a}=10^{8.7}$ $1 /$ mole-sec. If the recombination rate constant, $k_{-b}$ is assumed to be a factor of 5 larger than $k_{-a}$ (i.e., no resonance tightening in the transition state), then path $(b)$ reaction thermodynamics give a rate constant for decomposition of about: $k_{b}=10^{16.8} \times 10^{-81.5 / \theta} \mathrm{sec}^{-1}$. This suggests about 10 percent decomposition via path $(b)$, see [2].

\section{Experimental}

[1] Toluene carrier technique. Rates of the reaction were based on the $\mathrm{C}_{2} \mathrm{H}_{4}$ formation. Complete decomposition of the $n$-propyl radical (i.e., $\mathrm{C}_{3} \mathrm{H}_{7} \longrightarrow \mathrm{CH}_{3} \cdot+\mathrm{C}_{2} \mathrm{H}_{4}$ ) was reasonably assumed. The main products were $\mathrm{C}_{2} \mathrm{H}_{4}, \mathrm{CH}_{4}$, and $\left(\emptyset \mathrm{CH}_{2}\right)_{2}$. Ratios of products suggested side reactions: $\left(\mathrm{CH}_{4}+\mathrm{H}_{2}\right) / \mathrm{C}_{2} \mathrm{H}_{4} \simeq 0.7 ;\left(\mathrm{H}_{2} / \mathrm{CH}_{4}+\mathrm{H}_{2}\right) \simeq 0.2 ;\left(\emptyset \mathrm{CH}_{2}\right)_{2} / \mathrm{C}_{2} \mathrm{H}_{4}$ $=0.4$. The usual mechanism (see ethyl benzene) was assumed.

[2] Aniline carrier technique. Rate constants were based on $\mathrm{CH}_{4}$ formation. Overall decomposition rates were based on $\mathrm{C}_{2} \mathrm{H}_{4}$ formation. Accuracy was not sufficient to determine parameters of path $b$, which was thought to be competitive. Mechanism:

$$
\emptyset \mathrm{C}_{4} \mathrm{H}_{9} \stackrel{1}{\longrightarrow} \emptyset \mathrm{CH}_{2} \cdot+\mathrm{C}_{3} \mathrm{H}_{7} \cdot \quad \mathrm{C}_{3} \mathrm{H}_{7} \cdot \stackrel{2}{\longrightarrow} \mathrm{CH}_{3} \cdot+\mathrm{C}_{2} \mathrm{H}_{4}
$$

(Continued)

* See discussion on rotational barriers in radical preceding bond fission reactions. 
n-Butylbenzene (Continued)

$$
\begin{aligned}
& \mathrm{CH}_{3} \cdot+\emptyset \mathrm{NH}_{2} \stackrel{3}{\longrightarrow} \mathrm{CH}_{4}+\emptyset \mathrm{NH} \quad \emptyset \mathrm{C}_{4} \mathrm{H}_{9} \stackrel{4}{\longrightarrow} \emptyset \mathrm{C}_{2} \mathrm{H}_{4} \cdot+\mathrm{C}_{2} \mathrm{H}_{5} \cdot \\
& \mathrm{C}_{2} \mathrm{H}_{5} \cdot \stackrel{5}{\longrightarrow} \mathrm{H} \cdot+\mathrm{C}_{2} \mathrm{H}_{4} .
\end{aligned}
$$

Principal products were: $\mathrm{CH}_{4}, \mathrm{H}_{2}$, and $\mathrm{C}_{2} \mathrm{H}_{4}$. Yields of $\mathrm{C}_{2} \mathrm{H}_{6}, \mathrm{C}_{3} \mathrm{H}_{8}$, and $\mathrm{C}_{3} \mathrm{H}_{6}$ were less than 1 percent of the gaseous products. 
Reaction: Isopropylbenzene (cymene)

$\begin{array}{cccr} & & & \\ & \text { I } & \text { II }\left(\mathrm{V}=4^{*}\right) & \text { III } \\ \Delta H_{f(298)}^{\circ} & 0.9 & (36.6) & 34.0 \\ S_{(298)}^{\circ} & 92.9 & 84.1 & 46.4 \\ C_{p(298)}^{\circ} & 36.3 & 31.6 & 8.3 \\ C_{p(1000)}^{\circ} & 87.3 & 73.5 & 14.4\end{array}$

$\Delta S^{\circ}=37.6 \mathrm{~g} / \mathrm{mole}$

$\Delta H^{\circ}=(69.7) \mathrm{kcal} / \mathrm{mole}$

$\Delta C_{p}^{\circ}=3.6 \mathrm{~g} / \mathrm{mole}\left\langle\Delta \bar{C}_{p}^{\circ}=2.0\right\rangle_{298-900}$

\begin{tabular}{|c|c|c|c|c|c|}
\hline $\log A$ & $\boldsymbol{E}$ & $\log k_{T}$ & Conditions & System & \\
\hline
\end{tabular}

$\begin{array}{llll}13.3 \quad 61 \quad-0.88 & 865-935^{\circ} \mathrm{K} \quad \text { flow } \\ & 0.9-1.2 \text { torr }\end{array}$

$\begin{array}{lllll}14.3 & 66.0 \quad-1.04 & 892-989^{\circ} \mathrm{K} \quad \text { flow }\end{array}$ (7 torr total)

Preferred:

$\log k=15.45-71.0 / \theta$.

Comments: The heat of formation of the radical in parenthesis corresponds to a resonance energy of 13.5 $\mathrm{kcal} / \mathrm{mole}$ (as in the benzyl radical). The thermodynamics then predict an activation energy of $E \simeq 71.0$ $\mathrm{kcal} / \mathrm{mole}$, which is within experimental error of [2]. The higher (thermodynamic) value gives a more reasonable recombination rate constant $\left(k_{\text {rec }}=10^{8.6} \mathrm{l} / \mathrm{mole} \cdot \mathrm{sec}\right)$ and is therefore preferred.

\section{Experimental}

[1] Rates were based on the rate of $\mathrm{CH}_{4}$ produced. (See comments on $p$-isopropyl toluene.) The $A$-factor (10 $\left.{ }^{13.3}\right)$ was assumed.

[2] Rates were based on the $\mathrm{CH}_{4}$ production.

*See discussion on rotational barriers in radicals preceding bond fission reactions. 
Reaction: $t$-Butylbenzene

\begin{tabular}{|c|c|c|c|}
\hline$\Delta H_{f(298)}^{\circ}$ & $\begin{array}{c}\text { I } \\
-5.3\end{array}$ & $\begin{array}{l}\mathrm{II}(V=4) \\
\quad(26.2)\end{array}$ & $\begin{array}{l}\text { III } \\
34\end{array}$ \\
\hline$S_{(298)}^{\circ}$ & 95.0 & 89.6 & 46.5 \\
\hline$C_{p(298)}^{\circ}$ & 42.3 & 36.6 & 8.5 \\
\hline$C_{p(1000)}^{\circ}$ & 100 & 85.3 & 14.4 \\
\hline
\end{tabular}

$\Delta S^{\circ}=41.1 \mathrm{~g} / \mathrm{mole}$

$\Delta H^{\circ}=65.5 \mathrm{kcal} / \mathrm{mole}$

$\Delta C_{p}^{\circ}=2.8 \mathrm{~g} / \mathrm{mole}$

\begin{tabular}{|c|c|c|c|c|c|c|}
\hline $\log A$ & $E$ & $\begin{array}{c}\log \boldsymbol{k}_{T} \\
(900)\end{array}$ & Conditions & System & Surface & References \\
\hline 13.48 & 59.5 & -0.97 & $\begin{array}{l}865-935^{\circ} \mathrm{K} \\
0.9-1.2 \text { torr }\end{array}$ & flow & & 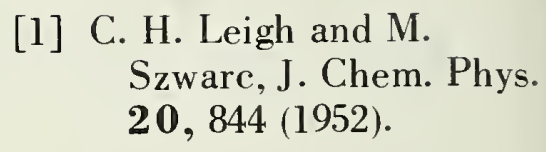 \\
\hline
\end{tabular}

\section{Preferred:}

$\log k=15.2-66.6 / \theta$.

Comments: The $A$-factor assumed by the authors is in error. The preferred parameters were obtained by scaling the reported parameters to the indicated reaction enthalpy, assuming a benzyl resonance energy of $13.5 \mathrm{kcal} / \mathrm{mole}$ for the product radical. Using the reaction entropy ${ }^{*}$ one obtains: $k_{\mathrm{rec}}=10^{7.8} \mathrm{l} / \mathrm{mole}$-sec.

\section{Experimental}

[1] Analysis and rates were based on $\mathrm{CH}_{4}$ formation. (See comments on $p$-isopropyl toluene.) The $A$-factor reported was assumed (by analogy) to be the same as that for ethylbenzene corrected for the different reaction path degeneracies.

*See discussion on rotational barriers in radicals preceding bond fission reactions. 
Reaction: $p$-Isopropyltoluene ( $p$-cymene)

\begin{tabular}{|c|c|c|c|}
\hline$\Delta H_{f(298)}^{\circ}$ & $\begin{array}{c}\text { I } \\
-7.0\end{array}$ & $\begin{array}{c}\mathrm{II}(V=4) \\
\quad(28.7)\end{array}$ & $\begin{array}{l}\mathrm{CH}_{3} \text {. } \\
34.0\end{array}$ \\
\hline$S_{(298)}^{\circ}$ & 101.3 & $92.5^{*}$ & 46.4 \\
\hline$C_{p(298)}^{\circ}$ & 41.9 & 37.2 & 8.3 \\
\hline$C_{p(1000)}^{\circ}$ & 99.2 & 85.4 & 14.4 \\
\hline
\end{tabular}

$\Delta S^{\circ}=37.6 \mathrm{~g} / \mathrm{mole}$

$\Delta H^{\circ}=69.7 \mathrm{kcal} / \mathrm{mole}$

$\Delta C_{p}^{\circ}=3.6 \mathrm{~g} / \mathrm{mole}$

\begin{tabular}{|c|c|c|c|c|c|c|}
\hline $\log A$ & $\boldsymbol{E}$ & $\begin{array}{c}\log \boldsymbol{k}_{T} \\
(900)\end{array}$ & Conditions & System & Surface & References \\
\hline 13.3 & 60 & -1.27 & $\begin{array}{l}865-935^{\circ} \mathrm{K} \\
0.9-1.2 \text { torr }\end{array}$ & flow & & 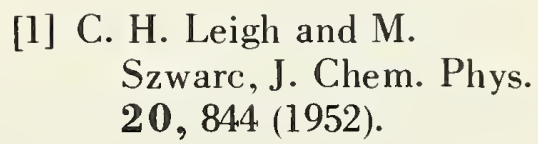 \\
\hline
\end{tabular}

Preferred:

$\log k=16.0-71.0 / \theta$.

Comments: Reported parameters are undoubtedly too low since the $A$-factor assumed was in error. The preferred values were obtained by scaling to the indicated reaction enthalpy. The latter is based on an assigned resonance energy in the product radical of $13.5 \mathrm{kcal} / \mathrm{mole}$. From the reaction entropy (see discussion on rotational barriers in radicals), one can calculate $k_{\text {rec }}=10^{9.1} \mathrm{l} / \mathrm{mole}-\mathrm{sec}$.

\section{Experimental}

[1] Rate constants were based on 5/7 the rate of $\mathrm{CH}_{4}$ reaction. This factor accounted for the $\mathrm{CH}_{4}$ produced ultimately by the reaction $\mathrm{H} \cdot+\varnothing \mathrm{CH}_{3} \longrightarrow \mathrm{CH}_{3} \cdot+\emptyset \mathrm{H}$, which was important under similar conditions in the toluene pyrolysis system. Reported Arrhenius parameters were calculated from the rate constants and were based on an $A$-factor assumed to be $2 \times A$ (ethylbenzene). (The factor of 2 corrects for the difference in reaction path degeneracies of the two reactions.) Reasonable agreement between the calculated Arrhenius line and the experimental data was demonstrated.

${ }^{*}$ See discussion on rotational barriers in radicals preceding bond fission reactions. 
Reaction: Dibenzyl (1,2-diphenylethane)

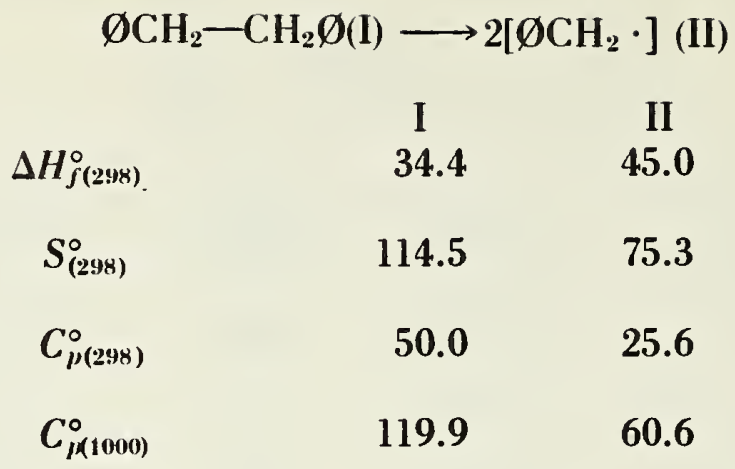

$\Delta S^{\circ}=36.1 \mathrm{~g} / \mathrm{mole}$

$\Delta H^{\circ}=55.6 \mathrm{kcal} / \mathrm{mole}$

$\Delta C_{p}^{\circ}=1.2 \mathrm{~g} / \mathrm{mole}$

\begin{tabular}{|c|c|c|c|c|c|c|}
\hline $\log A$ & $\boldsymbol{E}$ & $\begin{array}{l}\log k_{T} \\
(975)\end{array}$ & Conditions & System & Surface & References \\
\hline 9.3 & 48.0 & -1.46 & $\begin{array}{l}903-1047^{\circ} \mathrm{K} \\
.01-.4 \text { torr }\end{array}$ & flow & $<5 \%$ & $\begin{array}{l}\text { [1] C. Horrex and S. E. Miles, } \\
\text { Disc. Faraday Soc. 10, } \\
187 \text { (1951). }\end{array}$ \\
\hline
\end{tabular}

Preferred:

Not reliable.

Comments: Kinetic results are badly scattered and show sizable dependence on initial pressures and contact times. In addition, the reported rates of reaction are at least two orders of magnitude lower than those reasonably estimated from the thermodynamics and back reaction rate constant. The reason for this discrepancy probably arises from the dibenzyl $\rightleftharpoons$ benzyl equilibrium which should be rapidly achieved. The observed decomposition kinetics would therefore not apply to the bond-breaking step, but rather to the secondary free radical chain processes following and including the initial equilibrium. By assuming a recombination $A$-factor of $10^{8.1 \pm 0.5} 1 /$ mole-sec, an estimate of the decompositon kinetics can be made from the thermodynamics. $k_{\mathrm{est}}=10^{14.4 \pm 0.5-56.8 / \theta} \mathrm{sec}^{-1}$.

\section{Experimental}

[1] Analysis was by ordinary chemical techniques and by V.P. and U.V. Products were complex. Main products: styrene, toluene, benzene, and stilbene; minor products: $\mathrm{CH}_{4}, \mathrm{H}_{2}$. The overall stoichiometry employed in obtaining the rate constants was, $3 \emptyset \mathrm{CH}_{2} \mathrm{CH}_{2} \emptyset \longrightarrow 2 \emptyset \mathrm{CH}_{3}+\emptyset \mathrm{H}+\emptyset \mathrm{CH}=\mathrm{CH}_{2}+\emptyset \mathrm{CH}=\mathrm{CH} \emptyset$. The rate of toluene formation (taken as 55 percent of the weight of the liquid products) was equated to the rate of benzyl radical formation. 


\section{Reaction: $o \cdot X y l e n e$}

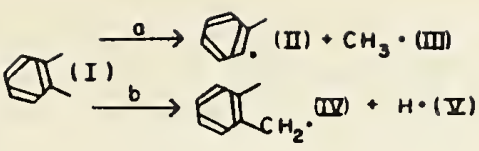

$\begin{array}{cccccc} & \text { I } & \text { II } & \text { III } & \text { IV } & \text { V } \\ \Delta H_{\text {(298) }}^{\circ} & 4.8 & 72.2 & 34.0 & 37.8 & 52.1 \\ S_{(298)}^{\circ} & 84.0 & 78.9 & 46.4 & 83.0 & 27.4 \\ C_{p(298)}^{\circ} & 31.3 & 23.4 & 8.5 & 31.0 & 5.0\end{array}$
a
b
$\Delta S^{\circ}=41.3$
$26.4 \mathrm{~g} / \mathrm{mole}$
$\Delta H^{\circ}=101.4$
$85.1 \mathrm{kcal} / \mathrm{mole}$
$\Delta C_{p}^{\circ}=0.6$
$4.7 \mathrm{~g} / \mathrm{mole}$

Path long $A \quad E \quad \begin{gathered}\log k_{T} \\ (1000)\end{gathered}$ Conditions System Surface $\quad$ References

$\begin{array}{llllll}a & 12.3 & 67.0 & -2.32 & 917-978^{\circ} \mathrm{K} \quad \text { flow }\end{array}$

[1] J. G. Burr and J. D. Strong, J. Am.

Chem. Soc., 86, 5065 (1964).

$b$

$13.7 \quad 74.8-2.64 \quad 1003-1110^{\circ} \mathrm{K}$ flow

[2] M. Szwarc, J. Chem.

Phys., 16, 128

(1948); Nature 160, 403 (1947).

Preferred:

Not reliable.

Comments: Both sets of Arrhenius parameters are quite incompatible thermodynamically with the processes indicated. The parameters should be very similar to those of the corresponding toluene reaction. Adjusting from the toluene parameters for differences in symmetry and for ortho substituent group effects one predicts for these reactions: $\log k_{a}=15.8-102 / \theta ; \log k_{b}=15.8-88.3 / \theta$.

Reaction $a$ is therefore much too slow at. reaction temperatures to be important. Since the reported rates are faster than those predicted (by factors of 4 to 10), some chain decomposition seems likely in both systems.

\section{Experimental}

[1] Hydrogen and deuterium carriers used on normal and methyl $d_{6}$ xylenes. Products were $\mathrm{CH}_{4}$ and $\emptyset \mathrm{CH}_{3}$. Proposed path $(a)$ as the primary split in xylene to explain the relatively low rate of $H-D$ exchange in the methyl groups of the recovered xylene. H-atom displacement of $\mathrm{CH}_{3}$ in xylene was ruled out. However, see $\mathrm{S}$. W. Benson and $\mathrm{R}$. Shaw, J. Chem. Phys. 47, 4052 (1967); J. Am. Chem. Soc. 89, 5351 (1967), for a detailed treatment of the mechanism.

[2] Principal products were $\mathrm{CH}_{4}$ and $\mathrm{H}_{2}$ in a $1 / 1$ ratio. $\mathrm{C}_{2}$ hydrocarbons represented 10 percent of the gaseous products. Large yields of $\mathrm{H}_{2}$ were taken as evidence for the $(\mathrm{C}-\mathrm{H})$ bond rupture in the primary process. Analogous product ratios on the xylene and toluene systems were given as evidence for similar reaction mechanisms. 
Reaction: $m$-Xylene

$\begin{array}{cccc} & \text { I } & \text { II } & \text { III } \\ \Delta H_{f(298)}^{\circ} & 4.2 & 37.2 & 52.1 \\ S_{(298)}^{\circ} & 84.8 & 83.7 & 27.4 \\ C_{p(298)}^{\circ} & 30.2 & 30.6 & 5.0\end{array}$

$\Delta S^{\circ}=26.3 \mathrm{~g} / \mathrm{mole}$

$\Delta H^{\circ}=85.1 \mathrm{kcal} / \mathrm{mole}$

$\Delta C_{p}^{\circ}=5.4 \mathrm{~g} / \mathrm{mole}$

\begin{tabular}{|c|c|c|c|c|c|c|}
\hline $\log A$ & $E$ & $\begin{array}{c}\log \mathbf{k}_{T} \\
(1077)\end{array}$ & Conditions & System & Surface & References \\
\hline 13.6 & 77.1 & $(-2.05)$ & $1013-1140^{\circ} \mathrm{K}$ & flow & none & $\begin{array}{l}\text { [1] M. Szwarc, J. Chem. } \\
\text { Phys., 16, } 128 \text { (1948). }\end{array}$ \\
\hline
\end{tabular}

Preferred:

Not reliable.

Comments: Rate constants are probably close to correct. By comparison with the toluene reaction, one would predict, $\log k_{\text {est }}=15.8-88.3 / \theta$.

\section{Experimental}

[1] The $\left(\mathrm{H}_{2} / \mathrm{CH}_{4}\right)$ ratios were identical to those found in the toluene pyrolysis, namely about $3 / 2$. See comments on $\boldsymbol{o}$-xylene. 
Reaction: $p$-Xylene
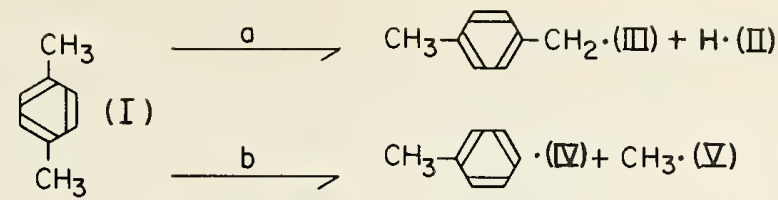

$\Delta H_{f(298)}^{\circ}$

I II

4.8

37.2

$\mathrm{CH}_{3}-\square \cdot(\mathrm{Z})+\mathrm{CH}_{3} \cdot(\mathrm{Z})$

$S_{(298)}^{\circ}$

84.0

83.7

III

IV

V

$\begin{array}{lll}52.1 & 72.2 & 34.0\end{array}$

$C_{p(298)}^{\circ}$

31.3

29.3

27.4

77.5

46.5

$a$ $b$

$\Delta S^{\circ}=27.1$

$40 \mathrm{~g} / \mathrm{mole}$

$\Delta H^{\circ}=84.5$

$101.4 \mathrm{kcal} / \mathrm{mole}$

$\Delta C_{p}^{\circ}=3.0$

$1.9 \mathrm{~g} / \mathrm{mole}$

$\begin{array}{lllllll}\text { Path } & \log A & \boldsymbol{E} & \log k_{T} & \text { Conditions } & \text { System } & \text { Surface }\end{array}$

$13.7 \quad 76.2-5.67 \quad 1018-1123^{\circ} \mathrm{K}$ flow

$\begin{array}{lll}5.0 & 24.7 \quad 8.5\end{array}$

$\begin{array}{cccc}13.97 \quad 76 & -5.35 \quad \begin{array}{ll}1073-1373^{\circ} \mathrm{K} \\ \end{array} \quad \text { flow } \\ & & & 0.2 \mathrm{~mm} \mathrm{Hg}\end{array}$

$a$

$\begin{array}{rll}13.97 \quad 76 \quad-5.35 \quad & 1073-1373^{\circ} \mathrm{K} \text { flow } \\ & \sim 0.2 \mathrm{~mm} \mathrm{Hg}\end{array}$

$b$

$a$
$b$

$b$

Preferred:

$\begin{array}{lllll}14.43 & 79.5 & -5.78 \quad 795-920^{\circ} \mathrm{K} \quad \text { flow }\end{array}$

$\begin{array}{lll}14.67 & 81.8 & -6.12\end{array}$

\section{References}

[1] M. Szwarc, J. Chem. Phys. 16, 128 (1948).

[2] L. A. Errede and F. DeMaria, J. Phys. Chem. 66, 2664 (1962). (as above)

[3] J. G. Burr and J. D. Strong, J. Am. Chem. Soc. 86, 5065 (1964).

[4] J. R. Schaefgen, J. Polymer Sci. 15, 203 (1955).

Rate constants for path $a$ are probably reliable. Parameters are low. Path $b$ rate constants are unreliable.

Comments: Estimate:

$\log k_{a}=15.8-88.3 / \theta$

$\log k_{b}=15.8-102 / \theta$.

(See comments on $o$ - and $m$-xylenes.)

\section{Experimental}

[1] The $\left(\mathrm{H}_{2} / \mathrm{CH}_{4}\right)$ ratios were about $6.5 / 3.5$, slightly higher than those obtained in the toluene pyrolysis. Rate constants were calculated on the basis of the $\left(\mathrm{CH}_{4}+\mathrm{H}_{2}\right)$ produced.

[2] Path $a$ parameters were determined on the basis of $p$-xylyl equivalents (defined as all products of the reaction other than toluene and benzene). Path $b$ parameters were obtained on the basis of the toluene and benzene production where it was assumed that toluene produced benzene by the same mechanism as xylene decomposed to toluene.

[3] See [1] for $o$-xylene.

[4] Rate constants for path $a$ were based on the rate of $\mathrm{H}_{2}$ formation, and those of path $b$ were based on the $\mathrm{CH}_{4}$ formation. Analysis by M.S. Qualitative product identification by I.R. Rate constants of path $b$ varied with contact time and pressure. 
Reaction: $\alpha$-Methylnaphthalene ( $\alpha=$ ortho)

$$
\beta \text {-Methylnaphthalene }(\beta=\text { meta })
$$

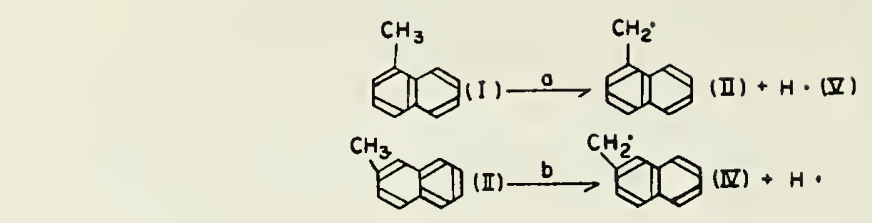

$\begin{array}{cccccc} & \text { I } & \text { II } & \text { III } & \text { IV } & \text { V } \\ \Delta H_{f(298)}^{\circ} & -29.0 & -29.0 & 4.0 & 4.0 & 52.1 \\ S_{(298)}^{\circ} & 81.7 & 81.7 & 80.6 & 80.6 & 27.4 \\ C_{p(298)}^{\circ} & & & & & 5.0\end{array}$

Path $a$

$$
\begin{aligned}
& \Delta S^{\circ}=26.3 \mathrm{~g} / \mathrm{mole} \\
& \Delta H^{\circ}=85.1 \mathrm{kcal} / \mathrm{mole} \\
& \Delta C_{p}^{\circ}=
\end{aligned}
$$

$\begin{array}{llllll}\text { Path } \quad \log A & E & \log k_{T} & \text { Conditions } & \text { System } & \text { Surface }\end{array}$ $a$

$13.2 \quad 73.5$

$13.2 \quad 73.5$

none given (flow)

$b$

\section{References}

[1] M. Szwarc and A. Shaw, unpublished, quoted by Szwarc, Chem. revs. 47 , 75 (1950).

Preferred:

$\log k_{a}=15.5-84.3 / \theta$

$\log k_{b}=15.5-84.3 / \theta$.

Comments: The rate constants are probably reliable, but the parameters are certainly low. One would expect the $A$-factor to be the same as that for toluene, and adjustment to that value gives the preferred parameters.

\section{Experimental}

See [2] for $o$-xylene. 
Reaction: Para, ortho, and meta fluorotoluenes

$$
\begin{aligned}
& \text { para } \mathrm{CH}_{3} \emptyset \mathrm{F}(\mathrm{I}) \stackrel{a}{\longrightarrow} \text { para } \cdot \mathrm{CH}_{2} \varnothing \mathrm{F}(\mathrm{II})+\mathrm{H} \cdot(\mathrm{III}) \\
& \text { ortho } \mathrm{CH}_{3} \emptyset \mathrm{F} \quad \stackrel{b}{\longrightarrow} \text { ortho } \cdot \mathrm{CH}_{2} \emptyset \mathrm{F}+\mathrm{H} \cdot \\
& \text { meta } \mathrm{CH}_{3} \varnothing \mathrm{F} \stackrel{c}{\longrightarrow} \text { meta } \cdot \mathrm{CH}_{2} \varnothing \mathrm{F}+\mathrm{H} \cdot \\
& \begin{array}{cccc} 
& \text { I } & \text { II } & \text { III } \\
\Delta H_{f(298)}^{\circ} & -34.3 & -1.3 & 52.1
\end{array} \\
& \begin{array}{llll}
S_{(298)}^{\circ} & 80.8 & 79.7 & 27.4
\end{array} \\
& \begin{array}{llll}
C_{p(298)}^{\circ} & 27.7 & 28.1 & 5.0
\end{array} \\
& \begin{array}{llll}
C_{p(1000)}^{\circ} & 64.2 & 62.3 & 5.0
\end{array}
\end{aligned}
$$

$\Delta S^{\circ}=26.3 \mathrm{~g} / \mathrm{mole}$

$\Delta H^{\circ}=85.1 \mathrm{kcal} / \mathrm{mole}$

$\Delta C_{p}^{\circ}=5.4 \mathrm{~g} / \mathrm{mole}$

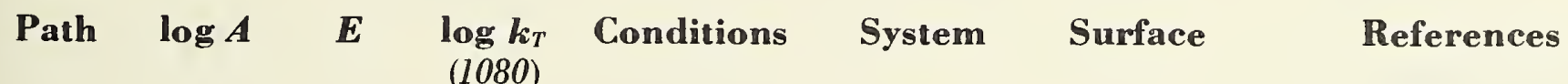

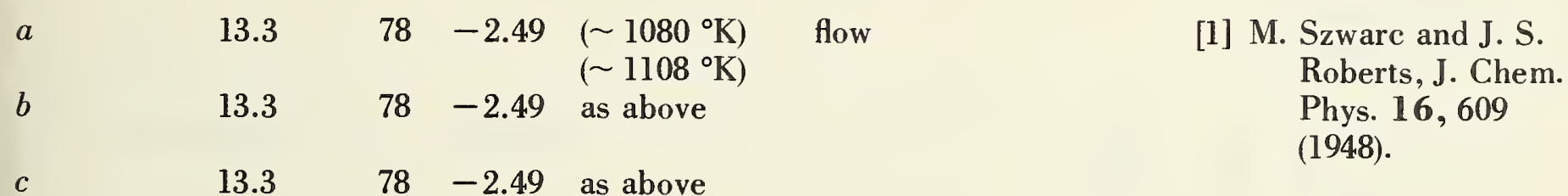

Preferred:

Rate constants probably reliable. Parameters are low.

$\log k=15.5-88.8 / \theta$.

Comments: Errors inherent in the toluene results have been introduced here. Scaling the reported parameters to the "corrected" toluene $A$-factor gives the preferred values above.

\section{Experimental}

[1] Analysis procedures and interpretation were identical to those employed in the toluene system. The observed rates were within 20 percent of the toluene rates at 1080 and at $1108^{\circ} \mathrm{K}$. Activation energies were calculated assuming an $A$-factor equivalent to that of the toluene decomposition. 
Reactants: $\alpha$-Picoline

$\beta$-Picoline

$\gamma$-Picoline

$\begin{array}{cccccccc} & \text { I } & \text { II } & \text { III } & \text { IV } & \text { V } & \text { VI } & \text { H } \\ \Delta H_{f(298)}^{\circ} & 23.6 & 25.3 & 25.3 & 54.6 & 55.6 & 56.6 & 52.1 \\ S_{(298)}^{\circ} & 77.8 & 77.8 & 76.4 & 76.7 & 76.7 & 75.3 & 27.4 \\ C_{p(298)}^{\circ} & 24.1 & 23.9 & 24.0 & 24.6 & 24.6 & 24.6 & 5.0\end{array}$

$$
\begin{aligned}
& \Delta S^{\circ}=26.3 \mathrm{~g} / \mathrm{mole} \\
& \Delta H^{\circ}=83.1 \mathrm{kcal} / \mathrm{mole} \\
& \Delta C_{p}^{\circ}=5.5 \mathrm{~g} / \mathrm{mole}
\end{aligned}
$$

\begin{tabular}{|c|c|c|c|c|c|c|c|}
\hline Path & $\log A$ & $\boldsymbol{E}$ & $\begin{array}{l}\log \boldsymbol{k}_{T} \\
(1054)\end{array}$ & Conditions & System & Surface & References \\
\hline & 13.3 & 75.5 & -2.36 & $\sim 1058^{\circ} \mathrm{K}$ & flow & & $\begin{array}{l}\text { [1] J. S. Roberts and } \\
\text { M. Szwarc J. Chem. }\end{array}$ \\
\hline & 13.3 & 76.5 & -2.56 & $\sim 1054^{\circ} \mathrm{K}$ & flow & & $\begin{array}{l}\text { Phys., 16, } 981 \\
\text { (1948). }\end{array}$ \\
\hline & 13.3 & 77.5 & -2.77 & $\sim 1056^{\circ} \mathrm{K}$ & flow & & \\
\hline
\end{tabular}

Preferred: gives:

Rate constants probably reliable but parameters are low. Adjustment to the preferred values for toluene

$\alpha$-picoline: $\log k=15.5-86.2 / \theta$;

$\beta$-picoline: $\log k=15.5-87.2 / \theta$;

$\gamma$-picoline: $\log k=15.5-88.2 / \theta$.

\section{Experimental}

[1] Procedures, analysis, and interpretation were identical to those for toluene [1]. The same $A$-factor as the toluene decomposition was assumed and the activation energy was calculated from the observed rate constants. 
Reaction: Phenylethanoic acid (phenylacetic acid)

\begin{tabular}{|c|c|c|c|}
\hline \multirow[b]{2}{*}{$\Delta H_{f(298)}^{\circ}$} & \multicolumn{3}{|c|}{$\begin{array}{l}\stackrel{a}{\longrightarrow} \emptyset \mathrm{CH}_{2} \cdot(\mathrm{II})+\cdot \mathrm{COOH}(\mathrm{III}) \\
\stackrel{b}{\mathrm{C}} \emptyset \mathrm{CH}=\mathrm{CO}+\mathrm{H}_{2} \mathrm{O}\end{array}$} \\
\hline & $\begin{array}{c}\text { I } \\
-75.8\end{array}$ & $\begin{array}{c}\text { II } \\
45.0\end{array}$ & $\begin{array}{c}\text { III } \\
(-53.3)\end{array}$ \\
\hline$S_{(298)}^{\circ}$ & 97.3 & 75.3 & 60.7 \\
\hline$C_{p(298)}^{\circ}$ & 34.3 & 25.6 & 10.3 \\
\hline$C_{p(1000)}^{\circ}$ & 76.9 & 60.4 & 16.8 \\
\hline
\end{tabular}

$\Delta S^{\circ}=38.7 \mathrm{~g} / \mathrm{mole}$

$\Delta H^{\circ}=67.5 \mathrm{kcal} / \mathrm{mole}$

$\Delta C_{p}^{\circ}=1.6 \mathrm{~g} / \mathrm{mole}\left\langle\overline{\Delta C}_{p}^{\circ} \simeq 1.0\right\rangle$

Path $\quad \log A \quad E \quad \log k_{T}$ Conditions System Surface References

(928)

$12.9 \quad 55.0 \quad-0.06 \quad 860-995^{\circ} \mathrm{K} \quad$ flow $\quad<5 \%$

$4.7-12.8$ torr

[1] M. H. Back and S. H.

Sehon, Can. J.

Chem. 38, 1261

(1960).

$\begin{array}{llll}a & 14.3 & 66 & -1.26\end{array}$

[2] J. W. Taylor and M.

Szware, M. Sci.

Thesis (J. W. Taylor), Univ. of Manchester (1951).

Preferred:

$\log k_{a}=14.3-68.1 / \theta$.

Comments: The observed $A$-factors imply recombination rate constants of about $10^{6.4} \mathrm{l} / \mathrm{mole}$-sec and $10^{7.8}$ $1 /$ mole-sec $\left([1,2]\right.$ respectively). A reasonable guess would be about $10^{8.6 \pm 0.5} \mathrm{l} / \mathrm{mole}$-sec (i.e., a mean between the $k_{\text {rec }}$ obtained for $\left(\mathrm{C}_{3} \mathrm{H}_{7} \cdot+\varnothing \mathrm{CH}_{2} \cdot\right)$ and $\left(\mathrm{CH}_{3} \cdot+\emptyset C \mathrm{CHCH}_{3}\right)$. The preferred parameters give $\Delta H_{f}^{\circ}$ $(\cdot \mathrm{COOH})=-53.3$ and $D H^{\circ}(\mathrm{H}-\mathrm{COOH})=87.3 \mathrm{kcal} / \mathrm{mole}$.

\section{Experimental}

[1] Toluene carrier technique. Products of the reaction were mainly $\mathrm{CO}_{2}$ and dibenzil with smaller yields of CO. $\mathrm{H}_{2}$ and $\mathrm{CH}_{4}$ were formed in amounts between 20 and 60 percent of the $\mathrm{CO}_{2}$. Phenylketone was also detected by hydrolysis and titration as the acid. Path (a) parameters were calculated from the rate of $\left(\mathrm{CO}+\mathrm{CO}_{2}\right)$ production. Rate constants were independent of $t c, P_{T}$ and $\delta P_{\text {reactant }}$.

Rate constants of path $b$ (not sufficiently accurate for an Arrhenius analysis) were obtained from the total acid rate of decomposition less the $\left(\mathrm{CO}+\mathrm{CO}_{2}\right)$ production rate. The standard toluene carrier mechanism was assumed. Some indication of oxalic acid as a product under certain conditions was noted. Formic acid was not observed. 
Reaction: Diphenylethanoic acid (diphenylacetic acid)

$$
\emptyset_{2} \mathrm{CHCOOH}(\mathrm{I}) \longrightarrow \emptyset_{2} \mathrm{CH} \cdot(\mathrm{II})+\cdot \mathrm{COOH}(\mathrm{III})
$$

$\begin{array}{cccc} & \text { I } & \text { II } & \text { III } \\ \Delta H_{f(298)}^{\circ} & -52.5 & (59.3) & {[-53.3]} \\ S_{(298)}^{\circ} & 123.7 & 100.2 & 60.7 \\ C_{p(298)}^{\circ} & 52.8 & 44.5 & 10.3 \\ C_{p(1000)}^{\circ} & 122.3 & 107.3 & 16.8\end{array}$

$\Delta S^{\circ}=37.2 \mathrm{~g} / \mathrm{mole}$

$\Delta H^{\circ}=58.5 \mathrm{kcal} / \mathrm{mole}$

$\Delta C_{p}^{\circ}=2.0 \mathrm{~g} / \mathrm{mole}$

\begin{tabular}{|c|c|c|c|c|c|c|}
\hline $\log A$ & $\boldsymbol{E}$ & $\begin{array}{c}\log k_{T} \\
(848)\end{array}$ & Conditions & System & Surface & References \\
\hline 12.9 & 52 & -0.50 & $788-909^{\circ} \mathrm{K}$ & flow & $\begin{array}{l}\text { (partly } \\
\text { hetero. } \\
\text { geneous } \\
\sim 11 \% \text { ) }\end{array}$ & $\begin{array}{l}\text { [1] M. H. Back and A. H. } \\
\text { Sehon, Can. J. Chem. } \\
\text { 38, 1271 (1960). }\end{array}$ \\
\hline
\end{tabular}

Preferred:

Suspect (see comments).

Comments: The partial heterogeneous nature of the secondary reactions could easily produce sizable errors in the Arrhcnius parameters. The very low $A$-factor reported implies an unreasonably low back reaction recombination rate of $k_{r} \leqslant 10^{4.2} 1 /$ mole-sec. Assuming that the rates observed do represent within a factor of 2 or 3 the reaction of interest, and taking a recombination rate of $k_{r} \simeq 10^{\$ .1} \mathrm{l} / \mathrm{mole}$-sec, one obtains more reasonable parameters. $\log k=14.8-59.4 / \theta, \Delta H_{f}^{\circ}\left(\emptyset_{2} \mathrm{CH}\right)=59.2 \pm 3 \mathrm{kcal} / \mathrm{mole}$. Reasonance energy $=$ $22.8 \mathrm{kcal} / \mathrm{mole}$.

\section{Experimental}

[1] Toluene carrier technique. Rates were based on $\mathrm{CO}$ and $\mathrm{CO}_{2}$ production. Products observed were $\mathrm{CO}, \mathrm{CO}$. $\mathrm{H}_{2}$ a ${ }^{\prime} d \mathrm{CH}_{4}$. Rate constants were apparently independent of $t c, P_{T}$, and $\delta P_{\text {reactant. }}$ Production of $\mathrm{CO}$ was increased in the packed vessel, therefore, a heterogeneous decomposition of $\cdot \mathrm{COOH}$ was suggested. 
Reaction: Peracetic acid

\begin{tabular}{cccc}
\multicolumn{4}{r}{$\mathrm{CH}_{3} \mathrm{COOOH}(\mathrm{I}) \longrightarrow \mathrm{CH}_{3} \mathrm{COO} \cdot(\mathrm{II})+\cdot \mathrm{OH}$ (III) } \\
& I & II & III \\
$\Delta H_{f(298)}^{\circ}$ & -80.9 & -49.7 & 8.0 \\
$S_{(298)}^{\circ}$ & 80.4 & 67.4 & 43.9 \\
$C_{p(298)}^{\circ}$ & 20.0 & 13.9 & 7.1
\end{tabular}

$\Delta S^{\circ}=30.9 \mathrm{~g} / \mathrm{mole}$

$\Delta H^{\circ}=39.2 \mathrm{kcal} / \mathrm{mole}$

$\Delta C_{p}^{\circ}=1.0 \mathrm{~g} / \mathrm{mole}$

\begin{tabular}{|c|c|c|c|c|c|c|}
\hline $\log A$ & $\boldsymbol{E}$ & $\begin{array}{c}\log \boldsymbol{k}_{T} \\
(516)\end{array}$ & Conditions & System & Surface & References \\
\hline 14.0 & $32 \pm 2$ & +0.46 & $\begin{array}{l}400-633^{\circ} \mathrm{K} \\
1.6-22.7 \text { torr }\end{array}$ & flow & $\begin{array}{l}\text { (appreci- } \\
\text { able) }\end{array}$ & $\begin{array}{l}\text { [1] C. Schmidt and A. H. } \\
\text { Sehon, Can. J. Chem. } \\
\text { 41, } 1819 \text { (1963). }\end{array}$ \\
\hline
\end{tabular}

\section{Preferred:}

Not reliable.

Comments: It seems likely that the homogeneous decomposition was never effectively isolated from the heterogeneous effects. The thermodynamics predict a considerably higher activation energy and though the heats of formation of the various species are somewhat uncertain, it is unlikely that they could be in error by the amount required by the reported kinetic parameters.

\section{Experimental}

[1] Toluene carrier technique. Rates were calculated from the amount of decomposed peracetic acid. Stoichiometry was: $\Delta(P) \simeq \mathrm{CO}_{2}+\mathrm{CH}_{3} \mathrm{COOH}$. It was necessary to make appreciable adjustments on observed $k$ 's in order to isolate the homogeneous from the heterogeneous reactions. Main products were $\mathrm{CO}_{2}, \mathrm{CH}_{3} \mathrm{COOH}, \mathrm{C}_{2} \mathrm{H}_{6}, \mathrm{H}_{2} \mathrm{CO}, \mathrm{O}_{2}$, traces of $\mathrm{CO}$, and $\left(\varnothing \mathrm{CH}_{2}\right)_{2}$. The rate constant parameters wère obtained by assuming $A=10^{14.0} \mathrm{sec}^{-1}$. Large scatter in the rate constants prohibited a more exact treatment. 
Reaction: Dimethyl ether

\begin{tabular}{cccr}
\multicolumn{4}{r}{$\mathrm{CH}_{3} \mathrm{OCH}_{3}(\mathrm{I}) \longrightarrow \mathrm{CH}_{3} \mathrm{O} \cdot(\mathrm{II})+\mathrm{CH}_{3} \cdot$ (III) } \\
$\Delta H_{f(298)}^{\circ}$ & I & II & III \\
$S_{(298)}^{\circ}$ & -43.7 & 3.9 & 34.0 \\
$C_{p(298)}^{\circ}$ & 63.7 & 54.6 & 46.4 \\
$C_{p(1000)}^{\circ}$ & 15.6 & 8.4 & 8.3 \\
& 34.2 & 18.6 & 14.4
\end{tabular}

$\Delta S^{\circ}=37.3 \mathrm{~g} / \mathrm{mole}$

$\Delta H^{\circ}=81.6 \mathrm{kcal} / \mathrm{mole}$

$\Delta C_{p}^{\circ}=1.1 \mathrm{~g} / \mathrm{mole}$

$\left.<\Delta C_{p}^{\circ}\right\rangle \simeq 0.3 \mathrm{~g} / \mathrm{mole}$

$\begin{array}{llllll}\log \boldsymbol{A} & \boldsymbol{E} & \log k_{T} & \text { Conditions } & \text { System } & \text { Surface }\end{array}$

$18.1 \quad 81.0 \quad-4.46 \quad 750-820^{\circ} \mathrm{K}$

$35-400$ torr

[1] S. W. Benson and D. V. S. Jain, J. Chem. Phys. 31, 1008 (1959).

$\begin{array}{llll}17.5 & 81.0 & -5.06 & \text { (as above) }\end{array}$

[2] K. H. Anderson and S. W. Benson, J. Chem. Phys. 39, 1677 (1963).

\section{Preferred:}

$\log k=16.0-81.0 / \theta$.

Comments: Rate constants are probably somewhat too high. The thermodynamics are consistent with the reported activation energy. The preferred $A$-factor and reaction thermodynamics predict a recombination rate constant of $k_{\text {rec }}=10^{9.9} \mathrm{l} / \mathrm{mole}$-sec. The reported $A$-factors could easily be in error.

\section{Experimental}

[1] Observed order dependence $\left(n=\frac{3}{2}\right)$. Deduced initiation kinetics from analysis of a complex mechanism..

[2] Slight revisions in reaction mechanism of [1] lead to the revised $A$-factor.

Notes: McKenney and Laidler, Can. J. Chem. 41, 1984 (1963) and McKenney, B. W. Wojciechowski, and K. J. Laidler, Can. J. Chem. 41, 2009 (1963) suggest that the above reaction is in its pressure-dependent region. F. O. Rice and W. R. Johnston, J. Am. Chem. Soc. 56, 214 (1934), by the Paneth technique, estimate $E=81.1 \mathrm{kcal} / \mathrm{mole}$. 
Reaction: Diethyl ether

\begin{tabular}{|c|c|c|c|}
\hline & \multicolumn{3}{|c|}{$\begin{array}{l}\stackrel{a}{\mathrm{I})} \stackrel{b}{\stackrel{b}{\longrightarrow}} \mathrm{C}_{2} \mathrm{H}_{5} \cdot(\mathrm{II})+\mathrm{C}_{2} \mathrm{H}_{5} \mathrm{O} \cdot(\text { III }) \\
\mathrm{C}_{2} \mathrm{H}_{5} \mathrm{OH}\end{array}$} \\
\hline & $\begin{array}{c}I \\
-60.7\end{array}$ & $\begin{array}{c}\text { II } \\
25.7\end{array}$ & $\begin{array}{c}\text { III } \\
-4.6\end{array}$ \\
\hline$S_{(298)}^{\circ}$ & 84.7 & 57.8 & 65.3 \\
\hline$C_{p(298)}^{\circ}$ & 25.7 & 12.1 & 14.0 \\
\hline$C_{p(1000)}^{\circ}$ & 58.9 & 27.2 & 31.3 \\
\hline
\end{tabular}

$\Delta S^{\circ}=38.4 \mathrm{~g} / \mathrm{mole}$

$\Delta H^{\circ}=81.8 \mathrm{kcal} / \mathrm{mole}$

$\Delta C_{p}^{\circ}=0.4 \mathrm{~g} / \mathrm{mole}$

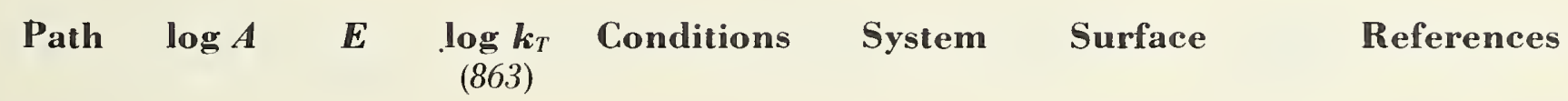

$b$

18.0

$\begin{array}{lll}84 & -3.28 & 833-913{ }^{\circ} \mathrm{K} \\ & 10-360 \text { torr }\end{array}$

[1] K. J. Laidler and

D. J. McKenney, Proc. Roy. Soc.

278A, 517 (1964).

$\begin{array}{lllll}a & 14.0 & 78 & -5.76 & 833-893^{\circ} \mathrm{K} \\ & & & \text { static }\end{array}$

[2] K. J. Laidler and

D. J. McKenney, Proc. Roy. Soc.

278A, 505 (1964).

\section{Preferred:}

Reaction $a$-rate constants probably reliable.

Reaction $b$-not reliable.

Comments: By transition state considerations, reaction a should have a larger, more positive entropy of activation than reaction $b$. Since the opposite has been reported, one must be suspicious of the mechanism assumed and the validity of the conclusions. A fairly reliable estimate for the parameters of reaction $a$ can be made from the thermodynamics by assuming a recombination rate constant of $k_{\text {rec }}=10^{9.7 \pm 1.0} 1 / \mathrm{mole}$-sec. One then obtains $\log k_{a}=16.3-81.8 / \theta$.

\section{Experimental}

[1] Inhibited reaction with NO. Complex mechanism was proposed from which the rate constant was derived.

[2] Uninhibited reaction. Interpreted order change from 1.0 (low pressures) to (3/2) (high pressures) to two terms in the rate expression (i.e., a molecular path and a free radical path). The observed kinetics parameters were fit by using those reported above for the initiation reaction. 
Reaction: Propanone (acetone)

\begin{tabular}{lccc}
\multicolumn{4}{r}{$\mathrm{CH}_{3} \mathrm{COCH}_{3}(\mathrm{I}) \longrightarrow \mathrm{CH}_{3} \cdot(\mathrm{II})+\mathrm{CH}_{3} \mathrm{COO}$ (III) } \\
& $\mathrm{I}$ & II & III \\
$\Delta H_{f(298)}^{\circ}$ & -51.7 & 34.0 & -5.4 \\
$S_{(298)}^{\circ}$ & 70.5 & 46.5 & 64.5 \\
$C_{p(298)}^{\circ}$ & 17.9 & 8.3 & 12.7 \\
$C_{p(1000)}^{\circ}$ & 39.2 & 14.4 & 23.8
\end{tabular}

$\Delta S^{\circ}=40.5 \mathrm{~g} / \mathrm{mole}$

$\Delta H^{\circ}=80.3 \mathrm{kcal} / \mathrm{mole}$

$\Delta C_{p}^{\circ}=3.1 \mathrm{~g} / \mathrm{mole}$

$<\Delta \mathrm{C}_{p}^{\circ} \simeq 1.1>_{298-1050}$

\begin{tabular}{|c|c|c|c|c|c|c|}
\hline $\log A$ & $\boldsymbol{E}$ & $\begin{array}{r}\log \boldsymbol{k}_{r} \\
(1050)\end{array}$ & Conditions & System & Surface & References \\
\hline 14.38 & 72.0 & -0.61 & $\begin{array}{l}993-1101{ }^{\circ} \mathrm{K} \\
0.07-0.26 \text { torr }\end{array}$ & flow & & $\begin{array}{l}\text { [1] M. Szwarc and J. W. } \\
\text { Taylor, J. Chem. Phy } \\
\text { 23, } 2310 \text { (1955). }\end{array}$ \\
\hline 14.15 & 70.9 & -0.61 & $\begin{array}{l}990-1110^{\circ} \mathrm{K} \\
1 \% \text { in toluene } \\
\quad \text { at } 13 \text { torr }\end{array}$ & & & $\begin{array}{l}\text { [2] D. Clark and H. O. } \\
\text { Pritchard, J. Chem. } \\
\text { Soc. } 2136 \text { (1956). }\end{array}$ \\
\hline
\end{tabular}

Preferred:

$\log k=16.26-81.0 / \theta$.

Comments: The reported parameters must be low because the thermodynamics of the reaction are well established. A pressure falloff could be responsible, although some chain decomposition (evidenced by the formation of ketene in appreciable yields) seems the more likely explanation. The rate constant parameters have been scaled to fit the reaction enthalpy. From $\Delta S^{\circ}$ and $A$, one obtains a recombination rate constant of $10^{9.1} \mathrm{l} /$ mole-sec.

\section{Experimental}

[1] Toluene carrier technique. It was assumed that all acetyl radicals decomposed to $\mathrm{CO}$ and the rate constants were calculated on the basis of $(\mathrm{CO})$ production. From 10 to 15 percent ketene was also detected. First-order rate constants were found to increase slightly with increasing total pressure. Rate constants were independent of $t_{c}$, percent conversion, and $\delta P$ of acetone.

[2] Toluene carrier techniques. Rate constants were based on (CO) yields (see acetophenone [1]). 
Reaction: Acetophenone

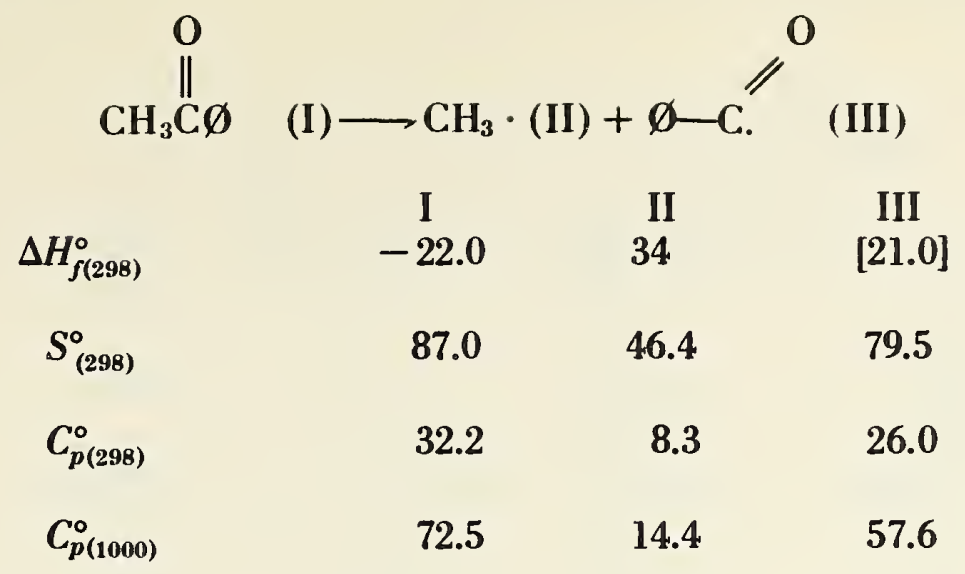

$$
\begin{aligned}
\Delta S^{\circ} & =38.9 \mathrm{~g} / \mathrm{mole} \\
\Delta H^{\circ} & =77.0 \mathrm{kcal} / \mathrm{mole} \\
\Delta C_{p}^{\circ} & =2.1 \mathrm{~g} / \mathrm{mole} \\
\left\langle\Delta C_{p}^{\circ}\right. & \simeq 0.8\rangle_{298-1000}
\end{aligned}
$$

\begin{tabular}{ccccccc}
$\log A$ & $E$ & $\begin{array}{c}\log k_{T} \\
(1045)\end{array}$ & Conditions & System & Surface & \multicolumn{1}{c}{ References } \\
15.71 & 77.6 & -0.52 & $\begin{array}{l}980-1110 \% \text { K } \\
1 \% \text { reactant in } \\
13 \pm 1 \mathrm{~mm} \text { of } \\
\text { toluene }\end{array}$ & flow & [1] D. Clark and H. O. \\
Pritchard, J. Chem. \\
Soc., 2136 (1956).
\end{tabular}

Preferred:

$\log k=15.7-77.6 / \theta$.

Comments: The reported parameters give $\Delta H_{f}^{\circ}(\emptyset \mathrm{CO})=21.0 \mathrm{kcal} / \mathrm{mole}$ (see benzil). Recombination rate constant of $k_{\text {rec }} \simeq 10^{8.9} \mathrm{1} / \mathrm{mole}$-sec can be estimated from the reaction entropy and $A$-factor. New values of $\Delta H_{f}^{\circ}(\varnothing \dot{\mathrm{C}} \mathrm{O})=29 \pm 1 \mathrm{kcal} / \mathrm{mole}$ cast some doubt on $E_{\text {obs }}$.

\section{Experimental}

[1] Toluene carrier technique. Rate constants were obtained from the rates of carbon monoxide production.Analysis of $\mathrm{CO}$ was made be oxidation to $\mathrm{CO}_{2}$ with $\mathrm{I}_{2} \mathrm{O}_{5}$. A multiple critical oscillator theory (see section $\mathrm{V}-5.0$ ) was invoked to explain the high-frequency factor for the reaction.

The following sequence of reactions was assumed: $\mathrm{CH}_{3} \mathrm{CO} \emptyset \longrightarrow \mathrm{CH}_{3} \cdot \emptyset \mathrm{CQO} ; \emptyset \mathrm{CO} \longrightarrow \emptyset \cdot+\mathrm{CO}$. 
Reaction: 1-Phenyl-2-propanone (benzyl methyl ketone)

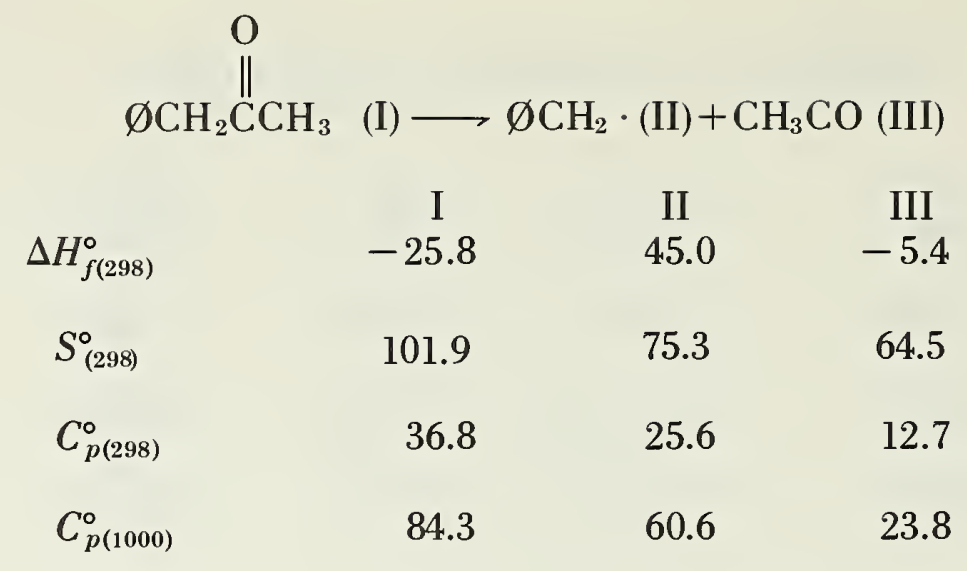

$\Delta S^{\circ}=37.9 \mathrm{~g} / \mathrm{mole}$

$\Delta H^{\circ}=65.4 \mathrm{kcal} / \mathrm{mole}$

$\Delta C_{p}^{\circ}=1.5 \mathrm{~g} / \mathrm{mole}$

\begin{tabular}{|c|c|c|c|c|c|c|}
\hline $\log A$ & $E$ & $\begin{array}{c}\log k_{T} \\
(900)\end{array}$ & Conditions & System & Surface & References \\
\hline 16.0 & 68.2 & -0.56 & {$\left[\bar{T} \simeq 900^{\circ} \mathrm{K}\right]$} & flow & & $\begin{array}{l}\text { [1] J. W. Taylor, Ph. D. } \\
\text { Thesis, Univ. of Man- } \\
\text { chester (1953). }\end{array}$ \\
\hline
\end{tabular}

Preferred:

$\log k=15.3-65.9 / \theta$.

Comments: The thermodynamics suggest that the reported parameters may be somewhat high. The preferred $A$-factor and reaction entropy give a reasonable recombination rate constant, $k_{\text {rec }}=10^{8.7} \mathrm{l} / \mathrm{mole}$-sec.

\section{Experimental}

[1] Toluene carrier technique. 
Reaction: Benzophenone

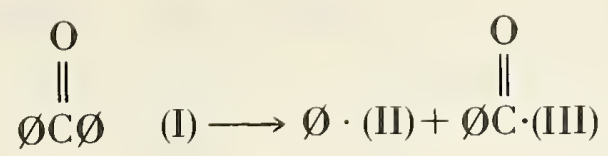

$\begin{array}{cccc} & \text { I } & \text { II } & \text { III } \\ \Delta H_{f(298)}^{\circ} & 12.5 & 80.0 & {[21.0] \text { or [29] }} \\ S_{(298)}^{\circ} & 104.2 & 69.2 & 79.5 \\ C_{p(298)}^{\circ} & 46.2 & 19.2 & 26.0 \\ C_{p(1000)}^{\circ} & 106 & 47.0 & 57.6\end{array}$

$$
\begin{aligned}
\Delta S^{\circ} & =44.5 \mathrm{~g} / \mathrm{mole} \\
\dot{\Delta} H^{\circ} & =88.5 \mathrm{kcal} / \mathrm{mole} \\
\Delta C_{p}^{\circ} & =-1.0 \mathrm{~g} / \mathrm{mole} \\
\left\langle\Delta C_{p}^{\circ}\right. & \simeq-1.27\rangle_{298-1100}
\end{aligned}
$$

\begin{tabular}{|c|c|c|c|c|c|c|}
\hline $\log A$ & $\boldsymbol{E}$ & $\begin{array}{l}\log \boldsymbol{k}_{T} \\
(1112)\end{array}$ & Conditions & System & Surface & References \\
\hline 16.2 & 87.5 & -0.99 & $\begin{array}{l}1075-1150^{\circ} \mathrm{K} \\
1 \% \text { reactant in } \\
13 \text { torr or }\end{array}$ & flow & none & $\begin{array}{l}\text { [1] D. Clark and H. O. } \\
\text { Pritchard, J. Chem. } \\
\text { Soc., } 2136 \text { (1956). }\end{array}$ \\
\hline
\end{tabular}

\section{Preferred:}

$\log k=16.2-87.5 / \theta$ or $16.2-95 / \theta$.

Comments: The activation energy has been used to obtain $\Delta H_{f}^{\circ}(\varnothing \mathrm{CO})=21.0 \mathrm{kcal} / \mathrm{mole}$ (see benzil). This may be too low. The reaction entropy along with $A$ gives $k_{\text {rec }} \simeq 10^{8.8} 1 /$ mole-sec.

\section{Experimental}

[1] See acetophenone. 
Reaction: 1,3-Diphenyl-1,2-propanone (dibenzilketone)

\begin{tabular}{|c|c|c|c|}
\hline$\Delta H_{f(\mathbf{2 9 8})}^{\circ}$ & $\begin{array}{l}\text { I } \\
2.5\end{array}$ & $\begin{array}{c}\text { II } \\
{[22.5]}\end{array}$ & $\begin{array}{c}\text { III } \\
45.0\end{array}$ \\
\hline$S_{(298)}^{\circ}$ & 130 & 95.6 & 75.3 \\
\hline$C_{p(298)}^{\circ}$ & 54.4 & 31.9 & 25.6 \\
\hline$C_{p(1000)}^{\circ}$ & 129.9 & 69.0 & 60.6 \\
\hline
\end{tabular}

$\Delta S^{\circ}=40.9 \mathrm{~g} / \mathrm{mole}$

$\Delta H^{\circ}=65.0 \mathrm{kcal} / \mathrm{mole}$

$\Delta C_{p}^{\circ}=3.1 \mathrm{~g} /$ mole

$\left\langle\Delta C_{p}^{\circ} \simeq 1.7\right\rangle_{298-860}$

\begin{tabular}{|c|c|c|c|c|c|c|}
\hline $\log A$ & $\boldsymbol{E}$ & $\begin{array}{c}\log k_{T} \\
(860)\end{array}$ & Conditions & System & Surface & References \\
\hline 17.25 & 71.8 & -1.00 & $\begin{array}{l}810-910^{\circ} \mathrm{K} \\
1 \% \text { reactant } \\
\text { in toluene } \\
\quad(13 \text { torr })\end{array}$ & flow & none & $\begin{array}{l}\text { [1] D. Clark and H. O. } \\
\text { Pritchard, J. Chem. Soc. } \\
2136 \text { (1956). }\end{array}$ \\
\hline
\end{tabular}

\section{Preferred:}

$\log k=16.13-67.5 / \theta$. Rate constants are probably reliable.

Comments: The heat of formation $\Delta H_{f}^{\circ}\left(\emptyset \mathrm{CH}_{2} \mathrm{CO}\right)$ has been estimated from the acetyl radical heat of formation ( $-5.4 \mathrm{kcal} / \mathrm{mole})$ and group additivities. On the basis of the thermodynamics, the reported parameters seem too high. Adjusting $A$ and $E$ to the reaction enthalpy gives a reasonable value for the back reaction rate constant, $k_{\text {rec }}=10^{8.7 \pm 1} \mathrm{l} / \mathrm{mole}-\mathrm{sec}$.

\section{Experimental}

[1] See acetophenone. 
Reaction: 1,2-Diphenyl-1,2-ethanedione (benzil)

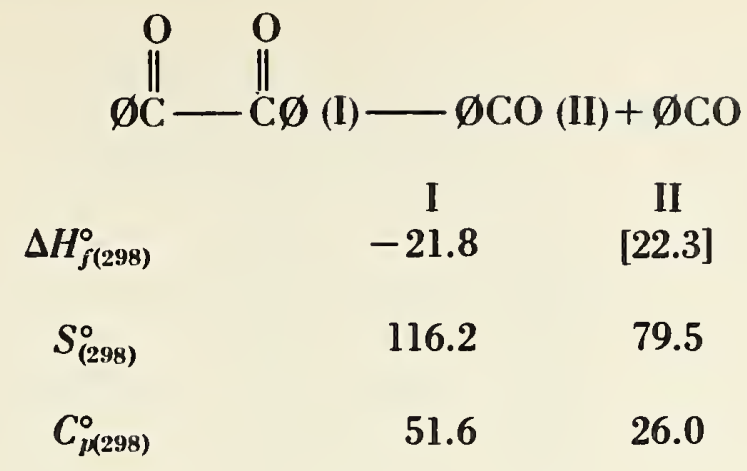

$\Delta S^{\circ}=42.8 \mathrm{~g} / \mathrm{mole}$

$\Delta H^{\circ}=66.4 \mathrm{kcal} / \mathrm{mole}$

$\Delta C_{p}^{\circ}=0.4 \mathrm{~g} / \mathrm{mole}$

\begin{tabular}{|c|c|c|c|c|c|c|}
\hline $\log A$ & $\boldsymbol{E}$ & $\begin{array}{c}\log k_{T} \\
(860)\end{array}$ & Conditions & System & Surface & References \\
\hline 16.3 & 66.4 & -0.57 & {$\left[\bar{T} \simeq 860^{\circ} \mathrm{K}\right]$} & flow & & $\begin{array}{l}\text { [1] Jacquiss, Ph.D. Thesis, } \\
\text { University of Man- } \\
\text { chester (1953). }\end{array}$ \\
\hline
\end{tabular}

\section{Preferred:}

$\log k=16.3-66.4 / \theta$.

Comments: The kinetics give $\Delta H_{f}^{\circ}(\varnothing \dot{\mathrm{C} O})=22.4 \mathrm{kcal} / \mathrm{mole}$. This should be compared to the value obtained from the acetophone and benzophenone decompositions of $21.0 \mathrm{kcal} / \mathrm{mole}$ respectively. A recombination rate constant of about $k_{\text {rec }} \simeq 10^{8.8} \mathrm{l} / \mathrm{mole}$-sec is obtained from the reaction entropy and reported $A$-factor. Recent data give $\Delta H_{f}^{\circ}(\emptyset \mathrm{CO})=29 \mathrm{kcal} / \mathrm{mole}$, which would give $E \simeq 78 \mathrm{kcal} / \mathrm{mole}$. 


\begin{tabular}{|c|c|c|c|c|}
\hline \multicolumn{2}{|c|}{$\mathrm{CF}_{3} \mathrm{COCH}_{3}$ (I) } & \multicolumn{3}{|c|}{$\begin{array}{l}\frac{a}{b} \mathrm{CF}_{3} \cdot(\mathrm{II})+\mathrm{CH}_{3} \mathrm{CO} \cdot(\mathrm{III}) \\
\underset{\mathrm{CH}_{3}}{\longrightarrow} \cdot(\mathrm{IV})+\mathrm{CF}_{3} \mathrm{CO} \cdot(\mathrm{V})\end{array}$} \\
\hline$\Delta H_{f(298)}^{\circ}$ & $\begin{array}{c}\text { I } \\
-200.2\end{array}$ & $\begin{array}{c}\text { II } \\
-112.5\end{array}$ & $\begin{array}{c}\text { III } \\
-5.4\end{array}$ & $\begin{array}{l}\text { IV } \\
34\end{array}$ \\
\hline$S_{(298)}^{\circ}$ & 83.9 & 62.4 & 64.5 & 46.4 \\
\hline$C_{p(298)}^{\circ}$ & 24.5 & 12.2 & 12.7 & 8.3 \\
\hline
\end{tabular}

$a$
$\Delta S^{\circ}=43.0 \mathrm{~g} / \mathrm{mole}$

$\Delta H^{\circ}=82.3 \mathrm{kcal} / \mathrm{mole}$

$\Delta C_{p}^{\circ}=0.4 \mathrm{~g} / \mathrm{mole}$

\begin{tabular}{|c|c|c|c|c|c|c|c|}
\hline Path & $\log A$ & $\boldsymbol{E}$ & $\begin{array}{c}\log \boldsymbol{k}_{T} \\
(1023)\end{array}$ & Conditions & System & Surface & References \\
\hline$a$ or $b$ & 13.48 & 67.8 & -1.01 & $\begin{array}{l}970-1075^{\circ} \mathrm{K} \\
1 \% \text { in } 13 \text { torr } \\
\text { of toluene. }\end{array}$ & flow & 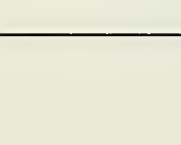 & $\begin{array}{l}\text { [1] D. Clark and H. O. } \\
\text { Pritchard, J. Chem. } \\
\text { Soc., } 2136 \text { (1956). }\end{array}$ \\
\hline
\end{tabular}

Preferred:

$\log k_{a}=16.6-81.9 / \theta$. Rate constants probably reliable.

Comments: Arrhenius parameters are certainly. low. The situation is similar to that of acetone where the activation energy obtained from the toluene carrier technique is $10 \mathrm{kcal} / \mathrm{mole}$ less than the bond dissociation energy in that molecule. Although the bond dissociation energy $\left(\mathrm{D}\left(\mathrm{CH}_{3} \mathrm{CO}-\mathrm{CF}_{3}\right)\right)$ is not known, it is unlikely to be much less than that for path $b$. The preferred parameters have been obtained by assuming the mean rate constant reported to be roughly valid, and setting the recombination rate constant equal to that calculated for acetone, (i.e., $k_{\text {rec }} \simeq 10^{9.1} 1 /$ mole-sec).

\section{Experimental}

[1] Toluene carrier technique. Rates were based on the CO formation. The path of the decomposition ( $a$ or $b$ above) was not ascertained. (See acetophenone.) 
Reaction: $\alpha, \alpha, \alpha$-Trifluoroacetophenone

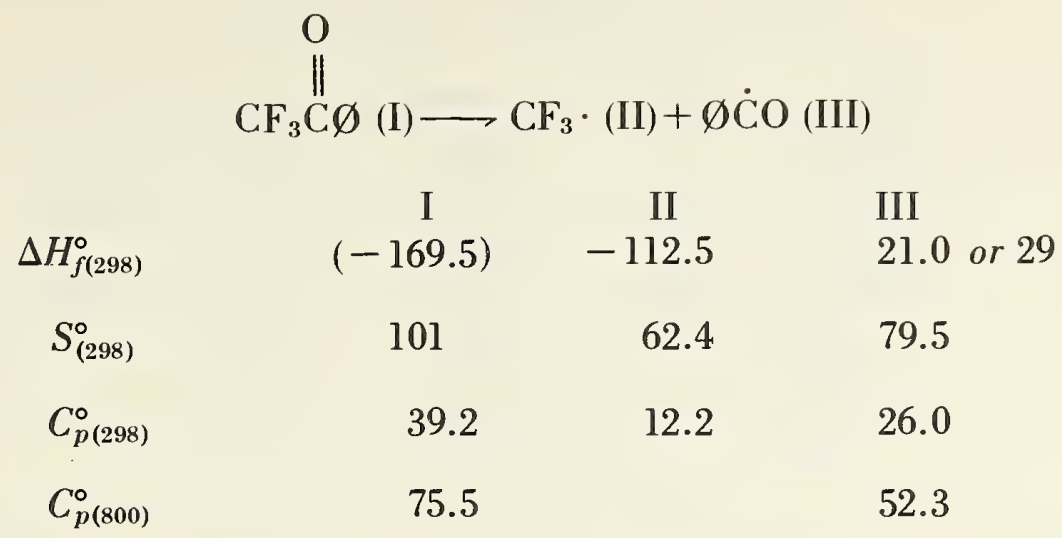

$\Delta S^{\circ}=40.9 \mathrm{~g} / \mathrm{mole}$

$\Delta H^{\circ}=78.0 \mathrm{kcal} /$ mole (or 86)

$\Delta C_{p}^{\circ}=-1.0 \mathrm{~g} / \mathrm{mole}$

\begin{tabular}{|c|c|c|c|c|c|c|}
\hline $\log A$ & $\boldsymbol{E}$ & $\begin{array}{c}\log \boldsymbol{k}_{T} \\
(817)\end{array}$ & Conditions & System & Surface & References \\
\hline 15.25 & 73.8 & -4.49 & $\begin{array}{l}787-847^{\circ} \mathrm{K} \\
1 \% \text { reactant } \\
\text { in } 13 \text { torr } \\
\text { of toluene }\end{array}$ & flow & none & $\begin{array}{l}\text { [1] D. Clark and H. O. } \\
\text { Pritchard, J. Chem. } \\
\text { Soc., } 2136 \text { (1956). }\end{array}$ \\
\hline
\end{tabular}

Preferred:

$\log k=15.25-73.8 / \theta$.

Comments: All heats of formation are uncertain to at least $\pm 2 \mathrm{kcal} /$ mole. The discrepancy between the observed activation energy and the estimated $\Delta H^{\circ}$ reaction is within these limits. From $A$ and $\Delta S^{\circ}$ one obtains $k_{\text {rec }} \simeq 10^{8.4} 1 /$ mole $=$ sec.

\section{Experimental}

[1] See acetophenone. 
Reaction: 2,3-Butanedione (biacetyl)

\begin{tabular}{ccc}
\multicolumn{2}{c}{$\mathrm{CH}_{3} \mathrm{CO}-\mathrm{COCH}_{3}(\mathrm{I}) \longrightarrow$} & $2 \mathrm{CH}_{3} \mathrm{CO}(\mathrm{II})$ \\
$\Delta H_{f(298)}^{\circ}$ & I & II \\
$S_{(298)}^{\circ}$ & -78.0 & -5.4 \\
$C_{(2298)}^{\circ}$ & 85.8 & 64.5 \\
$C_{p(800)}^{\circ}$ & 23.6 & 12.7 \\
& 44.0 & 21.4
\end{tabular}

$\Delta S^{\circ}=43.2 \mathrm{~g} / \mathrm{mole}$

$\Delta H^{\circ}=67.2 \mathrm{kcal} / \mathrm{mole}$

$\Delta C_{p}^{\circ}=1.8 \mathrm{~g} / \mathrm{mole}$

$\left\langle\Delta C_{p}^{\circ} \simeq 0.8\right\rangle$

$\begin{array}{llclll}\log A & \boldsymbol{E} & \begin{array}{c}\log k_{T} \\ (662)\end{array} & \text { Conditions } & \text { System } & \text { Surface } \\ 15.94 & 63.2 & -4.92 & \begin{array}{l}626-698^{\circ} \mathrm{K} \\ \sim 200 \text { torr }\end{array} & \text { static } & <10 \%\end{array}$

$15.7 \quad 66 \quad-6.09$

[2] J. W. Taylor, Ph. D. Thesis, Univ. of Manchester (1953).

\section{Preferred:}

$\log k=16.2-67.5 / \theta$.

Comments: Kinetics [2] and thermodynamics are fairly consistent. From the reaction entropy and the reported $A$-factor one obtains $k_{\text {rec }}=10^{8.5} 1 /$ mole-sec.

\section{Experimental}

[1] Rates were obtained from initial $\Delta \mathbf{P}$ data [1 a], and from the analysis of undecomposed biacetyl [1b]. Biacetyl was converted to dimethylglyoxime and precipitated as nickel dimethylglyoxime. Products were $\mathrm{CO}, \mathrm{CH}_{4}$, and $\mathrm{CH}_{2}=\mathrm{C}=0$. Rates were first order in the intial stages only. A free radical chain process was assumed since reaction rates were lower in the presence of propylene. The observed parameters were indentified with a Rice-Herzfeld chain mechanism.

[2] Toluene carrier technique. 
Reaction: Benzylethanoate (benzyl acetate)

$\begin{array}{cccc}\mathrm{CH}_{3} \mathrm{COO}-\mathrm{CH}_{2} \varnothing(\mathrm{I}) \longrightarrow & \mathrm{CH}_{3} \mathrm{CO}_{2} \cdot(\mathrm{II})+\varnothing \mathrm{CH}_{2} \cdot(\mathrm{III}) \\ \Delta H_{f(298)}^{\circ} & \text { I } & \text { II } & \text { III } \\ S_{(298)}^{\circ} & -72.2 & -49.7 & 45.0 \\ \Delta C_{p(298)}^{\circ} & 109.3 & 67.4 & 75.3 \\ & 38.5 & 13.9 & 25.2\end{array}$
$\Delta S^{\circ}=33.4 \mathrm{~g} / \mathrm{mole}$
$\Delta H^{\circ}=67.5 \mathrm{kcal} / \mathrm{mole}$
$C_{p}^{\circ}=0.6 \mathrm{~g} / \mathrm{mole}$

\begin{tabular}{|c|c|c|c|c|c|c|}
\hline $\log A$ & $\boldsymbol{E}$ & $\begin{array}{c}\log \boldsymbol{k}_{T} \\
(800)\end{array}$ & Conditions & System & Surface & References \\
\hline 14.5 & 67 & -3.81 & & flow & & $\begin{array}{l}\text { [1] J. W. Taylor (with M. } \\
\text { Szwarc), M. Sci. Thesis, } \\
\text { Univ. of Manchester } \\
\text { (1951); M. Szwarc and } \\
\text { J. Watson Taylor, J. } \\
\text { Chem. Phys. 21, } 1746 \\
\text { (1953). }\end{array}$ \\
\hline
\end{tabular}

Preferred:

Reliable. $\log k=14.5-67 / \theta$.

Comments: From $A$ and the reaction entropy one obtains $k_{\text {rec }}=10^{9.0} \mathrm{l} / \mathrm{mole}$-sec.

\section{Experimental}

[1] Toluene carrier technique. Products were principally $\mathrm{CO}_{2}$ and $\mathrm{CH}_{4}$, on whose rates of formation the reported parameters were based. $\mathrm{CO}$ and $\emptyset \mathrm{CH}=\mathrm{O}$ were also observed and attributed to a concerted elimination reaction of the benzyl acetate. However, some induced radical decomposition forming these products is more likely (see benzyl benzoate and as follows):

CO formation:

$$
\begin{aligned}
& \mathrm{R} \cdot+\mathrm{CH}_{3} \mathrm{CO}_{2} \mathrm{CH}_{2} \emptyset \longrightarrow \mathrm{RH}+\mathrm{CH}_{3} \mathrm{CO}_{2} \dot{\mathrm{C}} \boldsymbol{} \emptyset \\
& \mathrm{CH}_{3} \mathrm{CO}_{2} \dot{\mathrm{C}} \mathrm{H} \emptyset \longrightarrow \mathrm{CH}_{3} \dot{\mathrm{CO}}+\emptyset \mathrm{CH}=\mathrm{O} \\
& \mathrm{CH}_{3} \dot{\mathrm{CO}}+(\mathrm{M}) \longrightarrow \mathrm{CH}_{3} \cdot+\mathrm{CO}+(\mathrm{M}) .
\end{aligned}
$$


Reaction: Benzylbenzoate

\begin{tabular}{|c|c|c|c|}
\hline$\Delta H_{f(29 \dot{8})}^{\circ}$ & $\begin{array}{c}\text { I } \\
-43.1\end{array}$ & $\begin{array}{c}\text { II } \\
(-21.7)\end{array}$ & $\begin{array}{c}\text { III } \\
45.0\end{array}$ \\
\hline$S_{(298)}^{\circ}$ & 128 & 87.9 & 75.3 \\
\hline$C_{p(298)}^{\circ}$ & 53 & 28.9 & 25.6 \\
\hline
\end{tabular}

$\Delta S^{\circ}=35.2 \mathrm{~g} / \mathrm{mole}$

$\Delta H^{\circ}=66.4 \mathrm{kcal} / \mathrm{mole}$

$\Delta C_{p}^{\circ}=1.5 \mathrm{~g} / \mathrm{mole}$

\begin{tabular}{|c|c|c|c|c|c|c|}
\hline $\log A$ & $\boldsymbol{E}$ & $\begin{array}{c}\log \boldsymbol{k}_{T} \\
(950)\end{array}$ & Conditions & System & Surface & References \\
\hline 15.3 & 69 & -0.58 & $900-1000^{\circ} \mathrm{K}$ & flow & & $\begin{array}{l}\text { [1] M. Szwarc and J. Watson } \\
\text { Taylor, J. Chem. Phys. } \\
\text { 21, 1746 (1953). }\end{array}$ \\
\hline
\end{tabular}

Preferred:

$\log k=14.8-66.8 / \theta$.

Comments: The heat of formation of $\left(\varnothing \mathrm{CO}_{2}^{*}\right)$ has been estimated from the heat of formation of $\left(\mathrm{CH}_{3} \mathrm{CO}_{2} \cdot\right)$ with additivity relations. Reported parameters seem slightly high. From $A$ and the reaction entropy, one obtains, $k_{\text {rec }}=10^{8.9} \mathrm{l} /$ mole-sec.

\section{Experimental}

[1] Toluene carrier technique. Rates obtained from $\mathrm{CO}_{2}$ production. Rate constants were independent of $t c, P_{T}$ and $\delta P$ reactant. Liquid chromatographic analysis showed that $\left[\left(\mathrm{CO}_{2}\right)=\left(\varnothing \mathrm{CH}_{2}\right)_{2}=\Delta\right.$ (reactant) $] . \mathrm{CH}_{4}$ and $\mathrm{H}_{2}$ (with yields $\approx 25$ percent of $\mathrm{CO}_{2}$ ) were attributed to the decompostion of dibenzyl. Carbon monoxide and benzaldehyde (both minor products) were thought to arise as follows:

$$
\begin{aligned}
& \emptyset \cdot+\varnothing \mathrm{CO}_{2} \mathrm{CH}_{2} \mathrm{O} \longrightarrow \emptyset \mathrm{H}+\varnothing \mathrm{CO}_{2} \mathrm{CH} \emptyset \\
& \varnothing \mathrm{CO}_{2} \mathrm{CH} \emptyset \longrightarrow \emptyset \mathrm{CO}+\varnothing \mathrm{CHO} \\
& \varnothing \mathrm{CO} \longrightarrow \emptyset \cdot+\mathrm{CO}
\end{aligned}
$$


Reaction: Dimethylperoxide

\begin{tabular}{ccc}
\multicolumn{2}{r}{$\mathrm{CH}_{3} \mathrm{OOCH}_{3}(\mathrm{I}) \longrightarrow 2 \mathrm{CH}_{3} \mathrm{O} \cdot(\mathrm{II})$} \\
$\Delta H_{f(298)}^{\circ}$ & $\mathrm{I}$ & $\mathrm{II}$ \\
$S_{(298)}^{\circ}$ & -29.4 & {$[3.9]$} \\
$C_{p(298)}^{\circ}$ & 74.6 & 54.6 \\
$C_{, p(500)}^{\circ}$ & 17.6 & 8.4 \\
& 24.4 & 11.7
\end{tabular}

$\Delta S^{\circ}=34.6 \mathrm{~g} / \mathrm{mole}$

$\Delta H^{\circ}=137.2 \mathrm{kcal} / \mathrm{mole}$

$\Delta C_{p}^{\circ}=-0.8 \mathrm{~g} / \mathrm{mole}$

\begin{tabular}{|c|c|c|c|c|c|c|}
\hline $\log A$ & $\boldsymbol{E}$ & $\begin{array}{c}\log \boldsymbol{k}_{T} \\
(440)\end{array}$ & Conditions & System & Surface & References \\
\hline 15.61 & 36.9 & -2.74 & $427-452^{\circ} \mathrm{K}$ & static & & $\begin{array}{l}\text { [1] Y. Takezaki and C. } \\
\text { Takeuchi, J. Chem. } \\
\text { Phys. 22, } 1527 \text { (1954). }\end{array}$ \\
\hline 15.2 & 35.3 & -2.35 & $\begin{array}{l}393-435{ }^{\circ} \mathrm{K} \\
3-30 \text { torr }\end{array}$ & static & & $\begin{array}{l}\text { [2] P. L. Hanst and J. G. } \\
\text { Calvert, J. Phys. Chem. } \\
\text { 63, } 104 \text { (1959). }\end{array}$ \\
\hline
\end{tabular}

Preferred:

$\log k=15.6-36.9 / \theta$.

Comments: $k_{\text {rec }} \simeq 10^{9.9} \mathrm{l} /$ mole-sec, calculated from the reaction entropy and the preferred $A$-factor.

\section{Experimental}

[1] Decomposition was performed in excess methanol. The only major products were $\mathrm{CH}_{3} \mathrm{OH}$ and CO. Extensive correlations of stoichiometry and products with the mechanism were made. Rates followed by $\Delta \mathrm{P}$. Analysis for $\mathrm{H}_{2} \mathrm{CO}$ and ethylene glycol were made by titrating with $\mathrm{I}_{2}$ and $\mathrm{HIO}_{4}$ respectively.

[2] Rates were followed by time sampling and I.R. analysis on both the products and the residual peroxide. Stoichimetry: 2 Peroxide $\longrightarrow 3 \mathrm{ROH}+\mathrm{CO}$. The only important products were $\mathrm{CH}_{3} \mathrm{OH}$ and $\mathrm{CO}$. Addition of NO led to the formation of methyl nitrite as the only product. 
Reaction: Diethyl peroxide

\begin{tabular}{|c|c|c|}
\hline$\Delta H_{f(298)}^{\circ}$ & $\begin{array}{c}\text { I } \\
-47.0\end{array}$ & $\begin{array}{c}\text { II } \\
{[-4.9]}\end{array}$ \\
\hline$S_{(298)}^{\circ}$ & 93.4 & 65.9 \\
\hline$C_{p(298)}^{\circ}$ & 29.2 & 14.2 \\
\hline$C_{p(500)}^{\circ}$ & 42.8 & 20.9 \\
\hline
\end{tabular}

$\Delta S^{\circ}=38.4 \mathrm{~mole}$

$\Delta H^{\circ}=37.2 \mathrm{kcal} / \mathrm{mole}$

$\Delta C_{p}^{\circ}=-0.8 \mathrm{~g} / \mathrm{mole}$

$\left\langle\overline{\Delta C}_{p}^{\circ}=-0.9\right\rangle \mathrm{g} /$ mole

\begin{tabular}{|c|c|c|c|c|c|c|}
\hline $\log A$ & $\boldsymbol{E}$ & $\begin{array}{c}\log k_{T} \\
(435)\end{array}$ & Conditions & System & Surface & References \\
\hline 14.7 & 31.5 & -1.13 & $\begin{array}{l}413-4.57^{\circ} \mathrm{K} \\
2-29 \text { torr }\end{array}$ & static & none & $\begin{array}{l}\text { [1] E. J. Harris and A. C. } \\
\text { Edgerton, Proc. Roy. } \\
\text { Soc. (L) A168, } 1 \text { (1938). }\end{array}$ \\
\hline 13.2 & 31.7 & -2.73 & $479-518^{\circ} \mathrm{K}$ & flow & & $\begin{array}{l}\text { [2] R. E. Rebbert and K. J. } \\
\text { Laidler, J. Chem. Phys. } \\
\text { 20, 574 (1952). }\end{array}$ \\
\hline 12.04 & 29.9 & -2.99 & & & & $\begin{array}{l}\text { [3] K. Moriya, Rev. Phys. } \\
\text { Chem. (Japan), Shinkichi } \\
\text { Horiba } 143 \text { (1946). }\end{array}$ \\
\hline 14.2 & 34.1 & -2.94 & (recalculated) & & & $\begin{array}{l}\text { [4] P. L. Hanst and J. G. } \\
\text { Calvert, J. Phys. Chem. } \\
\text { 63, } 104 \text { (1959). }\end{array}$ \\
\hline & 37.3 & & & $\begin{array}{l}\text { flow in } \\
\text { excess NO }\end{array}$ & none & $\begin{array}{l}\text { [5] J. Thynne (personal } \\
\text { communication). }\end{array}$ \\
\hline
\end{tabular}

Preferred:

$\log k=15.6-37.3 / \theta$.

Comments: From additivity and $\Delta H_{f}^{\circ}\left(\mathrm{CH}_{3} \mathrm{O} \cdot\right)=3.9 \mathrm{kcal} / \mathrm{mole}$, one obtains $\Delta H_{f}^{\circ}\left(\mathrm{C}_{2} \mathrm{H}_{5} \mathrm{O} \cdot\right)=-4.6 \mathrm{kcal} / \mathrm{mole}$ in good agreement with the kinetics. From the reaction entropy and the $A \cdot$ factor, $k_{\text {rec }}=10^{8.9} \mathrm{l} / \mathrm{mole}$-sec.

\section{Experimental}

[1] Rates by pressure change. $\left(P_{f} / P_{o}\right) \sim 2.17 \pm 0.03$. Products observed were $\mathrm{H}_{2} \mathrm{CO}, \mathrm{CH}_{3} \mathrm{CHO}, \mathrm{C}_{2} \mathrm{H}_{5} \mathrm{OH}, \mathrm{CO}$, $\mathrm{H}_{2}, \mathrm{CH}_{4}$, and $\mathrm{C}_{2} \mathrm{H}_{6}$ with good mass balances. A mechanism was not proposed.

[2] Toulene flow system. Main products were $\mathrm{C}_{2} \mathrm{H}_{6}$ and $\mathrm{H}_{2} \mathrm{CO}$; minor products were $\left(\emptyset \mathrm{CH}_{2}\right)_{2}$ and $\mathrm{CH}_{4}$. Rates were calculated from the rate of change of $\left(\mathrm{C}_{2} \mathrm{H}_{6}+\frac{1}{2} \mathrm{CH}_{4}\right)$ determined gasometrically. Total decomposition of $\mathrm{C}_{2} \mathrm{H}_{5} \mathrm{O} \longrightarrow$ $\mathrm{CH}_{3}+\mathrm{CH}_{2} \mathrm{O}$ was observed.

[4] The combined results of [1], [2], and [3] when plotted together in the Arrhenius form gave the reported values. 
Reaction: di- $n$-Propel peroxide

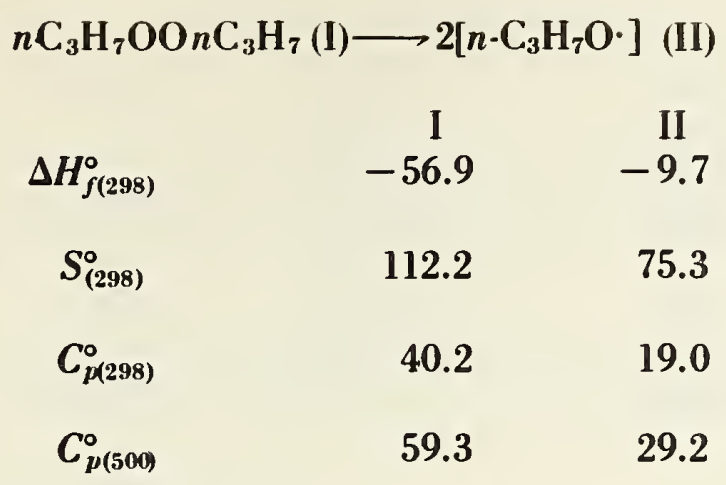

$\Delta S^{\circ}=38.4 \mathrm{~g} / \mathrm{mole}$

$\Delta H^{\circ}=37.5 \mathrm{kcal} / \mathrm{mole}$

$\Delta C_{p}^{\circ}=-2.2 \mathrm{~g} / \mathrm{mole}$

$\left\langle\overline{\Delta C}_{p}^{\circ} \simeq-1.5\right\rangle \mathrm{g} / \mathrm{mole}$

$\log A$

E $\quad \log k \boldsymbol{r}$ (434)

$\begin{array}{llllll}15.3 & 36.5 & -3.08 & 420-448^{\circ} \mathrm{K} & \text { static none }\end{array}$ $0.4-2.6$
Conditions System Surface

[1]

E. J. Harris, Proc.

Roy. Soc. (L) A173, 126 (1939).

Preferred:

$\log k=15.6-37.2 / \theta$.

Comments: The reaction kinetics give $\left[\Delta H_{f}^{\circ}\left(\mathrm{C}_{3} \mathrm{H}_{7} \mathrm{O} \cdot\right)=-10.0 \mathrm{kcal} / \mathrm{mole}\right] . k_{\mathrm{rec}}=10^{8.9} 1 / \mathrm{mole}-\mathrm{sec}$ is obtained from $A$ and the reaction entropy.

Experimental

[1] Rates were followed by pressure measurements with $P_{d} P_{o} \simeq 2.5$. Products identified were $\mathrm{C}_{3} \mathrm{H}_{7} \mathrm{OH}, \mathrm{C}_{2} \mathrm{H}_{5} \mathrm{CHO}$, $\mathrm{CH}_{2} \mathrm{O}, \mathrm{C}_{4} \mathrm{H}_{10}, \mathrm{CO}$, and $\mathrm{H}_{2}$. No mechanism was proposed.

429 
Reaction: di- $t$-Butyl peroxide

$\begin{array}{crr}\left(\mathrm{CH}_{3}\right)_{3} \mathrm{COOC}\left(\mathrm{CH}_{3}\right)_{3}(\mathrm{I}) \longrightarrow 2\left[\left(\mathrm{CH}_{3}\right)_{3} \mathrm{CO} \cdot\right](\mathrm{II}) \\ \Delta \mathrm{H}_{f(298)}^{\circ} & -80.5 & {[-21.5]} \\ S_{(298)}^{\circ} & 112.1 & 76.5 \\ C_{p(298)}^{\circ} & 52.5 & 25.7 \\ C_{p(500)}^{\circ} & 79.5 & 38.5\end{array}$

$$
\begin{aligned}
& \Delta S^{\circ}=40.9 \mathrm{~g} / \mathrm{mole} \\
& \Delta H^{\circ}=37.5 \mathrm{kcal} / \mathrm{mole} \\
& \Delta C_{p}^{\circ}=-1.1 \mathrm{~g} / \mathrm{mole} \\
& \left\langle\overline{\Delta C}_{p}^{\circ}=-1.5\right\rangle \mathrm{g} / \mathrm{mole}
\end{aligned}
$$

\begin{tabular}{|c|c|c|c|c|c|c|}
\hline $\log A$ & $\boldsymbol{E}$ & $\begin{array}{c}\log \boldsymbol{k}_{T} \\
(423)\end{array}$ & Conditions & System & Surface & References \\
\hline 16.51 & 39.1 & -3.70 & $\begin{array}{l}413-433^{\circ} \mathrm{K} \\
50-380 \text { torr }\end{array}$ & static & none & $\begin{array}{l}\text { [1] J. H. Raley, F. F. Rust, } \\
\text { and W. E. Vaughan, } \\
\text { J. Am. Chem. Soc. 70, } \\
88 \text { (1948). }\end{array}$ \\
\hline 14.74 & $36.0 \pm 1$ & $1-3.86$ & $\begin{array}{l}\text { (a) } 393-553^{\circ} \mathrm{K} \\
10-112 \text { torr }\end{array}$ & static & & $\begin{array}{l}\text { [2] J. Murawski, J. S. } \\
\text { Roberts, and }\end{array}$ \\
\hline$[13.3$ & $34.0]$ & -4.27 & $\begin{array}{l}\text { (b) } 724-832^{\circ} \mathrm{K} \\
0.03-3.5 \text { torr }\end{array}$ & flow & none & $\begin{array}{l}\text { M. Szwarc, J. } \\
\text { Chem. Phys. 19, } 698 \\
\text { (1951); M. Szwarc and } \\
\text { J. S. Roberts, J. Chem. } \\
\text { Phys. 18, 561 (1950). }\end{array}$ \\
\hline 16.4 & 38.7 & -3.60 & & & & $\begin{array}{l}\text { [3] A. R. Blake and K. O. } \\
\text { Kutschke, Can. J. } \\
\text { Chem. 3 7, } 1462 \text { (1959). }\end{array}$ \\
\hline 15.6 & 37.4 & -3.73 & $\begin{array}{l}403-443 \% \\
27-130 \text { torr }\end{array}$ & & & $\begin{array}{l}\text { [4] L. Batt and S. W. } \\
\text { Benson, J. Chem. Phys. } \\
\text { 36, } 895 \text { (1962). }\end{array}$ \\
\hline $15 \pm 1$ & $38 \pm 2$ & -4.64 & $\begin{array}{l}402-427^{\circ} \mathrm{K} \\
\sim 20 \text { torr }\end{array}$ & & & $\begin{array}{l}\text { [5] R. K. Burton and D. H. } \\
\text { Volman, J. Chem. Phys. } \\
\text { 20, } 25 \text { (1952). }\end{array}$ \\
\hline 15.85 & 38 & -3.79 & $\begin{array}{l}553-623 \% \\
P_{T} \simeq 5-13 \text { torr }\end{array}$ & flow & & $\begin{array}{c}\text { [6] F. P. Lossing and A. W. } \\
\text { Tickner, J. Chem. } \\
\text { Phys. 20, } 907 \text { (1952). }\end{array}$ \\
\hline
\end{tabular}

Preferred:

$\log k=15.6-37.4 / \theta$.

Comments: From the entropy one obtains $k_{\mathrm{rec}}=10^{8.3} \mathrm{l} / \mathrm{mole}-\mathrm{sec}$. Additivity relations give $\Delta H_{f}^{\circ}(t \mathrm{BuO} \cdot)=-21.3$ $\mathrm{kcal} / \mathrm{mole}$. 


\section{Experimental}

[1] Rates were followed manometrically using stoichiometry of $\mapsto 3$. Analysis by M. S. Products were $\mathrm{CH}_{3} \mathrm{COCH}_{3}$, $\mathrm{CH}_{4}, \mathrm{C}_{2} \mathrm{H}_{6}$ and $\mathrm{CH}_{3} \mathrm{COC}_{2} \mathrm{H}_{5}$.

[2] Pyrolysis in static and flow systems was performed in the presence of toluene. Rate constants were calculated in two ways and agreed within 20 percent. Parameters obtained assuming that all benzyl radicals produced by methyl- $\mathrm{H}$ abstraction dimerized are in brackets. Other parameters were obtained by assuming all benzyl radicals terminated with methyl radicals.

[3] Rate constants were based on the rate of $\mathrm{CH}_{4}+\mathrm{C}_{3} \mathrm{H}_{6}$ formation.

[4] Rates by pressure change. Total analysis of products by G.L.C. gave a stoichiometry of $1 \rightarrow 3$. The authors demonstrated that temperature gradients resulting from the reaction exothermicity could lead to appreciable error in the Arrhenius parameters.

[5] Analysis by M.S. of quenched reaction mixture.

[6] Analysis by M.S. The carrier gas was He. 
Reaction: Diethyanoyl peroxide (diacetyl peroxide)<smiles>CC(=O)C=O</smiles>

\begin{tabular}{ccc}
$\Delta H_{f(298)}^{\circ}$ & I & II \\
$S_{(298)}^{\circ}$ & -129.2 & $(-49.7)$ \\
$C_{p(298)}^{\circ}$ & 101.4 & 67.4 \\
$C_{p(500)}^{\circ}$ & 31.6 & 13.9 \\
\hline
\end{tabular}

$\Delta S^{\circ}=33.4 \mathrm{~g} / \mathrm{mole}$

$\Delta H^{\circ}=29.8 \mathrm{kcal} / \mathrm{mole}$

$\Delta C_{p}^{\circ}=-3.8 \mathrm{~g} / \mathrm{mole}$

$\left\langle\overrightarrow{\Delta C}_{p}^{\circ}=-3.5\right\rangle_{298-410}$

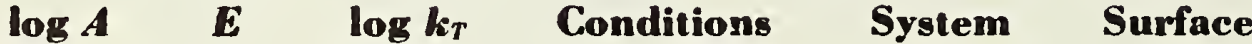

(413)

\section{$29.5-1.37$}

$363-463^{\circ} \mathrm{K}$ flow $0.03-0.156$ torr

\section{References}

[1] A. Rembaum and $M$. Szwarc, J. Am. Chem. Soc. 76, 5975 (1954).

\section{Preferred:}

$\log k=14.25-29.5 / \theta$.

Comments: The reaction entropy and observed $A$-factor give $k_{\text {rec }}=10^{8.7} 1 /$ mole-sec. The heat of formation of the acetoxy radical gives $D H^{\circ}\left(\mathrm{CH}_{3} \mathrm{CO}_{2}-\mathrm{H}\right)=106 \mathrm{kcal} / \mathrm{mole}$.

\section{Experimental}

[1] Toluene and benzene carriers employed. Standard gas analysis for $\mathrm{CO}_{2}, \mathrm{C}_{2} \mathrm{H}_{6}$, and $\mathrm{CH}_{4}$ confirmed the stoichiometry: $\left(\frac{\mathrm{CO}_{2}}{\mathrm{C}_{2} \mathrm{H}_{6}+\mathrm{CH}_{4}}\right)=2$. This is consistent with

$$
\left(\mathrm{CH}_{3} \mathrm{COO}\right)_{2} \longrightarrow 2 \mathrm{CO}_{2}+\mathrm{C}_{2} \mathrm{H}_{6} ;\left(\mathrm{CH}_{3} \mathrm{COO}\right)_{2}+\emptyset \mathrm{CH}_{3} \longrightarrow 2 \mathrm{CO}_{2}+\mathrm{CH}_{4}+\frac{1}{2}\left(\emptyset \mathrm{CH}_{2}\right)_{2} \text {. }
$$

Rate constants were independent of $P_{T}, \delta P_{\text {reactant }}$, and $t_{c}$. 
Reaction: Dipropanoyl peroxide (dipropionyl peroxide)

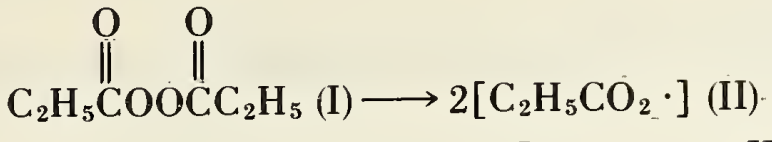

$$
\begin{aligned}
& \begin{array}{ccc} 
& \text { I } & \text { II } \\
\Delta H_{f(298)}^{\circ} & -139.8 & {[-55.0]}
\end{array} \\
& \begin{array}{lll}
S_{(298)}^{\circ} & 119.4 & 76.4
\end{array} \\
& \begin{array}{lll}
C_{p(298)}^{\circ} & 45.0 & 20.6
\end{array} \\
& \begin{array}{lll}
C_{p(500)}^{\circ} & 57.6 & 27.5
\end{array}
\end{aligned}
$$

$$
\begin{aligned}
& \Delta S^{\circ}=33.4 \mathrm{~g} / \mathrm{mole} \\
& \Delta H^{\circ}=29.8 \mathrm{kcal} / \mathrm{mole} \\
& \Delta C_{p}^{\circ}=-3.8 \mathrm{~g} / \text { mole }
\end{aligned}
$$

$\begin{array}{rlllr}\log A & E & \begin{array}{c}\log k_{T} \\ (418)\end{array} & \text { Conditions } & \text { Sy } \\ & & & & \\ 14.4 & 30.0 & -1.27 & \begin{array}{l}373-464^{\circ} \mathrm{K} \\ 7.6-20.6 \text { torr }\end{array} & \text { flow }\end{array}$

Surface

[1] A. Rembaum and M.

Szwarc, J. Chem. Phys. 23, 909 (1955).

\section{Preferred:}

$\log k=14.4-30.0 / \theta$.

Comments: A recombination rate constant of $k_{\text {rec }}=10^{8.8} 1 /$ mole-sec is obtained from the reaction entropy and the observed $A$-factor.

\section{Experimental}

[1] Toluene and benzene carriers were employed. Rates were equated to $\frac{1}{2}$ the rate of formation of $\mathrm{CO}_{2}$. Other products: $\mathrm{C}_{2} \mathrm{H}_{4}$ (8 percent), $\mathrm{C}_{2} \mathrm{H}_{6}$ (8.6 percent), $n-\mathrm{C}_{4} \mathrm{H}_{10}$ (80.9 percent). First-order rate constants were independent of $t_{c}$, $P_{T}$, and $\delta P_{\text {reactant }}$. 
Reaction: Dibutanoyl peroxide (dibutyrl peroxide)

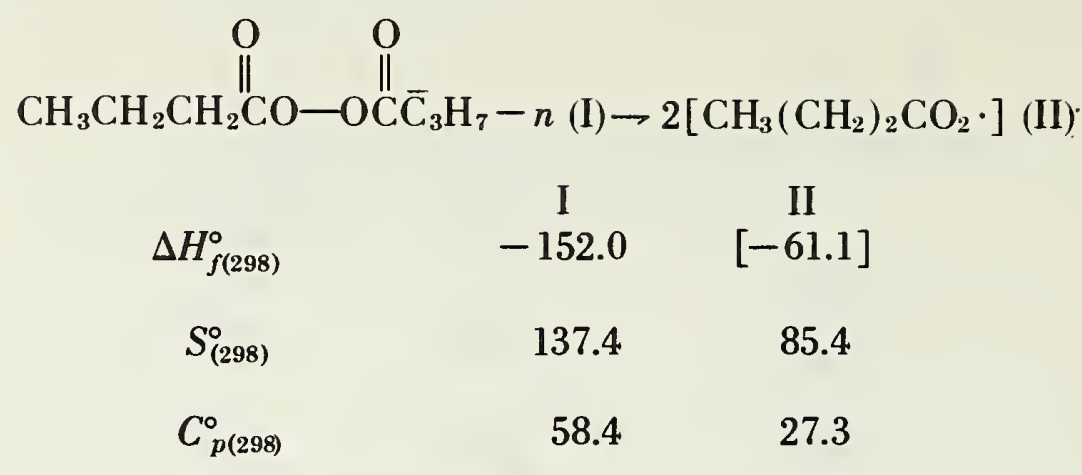

$\Delta S^{\circ}=33.4 \mathrm{~g} / \mathrm{mole}$

$\Delta H^{\circ}=29.8 \mathrm{kcal} / \mathrm{mole}$

$\Delta C_{p}^{\circ}=-3.8 \mathrm{~g} / \mathrm{mole}$

$\left\langle\Delta C_{p}^{\circ}=-3.5\right\rangle_{298-T} \mathrm{~g} /$ mole

$\begin{array}{lllllll}\log A & E & \begin{array}{c}\log k_{T} \\ (411)\end{array} & \text { Conditions } & \text { System } & \text { Surface } & \\ 14.28 & 29.6 & -1.46 & 370-452^{\circ} \mathrm{K} & \text { flow } & & \text { [1] A. }\end{array}$

\section{References}

1] A. Rembaum and M.

Szwarc, J. Chem. Phys. 23, 909 (1955).

\section{Preferred:}

$\log k=14.3-29.6 / \theta .^{*}$ Rate constant is reliable.

Comments: A recombination rate constant of $10^{8.7} \mathrm{l} / \mathrm{mole}$-sec is obtained from the reaction entropy and the observed $A$-factor.

\section{Experimental}

[1] Benzene and toluene carrier gases were employed. Stoichiometry of 1 peroxide $\longrightarrow 2 \mathrm{CO}_{2}$ was confirmed and the decomposition rate was obtained from the $\mathrm{CO}_{2}$ formation rate. Rate constants were independent of $t_{c}, P_{T}$, and $\delta P_{\text {reactani }}$.

*The isobutyl peroxide decomposition has been studied in solution of isooctane, J. Smid and M. Szwarc, J. Chem. Phys. 29, 432 (1958). Rate constants were consistent with $\log k=14.45-27.3 / \theta$. 
Reaction: Methyl hydroperoxide

$$
\mathrm{CH}_{3} \mathrm{OOH}(\mathrm{I}) \stackrel{(M)}{\longrightarrow} \mathrm{CH}_{3} \mathrm{O}(\mathrm{II})+\mathrm{OH}(\mathrm{III})
$$

$\begin{array}{cccc} & \text { I } & \text { II } & \text { III } \\ \Delta H_{f(298)}^{\circ} & -32.0 & (3.9) & 8.0 \\ S_{(298)}^{\circ} & 65.8 & 54.6 & 43.9 \\ C_{p(298)}^{\circ} & 14.7 & 8.4 & 7.1\end{array}$

$\Delta S^{\circ}=32.7 \mathrm{~g} / \mathrm{mole}$

$\Delta H^{\circ}=43.9 \mathrm{kcal} / \mathrm{mole}$

$\Delta C_{p}^{\circ}=0.8 \mathrm{~g} / \mathrm{mole}$

\begin{tabular}{ccccccc}
$\log \boldsymbol{A}$ & $\boldsymbol{E}$ & $\begin{array}{c}\log \boldsymbol{k}_{T} \\
(608)\end{array}$ & Conditions & System & Surface & \multicolumn{1}{c}{ References } \\
$11 \pm 2$ & $32 \pm 5$ & $(-0.5)$ & $\begin{array}{l}565-651^{\circ} \mathrm{K} \\
22-49 \text { torr }\end{array}$ & flow & (see below) & [1] A. D. Kirk, Can. J. \\
Chem. 43, 2236 (1965)。
\end{tabular}

Preferred:

$\log k=14.9-43.0 / \theta$. Rate constants may be slightly low.

Comments: The rate constants in the middle of the range are reasonable, but (as the author was aware) the thermodynamics coupled with a normal recombination rate $\left(k_{\text {rec }} \approx 10^{9.6} \mathrm{l} /\right.$ mole-sec $)$ predict considerably higher Arrhenius parameters.

Higher values have, in fact, been observed in solution and are preferred.

\section{Experimental}

[1] Toluene carrier technique was employed. Rates were obtained from the iodimetric titration of the remaining hydroperoxide and the ratio of $\left(\emptyset \mathrm{CH}_{2}\right)_{2} /\left(\mathrm{CH}_{3} \mathrm{O}_{2}\right)$. Products analysed by G.L.C.: major product, $\left(\emptyset \mathrm{CH}_{2}\right)_{2}$; minor products, $\mathrm{CH}_{4}, \mathrm{CO}, \mathrm{O}_{2}$, and $\mathrm{C}_{2} \mathrm{H}_{6}$. Heterogeneous decomposition in the low-temperature range was indicated since the ratio of $\left(\emptyset \mathrm{CH}_{2}\right)_{2} / \Delta\left(\mathrm{CH}_{3} \mathrm{OOH}\right)$ varied from $0.05-0.80$ from $290-380{ }^{\circ} \mathrm{C}$. 
Reaction: Ethyl hydroperoxide

\begin{tabular}{cccc}
\multicolumn{4}{c}{$\mathrm{C}_{2} \mathrm{H}_{5} \mathrm{OOH}(\mathrm{I}) \longrightarrow \mathrm{C}_{2} \mathrm{H}_{5} \mathrm{O} \cdot(\mathrm{II})+\cdot \mathrm{OH}(\mathrm{III})$} \\
& $\mathrm{I}$ & II & III \\
$\Delta H_{f(298)}^{\circ}$ & -40.5 & -4.9 & 8.0 \\
$S_{(298)}^{\circ}$ & 76.3 & 65.9 & 43.9 \\
$C_{p(298)}^{\circ}$ & 19.7 & 14.0 & 7.1
\end{tabular}

$\Delta S^{\circ}=33.5 \mathrm{~g} / \mathrm{mole}$

$\Delta H^{\circ}=43.6 \mathrm{kcal} / \mathrm{mole}$

$\Delta C_{p}^{\circ}=1.4 \mathrm{~g} / \mathrm{mole}$

\begin{tabular}{|c|c|c|c|c|c|c|}
\hline $\log A$ & $\boldsymbol{E}$ & $\underset{(605)}{\log \boldsymbol{k}_{T}}$ & Conditions & System & Surface & References \\
\hline 13.4 & 37.7 & -0.22 & $553-653^{\circ} \mathrm{K}$ & flow & $\begin{array}{l}\text { (consid- } \\
\text { erable) }\end{array}$ & $\begin{array}{l}\text { [1] A. D. Kirk and J. H. } \\
\text { Knox, Trans. Faraday } \\
\text { Soc. 56, } 1296(1960) ; \\
\text { A. D. Kirk and J. H. } \\
\text { Knox, Proc. Chem. Soc., } \\
\text { 384 (1959). }\end{array}$ \\
\hline
\end{tabular}

Preferred:

$\log k=15.35-43.0 / \theta$. Rate constants are probably reliable.

Comments: It is unlikely that the cross recombination is unimportant in this system. The thermodynamics indicate that the Arrhenius parameters are low. Assuming that the rate constants are valid, one obtains the preferred values and $k_{\text {rec }}=10^{9.7} \mathrm{l} / \mathrm{mole}$-sec.

\section{Experimental}

[1] Benzene carrier technique. Rate constants were calculated on the basis of the amount of diphenyl produced and the amount of peroxide decomposed, assuming further that all hydroxyl radicals formed abstracted $\mathrm{H}$ from benzene and that all methyl radicals combined to form ethane. Decomposition of the peroxide also occurred heterogeneously. Since the biphenyl yields were essentially invariant to the $(S / V)$ ratios, they were used as a measure of the homogeneous bond fission reaction.

Mechanism:

$$
\begin{aligned}
\mathrm{ROOH} & \stackrel{1}{\longrightarrow} \mathrm{RO} \cdot+\cdot \mathrm{OH} \\
\cdot \mathrm{OH}+\emptyset \mathrm{H} & \stackrel{2}{\longrightarrow} \mathrm{H}_{2} \mathrm{O}+\emptyset \cdot \\
\mathrm{RO} \cdot & \stackrel{3}{\longrightarrow} \mathrm{CH}_{3} \cdot+\mathrm{R}_{1} \mathrm{COR}_{2} \\
\mathrm{CH}_{3} \cdot+\mathrm{CH}_{3} \cdot & \stackrel{4}{\longrightarrow} \mathrm{C}_{2} \mathrm{H}_{6} \\
\emptyset \cdot+\emptyset \cdot & \stackrel{5}{\longrightarrow} \emptyset_{2 .} .
\end{aligned}
$$


Reaction: Isopropyl hydroperoxide

$\begin{array}{cccc}\left(\mathrm{CH}_{3}\right)_{2} \mathrm{CHOOH}(\mathrm{I}) \longrightarrow & \left(\mathrm{CH}_{3}\right)_{2} \mathrm{CHO} \cdot(\mathrm{II})+\cdot \mathrm{OH}(\mathrm{III}) \\ & \mathrm{I} & \mathrm{II} & \mathrm{III} \\ \Delta H_{f(298)}^{\circ} & -47.4 & (-12.4) & 8.0 \\ S_{(298)}^{\circ} & 82.6 & 72.2 & 43.9 \\ C_{p(298)}^{\circ} & 25.7 & 20.0 & 7.1\end{array}$

$\Delta S^{\circ}=33.5 \mathrm{~g} / \mathrm{mole}$

$\Delta H^{\circ}=43.0 \mathrm{kcal} / \mathrm{mole}$

$\Delta C_{p}^{\circ}=1.4 \mathrm{~g} / \mathrm{mole}$

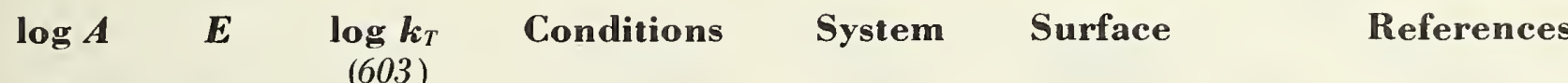

$\begin{array}{llll}15.2 & 40.0 & 0.70 & 553-653{ }^{\circ} \mathrm{K} \quad \text { flow }\end{array}$

(appre-

ciable)
[1] A. D. Kirk and J. H.

Knox, Trans. Faraday

Soc. 56, 1296 (1960);

*A. D. Kirk and J. H.

Knox, Proc. Chem.

Soc., 384 (1959).

Preferred:

Estimate $\log k=15.5-43.0 / \theta$.

Comments: The kinetics [1] $]^{*}$ and thermodynamics agree within experimental error. A recombination rate constant of $k_{\text {rec }}=10^{9.9} \mathrm{l} /$ mole-sec is obtained from the reaction entropy and preferred $A$-factor.

\section{Experimental}

[1] Benzene carrier technique was employed.

*See comments on ethyl hydroperoxide. 
Reaction: $t$-Butyl hydroperoxide

\begin{tabular}{crcc}
\multicolumn{4}{r}{$\left(\mathrm{CH}_{3}\right)_{3} \mathrm{COOH}(\mathrm{I}) \longrightarrow\left(\mathrm{CH}_{3}\right)_{3} \mathrm{CO} \cdot(\mathrm{II})+\cdot \mathrm{OH}(\mathrm{III})$} \\
$\Delta H_{f(298)}^{\circ}$ & I & II & III \\
$S_{(298)}^{\circ}$ & -56.5 & -21.3 & 8.0 \\
$C_{p(298)}^{\circ}$ & 86.4 & 76.5 & 43.9 \\
& 31.4 & 25.7 & 7.1
\end{tabular}

$\Delta S^{\circ}=34.0 \mathrm{~g} / \mathrm{mole}$

$\Delta H^{\circ}=43.2 \mathrm{kcal} / \mathrm{mole}$

$\Delta C_{p}^{\circ}=1.4 \mathrm{~g} / \mathrm{mole}$

\begin{tabular}{|c|c|c|c|c|c|c|}
\hline $\log A$ & $\boldsymbol{E}$ & $\begin{array}{c}\log \boldsymbol{k}_{T} \\
(603)\end{array}$ & Conditions & System & Surface & References \\
\hline 13.7 & 37.8 & 0.00 & $553-653^{\circ} \mathrm{K}$ & flow & $\begin{array}{l}\text { (appre- } \\
\text { ciable) }\end{array}$ & $\begin{array}{l}\text { [1] A. D. Kirk and J. H. } \\
\text { Knox, Trans. Faraday } \\
\text { Soc. 56, } 1296(1960) ; \\
\text { A. D. Kirk and J. H. } \\
\text { Knox, Proc. Chem. Soc., } \\
\text { 384 (1959). }\end{array}$ \\
\hline
\end{tabular}

Preferred:

$\log k=15.6-43.0 / \theta$.

Comments: Rate constant parameters have been scaled to give agreement with the $\Delta H_{f}^{\circ}$. This is consistent with a recombination rate constant of $k_{\mathrm{rec}}=10^{9.9} \mathrm{l} / \mathrm{mole}$-sec. The preferred value is also consistent with the rates found in dilute, non-polar solvents, R. R. Hiatt and K. C. Irwin, J. Org. Chem. 33, 1436 (1968).

\section{Experimental}

[1] Benzene carrier technique. See comments on ethyl hydroperoxide. 
Reaction: Hydrazine

\begin{tabular}{ccr}
\multicolumn{3}{r}{$\left.\mathrm{NH}_{2}-\mathrm{NH}_{2}(\mathrm{I}) \longrightarrow \mathrm{NH}_{2} \cdot\right](\mathrm{II})$} \\
$\Delta H_{f(298)}^{\circ}$ & $\mathrm{I}$ & $\mathrm{II}$ \\
$S_{(298)}^{\circ}$ & 22.8 & $* *(46.0)$ \\
$C_{p(298)}^{\circ}$ & 56.9 & 48.2 \\
$C_{p(1000)}^{\circ}$ & 12.2 & 8.1 \\
& 23.0 & 10.0
\end{tabular}

$\Delta S^{\circ}=39.5 \mathrm{~g} / \mathrm{mole}$

$\Delta H^{\circ}=(69.2) \mathrm{kcal} / \mathrm{mole}$

$\Delta C_{p}^{\circ}=4.0 \mathrm{~g} / \mathrm{mole}$

$\Delta C_{p}^{\circ}=-3.0 \mathrm{~g} / \mathrm{mole}$

\begin{tabular}{|c|c|c|c|c|c|c|}
\hline $\log A$ & $\boldsymbol{E}$ & $\begin{array}{c}\log \boldsymbol{k}_{T} \\
(980)\end{array}$ & Conditions & System & Surfaces & References \\
\hline 12.6 & 60 & $-0: 78$ & $\begin{array}{l}903-1053 \% \\
\delta P \simeq 0.05-.78 \\
P_{T}=5-15 \mathrm{~mm} \\
\quad \mathrm{Hg}\end{array}$ & flow & $\begin{array}{l}\text { none }{ }^{*} \\
(90 \% \\
\text { hetero- } \\
\text { geneous) }\end{array}$ & $\begin{array}{l}\text { [1] M. Szwarc, J. Chem. Phys. } \\
\text { 17, } 505 \text { (1949); Proc. } \\
\text { Roy. Soc. A198, } 267 \\
\text { (1949). }\end{array}$ \\
\hline 11.7 & 54.4 & -0.43 & $\begin{array}{l}887-1034 \% \\
6.7-28.5 \mathrm{~mm} \\
\quad \mathrm{Hg}\end{array}$ & flow & none & $\begin{array}{l}\text { [2] J. A. Kerr, R. C. Sekhar, } \\
\text { and A. F. Trotman- } \\
\text { Dickenson, J. Chem. } \\
\text { Soc. } 3217 \text { (1963); J. A. } \\
\text { Kerr, A. F. Trotman- } \\
\text { Dickenson and M. } \\
\text { Wolter, J. Chem. Soc. } \\
\text { 3584 (1964). }\end{array}$ \\
\hline 13.0 & 54.0 & +0.96 & $\begin{array}{l}970-1120 \% \\
2-9 \text { atm } \\
1 \% \text { in } \mathrm{Ar}\end{array}$ & S.P.Sh. & & $\begin{array}{l}\text { [3] E. T. McHale, B. E. Knox, } \\
\text { and H. P. Palmer, Tenth } \\
\text { Symposium Inter- } \\
\text { national on Combus- } \\
\text { tion, Butterworths } \\
\text { Publication, London, } \\
\text { 1965, p. } 341 .\end{array}$ \\
\hline
\end{tabular}

Preferred:

Suspect. $\log k=16.5-70.8 / \theta^{* *}$. Reference [3] rate scaled.

Comments: This reaction is certainly in its second-order region for all studies reported. Pressure dependence for the reaction has been demonstrated by several workers in addition to those referenced here (see $M$. Gilbert, Comb. \& Flames 2, 149 (1958); R. W. Diesen, J. Chem. Phys. 39, 2121 (1963)).

The best heat of formation for $\left(\mathrm{NH}_{2} \cdot\right)$ consistent with the kinetics of all of the hydrazine and amine decompositions, assuming that the rate constant parameters reported are correct, is $\Delta H_{f}^{\circ}\left(\mathrm{NH}_{2}{ }^{\circ}\right)=41.0$

(Continued)

*A decomposition into $\mathrm{N}_{2}$ and $\mathrm{H}_{2}$ was also observed which was strongly affected by the (S/V)ratio.

**Preferred parameters for this reaction (and the other similar studies) have beeri calculated on the basis of assumed recombination rate constants. (The analysis is discussed in detail in section $\mathrm{V}-\mathbf{8 . 0}$ ). 
Hydrazine (Continued)

$\mathrm{kcal} /$ mole. However, the recombination rate constants calculated for these reactions from the reported $A$-factors and estimated reaction entropies are anomolously low and very difficult to believe. In addition, the bond $(\mathrm{N}-\mathrm{H})$ dissociation energy in ammonia, using $41.0 \mathrm{kcal} / \mathrm{mole}$ for the $\mathrm{NH}_{2}$ heat of formation is the same as the first $(\mathrm{C}-\mathrm{H})$ bond energy in methane (i.e., $104 \mathrm{kcal} / \mathrm{mole}$ ), which does not make too much sense.

It seems very likely that the usual problem with the toluene carrier technique (i.e., "low" activation energies, probably due to a combination of induced decomposition and surface catalysis), is showing up in this and the other hydrazine and amine studies. If such is the case, the activation energies and $A$-factors for all studies should be higher.

\section{Experimental}

[1] Toluene carrier technique. Rates were based on dibenzyl formation.

[2] Toluene carrier technique. Heterogeneous decomposition observed and corrections made to the rate. Reaction rates were strongly pressure dependent indicating that the reaction was well into its falloff region. The parameters reported are for $P_{T} \simeq 14.5 \overline{\mathrm{mm}} \mathrm{Hg}$.

[3] There was no internal standard employed in the S.P.Sh. tube experiments. The ratio of $\left(\mathrm{NH}_{3} / \mathrm{N}_{2}\right) \simeq 2.15$ and indicates some chain reaction. Parameters reported were estimates for the high-pressure region reaction. 
Reaction: Methylhydrazine

\begin{tabular}{cccc}
\multicolumn{4}{r}{$\mathrm{CH}_{3} \mathrm{NHNH}_{2}(\mathrm{I}) \longrightarrow \mathrm{CH}_{3} \mathrm{NH} \cdot(\mathrm{II})+\cdot \mathrm{NH}_{2}(\mathrm{III})$} \\
$\Delta H_{f(298)}^{\circ}$ & I & II & III \\
$S_{(298)}^{\circ}$ & 22.2 & $(41.7)$ & $(46.0)$ \\
$C_{p(298)}^{\circ}$ & 66.9 & 58.2 & 48.2 \\
$C_{p(1000)}^{\circ}$ & 17.1 & 11.6 & 8.1 \\
& 34.6 & 24.2 & 10.0
\end{tabular}

$\Delta S^{\circ}=39.5 \mathrm{~g} / \mathrm{mole}$

$\Delta H^{\circ}=(65.5) \mathrm{kcal} / \mathrm{mole}$

$\Delta C_{p}^{\circ}=2.6 \mathrm{~g} / \mathrm{mole}$

$\Delta C_{p}^{\circ}=-0.4 \mathrm{~g} / \mathrm{mole}$

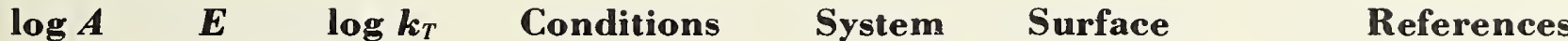

(805)

13.19

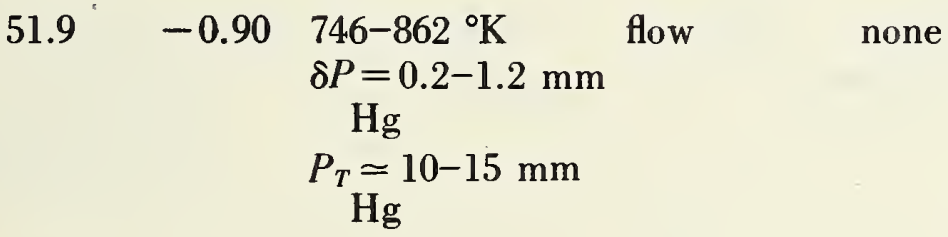
[1] J. A. Kerr, R. C. Sekhar, and A. F. Trotman- Dickenson, J. Chem. Soc. 3217 (1963).

\section{Preferred:}

$\log k=16.7-64.8 / \theta$.

Comments: Arrhenius parameters are suspect. Rate constants are probably reliable, although pressure falloff may have been important. (See hydrazine decomposition and section V-8.0.)

\section{Experimental}

[1] Toluene carrier technique. Rates were based on the production of ammonia. A heterogeneous decomposition into $\mathrm{CH}_{4}, \mathrm{H}_{2}$, and $\mathrm{N}_{2}$ was also observed. Homogeneous products were $\mathrm{CH}_{4}, \mathrm{H}_{2}, \mathrm{NH}_{3}$, and $\left(\emptyset \mathrm{CH}_{2}\right)_{2}$. 
Reaction: 1,1-Dimethylhydrazine

\begin{tabular}{cccc}
\multicolumn{4}{r}{$\left(\mathrm{CH}_{3}\right)_{2} \mathrm{NNH}_{2}(\mathrm{I}) \longrightarrow\left(\mathrm{CH}_{3}\right)_{2} \mathrm{~N} \cdot(\mathrm{II})+\cdot \mathrm{NH}_{2}(\mathrm{III})$} \\
$\Delta H_{f(298)}^{\circ}$ & I & II & III \\
$S_{(298)}^{\circ}$ & 20.4 & $(37.4)$ & $(46.0)$ \\
$C_{p(298)}^{\circ}$ & 71.8 & 65.4 & 48.4 \\
$C_{p(1000)}^{\circ}$ & 23.5 & 16.1 & 8.1 \\
& 47.8 & 35.1 & 10.1
\end{tabular}

$\Delta S^{\circ}=42.0 \mathrm{~g} / \mathrm{mole}$

$\Delta H^{\circ}=63.0 \mathrm{kcal} / \mathrm{mole}$

$\Delta C_{p}^{\circ}=0.7 \mathrm{~g} / \mathrm{mole}$

$\Delta C_{p}^{\circ}=-2.6 \mathrm{~g} / \mathrm{mole}$

\begin{tabular}{ccccccc}
$\log \boldsymbol{A}$ & $\boldsymbol{E}$ & $\begin{array}{c}\log \boldsymbol{k}_{T} \\
(770)\end{array}$ & Conditions & System & Surface & \multicolumn{1}{c}{ References } \\
13.2 & 49.6 & -0.88 & $\begin{array}{l}709-831^{\circ} \mathrm{K} \\
P_{T}=10-15 \mathrm{~mm} \\
\mathrm{Hg}\end{array}$ & flow & none & [1] J. A. Kerr, R. C. Sekhar \\
and A. F. Trotman- \\
Dickenson, J. Chem. \\
\end{tabular}

\section{Preferred:}

Suspect. Estimate, $\log k=16.9-62.7 / \theta$ (see below).

Comments: Arrhenius parameters are low. Rate constants are probably reliable. (See hydrazine decomposition and section $\mathrm{V}-8.0$.)

\section{Experimental}

[1] Toulene carrier technique. Rates were based on ammonia formation. Dimethylamine was not observed as a product. This implies that the $\left(\mathrm{CH}_{3}\right)_{2} \mathrm{~N} \cdot$ did not abstract hydrogen from toluene. 
Reaction: Benzylamine (phenylaminomethane)

\begin{tabular}{|c|c|c|c|}
\hline$\Delta H_{f(298)}^{\circ}$ & $\begin{array}{c}\text { I } \\
21.0\end{array}$ & $\begin{array}{c}\text { II } \\
45.0\end{array}$ & $\underset{(46.0)}{\text { III }}$ \\
\hline$S_{(298)}^{\circ}$ & 87.6 & 75.3 & 48.2 \\
\hline$C_{p(298)}^{\circ}$ & 30.1 & 25.6 & 8.1 \\
\hline$C_{p(1000)}^{\circ}$ & 70.1 & 60.6 & 10.0 \\
\hline
\end{tabular}

$\Delta S^{\circ}=35.9 \mathrm{~g} / \mathrm{mole}$

$\Delta H^{\circ}=70.0 \mathrm{kcal} / \mathrm{mole}$

$\Delta C_{p}^{\circ}=3.6 \mathrm{~g} / \mathrm{mole}$

$\Delta C_{p}^{\circ}=0.5 \mathrm{~g} / \mathrm{mole}$

\begin{tabular}{|c|c|c|c|c|c|c|}
\hline $\log A$ & $\boldsymbol{E}$ & $\begin{array}{c}\log \boldsymbol{k}_{T} \\
(945)\end{array}$ & Conditions & System & Surface & References \\
\hline 12.8 & 59 & -0.85 & $\begin{array}{l}923-1073 \\
P_{T}=7-15 \mathrm{~mm} \\
\mathrm{Hg}\end{array}$ & flow & none & $\begin{array}{l}\text { [1] M. Szwarc, J. Chem. } \\
\text { Phys. 17, } 505 \text { (1949); } \\
\text { Proc. Roy. Soc. A198, } \\
285 \text { (1949). }\end{array}$ \\
\hline 13.0 & 59.8 & -0.83 & $829-1061^{\circ} \mathrm{K}$ & flow & & $\begin{array}{l}\text { [2] J. A. Kerr, R. C. Sekhar, } \\
\text { and A. F. Trotman- } \\
\text { Dickenson, J. Chem. } \\
\text { Soc. } 3217 \text { (1963). }\end{array}$ \\
\hline
\end{tabular}

Preferred:

$\log k=15.3-69.8 / \theta$.

Comments: Reported parameters are suspect. (See discussion on the hydrazine decomposition and section V-8.0.)

\section{Experimental}

[1] Toluene carrier technique. $\Delta\left(\mathrm{NH}_{3}\right)=\left(\varnothing \mathrm{CH}_{2}\right)_{2}$. Rate was based on the ammonia production.

[2] Toluene carrier technique. Rates were based on ammonia formation. Product identifications by G.L.C. 
Reaction: N-Methylbenzylamine

\begin{tabular}{cccc}
\multicolumn{4}{r}{$\varnothing \mathrm{CH}_{2} \mathrm{NHCH}_{3}(\mathrm{I}) \longrightarrow \varnothing \mathrm{CH}_{2} \cdot(\mathrm{II})+\cdot \mathrm{NHCH}_{3}(\mathrm{III})$} \\
& $\mathrm{I}$ & II & III \\
$\Delta H_{f(298)}^{\circ}$ & 21.5 & 45.0 & $(41.7)$ \\
$S_{(298)}^{\circ}$ & 94.8 & 75.3 & 58.2 \\
$C_{p(298)}^{\circ}$ & 34.8 & 25.2 & 11.6 \\
$C_{p(1000)}^{\circ}$ & 83.4 & 60.6 & 24.2
\end{tabular}

$\Delta S^{\circ}=38.7 \mathrm{~g} / \mathrm{mole}$

$\Delta H^{\circ}=(65.2) \mathrm{kcal} / \mathrm{mole}$

$\Delta C_{p}^{\circ}=2.0 \mathrm{~g} / \mathrm{mole}$

$\Delta C_{p}^{\circ}=1.4 \mathrm{~g} / \mathrm{mole}$

\begin{tabular}{|c|c|c|c|c|c|c|}
\hline $\log A$ & $\boldsymbol{E}$ & $\begin{array}{c}\log k_{T} \\
(875)\end{array}$ & Conditions & System & Surface & References \\
\hline 12.86 & 57.7 & -1.55 & $\begin{array}{l}819-936^{\circ} \mathrm{K} \\
P_{T} \simeq 15 \mathrm{~mm} \mathrm{Hg} .\end{array}$ & flow & & $\begin{array}{l}\text { [1] J. A. Kerr, R. C. } \\
\text { Sekhar, and A. F. } \\
\text { Trotman-Dickenson, } \\
\text { J. Chem. Soc. } 3217 \\
\text { (1963). }\end{array}$ \\
\hline
\end{tabular}

Preferred:

$\log k=15.7-69.0 / \theta$.

Comments: Rate constants are probably reliable. The Arrhenius parameters are suspect. (See discussion on the hydrazine decomposition and also section $\mathrm{V}-8.0$.)

\section{Experimental}

[1] Toluene carrier technique. Rates based on formation of $\left(\mathrm{CH}_{3} \mathrm{NH}_{2}\right)$. 
Reaction: Phenylhydrazine

\begin{tabular}{cccc}
\multicolumn{4}{c}{$\emptyset \mathrm{NHNH}_{2}(\mathrm{I}) \longrightarrow \emptyset \mathrm{NH}_{-}(\mathrm{II})+\cdot \mathrm{NH}_{2}(\mathrm{III})$} \\
$\Delta H_{f(298)}^{\circ}$ & $\mathrm{I}$ & $\mathrm{II}$ & $\mathrm{III}$ \\
$S_{(298)}^{\circ}$ & 50.3 & $(55.0)$ & $(46.0)$ \\
$C_{p(298)}^{\circ}$ & 87.0 & 76.3 & 48.2 \\
$C_{p(800)}^{\circ}$ & $30.9^{\circ}$ & 25.2 & 8.1 \\
& 63.1 & 51.2 & 9.3
\end{tabular}

$\Delta S^{\circ}=37.5 \mathrm{~g} / \mathrm{mole}$ $\Delta H^{\circ}=50.7 \mathrm{kcal} / \mathrm{mole}$

$\Delta C_{p}^{\circ}=2.4 \mathrm{~g} / \mathrm{mole}$ $\Delta C_{p}^{\circ}=-2.6 \mathrm{~g} / \mathrm{mole}$

$\begin{array}{cccccc}\log A & \boldsymbol{E} & \begin{array}{c}\log \boldsymbol{k}_{T} \\ (655)\end{array} & \text { Conditions } & \text { System } & \text { Surface } \\ 11.8 & 40.0 & -1.55 & 600-712^{\circ} \mathrm{K} & \text { flow } & \end{array}$

\section{References}

[1] J. A. Kerr, Margaret Walter, and A. F. Trotman-Dickenson, J.

Chem. Soc. 3584 (1964).

\section{Preferred:}

$\log k=15.5-51.1 / \theta$.

Comments: Arrhenius parameters are highly suspect. Rate constants are probably reliable. (See discussion on the hydrazine decomposition, section $\mathrm{V}-8.0$.)

\section{Experimental}

[1] Aniline carrier technique. Rate constants based on ammonia formation. (See methylaniline for the mechanism.) 
Reaction: N-Methylaniline

\begin{tabular}{cccc}
\multicolumn{4}{c}{$\emptyset \mathrm{NHCH}_{3}(\mathrm{I}) \longrightarrow \varnothing \dot{\mathrm{NH}}(\mathrm{II})+\dot{\mathrm{C}} \mathrm{H}_{3}(\mathrm{III})$} \\
$\Delta H_{f(298)}^{\circ}$ & I & II & III \\
$S_{(298)}^{\circ}$ & 20.7 & $(55.0)$ & 34.0 \\
$C_{p(298)}^{\circ}$ & 86.2 & 76.3 & 46.4 \\
$C_{p(1000)}^{\circ}$ & 30.9 & 25.5 & 8.3 \\
& 74.5 & 56.8 & 14.4
\end{tabular}

$\Delta S^{\circ}=36.5 \mathrm{~g} / \mathrm{mole}$

$\Delta H^{\circ}=(68.3) \mathrm{kcal} / \mathrm{mole}$

$\Delta C_{p}^{\circ}=2.9 \mathrm{~g} / \mathrm{mole}$

$\Delta C_{p}^{\circ}=-3.3 \mathrm{~g} / \mathrm{mole}$

\begin{tabular}{|c|c|c|c|c|c|c|}
\hline $\log A$ & $E$ & $\begin{array}{c}\log k_{T} \\
(880)\end{array}$ & Conditions & System & Surface & References \\
\hline 13.4 & 60.0 & -1.50 & $\begin{array}{l}812-949^{\circ} \mathrm{K} \\
P_{T}=7-12.2 \mathrm{~mm} \\
\quad \mathrm{Hg}\end{array}$ & flow & none & $\begin{array}{l}\text { [1] G. L. Estelban, J. A. Kerr, } \\
\text { and A. F. Trotman- } \\
\text { Dickenson, J. Chem. } \\
\text { Soc. } 3879 \text { (1963) }\end{array}$ \\
\hline
\end{tabular}

Preferred:

$\log k=15.3-67.7 / \theta$.

Comments: Arrhenius parameters are suspect. The rate constants are probably reliable. (See discussion on the hydrazine decomposition and section $\mathrm{V}-\mathbf{8 . 0}$.)

\section{Experimental}

[1] Aniline carrier technique. Rate constants were based on the methane formation. Mechanism proposed:

$$
\begin{aligned}
& \emptyset \mathrm{NH}-\mathrm{CH}_{3} \longrightarrow \emptyset \mathrm{NH} \cdot+\mathrm{CH}_{3} \cdot \\
& \mathrm{CH}_{3} \cdot+\emptyset \mathrm{NH}_{2} \longrightarrow \mathrm{CH}_{4}+\emptyset \mathrm{NH} \\
& 2 \emptyset \mathrm{NH} \longrightarrow\left(\emptyset \mathrm{NH}_{2}\right)_{2} .
\end{aligned}
$$


Reaction: NN-Dimethylaniline

\begin{tabular}{cccc}
\multicolumn{4}{c}{$\emptyset \mathrm{N}\left(\mathrm{CH}_{3}\right)_{2}(\mathrm{I}) \longrightarrow \mathrm{NCH}_{3}(\mathrm{II})+\mathrm{CH}_{3} \cdot(\mathrm{III})$} \\
$\Delta H_{f(298)}^{\circ}$ & I & II & III \\
$S_{(298)}^{\circ}$ & ${ }^{\circ}(22.7)$ & {$[53.5]$} & 34.0 \\
$C_{p(298)}^{\circ}$ & 92.0 & 84.1 & 46.4 \\
$C_{p(1000)}^{\circ}$ & 36.2 & 31.1 & 8.3 \\
& 85.0 & 70.3 & 14.4
\end{tabular}

$\Delta S^{\circ}=37.4 \mathrm{~g} / \mathrm{mole}$

$\Delta H^{\circ}={ }^{*}(65.1) \mathrm{kcal} / \mathrm{mole}$

$\Delta C_{p}^{\circ}=0.9 \mathrm{~g} / \mathrm{mole}$

$\Delta C_{p}^{\circ}=-0.3 \mathrm{~g} /$ mole

\begin{tabular}{|c|c|c|c|c|c|c|}
\hline $\log A$ & $E$ & $\begin{array}{c}\log \boldsymbol{k}_{T} \\
(870)\end{array}$ & Conditions & System & Surface & References \\
\hline 12.9 & 57.0 & -1.10 & $\begin{array}{l}825-9544^{\circ} \mathrm{K} \\
P_{T}=6-12 \mathrm{~mm} \\
\quad \mathrm{Hg}\end{array}$ & flow & & $\begin{array}{l}\text { [1] J. A. Kerr, Margaret } \\
\text { Walter, and A. F. } \\
\text { Trotman-Dickenson, J. } \\
\text { Chem. Soc. 3584 (1964) }\end{array}$ \\
\hline
\end{tabular}

Preferred:

$\log k=15.0-65.2 / \theta$.

Comments: Arrhenius parameters are suspect. Rate constants are probably reliable. (See hydrazine decomposition and section $\mathrm{V}-8.0$.)

\section{Experimental}

[1] Aniline carrier technique. Rate constants were based on the $\mathrm{CH}_{4}$ formation (see methylaniline).

*The group values in the aryl amines do not look right. A better value for the reactant heat of formation is probably $\Delta H_{f}^{\circ}\left(\emptyset \mathrm{N}\left(\mathrm{CH}_{3}\right)_{2}\right.$ $=18.8 \mathrm{kcal} / \mathrm{mole}$. This would give $\Delta H_{f}^{\circ}\left(\emptyset \dot{\mathrm{N} C \mathrm{CH}_{3}}\right) \simeq 49.6 \mathrm{kcal} / \mathrm{mole}$. 


\begin{tabular}{cccc}
\multicolumn{4}{r}{$\mathrm{CH}_{3} \mathrm{~N}=\mathrm{NCH}_{3}(\mathrm{I}) \longrightarrow \mathrm{CH}_{3} \cdot(\mathrm{II})+\cdot \mathrm{N}=\mathrm{NCH}_{3}(\mathrm{III})$} \\
$\Delta H_{f(298)}^{\circ}$ & I & II & III \\
$S_{(298)}^{\circ}$ & 43.8 & 34.0 & $*[50.9]$ \\
$C_{p(298)}^{\circ}$ & 71.0 & 46.4 & 65.0 \\
$C_{p(800)}^{\circ}$ & 20.1 & 8.3 & 13.6
\end{tabular}

$$
\begin{aligned}
\Delta S^{\circ} & =39.6 \mathrm{~g} / \mathrm{mole} \\
\Delta H^{\circ} & =(52.5)^{*} \mathrm{kcal} / \mathrm{mole} \\
\Delta C_{p}^{\circ} & =1.9 \mathrm{~g} / \mathrm{mole} \\
\Delta C_{p}^{\circ} & =-0.9 \mathrm{~g} / \mathrm{mole} \\
\left\langle\Delta C_{p}^{\circ} \simeq 0.5\right\rangle &
\end{aligned}
$$

\begin{tabular}{|c|c|c|c|c|c|}
\hline $\log A$ & $\boldsymbol{E}$ & $\begin{array}{c}\log \boldsymbol{k}_{T} \\
(575)\end{array}$ & Conditions & System & Surface \\
\hline 16.5 & 52.5 & -3.45 & $\begin{array}{l}552-600{ }^{\circ} \mathrm{K} \\
36-435 \mathrm{~mm} \mathrm{Hg}\end{array}$ & static & none \\
\hline 14.2 & 46 & -3.28 & $\begin{array}{l}663-723^{\circ} \mathrm{K} \\
\left(P_{T} \simeq 15 \mathrm{~mm}\right. \\
\mathrm{Hg})\end{array}$ & flow & \\
\hline
\end{tabular}

\section{References}

$$
\begin{array}{cl}
51.2-3.76 & 502-594{ }^{\circ} \mathrm{K} \\
& 0.2-800 \mathrm{~mm} \mathrm{Hg} \\
& (130-170 \mathrm{~mm} \\
& \text { propylene })
\end{array}
$$

static

[3] C. Steel and A. F. Trotman-Dickenson, J. Chem. Soc., 975 (1959).
(16.5)
(53.3) $\quad-3.76$
shock

H. C. Ramsperger, J. Am. Chem. Soc. 49, 912 (1927).

[2] M. Page, H.O. Pritchard, and A. F. TrotmanDickenson, J. Chem. Soc., 3878 (1953).

$$
\begin{array}{llll}
17.3 & 55.4 & -3.76 & 523-573^{\circ} \mathrm{K} \\
& & & 164 \mathrm{~mm} \mathrm{Hg}
\end{array}
$$

[5] W. Forst and O. K. Rice, Can. J. Chem. 41, 562 (1963).
$\begin{array}{llll}14.5 & 49.4 & -4.28 & 1.06 \mathrm{~mm} \mathrm{Hg}\end{array}$ (pressure dependence)
$15.9-50.2 \quad-2.70 \quad 563-583^{\circ} \mathrm{K} \quad$ static $1.2-120 \mathrm{~mm} \mathrm{Hg}$ (100)

[6] O. K. Rice and D. V. Sickman, J. Chem. Phys. 4, 242 (1936).

(Continued)

\footnotetext{
${ }^{*}$ See sections $\mathrm{V}-7.0$ and $\mathrm{V}-4.1-\mathrm{c}$.
} 
(590)

\section{5}

$$
52.5
$$

static

(100-140 mm

$\mathrm{Hg})$
[7] H. A. Taylor and F. P. Jahn, J. Chem. Phys. 7, 470 (1939).

\section{Preferred:}

$\log k=16.5-52.5 / \theta$

$k_{\text {rec }}=10^{9.4} 1 /$ mole-sec.

\section{Experimental}

[1] Rates were obtained from pressure-time measurements. Products were found to be principally $\mathrm{C}_{2} \mathrm{H}_{6}$ and $\mathrm{N}_{2}$ with small yields of $\mathrm{CH}_{4}, \mathrm{C}_{2} \mathrm{H}_{4}$, and $\mathrm{H}_{2}$ also obtained.

[2] Toluene carrier technique. Rates based on $\mathrm{N}_{2}$ formation.

[3] Rate by $\mathrm{N}_{2}$ Production. High-pressure reaction indicated the presence of short chains. Propylene increased the rate at low pressures (due to pressure dependence of rate constant), but inhibited the reaction at high pressures by stopping chains.

[4] Rates based on $\mathrm{N}_{2}$ formation. Points scattered, but fell in the middle of the extrapolated Arrhenius plots of [3] and [5].

[5] Rates obtained from $\mathrm{N}_{2}$ formation. Inhibition by NO, ethylene, and propylene indicated the importance of short chains in the pure azomethane decomposition. Pressure dependence of the rate constant below $20 \mathrm{~mm} \mathrm{Hg}$ was also demonstrated.

[6] Rate constants were obtained from the pressure-time curves with an assumed stoichiometry of 1-2. Reported parameters are the extrapolated values for $P$ since the reaction was found to be in the falloff over the entire pressure range.

[7] Rates were followed manometrically with $P_{f} / P_{o}=2.15$. 
Reaction: Hexafluoroazomethane (perfluoroazomethane)

$$
\begin{aligned}
& \mathrm{CF}_{3} \mathrm{~N}=\mathrm{NCF}_{3}(\mathrm{I}) \stackrel{a}{\stackrel{b}{\longrightarrow}} \mathrm{CF}_{3} \cdot(\mathrm{III})+\mathrm{CF}_{3} \mathrm{~N}=\mathrm{N} \cdot(\mathrm{IV}) \\
& \begin{array}{cclcc} 
& \text { I } & \text { II } & \text { III } & \text { IV } \\
\Delta H_{f(298)}^{\circ} & {[-245.6]} & 0 & -112.5 & {[-77.1]}
\end{array} \\
& \begin{array}{lllll}
S_{(298)}^{\circ} & 95.2 & 45.8 & 62.4 & 77.1
\end{array} \\
& \begin{array}{lllll}
C_{p(298)}^{\circ} & 33.1 & 7.0 & 12.2 & 20.1
\end{array}
\end{aligned}
$$

\begin{tabular}{|c|c|c|c|c|c|c|c|}
\hline Path & $\log A$ & $\boldsymbol{E}$ & $\begin{array}{c}\log \boldsymbol{k}_{T} \\
(605)\end{array}$ & Conditions & System & Surface & References \\
\hline$a$ & 16.2 & 55.2 & -3.74 & $\begin{array}{l}572-634^{\circ} \mathrm{K} \\
0.3-73 \mathrm{~mm} \mathrm{Hg} \\
\quad(63 \mathrm{~mm} \mathrm{Hg})\end{array}$ & static & none & $\begin{array}{l}\text { [1] E. Leventhal, } \\
\text { C. R. Simonds, } \\
\text { and C. Steel, Can. } \\
\text { J. Chem. 40, } 930 \\
\text { (1962). }\end{array}$ \\
\hline$b$ & 13.9 & 48.5 & -3.62 & $\begin{array}{c}690-769^{\circ} \mathrm{K} \\
1 \% \text { of } P_{T}=13 \\
\mathrm{~mm} \mathrm{Hg}\end{array}$ & flow & & $\begin{array}{l}\text { [2] D. Clark and H. O. } \\
\text { Pritchard, J. } \\
\text { Chem. Soc. } 2136 \\
\text { (1956). }\end{array}$ \\
\hline
\end{tabular}

$$
\begin{aligned}
a & \\
\Delta S^{\circ} & =44.3 \mathrm{~g} / \mathrm{mole} \\
\Delta H^{\circ} & =56.0 \mathrm{kcal} / \mathrm{mole} \\
\Delta C_{p}^{\circ} & =-0.8 \mathrm{~g} / \mathrm{mole}
\end{aligned}
$$

Preferred:

$\log k_{a}=16.2-55.2 / \theta$.

Comments: In view of the large uncertainties associated with the estimated thermodynamics, the agreement with the observed kinetics is quite satisfactory. Clearly path $a$ is the principal reaction pathway. $k_{\text {rec }}=10^{8.6}$ l/mole-sec.

\section{Experimental}

[1] Rate by $\mathrm{N}_{2}$ formation. The rate constants were found to be pressure dependent below $60 \mathrm{~mm} \mathrm{Hg}$. The only noncondensable product formed was $\mathrm{N}_{2}$; the $\mathrm{CF}_{3}$. evidently added to the reactant.

[2] Toluene carrier technique. Rates were based on the $\mathrm{N}_{2}$ formation. The low Arrhenius parameters of [2] may in part be attributed to the pressure dependence of the decomposition below $60 \mathrm{~mm} \mathrm{Hg}$. 
Reaction: Azoethane

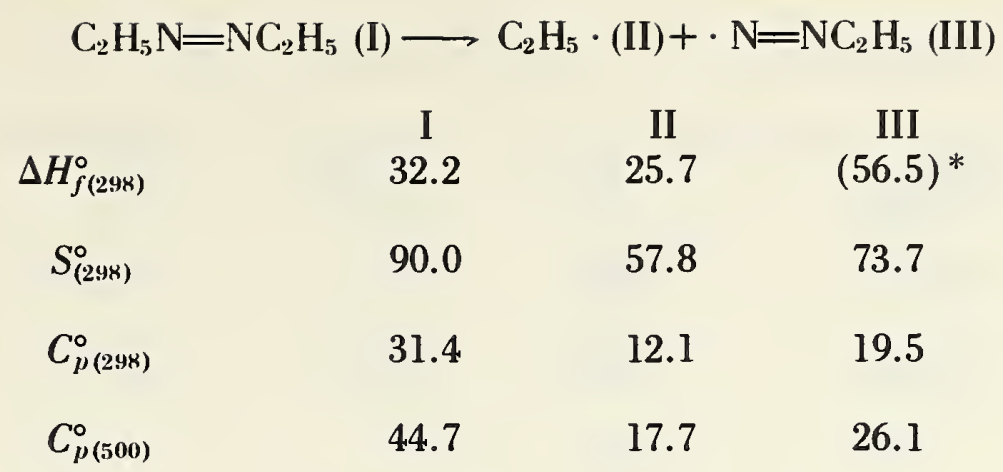

$$
\begin{aligned}
& \Delta S^{\circ}=41.5 \mathrm{~g} / \mathrm{mole} \\
& \Delta H^{\circ}=50.0^{*} \mathrm{kcal} / \mathrm{mole} \\
& \Delta C_{p}^{\circ}=0.2 \mathrm{~g} / \mathrm{mole} \\
& \Delta C_{p}^{\circ}=-0.9 \mathrm{~g} / \mathrm{mole}
\end{aligned}
$$

\section{$\begin{array}{ccccc}\log \boldsymbol{A} & \boldsymbol{E} & \begin{array}{c}\log \boldsymbol{k}_{T} \\ (575)\end{array} & \text { Conditions } & \text { Sys } \\ 16.0 & 51.2 & -3.46 & 551-600^{\circ} \mathrm{K} & \text { flow }\end{array}$ 0.3-708 mm Hg}

$\begin{array}{llll}15.7 & 48.5 & -2.73 & 533-583\end{array}{ }^{\circ} \mathrm{K} \quad$ static
System Surface References

[1] H. C. Ramsperger, J. Am. Chem. Soc. 49, 912 (1927).

[2] W. D. Clark, Ph. D. thesis, University of Oregon (1958).

\section{Preferred:}

$\log k=16.3-50.0 / \theta$.

Comments: $k_{\text {rec }}=10^{9.0} \mathrm{l} /$ mole-sec using the preferred $A$-factor and reaction entropy. (See section $\mathrm{V}-7.0$ and azomethane.)

\section{Experimental}

[1] Rates were obtained from pressure measurements.

[2] Mass spectrometric study particularly concerned with the pressure falloff of the rate constants. 
Reaction: Azobutane

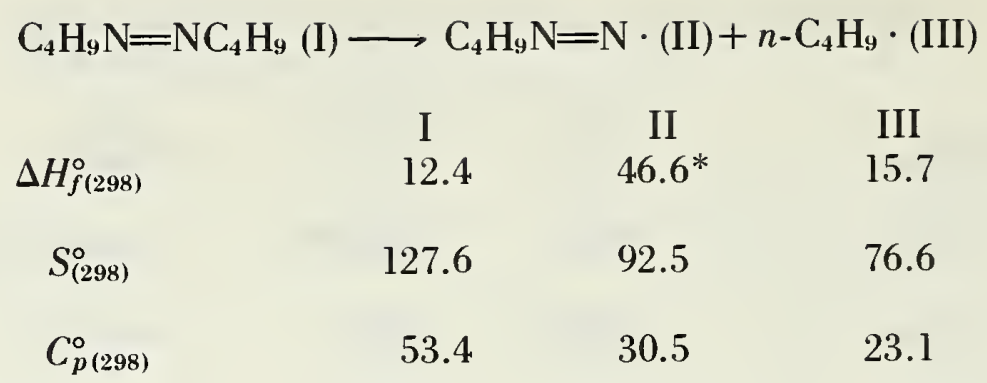

$\Delta S^{\circ}=41.5 \mathrm{~g} / \mathrm{mole}$

$\Delta H^{\circ}=*(49.9) \mathrm{kcal} / \mathrm{mole}$

$\Delta C_{p}^{\circ}=0.2 \mathrm{~g} / \mathrm{mole}$

\begin{tabular}{|c|c|c|c|c|c|c|}
\hline $\log A$ & $\boldsymbol{E}$ & $\begin{array}{c}\log \boldsymbol{k}_{T} \\
(565)\end{array}$ & Conditions & System & Surface & References \\
\hline 17.71 & 53.2 & -2.87 & $\begin{array}{l}473-673^{\circ} \mathrm{K} \\
0.9 \% \text { in } \mathrm{He}\end{array}$ & flow & & $\begin{array}{l}\text { [1] A. U. Blackham and } \\
\text { N. L. Eatough, } \\
\text { J. Am. Chem. Soc. 84, } \\
2922 \text { (1962). }\end{array}$ \\
\hline
\end{tabular}

Preferred:

$\log k=16.5-50.0 / \theta$.

Comments: From the preferred $A$-factor and reaction entropy one obtains $k_{\text {rec }}=10_{-0.3}^{9.2+0.2} 1 / \mathrm{mole}$-sec.

\section{Experimental}

[1] Both $\mathrm{He}$ and $\mathrm{H}_{2}$ carriers were employed. $\left(E_{\text {act }}\right.$ in $\mathrm{He}$ are reported here.) Rates were based on $\mathrm{N}_{2}$ formation. Analysis of the gaseous products was made by G.L.C. Products in decreasing order of importance were $\mathrm{C}_{2} \mathrm{H}_{4}, \mathrm{C}_{4} \mathrm{H}_{10}$, $\mathrm{C}_{3} \mathrm{H}_{8}, \mathrm{C}_{2} \mathrm{H}_{6}$, and $\mathrm{CH}_{4}$.

*See section $V-7.0$. 
Reaction: $1,1^{\prime}$-Azoisobutane

$\mathrm{CH}_{3} \mathrm{CH}\left(\mathrm{CH}_{3}\right) \mathrm{CH}_{2} \mathrm{~N}=\mathrm{NCH}_{2} \mathrm{CHCH}\left(\mathrm{CH}_{3}\right)_{2}(\mathrm{I}) \longrightarrow\left(\mathrm{CH}_{3}\right)_{2} \mathrm{CHCH}_{2} \mathrm{~N}=\mathrm{N} \cdot(\mathrm{II})+\left(\mathrm{CH}_{3}\right)_{2} \mathrm{CHCH}_{2}$ (III)

$\begin{array}{cccc}\Delta H_{f(298)}^{\circ} & \text { I } & \text { II } & \text { III } \\ S_{(298)}^{\circ} & 9.8 & 45.3^{*} & 13.7 \\ C_{p(298)}^{\circ} & 122.2 & 89.8 & 73.9 \\ & 52.8 & 30.2 & 22.9\end{array}$

$\Delta S^{\circ}=41.5 \mathrm{~g} / \mathrm{mole}$

$\Delta H^{\circ}=*(49.2) \mathrm{kcal} / \mathrm{mole}$

$\Delta C_{p}^{\circ}=0.3 \mathrm{~g} / \mathrm{mole}$

\begin{tabular}{|c|c|c|c|c|c|c|}
\hline $\log A$ & $\boldsymbol{E}$ & $\begin{array}{c}\log \boldsymbol{k}_{T} \\
(575)\end{array}$ & Conditions & System & Surface & References \\
\hline 16.23 & 49.0 & -2.39 & $\begin{array}{l}473-673^{\circ} \mathrm{K} \\
1-4 \% \text { in } \mathrm{He}\end{array}$ & flow & none & $\begin{array}{l}\text { [1] A. U. Blackham and } \\
\text { N. L. Eatough, } \\
\text { J. Am. Chem. Soc. 84, } \\
2922 \text { (1962). }\end{array}$ \\
\hline
\end{tabular}

Preferred:

$\log k=16.23-49.0 / \theta$.

Comments: This is consistent with a recombination rate constant of $k_{\text {rec }}=10^{9.0_{-0.3}^{+0.2}} \mathrm{~J} / \mathrm{mole}-\mathrm{sec}$ (i.e., using reaction entropy and reported $A$-factor).

\section{Experimental}

[1] Helium carrier. Rates were based on $\mathrm{N}_{2}$ formation. Products analyzed by G.L.C. (in decreasing order of importance) were $\mathrm{C}_{3} \mathrm{H}_{6}, \mathrm{~N}_{2}, \mathrm{C}_{2} \mathrm{H}_{6}, \mathrm{CH}_{4}, i-\mathrm{C}_{4} \mathrm{H}_{10}, i-\mathrm{C}_{4} \mathrm{H}_{8}$, and $\mathrm{C}_{3} \mathrm{H}_{8}$.

*See section V-7.0. 
Reaction: Azoisopropane

$\begin{array}{cccc}\left(\mathrm{CH}_{3}\right)_{2} \mathrm{CHN}=\mathrm{NCH}\left(\mathrm{CH}_{3}\right)_{2}(\mathrm{I}) \longrightarrow & \left(\mathrm{CH}_{3}\right)_{2} \mathrm{CH} \cdot(\mathrm{II})+\cdot \mathrm{N}=\mathrm{NCH}\left(\mathrm{CH}_{3}\right)_{2}(\mathrm{III}) \\ & \mathrm{I} & \begin{array}{c}\mathrm{II} \\ (V=4.0)\end{array} & \mathrm{III} \\ \Delta H_{f(298)}^{\circ} & 21.0 & 17.6 & (50.9)^{*} \\ S_{(298)}^{\circ} & 103.3 & 65.4 & 80.4 \\ C_{p(298)}^{\circ} & 41.9 & 17.7 & 24.7\end{array}$

$\Delta S^{\circ}=42.5 \mathrm{~g} / \mathrm{mole}$

$\Delta H^{\circ}=* 47.5 \mathrm{kcal} / \mathrm{mole}$

$\Delta C_{p}^{\circ}=0.5 \mathrm{~g} / \mathrm{mole}$

\begin{tabular}{|c|c|c|c|c|c|c|}
\hline $\log A$ & $\boldsymbol{E}$ & $\begin{array}{c}\log \boldsymbol{k}_{T} \\
(545)\end{array}$ & Conditions & System & Surface & References \\
\hline 13.75 & 40.9 & -2.66 & $\begin{array}{l}523-563^{\circ} \mathrm{K} \\
0.25-46 \mathrm{~mm} \mathrm{Hg}\end{array}$ & static & none & $\begin{array}{l}\text { [1] H. C. Ramsperger, } \\
\text { Proc. Nat. Acad. Sci. 13, } \\
849 \text { (1927); J. Am. Chem. } \\
\text { Soc. 50, 714 (1928). }\end{array}$ \\
\hline
\end{tabular}

\section{Preferred:}

$\log k=16.4-47.5 / \theta$.

Comments: From the preferred $A$-factor and reaction entropy, one obtains $k_{\text {rec }}=10^{8.5 \pm 0.3}$. $1 / \mathrm{mole}$-sec (see sections $\mathrm{V}-4.1-\mathrm{c}$ and $\mathrm{V}-7.0$ ).

\section{Experimental}

[1] Rates were obtained from pressure measurements. Slight falloff in the rate constants was observed at the lower pressures. Reaction products were believed to be principally $n$-hexane and $N_{2}$ with smaller amounts of propylene and propane.

*See section $\mathrm{V}-7.0$. 
Reaction: 2,2'-Azobutane

$\begin{array}{rccc}\mathrm{CH}_{3} \mathrm{CH}_{2} \mathrm{CH}\left(\mathrm{CH}_{3}\right) \mathrm{N}=\mathrm{Nsec} \text {-butyl }(\mathrm{I}) \longrightarrow \mathrm{CH}_{3} \mathrm{CH}_{2} \mathrm{CH}\left(\mathrm{CH}_{3}\right) \mathrm{N}=\mathrm{N} \cdot(\mathrm{II})+\mathrm{CH}_{3} \mathrm{CH}_{2} \dot{\mathrm{C}} \mathrm{HCH}_{3}(\mathrm{III}) \\ \Delta H_{f(298)}^{\circ} & \text { I } & \text { II } & \text { III } \\ S_{(298)}^{\circ} & 12.6 & * 46.7 & 12.6 \\ C_{p(298)}^{\circ} & 52.7 & 30.2 & 23.2\end{array}$

$\Delta S^{\circ}=43.9 \mathrm{~g} /$ mole

$\Delta H^{\circ}=*(46.7) \mathrm{kcal} / \mathrm{mole}$

$\Delta C_{p}^{\circ}=0.5 \mathrm{~g} / \mathrm{mole}$

\begin{tabular}{|c|c|c|c|c|c|c|}
\hline $\log A$ & $\boldsymbol{E}$ & $\begin{array}{c}\log k_{T} \\
(580)\end{array}$ & Conditions & System & Surface & References \\
\hline 17.28 & 48.4 & -0.96 & $\begin{array}{l}539-618^{\circ} \mathrm{K} \\
(4.1 \% \text { in } \mathrm{He})\end{array}$ & flow & & $\begin{array}{l}\text { [1] A. U. Blackham and } \\
\text { N. L. Eatough, J. Am. } \\
\text { Chem. Soc. 84, } 2922 \\
\text { (1962). }\end{array}$ \\
\hline
\end{tabular}

Preferred:

$\log k=16.6-46.7 / \theta$.

Comments: From the reaction entropy and the preferred $A$-factor one obtains $k_{\text {rec }} \simeq 10^{8.4} 1 /$ mole-sec.

\section{Experimental}

[1] Rates based on $\mathrm{N}_{2}$ formation. Products by G.L.C. analysis were found to be (in decreasing order of importance) $\mathrm{C}_{3} \mathrm{H}_{6}, \mathrm{~N}_{2}, n-\mathrm{C}_{4} \mathrm{H}_{10}, \mathrm{C}_{2} \mathrm{H}_{6}, 1-\mathrm{C}_{4} \mathrm{H}_{8}, 2 \cdot \mathrm{C}_{4} \mathrm{H}_{8}$, and $\mathrm{CH}_{4}$.

* See section V-7.0. 
Reaction: Methyl isopropyl diimide

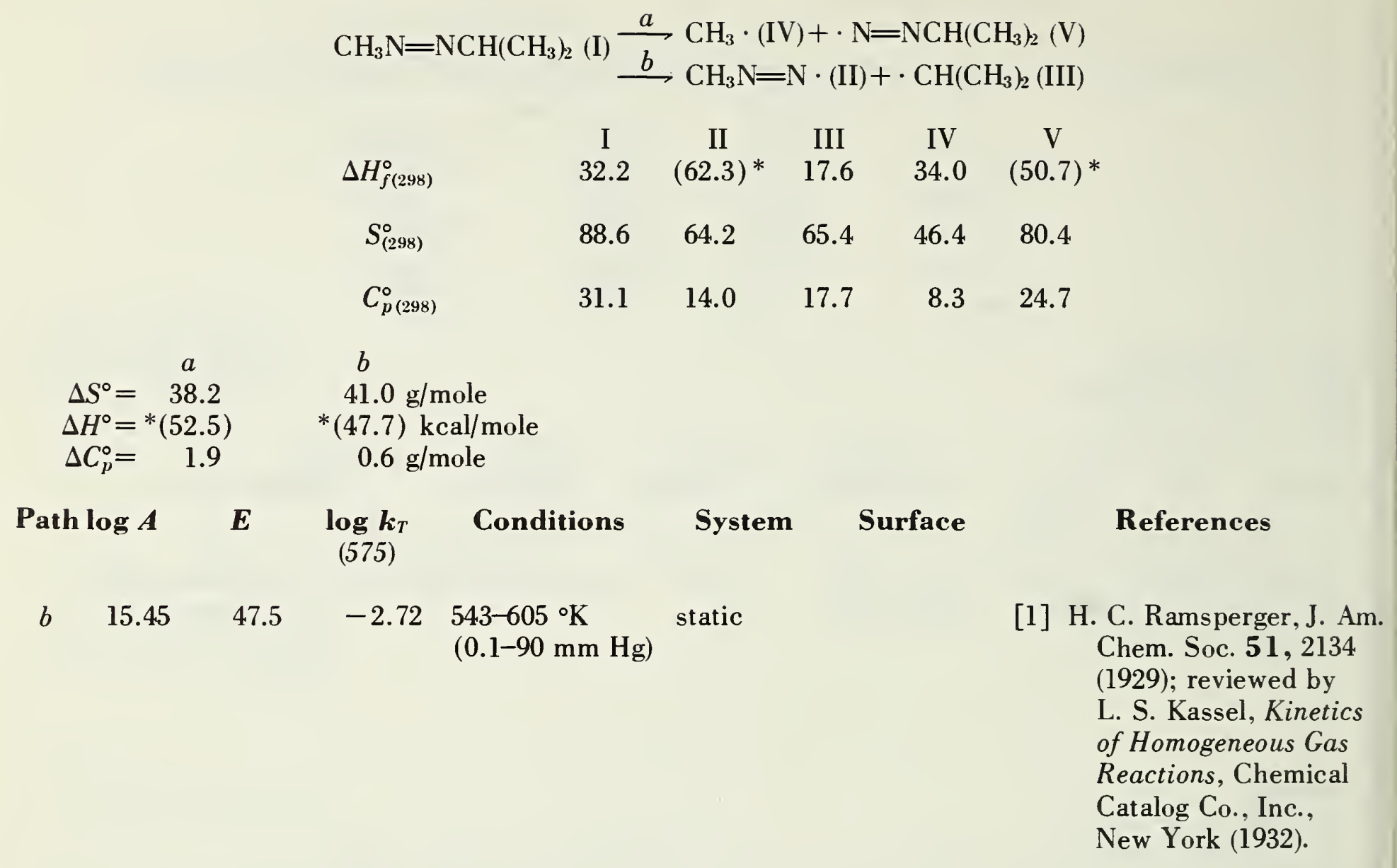

Preferred:

Suspect. The rate constant looks too low. We feel parameters should be like azoisopropane and prefer $\log k=16.4-47.5 / \theta$.

Comments: Path $b$ is favored by almost $5 \mathrm{kcal} / \mathrm{mole}$ in $E$ and about $3 \mathrm{~g} / \mathrm{mole}$ in the activation entropy. Reaction $a$ should therefore not compete.

\section{Experimental}

[1] Rates were followed manometrically. Falloff was observed over the entire pressure range and $k \infty$ values were obtained by extrapolation of plots of $1 / k$ versus $1 / p$.

*See section V-7.0. 
Reaction: 2,2'-Azoisobutane

\begin{tabular}{cccc}
$\left(\mathrm{CH}_{3}\right)_{3} \mathrm{CN}=\mathrm{N} t \mathrm{Bu}(\mathrm{I}) \longrightarrow$ & \multicolumn{3}{c}{$\mathrm{BuN}=\mathrm{N} \cdot(\mathrm{II})+\left(\mathrm{CH}_{3}\right)_{3} \mathrm{C} \cdot(\mathrm{III})$} \\
& $\mathrm{I}$ & $\mathrm{II}$ & $\mathrm{III}$ \\
$(V=4.0)$ \\
$H_{f(298)}^{\circ}$ & 7.4 & $(44.1)^{*}$ & 6.8 \\
$S_{(298)}^{\circ}$ & 109.3 & 83.4 & 70.3 \\
$C_{p(298)}^{\circ}$ & 53.9 & 30.8 & 22.6
\end{tabular}

$\Delta S^{\circ}=44.4 \mathrm{~g} / \mathrm{mole}$

$\Delta H^{\circ}=*(43.5) \mathrm{kcal} / \mathrm{mole}$

$\Delta C_{p}^{\circ}=-0.5 \mathrm{~g} / \mathrm{mole}$

\begin{tabular}{|c|c|c|c|c|c|c|}
\hline $\log A$ & $\boldsymbol{E}$ & $\begin{array}{c}\log \boldsymbol{k}_{T} \\
(575)\end{array}$ & Conditions & System & Surface & References \\
\hline 16.34 & 42.8 & +0.07 & $\begin{array}{l}453-4.93{ }^{\circ} \mathrm{K} \\
22 \mathrm{~mm} \mathrm{Hg}\end{array}$ & static & none & $\begin{array}{l}\text { [1] J. B. Levy and B. K. W. } \\
\text { Copeland, J. Am. } \\
\text { Chem. Soc. 82, } \\
\text { 5314 (1960). }\end{array}$ \\
\hline 17.15 & 43.0 & +0.81 & $\begin{array}{l}473-673^{\circ} \mathrm{K} \\
1-4 \% \text { in } \mathrm{He}\end{array}$ & flow & & $\begin{array}{l}\text { [2] A. U. Blackham and } \\
\text { N. L. Eatough, } \\
\text { J. Am. Chem. Soc. 84, } \\
2922(1962) .\end{array}$ \\
\hline
\end{tabular}

\section{Preferred:}

$\log k=17.15-43.5 / \theta$.

Comments: There is an appreciable discrepancy between the rates of the studies. We prefer the most recent values. From the $A$-factor and the reaction entropy, one obtains $k_{\text {rec }} \simeq 10^{8.9} \mathrm{l} / \mathrm{mole}$-sec.

\section{Experimental}

[1] Rates were obtained manometrically. M.S. analysis showed that $\mathrm{N}_{2}$ and $i-\mathrm{C}_{4} \mathrm{H}_{10}$ were the main products (i.e., $\simeq 90$ percent). The effect of $\mathrm{NO}$ and isobutene on the reaction was investigated.

[2] Rates were based on $\mathrm{N}_{2}$ production. Products by G.L.C. analysis (in decreasing order of importance) were $\mathrm{N}_{2}, i \cdot \mathrm{C}_{4} \mathrm{H}_{8}$, and $i \cdot \mathrm{C}_{4} \mathrm{H}_{10}$.

*See section V-7.0. 
Reaction: Azotoluene

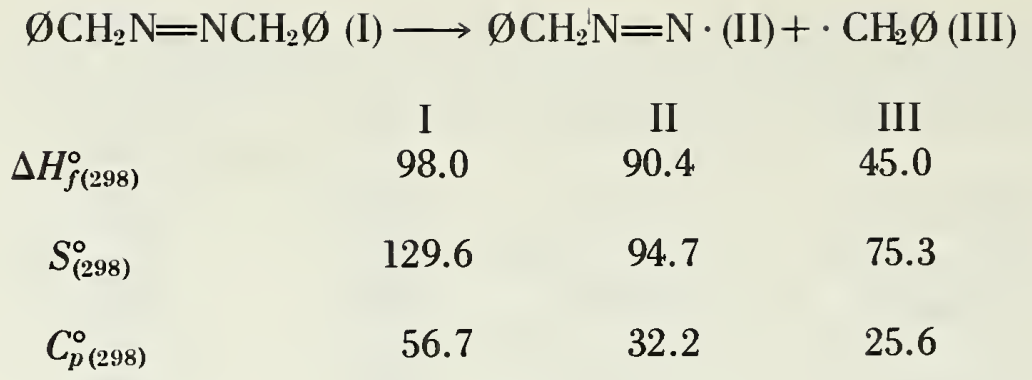

$\begin{aligned} \Delta S^{\circ} & =40.4 \mathrm{~g} / \mathrm{mole} \\ \Delta H^{\circ} & =37.4 \mathrm{kcal} / \mathrm{mole} \\ \Delta C_{p}^{\circ} & =1.1 \mathrm{~g} / \mathrm{mole}\end{aligned}$

\begin{tabular}{|c|c|c|c|c|c|c|}
\hline $\log A$ & $\boldsymbol{E}$ & $\begin{array}{c}\log k_{T} \\
(485)\end{array}$ & Conditions & System & Surface & References \\
\hline 14.1 & 35.0 & -1.67 & $\begin{array}{l}455-511^{\circ} \mathrm{K} \\
2.5-11 \mathrm{~mm} \mathrm{Hg}\end{array}$ & static & none & $\begin{array}{l}\text { [1] G. Williams and A. S. C. } \\
\text { Lawrence, Proc. Roy. } \\
\text { Soc. (L) A156, } 455 \\
\text { (1936). }\end{array}$ \\
\hline
\end{tabular}

Preferred:

$\log k=15.3-37.6 / \theta ; k_{\text {rec }}=10^{7.7} \mathrm{l} / \mathrm{mole} \cdot \mathrm{sec}$.

Comments: Heats of formation have been obtained from additivity values and are based on azomethane and the $\left(\mathrm{CH}_{3} \mathrm{~N}=\mathrm{N} \cdot\right)$ radical. Scaled parameters look reasonable and are therefore preferred; however, uncertainties do exist in the estimates (see V-7.0).

\section{Experimental}

[1] Rates were followed manometrically. The formation of $\mathrm{N}_{2}$ and dibenzyl was not quantitative $\left(P_{f} / P_{o}\right)=1.77$. Products were not analyzed. 


\section{Reaction: Tetramethyltetrazine}

$$
\begin{aligned}
& \left(\mathrm{CH}_{3}\right)_{2} \mathrm{NN}=\mathrm{NN}\left(\mathrm{CH}_{3}\right)_{2} \text { (I) } \stackrel{a}{b} 2\left[\left(\mathrm{CH}_{3}\right)_{2} \mathrm{~N} \cdot\right] \text { (II) }+\mathrm{N}_{2}(\mathrm{IV}) \\
& \stackrel{b}{\longrightarrow}\left(\mathrm{CH}_{3}\right)_{2} \mathrm{NN}=\mathrm{N} \cdot(\mathrm{III})+\cdot \mathrm{N}\left(\mathrm{CH}_{3}\right)_{2}
\end{aligned}
$$

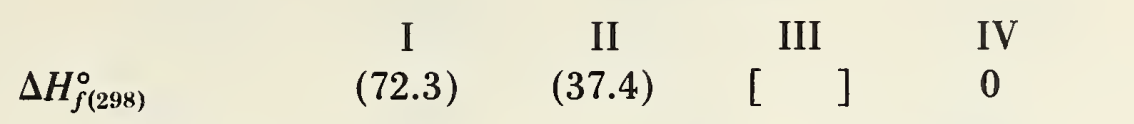

$\begin{array}{lllll}S_{(298)}^{\circ} & 103.2 & 65.4 & (77.0) & 45.8\end{array}$

$\begin{array}{lllll}C_{p(298)}^{\circ} & 39.9 & 16.1 & 24.3 & 7.0\end{array}$

$\begin{array}{ccl}a & b & \\ \Delta S^{\circ}=73.4 & 39.2 \mathrm{~g} / \mathrm{mole} \\ \Delta H^{\circ}=(2.5) & (\quad) \mathrm{kcal} / \mathrm{mole} \\ \Delta C_{p}^{\circ}=-0.7 & 0.5 \mathrm{~g} / \mathrm{mole}\end{array}$

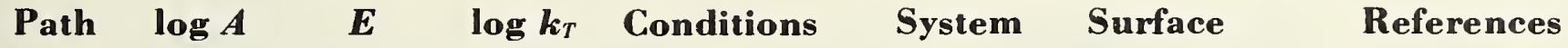
(450)

$11.4 \quad 31.9 \pm 3 \quad-4.10 \quad 399-421^{\circ} \mathrm{K} \quad$ static

[1] J. S. Watson and A. J. Waring, Can. J. Chem. 38, 298 (1960).

none

[2] B. G. Gowenlock and 6-12 $\mathrm{mm} \mathrm{Hg}$

P. P. Jones, Can.

J. Chem. 41, 1911 (1963); Chem. and Industry, 557 (1960).

Preferred:

$\log k_{b}=14.4-36.1 / \theta$.

Comments: The $A$-factor seems low by about a factor of 10 , since from the observed $A$-factor and reaction entropy one obtains $k_{\text {rec }} \simeq 10^{7.4} \mathrm{l} /$ mole-sec.

\section{Experimental}

[1] Rates were based on $\mathrm{N}_{2}$ formation.

[2] $\mathrm{SF}_{6}-\mathrm{CO}_{2}$ and NO- $\mathrm{CO}_{2}$ carriers were employed. Rates were based on the amount of $\mathrm{N}_{2}$ produced and upon G.L.C. analysis of the liquid products. Principal products were $\mathrm{N}_{2}$ and tetramethylhydrazine (in the $\mathrm{SF}_{6}-\mathrm{CO}_{2}$ carrier) and dimethylnitrosoamine (in the $\mathrm{NO} \cdot \mathrm{CO}_{2}$ carrier).

*Multiple bond rupture, path $a$, is not likely and path $b$ is preferred. (See section V-7.0.) 
Reaction: Tetraethyltetrazine

$\begin{array}{cccc}\left(\mathrm{C}_{2} \mathrm{H}_{5}\right)_{2} \mathrm{NN}=\mathrm{NN}\left(\mathrm{C}_{2} \mathrm{H}_{5}\right)_{2}(\mathrm{I}) \longrightarrow\left(\mathrm{C}_{2} \mathrm{H}_{5}\right)_{2} \mathrm{~N} \cdot(\mathrm{II})+\cdot \mathrm{N}=\mathrm{NN}\left(\mathrm{C}_{2} \mathrm{H}_{5}\right)_{2}(\mathrm{III}) \\ \Delta H_{f(298)}^{\circ} & \begin{array}{c}\mathrm{I} \\ \text { (II }\end{array} & \begin{array}{c}\text { II } \\ \text { III }\end{array} \\ S_{(298)}^{\circ} & 141 & 84.2 & 96.0 \\ C_{p(298)}^{\circ} & 62 & 27.2) & 35.3\end{array}$

$\Delta S^{\circ}=39.2 \mathrm{~g} / \mathrm{mole}$

$\Delta H^{\circ}=$

$\Delta C_{p}^{\circ}=0.4 \mathrm{~g} / \mathrm{mole}$

\begin{tabular}{ccccccc}
$\log \boldsymbol{A}$ & $\boldsymbol{E}$ & $\begin{array}{c}\log \boldsymbol{k}_{T} \\
(490)\end{array}$ & Conditions & System & Surface & \multicolumn{1}{c}{ References } \\
13.7 & 33.0 & -1.02 & $\begin{array}{l}4.71-508^{\circ} \mathrm{K} \\
9-29 \mathrm{~mm} \mathrm{Hg}\end{array}$ & flow & none & [1] B. G. Gowenlock, \\
& & & & & $\begin{array}{l}\text { P. P. Jones, } \\
\text { and D. R. Snelling, } \\
\text { Can. J. Chem. 41, }\end{array}$ \\
& & & & & 1911 (1963).
\end{tabular}

Preferred:

$\log k=14.4-34.5 / \theta$ (see below).

Comments: Tetramethyltetrazine should have a higher activation energy than tetraethyltetrazine by about 2 gauche repulsions (i.e., $\sim 1.6 \mathrm{kcal} / \mathrm{mole}$ ); therefore the two studies are in fair internal agreement. The $A$-factor of both reactions, however, seems low.

\section{Experimental}

[1] Rates were based on $\mathrm{N}_{2}$ formation. G.L.C., M.S., and spectroscopic analysis on the products were employed. Products included diethylamine, diethylmethylamine, ethylithlideneamine, tetraethylhydrazine and 1,3,5-triethylhexahydro-s-triazene. 


\begin{tabular}{|c|c|c|c|}
\hline \multirow[b]{2}{*}{$\Delta H_{f(298)}^{\circ}$} & \multicolumn{3}{|c|}{$\begin{array}{l}\stackrel{a}{\longrightarrow} \mathrm{CH}_{3} \cdot(\mathrm{II})+\cdot \mathrm{CH}_{2} \mathrm{CN} \\
\stackrel{b}{\longrightarrow} \mathrm{H}_{2}+\mathrm{CH}_{2}=\mathrm{CHCN} \\
\stackrel{c}{\longrightarrow} \mathrm{HCN}+\mathrm{C}_{2} \mathrm{H}_{4}\end{array}$} \\
\hline & $\begin{array}{c}I \\
12.7\end{array}$ & $\begin{array}{c}\text { II } \\
34.0\end{array}$ & $\begin{array}{c}\text { III } \\
{[51.1]}\end{array}$ \\
\hline$S_{(298)}^{\circ}$ & 68.4 & 46.5 & 58.5 \\
\hline$C_{p_{(298)}}^{\circ}$ & 17.3 & 8.8 & 10.4 \\
\hline$C_{p_{(1000)}}^{\circ}$ & 36.1 & 14.4 & 20.7 \\
\hline
\end{tabular}

$\begin{array}{ll}\Delta S^{\circ} & =36.6 \mathrm{~g} / \mathrm{mole} \\ \Delta H^{\circ} & =72.4 \mathrm{kcal} / \mathrm{mole} \\ \Delta C_{p}^{\circ} & =1.9 \mathrm{~g} / \mathrm{mole} \\ \Delta C_{p(1000)}^{\circ} & =-1.0 \mathrm{~g} / \mathrm{mole}\end{array}$

\section{$\begin{array}{lllllll}\text { Path } & \log A & \boldsymbol{E} & \log \boldsymbol{k}_{T} & \text { Conditions } & \text { System } & \text { Surface }\end{array}$}

$\begin{array}{llll}14.1 & 72.7 & 958-1038{ }^{\circ} \mathrm{K} \text { flow none } \\ & & 6.5-15.8 \mathrm{~mm} \mathrm{Hg}\end{array}$

[1] M. Hunt, J. A.

Kerr, and A. F.

Trotman-Dickenson, J. Chem. Soc. 5074 (1965)

(See molecular eliminations for discussion of paths $b$ and $c$.)

\section{Preferred:}

tentative, $\log k_{a}=14.1-72.7 / \theta$.

Comments: The kinetics give a resonance energy of about $12.5 \mathrm{kcal} / \mathrm{mole}$ for $\left(\cdot \mathrm{CH}_{2} \mathrm{CN}\right)$ and a recombination rate constant of $k_{\text {rec }}=10^{7.9} \mathrm{l} / \mathrm{mole}$-sec. The latter seems low and may be indicative of a pressure falloff. A calculation of the $M_{1 / 2}$ (the middle of the pressure falloff region) with $n=16$ and an assumed $A_{x}=10^{15.3}$ sec $^{-1}$ gives $M_{1 / 2} \simeq 5 \mathrm{~mm} \mathrm{Hg}$.

Note that if $\cdot \mathrm{CH}_{2} \mathrm{CN}$ resonance is of the order of $10 \mathrm{kcal}$, then $\emptyset \dot{\mathrm{N}} \mathrm{H}+\mathrm{E} t \mathrm{CN} \longrightarrow \emptyset \mathrm{NH}_{2}+\mathrm{CH}_{3} \dot{\mathrm{C}} \mathrm{HCN}$ is close to thermoneutral. Free radical chain decomposition of ethylcyanide would then be likely.

\section{Experimental}

[1] Aniline carrier technique. Path a: Rates were based on the methane formation. Path $b$ : Rates were based on the $\mathrm{H}_{2}$ production. Path $c$ : Rates were based on the $\mathrm{C}_{2} \mathrm{H}_{4}$ formation. Analysis was performed by standard gasometric techniques and checked by G.L.C. The mechanism assumed was,

$$
\begin{aligned}
& \mathrm{C}_{2} \mathrm{H}_{5} \mathrm{CN} \longrightarrow \mathrm{CH}_{3} \cdot+\cdot \mathrm{CH}_{2} \mathrm{CN} \\
& \mathrm{CH}_{3} \cdot+\emptyset \mathrm{NH}_{2} \longrightarrow \mathrm{CH}_{4}+\emptyset \dot{\mathrm{NH}} \\
& 2 \emptyset \mathrm{NH} \longrightarrow(\emptyset \mathrm{NH})_{2} \text { or polymer } \\
& \mathrm{C}_{2} \mathrm{H}_{5} \mathrm{CN} \longrightarrow \mathrm{H}_{2}+\mathrm{CH}_{2}=\mathrm{CHCN} \\
& \mathrm{C}_{2} \mathrm{H}_{5} \mathrm{CN} \longrightarrow \mathrm{C}_{2} \mathrm{H}_{4}+\mathrm{HCN}
\end{aligned}
$$


Reaction: 2,2-Dimethylpropanonitrile ( $t$-butyl cyanide)

\begin{tabular}{|c|c|c|c|}
\hline$\Delta H_{f(298)}^{\circ}$ & $\begin{array}{c}\text { I } \\
-2.0\end{array}$ & $\underset{34.0}{\text { II }}$ & $\begin{array}{c}\text { III } \\
{[33.8]}\end{array}$ \\
\hline$S_{(298)}^{\circ}$ & 76.6 & 46.5 & 74.0 \\
\hline$C_{p(298)}^{\circ}$ & 28.2 & 8.8 & 21.3 \\
\hline$C_{p(1000)}^{\circ}$ & 60.0 & 14.4 & 44.6 \\
\hline
\end{tabular}

$\Delta S^{\circ}=43.9 \mathrm{~g} / \mathrm{mole}$
$\Delta H^{\circ}=69.8 \mathrm{kcal} / \mathrm{mole}$
$\Delta C_{p}^{\circ}=1.9 \mathrm{~g} / \mathrm{mole}$
$\Delta C_{p(1000)}^{\circ}=-1.0 \mathrm{~g} / \mathrm{mole}$

$\log A \quad E \quad \log k$

(900)

Conditions

System

Surface

References

15.16

$70.2-1.89$

$875-925^{\circ} \mathrm{K} \quad$ flow

none

8.6-12.0 mm Hg

[1] M. Hunt, J. A. Kerr and A. F. TrotmanDickenson, J. Chem. Soc. 5074 (1965).

Preferred:

$\log k=15.16-70.2 / \theta$.

Comments: If the kinetics are taken as correct, the heat of formation of the radical in brackets is obtained. This is consistent with a resonance energy of $10.6 \mathrm{kcal} /$ mole. From the reaction entropy a recombination rate constant of $k_{\text {rec }}=10^{7.4} \mathrm{l} / \mathrm{mole}-\mathrm{sec}$ is obtained.

\section{Experimental}

[1] Aniline carrier technique. Rates were based on $\mathrm{CH}_{4}$ formation. Appreciable yields of $\mathrm{H}_{2}$ were tentatively ascribed to the decomposition of the cyano radical,<smiles>CC(C#N)=CNC(C)(C)C</smiles>

Otherwise the mechanism assumed was the usual free radical non-chain path to products (see ethanonitrile). 
Reaction: 2-Methyl-2-phenylpropanonitrile (cumyl cyanide)

\begin{tabular}{|c|c|c|c|}
\hline$\Delta H_{f(298)}^{\circ}$ & $\begin{array}{c}\text { I } \\
28.5\end{array}$ & $\begin{array}{c}\text { II } \\
{[54.4]}\end{array}$ & $\begin{array}{c}\text { III } \\
34.0\end{array}$ \\
\hline$S_{(298)}^{\circ}$ & 99.9 & 91.0 & 46.5 \\
\hline$C_{p(298)}^{\circ}$ & 43.1 & 35.0 & 8.8 \\
\hline$C_{p(1000)}^{\circ}$ & 93.8 & 78.6 & 14.4 \\
\hline
\end{tabular}

$$
\begin{array}{ll}
\Delta S^{\circ} & =37.6 \mathrm{~g} / \mathrm{mole} \\
\Delta H^{\circ} & =59.9 \mathrm{kcal} / \mathrm{mole} \\
\Delta C^{\circ} & =0.7 \mathrm{~g} / \mathrm{mole} \\
\Delta C^{\circ}{ }^{\circ}(1000) & =-0.8 \mathrm{~g} / \mathrm{mole}
\end{array}
$$

\begin{tabular}{|c|c|c|c|c|c|c|}
\hline $\log A$ & $\boldsymbol{E}$ & $\begin{array}{c}\log k_{r} \\
(845)\end{array}$ & Conditions & System & Surface & References \\
\hline 12.3 & 54.1 & -1.69 & $\begin{array}{l}794-897^{\circ} \mathrm{K} \\
5.3-13.7 \mathrm{~mm} \mathrm{Hg}\end{array}$ & flow & none & $\begin{array}{l}\text { [1] M. Hunt, J. A. Kerr } \\
\text { and A. F. Trotman- } \\
\text { Dickenson, J. Chem. } \\
\text { Soc. } 5074 \text { (1965). }\end{array}$ \\
\hline
\end{tabular}

Preferred:

$\log k=13.8-59.9 / \theta$.

Comments: Preferred parameters are based on the reaction entropy and a recombination rate constant of $k_{\text {rec }} \cong 10^{7.4} \mathrm{l} / \mathrm{mole}$-sec (i.e., this is the recombination rate constant found for the reverse of the $t$-butyl benzene reaction). The heat of formation of the cyano radical in brackets is then calculated and suggests a total resonance energy of $22.1 \mathrm{kcal} / \mathrm{mole}$. This is close to the sum of the benzyl and cyano resonance energies (i.e., $\simeq 13.5+10.6=24.1 \mathrm{kcal} / \mathrm{mole}$ ).

\section{Experimental}

[1] Aniline carrier technique. Rates were based on $\mathrm{CH}_{4}$ production. Since the $A$-factor was relatively low, the authors suggested that the $\mathrm{CH}_{4}$ formation could arise from a four-center molecular elimination reaction rather than the bond-breaking process indicated above.* Appreciable yields of hydrogen were again observed whose origin could not be determined.

*The proposed molecular elimination of methane is not considered here to be reasonable; it would involve pentavalent carbon in the transition state. 
Reaction: Nitritomethane (methyl nitrite)

\begin{tabular}{cccc}
\multicolumn{5}{c}{$\mathrm{CH}_{3} \mathrm{ONO}(\mathrm{I}) \longrightarrow \mathrm{CH}_{3} \mathrm{O} \cdot(\mathrm{II})+\mathrm{NO}(\mathrm{III})$} \\
$\Delta H_{f(298)}^{\circ}$ & $\mathrm{I}$ & $\mathrm{II}$ & $\mathrm{III}$ \\
$S_{(298)}^{\circ}$ & -15.6 & 3.9 & 21.6 \\
$C_{p(298)}^{\circ}$ & 72.3 & 54.6 & 50.3 \\
& 15.4 & 8.4 & 7.1
\end{tabular}

$\Delta S^{\circ}=32.6 \mathrm{~g} / \mathrm{mole}$

$\Delta H^{\circ}=41.1 \mathrm{kcal} / \mathrm{mole}$

$\Delta C_{p}^{\circ}=0.1 \mathrm{~g} / \mathrm{mole}$

\begin{tabular}{|c|c|c|c|c|c|c|}
\hline $\log A$ & $\boldsymbol{E}$ & $\begin{array}{c}\log \boldsymbol{k}_{T} \\
(485)\end{array}$ & Conditions & System & Surface & References \\
\hline 13.2 & 36.4 & -3.20 & $\begin{array}{c}463-513^{\circ} \mathrm{K} \\
5.2 \longrightarrow 51.4 \\
\mathrm{~mm} \mathrm{Hg}\end{array}$ & static & $<5 \%$ & $\begin{array}{l}\text { [1] E. W. R. Steacie and G. } \\
\text { T. Shaw, Proc. Roy. } \\
\text { Soc. A146, } 388 \text { (1934). }\end{array}$ \\
\hline 13.1 & 36.1 & -3.17 & $\begin{array}{l}483-513^{\circ} \mathrm{K} \\
0.05-50 \mathrm{~mm} \mathrm{Hg}\end{array}$ & static & & $\begin{array}{l}\text { [2] E. W. R. Steacie and D. S. } \\
\text { Calder, J. Chem. Phys. } \\
\text { 4, } 96 \text { (1936). }\end{array}$ \\
\hline$*(13.0)$ & $(36.6)$ & -3.49 & $453-513^{\circ} \mathrm{K}$ & static & (none) & $\begin{array}{l}\text { [3] L. Phillips, J. Chem. Soc., } \\
3082 \text { (1961). }\end{array}$ \\
\hline
\end{tabular}

\section{Preferred:}

$\log k=15.6-41.1 / \theta$

$\log k_{\exp }=13.0-36.6 / \theta$.** $^{*}$

Comments: The mechanism for nitrite (and nitrate) decompositions currently accepted, G. Baker and R. Shaw, J. Chem. Soc., 6965 (1965), relates the experimental first-order rate constant to the initial (N-O) split and to the recombination and disproportionation reactions of the alkoxy radical with $\mathrm{NO}\left(\right.$ and $\left.\mathrm{NO}_{2}\right)$. The mechanism proposed for nitrite:

$$
\begin{aligned}
& \mathrm{RONO} \stackrel{\frac{1}{2}}{\stackrel{2}{\leftrightarrows}} \mathrm{R} \cdot+\mathrm{NO} \\
& \mathrm{RO}++\mathrm{NO} \stackrel{3}{\longrightarrow} \mathrm{HNO}+\mathrm{R}_{-\mathrm{H}} \mathrm{O} \\
& 2 \mathrm{HNO} \stackrel{4}{\longrightarrow} \mathrm{H}_{2} \mathrm{O}+\mathrm{N}_{2} \mathrm{O} \\
& 2 \mathrm{RO} \cdot \stackrel{5}{\longrightarrow} \mathrm{ROH}+\mathrm{R}_{-\mathrm{H}} \mathrm{O}
\end{aligned}
$$

Steady state relations give, $k_{\exp }=\frac{k_{1} k_{3}}{k_{2}+k_{3}}$. Other studies (see Baker and Shaw for references) have obtained disproportionation to combination ratios $\left(\frac{k_{3}}{k_{2}}\right)$ for $\mathrm{NO}$ with $\mathrm{CH}_{3} \mathrm{O} \cdot(0.5)$, with $\mathrm{C}_{2} \mathrm{H}_{5} \mathrm{O} \cdot(0.3)$, and with $i \mathrm{PrO} \cdot(0.15)$. Therefore rate constants for the decompositions obtained by pressure measurements should be higher than those reported by about factors of 3-10. Although the activation energies for reactions

(Continued)

\footnotetext{
*Originally reported as $\log A=12.2, E=34.3$.

**A very plausible explanation for the "lower than predicted" experimental parameters in this and other nitrite and nitrate decom. positions is that some direct molecular elimination may also be taking place via a four-center transition state to $\mathrm{CH}_{2} \mathrm{O}+\mathrm{HNO}$.
} 


\section{Nitritomethane (Continued)}

2 and 3 are certainly low, they are not necessarily zero. The discrepancy in rates between disproportionation and recombination could arise from differences in activation energies as well as from differences in $A$-factors. Comparison of the observed activation energies to the reaction thermodynamics suggests that activation energy differences of $E_{2}-E_{3} \simeq 0-2 \mathrm{kcal} /$ mole are possible.

Rate constant analysis***: From the mechanism, $k_{1}=k_{\text {exp }}\left(\frac{k_{2}+k_{3}}{k_{3}}\right)$. We have assumed that $E_{2}-E_{3}=0$. Therefore, $A_{1}=A_{\text {exp }} \times\left(\frac{k_{2}+k_{3}}{k_{3}}\right)$. Since $E_{1}=\Delta H_{T}^{\circ}$, the preferred parameters have been obtained by scaling the "corrected" reported values to the reaction enthalpy. Thus for methylnitrite $\left(k_{\text {obs }}=10^{13.0-36.6 / \theta}\right)$ :

$$
\begin{aligned}
& A_{1(\exp )}=10^{13.0} \times 3=10^{13.48} . \\
& E_{1}=41.1 \mathrm{kcal} / \mathrm{mole}, \\
& A_{1(\text { corrected })}=10^{15.6} \mathrm{sec}^{-1} .
\end{aligned}
$$

therefore

From this follow the rate constants and preferred parameters given above. From $A$ and the reaction entropy one obtains $k_{-1(\mathrm{rec})}=10^{9.8} \mathrm{l} / \mathrm{mole-sec}$.

\section{Experimental}

[1] Rate by $\Delta \mathrm{P}$. Qualitative identification of $\mathrm{CH}_{2} \mathrm{O}$ and $\mathrm{CH}_{3} \mathrm{OH}$ as products.

[2] Rate by $\Delta P$. Most of the study was performed in an apparent falloff region.

[3] Analysis was by I.R. of the total product mixture. M.S. and Chem. were also made. Rate constants calculated from the pressure change and those calculated from methylnitrite disappearance were in good agreement. The mechanism proposed was:

$$
\begin{aligned}
& \mathrm{MeONO} \frac{1}{2} \mathrm{MeO} \cdot+\mathrm{NO} \\
& \mathrm{MeO}+\underset{\mathrm{NO}}{ } \stackrel{3}{\longrightarrow} \mathrm{CH}_{2} \mathrm{O}+\mathrm{HNO} \\
& 2 \mathrm{HNO} \stackrel{4}{\longrightarrow} \mathrm{H}_{2} \mathrm{O}+\mathrm{N}_{2} \mathrm{O} \\
& 2 \mathrm{MeO} \cdot \stackrel{5}{\longrightarrow} \mathrm{CH}_{2} \mathrm{O}+\mathrm{MeOH}
\end{aligned}
$$

The observed rate constant then was equated to, $k_{\mathrm{obs}}=\left(\frac{k_{1} k_{3}}{k_{2}+k_{3}}\right)$.

\footnotetext{
***Note added in proof: Preferred Arrhenius parameters on the data sheets for the higher alkyl nitrites and nitrates are slightly low, as they were originally estimated on the basis of different free radical heats of formation. On the other hand, the thermodynamics given on the data sheets represent our "best" thermochemical estimates at this time. Activation energies should therefore be higher by about $1 \mathrm{kcal} / \mathrm{mole}$, and $A$ - factors for dissociation and recombination should be higher by about $0.5 \mathrm{log}$ units.
} 
Reaction: Nitritoethane (ethyl nitrite)

\begin{tabular}{cccr}
\multicolumn{4}{c}{$\mathrm{C}_{2} \mathrm{H}_{5} \mathrm{ONO}(\mathrm{I}) \longrightarrow \mathrm{C}_{2} \mathrm{H}_{5} \mathrm{O} \cdot(\mathrm{II})+\mathrm{NO}$ (II) } \\
$\Delta H_{f(298)}^{\circ}$ & I & II & III \\
$S_{(298)}^{\circ}$ & -24.5 & -4.9 & 21.6 \\
$C_{p(298)}^{\circ}$ & 80.6 & 65.9 & 50.3 \\
& $(20.4)$ & 14.0 & 7.1
\end{tabular}

$\Delta S^{\circ}=35.6 \mathrm{~g} / \mathrm{mole}$

$\Delta H^{\circ}=41.2 \mathrm{kcal} / \mathrm{mole}$

$\Delta C_{p}^{\circ}=0.7 \mathrm{~g} / \mathrm{mole}$

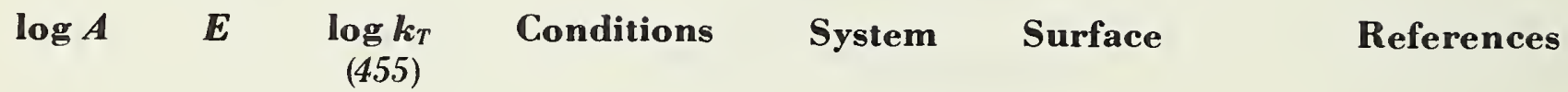

\begin{tabular}{|c|c|c|c|c|c|}
\hline 37.7 & -4.01 & $\begin{array}{l}484-505^{\circ} \mathrm{K} \\
10-210 \mathrm{~mm} \mathrm{Hg}\end{array}$ & static & none & $\begin{array}{l}\text { [1] E. W. R. Steacie and } \\
\text { G. T. Shaw, J. Chem. } \\
\text { Phys. 2, 345 (1934). }\end{array}$ \\
\hline 37.5 & -4.21 & $\begin{array}{l}434-474^{\circ} \mathrm{K} \\
10-50 \mathrm{~mm} \mathrm{Hg}\end{array}$ & static & none & $\begin{array}{l}\text { [2] J. B. Levy, J. Am. Chem. } \\
\text { Soc. 78, } 1780 \text { (1956). }\end{array}$ \\
\hline
\end{tabular}

Preferred:

$\log k=16.07-40.5 / \theta ; k_{\text {rec }}=10^{9.8} \mathrm{l} / \mathrm{mole}-\mathrm{sec}$

$\log k_{\text {exp }}=14.1-37.7 / \theta$ (see methyl nitrite).

\section{Experimental}

[1] Rates determined from pressure changes.

[2] Rates followed by I.R. analysis for disappearance of $\mathrm{C}_{2} \mathrm{H}_{5} \mathrm{ONO}$ and appearance of $\mathrm{CH}_{3} \mathrm{CHO}$ and $\mathrm{N}_{2} \mathrm{O}$. Observed that the rate of reaction in excess NO was equal to the initial rates for the pure nitrite decomposition. Mechanism proposed was:

$$
\begin{aligned}
& \mathrm{C}_{2} \mathrm{H}_{5} \mathrm{ONO} \stackrel{\frac{1}{-1}}{=} \mathrm{C}_{2} \mathrm{H}_{5} \mathrm{O} \cdot+\mathrm{NO} \\
& \mathrm{C}_{2} \mathrm{H}_{5} \mathrm{O} \cdot+\mathrm{NO} \stackrel{2}{\longrightarrow} \mathrm{HNO}+\mathrm{CH}_{3} \mathrm{CHO} \\
& 2 \mathrm{HNO} \stackrel{3}{\longrightarrow} \mathrm{N}_{2} \mathrm{O}+\mathrm{H}_{2} \mathrm{O} \\
& \mathrm{C}_{2} \mathrm{H}_{5} \mathrm{O} \cdot+\mathrm{HNO} \stackrel{4}{\longrightarrow} \mathrm{C}_{2} \mathrm{H}_{5} \mathrm{OH}+\mathrm{NO}
\end{aligned}
$$

where $k_{\mathrm{obs}}=\frac{k_{1} k_{2}}{k_{-1}}$. 
Reaction: 1-Nitritopropane ( $n$-propyl nitrite)

\begin{tabular}{cccc}
\multicolumn{4}{r}{$n \cdot \mathrm{C}_{3} \mathrm{H}_{7} \mathrm{ONO}(\mathrm{I}) \longrightarrow n \cdot \mathrm{C}_{3} \mathrm{H}_{7} \mathrm{O} \cdot(\mathrm{II})+\mathrm{NO}(\mathrm{III})$} \\
& $\mathrm{I}$ & II & III \\
$\Delta H_{f(298)}^{\circ}$ & $(-29.5)$ & -9.7 & 21.6 \\
$S_{(298)}^{\circ}$ & 90.0 & 75.3 & 50.3 \\
$C_{p(298)}^{\circ}$ & 25.9 & 19.5 & 7.1
\end{tabular}

$\Delta S^{\circ}=35.6 \mathrm{~g} / \mathrm{mole}$ $\Delta H^{\circ}=41.4 \mathrm{kcal} / \mathrm{mole}$ $\Delta C_{p}^{\circ}=0.7 \mathrm{~g} / \mathrm{mole}$

\begin{tabular}{|c|c|c|c|c|c|c|}
\hline $\log A$ & $\boldsymbol{E}$ & $\begin{array}{c}\log \boldsymbol{k}_{T} \\
(465)\end{array}$ & Conditions & System & Surface & References \\
\hline 14.4 & 37.7 & -3.32 & $\begin{array}{l}443-483^{\circ} \mathrm{K} \\
60-170 \mathrm{~mm} \mathrm{Hg}\end{array}$ & static & none & $\begin{array}{l}\text { [1] E. W. R. Steacie and } \\
\text { G. T. Shaw, J. Chem. } \\
\text { Phys. 3, 344 (1935). }\end{array}$ \\
\hline
\end{tabular}

Preferred:

$\log k=16.2-40.2 / \theta ; k_{\mathrm{rec}}=10^{10.1} \mathrm{l} / \mathrm{mole}-\mathrm{sec}$

$\log k_{\mathrm{exp}}=14.4-37.7 / \theta$

Comments: See methyl nitrite for a discussion of the mechanism and the method of rate constant evaluation used here.

\section{Experimental}

[1] Identified overall kinetic rate to that of the initiation step. Rates were followed manometrically. 
Reaction: 2-Nitritopropane (isopropyl nitrite)

\begin{tabular}{|c|c|c|c|}
\hline$\Delta H_{f(29 x)}^{\circ}$ & $\begin{array}{c}\mathrm{I} \\
-32.3\end{array}$ & $\begin{array}{c}\text { II } \\
-12.4\end{array}$ & $\begin{array}{c}\text { III } \\
21.6\end{array}$ \\
\hline$S_{(2 ! 98)}^{\circ}$ & 86.9 & 74.4 & 50.3 \\
\hline$C_{p(2,9 k)}^{\circ}$ & 26.4 & 20.0 & 7.1 \\
\hline
\end{tabular}

$\Delta S^{\circ}=37.8 \mathrm{~m} / \mathrm{mole}$

$\Delta H^{\circ}=41.5 \mathrm{kcal} / \mathrm{mole}$

$\Delta C_{p}^{\circ}=0.7 \% /$ mole

\begin{tabular}{|c|c|c|c|c|c|c|}
\hline $\log A$ & $\boldsymbol{E}$ & $\begin{array}{c}\log _{(46.5)} \boldsymbol{k}_{r} \\
(46.5)\end{array}$ & Conditions & System & Surface & References \\
\hline 14.1 & 37.0 & -3.30 & $\begin{array}{l}443-483^{\circ} \mathrm{K} \\
100-227 \mathrm{~mm} \mathrm{H} \underset{c}{ }\end{array}$ & static & none & $\begin{array}{l}\text { [1] E. W. R. Steacie and } \\
\text { C. T. Shaw, Proc. Roy. } \\
\text { Soc A151, } 685 \text { (1935); } \\
\text { E. W. R. Steacie and } \\
\text { S. Katz, J. Chem. } \\
\text { Phys. 5, } 125 \text { (1937). }\end{array}$ \\
\hline
\end{tabular}

Preferred:

$\log h=16.5-40.2 / \theta ; k_{\text {rec }}=10^{9.9} \mathrm{l} /$ mole-sec

$\log _{\text {exp }}=14.1-37.0 / \theta$ (see methyl nitrite).

Comments: For references to the mechanism of the reaction and the method of the rate constant evaluation used here, see methyl nitrite.

\section{Experimental}

[1] Rates were followed manometrically. 
Reaction: l-Nitritobutane ( $n$-butyl nitrite)

\begin{tabular}{|c|c|c|c|}
\hline$\Delta H_{f(298)}^{\circ}$ & $\begin{array}{c}\text { I } \\
-34.5\end{array}$ & $\begin{array}{c}\text { II } \\
-14.7\end{array}$ & $\begin{array}{c}\text { III } \\
21.6\end{array}$ \\
\hline$S_{(298)}^{\circ}$ & 99.8 & 84.3 & 50.3 \\
\hline$C_{p(298)}^{o}$ & 31.4 & 25.0 & 7.1 \\
\hline
\end{tabular}

$\Delta S^{\circ}=35.6 \because /$ mole

$\Delta H^{\circ}=41.4 \mathrm{kcal} / \mathrm{mole}$

$\Delta C_{p}^{\circ}=0.7 \mathrm{~g} / \mathrm{mole}$

\begin{tabular}{|c|c|c|c|c|c|c|}
\hline $\log A$ & $\boldsymbol{E}$ & $\underset{(465)}{\log k r}$ & Conditions & System & Surface & References \\
\hline 14.48 & 37.0 & -2.92 & $\begin{array}{l}443-485^{\circ} \mathrm{K} \\
50-400 \mathrm{~mm} \mathrm{H} \stackrel{x}{ }\end{array}$ & static & none & $\begin{array}{l}\text { [1] E. W. R. Steacie and } \\
\text { W. McF. Smith, } \\
\text { J. Chem. Phys. 4, } \\
\text { 504 (1936). }\end{array}$ \\
\hline
\end{tabular}

Preferred:

$\log k=16.3-40.5 / \theta ; k_{\text {rec }}=10^{10.1} \mathrm{l} /$ mole-sec

$\log k_{\exp }=14.48-37.0 / \theta$ (see methyl nitrite).

Comments: See methyl nitrite for a discussion of the mechanism and of the method of rate constant evaluation used here.

\section{Experimental}

[1] Rates were followed manometrically. The system was complicated by the formation of polymeric products. 
Reaction: Nitratomethane (methyl nitrate)

$\begin{array}{crcr} & \mathrm{CH}_{3} \mathrm{ONO}_{2}(\mathrm{I}) \longrightarrow \mathrm{CH}_{3} \mathrm{O} \cdot(\mathrm{II})+\mathrm{NO}_{2} \text { (III) } \\ \Delta H_{f(298)}^{\circ} & \mathrm{I} & \mathrm{II} & \text { III } \\ S_{(298)}^{\circ} & -28.6 & 3.9 & 8.1 \\ C_{p(298)}^{\circ} & 75.3 & 54.6 & 57.5 \\ & 18.3 & 8.4 & 8.7\end{array}$

$\Delta S^{\circ}=36.8 \mathrm{~g} / \mathrm{mole}$

$\Delta H^{\circ}=40.6 \mathrm{kcal} / \mathrm{mole}$

$\Delta C_{\mu}^{\circ}=-1.2 \mathrm{~g} / \mathrm{mole}$

\begin{tabular}{|c|c|c|c|c|c|c|}
\hline $\log A$ & $\boldsymbol{E}$ & $\underset{(500)}{\log \boldsymbol{k}_{\boldsymbol{\gamma}}}$ & Conditions & System & Surface & References \\
\hline 14.4 & 39.5 & -2.86 & $\begin{array}{l}483-513^{\circ} \mathrm{K} \\
5-15 \mathrm{~mm} \mathrm{Hg}\end{array}$ & static & none & $\begin{array}{l}\text { [1] A. Appin, J. Chariton, } \\
\text { and O. Todes, Acta } \\
\text { Physiochim., U.S.S.R., } \\
\text { 5, } 655 \text { (1936). }\end{array}$ \\
\hline
\end{tabular}

Preferred:

$\log k=15.5-39.8 / \theta ; k_{\text {rec }}=10^{9.6} \mathrm{l} / \mathrm{mole}-\mathrm{sec}$.

For $\mathrm{CH}_{3} \mathrm{O}+\mathrm{NO}_{2}, \frac{k_{\text {disp }}}{k_{\text {ree }}} \simeq 0.1$ (G. Baker and R. Shaw, J. Chem. Soc. 6965 (1965)).

$\log k_{\exp }=14.4-39.5 / \theta$ (see methyl nitrite).

Comments: It is quite possible that some direct five-center molecular elimination to $\mathrm{CH}_{2} \mathrm{O}+\mathrm{HONO}$ also occurs here. (See methyl nitrite for a discussion of the mechanism and method of rate constant evaluation.)

\section{Experimental}

[1] Rates were followed manometrically. Reaction products, $\mathrm{CH}_{2} \mathrm{O}, \mathrm{CH}_{3} \mathrm{OH}$, and $\mathrm{NO}_{2}$, were qualitatively identified. 
Reaction: Nitratoethane (ethyl nitrate)

\begin{tabular}{cccc}
\multicolumn{4}{c}{$\mathrm{C}_{2} \mathrm{H}_{5} \mathrm{ONO}_{2}(\mathrm{I}) \longrightarrow \mathrm{C}_{2} \mathrm{H}_{5} \mathrm{O} \cdot(\mathrm{II})+\mathrm{NO}_{2}(\mathrm{III})$} \\
& $\mathrm{I}$ & $\mathrm{II}$ & III \\
$\Delta H_{f(298)}^{\circ}$ & -36.7 & -4.9 & 8.1 \\
$S_{(298)}^{\circ}$ & 85.1 & 65.9 & 57.5 \\
$C_{p(298)}^{\circ}$ & $(23.3)$ & 14.0 & 8.7
\end{tabular}

$\Delta S^{\circ}=38.3 \mathrm{~g} / \mathrm{mole}$
$\Delta H^{\circ}=39.9 \mathrm{kcal} / \mathrm{mole}$
$\Delta C_{p}^{\circ}=-0.6 \mathrm{~g} / \mathrm{mole}$

$\begin{array}{lll}\log A & E & \begin{array}{ll}\log k_{T} \\ (455)\end{array}\end{array}$

Conditions System Surface

References

15.8

$39.9-3.36$

$453-488^{\circ} \mathrm{K} \quad$ static

static none

$30-50 \mathrm{~mm} \mathrm{Hg}$

$13.86 \quad 36.0-3.43$

$\begin{array}{llll}16.0 & 39.3 & -2.88 & 434-474\end{array}{ }^{\circ} \mathrm{K} \quad$ static

$16.9-41.2-2.89$

8.1

57.5

8.7

$\begin{array}{lllll}16.0 & 39.3 & -2.88 & 434-474^{\circ} \mathrm{K} & \text { static } \\ 16.9 & 41.2 & -2.89 & & \\ & & & & \\ 14.74 & 38.0 & -3.51 & 448-482^{\circ} \mathrm{K} & \text { static } \\ & & & 30-100 \mathrm{~mm} \mathrm{Hg} & \end{array}$
[2] L. Phillips, Nature 165 ,
564 (1950).

[3] J. B. Levy, J. Am. Chem. Soc. 76, 3254 (1954); 3790 (1954).

[4] F. H. Pollard, H. S. B. Marshall, and A. E. Pedler, Trans. Faraday Soc. 52, 59 (1956).

Preferred:

$\log k_{1}=16.5-39.4 / \theta ; k_{\text {rec }}=10^{9.8} 1 /$ mole-sec.

For $\mathrm{C}_{2} \mathrm{H}_{5} \mathrm{O}+\mathrm{NO}_{2}, \frac{k_{\text {disp }}}{k_{\text {rec }}}=0.46$ (G. Baker and R. Shaw, J. Chem. Soc., 6965 (1965)).

$\log k_{\exp }=16.0-39.3 / \theta$.

Comments: Agreement between the predicted and experimental kinetics tends to support exclusive initiation via the $\left(\mathrm{RO}-\mathrm{NO}_{2}\right)$ bond rupture. (See methyl nitrite for a discussion of the mechanism.)

\section{Experimental}

11] Rates were followed by pressure changes. Products identified were $\mathrm{CH}_{3} \mathrm{CHO}$, $\mathrm{CO}$, and $\mathrm{CO}_{2}$.

[3] Analysis by I.R. and visible absorption spectroscopy. Followed product buildup and reactant disappearance as a function of time. Found that the discrepancies between [1] and [2] resulted from calculating rate constants from different regions of the pressure-time curves. Also found that the mechanism of the decomposition was more complex than originally assumed.

[4] Rates were followed spectrophotometrically (in the $\mathrm{UV}$ on $\mathrm{NO}_{2}$ ) and by pressure changes. Identified initial rates of reaction to the above bond-breaking reactions. 
Reaction: 1,2-Dinitratoethane (ethylene glycol dinitrate) $\stackrel{a}{\longrightarrow}$

1,3-Dinitratopropane (trimethylene glycol dinitrate) $\stackrel{b}{\longrightarrow}$

1,2-Dinitratopropane (propylene glycol dinitrate) $\stackrel{c}{\longrightarrow}$

(product analyses were not made)

Nitroglycerine $\stackrel{d}{\longrightarrow}$

Trimethylol-nitromethane trinitrite $\stackrel{e}{\longrightarrow}$

$$
\begin{gathered}
\Delta H_{f(298)}^{\circ} \\
S_{(298)}^{\circ} \\
C_{p(298)}^{\circ}
\end{gathered}
$$

$\Delta S^{\circ}=$

\begin{tabular}{|c|c|c|c|c|c|c|c|}
\hline Path & $\log A$ & $\boldsymbol{E}$ & $\log k_{T}$ & Conditions* & System & Surface & References \\
\hline$a$ & 15.9 & 39 & $-7.14(370)$ & $358-378^{\circ} \mathrm{K}$ & static & & $\begin{array}{r}\text { [1] L. Phillips, Nature } \\
\quad 160,753 \text { (1947). }\end{array}$ \\
\hline & 15.2 & 38.1 & $-7.30(370)$ & $358-383^{\circ} \mathrm{K}$ & & & \\
\hline & 15.2 & 37.4 & $-7.20(365)$ & $353-373^{\circ} \mathrm{K}$ & & & \\
\hline & 17.1 & 40.3 & $-7.03(365)$ & $348-378^{\circ} \mathrm{K}$ & & & \\
\hline & 15.3 & 36.4 & $-6.80(360)$ & $348-368{ }^{\circ} \mathrm{K}$ & & & \\
\hline
\end{tabular}

$\Delta H^{\circ}=$

$\Delta C_{p}^{\circ}=$

Preferred:

Reasonable as reported.

Comments: The similarity in the Arrhenius parameters to those of the methyl and ethyl nitrate decompositions indicates, similar reaction mechanisms. The high $A$-factors suggest exclusive initial $\left(\mathrm{RO}-\mathrm{NO}_{2}\right)$ bond rupture.

\section{Experimental}

[1] Rate constants are those for the overall decomposition and were obtained from the pressure-time curves. Identification of the Arrhenius parameters with those of the initial $\left(\mathrm{RO}-\mathrm{NO}_{2}\right)$ bond split was considered likely.

* No other conditions were specified. 
Reaction: Nitromethane

$\begin{array}{cccc} & \mathrm{CH}_{3} \mathrm{NO}_{2}(\mathrm{I}) \stackrel{\mathrm{l}}{\longrightarrow} \mathrm{CH}_{3} \cdot(\mathrm{II})+\mathrm{NO}_{2}(\mathrm{III}) & \\ \Delta H_{f(298)}^{\circ} & \mathrm{I} & \mathrm{II} & \mathrm{III} \\ S_{(298)}^{\circ} & -17.9 & 34.0 & 8.1 \\ C_{p(298)}^{\circ} & 65.8 & 46.1 & 57.3 \\ & 16.8 & 8.8 & 8.7\end{array}$

$\Delta S^{\circ}=37.6 \mathrm{~g} / \mathrm{mole}$

$\Delta H^{\circ}=60.0 \mathrm{kcal} / \mathrm{mole}$

$\Delta C_{p}^{\circ}=0.7 \mathrm{~g} / \mathrm{mole}$

\begin{tabular}{|c|c|c|c|c|c|c|}
\hline $\log A$ & $\boldsymbol{E}$ & $\begin{array}{r}\log \boldsymbol{k}_{T} \\
(1000)\end{array}$ & Conditions & System & Surface & References \\
\hline 14.6 & 53.6 & +2.88 & $\begin{array}{l}653-703^{\circ} \mathrm{K} \\
200-400 \mathrm{~mm} \\
\mathrm{Hg}\end{array}$ & static & none & $\begin{array}{l}\text { [1] T. L. Cottrell, T. E. } \\
\text { Graham, and T. J. } \\
\text { Reid, Trans. Faraday } \\
\text { Soc. 47, 584 (1951). }\end{array}$ \\
\hline 13.6 & 50.6 & +2.54 & $\begin{array}{l}693-753^{\circ} \mathrm{K} \\
\delta \mathrm{P}_{0} \simeq 0.06-0.18 \\
\quad \mathrm{~mm} \mathrm{Hg}\end{array}$ & flow & none & $\begin{array}{l}\text { [2] L. J. Hildebrand, Jr., and } \\
\text { M. L. Kilpatrick, J. } \\
\text { Chem. Phys. 19, } 381 \\
\text { (1951). }\end{array}$ \\
\hline 13.43 & 50.0 & +2.50 & as above & flow & none & $\begin{array}{l}\text { [3] ibid.; J. Chem. Phys. 21, } \\
525 \text { (1953). }\end{array}$ \\
\hline 11.4 & 42.8 & +2.04 & $\sim 660^{\circ} \mathrm{K}$ & static & 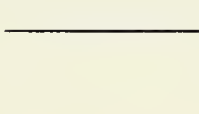 & $\begin{array}{l}\text { [4] C. Frejacques, Comp. } \\
\text { Rend. 231, } 1061 \text { (1950). }\end{array}$ \\
\hline 15.1 & 57 & +2.64 & $700-1300^{\circ} \mathrm{K}$ & $\begin{array}{l}\text { Sh.T-study } \\
\text { of ignition } \\
\text { delay in } \mathrm{O}_{2} \\
+\mathrm{CH}_{3} \mathrm{NO}_{2}\end{array}$ & none & $\begin{array}{l}\text { [5] A. A. Borisov, S. M. } \\
\text { Kogarko, and G. I. } \\
\text { Skachkov, Kinetika i } \\
\text { Kataliz. 7, } 589 \text { (1966). }\end{array}$ \\
\hline 13.73 & 49.2 & +2.98 & $\begin{array}{l}585-613^{\circ} \mathrm{K} \\
40 \text { atm }\end{array}$ & static & 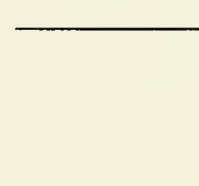 & $\begin{array}{l}\text { [6] A. Makovky and T. B. } \\
\text { Gruenwald, Trans. } \\
\text { Faraday Soc. 55, } 952 \\
\text { (1959). }\end{array}$ \\
\hline 16.6 & $61 \pm 3$ & +3.27 & $\begin{array}{l}553-593^{\circ} \mathrm{K} \\
25-200 \text { torr }\end{array}$ & static & $\begin{array}{l}\text { none in } \\
\text { early } \\
\text { stages }\end{array}$ & $\begin{array}{l}\text { [7] H. A. Taylor and V. V. } \\
\text { Vesselovsky, J. Phys. } \\
\text { Chem. 39, } 1095 \text { (1935). }\end{array}$ \\
\hline
\end{tabular}

Preferred:

$\log k=15.6-59.0 ! 00^{*}$

(Continued)

*Calculated from the kinetics of the back reaction (see [4] above) and from the reaction thermochemistry. 


\section{Experimental}

[1] Parameters were obtained from the initial rates of the pressure-time curves. Products found were NO, CO, $\mathrm{CH}_{4}, \mathrm{~N}_{2} \mathrm{O}, \mathrm{H}_{2} \mathrm{O}$, and $\mathrm{CO}_{2}$. Product ratios were temperature independent. A fairly complex mechanism was proposed. They assumed no chain on the basis of lack of inhibition by NO. This is not a valid criterion.

[2] Rates were obtained from polarographic analysis of the undecomposed nitromethane. High initial yields of $\mathrm{H}_{2} \mathrm{CO}$ were taken as an indication of some intramolecular reaction.

[3] Procedures as in [2]. Reproducible kinetics were obtained only after passing wet $\mathrm{N}_{2}$ carrier through the reaction chamber prior to each run.

[4] The kinetics of the back reaction have been measured by L. Phillips and R. Shaw, Tenth Int. Symp. on Comb., 453 (1964) (Williams and Williams, Balt., Md.). They found $k_{-1}=10^{9.2} \mathrm{l} / \mathrm{mole}$-sec. This has since been confirmed by V. YaShtern, Chemical Kinetics and Chain Reactions, 286 (see p. 313), (1966), U.S.S.R. Academy of Sciences, Moscow. From these numbers and the thermochemistry, we obtain: $k_{1}=10^{15.6-59.0 / \theta}$.

[5] A composite plot of all of the data from 600 to $1200^{\circ} \mathrm{K}$ was used to obtain the Arrhenius parameters.

[6] Main products were $\mathrm{NO}, \mathrm{HCN}, \mathrm{CO}_{2}, \mathrm{H}_{2} \mathrm{CO}$, and $\mathrm{H}_{2} \mathrm{O}$. Effects of additives $\left(\mathrm{NO}, \mathrm{NO}_{2}, \mathrm{O}_{2},\left(\mathrm{CH}_{3} \mathrm{CO}_{2}\right)_{2}\right)$ were investigated. $\mathrm{HCN}$ yields increased with initial pressures but were inhibited by $\mathrm{O}_{2}$. Induced free radical chain decomposition was invoked to rationalize the "low" Arrhenius parameters.

[7] Parameters obtained from one-fourth-lives. Below $400{ }^{\circ} \mathrm{C}$ these showed small inverse pressure dependence. They proposed split into $\mathrm{CH}_{3} \mathrm{NO}+\mathrm{O}$, which has $\Delta H=91 \mathrm{kcal}$ and thus impossibly slow. $\mathrm{He}, \mathrm{N}_{2}, \mathrm{NO}, \mathrm{CO}_{2}$ had no effect on $t-\Delta P$ curves. $\mathrm{O}_{2}$ changed $\Delta P_{x}$ slightly and reduced $t_{1 / 4}$ slightly. $\Delta P_{x} / p_{0} \sim 1.3$ in absence of $\mathrm{O}_{2}$. Surface increases reduced $\Delta P_{\infty} / P_{0}$ to 0.92 


\begin{tabular}{cccc}
\multicolumn{4}{c}{$\mathrm{C}_{2} \mathrm{H}_{5} \mathrm{NO}_{2}(\mathrm{I}) \longrightarrow \mathrm{C}_{2} \mathrm{H}_{5} \cdot(\mathrm{II})+\mathrm{NO}_{2}$ (I) } \\
$\Delta H_{f(298)}^{\circ}$ & I & II & III \\
$S_{(2,98)}^{\circ}$ & -23.9 & 25.7 & 8.1 \\
$C_{p(2998)}^{\circ}$ & 75.3 & 57.8 & 57.5 \\
& 20.0 & 12.1 & 8.7
\end{tabular}

$\Delta S^{\circ}=40.0 \mathrm{~g} / \mathrm{mole}$ $\Delta H^{\circ}=57.7 \mathrm{kcal} / \mathrm{mole}$ $\Delta C_{1}^{\circ}=0.8 \mathrm{~g} / \mathrm{mole}$

\begin{tabular}{ccccccc}
$\log \boldsymbol{A}$ & $\boldsymbol{E}$ & $\begin{array}{c}\log _{(785)} k_{T} \\
\text { (7) }\end{array}$ & \multicolumn{1}{c}{ Conditions } & System & Surface & \multicolumn{1}{c}{ References } \\
17.6 & $60.6 \pm 5$ & +0.72 & $763-808^{\circ} \mathrm{K}$ & flow & [1] K. A. Wilde, J. Phys.
\end{tabular}

Chem. 61, 385 (1957).

Preferred:

Unreliable.

Comments: G. N. Spokes and S. W. Benson, using a very low-pressure-high-temperature pyrolysis technique, have shown that the initiation steps in the pyrolysis of 1 and 2-nitro-propanes are molecular elimination of $\mathrm{HNO}_{2}$. Rate constants in the 800 to $1100{ }^{\circ} \mathrm{K}$ range coupled with the low-temperature data in the 600 to $800{ }^{\circ} \mathrm{K}$ range gave Arrhenius parameters which were in good agreement with the low-temperature molecular elimination mechanism. Thus the mechanistic change proposed cannot be supported.

The reaction at all temperatures appears to be $\mathrm{C}_{2} \mathrm{H}_{5} \mathrm{NO}_{2} \longrightarrow \mathrm{C}_{2} \mathrm{H}_{4}+\mathrm{HNO}_{2} ;$ presumably a five-center elimination; with $\log k=11.75-43 / \theta$.

\section{Experimental}

[1] Rates were based on G.L.C. analysis of the nitroethane remaining. Reaction products were $\mathrm{HNO}_{2}$ and $\mathrm{C}_{2} \mathrm{H}_{4}$. An increase in the slope of the Arrhenius equation at high temperatures led to postulation of the above initiation $\left(\mathrm{R}^{-} \mathrm{NO}_{2}\right)$ bond rupture process. 
Reaction: Nitrotrichloromethane

\begin{tabular}{|c|c|c|c|}
\hline \multirow[b]{3}{*}{$\Delta H_{f(2,98)}^{\circ}$} & $\mathrm{CCl}_{3} \mathrm{NO}_{2.2}(\mathrm{I}) \stackrel{a}{b}$ & $\begin{array}{l}\text { I) }+\cdot \text { I } \\
+\mathrm{NOC}\end{array}$ & \\
\hline & I & II & III \\
\hline & $(\quad)$ & 18.5 & 8.1 \\
\hline$S_{(298)}^{\circ}$ & 86.2 & 72 & 57.5 \\
\hline$C_{p(298)}^{\circ}$ & 25.5 & 14.7 & 8.7 \\
\hline
\end{tabular}

$\Delta S^{\circ}=43.3 \mathrm{~g} / \mathrm{mole}$

$\Delta H^{\circ}=$

$\Delta C_{p}^{\circ}=-0.1 \% /$ mole

\begin{tabular}{|c|c|c|c|c|c|c|}
\hline $\log A$ & $\boldsymbol{E}$ & $\underset{(425)}{\log k_{T}}$ & Conditions & System & Surface & References \\
\hline 15.69 & 37.7 & -3.70 & $\begin{array}{l}411-443^{\circ} \mathrm{K} \\
60-200 \mathrm{~mm} \mathrm{H} \mathrm{H}\end{array}$ & static & none & $\begin{array}{l}\text { [1] E. W. R. Steacie and } \\
\text { W. McF. Smith, } \\
\text { J. Chem. Phys. 6, } \\
\text { 145 (1938): Can. J. Res. } \\
\text { B16, } 222 \text { (1938). }\end{array}$ \\
\hline
\end{tabular}

Preferred:

Suspect as unimolecular reactions.

Comments: From the complexity of the reaction products one would not expect the reported parameters to be related simply to the bond fission reaction.

\section{Experimental}

[1] Rates were followed manometrically. The reaction products were phosgene $\left(\mathrm{COCl}_{2}, \mathrm{NO}, \mathrm{NOCl}\right.$, and $\mathrm{Cl}_{2}$. The $\mathrm{NO}$ and $\mathrm{Cl}_{2}$ were presumably formed from the secondary decomposition of $\mathrm{NOCl}$, although the amounts of $\mathrm{NO}$ and $\mathrm{Cl}_{2}$ were found to be in excess of those expected from the NOCl decomposition alone. 
Reaction: Tetranitromethane

$\mathrm{C}\left(\mathrm{NO}_{2}\right)_{4} \longrightarrow \cdot \mathrm{C}\left(\mathrm{NO}_{2}\right)_{3}+\mathrm{NO}_{2}$

$\Delta H_{f(298)}^{\circ}$

$S_{(298)}^{\circ}$

$C_{p(298)}^{\circ}$

$\Delta S^{\circ}=$

$\Delta H^{\circ}=$

$\Delta C_{p}=$

\begin{tabular}{|c|c|c|c|c|c|c|}
\hline $\log A$ & $\boldsymbol{E}$ & $\log k_{T}$ & Conditions & System & Surface & References \\
\hline 17.53 & 40.88 & & $\begin{array}{l}443-506^{\circ} \mathrm{K} \\
\delta \mathrm{P}=1.09 \text { torr } \\
\mathrm{P}_{T}=1 \text { atm }(\mathrm{He})\end{array}$ & $\begin{array}{l}\text { Stirred } \\
\text { flow } \\
\text { reactor }\end{array}$ & & $\begin{array}{l}\text { [1] J. M. Sullivan and } \\
\text { A. E. Axworthy, J. } \\
\text { Phys. Chem. 70, } \\
3366 \text { (1966). }\end{array}$ \\
\hline
\end{tabular}

Preferred:

Reasonable.

$\log k=17.53-40.88 / \theta$.

Comments: The $A$-factor is in the region expected for such a decomposition (see neopentane).

\section{Experimental}

[1] Analysis by G.L.C. On reactant. Products identified by M.S. and I.R. were NO.. NO, N.O, and CO.. Mass balances were not obtained. The rate deternining step was assumed to be the above bond fission. Subsequent fast. non-chain. reactions of the primary fission products were postulated. 
Reaction: Ethanaldoxime (acetaldoxime)

\begin{tabular}{|c|c|c|c|}
\hline$\Delta H_{f(298)}^{\circ}$ & $\begin{array}{c}\text { I } \\
-7.3\end{array}$ & $\begin{array}{c}\text { II } \\
{[33.1]}\end{array}$ & $\begin{array}{c}\text { III } \\
8.0\end{array}$ \\
\hline$S_{(298)}^{\circ}$ & 72.4 & 64.6 & 43.9 \\
\hline$C_{p(298)}^{\circ}$ & 18.8 & 13.1 & 7.1 \\
\hline
\end{tabular}

$\Delta S^{\circ}=36.1 \mathrm{~g} / \mathrm{mole}$

$\Delta H^{\circ}=48.4 \mathrm{kcal} / \mathrm{mole}$

$\Delta C_{p}^{\circ}=1.4 \mathrm{~g} / \mathrm{mole}$

$\begin{array}{llllll}\log \boldsymbol{A} & \boldsymbol{E} & \begin{array}{c}\log \boldsymbol{k}_{T} \\ (660)\end{array} & \text { Conditions } & \text { System } & \text { Surface } \\ 12.83 & 47 & -2.73 & 603-713^{\circ} \mathrm{K} & \text { static } & \sim 8 \%\end{array}$
[1] G. L. Pratt and J. H. Purnell, Trans. Fara- day Soc. 58, 692 (1962).

Comments: Very careful study; however, the reaction is extremely complex and the interpretation needs verification. The $A$-factor looks low by about a factor of 10 . If correct, the acetaldimene radical heat of formation is $\Delta H_{f}^{\circ}\left(\mathrm{CH}_{3} \mathrm{CH}=\mathrm{N}\right.$. $) \simeq 33.1 \mathrm{kcal} /$ mole. The similarity in activation energies of the acetaldoxime decomposition and of the nitric and nitrous acid decompositions (i.e., 47,49 , and $47 \mathrm{kcal} / \mathrm{mole}$ respectively) is probably more than fortuitous.

\section{Experimental}

[1] Rates were followed manometrically and by G.L.C. Complete mass balances were made on the following products: $\mathrm{N}_{2}, \mathrm{CO}, \mathrm{CH}_{4}, \mathrm{CO}_{2}, \mathrm{~N}_{2} \mathrm{O}, \mathrm{C}_{2} \mathrm{H}_{4}, \mathrm{C}_{2} \mathrm{H}_{6}, \mathrm{C}_{3} \mathrm{H}_{6}, \mathrm{C}_{3} \mathrm{H}_{4}, \mathrm{HCN}, \mathrm{CH}_{3} \mathrm{CHO}$, and $\mathrm{CH}_{3} \mathrm{CN}$. An initial slow rate (induction period) was followed by a fast reaction. The slow reaction kinetics were tentatively equated to the above dissociation (rate determining) on the basis of the thermochemistry. The mechanisms proposed were very complex. The fast reaction was inhibited by propylene and sensitized by $\mathrm{NO}$ and $\mathrm{O}_{2}$ in amounts greater than 1 percent. A four-center molecular elimination directly to $\mathrm{CH}_{3} \mathrm{CN}$ and $\mathrm{H}_{2} \mathrm{O}$ was also considered possible. On the basis of the molecular product yields, this accounted for less than 25 percent of the total oxime decomposition. No reasonable mechanism could be proposed for the formation of $\mathrm{CO}_{2}, \mathrm{~N}_{2}, \mathrm{CH}_{3} \mathrm{NO}_{2}, \mathrm{CH}_{3} \mathrm{CHO}$, and $\mathrm{CO}$. 
Reaction: Dimeric nitrosomethane<smiles>COCCCOC</smiles>

$\begin{array}{lcc}\Delta H_{f(298)}^{\circ} & \text { I } & \text { II } \\ S_{(298)}^{\circ} & 79 & 17 \\ C_{p(298)}^{\circ} & 63.5\end{array}$

$$
\begin{aligned}
& \Delta S^{\circ}=48 \mathrm{~g} / \mathrm{mole} \\
& \Delta H^{\circ}= \\
& \Delta C_{p}^{\circ}=2.4 \mathrm{~g} / \mathrm{mole}
\end{aligned}
$$

\begin{tabular}{|c|c|c|c|c|c|c|}
\hline $\log A$ & $\boldsymbol{E}$ & $\begin{array}{c}\log \boldsymbol{k}_{T} \\
(390)\end{array}$ & Conditions & System & Surface & References \\
\hline 13.4 & 23 & +0.51 & $\begin{array}{l}374-404^{\circ} \mathrm{K} \\
\delta P=10^{-3}-10^{-4} \\
\mathrm{~mm} \mathrm{Hg} \\
P_{T} \sim 4-8 \mathrm{~mm} \mathrm{Hg}\end{array}$ & flow & & $\begin{array}{l}\text { [1] L. Batt, B. G. Gowen- } \\
\text { lock, and J. Trotman, } \\
\text { J. Chem. Soc. } 2222 \\
\text { (1960). }\end{array}$ \\
\hline
\end{tabular}

Preferred:

Reasonable. $\log k=13.4-23 / \theta$.

Comments: Parameters should be slightly higher if the pressure falloff is real. The activation energy reported is in good agreement with solution studies which yield $E_{a}=20.9$ to $24.0 \mathrm{kcal} / \mathrm{mole}$ for a variety of dimers. (Anderson and Hammick, J. Chem. Soc. 30 (1935); Bamberger and Seligman, Ber. 36, 685 (1903); Hammick and Lister, J. Chem. Soc. 489 (1937)).

\section{Experimental}

[1] Nitrogen carrier. Analyses were made spectrophotometrically on the cis dimer. Monomeric nitrosoalkanes at liquid $\mathrm{N}_{2}$ temperatures form cis dimers exclusively when warmed to $-78{ }^{\circ} \mathrm{C}$. Increasing the total pressure (with $\mathrm{N}_{2}$ ) increased the reaction rates by about 35 percent although perfluorocyclohexane had no accelerating effect. 
Reaction: Dimeric 2-methyl-1-nitrosopropane (dimeric nitrosoisobutane)

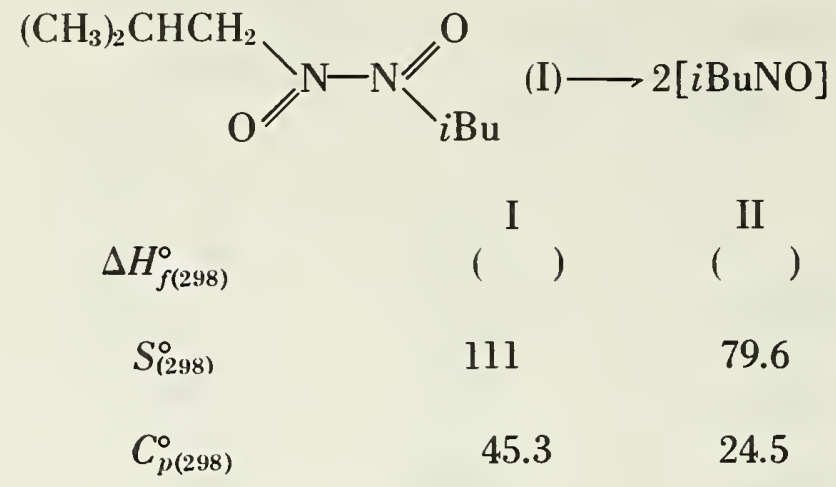

$\Delta S^{\circ}=48.2 \mathrm{~g} / \mathrm{mole}$

$\Delta H^{\circ}=$

$\Delta C_{p}^{\circ}=3.7 \mathrm{~g} / \mathrm{mole}$

\begin{tabular}{|c|c|c|c|c|c|c|}
\hline $\log A$ & $\boldsymbol{E}$ & $\begin{array}{c}\log \boldsymbol{k}_{T} \\
(390)\end{array}$ & Conditions & System & Surface & References \\
\hline 14.4 & 25.6 & +0.05 & $\begin{array}{l}374-402^{\circ} \mathrm{K} \\
\delta P \simeq 10^{-3}-10^{-4} \\
\quad \text { torr } \\
P_{T} \simeq 4-8 \text { torr }\end{array}$ & flow & & $\begin{array}{l}\text { [1] L. Batt, B. G. Gowenlock, } \\
\text { and J. Trotman, J. } \\
\text { Chem. Soc. } 2222 \text { (1960). }\end{array}$ \\
\hline
\end{tabular}

Preferred:

Reasonable. $\log k=14.4-25.6 / \theta$.

\section{Experimental}

[1] See dimeric nitrosomethane. 
Reaction: Diazomethane

\begin{tabular}{cccc}
\multicolumn{4}{r}{$\mathrm{CH}_{2}=\mathrm{N}=\mathrm{N}(\mathrm{I}) \longrightarrow \mathrm{CH}_{2}:(\mathrm{II})+\mathrm{N}_{2}(\mathrm{III})$} \\
$\Delta H_{f(298)}^{\circ}$ & $\mathrm{I}$ & $\mathrm{II}$ & $\mathrm{III}$ \\
$S_{(298)}^{\circ}$ & $(71)$ & $(90)$ & 0 \\
$C_{p(298)}^{\circ}$ & 58.1 & 43.3 & 45.8 \\
& 12.6 & 7.5 & 7.0
\end{tabular}
$\Delta S^{\circ}=31 \mathrm{~g} / \mathrm{mole}$
$\Delta H^{\circ}=19 \mathrm{kcal} / \mathrm{mole}$
$\Delta C_{p}^{\circ}=1.9 \mathrm{~g} / \mathrm{mole}$

\begin{tabular}{|c|c|c|c|c|c|c|}
\hline $\log A$ & $\boldsymbol{E}$ & $\begin{array}{c}\log \boldsymbol{k}_{T} \\
(610)\end{array}$ & Conditions & System & Surface & References \\
\hline 13.0 & 35 & +0.46 & $\begin{array}{l}498-723^{\circ} \mathrm{K} \\
25 \mathrm{~mm} \mathrm{Hg}\end{array}$ & static & $<10 \%$ & $\begin{array}{l}\text { [1] D. W. Setzer amd B. S. } \\
\text { Rabinovitch, Can. J. } \\
\text { Chem. 40, } 1425 \text { (1962). }\end{array}$ \\
\hline 11.9 & 31.75 & +0.52 & $603-773{ }^{\circ} \mathrm{K}$ & flow & & $\begin{array}{l}\text { [2] P. S. Shantarovich, } \\
\text { Doklady Akad. Nauk, } \\
\text { S.S.S.R. 116, } 255 \\
\text { (1957). }\end{array}$ \\
\hline 12.95 & 32 & +1.48 & $\begin{array}{l}566-666 \\
\delta P=4-95.5 \mathrm{~mm} \\
\mathrm{Hg} \\
P_{H_{2}}=151-716 \\
\mathrm{~mm} \mathrm{Hg} \\
\delta P=0.4-2 \mathrm{~mm} \\
\mathrm{Hg} \text { flow } \\
P_{H_{2}}=100-400 \\
\mathrm{~mm} \mathrm{Hg}\end{array}$ & static & & $\begin{array}{l}\text { [3] W. J. Dunning and C. C. } \\
\text { McCain, J. Chem. Soc. } \\
\text { (B) } 68 \text { (1966). }\end{array}$ \\
\hline
\end{tabular}

Preferred:

$\log k=13.0-35 / \theta$.

Comments: From the thermodynamics one can calculate a rate constant for methylene addition to nitrogen of $\log k_{(1 / \text { mole-sec })}=8.3-17.2 / \theta$.

\section{Experimental}

[1] Analysis by G.L.C. The experimental rate constants, given by $k_{25} \mathrm{~mm} \mathrm{Hg}=1.2 \times 10^{12} \times 10^{-34 / \theta}$, were shown to be in the pressure falloff region. Parameters reported above were the estimated $P_{x}$ values.

[3] Analysis by M.S. Decompositions performed in the presence of $\mathrm{H}_{2}$ and Ar. 
Reaction: Azidomethane (methyl azide)

$\begin{array}{crcc} & \mathrm{CH}_{3} \mathrm{~N}_{3}(\mathrm{I}) \longrightarrow \mathrm{CH}_{3} \ddot{\mathrm{N}}(\mathrm{II})+\mathrm{N}_{2}(\mathrm{III}) & \\ \Delta H_{f(298)}^{\circ} & \mathrm{I} & \mathrm{II} & \mathrm{III} \\ S_{(298)}^{\circ} & (66) & {[106.2]} & 0 \\ C_{p(298)}^{\circ} & 66.6 & 53.4 & 45.8 \\ & 15.9 & 8.5 & 7.0\end{array}$

$\Delta S^{\circ}=32.6 \mathrm{~g} / \mathrm{mole}$

$\Delta H^{\circ}=[40.2] \mathrm{kcal} / \mathrm{mole}$

$\Delta C_{p}^{\circ}=-0.4 \mathrm{~g} /$ mole

\begin{tabular}{|c|c|c|c|c|c|c|}
\hline $\log A$ & $\boldsymbol{E}$ & $\begin{array}{c}\log \boldsymbol{k}_{T} \\
(495)\end{array}$ & Conditions & System & Surface & References \\
\hline 15.48 & 43.5 & -3.73 & $\begin{array}{l}473-513^{\circ} \mathrm{K} \\
0.8-466 \mathrm{~mm} \mathrm{Hg} \\
\quad(>100 \mathrm{~mm} \mathrm{Hg})\end{array}$ & static & none & $\begin{array}{l}\text { [1] J. A. Leermakers, J. Am. } \\
\text { Chem. Soc. 55, } 3098 \\
\text { (1933). }\end{array}$ \\
\hline
\end{tabular}

Preferred:

Suspect.

Comments: The reaction complexities are apparent from the products formed. H-atom induced decomposition of the reactant is suggested by the presence of $\mathrm{HN}_{3}$. Identification of the observed kinetics with the initiation step above is questionable.

\section{Experimental}

[1] Rates were followed manometrically $\left(P_{d} / P_{o}=1.66\right)$. Products were $\mathrm{HN}_{3}, \mathrm{~N}_{2}, \mathrm{NH}_{3}, \mathrm{C}_{2} \mathrm{H}_{6}, \mathrm{C}_{2} \mathrm{H}_{4}$ and hexamethylene tetraamine $\left(\left(\mathrm{CH}_{2}\right)_{6} \mathrm{~N}_{4}\right)$. Other modes of scission were proposed on the basis of the products; however, the major decomposition path (i.e., $\sim 75$ percent) was believed to be the above. Rate constants exhibited pressure falloff below $100 \mathrm{~mm} \mathrm{Hg}$. 
Reaction: Azidoethane (ethyl azide)

\begin{tabular}{|c|c|c|c|}
\hline & \multicolumn{3}{|c|}{$\mathrm{C}_{2} \mathrm{H}_{5} \mathrm{~N}_{3}(\mathrm{I}) \longrightarrow \mathrm{C}_{2} \mathrm{H}_{5} \mathrm{~N}:(\mathrm{II})+\mathrm{N}_{2}(\mathrm{III})$} \\
\hline$\Delta H_{f(298)}^{\circ}$ & $\begin{array}{c}\text { I } \\
(59.4)\end{array}$ & $\underset{[99.6]}{\text { II }}$ & $\begin{array}{r}\text { III } \\
0\end{array}$ \\
\hline$S^{\circ}{ }_{(298)}$ & 76 & 62.8 & 45.8 \\
\hline$C_{p(298)}^{\circ}$ & 21.4 & 14.0 & 7.0 \\
\hline
\end{tabular}

$\Delta S^{\circ}=32.6 \mathrm{~g} / \mathrm{mole}$

$\Delta H^{\circ}=40.2 \mathrm{kcal} / \mathrm{mole}$

$\Delta C_{p}^{\circ}=-0.4 \mathrm{~g} / \mathrm{mole}$

\begin{tabular}{ccccccc}
$\log \boldsymbol{A}$ & $\boldsymbol{E}$ & $\begin{array}{c}\log \boldsymbol{k}_{T} \\
(495)\end{array}$ & Conditions & System & Surface & \multicolumn{1}{c}{ References } \\
14.3 & 39.74 & -3.25 & $\begin{array}{l}473-513^{\circ} \mathrm{K} \\
0.8-195 \mathrm{~mm} \mathrm{Hg}\end{array}$ & static & $<10 \%$ & [1] J. A. Leermakers, J. Am. \\
& & & & & $\begin{array}{c}\text { Chem. Soc. } \mathbf{5 5}, 2719 \\
\text { (1933). }\end{array}$
\end{tabular}

Preferred:

Suspect.

Comments: Obviously complex reaction; very likely with induced decomposition. (See methylazide.)

\section{Experimental}

[1] Rates were followed manometrically. Products formed were $\mathrm{N}_{2}, \mathrm{C}_{2} \mathrm{H}_{4}, \mathrm{C}_{4} \mathrm{H}_{10}, \mathrm{CH}_{3} \mathrm{CH}=\mathrm{NH}$, and $\mathrm{HN}_{3}$. A falloff in the rate constants was observed at pressures around $60 \mathrm{~mm} \mathrm{Hg}$. 
Reaction: Methanethiol (methyl mercaptan)

$\begin{array}{cccc} & \mathrm{CH}_{3} \mathrm{SH}(\mathrm{I}) \longrightarrow \mathrm{CH}_{3} \cdot(\mathrm{II})+\mathrm{HS} \cdot(\mathrm{III}) & \\ & \mathrm{I} & \mathrm{II} & \text { III } \\ \Delta H_{f(298)}^{\circ} & -5.5 & 34.0 & (35.5) \\ S_{(298)}^{\circ} & 59.9 & 46.5 & 46.7 \\ C_{p(298)}^{\circ} & 12.1 & 8.8 & 7.1 \\ C_{p(1000)}^{\circ} & 21.4 & 14.4 & 7.3\end{array}$

$\begin{array}{ll}\Delta S^{\circ} & =33.3 \mathrm{~g} / \mathrm{mole} \\ \Delta H^{\circ} & =75.0 \mathrm{kcal} / \mathrm{mole} \\ \Delta C_{p}^{\circ} & =3.8 \mathrm{~g} / \mathrm{mole} \\ \Delta C_{p(1000)}^{\circ} & =0.3 \mathrm{~g} / \mathrm{mole}\end{array}$

$\log A \quad E \quad \log k_{T}$

(1055)

Conditions System Surface

References

$13.48 * 67 \pm 2 \quad-0.40$

$1005-1102^{\circ} \mathrm{K}$
$\delta P \sim 0.5 \mathrm{~mm} \mathrm{Hg}$
$P \sim 12 \mathrm{~mm} \mathrm{Hg}$

none

$P_{T} \approx 12 \mathrm{~mm} \mathrm{Hg}$

[1] A. H. Sehon and B. deB. Darwent, J. Am. Chem. Soc. 76, 4806 (1954).

\section{Preferred:}

$\log k=15.5-76.6 / \theta$. Rate constants are probably low.

Comments: From $A$ and the reaction entropy one obtains $k_{\text {rec }}=10^{9.6} 1 /$ mole-sec. Even this value for recombination seems low. RRK estimates indicate that the reaction could well be in its falloff region (i.e., RRK $-M_{1 / 2}=3 \mathrm{~atm}$, for $n \approx 7$ ). The heat of formation of (HS ·) used comes from recent mass-spectrometric studies on sulfides.

(See J. A. Kerr, Chem. Rev. 66, 465 (1966).)

\section{Experimental}

[1] Toluene carrier technique. Rates were calculated on the basis of the $\mathrm{H}_{2} \mathrm{~S}$ formed, which - was analyed by iodometric titration in acid solution. The products were $\mathrm{CH}_{4}, \mathrm{H}_{2} \mathrm{~S}, \mathrm{H}_{2}$, and $\left(\varnothing \mathrm{CH}_{2}\right)_{2}$. The ratio of dibenzl to $\mathrm{H}_{2} \mathrm{~S}$ varied irregularly and at the higher temperatures appreciable decomposition of toluene also occurred. The experimental results are therefore somewhat suspect.

*The activation energy was calculated assuming $A=3 \times 10^{13} \mathrm{sec}^{-1}$, which is equivalent to a recombination rate constant of about $10^{8} \mathrm{l} / \mathrm{mole}$-sec. This is certainly too low. 
Reaction: Ethanethiol (ethyl mercaptan)

$$
\begin{aligned}
& \begin{aligned}
\mathrm{C}_{2} \mathrm{H}_{5} \mathrm{SH}(\mathrm{I}) \stackrel{a}{\stackrel{b}{\longrightarrow}} \mathrm{C}_{2} \mathrm{H}_{5} \cdot(\mathrm{II})+\mathrm{HS} \cdot(\mathrm{III}) \\
\stackrel{\mathrm{C}_{2} \mathrm{H}_{4}}{(\mathrm{IV})}+\mathrm{H}_{2} \mathrm{~S}(\mathrm{~V})
\end{aligned} \\
& \begin{array}{cccccc} 
& \text { I } & \text { II } & \text { III } & \text { IV } & \text { V } \\
\Delta H_{f(298)}^{\circ} & -11.0 & 25.7 & \cdot(35.5) & 12.5 & -4.8
\end{array} \\
& \begin{array}{llllll}
S_{(298)}^{\circ} & 70.4 & 57.8 & 46.7 & 52.5 & 49.2
\end{array} \\
& \begin{array}{llllll}
C_{p(298)}^{\circ} & 17.2 & 11.9 & 7.1 & 10.4 & 8.1
\end{array}
\end{aligned}
$$

$a$

$\Delta S^{\circ}=34.1 \mathrm{~g} / \mathrm{mole}$

\begin{tabular}{|c|c|c|c|c|}
\hline$a$ & 13.48 & $63 \pm 1$ & $\begin{array}{l}785-938^{\circ} \mathrm{K} \\
\delta P \sim 0.7 \mathrm{~mm} \\
\mathrm{Hg} \\
P_{T} \sim 12 \mathrm{~mm} \mathrm{Hg}\end{array}$ & flow \\
\hline & 13.0 & $51.5 \pm 3.5$ & (as above) & $\begin{array}{l}\text { see ex- } \\
\quad \text { perimental }\end{array}$ \\
\hline
\end{tabular}

$\Delta H^{\circ}=71.0 \mathrm{kcal} / \mathrm{mole}$

$\Delta C_{p}^{\circ}=1.8 \mathrm{~g} / \mathrm{mole}$

\section{$\begin{array}{llllll}P a t h & \log A & \boldsymbol{E} & \log k_{T} & \text { Conditions } & \text { System }\end{array}$}

Preferred:

$\log k_{a}=15.8-72.2 / \theta$. Rate constants in the middle of the range are probably reliable.

Comments: See molecular eliminations for path $b$ evaluation. The frequency factor assumed for path $a$ is undoubtedly low. Scaling to the calculated activation energy obtained from the suggested reaction enthalpy gives the preferred values. One also obtains a reasonable recombination rate constant of $k_{-\prime \prime}=10^{10.2} \mathrm{l} / \mathrm{mole}$. sec.

\section{Experimental}

[1] Toluene carrier technique. (For the mechanism assumed see benzyl mercaptan.)

Path $b$ predominated at the lower temperatures since below $787^{\circ} \mathrm{K}$ no dibenzyl was formed and the products were exclusively $\mathrm{C}_{2} \mathrm{H}_{4}$ and $\mathrm{H}_{2} \mathrm{~S}$. At the higher temperatures, $\mathrm{H}_{2} \mathrm{~S}$ and $\mathrm{C}_{2} \mathrm{H}_{4}$ were the major products. but $\mathrm{H}_{2}, \mathrm{CH}_{4}$, and dibenzyl were also formed. Rate constants of path $a$ were equated to the production of $\left(\mathrm{H}_{2}+\mathrm{CH}_{4}\right)$, and those of path $b$ were obtained from $\left[\mathrm{H}_{2} \mathrm{~S}-\left(\mathrm{H}_{2}+\mathrm{CH}_{4}\right)\right]$. The molecular reaction appeared to be somewhat surface dependent at the lower temperatures. Rate constant parameters were deduced by assuming the frequency factors reported and calculating the activation energies from the rate constants. 
Reaction: Phenyl methanethiol (benzyl mercaptan)

\begin{tabular}{|c|c|c|c|}
\hline$\Delta H_{f(298)}^{\circ}$ & $\begin{array}{c}\text { I } \\
21.9\end{array}$ & $\begin{array}{c}\text { II } \\
45.0\end{array}$ & $\underset{(35.5)}{\text { III }}$ \\
\hline$S_{(298)}^{\circ}$ & 90.8 & 75.3 & 46.7 \\
\hline$C_{p(298)}^{\circ}$ & 29.9 & 25.6 & 7.1 \\
\hline$C_{p(298)}^{\circ}$ & 66.7 & 60.6 & 7.3 \\
\hline
\end{tabular}

$\Delta S^{\circ}=31.2 \mathrm{~g} / \mathrm{mole}$
$\Delta H^{\circ}=58.6 \mathrm{kcal} / \mathrm{mole}$
$\Delta C_{p}^{\circ}=2.8 \mathrm{~g} / \mathrm{mole}$
$\Delta C_{p(1000)}^{\circ}=1.2 \mathrm{~g} / \mathrm{mole}$

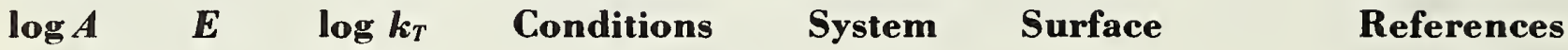

$\begin{array}{cc}53+0.46 \quad \begin{array}{l}760-1020^{\circ} \mathrm{K} \\ 0.065-1.47 \mathrm{~mm} \\ \mathrm{Hg}\end{array} & \text { flow none }\end{array}$

[1] A. H. Sehon and B. deB. Darwent, J. Am. Chem. Soc. 76, 4806 (1954).

Preferred:

$\log k=15.1-59.7 / \theta$. Rate constants in the middle of the temperature range are probably reliable.

Comments: Parameters are probably low, typical of the toluene carrier technique results. From the preferred $A$-factor and the reaction entropy one obtains $k_{\text {rec }}=10^{9.7} \mathrm{l} /$ mole-sec. This is just about the mean of the recombination rate constants for $\mathrm{H}$ and $\mathrm{CH}_{3}$ with $\emptyset \mathrm{CH}_{2} \cdot$ radicals-i.e., $10^{10.5} \mathrm{l} / \mathrm{mole}$-sec and $10^{9.0} \mathrm{l} / \mathrm{mole}$-sec, respectively.

\section{Experimental}

[1] Toluene carrier technique. Rates were based on the $\mathrm{H}_{2} \mathrm{~S}$ production which was analyzed by iodometric titration in acid solution. Products of the reaction were principally $\mathrm{H}_{2} \mathrm{~S}$ and dibenzl with minor amounts of $\mathrm{CH}_{4}, \mathrm{H}_{2}$, and stilbene. The mechanism assumed was.

$$
\begin{aligned}
& \emptyset \mathrm{CH}_{2} \mathrm{SH} \longrightarrow \emptyset \mathrm{CH}_{2} \cdot+\mathrm{SH} \cdot \\
& \mathrm{HS} \cdot+\emptyset \mathrm{CH}_{3} \longrightarrow \mathrm{H}_{2} \mathrm{~S}+\emptyset \mathrm{CH}_{2} \cdot \\
& 2 \varnothing \mathrm{CH}_{2} \cdot \longrightarrow\left(\emptyset \mathrm{CH}_{2}\right)_{2}
\end{aligned}
$$


Reaction: Thioanisole (phenylmethyl sulfide)

\begin{tabular}{|c|c|c|c|}
\hline$\Delta H_{f(298)}^{\circ}$ & $\begin{array}{c}\text { I } \\
23.5\end{array}$ & $\begin{array}{c}\text { II } \\
{[49.5]}\end{array}$ & $\begin{array}{c}\text { III } \\
34.0\end{array}$ \\
\hline$S_{(298)}^{\circ}$ & 87.7 & 76.5 & 46.4 \\
\hline$C_{p(298)}^{\circ}$ & 30.0 & 23.7 & 8.5 \\
\hline
\end{tabular}

$\Delta S^{\circ}=35.2 \mathrm{~g} / \mathrm{mole}$

$\Delta H^{\circ}=60.0 \mathrm{kcal} / \mathrm{mole}$

$\Delta C_{p}^{\circ}=2.2 \mathrm{~g} / \mathrm{mole}$

\begin{tabular}{|c|c|c|c|c|c|c|}
\hline $\log A$ & $\boldsymbol{E}$ & $\begin{array}{c}\log k_{T} \\
(900)\end{array}$ & Conditions & System & Surface & References \\
\hline 14.48 & 60.0 & -0.09 & $823-979^{\circ} \mathrm{K}$ & flow & & $\begin{array}{l}\text { [1] M. H. Back and A. H. } \\
\text { Sehon, Can. J. Chem. } \\
\mathbf{3 8 , 1 0 7 6 ~ ( 1 9 6 0 ) . ~}\end{array}$ \\
\hline
\end{tabular}

Preferred:

Reasonable. $\log k=14.48-60.0 / \theta$.

Comments: This gives, from $A$ and the reaction entropy, $k_{\mathrm{rec}}=10^{8.7} 1 / \mathrm{mole-sec}$ (assuming $\overline{\Delta C}_{p}^{\circ}=0$ ). The reported parameters, however, could be low since the $\emptyset S \cdot$ radical would be expected to have a stability similar to $\varnothing \mathrm{CH}_{2} \cdot$ and therefore participate in radical termination processes. One would expect that the rate of methane formation would be a better measure of the reaction rate.

\section{Experimental}

[1] Toluene carrier technique. Mercaptan product $(\varnothing \mathrm{SH})$ was analyzed by potentiometric titration with $\mathrm{AgNO}_{3}$. Noncondensable products were analyzed by combustion over $\mathrm{CuO}$ at $300{ }^{\circ} \mathrm{C}$. Major products were $\varnothing \mathrm{SH}, \mathrm{CH}_{4}$, and $\left(\emptyset \mathrm{CH}_{2}\right)_{2}$ with smaller yields of $\mathrm{H}_{2}$. Neither $\mathrm{C}_{2} \mathrm{H}_{6}$ nor $\mathrm{CH}_{3} \mathrm{SH}$ were detected. Rate constants were calculated from rate of formation of thiophenol. The usual mechanism was assumed (see benzyl mercaptan). 
Reaction: Methyl benzyl sulfide

\begin{tabular}{cccc}
\multicolumn{4}{r}{$\varnothing \mathrm{CH}_{2} \mathrm{SCH}_{3}(\mathrm{I}) \longrightarrow \mathrm{CH}_{2} \cdot(\mathrm{II})+\mathrm{CH}_{3} \mathrm{~S} \cdot(\mathrm{III})$} \\
$\Delta H_{f(298)}^{\circ}$ & $\mathrm{I}$ & $\mathrm{II}$ & III \\
$S_{(298)}^{\circ}$ & 19.0 & 45.0 & {$[28.0]$} \\
$C_{p(298)}^{\circ}$ & 99.5 & 75.3 & 57.2 \\
$C_{p(1000)}^{\circ}$ & 35.5 & 25.6 & 9.6 \\
& 80.3 & 60.6 & 19.4
\end{tabular}

$\Delta S^{\circ} \quad=33.0 \mathrm{~g} / \mathrm{mole}$

$\Delta H^{\circ} \quad=54.0 \mathrm{kcal} / \mathrm{mole}$

$\Delta C_{p}^{\circ} \quad=-0.3 \mathrm{~g} / \mathrm{mole}$

$\Delta C_{p(1000)}^{\circ}=-0.3 \mathrm{~g} / \mathrm{mole}$

\begin{tabular}{cccccc}
$\log A$ & $\boldsymbol{E}$ & $\begin{array}{c}\log \boldsymbol{k}_{T} \\
(830)\end{array}$ & \multicolumn{1}{c}{ Conditions } & System & Surf \\
13.48 & $51.5 \pm 2$ & -0.08 & $\begin{array}{l}818-844^{\circ} \mathrm{K} \\
0.1-1.3 \mathrm{~mm} \mathrm{Hg}\end{array}$ & flow & none \\
& & & &
\end{tabular}

\section{References}

[1] E. H. Braye, A. H. Sehon, and B. de B. Darwent, J. Am. Chem. Soc. 77, 5282 (1955).

Preferred:

$\log k=14.1-53.8 / \theta$. Rate constants are probably reliable in the middle of the temperature range.

Comments: The preferred parameters have been obtained from fitting the $A$-factor to the reaction entropy and with an assumed recombination rate constant of $k_{\text {rec }}=10^{8.8} \mathrm{l} / \mathrm{mole}$-sec (i.e., an average of the recombination rate constants of $\left(\mathrm{CH}_{3}+\emptyset \mathrm{CH}_{2} \cdot\right)$ and $\left.\left(\mathrm{C}_{2} \mathrm{H}_{5} \cdot+\emptyset \mathrm{CH}_{2} \cdot\right)\right)$. The heat of formation obtained for $\left(\mathrm{CH}_{3} \mathrm{~S} \cdot\right)$ is that given in brackets. From an analysis of all available thermal data, Kerr suggests $\Delta H_{f}^{\circ}\left(\mathrm{CH}_{3} \mathrm{~S} \cdot\right)=30$ $\mathrm{kcal} / \mathrm{mole}$ [J. A. Kerr, Chem. Revs. 66, 465 (1077)]. This value would give an even higher activation energy than the one preferred here, which is quite likely.

\section{Experimental}

[1] Toluene carrier technique. (For the mechanism assumed, see benzyl mercaptan.) Products of the decomposition were principally $\mathrm{CH}_{3} \mathrm{SH}$ and $\left(\emptyset \mathrm{CH}_{2}\right)_{2}$, with less than 17 percent $\mathrm{H}_{2} \mathrm{~S}$ and minor amounts of $\mathrm{H}_{2}$ and $\mathrm{CH}_{4}$. The origin of $\mathrm{H}_{2} \mathrm{~S}$ as a product was uncertain although it was thought to arise from the $\mathrm{CH}_{3} \mathrm{~S}$. radical. Rate constants were calculated from the $\left(\mathrm{CH}_{3} \mathrm{SH}+\mathrm{H}_{2} \mathrm{~S}\right)$ yields analyzed by standard chemical methods. 
Reaction: Methanylsulfonylphenylmethane (benzyl methyl sulfone)

\begin{tabular}{cccc}
\multicolumn{4}{c}{$\mathrm{CH}_{3} \mathrm{SO}_{2} \mathrm{CH}_{2} \varnothing(\mathrm{I}) \longrightarrow \mathrm{CH}_{2} \cdot(\mathrm{II})+\mathrm{CH}_{35} \mathrm{SO}_{2} \cdot(\mathrm{III})$} \\
$\Delta H_{f(298)}^{\circ}$ & -68.0 & II & III \\
$S_{(298)}^{\circ}$ & 104 & 75.0 & {$[-63.3]$} \\
$C_{p(298)}^{\circ}$ & 41.1 & 25.6 & 16.6 \\
$C_{p(800)}^{\circ}$ & 84.7 & 54.4 & 28.4
\end{tabular}

$\Delta S^{\circ} \quad=36.9 \mathrm{~g} / \mathrm{mole}$

$\Delta H^{\circ} \quad=49.7 \mathrm{kcal} / \mathrm{mole}$

$\Delta C_{\mu}^{\circ}=1.1 \mathrm{~g} / \mathrm{mole}$

$\Delta C_{\mu(800)}^{\circ}=-1.9 \mathrm{~g} / \mathrm{mole}$

\begin{tabular}{|c|c|c|c|c|c|c|}
\hline $\log A$ & $\boldsymbol{E}$ & $\begin{array}{c}\log \boldsymbol{k}_{r} \\
(705)\end{array}$ & Conditions & System & Surface & References \\
\hline 14.52 & 51.25 & -1.37 & $\begin{array}{l}660-750^{\circ} \mathrm{K} \\
0.34 \rightarrow 0.44 \mathrm{~mm} \\
\mathrm{Hg}\end{array}$ & flow & none & $\begin{array}{c}\text { [1] W. K. Busfield and K. J. } \\
\text { Ivin, Trans. Faraday } \\
\text { Soc. 57, 1044 (1961). }\end{array}$ \\
\hline
\end{tabular}

Preferred:

$\log k=14.52-51.25 / \theta$. (However, see dimethylsulfone.)

Comments: The kinetics are in reasonably good agreement with the thermodynamics which confirms the heat of formation of the benzyl radical and the benzyl resonance energy. A recombination rate constant of $k_{\text {rec }}=10^{8.2} \mathrm{l} /$ mole-sec is obtained from the reaction entropy and reported $A$-factor.

\section{Experimental}

[1] Toluene carrier technique. Rates were based on the iodometric titration of $\mathrm{SO}_{2}$ produced in the subsequent decomposition of the methyl sulfonyl radical $\left(\mathrm{CH}_{3} \dot{\mathrm{SO}}_{2} \longrightarrow \mathrm{CH}_{3}+\mathrm{SO}_{2}\right)$. 
Reaction: Methanesulfonylmethane (dimethyl sulfone)

\begin{tabular}{cccc}
\multicolumn{4}{r}{$\mathrm{CH}_{3} \mathrm{SO}_{2} \mathrm{CH}_{3}(\mathrm{I}) \longrightarrow \mathrm{CH}_{3} \mathrm{SO}_{2} \cdot(\mathrm{II})+\mathrm{CH}_{3} \cdot(\mathrm{III})$} \\
& $\mathrm{I}$ & $\mathrm{II}$ & $\mathrm{III}$ \\
$\Delta H_{f(298)}^{\circ}$ & -89.9 & {$[-63.3]$} & 34 \\
$S_{(298)}^{\circ}$ & 72.6 & 65.6 & 46.4 \\
$C_{p(298)}^{\circ}$ & 22.3 & 16.6 & 8.3 \\
$C_{p(800)}^{\circ}$ & 43.4 & 28.4 & 12.7
\end{tabular}

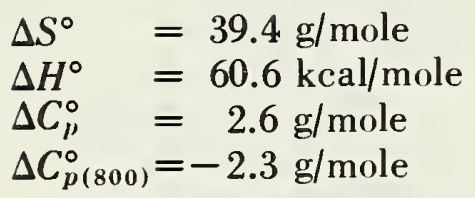

$\begin{array}{llllll}\log \boldsymbol{A} & \boldsymbol{E} & \log \boldsymbol{k}_{T} & \text { Conditions } & \text { System } & \text { Surface }\end{array}$

$\begin{array}{llllll}14.33 & 60.6 & -1.25 & 783-913^{\circ} \mathrm{K} & \text { flow } & \text { none }\end{array}$ $0.2-0.7 \mathrm{~mm} \mathrm{Hg}$
[1] W. K. Busfield and K. J. Ivin, Trans. Faraday Soc. 57, 1044 (1961).

\section{Preferred:}

$\log k=14.3-60.6 / \theta$. However, see below.

Comments: From the kinetics the heat of formation of radical $\left(\mathrm{CH}_{3} \mathrm{SO}_{2} \cdot\right)$ in brackets is obtained. One also can calculate $k_{r e c}=10^{7.8} \mathrm{l} / \mathrm{mole}-\mathrm{sec}$. The three sulfone pyrolysis by these workers are mutually consistent. That is, the heats of formation of benzyl and allyl radicals calculated from the heat of formation of $\left(\mathrm{CH}_{3} \dot{\mathrm{SO}}_{2}\right)$ obtained from the methyl sulfone pyrolysis are in reasonably good agreement with other determinations. However, because of the general systematic trends toward low Arrhenius parameters in the toluene carrier systems, and because of the relatively low value for the calculated recombination rate constant, the heat of formation of $\left(\mathrm{CH}_{3} \mathrm{SO}_{2}\right)$ should not be considered to be known with certainty. In fact, intuitively, one would be tempted to place the recombination rate constant closer to that of $\left(\mathrm{CH}_{3} \cdot+t \mathrm{Bu} \cdot\right)$ or about $10^{9 \cdot 3} 1 / \mathrm{mole}$-sec. This would raise the heat of formation of $\left(\mathrm{CH}_{3} \mathrm{SO}_{2} \cdot\right)$ to about $\Delta H_{f}^{\circ}\left(\mathrm{CH}_{3} \dot{\mathrm{SO}} 2\right) \simeq-58.0 \mathrm{kcal} / \mathrm{mole}$.

\section{Experimental}

Toluene carrier technique.

[1] Rates were based on the production of $\mathrm{SO}_{2}$, which was determined by iodometric titration. Other products were $\mathrm{CH}_{4} . \mathrm{CH}_{3} \mathrm{CH}_{2} \emptyset$, $\left(\emptyset \mathrm{CH}_{2}\right)_{2}$, and small amounts of $\mathrm{C}_{2} \mathrm{H}_{6}\left(\mathrm{CH}_{4} / \mathrm{SO}_{2}\right)$ ratios varied randomly between 1.24 and 1.46 . The fraction of $\mathrm{CH}_{3}$. radicals forming $\mathrm{CH}_{4}$ changed from 25 percent $\left(370^{\circ} \mathrm{C}\right)$ up to 70 percent $\left(600{ }^{\circ} \mathrm{C}\right)$. Analyses of ethylbenzene and dibenzyl were made by I.R. (qualitative) and by G.L.C. (semi quantitative). The mechanism assumed was,

$$
\begin{aligned}
& \mathrm{CH}_{3} \mathrm{SO}_{2} \mathrm{CH}_{3} \longrightarrow \mathrm{CH}_{3} \mathrm{SO}_{2} \cdot+\mathrm{CH}_{3} \cdot \\
& \mathrm{CH}_{3} \mathrm{SO}_{2} \cdot \longrightarrow \mathrm{CH}_{3} \cdot+\mathrm{SO}_{2} \\
& \mathrm{CH}_{3} \cdot+\emptyset \mathrm{CH}_{3} \longrightarrow \mathrm{CH}_{4}+\emptyset \mathrm{CH}_{2} \cdot \\
& \mathrm{CH}_{3} \cdot+\emptyset \mathrm{CH}_{2} \cdot \longrightarrow \emptyset \mathrm{CH}_{2} \mathrm{CH}_{3} \\
& 2 \emptyset \mathrm{CH}_{2} \cdot \longrightarrow\left(\emptyset \mathrm{CH}_{2}\right)_{2} .
\end{aligned}
$$


Reaction: 3-Methanesulfonyl-1-propene (allyl methyl sulfone)

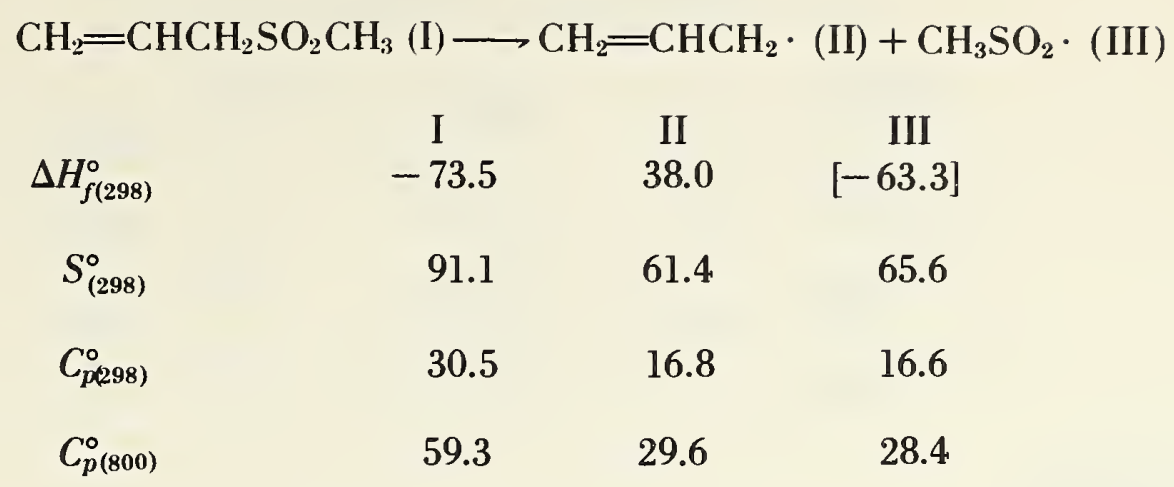

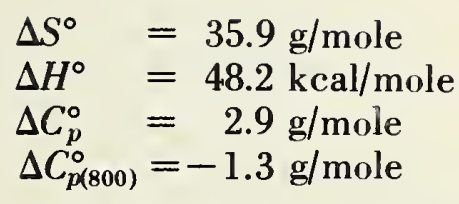

\begin{tabular}{|c|c|c|c|c|c|c|}
\hline $\log A$ & $\boldsymbol{E}$ & $\begin{array}{c}\log \boldsymbol{k}_{T} \\
(685)\end{array}$ & Conditions & System & Surface & References \\
\hline 14.1 & 47.7 & -1.12 & $\begin{array}{l}633-733^{\circ} \mathrm{K} \\
0.26-1.65 \mathrm{~mm} \\
\quad \mathrm{Hg}\end{array}$ & flow & none & $\begin{array}{c}\text { [1] W. K. Busfield and K. J. } \\
\text { Ivin, Trans. Faraday } \\
\text { Soc. } 57,1044 \text { (1961). }\end{array}$ \\
\hline
\end{tabular}

Preferred:

$\log k=14.1-47.7 / \theta$. Tentatively acceptable; however, see comments on dimethyl sulfone.

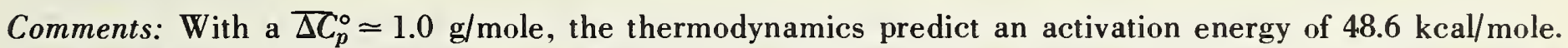
The discrepancy is within experimental error. A recombination rate constant of $k_{\text {rec }} \simeq 10^{7.8} 1 / \mathrm{mole}-\mathrm{sec}$ is obtained from the entropy and reported $A$-factor.

\section{Experimental}

[1] Toluene carrier technique. Rates were based on the iodometric titration of $\mathrm{SO}_{2}$. In addition to the expected products, small amounts of diallyl and l-butene were observed. The general reaction mechanism therefore was extended to include.

$$
\begin{aligned}
& 2 \mathrm{CH}_{2}=\mathrm{CHCH}_{2} \cdot \longrightarrow\left(\mathrm{CH}_{2}=\mathrm{CHCH}_{2}\right)_{2} \\
& \mathrm{CH}_{3} \cdot+\mathrm{CH}_{2}=\mathrm{CHCH}_{2} \cdot \longrightarrow \mathrm{CH}_{2}=\mathrm{CHCH}_{2} \mathrm{CH}_{3} .
\end{aligned}
$$

An expected termination product, 4-phenyl-1-butene, was not detected. 
Reaction: Perfluoroethane

$\begin{array}{crr} & \mathrm{C}_{2} \mathrm{~F}_{6}(\mathrm{I}) \longrightarrow 2 \mathrm{CF}_{3} \cdot(\mathrm{II}) \\ \Delta H_{f(298)}^{\circ} & \mathrm{I} & \mathrm{II} \\ S_{(298)}^{\circ} & -316.8 & (-112.5) \\ C_{p(298)}^{\circ} & 79.4 & 62.4 \\ C_{p(1500)}^{\circ} & 25.4 & 12.2 \\ & 41.4 & 19.1\end{array}$

$\begin{array}{ll}\Delta S^{\circ} & =45.4 \mathrm{~g} / \mathrm{mole} \\ \Delta H^{\circ} & =91.8 \mathrm{kcal} / \mathrm{mole} \\ \Delta C_{p}^{\circ}, & =-1.0 \mathrm{~g} / \mathrm{mole} \\ \Delta C_{p(1500)}^{\circ} & =-3.2 \mathrm{~g} / \mathrm{mole}\end{array}$

\begin{tabular}{|c|c|c|c|c|c|c|}
\hline $\log A$ & $\boldsymbol{E}$ & $\begin{array}{l}\log \boldsymbol{k}_{T} \\
(1450)\end{array}$ & Conditions & System & Surface & References \\
\hline 17.63 & $94.4 \pm 4$ & +3.40 & $\begin{array}{c}1300-1600{ }^{\circ} \mathrm{K} \\
\mathrm{Ar} / \mathrm{H}_{2} / \mathrm{C}_{2} \mathrm{~F}_{6} \sim \\
\quad(7500 / 17.4 / 1)\end{array}$ & S.P. shock & & $\begin{array}{l}\text { [1] E. Tschouikow-Roux, J. } \\
\text { Chem. Phys. 43, } 2251 \\
\text { (1965); J. Phys. Chem. } \\
\mathbf{6 9 ,} 1075 \text { (1965). }\end{array}$ \\
\hline
\end{tabular}

Preferred:

$\log k=17.27-92 / \theta$.

Comments: Initiation kinetics were deduced from the reaction mechanism and depend on the activation energy of $\mathrm{CF}_{3}+\mathrm{H}_{2}$. The author used the experimental $E$ of this reaction. However, the reaction was measured at $500{ }^{\circ} \mathrm{K}$ and should be corrected to $\sim 1450^{\circ} \mathrm{K}$. When the heat capacity correction is applied, $E_{\text {dissociation }}$ $\simeq 92 \mathrm{kcal} / \mathrm{mole}$. The kinetics agree with the thermodynamics within the error limits noted. From the entropy and the reported $A$-factor, one obtains $k_{\text {rec }}=10^{10.5} \mathrm{l} / \mathrm{mole}$-sec. (observed $k_{\text {rec }}=10^{10.36} \mathrm{l} / \mathrm{mole-sec}-\mathrm{P}$. B. Ayscough, J. Chem. Phys. 24, 944 (1956).) The heat of formation of $\mathrm{CF}_{3}$. has been determined by Whittle, using competitive bromination techniques - Trans. Faraday Soc. 61, 1182 (1965).

\section{Experimental}

[1] Analysis by G.L.C. Products were $\mathrm{HCF}_{3}$ and $\mathrm{C}_{2} \mathrm{~F}_{4}$. The reaction scheme proposed was quite complex, and included the following steps to explain the products:

$$
\begin{aligned}
& \mathrm{C}_{2} \mathrm{~F}_{6} \longrightarrow 2 \mathrm{CF}_{3} \cdot \\
& \mathrm{CF}_{3} \cdot+\mathrm{H}_{2} \longrightarrow \mathrm{CF}_{3} \mathrm{H}+\mathrm{H} \cdot \\
& \mathrm{H} \cdot+\mathrm{C}_{2} \mathrm{~F}_{6} \longrightarrow \mathrm{C}_{2} \mathrm{~F}_{5} \cdot+\mathrm{HF} \\
& \mathrm{C}_{2} \mathrm{~F}_{5} \cdot+\mathrm{H}_{2} \longrightarrow \mathrm{C}_{2} \mathrm{~F}_{5} \mathrm{H}+\mathrm{H} . \\
& \mathrm{C}_{2} \mathrm{~F}_{5} \mathrm{H} \longrightarrow \mathrm{C}_{2} \mathrm{~F}_{4}+\mathrm{HF} \\
& \mathrm{CF}_{3} \mathrm{H} \stackrel{(M)}{\longrightarrow} \mathrm{CF}_{7}:+\mathrm{HF} \\
& \mathrm{CF}_{2}:+\mathrm{CF}_{3} \mathrm{H} \longrightarrow\left(\mathrm{C}_{2} \mathrm{~F}_{5} \mathrm{H}\right)^{*} \longrightarrow \mathrm{C}_{2} \mathrm{~F}_{4}+\mathrm{HF} .
\end{aligned}
$$


Reaction: Perfluoropropene

\begin{tabular}{crcr}
\multicolumn{4}{c}{$\mathrm{CF}_{3} \mathrm{CF}=\mathrm{CF}_{2}(\mathrm{I}) \longrightarrow \mathrm{C}_{2} \mathrm{~F}_{4}(\mathrm{II})+\mathrm{CF}_{2}:(\mathrm{III})$} \\
$\Delta H_{f(298)}^{\circ}$ & $\mathrm{I}$ & $\mathrm{II}$ & \multicolumn{1}{l}{$\mathrm{III}$} \\
$S_{(298)}^{\circ}$ & $(-269.0)$ & -155.6 & -39.7 \\
$C_{p(298)}^{\circ}$ & 90.0 & 71.6 & 59.6 \\
& 26.4 & 18.4 & 9.1
\end{tabular}

$\Delta S^{\circ}=141.2 \mathrm{~g} / \mathrm{mole}$

$\Delta H^{\circ}=(73.7) \mathrm{kcal} / \mathrm{mole}$

$\Delta C_{p}^{\circ}=1.1 \mathrm{~g} / \mathrm{mole}$

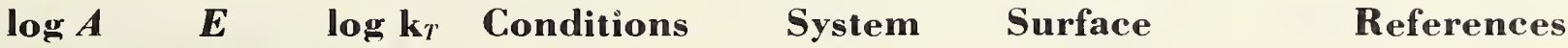 \\ $(885)$ \\ $13.0 \leqslant 75 \quad-5.52 \quad 823-948^{\circ} \mathrm{K} \quad$ static \\ [1] B. Atkinson and V. \\ Atkinson, J. Chem. Soc., 2086 (1957).}

Preferred:

Very questionable reaction.

Comments: Perhaps a more reasonable mechanism also consistent with the kinetics is

$$
\mathrm{CF}_{3} \mathrm{CF}=\mathrm{CF}_{2} \rightleftharpoons \triangle_{\mathrm{F}_{6}} ; \triangle_{\mathrm{F}_{6}} \longrightarrow \mathrm{C}_{2} \mathrm{~F}_{4}+\mathrm{CF}_{2}:
$$

\section{Experimental}

[1] Continuous reactant-product time curves were obtained by I.R. analysis. The mechanism assumed fit a $3 / 2$ order production of perfluoroisobutene and was consistent with an apparent induction period in the total pressure curve.

Mechanism proposed:

$$
\begin{aligned}
& \mathrm{CF}_{3} \mathrm{CF}=\mathrm{CF}_{2} \longrightarrow \mathrm{C}_{2} \mathrm{~F}_{4}+\mathrm{CF}_{2}: \\
& \mathrm{C}_{2} \mathrm{~F}_{4}+\mathrm{CF}_{2}: \longrightarrow \mathrm{CF}_{3} \mathrm{CF}=\mathrm{CF}_{2} \\
& \mathrm{CF}_{2}:+\mathrm{CF}_{3} \mathrm{CF}=\mathrm{CF}_{2} \longrightarrow\left(\mathrm{CF}_{3}\right)_{2} \mathrm{C}=\mathrm{CF}_{2} \\
& \mathrm{C}_{2} \mathrm{~F}_{4}+\mathrm{CF}_{3} \mathrm{CF}=\mathrm{CF}_{2} \longrightarrow\left(\mathrm{CF}_{3}\right)_{2} \mathrm{C}=\mathrm{CF}_{2}+\mathrm{CF}_{2}:
\end{aligned}
$$


Reaction: Perfluoro-2-methyl-1-propene (perfluoroisobutene)

$\begin{array}{crcr}\left(\mathrm{CF}_{3}\right)_{2} \mathrm{C}=\mathrm{CF}_{2}(\mathrm{I}) \longrightarrow \mathrm{CF}_{3} \cdot(\mathrm{II})+\mathrm{CF}_{3} \mathrm{C}=\mathrm{CF}_{2}(\mathrm{III}) \\ \Delta H_{f(298)}^{\circ} & \mathrm{I} & \mathrm{II} & \mathrm{III} \\ S_{(298)}^{\circ} & 104.4 & 62.4 & 86.6 \\ C_{1(298)}^{\circ} & 34.4 & 12.2 & 23.4\end{array}$

$\Delta S^{\circ}=44.6 \mathrm{~g} / \mathrm{mole}$

$\Delta H^{\circ}=91.5 \mathrm{kcal} / \mathrm{mole}$

$\Delta C_{p}^{\circ}=1.2 \mathrm{~g} / \mathrm{mole}$

\begin{tabular}{|c|c|c|c|c|c|c|}
\hline $\log A$ & $\boldsymbol{E}$ & $\begin{array}{r}\log \boldsymbol{k}_{V} \\
(1000)\end{array}$ & Conditions & System & Surface & References \\
\hline 14.04 & 82.7 & -4.04 & $973-1023$ \% & static & & $\begin{array}{l}\text { [1] B. Atkinson and V. } \\
\text { Atkinson, J. Chem. } \\
\text { Soc., } 2086 \text { (1957). }\end{array}$ \\
\hline
\end{tabular}

Preferred:

Suspect.

Comments: Arrhenius parameters are certainly low for this reaction. Also, the kinetic interpretation is complex enough to entail grave uncertainties. However, if the rate constant is accepted along with a reasonable recombination rate of $k_{\text {rec }} \simeq 10^{4.0} \mathrm{l} /$ mole-sec, one obtains $\log k=15.9-91.4 / \theta$ and $\Delta H_{f}^{\circ}\left(\mathrm{CF}_{3} \mathrm{C} \cdot=\mathrm{CF}_{2}\right)=$ $-176.0 \mathrm{kcal} / \mathrm{mole}$.

\section{Experimental}

[1] Products were perfluoroethane and polymer. Rates of reaction were followed by IR analyses and were shown to be first order. The mechanism proposed was

$$
\begin{aligned}
& \left(\mathrm{CF}_{3}\right)_{2} \mathrm{C}=\mathrm{CF}_{2} \longrightarrow \mathrm{CF}_{3} \cdot+\mathrm{CF}_{3} \mathrm{CF}=\mathrm{CF} . \\
& \mathrm{CF}_{3} \cdot+\left(\mathrm{CF}_{3}\right)_{2} \mathrm{C}=\mathrm{CF}_{2} \longrightarrow \mathrm{C}_{2} \mathrm{~F}_{6}+\mathrm{CF}_{3} \mathrm{CF}=\mathrm{CF} . \\
& \mathrm{CF}_{3}[\mathrm{CF}=\mathrm{CF}]_{n}+\mathrm{CF}_{3}[\mathrm{CF}=\mathrm{CF}]_{m} \longrightarrow \mathrm{CF}_{3}[\mathrm{CF}=\mathrm{CF}]_{n+m}+\mathrm{CF}_{3} . \\
& \mathrm{CF}[\mathrm{CF}=\mathrm{CF}]_{p}+\mathrm{CF}_{3}[\mathrm{CF}=\mathrm{CF}]_{q} \longrightarrow \mathrm{CF}_{3}[\mathrm{CF}=\mathrm{CF}]_{p+q} \mathrm{CF}_{3} .
\end{aligned}
$$

The parameters reported were considered minimum values for the initial split. 
Reaction: Bromomethane (methyl bromide)

$\begin{array}{cccc} & \mathrm{CH}_{3} \mathrm{Br}(\mathrm{I}) \longrightarrow \mathrm{CH}_{3} \cdot(\mathrm{II})+\mathrm{Br} \cdot(\mathrm{III}) & \\ & \mathrm{I} & \text { II } & \text { III } \\ \Delta H_{f(298)}^{\circ} & -9.3 & 34.0 & 26.7 \\ S_{(298)}^{\circ} & 58.3 & 46.5 & 41.8 \\ C_{p(298)}^{\circ} & 10.3 & 8.4 & 5.0 \\ C_{p(1000)}^{\circ} & 17.5 & 14.5 & 5.0\end{array}$

$\begin{array}{ll}\Delta S^{\circ} & =30.0 \mathrm{~g} / \text { mole } \\ \Delta H^{\circ} & =70.0 \mathrm{kcal} / \mathrm{mole} \\ \Delta C_{p}^{\circ} & =3.1 \mathrm{~g} / \mathrm{mole} \\ \Delta C_{p(1000)}^{\circ} & =2.0 \mathrm{~g} / \mathrm{mole}\end{array}$

$\log A \quad E \quad \log k$ $(1030)$

Conditions System Surface

References

13.3

$\begin{aligned} 67.5-1.02 \quad & 977-1080^{\circ} \mathrm{K} \quad \text { flow } \quad<5 \% \\ & \delta P_{o} \simeq 0.7 \mathrm{~mm} \mathrm{Hg} \\ & P_{T} \sim 5-20 \mathrm{~mm} \mathrm{Hg}\end{aligned}$
[1] A. H. Sehon and M. Szwarc, Proc. Roy. Soc. (L) A209, 110 (1951).

Preferred:

$\log k_{\text {(minimum) }}=14.2-71.7 / \theta$ (see below). Rate constants are probably low.

Comments: An RRK calculation with all possible oscillators active (i.e., $\sim n=9$ ) indicates that for the reaction conditions reported, the $\mathrm{CH}_{3} \mathrm{Br}$ decomposition is probably well into the pressure falloff region $\mathrm{RRK}$ $\left(\mathrm{M}_{1 / 2} \simeq 20 \mathrm{~mm} \mathrm{Hg}\right)$. The parameters reported, then, are certainly low as the thermodynamics would suggest. If a minimum high-pressure rate constant of twice those reported is used and the parameters are scaled to agree with $\Delta H_{r}^{\circ}$, fairly reasonable Arrhenius values are obtained.

The $A$-factor must be at least $\left(\frac{e k T}{h}\right)$, and $\Delta S^{\ddagger} \simeq 2-4 \mathrm{~g} /$ mole seems quite possible for a transition state in which the $(\mathrm{H}-\mathrm{C}-\mathrm{Br})$ bending modes have been loosened. The preferred parameters give a minimum recombination rate constant of

$$
k_{\text {rec (minimum) }} \simeq 10^{8.8} \mathrm{l} / \mathrm{mole}-\mathrm{sec},
$$

which is still about a factor of 10 lower than one would expect.

\section{Experimental}

[1] Toluene carrier technique. Rates were calculated on the basis of the $\mathrm{HBr}$ formation. Ratios of $\frac{\left(\emptyset \mathrm{CH}_{2}\right)_{2}}{(\mathrm{HBr})}$ were always less than unity and varied with temperature. The $\mathrm{HBr}$ rates of production increased with increasing $\delta P_{0}$ and $P_{T}$. The usual Arrhenius plots were therefore considered unreliable and the rate constant parameters were obtained from the rate constants and an assumed A-factor. Mechanism proposed:

$$
\begin{aligned}
& \mathrm{CH}_{3} \mathrm{Br} \longrightarrow \mathrm{CH}_{3} \cdot+\mathrm{Br} \cdot \\
& \mathrm{R}+\emptyset \mathrm{CH}_{3} \longrightarrow \mathrm{RH}+\emptyset \mathrm{CH}_{2} . \\
& 2 \emptyset \mathrm{CH}_{2} \longrightarrow\left(\emptyset \mathrm{CH}_{2}\right)_{2}
\end{aligned}
$$


Reaction: Dibromomethane (methylene dibromide)

\begin{tabular}{cccc}
\multicolumn{5}{c}{$\mathrm{CH}_{2} \mathrm{Br}_{2}(\mathrm{I}) \longrightarrow \mathrm{CH}_{2} \mathrm{Br} \cdot(\mathrm{II})+\mathrm{Br} \cdot(\mathrm{III})$} \\
$\Delta H_{f(298)}^{\circ}$ & $(\mathrm{I}$ & $\mathrm{II}$ & $\mathrm{III}$ \\
$S_{(298)}^{\circ}$ & 70.6 & 60.0 & 41.8 \\
$C_{p(298)}^{\circ}$ & 13.1 & 8.6 & 5.0
\end{tabular}

$\Delta S^{\circ}=31.2 \mathrm{~g} / \mathrm{mole}$

$\Delta H^{\circ}=(\quad) \mathrm{kcal} / \mathrm{mole}$

$\Delta C_{p}^{\circ}=0.5 \mathrm{~g} / \mathrm{mole}$

\begin{tabular}{|c|c|c|c|c|c|c|}
\hline $\log A$ & $\boldsymbol{E}$ & $\begin{array}{c}\log \boldsymbol{k}_{T} \\
(970)\end{array}$ & Conditions & System & Surface & References \\
\hline 13.3 & 62.5 & -0.78 & $\begin{array}{l}921-1021 \% \\
\delta P_{o} \sim 0.5 \mathrm{~mm} \\
\mathrm{Hg} \\
P_{T} \sim 5-20 \mathrm{~mm} \\
\quad \mathrm{Hg}\end{array}$ & flow & none & $\begin{array}{l}\text { [1] A. H. Sehon and M. } \\
\text { Szwarc, Proc. Roy. Soc. } \\
\text { (L) A209, } 110 \text { (1951). }\end{array}$ \\
\hline
\end{tabular}

Preferred:

$\log k_{\min }=14.0-65.6 / \theta$.

Comments: Arrhenius parameters are low. Reaction is probably in the pressure falloff region. (See comments on chlorobromomethane.)

\section{Experimental}

[1] Toluene carrier technique. 
Reaction: Tetrabromomethane (carbon tetrabromide)

\begin{tabular}{cccc}
\multicolumn{4}{c}{$\mathrm{CBr}_{4}(\mathrm{I}) \longrightarrow \mathrm{CBr}_{3} \cdot(\mathrm{II})+\mathrm{Br} \cdot(\mathrm{III})$} \\
$\Delta H_{f(298)}^{\circ}$ & $\mathrm{I}$ & $\mathrm{II}$ & $\mathrm{III}$ \\
$S_{(298)}^{\circ}$ & 38.0 & $(63.7 \pm 4)$ & 26.7 \\
$C_{p(298)}^{\circ}$ & 85.4 & 80.8 & 41.8 \\
& 21.8 & 16.1 & 5.0
\end{tabular}

$\Delta S^{\circ}=37.2 \mathrm{~g} / \mathrm{mole}$

$\Delta H^{\circ}=(52.4) \mathrm{kcal} / \mathrm{mole}$

$\Delta C_{p}^{\circ}=-0.7 \mathrm{~g} / \mathrm{mole}$

\begin{tabular}{|c|c|c|c|c|c|c|}
\hline $\log A$ & $\boldsymbol{E}$ & $\begin{array}{c}\log \boldsymbol{k}_{T} \\
(740)\end{array}$ & Conditions & System & Surface & References \\
\hline 13.3 & 49.0 & -1.17 & $\begin{array}{l}687-792^{\circ} \mathrm{K} \\
\delta P_{o} \simeq 0.5 \mathrm{~mm} \\
\quad \mathrm{Hg} \\
P_{T} \sim 5-20 \mathrm{~mm} \\
\quad \mathrm{Hg}\end{array}$ & flow & $\sim 32 \%$ & $\begin{array}{l}\text { [1] A. H. Sehon and M. } \\
\text { Szwarc, Proc. Roy. Soc. } \\
\text { (L) A209, } 110 \text { (1951). }\end{array}$ \\
\hline
\end{tabular}

Preferred:

$\log k_{\text {minimum }}=14.3-52.4 / \theta$. Rate constants may be low.

Comments: Arrhenius parameters are low. Reaction is probably in the pressure falloff region. (See comments on $\mathrm{CH}_{3} \mathrm{Br}$.)

\section{Experimental}

[1] Toluene carrier technique. 
Reaction: Chlorobromomethane

\begin{tabular}{cccc}
\multicolumn{4}{c}{$\mathrm{CH}_{2} \mathrm{ClBr}(\mathrm{I}) \longrightarrow \cdot \mathrm{CH}_{2} \mathrm{Cl}(\mathrm{II})+\mathrm{Br} \cdot(\mathrm{III})$} \\
& $\mathrm{I}$ & II & III \\
$\Delta H_{f(298)}^{\circ}$ & -9.0 & {$[25.3]$} & 26.7 \\
$S_{(298)}^{\circ}$ & 68.6 & 57.0 & 41.8 \\
$C_{p(298)}^{\circ}$ & 12.3 & 8.4 & 5.0
\end{tabular}

$\Delta S^{\circ}=30.2 \mathrm{~g} / \mathrm{mole}$

$\Delta H^{\circ}=61 \pm 4 \mathrm{kcal} / \mathrm{mole}$

$\Delta C_{p}^{\circ}=1.1 \mathrm{~g} / \mathrm{mole}$

\begin{tabular}{|c|c|c|c|c|c|c|}
\hline $\log A$ & $\boldsymbol{E}$ & $\begin{array}{c}\log \boldsymbol{k}_{T} \\
(755)\end{array}$ & Conditions & System & Surface & References \\
\hline 13.3 & 61.0 & -0.66 & $\begin{array}{l}909-1000{ }^{\circ} \mathrm{K} \\
\delta P_{o} \sim 0.5 \mathrm{~mm} \\
\mathrm{Hg} \\
P_{T}=5-20 \mathrm{~mm} \\
\quad \mathrm{Hg}\end{array}$ & (flow) & none & $\begin{array}{l}\text { [1] A. H. Sehon and M. } \\
\text { Szware, Proc. Roy. Soc. } \\
\text { (L) A209, } 110 \text { (1951). }\end{array}$ \\
\hline
\end{tabular}

Preferred:

$\log k_{\min }=13.7-62.8 / \theta$.

Comments: Arrhenius parameters are low. Reaction is probably in the pressure falloff region. (See comments on $\mathrm{CH}_{3} \mathrm{Br}$.)

\section{Experimental}

[1] Toluene carrier technique. Rates (based on $\mathrm{HBr}$ formation) were slightly total pressure dependent. $A$-factor was assumed. The mechanism proposed was the same as that for $\mathrm{CH}_{3} \mathrm{Br}$. 
Reaction: Dichlorobromomethane

\begin{tabular}{cccc}
\multicolumn{4}{c}{$\mathrm{CHCl}_{2} \mathrm{Br}(\mathrm{I}) \longrightarrow \mathrm{CHCl}_{2} \cdot(\mathrm{II})+\mathrm{Br} \cdot(\mathrm{III})$} \\
$\Delta H_{f(298)}^{\circ}$ & $(\mathrm{I})$ & $(\mathrm{II})$ & III \\
$S_{(298)}^{\circ}$ & 75.4 & 65.6 & 41.8 \\
$C_{p(298)}^{\circ}$ & 16.1 & 11.3 & 5.0
\end{tabular}

$\Delta S^{\circ}=32 \mathrm{~g} / \mathrm{mole}$

$\Delta H^{\circ}=(\quad) \mathrm{kcal} / \mathrm{mole}$

$\Delta C_{p}^{\circ}=0.2 \mathrm{~g} / \mathrm{mole}$

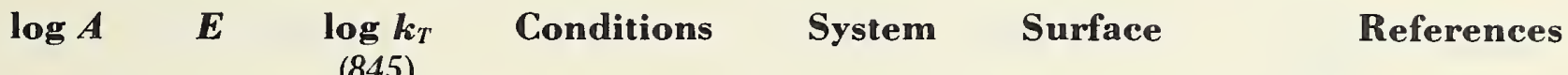

$13.3 \quad 53.5 \quad-0.54 \quad 800-891^{\circ} \mathrm{K} \quad$ flow $\quad \sim 8 \%$

$\delta P_{o} \simeq 0.5 \mathrm{~mm} \mathrm{Hg}$

$P_{T} \sim 5-20 \mathrm{~mm}$

$\mathrm{Hg}$

[1] A. H. Sehon and M.

Szwarc, Proc. Roy.

Soc. (L) A209, 110

(1951)

Preferred:

$\log k_{\min }=13.7-55.0 / \theta$.

Comments: Arrhenius parameters are low. Reaction is probably in the pressure falloff region. (See comments on $\mathrm{CH}_{3} \mathrm{Br}$.)

\section{Experimental}

[1] Toluene carrier technique. 
Reaction: Trichlorobromomethane

$\begin{array}{cccc} & \mathrm{CCl}_{3} \mathrm{Br}(\mathrm{I}) \longrightarrow \mathrm{CCl}_{3} \cdot(\mathrm{II})+\mathrm{Br} \cdot(\mathrm{III}) \\ \Delta H_{f(298)}^{\circ} & \mathrm{I} & \mathrm{II} & \mathrm{III} \\ S_{(298)}^{\circ} & -9.4 & 18.5 & 26.7 \\ C_{p(298)}^{\circ} & 79.9 & 72 & 41.8 \\ & 20.5 & 14.7 & 5.0\end{array}$

$\Delta S^{\circ}=33.9 \mathrm{~g} / \mathrm{mole}$

$\Delta H^{\circ}=54.6 \mathrm{kcal} / \mathrm{mole}$

$\Delta C_{p}^{\circ}=-0.8 \mathrm{~g} / \mathrm{mole}$

\begin{tabular}{|c|c|c|c|c|c|c|}
\hline $\log A$ & $\boldsymbol{E}$ & $\begin{array}{c}\log \boldsymbol{k}_{T} \\
(770)\end{array}$ & Conditions & System & Surface & References \\
\hline 13.3 & $50 \pm 2$ & -0.89 & $\begin{array}{l}718-827^{\circ} \mathrm{K} \\
\delta P^{\circ} \simeq 0.5 \mathrm{~mm} \\
\quad \mathrm{Hg} \\
P_{T} \simeq 5-20 \mathrm{~mm} \\
\quad \mathrm{Hg}\end{array}$ & flow & $\sim 11 \%$ & $\begin{array}{l}\text { [1] A. H. Sehon and M. } \\
\text { Szwarc Proc. Roy. } \\
\text { Soc. A209, } 110(1951) \text {; } \\
\text { J. Chem. Phys. 18, } \\
1685(1950)\end{array}$ \\
\hline
\end{tabular}

Preferred:

$\log k_{\text {(minimum })}=14.3-53.7 / \theta ; \log k_{\text {rec }(\text { minimum })}=10^{9.5} \mathrm{l} / \mathrm{mole}-\mathrm{sec}$.

Comments: Reaction is in the pressure falloff region.

\section{Experimental}

[1] Toluene carrier technique. Kinetics were followed by the rate of $\mathrm{HBr}$ formation. The above rate constant parameters have been corrected for the heterogeneous contribution to the rate of reaction. Rate constants were total pressure dependent (i.e., a factor of 4 increase in total pressure increased the rate constants by about 20 percent at the lower temperatures and nearly 60 percent at the higher temperatures). 
Reaction: Trifluorobromomethane

$\begin{array}{crcc} & \mathrm{CF}_{3} \mathrm{Br}(\mathrm{I}) \longrightarrow \mathrm{CF}_{3} \cdot(\mathrm{II})+\mathrm{Br} \cdot(\mathrm{III}) & \\ \Delta H_{f(298)}^{\circ} & \mathrm{I} & \mathrm{II} & \text { III } \\ S_{(298)}^{\circ} & -152.4 & -112.5 & 26.7 \\ C_{p(298)}^{\circ} & 71.2 & 58.8 & 41.8 \\ & 16.6 & 11.2 & 5.0\end{array}$

$\Delta S^{\circ}=29.4 \mathrm{~g} / \mathrm{mole}$

$\Delta H^{\circ}=66.6 \mathrm{kcal} / \mathrm{mole}$

$\Delta C_{p}^{\circ}=-0.4 \mathrm{~g} / \mathrm{mole}$

\begin{tabular}{|c|c|c|c|c|c|c|}
\hline $\log A$ & $\boldsymbol{E}$ & $\begin{array}{c}\log \boldsymbol{k}_{T} \\
(1055)\end{array}$ & Conditions & System & Surface & References \\
\hline 13.3 & 64.5 & -0.06 & $\begin{array}{l}102 \mathrm{l}-1090^{\circ} \mathrm{K} \\
\delta P \simeq 0.5 \mathrm{~mm} \mathrm{Hg} \\
P_{T} \sim 5-20 \mathrm{~mm} \\
\quad \mathrm{Hg}\end{array}$ & flow & $<5 \%$ & $\begin{array}{l}\text { [1] A. H. Sehon and M. } \\
\text { Szware, Proc. Roy. } \\
\text { Soc. (L) A209, } 110 \\
\text { (1951). }\end{array}$ \\
\hline
\end{tabular}

Preferred:

$\log k_{\text {minimum }}=13.7-66.3 / \theta ; k_{\text {rec (minimum) }}=10^{9.0} \mathrm{l} / \mathrm{mole}-\mathrm{sec}$.

Comments: Arrhenius parameters are low. Reaction is probably in the pressure falloff region. (See comments on $\mathrm{CH}_{3} \mathrm{Br}$.)

\section{Experimental}

[1] Toluene carrier technique. 
Reaction: Tribromomethane

$$
\begin{gathered}
\mathrm{CHBr}_{3}(\mathrm{I}) \longrightarrow \mathrm{CHBr}_{2} \cdot(\mathrm{II})+\mathrm{Br} \cdot(\mathrm{III}) \\
\Delta H_{f(298)}^{\circ} \\
S_{(298)}^{\circ} \\
C_{p(298)}^{\circ}
\end{gathered}
$$

\begin{tabular}{|c|c|c|c|c|c|c|}
\hline $\log A$ & $\boldsymbol{E}$ & $\begin{array}{c}\log k_{T} \\
(840)\end{array}$ & Conditions & System & Surface & References \\
\hline 13.3 & 55.5 & -1.14 & $\begin{array}{l}786-899^{\circ} \mathrm{K} \\
\delta P=0.5 \text { torr } \\
P_{T}=5-20 \text { torr }\end{array}$ & flow & $\sim 12 \%$ & $\begin{array}{l}\text { [1] A. H. Sehon and M. } \\
\text { Szwarc, Proc. Roy. } \\
\text { Soc. (L) A209, } 110 \\
\text { (1951). }\end{array}$ \\
\hline
\end{tabular}

$$
\Delta S^{\circ}=
$$

$\Delta H^{\circ}=$

$\Delta C_{p}^{\circ}=$

\section{Preferred:}

$\log k_{\text {minimum }}=14.2-59.0 / \theta$.

Reported parameters are probably low.

\section{Experimental}

[1] Toluene carrier technique. (See comments on $\mathrm{CH}_{3} \mathrm{Br}$.) 
Reaction: Bromobenzene

\begin{tabular}{cccc}
\multicolumn{4}{c}{$\emptyset \mathrm{Br}(\mathrm{I}) \longrightarrow \emptyset \cdot(\mathrm{II})+\mathrm{Br} \cdot(\mathrm{III})$} \\
$\Delta H_{f(298)}^{\circ}$ & $\mathrm{I}$ & $\mathrm{II}$ & $\mathrm{III}$ \\
$S_{(298)}^{\circ}$ & 25.8 & 80.0 & 26.7 \\
$C_{p(298)}^{\circ}$ & 77.9 & 67.9 & 41.8 \\
& 22.7 & 18.5 & 5.0
\end{tabular}

$\Delta S^{\circ}=31.8 \mathrm{~g} / \mathrm{mole}$

$\Delta H^{\circ}=80.9 \mathrm{kcal} / \mathrm{mole}$

$\Delta C_{p}^{\circ}=0.8 \mathrm{~g} / \mathrm{mole}$

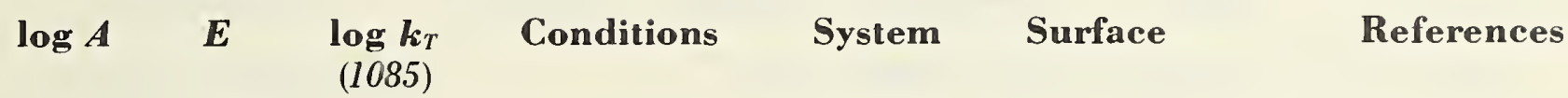

$13.3 \quad 70.9 \quad-0.98 \quad 1030-1143{ }^{\circ} \mathrm{K} \quad$ flow

$\delta P_{0} \simeq 0.12-1.24$

[1] D. Williams and M.

$\mathrm{mm} \mathrm{Hg}$

$P_{T} \simeq 15 \mathrm{~mm} \mathrm{Hg}$

Szwarc, J. Chem. Phys.

20, 1171 (1952).

$13.3 \quad 70.9 \quad-0.98 \quad 1023-1143^{\circ} \mathrm{K} \quad$ flow

$\delta P_{o} \simeq 0.13-0.78$

$\mathrm{mm} \mathrm{Hg}$

$P_{T} \simeq 12-15 \mathrm{~mm}$

$\mathrm{Hg}$

[2] M. Ladacki and M.

Szwarc, Proc. Roy.

Soc. (L) A219,

341 (1953).

Preferred:

$\log k=15.3-80.9 / \theta$.

Comments: The thermodynamics indicate that the reported parameters are low. If one assumes that the observed rate constants are roughly representative of the decomposition rate and adjusts the Arrhenius parameters to the reaction enthalpy, one obtains the preferred values. These are consistent with $k_{\text {rec }}=10^{10.4}$ l/mole-sec.

\section{Experimental}

[1] Toluene carrier technique. Rates were based on $\mathrm{HBr}$ formation. 
Reaction: Substituted bromobenzenes

$$
\begin{gathered}
(X-\varnothing \mathrm{Br}) \longrightarrow X-\emptyset \cdot+\mathrm{Br} \cdot \\
\Delta H_{f(298)}^{\circ} \\
S_{(298)}^{\circ} \\
C_{p(298)}^{\circ}
\end{gathered}
$$

\begin{tabular}{|c|c|c|c|c|c|c|}
\hline $\boldsymbol{X}$ & $\log A$ & $\boldsymbol{E}$ & $\log k_{T}$ & Conditions & System & Surface \\
\hline$p-\mathrm{F}$ & 13.3 & 70.4 & $-1.01(1075)$ & $1053-1098^{\circ} \mathrm{K}$ & flow & \\
\hline$p-\mathrm{Cl}$ & 13.3 & 70.3 & $-0.93(1080)$ & $1044-1119^{\circ} \mathrm{K}$ & & \\
\hline$m-\mathrm{Cl}$ & 13.3 & 69.9 & $-0.90(1070)$ & $1033-1107^{\circ} \mathrm{K}$ & & \\
\hline$o-\mathrm{Cl}$ & 13.3 & 69.7 & $-0.81(1080)$ & $1043-1113^{\circ} \mathrm{K}$ & & \\
\hline$p-\mathrm{Br}$ & 13.6 & 70.6 & $-0.69(1080)$ & $1047-1113^{\circ} \mathrm{K}$ & & \\
\hline$o-\mathrm{Br}$ & 13.6 & 69.1 & $-0.45(1075)$ & $1041-1111^{\circ} \mathrm{K}$ & & \\
\hline$p-\mathrm{CH}_{3}$ & 13.3 & 70.7 & $-1.08(1075)$ & $1046-1100^{\circ} \mathrm{K}$ & & \\
\hline$m-\mathrm{CH}_{3}$ & 13.3 & 70.7 & $-1.14(1070)$ & $1044-1098^{\circ} \mathrm{K}$ & & \\
\hline$o-\mathrm{CH}_{3}$ & 13.3 & 70.1 & $-1.02(1070)$ & $1043-1096^{\circ} \mathrm{K}$ & & \\
\hline$p-\emptyset$ & 13.3 & 70.7 & $-1.14(1070)$ & $1036-1100^{\circ} \mathrm{K}$ & & \\
\hline$m-\varnothing$ & 13.3 & 70.1 & $-0.82(1085)$ & $1047-1119^{\circ} \mathrm{K}$ & & \\
\hline$o-\varnothing$ & 13.3 & 68.2 & $-0.70(1065)$ & $1031-1103^{\circ} \mathrm{K}$ & & \\
\hline$p-\mathrm{CN}$ & 13.3 & 70.6 & $-1.06(1075)$ & $1042-1109^{\circ} \mathrm{K}$ & & \\
\hline$m-\mathrm{CN}$ & 13.3 & 70.1 & $-1.09(1065)$ & $1042-1090^{\circ} \mathrm{K}$ & & \\
\hline$o-\mathrm{CN}$ & 13.3 & 70.3 & $-0.99(1075)$ & $1048-1098^{\circ} \mathrm{K}$ & & \\
\hline$p-\mathrm{OH}$ & 13.3 & 67.0 & $-0.45(1065)$ & $1035-1093^{\circ} \mathrm{K}$ & & \\
\hline$o-$ & 13.3 & 67.1 & $-1.01(1025)$ & $1005-1043^{\circ} \mathrm{K}$ & & \\
\hline
\end{tabular}

[1] M. Szwarc and D. Williams, Proc. Roy. Soc. (L) A219, 353 (1953).

Preferred:

Reported Arrhenius parameters are suspect (see bromobenzene).

Comments: The assumption regarding the $A$-factors of these reactions (see below) would seem to be reasonable except for compounds with ortho substituents where the thermodynamic and kinetic properties should reflect the interraction of neighboring groups. The kinetic parameters reported for the substituted bromobenzenes should have the same reliability as those for the parent compound (i.e., bromobenzene). It would appear that they are all low, although the rate constants should be nearly correct in the middle of the temperature range.

\section{Experimental}

[1] Toluene carrier technique. Partial pressures of the individual compounds were not varied appreciably from about $0.2 \mathrm{~mm} \mathrm{Hg}$. Rates were based on $\mathrm{HBr}$ formation. $A$-factors were assumed to be the same as that observed for bromobenzene. 


\section{Reaction: 3-Bromopyridene (a)}

2-Bromopyridene $(b)$

$$
\begin{aligned}
& \mathrm{C}_{N}^{\mathrm{Br}}(\mathrm{I}) \stackrel{\mathrm{a}}{\mathrm{F}_{N}}(\mathrm{II})+\mathrm{Br} \text { (III) } \\
& \left(\mathrm{Cl}_{\mathrm{Br}}\right) \\
& \text { (I) } \stackrel{b}{\text { (II) }}+\mathrm{Br}_{\mathrm{N}} \text {. (III) } \\
& \Delta H_{f(298)}^{\circ} \\
& \text { I } \\
& \text { II } \\
& \text { III } \\
& {\left[\begin{array}{lll}
{[39.5]} & (\quad) & 26.7
\end{array}\right.} \\
& S_{(298)}^{\circ}
\end{aligned}
$$

$$
\begin{aligned}
& a \\
& \Delta S^{\circ}=33.9 \mathrm{~g} / \mathrm{mole} \\
& \Delta H^{\circ}= \\
& \Delta C_{\underline{!}}^{\circ}=0.8 \mathrm{~g} / \mathrm{mole}
\end{aligned}
$$

$\begin{array}{lllllll}\text { Path } & \log A & \boldsymbol{E} & \log k_{T} & \text { Conditions } & \text { System } & \text { Surface }\end{array}$ $(1200)$

$a$

$\begin{array}{ccc}13.3 \quad 75.9-0.53 \quad & 1179-1220^{\circ} \mathrm{K} \\ & \delta P_{o} \simeq 0.5 \mathrm{~mm} \\ & & \text { flow }\end{array}$

$(1085)$

$b$

13.3

$$
\begin{aligned}
& 71.5-1.10 \quad 1054-1115^{\circ} \mathrm{K} \\
& \delta P_{o} \simeq 0.25 \mathrm{~mm} \mathrm{Hg}
\end{aligned}
$$

Preferred:

The Arrhenius parameters are undoubtedly low (see bromobenzene). However, the rate constants around $\bar{T}$ should be reasonably reliable.

\section{Experimental}

[1] Toluene carrier technique. Rates were based on $\mathrm{HBr}$ formation. An $A$-factor equal to that of bromobenzene was assumed. 
Reaction: 2-Bromonaphthalene ( $\beta$-bromonaphthalene) ( $a$ )

1-Bromonaphthalene ( $\alpha$-bromonaphthalene) (b)

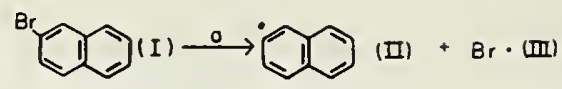

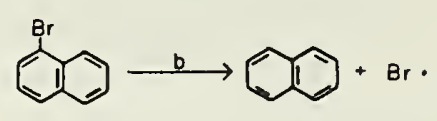

$\Delta H_{f(298)}^{\circ}$
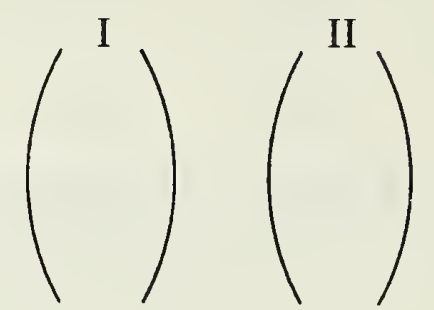

III

26.7

$S_{(298)}^{\circ}$

$C_{p(298)}^{\circ}$

$\Delta S^{\circ}=$

$\Delta H^{\circ}=$

$\Delta C_{p}^{\circ}=$

\begin{tabular}{|c|c|c|c|c|c|c|c|}
\hline Path & $\log A$ & $\boldsymbol{E}$ & $\begin{array}{l}\log \boldsymbol{k}_{T} \\
(1075)\end{array}$ & Conditions & System & Surface & References \\
\hline$a$ & 13.2 & 70.0 & -1.03 & $1022-1133^{\circ} \mathrm{K}$ & flow & none & $\begin{array}{l}\text { [1] M. Ladaki and M. } \\
\text { Szwarc, Proc Roy. }\end{array}$ \\
\hline$b$ & 13.5 & 70.9 & -0.92 & $\begin{array}{l}1014-1133^{\circ} \mathrm{K} \\
\delta P \simeq 0.3 \text { torr } \\
P_{T} \simeq 10-15 \text { tor }\end{array}$ & & & $\begin{array}{l}\text { Soc. (L) A2 19, } 341 \\
\text { (1953); J. Chem. } \\
\text { Phys. 20, 1814 } \\
\text { (1952). }\end{array}$ \\
\hline
\end{tabular}

46.1

5.0

\section{Preferred:}

These reactions, as noted by the authors, should be very similar to bromobenzene. Since slightly higher parameters are more reasonable for bromobenzene, the above should also probably be scaled correspond. ingly upward. Rate constants for decomposition are probably reliable around $T_{m}$.

\section{Experimental}

[1] Toluene carrier technique. $\delta P$ were varied over a factor of 3 to 5 with no effect on the rates. Rates based on $\mathrm{HBr}$ formation. Some difficulties in obtaining the absolute value of the frequency factors was noted, although activation energies were considered reliable. 
Reaction: 9-Bromophenanthrene (a)

9-Bromoanthracene $(b)$
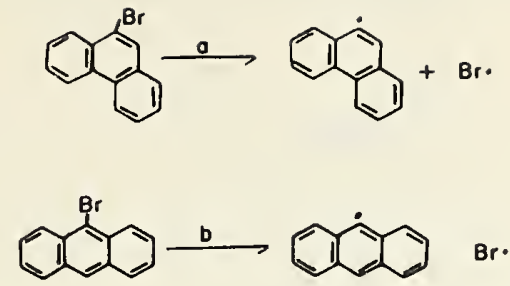

$$
\begin{gathered}
\Delta H_{f(298)}^{\circ} \\
S_{(298)}^{\circ} \\
C_{p(298)}^{\circ}
\end{gathered}
$$

$$
\begin{aligned}
& \Delta S^{\circ}= \\
& \Delta H^{\circ}= \\
& \Delta C_{p}^{\circ}=
\end{aligned}
$$

$\begin{array}{llllll}\text { Path } & \log A & \boldsymbol{E} & \log \boldsymbol{k}_{T} & \text { Conditions } & \text { System }\end{array}$ (1065)

13.0

$$
67.7-0.90 \quad 1002-1125^{\circ} \mathrm{K} \quad \text { flow }
$$

none

\section{References}

[1] M. Ladaki and M. Szware, Proc. Roy. Soc. (L) A219, 341 (1953); J. Chem. Phys. 20, 1814 (1952).

(1015)
$b$
13.2
$65.6-0.93$
$964-1062^{\circ} \mathrm{K}$
$\delta P \simeq 0.3$ torr
$P_{T} \simeq 10-15$

torr

Preferred:

These reactions, as noted by the authors, should be very similar to bromobenzene. Since slightly higher parameters are more reasonable for bromobenzene, the above should also be scaled correspondingly upward. Rate constants for decomposition are probably realiable.

\section{Experimental}

[1] Toluene carrier technique. $\delta P$ were varied over a factor of 3 to 5 with no effect on the rates. Rates based on $\mathrm{HBr}$ formation. Some difficulties in obtaining the absolute value of the frequency factors was noted, although activation engeries were considered reliable. 
Reaction: 2-Bromothiophene

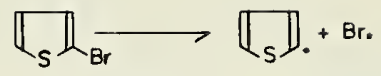

$\Delta H_{f(298)}^{c}$

$S_{(298)}^{\circ}$

$C_{p(298)}^{\circ}$

$\Delta S^{\circ}=$
$\Delta H^{\circ}=$
$\Delta C_{p}^{\circ}=$

$\log A$

$\begin{array}{rrrr}\log k_{T} & \text { Conditions } & \text { System } & \text { Surface } \\ (1055) & & & \end{array}$

References

$$
\begin{array}{lll}
13.3 \quad 68.5-0.89 & 1028-1082^{\circ} \mathrm{K} \\
& & \delta P_{o}=0.5 \mathrm{~mm} \mathrm{Hg}
\end{array}
$$

[1] M. Szwarc and D.

Williams, Proc. Roy.

Soc. A219, 353 (1953).

Preferred:

The Arrhenius parameters are undoubtedly low (see bromobenzene). Rate constants around $\bar{T}$ are probably reliable.

\section{Experimental}

[1] Toluene carrier technique. Rates were based on $\mathrm{HBr}$ formation. An $A$ factor equal to that of bromobenzene was assumed. 
Reaction: 3-Bromo-1-propene (allylbromide)

$\begin{array}{cccc}\mathrm{CH}_{2}=\mathrm{CHCH}_{2} \mathrm{Br}(\mathrm{I}) \longrightarrow \mathrm{CH}_{2}=\mathrm{CHCH}_{2} \cdot(\mathrm{II})+\mathrm{Br} \cdot(\mathrm{III}) \\ \Delta H_{f(298)}^{\circ} & \mathrm{I} & \text { II } & \text { III } \\ S_{(298)}^{\circ} & 10.9 & 40.6 & 26.7 \\ C_{p(298)}^{\circ} & 75.8 & 61.4 & 41.8 \\ & 18.7 & 14.4 & 5.0\end{array}$

\begin{abstract}
$\Delta S^{\circ}=27.4 \mathrm{~g} / \mathrm{mole}$
$\Delta H^{\circ}=56.4 \mathrm{kcal} / \mathrm{mole}$

$\Delta C_{p}^{\circ}=0.7 \mathrm{~g} / \mathrm{mole}$
\end{abstract}

\begin{tabular}{|c|c|c|c|c|c|c|}
\hline $\log A$ & $\boldsymbol{E}$ & $\begin{array}{l}\log \boldsymbol{k}_{T} \\
(800)\end{array}$ & Conditions & System & Surface & References \\
\hline 12.7 & $47.5 \pm 2$ & -0.28 & $732-865^{\circ} \mathrm{K}$ & flow & $<5 \%$ & $\begin{array}{l}\text { [1] M. Szware, B. N. Ghosh, } \\
\text { and A. H. Sehon, } \\
\text { J. Chem. Phys. 18, } \\
\text { 1142 (1950); M. Szwarc } \\
\text { and B. N. Ghosh, J. } \\
\text { Chem. Phys. 17, } 744 \\
\text { (1949). }\end{array}$ \\
\hline 12.3 & 45.5 & -0.13 & $593-653^{\circ} \mathrm{K}$ & static & $<1 \%$ & $\begin{array}{l}\text { [2] A. Maccoll, J. Chem. } \\
\text { Phys. 17, } 1350 \text { (1949). }\end{array}$ \\
\hline
\end{tabular}

\title{
Preferred:
}

Unreliable. We favor: $\log k=14.3-56.4 / \theta$.

Comments: The reaction rates of both studies are faster (by about a factor of 10-100) than is reasonable on the basis of the thermodynamics and a reasonable recombination rate constant. Chain decomposition of the allylbromide is therefore indicated. This is supported by the poor mass balances in the system and the deficiencies of termination product yields relative to $\mathrm{HBr}$ yields.

A very reasonable chain process for $\mathrm{HBr}$ formation exists in this system:

$$
\begin{aligned}
& \mathrm{R} \cdot+\mathrm{CH}_{2}=\mathrm{CHCH}_{2} \mathrm{Br} \longrightarrow \mathrm{RCH}_{2} \mathrm{CHCH}_{2} \mathrm{Br} \\
& \mathrm{RCH}_{2} \mathrm{CHCH}_{2} \mathrm{Br} \longrightarrow \mathrm{RCH}_{2} \mathrm{CH}=\mathrm{CH}_{2}+\mathrm{Br} .
\end{aligned}
$$

where $\mathrm{R} \cdot=\varnothing \mathrm{CH}_{2} \cdot$ or $\mathrm{CH}_{2}=\mathrm{CHCH}_{2}$.

Since $\mathrm{HBr}$ is a better radical trap than toluene (by at least a factor of $10^{2}$ ) most of the propylene formed probably came from the reaction:

$$
\mathrm{CH}_{2}=\mathrm{CHCH}_{2}+\mathrm{HBr} \longrightarrow \mathrm{CH}_{2}=\mathrm{CHCH}_{3}+\mathrm{Br} \text {. }
$$

It is also quite likely that all the allyl radicals formed were trapped as propylene, and that the propylene would have been the better measure of the rate of the $(\mathrm{C}-\mathrm{Br})$ dissociation. With regard to study [2], the observed first-order kinetics were equated to the initiation step of a complex free radical mechanism which could not be given in any detail. It was assumed that no chain decomposition occurred since addition 
of propylene did not appreciably alter the reaction rate. However, if the chain process suggested were operative, no inhibition would be expected and this assumption regarding chains would then be unjustified.

\section{Experimental}

[1] Toluene carrier technique. Rates were based on $\mathrm{HBr}$ formation. The (dibenzyl/HBr) ratios varied from $\sim 0.2$ $\left(766^{\circ} \mathrm{K}\right)$ to $\sim 0.4\left(865^{\circ} \mathrm{K}\right)$. Large amounts of propylene were observed but very little allene $(<1$ percent propylene). Propylene yields. however, were considerably smaller ( $\sim 20$ percent $)$ than those of $\mathrm{HBr}$. The usual mechanism was assumed with the addition of the two termination reactions: 2 allyl $\longrightarrow$ diallyl; allyl $+\emptyset \mathrm{CH}_{2} \cdot \longrightarrow$ products.

[2] Rates were followed manometrically and by $\mathrm{HBr}$ formation. Rate constants were deduced from an assumed free radical mechanism. Products identified were isopropyl bromide, benzene, and 1-bromopropene. 
Reaction: Bromophenylmethane (benzyl bromide)

\begin{tabular}{cccc}
\multicolumn{3}{r}{$\emptyset \mathrm{CH}_{2} \mathrm{Br}(\mathrm{I})$} & \multicolumn{5}{c}{$\varnothing \mathrm{CH}_{2} \cdot(\mathrm{II})+\mathrm{Br} \cdot(\mathrm{III})$} \\
& $\mathrm{I}$ & $\mathrm{II}$ & III \\
$\Delta H_{f(298)}^{\circ}$ & 16.0 & 45.0 & 26.7 \\
$S_{(298)}^{\circ}$ & 88.4 & 75.3 & 41.8 \\
$C_{p(298)}^{\circ}$ & 28.2 & 25.6 & 5.0 \\
$C_{p(1000)}^{\circ}$ & $(64.5)$ & 60.6 & 5.0
\end{tabular}

$\Delta S^{\circ}=28.7 \mathrm{~g} / \mathrm{mole}$

$\Delta H^{\circ}=55.7 \mathrm{kcal} / \mathrm{mole}$

$\Delta C_{p}^{\circ}=2.4 \mathrm{~g} / \mathrm{mole}$

$\Delta C_{p}^{\circ}=1.1 \mathrm{~g} / \mathrm{mole}$

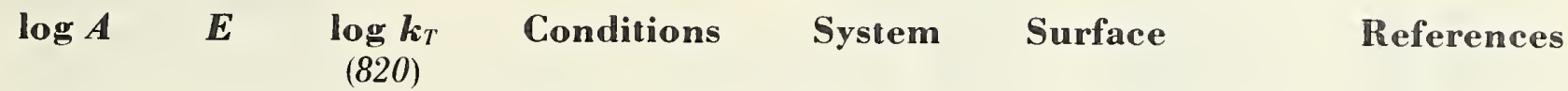

$\begin{array}{lllll}13.0 & 50.5 & -0.46 & 768-872^{\circ} \mathrm{K} \quad \text { flow } & \text { none }\end{array}$

$\delta P=0.01-2.3$

$\mathrm{mm} \mathrm{Hg}$

$P_{T} \simeq 8.7-15.2$

$\mathrm{mm} \mathrm{Hg}$

\section{Preferred:}

$\log k=14.6-56.7 / \theta$.

Comments: The reaction enthalpy has been well established; thus the reported parameters are low. Assuming valid rate constants and scaling to the reaction enthalpy gives the preferred values. These are consistent with a recombination rate constant of $k_{\text {rec }}=10^{9.9} \mathrm{l} /$ mole-sec.

\section{Experimental}

[1] Toluene carrier technique. Rates were based on the rate of formation of HBr. The mechanism proposed was:

$$
\begin{aligned}
& \emptyset \mathrm{CH}_{2} \mathrm{Br} \longrightarrow \varnothing \mathrm{CH}_{2} \cdot+\mathrm{Br} \cdot \\
& \mathrm{Br} \cdot+\varnothing \mathrm{CH}_{3} \longrightarrow \mathrm{HBr}+\emptyset \mathrm{CH}_{2} \cdot \\
& 2 \varnothing \mathrm{CH}_{2} \cdot \longrightarrow\left(\varnothing \mathrm{CH}_{2}\right)_{2}
\end{aligned}
$$

$\left(\mathrm{HBr} /\left(\varnothing \mathrm{CH}_{2}\right)_{2}\right)$ ratios were unity within experimental error; $\left(\mathrm{H}_{2} / \mathrm{CH}_{4}\right) \simeq 3 / 2$, as found in the toluene pyrolysis. The latter is indicative of secondary reactions. 
Reaction: Substituted bromophenylmethanes (substituted benzylbromides)

$$
\begin{gathered}
\left(X-\varnothing \mathrm{CH}_{2} \mathrm{Br}\right)(\mathrm{I}) \longrightarrow \\
\Delta H_{f(298)}^{\circ} \\
S_{(298)}^{\circ} \\
C_{p(298)}^{\circ}
\end{gathered}
$$

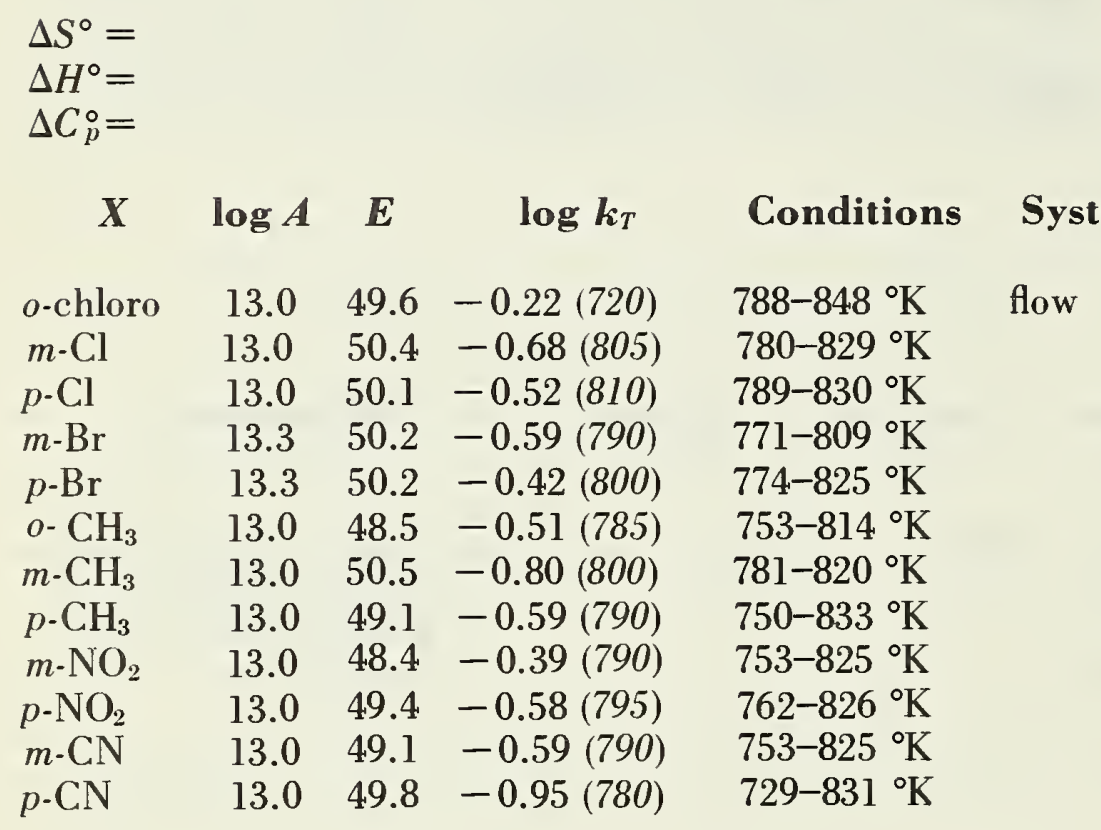

References

[1] C. H. Leigh, A. H. Sehon, and M. Szwarc, Proc. Roy. Soc. (L) A2 09, 97 (1951).

\section{Preferred:}

The above parameters should all be scaled up a factor of 40 in $A$ and about $6.0 \mathrm{kcal} / \mathrm{mole}$ in $E$ (see bromotoluene). Rate constants are probably reliable around $\bar{T}$.

\section{Experimental}

[1] Toluene carrier technique. Rates were found to be independent of the substituted benzylbromide and toluene pressures. Arrhenius parameters were obtained from the rate constants using the assumption that all $A$-factors should be the same as that for the benzyl bromide decomposition. 
Reaction: $p$-Bromomethyltoluene ( $p$-xylyl bromide) $(a)$

$\omega, \omega^{\prime}$-dibromo para-xylene (b)

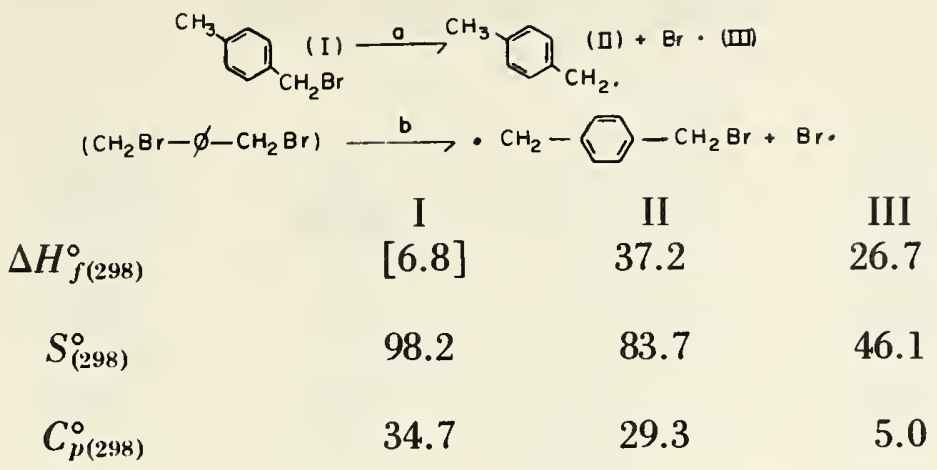

$$
\begin{aligned}
& a \\
& \Delta S^{\circ}=31.6 \mathrm{~g} / \mathrm{mole} \\
& \Delta H^{\circ}= 57.1 \mathrm{kcal} / \mathrm{mole} \\
& \Delta C_{p}^{\circ}=-0.4 \mathrm{~g} / \mathrm{mole}
\end{aligned}
$$

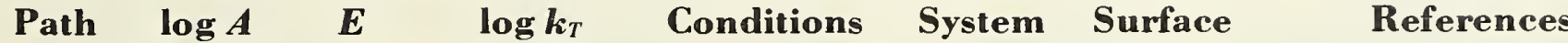 (780)}

$$
a
$$

$50.5-1.15$

$748-814^{\circ} \mathrm{K}$ $\delta P \simeq 0.05-1.0$

$\mathrm{mm} \mathrm{Hg}$

[1] M. Levy, M. Szwarc and J. Throssell,

J. Chem. Phys. 22, 1904 (1954).

(775)

$$
\begin{array}{cccc}
b \quad & 13.3 & 50.5 \quad-0.94 \quad & 748-802{ }^{\circ} \mathrm{K} \\
& & & \delta P \simeq 0.05 \mathrm{~mm} \\
& & \\
& & \mathrm{Hg}
\end{array}
$$

\section{Preferred:}

The parameters should be similar to those of benzylbromide:

$\log k_{a}=14.6-56.6 / \theta$.

$\log k_{b}=14.9-56.6 / \theta$.

Comments: The authors appear to have discounted their rate constants at the lower temperature. If all the data are used, an activation energy of $54.5 \mathrm{kcal} / \mathrm{mole}$ is obtained.

\section{Experimental}

[1] Toluene carrier technique. Rates were based on $\mathrm{HBr}$ formation. 
Reaction: Benzoyl bromide

$\begin{array}{cccc} & \emptyset \mathrm{COBr}(\mathrm{I}) \longrightarrow \emptyset \mathrm{CO}(\mathrm{II})+\mathrm{Br} .(\mathrm{III}) & \\ & & & \\ \Delta H_{f(298)}^{\circ} & \mathrm{I} & \mathrm{II} & \mathrm{III} \\ \mathrm{S}_{(\mathbf{2 9 8 )}}^{\circ} & -12.3 & {[21.0]} & 26.7 \\ C_{p(298)}^{\circ} & 94.3 & 79.5 & 46.1 \\ C_{p(1000)}^{\circ} & 31.3 & 26.0 & 5.0 \\ & (63.9) & 57.6 & 5.0\end{array}$

$\Delta S^{\circ}=31.3 \mathrm{~g} / \mathrm{mole}$

$\Delta H^{\circ}=60.0 \mathrm{kcal} / \mathrm{mole}$

$\Delta C_{p}^{\circ}=-0.3 \mathrm{~g} / \mathrm{mole}$

$\left\langle\Delta C_{\mu}^{\circ}\right\rangle=(-1.3) \mathrm{g} / \mathrm{mole}$

\begin{tabular}{|c|c|c|c|c|c|c|}
\hline $\log A$ & $\boldsymbol{E}$ & $\begin{array}{l}\log \boldsymbol{k}_{T} \\
(880)\end{array}$ & Conditions & System & Surface & References \\
\hline 13.7 & 57.0 & -0.46 & $\begin{array}{l}814-946^{\circ} \mathrm{K} \\
\Delta P=0.1-2.45 \\
\quad \mathrm{~mm} \mathrm{Hg} \\
P_{T}=5-16 \mathrm{~mm}\end{array}$ & flow & none & $\begin{array}{l}\text { [1] M. Ladaki, C. H. Leigh, } \\
\text { and M. Szwarc, Proc. } \\
\text { Roy. Soc. A2 14, } 273 \\
\text { (1952). }\end{array}$ \\
\hline
\end{tabular}

Preferred:

$\log k=14.3-59.7 / \theta$ (see below).

Comments: Parameters have been adjusted to the estimated reaction enthalpy. The recombination rate constant calculated from the $A$-factor and reaction entropy is then quite reasonable. $k_{\text {rec }}=10^{9.5} \mathrm{l} / \mathrm{mole}$-sec.

\section{Experimental}

[1] Toluene carrier technique. Rate by $(\mathrm{CO})$ production. $\Delta(\mathrm{CO})=\Delta(\mathrm{HBr})$. 
Reaction: Chlorophenylmethane (benzyl chloride)

\begin{tabular}{cccc}
\multicolumn{5}{c}{$\emptyset \mathrm{CH}_{2} \mathrm{Cl}(\mathrm{I}) \longrightarrow \varnothing \mathrm{CH}_{2} \cdot(\mathrm{II})+\mathrm{Cl} \cdot(\mathrm{III})$} \\
& I & II & III \\
$\Delta H_{f(298)}^{\circ}$ & $(7.0)$ & 45.0 & 29.0 \\
$S_{(298)}^{\circ}$ & 85.4 & 75.3 & 39.5 \\
$C_{p(2 ! 18)}^{\circ}$ & 28.8 & 25.2 & 5.2
\end{tabular}

$\Delta S^{\circ}=29.4 \mathrm{~g} / \mathrm{mole}$

$\Delta H^{\circ}=67.0 \mathrm{kcal} / \mathrm{mole}$

$\Delta C_{p}^{\circ}=1.6 \mathrm{~g} / \mathrm{mole}$

\begin{tabular}{|c|c|c|c|c|c|c|}
\hline $\log A$ & $\boldsymbol{E}$ & $\underset{(990)}{\log \boldsymbol{k}_{T}}$ & Conditions & System & Surface & References \\
\hline 14.83 & 68.0 & -0.18 & $\begin{array}{l}922-1020^{\circ} \mathrm{K} \\
\delta P \simeq 0.12-0.66 \\
\quad \mathrm{~mm} \mathrm{Hg}\end{array}$ & flow & $\sim 10 \%$ & $\begin{array}{l}\text { [1] M. Szwarc and J. W. } \\
\text { Taylor, J. Chem. } \\
\text { Phys. 22, } 270 \text { (1954). }\end{array}$ \\
\hline
\end{tabular}

Preferred:

Reliable. $\log k=14.8-68.0 / \theta$.

From $A$ and the reaction entropy one obtains: $k_{\text {rec }}=10^{9.6} \mathrm{l} / \mathrm{mole}-\mathrm{sec}$.

Experimental

[1] Toluene carrier technique. Rates based on $\mathrm{HCl}$ formation. $\Delta(\mathrm{HCl})=\Delta\left(\varnothing \mathrm{CH}_{2}\right)_{2}$ as required by the usual mechanism (see benzyl bromide). 
Reaction: Benzoyl chloride

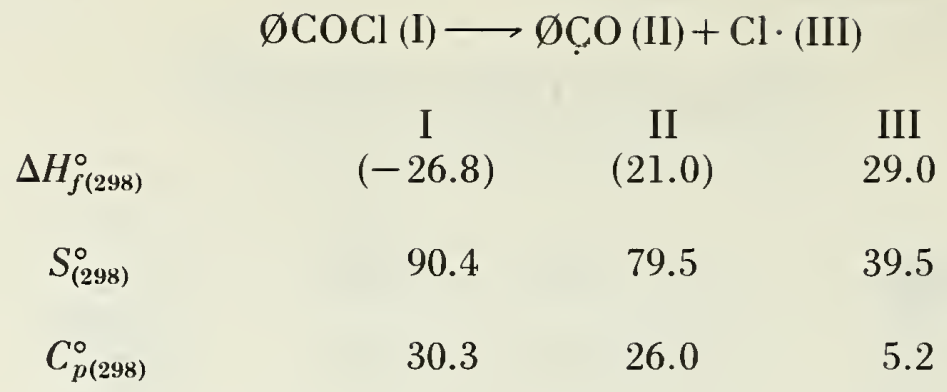

$\Delta S^{\circ}=28.6 \mathrm{~g} / \mathrm{mole}$

$\Delta H^{\circ}=76.8 \mathrm{kcal} / \mathrm{mole}$

$\Delta C_{p}^{\circ}=0.9 \mathrm{~g} / \mathrm{mole}$

\begin{tabular}{|c|c|c|c|c|c|c|}
\hline $\log A$ & $\boldsymbol{E}$ & $\begin{array}{c}\log \boldsymbol{k}_{T} \\
(1025)\end{array}$ & Conditions & System & Surface & References \\
\hline 15.38 & 73.6 & -0.32 & $\begin{array}{l}971-1080^{\circ} \mathrm{K} \\
\delta P \simeq 0.03-0.28 \\
\mathrm{~mm} \mathrm{Hg} \\
P_{T} \simeq 6.7-21 \\
\mathrm{~mm} \mathrm{Hg}\end{array}$ & flow & $-6 \%$ & $\begin{array}{l}\text { [1] M. Szwarc and J. W. } \\
\text { Taylor, J. Chem. } \\
\text { Phys. 22, } 270 \text { (1954). }\end{array}$ \\
\hline
\end{tabular}

Preferred:

$\log k=15.38-73.6 / \theta$.

Comments: Although the observed activation energy is in only fair agreement with the estimated thermodynamics, the difference is not outside the error limits. From $A$ and the reaction entropy, one obtains, $k_{\text {rec }} \simeq 10^{10.9} \mathrm{l} /$ mole-sec (which seems slightly high).

\section{Experimental}

[1] Toluene carrier technique. Rates were based on CO formation. Mass balances were good.

$$
\Delta(\emptyset \mathrm{COCl})=\Delta(\mathrm{HCl})=\Delta(\mathrm{CO})
$$


Reaction: Iodoethane (ethyl iodide)

$\begin{array}{cccc} & \mathrm{C}_{2} \mathrm{H}_{5} \mathrm{I}(\mathrm{I}) \longrightarrow \mathrm{C}_{2} \mathrm{H}_{5} \cdot(\mathrm{II})+\mathrm{I} \cdot(\mathrm{III}) & \\ \Delta H_{f(298)}^{\circ} & \mathrm{I} & \mathrm{II} & \mathrm{III} \\ S_{(298)}^{\circ} & -2.2 & 25.7 & 25.5 \\ C_{p(298)}^{\circ} & 70.7 & 57.8 & 43.2 \\ C_{p(800)}^{\circ} & 15.4 & 12.1 & 5.0 \\ & 32.0 & 24.0 & 5.0\end{array}$

$\Delta S^{\circ}=30.3 \mathrm{~g} / \mathrm{mole}$

$\Delta H^{\circ}=53.4 \mathrm{kcal} / \mathrm{mole}$

$\Delta C_{p}^{\circ}=1.7 \mathrm{~g} / \mathrm{mole}$

$\Delta \mathrm{C}_{p}^{\circ}=-3.0 \mathrm{~g} / \mathrm{mole}$

$\log A \quad \boldsymbol{E} \quad \log \boldsymbol{k}_{T}$

Conditions

System

Surface

References

(740)

13.65

$$
\begin{array}{cc}
50.0 \quad(-1.11) \quad & 704-774^{\circ} \mathrm{K} \\
& \delta P=0.6-0.4 \\
& \mathrm{~mm} \mathrm{Hg} \\
& P_{T} \simeq 2-18 \\
& \mathrm{~mm} \mathrm{Hg}
\end{array}
$$

none

[1] Joe-Hyun Yang and

D. C. Conway, J. Chem. Phys. 43, 1296 (1965).

Preferred:

$\log k=14.6-53.2 / \theta$ (based on the reaction enthalpy).

Comments: This gives a back reaction rate constant of: $k_{b}=10^{9.8} 1 /$ mole-sec. An RRK calculation of the pressure falloff region with $s=16$ gives an $M_{1 / 2} \simeq 10 \mathrm{~mm} \mathrm{Hg}$. Therefore some pressure falloff in the reaction rates under the above conditions is possible.

\section{Experimental}

[1] Toluene carrier technique. Rates were determined from scintillation counter measurements of the $\mathrm{C}^{14}$ content in ethane (ethyl iodide was $\mathrm{C}^{14}$ labeled). Hydrogen halide elimination was competitive (see HI elimination reactions). 
Reaction: Dimethylmercury

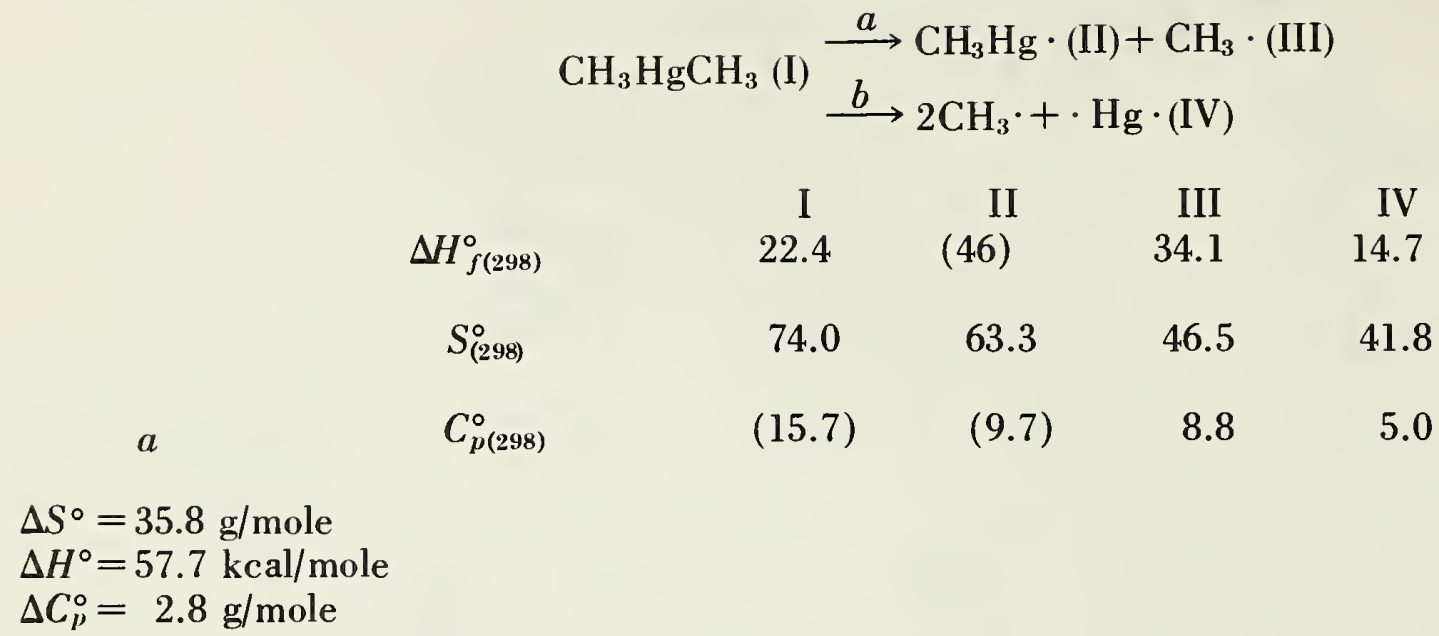

\begin{tabular}{|c|c|c|c|c|c|c|}
\hline $\log A$ & $\boldsymbol{E}$ & $\begin{array}{c}\log \boldsymbol{k}_{T} \\
(645)\end{array}$ & Conditions & System & Surface & References \\
\hline 15.02 & 58 & -4.63 & $\begin{array}{l}588-704^{\circ} \mathrm{K} \\
10-575 \mathrm{~mm} \mathrm{Hg}\end{array}$ & static & $(*)$ & $\begin{array}{l}\text { (1) A. S. Kallend and J. H. } \\
\text { Purnell, Trans. Faraday } \\
\text { Soc. 60, } 103(1964) \text {; } \\
\text { ibid. 60, } 93(1964) .\end{array}$ \\
\hline 14.8 & 53.7 & -3.40 & $\begin{array}{l}693-800{ }^{\circ} \mathrm{K} \\
P_{T} \simeq 3.6-260 \\
\text { mm Hg }\end{array}$ & flow & & $\begin{array}{l}\text { (2) M. Krech and S. J. Price, } \\
\text { Can. J. Chem. 41, 224 } \\
\text { (1963). }\end{array}$ \\
\hline 15.7 & 57.9 & -3.92 & $\begin{array}{l}555-621^{\circ} \mathrm{K} \\
59-72 \mathrm{~mm} \mathrm{Hg}\end{array}$ & static & & $\begin{array}{l}\text { (3) M. E. Russell and R. B. } \\
\text { Bernstein, J. Chem. } \\
\text { Phys. 30,607 (1959) }\end{array}$ \\
\hline 13.1 & 50.1 & -3.88 & $\begin{array}{l}738-881^{\circ} \mathrm{K} \\
(16 \mathrm{~mm} \mathrm{Hg})\end{array}$ & flow & & $\begin{array}{l}\text { (4) S. J. W. Price and } \\
\text { A. F. Trotman-Dicken- } \\
\text { son, Trans. Faraday } \\
\text { Soc. 53, } 939 \text { (1957). }\end{array}$ \\
\hline 14.3 & 51.3 & -3.08 & $\begin{array}{l}567-606^{\circ} \mathrm{K} \\
70-80 \mathrm{~mm} \mathrm{Hg}\end{array}$ & static & $<2 \%$ & $\begin{array}{l}\text { (5) C. M. Laurie and L. H. } \\
\text { Long, Trans. Faraday } \\
\text { Soc. } 51,665 \text { (1955) }\end{array}$ \\
\hline$(16.9)$ & 57.0 & -2.42 & $\begin{array}{l}579-615^{\circ} \mathrm{K} \\
10-70 \mathrm{~mm} \mathrm{Hg}\end{array}$ & static & & $\begin{array}{l}\text { (6) L. M. Yeddanapalli, R. } \\
\text { Srinivason, and V. J. } \\
\text { Paul, J. Sci. Industr. } \\
\text { Res. 13B, } 232 \text { (1954). }\end{array}$ \\
\hline rred: & 51.5 & -3.95 & $\begin{array}{l}762-825^{\circ} \mathrm{K} \\
(10 \mathrm{~mm} \mathrm{Hg})\end{array}$ & flow & $<10 \%$ & $\begin{array}{l}\text { (7) B. G. Gowenlock, J. C. } \\
\text { Polanyi, and E. War- } \\
\text { hurst, Proc. Roy. Soc. } \\
\text { A2 18, } 269 \text { (1953). }\end{array}$ \\
\hline
\end{tabular}

Comments: $D_{1}+D_{2}=57.3 \pm 4$ (Mortimer, Pritchard, and Skinner, Trans. Faraday Soc. 48, 220 (1952)). $D_{1}+D_{2}=57.1 \pm 2$ (Carson, Carson, and Wilmhurst, Nature 1 70, 320 (1952)). However, more recent thermo- 


\section{Dimethylmercury (Continued)}

dynamic data give $D_{1}+D_{2}=\Delta H_{b}^{\circ}=62.5 \mathrm{kcal} / \mathrm{mole}$. Clearly path $b$ is not compatible with any observed activation energies.

\section{Experimental}

[1] Rates obtained from G.L.C. product analysis. Products were $\mathrm{CH}_{4}, \mathrm{C}_{2} \mathrm{H}_{6}, \mathrm{C}_{3} \mathrm{H}_{8}, \mathrm{C}_{2} \mathrm{H}_{4}, \mathrm{C}_{3} \mathrm{H}_{6}$, and $\mathrm{Hg}+$ polymer. Mechanism proposed was consistent with product studies and explained many of the earlier noted experimental anomalies. Decomposition was shown to be in the falloff region at the $P$ and $T$ of many of the earlier studies.

[2] Benzene carrier employed. Rates were based on carbon balance of the products which were analyzed by G.L.C. Believed high-pressure rates were achieved at $P>100 \mathrm{~mm} \mathrm{Hg}$ and that the rate parameters reported pertained to this region. At $P=16 \mathrm{~mm} \mathrm{Hg}, \log k=13.6-51.0 / \theta$.

[3] M.S. analysis on all products. Found rate constants under maximum inhibition of free radical chains with cyclopentene to be pressure dependent in the regions of study. Reported parameters are those obtained from the usual extrapolation to $P_{x}$.

[4] Analysis by G.L.C. on hydrocarbon products. Toluene carrier technique.

[5] Rate based on $\mathrm{CH}_{4}$ and $\mathrm{C}_{2} \mathrm{H}_{6}$ formation.

[6] Rates were based on gaseous product analysis (Blacet Leighton micro gas analysis). The kinetics were shown to follow more appropriately a $3 / 2$ order rather than a 1 order mechanism. The reported activation energy was deduced from the mechanism (as was the $A$-factor for this monograph). (Polymer formed and disappeared in presence of $\mathrm{H}_{2}$.)

[7] Rates based on gas analysis $\left(\mathrm{CH}_{4}, \mathrm{C}_{2} \mathrm{H}_{6}\right.$, and $\left.\mathrm{C}_{2} \mathrm{H}_{4}\right)$. Pressure dependence of the rate constants was observed. Toluene in $\mathrm{N}_{2}$ and $\mathrm{CO}_{2}$ carriers was used. The $A$-factor of $10^{13.5}$ was assumed.

\footnotetext{
* Surface decomposition was shown to occur, altering both the rate and the product distribution.
} 
Reaction: Diethylmercury

\begin{tabular}{cccc}
\multicolumn{4}{r}{$\mathrm{C}_{2} \mathrm{H}_{5} \mathrm{HgC}_{2} \mathrm{H}_{5}(\mathrm{I}) \longrightarrow \mathrm{C}_{2} \mathrm{H}_{5} \cdot(\mathrm{II})+\cdot \mathrm{HgC}_{2} \mathrm{H}_{5}(\mathrm{III})$} \\
$\Delta H_{f(298)}^{\circ}$ & $\mathrm{I}$ & II & III \\
$S_{(298)}^{\circ}$ & 15.9 & 25.7 & {$[36.7]$} \\
$C_{p(298)}^{\circ}$ & 93.8 & 57.8 & 73 \\
& $(26.7)$ & 12.1 & $(15.2)$
\end{tabular}

$\Delta S^{\circ}=37.0 \mathrm{~g} /$ mole

$\Delta H^{\circ}=46.5 \mathrm{kcal} / \mathrm{mole}$

$\Delta C_{p}^{\circ}=0.6 \mathrm{~g} / \mathrm{mole}$

\begin{tabular}{rrrlll}
$\log \boldsymbol{A}$ & $\boldsymbol{E}$ & $\begin{array}{c}\log \boldsymbol{k}_{T} \\
(620)\end{array}$ & \multicolumn{1}{c}{ Conditions } & System & Surface \\
14.1 & 41.5 & -0.53 & $\begin{array}{l}593-643^{\circ} \mathrm{K} \\
4(10 \mathrm{~mm} \mathrm{Hg})\end{array}$ & flow
\end{tabular}

$\begin{array}{llll}14.1 & 42.5 \pm 2 & -0.88 & \begin{array}{l}593-690^{\circ} \mathrm{K} \\ (10 \mathrm{~mm} \mathrm{Hg})\end{array} \quad \text { flow }\end{array}$

\section{References}

[1] B. G. Gowenlock, J. C. Polanyi, and E. Warhurst, Proc. Roy. Soc. A2 18, 269 (1953).

[2] H. V. Carter, E. I. Chapell, and E. Warhurst, J. Chem. Soc., 106 (1956).

\section{Preferred:}

Parameters are too low. We prefer $\log k=15.5-46.5 / \theta$.

Comments: Pressure dependence of the rate constants [1] indicates that the reaction could well be in its fall-off region. Free radical chain decomposition is also quite possible. Arrhenius parameters are clearly low (i.e., compare to dimethyl mercury, di- $n$-propyl mercury, etc., with $A>10^{15.0} \mathrm{sec}^{-1}$ ). $D_{1}+D_{2}=50.2$ $\mathrm{kcal} / \mathrm{mole}$.

\section{Experimental}

[1] $\mathrm{N}_{2}, \mathrm{CO}_{2}$, and $\mathrm{H}_{2}$ carriers with added toluene. Rates were based on the yields of gaseous products $\left(\mathrm{C}_{4} \mathrm{H}_{10}, \mathrm{C}_{2} \mathrm{H}_{6}\right.$, and $\mathrm{C}_{2} \mathrm{H}_{4}$ ) and checked reasonably well with the $\mathrm{Hg}$ produced. Rate constants were dependent on the total pressure.

[2] $\mathrm{N}_{2}$ carrier with and without added toluene. Rates were based on the hydrocarbon product yields and from the yields of $\mathrm{Hg}$. Ratios $\left(\mathrm{C}_{2} \mathrm{H}_{6} / \mathrm{C}_{2} \mathrm{H}_{4}\right)=1$ and $\left.\mathrm{C}_{4} / \mathrm{C}_{2}\right)=4$ were essentially temperature independent. 
Reaction: di- $n$-Propylmercury

\begin{tabular}{cccc}
\multicolumn{4}{r}{$\mathrm{C}_{3} \mathrm{H}_{7} \mathrm{HgC}_{3} \mathrm{H}_{7}(\mathrm{I}) \longrightarrow \mathrm{C}_{3} \mathrm{H}_{7} \cdot(\mathrm{II})+\cdot \mathrm{HgC}_{3} \mathrm{H}_{7}$ (III) } \\
& I & II & III \\
$\Delta H_{f(298)}^{\circ}$ & 4.2 & 20.7 & {$[30.6]$} \\
$S_{(298)}^{\circ}$ & 112.6 & 67.2 & 82.5 \\
$C_{p(298)}^{\circ}$ & $(37.7)$ & 17.6 & $(20.7)$
\end{tabular}

$$
\begin{aligned}
& \Delta S^{\circ}=37.1 \mathrm{~g} / \mathrm{mole} \\
& \Delta H^{\circ}=47.1 \mathrm{kcal} / \mathrm{mole} \\
& \Delta C_{\nu}^{\circ}=0.6 \mathrm{~g} / \mathrm{mole}
\end{aligned}
$$

\begin{tabular}{|c|c|c|c|c|c|c|}
\hline $\log A$ & $\boldsymbol{E}$ & $\begin{array}{c}\log _{(640)} \boldsymbol{k}_{T} \\
(64)\end{array}$ & Conditions & System & Surface & References \\
\hline 15.5 & 47.1 & -0.59 & $\begin{array}{l}605-677^{\circ} \mathrm{K} \\
(4.4 \mathrm{~mm} \mathrm{Hg})\end{array}$ & flow & none & $\begin{array}{l}\text { [1] H. T. J. Chilton and B. G. } \\
\text { Gowenlock, Trans. } \\
\text { Faraday Soc. 50, } 824 \\
\text { (1954). }\end{array}$ \\
\hline
\end{tabular}
Preferred:
Reliable. $\log k=15.5-47.1 / \theta$
$D_{1}+D_{2}=50.2 \mathrm{kcal} / \mathrm{mole}$.

\section{Experimental}

[1] $\mathrm{N}_{2}$ and $\mathrm{NO}$ carriers employed. Rates were based on $\mathrm{Hg}$ formation. The rate constants were observed to be total pressure dependent. Products ịn the $N_{2}$ carrier system were $C_{2}, C_{B}$, and $C_{6}$ hydrocarbons. 
Reaction: di-n-Butyl mercury

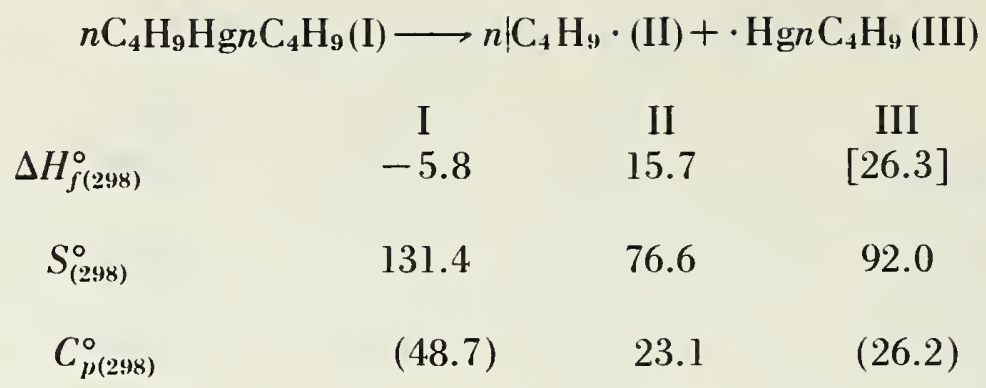

$\Delta S^{\circ}=37.2 \mathrm{~g} / \mathrm{mole}$

$\Delta H^{\circ}=47.8 \mathrm{kcal} / \mathrm{mole}$

$\Delta C_{p}^{\circ}=0.6 \mathrm{~g} / \mathrm{mole}$

\begin{tabular}{|c|c|c|c|c|c|c|}
\hline $\log A$ & $\boldsymbol{E}$ & $\begin{array}{c}\log \boldsymbol{k}_{T} \\
(650)\end{array}$ & Conditions & System & Surface & References \\
\hline 15.8 & $47.8 \pm 2$ & -0.27 & $617-686^{\circ} \mathrm{K}$ & flow & none & $\begin{array}{l}\text { [1] B. H. M. Billinge and } \\
\text { B. G. Gowenlock, Trans. } \\
\text { Faraday Soc. 59, 690, } \\
\text { (1963). }\end{array}$ \\
\hline
\end{tabular}

Preferred:

Reliable.

$\log k=15.8-47.8 / \theta$.

\section{Experimental}

[1] $\mathrm{CO}_{2}, \mathrm{SF}_{6}, \mathrm{~N}_{2}$, and $\mathrm{NO}$ carriers employed. Rates were based on $\mathrm{Hg}$ formation. 
Reaction: Diisopropylmercury

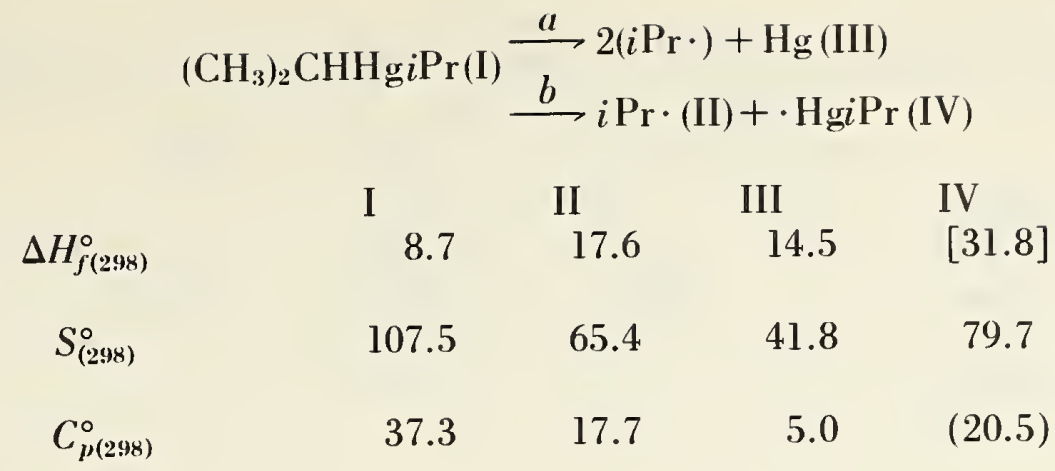

$b$

$\Delta S^{\circ}=37.6 \mathrm{~g} / \mathrm{mole}$

$\Delta H^{\circ}=40.7 \mathrm{kcal} / \mathrm{mole}$

$\Delta C_{p}^{\circ}=0.9 \mathrm{~g} /$ mole
References

[1] H. T. J. Chilton and B. G. Gowenlock, Trans. Faraday Soc. 49, 1451 (1953).

[2] B. H. M. Billinge and B. G. Gowenlock, Trans. Faraday Soc. 59, 690 (1963), Proc. Chem. Soc., 24 (1962).

Preferred:

$\log k_{b}=16.4-40.7 / \theta$.

Comments: Although $D_{1}+D_{2}=\Delta H_{a}^{\circ}=41.0$, the simultaneous bond rupture is not considered here to be probable. Identification of the observed kinetics with path $b$ is preferred.

\section{Experimental}

[1] $\mathrm{N}_{2}$ and $\mathrm{NO}$ carriers. Rates were based on $\mathrm{Hg}$ formation. Sensitivity to total pressure was observed.

[2] $\mathrm{NO}, \mathrm{SF}_{6}, \mathrm{CO}_{2}$, and $\mathrm{N}_{2}$ used as carrier gases. Rates were pressure independent in the ranges studied. Rates were based on G.L.C. analysis of the gaseous products. Path $b$ and $a$ were suggested to be examples of single and multiple critical oscillator reactions. 
Reaction: Divinylmercury

$\begin{array}{cccc}\mathrm{CH}_{2}=\mathrm{CHHgCH}=\mathrm{CH}_{2} & (\mathrm{I}) \longrightarrow \mathrm{CH}_{2}=\mathrm{CH} \cdot(\mathrm{II})+\cdot \mathrm{HgCH}=\mathrm{CH}_{2}(\mathrm{III}) \\ \Delta H_{f(298)}^{\circ} & (\mathrm{I} & \text { II } & \text { III } \\ S_{(298)}^{\circ} & 88.6 & 56.6 & (70.4) \\ C_{p(298)}^{\circ} & (\quad) & 9.7 & (\quad)\end{array}$

$\begin{array}{ll}\Delta S^{\circ}=38.4 \mathrm{~g} / \mathrm{mole} \\ \Delta H^{\circ}= & \mathrm{kcal} / \mathrm{mole} \\ \Delta C_{p}^{\circ}= & \mathrm{g} / \mathrm{mole}\end{array}$

\begin{tabular}{ccccccc}
$\log \boldsymbol{A}$ & $\boldsymbol{E}$ & $\begin{array}{c}\log \boldsymbol{k}_{T} \\
(845)\end{array}$ & Conditions & System & Surface & \multicolumn{1}{c}{ References } \\
11.94 & 48.3 & -0.55 & $\begin{array}{l}775-915 \% \\
16-24 \mathrm{~mm} \mathrm{Hg}\end{array}$ & flow & none & [1] A. F. Trotman-Dickenson, \\
J. Chem. Soc. 2580 \\
(1961).
\end{tabular}

Preferred:

Parameters are not reliable. We favor $\log k \simeq 16-64 / \theta$. Rate constants are probably reliable around $\bar{T}$ of study.

Comments: Since the entropy of activation is certainly positive for this reaction, the reported parameters are clearly too low. The reported $A$-factor implies an entropy of activation of $\Delta S^{\ddagger}=-8.3 \mathrm{~g} / \mathrm{mole}$, which is not possible for the bond fission reaction.

\section{Experimental}

[1] Toluene carrier technique. Rates based on $\mathrm{Hg}$ production were concordant with measurements of ethylene, acetylene, and 1,3-butadiene formed at the lower temperatures. At the higher temperatures faster rates were calculated on the basis of the $\mathrm{Hg}$ production. Heterogeneous polymerization of some of the gaseous products was considered the likely cause of this discrepancy. $\emptyset \mathrm{CH}_{2} \cdot$ radicals can initiate a displacement chain in the system. 
Reaction: Diphenylmercury

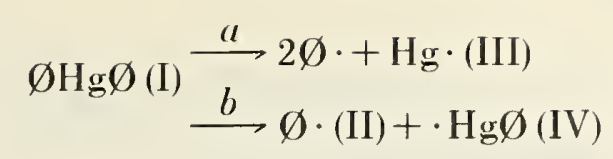

$\begin{array}{ccccc} & \text { I } & \text { II } & \text { III } & \text { IV } \\ \Delta H_{f(298)}^{\circ} & (88.0) & 80.0 & 14.5 & ([76])\end{array}$

$S_{(298)}^{\circ}$

69.2

41.8

$C_{p(298)}^{\circ}$

19.2

5.0

$\begin{aligned} & b \\ & \Delta S^{\circ}=39.2 \\ & \Delta H^{\circ}=68 \\ & \Delta C_{p}^{\circ}=\end{aligned}$

"

$\mathrm{g} / \mathrm{mole}$

$86.5 \mathrm{kcal} / \mathrm{mole}$

$\log \boldsymbol{E} \quad \log \boldsymbol{k}_{T}$

Conditions

System

Surface

References

$16.0 \quad 68 \pm 4+0.02 \begin{aligned} & 896-960{ }^{\circ} \mathrm{K} \\ & (10 \pm \mathrm{mm} \mathrm{Hg})\end{aligned} \quad$ flow $\quad$ none

[1] H. V. Carter, E. I.

Chappell, and

E. Warhurst, J. Chem.

Soc. 106 (1956).

\section{Preferred:}

$$
\log k_{b}=16.0-68 / \theta \text {. }
$$

Comments: We prefer to identify the observed kinetic parameters with path $b$ since $D_{1}+D_{2} \simeq 86.5$ $\mathrm{kcal} / \mathrm{mole}$ and since the high entropy of activation for path $b$ is consistent with $A$ observed.

\section{Experimental}

[1] $\mathrm{N}_{2}$ carrier with added toluene. Rates were based on yields of $\mathrm{Hg} \cdot$ (by weight). Path $a$ was considered the most likely primary split. 
Reaction: Phenylmercuric chloride

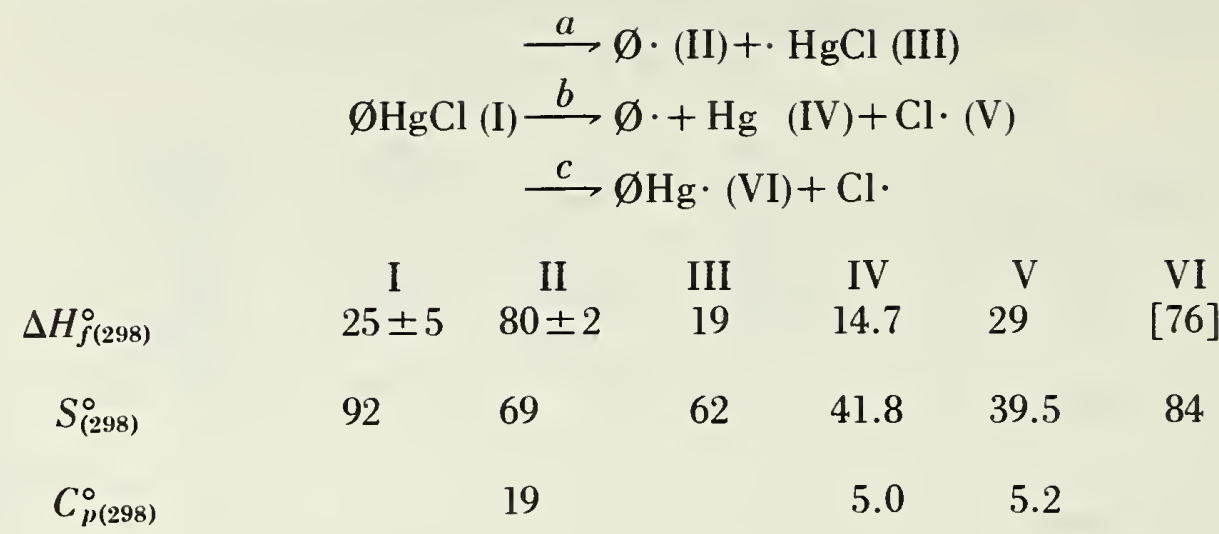

\begin{tabular}{rll}
$\quad$ & \multicolumn{1}{c}{$b$} & \multicolumn{1}{c}{$c$} \\
$\Delta S^{\circ}=39$ & 58.3 & $31.5 \mathrm{~g} / \mathrm{mole}$ \\
$\Delta H^{\circ}=74$ & 98.7 & $80 \mathrm{kcal} / \mathrm{mole}$ \\
$\Delta C_{p}^{\circ}=$ & &
\end{tabular}

$\begin{array}{rlrllll}\text { Path } & \log \boldsymbol{A} & \boldsymbol{E} & \begin{array}{c}\log k_{T} \\ (1015)\end{array} & \text { Conditions } & \text { System } & \text { Surface } \\ 13.0 & 59 \pm 3+0.30 & \begin{array}{l}975-1055^{\circ} \mathrm{K} \\ (10 \mathrm{~mm} \mathrm{Hg})\end{array} & \text { flow } & \text { none }\end{array}$

\section{References}

[1] H. V. Carter, E. I. Chappell, and E. Warhurst, J. Chem. Soc. 106 (1956).

\section{Preferred:}

Parameters are suspect.

$\log k_{u}=15.6-71 / \theta$. Rate constants are probably reliable around $\bar{T}$ of study.

Comments: The observed activation energy is well below any reasonable estimate which can be made for path $a$. Paths $b$ and $c$ are prohibitively endothermic. If a recombination rate constant of about $k_{-a}=10^{9.0}$ 1/mole-sec is assumed, one obtains from the reaction entropy, $A_{a}=10^{15.6} \mathrm{sec}^{-1}$. Adjusting the reported parameters to this value gives a more reasonable rate constant expression in terms of the thermodynamics.

\section{Experimental}

[1] $\mathrm{N}_{2}$ carrier with added toluene. Rates were based on the yields of $\mathrm{Hg}$ and $\mathrm{Hg}_{2} \mathrm{Cl}_{2}$. The latter product was analyzed by reaction with hot $\mathrm{KI}$ solution. $\left(\mathrm{KI}+\mathrm{HgX}_{2} \longrightarrow \mathrm{Hg}+\mathrm{HgX}_{4}=\right)$ Changes in substrate concentration and time of contact did not affect the rate constants. Path $a$ was preferred. 
Reaction: Phenylmercuric bromide

$$
\begin{aligned}
& \begin{aligned}
\emptyset \mathrm{HgBr}(\mathrm{I}) \stackrel{a}{\longrightarrow} \emptyset \cdot(\mathrm{II})+\cdot \mathrm{HgBr}(\mathrm{III}) \\
\stackrel{b}{\longrightarrow} \emptyset \cdot+\mathrm{Hg} \cdot(\mathrm{IV})+\mathrm{Br} \cdot(\mathrm{V})
\end{aligned} \\
& \begin{array}{cccccc} 
& \text { I } & \text { II } & \text { III } & \text { IV } & \text { V } \\
\Delta H_{f(298)}^{\circ} & (33 \pm 5) & 80 \pm 2 & 23 & 14.7 & 26.7
\end{array} \\
& \begin{array}{llllll}
S_{(298)}^{\circ} & 95 & 69 & 65 & 41.8 & 41.8
\end{array} \\
& \begin{array}{llll}
C_{p(298)}^{\circ} & 19 & 5.0 & 5.0
\end{array}
\end{aligned}
$$

$\begin{array}{rl}a & b \\ \Delta S^{\circ}=39 & 57.6 \mathrm{~g} / \mathrm{mole} \\ \Delta H^{\circ}=70 & 88.4 \mathrm{kcal} / \mathrm{mole} \\ \Delta C_{p}^{\circ}= & \end{array}$

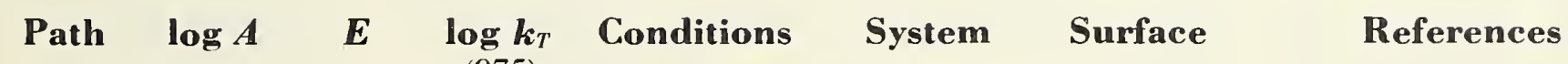

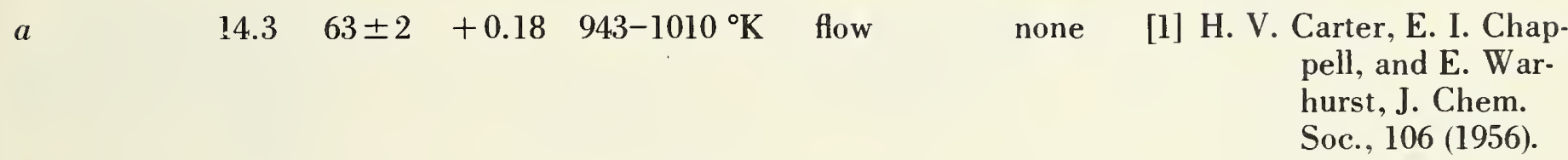

Preferred:

From the thermodynamics, the parameters seem low. We favor $\log k_{a}=15.6-68.8 / \theta$.

\section{Experimental}

[1] $\mathrm{N}_{2}$ carrier with added toluene. Rates were based on yields of $\mathrm{Hg}$ and $\mathrm{Hg}_{2} \mathrm{Br}_{2}$ (see $\emptyset \mathrm{HgCl}$ ). 
Reaction: Phenylmercuric iodide

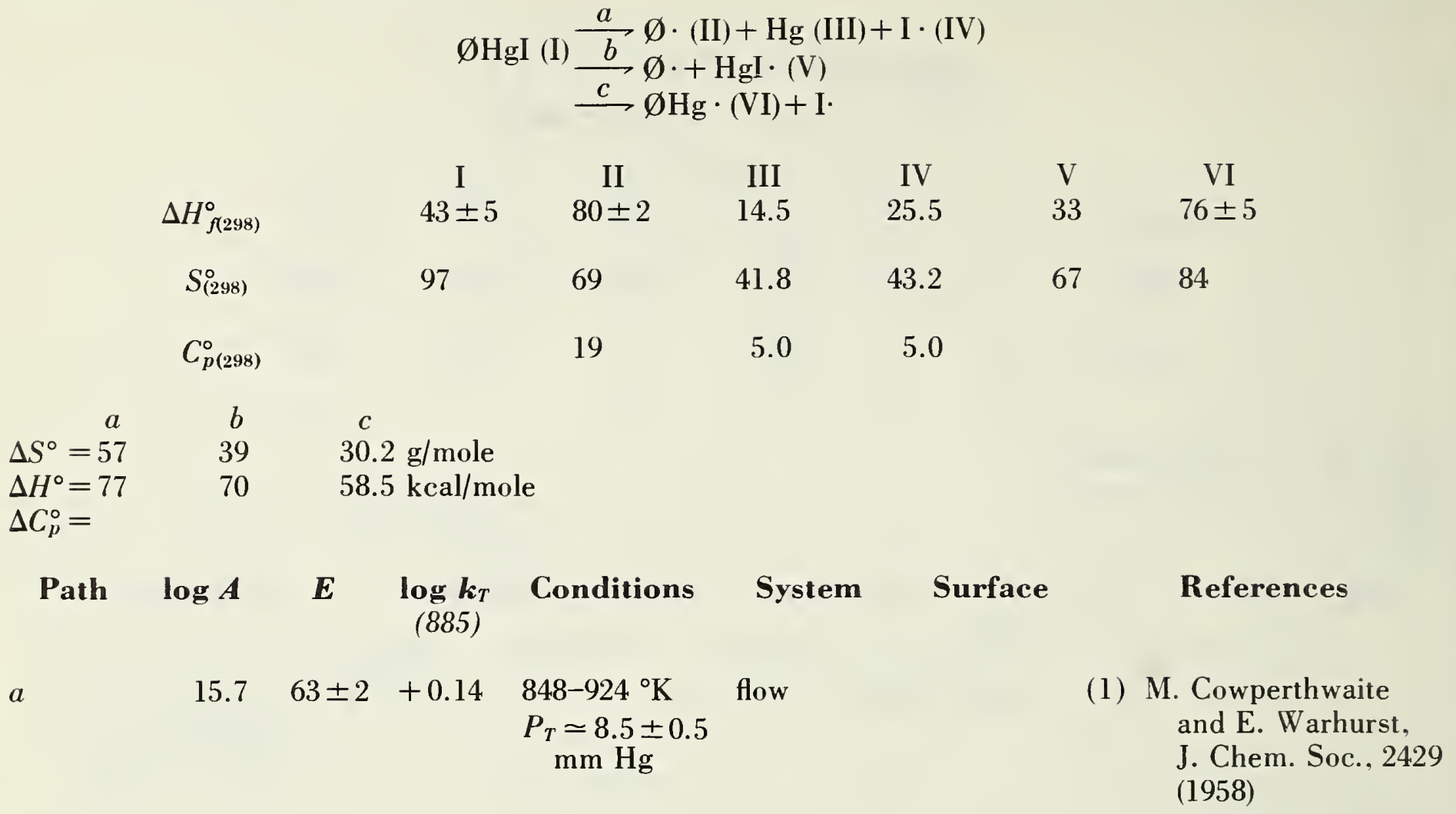

Preferred:

Suspect. $\log k_{b+c}=15.7-63 / \theta$ (see below).

Comments: The parameters look reasonable, although they are not in agreement with the estimated thermodynamics. One can estimate that $A_{b}=10^{15.7} \mathrm{sec}^{-1}$ (as observed) and $A_{c}=10^{14.0} \mathrm{sec}^{-1}$. Thus, in view of the reaction enthalpies of paths $b$ and $c$, along with their possible errors, both $b$ and $c$ look like possible competitive processes. Path $a$ is prohibitively endothermic.

\section{Experimental}

(1) $\mathrm{N}_{2}$ carrier with added toluene. Rates were based on undecomposed $\emptyset \mathrm{HgI}$. Products were $\mathrm{Hg}, \mathrm{Hg}_{2} \mathrm{I}_{2}$, and hydrocarbons. Path $a$ was favored. 
Reaction: Dimethylcadmium

$\mathrm{CH}_{3} \mathrm{CdCH}_{3}$ (I), $\stackrel{a}{\longrightarrow} \mathrm{CH}_{3} \cdot(\mathrm{II})+\mathrm{CH}_{3} \mathrm{Cd} \cdot($ III)

$\begin{array}{ccccc} & \text { I } & \text { II } & \text { III } & \text { IV } \\ \Delta H_{f(298)}^{\circ} & 25.2 & 34.0 & {[45.6]} & 26.8 \\ S_{(2998)}^{\circ} & 72.5 & 46.5 & 62 & 40.1 \\ C_{p(298)}^{\circ} & & 8.8 & & 5\end{array}$

$\Delta S^{\circ}=36.0 \mathrm{~g} / \mathrm{mole}$

$\Delta H^{\circ}=[54.4]$

$\Delta C_{p}^{\circ}=$

\begin{tabular}{|c|c|c|c|c|c|c|c|}
\hline Path & $\log A$ & $\boldsymbol{E}$ & $\begin{array}{c}\log \boldsymbol{k}_{T} \\
(770)\end{array}$ & Conditions & System & Surface & References \\
\hline$a$ & 13.4 & 48.8 & -0.45 & $\begin{array}{l}743-800^{\circ} \mathrm{K} \\
16.3-162 \mathrm{~mm} \mathrm{Hg}\end{array}$ & flow & none & $\begin{array}{l}\text { [1] M. Krech and S. J. Price, } \\
\text { Can. J. Chem. 43, } 1929 \\
\text { (1965) }\end{array}$ \\
\hline
\end{tabular}

$a$

$11.9 \quad 45.8-1.10 \quad 742-844^{\circ} \mathrm{K} \quad$ flow

$4.4-25 \mathrm{~mm} \mathrm{Hg}$

[2] S. J. W. Price and A. F. Trotman-Dickenson,

(18 $\mathrm{mm})$

Trans. Faraday Soc. 53, 939 (1957)

$a$

(13.0) $43.5+0.65531^{\circ} \mathrm{K} \quad$ static

(20 $\mathrm{mm} \mathrm{Hg}$ )

(*) [3] C. M. Laurie and R. H. Long, Trans. Faraday Soc. 53, 1431 (1957)

Preferred:

Parameters are suspect.

Estimate: $\log k=15.0-54.4 / \theta$.

Comments: Although the authors of [1] claim to have reached the high-pressure region, the "close-tonormal" $A$-factor reported is still probably 1 to 2 orders of magnitude too low. $\left(D_{1}+D_{2}=69.6 \mathrm{kcal} / \mathrm{mole}\right.$.)

\section{Experimental}

[1] Benzene carrier technique. Rates were based on hydrocarbon $\left(\mathrm{CH}_{4}, \mathrm{C}_{2} \mathrm{H}_{6}, \mathrm{C}_{2} \mathrm{H}_{4}, \mathrm{C}_{3} \mathrm{H}_{8}\right)$ product analysis (G.L.C. $)$. The reaction was pressure dependent below $80 \mathrm{~mm} \mathrm{Hg}$. Subsequent decomposition of $\left(\mathrm{CH}_{3} \mathrm{Cd} \cdot\right)$ was fast.

[2] Toluene carrier technique. Rates were based on $\mathrm{CH}_{4}$ and ethane yields, and were found to be strongly pressure dependent.

[3] A large heterogeneous contribution was observed. The $A$-factor was assumed and rates were based on both $\mathrm{Cd}$ and $\mathrm{CH}_{4}+\mathrm{C}_{2} \mathrm{H}_{6}$ produced.

* The homogeneous reaction was isolated by extrapolating the rates to zero $(S / V)$ ratios. 
Reaction: Dimethylzinc

\begin{tabular}{ccccc} 
& $\left(\mathrm{CH}_{3}\right)_{2} \mathrm{Zn}(\mathrm{I})$ & \multicolumn{4}{c}{$\mathrm{ZnCH}_{3}(\mathrm{III})+\mathrm{CH}_{3} \cdot(\mathrm{II})$} \\
$\Delta H_{f(298)}^{\circ}$ & I & II & III & $\mathrm{Zn}$ \\
$S_{(298)}^{\circ}$ & $(13.0)$ & 34.0 & $(\quad)$ & 31.2 \\
$C_{p(298)}^{\circ}$ & 74 & 46.5 & 63 & 38.5 \\
& $(\quad)$ & 8.8 & $(\quad)$
\end{tabular}

$\Delta S^{\circ}=35.5 \mathrm{~g} / \mathrm{mole}$

$\Delta H^{\circ}=$

$\Delta C_{1}^{\circ}=$

\begin{tabular}{|c|c|c|c|c|c|c|}
\hline $\log A$ & $\boldsymbol{E}$ & $\begin{array}{c}\log \boldsymbol{k}_{T} \\
(920)\end{array}$ & Conditions & System & Surface & References \\
\hline 11.25 & 47.2 & +0.04 & $\begin{array}{l}843-1000^{\circ} \mathrm{K} \\
(16 \mathrm{~mm} \mathrm{Hg})\end{array}$ & flow & none & $\begin{array}{l}\text { [1] S. J. W. Price and } \\
\text { A. F. Trotman- } \\
\text { Dickenson, Trans. } \\
\text { Faraday Soc. } \mathbf{5 3}, \\
1208 \text { (1957). }\end{array}$ \\
\hline
\end{tabular}

\section{Preferred:}

Unreliable.

Comments: Not representative of the high-pressure rate constant. The low $A$-factor reported is indicative of free radical chain processes and/or of a unimolecular reaction well into its fall-off region. The "high pressure" $A$-factor should be similar to that of mercury dimethyl (i.e., $\log A=15.0$ ).

\section{Experimental}

[1] Toluene carrier technique. Gaseous product was principally $\mathrm{CH}_{4}$, with smaller amounts of $\mathrm{C}_{2} \mathrm{H}_{6}, \mathrm{H}_{2}$, and $\mathrm{C}_{2} \mathrm{H}_{4}$ also observed. Toluene decomposition was corrected for from the $\mathrm{H}_{2}$ yields. Decomposition was believed to occur in two steps:

$$
\begin{aligned}
& \mathrm{Zn}\left(\mathrm{CH}_{3}\right)_{2} \stackrel{1}{\longrightarrow} \cdot \mathrm{ZnCH}_{3}+\mathrm{CH}_{3} \\
& \cdot \mathrm{ZnCH}_{3} \stackrel{2}{\longrightarrow} \mathrm{Zn}+\mathrm{CH}_{3} \text { (see free radical reactions). }
\end{aligned}
$$

The second step was much slower than the first at all temperatures. Rate constants were obtained by employing the method of consecutive reaction rates at intermediate temperatures, while at the higher temperatures the kinetics were interpreted in terıns of the second step only (i.e., instantaneous decomposition of the reactant was assumed). All processes were found to be "markedly" pressure dependent. Parameters reported for the second step were $\log k_{2}\left(\mathrm{sec}^{-1}\right)=6.8-35 / \theta(16 \mathrm{~mm} \mathrm{Hg})$. 


$\begin{array}{rrrrr} & \left(\mathrm{CH}_{3}\right)_{3} \mathrm{As}(\mathrm{I}) \longrightarrow \mathrm{CH}_{3} \cdot(\mathrm{II})+\left(\mathrm{CH}_{3)_{2}} \mathrm{As} \cdot(\mathrm{III})\right. & \\ \Delta H_{f(298)}^{\circ} & \text { I } & \text { II } & \text { III } & \mathrm{As} \\ S_{(2998)}^{\circ} & 8.7 & 34.0 & (\quad) & (\quad) \\ C_{\text {l(298) }}^{\circ} & 27.1 & 46.5 & (\quad) & (\quad) \\ & 27.5 & 8.8 & (\quad) & (\quad)\end{array}$

$\Delta C_{p}^{\circ}=$

$\begin{array}{ccccccc}\log \boldsymbol{A} & \boldsymbol{E} & \begin{array}{c}\log \boldsymbol{k}_{T} \\ (705)\end{array} & \text { Conditions } & \text { System } & \text { Surface } & \text { References } \\ 12.8 & 54.6 & -4.13 & \begin{array}{l}683-723^{\circ} \mathrm{K} \\ 55-193 \mathrm{~mm} \mathrm{Hg} \\ (100 \mathrm{~mm} \mathrm{Hg})\end{array} & \text { static } & \text { none } & \text { [1] P. B. Ayscough and } \\ & & & & \begin{array}{l}\text { H. J. Eméleus, J. Chem. } \\ \text { Soc., 3381 (1954). }\end{array}\end{array}$

Preferred:

Unreliable.

Comments: The very large methane yields indicate that extensive $\mathrm{H}$-abstraction from the parent alkyl occured (i.e., chain decomposition), thus it is unlikely that the reported parameters can be associated with the initial bond rupture. The same conclusion concerning this reaction has been reached by Price and Trotman. Dickenson by analogy with the kinetic complexities of the trimethyl antimony reaction (see $\left.\left(\mathrm{CH}_{3}\right)_{3} \mathrm{Sb}\right)$.

\section{Experimental}

[1] Rates were based on pressure changes in the initial stages of reaction. $\left(P_{f} \mid P_{0}\right) \simeq 2.0$. M.S. product analysis ( $\mathrm{CH}_{4}$ (90 percent), $\mathrm{C}_{2} \mathrm{H}_{6}$ (4 percent), $\mathrm{C}_{2} \mathrm{H}_{4}, \mathrm{C}_{3} \mathrm{H}_{6}$, and $\mathrm{H}_{2}$ ) was stoichiometric in the initial stages. (No primary split was suggested). 
Reaction: tris-(Trifluoromethyl)arsine

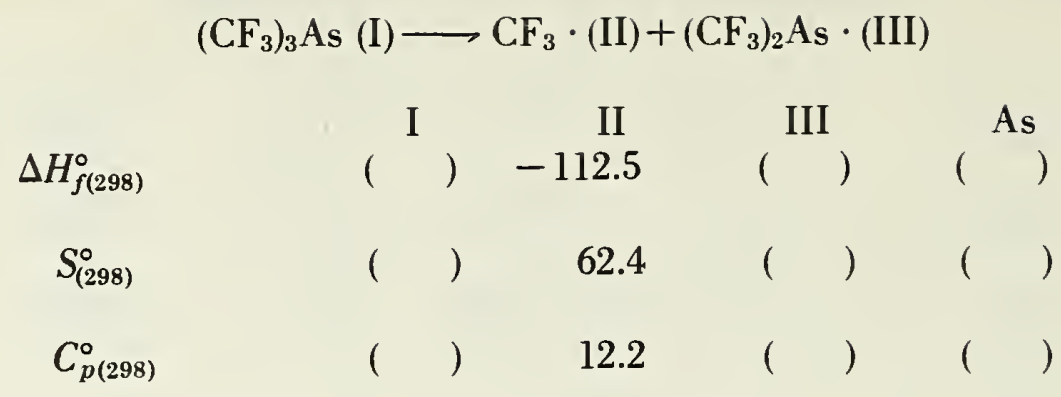

$\Delta S^{\circ}=$

$\Delta H^{\circ}=$

$\Delta C_{p}^{\circ}=$

$\begin{array}{ccccccc}\log \boldsymbol{A} & \boldsymbol{E} & \begin{array}{c}\log \boldsymbol{k}_{T} \\ (655)\end{array} & \text { Conditions } & \text { System } & \text { Surface } & \text { References } \\ 15.4 & 57.4 & -3.75 & \begin{array}{l}623-683^{\circ} \mathrm{K} \\ 51-194 \mathrm{~mm} \mathrm{Hg}\end{array} & \text { static } & <5 \% & \text { [1] P. B. Ayscough and H. J. } \\ & & & & & \begin{array}{l}\text { Emeléus, J. Chem. } \\ \text { Soc., 3381 (1954) }\end{array}\end{array}$

Preferred:

$\log k=15.4-57.4 / \theta$.

Comments: Although subsequent reactions of products with the silica surface are evident, the reported parameters are quite reasonable.

\section{Experimental}

[1] Rates were obtained from initial pressure changes. The reaction corresponded roughly to $\mathrm{As}\left(\mathrm{CF}_{3}\right)_{3} \longrightarrow \mathrm{As}$ $+3 / 2 \mathrm{C}_{2} \mathrm{~F}_{6}$ although up to 10 percent $\mathrm{C}_{3} \mathrm{~F}_{8}$ and $\mathrm{C}_{4} \mathrm{~F}_{10}$ were also formed. Identification and analysis was by $\mathrm{I} \cdot \mathrm{R}$. $\mathrm{SiF}_{4}$ and $\mathrm{CO}_{2}$ were also observed. The ratio of $\left(\mathrm{SiF}_{4}+\mathrm{CO}_{2}\right) /$ fluorocarbon was strongly dependent on the nature of the surface, varying between 10 and 30 percent. 
Reaction: Trimethylbismuth

\begin{tabular}{|c|c|c|c|c|}
\hline$\Delta H_{f(298)}^{\circ}$ & $\begin{array}{c}\text { I } \\
-29.2\end{array}$ & $\begin{array}{c}\text { II } \\
34.0\end{array}$ & $\begin{array}{c}\text { III } \\
{[-19.2]}\end{array}$ & $\begin{array}{c}\mathrm{Bi} \\
49.5\end{array}$ \\
\hline$S_{(298)}^{\circ}$ & 85 & 46.5 & 75.3 & 44.7 \\
\hline$C_{p(298)}^{\circ}$ & 28 & 8.8 & & 5 \\
\hline
\end{tabular}

$a$

$\Delta S^{\circ}=36.8 \mathrm{~g} / \mathrm{mole}$

$\Delta H^{\circ}=[44] \mathrm{kcal} / \mathrm{mole}$

$\Delta C_{p}^{\circ}=$

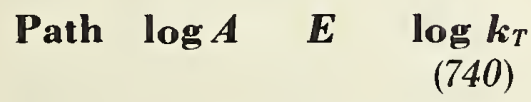

Conditions System Surface

References

$14.044 .0+1.01619-857^{\circ} \mathrm{K}$ flow none

$P_{T}-16 \mathrm{~mm} \mathrm{Hg}$

[1] S. J. W. Price and A. F.

Trotman-Dickenson,

Trans. Faraday Soc. 54, $1630(1958)$

\section{Preferred:}

Suspect. However, see below.

Comments: In terms of the entropy of activation, the $A$-factor looks low by about an order of magnitude. However, there is considerable uncertainty in all the thermodynamic values and the reported parameters are not unreasonable.

\section{Experimental}

[1] Toluene carrier technique. Rates were based on yields of ethane and methane. (Very small yields of $\mathrm{H}_{2}$ and $\mathrm{C}_{2} \mathrm{H}_{4}$ also obtained were considered unimportant.) Reaction rates were shown to be independent of the contact time, the metal alkyl concentration, the $S / V$ ratio, and the total pressure above $10 \mathrm{~mm} \mathrm{Hg}$. Total decomposition to free metal and methyls occurred; thus subsequent decompositions of the metal alkyl radicals were fast relative to the initial bond rupture. 
Reaction: Trimethylgallium

$\begin{array}{ccccc} & \left(\mathrm{CH}_{3}\right)_{3} \mathrm{Ga}(\mathrm{I}) \longrightarrow \mathrm{CH}_{3} \cdot(\mathrm{II})+\cdot \mathrm{Ga}\left(\mathrm{CH}_{3}\right)_{2}(\mathrm{III}) \\ \Delta H_{f(298)}^{\circ} & \text { I } & \text { II } & \text { III } & \mathrm{Ga} \\ S_{(298)}^{\circ} & -6.7 & 34.0 & {[18.8]} & 66 \\ \mathrm{C}_{p(298)}^{\circ} & 83.6 & 46.5 & (75) & 40.4 \\ & 25.9 & 8.8 & & 5\end{array}$

$\Delta S^{\circ}=37.9 \mathrm{~g} / \mathrm{mole}$

$\Delta H^{\circ}=59.5 \mathrm{kcal} / \mathrm{mole}$

$\Delta C_{p}^{\circ}=$

$\begin{array}{cccccc}\log A & \boldsymbol{E} & \begin{array}{c}\log \boldsymbol{k}_{T} \\ (840)\end{array} & \text { Conditions } & \text { System } & \text { Surface } \\ 15.54 & 59.5 & +0.06 & \begin{array}{l}686-983^{\circ} \mathrm{K} \\ 6.1-31.1 \mathrm{~mm} \mathrm{Hg}\end{array} & \text { flow } & <10 \%\end{array}$

\section{References}

[1] M. G. Jacko and S. J. W. Price, Can. J. Chem. 41, 1560 (1963).

Preferred:

$\log k_{1}=15.54-59.5 / \theta$.

$D_{1}+D_{2}+D_{3}=174.7 \mathrm{kcal} / \mathrm{mole}$

Comments: The Arrhenius parameters are reasonable in terms of both reaction entropy and reaction enthalpy.

\section{Experimental}

[1] Toluene carrier technique. Rates were obtained from $\mathrm{CH}_{4}, \mathrm{C}_{2} \mathrm{H}_{6}$, and $\mathrm{C}_{2} \mathrm{H}_{4}$ product yields (G.L.C. analysis). Decomposition appeared to proceed principally in two steps with the second step much slower than the first.

$$
\begin{aligned}
& \left(\mathrm{CH}_{3}\right)_{3} \mathrm{Ga} \stackrel{1}{\longrightarrow}\left(\mathrm{CH}_{3}\right)_{2} \mathrm{Ga} \cdot+\mathrm{CH}_{3} \cdot \\
& \left(\mathrm{CH}_{3}\right)_{2} \mathrm{Ga} \stackrel{2}{\longrightarrow}\left(\mathrm{CH}_{3} \mathrm{Ga}\right)+\mathrm{CH}_{3} .
\end{aligned}
$$

High-temperature decomposition was assumed to be controlled entirely by reaction 2 (see free radical reaction). and the meihod of consecutive reactions was used at lower temperatures to obtain the parameters of reaction $l$ (see also dimethyl zinc).

Rate constants declined markedly for (toluene/reactant) ratios less than 45 indicating a tendency for chain decomposition. Rate constant $\left(k_{1}\right)$ was slightly total pressure dependent, while $k_{2}$ was strongly pressure dependent. Both constan+3 were highly sensitive to the nature of the surface in the reaction zone. Analysis of a black polymer formed gave an empirical formula $\left(\mathrm{GaCH}_{3}\right)$. 
Reaction: Trimethylindium

\begin{tabular}{|c|c|c|c|c|}
\hline & I & II & III & In \\
\hline$\Delta H_{f(298)}^{\circ}$ & $(\quad)$ & 34.0 & $(\quad)$ & 58.2 \\
\hline$S_{(298)}^{\circ}$ & 83.8 & 46.5 & (75) & 41.5 \\
\hline$C_{p(298)}^{\circ}$ & 27.8 & 8.8 & ( & 5 \\
\hline
\end{tabular}

$\Delta S^{\circ}=$

$\Delta H^{\circ}=$

$\Delta C_{p}^{\circ}=$

\begin{tabular}{|c|c|c|c|c|c|c|}
\hline $\log A$ & $\boldsymbol{E}$ & $\underset{(665)}{\log \boldsymbol{k}_{T}}$ & Conditions & System & Surface & References \\
\hline 15.7 & 47.2 & +0.18 & $\begin{array}{l}550-781^{\circ} \mathrm{K} \\
P_{T} \simeq 6-33.5 \mathrm{~mm} \\
\quad \mathrm{Hg} \\
(13 \mathrm{~mm} \mathrm{Hg})\end{array}$ & flow & $<15 \%$ & $\begin{array}{l}\text { [1] M. G. Jacko and S. J. W } \\
\text { Price, Can. J. Chem. } \\
\text { 42, } 1198 \text { (1965). }\end{array}$ \\
\hline
\end{tabular}

Preferred:

$\log k_{1}=15.7-47.2 / \theta$.

Parameters are reasonable.

\section{Experimental}

[1] Toluene carrier technique. Rates based on G.L.C. analysis of hydrocarbon products $\left(\mathrm{CH}_{4}, \mathrm{C}_{2} \mathrm{H}_{6}, \mathrm{C}_{2} \mathrm{H}_{4}, \mathrm{C}_{3} \mathrm{H}_{6}\right.$, $\mathrm{C}_{2} \mathrm{H}_{5} \emptyset$, and $\left.\left(\varnothing \mathrm{CH}_{2}\right)_{2}\right)$. Decomposition occurred in three steps, the second being very fast and the third very slow.

$$
\begin{aligned}
& \text { In }\left(\mathrm{CH}_{3}\right)_{3} \stackrel{1}{\longrightarrow} \cdot \operatorname{In}\left(\mathrm{CH}_{3}\right)_{2}+\mathrm{CH}_{3} \cdot \\
& \text { - In }\left(\mathrm{CH}_{3}\right)_{2} \stackrel{2}{\longrightarrow}: \operatorname{In}\left(\mathrm{CH}_{3}\right)+\mathrm{CH}_{3} \cdot \\
& \text { : In }\left(\mathrm{CH}_{3}\right) \stackrel{3}{\longrightarrow} \text { In }+\mathrm{CH}_{3} .
\end{aligned}
$$

Kinetics of step 3 were studied at high temperatures where steps 1 and 2 were essentially instantaneous (see free radical reactions). At lower temperatures a white polymer of composition $\left(\operatorname{In}\left(\mathrm{CH}_{3}\right)\right) n$ was formed. Rate constant $k_{1}$ was slightly total pressure dependent, while $k_{3}$ was strongly total pressure dependent. $k_{1}$ and $k_{3}$ both declined rapidly for (toluene/ alkyl) ratios of less than 150 . 
Reaction: Trimethylantimony

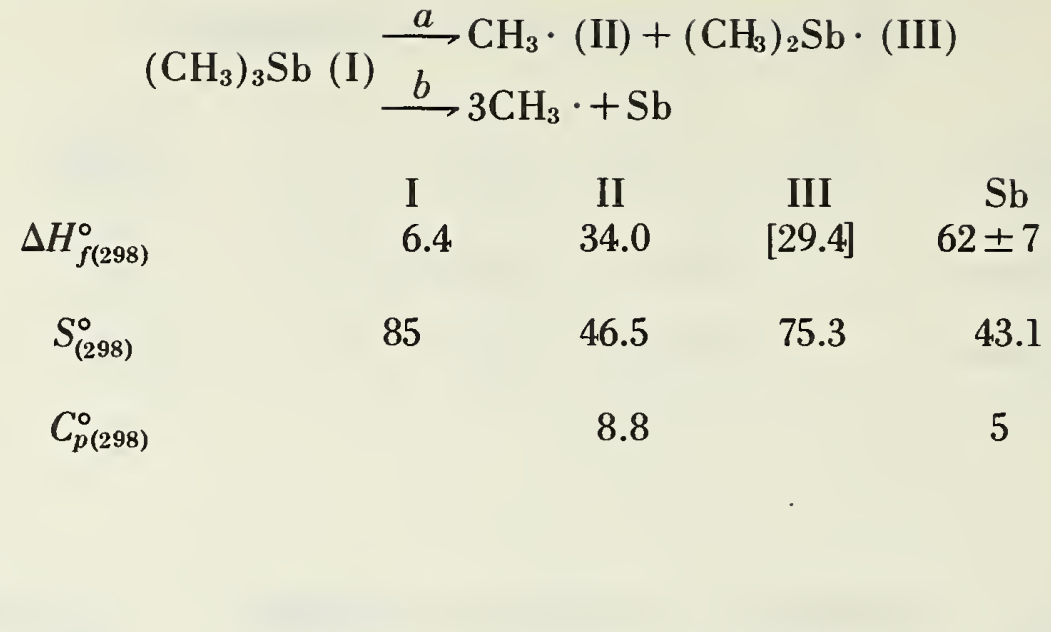

$\Delta S^{\circ}=36.8 \mathrm{~g} / \mathrm{mole}$

$\Delta H^{\circ}=[57] \mathrm{kcal} / \mathrm{mole}$

$\Delta C_{p}^{\circ}=$

Path $\log A$

$\boldsymbol{E}$

$\log \boldsymbol{k}_{T} \quad$ Conditions

(840)

System

Surface

References

$a$

15.2

$\begin{array}{rl}57.0+0.37 & 748-937^{\circ} \mathrm{K} \\ & (16.5 \mathrm{~mm} \mathrm{Hg})\end{array}$

none

[1] S. J. W. Price and

A. F. Trotman-

Dickenson, Trans.

Faraday Soc. 54,

1630 (1958).

\section{Preferred:}

Suspect. However, see below.

Comments: In view of the obvious complexities of this system, little confidence can be placed in the reported Arrhenius parameters. However, the $A$-factor is about right for the reaction entropy and the activation energy is not unreasonable.

$$
\begin{gathered}
D_{1}+D_{2}+D_{3}=157.6 \mathrm{kcal} / \mathrm{mole} ; \bar{D}=52.5 \mathrm{kcal} / \mathrm{mole} \text {. } \\
\text { Experimental }
\end{gathered}
$$

[1] Toluene carrier technique. The reaction was shown to be homogeneous, but otherwise quite complex. Yields of $\mathrm{CH}_{4}$ and $\mathrm{C}_{2} \mathrm{H}_{6}$ were always far below the amount expected on the basis of the metal alkyl decomposed. Polymer formation was large and attributed to a polymer of the type $(\mathrm{SbMe})_{n}$. First-order rate constants based on the amount of metal produced, or on the amount of gaseous products produced, were both total pressure dependent. Parameters reported are those based on the rate of metal production and were attributed to the initial split. 
Reaction: Trimethylthallium

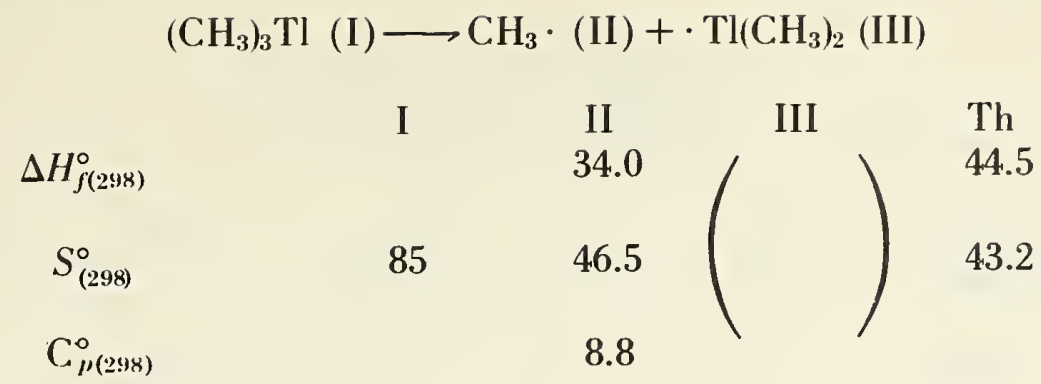

$\Delta S^{\circ}=$

$\Delta H^{\circ}=$

$\Delta C_{p}^{\circ}=$

\begin{tabular}{|c|c|c|c|c|c|c|}
\hline $\log A$ & $\boldsymbol{E}$ & $\begin{array}{c}\log \boldsymbol{k}_{T} \\
(525)\end{array}$ & Conditions & System & Surface & References \\
\hline 10.8 & 27.4 & -0.61 & $\begin{array}{l}458-591^{\circ} \mathrm{K} \\
P_{T}=5.6-33.0 \\
\quad \text { mm Hg }\end{array}$ & flow & $(*)$ & $\begin{array}{l}\text { [1] M. G. Jacko and S. J. W } \\
\text { Price, Can. J. Chem. } \\
\text { 43, } 1961 \text { (1965). }\end{array}$ \\
\hline
\end{tabular}

Preferred:

Unreliable.

Comments: The excessively low $A$-factor is not reasonable, and suggests that the homogeneous reaction has not been effectively isolated.

\section{Experimental}

[1] Toluene carrier technique. Rates based on G.L.C. product analysis for $\mathrm{CH}_{4}, \mathrm{C}_{2} \mathrm{H}_{6}, \mathrm{C}_{2} \mathrm{H}_{4}$, and $\mathrm{C}_{2} \mathrm{H}_{5} \emptyset$ and (or) by direct $\mathrm{Tl}$ analysis. The latter indicates that decomposition of the thallium alkyl radicals follow rapidly the initial bond rupture.

*Extensive heterogeneities were found. Even after consistent pretreatment of the reaction vessels with hot 50 percent $\mathrm{HF}$, reaction rates for initial bond rupture were estimated to be 27 percent heterogeneous at the higher temperature and 13 percent at the lower. A slight total pressure effect was also observed. 
Reaction: Germane

\begin{tabular}{|c|c|c|c|}
\hline \multirow[b]{3}{*}{$\Delta H_{f(298)}^{\circ}$} & \multicolumn{2}{|c|}{$\begin{array}{r}\mathrm{GeH}_{4}(\mathrm{I}) \stackrel{a}{\longrightarrow} \mathrm{GeH}_{2}+\mathrm{H}_{2}(\mathrm{III}) \\
\stackrel{b}{\longrightarrow} \mathrm{GeH}_{3}+\mathrm{H} \text { (II) }\end{array}$} & \multirow{3}{*}{$\begin{array}{c}\text { III } \\
0\end{array}$} \\
\hline & I & II & \\
\hline & $(\quad)$ & $(\quad)$ & \\
\hline$S_{(298)}^{\circ}$ & 51.2 & $(\quad)$ & 31.2 \\
\hline$C_{p(298)}^{\circ}$ & $(\quad)$ & $(\quad)$ & 7.0 \\
\hline
\end{tabular}

$\Delta S^{\circ}=$

$\Delta H^{\circ}=$

$\Delta C_{p}^{\circ}=$

\begin{tabular}{|c|c|c|c|c|c|}
\hline Path & $\log A$ & $\boldsymbol{E}$ & $\begin{array}{c}\log k_{T} \\
(575)\end{array}$ & Conditions & System \\
\hline$b(?)$ & 15.3 & 51.4 & -4.23 & $\begin{array}{l}551-608{ }^{\circ} \mathrm{K} \\
2-40 \mathrm{~mm} \mathrm{Hg}\end{array}$ & static \\
\hline
\end{tabular}

[1] Kinzi Tamaru, M. Boudart, and H. Taylor, J. Phys. (1955); P. J. Fensh am, K. Tam aru, M. Boudart, and H. Taylor, J. Phys. Chem. 59, 806 (1955).

Preferred:

Observed kinetics are reasonable; however, see below.

Comments: The parameters of $\mathrm{H}$-abstraction by $\mathrm{H}$-atoms from germane are not known; however $\mathrm{H}+\mathrm{D}_{2}$ has a $9 \mathrm{kcal} / \mathrm{mole}$ activation energy. If, as seems reasonable, $\mathrm{H}$-abstraction from $\mathrm{GeH}_{4}$ has a much lower activation energy, the absence of $\mathrm{HD}$ in the $D_{2}-\mathrm{GeH}_{4}$ experiments is expected. Large amounts of $\mathrm{HD}$ in the $\mathrm{GeH}_{4}-\mathrm{GeD}_{4}$ pyrolysis, on the other hand, with little or no isotopic mixing in the germanes, strongly suggests a free radical chain process. We favor path $b$ and identify the reported parameters with a chain process.

\section{Experimental}

[1] Rates followed manometrically. Complex system. A zero-order surface reaction took place on deposited germanium concurrently with the homogeneous reaction. Stoichiometry $(1 \rightarrow 2)$ was assumed. Eventual products of the decomposition were $\mathrm{Ge}$ and $\mathrm{H}_{2}$; no $\left(\mathrm{GeH}_{2}\right)_{n}$ polymer was observed. The surface reaction was catalyzed by $\mathrm{O}_{2}$. $\mathrm{GeH}_{4}$ pyrolyzed in $\mathrm{D}_{2}$ gave no $\mathrm{HD}$. Mixtures of $\mathrm{GeH}_{4}$ and $\mathrm{GeD}_{4}$ gave appreciable HD production. Since no HD was formed in the presence of $\mathrm{D}_{2}$, the authors concluded that the initiation step was path $a$. 


\begin{tabular}{|c|c|c|c|c|}
\hline \multirow[b]{2}{*}{$\Delta H_{f(298)}^{\circ}$} & \multicolumn{4}{|c|}{$\begin{array}{rl}\mathrm{Ge}\left(\mathrm{C}_{2} \mathrm{H}_{5}\right)_{4}(\mathrm{I}) \stackrel{a}{\longrightarrow} \mathrm{b} & \mathrm{Ge}+4 \mathrm{C}_{2} \mathrm{H}_{5} \cdot \\
\stackrel{\mathrm{Ge}}{\longrightarrow}\left(\mathrm{C}_{2} \mathrm{H}_{5}\right)_{3}(\mathrm{II})+\mathrm{C}_{2} \mathrm{H}_{5} \cdot(\mathrm{III})\end{array}$} \\
\hline & $\begin{array}{c}\text { I } \\
(-35.0)\end{array}$ & $\left({ }^{\text {II }}\right)$ & $\begin{array}{c}\text { III } \\
25.7\end{array}$ & $\begin{array}{c}\mathrm{Ge} \\
78.4\end{array}$ \\
\hline$S_{(298)}^{\circ}$ & 129.6 & (113) & 57.8 & 40.1 \\
\hline$C_{p(298)}^{\circ}$ & 54.0 & $(\quad)$ & 12.1 & 5 \\
\hline
\end{tabular}

$\Delta S^{\circ}=41.2 \mathrm{~g} / \mathrm{mole}$

$\Delta H^{\circ}=$

$\Delta C_{p}^{\circ}=$

\begin{tabular}{lllllll}
$\log \boldsymbol{A}$ & $\boldsymbol{E}$ & $\begin{array}{c}\log \boldsymbol{k}_{T} \\
(710)\end{array}$ & Conditions & System & Surface & \multicolumn{1}{c}{ References } \\
14.23 & 51.0 & -1.47 & $\begin{array}{l}693-723^{\circ} \mathrm{K} \\
17-465 \mathrm{~mm} \mathrm{Hg}\end{array}$ & static & $<5 \%$ & [1] R. L. Geddes and E. \\
& & & & & $\begin{array}{l}\text { Mack, J. Am. Chem. } \\
\text { Soc. 52, 4372 (1930) }\end{array}$
\end{tabular}

Preferred:

Unreliable.

Comments: The mechanism of this reaction is undoubtedly complex (see $\left.\mathrm{Pb}\left(\mathrm{C}_{2} \mathrm{H}_{5}\right)_{4}\right)$. Free radical chain processes must be important. An identification of the reported parameters with an initial split is not possible.

\section{Experimental}

[1] Rates were followed manometrically. Gaseous products were similar to those of the $n$-butane pyrolysis being 60 to 70 percent ethane and ethylene. First-order rate constants fell off appreciably below $70 \mathrm{~mm} \mathrm{Hg}$ but were unaffected by the addition of inert gases. 
Reaction: Tetracarbonyl nickel (o) (nickel c arbonyl)

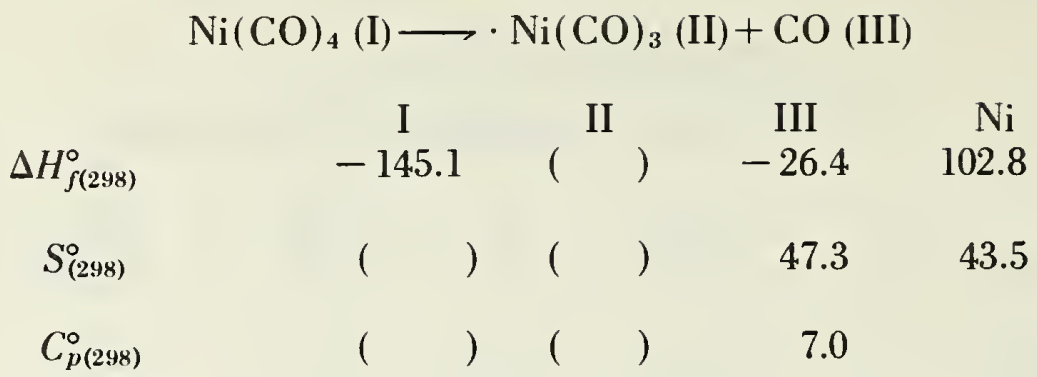

$\Delta S^{\circ}=$
$\Delta H^{\circ}=$
$\Delta C_{p}^{\circ}=$

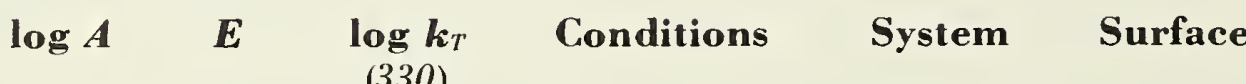

References

$9.5 \quad 19.1 \quad-3.15 \quad 308-353^{\circ} \mathrm{K} \quad$ static

[1] R. K. Chan and R.

McIntosh, Can. J. Chem. 40, 845 (1962).

Preferred:

Unreliable.

Comments: This reaction is obviously complex with appreciable surface catalysis. The abnormally low $A$-factor is untenable in terms of transition state theory.

\section{Experimental}

[1] Rates were based on pressure measurements. The homogeneous mechanism of Bawn (Trans. Faraday Soc. 31,440 (1935) was used.

$$
\begin{aligned}
& \mathrm{Ni}(\mathrm{CO})_{4} \stackrel{1}{\longrightarrow} \mathrm{Ni}(\mathrm{CO})_{3}+\mathrm{CO} \\
& \mathrm{Ni}(\mathrm{CO})_{3} \stackrel{2}{\longrightarrow} \mathrm{Ni}+3 \mathrm{CO} \\
& \mathrm{CO}+\mathrm{Ni}(\mathrm{CO})_{3} \stackrel{3}{\longrightarrow} \mathrm{Ni}(\mathrm{CO})_{4}
\end{aligned}
$$

*A large heterogeneous component to the rate was observed and the overall kinetics were separated into homogeneous and heterogeneous parts. The heterogeneous part was assumed to be proportional to adsorbed $\mathrm{Ni}(\mathrm{CO})_{4}$ which competes for adsorption sites with CO. 
Reaction: Tetraethyl lead

\begin{tabular}{ccccc}
\multicolumn{5}{r}{$\left(\mathrm{C}_{2} \mathrm{H}_{5}\right)_{4} \mathrm{~Pb}(\mathrm{I}) \longrightarrow \mathrm{C}_{2} \mathrm{H}_{5} \cdot(\mathrm{II})+\cdot \mathrm{Pb}\left(\mathrm{C}_{2} \mathrm{H}_{5}\right)_{33}(\mathrm{III})$} \\
& $\mathrm{I}$ & $\mathrm{II}$ & III & $\mathrm{Pb}$ \\
$\Delta H_{f(298)}^{\circ}$ & 23.0 & 25.7 & $(\stackrel{ }{\circ})$ & 46.8 \\
$S_{(298)}^{\circ}$ & $(\quad)$ & 57.8 & & 41.9 \\
$C_{p(298)}^{\circ}$ & & 12.1 & $(\quad)$ & 5
\end{tabular}

$\Delta S^{\circ}=$

$\Delta H^{\circ}=$

$\Delta C_{p}^{\circ}=$

\begin{tabular}{|c|c|c|c|c|c|c|}
\hline $\log A$ & $\boldsymbol{E}$ & $\begin{array}{c}\log \boldsymbol{t}_{T} \\
(525)\end{array}$ & Conditions & System & Surface & References \\
\hline 12.6 & 37.0 & -2.80 & $\begin{array}{l}506-548^{\circ} \mathrm{K} \\
2-13 \mathrm{~mm} \mathrm{Hg}\end{array}$ & static & $<5 \%$ & $\begin{array}{l}\text { [1] G. L. Pratt and J. H. } \\
\text { Purnell, Trans. Fara- } \\
\text { day Soc. 60, } 519 \\
\text { (1964). }\end{array}$ \\
\hline 12.1 & 36.9 & -3.26 & $\begin{array}{l}518-548^{\circ} \mathrm{K} \\
13-52 \mathrm{~mm} \mathrm{Hg}\end{array}$ & static & $<5 \%$ & $\begin{array}{l}\text { [2] J. A. Leernakers, J. } \\
\text { Am. Chem. Soc. 55, } \\
4508 \text { (1933). }\end{array}$ \\
\hline
\end{tabular}

Preferred:

Suspect.

Comments: $D_{1}+D_{2}+D_{3}+D_{4}=126.6 \mathrm{kcal} / \mathrm{mole} ; \bar{D}=31.7 \mathrm{kcal} / \mathrm{mole}$.

Reference [1] represents one of the most careful studies made on any metal alkyl reaction. However, the complexities of the reaction are apparent and a negative activation entropy is not reasonable. Free radical chain decomposition is apparently responsible for the low parameters.

\section{Experimental}

[1] G.L.C. analysis of all products. Approximately 17 products were observed, although the major products (identified also as the only initial products) were $n-\mathrm{C}_{4} \mathrm{H}_{10}, \mathrm{C}_{2} \mathrm{H}_{4}, \mathrm{C}_{2} \mathrm{H}_{6}$, and $\mathrm{H}_{2}$. Rates were obtained from initial rates of product formation. Product ratios were shown to vary with percent reaction and a chain decomposition (length $\sim 4.6$ ) was evident. Product yield versus time curves for the four major products were constructed and individual reaction orders for their formation were obtained.

[2] Rates were based on pressure measurements. 
Reaction: Silane

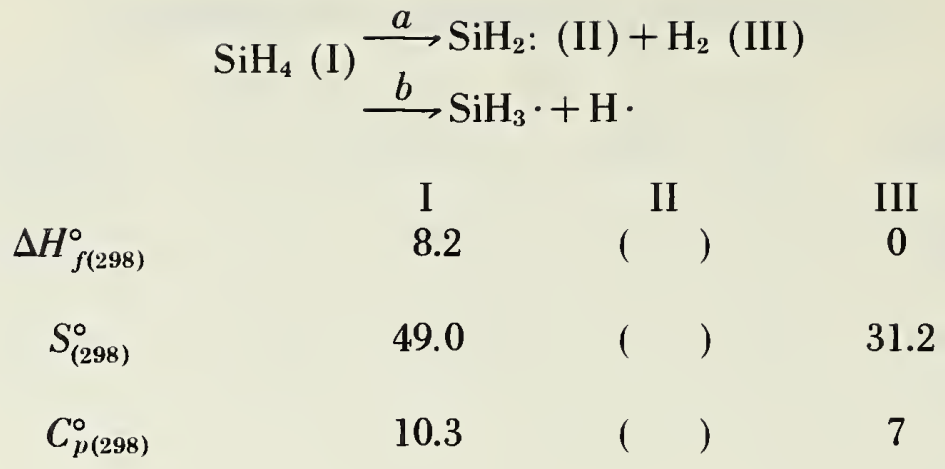

$\Delta S^{\circ}=$
$\Delta H^{\circ}=$
$\Delta C_{p}^{\circ}=$

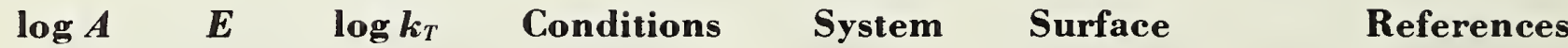

$15.2 \quad 55.9 \quad-2.90 \quad 646-703^{\circ} \mathrm{K} \quad$ static none 35-230 torr

[1] J. H. Purnell and R.

Walsh, Proc. Roy. Soc. A293, 543 (1966).

\section{Preferred:}

Overall kinetics are reliable; however, identification of the parameters with any initial step is questionable.

Comments: Electron impact studies have placed $\Delta H^{\circ}\left(\mathrm{SiH}_{3}-\mathrm{H}\right)$ at about $94 \pm 3 \mathrm{kcal} / \mathrm{mole}$ while the average $E(\mathrm{Si}-\mathrm{H})$ in silane is $78 \mathrm{kcal} / \mathrm{mole}$. The authors correctly showed that the observed activation energy is too low for mechanism $B$ (path $b$ ) if electron impact numbers are used and can only barely be reconciled with the average bond energies. They therefore tentatively attributed the kinetics to mechanism $A$. However, in the copyrolysis of $\mathrm{SiH}_{4}$ and $\mathrm{SiD}_{4}$, Ring, et al. (private communication) have shown that $\mathrm{H}_{2} / \mathrm{HD} / \mathrm{D}_{2}=4 / 3 / 1$ in the early stages of pyrolysis where $\mathrm{H}, \mathrm{D}$ exchange in the silanes was negligible. This is consistent with a free radical formation of hydrogen involving $\mathrm{H}$-atoms and cannot be reconciled with the silene mechanism. The mechanism of the $\mathrm{SiH}_{4}$ decomposition, and particularly the initiation reaction, is therefore still unknown, but. we favor path $b$ initiation.

\section{Experimental}

[1] Rates were determined by complete G.L.C. analysis of the products. Principal products were $\mathrm{Si}_{2} \mathrm{H}_{6}$ and $\mathrm{H}_{2}$ $\mathrm{Si}_{3} \mathrm{H}_{8}$ was a minor product and a solid polymer of formula $\left(\mathrm{SiH}_{2} h_{n}\right.$ which decomposed eventually to $\mathrm{Si}$, and $\mathrm{H}_{2}$ was also formed. The reaction order in silane for the initial stages of reaction (0-2 percent) was 1.5 and was attributed to a pressure falloff in the above initiation reaction. Additions of the inert gas $\left(\mathrm{SF}_{6}\right)$ accelerated reaction rates but did not affect the order of the reaction relative to $\mathrm{SiH}_{4}$. Latter stages of reaction (10-20 percent) were shown to be kinetically complex, involving principally the decomposition of polymer and $\mathrm{Si}_{2} \mathrm{H}_{6}$ which rapidly reached steady state. Rates were first order in silane and agreed with earlier studies by Stockland and Hodgness et al. (references cited by Purnell and Walsh).

$$
\log k_{\text {later stames }} \simeq 13.2-51.2 / \theta \text {. }
$$

The two mechanisms considered were:

$$
\text { A. } \begin{aligned}
& \mathrm{SiH}_{4} \longrightarrow \mathrm{SiH}_{2}+\mathrm{H}_{2} \text { slow } \\
& \mathrm{SiH}_{2}+\mathrm{SiH}_{4} \rightleftharpoons \mathrm{Si}_{2} \mathrm{H}_{6} \text { fast } \\
& \\
& \mathrm{SiH}_{2}+\mathrm{Si}_{2} \mathrm{H}_{6} \rightleftharpoons \mathrm{Si}_{3} \mathrm{H}_{8}
\end{aligned}
$$

B. $\mathrm{SiH}_{4} \longrightarrow \mathrm{H}+\mathrm{SiH}_{3}$ $\mathrm{H}+\mathrm{SiH}_{4} \rightleftharpoons \mathrm{H}_{2}+\mathrm{SiH}_{3}$ $\mathrm{SiH}_{3}+\mathrm{SiH}_{4} \rightleftharpoons \mathrm{Si}_{2} \mathrm{H}_{6}+\mathrm{H}$ $2 \mathrm{SiH}_{3} \longrightarrow \mathrm{Si}_{2} \mathrm{H}_{6}$ 
Reaction: Tetramethylsilane (silicon tetramethyl)

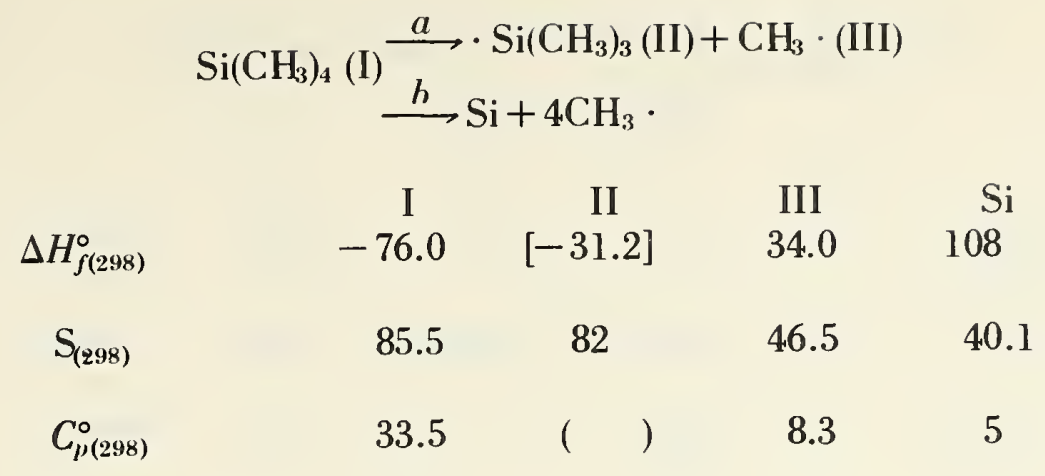

$\Delta S^{\circ}=43 \mathrm{~g} / \mathrm{mole}$

$\Delta H^{\circ}=[78.8] \mathrm{kcal} / \mathrm{mole}$

$\Delta C_{p}^{\circ}=(\quad) \mathrm{g} / \mathrm{mole}$

$\begin{array}{clcllll}\log A & \boldsymbol{E} & \begin{array}{c}\log \boldsymbol{k}_{T} \\ (965)\end{array} & \text { Conditions } & \text { System } & \text { Surface } & \\ 15.1 & 78.8 & -2.75 & \begin{array}{l}933-933^{\circ} \mathrm{K} \\ 4-438 \mathrm{~mm} \mathrm{Hg}\end{array} & \text { static } & \text { none } & \text { [1] }\end{array}$

\section{References}

1] D. F. Helm and E. Mack. J. Am. Chem. Soc. 59, $60(1937)$

[2] T. V. Sathyamurthy, S.

Swaminathan, and L. M.

Yeddanapalli, J. Ind.

Chem. Soc. 27, 509

(1950), C.A.-6469a (1951)

\section{Preferred:}

$\log k_{a}=15.1-78.8 / \theta$.

Comments: This reaction must be a chain reaction with path $a$ as the initiation reaction. Although the reaction must be quite complex, the parameters reported look fairly reasonable for path $a$.

\section{Experimental}

[1] Rates were followed by pressure changes. Products were silicon, carbon, and hydrocarbons similar to those found in the ethane decomposition. First-order constants were obtained for initial pressures greater than $100 \mathrm{~mm} \mathrm{Hg}$; higher orders $(\sim 3 / 2)$ were observed for lower initial pressures $\left(P_{x} / P_{o}\right) \simeq 2.95-3.01$.

[2] Re-analyses of rate data [1]. Free radical mechanism proposed. First-order kinetics was predicted at pressures above 149 torr. 
Reaction: Tetraethylsilane (silicon tetraethyl)

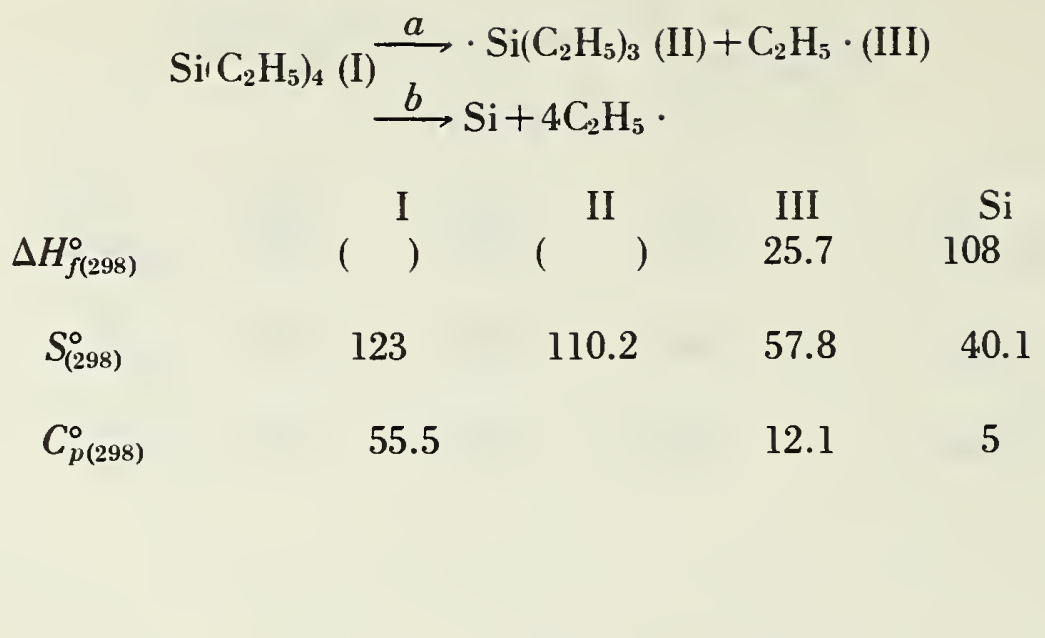

$\begin{array}{llllll}\log A & E & \log k_{T} & \text { Conditions } & \text { System } & \text { Surface }\end{array}$

none $793-873^{\circ} \mathrm{K}$
$20-200 \mathrm{~mm} \mathrm{Hg}$

\section{References}

[1] C. E. Waring, Trans. Faraday Soc. 36, $1142(1940)$

Preferred:

Unreliable.

Comments: At the reaction temperatures, extensive chain decomposition must take place, mainly promoted by $\mathrm{H}$-atom reactions with the reactant. No identification of the parameters reported with an initial decomposition is possible.

\section{Experimental}

[1] Rates were obtained on the basis of initial pressure increases. The ratio $\left(P_{f} / P_{0}\right)$ varied with $P_{o}$. Products were ethylene, $\mathrm{CH}_{4}, \mathrm{H}_{2}$, and $\mathrm{Si}$. Addition of small quantities of $\mathrm{NO}$ did not appreciably affect the initial reaction rates, although inhibition in later stages was observed. Heterogeneous decomposition was appreciable in clean reaction cells but unimportant in the initial stages for Si-coated cells. 
Reaction: Tetra- $n$-propylsilane (silicon tetra $n$-propyl)

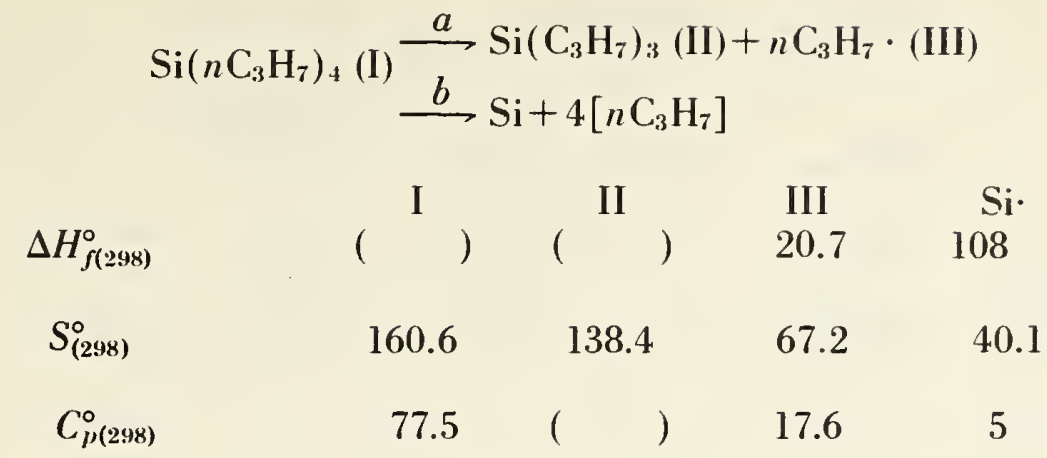
$\Delta S^{\circ}=(45.0) \mathrm{g} / \mathrm{mole}$
$\Delta H^{\circ}=\mathrm{kcal} / \mathrm{mole}$
$\Delta C_{p}^{\circ}=\quad \mathrm{g} / \mathrm{mole}$

\begin{tabular}{ccccccc}
$\log \boldsymbol{A}$ & $\boldsymbol{E}$ & $\log \boldsymbol{k}_{T}$ & Conditions & System & Surface & \multicolumn{1}{c}{ References } \\
14.14 & 46.0 & $(-2.11)$ & $\begin{array}{l}793-843^{\circ} \mathrm{K} \\
20-160 \mathrm{~mm} \mathrm{Hg}\end{array}$ & static & $\begin{array}{c}\text { none (when } \\
\text { condi- } \\
\text { tioned) }\end{array}$ & $\begin{array}{c}\text { [1] C. E. Waring, Trans. } \\
\text { Faraday Soc. 36, } 1142 \\
\text { (1940). }\end{array}$
\end{tabular}

Preferred:

Unreliable.

Comments: Must be a chain reaction.

\section{Experimental}

[1] Products were $\mathrm{C}_{3} \mathrm{H}_{6}, \mathrm{C}_{2} \mathrm{H}_{4}, \mathrm{CH}_{4}, \mathrm{H}_{2}$, and Si. See comments on $\mathrm{Si}\left(\mathrm{C}_{2} \mathrm{H}_{5}\right)_{4}$. 
Reaction: Hexamethyldisilane

$\begin{array}{ccc}\left(\mathrm{CH}_{3}\right)_{3} \mathrm{Si}-\mathrm{Si}\left(\mathrm{CH}_{3}\right)_{3} & (\mathrm{I}) \longrightarrow 2\left(\mathrm{CH}_{3}\right)_{3} \mathrm{Si} \cdot(\mathrm{II}) \\ \Delta H_{f(298)}^{\circ} & \mathrm{I} & \mathrm{II} \\ S_{(298)}^{\circ} & (\quad) & (-3.12) \\ C_{p(298)}^{\circ} & (99) & (73) \\ & (50) & (27)\end{array}$

$$
\begin{aligned}
& \Delta S^{\circ}=47.0 \mathrm{~g} / \mathrm{mole} \\
& \Delta H^{\circ}= \\
& \Delta C_{p}^{\circ}=4.0 \mathrm{~g} / \text { mole }
\end{aligned}
$$

\begin{tabular}{|c|c|c|c|c|c|c|}
\hline $\log A$ & $\boldsymbol{E}$ & $\begin{array}{c}\log \boldsymbol{k}_{T} \\
(980)\end{array}$ & Conditions & System & Surface & References \\
\hline $12.2 \pm 1$ & $49.5 \pm 6$ & +1.16 & $\begin{array}{l}940-1020^{\circ} \mathrm{K} \\
P_{T} \simeq 10^{-3}-10^{-5} \\
\quad \text { torr }\end{array}$ & flow & none & $\begin{array}{l}\text { [1] J. A. Connor, R. N. Has- } \\
\text { zeldine, G. J. Leigh, and } \\
\text { R. D. Sedgwick, J. } \\
\text { Chem. Soc. A, } 768 \\
\text { (1967). }\end{array}$ \\
\hline & $58 \pm 4$ & & $\begin{array}{l}923-983^{\circ} \mathrm{K} \\
\delta P \simeq 0.1-1 \text { torr } \\
\frac{P_{\text {toluene }}}{\delta P}=20-170\end{array}$ & flow & & $\begin{array}{l}\text { [2] J. A. Connor, G. Finney, } \\
\text { G. J. Leigh, R. N. Has- } \\
\text { zeldine, P. J. Robinson, } \\
\text { R. D. Sedgwick, and } \\
\text { R. F. Simmons, Chem. } \\
\text { Comm., } 178 \text { (1966). }\end{array}$ \\
\hline
\end{tabular}

Preferred:

Parameters seem low. We estimate, $\log k=16.0-62.0 / \theta$; however, see below.

Comments: Although there are large uncertainties in the thermodynamics, the entropy of activation for this system must be sizable. Certainly a negative activation entropy is quite impossible. The rate constants of [1] are undoubtedly pressure dependent and do not represent the reaction in its first order region. Unforturately, no rate constants were given in [2], although the activation energy looks more reasonable. The bond dissociation energies of silicon-silicon bonds are still very much in doubt.

\section{Experimental}

[1]Straight flow technique. Analysis by M.S. directly on the products leaving the reaction zone. Similar parameters were obtained with and without toluene. Products in the presence of toluene were bibenzyl, triethylsilane and traces of other alkyl-silanes, methane and hydrogen.

[2] Toluene carrier technique. Products were those of [1]. 
Reaction: Tetramethyltin

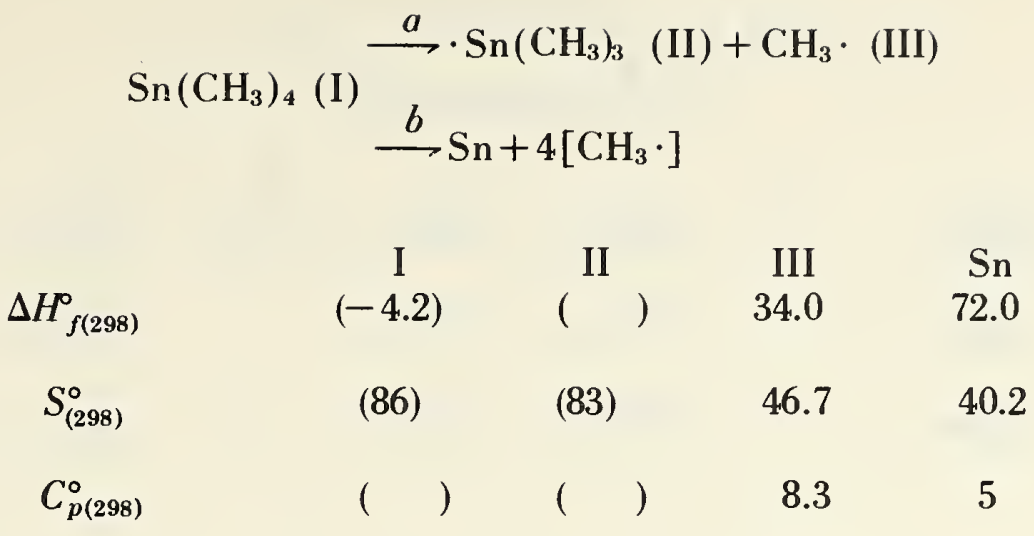

$\Delta S^{\circ}=$

$\Delta H^{\circ}=$

$\Delta C_{p}^{\circ}=$

$\log$ $\boldsymbol{E} \quad \log \boldsymbol{k}_{T}$
$(740)$

Conditions

System

Surface

References

21.92

\section{4}

$\begin{array}{ll}-2.42 & 713-766^{\circ} \mathrm{K} \\ & 5-185 \mathrm{~mm} \mathrm{Hg}\end{array}$

static $<5 \%$ *

[1] C. E. Waring and W. S.

Horton, J. Am. Chem.

Soc. 67, 540 (1945).

Preferred:

Unreliable.

Comments: The reaction is far too complex to be studied by simple manometric methods. It is unlikely that the reported parameters have any relevance to the initial bond breaking reactions $a$. Reaction $b$ is obviously not possible.

\section{Experimental}

[1] Rates were followed manometrically. Gaseous products were $\mathrm{CH}_{4}(80-90$ percent $), \mathrm{C}_{2} \mathrm{H}_{4}, \mathrm{H}_{2}$, and higher olefins. Initial reaction rates were used to obtain the rate constants. NO catalyzed the decomposition.

*In clean reaction vessels the decomposition was predominantly heterogeneous. 
Reaction: Dichlorodimethylstannane (dimethyl tin dichloride)

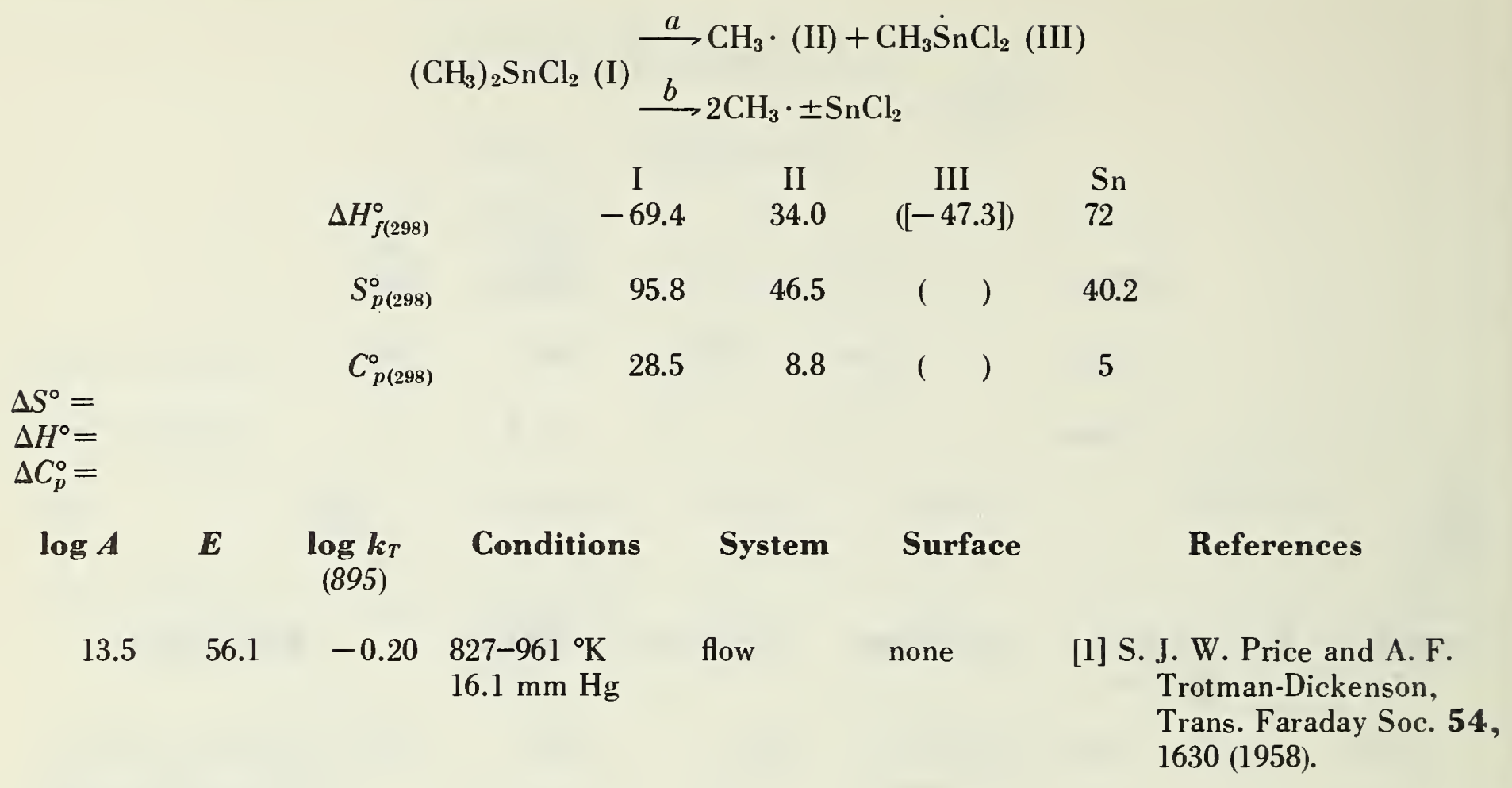

Preferred:

Suspect.

Comments: The total pressure dependence on the reaction rates indicates pressure falloff behavior and/or mechanistic complexities.

\section{Experimental}

[1] Toluene carrier technique. Rates were based on yields of $\mathrm{CH}_{4}$ and $\mathrm{C}_{2} \mathrm{H}_{6}$.

$$
\text { Mechanism: } \begin{aligned}
& \left(\mathrm{CH}_{3}\right)_{2} \mathrm{SnCl} \longrightarrow \mathrm{CH}_{3} \dot{\mathrm{SnCl}}_{2}+\mathrm{CH}_{3} \cdot \\
& \mathrm{CH}_{3} \mathrm{SnCl}_{2} \longrightarrow \mathrm{CH}_{3} \cdot+\mathrm{SnCl}_{2} \\
& \mathrm{CH}_{3} \cdot+\mathrm{CH}_{3} \emptyset \longrightarrow \mathrm{CH}_{4}+\cdot \mathrm{CH}_{2} \emptyset \\
& 2 \mathrm{CH}_{3} \cdot \longrightarrow \mathrm{C}_{2} \mathrm{H}_{6} \\
& 2 \emptyset \mathrm{CH}_{2} \cdot \longrightarrow\left(\emptyset \mathrm{CH}_{2}\right)_{2}
\end{aligned}
$$

Rate constants were shown to be independent of the contact time and of the pressure of the metal alkyl, but slightly dependent on the total pressure. Under conditions of total decomposition, $\Delta\left(\mathrm{CH}_{3}\right)_{2} \mathrm{SnCl}_{2}=\Delta \mathrm{C}_{2} \mathrm{H}_{6}+\frac{1}{2} \Delta \mathrm{CH}_{4}$. Tin (II) chloride yields were not reported. 


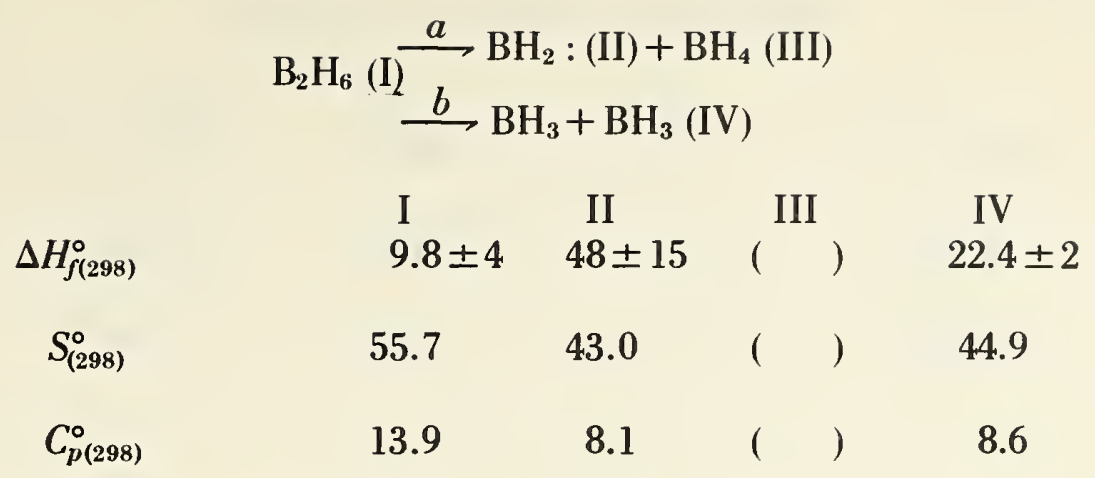

$b$

$\Delta S^{\circ}=34.1 \mathrm{~g} / \mathrm{mole}$

$\Delta H^{\circ}=35.0 \mathrm{kcal} / \mathrm{mole}$

$\Delta C_{p}^{\circ}=3.3 \mathrm{~g} / \mathrm{mole}$

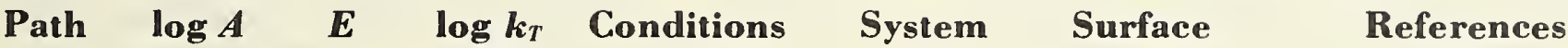

$\begin{array}{lllll}a & 18.0 & 44 & -3.13 & \text { (see comments) }\end{array}$

[1] T. P. Fehlner, J. Am.

Chem. Soc. 87, 4200 (1965)

$b \quad 35 \pm 3 \quad$ (see comments)

[2] M. E. Garabedian and

S. W. Benson, J. Am. Chem. Soc.

86, 176 (1964)

Preferred:

$\log k_{b}=13.0-35 / \theta$.

Comments: The activation energy of [2] has been confirmed by the kinetics and thermochemistry of the $\left(\mathrm{BH}_{3} \mathrm{PH}_{3}\right)$ decomposition.

\section{Experimental}

[1] Reinterpretation of existing kinetic data. Arrhenius constants deduced from the overall kinetics and assumed parameters of the chain reactions. Mechanism proposed was a complex chain.

[Prior studies: J. K. Bragg, L. V. McCarty, and F. J. Norton, J. Am. Chem. Soc. 73, 2134 (1951); K. Borer, A. B. Littlewood, and C. S. Phillips, J. Inorg. Nucl. Chem. 15, 316 (1960); R. P. Pease and R. N. Pease, J. Am. Chem. Soc. 73, 2132 (1951); R. E. Enrioni and R. Schaeffer, J. Inorg. Nucl. Chem. 18, 103 (1961); S. H. Bauer, J. Am. Chem. Soc. 78,5775 (1956).]

Thermodynamics of $\mathrm{B}_{2} \mathrm{H}_{6}$ and $\mathrm{BH}_{3}$ are from A. Shepp and S. H. Bauer, J. Am. Chem. Soc. 76, 265 (1954).

[2] Reinterpretation of the existing data on this reaction and on the $\mathrm{BH}_{3} \mathrm{CO}$ decomposition. 
Reaction: Borine carbonyl

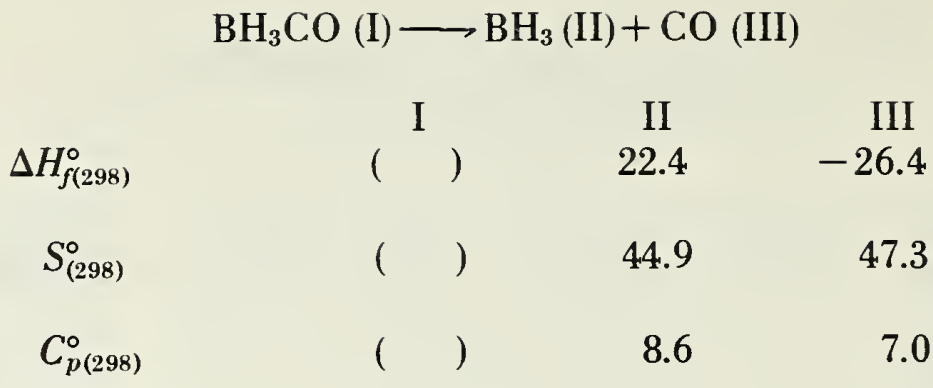

$\Delta S^{\circ}=$
$\Delta H^{\circ}=$
$\Delta C_{p}^{\circ}=$

$\begin{array}{llllll}\log A & E & \log k_{T} & \text { Conditions } & \text { System } & \text { Surface }\end{array}$ (425)

$14.42 \quad 23.7+2.23$ (see Experimental)

[1] M. E. Garabedian and S. W. Benson, J. Am. Chem. Soc. 86, 176 (1964)

$\begin{array}{lll}14 \pm 0.5 \quad 23.3+2.01 \quad 388-462{ }^{\circ} \mathrm{K} \\ & & 0.9-22 \times 10^{-3} \\ & & \\ & & \end{array}$

[2] T. P. Fehlner and W. S. Koski, J. Am. Chem. Soc. 87, 409 (1965)

$\begin{array}{lll}k_{90} & 11.7 \quad 19.6\end{array}$ 267-305 ${ }^{\circ} \mathrm{K} \quad$ static

torr $P_{T} \sim 90$ torr

[3] J. Grotewald, E. A. Lissi, and A. E. Villa, J. Chem. Soc. A, 1038 (1966)

$k_{\infty} \quad 13.4 \quad 21.4$

Preferred:

$\log k=14.4-23.7 / \theta$.

\section{Experimental}

[1] Based on a reanalysis of the data of A. B. Burg, J. Am. Chem. Soc. 74, 3482 (1952), J. Am. Chem. Soc. 59, 780 (1937), and of Y. C. Fu and G. R. Hill, J. Am. Chem. Soc. 84, 353 (1962).

[2] Analysis by M.S.

[3] Triethylamine was added to scavenge all $\mathrm{BH}_{3}$ so that the initial fission kinetics could be studied. Rates were followed by CO analysis (chromatography on molecular sieves). Mechanism:

$$
\begin{aligned}
(\mathrm{M})+\mathrm{BH}_{3} \mathrm{CO} & \rightarrow \mathrm{BH}_{3}+\mathrm{CO}+(\mathrm{M}) \\
\mathrm{BH}_{3}+\mathrm{Et}_{3} \mathrm{~N} & \rightarrow \mathrm{Et}_{3} \mathrm{~N}: \mathrm{BH}_{3} \\
\mathrm{BH}_{3} \mathrm{CO}+\mathrm{Et}_{3} \mathrm{~N} & \rightarrow \text { complex }
\end{aligned}
$$

The $P_{1 / 2}$ was found to be about 90 torr from a study of total pressure effect on the reaction rate. 
Reaction: Trifluorophosphine borane

\begin{tabular}{crcr}
\multicolumn{4}{c}{$\mathrm{BH}_{3} \mathrm{PF}_{3}(\mathrm{I}) \longrightarrow \mathrm{BH}_{3}(\mathrm{II})+: \mathrm{PF}_{3}(\mathrm{III})$} \\
$\Delta H_{\text {f(298) }}^{\circ}$ & I & II & \multicolumn{1}{l}{ III } \\
$S_{(298)}^{\circ}$ & $(733)$ & 44.9 & 65.2 \\
$C_{p(298)}^{\circ}$ & $(\quad)$ & 8.6 & 14.0
\end{tabular}

$\Delta S^{\circ}=36.8 \mathrm{~g} / \mathrm{mole}$

$\Delta H^{\circ}=23.5 \mathrm{kcal} / \mathrm{mole}$

$\Delta C_{p}^{\circ}=$

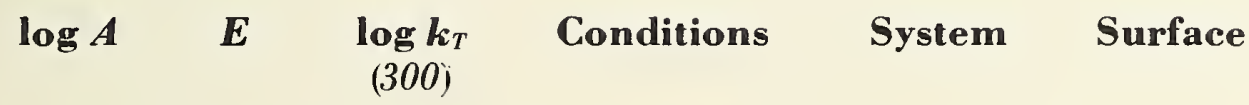

References

$13.94 \quad 23.45 \quad-3.14 \quad 273-328^{\circ} \mathrm{K} \quad$ static

(10-28 torr,

170-308 torr)

[1] A. B. Burg and Yuan-Chin Fu, J. Am. Chem. Soc. 88, 1147 (1966).

Preferred:

Reliable. $\quad \log k=13.94-23.45 / \theta$.

\section{Experimental}

[1] Rates were based on the formation of $\mathrm{PF}_{3}$ which was followed by I.R. analysis. The equilibrium

$$
2 \mathrm{BH}_{3} \mathrm{PF}_{3} \rightleftharpoons \mathrm{B}_{2} \mathrm{H}_{6}+2 \mathrm{PF}_{3}
$$

was also studied,

$$
\log K_{(\mathrm{atm})}=8.67-11.87 / \theta
$$

The mechanism proposed was:

$$
\begin{aligned}
& \mathrm{BH}_{3} \mathrm{PF}_{3} \frac{\mathrm{l}}{2} \mathrm{BH}_{3}+: \mathrm{PF}_{3} \\
& \mathrm{BH}_{3}+\mathrm{BH}_{3} \mathrm{PF}_{3} \frac{3}{4} \mathrm{~B}_{2} \mathrm{H}_{6}+\mathrm{PF}_{3} .
\end{aligned}
$$

From the kinetics, $\log \left(k_{1} k_{3} / k_{2}\right)=15.84-29.3 / \theta$, and from the thermodynamics (with equilibria in units of moles/1),

$$
\mathrm{B}_{2} \mathrm{H}_{6} \underset{6}{\stackrel{5}{6}} 2 \mathrm{BH}_{3}, \Delta G_{(\mathrm{kcal} / \mathrm{mole})}^{\mathrm{o}}=35.0-T(0.0342) \text {. }
$$

Thus the activation energy for diborane decomposition was set at $35 \pm 1 \mathrm{kcal} / \mathrm{mole}$ assuming $E_{6}=0$. In addition,

$$
\begin{aligned}
& \log k_{1} / k_{2}=8.06-23.45 / \theta \\
& \log \frac{k_{1} k_{3}}{k_{2} k_{4}}=8.67-11.88 / \theta \\
& \log k_{5} / k_{6}=7.47-35.0 / \theta
\end{aligned}
$$


Trifluorophosphine borane (Continued)

The decomposition kinetics of $\mathrm{BH}_{3} \cdot \mathrm{CF}_{3} \mathrm{PF}_{2}$ and $\mathrm{BH}_{3} \cdot\left(\mathrm{CF}_{3}{ }_{2} \mathrm{PF}\right.$ were also studied and found to conform to mechanisms similar to that for $\mathrm{PF}_{3} \cdot \mathrm{BH}_{3}$.

$$
\begin{aligned}
\left(\mathrm{BH}_{3} \cdot \mathrm{CF}_{3} \mathrm{PF}_{2}\right) \log \left(\frac{k_{1} k_{3}}{k_{2}}\right) & =11.02-24.47 / \theta \\
\left(\mathrm{BH}_{3} \cdot\left(\mathrm{CF}_{3}\right)_{2} \mathrm{PF}\right) \log \left(\frac{k_{1} k_{3}}{k_{2}}\right) & =11.70-21.54 / \theta
\end{aligned}
$$


Reaction: Dinitrogen oxide (nitrous oxide)

$\begin{array}{cccc} & \mathrm{N}_{2} \mathrm{O}(\mathrm{I}) \longrightarrow \mathrm{N}_{2}(\mathrm{II})+\mathrm{O}(\mathrm{III}) \\ \Delta H_{f(298)}^{\circ} & \mathrm{I} & \mathrm{II} & \text { III } \\ S_{(298)}^{\circ} & 19.5 & 0 & 59.2 \\ C_{p(298)}^{\circ} & 52.6 & 45.8 & 38.5 \\ & 9.3 & 7.0 & 5.2\end{array}$

$\Delta S^{\circ}=31.7 \mathrm{~g} / \mathrm{mole}$

$\Delta H^{\circ}=39.7 \mathrm{kcal} / \mathrm{mole}$

$\Delta C_{p}^{\circ}=2.9 \mathrm{~g} / \mathrm{mole}$

\begin{tabular}{|c|c|c|c|c|c|c|}
\hline $\log A$ & $\boldsymbol{E}$ & $\begin{array}{c}\log k_{T} \\
(720)\end{array}$ & Conditions & System & Surface & References \\
\hline 12.38 & 65.0 & -3.06 & $\begin{array}{l}840-999{ }^{\circ} \mathrm{K} \\
0.25-40 \text { atm }\end{array}$ & static & & $\begin{array}{l}\text { [1] E. Hunter, Proc. Roy. } \\
\text { Soc. (L) A144, } 386 \\
\text { (1934). }\end{array}$ \\
\hline 11.7 & 60.6 & -2.70 & $\begin{array}{l}\text { (reanalysis of all } \\
\text { existing data) } \\
(40 \mathrm{~atm})\end{array}$ & & & $\begin{array}{l}\text { [2] H. S. Johnston, J. Chem. } \\
\text { Phys. 19, } 663 \text { (1951). }\end{array}$ \\
\hline
\end{tabular}

Preferred:

Not reliable.

Comments: The very low Arrhenius $A$-factor supports Johnston's analysis [2], as a second-order pressure dependent unimolecular reaction.

\section{Experimental}

[1] Rates followed by pressure change. Above $30 \mathrm{~atm}$, the rate constants were essentially pressure independent. Mechanism:

$$
\begin{aligned}
& \mathrm{N}_{2} \mathrm{O} \longrightarrow \mathrm{N}_{2}+\mathrm{O} ; 2 \mathrm{O}+\mathrm{M} \longrightarrow \mathrm{O}_{2} \\
& \mathrm{O}+\mathrm{N}_{2} \mathrm{O} \longrightarrow \mathrm{O}_{2}+\mathrm{N}_{2} \text { (only in initial stages). }
\end{aligned}
$$

[2] Corrected all existing rate constants for the heterogeneous reaction at low pressures. Showed that even at $40 \mathrm{~atm}$, the rate constants were still increasing with increasing pressure; therefore, the reaction was probably not in its high-pressure region.

For earlier references to this reaction see H. J. Schumacher, Chemische Gasreaktionen, Photo-Lithoprint Reproduction, Edwards Brothers, Inc., Lithoprinters, Ann Arbor, Michigan, 1943, p. 131. 
Reaction: Dinitrogen tetroxide

$\begin{array}{ccc} & \mathrm{N}_{2} \mathrm{O}_{4}(\mathrm{I}) \longrightarrow 2 \mathrm{NO}_{2}(\mathrm{II}) & \\ & \mathrm{I} & \mathrm{II} \\ \Delta H_{f(298)}^{\circ} & 2.3 & 8.1 \\ S_{(298)}^{\circ} & 72.7 & 57.5 \\ C_{p(298)}^{\circ} & 18.9 & 8.7\end{array}$

$\Delta S^{\circ}=42.3 \mathrm{~g} / \mathrm{mole}$

$\Delta H^{\circ}=13.9 \mathrm{kcal} / \mathrm{mole}$.

$\Delta C_{p}^{\circ}=-1.5 \mathrm{~g} /$ mole

\begin{tabular}{|c|c|c|c|c|c|c|}
\hline $\log A$ & $\boldsymbol{E}$ & $\begin{array}{c}\log k_{T} \\
(275)\end{array}$ & Conditions & System & Surface & References \\
\hline 16.0 & 13.1 & +5.59 & $\begin{array}{l}253-301{ }^{\circ} \mathrm{K} \\
P_{T} \simeq 0.5-7 \text { atm } \\
1 \% \mathrm{~N}_{2} \mathrm{O}_{4} \text { in } \mathrm{N}_{2} \\
\quad \text { or } \mathrm{CO}\end{array}$ & shock & & $\begin{array}{l}\text { [1] T. Carrington and N. } \\
\text { Davidson, J. Phys. } \\
\text { Chem. 57, } 418 \text { (1953). }\end{array}$ \\
\hline
\end{tabular}

Preferred:

$\log k=16.0-13.1 / \theta ; A_{\text {rec }} \simeq 10^{8.2} 1 /$ mole-sec.

\section{Experimental}

[1] Rates were followed spectrophotometrically. For earlier references see H. J. Schumacher, Chemische Gasreaktionen, Photo-Lithoprint Reproduction, Edwards Brothers, Inc., Lithoprinters, Ann Arbor, Mich., 1943. $A$-factor was estimated by extrapolation of data to infinite pressure. 
Reaction: Dinitrogen pentoxide

$\begin{array}{cccc} & \mathrm{N}_{2} \mathrm{O}_{5}(\mathrm{I}) \longrightarrow \mathrm{NO}_{2}(\mathrm{II})+\mathrm{NO}_{3}(\mathrm{III}) & \\ & \mathrm{I} & \mathrm{II} & \mathrm{III} \\ \Delta H_{f(298)}^{\circ} & 2.7 & 8.1 & 17.0 \\ S_{(298)}^{\circ} & 82.8 & 57.5 & 60.4 \\ C_{1(298)}^{\circ} & 23.0 & 8.7 & 11.2\end{array}$

$\Delta S^{\circ}=35.1 \mathrm{~g} / \mathrm{mole}$

$\Delta H^{\circ}=22.4 \mathrm{kcal} / \mathrm{mole}$

$\Delta C_{p}^{\circ}=-3.1 \mathrm{~g} / \mathrm{mole}$

\begin{tabular}{|c|c|c|}
\hline $\log A$ & $\boldsymbol{E}$ & $\begin{array}{c}\log \boldsymbol{k}_{T} \\
(275)\end{array}$ \\
\hline
\end{tabular}

$12.78 \quad 19 \quad-2.32 \quad 264-283^{\circ} \mathrm{K} \quad$ static

88-133 mm Hg Daniels J. Am. Chem.

Soc. 74, 6209 (1952).

13.1 $20 \quad-2.80 \quad 293-303^{\circ} \mathrm{K} \quad$ static $\quad$ (none)

[2] I. C. Hisatsune, Bryce Crawford, Jr., and R. A. Ogg, Jr., J. Am. Chem.

Soc. 79, 4648 (1957).

$\begin{array}{lll}14.78 & 21 & -1.91\end{array}$

[3] S. W. Benson, Foundation of Chemical Kinetics, McGraw-Hill Book Co., Inc., New York, 1960, p. 408.

Preferred:

$\log k=14.78-21.0 / \theta ; k_{\text {rec }}=10^{8.6} \mathrm{l} /$ mole-sec.

\section{Experimental}

[1] Decomposed $\mathrm{N}_{2} \mathrm{O}_{5}$ in labeled $\mathrm{N}^{15} \mathrm{O}_{2}$. The rate of exchange was followed by M.S. analysis of the $\mathrm{N}_{2} \mathrm{O}_{5}$.

[2] Analysis by fast scanning I.R.

[3] Rate constants were calculated from the data of R. L. Mills and H. S. Johnston, J. Am. Chem. Soc. 73, 938 (1951). Also see [3] for extensive referencing of the kinetics of this reaction.

The equilibrium constant has been measured as $K_{c}=10^{4.97-20.1 \%} \mathrm{~mole} / \mathrm{l}$. 
Reaction: Nitryl chloride

$\begin{array}{cccc} & \mathrm{NO}_{2} \mathrm{Cl}(\mathrm{I}) \longrightarrow \mathrm{NO}_{2}(\mathrm{II})+\mathrm{Cl}(\mathrm{III}) & \\ \Delta H_{f(298)}^{\circ} & \mathrm{I} & \mathrm{II} & \mathrm{III} \\ S_{(298)}^{\circ} & (2.9) & 8.1 & 29.0 \\ C_{p(298)}^{\circ} & (65) & 57.5 & 39.5 \\ & (12.7) & 8.7 & 5.2\end{array}$

$\Delta S^{\circ}=32 \mathrm{~g} / \mathrm{mole}$

$\Delta H^{\circ}=34.2 \mathrm{kcal} / \mathrm{mole}$

$\Delta C_{p}^{\circ}=1.2 \mathrm{~g} / \mathrm{mole}$

\begin{tabular}{|c|c|c|c|}
\hline $\log A$ & $\boldsymbol{E}$ & $\begin{array}{c}\log k_{T} \\
(390)\end{array}$ & Conditions \\
\hline 12.36 & 27.0 & -2.77 & $\begin{array}{l}373-403^{\circ} \mathrm{K} \\
103-5900 \text { torr } \\
11.4-430 \text { torr }\end{array}$ \\
\hline
\end{tabular}

(See below)

[2] H. F. Cordes and H. S.

Johnston, J. Am. Chem. Soc. 76, 4264 (1954)

\section{Preferred:}

$\log k_{x}=15.0-33 / \theta$.

$\log k_{\text {rec }} \simeq 9.5$. Rate constants [1] are probably reliable.

\section{Experimental}

[1] Rates were followed by pressure change. Reaction products were $\mathrm{NO}_{2}$ and $\mathrm{Cl}_{2}$. Rate constants were pressure dependent. $k_{\infty}$ values were obtained from $(1 / k$ versus $1 / p)$ plot extrapolations.

Chain length is 2.

$$
\text { Mechanism: } \begin{aligned}
& \mathrm{NO}_{2} \mathrm{Cl} \rightleftharpoons \\
& \mathrm{Cl}+\mathrm{NO}_{2} \mathrm{Cl} \rightleftharpoons \mathrm{NO}_{2} \mathrm{Cl} \\
& \mathrm{Cl}_{2}+\mathrm{M} \rightleftharpoons 2 \mathrm{Cl}+\mathrm{NO}
\end{aligned}
$$

[2] The reaction was shown to be in the second order region with first order rate constants of any single run being total pressure dependent. Inert gas effects and relative collision efficiencies were studied. 
Reaction: Nitroxyl fluoride (fluorine nitrate)

$\begin{array}{cccc} & \mathrm{NO}_{3} \mathrm{~F}(\mathrm{I})-\mathrm{NO}_{3}(\mathrm{II})+\mathrm{F}(\mathrm{III}) & \\ \Delta H_{f(298)}^{\circ} & \mathrm{I} & \mathrm{II} & \mathrm{III} \\ S_{(2.98)}^{\circ} & 2.5 & 17.1 & 18.3 \\ C_{\boldsymbol{p}(2.98)}^{\circ} & 70.0 & 59.4 & 37.9 \\ & 15.6 & 12.0 & 5.4\end{array}$

$\Delta S^{\circ}=27.3 \mathrm{~g} / \mathrm{mole}$

$\Delta H^{\circ}=32.9 \mathrm{kcal} / \mathrm{mole}$

$\Delta C_{p}^{\circ}=1.8 \mathrm{~g} / \mathrm{mole}$

\begin{tabular}{|c|c|c|c|}
\hline $\log A$ & $\boldsymbol{E}$ & $\begin{array}{c}\log \boldsymbol{k}_{T} \\
(385)\end{array}$ & References \\
\hline
\end{tabular}

$15.39 \quad 32.3 \quad-2.95 \cdot 363-403^{\circ} \mathrm{K}$

0.6-400 $\mathrm{mm} \mathrm{Hy}$

[1] L. Viscido. J. E. Sicre. and H. J. Schumacher, Zeit. für Physik. Chemie. N.F. 33, 206 (1962)

13.76

$29.7-3.11 \quad 353-379^{\circ} \mathrm{K}$

$<1 \%$ $2-211 \mathrm{~mm} \mathrm{Hg}$

[2] W. E. Skeins and G. H. Cady, J. Am. Chem. Soc. 80, 5640 (1958)

Preferred:

$\log k=15.39-32.3 / \theta$.

Comments: The $A$-factor and reaction entropy give a recombination rate which is close to collision frequencies, $k_{\text {rec }}=10^{10.9} \mathrm{l} /$ mole-sec.

\section{Experimental}

[1] Rates were followed manometrically. Products were $\mathrm{O}_{2}$ and $\mathrm{NO}_{2} \mathrm{~F}$ in stoichiometric amounts. $\left(\mathrm{NO}_{3} \mathrm{~F} \rightarrow \mathrm{NO}_{2} \mathrm{~F}\right.$ $+1 / 2 \mathrm{O}_{2}$ ) Identification by I.R. Falloff was observed. and at $373^{\circ} \mathrm{K}$ the $P_{1 / 2} \simeq 32 \mathrm{~mm} \mathrm{Hg}$. The mechanism proposed was:

$$
\begin{aligned}
& \mathrm{NO}_{3} \mathrm{~F} \longrightarrow \mathrm{NO}_{3}+\mathrm{F} \\
& 2 \mathrm{NO}_{3} \longrightarrow 2 \mathrm{NO}_{2}+\mathrm{O}_{2} \\
& \mathrm{NO}_{2}+\mathrm{F}+\mathrm{M} \longrightarrow \mathrm{NO} \mathrm{O}_{2} \mathrm{~F}+\mathrm{M} .
\end{aligned}
$$

[2] Rates were followed by pressure changes. Products were identified by I.R., M.S., and gas density as $\mathrm{O}_{2}$ and $\mathrm{NO}_{2} \mathrm{~F}$. Falloff observed at $P<50 \mathrm{~mm} \mathrm{Hg}$. The mechanism above was favored. 
Reaction: Chlorine nitrate (nitroxyl chloride)

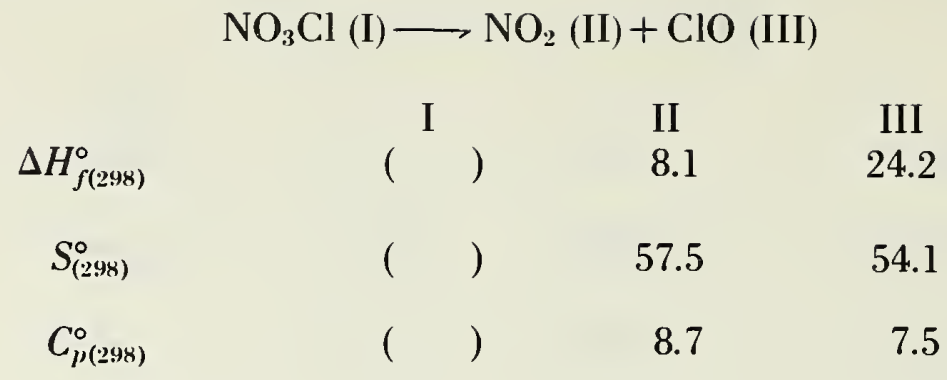

$\Delta S^{\circ}=$

$\Delta H^{\circ}=$

$\Delta C_{p}^{\circ}=$

$\log A$

E $\quad \log k$

Conditions

System

Surface

References

$\begin{array}{lllll}14.2 & 30 & -2.84 & 363-403 \% & \mathrm{~K}\end{array}$ 8-200 $\mathrm{mm} \mathrm{Hg}$

[1] L. F. R. Cafferata, J. E. Sicre, and H. J. Schumacher, Zeit für Physik. Chemie. N.F. 29, 188 (1961).

Preferred:

$\log k=14.2-30 / \theta$.

\section{Experimental}

[1] The reaction was followed manometrically. The stoichiometry was found to be $\mathrm{NO}_{3} \mathrm{Cl} \longrightarrow \mathrm{NO}_{2}+1 / 2 \mathrm{Cl}_{2}+1 / 2 \mathrm{O}_{2}$. Rate constants were pressure dependent.

The mechanism proposed was:

$$
\begin{aligned}
& \mathrm{NO}_{3} \mathrm{Cl} \stackrel{\mathrm{l}}{\underset{2}{\rightleftarrows}} \mathrm{NO}_{2}+\mathrm{OCl} \\
& \mathrm{ClO} \stackrel{\text { wall }}{\rightleftharpoons} 1 / 2 \mathrm{Cl}_{2}+\mathrm{l} / 2 \mathrm{O}_{2} \\
& \mathrm{ClO}+\mathrm{NO}_{3} \mathrm{Cl} \stackrel{4^{*}}{\rightleftharpoons} \mathrm{NO}_{2}+\mathrm{O}_{2}+\mathrm{Cl}_{2}
\end{aligned}
$$

*Reaction 4 was not meant to represent an elementary process. 
Reaction: Difluorine dioxide

$$
\begin{array}{lcc} 
& \mathrm{F}_{2} \mathrm{O}_{2}(\mathrm{I}) \stackrel{a}{\underline{b}} \stackrel{2 \mathrm{FO} \cdot(\mathrm{II})}{\longrightarrow} \mathrm{FO}_{\dot{2}}+\mathrm{F} \\
& \\
& \mathrm{I} & \mathrm{II} \\
\Delta H_{f(298)}^{\circ} & 18 \pm 5 & (27) \\
S_{(298)}^{\circ} & & \\
C_{p(298)}^{\circ} &
\end{array}
$$

\begin{tabular}{|c|c|c|c|c|c|c|}
\hline $\log A$ & $\boldsymbol{E}$ & $\begin{array}{c}\log \boldsymbol{k}_{T} \\
(230)\end{array}$ & Conditions & System & Surface & References \\
\hline 12.77 & 17.3 & -3.67 & $\begin{array}{l}213-248^{\circ} \mathrm{K} \\
2.5-500 \mathrm{~mm} \mathrm{Hg}\end{array}$ & static & & $\begin{array}{l}\text { [1] H. J. Schumacher and P. } \\
\text { Frisch, Z. Physik. } \\
\text { Chem. B3 7, } 1 \text { (1937). }\end{array}$ \\
\hline
\end{tabular}

Preferred:

Unreliable as a unimolecular reaction (see below).

Comments: Kinetic parameters are reliable; however, the proposed mechanism is not very reasonable. The kinetic data are beautifully first order over 3 half-lives, which implies that the reactant collision efficiency $\left(\alpha_{\mathrm{F}_{2} \mathrm{O}_{2}}\right)$ for activation is just twice the sum of the product collision efficiencies. However, the authors give $\left(\alpha_{\mathrm{O}_{2}}+\alpha_{\mathrm{F}_{2}}=1.5 \alpha_{\mathrm{F}_{2} \mathrm{O}_{2}}\right.$. The proposed split (path $\left.a\right)$ is also not favored by the reaction enthalpy. Thus we have,

$$
\Delta H_{f}^{\circ}\left(\mathrm{F}_{2} \mathrm{O}\right)=5.9 \mathrm{kcal} / \mathrm{mole}
$$

$$
\begin{array}{ll}
\mathrm{F}_{2} \mathrm{O} \stackrel{\mathrm{l}}{\longrightarrow} 2 \mathrm{~F} \cdot+\mathrm{O} \quad \Delta H_{1}^{\circ} \simeq 91 \mathrm{kcal} / \mathrm{mole} \\
\text { If } & D H^{\circ}(\mathrm{FO}-\mathrm{F}) \simeq 40 \pm 3 \mathrm{kcal} / \mathrm{mole} \\
\text { then } & D H^{\circ}(\mathrm{F}-\mathrm{O}) \simeq 51 \pm 3 \mathrm{kcal} / \mathrm{mole} \\
\text { and } & \Delta H_{f}^{\circ}(\cdot \mathrm{OF}) \simeq 27 \mathrm{kcal} / \mathrm{mole}
\end{array}
$$

Note also that,

$$
\mathrm{F}_{2} \mathrm{O}_{2} \stackrel{2}{\longrightarrow} 2 \mathrm{~F}+\mathrm{O}_{2} \quad \Delta H_{2}^{\circ} \simeq 20 \mathrm{kcal} / \mathrm{mole}
$$

In view of the above thermodynamics, a more reasonable interpretation of the reaction is as follows:

$$
\begin{aligned}
& \mathrm{M}+\mathrm{F}_{2} \mathrm{O}_{2} \rightleftharpoons \mathrm{F}+\mathrm{FO}_{2}+\mathrm{M} \\
& \mathrm{M}+\mathrm{FO}_{2} \longrightarrow \mathrm{F}+\mathrm{O}_{2}+\mathrm{M}
\end{aligned}
$$


Difluorine dioxide (Continued)

$$
\begin{aligned}
& \mathrm{F}+\mathrm{F}_{2} \mathrm{O}_{2} \longrightarrow \mathrm{F}_{2}+\mathrm{FO}_{2} \\
& \mathrm{~F} \cdot \stackrel{\text { wall }}{\longrightarrow} 1 / 2 \mathrm{~F}_{2} \\
& 2 \mathrm{~F} \cdot+\mathrm{M} \rightleftarrows \mathrm{F}_{2}+\mathrm{M}
\end{aligned}
$$

Path $b$ seems the more likely initiation step, and it should be pressure dependent.

\section{Experimental}

[1] Rates were followed by pressure change. Products were $\mathrm{F}_{2}$ and $\mathrm{O}_{2}$. The reaction was in the falloff region under experimental conditions and $k_{x}$ values were obtained from $1 / k$ versus $1 / p$ plot extrapolations. $P_{x}$ was estimated at around $10^{3} \mathrm{~mm} \mathrm{Hg}$. Path $a$ was proposed as the rate-determining initiation step.

The proposed mechanism was:

$$
\begin{aligned}
& \mathrm{F}_{2} \mathrm{O}_{2} \longrightarrow 2 \mathrm{FO} . \\
& 2 \mathrm{FO} \longrightarrow \mathrm{F}_{2}+\mathrm{O}_{2} .
\end{aligned}
$$


Reaction: Chloryl fluoride

$$
\mathrm{FClO}_{2} \longrightarrow \mathrm{FClO}+\mathrm{O}
$$

$\Delta H_{f(298)}^{\circ}$

$$
\mathrm{S}_{(298)}^{\circ}
$$

$\mathrm{C}_{\text {(298) }}^{\circ}$

$\Delta S^{\circ}=$

$\Delta H^{\circ}=$

$\Delta C_{p}^{\circ}=$

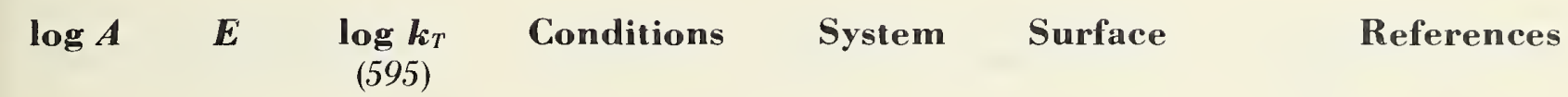

$\begin{array}{llll}13.36 & 45.0 & -3.17 & 573-613^{\circ} \mathrm{K} \\ & & 5-450 \mathrm{~mm} \mathrm{Hg}\end{array}$ static

[1] M. J. Heras, P. J.

Aymonino, and H. J.

Schumacher, Zeit für

Physik. Chemie, N.F.

22, 161 (1959).

\section{Preferred:}

Probably reliable. $\log k=13.36-45.0 / \theta$.

\section{Experimental}

[1] Rates were followed by pressure change. Products were $\mathrm{FCl}$ and $\mathrm{O}_{2}$. Reaction of $\mathrm{FCl}$ with the glass walls also produced $\mathrm{Cl}_{2}$ and $\mathrm{SiF}_{4}$.

The mechanism proposed was:

$$
\begin{aligned}
& \mathrm{FClO}_{2} \longrightarrow \mathrm{FClO}+\mathrm{O} \\
& \mathrm{O}+\mathrm{FClO}_{2} \longrightarrow \mathrm{FClO}+\mathrm{O}_{2} \\
& 2 \mathrm{FClO} \longrightarrow 2 \mathrm{FCl}+\mathrm{O}_{2} \\
& \mathrm{FClO} \stackrel{\text { wall }}{\longrightarrow} \mathrm{FCl}+1 / 2 \mathrm{O}_{2} .
\end{aligned}
$$


Reaction: Perchloryl fluoride

$\begin{array}{cccc} & \mathrm{FClO}_{3}(\mathrm{I}) \longrightarrow \mathrm{FClO}_{2}(\mathrm{II})+\mathrm{O}(\mathrm{III}) & \\ \Delta H_{f(298)}^{\circ} & \mathrm{I} & \mathrm{II} & \mathrm{III} \\ S_{(298)}^{\circ} & -5.1 & (\stackrel{ }{ }) & 59.2 \\ C_{p(298)}^{\circ} & 66.7 & (63) & 38.5 \\ & 15.5 & (7.2) & 5.2\end{array}$

$\Delta S^{\circ}=$
$\Delta H^{\circ}=$
$\Delta C_{p}^{\circ}=$

$\begin{array}{lllll}\log A & E & \log k_{T} & \text { Conditions } & \text { System }\end{array}$ (755)

$13.59 \quad 58.4 \quad-3.32 \quad 738-768^{\circ} \mathrm{K} \quad$ static none

[1] R. Gatti, J. E. Sicre, and H. J. Schumacher, Zeit. für Physik. Chemie., N.F. 23, $164(1960)$.

Preferred: Probably reliable. $\log k=13.59-58.4 / \theta$.

\section{Experimental}

[1] The rate of reaction was followed manometrically. The products were $\mathrm{FCl}$ and $\mathrm{O}_{2}$. The reaction mechanism proposed was:

$$
\begin{aligned}
& \mathrm{FClO}_{3} \stackrel{\text { slow }}{\longrightarrow} \mathrm{O}+\mathrm{FClO}_{2} \\
& \mathrm{O}+\mathrm{FClO}_{3} \underset{\mathrm{FClO}_{2}}{\longrightarrow} \mathrm{FCl}+\mathrm{O}_{2}
\end{aligned}+\mathrm{O}_{2}
$$


Reaction: Dichlorine heptoxide

$\begin{array}{cccc} & \mathrm{Cl}_{2} \mathrm{O}_{7}(\mathrm{I}) \longrightarrow \mathrm{ClO}_{3}(\mathrm{II})+\mathrm{ClO}_{4}(\mathrm{III}) & \\ \Delta H_{f(298)}^{\circ} & \mathrm{I} & \mathrm{II} & \mathrm{III} \\ S_{(298)}^{\circ} & (65.0) & 37 & (\quad) \\ C_{p(298)}^{\circ} & (\quad) & 61.3 & (\quad) \\ & (\quad) & 12.1 & (\quad)\end{array}$

$\Delta S^{\circ}=$

$\Delta H^{\circ}=$

$\Delta C_{p}^{\circ}=$

$\log$

$\boldsymbol{E} \quad \log \boldsymbol{k}_{T}$
$(385)$

Conditions

System

Surface

static

none

$1-80 \mathrm{~mm} \mathrm{Hg}$

total $P \sim 400$

$\mathrm{mm} \mathrm{Hg}$
References

[1] E. Coloccia, R. V.

Figini, and H. J.

Schumacher, Angew.

Chem. 68, 492 (1956).

Preferred:

Reasonable. $\log k=15.72-33.5 / \theta$.

Comments: With $n_{\max }=20$, an $\mathrm{RRK}-M_{1 / 2} \simeq 76 \mathrm{~mm} \mathrm{Hg}$ is calculated. Thus falloff would be predicted near the pressure range observed.

\section{Experimental}

[1] Rates were followed manometrically. Products were $\mathrm{Cl}_{2}$ and $\mathrm{O}_{2}$. Evidence for $\mathrm{ClO}_{2}$ as an intermediate was obtained by pyrolysis in fluorine in which $\mathrm{FClO}_{2}$ was obtained as a product. Falloff of the reaction rate was observed at pressures below $10 \mathrm{~mm} \mathrm{Hg}$. At $1 \mathrm{~mm} \mathrm{Hg}$ the reaction rate constant was $1 / 2 k_{x}$. This may be a chemical effect, not a genuine pressure effect. 
Reaction: Perchloric acid

\begin{tabular}{cccc}
\multicolumn{4}{c}{$\mathrm{HOClO}_{3}(\mathrm{I}) \longrightarrow \mathrm{HO} \cdot(\mathrm{II})+\cdot \mathrm{ClO}_{3}(\mathrm{III})$} \\
$\Delta H_{f(298)}^{\circ}$ & $\mathrm{I}$ & $\mathrm{II}$ & $\mathrm{III}$ \\
$S_{(298)}^{\circ}$ & $4 \pm 4$ & 9.4 & 37 \\
$C_{p(298)}^{\circ}$ & 69 & 43.9 & 61.3 \\
& 17.6 & 7.2 & 12.1
\end{tabular}

$\Delta S^{\circ}=36.2 \mathrm{~g} / \mathrm{mole}$

$\Delta H^{\circ}=42.4 \pm 4 \mathrm{kcal} / \mathrm{mole}$

$\Delta C_{p}^{\circ}=1.7 \mathrm{~g} / \mathrm{mole}$

$\begin{array}{rllclcl}\log \boldsymbol{A} & \boldsymbol{E} & \log \boldsymbol{k}_{T} & \text { Conditions } & \text { System } & \text { Surface } & \text { References } \\ 13.8 & 45.1 & & 623-712^{\circ} \mathrm{K} & \text { flow } & <5 \% & \text { [1] J. B. Levy, J. Phys. } \\ & & & & & & \text { Chem. 66, 1092 (1962). }\end{array}$

Preferred:

Reasonable. $\log k=13.8-45.1 / \theta$.

Comments: The energetics are consistent with the thermodynamics, however, a rate constant for recombination of about, $k_{\mathrm{rec}}=10^{7.7} \mathrm{l} / \mathrm{mole}$-sec is calculated from $A$ and $\Delta S^{\circ}$ which seems low.

\section{Experimental}

[1] $\mathrm{N}_{2}$ carrier was employed. Products were $\mathrm{H}_{2} \mathrm{O}, \mathrm{O}_{2}$, and $\mathrm{Cl}_{2}$. Analysis was for chloride by the Volhard and iodometric methods. The mechanism proposed was:

$$
\begin{aligned}
& \mathrm{HOClO}_{3} \stackrel{\mathrm{l}}{\longrightarrow} \mathrm{HO} \cdot+\mathrm{ClO}_{3} . \\
& \mathrm{HO}+\mathrm{HOClO}_{3} \stackrel{2}{\longrightarrow} \mathrm{H}_{2} \mathrm{O}+\mathrm{ClO}_{4} \cdot \\
& \mathrm{ClO}_{4} \cdot \stackrel{3}{\longrightarrow} 1 / 2 \mathrm{Cl}_{2}+2 \mathrm{O}_{2} \\
& \mathrm{ClO}_{3} \cdot \stackrel{4}{\longrightarrow} 1 / 2 \mathrm{Cl}_{2}+3 / 2 \mathrm{O}_{2} .
\end{aligned}
$$

The observed kinetics were identified with the initiation step; subsequent reactions of indeterminate nature were considered fast and non-chain producing. The reaction was heterogeneous at $T<623^{\circ} \mathrm{K}$. 
5. Free Radical Reactions 


\begin{tabular}{ccccr}
\multicolumn{5}{c}{$\mathrm{C}_{2} \mathrm{H}_{5} \cdot(\mathrm{I}) \longrightarrow \mathrm{H} \cdot \mathrm{C}_{2} \mathrm{H}_{4}(\mathrm{II})$} \\
& \multicolumn{2}{c}{$\mathrm{I}$} & $\mathrm{H}$ & $\mathrm{II}$ \\
& $\begin{array}{cccc}V=0 \\
\Delta H_{f(298)}^{\circ}\end{array}$ & $V=4$ & & \\
$S_{(298)}^{\circ}$ & 59.7 & 25.7 & 52.1 & 12.5 \\
$C_{p(298)}^{\circ}$ & 10.9 & 57.8 & 27.4 & 52.5 \\
$C_{p(500),}^{\circ}$ & 16.7 & 17.7 & 5.0 & 10.4 \\
& & & 5.0 & 15.0
\end{tabular}

$V=0$
$\Delta S^{\circ}=20.3$
$\Delta H^{\circ}=38.9$
$\Delta C_{\nu}^{\circ}=4.5$

$\log A$

14.0

40

$V=4$

$22.1 \mathrm{~g} / \mathrm{mole}$

$38.9 \mathrm{kcal} / \mathrm{mole}$

$3.3 \mathrm{~g} / \mathrm{mole}$

$\log k_{T}$
$(750)$
I

2.5

2.5

0.4

15.0
$11.2 \quad 31$
$\begin{array}{ll}2.16 & 623-778^{\circ} \mathrm{K} \\ & P_{T} \sim 10 \text { torr }\end{array}$
(see Experimental)

$\begin{array}{llll}13.0 & 40.0 \quad 1.34 & \begin{array}{l}693-803^{\circ} \mathrm{K} \\ 10-150 \text { torr }\end{array}\end{array} \quad$ (see Experimental)

Conditions

System Surface

References

$\operatorname{Hg}\left({ }^{3} P_{1}\right)$

[1] S. Bywater and E. W. R. Steacie, J. Chem. Phys. 19, 326 (1951).

[2] J. A. Kerr and A. F. Trotman-Dickenson, r. Chem. Soc. 1611 (1960).

[3] J. H. Purnell and C. P. Quinn, Proc. Roy. Soc. A2 70, 267 (1962).

\section{Preferred:}

$\log k_{a} \simeq 13.5-40.7 / \theta$.

Comments: Kinetics of the back reaction have been reported by a number of workers: K. R. Jennings and R. J. Cvetanovic, J. Chem. Phys. 35, 1233 (1961); H. W. Melville and J. C. Robb, Proc. Roy. Soc. (L) A202, 181 (1950); Ibid. A2 18, 311 (1953); B. deB. Darwent and R. Roberts, Disc. Faraday Soc. 14, 15 (1953); K. Yang, J. Am. Chem. Soc. 84, 3795 (1962). Activation energies range from 2.5 to $5.0 \mathrm{kcal} / \mathrm{mole}$ with variations in absolute rates of up to an order of magnitude. We favor $k_{a}(1 / \mathrm{mole}-\mathrm{sec}) \simeq 10^{10.6-3.0 / \theta}$ (K. Yang). From the reaction thermodynamics one then estimates $\left(T \sim 600^{\circ} \mathrm{K}\right)$ :

$\log k_{a}(V=0) \simeq 13.4-40.7 / \theta$;

$\log k_{a}(V=4) \simeq 13.7-40.7 / \theta$.

\section{Experimental}

[1] Photosensitized decomposition of ethane at high temperatures. At low temperatures ethyl radicals formed in the reactions $\left(\mathrm{C}_{2} \mathrm{H}_{6}+\mathrm{Hg}\left({ }^{3} \mathrm{P}_{1}\right) \longrightarrow \mathrm{Hg}\left({ }^{1} \mathrm{~S}_{0}\right)+\mathrm{C}_{2} \mathrm{H}_{5}+\mathrm{H} ; \mathrm{H}+\mathrm{C}_{2} \mathrm{H}_{6} \longrightarrow \mathrm{H}_{2}+\mathrm{C}_{2} \mathrm{H}_{5}\right)$ recombine. At higher temperatures the ethyl radical decomposition competes with recombination. Rate constants were based on the $\mathrm{H}_{2}$ quantum yields at high temperatures. 
[2] System-photoinitiated chain decomposition of propionaldehyde in the presence of ethylene. Above $350{ }^{\circ} \mathrm{C}$ the rate of ethylene formation increased appreciably, indicating ethyl radical decomposition. The monitoring reaction was the ethyl recombination.

[3] Kinetics deduced from a detailed treatment of the $n$-butane decomposition kinetics. Total product analysis by G.L.C.

Mechanism:

$$
\begin{gathered}
\mathrm{C}_{4} \mathrm{H}_{10} \stackrel{1 b}{\longrightarrow} \mathrm{C}_{3} \mathrm{H}_{7} \cdot+\mathrm{CH}_{3} ; \mathrm{C}_{4} \mathrm{H}_{10} \stackrel{1 c}{\longrightarrow} \mathrm{C}_{2} \mathrm{H}_{5} \cdot+\mathrm{C}_{2} \mathrm{H}_{5} \cdot \\
s-\mathrm{C}_{4} \mathrm{H}_{9} \cdot \stackrel{2}{\longrightarrow} \mathrm{C}_{2} \mathrm{H}_{4}+\mathrm{C}_{2} \mathrm{H}_{5} \cdot \\
s-\mathrm{C}_{4} \mathrm{H}_{9} \cdot \stackrel{3}{\longrightarrow} \mathrm{C}_{3} \mathrm{H}_{6}+\mathrm{CH}_{3} \cdot \\
\mathrm{C}_{2} \mathrm{H}_{5} \cdot+\mathrm{C}_{4} \mathrm{H}_{10} \stackrel{4}{\longrightarrow} \mathrm{C}_{2} \mathrm{H}_{6}+\mathrm{C}_{4} \mathrm{H}_{9} \cdot \quad(n \text { or } s) \\
\mathrm{CH}_{3} \cdot+\mathrm{C}_{4} \mathrm{H}_{10} \stackrel{5}{\longrightarrow} \mathrm{CH}_{4}+\mathrm{C}_{4} \mathrm{H}_{9} \cdot \quad(n \text { or } s) \\
\mathrm{H}+\mathrm{C}_{4} \mathrm{H}_{10} \stackrel{6}{\longrightarrow} \mathrm{H}_{2}+\mathrm{C}_{4} \mathrm{H}_{9} \cdot \\
\mathrm{C}_{2} \mathrm{H}_{5} \cdot \stackrel{7}{\longrightarrow} \mathrm{C}_{2} \mathrm{H}_{4}+\mathrm{H} \cdot \\
2 \mathrm{C}_{2} \mathrm{H}_{5} \cdot \stackrel{8 a}{\longrightarrow} \mathrm{C}_{4} \mathrm{H}_{10} \\
\stackrel{8 b}{\longrightarrow} \mathrm{C}_{2} \mathrm{H}_{4}+\mathrm{C}_{2} \mathrm{H}_{6}
\end{gathered}
$$

Values of $k_{7}$ were obtained from the (ethylene/ethane) $=\alpha$ ratio, using the steady state relation $(\alpha-1)\left[\mathrm{C}_{4} \mathrm{H}_{10}\right]=2 k_{7} / k_{4}$ and $k_{4}=5.9 \times 10^{8-15.2 / \theta} \mathrm{l} / \mathrm{mole}$-sec. The decomposition was shown to be pressure dependent under reaction conditions and was fit to an RRK-pressure falloff curve with $s=7$. 
Reaction: $n$-Propyl radical

\begin{tabular}{|c|c|c|c|c|c|c|c|}
\hline & & $\mathrm{CH}_{3} \mathrm{C}$ & (I) & $\begin{array}{l}+\mathrm{CH}_{3} \\
\cdot(\mathrm{III})\end{array}$ & $\begin{array}{l}\mathrm{H}_{2} \text { (II) } \\
\text { (IV) }\end{array}$ & & \\
\hline & & I & & & & & \\
\hline$\Delta H_{f(298)}^{\circ}$ & $\begin{array}{l}\stackrel{V=0}{\longleftarrow} \\
\longleftarrow\end{array}$ & $\begin{array}{c}V=4 \\
20.7\end{array}$ & $\begin{array}{l}V=8 \\
\longrightarrow\end{array}$ & $\begin{array}{c}\mathrm{H} \cdot \\
52.1\end{array}$ & $\begin{array}{l}\text { II } \\
4.9\end{array}$ & $\begin{array}{l}\text { III } \\
34.0\end{array}$ & $\begin{array}{c}\text { IV } \\
12.5\end{array}$ \\
\hline$S_{(298)}^{\circ}$ & 69.2 & 67.2 & 66.4 & 27.4 & 63.8 & 46.4 & 52.5 \\
\hline$C_{p(298)}^{\circ}$ & 16.4 & 17.6 & 17.4 & 5.0 & 15.3 & 8.3 & 10.4 \\
\hline$C_{p(500)}^{\circ}$ & 25.0 & 26.0 & 26.1 & 5.0 & 24.4 & 10.1 & 15.0 \\
\hline$C_{p(800)}^{\circ}$ & 34.1 & 35.1 & 35.3 & 5.0 & 30.8 & 12.7 & 20.1 \\
\hline $\begin{array}{l}\quad a(V=4) \\
\Delta S^{\circ}=24.0 \\
\Delta H^{\circ}=36.3 \\
\Delta C_{p}^{\circ}=2.7\end{array}$ & $\begin{array}{r}b \\
31.7 \\
25.8 \\
1.7\end{array}$ & $\begin{array}{l}\text { le } \\
\text { mole }\end{array}$ & & & & & \\
\hline
\end{tabular}

\begin{tabular}{lrrrr} 
Path & $\log \boldsymbol{A}$ & $\boldsymbol{E}$ & \multicolumn{1}{l}{$\begin{array}{c}\log k_{T} \\
(510)\end{array}$} & \multicolumn{1}{c}{ Conditions } \\
& & & \multicolumn{1}{c}{} \\
$a$ & 13.6 & 35.0 & $-1.40(430)$ & $298-(737){ }^{\circ} \mathrm{K}$ \\
$b$ & 11.7 & 25.2 & -1.11 & $10-40 \mathrm{~mm} \mathrm{Hg}$
\end{tabular}

System Surface

References

[1] J. A. Kerr and A. F. Trotman-Dickenson, Trans.

Faraday Soc. 55, 572 (1959).

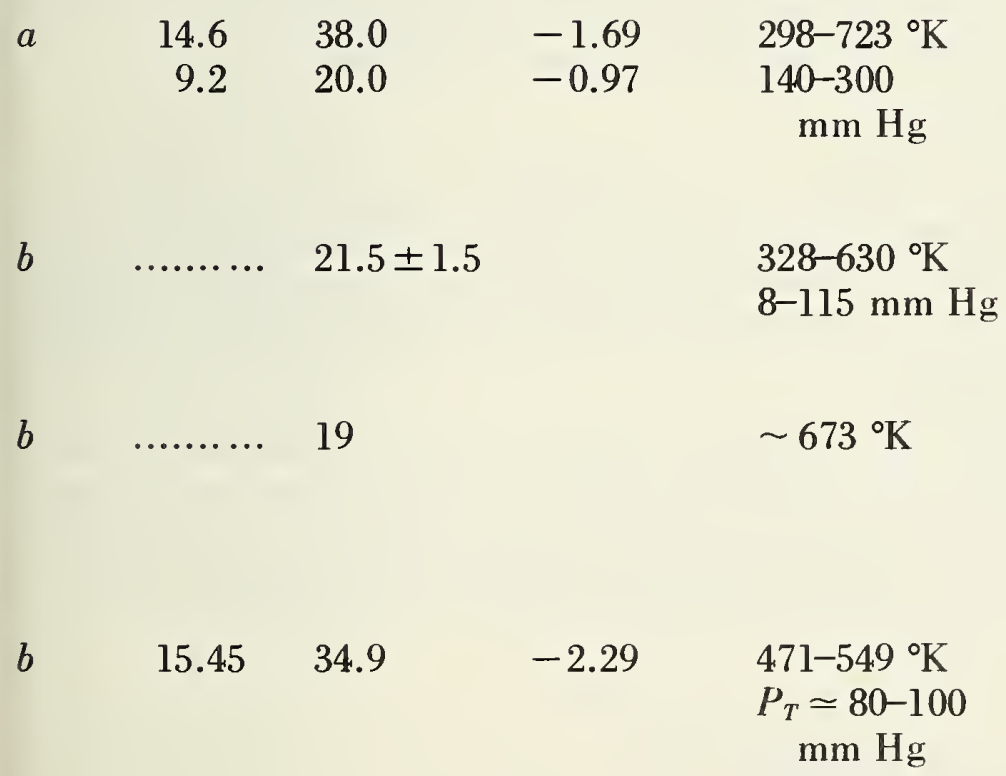

[2] S. Bywater and E. W. R. Steacie, J. Chem. Phys. 19, 319 (1951).

[3] C. R. Masson, J. Am. Chem. Soc. 74, 4731 (1952).

[4] R. W. Durham, G. R. Martin, and H. C. Sutton, Nature 164, 1052 (1949).

[5] J. G. Calvert and W. C. Sleppy, J. Am. Chem. Soc. 81, 1544 (1959). 


\begin{tabular}{|c|c|c|c|c|c|c|c|}
\hline Path & $\log A$ & $\boldsymbol{E}$ & $\underset{(510)}{\log \boldsymbol{k}_{T}}$ & Conditions & System & Surface & References \\
\hline$b$ & 15.36 & 34.5 & -2.18 & $\begin{array}{c}297-564^{\circ} \mathrm{K} \\
P_{T} \simeq 13-25 \\
\mathrm{~mm} \mathrm{Hg}\end{array}$ & & & $\begin{array}{l}\text { [6] J. A. Kerr and J. G. } \\
\text { Calvert, J. Am. } \\
\text { Chem. Soc. 83, } \\
\text { 3391 (1961). }\end{array}$ \\
\hline$a$ & $\begin{array}{l}14.1 \\
13.9\end{array}$ & $\begin{array}{l}37.0 \\
31.0\end{array}$ & $\begin{array}{l}-1.76 \\
-1.86\end{array}$ & $\begin{array}{l}569-693^{\circ} \mathrm{K} \\
P_{T} \simeq 50 \\
\quad \mathrm{~mm} \mathrm{Hg}\end{array}$ & & & $\begin{array}{l}\text { [7] W. M. Jackson and } \\
\text { J. R. McNesby, J. } \\
\text { Am. Chem. Soc. } \\
\text { 83, } 4891 \text { (1961). }\end{array}$ \\
\hline
\end{tabular}

Preferred:

$\log k_{a}=13.8-38 / \theta$

$\log k_{b}=13.6-33.1 / \theta$.

Comments: A large number of measurements for both back reactions are available. We favor the following: $\log k_{-a}(1 /$ mole-sec $)=10.6-3.0 / \theta$ [K. Yang, J. Am. Chem. Soc. 84, 3795 (1962).]

$\log \mathrm{k}_{-b}(\mathrm{l} / \mathrm{mole-sec})=9.07-8.66 / \theta$ [R. K. Brinton, J. Chem. Phys. 29, 781 (1958).]

$\log k_{-b}(1 /$ mole-sec $)=8.6-7.9 / \theta$ [R. J. Cvetanovic and R. S. Irwin, J. Chem. Phys. 46, 1694 (1967)].

Calculations of the decomposition parameters from the above and the reaction thermodynamics $(V=2.0$ $\mathrm{kcal} / \mathrm{mole}): \Delta H_{T}^{\circ}(a)=37.2 \mathrm{kcal} / \mathrm{mole} ; \Delta S_{T}^{\circ}(a)=25.6 \mathrm{~g} / \mathrm{mole} ; \Delta H_{T}^{\circ}(b)=25.7 \mathrm{kcal} / \mathrm{mole} ; \Delta S_{T}^{\circ} \simeq 31.2 \mathrm{~g} / \mathrm{mole}$, $T=600^{\circ} \mathrm{K}$ gives

$\log k_{a}=14.1-39 / \theta$.

$\log k_{b}=13.8-33.2 / \theta$.

It is apparent that there is an absolute discrepancy in reaction rates observed and predicted by about a factor of 10, the observed rate constants being the higher. Agreements in activation energies are acceptable with "best" values of $E_{a} \simeq 38 \pm 2 \mathrm{kcal} / \mathrm{mole}$ and $E_{b} \simeq 33 \pm 2 \mathrm{kcal} / \mathrm{mole}$. However, scaled $A$-factors (observed) for the decompositions do not agree with those calculated.

It is almost impossible to trace the origin of the discrepancy since assumptions regarding other "reference" reactions are involved. The error could be in the forward and reverse $A$-factors, or in the estimated entropies; however, the former seem more likely. We favor parameters close to those calculated since they are close to what might be predicted by transition state calculations (see section $\mathrm{V}-10.0$ ).

\section{Experimental}

[1] Photolysis of $n$-butyraldehyde in the presence of ethylene or propionaldehyde. Analysis by G.L.C. Path $b$ decomposition was measured relative to the $n$-propyl radical recombination $\left(k_{\mathrm{rec}}=10^{11} \mathrm{l} / \mathrm{mole}-\mathrm{sec}\right.$-assumed $)$ from both the production of methane and of ethylene. Similar results were obtained from both products. Above $300{ }^{\circ} \mathrm{C}$. propylene production increased markedly and was attributed to path $a$. The monitoring reaction was again the $n$-propyl recombination reaction. which was calculated at the higher temperatures from the known relative rate constants for $n$-propyl radical $\mathrm{H}$-abstraction from the aldehyde and for recombination.

[2| $\mathrm{Hg}\left({ }^{3} P_{1}\right)$ photosensitized decomposition of propane. Decompositions (paths $a$ and $b$ ) occurred above $300{ }^{\circ} \mathrm{C}$. $\mathrm{C}_{1}-\mathrm{C}_{\overline{5}}$ products were separated by fractional sublimation and analyzed with a Blacet-Leighton apparatus. Hexanes were analyzed by M.S. Both paths $a$ and $b$ were calculated from the high-temperature chain production of $\mathrm{H}_{2}$ and $\mathrm{CH}_{4}$, respectively. relative to the recombination reaction. Lower pressure experinents led to erratic results. It should be noted that both $n$-propyl and isopropyl radicals are produced in this reaction and both can decompose to give $\mathrm{H}_{2}$, as ultimate product.

[3] System-Photolysis of di-n-propyl ketone (3130 A). Primary processes are:

$$
\mathrm{C}_{3} \mathrm{H}_{\overline{7}} \mathrm{COC}_{33} \mathrm{H}_{\overline{7}} \stackrel{h \nu}{\mathrm{C}_{2} \mathrm{H}_{4}+\mathrm{CH}_{3} \mathrm{COC}_{3} \mathrm{H}_{\overline{7}}} \underset{2 \mathrm{C}_{3} \mathrm{H}_{\overline{7}} \cdot+\mathrm{CO} .}{ }
$$

(Continued) 
The rate of $n$-propyl decomposition was monitored two ways, (1) relative to $\mathrm{H}$-abstraction from the ketone

$$
\mathrm{C}_{3} \mathrm{H}_{7} \longrightarrow \mathrm{C}_{3} \mathrm{H}_{8}+\mathrm{K}_{-\mathrm{H}},
$$

and (2) relative to the $n$-propyl radical recombination reaction. Insufficient data are provided for an $A$-factor estimate.

[4| System $-n$-propyl radicals produced by the Polanyi sodium flame technique (H. Hartel and M. Polanyi, Z. Physik Chem. B, 11, $97(1930)$ ) were passed through a heated furnace along with radioactive ( $\left.I_{2}^{131}\right)$. The resulting alkyl iodides were trapped, separated, and analyzed by $\beta$-scintillation techniques. Data not amenable to the determination of $A$.

[5] $n$-propyl radicals were produced in the photolysis of azomethane $(3660 \mathrm{~A})$ in $n$-butyraldehyde-azomethane mixtures. The $\mathrm{C}_{3} \mathrm{H}_{7} \cdot$ decomposition was monitored relative to the two radical-radical recombination reactions: $\left(\mathrm{CH}_{3}+n \mathrm{C}_{3} \mathrm{H}_{7} \stackrel{5}{\longrightarrow} \mathrm{C}_{4} \mathrm{H}_{10}\right.$, and $2 \mathrm{CH}_{3} \cdot \stackrel{6}{\longrightarrow} \mathrm{C}_{2} \mathrm{H}_{6}$ with $k_{5}=k_{6}=2.2 \times 10^{10} \mathrm{l} / \mathrm{mole}$-sec $)$. Analysis by G.L.C.

[6] $n$-propyl radicals were produced in the photolysis of azo-n-propane. at $3660 \AA$. The $\mathrm{C}_{3} \mathrm{H}_{7} \cdot$ decomposition was monitored relative to the $n \cdot \mathrm{C}_{3} \mathrm{H}_{7} \cdot$ radical recombination reaction. Total product analyses were made. good mass balances were obtained. A total pressure effect on the decomposition was demonstrated. Analysis by C.L.C.

[7] Photolysis of acetone $\left(d_{6}\right)$ in propane-2,2- $d_{2}$. The relative rates of the two decomposition paths were determined by the rates of formation $\mathrm{CH}_{4}$ and $\mathrm{CH}_{3} \mathrm{D}$ (i.e., $\mathrm{CH}_{3} \mathrm{CD}_{2} \mathrm{CH}_{2} \cdot \longrightarrow \mathrm{CH}_{3} \cdot \mathrm{CH}_{2}=\mathrm{CD}_{2}$, the only source of $\mathrm{CH}_{3}$ radicals), and of $\mathrm{HD}$ and $\mathrm{D}_{2}$ (i.e., $\mathrm{CH}_{3} \mathrm{CD}_{2} \mathrm{CH}_{2} \longrightarrow \mathbb{N}+\mathrm{D} \cdot$, the only source of $\mathrm{D}$ atoms). Analysis by M.S. Rate constants for $\mathrm{D}$ atom elimination (path $a$ ) were corrected for the deuterium isotope effect. Absolute parameters were obtained on the basis of thermochemical data, assumption regarding the back activation energies (i.e., $E_{-a}=2 \mathrm{kcal} / \mathrm{mole}$ and $E_{-b}=7$ $\mathrm{kcal} / \mathrm{mole}$ ), and with the assumption that $k_{a}$ and $k_{b}$ were the values determined in reference [1].

NoTE added in proof: Methyl additions to a large number of olefins have been studied recently by R. J. Cvetanovic and R. S. Irwin. Arrhenius parameters are reported based on H-abstraction rates of methyl from isobutane, using $E_{-H}=7.6$ $\mathrm{kcal} / \mathrm{mole}$. Slightly better agreement between the reaction thermodynamics and the observed kinetics of the dissociation and addition reactions are obtained with these addition parameters. 
Reaction: 2-Deuteroisopropyl and isopropyl radical

\begin{tabular}{|c|c|c|c|c|c|c|c|c|c|}
\hline & & & $\mathrm{CH}_{3} \dot{\mathrm{CD}}$ & $\mathrm{CCH}_{3}(\mathrm{I}) \frac{a}{b}$ & $\begin{array}{l}\rightarrow \mathrm{CH}_{3} \mathrm{C} \\
-\mathrm{CH}_{2}=\end{array}$ & $\begin{array}{l}\mathrm{D}=\mathrm{CH}_{2} \\
=\mathrm{CHD}\end{array}$ & $\begin{array}{l}\text { I) }+\mathrm{H} \cdot \\
+\mathrm{CH}_{3}\end{array}$ & & \\
\hline & & $\Delta H_{f(298)}^{\circ}$ & & $\begin{array}{c}\mathrm{I}(V=4) \\
17.6\end{array}$ & $\begin{array}{l}\text { II } \\
4.8\end{array}$ & $\begin{array}{c}\mathrm{H} \cdot \\
52.1\end{array}$ & $\begin{array}{c}\text { III } \\
12.5\end{array}$ & $\begin{array}{l}\mathrm{CH}_{3} . \\
34.0\end{array}$ & \\
\hline & & $S_{(298)}^{\circ}$ & & 65.4 & 63.7 & 27.6 & 52.5 & 46.4 & \\
\hline & & $C_{p(298)}^{\circ}$ & & 17.7 & 15.3 & 5.0 & 10.5 & 8.3 & \\
\hline & & $C_{p(800)}^{\circ}$ & & 34.7 & 30.7 & 5.0 & 20.2 & 12.7 & \\
\hline $\begin{array}{c}a \\
\Delta S^{\circ}=25.9 \\
\Delta H^{\circ}=39.3 \\
\Delta C_{p}^{\circ}=2.6\end{array}$ & & $\begin{array}{c}b \\
33.5 \mathrm{~g} / \mathrm{mole} \\
28.9 \mathrm{kcal} / \mathrm{mo} \\
1.1 \mathrm{~g} / \mathrm{mole}\end{array}$ & & & & & & & \\
\hline Path & $\log A$ & $\boldsymbol{E}$ & $\begin{array}{c}\log k_{T} \\
(510)\end{array}$ & Conditis & ons & System & Surface & e & References \\
\hline$a$ & 13.1 & $35 \pm 1$ & -1.90 & $680-777^{\circ}$ & & & & {$[1]$} & C. A. Heller and \\
\hline$b$ & 12.0 & $32.5 \pm 2$ & -1.93 & $P_{T} \sim 10-2$ & 20 torr & & & 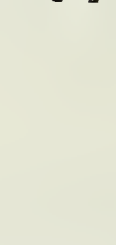 & $\begin{array}{l}\text { A. S. Gordon, J. } \\
\text { Phys. Chem. 62, } \\
709 \text { (1958); J. Phys. } \\
\text { Chem. 60, } 1315 \\
\text { (1956). }\end{array}$ \\
\hline$a$ & 13.8 & 36.9 & -2.02 & $673-773^{\circ}$ & & & & {$[2]$} & J. A. Kerr and A. F. \\
\hline$b$ & 19.6 & 29.5 & -2.04 & $P_{T} \sim 10-2$ & 20 torr & & & 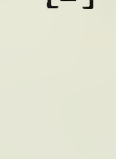 & $\begin{array}{l}\text { Trotman-Dicken- } \\
\text { son, Trans. Faraday } \\
\text { Soc. 55, } 921 \text { (1959). }\end{array}$ \\
\hline$a$ & 14.6 & 38 & -1.69 & $298-723^{\circ}$ & & & & [3] & S. Bywater and E. W. \\
\hline$b$ & 9.2 & 20 & 0.63 & $140-300 \mathrm{t}$ & & & & 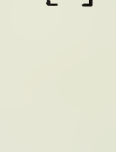 & $\begin{array}{l}\text { R. Steacie, J. } \\
\text { Chem. Phys. 19, } \\
319 \text { (1951). }\end{array}$ \\
\hline$b$ & & $>45$ & & $\begin{array}{l}745-826^{\circ} \mathrm{K} \\
\sim 50 \mathrm{torr}\end{array}$ & & & & {$[4]$} & $\begin{array}{l}\text { W. M. Jackson and } \\
\text { J. R. McNesby, J. } \\
\text { Chem. Phys. 36, } \\
2272 \text { (1962). }\end{array}$ \\
\hline
\end{tabular}

Preferred:

Reaction $a: \log k_{a} 14.3-41.3 / \theta$ (from $k_{-a}$ and thermodynamics)

$\log k_{-a}(1 /$ mole-sec $)=10.5-2.6 / \theta$ (K. Yang, J. Am. Chem. Soc. 84, 3795 (1962).)

Reaction $b$ : Suspect.

Comments: It has been argued* that reaction $b$ must involve simultaneous isomerization and decomposition, since in the decomposition of diisopropyl mercury, no $n$-hexane or 2-methyl pentane (i.e.. products of $n$-propyl radical recombination reactions) were found ${ }^{*}$ and that in the photolysis of diisopropyl ketone, the $\mathrm{C}_{6}$ hydro-

(Continued)

* B. H. M. Billinge and B. G. Gowenlock, J. Chem. Soc. 3252 (1962). 


\section{Deuteroisipropyl and isopropyl nadical (Continued)}

carbon product formed was $>95$ percent 2,3-dimethylbutane.** However, it should be noted that since $n$-propyl radicals decompose about $10^{*}$ times faster than isopropyl radicals at $700^{\circ} \mathrm{K}$, the principal radical in both systems should be isopropyl. Absence of the $n$-propyl termination products, therefore, does not exclude the possibility of isomerization followed by decomposition homogeneously. However, the displacement reaction $\mathrm{H} \cdot+\mathrm{CH}_{3} \mathrm{CD}=\mathrm{CH}_{2} \longrightarrow \mathrm{CH}_{3} \mathrm{CHDCH}_{2} \cdot \longrightarrow \mathrm{CH}_{3}+\mathrm{CHD}=\mathrm{CH}_{2}$ could equally well account for the production of $\mathrm{CHD}=\mathrm{CH}_{2}$. From reference [4], with isotopically labeled radicals, the minimum activation energy for the isomerization would be $45 \mathrm{kcal}$ and is thus not observable. This seems quite reliable work.

\section{Experimental}

[1] Photolysis of diisopropyl ketone. Isopropyl radicals were presumed to be formed photochemically (I $+h \nu$ $\rightarrow i \operatorname{Pr} \cdot+i \operatorname{PrCO})$ and as a result of chain decomposition of the ketone $(\mathrm{R}+\mathrm{K} \rightarrow \mathrm{RD}+>\operatorname{co}\langle(\mathrm{K} \cdot) ; \mathrm{K} \cdot \rightarrow i \mathrm{Pr} \cdot++\rangle=\mathrm{c}=0)$. Reaction $a$ was determined from the propylene formed (after correcting for that produced by radical disproportionation and decomposition of $(\cdot>-c<)$ relative to isopropyl radical-radical recombination. Reaction $b$ was determined by the ethylene and by the methyl radical products. Other secondary reactions capable of producing methane and ethylene were proposed, discussed, and discarded. $A$-factors reported here are based on an isopropyl radical recombination rate of $k\left(i_{\mathrm{Pr}}-\mathbb{r}_{\mathrm{r}}\right), 10^{10} \mathrm{l} / \mathrm{mole}$-sec. Products were analyzed on G.L.C. and M.S.

[2] Photolysis of isobutyraldehyde. A complex but reasonably straightforward Rice-Herzfeld mechanism was proposed. The rate of reaction $a$ was monitored by the recombination reaction $\left(2 i \operatorname{Pr} \rightarrow \mathrm{C}_{6} \mathrm{H}_{14}\right)$. The amount of propylene formed in the decomposition was estimated by subtracting from the total amount formed at the higher temperatures of decomposition the amount of propylene produced by disproportionation of isopropyl radicals and that produced in the decomposition of the aldehydic radical $>\mathrm{CHO} \rightarrow+\mathrm{CHO}$. This latter reaction was assumed to follow rapidly after $\mathrm{H}$-abstraction from the aldehyde and was estimated from the increase in propylene yields in the lower temperature range from 280 to $400{ }^{\circ} \mathrm{C}$. Hexane produced by recombination was also estimated from the measured rate constant ratios of recombination, disproportionation, and $\mathrm{H}$-abstraction involving $(i \mathrm{Pr} \cdot)$ radicals at "low" temperatures. Reaction $b$ was estimated relative to the increased methane and ethylene yields at $T>400{ }^{\circ} \mathrm{C}$ and, as before, monitored by "estimated" yields of hexane via recombination. Both reported rate const ants were based on an assumed $i \operatorname{Pr}+i \operatorname{Pr}$ recombination rate constant of $K_{\text {rec }}=10^{11} 1 /$ mole-sec with $E_{\text {rec }}=0$. Analysis of the products was by G.L.C.

[3] (See comments on $n$-propyl radical decomposition.) 
Reaction: Cyclobutyl radical

$\begin{array}{ccc} & \square(\mathrm{I}) \stackrel{\mathrm{O}}{\longrightarrow}{ }^{\circ} \mathrm{CH}_{2} \mathrm{CH}_{2} \mathrm{CH} \mathrm{CH}_{2} & \text { (II) } \\ \Delta H_{f(298)}^{\circ} & \text { I } & \text { II } \\ S_{(2998)}^{\circ} & 49.4 & 46.0 \\ C_{p(298)}^{\circ} & 65.4 & 74.9 \\ & 17.0 & 20.3\end{array}$

$\Delta S^{\circ}=9.5 \mathrm{~g} / \mathrm{mole}$

$\Delta H^{\circ}=-3.4 \mathrm{kcal} / \mathrm{mole}$

$\Delta C_{p}^{\circ}=3.3 \mathrm{~g} / \mathrm{mole}$

\begin{tabular}{|c|c|c|c|c|c|c|}
\hline $\log A$ & $\boldsymbol{E}$ & $\log k_{T}$ & Conditions & System & Surface & References \\
\hline 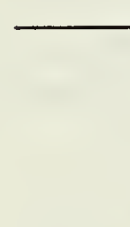 & 18.1 & & $\begin{array}{l}456-698^{\circ} \mathrm{K} \\
P_{T}=75-115 \text { torr }\end{array}$ & & & $\begin{array}{l}\text { [1] A. S. Gordon. S. R. } \\
\text { Smith, and C. M. } \\
\text { Drew. J. Chem. } \\
\text { Phys. 36, 824 } \\
\text { (1962). }\end{array}$ \\
\hline
\end{tabular}

Preferred:

Suspect.

Comments: In a private communication, R. Walsh informs us that an approximate calculation of the $A$-factor for this reaction from the reported data [1] gives $\log A \simeq 7.7$. Using a much more reasonable $\log A=13.5$, he estimates that the activation energy for the cyclobutyl radical decompositions should be about $34 \pm 6$ $\mathrm{kcal} / \mathrm{mole}$; this seems reasonable.

\section{Experimental}

[1] Cyclobutyl radicals were generated by the photolysis of acetone $d_{6}$ in cyclobutane. Analysis by M.S. The mechanism assumed was

$$
\begin{array}{ll}
\mathrm{CO}_{3}+\square \stackrel{1}{\longrightarrow} \mathrm{CO}_{3} \mathrm{H}+\square^{\cdot} & \mathrm{CO}_{3}+\mathrm{CO}_{3} \mathrm{COCO}_{3} \stackrel{2}{\longrightarrow} \mathrm{CO}_{4}+\cdot \mathrm{CO}_{2} \mathrm{COCO}_{3} \\
\mathrm{CD}_{3}^{+}+\square^{\cdot} \longrightarrow \mathrm{CO}_{3}^{-} \square & \square^{\cdot} \stackrel{4}{\longrightarrow} \mathrm{CH}_{2}=\mathrm{CHOH}_{2} \mathrm{CH}_{2}{ }^{\cdot}
\end{array}
$$

This gives

$$
\frac{\left[\text { yield totol } \mathrm{CH}_{4}\right][\text { yield butenyl products }]}{\left[Y\left(\square^{C D_{3}}\right)\right]\left[\square+\mathrm{CD}_{3} \operatorname{COCD}_{3}\right] \times t}=\frac{k_{1,2} k_{4}}{k_{3}}
$$

The dat a reported were not sufficient to determine $A_{3}$. 
Reaction: 1-Buten-4-yl radical

- $\mathrm{CH}_{2} \mathrm{CH}_{2} \mathrm{CH}=\mathrm{CH}_{2}(\mathrm{I}) \longrightarrow \mathrm{CH}_{3} \dot{\mathrm{C}} \mathrm{HCH}=\mathrm{CH}_{2}$ (II) (resonance stabilized)

$\begin{array}{ccc}\Delta H_{f(298)}^{\circ} & \text { I } & \text { II } \\ S_{(298)}^{\circ} & 46.0 & 30.5 \\ C_{p(298)}^{\circ} & 74.9 & 71.8\end{array}$

$\Delta S^{\circ}=-3.1 \mathrm{~g} / \mathrm{mole}$

$\Delta H^{\circ}=-15.5 \mathrm{kcal} / \mathrm{mole}$

$\Delta C_{p}^{\circ}=0.8 \mathrm{~g} / \mathrm{mole}$

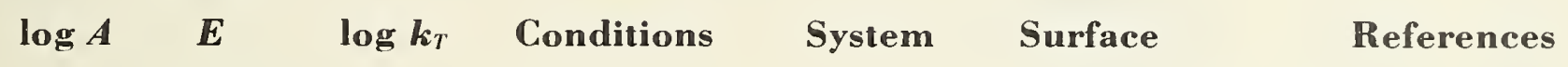

$$
\begin{array}{ll}
20 \pm 0.7 & 456-698^{\circ} \mathrm{K} \\
& P_{T}=75-115 \text { torr }
\end{array}
$$

[1] A. S. Gordon, S. R. Smith, and C. M. Drew, J. Chem. Phys. 36, 824 (1962).

\section{Preferred:}

Seems unreasonable.

Comments: With a minimum $A$-factor of $10^{12}$ for the isomerization, $E=20 \mathrm{kcal}$ would lead to 100 percent isomerization compared to the much slower recombination with $\mathrm{CH}_{3}$. A much more likely process is loss of $\mathrm{H}$-atoms to form butadiene with $k \sim 10^{13.3-34 / \theta}$. At $700^{\circ} \mathrm{K}$ this should be faster than recombination. Reactions of butadiene with $\mathrm{H}$ and $\mathrm{CH}_{3}$ could then account for the other products. Note that butadiene should also be formed by disproportionation of radicals.

\section{Experimental}

[1] Radicals were generated by the photolysis of acetone $d_{6}$ in the presence of cyclobutane. (See cyclobutyl decomposition for the mechanism.) Analysis by M.S. The above reaction occurred above $623^{\circ} \mathrm{K}$. The methallyl radical product was monitored by the formation of 3-methyl but-1-ene and pent-2-ene (products of recombination with the dominant $\mathrm{CH}_{3}$ radicals). By comparing the extent of $d$ label in the products with the $d$ label in 1-butene (i.e., $\cdot \mathrm{CH}_{2} \mathrm{CH}_{2} \mathrm{CH}=\mathrm{CH}_{2}$ $+\square$ or $\mathrm{CD}_{3} \mathrm{COCD}_{3} \longrightarrow$ ) it was deduced that less than 15 percent of the methallyl products were formed by the reactions

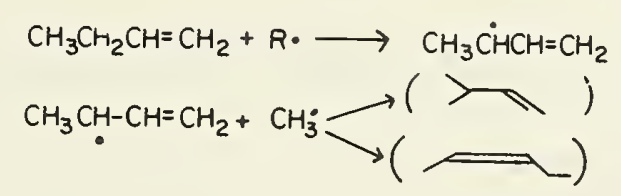


Reaction: $n$-Butyl radical

\begin{tabular}{|c|c|c|c|c|c|c|}
\hline \multirow[b]{2}{*}{$\Delta H_{f^{\prime}(298)}^{\circ}$} & & \multicolumn{5}{|c|}{$\begin{aligned} \mathrm{CH}_{3} \mathrm{CH}_{2} \mathrm{CH}_{2} \mathrm{CH}_{2} \cdot(\mathrm{I}) \stackrel{\frac{a}{b}}{\stackrel{b}{c}} \mathrm{C}_{2} \mathrm{H}_{5} \cdot(\mathrm{II})+\mathrm{C}_{2} \mathrm{H}_{4}(\mathrm{III}) \\
\stackrel{\mathrm{CH}_{3}}{\longrightarrow} \mathrm{H}_{2}+\left(\mathrm{CH}_{3} \mathrm{CHCH}=\mathrm{CHCH}_{3} \text { (IV) allylic re }\right.\end{aligned}$} \\
\hline & & $\begin{array}{c}\mathrm{I} \\
15.7\end{array}$ & $\begin{array}{c}\text { II } \\
25.7\end{array}$ & $\begin{array}{c}\text { III } \\
12.5\end{array}$ & $\begin{array}{l}\mathrm{CH}_{3} \\
34.0\end{array}$ & $\begin{array}{l}\text { IV } \\
4.8\end{array}$ \\
\hline$S_{(298)}^{\circ}$ & & 76.6 & 57.8 & 52.5 & 46.4 & 63.7 \\
\hline$C_{p(298)}^{\circ}$ & & 23.1 & 12.1 & 10.5 & 8.3 & 15.3 \\
\hline $\begin{array}{l}\Delta S^{\circ}= \\
\Delta H^{\circ}= \\
\Delta C^{\circ}=-\end{array}$ & $\begin{array}{l}a \\
3.7 \\
2.5 \\
0.5\end{array}$ & $\begin{array}{r}b \\
33.5 \\
23.1 \\
0.5\end{array}$ & $\begin{array}{r}c \\
26.4 \mathrm{~g} \\
14.8 \mathrm{k} \\
5.0 \mathrm{~g}\end{array}$ & $\begin{array}{l}\text { mole } \\
\text { al/mole } \\
\text { mole }\end{array}$ & & \\
\hline$\left\langle\Delta C_{p}^{\circ}\right\rangle=$ & -1.0 & & & & & \\
\hline Path & $\log A$ & $\boldsymbol{E}$ & $\begin{array}{c}\log \boldsymbol{k}_{T} \\
(650)\end{array}$ & Conditions & System & Surface \\
\hline $\begin{array}{l}a \\
b\end{array}$ & $\begin{array}{l}11.2 \\
12.1\end{array}$ & $\begin{array}{l}22.0 \\
27.1\end{array}$ & $\begin{array}{l}3.80 \\
2.99\end{array}$ & $334-689^{\circ} \mathrm{K}$ & & \\
\hline$a$ & & 23 & & $\begin{array}{l}523-673^{\circ} \mathrm{K} \\
200 \text { torr }\end{array}$ & & \\
\hline$c$ & 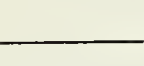 & 30 & & $523-673^{\circ} \mathrm{K}$ & & \\
\hline
\end{tabular}

Preferred:

$\log k_{u}=13.6-29 / \theta$ (from adjusted $k_{n}$ and the thermodynamics)

$\log k_{-\imath}=9.05-8.6 / \theta$ (J. A. Kerr and A. F. Trotman-Dickenson. J. Chem. Soc. 1611 (1960).)

Comments: Reaction $b$, if valid, must involve isomerization from $n$-butyl to sec-butyl radical. The observed rate of decomposition of the sec-butyl radical is much faster than reaction $b$, while the calculated rate is about the same. The reported parameters [1] are not unreasonable for a four-center internal H-abstraction and, therefore, could represent the radical isomerization reaction. However, the data seem too inconclusive. Reaction $c 1,2 \cdot \mathrm{H}_{2}$ elimination from molecules appears to proceed with activation energies of the order of $60 \mathrm{kcal} / \mathrm{mole}$ (see four-center reactions). The only difference between reaction $c$ and such molecular elimination is the resulting allyllic resonance in the radical product (i.e., $R_{\text {allyl }} \simeq 12.6 \mathrm{kcal} / \mathrm{mole}$ ). Thus it is unlikely that $E_{c}$ is much less than $45 \mathrm{kcal} /$ mole, plus a low $A$-factor. This would make it too slow to be observable.

\section{Experimental}

[1] Radicals were produced in the photolysis of $n$-valeraldehyde and products were analyzed by G.L.C. It was assumed that ethane and ethylene were formed principally from reaction $a$. Corrections for propylene formation from type II elimination from the aldehyde were made in order to calculate the rate of reaction $b$. Both reactions were moni-

(Continued) 


\section{n-Butyl radical (Continued)}

tored relative to the $n$-butyl radical recombination which was assigned a rate of $10^{11} \mathrm{l} /$ mole-sec.

[2] $\mathrm{Hg}\left({ }^{3} P_{1}\right)$ photosensitized decomposition of $n$-butane. Analysis by fractional distillation, Blacet-Leighton microanalysis apparatus, and M.S. Above $250^{\circ} \mathrm{C}$ appreciable additional amounts of methane, ethane, ethylene, and propylene were formed. These were attributed to the $n$-butyl and sec-butyl radical decompositions. Activation energy was calculated from the total rate of $\mathrm{C}_{2}$ production at constant light intensity.

[3] Photolysis of several hydrocarbons (cyclic $\mathrm{C}_{4}-\mathrm{C}_{7}, \mathrm{C}_{2} \mathrm{H}_{6}, \mathrm{C}_{3} \mathrm{H}_{8}$, and $\mathrm{C}_{4} \mathrm{H}_{10}$ ) in presence of acetone $d_{6}$. Reaction proposed on the basis of the much greater $\left(\mathrm{H}_{2} / \mathrm{HD}\right)$ ratios as opposed to the $\left(\mathrm{CD}_{3} \mathrm{H} / \mathrm{CD}_{4}\right)$ ratios. The standard $\left[\left(\mathrm{H}_{2} / \mathrm{HD}\right) /\left(\mathrm{CD}_{3} \mathrm{H} / \mathrm{CD}_{4}\right)\right]$ ratio was assumed to be that given by $\mathrm{C}_{2} \mathrm{H}_{6}$ where no such $\mathrm{H}_{2}$ elimination from the radical is possible. The reported activation energy is an estimate. 
Reaction: sec-Butyl radical (1-methyl-1-propyl radical)

\begin{tabular}{|c|c|c|c|}
\hline \multirow{3}{*}{$\mathrm{CH}_{3} \dot{\mathrm{C}}$} & \multicolumn{3}{|c|}{$\stackrel{a}{\longrightarrow} \mathrm{CH}_{3} \cdot+\mathrm{CH}_{3} \mathrm{CH}=\mathrm{CH}_{2}$ (II) } \\
\hline & \multicolumn{3}{|c|}{ 1) $b$} \\
\hline & $\mathrm{I}(V=4.0)$ & $\mathrm{CH}_{3}$. & II \\
\hline$\Delta H_{f(298)}^{\circ}$ & 12.6 & 34.0 & 4.8 \\
\hline$S^{\circ}$ & 76.2 & 46.4 & 63.7 \\
\hline$C_{p(298)}^{\circ}$ & 23.2 & 8.3 & 15.3 \\
\hline
\end{tabular}

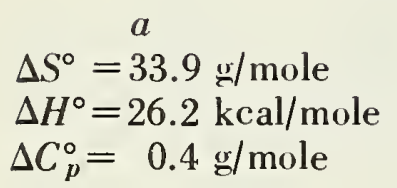

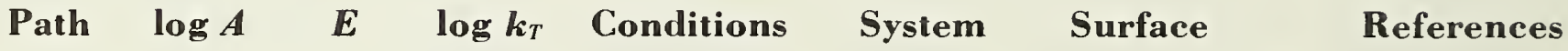
(570)

$a$

$11.81 \quad 24$

$15.32 \quad 30.6$

2.61

3.59

$523-622^{\circ} \mathrm{K}$

$a$ 23

$<1 \%$ of

path $a$ $523-673^{\circ} \mathrm{K}$

200 torr

$638-779{ }^{\circ} \mathrm{K}$

$\sim 32$ torr
[1] a. J. T. Gruver and J. G. Calvert, J. Am. Chem. Soc. 78, 5208 (1956). b. J. G. Calvert, Chem. Rev. 59, 569 (1959).

[2] S. Bywater and E. W. R. Steacie, J. Chem: Phys. 19, 172 (1951).

[3] J. R. McNesby, C. M. Drew, and A. S. Gordon, J. Chem. Phys. 24, 1260 (1956).

\section{Preferred:}

$\log k_{\text {in }}(\mathrm{calc})=14.16-33.9 / \theta$

$\log k_{-a}(1 /$ mole-sec $)=8.88-8.8 / \theta$; M. Mujoski and R. K. Brinton, J. Chem. Phys. 36, 3019 (1962).

Comments: The reported reaction rate constant is more than two orders of magnitude larger than the calculated value. The $A$-factor discrepancy can be reconciled from the fact that $k_{\text {rec }}$ (sec-butyl) $\simeq 10^{9.8} 1 / \mathrm{mole}$-sec is perhaps a better value than the one assumed by Calvert. However, the $4 \mathrm{kcal} / \mathrm{mole}$ difference in activation energies is more difficult to explain. Perhaps the secondary butyl radical is less stable than indicated and the activation energy reported should be slightly higher (e.g., $E_{b} \simeq 32 \mathrm{kcal} / \mathrm{mole}$ ). 


\section{sec-Butyl radical (Continued)}

\section{Experimental}

[1a] Radicals were generated by the photolysis of 2-methylbutanol (3130 $)$. Primary dissociations were $(\mathrm{R}=$ sec-butyl)

$$
\begin{array}{ll}
\mathrm{RCHO} \stackrel{1}{\longrightarrow} \mathrm{R} \cdot+\cdot \mathrm{CHO} & \Phi_{1}=0.8 \\
\mathrm{RCHO} \stackrel{2}{\longrightarrow} \mathrm{C}_{2} \mathrm{H}_{4}+\mathrm{C}_{2} \mathrm{H}_{5} \mathrm{CHO} & \Phi_{2}=0.2
\end{array}
$$

Rates were calculated on the basis of methane formation. The recombination reference reaction was assumed to be $k_{\text {rec }}($ sec-butyl $)=10^{11.3} \mathrm{l} /$ mole-sec.

[1b] Revised kinetic treatment of ref. [1a] using original data. The rate of propylene formation was equated to the rate of decomposition rather than the rate of methane formation. Methane was believed to be in error due to a possible "down chain" temperature dependent primary process decomposition of the aldehyde

$$
1 \succ-\mathrm{CHO}^{*} \longrightarrow-\mathrm{CHO}^{*} \mathrm{CH}_{3} \cdot
$$

Evidence for similar splits in propionaldehyde and isobutyraldehyde was given.

[2] See comments on $n$-butyl radical (radical produced simultaneously along with $n$-butyl radicals in the $\mathrm{Hg}\left({ }^{3} P_{1}\right)$ photosensitized decomposition of $n$-butane).

[3] They used photolysis of acetone and $\mathrm{CH}_{3} \mathrm{CD}_{2} \mathrm{CO}_{2} \mathrm{CH}_{3}$ to produce $\mathrm{CH}_{2} \mathrm{CD}_{2} \mathrm{CO}_{2} \mathrm{CH}_{3}$ and $\mathrm{CH}_{3} \dot{\mathrm{CDCD}}_{2} \mathrm{CH}_{3}$ radicals. They found only normal products from fission without rearrangement, showing absence of $\mathrm{H}$-shifts in the radicals. 
Reaction: $t$-Butyl radical

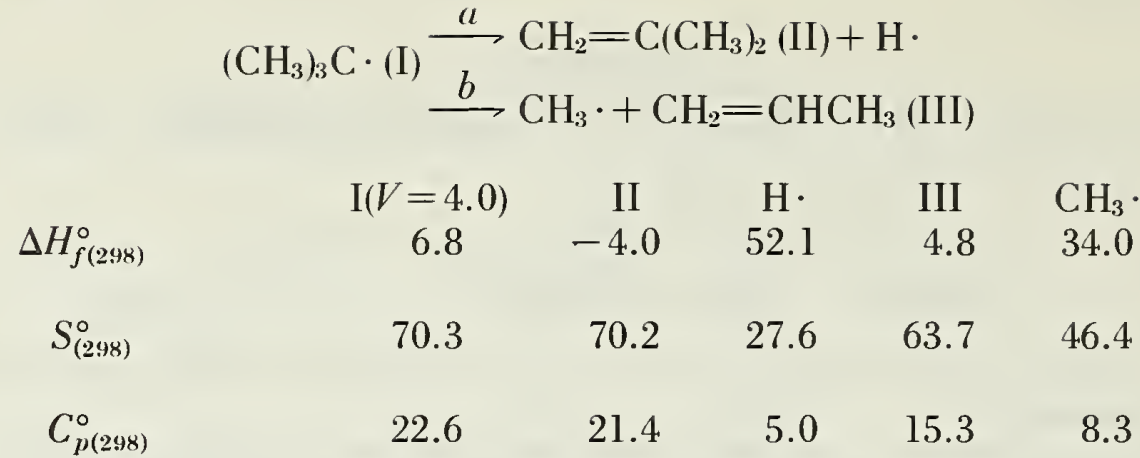

$\begin{array}{cc}\quad a(V=0) & b \\ \Delta S^{\circ}=27.5 & 39.8 \mathrm{~g} / \mathrm{mole} \\ \Delta H^{\circ}=41.3 & 32.0 \mathrm{kcal} / \mathrm{mole} \\ \Delta C_{p}^{\circ}=3.8 & 1.0 \mathrm{~g} / \mathrm{mole} \\ \left.<\Delta C_{p}^{\circ}\right\rangle \simeq 2.5 & \end{array}$

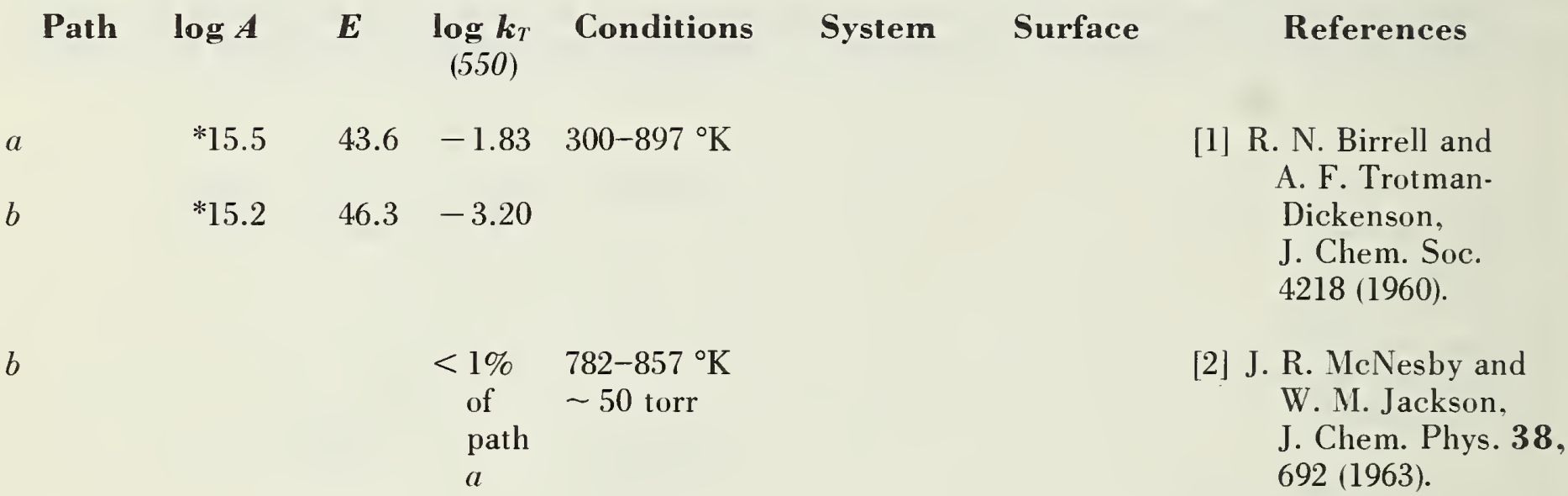

Preferred:

Path $b$ : Unreliable.

Path $a$ : Reported [1] and calculated rates are in reasonable agreement.

$\log k_{a}=14.6 \pm 0.7-43.1 / \theta$ (from $k_{-a}$ and the reaction thermodynamics).

$\log k_{-a}(1 /$ mole-sec) $10.5-2.2 / \theta$.

Comments: The path $b$ reaction involves radical isomerization $(t \mathrm{Bu} \cdot \longrightarrow$ isobutyl $\cdot)$ followed by decomposition. Similar 1,2-H-migration isomerizations are also reported for the isopropyl and $n$-butyl radicals. Reported parameters should apply to the isomerization only, since decomposition of isobutyl is relatively fast. It is more likely that the isomerization does not occur and that the $\mathrm{C}_{3} \mathrm{H}_{6}$ formation arises from

$$
\mathrm{H} \cdot+i \text {-butene } \underset{\text { Experimental }}{\longrightarrow} \text { isobutyl } \mathrm{CH}_{3} \cdot+\mathrm{C}_{3} \mathrm{H}_{6} .
$$

[1] Radical source was the photolysis of pivaldehyde. Analysis was by G.L.C. Above $663{ }^{\circ} \mathrm{K}$ the rate of formation of isobutene increased rapidly. This was attributed to path $a$ decomposition. The reference reaction, $2 t \mathrm{Bu} \longrightarrow(t \mathrm{Bu})_{2}$, was assigned a recombination rate of $k_{\mathrm{rec}} \simeq 10^{11.0} \mathrm{l} / \mathrm{mole}$-sec. ${ }^{*}$ Above $743^{\circ} \mathrm{K}$ the rate of propane formation increased sharply and was accompanied by a corresponding increase in methane. Parameters were based on the propene produced in three runs only, and consequently were not considered particularly reliable.

[2] McNesby and Jackson have shown (see discussion on isobutyl radical) that there is negligible isomerization of $i$-butyl to $t$-butyl. Path $b$, which is the reverse reaction and uphill thermodynamically, is even less likely.

\footnotetext{
* This is too high by 1.5 powers of 10 : therefore, the reported $A$-factors have been lowered here by $-0.8 \log$ units.
} 
Reaction: Isobutyl radical

$$
\begin{aligned}
& \stackrel{a}{\longrightarrow} \mathrm{CH}_{2}=\mathrm{CHCH}_{3}(\mathrm{II})+\mathrm{CH}_{3} . \\
& \left(\mathrm{CH}_{3}\right)_{2} \mathrm{CHCH}_{2} \cdot(\mathrm{I}) \\
& \stackrel{b}{\longrightarrow} \mathrm{H} \cdot+\mathrm{CH}_{2}=\left(\mathrm{CH}_{3}\right)_{2} \mathrm{CHCH}_{2} \cdot(\mathrm{I}) \\
& \begin{array}{cccccc} 
& \mathrm{I}(V=4.0) & \mathrm{II} & \mathrm{CH}_{3} \cdot & \mathrm{H} \cdot & \text { III } \\
\Delta H_{f(298)}^{\circ} & 13.7 & 4.8 & 34.0 & 52.1 & -4.0
\end{array} \\
& \begin{array}{llllll}
S_{(298)}^{\circ} & 73.9 & 63.7 & 46.4 & 27.6 & 70.2
\end{array} \\
& \begin{array}{llllll}
C_{p(298)}^{\circ} & 22.8 & 15.3 & 8.3 & 5.0 & 21.4
\end{array}
\end{aligned}
$$

$$
\begin{array}{cc}
a & b \\
\Delta S^{\circ}=36.2 & 23.9 \mathrm{~g} / \mathrm{mole} \\
\Delta H^{\circ}=25.1 & 34.4 \mathrm{kcal} / \mathrm{mole} \\
\Delta C_{p}^{\circ}=0.8 & 3.6 \mathrm{~g} / \mathrm{mole}
\end{array}
$$

$\begin{array}{llllll}\text { Path } & \log \boldsymbol{A} \quad \boldsymbol{E} \quad \log \boldsymbol{k}_{T} & \text { Conditions } & \text { System } & \text { Surface } & \text { References }\end{array}$

$\begin{array}{lll}a & 18.5 & 523-673^{\circ} \mathrm{K} \\ b & 40.0 & 200 \text { torr }\end{array}$

$782-857^{\circ} \mathrm{K}$

$$
\text { decomposing mode) }
$$

$b$

$a$

(not an important
decomposing mode)

$$
<1 \% \text { of } \quad 782-857^{\circ} \mathrm{K}
$$$$
\text { path } a \quad \sim 50 \text { torr }
$$

$a$

$\begin{array}{llll}12.4 & 31.0 & 0.51 \quad \begin{array}{l}540-598 \\ { }^{\circ} \mathrm{K} \\ 10-50 \text { torr }\end{array}\end{array}$

[1] S. Bywater and

E. W. R. Steacie, J. Chem. Phys. 19, 172 (1951).

[2] W. M. Jackson, J. R. McNesby, and B. deB. Darwent, J. Chem. Phys. 37 , 2256 (1962).

[3] E. L. Metcalfe and A. F. TrotmanDickenson, J. Chem. Soc. 5072 (1960).

[4] J. R. McNesby and W. M. Jackson; J. Chem. Phys. 38, 692 (1963).

[5] D. H. Slater, S. S. Collier, and J. G. Calvert, J. Am. Chem. Soc. 90, 268 (1968).

\section{Preferred:}

$\log k_{a}=14.2-32.7 / \theta$ (calculated from $k_{-a}$ and the reaction thermodynamics),

$\log k_{-a}=8.4-8.8 / \theta$, approximately $(1 / 3)$ addition anti-Markonikov (see sec-butyl radical), $\log k_{b}=13.7-36.6 / \theta$,

$\log k_{-b}=10.2-2.2 / \theta$, same approximation as for $k_{-a}$. 


\section{Experimental}

[1] (See experimental on $n$-butyl radical.)

[2] Relative rates of reactions $a$ and $b$ were determined in the pyrolysis of deuterium-labeled isobutane $\left(\mathrm{CH}_{3}\right)_{3} \mathrm{CD}$. Isotopic analysis of the hydrogen, methane, and isobutene fractions was made by M.S.

$$
\begin{array}{ll}
\cdot \mathrm{CH}_{2} \mathrm{CD}\left(\mathrm{CH}_{3}\right)_{2} \stackrel{5}{\underline{6}} \underset{\mathrm{CH}_{2}=\mathrm{CDCH}_{3}+\mathrm{CH}_{3} .}{\longrightarrow} \mathrm{CH}_{2}=\mathrm{C}\left(\mathrm{CH}_{3}\right)_{2}+\mathrm{D} .
\end{array}
$$

From the yields of $\mathrm{D}_{2}$ and $\mathrm{HD}$ (from 6 and $\mathrm{H}$-abstraction) relative to $\mathrm{CH}_{3} \mathrm{D}$ and $\mathrm{CH}_{4}$ (from 5 and $\mathrm{H}$-abstraction), the authors concluded that maximum values of $\left(k_{5} / k_{6}\right)$ were $0.008\left(509{ }^{\circ} \mathrm{C}\right)$ and $0.013\left(584{ }^{\circ} \mathrm{C}\right)$. These maximum values are about a factor of 10 lower than reported ratios of references [1] and [2]. The conclusion is that the importance of path $b$ has been badly overestimated in the prior studies. This conclusion was also confirmed in a similar follow-up study on the pyrolysis of $\left(\mathrm{CH}_{3}\right)_{2} \mathrm{CDCH}_{3}$ (Ref. [4]).

[3] Photolysis of isovaleraldehyde was the source of isobutyl radicals. Analysis of the hydrocarbon products was made by G.L.C. Above $279^{\circ} \mathrm{C}$ the rate of formation of propene increased. This was attributed to reaction $a$. Above $329^{\circ} \mathrm{C}$ the rate of isobutene increased. This was attributed to path $b$. The reference reaction in both cases was the recombination of isobutyl radicals and assigned a reaction rate of $k_{\text {rec }}\left(\right.$ iso $\left.\mathrm{C}_{4} \mathrm{H}_{9}\right)=10^{102} \mathrm{l} / \mathrm{mole}$-sec.

[4] See reference [3].

[5] Followed photolysis of azoisobutane. $\mathrm{C}_{3} \mathrm{H}_{6}$ was formed above $500{ }^{\circ} \mathrm{K}$ (G.L.C.). They measured $\mathrm{C}_{3} \mathrm{H}_{6}$ relative to recombination of $i$ - $\mathrm{Bu}$ radicals and assumed $10^{11}$ for recombination. However, these measurements were clearly in the pyrolysis range of the azo compound and may be subject to serious corrections for thermal reactions. 
[2] Radicals were produced by $\mathrm{Hg}\left({ }^{3} P_{1}\right)$ photosensitized reaction with cyclopentane. Total products analyzed by G.L.C. The activation energy for path $a$ was determined by five independent relations with a standard deviation of $1.9 \mathrm{kcal} / \mathrm{mole}$. Path $c$ was deemed important above $300^{\circ} \mathrm{C}$. Products were $\mathrm{H}_{2}, \mathrm{C}_{2} \mathrm{H}_{4}, \square$ biallyl, allylcyclopentane,

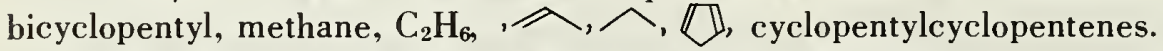

[3] Cyclopentyl radicals were generated by the decomposition of cyclopentylmethyl nitrite:

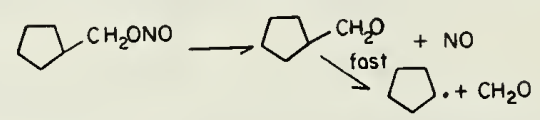

Analysis by M.S. It was estimated that at $700{ }^{\circ} \mathrm{C}$, contributions of the various reaction paths were: path $a, 66$ percent; path $b,<2$ percent; path $c, 34$ percent. Mass balances were good to \pm 2 percent. Path $c$ increased in importance with decreasing temperature.

Note: The mechanism involves the steps:

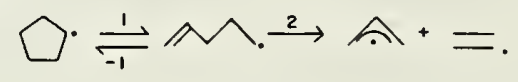

$\Delta H_{1}=16.6$ and $\Delta H_{2}=12.1 \mathrm{kcal}$. If $k_{2}<k_{-1}$, then $E_{-2}=9 \mathrm{kcal}$, a reasonable result, and $E_{-1} \leqslant 11 \mathrm{kcal}$, a not unreasonable result. If, however, $k_{2}>k_{-1}$ and siep 1 is rate determining, this implies $E_{-1}=21 \mathrm{kcal}$, which seems much too high. 
Reaction: Neopentyl radical

\begin{tabular}{cccc}
\multicolumn{4}{r}{$\left(\mathrm{CH}_{3}\right)_{3} \mathrm{CCH}_{2} \cdot(\mathrm{I}) \stackrel{a}{\longrightarrow} \mathrm{CH}_{3} \cdot+\left(\mathrm{CH}_{3}\right)_{2} \mathrm{C}=\mathrm{CH}_{2}(\mathrm{II})$} \\
$\Delta H_{f(298)}^{\circ}$ & $\mathrm{I}(V=0)$ & $\mathrm{CH}_{3} \cdot$ & $\mathrm{II}$ \\
$S_{(298)}^{\circ}$ & 6.0 & 34.0 & -4.0 \\
$C_{p(298)}^{\circ}$ & 79.2 & 46.4 & 70.2 \\
& 28.7 & 8.3 & 21.4
\end{tabular}

$\Delta S^{\circ}=37.4 \mathrm{~g} / \mathrm{mole}$

$\Delta H^{\circ}=24.0 \mathrm{kcal} / \mathrm{mole}$

$\Delta C_{p}^{\circ}=1.0 \mathrm{~g} / \mathrm{mole}$

$\begin{array}{llrrrr}\log \boldsymbol{A} & \boldsymbol{E} & \begin{array}{c}\log \boldsymbol{k}_{T} \\ (762)\end{array} & \text { Conditions } & \text { System } & \text { Surface } \\ 13 \pm 1 & 34 \pm 3.5 & 3.22 & 762^{\circ} \mathrm{K}\end{array}$

[1] K. H. Anderson and

S. W. Benson, J. Chem.

Phys. 40, 3747 (1964).

Preferred:

$\log k=14.0-37.5 / \theta$.

Rate constant is reliable.

Comments: In solution, the rate of methyl addition to isobutene has about the same parameters as methyl addition to ethylene. M. Feld and M. Szwarc, J. Am. Chem. Soc. 82, 3791 (1960). Assuming anti-Markonikov addition to be about one fifth the "normal" rate with equivalent activation energies, therefore, gives $k_{-a}$ $(1 /$ mole-sec $) \simeq 8.4-8.3 / \theta$. With the reaction thermodynamics one then estimates $\log k_{a} \simeq 14.4-32.3 / \theta$. This is so much faster than the observed rate that it appears the assumption on activation energies must be in error and that the activation energy for the reverse step must be closer to $12 \mathrm{kcal}$.

\section{Experimental}

[1] Rates of decomposition of neopentane in the presence and absence of $\mathrm{HCl}$ were used to obtain the relative rates of the chain steps:

$$
\begin{aligned}
& \mathrm{CH}_{3} \cdot+\mathrm{NP} \stackrel{2}{\longrightarrow} \mathrm{CH}_{4}+\mathrm{NP} . \\
& \mathrm{NP} \cdot \stackrel{3}{\longrightarrow} \text { isobutene }+\mathrm{CH}_{3} .
\end{aligned}
$$

Thus, $\frac{\text { Rate (with } \mathrm{HCl})}{\text { Rate (without } \mathrm{HCl})}=\frac{k_{3}}{k_{2}}(\mathrm{NP})_{0}$. Parameters were assigned from the rate constant assuming $A_{3} \simeq 10^{13.0} \mathrm{sec}^{-1}$. 
Reaction: 1-Methyl-1-pentyl radical (2-hexyl radical)

$\begin{array}{cccc}\mathrm{CH}_{3} \dot{\mathrm{C}} \mathrm{H}\left(\mathrm{CH}_{2}\right)_{3} \mathrm{CH}_{3}(\mathrm{I}) \longrightarrow \mathrm{C}_{3} \mathrm{H}_{6}(\mathrm{II})+\mathrm{CH}_{3} \mathrm{CH}_{2} \mathrm{CH}_{2} \cdot(\mathrm{III}) \\ \Delta H_{f(2.98)}^{\circ} & \mathrm{I}(V=4.0) & \mathrm{II} & \mathrm{III}(V=4.0) \\ S_{(298)}^{\circ} & 2.7 & 4.9 & 20.7 \\ C_{p(298)}^{\circ} & 95 & 63.8 & 67.2 \\ C_{p(800)}^{\circ} & 34.2 & 15.3 & 17.6 \\ & 67.9 & 30.8 & 35.1\end{array}$

$\Delta S^{\circ}=36 \mathrm{~g} / \mathrm{mole}$

$\Delta H^{\circ}=22.9 \mathrm{kcal} / \mathrm{mole}$

$\Delta C_{p}^{\circ}=-1.3 \mathrm{~g} / \mathrm{mole}$

$\begin{array}{ccccccc}\log \boldsymbol{A} & \boldsymbol{E} & \begin{array}{c}\log \boldsymbol{k}_{T} \\ (822)\end{array} & \text { Conditions } & \text { System } & \text { Surface } & \text { References } \\ 13.48 & 22.4 & +7.51 & \begin{array}{l}822^{\circ} \mathrm{K} \\ 92 \text { torr }\end{array} & \text { static } & \text { [1] C. P. Quinn, Trans. Fara- } \\ \text { day Soc. 59, 2543 } \\ \text { (1963). }\end{array}$

Preferred:

$\log k=14.3-27.4 / \theta$.

Comments: If one assumes a back reaction rate similar to $\left(\mathrm{CH}_{3}+\mathbb{N}\right)$ of $\log k_{-n}(1 / \mathrm{mole}-\mathrm{sec}) \simeq 9.1-8.6 / \theta$, then from the reaction thermodynamics, one can calculate $\log k_{11} \simeq 14.3-29 / \theta$ (i.e., a factor of 10 slower than the value reported). However, $K_{8}\left(800^{\circ} \mathrm{K}\right) \simeq 3$; therefore, the agreement between reported and calculated rates is fairly good (i.e., $\sim$ factor of 3 ). We prefer the calculated $A$-factor and an activation energy adjusted to give the proper rate at $800^{\circ} \mathrm{K}$.

\section{Experimental}

[1] Pyrolysis of ethane. Analyses of all products as a function of time by G.L.C. The rate of secondary methane formation (i.e., not from the primary free radical chain reactions of the ethane decomposition) was interpreted in terms of the following mechanism involving the isomerization of $n$-hexyl radicals:

$$
\begin{gathered}
\mathrm{C}_{2} \mathrm{H}_{3} \cdot+\mathrm{C}_{2} \mathrm{H}_{4} \stackrel{6}{\rightleftharpoons} \mathrm{CH}_{3} \mathrm{CH}_{2} \mathrm{CH}_{2} \mathrm{CH}_{2} \cdot \\
\mathrm{CH}_{3} \mathrm{CH}_{2} \mathrm{CH}_{2} \mathrm{CH}_{2} \cdot+\mathrm{C}_{2} \mathrm{H}_{4} \stackrel{7}{\rightleftharpoons} \mathrm{CH}_{3}\left(\mathrm{CH}_{2}\right)_{4} \mathrm{CH}_{2} \cdot \\
\mathrm{CH}_{3}\left(\mathrm{CH}_{2}\right)_{4} \mathrm{CH}_{2} \cdot \stackrel{8}{\rightleftharpoons} \mathrm{CH}_{3} \mathrm{CH}_{2}\left(\mathrm{CH}_{2}\right)_{3} \mathrm{CH}_{3} \\
\mathrm{CH}_{3} \mathrm{CH}\left(\mathrm{CH}_{2}\right)_{3} \mathrm{CH}_{3} \stackrel{9}{\longrightarrow} \mathrm{C}_{3} \mathrm{H}_{6}+\mathrm{CH}_{3} \mathrm{CH}_{2} \mathrm{CH}_{2} \cdot
\end{gathered}
$$

A steady-state treatment gave $K_{8} k_{9}=3 \times 10^{7} \mathrm{sec}^{-1}$. On the assumptions that $K_{8} \simeq 1$ and $A_{9} \simeq 3 \times 10^{13} \mathrm{sec}^{-1}$, the reported parameters were calculated. 
Reaction: Formyl radical

$\begin{array}{cccc} & \mathrm{HCO}(\mathrm{I}) \longrightarrow \mathrm{H} \cdot+\mathrm{CO}(\mathrm{II}) & \\ \Delta H_{f(298)}^{\circ} & \mathrm{I} & \mathrm{H} & \mathrm{II} \\ S_{(298)}^{\circ} & 7.2 & 52.1 & -26.4 \\ C_{p(298)}^{\circ} & 53.7 & 27.6 & 47.3 \\ C_{p(500)}^{\circ} & 8.3 & 5.0 & 7.0 \\ & 9.2 & 5.0 & 7.2\end{array}$

$\Delta S^{\circ}=21.2 \mathrm{~g} / \mathrm{mole}$

$\Delta H^{\circ}=18.5 \mathrm{kcal} / \mathrm{mole}$

$\Delta C_{p}^{\circ}=3.7 \mathrm{~g} / \mathrm{mole}$

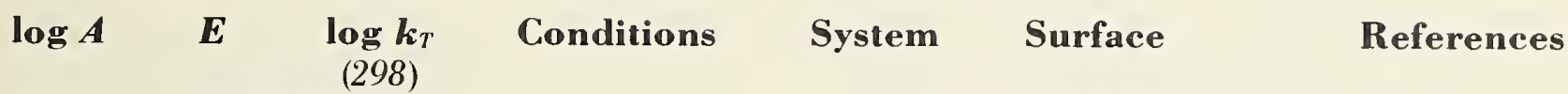

$13 \quad 14.4 \quad 2.51 \quad 298^{\circ} \mathrm{K}$

$P_{T} \sim 200$ torr

$>13$

14.0 $423-623^{\circ} \mathrm{K}$

$P_{T} \sim 150-450$ torr

$393-498{ }^{\circ} \mathrm{K}$

$P_{T} \sim 130$ torr
[1] R. J. Cvetanovic, Can. J. Chem. 33, 1684 (1955).

[2] J. G. Calvert and E. W. R. Steacie, J. Chem. Phys. 19, 176 (1951).

[3] F. B. Marcotte, W. A. Noyes, Jr., J. Am. Chem. Soc. 74, 783 (1952).

Preferred:

$\log k=13.7-19.0 / \theta$.

Comments: This reaction must be pressure-dependent at normal temperature and pressure conditions (J. E. Longfield and W. D. Walters, J. Am. Chem. Soc. 77, 6098 (1955)). None of the reported parameters are reliable. A reasonable estimate can be made by assuming the following parameters for the back reaction, $k_{b}=10^{10.8-1.0 / \theta} \mathrm{I} / \mathrm{mole}-\mathrm{sec}$, and using the indicated thermodynamics. One then obtains $\log k_{\text {est }}=13.7-19.0 / \theta$. Calvert has discussed the experimental data from all sources on this radical (J. Phys. Chem. 61, 120 (1957)) and has concluded that $\mathrm{D}(\mathrm{H}-\mathrm{CO}) \simeq 15 \mathrm{kcal} / \mathrm{mole}$. The thermodynamics are based on the reliable measurement of $\Delta H_{f}^{\circ}(\cdot \mathrm{CHO})$ by Walsh and $\mathrm{S}$. W. Benson.

\section{Experimental}

[1] $\mathrm{Hg}\left({ }^{3} P_{1}\right)$ photosensitized decomposition of ethylene oxide. The rate of the decomposition reaction was estimated from the observed stoichiometry relative to the radical termination reaction, $\mathrm{CH}_{3}+\mathrm{HCO} \longrightarrow \mathrm{CH}_{4}+\mathrm{CO}$, and $2 \mathrm{CH}_{3} \longrightarrow \mathrm{C}_{2} \mathrm{H}_{6}$. The $A$-factor of $10^{13}$ was assumed for this single temperature study.

[2] Vapor phase photolysis of $\mathrm{H}_{2} \mathrm{CO}$. Activation energy was obtained from the Arrhenius plot of the rate of $\left(\mathrm{H}_{2}\right)$ production. Prior values of 16.4 (Akeroyd and Norrish, J. Chem. Soc., 890 (1936) and 12 (Style and Summers, Trans. Faraday Soc. 35, 899 (1939)) obtained in similar systems were quoted.

[3] Photolysis of acetone in the presence of $\mathrm{O}_{2}$. Formyl radicals were produced by the reaction

$$
\mathrm{CH}_{3}+\mathrm{O}_{2} \longrightarrow \mathrm{CH}_{3} \mathrm{O}+\mathrm{H}_{2} \mathrm{O} \text {. }
$$

The decomposition kinetics were monitored by the competitive trapping reaction:

$$
\begin{aligned}
& \mathrm{HCO} \longrightarrow \mathrm{H}+\mathrm{CO} \\
& \mathrm{HCO}+\mathrm{O}_{2} \longrightarrow \text { (other products) }
\end{aligned}
$$

No $A$-factors could be determined. 
Reaction: Hydroxymethyl radical

$\begin{array}{cccc} & \cdot \mathrm{CH}_{2} \mathrm{OH}(\mathrm{I}) \longrightarrow \mathrm{CH}_{2} \mathrm{O}(\mathrm{II})+\mathrm{H} \cdot(\mathrm{III}) & \\ & \mathrm{I} & \mathrm{II} & \mathrm{III} \\ \Delta H_{f(298)}^{\circ} & -4 & -27.7 & 52.1 \\ S_{(298)}^{\circ} & 58.6 & 52.3 & 27.4 \\ C_{p(298)}^{\circ} & 10.2 & 8.5 & 5.0\end{array}$

$\Delta S^{\circ}=21.1 \mathrm{~g} / \mathrm{mole}$

$\Delta H^{\circ}=28.4 \mathrm{kcal} / \mathrm{mole}$

$\Delta C_{p}^{\circ}=3.3 \mathrm{~g} / \mathrm{mole}$

\begin{tabular}{|c|c|c|c|c|c|c|}
\hline $\log A$ & $\boldsymbol{E}$ & $\begin{array}{c}\log \boldsymbol{k}_{T} \\
(725)\end{array}$ & Conditions & System & Surface & References \\
\hline 13.05 & 29 & 4.31 & $\begin{array}{l}673-773^{\circ} \mathrm{K} \\
30-100 \text { torr }\end{array}$ & static & & $\begin{array}{l}\text { [1] M. K. Phibbs and B. } \\
\text { deB. Darwent, J. } \\
\text { Chem. Phys. 18, } \\
495 \text { (1950). }\end{array}$ \\
\hline
\end{tabular}

Preferred:

$\log k_{a}=13.05-29 / \theta$.

Comments: A back reaction sim:lar to $\mathrm{H}+\mathrm{C}_{2} \mathrm{H}_{4}$ is reasonable. If one assumes, therefore, $\log k_{-a}(1 / \mathrm{mole}$ $\sec )=10.6-3.0 / \theta$, then from the reaction thermodynamics, $\log k_{a}($ est $) \simeq 13.3-31 / \theta$. The agreement is good. It is probable, however, that the measured rate was in a pressure-dependent region.

\section{Experimental}

[1] $\mathrm{Hg}\left({ }^{3} P_{1}\right)$ photosensitized decomposition of methyl alcohol. The decomposition rate was determined relative to the recombination reaction (calculated)

$$
\begin{gathered}
2 \dot{\mathrm{C}} \mathrm{H}_{2} \mathrm{OH} \stackrel{3}{\longrightarrow}\left(\mathrm{CH}_{2} \mathrm{OH}\right)_{2} \\
\dot{\mathrm{C}} \mathrm{H}_{2} \mathrm{OH} \stackrel{4}{\longrightarrow} \mathrm{CH}_{2} \mathrm{O}+\mathrm{H} \\
\frac{k_{1}}{k_{3}^{1 / 2}}=10^{8.05-29 / \theta}\left(\frac{\text { moles }}{\text { l. sec }}\right)^{1 / 2}
\end{gathered}
$$

Analysis was performed on the noncondensible products by standard gas techniques and formaldehyde was titrated. The $A$-factor reported here assumes $k_{3} \simeq 10^{10} \mathrm{l} /$ mole-sec. 
Reaction: Acetyl radical

$\begin{array}{cccc} & \mathrm{CH}_{3} \mathrm{CO}(\mathrm{I}) \longrightarrow \mathrm{CH}_{3} \cdot(\mathrm{II})+\mathrm{CO}(\mathrm{III}) \\ \Delta H_{f(298)}^{\circ} & -5.4 & 34.0 & -26.4 \\ S_{(298)}^{\circ} & 64.5 & 46.4 & 47.3 \\ C_{p(298)}^{\circ} & 12.7 & 8.3 & 7.0 \\ C_{p(500)}^{\circ} & 16.4 & 10.1 & 7.2\end{array}$

$\Delta S^{\circ}=29.2 \mathrm{~g} / \mathrm{mole}$

$\Delta H^{\circ}=13.0 \mathrm{kcal} / \mathrm{mole}$

$\Delta C_{p}^{\circ}=2.6 \mathrm{~g} / \mathrm{mole}$

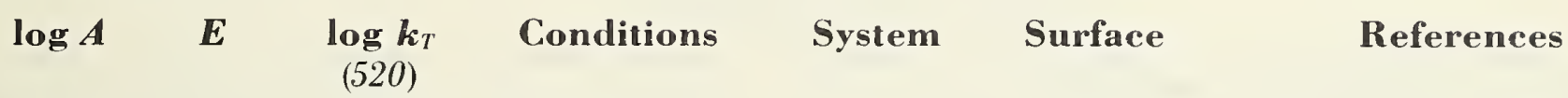

$10.22 \quad 13.5 \quad 4.55 \quad 296-400^{\circ} \mathrm{K}$

$P_{T} \sim 25-65$ torr

$10.3 \quad 15.0 \pm 1 \quad 3.99 \quad 473-568^{\circ} \mathrm{K}$

$P_{T} \sim 12-150 \mathrm{~mm}$

$\mathrm{Hg}$

$16.0 \pm 2 \quad 393-498^{\circ} \mathrm{K}$

$P_{T} \sim 130$ torr

$13.5 \pm 2 \quad 300-390{ }^{\circ} \mathrm{K}$
[1] J. G. Calvert and J. T. Gruver, J. Am. Chem. Soc. 80, 1313 (1958).

[2] E. O'Neal and S. W. Benson, J. Chem. Phys. 36, 2196 (1962) .

[3] F. B. Marcotte and W. A.

Noyes, Jr., J. Am.

Chem. Soc. 74, 783 (1952).

[4] D. H. Volman and W. M. Graven, J. Am. Chem. Soc. 75, 3111 (1953).

Preferred:

$\log k=10.3-15.0 / \theta$. These parameters are in reasonably good agreement with the thermodynamics and the back reaction kinetics. However, the low $A$-factor is in serious disagreement with unimolecular rate theory and poses a problem which needs more study.

Comments: Measurements on the back reaction: $\mathrm{CH}_{3} \cdot+\mathrm{CO} \longrightarrow \mathrm{CH}_{3} \mathrm{CO}$

$k_{-a}=1.41 \times 10^{5} \times 10^{-3.84 / \theta} \mathrm{l} / \mathrm{mole}-\mathrm{sec}$ (ref. [1]).

$k_{-a}=10^{5.56-3.9 / \theta}$ 1/mole-sec (J. A. Kerr and J. G. Calvert, J. Phys. Chem. 69, 1922 (1965)).

The reaction thermodynamics $\left(\Delta S_{T}^{\circ} \simeq 30.1 \mathrm{~g} / \mathrm{mole}, \Delta H_{T}^{\circ} \simeq 13.4 \mathrm{kcal} / \mathrm{mole}\right)$ and the back reaction kinetics predict $k_{a}=10^{10.1-16.3 / \theta} \mathrm{sec}^{-1}$, in reasonably good agreement with references [2] and [3]. Note that the $A$-factor is abnormally low. The reaction is pressure-dependent at normal experimental pressures.

\section{Experimental}

[1] Photolysis of azomethane in the presence of acetaldehyde. Analysis by M.S. and G.L.C. The mechanism was 


$$
\begin{aligned}
& \mathrm{CH}_{3} \cdot+\mathrm{CH}_{3} \mathrm{CHO} \stackrel{3}{\longrightarrow} \mathrm{CH}_{4}+\mathrm{CH}_{3} \mathrm{CO} \\
& \mathrm{CH}_{3} \mathrm{CO} \stackrel{1}{=} \mathrm{CH}_{3} \cdot+\mathrm{CO} \\
& \mathrm{CH}_{3}+\mathrm{CH}_{3} \mathrm{COO} \stackrel{2}{\longrightarrow} \mathrm{CH}_{3} \mathrm{COCH}_{3} \\
& 2 \mathrm{CH}_{3} \cdot \stackrel{5}{\longrightarrow} \mathrm{C}_{2} \mathrm{H}_{6}
\end{aligned}
$$

This gives $\mathbf{R}_{\mathrm{CO}_{0}} \mathrm{R}_{\mathrm{C}_{2} \mathrm{H}_{\mathrm{i}} / \mathbf{R}_{\text {acetone }}}=k_{1} k_{5}^{1 / 2} / k_{4}$.

The back reaction rate was also determined through photolysis of azomethane in CO with the result $k^{2}(1 / \mathrm{mole}$-sec) $=10^{5.1 .5-3.8 / 8}$.

[2] Photolysis of acetone in the presence of (HI). The decomposition was studied as a function of pressure relative to the radical trapping reaction with $\mathrm{HI}$.

$$
\begin{aligned}
& \mathrm{CH}_{3} \mathrm{CO}+(\mathrm{M}) \stackrel{d}{\stackrel{3}{\longrightarrow}} \mathrm{CH}_{3} \cdot+\mathrm{CO}+(\mathrm{M}) \\
& \mathrm{CH}_{3} \stackrel{\mathrm{CO}}{\longrightarrow}+\mathrm{HI} \stackrel{\mathrm{CHO}+\mathrm{I} .}{\longrightarrow}
\end{aligned}
$$

The parameters are based on an assumed trapping reaction constant of $k_{3}=10^{9.2-1.5 / \theta} \mathrm{l} / \mathrm{mole}$-sec, a value supported by other kinetic studies on the reaction of $\mathrm{HI}+\mathrm{CH}_{3} \mathrm{COI} \longrightarrow \mathrm{CH}_{3} \mathrm{CHO}+\mathrm{I}_{2}$. Analysis by G.L.C. $\left(\mathrm{CH}_{3} \mathrm{CHO}\right)$ and standard methods for $\mathrm{CH}_{4}$ and $\mathrm{CO}$. with $\mathrm{O}_{2}$

[3] Photolysis of acetone in the presence of $\mathrm{O}_{2}$. The decomposition was monitored relative to the trapping reaction

$$
\begin{aligned}
& \mathrm{CH}_{3} \mathrm{CO} \stackrel{2}{\longrightarrow} \mathrm{CH}_{3} \cdot+\mathrm{CO} \\
& \mathrm{CH}_{3} \mathrm{C} \cdot \mathrm{O}+\mathrm{O}_{2} \stackrel{3}{\longrightarrow} \text { (other products) }
\end{aligned}
$$

The Arrhenius $A$-factor was not determined and it was assumed that $E_{3} \simeq 0$. Analysis on the noncondensible gas products by standard methods.

[4] Photolysis of acetone in the presence of butadiene. Decomposition was measured relative to the acetyl addition to butadiene.

$$
\begin{aligned}
& \mathrm{CH}_{3} \mathrm{CO}+\stackrel{12}{\longrightarrow} \text { oddn product } \\
& \mathrm{CH}_{3} \mathrm{CO} \stackrel{8}{\longrightarrow} \mathrm{CH}_{3}^{+}+\mathrm{CO}
\end{aligned}
$$

$k_{12} / k_{8} \simeq 720, E_{8}-E_{12}=8.1 \mathrm{kcal} /$ mole. The activation energy $E_{12} \simeq 5.4$ was also measured in the study by following the rate of butadiene polymerization with $\Delta \mathrm{P}$ measurements. 
Reaction: Epoxyethyl radical<smiles>CC(=O)CCC1CO1</smiles>

$\begin{array}{cccr} & \text { I } & \text { II } & \text { III } \\ \Delta H_{f(298)}^{\circ} & (36.2) & 34.0 & -26.4 \\ S_{(298)}^{\circ} & 59.4 & 46.4 & 47.3 \\ C_{p(298)}^{\circ} & 12.1 & 8.3 & 7.0\end{array}$

$\Delta S^{\circ}=34.3 \mathrm{~g} / \mathrm{mole}$

$\Delta H^{\circ}=-28.6 \mathrm{kcal} / \mathrm{mole}$

$\Delta C_{p}^{\circ}=3.2 \mathrm{~g} / \mathrm{mole}$

\begin{tabular}{|c|c|c|c|c|c|c|}
\hline $\log A$ & $\boldsymbol{E}$ & $\begin{array}{c}\log \boldsymbol{k}_{T} \\
(1050)\end{array}$ & Conditions & System & Surface & References \\
\hline 13 & 25 & 780 & $\begin{array}{l}900-1200^{\circ} \mathrm{K} \\
\left(\mathrm{Ar}, \mathrm{CO}_{2}, \text { and }\right. \\
\left.\mathrm{N}_{2} \text { carriers }\right)\end{array}$ & flow & & $\begin{array}{l}\text { [1] L. Cracco, I. Glassmann, } \\
\text { and I. E. Smith, J. } \\
\text { Chem. Phys. 31, } 506 \\
\text { (1959). }\end{array}$ \\
\hline
\end{tabular}

Preferred:

Not reliable.

Comments: Kinetic scheme is oversimplified and $k_{3}$ is assigned at least 100 -fold too fast compared to known rate. Reaction involves hot molecules and hot radicals. $k_{3}$ (measured) $=10^{8.1-10 / \theta}$.

\section{Experimental}

[1] Nitrogen carrier flow decomposition of ethylene oxide at high temperatures. Rate of reaction based on the temperature profile in the reaction zone (a unique system). Above parameters were based on an assumed mechanism fit to observed kinetics:

$$
\begin{aligned}
& \nabla+\text { rodicals } k_{1} \sim 10^{13-5.5 / \theta} \sec ^{-1} \\
& \nabla^{\circ} \stackrel{2}{\longrightarrow} \mathrm{CH}_{3}+\mathrm{CO} \\
& 8+\mathrm{CH}_{3} \stackrel{3}{\longrightarrow} \mathrm{CH}_{4}+\nabla^{0} \quad \mathrm{k}_{3} \sim 6 \times 10^{9-5 / \theta} \mathrm{1} / \mathrm{mole}-\mathrm{sec} \\
& \mathrm{CH}_{3}+\nabla^{\circ} \stackrel{4}{\longrightarrow} \text { termination } \mathrm{k}_{4} \sim 6 \times 10^{10} 1 / \text { mole-sec }
\end{aligned}
$$


Reaction: Ethoxy radical

\begin{tabular}{|c|c|c|c|}
\hline$\Delta H_{f(298)}^{\circ}$ & $\begin{array}{c}\mathrm{I} \\
(-4.9)\end{array}$ & $\begin{array}{c}\text { II } \\
34.0\end{array}$ & $\begin{array}{c}\text { III } \\
-27.7\end{array}$ \\
\hline$S_{(298)}^{\circ}$ & 65.9 & 46.1 & 52.3 \\
\hline$C_{p(298)}^{\circ}$ & 14.0 & 8.3 & 8 \\
\hline
\end{tabular}

$\Delta S^{\circ}=32.5 \mathrm{~g} / \mathrm{mole}$

$\Delta H^{\circ}=11.2 \mathrm{kcal} / \mathrm{mole}$

$\Delta C_{p}^{\circ}=2.8 \mathrm{~g} / \mathrm{mole}$

\section{$\begin{array}{llllll}\log A & E & \log k_{T} & \text { Conditions } & \text { System } & \text { Surface }\end{array}$ \\ (375)}

$\begin{array}{llll}10.45 & 13 & 2.87 & 488-468^{\circ} \mathrm{K} \\ 10.1 & 12.5 & 2.82\end{array}$

[1] M. H. J. Wijnen, J. Am. Chem. Soc. 82, 3034 $(1960)^{*}$; M. H. J.

Wijnen, J. Am. Chem. Soc. 80, $2394(1958)^{* *}$.

Preferred:

$\log k=13.4-17.5 / \theta$.

Comments: Kinetics of the back reaction are not known; however, one might guess that methyl addition to formaldehyde will have parameters intermediate between those for $\mathrm{CH}_{3}+\mathrm{CO} \longrightarrow \mathrm{CH}_{3} \mathrm{CO}$ and $\mathrm{CH}_{3}$ $+\mathrm{C}_{2} \mathrm{H}_{4} \longrightarrow \mathrm{C}_{3} \mathrm{H}_{7}$. From the kinetics of the former (see acetyl radical), one obtains $\log k_{a} \simeq 10.9-13.5 / \theta$, while those of the latter give $\log k_{a}=14.4-19.2 / \theta$. The isopropoxy and $t$-butoxy radical decompositions have both been shown to have some pressure dependence. One would therefore also expect the above reaction to be in its pressure falloff region and thus account for the low experimental parameters.

\section{Experimental}

*Reinvestigation of ethyl propionate photolysis. Same conditions, but analysis was complete (except for CH 2 O) by G.L.C. Products were $\mathrm{CO}, \mathrm{CO}_{2}, \mathrm{CH}_{2} \mathrm{O}, \mathrm{CH}_{4}, \mathrm{C}_{2} \mathrm{H}_{6}, \mathrm{C}_{2} \mathrm{H}_{4}, \mathrm{C}_{3} \mathrm{H}_{8}, \mathrm{C}_{4} \mathrm{H}_{10}, \mathrm{C}_{2} \mathrm{H}_{5} \mathrm{OH}, \mathrm{CH}_{3} \mathrm{CHO}$, and $\mathrm{C}_{2} \mathrm{H}_{5} \mathrm{OC}_{2} \mathrm{H}_{5}$. Decomposition was again measured relative to ethoxy $\mathrm{H}$-abstraction from the parent ester, and the kinetics of the $\mathrm{H}$-abstraction were determined relative to the radical recombination reactions:

$$
\begin{gathered}
2 \mathrm{C}_{2} \mathrm{H}_{5} \cdot \frac{2}{4} \cdot \mathrm{C}_{4} \mathrm{H}_{10} \\
\mathrm{C}_{2} \mathrm{H}_{5} \cdot+\mathrm{C}_{2} \mathrm{H}_{5} \mathrm{O} \cdot \frac{4}{16} \mathrm{C}_{2} \mathrm{H}_{5} \mathrm{OC}_{2} \mathrm{H}_{5} \\
\mathrm{C}_{2} \mathrm{H}_{5} \mathrm{O} \cdot+\mathrm{P} \stackrel{16}{\mathrm{C}_{2} \mathrm{H}_{5} \mathrm{OH}+\mathrm{P}_{-H}}
\end{gathered}
$$

at $19^{\circ} \mathrm{C}, k_{16} k_{2}^{1 / 2} / k_{4}=31 \times 10^{-12}\left(\frac{\mathrm{cc}}{\text { molec }}\right)^{1 / 2} \sec ^{-1 / 2}$. Assuming that $k_{2}=3 \times 10^{10} \mathrm{l} / \mathrm{mole}$-sec and $k_{4}=10^{10} \mathrm{l} / \mathrm{mole} \cdot \mathrm{sec}$, one then obtains $k_{16}\left(19^{\circ} \mathrm{C}\right)=4.42 \times 10^{4} \mathrm{l} / \mathrm{mole}$-sec. The Arrhenius plot of $\left(k_{16} k_{2}{ }^{1 / 2} / k_{4}\right)$ gave $E \simeq E_{16}=5.5 \mathrm{kcal} / \mathrm{mole}$; one obtains $k_{16}=10^{8.75-5.5 / \theta} \mathrm{l} / \mathrm{mole}-\mathrm{sec}$. Also, for the decomposition reaction ( 8 below), the data gave $k_{16} / k_{8}=10^{-1.7} \times 10^{7.5 / \theta}(1 / \mathrm{moles})$.

**The photolysis of ethyl propionate. Analysis of gaseous products by M.S. A usual Rice-Herzfeld scheme was proposed. The decomposition reaction was estimated relative to the $\mathrm{H}$-abstraction reaction:

$$
\begin{gathered}
\mathrm{C}_{2} \mathrm{H}_{5} \mathrm{O} \cdot \frac{8}{15} \mathrm{CH}_{3} \cdot+\mathrm{CH}_{2} \mathrm{O} \\
\mathrm{C}_{2} \mathrm{H}_{5} \mathrm{O} \cdot+\mathrm{A} \frac{15}{7} \mathrm{C}_{2} \mathrm{H}_{5} \mathrm{OH}+\mathrm{A}_{-H} \\
2 \mathrm{C}_{2} \mathrm{H}_{5} \mathrm{O} \cdot \stackrel{\mathrm{C}_{2} \mathrm{H}_{5} \mathrm{OH}+\mathrm{CH}_{3} \mathrm{CHO}}{\longrightarrow}
\end{gathered}
$$

Results were:

$$
\frac{k_{8}}{k_{15}}=10^{1.34-7 / \theta} ; k_{8} / k_{7^{1 / 2}} \simeq 2.3 \times 10^{-5}\left(\frac{\mathrm{mole}}{\mathrm{l}-\mathrm{sec}}\right)^{1 / 2} \text { at } 30^{\circ} \mathrm{C}
$$


Reaction: 2-Oxo-1-propyl radical (acetonyl radical)

$\cdot \mathrm{CH}_{2} \mathrm{COCH}_{3}(\mathrm{I}) \longrightarrow \mathrm{CH}_{3} \cdot(\mathrm{II})+\mathrm{CH}_{2} \mathrm{CO}(\mathrm{III})$

\begin{tabular}{l|ccc}
$\Delta H_{f(298)}^{\circ}$ & I & II & III \\
$S_{(298)}^{\circ}$ & $\left(\begin{array}{c}-6 \\
72.5\end{array}\right.$ & 34.0 & -14.6 \\
$C_{p(298)}^{\circ}$ & 46.4 & 57.1 \\
17.8 & 8.3 & 11.4
\end{tabular}

$\Delta S^{\circ}=31.0 \mathrm{~g} / \mathrm{mole}$

$\Delta H^{\circ}=25.6 \mathrm{kcal} / \mathrm{mole}$

$\Delta C_{p}^{\circ}=1.9 \mathrm{~g} / \mathrm{mole}$

\section{$\begin{array}{llllll}\log A & E & \log k_{T} & \text { Conditions } & \text { System } & \text { Surface }\end{array}$}

$41 \quad 365-435^{\circ} \mathrm{K}$

$P_{T} \simeq 50$ torr

[1] R. K. Brinton, J. Am.

Chem. Soc. 83, 1541

(1961).

\section{Preferred:}

$\log k_{a}=12.5-40 / \theta$

This assumes a back reaction with parameters $\log k_{-a}(1 /$ mole-sec $)=7.7-15 / \theta$.

\section{Experimental}

[1] High-temperature photolysis of acetone. The decomposition was monitored relative to the acetonyl radical recombination reaction.

$$
\begin{array}{r}
\cdot \mathrm{CH}_{2} \mathrm{COCH}_{3} \stackrel{7}{\longrightarrow} \mathrm{CH}_{2} \mathrm{CO}+\mathrm{CH}_{3} \\
2 \cdot \mathrm{CH}_{2} \mathrm{COCH}_{3} \stackrel{6}{\longrightarrow}\left(\mathrm{CH}_{3} \mathrm{COCH}_{2}\right)_{2}
\end{array}
$$

Analysis was by a variety of methods including M.S., G.L.C., and chemicals. The rate constant ratios $\left(k_{7} / k_{6}^{1 / 2}\right)$ were found to increase with increasing pressure. The $A$-factor reported above has been calculated, assuming $k_{6} \simeq 10^{10} \mathrm{l} / \mathrm{mole}$ sec. 
Reaction: Isopropoxy radical

\begin{tabular}{cccr}
\multicolumn{4}{r}{$\left(\mathrm{CH}_{3}\right)_{2} \mathrm{CHO} \cdot(\mathrm{I}) \longrightarrow \mathrm{CH}_{3} \mathrm{CHO}(\mathrm{II})+\mathrm{CH}_{3} \cdot(\mathrm{III})$} \\
& $\mathrm{I}$ & $\mathrm{II}$ & III \\
$\Delta H_{f(298)}^{\circ}$ & $(-12.4)$ & -39.8 & 34.0 \\
$S_{(298)}^{\circ}$ & 72.2 & 63.2 & 46.4 \\
$C_{p(298)}^{\circ}$ & 20.0 & 13.1 & 8.3
\end{tabular}

$\Delta S^{\circ}=37.4 \mathrm{~g} / \mathrm{mole}$

$\Delta H^{\circ}=(6.6) \mathrm{kcal} / \mathrm{mole}$

$\Delta C_{p}^{\circ}=1.4 \mathrm{~g} / \mathrm{mole}$

\begin{tabular}{|c|c|c|c|c|c|c|}
\hline $\log A$ & $\boldsymbol{E}$ & $\begin{array}{c}\log k_{T} \\
(460)\end{array}$ & Conditions & System & Surface & References \\
\hline 10.65 & 16.0 & 3.05 & $\begin{array}{l}448-473^{\circ} \mathrm{K} \\
P \sim 35 \mathrm{~mm} \mathrm{Hg}\end{array}$ & & & $\begin{array}{l}\text { [1] J. M. Ferguson and } \\
\text { L. Phillips, J. Chem. } \\
\text { Soc., } 4416 \text { (1965). }\end{array}$ \\
\hline 11.8 & 17.3 & 3.58 & $\begin{array}{l}433-473^{\circ} \mathrm{K} \\
P \sim 20-230 \\
\quad \text { torr }\end{array}$ & & & $\begin{array}{l}\text { [2] D. L. Cox, R. A. Liver- } \\
\text { more, and L. Phillips, } \\
\text { J. Chem. Soc. B, } 245 \\
\text { (1966). }\end{array}$ \\
\hline 9.32 & 11.5 & 3.86 & $\begin{array}{l}301-338^{\circ} \mathrm{K} \\
15-30 \mathrm{~mm} \mathrm{Hg}\end{array}$ & & & $\begin{array}{l}\text { [3] M. J. H. Wijnen, J. Am. } \\
\text { Chem. Soc. 82, } 1847 \\
\text { (1960). }\end{array}$ \\
\hline
\end{tabular}

Preferred:

All results are suspect.

Comments: The kinetics and thermodynamics for this reaction are incompatible. Thus

$$
A_{a} \simeq A_{-a}\left(\mathrm{~atm}^{-1} \mathrm{sec}^{-1}\right) \times 10 \frac{\Delta S^{\circ}}{4.575}
$$

and from [2], using $A_{a}=10^{11.8} \mathrm{sec}^{-1}$, one obtains $A_{-a} \simeq 10^{5.5} \mathrm{l} / \mathrm{mole}-\mathrm{sec}$, which compares to the "abnormally" low value found for $\mathrm{CH}_{3}+\mathrm{CO}$ addition. However, $E_{-a} \simeq E_{a}-\Delta E^{\circ}=17.3-5.7=11.6 \mathrm{kcal} / \mathrm{mole}$, which is appreciably higher than the "normal" value for $\mathrm{CH}_{3}+\mathrm{C}_{2} \mathrm{H}_{4}$ addition. Such a low $A$ factor and high activation energy for the back reaction $(-a)$ are particularly unreasonable. There also exists a rather uncomfortable trend in absolute decomposition rate constants in the series $\mathrm{C}_{2} \mathrm{H}_{5} \mathrm{O}, i-\mathrm{C}_{3} \mathrm{H}_{7} \mathrm{O}$, and $t-\mathrm{C}_{4} \mathrm{H}_{9} \mathrm{O}$. Thus $1 / 10 k_{t \text {-Buo }} \simeq 10 k_{\text {ipro }} \simeq k_{\mathrm{E} t \mathrm{O}}$ at $T \sim 400^{\circ} \mathrm{K}$. The above reported decomposition appears to be much too slow. It is very likely pressure dependent.

\section{Experimental}

[1] Pyrolysis of isopropyl nitrite in static system. The decomposition was measured relative to the rate of reaction with NO.

$$
\begin{gathered}
\mathrm{NO}+i \mathrm{PrO} \stackrel{2}{\longrightarrow} \mathrm{CH}_{3} \mathrm{COCH}_{3}+\mathrm{HNO} \\
i \mathrm{PrO} \stackrel{4}{\longrightarrow} \mathrm{CH}_{3}+\mathrm{CH}_{3} \mathrm{CHO}
\end{gathered}
$$

$$
k_{2}=10^{7} \mathrm{l} / \mathrm{mole}-\mathrm{sec}
$$

Continuous analysis of reaction was by I.R. The NO was determined by M.S.

(Continued) 
[2] Decomposition of isopropyl nitrite in NO. The decomposition was found to be total-pressure dependent. By employing a classical Hinshelwood-Lindemann treatment of the data (i.e., $k_{\text {uni }}=k_{0} M /\left(1+k_{0} M / k_{\infty}\right)$ ), the high- and low-pressure limiting rate constants were estimated. The low-pressure constant reported was $k_{0}=10^{10.1-8.3 \theta^{1}} / \mathrm{mole}$ sec, giving an RRK $-s \sim 20$. Energy transfer efficiencies of various third bodies were studied.

[3] Photolysis of isopropylpropionate. $i \mathrm{PrOCOC}_{2} \mathrm{H}_{5} \stackrel{h \nu}{\longrightarrow} \mathrm{C}_{2} \mathrm{H}_{5} \mathrm{CO}+i \mathrm{PrO}$. The rate of decomposition was monitored relative to the $\mathrm{H}$-abstraction from the parent molecule $\left(E_{7}-E_{6}\right)=7 \mathrm{kcal} / \mathrm{mole}$

$$
\begin{aligned}
& i \mathrm{PrO}+\mathrm{A} \stackrel{6}{\longrightarrow} i \mathrm{PrOH}+\mathrm{R}_{-\mathrm{H}} \\
& i \mathrm{PrO} \stackrel{7}{\longrightarrow} \mathrm{CH}_{3} \mathrm{CHO}+\mathrm{CH}_{3}
\end{aligned}
$$

Production of acetaldehyde via spontaneous decomposition of "hot" $i \mathrm{PrO}$. radicals was proposed and an appropriate correction made. Formation of $i \mathrm{PrOH}$ via radical disproportionation was believed to be unimportant. The relative rate constant ratios reported were $k_{7} / k_{6}=1.0 \times 10^{17}(\mathrm{molec} / \mathrm{cc}) 2{ }^{\circ} \mathrm{C}$, and $5 \times 10^{17} \mathrm{molec} / \mathrm{cc}\left(65^{\circ} \mathrm{C}\right)$, from which one obtains $k_{7} / k_{6}($ moles $/ 1)=3.7 \times 10^{-6 / \theta}$. The parameters reported here are for an assumed rate of $\mathrm{H} \cdot$ abstraction of $k_{6}$ $=10^{8.75-55 / \theta} \mathrm{l} / \mathrm{mole}$-sec (same as observed for $\mathrm{H}$-abstraction by $\mathrm{C}_{2} \mathrm{H}_{5} \mathrm{O} \cdot$ from ethyl propionate: see ethoxy radical decomposition). 
Reaction:sec-Butoxy radical

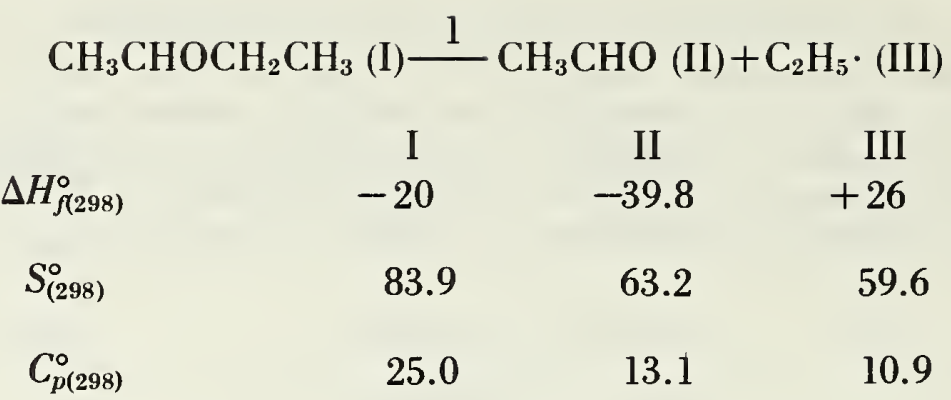

$\Delta S^{\circ}=38.9 \mathrm{~g} / \mathrm{mole}$

$\Delta H^{\circ}=6.2 \mathrm{kcal} / \mathrm{mole}$

$\Delta C_{p}^{\circ}=-1.0 \mathrm{~g} / \mathrm{mole}$

$\begin{array}{ccccccc}\log \boldsymbol{A} & \boldsymbol{E} & \begin{array}{c}\log \boldsymbol{k}_{T} \\ (460)\end{array} & \text { Conditions } & \text { System } & \text { Surface } & \text { References } \\ \sim 14 & \sim 17.5 & 5.69 & 423^{-463^{\circ} \mathrm{K}} & \text { static } & \begin{array}{c}\text { very little } \\ \text { effect }\end{array} & \begin{array}{r}{[1] \text { R. L. East and L. }} \\ \text { Phillips, J. Chem. } \\ \text { Soc. A, 1939 (1967). }\end{array}\end{array}$

Preferred:

Reported values are estimates and very likely lower limits to correct high-pressure values.

\section{Experimental}

[1] sec-BuÓ radicals were generated by pyrolysis of the nitrite in NO. Relative rates of radical decompositior (l) to disproportionation (2) were followed in terms of the yield ratios of $\mathrm{CH}_{3} \mathrm{CHO}$ and $\mathrm{CH}_{3} \mathrm{COC}_{2} \mathrm{H}_{5}$. The latter wer total pressure dependent. High-pressure parameters reported here were obtained from a simple Hinshelwood-Linde mann treatment assuming $E_{2}=0$ and $A_{1}=10^{14} \mathrm{sec}^{-1}$.

$$
\begin{aligned}
(\mathrm{M})+\mathrm{CH}_{3} \mathrm{CHOCH}_{2} \mathrm{CH}_{3} \stackrel{\mathrm{l}}{\longrightarrow} \mathrm{CH}_{3} \mathrm{CHO}+\mathrm{C}_{2} \mathrm{H}_{5} \cdot+(\mathrm{M}) \\
\mathrm{NO}+\text { sec }-\mathrm{BuO} \cdot \stackrel{2}{\longrightarrow} \mathrm{HNO}+\mathrm{CH}_{3} \mathrm{COC}_{2} \mathrm{H}_{5}
\end{aligned}
$$


Reaction: $t$-Butoxy radical

\begin{tabular}{cccr}
\multicolumn{4}{r}{$\left(\mathrm{CH}_{3}\right)_{3} \mathrm{CO} \cdot(\mathrm{I}) \longrightarrow \mathrm{CH}_{3} \mathrm{COCH}_{3}(\mathrm{II})+\mathrm{CH}_{3} \cdot(\mathrm{III})$} \\
& I & II & III \\
$\Delta H_{f(298)}^{\circ}$ & $(-21.5)$ & -51.7 & 34.0 \\
$S_{(298)}^{\circ}$ & 76.5 & 70.5 & 46.4 \\
$C_{\nu(298)}^{\circ}$ & 25.7 & 17.9 & 8.3
\end{tabular}

$\Delta S^{\circ}=4.0 .4 \mathrm{~g} / \mathrm{mole}$ $\Delta H^{\circ}=(3.8) \mathrm{kcal} / \mathrm{mole}$ $\Delta C_{p}^{\circ}=0.5 \mathrm{~g} / \mathrm{mole}$

$\begin{array}{rrrlrr}\log A & E & \begin{array}{r}\log \mathbf{k}_{T} \\ (425)\end{array} & \text { Conditions } & \text { System } & \text { Surface } \\ 9.7 & 13.2 & 2.91 & \begin{array}{l}400-4400^{\circ} \mathrm{K} \\ P_{T} \sim 150-550 \text { torr }\end{array} & \end{array}$

\section{References}

1] F. W. Birss, C. J. Danby, and C. N. Hinshelwood, Proc. Roy. Soc. (L) A239, 154(1957).

$\begin{array}{llll}11.2 \quad 11.0 \quad 5.54 & \begin{array}{l}298-352 \\ P \sim 20 \text { tor }\end{array}\end{array}$

[2] G. R. McMillan, J. Am. Chem. Soc. 82, 2422 (1960).
$13.71 \quad 17.0$
4.97
403-427 ${ }^{\circ} \mathrm{K}$
Pinene $0-200$ torr
$P \sim 15-20$ torr

[3] R. K. Brinton and D. H. Volman, J. Chem. Phys. 20, 25 (1952).
11.2
$300-390^{\circ} \mathrm{K}$
$P_{T} \sim 55$ torr

[4] D. H. Volman and W. M Graven, J. Am. Chem. Soc. 75, 3111 (1953).
10.179
5.54 298-352 \%
$P_{T} \sim 15$ torr

[5] G. McMillan and M. H. J. Wijnen, Can. J.

Chem. 36, 1227 (1958)
$13.5 \quad 16.5 \pm 1 \quad 5.01 \quad 393-453 \%$
$P_{T} \sim 100$ torr

[6] L. Batt and F. R. Cruickshank, J. Phys. Chem. 71, 1836 (1967).
$14.7 \quad 22.8$
398-436 ${ }^{\circ} \mathrm{K}$ 10-60 torr

[7] M. Y. Quee and J. C. J. Thynne, Trans. Faraday Soc 63, 2970 (1967).

\section{Preferred:}

Tentatively, $\log k=13.5-16.5 / \theta$.

Comments: As in the isopropoxy radical decomposition, there is an apparent serious discrepancy between the kinetics and the reaction thermodynamics. If one accepts the most intuitively appealing parameters (ref. [6] or [3]), then one calculates $\log k_{-a}(1 /$ mole-sec $)=6.6-13.5 / \theta$. The back reaction kinetics have been measured by $\mathrm{O}^{\prime} \mathrm{Neal}$ and Lubin (to be published), who found $\log _{-a}(1 / \mathrm{mole} \cdot \mathrm{sec})=6.17-5.0 / \theta$. Clearly there is a serious error in $k_{-a}, k_{a}, \Delta H^{\circ}$, or all three.

(Continued) 
The pressure dependence of this decomposition and an analysis of earlier results have been given by H. Hershenson and S. W. Benson, J. Chem. Phys. 37, 1889 (1962). See also D. J. Williams and M. F. R. Malcahy, Aus. J. Chem. 17, 1291 (1964) with regard to the experimental verification of the pressure dependence.

\section{Experimental}

[1] Decomposition of di-t-butyl peroxide in the presence of NO. Rates of reaction were measured by $\Delta \mathrm{P}$ and analysis of products by M.S. The decomposition was measured relative to the trapping reaction, $t \mathrm{BuO}+\mathrm{NO} \longrightarrow t \mathrm{BuONO}$. The rate of the trapping reaction was assumed to be the same as the rate of $\mathrm{CH}_{3} \mathrm{O}+\mathrm{NO}$, which was measured relative to the methyl radical recombination reaction to be $k_{4}(1 /$ mole-sec $) \simeq 10^{9.08}$.

[2] Photolysis (full $\mathrm{Hg}$ arc) of di-t-butyl peroxide with azomethane. The important steps in the mechanism assumed were:

$$
\begin{aligned}
& \mathrm{ROOR} \stackrel{h \nu}{\longrightarrow} 2 t \mathrm{BuO} \cdot ; \mathrm{CH}_{3} \mathrm{~N}=\mathrm{NCH}_{3} \stackrel{h \nu}{\longrightarrow} 2 \mathrm{CH}_{3} \cdot+\mathrm{N}_{2} \\
& t \cdot \mathrm{BuO} \cdot \stackrel{2}{\longrightarrow} \mathrm{CH}_{3} \mathrm{COCH}_{3}+\mathrm{CH}_{3} \cdot \\
& \mathrm{CH}_{3} \cdot+t \mathrm{BuO} \cdot \stackrel{5}{\longrightarrow} \mathrm{CH}_{3} \mathrm{O}-t \mathrm{Bu} \\
& \mathrm{CH}_{3} \cdot+\mathrm{CH}_{3} \cdot \stackrel{8}{\longrightarrow} \mathrm{C}_{2} \mathrm{H}_{6}
\end{aligned}
$$

Recombination rate constants. for 5 and 8 were taken as $k_{\text {rec }} \simeq 2.2 \times 10^{10} \mathrm{l} / \mathrm{mole}$-sec. The relative rates of decomposition (2) and H-abstraction of $t$ - $\mathrm{BuO}$ from isobutane $\left(t \mathrm{BuO}+\left(\mathrm{CH}_{3}\right)_{3} \mathrm{CH} \stackrel{16}{\longrightarrow} t \mathrm{BuOH}+t \mathrm{Bu} \cdot\right)$ were also determined in the photolysis of mixtures of the peroxide with isobutane. The result was $k_{16} / k_{2}=10^{-3.4+7 / \theta}(\mathrm{l} / \mathrm{moles})$ with $E_{2}-E_{16} \simeq 7$ $\mathrm{kcal} / \mathrm{mole}$, which implies a $4 \mathrm{kcal} / \mathrm{mole}$ activation energy for tertiary hydrogen $\mathrm{H} \cdot \mathrm{abstraction}$ by $t \mathrm{BuO} \cdot$. Analysis by G.L.C.

[3] Thermal decomposition of di-t-butyl peroxide in presence of ethylenimine. The relative rates of $\mathrm{H}$-abstraction from the imine and decomposition were determined.

$$
\begin{aligned}
& t \mathrm{BuO} \cdot \stackrel{2}{\longrightarrow} \mathrm{CH}_{3} \mathrm{COCH}_{3}+\mathrm{CH}_{3} . \\
& t \mathrm{BuO} \cdot+\left(\mathrm{CH}_{3}\right)_{2} \mathrm{NH} \stackrel{5}{\longrightarrow} t \mathrm{BuOH}+\left(\mathrm{CH}_{3}\right)_{2} \mathrm{~N} .
\end{aligned}
$$

$k_{2} / k_{5}=10^{+6.05-12 / \theta}($ moles $/ \mathrm{l})$. The parameters reported have been calculated assuming $k_{5}(1 / \mathrm{mole}-\mathrm{sec})=10^{\tau .66-5 / \theta}$.

[4] Photolysis of di-t-butyl peroxide in butadiene. Analysis of products (acetone, ethane, $\mathrm{CO}, \mathrm{CH}_{4}\left(\mathrm{CH}_{3} \mathrm{CO}\right)_{2}$, and $t \mathrm{BuOH})$ by M.S. Yields were corrected for the subsequent decomposition of acetone to $\mathrm{C}_{2} \mathrm{H}_{6}+\mathrm{CO}$. Rate of decomposition was measured relative to the rate of addition to butadiene.

$$
\stackrel{10}{\longrightarrow} \text { }{ }_{48400}^{\longrightarrow} \stackrel{2}{\longrightarrow} \text { ocetone }+\mathrm{CH}_{3}
$$

The data give $k_{2} / h_{10}=23 \times 10^{--5.8 / \theta}($ moles $/ \mathrm{l})$. The reported activation energy is based on $E_{10} \simeq 5.4 \mathrm{kcal} / \mathrm{mole}$ (measured in the study).

[5] Photolysis of di-t-butyl peroxide. Pertinent reactions were

$$
\begin{aligned}
t \mathrm{BuO} \cdot \stackrel{2}{\longrightarrow} \text { acetone }+\mathrm{CH}_{3} . \\
t \mathrm{BuO} \cdot+\mathrm{CH}_{3} \cdot \stackrel{5}{\longrightarrow} t \mathrm{BuOCH}_{3} \\
t \mathrm{BuO}+\mathrm{P} \stackrel{6}{\longrightarrow} t \mathrm{BuOH}+\mathrm{P}_{-\mathrm{H}} \\
\mathrm{CH}_{3}+\mathrm{CH}_{3} \cdot \stackrel{8}{\longrightarrow} 2 \mathrm{C}_{2} \mathrm{H}_{6}
\end{aligned}
$$

Analysis of products by M.S. and G.L.C. The results were $h_{2} h_{8} \cdot 1 / 2 / h_{5}=2.44 \times 10^{-2}(\mathrm{moles} / \mathrm{l}-\mathrm{sec})^{1 / 2}=10^{+. .99-9 / / t}$. (Indicatec parameters and based on $k_{5} \simeq k_{8}=2.2 \times 10^{10} \mathrm{l} / \mathrm{mole}^{-s e c}$.) In addition. $k_{2} / k_{6}=3.6 \times 10^{-3 / \theta}$ (moles/l). If H-abstraction is given the same parameters as found for ethoxy, adjusting for the different numbers of abstractable hydrogens. $k_{i i} \simeq 10^{9-6 / 4}$ $1 /$ mole-sec, one obtains $k_{2}=10^{9.56-9 / \theta} \sec ^{-1}$. 


\section{$t$-Butoxy radical (Continued)}

[6] Decomposition of di-t-butyl peroxide in the presence of $\mathrm{HBr}$. The radical decomposition was measure relative to its $\mathrm{H}$-abstraction from $\mathrm{HBr}$. Parameters of the trapping reaction with $\mathrm{HBr}(t \mathrm{BuO}+\mathrm{HBr} \stackrel{t}{\longrightarrow} t \mathrm{BuOH}+\mathrm{Br})$ were assumed to be $k_{t}=10^{\times .95-2 / \theta t} \mathrm{l} /$ mole-sec and the $A$-factor for decomposition was also assumed (i.e., $10^{13.5}$ sec $^{-1}$, as reported).

[7] Thermal decomposition of di-t-butyl peroxide in the presence of NO. Reaction was found to be pressure dependent and the high-pressure Arrhenius parameters were estimated by a Hinshelwood-Lindemann treatment. $M_{1 / 2} \simeq 112$ torr at $436^{\circ} \mathrm{K}$, and $n \simeq 23$. 
Reaction: 1-Ethoxy-1-ethyl radical

\begin{tabular}{|c|c|c|c|}
\hline$\Delta H_{f(298)}^{\circ}$ & $\begin{array}{c}\mathrm{I} \\
(-17.8)\end{array}$ & $\begin{array}{c}\text { II } \\
-39.8\end{array}$ & $\begin{array}{c}\text { III } \\
25.7\end{array}$ \\
\hline$S_{(298)}^{\circ}$ & 88.1 & 63.2 & 59.8 \\
\hline$C_{p(298)}^{\circ}$ & 25.4 & $13.1^{\circ}$ & 11.1 \\
\hline
\end{tabular}

$\Delta S^{\circ}=34.9 \mathrm{~g} / \mathrm{mole}$

$\Delta H^{\circ}=3.7 \mathrm{kcal} / \mathrm{mole}$

$\Delta C_{p}^{\circ}=-1.2 \mathrm{~g} /$ mole

\begin{tabular}{|c|c|c|c|c|c|c|}
\hline $\log A$ & $\boldsymbol{E}$ & $\begin{array}{r}\log k_{T} \\
(435)\end{array}$ & Conditions & System & Surface & References \\
\hline 10.91 & $23.5 \pm 2$ & -0.89 & $418-453^{\circ} \mathrm{K}$ & & & $\begin{array}{l}\text { [1] J. Long and G. Skirro } \\
\text { Trans. Faraday Soc } \\
\mathbf{5 8 , ~ 1 4 0 3 ~ ( 1 9 6 2 ) . ~}\end{array}$ \\
\hline 6.35 & 17.0 & -2.19 & $\begin{array}{l}540-638^{\circ} \mathrm{K} \\
P_{T} \simeq 6-25 \text { torr }\end{array}$ & & & $\begin{array}{l}\text { [2] M. H. Wijnen and } \\
\text { E. W. R. Steacie, } \\
\text { Can. J. Chem. 29, } \\
\text { } 1092 \text { (1951). }\end{array}$ \\
\hline
\end{tabular}

Preferred:

$\log k_{a}=10.91-23.5 / \theta$, although the $A$-factor seems too low.

Comments: The reaction thermodynamics and $k_{a}$ predict

$$
\begin{gathered}
\log \left[k_{-a}(1 / \text { mole-sec })\right] \simeq 5.6-20 / \theta \\
\text { Experimental }
\end{gathered}
$$

[1] Decomposition of di-t-butyl peroxide in the presence of diethyl ether. Analysis of all products $\left(\mathrm{CO}_{2} \mathrm{CH}_{4}, \mathrm{C}_{2} \mathrm{H}_{6}\right.$, $\mathrm{C}_{3} \mathrm{H}_{8}, \mathrm{CH}_{3} \mathrm{CHO}$ ) by G.L.C.

Mechanism:

$$
\begin{gathered}
\mathrm{CH}_{3}+\left(\mathrm{C}_{2} \mathrm{H}_{5}\right)_{2} \mathrm{O} \stackrel{1}{\longrightarrow} \mathrm{CH}_{4}+\mathrm{CH}_{3} \mathrm{CHOCH}_{2} \mathrm{CH}_{3}(\mathrm{R} \cdot) \\
\mathrm{R} \cdot+\mathrm{CH}_{3} \cdot \stackrel{4}{\longrightarrow} i \mathrm{PrOEt} \\
\mathrm{R} \cdot \stackrel{3}{\longrightarrow} \mathrm{CH}_{3} \mathrm{CHO}+\mathrm{C}_{2} \mathrm{H}_{5} \cdot \\
\mathrm{CH}_{3}+\mathrm{C}_{2} \mathrm{H}_{5} \stackrel{5}{\longrightarrow} \mathrm{C}_{3} \mathrm{H}_{8} \\
\mathrm{CH}_{3}+\mathrm{CH}_{3} \stackrel{2}{\longrightarrow} \mathrm{C}_{2} \mathrm{H}_{6}
\end{gathered}
$$

The decomposition was followed relative to reactions (2) and (4), giving

$$
\frac{k_{3} k_{2}^{1 / 2}}{k_{4}}\left(\frac{\text { moles }}{\text { cc-sec }}\right)^{1 / 2}=10^{7.11-23.5 \pm 2 / \theta} .
$$

The $A$-factor given above was reestimated, using $A_{2}=2 \times 10^{10} \mathrm{l} /$ mole-sec and $k_{4}=10^{10} \mathrm{l} / \mathrm{mole}$-sec.

[2] Photolysis of 2,2',4,4'-tetradeuterodiethyl ketone. Analysis for $\mathrm{CO}, \mathrm{C}_{2} \mathrm{H}_{4}$, and $\mathrm{C}_{2} \mathrm{H}_{6}$, using fractional distillation,

(Continued) 


\section{'1-Ethoxy-1-ethyl radical (Continued)}

M.S., and a Blacet-Leighton apparatus. The decomposition reaction was monitored relative to the pentanonyl radical recombination with ethyl $d_{2}$ radicals and the $\mathrm{D}$-abstraction of ethyl $d_{2}$ radicals from the ketone:

$$
\begin{gathered}
\left(k_{6} k_{4} / k_{9}\right) \simeq 10^{4.05-25.7 / \theta} \sec ^{-1} \\
\mathrm{CH}_{3} \mathrm{CD}_{2}+\mathrm{K} \stackrel{9}{\longrightarrow} \mathrm{X} \\
\mathrm{CH}_{3} \mathrm{CD}_{2}+\mathrm{K} \stackrel{4}{\longrightarrow} \mathrm{C}_{2} \mathrm{H}_{3} \mathrm{D}_{3}+\mathrm{K}_{-\mathrm{D}} \\
\mathrm{CH}_{3} \mathrm{CDCOCD}_{3} \mathrm{CH}_{3} \stackrel{6}{\longrightarrow} \mathrm{C}_{2} \mathrm{H}_{3} \mathrm{D}+\mathrm{CO}+\mathrm{CD}_{2} \mathrm{CH}_{3}
\end{gathered}
$$

The reported $A$-factor was obtained by assuming $k_{9} \simeq 10^{10} \mathrm{l} /$ mole-sec and using the calculated $k_{4}=10^{7.73-8.7 / \theta} \mathrm{I} / \mathrm{mole}$-sec. 
Reaction: 4-Oxo-2-hepty! radical

\begin{tabular}{cccc}
\multicolumn{4}{c}{$\mathrm{CH}_{3} \mathrm{CHCH}_{2} \mathrm{COC}_{3} \mathrm{H}_{7}(\mathrm{I}) \longrightarrow \mathrm{C}_{3} \mathrm{H}_{6}(\mathrm{II})+\mathrm{C}_{3} \mathrm{H}_{7} \mathrm{CO}(\mathrm{III})$} \\
$\Delta H_{f(298)}^{\circ}$ & I & II & III \\
$S_{(298)}^{\circ}$ & -27.7 & 4.9 & -15.4 \\
$C_{p(298)}^{\circ}$ & 110.7 & 63.8 & 83.5 \\
& 41.1 & 15.3 & 24.4
\end{tabular}

$\Delta S^{\circ}=\quad 56.6 \mathrm{~g} / \mathrm{mole}$

$\Delta H^{\circ}=17.2 \mathrm{kcal} / \mathrm{mole}$

$\Delta C_{p}^{\circ}=-1.4 \mathrm{~g} / \mathrm{mole}$

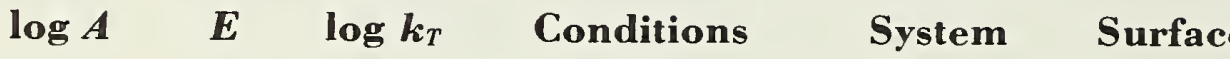

References

$10 \quad \begin{aligned} & 328-630^{\circ} \mathrm{K} \\ & P_{T}=8-115 \text { torr }\end{aligned}$

[1] C. R. Masson, J. Am. Chem. Soc. 74, 4731 (1952).

Preferred:

Unreliable.

Comments: The thermodynamics cannot be greatly in error, and they show that the above decomposition must have an activation energy greater than $17 \mathrm{kcal} / \mathrm{mole} . \Delta H_{f}^{\circ}\left(\mathrm{C}_{3} \mathrm{H}_{7} \dot{\mathrm{CO}}\right)$ is based on $\Delta H_{f}^{\circ}\left(\mathrm{CH}_{3} \mathrm{CO}\right)$ and group additives; $\Delta H_{f}^{\circ}$ (reactant) has been calculated assuming $D H^{\circ}(\mathrm{C}-\mathrm{H})=95 \mathrm{kcal} / \mathrm{mole}$ in the di-n-propyl ketone.

\section{Experimental}

[1] Photolysis of di-n-propyl ketone (3130 ̊). Above $160{ }^{\circ} \mathrm{C}$ a chain reaction became important and was attributed to the above reaction. The decomposition was measured relative to the heptanonyl radical recombination reaction, which, in turn, was estimated from mass balances. Products were $\mathrm{CO}, \mathrm{CH}_{4}, \mathrm{C}_{2} \mathrm{H}_{6}, \mathrm{C}_{2} \mathrm{H}_{4}, \mathrm{C}_{3} \mathrm{H}_{6}, \mathrm{C}_{3} \mathrm{H}_{8}, n \cdot \mathrm{C}_{6} \mathrm{H}_{14}$, and a $\mathrm{C}_{4}$-hydrocarbon. Data are insufficient to calculate the $A$-factor. Products were separated by low-temperature distillation and analyses were performed by a variety of techniques including oxidation over CuO, M.S., and absorption of unsaturates, using a Blacet-Leighton apparatus. 
Reaction: Methyl peroxymethyl radical

\begin{tabular}{cccc}
\multicolumn{4}{r}{$\mathrm{CH}_{3} \mathrm{OOCH}_{2} \cdot(\mathrm{I}) \longrightarrow \mathrm{CH}_{2} \mathrm{O}(\mathrm{II})+\mathrm{CH}_{3} \mathrm{O} \cdot(\mathrm{III})$} \\
$\Delta H_{f(298)}^{\circ}$ & $\mathrm{I}$ & II & III \\
$S_{(298)}^{\circ}$ & 13.5 & -27.7 & 3.9 \\
$C_{p(298)}^{\circ}$ & 77.3 & 52.3 & 54.6 \\
& 17.3 & 8.5 & 8.4
\end{tabular}

$\Delta S^{\circ}=29.6 \mathrm{~g} / \mathrm{mole}$

$\Delta H^{\circ}=-37.3 \mathrm{kcal} / \mathrm{mole}$

$\Delta C_{p}^{\circ}=-0.4 \mathrm{~g} / \mathrm{mole}$

$\begin{array}{llllll}\log A & \boldsymbol{E} & \log \boldsymbol{k}_{T} & \text { Conditions } & \text { System } & \text { Surface }\end{array}$

5.8

394- $453^{\circ} \mathrm{K}$

$P_{T} \sim 9-150$ torr
[1] Y. Takezake, T. Mizazaki, and N. Nakohara, J. Chem. Phys. 25, 536 (1956).

\section{Preferred:}

Unreliable, because of implausible mechanism.

Comments: In view of the reaction exothermicity, the activation energy would be expected to be quite low.

\section{Experimental}

[1] Photolysis of dimethylperoxide (2537 $)$. Major products were $\mathrm{CO}, \mathrm{CH}_{3} \mathrm{OH}$, and $\mathrm{CH}_{2} \mathrm{O}$. A complete and detailed treatment of the photochemically induced decomposition is presented. The overall rate, measured manometrically, was related to the reaction mechanism from which the activation energy relation, $E_{2}+E_{3}-E_{4}$, was deduced. Thus,

$$
\begin{gathered}
\mathrm{CH}_{3} \mathrm{O}+\mathrm{P} \stackrel{2}{\longrightarrow} \mathrm{CH}_{3} \mathrm{OH}+\cdot \mathrm{CH}_{2} \mathrm{OOCH}_{3} \\
\mathrm{CH}_{3} \mathrm{OOCH}_{2} \cdot \stackrel{3}{\longrightarrow} \mathrm{CH}_{2} \mathrm{O}+\mathrm{CH}_{3} \mathrm{O} \cdot \\
\mathrm{CH}_{3} \mathrm{O}+\mathrm{CH}_{3} \mathrm{OOCH}_{2} \stackrel{4}{\longrightarrow} 2 \mathrm{CH}_{2} \mathrm{O}+\mathrm{CH}_{3} \mathrm{OH} \cdot
\end{gathered}
$$

From the nature of the reactions, it is likely that $E_{2} \simeq E_{4}$. The $A$-factor could not be obtained from the data. Reaction 4 is not a likely one. 
Reaction: Perfluoro- $t$-butoxy radical

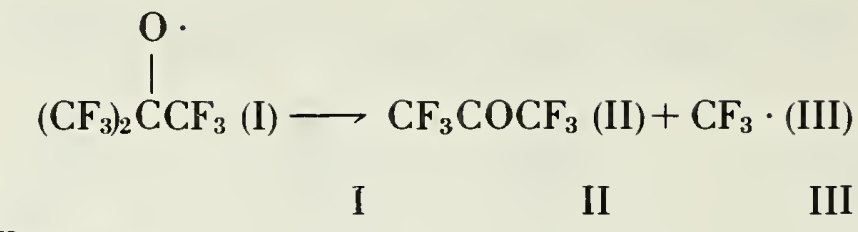

$\Delta H_{f(298)}^{\circ}$

$$
\begin{aligned}
& S_{(298)}^{\circ} \\
& C_{p(298)}^{\circ}
\end{aligned}
$$

$\Delta S^{\circ}=$
$\Delta H^{\circ}=$
$\Delta C_{p}^{\circ}=$

$\begin{array}{llllll}\log A & E & \log k_{T} & \text { Conditions } & \text { System } & \text { Surface }\end{array}$

References

$$
\begin{array}{ll}
30.6 & 323-610^{\circ} \mathrm{K} \\
& 25-180 \text { torr }
\end{array}
$$

[1] A. S. Gordon, J. Chem.

Phys. 36, 1330 (1962).

Preferred:

Reasonable.

Comments: $\Delta H^{\circ}$ estimated by author was $21 \mathrm{kcal}$ from $E_{\text {act }}$ for forward and reverse rate. This is a significantly stronger bond than in the $\mathrm{H}$-analog.

\section{Experimental}

[1] Photolysis of perfluoroacetone.

Mechanism:

$$
\begin{gathered}
\mathrm{CF}_{3} \mathrm{COCF}_{3} \stackrel{h \nu}{\longrightarrow} 2 \mathrm{CF}_{3} \cdot+\mathrm{CO} \\
\mathrm{CF}_{3} \cdot+\mathrm{A} \stackrel{1}{\underset{-1}{\rightleftharpoons}}\left(\mathrm{CF}_{3}\right)_{2} \mathrm{COCF}_{3} \\
\stackrel{\mathrm{O}}{\mid} \\
\mathrm{CF}_{3} \cdot+\left(\mathrm{CF}_{3}\right)_{2} \mathrm{CCF}_{3} \stackrel{2}{\longrightarrow}\left(\mathrm{CF}_{3}\right)_{3} \mathrm{COCF}_{3} \\
2 \mathrm{CF}_{3} \cdot \stackrel{3}{\longrightarrow} \mathrm{C}_{2} \mathrm{~F}_{6}
\end{gathered}
$$

Rates of addition (1) were obtained at low temperatures from the yields of ether and perfluoroethane. Rates of the decom position reaction $(-1)$ were obtained from the high-temperature data where decomposition becomes important. $E_{1} \simeq 9 . i$ $\mathrm{kcal} / \mathrm{mole}$. Analysis of the products was by G.L.C. Note radical structure is not certain. 
Reaction: 2-Chloro-1-ethyl radical

$\begin{array}{cccc} & \cdot \mathrm{CH}_{2} \mathrm{CH}_{2} \mathrm{Cl}(\mathrm{I}) \longrightarrow \mathrm{C}_{2} \mathrm{H}_{4}(\mathrm{II})+\mathrm{Cl}(\mathrm{III}) \\ \Delta H_{(298)}^{\circ} & \mathrm{I} & \text { II } & \text { III } \\ S_{(298)}^{\circ} & 20.2 & 12.5 & 29.0 \\ C_{(298)}^{\circ} & 68.2 & 52.5 & 39.5 \\ & 14.8 & 10.4 & 5.2\end{array}$

$\Delta S^{\circ}=23.8 \mathrm{~g} / \mathrm{mole}$ $\Delta H^{\circ}=21.3 \mathrm{kcal} / \mathrm{mole}$

$\Delta C_{p}^{\circ}=0.8 \mathrm{~g} / \mathrm{mole}$

$\begin{array}{llllll}\log A & \boldsymbol{E} & \log k_{T} & \text { Conditions } & \text { System } & \text { Surface }\end{array}$

$13 \quad 23.9$

$(*)$

[1] R. Eckling, P. Gold-

finger, G. Huybrechts, G. Martens, L. Meyers, and S. Smoes, Chem. Ber. 93, 3014 (1960).

\section{Preferred:}

$\log k_{a}=13.6-22.2 / \theta$.

Comments: Photochlorination study made by F. S. Dainton, D. A. Lomax, and M. Weston, Trans. Faraday Soc. 58, $308(1962)$. If $\log k_{-a}(1 /$ mole-sec $)=10.3-1.5 / \theta$, as suggested by P. B. Ayscough, A. J. Cocker, F. S. Dainton, and S. Hirst, Trans. Faraday Soc. 58, 318 (1962), then from the reaction thermodynamics, one obtains the preferred parameters.

\section{Experimental}

*[1] Calculated from various data sources. 
Reaction: 1,2-Dichloro-1-ethyl radical

\begin{tabular}{cccc}
\multicolumn{4}{r}{$\mathrm{CH}_{2} \mathrm{ClCHCl} \cdot(\mathrm{I}) \longrightarrow \mathrm{CH}_{2}=\mathrm{CHCl}$ (II) $+\mathrm{Cl}$ (III) } \\
& I & II & III \\
$\Delta H_{f(298)}^{\circ}$ & $(14.7)$ & 8.4 & 29.0 \\
$S_{(298)}^{\circ}$ & 76.9 & 63.0 & 39.5 \\
$C_{p(298)}^{\circ}$ & 17.5 & 13.0 & 5.2
\end{tabular}

$\Delta S^{\circ}=25.6 \mathrm{~g} / \mathrm{mole}$

$\Delta H^{\circ}=(22.7) \mathrm{kcal} / \mathrm{mole}$

$\Delta C_{p}^{\circ}=0.7 \mathrm{~g} / \mathrm{mole}$

$\begin{array}{cccccc}\log \boldsymbol{A} & \boldsymbol{E} & \begin{array}{c}\log \boldsymbol{k}_{T} \\ (315)\end{array} & \text { Conditions } & \text { System } & \begin{array}{c}\text { Surface } \\ \text { 10 }\end{array} \\ & 22 & -5.27 & \begin{array}{c}703-741^{\circ} \mathrm{K} \\ P_{T} \sim 1-80 \mathrm{~mm} \mathrm{Hg}\end{array} & \text { static } & \text { [1] K. E. Howlett, Trans. } \\ \text { Faraday Soc. 48, } \\ \end{array}$

Preferred:

$\log k=13.5-24.5 / \theta$.

\section{Comments:}

$\log k_{-a}=9.4-0.9 / \theta$. P. B. Ayscough, A. J. Cocker, F. S. Dainton, S. Hirst, and M. Weston, Proc. Chem Soc., 244 (1961).

$\log k_{a}(\mathrm{est})=13.1-23.0 / \theta$.

$\Delta H_{f}^{\circ}(\mathrm{R} \cdot)$ based on $D H^{\circ}(\mathrm{C}-\mathrm{H})=98 \mathrm{kcal} /$ mole (see $\mathrm{C}_{2} \mathrm{Cl}_{5} \cdot$ decomposition). This puts the $\mathrm{Pi}$ bond strengt] in $\mathrm{CHCl}=\mathrm{CHCl}$ at $1 \mathrm{kcal}$ less than butene-2.

\section{Experimental}

[1] Pyrolysis of 1,2-dichloroethylene. Rate was followed by pressure change with stoichiometry $\left(\mathrm{C}_{2} \mathrm{H}_{4} \mathrm{Cl}_{2} \longrightarrow\right.$ $\mathrm{C}_{2} \mathrm{H}_{3} \mathrm{Cl}+\mathrm{HCl}$ ). The mechanism proposed was

$$
\begin{gathered}
\mathrm{C}_{2} \mathrm{H}_{4} \mathrm{Cl}_{2} \stackrel{\mathrm{l}}{\longrightarrow} \cdot \mathrm{C}_{2} \mathrm{H}_{4} \mathrm{Cl}+\mathrm{Cl} \\
\mathrm{Cl}+\mathrm{C}_{2} \mathrm{H}_{4} \mathrm{Cl}_{2} \stackrel{2}{\longrightarrow} \cdot \mathrm{C}_{2} \mathrm{H}_{3} \mathrm{Cl}_{2}+\mathrm{HCl} \\
\cdot \mathrm{C}_{2} \mathrm{H}_{3} \mathrm{Cl}_{2} \stackrel{3}{\longrightarrow} \mathrm{C}_{2} \mathrm{H}_{3} \mathrm{Cl}+\mathrm{Cl} \\
\mathrm{Cl}+\cdot \mathrm{C}_{2} \mathrm{H}_{3} \mathrm{Cl} \stackrel{4}{\longrightarrow} \mathrm{C}_{2} \mathrm{H}_{3} \mathrm{Cl}+\mathrm{HCl}
\end{gathered}
$$

From the above, $k=\left(\frac{k_{1} k_{2} k_{3}}{k_{4}}\right)^{1 / 2}=10^{10.8-47.0 / \theta} \mathrm{sec}^{-1}$. An induction period was also observed and accounted for qual titatively. A pressure falloff of the rate was attributed to the unimolecular falloff of $k_{1}$, occurring at $P<20$ torr. Vari tion of $S / V$ had no effect.

[2] Calculated from a number of data sources, photochlorination studies of vinylchloride performed by F. S. Daiı ton, P. A. Lomax, and M. Weston, Trans. Faraday Soc. 58, 308 (1962).

*Temperature range was $25-55^{\circ} \mathrm{C}$. Rotating sector technique employed. 
Reaction: 1,2,2-Trichloro-1-ethyl radical

$$
\mathrm{CHCl}_{2} \mathrm{CHCl} \cdot(\mathrm{I}) \longrightarrow \mathrm{CHCl}=\mathrm{CHCl}(\mathrm{II})+\mathrm{Cl}(\mathrm{III})
$$

\begin{tabular}{|c|c|c|c|}
\hline$\Delta H_{f(298)}^{\circ}$ & $\begin{array}{c}\text { I } \\
13.1\end{array}$ & $\begin{array}{l}\text { II } \\
4.2\end{array}$ & $\begin{array}{c}\text { III } \\
29.0\end{array}$ \\
\hline$S_{(298)}^{\circ}$ & 83.9 & 69.4 & 39.5 \\
\hline$C_{p(298)}^{\circ}$ & 20.7 & 15.8 & 5.2 \\
\hline
\end{tabular}
$\Delta S^{\circ}=25.0 \mathrm{~g} / \mathrm{mole}$
$\Delta H^{\circ}=20.1 \mathrm{kcal} / \mathrm{mole}$
$\Delta C_{p}^{\circ}=0.3 \mathrm{~g} / \mathrm{mole}$

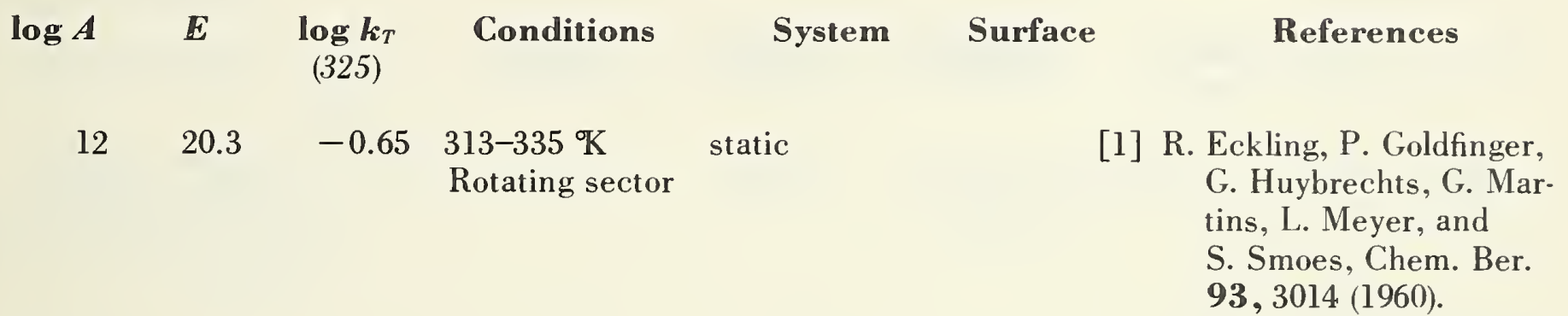

Preferred:

$\log k_{a}(\mathrm{est}) \simeq 13.1-20.4 / \theta$.

$\log k_{-a}=9.5-1.0 / \theta$. P. B. Ayscough, A. J. Cocker, F. S. Dainton, S. Hirst, and M. Weston, Proc. Chem. Soc., 244 (1961).

\section{Experimental}

[1] Calculated from data of various sources. Photochlorination study made by P. B. Ayscough, A. J. Cocker, F. S.Daiton, and S. Hirst, Trans. Faraday Soc. 58, 295 (1962). Rotating sector technique employed. 
Reaction: 1,1,2,2-Tetrachloro-1-ethyl radical

$\mathrm{CHCl}_{2} \mathrm{CCl}_{2} \cdot(\mathrm{I}) \longrightarrow \mathrm{Cl}(\mathrm{II})+\mathrm{CHCl}=\mathrm{CCl}_{2}(\mathrm{III})$

\begin{tabular}{c|ccc}
$\Delta H_{f(298)}^{\circ}$ & I & II & III \\
$S_{(298)}^{\circ}$ & $\left(\begin{array}{c}{ }^{\circ} \\
89.3\end{array}\right.$ & 29.0 & 0.3 \\
$C_{p(298)}^{\circ}$ & 39.5 & 77.5 \\
23.9 & 5.2 & 19.3
\end{tabular}

$\Delta S^{\circ}=27.7 \mathrm{~g} / \mathrm{mole}$

$\Delta H^{\circ}=19.8 \mathrm{kcal} / \mathrm{mole}$

$\Delta C_{p}^{\circ}=0.6 \mathrm{~g} / \mathrm{mole}$

\begin{tabular}{|c|c|c|c|c|c|c|c|}
\hline $\log A$ & $\boldsymbol{E}$ & $\begin{array}{c}\log \boldsymbol{k}_{T} \\
(468)\end{array}$ & Conditions & System & Surface & & References \\
\hline 13.7 & 20.4 & 4.11 & $\begin{array}{l}433-497{ }^{\circ} \mathrm{K} \\
P_{T} \simeq 60-110 \\
\mathrm{~mm} \mathrm{Hg}\end{array}$ & static & & {$[1]$} & $\begin{array}{l}\text { G. Huybrechts, L. Mey- } \\
\text { ers, and G. Verbeke, } \\
\text { Trans. Faraday Soc. 58, } \\
1128 \text { (1962). }\end{array}$ \\
\hline
\end{tabular}

Preferred:

Parameters are reasonable. $\log k=13.7-20.4 / \theta$.

Comments: Kinetics are in reasonable agreement with the estimated thermodynamics. The back-reaction kinetics have also been determined by Dainton et al., Proc. Chem. Soc., 244 (1961), to be $\log k_{-a}(1 / \mathrm{mole}$ sec) $=9.6-0.7 / \theta$. See also F. S. Dainton, D. A. Lomax, and M. Weston, Trans. Faraday Soc. 53, 460 (1947) for earlier work on the photochlorination of $\mathrm{C}_{2} \mathrm{HCl}_{3}$.

$\Delta H_{f}^{\circ}(\mathrm{R} \cdot)$ based on $D H(\mathrm{C}-\mathrm{H})=98 \mathrm{kcal} /$ mole $\left(\right.$ see $\mathrm{C}_{2} \mathrm{Cl}_{5} \cdot$ decomposition).

\section{Experimental}

[1] Photochlorination of trichloroethylene. Analysis of the chlorinated products was made by M.S. as a function of time. The kinetics for the chlorination mechanism gave a measure of the reverse addition reaction, $\log k_{2}(1 / \mathrm{mole}$ sec $)=9.75$, and the equilibrium constant for dissociation, $\left(k_{4} / k_{2}\right)=10^{3.97-20.4 / \theta}$ moles $/$, from which the decomposition kinetics were calculated.

Mechanism:

$$
\begin{gathered}
\mathrm{Cl}_{2} \stackrel{h \nu}{\rightleftharpoons} 2 \mathrm{Cl} \\
\mathrm{Cl}+\mathrm{C}_{2} \mathrm{HCl}_{3} \underset{4}{\stackrel{2}{\rightleftharpoons}} \cdot \mathrm{C}_{2} \mathrm{HCl}_{4} \\
\cdot \mathrm{C}_{2} \mathrm{HCl}_{4}+\mathrm{Cl}_{2} \underset{5}{\stackrel{3}{\rightleftharpoons}} \mathrm{C}_{2} \mathrm{HCl}_{5}+\mathrm{Cl} \\
2 \mathrm{Cl}+\mathrm{M} \stackrel{6}{\stackrel{6}{\longrightarrow}} \mathrm{Cl}_{2}+\mathrm{M} \\
\cdot \mathrm{C}_{2} \mathrm{HCl}_{4}+\mathrm{Cl} \stackrel{\frac{7}{\longrightarrow} \text { termination products }}{2} \text { termination products }
\end{gathered}
$$


Reaction: Pentachloroethyl radical

$\begin{array}{cccc} & \mathrm{C}_{2} \mathrm{Cl}_{5} \cdot(\mathrm{I}) \longrightarrow \mathrm{C}_{2} \mathrm{Cl}_{4}(\mathrm{II})+\mathrm{Cl}(\mathrm{III}) & \\ & \mathrm{I} & \mathrm{II} & \text { III } \\ \Delta H_{f(298)}^{\circ} & 7.9 & -3.6 & 29.0 \\ S_{(298)}^{\circ} & 94.5 & 81.4 & 39.5 \\ C_{p(298)}^{\circ} & 28.1 & 22.8 & 5.2\end{array}$

$\Delta S^{\circ}=26.4 \mathrm{~g} / \mathrm{mole}$

$\Delta H^{\circ}=17.5 \mathrm{kcal} / \mathrm{mole}$

$\Delta C_{p}^{\circ}=-0.1 \mathrm{~g} / \mathrm{mole}$

\begin{tabular}{|c|c|c|c|c|c|c|}
\hline $\log A$ & $\boldsymbol{E}$ & $\begin{array}{c}\log \boldsymbol{k}_{T} \\
(345)\end{array}$ & Conditions & System & Surface & References \\
\hline 12.8 & 16.8 & 2.15 & $30-388^{\circ} \mathrm{K}$ & static & & $\begin{array}{l}\text { [1] (a) J. Adam, P. Goldfinger, } \\
\text { and P. A. Gosselain, } \\
\text { Bull. Soc. Chim. Belges } \\
\text { 65, 549 (1956); } \\
\text { (b) P. Goldfinger, } \\
\text { M. Jeunehomme, and } \\
\text { G. Martens, J. Chem. } \\
\text { Phys. 29, 456 (1958); } \\
\text { (c) R. Eckling, } \\
\text { P. Goldfinger, } \\
\text { G. Huybrechts, } \\
\text { G. Martens, L. Meyers, } \\
\text { and S. Smoes, Chem. } \\
\text { Ber. 93, 3014 (1960). }\end{array}$ \\
\hline
\end{tabular}

Preferred:

$\log k_{a}=13.5-17.8 / \theta$.

Comments: The reaction enthalpy and radical heat of formation have been obtained from the kinetics. This also gives $\mathrm{DH}^{\circ}(\mathrm{C}-\mathrm{H})$ in $\mathrm{C}_{2} \mathrm{HCl}_{5} \simeq 98.9 \mathrm{kcal} /$ mole and suggests that the $\mathrm{DH}^{\circ}(\mathrm{C}-\mathrm{H})$ in chlorinated ethanes is not appreciably affected by the chlorine substitution. The back reaction rate, $\log k_{-a}(1 / \mathrm{mole}-\mathrm{sec})$ =9.4, has been measured by P. Goldfinger, G. Huybrechts, and G. Martens, Trans. Faraday Soc. 58, 2210 (1961). See also reference [1c] above. This gives $A_{a(\text { est })}=10^{13.4} \sec ^{-1}$ (using the estimated $\Delta S^{\circ}$ reaction), in reasonable agreement with the value reported.

\section{Experimental}

[1] Photochemical chlorination of tetrachloroethylene. Mechanism:

$$
\begin{array}{cl}
\mathrm{Cl}_{2} \stackrel{h \nu}{\stackrel{2}{\rightleftarrows}} 2 \mathrm{Cl} \cdot & \text { (Deduced rate constants) } \\
\mathrm{Cl}+\mathrm{C}_{2} \mathrm{Cl}_{4} \underset{-2}{\stackrel{2}{\rightleftarrows}} \mathrm{C}_{2} \mathrm{Cl}_{5} . & \log k_{-2} \simeq 9.6 \\
\mathrm{C}_{2} \mathrm{Cl}_{5}+\mathrm{Cl}_{2} \stackrel{3}{\stackrel{-3}{\rightleftarrows}} \mathrm{C}_{2} \mathrm{Cl}_{6}+\mathrm{Cl} \cdot & \log k_{3} \simeq 8.31-5.4 / \theta \\
\mathrm{Cl} \cdot+\mathrm{C}_{2} \mathrm{Cl}_{5} \cdot \stackrel{4}{\longrightarrow} \mathrm{C}_{2} \mathrm{Cl}_{6} & \log k_{-3} \simeq 11.6-19.5 / \theta \\
2 \mathrm{Cl} \cdot+\mathrm{M} \stackrel{5}{\longrightarrow} \mathrm{Cl}_{2}+\mathrm{M} & \log k_{4} \simeq 11.31 \\
2 \mathrm{C}_{2} \mathrm{Cl}_{5} \cdot \stackrel{6}{\longrightarrow} \mathrm{C}_{2} \mathrm{Cl}_{4}+\mathrm{C}_{2} \mathrm{Cl}_{6} & \log k_{6} \simeq 8.66
\end{array}
$$


For a complete summary of references to photochlorinated studies and a discussion of mechanisms for these reactions under varied conditions, see R. J. Cvetanovic, Advances in Photochemistry, Vol. 1, p. 115 (Interscience Publ., New York, 1963).

Reaction: 4-Chlorodifluoromethyl-2,5-cyclohexadienyl radical (resonance stabilized)

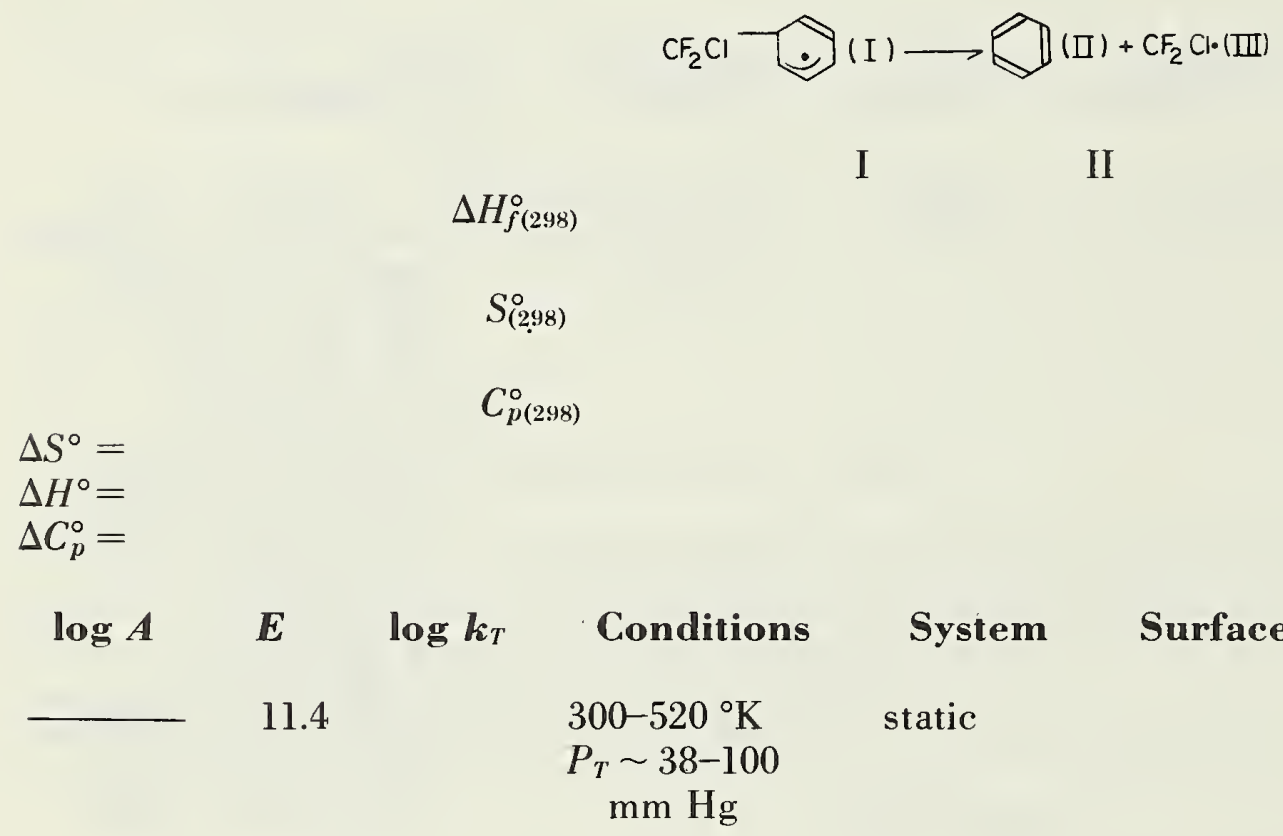

Suspect.

Comments: The value is only slightly larger than the estimated endothermicity of $10 \pm 2 \mathrm{kcal}$ and suggests too low an activation energy for the reverse reaction.

\section{Experimental}

[1] Photodecomposition of $\left(\mathrm{CF}_{2} \mathrm{ClCOCF}_{2} \mathrm{Cl}\right)$ in the presence of benzene. Products analyzed (M.S. and (;.L.C.) were $\mathrm{C}_{2} \mathrm{~F}_{4} \mathrm{Cl}_{2}, \mathrm{CF}_{2} \mathrm{Cl}_{2}, \mathrm{C}_{3} \mathrm{~F}_{6} \mathrm{Cl}_{2}, \mathrm{CF}_{2} \mathrm{ClH}$, and $\mathrm{CO}$. Higher molecular weight products were believed to be too involatile. The rate of addition (reverse of above) was measured from the decrease in rate of the $\left(\mathrm{CF}_{2} \mathrm{Cl}\right)$ radical products $\left(R_{f}\right)$ relative to $\mathrm{CO}$ formation caused by the addition of benzene compared to the pure ketone system. At higher temperatures (above $\left.120^{\circ} \mathrm{C}\right)$, the $\left(R_{f} / \mathrm{CO}\right)$ ratios reached a minimum and then increased with increasing temperature. This was interpreted in terms of the above decomposition. An Arrhenius plot of the $\left(R_{f} / \mathrm{CO}\right)$ ratios gave at low temperatures, $E_{\text {addn }} \sim 5.3$ $\mathrm{kcal} / \mathrm{mole}$, and at high temperatures, $E_{\mathrm{dec}} \simeq 11.4$, as reported. In a similar study, $E_{\mathrm{dec}} \simeq 10.7 \mathrm{kcal} / \mathrm{mole}$ for $\mathrm{cF}_{2} \mathrm{cl} /(\mathbb{)})_{\mathrm{d}_{6}}$
(Trans. Faraday Soc. 61, $122(1965)$. 
Reaction: 2-Bromo-1-ethyl radical

- $\mathrm{CH}_{2} \mathrm{CH}_{2} \mathrm{Br}(\mathrm{I}) \longrightarrow \mathrm{C}_{2} \mathrm{H}_{4}(\mathrm{II})+\mathrm{Br}(\mathrm{III})$

$\begin{array}{cccc} & \text { I } & \text { II } & \text { III } \\ \Delta H_{f(298)}^{\circ} & 30.4 & 12.5 & 26.7 \\ S_{(298)}^{\circ} & 70.2 & 52.5 & 41.8 \\ C_{p(298)}^{\circ} & 15.0 & 10.4 & 5.0\end{array}$

$\Delta S^{\circ}=24.1 \mathrm{~g} / \mathrm{mole}$

$\Delta H^{\circ}=8.8 \mathrm{kcal} / \mathrm{mole}$

$\Delta C_{p}^{\circ}=0.4 \mathrm{~g} / \mathrm{mole}$

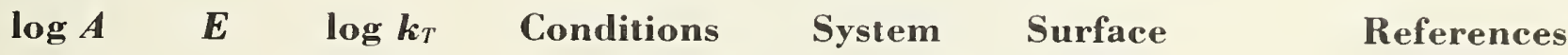 (310)}

$\begin{array}{llr}13 \pm 2 & 333-353^{\circ} \mathrm{K} & \text { static } \\ & P_{T} \sim 200 \text { torr } & \\ & \left(\mathrm{Br}_{2} /=\right) \sim 1.0 & \\ & & \\ 14 \pm 2 & 298-327^{\circ} \mathrm{K} & \text { static } \\ & & \\ \geqslant 7.4 & & \\ & & \\ & & \\ & & \\ & & \end{array}$

[1] H. Schmitz, H. J. Schumacher, and A. Jager, Chem. Ber. B51, 281 (1942).

[2] D. A. Armstrong and J. W. T. Spinkes, Can. J. Chem. 37, 1210 (1959).

[3] R. Barker and A. Maccoll, J. Chem. Soc., 2839 (1963).

$\begin{array}{llll}12.9 & 11.1 & 5.07 & \text { (ref. [2]) }\end{array}$

(4) E. O'Neal and S. W. Benson (unpublished calculations).

Preferred:

$\log k_{a}=12.9-11.1 / \theta$.

Comments: The back reaction rate has been measured at $60^{\circ} \mathrm{C}(\mathrm{R}$. J. Cuetanovic, Advances in Photochemistry, Vol. 1, p. 173, Interscience Publ., New York, 1963) to be $k_{-a}(1 /$ mole-sec $) \simeq 4 \times 10^{7}$. Assuming $A_{-a} \simeq 10^{9.5}$, one then calculates $E_{-a} \simeq 2.9 \mathrm{kcal} / \mathrm{mole}$, and $\log _{a} k_{a} \simeq 12.9-11.1 / \theta$.

\section{Experimental}

[1] Photolysis of ethylene with bromine. The rate was followed manometrically.

\section{Mechanism:}

$$
\begin{gathered}
\mathrm{Br}_{2} \stackrel{h \nu}{\rightleftharpoons} 2 \mathrm{Br} \\
\mathrm{C}_{2} \mathrm{H}_{4}+\mathrm{Br} \stackrel{\frac{2}{3}}{\rightleftharpoons} \mathrm{C}_{2} \mathrm{H}_{4} \mathrm{Br} \\
\mathrm{C}_{2} \mathrm{H}_{4} \mathrm{Br}+\mathrm{Br}_{2} \stackrel{4}{\stackrel{4}{\longrightarrow}} \mathrm{C}_{2} \mathrm{H}_{4} \mathrm{Br}_{2}+\mathrm{Br} \\
2 \mathrm{Br}+\mathrm{M} \stackrel{5}{\longrightarrow} \mathrm{Br}_{2}+\mathrm{M}
\end{gathered}
$$

[2] Gas phase addition of $\mathrm{HBr}$ to ethylene induced by $\mathrm{Co}^{60} \gamma$-rays. Relative rates of the decomposition and trapping (Continued) 
2-Bromo-1-ethyl radical (Continued)

reaction were obtained.

$$
\begin{gathered}
\cdot \mathrm{C}_{2} \mathrm{H}_{4} \mathrm{Br} \stackrel{1}{\longrightarrow} \mathrm{C}_{2} \mathrm{H}_{4}+\mathrm{Br} \\
\cdot \mathrm{C}_{2} \mathrm{H}_{4} \mathrm{Br}+\mathrm{HBr} \stackrel{2}{\longrightarrow} \mathrm{C}_{2} \mathrm{H}_{5} \mathrm{Br}+\mathrm{Br}
\end{gathered}
$$

[3] Photolysis of ethylbromide. Products were $\mathrm{C}_{2} \mathrm{H}_{4}$ and $\mathrm{HBr}$. Reaction rate was followed by $\Delta \mathrm{P}$ and checked by $\mathrm{HBr}$ titration.

Mechanism proposed:

$$
\begin{gathered}
\mathrm{C}_{2} \mathrm{H}_{5} \mathrm{Br}+h \nu \stackrel{1}{\mathrm{~B}} \mathrm{C}_{2} \mathrm{H}_{5}+\mathrm{Br} \\
\mathrm{Br}+\mathrm{C}_{2} \mathrm{H}_{5} \mathrm{Br} \frac{2}{\longrightarrow} \mathrm{HBr}+\cdot \mathrm{C}_{2} \mathrm{H}_{4} \mathrm{Br} \\
\cdot \mathrm{C}_{2} \mathrm{H}_{4} \mathrm{Br} \stackrel{3}{\longrightarrow} \mathrm{C}_{2} \mathrm{H}_{4}+\mathrm{Br} \\
\cdot \mathrm{C}_{2} \mathrm{H}_{4} \mathrm{Br}+\mathrm{Br} \stackrel{4}{\longrightarrow} \text { chain ending } \\
\mathrm{C}_{2} \mathrm{H}_{5}+\mathrm{Br} \stackrel{5}{\longrightarrow} \text { chain ending } \\
2 \mathrm{Br}+\mathrm{M} \stackrel{6}{\longrightarrow} \mathrm{Br}_{2}+\mathrm{M} \\
\mathrm{Br}+\mathrm{W} \stackrel{7}{\longrightarrow} 1 / 2 \mathrm{Br}_{2}+\mathrm{W}
\end{gathered}
$$

From the mechanism at high pressures, rate $=\left(\frac{k_{1} k_{i} k_{3}}{2 k_{4}}\right)^{1 / 2}\left(\mathrm{C}_{2} \mathrm{H}_{5} \mathrm{Br}_{0} \mathrm{I}_{0}^{1 / 2}\right.$, from which $E=1 / 2\left(E_{1}+E_{2}+E_{3}-E_{4}\right)=10.5$; The reported activation energy follows from $E_{2}=13.6 \mathrm{kcal} / \mathrm{mole}$ (Anderson and Van Artsdalen, J. Chem. Phys. 12 , 479 (1944)).

[4] Reanalysis of data of reference [2] on basis of "the wrong radical" mechanism.

\section{Mechanism:}

$$
\begin{gathered}
\mathrm{C}_{2} \mathrm{H}_{5} \mathrm{Br}+h \nu \stackrel{1}{\longrightarrow} \mathrm{C}_{2} \mathrm{H}_{5} \cdot+\mathrm{Br} \\
\mathrm{Br}+\mathrm{C}_{2} \mathrm{H}_{5} \mathrm{Br} \stackrel{2}{\stackrel{2}{\rightleftharpoons}} \cdot \mathrm{CH}_{2} \mathrm{CH}_{2} \mathrm{Br}+\mathrm{HBr} \\
\mathrm{M}+\mathrm{C}_{2} \mathrm{H}_{4} \mathrm{Br} \stackrel{3}{\stackrel{4}{\longrightarrow}} \mathrm{C}_{2} \mathrm{H}_{4}+\mathrm{Br}+\mathrm{M} \\
\mathrm{Br}+\mathrm{C}_{2} \mathrm{H}_{5} \mathrm{Br} \stackrel{4}{\stackrel{4}{\rightleftharpoons}} \mathrm{HBr}+\mathrm{CH}_{3} \cdot \dot{\mathrm{C}} \mathrm{HBr} \\
\mathrm{Br}+\mathrm{CH}_{3} \mathrm{CHBr} \stackrel{t}{\longrightarrow} \mathrm{CH}_{3} \mathrm{CHBr}_{2}
\end{gathered}
$$

The decomposition is pressure dependent (low-pressure region). 
Reaction: $p$-(Bromomethyl) benzyl radical

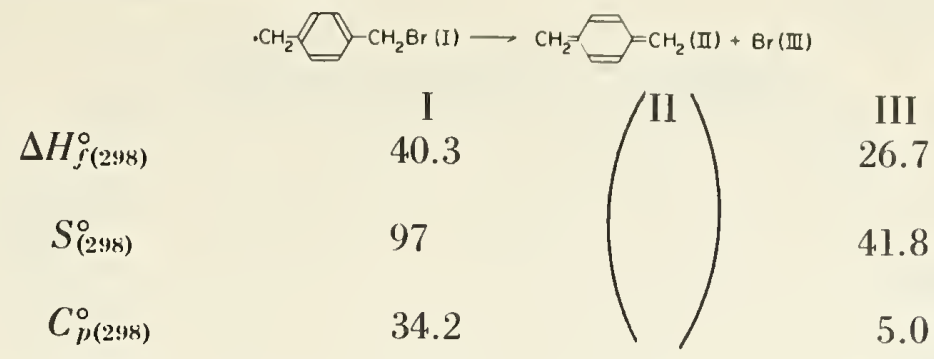

$\Delta S^{\circ}=$

$\Delta H^{\circ}=$

$\Delta C_{p}^{\circ}=$

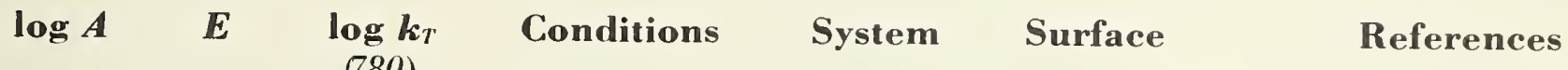

13
$1.79 \quad 748-814^{\circ} \mathrm{K} \quad$ flow $\delta P \simeq 0.03-0.06$
torr

[1] M. Levy, M. Szwarc, and J. Throssell, J. Chem. Phys. 22, 1904 (1954).

Preferred:

$\log k=13-40 / \theta$ (however, see comments).

Comments: Parameters are not unreasonable, but $E_{\text {act }}$ could well be about 10 kcal too low.

\section{Experimental}

[1] Toluene carrier technique in the pyrolysis of $\mathrm{w}, \mathrm{w}^{\prime}$-dibromo para-xylene.

Mechanism:

$$
\begin{aligned}
& \mathrm{BrCH}_{2} \emptyset \mathrm{CH}_{2}-\mathrm{Br} \stackrel{2}{\longrightarrow} \mathrm{Br}+\cdot \mathrm{CH}_{2} \varnothing \mathrm{CH}_{2} \mathrm{Br}(\mathrm{R} \cdot) \\
& \mathrm{R} \cdot+\emptyset \mathrm{CH}_{3} \stackrel{3}{\longrightarrow} \emptyset \mathrm{CH}_{2} \cdot+\mathrm{CH}_{3} \emptyset \mathrm{CH}_{2} \mathrm{Br}(\mathrm{P}) \\
& \mathrm{P} \stackrel{1}{\longrightarrow} \mathrm{CH}_{3} \varnothing \mathrm{CH}_{2} \cdot+\mathrm{Br} \\
& \mathrm{R} \cdot \stackrel{4}{\longrightarrow} \mathrm{Br}+\mathrm{CH}_{2}=\Longrightarrow=\mathrm{CH}_{2}
\end{aligned}
$$

termination by benzyl and paramethylbenzyl radicals.

Rates were based on the rate of $\mathrm{HBr}$ production. By equating the rates of reactions (2) and (1), it was possible to deduce the rate of reaction (4) relative to (3) from the steady-state relations. Parameters reported are for an assumed $A_{4}$ and an assigned $E_{3}=10 \mathrm{kcal} / \mathrm{mole}$. 
Reaction: Dimethylgallium radical

$$
\cdot \mathrm{Ga}\left(\mathrm{CH}_{3}\right)_{2}(\mathrm{I}) \longrightarrow \cdot \mathrm{Ga}\left(\mathrm{CH}_{3}\right)(\mathrm{II})+\mathrm{CH}_{3} \cdot(\mathrm{III})
$$

I II III

$\Delta H_{f(298)}^{\circ}$

$S_{(298)}^{\circ}$

$C_{p(298)}^{\circ}$

$\Delta S^{\circ}=$
$\Delta H^{\circ}=$
$\Delta C_{p}^{\circ}=$

$\begin{array}{llllll}\log A & \boldsymbol{E} & \log k_{T} & \text { Conditions } & \text { System } & \text { Surface }\end{array}$ (835)

$7.94 \quad 35.4 \quad-.1 .33 \quad\left(\right.$ see $\left.\mathrm{Ga}\left(\mathrm{CH}_{3}\right)_{3}\right) \quad$ flow $686-983{ }^{\circ} \mathrm{K}$

$P_{T} \simeq 13$ torr

[1] M. G. Jacko and S. W. Price, Can. J. Chem. 4.1, 1560 (1963).

Preferred:

Suspect.

Comments: In order for such a low $A$-factor to be valid, this decomposition must not only be in the falloff region, but it also must be non-adiabatic (i.e., require electronic state crossing).

\section{Experimental}

[1] See $\mathrm{Ga}\left(\mathrm{CH}_{3}\right)_{3}$ for experimental details. From the methyl balance relative to decomposed $\mathrm{Ga}\left(\mathrm{CH}_{3}\right)_{3}$, it was proposed that at low temperatures only the first methyl group is eliminated. At much higher temperatures (above $830{ }^{\circ} \mathrm{K}$ ). the above reaction was postulated. Treatment of the data using the theory of successive reactions gave the parameters reported. The reaction was found to be pressure-dependent and extremely sensitive to the nature of the surface. 
Reaction: Methylindium diradical

$: \mathrm{In}\left(\mathrm{CH}_{3}\right)(\mathrm{I}) \longrightarrow \mathrm{In}(\mathrm{II})+\mathrm{CH}_{3}(\mathrm{III})$

I II

$\Delta H_{f(298)}^{\circ}$

$S_{(298)}^{\circ}$

$C_{p(298)}^{\circ}$

$$
\begin{aligned}
& \Delta S^{\circ}= \\
& \Delta H^{\circ}= \\
& \Delta C_{p}^{\circ}=
\end{aligned}
$$

\begin{tabular}{|c|c|c|c|c|c|c|}
\hline $\log A$ & $\boldsymbol{E}$ & $\begin{array}{c}\log \boldsymbol{k}_{T} \\
(665)\end{array}$ & Conditions & System & Surface & References \\
\hline 10.91 & 38.7 & -1.81 & $\begin{array}{l}\left(\text { see } \operatorname{In}\left(\mathrm{CH}_{3}\right)_{3}\right) \\
550-781^{\circ} \mathrm{K} \\
P_{T} \simeq 13 \text { torr }\end{array}$ & flow & & $\begin{array}{l}\text { [1] M. G. Jacko and S. J. W. } \\
\text { Price, Can. J. Chem. } \\
\text { 42, } 1198 \text { (1963). }\end{array}$ \\
\hline
\end{tabular}

\section{Preferred:}

Reasonable.

Comments: The $A$-factor is in the region anticipated for a pressure-dependent unimolecular decomposition.

\section{Experimental}

[1] See $\mathrm{In}\left(\mathrm{CH}_{3}\right)_{3}$ for experimental details. At temperatures below $670^{\circ} \mathrm{K}$, two methyls were released. At higher temperatures, the above decomposition became important. Method of successive reactions was used to determine the Arrhenius parameters. From $(S / V)$ effects, the reaction was judged 97 percent homogeneous. The rate constant was also strongly pressure-dependent, indicating a unimolecular reaction well into its second-order region. 
Reaction: Methylzinc radical

$$
\mathrm{CH}_{3} \mathrm{Zn} \cdot(\mathrm{I}) \longrightarrow \mathrm{CH}_{3} \cdot(\mathrm{II})+\mathrm{Zn}(\mathrm{III})
$$

I II

III

$\Delta H_{f(298)}^{\circ}$

$S_{(298)}^{\circ}$

$C_{p(298)}^{\circ}$

$\Delta S^{\circ}=$

$\Delta H^{\circ}=$

$\Delta C_{p}^{\circ}=$

$\begin{array}{llllll}\log A & E & \log k_{T} & \text { Conditions } & \text { System } & \text { Surface }\end{array}$ (920)

flow

$\begin{array}{rll}6.8-35.0-1.52 & \left(\text { see } \mathrm{Zn}\left(\mathrm{CH}_{3}\right)_{2}\right) \quad \text { flow } \\ & 843-1000^{\circ} \mathrm{K} \\ & P_{T}=16 \text { torr }\end{array}$

[1] S. J. W. Price and A. F. Trotman-Dickenson, Trans. Faraday Soc. 53, 1208 (1957).

Preferred:

Pressure-dependent; not reliable.

See comments on the $\left(\mathrm{CH}_{3}\right)_{2} \mathrm{Ga} \cdot$ decomposition.

\section{Experimental}

[1] See $\left(\mathrm{CH}_{3}\right)_{2} \mathrm{Zn}$ for details. Above reaction was observed at $T>1000{ }^{\circ} \mathrm{K}$. The method of successive reactions was applied to the data in order to determine the above parameters. The rate was directly proportional to pressure, and therefore was in its second-order region. 


\section{Index to Reactants}

Acetaldoxime.......................................... 478

Acetamide........................................... 118

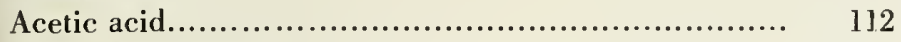

Acetic anhydride........................................ 216

Acetone.............................................. 416

Acetonyl radical..................................... 593

Acetophenone.......................................... 417

endo-2-Acetoxybornane................................. 180

exo-2-Acetoxybornane................................. 181

Acetyl radical.......................................... 589

Allylbromide......................................... $\quad 509$

Allylcarbinol...................................... $\quad 135$

Allyldimethylcarbinol.................................... 137

Allylmethylcarbinol................................... 136

Allyl methyl sulfone.................................... 491

$t$-Amyl acetate...................................... $\quad 190$

$t$-Amyl alcohol...................................... $\quad 110$

t-Amyl bromide.................................... 102

t-Amyl clıloride........................................ 73

1-Arylethyl ethanoates (substituted).................... 185

2-Arylethyl ethanoates (substituted)...................... 168

Arylethyl methyl carbonates ............................. $\quad 215$

Azidoethane.......................................... 483

Azidomethane........................................ 482

Azobutane.......................................... 452

$2,2^{\prime}$-Azobutane ....................................... 455

Azoethane................................................. 451

$1, l^{\prime}$-Azoisobutane..................................... 453

$2,2^{\prime}$-Azoisobutane ...................................... 457

Azoisopropane .......................................... 454

Azomethane............................................ 448

Azotoluene.....

B

Benzil.

Benzophenone.

Benzoyl bromide

Benzoyl chloride .......................................

Benzyl acetate.

Benzylamine...

Benzylbenzoate.

Benzyl bromide

Benzyl bromides, substituted............................ $\quad 512$

Benzyl chloride.

Benzylethanoate.

Benzyl mercaptan.

Benzyl methyl ketone...

Benzyl methyl sulfone.

Biacetyl.

Bicyclo[1,1,0]butane.

Bicyclo[2,2,1]-hepta-2,5-diene.

Bicyclo[3,2,0]heptane.

Bicyclo $[2,2,1]$-hept-2-ene $\ldots$

Bicyclo[3,2,0]-hept-6.ene

endo-Bicyclo[2,2,1]-hept-5-ene-2-carboxaldehyde..........

Bicyclo[2,1,1]hexane.

Bicyclo[2,2,0]hexane

Bicyclo[3,1,0]hexane.

Bicyclo[3,1,0]hex-2-ene.

Bicyclo[4,2,0]-oct-7-ene.

Bicyclo[5,1,0]-oct-2-ene.

Bicyclo[1,1,1]pentane.

Bicyclo[2,1,0]pentane.
Bicyclo[2,1,0]pent-2-ene............................. 325

Bicyclopropyl........................................... 236

Borine carbonyl....................................... $\quad 550$

Bornyl acetate........................................ 180

Bornyl chloride........................................... $\quad 79$

9-Bromoanthracene................................... $\quad 507$

Bromobenzene.......................................... 503

Bromobenzenes, substituted............................ 504

1-Bromobutane....................................... 91

2-Bromobutane....................................... 96

Bromocyclohexane.................................. 99

Bromocyclopentane.................................... 98

Bromocyclopropane.................................... $\quad 259$

Bromoethane.......................................... $\quad 89$

2-Bromo-1-ethyl radical................................ 611

1-Bromohexane ........................................ 93

Bromomethane....................................... $\quad 495$

$p$-(Bromomethyl) benzyl radical.......................... 613

2-Bromo-2-methylbutane.............................. $\quad 102$

1-Bromo-2-methylpropane............................ 94

2-Bromo-2-methylpropane.............................. 101

$p$-Bromomethyltoluene.................................. $\quad 513$

$\alpha$ - and $\beta$-Bromonaphthalene........................... 506

1 - and 2-Bromonaphthalene.......................... 506

1.Bromopentane....................................... $\quad 92$

4-Bromo-1-pentene....................................... 97

9.Bromophenanthrene................................. $\quad 507$

1-Bromo-1-phenylethane ............................. $\quad 100$

Bromophenylmethane............................... 511

l-Bromopropane..................................... $\quad 90$

2-Bromopropane....................................... 95

3-Bromo-1-propene........................................ 509

2- and 3-Bromopyridene................................. 505

Bromo-substituted phenylmethanes...................... 512

2-Bromothiophene...................................... 508

$n$-Butane.......................................... $\quad 385$

2,3-Butanedione .......................................... 424

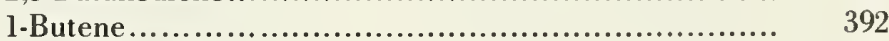

cis-2-Butene................................................ 351

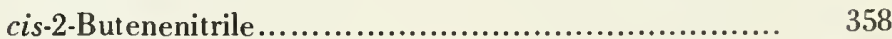

3-Butene-1-ol........................................ 135

3-Butenoic acid........................................... 141

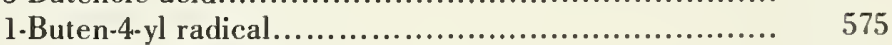

1-Buten-3-yl vinyl ether.................................. $\quad 366$

sec-Butoxy radical...................................... $\quad 596$

$t$-Butoxy radical........................................... 597

$n$-Butyl acetate..................................... 160

sec-Butyl acetate........................................ 170

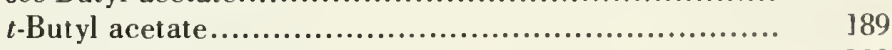

$t$-Butyl alcohol...................................... 109

$t$-Butyl amine........................................115, 116

$n$-Butylbenzene........................................... 399

$t$-But ylbenzene...................................... 402

$n$-Butyl bromide......................................... 91

sec-Butyl bromide........................................... 96

$t$-Butyl bromide.................................... 101

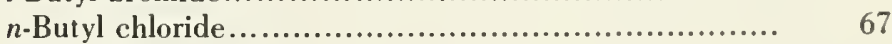

sec-Butyl chloride.......................................... 72

$t$-Butyl chloride........................................ 71

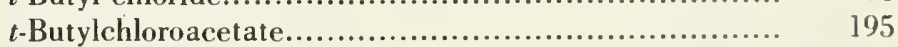

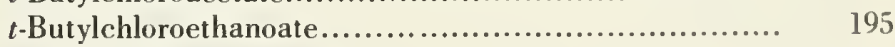

sec-Butyl chloroformate................................... 153

sec-Butyl chloromethanoate............................... 153

$t$-Butyl cyanide..................................... 462 


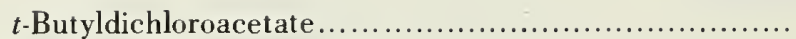

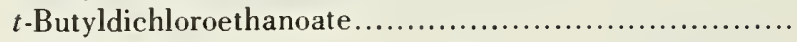

n-Butyl ethanoate.

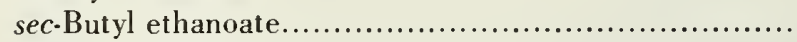

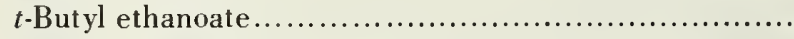

$t$-Butyl formate.

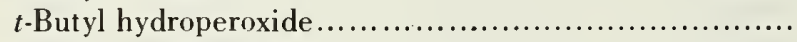

Butylidene diacetate..

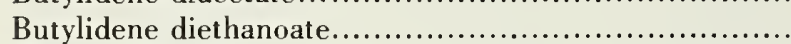

sec-Butyl iodide.

$t$-Butyl iodide...

$t$-Butyl mercaptan...

di- $n$-Butyl mercury.

$t$-Butyl methanoate.

$n$-Butyl nitrite.

di- $t$-Butyl peroxide.

$t$-Butyl propanoate.

$t$-Butyl propionate.

$n$-Butyl radical..

sec-Butyl radical..

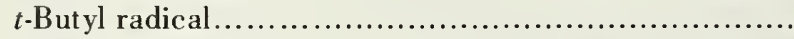

$n$-Butyl vinyl ether...

para-n-Butyraldehyde

\section{C}

Carbon tetrabromide

Chlorine nitrate .........................................

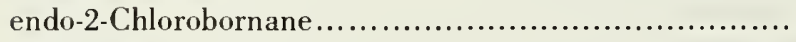

exo-2-Chlorobornane.

Chlorobromomethane

1. Chlorobutane

2-Chlorobutane

Chlorocyclohexane.

Chlorocyclopentane.....

Chlorocyclopropane

4. Chlorodifluoromethyl-2.5-cyclohexadienyl radica.......

Chloroethane...

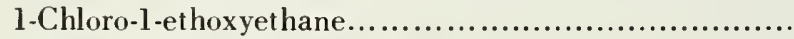

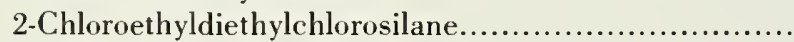

2-Chloroethylethyldichlorosilane..........................

$\alpha$-Chloroethyl ethyl ether.

$\alpha$-Chloroethyl methyl ether.

2-Chloro-1-ethyl radical...................................

2-Chloroethyltrichlorosilane.

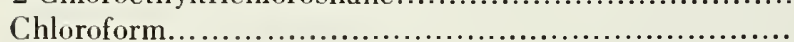

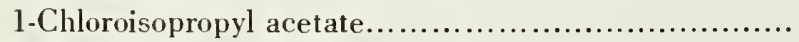

1-Chloro-1-methoxyethane................................

2-Chloro-2-methylbutane...

1-Chloro-2-methylpropane...............................

2-Chloro-2-methylpropane...............................

1-Chloropentane....

1-Chloro-1-phenylethane................................

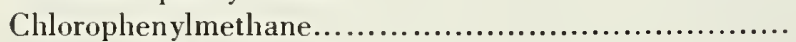

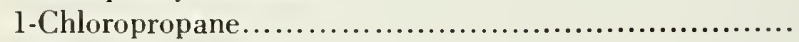

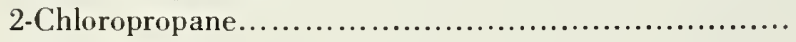

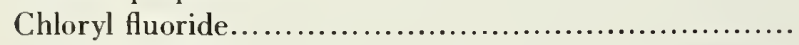

cis-Crotonitrile............................................

Crotonylidene diacetate...................................

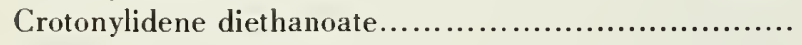

Cymene.......

p-Cumene.

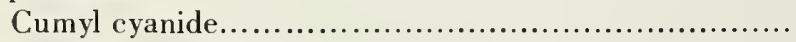

cis- $\beta$-Cyanost yrene.

Cyclobutane.

Cyclobutane carboxaldehyde...............................

Cyclobutanone..

497
558
Cyclobutene.

Cyclobutyl radical.

Cycloheptatriene.

1,4-Cyclohexadiene

Cyclohexene.

Cyclohexyl acetate.

Cyclohexyl bromide.

Cyclohexyl chloride

Cyclohexyl ethanoate.

1,5-Cyclooctadiene.

1,4-Cyclooctadiene

Cyclopentene.

Cyclopentyl bromide

Cyclopentyl chloride

Cyclopentyl radical....

Cyclopropane...

trans-1-Cyclopropyl-1-butene

1-Cyclopropyl-1-cyclopentene.

1-Cyclopropyl-2-methyl-1,1-propene

\section{D}

Decafluoroallylcyclopropane.

Decafluoro-1,2-dimethylcyclobutene.

Diacetyl peroxide.

(1)-2,2' -Diamino-6,6' -dimethyldiphenyl ...
1,2-Diarylethyl acetates..

1,2-Diarylethyl ethanoates................................

2,3-Diazobicyclo[2,2,1]-hept-2-ene.

2,3-Diazobicyclo[2,2,2]-oct-2-ene.

Diazomethane..

Dibenzyl.....

Dibenzil ketone.

Diborane....

1,1-Dibromoethane

Dibromomethane...

$\omega, \omega^{\prime}$-Dibromo-para-xylene.

Dibutanoyl peroxide.

Dibutyrl peroxide...

Dichlorine heptoxide.

Dichlorobromomethane.......

Dichlorodimethylstannane.

1,1-Dichloroethane...

trans-1,2-Dichlorethene.

cis-1,2-Dichloroethene...

1,2-Dichloro-1-ethyl radical.

cis-1,2-Dichlorohexafluorocyclobutane.

2,2-Dichloro-1-methyl-1-vinylcyclopropane.

1,1-Dichlorocyclopropane.

1,1-Dichloropropane.

1.2-Dichloropropane.

2.2-Dichloropropane...

endo-Dicyclopentadiene.

exo-Dicyclopentadiene.

1.1-Dicyclopropylethene

trans-1,2-Dideuterocyclopropane.

cis-1,2-Dideuteroethene.

cis, trans-1.2-Dideutero-3-methylcyclopropane

Diethanoyl peroxide.

Diethyl carbonate.

1,1-Diethylcyclopropane....

Diethyl ether.

3,3-Diethyldiazirine.

Diethyl mercury....

Diethyl peroxide. (cis or trans) 1.2-Dichlorohexafluorocyclobutane.......

Difluorine dioxide.
307,308 
1,1-Difluorocyclopropane......

Difluorodiazirine...

trans-Difluorodiazine

2,2-Difluoroethyltrifluorosilane....

2,5-Dihydrofuran.

Diisopropyl mercury

Dimeric-2-methyl-1-nitrosopropane.

Dimeric nitrosoisobutane.

Dimeric nitrosomethane.

2-Dimethylamino-1-methylethyl ethanoate................

$\beta$-Dimethylamino-2-propyl acetate......................

$\mathrm{N}, \mathrm{N}$-Dimethylaniline.

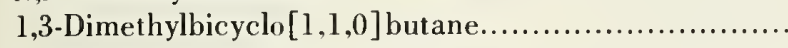

1,3-Dimethylbicyclo $[1,1,1]$ pentane.....................

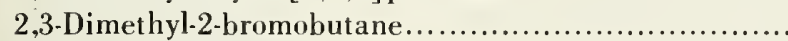

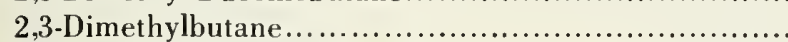

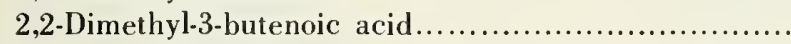

1,1-Dimethylbutyl acetate..

2,3-Dimethyl-2-butyl acetate.

$\alpha, \beta$-Dimethyl- $n$-butyl acetate...........................

1,1-Dimethylbutyl ethanoate.

1,2-Dimethylbutyl ethanoate.

Dimethylcadmium

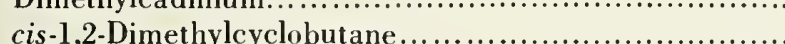

trans-1,2-Dimethylcyclobutane

1,2-Dimethyl-1-cyclobutene.

1,3-Dimethyl-1-cyclobutene.

2,3-Dimethyl-1-cyclobutene

1,1-Dimethylcyclopropane.....

cis-1,2-Dimethylcyclopropane

trans-1,2-Dimethylcyclopropane....

(2,2-Dimethylcyclopropyl)-1-ethanone ...................

$\beta, \beta$-Dimethylcyclopropyl methyl ketone

Dimethyldiazirine.

1,1-Dimethyl-1-ethanesulfonyl chloride...................

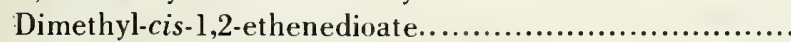

Dimethyl ether.

2,2-Dimethyl-3-ethyl-3-pentenoic acid

Dimethyl gallium radical.

1,1-Dimethylhydrazine.

3,3-Dimethylketane.

Dimethyl maleate.

Dimethyl mercury.......................................

3,3-Dimethyloxetane

3,7-Dimethyl-1,6-oct adiene.

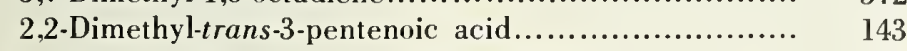

Dimethylperoxide.

2,2-Dimethylpropane.

2,2-Dimethylpropanonit rile.

3,3-Dimethyl pyrazoline.

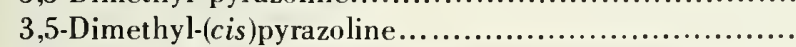

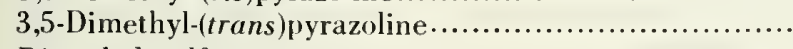

Dimethyl sulfone.

Dimethyl tin dichloride.

Dimethyl zinc.

Dinitrates....

1,2-Dinitratoethane

1,2-Dinitratopropane

1,3-Dinitratopropane.

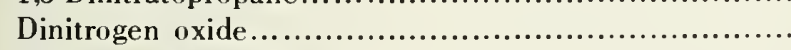

Dinitrogen pentoxide

Dinitrogen tetroxide.

1,4-Dioxane.

Diphenylacetic acid.

1,2-Diphenyl-1,2-ethanedione

1,2-Diphenylethane..

Diphenylethanoic acid

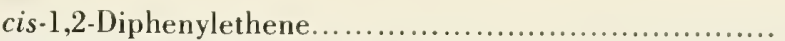

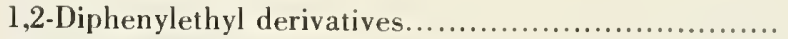

Diphenyl mercury.

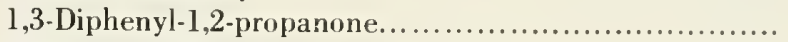

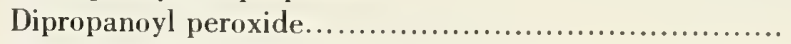

Dipropionyl peroxide..

Divinyl mercury.

\section{$\mathbf{E}$}

Epoxyethyl radical.................................... 591

Ethanoldoxime...................................... 478

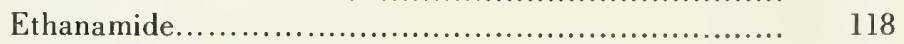

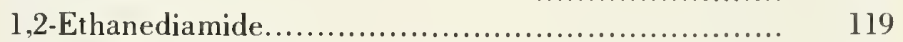

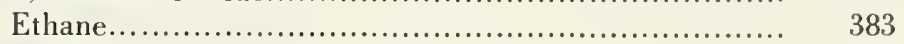

1,2-Ethanedioic acid................................... 113

Ethanethiol.............................................120, 485

Ethanoic acid............................................ 112

Ethanoic anhydride..................................... 216

$\beta$-ethanoxyethyl acetate............................... 167

Ethanoylcyclobutane.................................... $\quad 280$

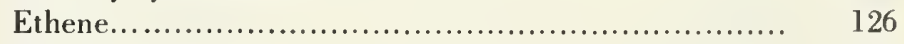

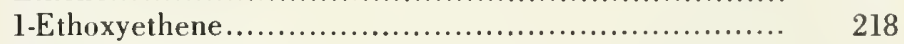

$\beta$-Ethoxyethyl acetate................................... 167

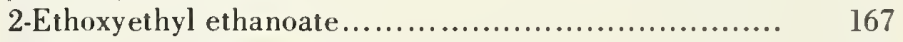

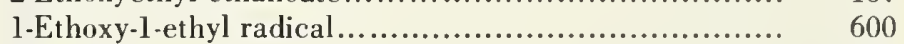

Ethoxy radical......................................... 592

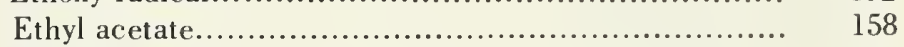

Ethyl azide........................................... 483

Ethylbenzene........................................... $\quad 397$

Ethyl bromide.......................................... $\quad 89$

$\beta$-Ethylbutyl acetate.................................. $\quad 165$

2-Ethylbutyl ethanoate................................. 165

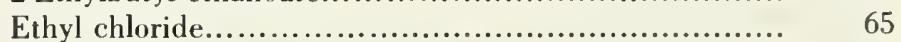

Ethyl chloroethanoate...................................... $\quad 149$

Ethyl chloroformate...................................... $\quad 149$

Ethyl cyanide............................................. 114, 461

Ethylcyclobutane......................................... 273

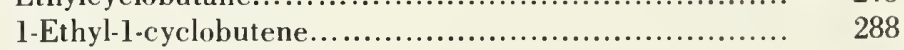

Ethyl cyclobutyl ketone................................... 281

Ethylcyclopropane..................................... 231

Ethyl-2,2-dimethylpropanoate............................... 199

Ethylene dichloride..................................... 81

Ethylene glycol dinitrate................................ 472

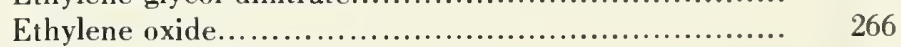

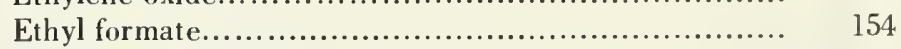

Ethyl hydroperoxide..................................... 436

Ethylidenecyclopropane.................................. 265

Ethylidene diacetate..................................... 202

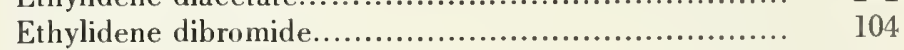

Ethylidene dibutanoate.................................... 211

Ethylidene dibutyrate................................... 211

Ethylidene diethanoate.................................... 202

Ethylidene dipropanoate................................ $\quad 209$

Ethylidene dipropionate................................... 209

Ethyl iodide............................................. 105, 517

Ethyl mercaptan........................................120, 485

Ethyl methanoate...................................... 154

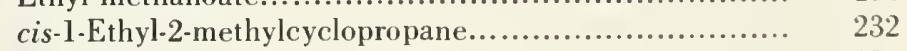

trans-1-Ethyl-2-methylcyclopropane......................... 232

1-Ethyl-1-methylpropyl acetate........................... 193

l-Ethyl-1-methylpropyl ethanoate....................... 193

Ethyl nitrate........................................ 471

Ethyl nitrite.......................................... 466

l-Ethylpentyl ethanoate................................... 178

Ethyl propanoate.......................................... 197

Ethyl propionate.................................... 197 
1-Ethylpropyl ethanoate......

Ethyl radical...

Ethyl trimethyl acetate.

Ethyl vinyl ether.

$\mathbf{F}$

Fluorine nitrate.

Fluorocyclopropane .....................................

Fluorotoluenes, $p, o, m$ substituted......................

Formaldoxime.

Formic acid....

Formyl fluoride.

Formyl radical.

Furfurylidene diacetate................................

Furfurylidene diethanoate.

G

Germane

1,5-Heptadiene

1,2,6-Heptatriene.

2-Heptyl acetate.

3-Heptyl acetate

4-Heptyl acetate.

Heptylidene diacetate

Heptylidene diethanoate

cis-1,3-Hexadiene ..........................................

$1,1^{\prime}, 6,6^{\prime}-d_{4}-1,5$-Hexadiene.

cis-1,3,5-Hexatriene...

Hexafluoroazomethane.

Hexafluorobutadiene...

Hexafluorocyclopropane.................................

cis-Hexafluoro-1,2-bis-trifluoromethylcyclobutane.........

trans-Hexafluoro-1,2-bis-trifluoromethylcyclobutane......

Hexamethyldisilane.

Hexamethyl ethane

$n$-Hexyl bromide.

2.Hexyl radical.

Hydrazine..

Hydroxymethyl radical....................................

4.Hydroxy-4-methyl-2-pentanone.

I

2-Iodobutane.

Iodoethane

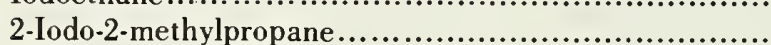

2-Iodopropane...

Isobornyl acetate.

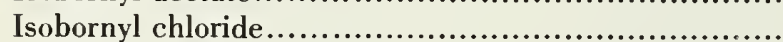

Isobutene

Isobutyl acetate.

Isobut yl bromide...

Isobutyl chloride....

Isobutyl chloroformate.

Isobutyl chloromethanoate.

Isobutyl ethanoate.

Isobutyl radical.

para-Isobutyraldehyde

Isopropenyl allyl ether.

Isopropenylcyclobutane.

Isopropenylcyclopropane...............................

1-Isopropenyl-1-methylcyclopropane.

3-Isopropenoxy-1-propene..

Isopropoxyethene.

Isopropoxy radical.
Isopropyl acetate.

Isopropylbenzene.....

Isopropyl bromide.

Isopropyl chloride.

Isopropyl chloroformate.

Isopropyl chloromethanoate.

Isopropylcyclobutane.

Isopropyl ethanoate.

Isopropyl ether.

Isopropyl formate.

Isopropyl hydroperoxide.

Isopropyl iodide...

Isopropyl methanoate.

$\alpha$-Isopropyl $\beta$-methyl- $n$-propyl acetate

1-Isopropyl-2-methylpropyl ethanoate.

Isopropyl nitrite..

$p$-Isopropyltoluene.

Isostilbene...

(-)Menthyl benzoate.

(-)Menthyl chloride..........

2-Methallyl vinyl ether.

Methane.

Methanesulfonylmethane..................................

Methanesulf onylphenylmethane.

3-Methanesulfonyl-1-propene.

Methanethiol..

Methanoic acid.

Methanoloxime....

2-Methoxyethyl ethanoate.................................

$\beta$-Methoxyethyl acetate.

1-Methoxy-2-propyl acetate............................

2-Methyl-2-aminopropane.

$\mathrm{N}$-Methylaniline.

Methyl azide.

$\mathrm{N}$-Methylbenzylamine.

Methyl benzyl sulfide.

cis-2-Methyl[2,1,0]bicyclopent ane.

trans-2-Methyl[210]bicyclopentane

Methyl bromide.

2-Methyl-2-butanol..

2-Methyl-1-butene...

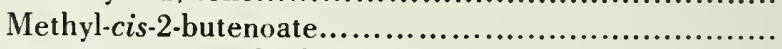

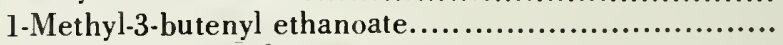

2-Methylbutyl acetate.

$\gamma$-Methylbutyl acetate...................................

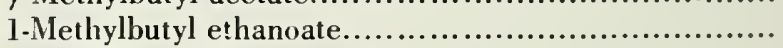

2-Methylbutyl ethanoate..................................

3-Methylbutyl ethanoate..................................

1-Methyl-2-chloroethyl ethanoate..........................

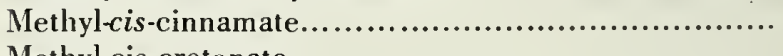

Methyl-cis-crotonate.

Methylcyclobutane.

Methyl cyclobutane carboxylate.

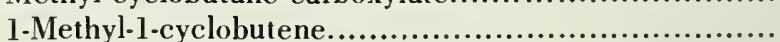

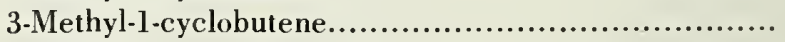

Methyl cyclobutyl ketone.

2-Methyl-2-(1-cycloheptenyl) propionic acid..................

1-Methyl-1,4-cyclohexadiene.

4-Methyl cyclohexene.

2-Methyl-2-(1-cyclohexenyl) propionic acid.................

1-Methylcyclohexyl acetate....

1-Methylcyclohexyl ethanoate..

2-Methyl-2-(1-cyclopent yl) propionic acid..................

Methylcyclopropane.

1-Methyl-2-dimethylaminoethyl ethanoate. 
Methylene bromide..................................... $\quad 496$

Methylenecyclobutane................................... 284

Methylene diacetate..................................... 201

Methylene dibutanoate................................ $\quad 210$

Methylene dibutyrate.................................. $\quad 210$

Methylene diethanoate.................................. 201

Methylene dipropanoate................................ 208

Methylene dipropionate............................... 208

endo-Methylene-2,5-tet rahydrobenzaladehyde............ 345

Me. iyl ethanoate...................................... 158

Methyl ethyl carbonaies.............................. 213

3-Meihyl-1,5-hexadiene................................. $\quad 362$

1-Methylhexyl ethanoate............................... 177

Methyl hydrazine........................................ 441

Methyl hydroperoxide.................................... 435

Methyl indium diradical................................... 615

Methyl isocyanide...................................... 373

Methyl isopropyl diimide............................... $\quad 456$

Methylmercaptan........................................ 484

2-Methyl-1-methylene cyclopropane....................... 265

1-Methyl-2-methoxyethyl ethanoate..................... $\quad 182$

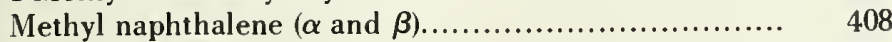

Methyl nitrate........................................ $\quad 470$

Methyl nitrite......................................... 464

1-Methyl-3-oxobutyl ethanoate (acetate)................ 183

2-Methyl-cis-1,3-pentadiene............................. $\quad 370$

4-Methyl-1,3-pentadiene................................. 371

2-Methyl-4-pentene-2-ol............................... 137

1-Methyl-1-pentyl radical................................ 586

Methyl peroxymethyl radical............................ 603

2-Methyl-2-phenylpropanonit rile........................ 463

2-Methyl-2-propanethiol................................. 121

2-Methyl-2-propanol................................... 109

2.Methyl propene...................................... 393

2-Methylpropyl acetate................................. 161

1-Methyl-1-propyl radical............................... 578

3-Methyl pyrazoline..................................... 306

4.Methyl pyrazoline................................... 306

Methyl-substituted pyrazolenes............................. 306

1-Methyl-1-vinylcyclopropane............................ 240

cis-1-Methyl-2-vinylcyclopropane........................ 238

trans-1-Methyl-2-vinylcyclopropane....................... 239

1-Methyl-1-vinyl-2,2-dichlorocyclopropane............... 258

2-Methyl-3-vinyloxy-1-propene........................... 365

Methyl zinc radical.

616

\section{$\mathbf{N}$}

$\alpha$ - and $\beta$-Metanaphthalene.

1,2-Metanaphthalene.

Neomenthyl chloride.

Neopentane

Neopentyl radical.

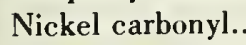

Nitratoethane...

Nitratomethane.

l-Nitritobutane.

Nitritoethane....

Nitritomethane.

1-Nitritopropane.

2-Nitritopropane.

Nitroethane.

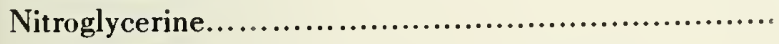

Nitromethane.

1-Nitropropane.

2-Nitropropane.

Nitrosomethane.

Nitrotrichloromethane.
Nitrous oxide...................................... 553

Nitroxyl chloride....................................... 558

Nitroxyl fluoride.................................... $\quad 557$

Nitryl chloride.......................................... $\quad 556$

endo-5-Norbornene-2-carboxaldehyde................. $\quad 345$

\section{O}

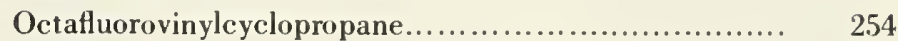

Oxetane............................................... 277

Oxirane................................................ 266

Oxalic acid............................................... 113

Oxamide................................................ 119

2-Oxo-1-propyl radical..................................... 593

4-Oxo-2-heptyl radical.................................. $\quad 602$

\section{$\mathbf{P}$}

Paraldehyde.

Pentachloroethyl radical................................ $\quad 609$

3,3-Pentamethylenediazirine.............................. 263

4-Pentene-2-ol........................................... 136

$n$-Pentyl acetate..................................... 162

2-Pentyl acetate....................................... 172

3-Pentyl acetate........................................... 173

$n$-Pentyl bromide......................................... $\quad 92$

$n$-Pentyl chloride..................................... $\quad 68$

Pentyl ethanoate....................................... 162

$t$-Pentyl ethanoate.................................... 190

Peracetic acid.......................................... $\quad 413$

Perchloric acid......................................... 564

Perchloryl fluoride......................................... $\quad 562$

Perfluoroallylcyclopropane................................... $\quad 255$

Perfluoroazomethane...................................... $\quad 450$

Perfluorobutadiene........................................ $\quad 352$

cis-Perfluoro-2-butene..................................... $\quad 353$

trans-Perfluoro-2-butene................................ 353

Perfluoro-t-butoxy radical.................................. 604

Perfluorocyclobutane..................................... 295

Perfluorocyclobutene...................................... 294

Perfluorocyclopropane................................... 251

Perfluoroethane........................................ 492

Perfluoroisobutene........................................ 494

Perfluoro-2-methyl-1-propene............................... 494

Perfluorooxirane ........................................ 268

Perfluoropropene.......................................... 493

Perfluorovinylcyclopropane.............................. 254

Phenylacetic acid........................................ 411

Phenylaminomethane.................................... 443

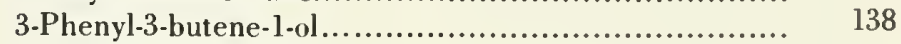

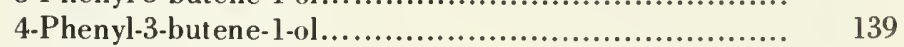

Phenylethanoic acid........................................ 411

$\alpha$-Phenylethyl acetate................................... 184

$2 \cdot$ Phenylethyl acetates (substituted)......................... 168

$\alpha$-Phenylethyl bromide....................................... 100

$\alpha$-Phenylethyl chloride.................................... $\quad 76$

1-Phenylethyl derivatives.................................. 185

1-Phenylethyl ethanoate.............................. 184

1.Phenyl-4-ethyl-4-hydroxyhex-1-ene..................... 140

Phenylethyl methyl carbonates......................... 215

Phenylhydrazine........................................ 445

Phenylmercuric bromide.................................. $\quad 527$

Phenylmercuric chloride................................ $\quad 526$

Phenylmercuric iodide.................................. 528

Phenylmethanethiol...................................... 486

Phenylmethyl sulfide .................................... 487

1-Phenyl-2-propanone................................... 418

Picolines................................................ 410

$\beta$-Pinene.......................................... 341 
$\begin{array}{ll} & \text { Page } \\ \text { Propanone } \ldots \ldots \ldots \ldots \ldots \ldots \ldots \ldots \ldots \ldots \ldots \ldots \ldots \ldots \ldots \ldots \ldots \ldots \ldots \ldots \ldots \ldots \ldots & 416\end{array}$

Propanonitrile......................................114, 461

Propene................................................ 391

Propionylcyclobutane.................................. 281

$n$-Propyl acetate...................................... $\quad 159$

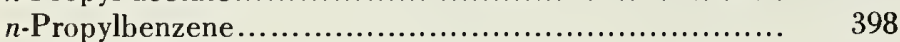

$n$-Propyl bromide.................................... 9

1-Propylbutyl ethanoate................................. 179

$n$-Propyl chloride..................................... $\quad 66$

$n$-Propylcyclobutane................................ 274

Propylene............................................. 391

Propylene glycol dinitrate.............................. 472

$n$-Propyl ethanoate.................................. 159

$n$-Propyl formate..................................... 155

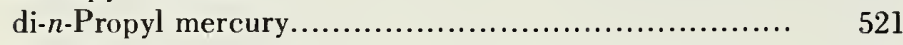

Propyl methanoate................................... $\quad 155$

$n$-Propylnitrite ...................................... 467

di- $n$-Propyl peroxide ................................. 429

$n$-Propyl radical........................................ 569

Pyrazoline..........................................305, 306

\section{Q}

Quadricyclo[2,2,1,02,6, $\left.0^{3,5}\right]$ heptane.

\section{S}

Silane

Silicon tetraethyl....

Silicon tetramethyl.

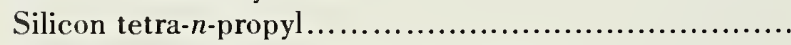

Spiropentane.

Substituted arylethyl methyl carbonates.....................

Substituted benzyl bromides..............................

Substituted bromobenzenes..............................

Substituted phenylethyl acetates.........................

Substituted pyrazolines...

\section{$\mathbf{T}$}

Tetrabromomethane

1,1,2,2-Tetrachloro-1-ethyl radical.

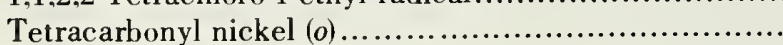

Tetraethyl germane.....................................

Tetraethyl lead..

Tetraethylsilane.

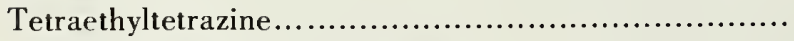

1,1,2,2-Tetrafluorocyclopropane.

Tetrafluoroethylene oxide.

Tetrafluorooxirane....

2.2.3.3-Tetramethylbutane.

cis-1.2.3.4-Tetramethyl-1-cyclobutene .....................

trans-1.2.3,4-Tetramethyl-1-cyclobutene...................

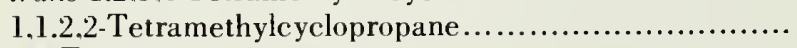

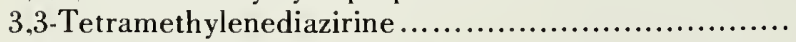

3.3.5.5-Tetramethyl pyrazoline ...........................

Tetramethylsilane.

Tetramethyltetrazine......................................

Tetramethyl tin .........................................

Tetranitromethane .......................................

Tetra-n-propylsilane.
497

608

540

539

541

544

460

250

268

268

390

293

292

235

262

306

543

459

547

477

545

Thioanisole

Toluene.

$p$-Tolyl isocyanide

Tribromomethane........................................

Trichlorobromomethane.

1,1,1-Trichloroethane.

1,2,2-Trichloro-1-ethyl radical.

2,2,2-Trichloroethylidene diacetate

2,2,2-Trichloro-1-ethylidene dibutanoate.

2,2,2-Trichloro-1-ethylidene dibutyrate.

2,2,2-Trichloroethylidene diethanoate.

Trichloromethane.

Trichloromethyl chloroformate.

Trichloromethyl chloromethanoate

Tricyclo $\left[3,3,0,0^{2,6}\right]$ octane.

Trifluoroacetone..

$\alpha, \alpha, \alpha$-Trifluoroacetophenone.

Trifluorobromomethane.

1,1,2-Trifluorocyclopropane.

2,2,2-Trifluoro-1-ethylcyclopropane.

tris(Trifluoromethyl) arsine.

Trifluoromethylcyclopropane.

Trifluorophosphine borane.

1,1,1-Trifluoro-2-propanone.

2,4,6-Tri-isopropyl-1,3,5-trioxane.

Trimethyl antimony.

Trimethyl arsine...

Trimethyl bismuth.

2,2,3-Trimethylbutane.

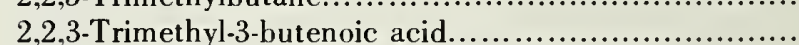

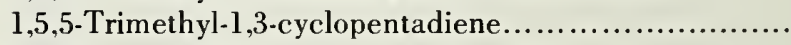

2,5,5-Trimethyl-1,3-cyclopentadiene.

cis,trans-1,2,3,-Trimethylcyclopropane.

Trimethylene glycol dinitrate.

Trimethylene oxide.

Trimethyl gallium

Trimethyl indium

Trimethylmethanesulfonyl chloride

Trimethylol-nitromethane trinitrite

1,1,1-T-rimethylpropyl ethanoate.

3,3,5-Trimethyl pyrazoline..

Trimethyl thallium........................................

2,4,6-Trimethyl-1,3,5-trioxane.

1,3,5-Trioxane.

2,4,6-Tri-n-propyl-1,3,5-trioxane..

$\mathbf{v}$

Vinyl allyl ether...

4-Vinylcyclohexene.

Vinylcyclopropane....

Vinyl isopropyl ether

3-Vinyloxy-1-propene.

363

311

237

219

363

$\mathbf{X}$

$m$-Xylene.

406

$o$-Xylene.

p-Xylene.

p-Xylyl bromide
405

407

513 


\section{Index by Formula}

$\mathrm{BH}_{3} \mathrm{PF}_{3}$

Trifluorophosphine borane.

$\mathrm{B}_{2} \mathrm{H}_{6}$

Diborane

$\mathrm{CBrCl}_{3}$

Trichloromethane................................. 500

$\mathrm{CBrF}_{3}$

Trifluorobromomethane

$\mathrm{CBr}_{4}$

Tetrabromomethane

$\mathrm{CCl}_{3} \mathrm{NO}_{2}$

Nitrotrichloromethane.................................. 476

$\mathrm{CF}_{2} \mathrm{~N}_{2}$

Difluorodiazirin

$\mathrm{CHBrCl}_{2}$

Dichlorobromomethane .................................. 499

$\mathrm{CHBr}_{3}$

Tribromomethane

$\mathrm{CHClF}_{2}$

Chlorodifluoromethane.................................... 129

$\mathrm{CHCl}_{3}$

Trichloromethane

CHFO

Formyl fluoride.

$\mathrm{CHO}$

Formyl radical

$\mathrm{CH}_{2} \mathrm{Br}_{2}$

Dibromomethane

$\mathrm{CH}_{2} \mathrm{BrCl}$

Chlorobromomethane ................................. 498

$\mathrm{CH}_{2} \mathrm{~N}_{2}$

Diazomethane

$\mathrm{CH}_{2} \mathrm{O}_{2}$

Methanoic acid

$\mathrm{CH}_{3} \mathrm{BO}$

Borine carbonyl

$\mathrm{CH}_{3} \mathrm{Br}$

Bromomethane...

$\mathrm{CH}_{3} \mathrm{In}$

Methylindium diradical

$\mathrm{CH}_{3} \mathrm{NO}$

Nitrosomethane

$\mathrm{CH}_{3} \mathrm{NO}$

Methanoloxime

$\mathrm{CH}_{3} \mathrm{NO}_{2}$

Nitritomethane

$\mathrm{CH}_{3} \mathrm{NO}_{3}$

Nitratomethane ........................................ 470

$\mathrm{CH}_{3} \mathrm{~N}_{3}$

Azidomethane

$\mathrm{CH}_{3} \mathrm{O}$

Hydroxymethyl radical................................. 588

$\mathrm{CH}_{3} \mathrm{Zn}$

Methylzinc radical

$\mathrm{CH}_{4}$

Methane...

$\mathrm{CH}_{4} \mathrm{O}_{2}$

Methyl hydroperoxide.................. 435

$\mathrm{CH}_{4} \mathrm{~S}$

Methanethiol

$\mathrm{CH}_{6} \mathrm{~N}_{2}$

Methylhydrazine

$\mathrm{CN}_{4} \mathrm{O}_{8}$

Tetranitromethane................................. 477

$\mathrm{C}_{2} \mathrm{D}_{2} \mathrm{H}_{2}$

cis-1.2.Dideuteroethene ............................. 349

$\mathrm{C}_{2} \mathrm{Cl}_{4} \mathrm{O}_{2}$

Trichloromethyl chloromethanoate.................. 88

$\mathrm{C}_{2} \mathrm{Cl}_{5}$

Pentachloroethyl radical............................... 609

$\mathrm{C}_{2} \mathrm{~F}_{4} \mathrm{O}$

Perfluorooxirane
$\mathrm{C}_{2} \mathrm{~F}_{6}$

Perfluoroethane ............................................. $\quad{ }_{492}$

$\mathrm{C}_{2} \mathrm{~F}_{6} \mathrm{~N}_{2}$

Hexafluoroazomethane..................................... 450

$\mathrm{C}_{2} \mathrm{HCl}_{4}$

1,1,2,2-Tetrachloro-1-ethyl radical....................... 608

$\mathrm{C}_{2} \mathrm{H}_{2} \mathrm{Cl}_{2}$

trans-1,2-Dichloroethene.......................... 350

$\mathrm{C}_{2} \mathrm{H}_{2} \mathrm{Cl}_{3}$

1,2,2-Trichloro-1-ethyl radical............................ 607

$\mathrm{C}_{2} \mathrm{H}_{2} \mathrm{O}_{4}$

1,2-Ethanedioic acid.

$\mathrm{C}_{2} \mathrm{H}_{3} \mathrm{Cl}_{2}$

1,2-Dichloro-1-ethyl radical............................... 606

$\mathrm{C}_{2} \mathrm{H}_{3} \mathrm{Cl}_{3}$

$1,1,1-$ Trichloroethane ................................ 85

$\mathrm{C}_{2} \mathrm{H}_{3} \mathrm{~F}_{5} \mathrm{Si}$

2,2-Difluoroethyltrifluorosilane ....................... 122

$\mathrm{C}_{2} \mathrm{H}_{3} \mathrm{O}$

Acetyl radical.

589

Epoxyethyl radical..................................... 591

$\mathrm{C}_{2} \mathrm{H}_{3} \mathrm{~N}$

Methyl isocyanide..................................... 373

$\mathrm{C}_{2} \mathrm{H}_{4}$

Ethene............................... 126

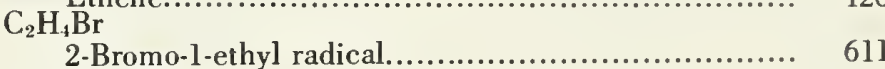

$\mathrm{C}_{2} \mathrm{H}_{4} \mathrm{Br}_{2}$

1,1-Dibromoethane ................................. 104

$\mathrm{C}_{2} \mathrm{H}_{4} \mathrm{Cl}$

2-Chloro-1-ethyl radical .............................. 605

$\mathrm{C}_{2} \mathrm{H}_{4} \mathrm{Cl}_{2}$

1,1-Dichloroethane.

$\mathrm{C}_{2} \mathrm{H}_{4} \mathrm{Cl}_{4} \mathrm{Si}$

2-Chloroethyltrichlorosilane........................... 123

$\mathrm{C}_{2} \mathrm{H}_{4} \mathrm{~N}_{2} \mathrm{O}_{2}$

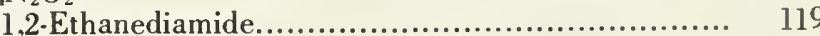

$\mathrm{C}_{2} \mathrm{H}_{4} \mathrm{O}$

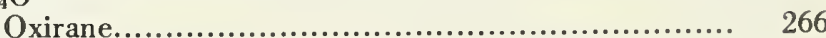

$\mathrm{C}_{2} \mathrm{H}_{4} \mathrm{O}_{2}$

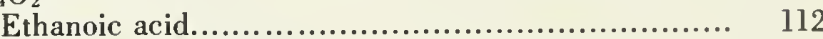

Methanoic acid dimer................................ 111

$\mathrm{C}_{2} \mathrm{H}_{4} \mathrm{O}_{3}$

Peracetic acid....................................... 413

$\mathrm{C}_{2} \mathrm{H}_{5}$

Ethyl radical....................................... 567

$\mathrm{C}_{2} \mathrm{H}_{5} \mathrm{Br}$

Bromoethane......................................... 89

$\mathrm{C}_{2} \mathrm{H}_{5} \mathrm{Cl}$

Chloroethane....................................... 65

$\mathrm{C}_{2} \mathrm{H}_{5} \mathrm{I}$

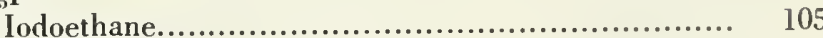

Iodoethane.......................................... 517

$\mathrm{C}_{2} \mathrm{H}_{5} \mathrm{NO}$

Ethanamide ........................................ 117

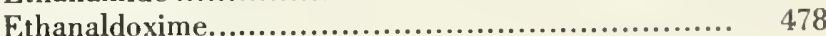

$\mathrm{C}_{2} \mathrm{H}_{5} \mathrm{NO}_{2}$

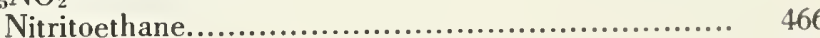

Nitroethane........................................... 130

Nitroethane.......................................... 475

$\mathrm{C}_{2} \mathrm{H}_{5} \mathrm{NO}_{3}$

Nitratoethane ....................................... 471

$\mathrm{C}_{2} \mathrm{H}_{5} \mathrm{~N}_{3}$

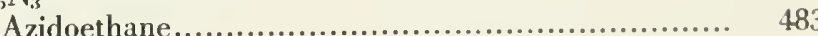

$\mathrm{C}_{2} \mathrm{H}_{5} \mathrm{O}$

Ethoxy radical ..................................... 592

$\mathrm{C}_{2} \mathrm{H}_{3} \mathrm{O}_{2}$

Methyl peroxymethyl radical........................ 603

$\mathrm{C}_{2} \mathrm{H}_{6}$

Ethane............................................. 383

$\mathrm{C}_{2} \mathrm{H}_{6 i} \mathrm{Cd}$

Dimethylcadmium 


\section{$\mathrm{C}_{2} \mathrm{H}_{6} \mathrm{Cl}_{2} \mathrm{Sn}$}

Dichlorodimethylstannane

$\mathrm{C}_{2} \mathrm{H}_{6} \mathrm{Hg}$

Dimethylmercury

$\mathrm{C}_{2} \mathrm{H}_{6} \mathrm{~N}_{2}$

Azomethane

$\mathrm{C}_{2} \mathrm{H}_{6} \mathrm{~N}_{2} \mathrm{O}_{2}$

Dimeric nitrosomethane.

$\mathrm{C}_{2} \mathrm{H}_{6} \mathrm{O}$

${ }_{6} \mathrm{O}$ imethyl ether

$\mathrm{C}_{2} \mathrm{H}_{6} \mathrm{O}_{2}$

Dimethylperoxide.

$\mathrm{C}_{2} \mathrm{H}_{6} \mathrm{~S}$

Ethyl hydroperoxide

Ethanethiol.

Ethanethiol.

$\mathrm{C}_{2} \mathrm{H}_{6} \mathrm{SO}_{2}$

$\mathrm{C}_{2} \mathrm{H}_{6} \mathrm{Zn}$

Methanesulfonylmethane............................ 490

Dimethylzinc.

$\mathrm{C}_{2} \mathrm{H}_{8} \mathrm{~N}_{2}$

1,1-Dimethylhydrazine

$\mathrm{C}_{3} \mathrm{AsF}_{\mathrm{g}}$

$\mathrm{C}_{3} \mathrm{~F}_{6}$

tris-(Trifluoromethyl) arsine.......................... 532

Hexafluorocyclopropane............................. 251

Perfluoropropene....................................... 493

$\mathrm{C}_{3} \mathrm{H}_{2} \mathrm{~F}_{4}$

$1,1,2,2$-Tetrafluorocyclopropane........................ 250

$\mathrm{C}_{3} \mathrm{H}_{3} \mathrm{~F}_{3}$

$1,1,2$-Trifluorocyclopropane............................. 249

$\mathrm{C}_{3} \mathrm{H}_{3} \mathrm{OF}_{3}$

$1,1,1$-Trifluoro-2-propanone............................ 422

$\mathrm{C}_{3} \mathrm{H}_{4} \mathrm{Br}$

3-Bromo-1-propene

$\mathrm{C}_{3} \mathrm{H}_{4} \mathrm{Cl}_{2}$

1,1-Dichlorocyclopropane............................. 257

$\mathrm{C}_{3} \mathrm{H}_{4} \mathrm{D}_{2}$

trans-1,2-dideuterocyclopropane....................... 224

$\mathrm{C}_{3} \mathrm{H}_{4} \mathrm{~F}_{2}$

1,1-Difluorocyclopropane.

$\mathrm{C}_{3} \mathrm{H}_{5} \mathrm{Br}$

Bromocyclopropane............................... 259

$\mathrm{C}_{3} \mathrm{H}_{5} \mathrm{Cl}$

Chlorocyclopropane.................................. 256

$\mathrm{C}_{3} \mathrm{H}_{5} \mathrm{ClO}_{2}$

Ethyl chloroethanoate................................. 149

$\mathrm{C}_{3} \mathrm{H}_{5} \mathrm{~F}$

Flurocyclopropane.

$\mathrm{C}_{4} \mathrm{H}_{5} \mathrm{~F}_{3}$

Trifturomethylcyclopropane......................... 252

$\mathrm{C}_{3} \mathrm{H}_{5} \mathrm{~N}$

Propanonitrile

Propanonitrile

$\mathrm{C}_{3} \mathrm{H}_{5} \mathrm{O}$

2-Oxo-1-propyl radical.

$\mathrm{C}_{3} \mathrm{H}_{6}$

Cyclopropane.

$\mathrm{C}_{3} \mathrm{H}_{6} \mathrm{Cl}_{2}$

1,1-Dichloropropane.

1,2-Dichloropropane.

2,2-Dichloropropane.

$\mathrm{C}_{3} \mathrm{H}_{6} \mathrm{D}$

2-Deuteroisopropyl radical............................. 572

$\mathrm{C}_{3} \mathrm{H}_{6} \mathrm{Ga}$

Dimethylgallium radical

$\mathrm{C}_{3} \mathrm{H}_{6} \mathrm{O}$

Oxetane.

(1) 277

$\mathrm{C}_{3} \mathrm{H}_{6} \mathrm{O}_{2}$

Ethylmethanoate
$\mathrm{C}_{3} \mathrm{H}_{6} \mathrm{O}_{3}$

1,3,5-Trioxane

$\mathrm{C}_{3} \mathrm{H}_{6} \mathrm{~N}_{2}$

Dimethyldiazirine

Pyrazoline.

$\mathrm{C}_{3} \mathrm{H}_{7}$

$n$-Propyl radical

$\mathrm{C}_{3} \mathrm{H}_{7} \mathrm{Br}$

1-Bromopropane...

$\mathrm{C}_{3} \mathrm{H}_{7} \mathrm{Cl}$

2-Bromopropane.

1.Chloropropane

2-Chloropropane

$\mathrm{C}_{3} \mathrm{H}_{7} \mathrm{ClO}$

1-Chloro-1-methoxyethane............................ 86

$\mathrm{C}_{3} \mathrm{H}_{7} \mathrm{I}$

2-Iodopropane................................... 106

$\mathrm{C}_{3} \mathrm{H}_{7} \mathrm{NO}_{2}$

1-Nitropropane.

1-Nitritopropane

2-Nitropropane.

2-Nitritopropane

$\mathrm{C}_{3} \mathrm{H}_{7} \mathrm{O}$

Isopropoxy radical.

$\mathrm{C}_{3} \mathrm{H}_{9} \mathrm{As}$

Trimethylarsine.

$\mathrm{C}_{3} \mathrm{H}_{9} \mathrm{Bi}$

Trimethylbismuth

$\mathrm{C}_{3} \mathrm{H}_{9} \mathrm{Ga}$

Trimethylgallium.

$\mathrm{C}_{3} \mathrm{H}_{9}$ In

Trimethylindium.

$\mathrm{C}_{3} \mathrm{H}_{9} \mathrm{Sb}$

Trimethylantimony

$\mathrm{C}_{3} \mathrm{H}_{9} \mathrm{Tl}$

Trimethylthallium

$\mathrm{C}_{4} \mathrm{Cl}_{2} \mathrm{~F}_{6}$

cis-1,2-Dichlorohexafluorocyclobutane..................

cis or trans-1,2-Dichlorohexafluorocyclobutane

$\mathrm{C}_{4} \mathrm{~F}_{6}$

Perfluorobutadiene...

$\mathrm{C}_{4} \mathrm{~F}_{8}$

Perfluorocyclobutene.

Perfluorocyclobutane

Perfluoro-2-methyl-1-propene

trans-Perfluoro-2-butene

$\mathrm{C}_{4} \mathrm{~F}_{9} \mathrm{O}$

Perfluoro-t-butoxy radical

$\mathrm{C}_{4} \mathrm{H}_{3} \mathrm{BrS}$

2-Bromothiophene

$\mathrm{C}_{4} \mathrm{H}_{5} \mathrm{~N}$

cis-2-Butenenitrile

$\mathrm{C}_{4} \mathrm{H}_{6}$

Bicyclo $[1,1,0]$ butane

Cyclobutene.

$\mathrm{C}_{4} \mathrm{H}_{6} \mathrm{D}_{2}$

cis-1,2-Dideutero-3-methylcyclopropane................

trans-1,2-Dideutero-3-methylcyclopropane................

cis or trans-1,2-Dideutero-3-methylcyclopropane .......

$\mathrm{C}_{6} \mathrm{H}_{6} \mathrm{D}_{4}$

$1,1^{\prime}, 6,6^{\prime}-d_{4}-1,5-$ Hexadiene

$\mathrm{C}_{4} \mathrm{H}_{6} \mathrm{Hg}$

Divinylmercury.

$\mathrm{C}_{4} \mathrm{H}_{6} \mathrm{O}$

Cyclobutanone.

2,5-Dihydrofuran.

$\mathrm{C}_{4} \mathrm{H}_{6} \mathrm{O}_{2}$

2,3-Butanedione

3-Butenoic acid.

$\mathrm{C}_{4} \mathrm{H}_{6} \mathrm{O}_{3}$

Ethanoic anhydride

$\mathrm{C}_{4} \mathrm{H}_{6} \mathrm{O}_{4}$

Diethyanoyl peroxide
Page 


\section{Index by Formula-Continued}

$\mathrm{C}_{4} \mathrm{H}_{7}$

1-Buten-4-yl-radical..

$\mathrm{C}_{4} \mathrm{H}_{7} \mathrm{ClO}_{2}$

Isopropyl chloromethanoate........................ 151

$\mathrm{C}_{5} \mathrm{H}_{7} \mathrm{~F}_{3}$

2,2,2-Trifluoro-1-ethylcyclopropane ................... 253

$\mathrm{C}_{4} \mathrm{H}_{8}$

1 -Butene........................................... 392

cis-2-Butene........................................ 351

Cyclobutane............................................ 269

Methylcyclopropane................................ 225

2-Methyl-1-butene.................................... 393

$\mathrm{C}_{4} \mathrm{H}_{8} \mathrm{~N}_{2}$

3-Methyl-pyrazoline................................... 306

4-Methyl-pyrazoline................................. 306

$\mathrm{C}_{4} \mathrm{H}_{8} \mathrm{O}$

3-Butene-1-ol........................................ 135

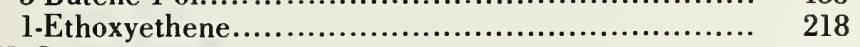

$\mathrm{C}_{4} \mathrm{H}_{8} \mathrm{O}_{2}$

1,4-Dioxane...................................... 313

Ethyl ethanoate......................................... 158

Isopropyl hydroperoxide................................ 437

Isopropyl methanoate............................... 156

$n$-Propyl methanoate................................ 155

$\mathrm{C}_{4} \mathrm{H}_{8} \mathrm{O}_{2} \mathrm{~S}$

3-Methanesulfonyl-1-propene.......................... 491

$\mathrm{C}_{4} \mathrm{H}_{8} \mathrm{O}_{3}$

Methyl ethyl carbonate ............................. 213

$\mathrm{C}_{4} \mathrm{H}_{9}$

n-Butyl radical.

sec-Butyl radical.

$t$-Butyl radical...

Isobutyl radical.

$\mathrm{C}_{4} \mathrm{H}_{9} \mathrm{Br}$

1-Bromobutane

2-Bromobutane....................................... 96

l-Bromo-2-methylpropane ................................ 94

2-Bromo-2-methylpropane......................... 101

$\mathrm{C}_{4} \mathrm{H}_{9} \mathrm{Cl}$

1-Chlorobutane

2-Chlorobutane.

1-Chloro-2-methylpropane.

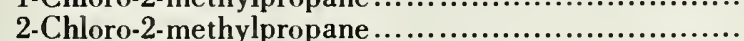

$\mathrm{C}_{4} \mathrm{H}_{9} \mathrm{ClO}$

1-Chloro-1-ethoxyethane

$\mathrm{C}_{4} \mathrm{H}_{9} \mathrm{ClO}_{2} \mathrm{~S}$

1,1-Dimethyl-1-ethanesulfonyl chloride................ 134

$\mathrm{C}_{4} \mathrm{H}_{9} \mathrm{Cl}_{3} \mathrm{Si}$

2-Chloroethyl ethyldichlorosilane..................... 124

$\mathrm{C}_{4} \mathrm{H}_{9} \mathrm{l}$

2-Iodobutane.......................................... 107

2-Iodo-2-methylpropane ............................... 108

$\mathrm{C}_{4} \mathrm{H}_{9} \mathrm{NO}_{2}$

1-Nitritobutane.

$\mathrm{C}_{4} \mathrm{H}_{9} \mathrm{O}$

sec-Butoxy radical

$t$-Butoxy radical....

l-Ethoxy-l-ethyl radical................................... 600

$\mathrm{C}_{4} \mathrm{H}_{10}$

$n$-Butane

$\mathrm{C}_{4} \mathrm{H}_{10} \mathrm{Hg}$

Diethylmercury

$\mathrm{C}_{4} \mathrm{H}_{10} \mathrm{~N}_{2}$

Azoethane.

Methyl isopropyl di.............

$\mathrm{C}_{4} \mathrm{H}_{10} \mathrm{O}$

Diethyl ether

2-Methyl-2-propanol

$\mathrm{C}_{4} \mathrm{H}_{10} \mathrm{O}_{2}$

Diethyl peroxide.

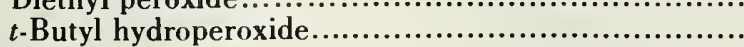

$\mathrm{C}_{4} \mathrm{H}_{10} \mathrm{~S}$

2-Methyl-2-propanethiol.

335-876 O-70-41

121
$\mathrm{C}_{4} \mathrm{H}_{11} \mathrm{~N}$

2-Methyl-2-aminopropane........................... 115

$\mathrm{C}_{4} \mathrm{H}_{12} \mathrm{~N}_{4}$

2-Methyl-2-aminopropane.......................... 116

Tetramethyltetrazine ................................. 459

$\underset{\text { Tetramethylsilane. }}{\mathrm{C}_{4} \mathrm{H}_{12} \mathrm{Si}}$

$\mathrm{C}_{4} \mathrm{H}_{12} \mathrm{Sn}$

Tetramethyltin...................................... 547

$\mathrm{C}_{4} \mathrm{NiO}_{4}$

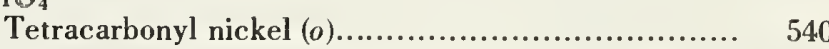

$\mathrm{C}_{5} \mathrm{~F}_{8}$

Octofluorovinylcyclopropane............................. 254

$\mathrm{C}_{5} \mathrm{H}_{4} \mathrm{BrN}$

3-Bromopyridene.

$\mathrm{C}_{5} \mathrm{H}_{6}$

505

Bicyclo[2,1,0]pent-2-ene.............................. 325

$\mathrm{C}_{5} \mathrm{H}_{8}$

trans-1,2-Dimethylcyclopropane...................... 229

Bicyclo[1,1,1]pentane.............................. 323

Bicyclo $[2,1,0]$ pentane ................................. 326

Cyclopentene............................................ 301

Ethylidenecyclopropane

2-Methyl-1-methylenecyclopropane.................. 265

Methylenecyclobutane................................... 284

l-Methyl-1-cyclobutene............................. 286

3-Methyl-1-cyclobutene............................... 287

Spiropentane....................................... 320

$\mathrm{C}_{5} \mathrm{H}_{8} \mathrm{~N}_{2}$

Vinylcyclopropane ................................... 237

$\mathrm{C}_{5} \mathrm{H}_{8} \mathrm{O}$

Cyclobutane carboxaldehyde........................ 279

$\mathrm{C}_{5} \mathrm{H}_{8} \mathrm{O}_{2}$

3-Vinyloxy-1-propene................................. 363

Methyl cis-2-butenoate................................. 356

Methyl trans-2-butenoate............................... 356

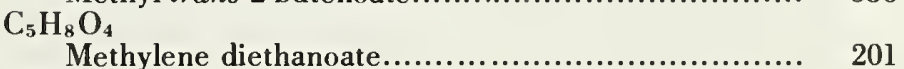

$\mathrm{C}_{5} \mathrm{H}_{9}$

Methylene diethanoate............................... 201

$\mathrm{C}_{5} \mathrm{H}_{9} \mathrm{Br}$

Bromocyclopentane

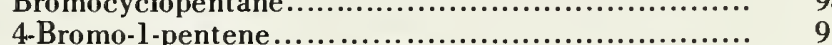

$\mathrm{C}_{5} \mathrm{H}_{9} \mathrm{Cl}$

Chlorocyclopentane.................................. 74

$\mathrm{C}_{5} \mathrm{H}_{9} \mathrm{ClO}_{2}$

Isobutyl chloromethanoate............................ 152

sec-Butyl chloromethanoate............................ 153

$\mathrm{C}_{5} \mathrm{H}_{9} \mathrm{~N}$

l-Methyl-2-chloroethyl ethanoate........................ 187

$\mathrm{C}_{5} \mathrm{H}_{9} \mathrm{~N}_{2}$

2,2-Dimethylpropanonitrile........................... 462

$\mathrm{C}_{5} \mathrm{H}_{10}$

3,3 -Tetramethylenediazirine ....................... 262

1,1-Dimethycyclopropane............................ 230

cis-1,2-Dimethylcyclopropane............................ 228

Ethylcyclopropane........................................ 231

Methylcyclobutane...................................... 270

$\mathrm{C}_{5} \mathrm{H}_{10} \mathrm{~N}_{2}$

3,3-Diethyldiazirine.................................... 261

3,3-Dimethylpyrazoline................................ 306

3,5-Dimethylpyrazoline................................. 306

$\mathrm{C}_{5} \mathrm{H}_{10} \mathrm{O}$

3,3-Dimethyloxetane................................ 278

Isopropoxyethene.................................... 219

4-Pentene-2-ol........................................ 136

$\mathrm{C}_{5} \mathrm{H}_{10} \mathrm{O}_{2}$

t.Butyl methanoate................................... 157

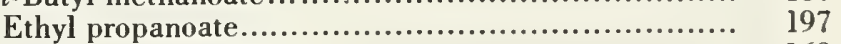

Isopropyl ethanoate.................................. 169

$\mathrm{C}_{5} \mathrm{H}_{10} \mathrm{O}$

1-Propyl ethanoate.................................... 159

Diethyl carbonate................................... 214 


\section{Index by Formula - Continued}

$\mathrm{C}_{5} \mathrm{H}_{10} \mathrm{O}_{3}$

$\mathrm{C}_{5} \mathrm{H}_{11}$

-Methoxyethyl ethanoate........................... 166

Neopentyl radical.

$\mathrm{C}_{5} \mathrm{H}_{11} \mathrm{Br}$

1.Bromopentane.

$\mathrm{C}_{5} \mathrm{H}_{11} \mathrm{Cl}$

2-Chloro-2-methylbutane...............................

$\mathrm{C}_{5} \mathrm{H}_{12}$

1-Chloropentane

2,2-Dimethylpropane

$\mathrm{C}_{5} \mathrm{H}_{12} \mathrm{O}$

2-Methyl-2-butanol.

$\mathrm{C}_{6} \mathrm{~F}_{10}$

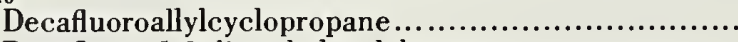

$\mathrm{C}_{6} \mathrm{~F}_{12}$

Decafluoro-1,2-dimethylcyclobutene.................... 300

trans-Hexafluoro-1,2-bis-trifluoromethylcyclobutane ... 299

cis-Hexafluoro-1,2-bis-trifluorocyclobutane............. 298

$\mathrm{C}_{6} \mathrm{H}_{4} \mathrm{Br}-X$

Substituted $(X)$-Bromobenzenes

$\mathrm{C}_{6} \mathrm{H}_{5} \mathrm{Br}$

Bromobenzene

$\mathrm{C}_{6} \mathrm{H}_{5} \mathrm{BrHg}$

Phenylmercuric bromide

$\mathrm{C}_{6} \mathrm{H}_{5} \mathrm{ClHg}$

Phenylmercuric chloride............................ 526

$\mathrm{C}_{6} \mathrm{H}_{5} \mathrm{HgI}$

Phenylmercuric iodide

$\mathrm{C}_{6} \mathrm{H}_{7} \mathrm{Cl}_{3} \mathrm{O}_{4}$

2,2,2-Trichloro-1-ethylidene diethanoate.............. 207

$\mathrm{C}_{6} \mathrm{H}_{7} \mathrm{~N}$

2-Methyl-2-phenylpropanonitrile........................ 463

$\mathrm{C}_{6} \mathrm{H}_{8}$

$\alpha, \beta$, and $\gamma$-Picoline................................... 410

Bicyclo[3,1,0]hex-2-ene................................ 329

1,4-Cyclohexadiene........................................ 312

cis-1,3,5-Hexatriene................................... 368

4-Vinylcyclohexene..................................... 312

$\mathrm{C}_{6} \mathrm{H}_{8} \mathrm{Cl}_{2}$

2,2-Dichloro-1-methyl-1-vinylcyclopropane............. 258

$\mathrm{C}_{6} \mathrm{H}_{8} \mathrm{~N}_{2}$

Phenylhydrazine

$\mathrm{C}_{6} \mathrm{H}_{8} \mathrm{O}_{4}$

$\mathrm{C}_{6} \mathrm{H}_{10}$

Dimethyl-cis-1,2-ethenedioate....................... 355

Bicyclopropyl.

Bicyclo[2,2,0]hexane.

Bicyclo[3,0]hexane.

Bicyclo $[3,1,0]$ hexane.

Cyclohexene....

Cyclohexene.

2-Dimethyl-1-cyclobutene...........

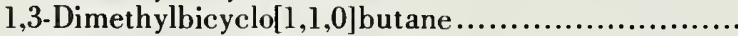

1,3-Dimethyl-1-cyclobutene............................

2,3-Dimethyl-1-cyclobutene ............................

1-Ethly-1-cyclobutene.

sopropenylcyclopropane...............................

1-Methyl-1-vinylcyclopropane.......................... 240

cis-1-Methyl-2-vinylcyclopropane......................... 238

trans-1-Methyl-2-vinylcyclopropane.................... 239

2-Methyl-cis-1,3-pentadiene.............................. 370

4-Methyl-1,3-pentadiene. ............................. 371

cis-2-Methyl[2,1,0]bicyclopentane..................... 327

trans-2-Methyl[2,1,0]bicyclopentane................. 327

$\mathrm{C}_{6} \mathrm{H}_{10} \mathrm{Cl}_{2} \mathrm{O}$

$t$-Butyl dichloroethanoate........................... 196

$\mathrm{C}_{6 i} \mathrm{H}_{10} \mathrm{~N}_{2}$

2,3-Diazobicyclo[2,2,2] oct-2-ene................... 338

$\mathrm{C}_{6} \mathrm{H}_{\mathrm{t} 0} \mathrm{O}$

1-Buten-3-yl vinyl ether

Ethanoylcyclobutane.
3-Isopropenoxy-1-propene......

2-Methyl-3-vinyloxy-1-propene.

$\mathrm{C}_{6} \mathrm{H}_{10} \mathrm{O}_{2}$

2,2-Dimethyl-3-butenoic acid.....

Methyl cyclobutanecarboxylate.

$\mathrm{C}_{6} \mathrm{H}_{12} \mathrm{O}_{33}$

2,4,6-Trimethyl-1,3,5-trioxane.

$\mathrm{C}_{6} \mathrm{H}_{10} \mathrm{O}_{4}$

Dipropionyl peroxide.

Ethylidene diethanoate.

$\mathrm{C}_{6} \mathrm{H}_{11} \mathrm{Br}$

Bromocyclohexane

$\mathrm{C}_{6} \mathrm{H}_{11} \mathrm{Cl}$

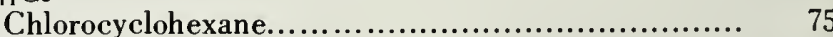

$\mathrm{C}_{6} \mathrm{H}_{11} \mathrm{ClO}$.

$t$-Butyl chloroethanoate.

$\mathrm{C}_{6} \mathrm{H}_{11} \mathrm{~N}_{2}$

3,3-Pentamethylenediazirine

$\mathrm{C}_{6} \mathrm{H}_{12}$

cis-1,2-Dimethylcyclobutane...........................

trans-1,2-Dimethylcyclobutane........................

Ethylcyclobutane.....................................

cis-1-Ethyl-2-methylcyclopropane.

trans-1-Ethyl-2-methylcyclopropane....................

cis,cis-1,2,3-Trimethylcyclopropane.....................

cis,trans-1,2,3-Trimethylcyclopropane.

$\mathrm{C}_{6} \mathrm{H}_{1 \cdot 2} \mathrm{~N}$

3,3,5-Trimethylpyrazoline.......................... 306

$\mathrm{C}_{6} \mathrm{H}_{12} \mathrm{O}$

$n$-Butyl vinyl ether.

2-Methyl-4-pentene-2-ol.

$\mathrm{C}_{6} \mathrm{H}_{12} \mathrm{O}$.

l-Butyl ethanoate

$t$-Butyl ethanoate.

sec-Butyl ethanoate.

$\mathrm{C}_{6 ;} \mathrm{H}_{12} \mathrm{O}_{3}$

sobutyl ethanoate.

1-Methyl-2-methoxyethyl ethanoate....................

l-Methyl-1-pentyl radical.

$\mathrm{C}_{6} \mathrm{H}_{13} \mathrm{Br}$

1-Bromohexane.

$\mathrm{C}_{6} \mathrm{H}_{1+}$

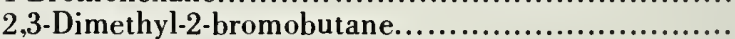

2,3-Dimethylbutane

$\mathrm{C}_{6} \mathrm{H}_{14} \mathrm{Cl}, \mathrm{Si}$

2-Chloroethyldiethylchlorosilane..................... 125

$\mathrm{C}_{6} \mathrm{H}_{1+} \mathrm{Hg}$

Diisopropylmercury

di- $n$-Propylmercury.

$\mathrm{C}_{6,} \mathrm{H}_{1+} \mathrm{N}_{2}$

Azoisopropane.

$\mathrm{C}_{6} \mathrm{H}_{14} \mathrm{O}$

Isopropyl ether

$\mathrm{C}_{6} \mathrm{H}_{14} \mathrm{O}_{2}$

di- $n$-Propyl peroxide.............................. 429

$\mathrm{C}_{6} \mathrm{H}_{1 \times} \mathrm{Si}$

Hexamethyldisilane.

$\mathrm{C}_{7} \mathrm{H}_{5} \mathrm{BrO}$

Benzoyl bromide.

$\mathrm{C}_{7} \mathrm{H}_{5} \mathrm{Cl}$.

Benzoyl chloride

$\mathrm{C}_{8} \mathrm{H}_{3} \mathrm{FO}_{3}$

$\alpha, \alpha, \alpha$-Trifluoroacetophenone.

$\mathrm{C}_{7} \mathrm{H}_{6} \mathrm{Br}-X$

Substituted $(X)$-bromo-phenylmethanes

$\mathrm{C}_{7} \mathrm{H}_{6} \mathrm{ClF}_{2}$

4-Chlorodifluoromethyl-2,5-cyclohexadienyl radical...

$\mathrm{C}_{7} \mathrm{H}_{7} \mathrm{Br}$

Bromophenylmethane

$\mathrm{C}_{7} \mathrm{H}_{7} \mathrm{Cl}$

Chlorophenylmethane 


\section{Index by Formula-Continued}

$\mathrm{C}_{7} \mathrm{H}_{8}$

Bicyclo[2,2,1]hepta-2,5-diene......................... 335

Cycloheptatriene..................................... 318

Quadricyclo $\left[2,2,1,0,{ }^{2,6} 0^{3,5}\right]$ heptane....................... 344

$\mathrm{C}_{7} \mathrm{H}_{8} \mathrm{~F}$

Toluene....................................... 394, 396

$\mathrm{C}_{7} \mathrm{H}_{8} \mathrm{~S}$

rtho, meta, and para-Fluorotoluenes................ 409

Phenyl methanethiol................................... 486

$\mathrm{C}_{7} \mathrm{H}_{9} \mathrm{~N}$

Phenylmethyl sulfide............................... 487

Benzylamine.

$\mathrm{C}_{7} \mathrm{H}_{10}$

$N$-Methylaniline

443

446

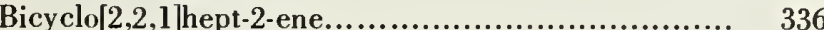

Bicyclo[3,2,0]hept-6-ene............................... 333

$1,2,6$-Heptatriene...................................... 367

$\mathrm{C}_{7} \mathrm{H}_{1}$

1-Methyl-1,4-cyclohexadiene............................ 309

Bicyclo|3,2,0]heptane................................. 332

trans-1-Cyclopropyl-1-butene............................ 242

1-Cyclopropyl-2-methyl-1-propene..................... 243

1,3-Dimethylbicyclo[1,1,1]pentane..................... 324

1,5-Heptadiene........................................... 361

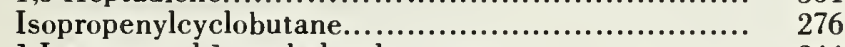

1-Isopropenyl-1-methylcyclopropane................... 244

4-Methylcyclohexene.................................... 310

3-Methyl-1,5-hexadiene............................... 362

$\mathrm{C}_{7} \mathrm{H}_{12} \mathrm{O}$

$\beta, \beta$-Dimethylcyclopropyl methyl ketone.............. 267

$\mathrm{C}_{7} \mathrm{H}_{12} \mathrm{O}_{2}$

Propionylcyclobutane................................. 281

2,2-Dimethyl-trans-3-pentenoic acid................... 143

l-Methyl-3-butenyl ethanoate.......................... 171

$\mathrm{C}_{7} \mathrm{H}_{12} \mathrm{O}_{3}$

1-Methyl-3-oxobutyl ethanoate...

$\mathrm{C}_{7} \mathrm{H}_{12} \mathrm{O}_{4}$

$\mathrm{C}_{7} \mathrm{H}_{1: 3} \mathrm{O}$

Methylene dipropanoate................................ 208

$\mathrm{C}_{7} \mathrm{H}_{14}$

4-Oxo-2-heptyl radical................................. 602

1,1-Diethylcyclopropane............................... 234

Isopropylcyclobutane.................................... 275

$\mathrm{C}_{7} \mathrm{H}_{1+}$

$n$-Propylcyclobutane................................. 274

1,1,2,2-Tetramethylcyclopropane...................... 235

$\mathrm{C}_{7} \mathrm{H}_{14} \mathrm{~N}_{2}$

3,3,5,5-Tetramethylpyrazoline.

$\mathrm{C}_{7} \mathrm{H}_{14} \mathrm{O}_{2}$

$t$-Butyl propanoate

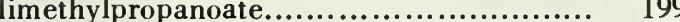

1-Ethylpropyl ethanoate.............................. 173

1-Methylbutyl ethanoate............................. 172

3-Methylbutyl ethanoate................................ 164

2-Methylbutyl ethanoate.................................. 163

1-Pentyl ethanoate....................................... 162

$t$-Pentyl ethanoate.................................... 190

$\mathrm{C}_{7} \mathrm{H}_{15} \mathrm{NO}_{2}$

1-Methyl-2-dimethylaminoethyl ethanoate............. 188

$\mathrm{C}_{7} \mathrm{H}_{16}$

$2,2,3$-Trimethylbutane............................... 389

$\mathrm{C}_{8} \mathrm{H}_{7} \mathrm{~N}$

$p$-Tolyl isocyanide

$\mathrm{C}_{8} \mathrm{H}_{8} \mathrm{Br}$

$p$-(Bromomethyl)benzyl radical......................... 613

$\mathrm{C}_{8} \mathrm{H}_{8} \mathrm{O}$

Acetophenone.

$\mathrm{C}_{8} \mathrm{H}_{8} \mathrm{O}$.

Phenylethanoic acid

$\mathrm{C}_{x} \mathrm{H}_{9} \mathrm{Br}$

p-Bromomethyltoluene.

1-Bromo-1-phenylethane.
$\mathrm{C}_{8} \mathrm{H}_{9} \mathrm{Cl}$

1-Chloro-1-phenylethane.

Page

$\mathrm{C}_{8} \mathrm{H}_{10}$

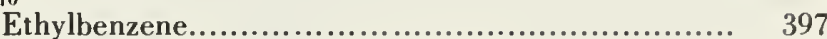

$\sigma$-Xylene............................................... 405

m-Xylene............................................ 406

$\mathrm{C}_{8} \mathrm{H}_{10} \mathrm{O}$

-Xylene......................................... 407

Endo-bicyclo[2,2,1]hept-5-ene-2-carboxaldihyde........ 345

$\mathrm{C}_{8} \mathrm{H}_{10} \mathrm{~S}$

Methyl benzyl sulfide................................... 488

$\mathrm{C}_{8} \mathrm{H}_{10} \mathrm{SO}_{2}$

Methanylsulfonylphenylmethane....................... 489

$\mathrm{C}_{8} \mathrm{H}_{11} \mathrm{~N}$

$N, N$-Dimethylaniline............................... 447

$\mathrm{C}_{8} \mathrm{H}_{1,2}$

V-Methylbenzylamine................................ 444

Bicyclo[4,2,0]oct-7-ene............................... 334

Bicylco $[5,1,0]$ oct-2-ene cycloocta-1,4-diene................ 339

1,5-Cyclooctadiene.................................. 319

1-Cyclopropyl-1-cyclopentene.......................... 246

1,1-Dicyclopropylethene............................. 245

Tricyclo $\left[3,3,0,0^{2,6}\right]$ octane............................ 340

1,5,5-Trimethyl-1,3-cyclopentadiene................... 302

2,5,5-Trimethyl-1,3-cyclopentadiene.................. 303

$\mathrm{C}_{8} \mathrm{H}_{12} \mathrm{O}_{2}$

-Vinylcyclohexene

Benzlbenzoate......................................... 426

$\mathrm{C}_{8} \mathrm{H}_{12} \mathrm{O}_{4}$

Crotonylidene diethanoate............................. 205

$\mathrm{C}_{8} \mathrm{H}_{14}$

cis-1,2,3,4-Tetramethyl-1-cyclobutene.................. 293

trans-1,2,3,4-Tetramethyl-1-cyclobutene.............. 292

$\mathrm{C}_{8} \mathrm{H}_{14} \mathrm{O}_{2}$

Cyclohexyl ethanoate.

$\mathrm{C}_{8} \mathrm{H}_{14} \mathrm{O}_{4}$

Butylidene diethanoate................................. 203

Dibutanoyl peroxide........................................ 434

Ethylidene dipropanoate................................... 209

$\mathrm{C}_{8} \mathrm{H}_{16} \mathrm{O}_{2}$

1,1-Dimethylbutyl ethanoate............................ 192

1,2-Dimethylbutyl ethanoate............................. 175

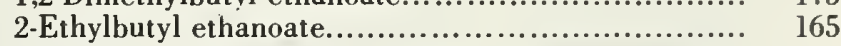

1-Ethyl-1-methylpropyl ethanoate..................... 193

$\mathrm{C}_{8} \mathrm{H}_{18}$

$1,1,2$-Trimethylpropyl ethanoate....................... 191

$2,2,3,3$-Tetramethylbutane............................. 390

$\mathrm{C}_{8} \mathrm{H}_{18} \mathrm{Hg}$

di- $n$-Butyl mercury............................... 522

$\mathrm{C}_{4} \mathrm{H}_{18} \mathrm{~N}_{2}$

$1,1^{\prime}$-Azoisobutane................................... 453

$\mathrm{C}_{8} \mathrm{H}_{18} \mathrm{~N}_{2}$

$2,2^{\prime}$-A zoisobutane..................................... 457

$2,2^{\prime}$-A zobutane......................................... 455

$\mathrm{C}_{8} \mathrm{H}_{18} \mathrm{~N} . \mathrm{O}_{2}$

Dimeric 2-methyl-1-nitrosopropane.................... 480

$\mathrm{C}_{8} \mathrm{H}_{18} \mathrm{~N}_{2}$

Azobutane

$\mathrm{C}_{8} \mathrm{H}_{18} \mathrm{O}_{2}$

di- $t$-Butyl peroxide

$\mathrm{C}_{8} \mathrm{H}_{210} \mathrm{Ge}$

Tetraethyl germane.................................. 539

$\mathrm{C}_{8} \mathrm{H}_{20} \mathrm{~N}_{4}$

Tetraethyltetrazine................................ 460

$\mathrm{C}_{8} \mathrm{H}_{20} \mathrm{~Pb}$

Tetraethyl lead..................................... 541

$\mathrm{C}_{8} \mathrm{H}_{20} \mathrm{Si}$

Tetraethylsilane....................................... 54

$\mathrm{C}_{4} \mathrm{H}_{7} \mathrm{~N}$

cis- $\beta$-Cyanostyrene.................................. 359

$\mathrm{C}_{4} \mathrm{H}_{10} \mathrm{O}$

1-Phenyl-2-propanone.............................. $\$ 18$

$\mathrm{C}_{9} \mathrm{H}_{10} \mathrm{O}$.,

Benzylethanoate. 


\section{Index by Formula-Continued}

$\mathrm{C}_{9} \mathrm{H}_{10} \mathrm{O}_{5}$

Furfurylidene diethanoate.

$\mathrm{C}_{9} \mathrm{H}_{12}$

Isopropylbenzene

$n$-Propylbenzene.

$\mathrm{C}_{9} \mathrm{H}_{1+} \mathrm{O}$

2-Methyl-2-(1-cyclopentyl) propionic acid............... 146

$\mathrm{C}_{9} \mathrm{H}_{16} \mathrm{O}_{2}$

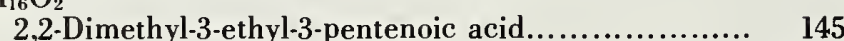

l-Methylcyclohexyl ethanoate......................... 194

$\mathrm{C}_{9} \mathrm{H}_{16} \mathrm{O}_{4}$

Methylene dibutanoate.............................. 210

$\mathrm{C}_{9} \mathrm{H}_{18} \mathrm{O}$

4-Hydroxy-4-methyl-2-pentanone..................... 217

$\mathrm{C}_{9} \mathrm{H}_{18} \mathrm{O}_{2}$

1-Ethylpentyl ethanoate............................. 178

1-Isopropyl-2-methylpropyl ethanoate................ 176

l-Methylhexyl ethanoate............................... 177

$\mathrm{C}_{10} \mathrm{H}_{7} \mathrm{Br}$

1-Propylbutyl ethanoate............................... 179

2-Bromonapthalene)

1-Bromonapthalene

$\mathrm{C}_{10} \mathrm{H}_{10} \mathrm{O}$

Methyl-cis-cinnamate

$\mathrm{C}_{10} \mathrm{H}_{11} \mathrm{O}_{2}-\mathrm{X}$

Substituted $(\mathrm{X})-\mathrm{l}$-arylethyl ethanoates...

$\mathrm{C}_{10} \mathrm{H}_{11} \mathrm{O}_{2}-\mathrm{X}$

(meta or para substituted, $\mathrm{X}) 2$-arylethyl ethanoates...

$\mathrm{C}_{10} \mathrm{H}_{11} \mathrm{O}_{3}-\mathrm{X}$

$X$-ortho, meta or para substituted

$\mathrm{C}_{10} \mathrm{H}_{12}$

1-Phenylethyl methyl carbonates

Endo-Dicyclopentadiene.

exo-Dicyclopentadiene.

$\mathrm{C}_{10} \mathrm{H}_{12} \mathrm{O}$

3-Phenyl-3-butene-1-ol

$\mathrm{C}_{10} \mathrm{H}_{12} \mathrm{O}$

1-Phenylethyl ethanoate............................ 184

$\mathrm{C}_{10} \mathrm{H}_{14}$

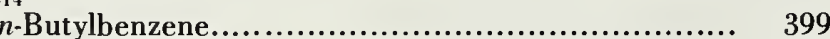

$t$-Butylbenzene......................................... 402

$p$-Isopropyltoluene................................... 403

$\mathrm{C}_{10} \mathrm{H}_{15} \mathrm{Cl}_{3} \mathrm{O}_{4}$

$2,2,2$-Trichloro-1-ethylidene dibutanoate.............. 212

$\mathrm{C}_{10} \mathrm{H}_{16}$

$\beta$-Pinene

$\mathrm{C}_{10} \mathrm{H}_{16} \mathrm{O}_{2}$

2-Methyl-2-(1-cyclohexenyl) propionic acid............ 147

$\mathrm{C}_{10} \mathrm{H}_{17} \mathrm{Cl}$

endo-2-Chlorobornane.

$\mathrm{C}_{10} \mathrm{H}_{18}$

3,7-Dimethly-1,6-octadiene........................... 372

$\mathrm{C}_{10} \mathrm{H}_{18} \mathrm{O}$

Ethylidene dibutanoate............................... 211

$\mathrm{C}_{11} \mathrm{H}_{18} \mathrm{O}_{2}$

endo-2-Acetoxybornane

exo-2-Acetoxybornane......................................

2-Methyl-2-(1-cycloheptyl) propionic acid

$\mathrm{C}_{10} \mathrm{H}_{19} \mathrm{Cl}$

Neomenthyl chloride.

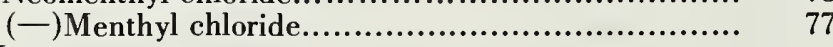

$\mathrm{C}_{11} \mathrm{H}_{10}$

$\alpha$-Methylnaphthalene

$\beta$-Methylnaphthalene
$\mathrm{C}_{11} \mathrm{H}_{20} \mathrm{O}_{4}$

Heptylidene diethanoate

$\mathrm{C}_{12} \mathrm{H}_{10} \mathrm{Hg}$

Diphenylmercury

$\mathrm{C}_{12} \mathrm{H}_{24} \mathrm{O}_{3}^{-}$

2,4,6-Tri-isopropyl-1,3,5-trioxane

$2,4,6$-Tri- $n$-propyl-1,3,5-trioxane

$\mathrm{C}_{12} \mathrm{H}_{28} \mathrm{Si}$

Tetra- $n$-propylsilane

$\mathrm{C}_{13} \mathrm{H}_{10} \mathrm{O}$

Benzophenone

$\mathrm{C}_{14} \mathrm{H}_{9} \mathrm{Br}$

9-Bromophenanthrene)

9-Bromoanthracene

$\mathrm{C}_{14} \mathrm{H}_{10} \mathrm{O}_{2}$

1,2-Diphenyl-1,2-ethanedione....................... 421

$\mathrm{C}_{14} \mathrm{H}_{12}$

cis-1,2-Diphenylethene.

$\mathrm{C}_{14} \mathrm{H}_{14}$

Dibenzyl

$\mathrm{C}_{14} \mathrm{H}_{14} \mathrm{~N}_{2}$

Azotoluene.

$\mathrm{C}_{14} \mathrm{H}_{16} \mathrm{~N}_{2}(\mathrm{l})$

2,2'-Diamino-6,6'-dimethyldiphenyl

$\mathrm{C}_{1 \ddagger} \mathrm{H}_{20} \mathrm{O}$

1-Phenyl-4-ethyl-4-hydroxyhex-1-ene

$\mathrm{C}_{14} \mathrm{H}_{12} \mathrm{O}$

Diphenylethanoic acid.

$\mathrm{C}_{15} \mathrm{H}_{14} \mathrm{O}$

1,3-Diphenyl-1,2-propanone.

$\mathrm{C}_{16} \mathrm{H}_{14} \mathrm{O}_{2}-\mathrm{X}, \mathrm{Y}$

(meta or para substituted X, Y

1,2-Diarylethyl ethanoates $\}$

$\mathrm{C}_{17} \mathrm{H}_{21} \mathrm{O}$

(-) Menthyl benzoate

CIFO.

Chloryl fluoride

$\mathrm{ClFO}_{3}$

Perchloryl fluoride

$\mathrm{ClHO}_{3}$

Perchloric acid

$\mathrm{ClNO}_{2}$

Nitryl chloride

$\mathrm{ClNO}_{3}$

Chlorine nitrate

$\mathrm{Cl}_{2} \mathrm{O}_{7}$

Dichlorine heptoxide

$\mathrm{FNO}_{3}$

Nitroxyl fluoride

$\mathrm{F}_{2} \mathrm{O}_{2}$

Difluorine dioxide.

$\mathrm{GeH}_{4}$

Germane

$\mathrm{N}_{2} \mathrm{~F}_{2}$

trans-Difluorodiazine

$\mathrm{N}_{2} \mathrm{H}_{4}$

$\mathrm{H}_{y}$ drazine

$\mathrm{N}_{2} \mathrm{O}$

Dinitrogen oxide

$\mathrm{N}_{2} \mathrm{O}_{4}$

Dinitrogen tetroxide

$\mathrm{N}_{2} \mathrm{O}_{5}$

Dinitrogen pentoxide

$\mathrm{SiH}_{4}$

Silane. 
THE NATIONAL ECONOMIC GOAL

Sustained maximum growth in a free market economy, without inflation, under conditions of full employment and equal opportunity

\section{THE DEPARTMENT OF COMMERCE}

The historic mission of the Department is "to foster, promote and develop the foreign and domestic commerce" of the United States. This has evolved, as a result of legislative and administrative additions, to encompass broadly the responsibility to foster, serve and promote the nation's economic development and technological advancement. The Department seeks to fulfill this mission through these activities:

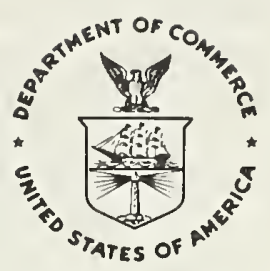

MISSION AND FUNCTIONS OF THE DEPARTMENT OF COMMERCE

\author{
"to foster, serve and \\ promote the nation's \\ economic development \\ and technological \\ advancement"
}

Participating with other government agencies in the creation of national policy, through the President's Cabinet and its subdivisions.

- Cabinet Committee on Economic Policy

Promoting progressive Assisting states, business policies and communities and growth.

- Business and Defense Services Administration

- Office of Field Services

\section{- Urban Affairs} Council

-Environmental Quality Council individuals toward economic progress.

\section{- Economic} Development Administration

Strengthening the international economic position of the United

States.

\section{- Bureau of International Commerce}

- Regional Planning Commissions

- Office of Minority Business Enterpriso

Commercial

Services
NOTE: This schematic is neither an organization chart nor a program outline for budget purposes. It is a general statement of the Department's mission in relation to the national goal of economic development.

JULY 1969
Assuring effective Acquiring, analyzing use and growth of the and disseminating nation's scientific information concernand technical ing the nation and resources. the economy to help achieve increased

- Environmental social and economic Science Services benefit.

Administration

- Patent Office

- Bureau of

- Office of Foreign

- National Bureau of Standards the Census

- Office of Foreign Direct Investments - Office of

Telecommunications

- United States Travel Service

- Maritime Administration
- Office of State Technical Services
- Office of Business Economics 


\section{NATIONAL BUREAU OF STANDARDS}

The National Bureau of Standards ' was established by an act of Congress March 3, 1901. Today, in addition to serving as the Nation's central measurement laboratory, the Bureau is a principal focal point in the Federal Government for assuring maximum application of the physical and engineering sciences to the advancement of technology in industry and commerce. To this end the Bureau conducts research and provides central national services in four broad program areas. These are: (1) basic measurements and standards, (2) materials measurements and standards, (3) technological measurements and standards, and (4) transfer of technology.

The Bureau comprises the Institute for Basic Standards, the Institute for Materials Research, the Institute for Applied Technology, the Center for Radiation Research, the Center for Computer Sciences and Technology, and the Office for Information Programs.

THE INSTITUTE FOR BASIC STANDARDS provides the central basis within the United States of a complete and consistent system of physical measurement; coordinates that system with measurement systems of other nations; and furnishes essential services leading to accurate and uniform physical measurements throughout the Nation's scientific community, industry, and commerce. The Institute consists of an Office of Measurement Services and the following technical divisions:

Applied Mathematics-Electricity-Metrology-Mechanics-Heat-Atomic and Molecular Physics-Radio Physics "-Radio Engineering "- Time and Frequency"-Astrophysics "- Cryogenics."

THE INSTITUTE FOR MATERIALS RESEARCH conducts materials research leading to improved methods of measurement standards, and data on the properties of well-characterized materials needed by industry, commerce, educational institutions, and Government; develops, produces, and distributes standard reference materials; relates the physical and chemical properties of materials to their behavior and their interaction with their environments; and provides advisory and research services to other Government agencies. The Institute consists of an Office of Standard Reference Materials and the following divisions:

Analytical Chemistry-Polymers-Metallurgy-Inorganic Materials-Physical Chemistry.

THE INSTITUTE FOR APPLIED TECHNOLOGY provides technical services to promote the use of available technology and to facilitate technological innovation in industry and Government; cooperates with public and private organizations in the development of technological standards, and test methodologies; and provides advisory and research services for Federal, state, and local government agencies. The Institute consists of the following technical divisions and offices:

Engineering Standards-Weights and Measures - Invention and Innovation - Vehicle Systems Research-Product Evaluation-Building Research-Instrument Shops-Measurement Engineering-Electronic Technology-Technical Analysis.

THE CENTER FOR RADIATION RESEARCH engages in research, measurement, and application of radiation to the solution of Bureau mission problems and the problems of other agencies and institutions. The Center consists of the following divisions:

Reactor Radiation-Linac Radiation-Nuclear Radiation-Applied Radiation.

THE CENTER FOR COMPUTER SCIENCES AND TECHNOLOGY conducts research and provides technical services designed to aid Government agencies in the selection, acquisition, and effective use of automatic data processing equipment; and serves as the principal focus for the development of Federal standards for automatic data processing equipment, techniques, and computer languages. The Center consists of the following offices and divisions:

Information Processing Standards-Computer Information - Computer Services - Systems Development-Information Processing Technology.

THE OFFICE FOR INFORMATION PROGRAMS promotes optimum dissemination and accessibility of scientific information generated within NBS and other agencies of the Federal government; promotes the development of the National Standard Reference Data System and a system of information analysis centers dealing with the broader aspects of the National Measurement System, and provides appropriate services to ensure that the NBS staff has optimum accessibility to the scientific information of the world. The Office consists of the following organizational units:

Office of Standard Reference Data-Clearinghouse for Federal Scientific and Technical

Information "-Office of Technical Information and Publications-Library-Office of Public Information-Office of International Relations.

\footnotetext{
1 Headquarters and Laboratories at Gaithersburg, Maryland, unless otherwise noted; mailing address Washington, D.C. 20234. 


\section{PERIODICALS}

JOURNAL OF RESEARCH reports National Burcau of Standards research and development in physics, mathematics, chemistry, and engineering. Comprehensive scientific papers give complete details of the work, including laboratory data, experimental procedures, and theoretical and mathematical analyses. Illustrated with photographs, drawings, and charts.

\section{Published in three sections, available separately:}

\section{Physics and Chemistry}

Papers of interest primarily to scientists working in these fields. This section covers a broad range of physical and chemical research, with major emphasis on standards of physical measurement, fundamental constants, and properties of matter. Issued six times a year. Annual subscription: Domestic, $\$ 9.50$; foreign, $\$ 11.75^{*}$.

\section{Mathematical Sciences}

Studies and compilations designed mainly for the mathematician and theoretical physicist. Topics in mathematical statistics, theory of experiment design, numerical analysis, theoretical physics and chemistry, logical design and programming of computers and computer systems. Short numerical tables. Issued quarterly. Annual subscription: Domestic, $\$ 5.00$; foreign, $\$ 6.25 *$.

\section{- Engineering and Instrumentation}

Reporting results of interest chiefly to the engineer and the applied scientist. This section includes many of the new developments in instrumentation resulting from the Bureau's work in physical measurement, data processing, and development of test methods. It will also cover some of the work in acoustics, applied mechanics, building research, and cryogenic engineering. Issued quarterly. Annual subscription: Domestic, $\$ 5.00$; foreign, $\$ 6.25 *$.

\section{TECHNICAL NEWS BULLETIN}

The best single source of information concerning the Bureau's research, developmental, cooperative and publication activities, this monthly publication is designed for the industry-oriented individual whose daily work involves intimate contact with science and technology-for engineers, chemists, physicists, research managers, product-development managers, and company executives. Annual subscription: Domestic, $\$ 3.00$; foreign, $\$ 4.00 *$.

- Difference in price is due to extra cost of foreicn mallins.

\section{NONPERIODICALS}

Applied Mathematics Series. Mathematical tables, manuals, and studies.

Building Science Series. Research results, test methods, and performance criteria of building materials, components, systems, and structures.

Handbooks. Recommended codes of engineering and industrial practice (including safety codes) developed in cooperation with interested industries, professional organizations, and regulatory bodies.

Special Publications. Proceedings of NBŚ conferences, bibliographies, annual reports, wall charts, pamphlets, etc.

Monographs. Major contributions to the technical literature on various subjects related to the Bureau's scientific and technical activities.

National Standard Reference Data Series. NSRDS provides quantitive data on the physical and chemical properties of materials, compiled from the world's literature and critically evaluated.

Product Standards. Provide requirements for sizes, types, quality and methods for testing various industrial products. These standards are developed cooperatively with interested Government and industry groups and provide the basis for common understanding of product characteristics for both buyers and sellers. Their use is voluntary.

Technical Notes. This series consists of communications and reports (covering both other agency and NBS-sponsored work) of limited or transitory interest.

Federal Information Processing Standards Publications. This series is the official publication within the Federal Government for information on standards adopted and promulgated under the Public Law 89-306, and Bureau of the Budget Circular A-86 entitled, Standardization of Data Elements and Codes in Data Systems.

\section{CLEARINGHOUSE}

The Clearinghouse for Federal Scientific and Technical Information, operated by NBS, supplies unclassified information related to Government-generated science and technology in defense, space, atomic energy, and other national programs. For further information on Clearinghouse services, write:

Clearinghouse

U.S. Department of Commerce

Springfield, Virginia 22151

Order NBS publications from:

Superintendent of Documents

Government Printing Office

Washington, D.C. 20402 


\section{Announcement of New Publications in \\ National Standard Reference Data Series}

Superintendent of Documents,

U.S. Government Printing Office,

Washington, D.C. 20402

Dear Sir:

Please add my name to the announcement list of new publications to be issued in the series: National Standard Reference Data Series-National Bureau of Standards.

Name

Company.

Address.

City State .Zip Code

(Notification key $\mathrm{N}-337$ ) 


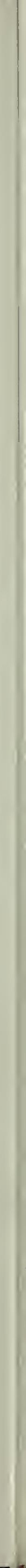




\section{Publications in the National Standard Reference Data Series National Bureau of Standards}

You may use this listing as your order form by checking the proper box of the publication(s) you desire or by providing the full identification of the publication you wish to purchase. The full letter symbols with each publications number and full title of the publication and author must be given in your order, e.g. NSRDS-NBS-17, Tables of Molecular Vibratiorial Frequencies, Part 3, by T. Shimanouchi.

Pay for publications by check, money order, or Superintendent of Documents coupons or deposit account. Make checks and money orders payable to Superintendent of Documents. Foreign remit-

NSRDS-NBS 1, National Standard Reference Data SystemPlan of Operation, by E. L. Brady and M. B. Wallenstein, 1964 (15 cents).

NSRDS-NBS 2, Thermal Properties of Aqueous Uni-univalent Electrolytes, by V. B. Parker, 1965 (45 cents).

NSRDS-NBS 3, Sec. 1, Selected Tables of Atomic Spectra, Atomic Energy Levels and Multiplet Tables, Si II, Si III, Si IV, by C. E. Moore, 1965 (35 cents).

NSRDS-NBS 3, Sec. 2, Selected Tables of Atomic Spectra, Atomic Energy Levels and Multiplet Tables, Si I, by C. E. Moore, 1967 (20 cents).

NSRDS-NBS 4, Atomic Transition Probabilities, Volume 1, Hydrogen Through Neon, by W. L. Wiese, M. W. Smith and B. M. Glennon, $1966(\$ 2.50)$.

NSRDS.NBS 5, The Band Spectrum of Carbon Monoxide, by P. H. Krupenie, 1966 (70 cents).

NSRDS-NBS 6, Tables of Molecular Vibrational Frequencies, Part 1, by T. Shimanouchi, 1967 (40 cents).

NSRDS.NBS 7, High Temperature Properties and Decomposition of Inorganic Salts, Part 1, Sulfates, by K. H. Stern and E. L. Weise, 1966 (35 cents).

NSRDS-NBS 8, Thermal Conductivity of Selected Materials, by R. W. Powell, C. Y. Ho, and P. E. Liley, $1966(\$ 1)$.

NSRDS-NBS 9, Bimolecular Gas Phase Reactions (rate coefficients), by A. F. Trotman-Dickenson and G. S. Milne, 1967 $(\$ 2)$.

NSRDS-NBS 10, Selected Values of Electric Dipole Moments for Molecules in the Gas Phase, by R. D. Nelson, Jr., D. R. Lide, Jr., and A. A. Maryott, 1967 (40 cents).

NSRDS-NBS 11, Tables of Molecular Vibrational Frequencies, Part 2, by T. Shimanouchi, 1967 (30 cents).

NSRDS-NBS 12, Tables for the Rigid Asymmetric Roto: Transformation Coefficients from Symmetric to Asymmetric Bases and Expectation Values of $\mathbf{P}_{\mathbf{z}}^{2}, \mathbf{P}_{\mathbf{z}}^{\mathbf{4}}$, and $\mathbf{P}_{\mathbf{z}}^{\mathbf{6}}$, by R. H. Schwendeman, 1968 (60 cents).

NSRDS-NBS 13, Hydrogenation of Ethylene on Metallic Catalysts, by J. Horiuti and K. Miyahara, 1968 (\$1).

NSRDS-NBS 14, X-Ray Wavelengths and ${ }^{5}$. Levels, by J. A. Bearden, 1967 (40 cents).

NSRDS-NBS 15, Molten Salts, Vol. 1, Electrical Conductance, Density, and Viscosity Data, by G. Janz, F. W. Dampier, G. R. Lakshminarayanan, P. K. Lorenz, and R. P. T. Tomkins, 1968 (\$3). tances should be made either by international money order or draft on an American bank. Postage stamps are not acceptable.

No charge is made for postage to destinations in the United States and possessions, Canada, Mexico, and certain Central and South American countries. To other countries, payments for documents must cover postage. Therefore, one-fourth of the price of the publication should be added for postage.

Send your order together with remittance to Superintendent of Documents, Government Printing Office, Washington, D.C. 20402.

NSRDS-NBS 16, Thermal Conductivity of Selected Materials, Part 2, by C. Y. Ho, R. W. Powell, and P. E. Liley, 1968 ( $\$ 2)$. NSRDS-NBS 17, Tables of Molecular Vibration Frequencies, Part 3, by T. Shimanouchi, 1968 (30 cents).

NSRDS-NBS 18, Critical Analysis of the Heat-Capacity Data of the Literature and Evaluation of Thermodynamic Properties of Copper, Silver, and Gold From 0 to $300 \mathrm{~K}$, by G. T. Furukawa, W. G. Saba, and M. L. Reilly, 1968 (40 cents).

NSRDS-NBS 19, Thermodynamic Properties of Ammonia as an Ideal Gas, by L. Haar, 1968 (20 cents).

NSRDS-NBS 20, Gas Phase Reaction Kinetics of Neutral Oxygen Species, by H. S. Johnson, 1968 (45 cents).

NSRDS-NBS 22, Atomic Transition Probabilities, Vol. II, Sodium Through Calcium, A Critical Data Compilation, by W. L. Wiese, M. W. Smith, and B. M. Miles (\$4.50).

NSRDS-NBS 23, Partial Grotrian Diagrams of Astrophysical Interest, by C. E. Moore and P. W. Merrill, 1968 (55 cents).

NSRDS-NBS 24, Theoretical Mean Activity Coefficients of Strong Electrolytes in Aqueous Solutions from 0 to $100^{\circ} \mathrm{C}$, by Walter J. Hamer, $1968(\$ 4.25)$.

NSRDS-NBS 25, Electron Impact Excitation of Atoms, by B. L. Moiseiwitsch and S. J. Smith, 1968 (\$2).

NSRDS-NBS 26, Ionization Potentials, Appearance Potentials, and Heats of Formation of Positive lons, by J. L. Franklin, J. G. Dillard, H. M. Rosenstock, J. T. Herron, K. Draxl, and F. H. Field (\$4).

NSRDS-NBS 27, Thermodynamic Properties of Argon from the Triple Point to $300 \mathrm{~K}$ at Presures to 1000 Atmospheres, by A. L. Gosman, R. D. McCarty, and J. G. Hust (\$1.25).

NSRDS-NBS 28, Molten Salts, Vol. 2, Section 1, Electrochemistry of Molten Salts: Gibbs Free Energies and Excess Free Energies From Equilibrium-Type Cells, by G. J. Janz and C. G. M. Dijkhuis. Section 2, Surface Tension Data, by G. J. Janz, G. R. Lakshminarayanan, R. P. T. Tomkins, and J. Wong (\$2.75).

NSRDS-NBS 29, Photon Cross Sections, Attenuation Coefficcients and Energy Absorption Coefficients From $10 \mathrm{keV}$ to $100 \mathrm{GeV}$, J. H. Hubbell (75 cents).

NSRDS-NBS 30, High Temperature Properties and Decomposition of Inorganic Salts. Part 2. Carbonates, by K. H. Stern and E. L. Weise, 1969 (45 cents). 

$$
\text { . }
$$ 
